\title{
Measurement of inclusive proton double -spin asymmetries and polarized structure functions
}

\author{
Robert G. Fersch \\ College of William \& Mary - Arts \& Sciences
}

Follow this and additional works at: https://scholarworks.wm.edu/etd

Part of the Physics Commons

\section{Recommended Citation}

Fersch, Robert G., "Measurement of inclusive proton double -spin asymmetries and polarized structure functions" (2008). Dissertations, Theses, and Masters Projects. Paper 1539623531.

https://dx.doi.org/doi:10.21220/s2-dbjh-xt87

This Dissertation is brought to you for free and open access by the Theses, Dissertations, \& Master Projects at W\&M ScholarWorks. It has been accepted for inclusion in Dissertations, Theses, and Masters Projects by an authorized administrator of W\&M ScholarWorks. For more information, please contact scholarworks@wm.edu. 


\title{
Measurement of Inclusive Proton Double-Spin Asymmetries and Polarized Structure Functions
}

\author{
Robert G. Fersch, Jr.
}

Annapolis, MD

B.A. Physics Education, University of Delaware, 1996 M.S. Physics, College of William and Mary, 2003

\begin{abstract}
A Dissertation presented to the Graduate Faculty of the College of William and Mary in Candicacy for the Degree of Doctor of Philosophy
\end{abstract}

Physics

The College of William and Mary

August, 2008 


\section{COPYRIGHT PAGE}

(C)2008, Robert G. Fersch, Jr. 


\section{APPROVAL PAGE}

This Dissertation is submitted in partial fulfillment of the requirements for the degree of

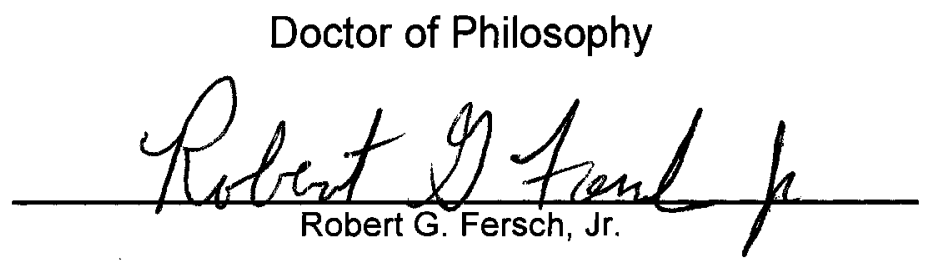

Approved by the Committee, May, 2008
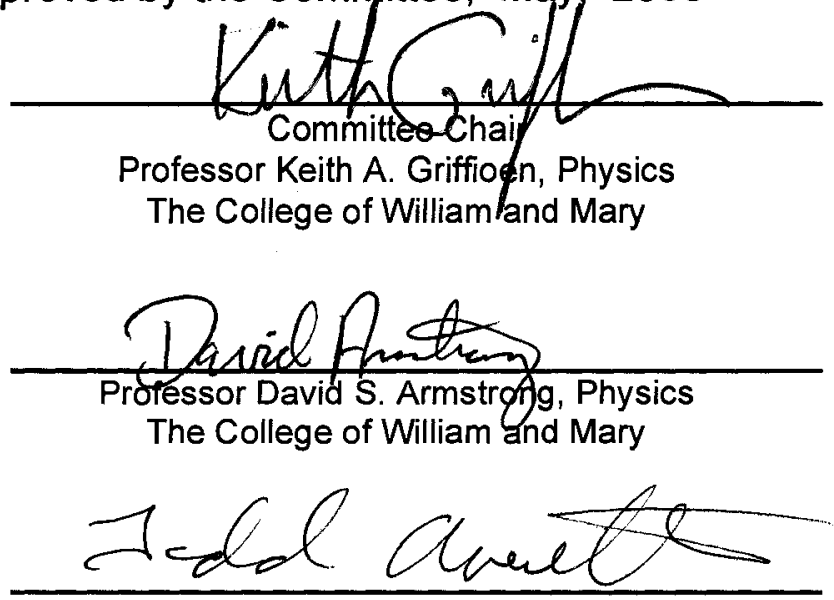

Associate Professor Todd D. Averett, Physics

The College of William and Mary

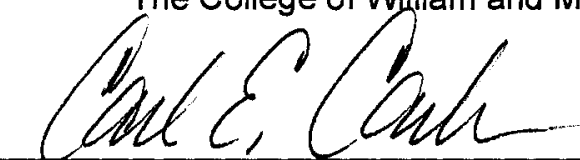

Professor Carl E. Carlson, Physics

The College of William and Mary

\section{Betr Borte}

Adjunct Professor Peter E. Bosted, Physics

University of Massachusetts

Senior Staff Scientist, Jefferson Laboratory 


\section{ABSTRACT PAGE}

The scattering of polarized electrons from a polarized proton target provides a means for studying the internal spin structure of the proton. The CLAS (CEBAF Large Acceptance Spectrometer) EG1b experiment in Hall-B at Jefferson Laboratory measured double-spin inclusive and exclusive electron-nucleon scattering asymmetries using longitudinally polarized frozen $\mathrm{NH}_{3}$ and $\mathrm{ND}_{3}$ targets and a longitudinally polarized electron beam at 4 different energies $(1.6,2.5,4.2,5.6 \mathrm{GeV})$. Extraction of the virtual photon asymmetry $A_{1}^{p}$ (for $0.05 \mathrm{GeV}^{2}<Q^{2}<5.0 \mathrm{GeV}^{2}$ ) provides precision measurements of the polarized proton spin-structure function $g_{1}^{p}$ in and above the resonance region. Linear regression of data between the varying energies yields new constraints on the virtual photon asymmetry $A_{2}^{p}$ (and thus the structure function $g_{2}^{p}$ ) in the resonance region (for $0.3 \mathrm{GeV}^{2}<Q^{2}<1.0$ $\mathrm{GeV}^{2}$ ). Measurements of these structure functions and their moments allows testing of perturbative Quantum Chromodynamics (PQCD) models and evaluation of moments of the structure functions in the Operator Product Expansion. Testing of Chiral Perturbation Theory $(\chi \mathrm{PT})$ at $Q^{2}<0.2 \mathrm{GeV}^{2}$ is enabled by the new data. Other applications of polarized structure functions include measurement of fowardspin polarizability, evaluation of high-order corrections in ${ }^{1} \mathrm{H}$ hyperfine splitting, and testing of quark-hadron duality. 


\section{Contents}

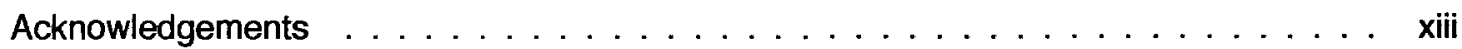

Preface ......................... . . . . . .

1 Background and Motivation 1

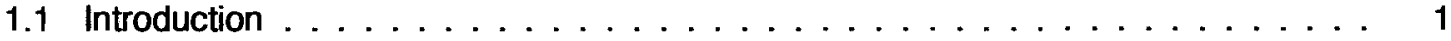

1.1 .1 Electron-proton scattering . . . . . . . . . . . . . . . 2

1.1 .2 Models of the proton . . . . . . . . . . . . . . . . 3

1.1 .3 Kinematic Definitions . . . . . . . . . . . . . . . . . . . . . . 5

1.1.4 A Motivation for Studying Proton Spin . . . . . . . . . . . . . . . . 9

1.2 Structure functions . . . . . . . . . . . . . . . . . . . . 10

1.2.1 Electron scattering and structure functions . . . . . . . . . . . . . 10

1.2.2 Unpolarized structure functions $\left(F_{1}, F_{2}, R\right) \ldots \ldots \ldots \ldots$

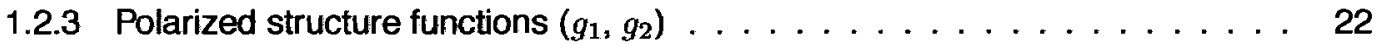

$1.2 .4 Q^{2}$ evolution and scaling violations $\ldots \ldots \ldots \ldots \ldots \ldots$

1.3 Moments and sum rules . . . . . . . . . . . . . . . . . 33

1.3.1 The Operator Product Expansion . . . . . . . . . . . . . . . . . . 33

1.3.2 First moment of $g_{1}$ and its sum rules . . . . . . . . . . . . 46

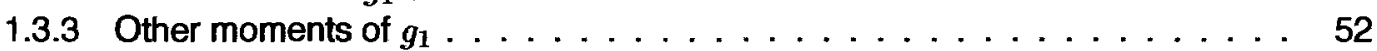

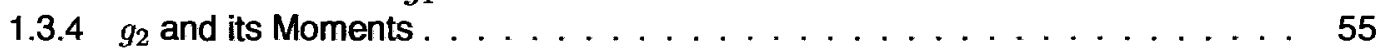

1.4 Asymmetries . . . . . . . . . . . . . . . . . . . . 57

1.4.1 Measuring asymmetries . . . . . . . . . . . . . . . . . . 57

1.4.2 Asymmetries to polarized structure functions . . . . . . . . . . . 59

1.4.3 Behavior of $A_{1}$ in the resonance region . . . . . . . . . . 65

1.5 Existing Measurements . . . . . . . . . . . . . . . . . . 68

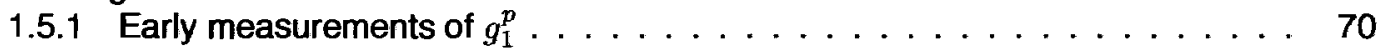

1.5 .2 The spin crisis . . . . . . . . . . . . . . . . . . . 72

1.5.3 Second generation SLAC and HERMES polarized experiments . . . . . . . . 73

1.5.4 The missing kinematic region: motivation for EG1 . . . . . . . . . 75

1.5.5 Current measurements of $g_{2}^{p}$ (E155x and RSS) . . . . . . . . . 77

2 Experimental Apparatus and Models $\quad 81$

2.1 The EG1b Experiment: Introduction . . . . . . . . . . . . . . . . . . 81

2.2 The CEBAF Electron Accelerator . . . . . . . . . . . . . . . . . . . . . . . 82

2.2 .1 Beam Injector . . . . . . . . . . . . . . . . . . 83

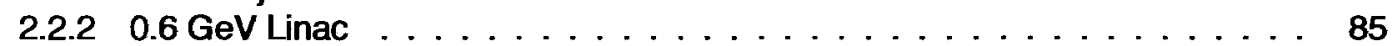

2.2.3 Recirculation Arcs and Beam Optics . . . . . . . . . . . . . . . . 86

2.3 Hall B Beam Line Devices . . . . . . . . . . . . . . . . . . . . . . . . . 88 
2.3.1 Møller Polarimeter . . . . . . . . . . . . . . . . . . . . 88

2.3.2 Beam Position Monitors (BPM) . . . . . . . . . . . . . . . . . . . . 92

2.3.3 Harp Beam Profile Monitors . . . . . . . . . . . . . . . . . . . . . 92

2.3 .4 Faraday Cup . . . . . . . . . . . . . . . . . . . . . 93

2.4 The EG1 Polarized Target . . . . . . . . . . . . . . . . . . . . . . . . 95

2.4.1 Preparation, Annealing and Rastering of the Target . . . . . . . . . . . . 95

2.4.2 Dynamic Nuclear Polarization: Overview . . . . . . . . . . . . . . . . . . . 96

2.4.3 DNP, Neglecting Spin-Spin Interactions . . . . . . . . . . . . . . . . 97

2.4.4 DNP in a real solid: Equal Spin Temperature (EST) Theory . . . . . . . . . 100

$2.4 .5{ }^{15} \mathrm{NH}_{3}$ as a Polarized Target . . . . . . . . . . . . . . . . . . 103

2.4 .6 Polarized Target System . . . . . . . . . . . . . . . . . . . . . . . . . 103

2.4.7 Modeling the Target for Background and Radiative Corrections . . . . . . . 110

2.5 The CLAS Detector . . . . . . . . . . . . . . . . . . . . . . . . . . 114

2.5 .1 Torus Magnet . . . . . . . . . . . . . . . . . . . . . . . . . 114

2.5 .2 Drift Chambers . . . . . . . . . . . . . . . . . . . . . 117

2.5.3 Cherenkov Counters . . . . . . . . . . . . . . . . . . . . . . . . 120

2.5 .4 Scintillation Counters . . . . . . . . . . . . . . . . . . . . . . . 124

2.5.5 Electromagnetic Calorimeters . . . . . . . . . . . . . . . . . . . . 132

2.6 Data Collection . . . . . . . . . . . . . . . . . . . . . . . . 136

2.6 .1 Data Acquisition . . . . . . . . . . . . . . . . . . . . 137

2.6.2 The Simple Event Builder (SEB) . . . . . . . . . . . . . . . . . . . . 139

2.6.3 The EG1b Data Set: Preparation for Analysis . . . . . . . . . . . . . . . 140

2.7 Structure function and asymmetry models . . . . . . . . . . . . . . . . 143

2.7.1 Model of unpolarized structure functions $F_{1}^{p}$ and $R^{p} \ldots \ldots \ldots \ldots$

2.7.2 Model of virtual photon asymmetries $A_{1}^{p}$ and $A_{2}^{p} \ldots \ldots \ldots \ldots . \ldots 145$

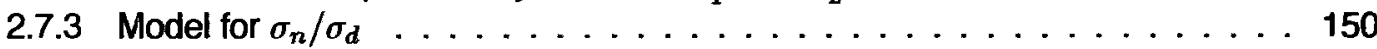

2.8 Radiated nuclear cross-section models $\ldots \ldots \ldots \ldots \ldots \ldots \ldots$. . . . . . . 151

3 Helicity and Particle Identification 157

3.1 Reading the Data Summary Tapes (DSTs) . . . . . . . . . . . . . . . . . 157

3.1.1 DST organization . . . . . . . . . . . . . . . . . . . . . 157

3.1.2 Reading particle data from the DST . . . . . . . . . . . . . . . 158

3.1.3 Tree File Structure . . . . . . . . . . . . . . . . . . . . . . . . . . 159

3.2 Removal of Problematic Helicity Buckets . . . . . . . . . . . . . . . . . . . 160

3.2.1 Identifying helicity buckets for removal . . . . . . . . . . . . . . . 160

3.2.2 Further helicity bucket problems . . . . . . . . . . . . . . . . . . . . 162

3.3 Quality Checks . . . . . . . . . . . . . . . . . . . . . . . . . . . . . . 164

3.3.1 Beam charge asymmetry check . . . . . . . . . . . . . . . . . 164

3.3.2 Event rate check . . . . . . . . . . . . . . . . . . . . . . . . . . 165

3.3.3 Target polarization and half-wave plate check . . . . . . . . . . . . . 167

3.3.4 Raster pattern check . . . . . . . . . . . . . . . . . . . . . . . 170

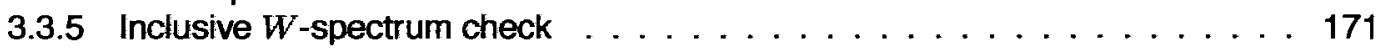

3.3.6 Miscellaneous issues . . . . . . . . . . . . . . . . . . . . . . . . . 173

3.3.7 Data Organization and Tests of Quality Checks . . . . . . . . . . . . . 176

3.4 Electron Identification . . . . . . . . . . . . . . . . . . . . 176

3.4.1 Cherenkov Counter Cuts . . . . . . . . . . . . . . . . . . . . . 177

3.4.2 Electromagnetic Calorimeter Cuts . . . . . . . . . . . . . . . . . . . 178

3.4.3 Particle identification parameters for elastic $e p$ events . . . . . . . . . . . 182 
3.5 Elastic $e p$ Event Selection . . . . . . . . . . . . . . . . . . . . . . . . . 183

3.6 Miscellaneous Minor Cuts . . . . . . . . . . . . . . . . . . . . . . . 186

3.6 .1 vertex cut . . . . . . . . . . . . . . . . . . . 186

3.6 .2 Trigger bits . . . . . . . . . . . . . . . . . . . . . 188

3.6 .3 Status flags . . . . . . . . . . . . . . . . . . . . 188

3.6 .4 EC energy sum correction . . . . . . . . . . . . . . . . . . . . . 189

3.6.5 Minor kinematic and geometric cuts . . . . . . . . . . . . . . 190

4 Precision Cuts and Corrections 192

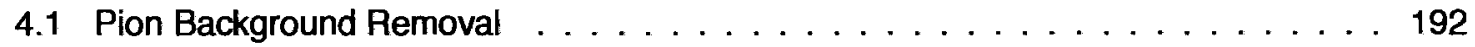

4.1 .1 Remaining pion background . . . . . . . . . . . . . . . . . . . . . . 192

4.1 .2 Geometric cuts on accepted CC triggers . . . . . . . . . . . . . . . . . . 194

4.1.3 PMT cuts on accepted CC triggers . . . . . . . . . . . . . . . . . . . 194

4.1 .4 Timing cuts on accepted CC triggers . . . . . . . . . . . . . . . . 196

4.1.5 Results of Osipenko's cuts and Implementation . . . . . . . . . . . . . . 198

4.2 Kinematics Corrections . . . . . . . . . . . . . . . . . . . . . . . . . . . 201

4.2.1 The kinematics correction package . . . . . . . . . . . . . . . . . . . . 201

4.2 .2 Raster correction . . . . . . . . . . . . . . . . . . . . . . . . . . . . 202

4.2 .3 Torus scaling correction . . . . . . . . . . . . . . . . . . . . . . . . . 205

4.2 .4 Beam energy correction . . . . . . . . . . . . . . . . . . . . . . 207

4.2 .5 Multiple scattering correction . . . . . . . . . . . . . 211

4.2.6 Stray magnetic field correction . . . . . . . . . . . . . . . 213

4.2.7 Energy loss $(d E / d x)$ correction . . . . . . . . . . . . . . . . 214

4.2 .8 Momentum corrections . . . . . . . . . . . . . . . . . . . 216

4.3 Momentum Correction at Low $\theta \ldots \ldots \ldots$

4.4 Fiducial Cuts . . . . . . . . . . . . . . . . . . . . . . . . . . . 229

4.4.1 Determination of Inefficient CC Regions . . . . . . . . . . . . . . 231

4.4.2 Determining the Fiducial Cut Boundaries . . . . . . . . . . . . . . . 233

4.4.3 Interpolation of Cut Parameters Between Momentum Bins . . . . . . . . 237

4.4 .4 Application and Effects of Fiducial Cuts . . . . . . . . . . . . . . . . 239

4.5 Faraday Cup Corrections . . . . . . . . . . . . . . . . . . . . . . . 242

4.5.1 Correction for multiple scattering divergence . . . . . . . . . . . . . 242

4.5 .2 Bit factor correction . . . . . . . . . . . . . . . . . . . . 245

4.6 Generation of Skims . . . . . . . . . . . . . . . . . . . . 246

5 Dilution Factors: Removal of Unpolarized Background 248

5.1 Calculation of Background-subtracted Asymmetries . . . . . . . . . . . . 248

5.2 Combining Data from Different Runs . . . . . . . . . . . . . . . . . . . . . . . 249

5.2.1 Corrections to empty $(\mathrm{LHe})$ target counts . . . . . . . . . . . . . . . 252

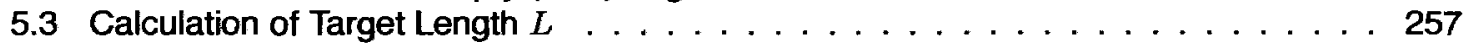

5.3.1 Calculation of $L$ from data . . . . . . . . . . . . . . . . . . . . . 259

5.3.2 Calculation of $L$ from radiated cross-sections . . . . . . . . . . . . . 260

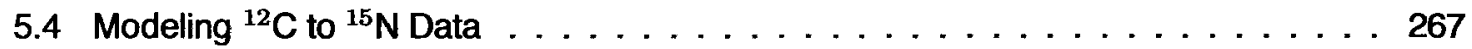

5.4 .1 Development of the model . . . . . . . . . . . . . . . . . . . . 267

5.4.2 Comparison to the older fit method . . . . . . . . . . . . . . . . . 272

5.4.3 Direct determination of $\ell_{N}$ with the model . . . . . . . . . . . . 278

5.5 Calculation of Ammonia Target Length $\ell_{A} \ldots \ldots \ldots \ldots \ldots \ldots$

5.5.1 Calculation of $\ell_{A}$ from data . . . . . . . . . . . . . 280 
5.5.2 Calculation of $\ell_{A}$ from radiated cross-sections $\ldots \ldots \ldots \ldots . \ldots 283$

5.6 Dilution Factors . . . . . . . . . . . . . . . . . . . . . . . . . . . . . . 284

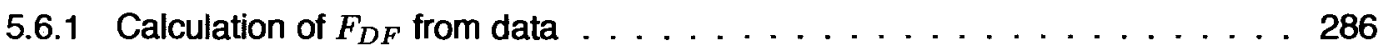

5.6.2 Calculation of $F_{D F}$ from radiated cross-sections $\ldots \ldots \ldots \ldots . \ldots . \ldots 293$

5.6.3 Application of dilution factors . . . . . . . . . . . . . . . . . . . 295

6 Removal of Polarized and Other Target Background Events 296

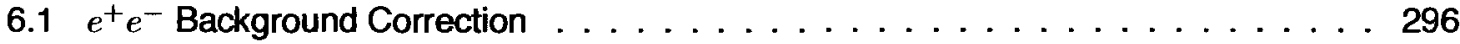

6.1.1 Measurement of pair-production rates and asymmetries . . . . . . . . . 299

6.1 .2 Application of pair symmetric correction . . . . . . . . . . . . 300

6.2 Beam $\times$ Target Polarizations . . . . . . . . . . . . . . . . . . . 304

6.2 .1 Inclusive method . . . . . . . . . . . . . . . . . . . . . 307

6.2 .2 Exclusive $e p$ method . . . . . . . . . . . . . . . . . . . . . . 314

6.2 .3 Final determination of $P_{b} P_{t}$ values $\ldots \ldots \ldots \ldots \ldots \ldots \ldots \ldots$

6.2 .4 Comparison to target NMR . . . . . . . . . . . . . . . . . . 325

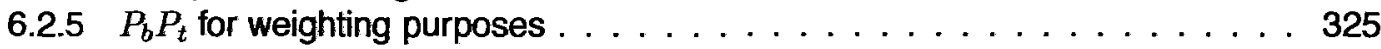

6.3 Polarized Nitrogen Correction . . . . . . . . . . . . . . . . . . . 330

6.4 Radiative corrections . . . . . . . . . . . . . . . . . . . . . 333

6.4.1 Basic methodology of radiative corrections . . . . . . . . . . . 335

6.4 .2 Internal radiative corrections . . . . . . . . . . . . . . . . 337

6.4 .3 External radiative corrections . . . . . . . . . . . . . . . . . . . 340

6.4 .4 Application of radiative corrections . . . . . . . . . . . . . . . . 342

7 Combination of Data Sets and Calculation of Systematic Errors 344

7.1 Evaluation and Combination of $A_{\|}$Values . . . . . . . . . . . 344

7.1.1 Combining asymmetries from opposite HWP status . . . . . . . . . . 346

7.1.2 Combining asymmetries from opposite target polarizations . . . . . . . . 347

7.1.3 Combining asymmetries from opposite torus currents . . . . . . . . . . . 352

7.1.4 Combining asymmetries from slightly differing beam energies . . . . . . . 353

7.2 Systematic error calculation . . . . . . . . . . . . . . . . . 355

7.2.1 Target model errors (1-9) . . . . . . . . . . . . . . . . . . . . 357

7.2 .2 Errors in dilution factor model (28) . . . . . . . . . . . . . . . . . 362

7.2.3 Uncertainties in determination of beam $\times$ target polarization $(10,13,17-21) \quad$ - 367

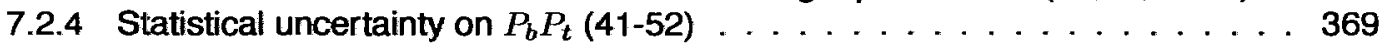

7.2.5 Miscellaneous background subtraction errors (22-27) $\ldots \ldots \ldots$

7.2.6 Kinematics resolution errors (29-32) . . . . . . . . . . . . . . . . 374

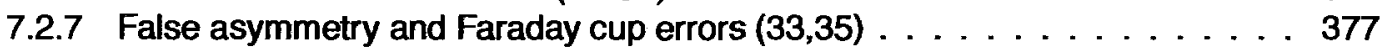

7.2 .8 Models errors $(36-40) \ldots \ldots \ldots \ldots$. . . . . . . . . . . . . . . . . . . . . . . .

7.2.9 Uncertainties with no effects on asymmetry analysis $(11-12,14-16,20,34) \quad \ldots 382$

7.2.10 Combination of systematic errors . . . . . . . . . . . . . . . . 382

8 Final Results 387

8.1 Virtual Photon Asymmetry Results . . . . . . . . . . . . . . . . . 389

8.1 .1 Extraction of $A_{1} \ldots \ldots \ldots \ldots \ldots \ldots \ldots \ldots$

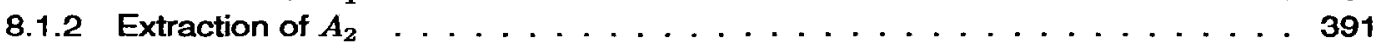

8.1.3 Refining Accuracy of $A_{1}$ and $A_{2}$ Measurements . . . . . . . . . . . 397

8.2 Spin Structure Function Results . . . . . . . . . . . . . . . . . . . 398

8.2 .1 Results for $g_{1} \ldots \ldots \ldots \ldots \ldots \ldots \ldots \ldots \ldots \ldots$ 


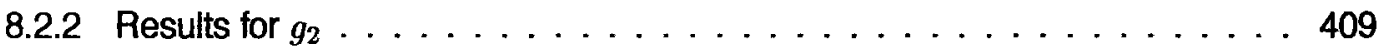

8.3 Spin Structure Function Moments . . . . . . . . . . . . . . . . . . . . . . 413

8.3.1 Procedure for calculating moments . . . . . . . . . . . . . . . . . 413

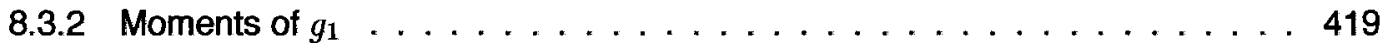

8.3.3 Moments of $g_{2} \ldots \ldots \ldots \ldots \ldots \ldots \ldots \ldots$

8.4 Applications . . . . . . . . . . . . . . . . . . . . . 424

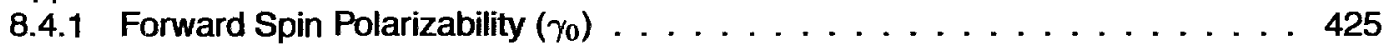

$8.4 .2{ }^{1}$ H Atomic Hyperfine Splitting . . . . . . . . . . . . . . . . . . . . 429

8.4 .3 Bloom-Gilman Duality . . . . . . . . . . . . . . . . . . . . 435

8.5 Future Directions . . . . . . . . . . . . . . . . . . . . . . . . . . . 443

8.5 .1 Outstanding Details . . . . . . . . . . . . . . . . . . 443

8.5.2 Upcoming Experiments and Data . . . . . . . . . . . . . . . . . 443

8.5 .3 Testing Quark Models . . . . . . . . . . . . . . . . . . . . . . . . . 446

A Glossary of Acronyms $\quad 451$

B Additional Tables $\quad 456$

B.1 Kinematic Bin Tables . . . . . . . . . . . . . . . . . . . . . . . . . 456

B.2 Pion Background Cut Parameters . . . . . . . . . . . . . . . . . . . . . . . . . 459

B.3 Fiducial Cut Parameters . . . . . . . . . . . . . . . . . . . . . 466

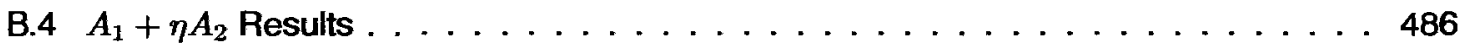

B.5 Virtual Photon Asymmetry Results . . . . . . . . . . . . . . 515

B.6 Structure Function Results . . . . . . . . . . . . . . . . . . . . 526

B.7 Moments and Integrals . . . . . . . . . . . . . . . . . 543

$\begin{array}{ll}\text { Bibliography } & 553\end{array}$ 


\section{List of Tables}

1.1 Flavors of quarks and their properties $\ldots \ldots \ldots \ldots \ldots \ldots$

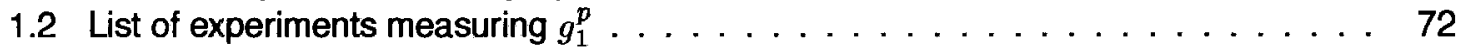

2.1 EG1 target material properties . . . . . . . . . . . . . . . . 113

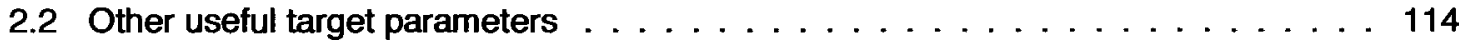

2.3 Radiation lengths of target materials . . . . . . . . . . . . . . . . . 155

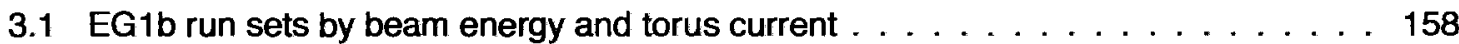

3.2 Helicity label definitions. . . . . . . . . . . . . . . . . . . . . . 161

3.3 Helicity pairing (HeLP) table example . . . . . . . . . . . . . . 163

3.4 Particle identification (PID) cuts . . . . . . . . . . . . . . . . . . . . . 182

3.5 Elastic $e p$ kinematic cuts . . . . . . . . . . . . . . . . . . . . . . . . 186

4.1 Raster $A D C$ to $\mathrm{cm}$ translation parameters . . . . . . . . . . . . . . . . . . . 202

4.2 Nominal vs. actual beam energies for EG1b . . . . . . . . . . . . . . . . . . . . 210

4.3 Sector-dependent Momentum Correction Parameters for EG1b. . . . . . . . . . . . . 224

4.4 Torus current-dependent parameter $T_{\text {set }}$ for outbending sets. . . . . . . . . . . . . 224

4.5 Forward angle momentum correction coefficients for EG1b . . . . . . . . . . . . 229

4.6 Loose fiducial cut parameters for inbending runs . . . . . . . . . . . . . 241

4.7 Sample tight fiducial cuts parameters for inbending runs . . . . . . . . . . . . . 241

4.8 Sample tight fiducial cut parameters for outbending runs . . . . . . . . . . . . . . . 242

4.9 Faraday Cup normalization factors for multiple scattering correction . . . . . . . . 245

5.1 Run bracket labels for EG1b . . . . . . . . . . . . . . . . . . . . . . . . 249

5.2 Run bracket labels for ${ }^{15} \mathrm{~N} /{ }^{12} \mathrm{C}$ data . . . . . . . . . . . . . . . . . . 250

5.3 Experimental $L$ measurements for empty target anomaly analysis . . . . . . . . 257

5.4 Empty target anomaly correction ratios . . . . . . . . . . . . . . . . . 257

5.5 Target cell length $L$ for each bracket . . . . . . . . . . . . . . . . . . . . 264

5.6 Normalization factors for ${ }^{15} \mathrm{~N} /{ }^{12} \mathrm{C}$ ratios . . . . . . . . . . . . . . . . . . . . 272

5.7 Fit coefficients $a$ and $b$ for ${ }^{15} \mathrm{~N} /{ }^{12} \mathrm{C}$ cross-section ratios $\ldots \ldots \ldots \ldots \ldots \ldots \ldots . \ldots \ldots$

5.8 Frozen nitrogen target lengths $\ell_{N}$ for ${ }^{15} \mathrm{~N}$ run brackets $\ldots \ldots \ldots \ldots \ldots \ldots \ldots$

5.9 Frozen ammonia effective target lengths $\ell_{A}$ for each bracket . . . . . . . . . . 285

5.10 High- $W$ cutoffs for dilution factor measurement . . . . . . . . . . . . . . 287

6.1 Fit parameters for $e^{+} e^{-}$background correction . . . . . . . . . . . . 303

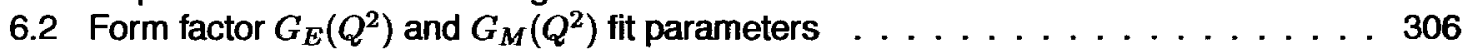

6.3 Carbon target scaling $W$-limits and scaling factors for elastic backgrounds . . . . . 309

6.4 Inclusive elastic $W$-limits . . . . . . . . . . . . . . . . . . . . . . . . . . 309 
6.5 Carbon scaling factors for exclusive $e p$ background . . . . . . . . . . . . 315

6.6 Exclusive $e p$ elastic $W$-bounds . . . . . . . . . . . . . . . . . . 316

$6.7 \quad P_{b} P_{t}$ measurements by half-wave-plate status and target polarization . . . . . . . . 319

$6.8 P_{b} P_{t}$ measurements by target polarization $\ldots \ldots \ldots \ldots \ldots \ldots \ldots \ldots$

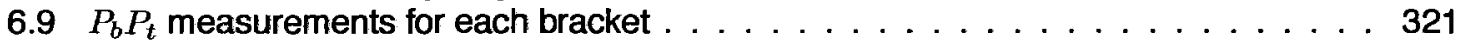

$6.10 P_{b} P_{t}$ measurements used for data weighting $\ldots \ldots \ldots \ldots \ldots \ldots \ldots \ldots$

6.11 Møller beam polarizations and ${ }^{15} \mathrm{~N}$ polarization $\ldots \ldots \ldots \ldots \ldots . \ldots . \ldots 33$

7.1 Students $t$-test results for half-wave-plate status combination . . . . . . . . . . 349

7.2 Students $t$-test results for target polarization combination . . . . . . . . . 351

7.3 Students $t$-test results for opposing torus current combination . . . . . . . . . . 353

7.4 Students $t$-test results for close beam energy combination . . . . . . . . . . . . 355

7.5 Systematic error index key . . . . . . . . . . . . . . . . . . . . . . . . . 359

7.6 Fit parameters for dilution factor model systematic error . . . . . . . . . . . . 366

8.1 World data reference sources . . . . . . . . . . . . . . . . . . . . . 389

8.2 Students $t$-test results for $A_{1}$ combinations from different beam energies . . . . . . 391

B.1 Standard $Q^{2}$ bins for EG1b . . . . . . . . . . . . . . . . . . 457

B.2 $\theta$ and momentum $(p)$ bins for $e^{+} e^{-}$background removal. . . . . . . . . . 458

B.3 Pion background cut parameters . . . . . . . . . . . . . . . 460

B.4 Inbending fiducial cut parameters . . . . . . . . . . . . . . . . 466

B.5 Outbending fiducial cut parameters . . . . . . . . . . . . . . . . 468

B.6 Results for $A_{1}+\eta A_{2}, E=1.6 \mathrm{GeV} \ldots \ldots \ldots \ldots \ldots \ldots$

B.7 Results for $A_{1}+\eta A_{2}, E=2.5 \mathrm{GeV} \ldots \ldots \ldots \ldots \ldots$. . . . . . . . . 491

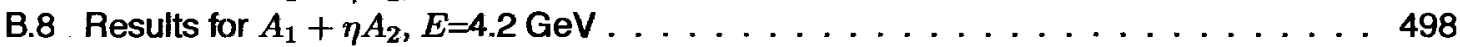

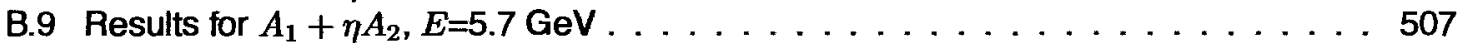

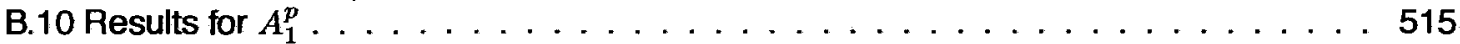

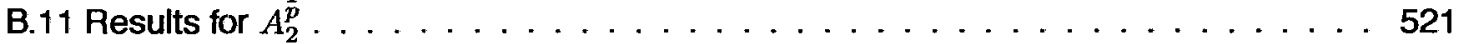

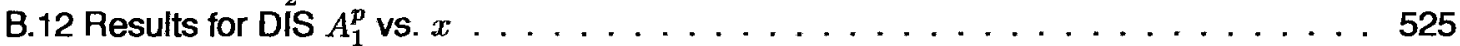

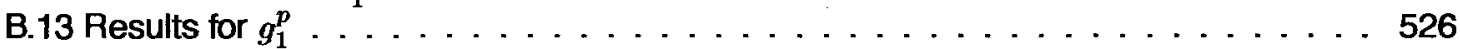

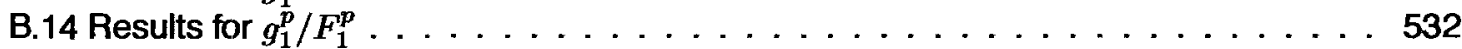

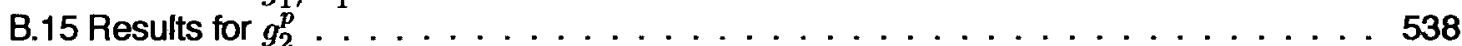

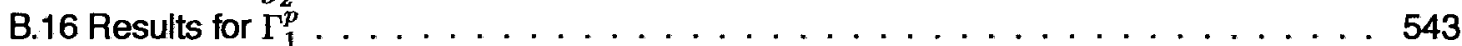

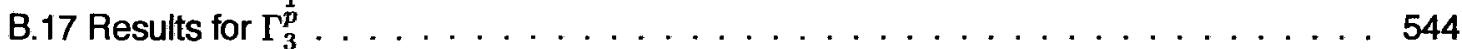

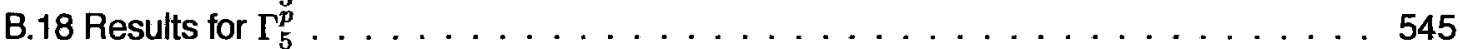

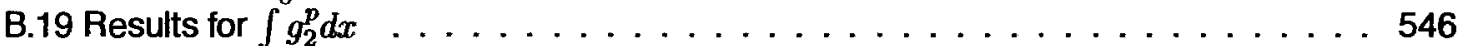

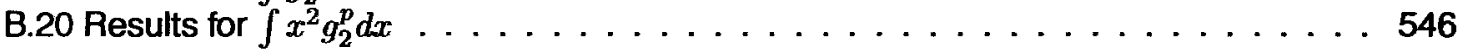

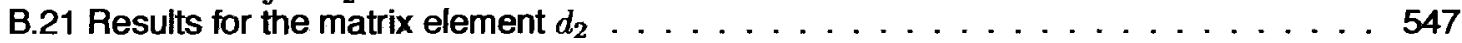

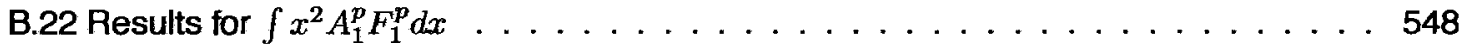

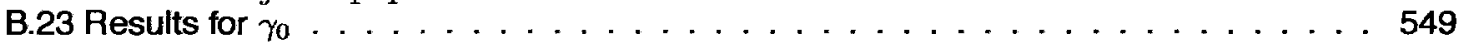

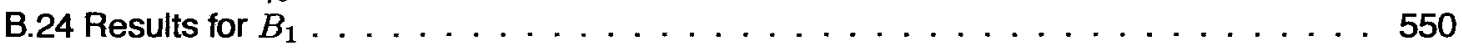

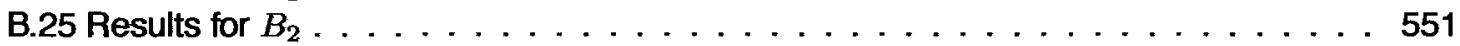

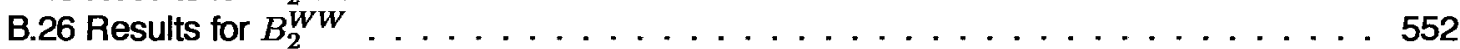




\section{List of Figures}

1.1 Pictures of the proton at varying probe distances $\ldots \ldots \ldots \ldots \ldots$

1.2 Inclusive $e p$ scattering kinematics . . . . . . . . . . . . . . . 6

1.3 Unpolarized inclusive scattering in the lab frame . . . . . . . . . . . . . . 12

1.4 Polarization transformations upon reflections and Lorentz boosts . . . . . . . . . 23

1.5 Scaling behavior of spin-flip transitions . . . . . . . . . . . . . . 28

1.6 Contour of integration in the Optical Theorem . . . . . . . . . . . . . . 43

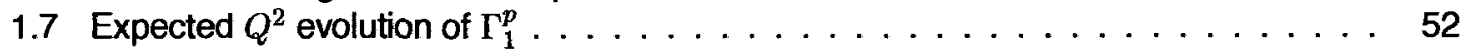

1.8 Expected $W$ evolution of $A_{1}^{p} \ldots \ldots \ldots \ldots \ldots \ldots \ldots \ldots \ldots$

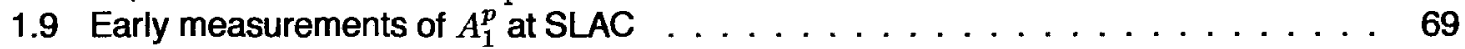

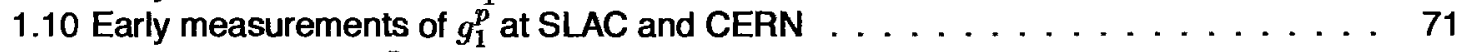

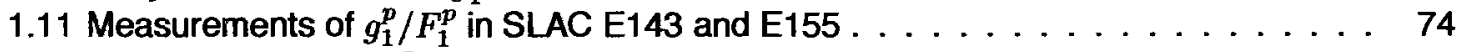

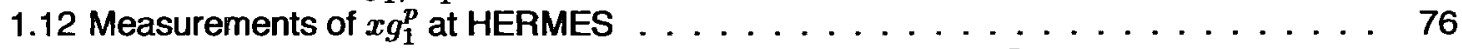

1.13 Comparison of abundances of world data vs. EG1 data for $g_{1}^{p} \ldots \ldots \ldots$

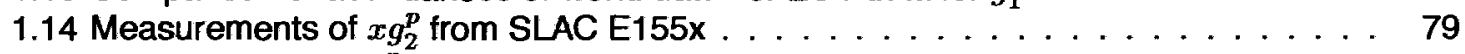

1.15 Measurements of $g_{2}^{p}$ from JLab RSS (Hall-C) $\ldots \ldots \ldots \ldots \ldots$

2.1 Schematic of CEBAF electron accelerator at Jefferson Lab $\ldots \ldots \ldots \ldots$

2.2 Schematic of photoelectron gun . . . . . . . . . . . . . . . . . . 84

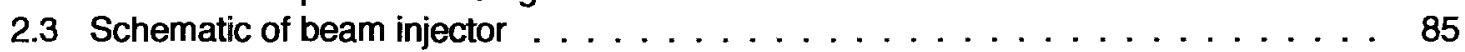

2.4 Photograph of cryounit . . . . . . . . . . . . . . . . . . 85

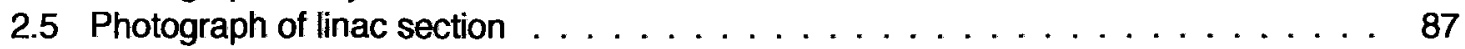

2.6 Photograph of recirculation arc . . . . . . . . . . . . . . . . . . 89

2.7 Schematic of Hall-B and beam line monitoring devices . . . . . . . . . . . . 90

2.8 Photograph of Moller polarimeter . . . . . . . . . . . . . . . . . . . 90

2.9 Schematic of Møller polarimeter . . . . . . . . . . . . . . . . . . . . . 91

2.10 Beam Position Monitor (BPM) measurements . . . . . . . . . . . . . . . . . 92

2.11 Results of harp scan measurement . . . . . . . . . . . . . . . . . 93

2.12 Faraday Cup and Synchrotron Light Monitor measurements comparison . . . . . . . 94

2.13 Photograph of frozen $\mathrm{NH}_{3}$ target beads . . . . . . . . . . . . . 96

2.14 Energy levels in magnetized proton target prior to DNP . . . . . . . . . . . . . . . 99

2.15 Energy level transitions in Dynamic Nuclear Polarization (DNP) . . . . . . . . . . 101

2.16 Distribution of energy levels in Equal Spin Temperature (EST) theory . . . . . . . . 102

2.17 Relative polarizations of ${ }^{15} \mathrm{~N}$ vs. $\mathrm{p}$ in SMC . . . . . . . . . . . . . . . 104

2.18 Schematic of polarized target cryostat . . . . . . . . . . . . . . . 105

2.19 Photograph of target assembly . . . . . . . . . . . . . . . . . 105

2.20 Online NMR polarization monitoring . . . . . . . . . . . . . . . . . 106

2.21 Photograph of target insert strip . . . . . . . . . . . . . . . . . . . 107 
2.22 Photograph of target stick and vacuum flange . . . . . . . . . . . . . 108

2.23 Photograph of filled target immersed in $\mathrm{LN}_{2} \ldots \ldots \ldots \ldots$. . . . . . . . . . 109

2.24 Photograph of Kapton foil . . . . . . . . . . . . . . . . . . . 110

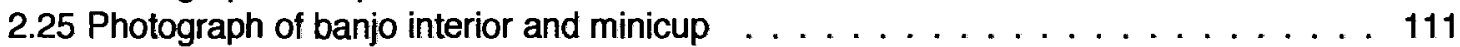

2.26 Photograph of assembled polarized target . . . . . . . . . . . . . . . . 112

2.27 Schematic of CLAS (parallel cutaway) . . . . . . . . . . . . . . . 115

2.28 Schematic of CLAS (perpendicular cutaway) $\ldots \ldots \ldots \ldots \ldots \ldots \ldots$

2.29 Schematic of torus magnet frame . . . . . . . . . . . . . . . . . . 117

2.30 Schematic of drift chamber cells . . . . . . . . . . . . . . 118

2.31 Photograph of outermost (Region 3) drift chamber . . . . . . . . . . . . . 119

2.32 Time-based tracking in the drift chamber . . . . . . . . . . . . . . . . . . 121

2.33 Distance of closest approach (DOCA) vs. drift time . . . . . . . . . . . . 121

2.34 Drift chamber residuals . . . . . . . . . . . . . . . . . . . . . . . . . . . 122

2.35 Schematic of Cherenkov Counter sector . . . . . . . . . . . . . . . . . . . 122

2.36 Schematic of Cherenkov Counter segment . . . . . . . . . . . . . . . . 124

2.37 Photograph of Cherenkov Counter . . . . . . . . . . . . . . . . 125

2.38 Schematic of Scintillator Counter . . . . . . . . . . . . . . . . . . . . . 126

2.39 Scintillation Counter PMT ADC channels . . . . . . . . . . . . . . . . . . . . 126

2.40 Time-walk evolution in Scintillation Counter PMT . . . . . . . . . . . . . . . 127

2.41 Time-of-flight left-right alignment plots . . . . . . . . . . . . . . . . . . . 129

2.42 Attentuation length calibration plot for Scintillation Counters . . . . . . . . . . 130

2.43 RF bunch timing offsets . . . . . . . . . . . . . . . 131

2.44 Paddle-to-paddle time-of-flight calibration . . . . . . . . . . . . . . . . 132

2.45 Schematic of Electromagnetic Calorimeter sector . . . . . . . . . . . . . . . . . 133

2.46 Vertical cross section of Electromagnetic Calorimeter . . . . . . . . . . . . . . . 134

2.47 Electromagnetic Calorimeter timing calibration . . . . . . . . . . . . . 135

2.48 Photograph of partially assembled CLAS . . . . . . . . . . . . . 136

2.49 Data Acquisition System flowchart . . . . . . . . . . . . . . . . . . . 138

2.50 Online data reconstruction . . . . . . . . . . . . . . . . 138

2.51 Time-of-flight reconstructed mass spectrum . . . . . . . . . . . . . . . . . . 141

2.52 Kinematic coverage of EG1b . . . . . . . . . . . . . . . . . . . . . . . . 142

2.53 Event timing quality check plot . . . . . . . . . . . . . . . . 144

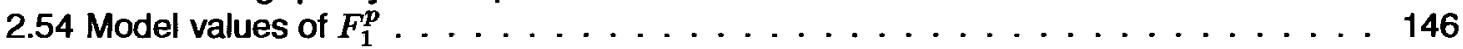

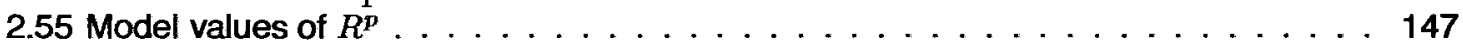

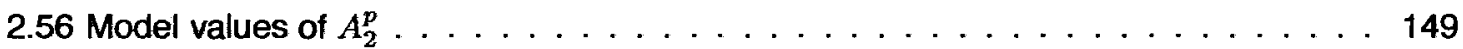

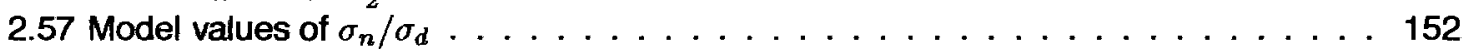

2.58 Mass thickness external to target vs. $\theta \ldots \ldots \ldots \ldots \ldots \ldots$

3.1 Helicity labeling example . . . . . . . . . . . . . . . . . 161

3.2 Online monitoring of helicity bits . . . . . . . . . . . . . . . . . 162

3.3 Beam charge asymmetry quality check . . . . . . . . . . . . . . . . 165

3.4 Inclusive count rates in each sector . . . . . . . . . . . . . . . . . 166

3.5 Count rate quality check example . . . . . . . . . . . . . . . . . 167

3.6 Identification of count rate change causes . . . . . . . . . . . . . . . 167

3.7 Target polarization and half-wave-plate status checks $\ldots \ldots \ldots \ldots \ldots$

3.8 Raster event rate density . . . . . . . . . . . . . . . . . . . . 170

3.9 Raster pattern quality comparison . . . . . . . . . . . . . . . . . . 172

3.10 Raster pattern anomaly for empty target . . . . . . . . . . . . . . 173 
3.11 Count spectra vs. $W$ for individual runs $\ldots \ldots \ldots \ldots \ldots \ldots \ldots$

3.12 Cherenkov Counter photoelectron spectra . . . . . . . . . . . . . . . . . . . . 179

3.13 Particle Identification by Electromagnetic Calorimeter energy . . . . . . . . . . . . 180

3.14 Particle Identification by Electromagnetic Calorimeter energy and momentum . . . . 181

3.15 Elastic ep event kinematic cuts . . . . . . . . . . . . . . . . . . . . . 185

3.16 Vertex position $v_{z}$ vs. run number . . . . . . . . . . . . . . . 187

3.17 Consistency of internal Electromagnetic Calorimeter signals . . . . . . . . . . . . 189

3.18 Anomalous vertex reconstruction in Sector $5 \ldots \ldots \ldots$. . . . . . . . . 191

4.1 Cherenkov photoelectron spectra of electrons and pions . . . . . . . . . . . 193

4.2 Cherenkov Counter projection plane definition . . . . . . . . . . . . . . . . . . 195

4.3 Cherenkov Counter and Scintillation Counter geometric discrepancy . . . . . . . . . 196

4.4 Cherenkov Counter and Scintillation Counter timing discrepancy . . . . . . . . . 197

4.5 Effect of precision Cherenkov cuts on photoelectron spectra . . . . . . . . . . . . 199

4.6 Determination of residual pion contamination after precision cuts . . . . . . . . . 200

4.7 Schematic of raster correction geometry (front view) . . . . . . . . . . . . 204

4.8 Schematic of raster correction geometry (longitudinal view) . . . . . . . . . 204

4.9 Effect of raster corrections on reconstructed $z$-vertex . . . . . . . . . . . . . 206

4.10 Effect of torus current correction . . . . . . . . . . . . . . . . . . 208

4.11 Visualization of multiple scattering . . . . . . . . . . . . . . . . . . 212

4.12 Summary of kinematic correction effects . . . . . . . . . . . . . . . 220

4.13 Distribution in $\theta$ of elastic $e p$ events . . . . . . . . . . . . . . . . . . . . . . 222

4.14 Azimuthal dependence of momentum corrections . . . . . . . . . . . . . . . 225

4.15 Low $\theta$ elastic peak positions prior to final corrections . . . . . . . . . . . . . 227

4.16 Low $\theta$ momentum correction fit . . . . . . . . . . . . . . . . . . . . . . 228

4.17 Effects of final low- $\theta$ momentum corrections . . . . . . . . . . . . . . . . . . 229

4.18 Net effects of kinematic corrections on inclusive spectra . . . . . . . . . . . . 230

4.19 Effects of target solenoid field on scattering angle measurement . . . . . . . . 232

4.20 Inbending fiducial cuts (low momentum) . . . . . . . . . . . . . 235

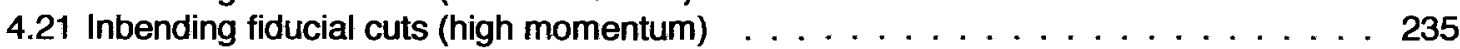

4.22 Outbending fiducial cuts . . . . . . . . . . . . . . . . . . . . 238

4.23 Interpolation of fiducial cut parameters . . . . . . . . . . . . . . . . . . . . 239

4.24 Loose inbending fiducial cut for asymmetry measurement . . . . . . . . . . . 240

4.25 Effects of fiducial cuts on pion contamination . . . . . . . . . . . . 243

5.1 Inclusive count rates for each beam energy . . . . . . . . . . . . . 253

5.2 Empty target anomaly raster pattern cuts . . . . . . . . . . . . . . 256

5.3 Target length $L$ measurement from data . . . . . . . . . . . . . . . . . . 261

5.4 Empty to carbon target count ratios for each bracket . . . . . . . . . . . . . . 262

5.5 Target length $L$ measurement from model . . . . . . . . . . . . . . . . 265

5.6 Target length $L$ for each bracket . . . . . . . . . . . . . . . . . 266

5.7 Count rate change during draining of minicup . . . . . . . . . . . . . . 268

5.8 Frozen nitrogen to carbon target count ratios ( $2.3 \mathrm{GeV}$ beam energy) . . . . . . . . 270

5.9 Frozen nitrogen to carbon target count ratios (other beam energies) . . . . . . . 273

5.10 Fit of carbon to frozen nitrogen inclusive spectra . . . . . . . . . . . . 277

5.11 Measurement of ${ }^{15} \mathrm{~N}$ frozen target length $\ell_{N} \ldots \ldots \ldots \ldots \ldots \ldots . \ldots . \ldots 279$

5.12 Measurement of ammonia target length $\ell_{A}$ from data . . . . . . . . . . 281

5.13 Measurement of ammonia target length $\ell_{A}$ from model . . . . . . . . . . . . 284 
5.14 Dilution factors from data plotted vs. $W \ldots \ldots$. . . . . . . . . . . . 289

5.15 Dilution factors plotted individually by sector and polarization status . . . . . . . . 290

5.16 Subtracted background using dilution factors $\ldots \ldots \ldots \ldots \ldots \ldots \ldots$

5.17 Dilution factors (from data) plotted vs. $Q^{2} \ldots \ldots \ldots \ldots . \ldots . \ldots . \ldots . \ldots 292$

5.18 Dilution factors from data and model compared . . . . . . . . . . . . . . . . 294

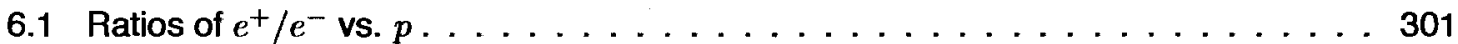

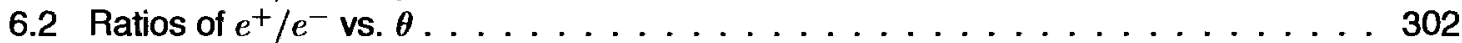

6.3 Positron asymmetries as a function of $p \ldots \ldots \ldots$. . . . . . . . . . . . 304

6.4 Background-subtracted inclusive elastic spectra . . . . . . . . . . . . . 310

6.5 Elastic $e p$ events in terms of $\phi_{p}-\phi_{e} \ldots \ldots \ldots \ldots \ldots$. . . . . . . . . 316

6.6 Background-subtracted elastic $e p$ spectra $\ldots \ldots \ldots \ldots$. . . . . . . . . . . 317

$6.7 \quad P_{b} P_{t}$ values divided by half-wave-plate status and target polarization $\ldots \ldots \ldots . .321$

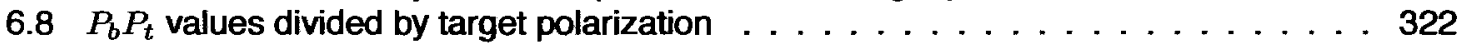

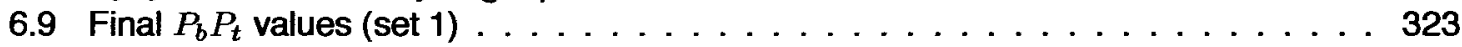

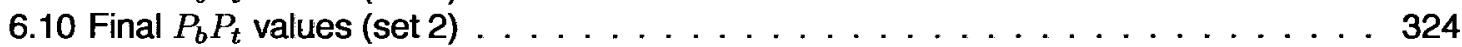

6.11 Comparison of measured $P_{b} P_{t}$ and Møller $\times$ NMR measurements $\ldots \ldots \ldots \ldots 326$

$6.12 P_{b} P_{t}$ inel values used for relative weighting of opposite polarizations $\ldots \ldots \ldots$

6.13 Parametrization of ${ }^{15} \mathrm{~N}$ vs. p polarizations . . . . . . . . . . . . 331

7.1 Raw asymmetries by half-wave-plate status and target polarization . . . . . . . 345

7.2 Raw asymmetries by target polarization . . . . . . . . . . . . . 348

7.3 Final combined raw asymmetry . . . . . . . . . . . . . . . . . . 351

7.4 Measurement of $A_{\|}$(before radiative corrections) . . . . . . . . . . . 352

7.5 Measurements of $A_{\|}$with radiative corrections . . . . . . . . . . . . . 354

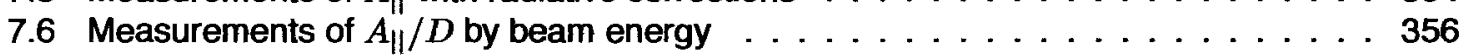

7.7 Systematic errors on $A_{1}+\eta A_{2}$ by source $\ldots \ldots \ldots \ldots \ldots \ldots \ldots$

7.8 Dilution factor systematic errors due to target model $\ldots \ldots \ldots \ldots 363$

7.9 Dilution factor model error determination . . . . . . . . . . . . . . 365

7.10 Systematic errors on $A_{1}+\eta A_{2}$ due to systematic $P_{b} P_{t}$ effects $\ldots \ldots \ldots \ldots \ldots$

7.11 Systematic errors on $A_{1}+\eta A_{2}$ due to statistical $P_{b} P_{t}$ effects $\ldots \ldots \ldots \ldots \ldots$

7.12 Systematic errors on $A_{1}+\eta A_{2}$ due to miscellaneous backgrounds . . . . . . . 375

7.13 Systematic errors on $A_{1}+\eta A_{2}$ due to kinematic errors . . . . . . . . . 378

7.14 Systematic errors on $A_{1}+\eta A_{2}$ due to models errors . . . . . . . . . . . 381

7.15 Local bins used for systematic error smoothing . . . . . . . . . . . . . . 383

7.16 Measurement of $A_{\|} / D$ showing total systematic errors (magnified) $\ldots \ldots \ldots \ldots 385$

7.17 Measurement of $A_{\|} / D$ showing total systematic errors (actual size) $\ldots \ldots \ldots$

8.1 Key to world data plots . . . . . . . . . . . . . . . 388

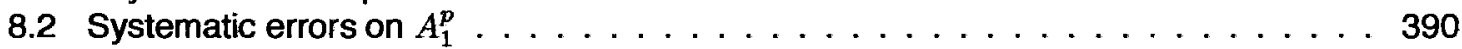

8.3 Results for $A_{1}^{p}$ vs. $W \ldots \ldots \ldots \ldots$

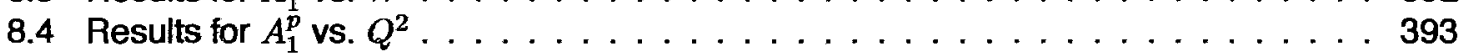

8.5 Results for $A_{1}^{p}$ vs. $x$ demonstrating duality . . . . . . . . . . . . . . 394

8.6 Kinematic coverage and examples of linear regression analysis . . . . . . . . . . 395

8.7 Results for $A_{1}^{p}$ vs. $W$ without use of the $A_{2}^{p}$ model . . . . . . . . . . . . . . 396

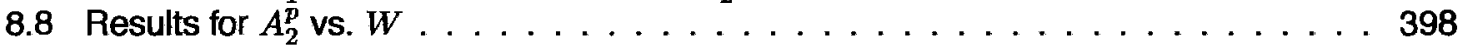

8.9 Results for $A_{2}^{p}$ vs. $x \ldots \ldots \ldots \ldots \ldots \ldots \ldots$

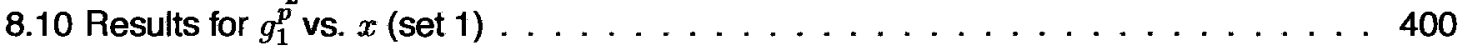




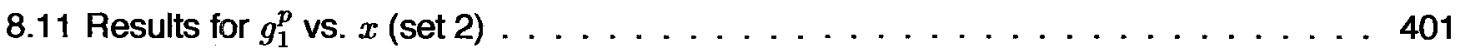

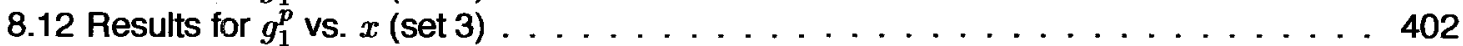

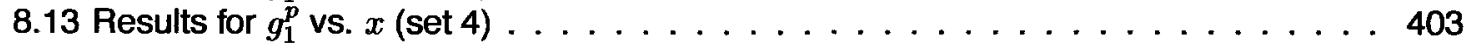

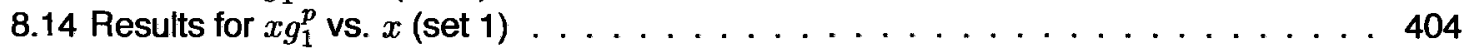

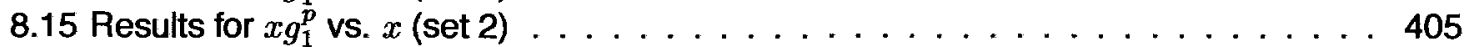

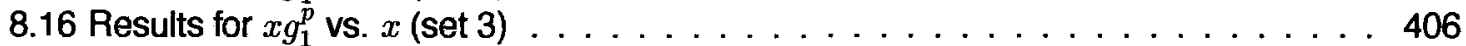

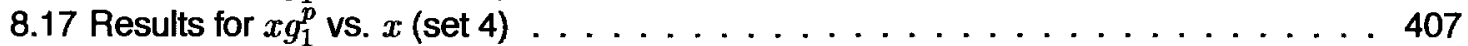

8.18 Results for $g_{1}^{p} / F_{1}^{p}$, averaged over all $Q^{2} \ldots \ldots \ldots \ldots \ldots \ldots$. . . . . 409

8.19 Results for $g_{1}^{p} / F_{1}^{p}$ vs. $Q^{2}$, binned in $x \ldots \ldots \ldots \ldots \ldots \ldots \ldots \ldots \ldots$

8.20 Results for $g_{1}^{p} / F_{1}^{p}$ vs. $Q^{2}$ compared between beam energies and analyses . . . . 411

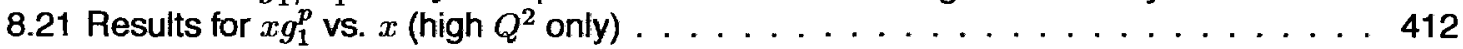

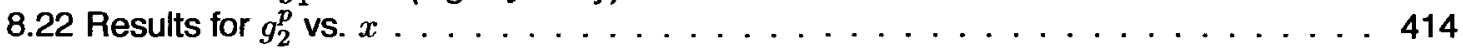

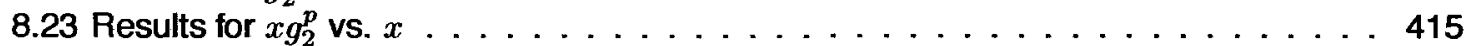

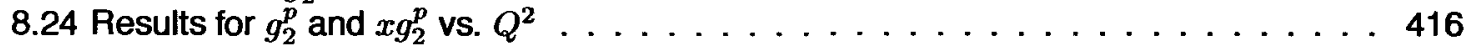

8.25 Results for $g_{2}^{p}$ and $x g_{2}^{p}$ vs. $Q^{2}$, binned in $\ldots \ldots \ldots \ldots \ldots \ldots \ldots \ldots \ldots \ldots$

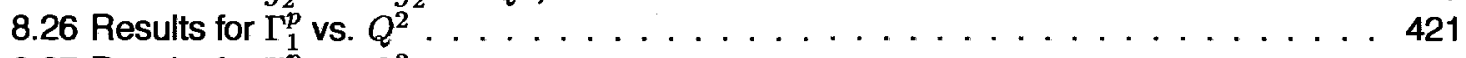

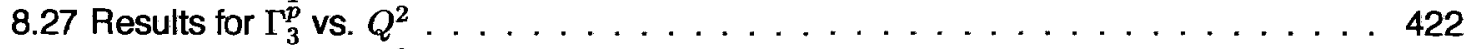

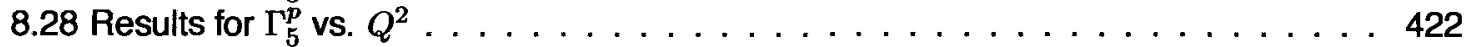

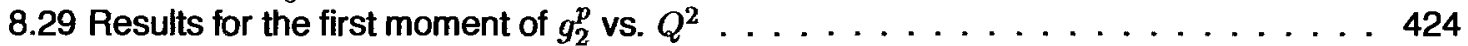

8.30 Results for the third moment of $g_{2}^{p}$ vs. $Q^{2} \ldots \ldots \ldots \ldots \ldots \ldots \ldots \ldots$

8.31 Results for the matrix element $d_{2}^{p}$ vs. $Q^{2} \ldots \ldots \ldots \ldots \ldots \ldots \ldots \ldots$

8.32 Results for the forward spin polarizability integral vs. $Q^{2} \ldots \ldots \ldots \ldots \ldots \ldots$

8.33 Results for the forward spin polarizability $\gamma_{0}$ vs. $Q^{2} \ldots \ldots \ldots \ldots \ldots$. . . . . 430

8.34 Results for the hyperfine splitting contribution $B_{1}$ vs. $Q^{2} \ldots \ldots \ldots \ldots \ldots$. . . 436

8.35 Results for the hyperfine splitting contribution $B_{2}$ vs. $Q^{2} \ldots \ldots \ldots \ldots \ldots$. . . 437

8.36 Results for the $B_{2}$ leading-twist term $B_{2}^{W W}$ vs. $Q^{2} \ldots \ldots \ldots \ldots \ldots \ldots$

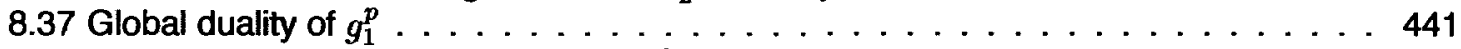

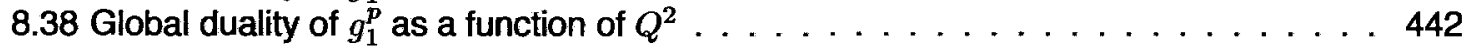

8.39 Projection of EG4 analysis results . . . . . . . . . . . . . . . 445

8.40 Projected kinematic coverage of SANE . . . . . . . . . . . . . . 446

8.41 Projected SANE results of the $d_{2}^{p}$ matrix element $\ldots \ldots \ldots$. . . . . . . . . 447

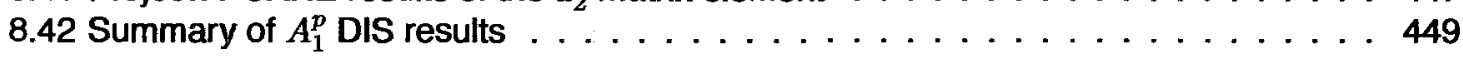

8.43 Projected $x$-coverage of CLAS12 DIS data . . . . . . . . . . . . . 450 


\section{Acknowledgements}

No research project of this magnitude takes place in a vacuum, nor can any advancement in collective knowledge be made without building on the work and experience of others. Therefore, credit must be given both to those who helped set the stage and lay the foundation for the work presented in this dissertation, and to the experienced researchers who taught me the necessary physics and assisted in the more daunting aspects of this research.

A special thanks goes to Renee Fatemi, Vipuli Dharmawardane, and Yelena Prok, whose past work and dissertations in inclusive analysis of the EG1a and EG1b experimental data built a solid framework for the continuation of this research. Appreciation also goes to Cornel Buttuceanu and Stepan Stepanyan, whose help was essential in the early stages of work in time-of-flight calibration and experimental event reconstruction. Credit is shared with the many members of the EG1 collaboration. The extensive experience of Professors Sebastian Kuhn and Gail Dodge from Old Dominion University provided much-needed structure and guidance throughout the analysis process. Also, the collaborative nature of this work must be emphasized, to which end I must thank the efforts of Josh Pierce (for his polarized target experience and programming of the DST data translator framework), Nevzat Guler (primarily for his development of the kinematic correction package, precision pion background and pair-symmetric background removal code and help with the $\boldsymbol{P}_{b} \boldsymbol{P}_{t}$ calculations and time-of-flight calibrations, among other tasks, including continued development of the structure function models package with S. Kuhn), Sharon Careccia (for work in calorimeter timing calibration and particle identification cuts), and Shifeng Chen (for drift chamber calibration and general database management). Harut Avakian deserves additional credit for his knowledge of the simple event builder details and target solenoid field corrections. Thanks also goes to Volker Burkert, Tony Forest, and Ralph Minehart for their many constructive critiques and contributions, which are too numerous to list here.

A very special thanks goes to Peter Bosted, whose many years of experience and parallel analyses of EG1 data provided an indispensable asset in producing results of high integrity. His invaluable hours of assistance and development of the radiated cross-section model, kinematic corrections, and general leadership in our collaborative effort in refining the details of the ${ }^{15} \mathrm{~N} /{ }^{12} \mathrm{C}$ 
cross-section ratio model, made him the most essential contributor to the analysis as a whole.

I would also like to thank my advisor, Professor Keith Griffioen at the College of William and Mary, for giving me the chance to get involved with the EG1 working group, for providing countless hours of his professional time toward advancing this research, maintaining high standards of writing and productivity, and sustaining the level of patience necessary to train me with the skills necessary to be successful in the field of experimental hadronic physics research. This thanks extends to all the members of my Defense Committee, whose input throughout the years has improved my ability to present physics work at conferences and other venues. All members of the CLAS Collaboration and Deep Processes Working Group at Jefferson Laboratory, not mentioned in these short paragraphs, are also credited with their many contributions.

None of this would be possible without the physics knowledge imparted by the many great teachers who have helped me build a base education for the subject, especially J. D. Walecka, Shiwei Zhang, Carl Carlson, Chris Carone, and Marc Vanderhaegen at the College of William and Mary, Mark Sharnoff, George Watson and Ed Eyler at the University of Delaware, and Roger Volrath of Glenelg High School in Maryland, who, during my formative years, started me in an ongoing educational journey by sparking an interest in this subject.

As a final note, I remark that besides professional and educational help, that there are those in my life who were crucial in helping me in maintaining my sanity during the sometimes arduous process of completing a disseration (sometimes at the cost of some of their own sanity). Without the support of my friends and family, especially my loving wife Theresa (who patiently endured my long work hours and repetitive remarks of "I'm almost done" for many months), I would not have maintained a positive outlook and happiness throughout the last several years of my life. I thank especially my wonderful parents, Robert (Sr.) and Barbara Fersch, who always taught me to maintain high ambitions without pressuring me for specific outcomes, allowing me to define my own educational journey and goals. 


\section{Preface}

This thesis is intended as a thorough documentation of the analysis procedures used in the analysis of inclusive proton data in the EG1 experiment. Where possible, explicit details of calculations and procedures are provided, so that this work can be employed as a reference work for future CLAS analyses, especially inclusive electron scattering analysis, though certain aspects of this analysis also have applications to exclusive and semi-inclusive event analysis, as well. An attempt was made to firmly clarify the motivation and theory behind the research goals, as well as to investigate applications of the evaluated $g_{1}$ and $g_{2}$ structure functions for the proton, in addition to specifying the experimental procedure.

Chapter 1 introduces electron scattering experiments, defines kinematic values, explores the theory behind structure functions and asymmetries, and provides a list and brief explanation of past accelerator experiments that specifically measure the $g_{1}$ and $g_{2}$ polarized structure functions of the proton. Chapter 2 details the JLab accelerator and CLAS detector apparatuses, the EG1 polarized target functionality and physics, and delineates the kinematic coverage of EG1. A description of the models used to parametrize structure functions and asymmetries not directly measured in EG1 is also presented.

Chapters 3 and 4 focus on early analysis tasks, mainly cuts and corrections on data. Chapter 3 focuses on basic particle and helicity identification and file quality checks, while Chapter 4 focuses on more refined and precise cuts and corrections, such as momentum and other kinematic corrections, and fiducial and pion removal cuts.

Chapters 5 through 7 contain the "main" analysis of the data. Chapter 5 contains all aspects of unpolarized background removal, including determination of target material thicknesses and dilution factor calculation. Chapter 6 concentrates on removal of all other backgrounds, namely beam and target polarization correction, pair-symmetric background, polarized nitrogen and radiative corrections. Chapter 7 summarizes the combination and compatibility testing of various data sets, and the determination of systematic error effects.

The final chapter (Chapter 8) presents all virtual photon asymmetry $\left(A_{1}\right.$ and $\left.A_{2}\right)$ and spin structure function ( $g_{1}$ and $g_{2}$ ) results, and their various moments in the Operator Product Expansion. 
Selected physics applications of the data are also explored. Extensive data tables (Appendix B) of all these quantities are provided at the end of this thesis, prior to the Bibliography. For convenience and utility to those unfamiliar with spin physics and/or CLAS jargon, a glossary of acronyms is included in Appendix A.

The result of a detailed documentation of an extensive analysis project is a very lengthy dissertation. Effort has thus been made to make this work useful as a reference by making chapters and sections as self-contained as possible, with cross-references supplied as needed. Those interested only in the theoretical motivation and new results, but not the experimental procedure, can easily read Chapters 1 and 8 and skip the bulk of the thesis; while those only interested in a summary of the CLAS detector and basic particle identification can read Chapters 2 and 3 , for example.

It is my hope that this thesis can be useful as a document for those wishing to become familiar with spin-structure functions, or those attempting to tackle various aspects of CLAS analysis (or both), especially newer graduate students at Jefferson Lab. With this intention in mind, I made an earnest attempt to explain and clarify subjects on a level assuming a standard two-year graduate physics education (with appropriate background in field theory and particle physics), but with little to no background in JLab or spin physics. Attempts were made to be as explicit as reasonably possible in derivations, or to provide specific references (listed in the Bibliography) where more information can be found. I sincerely hope that this thesis will find its intended future utility in the field of polarized physics experiments, so that others can continue to build and extend the reach of this and related research. 
Part 1 


\section{Chapter 1}

\section{Background and Motivation}

\subsection{Introduction}

The scattering of high-energy electrons from a stationary target provides an effective method for investigating the internal structure of nucleons, that is, the protons and neutrons that compose the atomic nucleus. In particular, the study of scattered electrons over a wide kinematic range provides measurements of the structure functions that quantify the internal dynamic behavior of the nucleon and the evolution of the perceived structure over varying short-distance scales. This thesis deals specifically with the study of the proton through analysis of data from the EG1b experiment at Jefferson Laboratory, focusing on the scattering of electrons from a stationary $\mathrm{NH}_{3}$ target. ${ }^{1}$ More specifically, we seek to study the spin structure of the proton, which requires the use of both a polarized electron beam and a polarized target.

In this chapter, the theory and formalism behind the study of spin structure functions are introduced, and a brief history of existing data utilizing polarized leptons scattered from polarized protons is reviewed, as a prelude to the discussion of the EG1 experiment.

\footnotetext{
${ }^{1} \mathrm{An} \mathrm{ND}_{3}$ target was used for the study of deuteron/neutron structure, as well; and although most of the analysis of this target data is similar to the $\mathrm{NH}_{3}$ analysis, it is not examined in this thesis. See, for example, Ret. [2].
} 


\subsubsection{Electron-proton scattering}

High-energy electrons are an excellent probe for the study of the nucleon. First, the interaction of electrons with protons is governed exclusively by the (well-understood) electromagnetic interaction, making this a very "clean" probe of the nucleon. The interaction occurs with the electromagnetic current density of the nucleon. ${ }^{2}$ Also, the strength of the electromagnetic coupling constant $\alpha=\frac{1}{137}$ is very small, so that perturbative techniques can be used to describe the interaction [1].

Experiments using stationary nuclear targets are ideal for making high-precision measurements of subatomic structure. While the high collision energies achievable using two colliding beams [3] cannot be reached with a fixed target, a much higher luminosity allows for collection of a much larger number of scattering events. The development of polarized electron beams (like that at the CEBAF accelerator at Jefferson Laboratory) and polarized targets (like the $\mathrm{NH}_{3}$ and $\mathrm{ND}_{3}$ targets used in the EG1 experiment) allows us to study the spin distributions of the quarks in nucleon, in addition to the unpolarized momentum distributions that were previously measurable with unpolarized beams/targets. Finally, the construction of fast response detector systems with large acceptances (that is, coverage over a large spatial geometry) such as the CEBAF Large Acceptance Spectrometer (CLAS), allows us to collect billions of particle events, and thus good statistical measurement of the quantities in question.

To consider how high energy electrons are useful for probing protons, we consider an optical analogy [1]. If radiation of a certain wavelength passes through a circular aperture of width $a$, and is projected on a distant plane, the angle $\theta$ from the central axis to the first minimum (i.e. the Airy disc) is given by

$$
\theta \approx \frac{0.61 \lambda}{a}
$$

If we use the standard terminology for the wave number in terms of wavelength $k=2 \pi / \lambda$, the momentum transfer $\kappa$ is given by

$$
\kappa \approx \frac{2 \pi \theta}{\lambda}
$$

\footnotetext{
2 The weak interaction also makes a small contribution to the interaction, but in the measurement of the asymmetries dealt with here, it is a very small contribution indeed, and is greatly overshadowed by other systematic errors; this effect is discussed in Section 7.2.7.
} 
and

$$
a \approx \frac{1.22 \pi}{\kappa}
$$

This implies that the larger the transfer of momentum, the shorter the resolved distance. Quantum mechanics asserts that electrons have a wavelength inversely proportional to their momenta, given by the deBroglie wavelength:

$$
\lambda=\frac{h}{p} \approx \frac{h c}{\nu}
$$

where $\nu$ is the energy transferred from the electron to the proton. Here the approximate equality $(\approx)$ assumes the electron mass is insignificant compared to that of the target, a valid approximation at ultra-relativistic velocities. Therefore, the higher the energy of the incident electron beam, the smaller the distance scale within the nucleon that can be resolved.

If the electron-nucleon interaction amplitude is determined only by the charge distribution, $\rho(r)$, and the scattered plane-wave amplitude at a point $\mathbf{r}$ is equal to $\exp (i \kappa \cdot \mathbf{r})$, then the total scattering amplitude $\mathscr{A}_{e l}$ is given by integrating over the volume of the incident nucleus $V$ [1]:

$$
\mathscr{A}_{e l}=\int_{V} d^{3} r \rho(r) e^{i \kappa \cdot \mathbf{r}}
$$

This is just the Fourier transform of the spatial charge distribution. We see now (semi-)quantitatively that the macroscopic measureable amplitude due to the scattering of electrons from nucleons reveals microscopic information about the charge distribution of the nucleon.

\subsubsection{Models of the proton}

Figure 1.1 qualitatively demonstrates a model of the proton as it is understood today, in terms of the squared 4-momentum transferred by the electron $\left(Q^{2}\right)$. How one pictures the proton depends upon the distance scale used to probe it. Equation 1.4 provides an approximate means of equating distance scales $(\sim \lambda)$ to transferred energy $(\nu)$.

At distances greater than $1 \mathrm{fm}=10^{-15} \mathrm{~m}$ (which corresponds to a transferred momentum of $\nu \sim$ $0.2 \mathrm{GeV}$ ), the proton behaves as a coherent object with overall charge $+e$ and angular momentum 


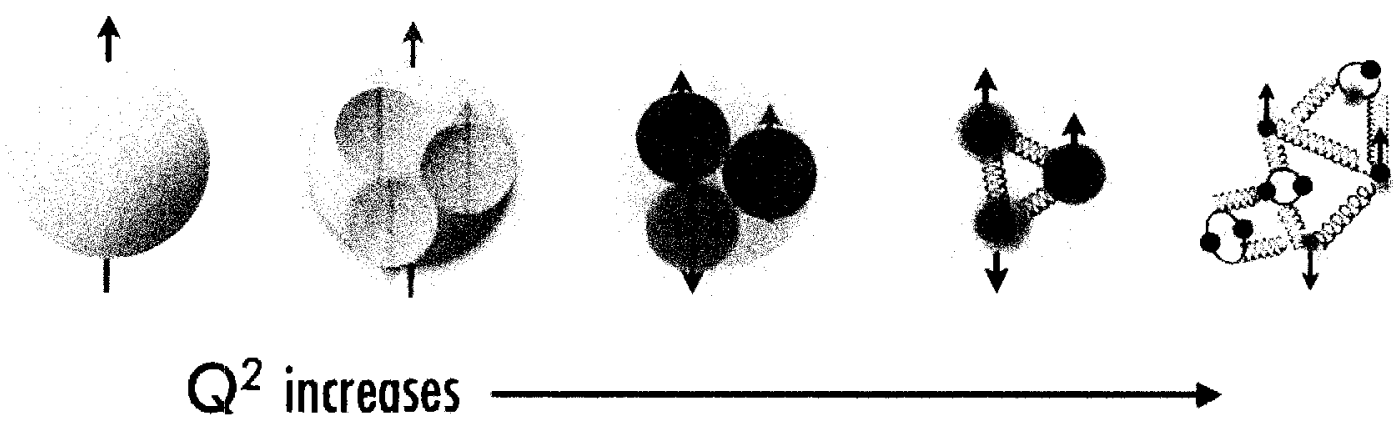

Figure 1.1: A qualitative picture of the proton model, starting at $Q^{2} \rightarrow 0$ on the left, $Q^{2}$ increasing with each diagram. The text explains in greater detail the evolution of the proton as a simple object (left) into a complex assembly of quarks, antiquarks, and gluons (right).

$\hbar / 2$, with a resultant magnetic moment

$$
\mu_{p}=\frac{2.79 e \hbar}{2 M}
$$

in the direction of the proton spin. However, quantum mechanics predicts, for a point-like particle, a value of $\mu_{p}=e / M$, thus providing the first hint that there must be some kind of structure to the proton.

At shorter distances, on the order of 1.0 to $0.1 \mathrm{fm},(0.2 \mathrm{GeV} \lesssim \nu \lesssim 2.0 \mathrm{GeV})$, one begins to resolve the structure of the constituents, or partons, within the proton, into 3 constituent quarks with fractional charge. Table 1.1 lists the 6 known flavors of quarks, and their basic properties. Specifically, two up quarks (charge $+\frac{2}{3} e$ ) and one down quark (charge $-\frac{1}{3} e$ ) make up a proton. The quarks are bound together by the strong interaction, which is governed by the theory of quantum chromodynamics (QCD). The discovery of parton structure by early deep inelastic scattering (DIS) experiments at the Stanford Linear Accelerator in the 1960's was a vindication of the Eightfold Way previously proposed by Murray Gell-Mann, which described the observed diversity of hadrons, including nucleons, as constructions of pairs or trios of up, down and strange quarks bound by the strong force [7]. Free quarks have never been observed due to the phenomenon of confinement, which, via the high binding energy of the strong force, restricts quarks to $q q q$ trios (known as baryons) or $q \bar{q}$ quark-antiquark pairs (known as mesons). 
At distances less than $0.1 \mathrm{fm}$ (or momentum transfer greater than $2.0 \mathrm{GeV} / c$ ), the simple picture of the proton, built of 3 constituent quarks, begins to break down. At these distances, QCD predicts (non-intuitively) that the force between quarks. Although incredibly strong at long distances, this binding force is small at short distance scales, allowing quarks significant freedom of motion within the confines of the nucleon. Thus, quarks can be observed as effectively free particles as $Q^{2} \rightarrow \infty$. This phenomenon is known as asymptotic freedom. At the shortest distance scales, not only can the incident electron scatter from one of the 3 point-like valence quarks, there is a possibility that scattering from one of the sea quarks in the proton medium can occur. Sea quarks are quark-antiquark pairs generated from hard (i.e. high-energy) gluons, carrier particles of the strong force between quarks; these quark-antiquark pairs can actively participate in a scattering event, as well. The gluons and sea quarks contribute to higher order perturbations in QCD theory at short distances. Quantification of the contributions of the gluons and sea quarks to the distributions of charge, linear momentum and angular momentum (spin) of the proton are main topics of medium and high energy physics research. The distribution of the spin, in particular, is the subject of the polarized scattering analysis presented in this thesis.

Table 1.1: The six known flavors of quarks and their basic properties. The valence mass is the actual mass of the bare quark. The constituent mass includes all the glwons and $q \bar{q}$ pairs that surround the bare quark, and constitute (approximate) effective mass in a hadron/meson. From Ref. [5] and [7].

\begin{tabular}{|c|c|c|c|c|}
\hline Flavor & Symbol & Charge & Valence Mass $\left(\mathrm{MeV} / c^{2}\right)$ & Constituent Mass $\left(\mathrm{MeV} / c^{2}\right)$ \\
\hline \hline down & $d$ & $-\frac{1}{3} e$ & $4 .-8$. & $363 / 310$ \\
\hline up & $u$ & $+\frac{2}{3} e$ & $1.5-4.0$ & $363 / 310$ \\
\hline strange & $s$ & $-\frac{1}{3} e$ & $80-130$ & $538 / 483$ \\
\hline charm & $c$ & $+\frac{2}{3} e$ & $1150-1350$ & $\sim 1500$ \\
\hline bottom & $b$ & $-\frac{1}{3} e$ & $4100-4400$ & $\sim 4700$ \\
\hline top & $t$ & $+\frac{2}{3} e$ & $169100-172700$ & not found as constituents \\
\hline
\end{tabular}

\subsubsection{Kinematic Definitions}

The formalism for describing electron-proton scattering uses several conventional variables that are common to the relevant literature [4]. Figure 1.2 shows the basic process of $e p$ scattering. 


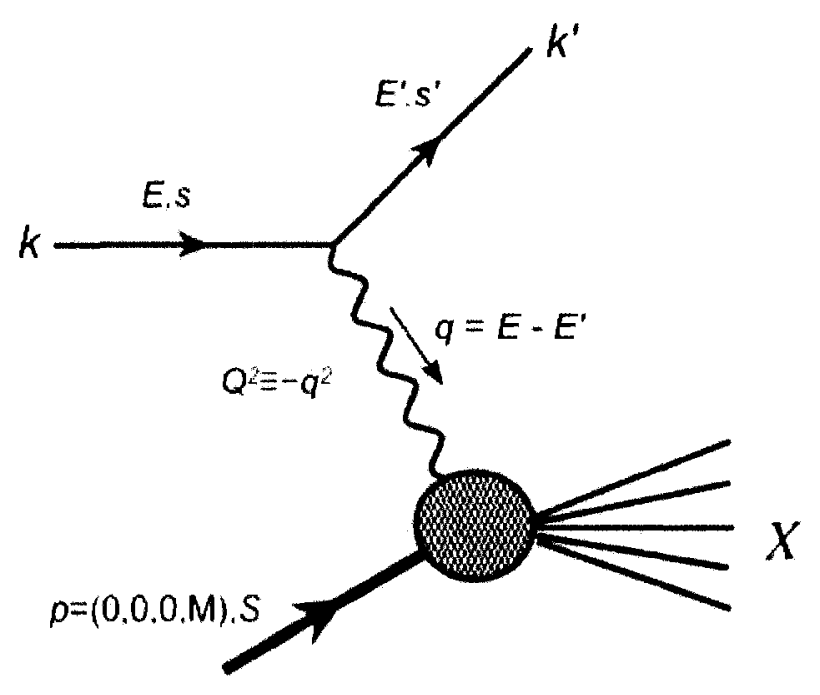

Figure 1.2: A diagram of inclusive electron-proton $(e p)$ scattering, as studied in this thesis. An incoming electron with momentum $E$ and spin $s$ exchanges a virtual photon of momentum $q$ with a stationary proton of mass $M$ and spin $S$, causing the scattered electron to recoil with momentum $E^{\prime}$ and spin $s^{\prime}$, with one or more reaction products released $(X)$. Measurements of inclusive scattering events only concern themselves with precision measurements of the scattered electron.

This process is described, in the lab reference frame, as an incident electron with energy (and momentum) $E, 4$-momentum $k$ and spin $s$ interacting by way of a virtual photon with energy $\nu$ and 4-momentum $q$, with a stationary proton with 4-momentum $p=(M, 0,0,0)$ and $\operatorname{spin} S$, where $M=0.938 \mathrm{GeV} / c^{2}$ is the proton mass. The incident electron recoils with energy (and momentum) $E^{\prime}, 4$-momentum $k^{\prime}$ and spin $s^{\prime}$. The virtual photon is an internal particle to the Feynman diagram, and as such, it need not be massless, nor, consequently, transversely polarized, as is a real photon [7]. In fact, later, we shall distinguish between the response functions corresponding to transverse and longitudinal virtual photon modes.

For convenient reference, here follows a list of commonly used variable definitions (that recur frequently in this thesis), with relevant descriptions to follow. Defining the beam axis along the $\hat{z}$-direction, and thus labeling the polar scattering angle $\theta$, one obtains

$$
Q^{2}=-q^{2}=4 E E^{\prime} \sin ^{2} \frac{\theta}{2}=2 E E^{\prime}(1-\cos \theta)
$$




$$
\begin{aligned}
& \nu=E-E^{\prime}=\frac{p \cdot q}{M} \\
& W=\sqrt{(p+q)^{2}}=\sqrt{M^{2}+2 M \nu-Q^{2}} \\
& s=(k+p)^{2}=2 M E+M^{2} \\
& x=\frac{Q^{2}}{2 p \cdot q}=\frac{Q^{2}}{2 M \nu} \\
& y=\frac{p \cdot\left(k-k^{\prime}\right)}{p \cdot k}=\frac{E-E^{\prime}}{E}=\frac{\nu}{E} \\
& \omega=\frac{2 p \cdot q}{Q^{2}}=\frac{1}{x} \\
& \gamma=\frac{2 M x}{\sqrt{Q^{2}}}=\frac{\sqrt{Q^{2}}}{\nu} \\
& \tau=\frac{\nu^{2}}{Q^{2}}=\frac{1}{\gamma^{2}} \\
& \epsilon=\left(1+2[1+\tau] \tan ^{2} \frac{\theta}{2}\right)^{-1} \\
& \eta=\frac{\epsilon \sqrt{Q^{2}}}{E-E^{\prime} \epsilon} \\
& \zeta=\frac{\eta(1+\epsilon)}{2 \epsilon} \\
& D=\frac{1-E^{\prime} \epsilon / E}{1+\epsilon R} \\
& d=D \sqrt{\frac{2 \epsilon}{1+\epsilon}} \\
& d^{\prime}=\frac{(1-\epsilon)(2-y)}{y(1+\epsilon R)}
\end{aligned}
$$

where $R$ is a ratio of unpolarized structure functions of $\nu$ and $Q^{2}$, defined later in section 1.2.2.

Equations 1.7 through 1.13 can be derived directly from Lorentz invariant 4-vector products defined in Figure 1.2; 1.14 through 1.18 are conventional kinematic definitions derived from the basic Lorentz invariants; and 1.19 through 1.21 are depolarization factors - described in more detail in Section 1.4.2. 
The first few of these quantities warrant discussion about their specific interpretations. The quantity $Q^{2}$ is the positive definite square of the virtual photon 4-momentum; it is fully analogous to $\kappa^{2}$ in the optical example in the Introduction. $Q^{2} \rightarrow 0$ indicates the limit of scattering by a real photon, while $Q^{2} \rightarrow \infty$ indicates the approach toward an infinitesimal distance resolution. The virtual photon energy $\nu$ is equal to the electron energy absorbed by the proton.

The variable $W$ is the Lorentz-invariant missing mass of the struck proton. In elastic $e p$ scattering, $W=M=0.938 \mathrm{GeV}$, but for the inelastic reaction $e p \rightarrow e p \pi^{+} \pi^{-}$, we find $W \geq M+2 m_{\pi} \geq 1.218$ GeV. ${ }^{3}$ The value of $W=M+m_{\pi}=1.077 \mathrm{GeV}$ is known as the inelastic threshold, below which there is not enough energy in the collision to create any new particles while conserving baryon and lepton number. Interactions where $W>2.0 \mathrm{GeV}$ (and $Q^{2}>1.0 \mathrm{GeV}^{2}$ ) are conventionally labeled as Deep Inelastic Scattering, or DIS events. The quantity $s$ is a Lorentz-invariant Mandelstam variable [3]; in this configuration it remains constant so long as the beam energy $E$ is constant.

The quantitities $x$ and $y$ are known as Bjorken Scaling Variables. ${ }^{4}$ Results for DIS are often expressed in terms of $Q^{2}$ and $x$; both $x$ and $y$ are commonly used in Perturbative QCD (pQCD) calculations. The interpretation of the meaning of $x$ is important; it requires definition of the Breit reference frame. Kinematically, $|\mathbf{q}|>\nu$, so that we can always find a reference frame for which $\nu=0$; we define this as the Breit frame [6]. In this reference frame, we have (for the proton momentum)

$$
P^{\mu}=(M|\mathbf{q}| / Q, 0,0,-M \nu / Q=0) \quad \text { (Breit frame) }
$$

In the Breit frame, the virtual photon carries no energy, so that the overall longitudinal ( $z$-direction) momentum of an (asymptotically free) struck quark in the nucleon must be of equal magnitude before and after the collision. That is, the quark 4-momentum is given by

$$
p_{f}^{q}(z)=p_{i}^{q}(z)+Q=-p_{i}^{q}(z) \quad \text { (Breit frame) }
$$

with the subscripts $f$ and $i$ representing final and initial momenta, respectively, and with $Q$ representing the magnitude of the momentum transfer. This relation requires that $p_{i}^{q}(z)=-Q / 2$;

\footnotetext{
${ }^{3}$ This reaction is associated with the well-known $\Delta^{+}$resonance.

${ }^{4} \xi$ is used in place of $x$ in many discussions. In this thesis, $\xi$ is reserved for a scaling replacement for $x$ after approximate target mass corrections (TMC), as explained at the end of this thesis, in Section 8.4.3.
} 
combining this with Eq. 1.11 yields

$$
x \equiv \frac{Q^{2}}{2 M \nu}=\frac{-Q p_{i}^{q}(z)}{M \nu}=\frac{p_{i}^{q}(z)}{-M \nu / Q}=\frac{p_{i}^{q}(z)}{p_{i}(z)} \quad \text { (Breit frame) }
$$

where $p_{i}(z)$ is defined by the $z$-component of the whole proton momentum, above. So, we see that $x$ is interpreted as the momentum ratio of the struck quark to that of the whole proton (in the Breit frame, where $\nu=0$ ). It is also useful to note (for finite $x$ ) that as $Q^{2} \rightarrow \infty, p_{i}(x) \rightarrow \infty$. For this special case (of scaling) the Breit reference frame is often referred to as the infinite momentum reference frame.

For electron-proton scattering, $x$ is constrained to

$$
0<x \leq 1
$$

with $x \rightarrow 0$ representing the short-distance (infinite $Q^{2}$ ) limit and $x=1$ representing a purely elastic scattering event. ${ }^{5}$

\subsubsection{A Motivation for Studying Proton Spin}

As explained in Section 1.1, electron scattering is used to investigate the electromagnetic structure of the proton. The qualitative picture of the proton shown in Figure 1.1 is the result of a culmination of decades of theoretical research and experimental data collection and analysis at worldwide accelerator facilities. The original "simple" proton model of 3 quarks bound together by the strong, or "color" force worked wonderfully well in predicting new excited baryon and meson particle states, as well as in modeling the observed magnetic moments of these particles [6]. Measurement of total quark momentum fractions demonstrated the need for a sophisticated model of the proton containing additional quark, antiquark and gluon "partons" within the internal nucleon structure, maintaining consistency with the quark model.

Given the success of the quark model in describing magnetic moments based on quark charges and spins [7], one might expect an experimental probing of the spin distributions to conform to the

\footnotetext{
${ }^{5}$ Scattering from larger nuclei can produce values of $x>1$. Specitically, in that case, $0<x \leq A$, where $A$ is the atomic mass. $x=A$ then represents quasi-elastic scattering from the nucleus.
} 
expectations of a constituent quark model. However, polarized beam and target experiments performed at SLAC and CERN beginning in the 1970's proved that this was far from the case. In fact, data from these experiments showed that only a very small portion of the proton spin was carried by its constituent quarks. This was known as the spin crisis, and is described more quantitatively later in Section 1.5. Experimental attempts to resolve the so-called spin crisis, along with unpolarized experimental data, have helped in constructing the picture of the proton we have today. However, as we shall see, the lack of precision meaurements of many of the essential quantities used to describe this picture still leaves many open questions.

The bulk of this chapter is dedicated to explaining how laboratory measurements have provided us with this insight into the structure of the proton, and providing definitions of the physical quantities necessary for extracting this information, namely the structure functions of the nucleon.

\subsection{Structure functions}

The measurement of structure functions, in terms of the kinematic quantities defined in Section 1.1.3, provides insight into the distribution and behavior of the constituent particles, or partons, within the proton. The scattering cross-sections of particles can be expressed in terms of these structure functions. In this section, the physical interpretation of both unpolarized structure functions $\left(F_{1}\right.$ and $\left.F_{2}\right)$ and polarized structure functions $\left(g_{1}\right.$ and $\left.g_{2}\right)$ are briefly explained, along with their physical context in electron scattering measurements.

\subsubsection{Electron scattering and structure functions}

To understand the significance of structure functions, one must become familiar with the terminology used to describe how measurable quantities in scattering relate to quantities that describe the physics of the proton. Namely, the significance of cross-sections must be expounded upon. Also, a description of the target response surface and the expression of the hadronic tensor in terms of the structure functions are detailed. 


\section{Cross-sections and internal structure measurements in scattering}

The cross-section of a particular scattering event, like that shown in Figure 1.2, is a directly measurable laboratory quantity. The overall cross-section $\sigma$ of a particular type of scattering event is defined in a straightforward way [8] as

$$
\sigma \equiv \frac{P(\delta x) / \delta x}{N}
$$

where $P(\delta x) / \delta x$ is the probability of a scattering event per unit distance, and $N$ is the number density of scattering centers. This gives the cross-section units of area, so that the cross-section can be loosely interpreted as the effective area of the response surface of the interaction.

In practice, in order to evaluate the model at various distance scales, as outlined in Section 1.1.2, one must know how the cross-section changes as a function of the angle and momentum of the scattered electron. Thus, scattering experiments typically measure the scattering cross-section in bins that cover small subdivisions of the total range of measured kinematic quantities $Q^{2}$ and $W$. This quantity, the differential cross-section

$$
\frac{d^{2} \sigma}{d Q^{2} d W}
$$

can then be integrated (which in this case, amounts to a simple summation over bins) to find the total cross-section $\sigma$. At a fixed beam energy $E$, the kinematics of inclusive scattering can be described completely by only two degrees of freedom, expressed by kinematic variables (Section 1.1.3). The (unpolarized) differential cross-section can thus be defined completely as a function of any 2 of these variables; e.g. $d \sigma / d Q^{2} d x, d \sigma / d \nu d x, d \sigma / d Q^{2} d W$, etc. Any one of these different differential cross-sections completely parametrizes the inclusive scattering behavior, as differing pairings of these kinematic variables simply correspond to different coordinate definitions of the same phase space. Differential cross-sections are commonly written in terms of the solid scattering angle $\Omega$ and recoil momentum $E^{\prime}$ as $d \sigma / d \Omega d E^{\prime}$. In the special case of inclusive scattering, the scattering event can be described completely in a two-dimensional plane in the $\phi$-direction (Figure 1.3), so 


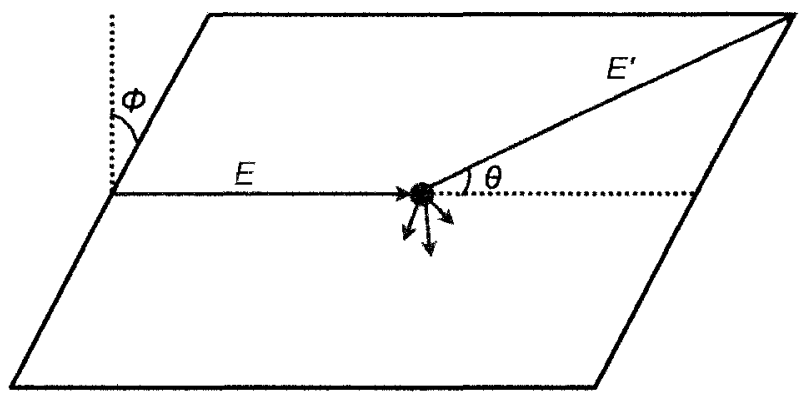

Figure 1.3: Unpolarized inclusive scattering viewed from the lab frame. The symbol $\phi$ refers to the angle of the scattering plane, and $\theta$ is the angle of the scattered electron with respect to the beamline axis. As long as the inelastic scattering products (red) are not tracked, the scattering event takes place in an azimuthal plane, so that $\phi$ need not be considered.

symmetry allows one to write

$$
\frac{1}{2 \pi} \int_{0}^{2 \pi} \frac{d^{3} \sigma}{d \Omega d E^{\prime}} d \phi=\frac{d^{2} \sigma}{d \theta d E^{\prime}}
$$

which also completely parametrizes the scattering event. Because, in reality, data exists in finite quantities, and hence, finite bin sizes are required. The measured cross-sections become

$$
\frac{d^{2} \sigma}{d \theta d E^{\prime}} \rightarrow \frac{\Delta \sigma}{\Delta \theta \Delta E^{\prime}} \quad ; \quad \frac{d^{2} \sigma}{d Q^{2} d W} \rightarrow \frac{\Delta \sigma}{\Delta Q^{2} \Delta W} \quad ; \quad \text { etc. }
$$

where $\Delta \theta, \Delta W$, etc. represent the finite bin size used to demarcate the data. Total cross-sections are then found by summation over the bins, rather than integration.

\section{Leptonic and hadronic cross-sections; their relation to the cross-section}

According to Fermi's Golden Rule [9] the partial cross-section can be written (to lowest order) [6] as

$$
\frac{\Delta \sigma}{\Delta \Phi}=\frac{1}{j} \frac{2 \pi}{\hbar}\left|\mathscr{M}_{f i}\right|^{2}
$$

where $j$ is the electron beam current density, $\Delta \Phi$ is the complete phase space (described by two kinematic variables as per the previous explanation) and $\mathscr{M}_{f i}=\left\langle\psi_{f}\left|\mathscr{K}_{\text {int }}\right| \psi_{i}\right\rangle$ is the transition amplitude between the initial and final wavefunctions of the electron-nucleon configuration. The 
amplitude of the interaction Hamiltonian $\mathscr{M}_{f i}$ contains all the physical dynamics of the interaction.

For the simple case of scattering of an electron from a point particle, the transition amplitude $\mathscr{M}_{f i}$ can be derived completely using the covariant Feynman Rules for quantum electrodynamics and techniques of Casimir et al. ${ }^{6}$ The (spin-averaged) result is given by ${ }^{7}$

$$
\left\langle|\mathscr{M}|^{2}\right\rangle=\frac{(4 \pi \alpha)^{2}}{q^{4}} L_{e}^{\mu \nu} L_{p \mu \nu}
$$

where $\alpha=\frac{1}{137}$ is the fine coupling constant, $q$ is the virtual photon amplitude (see Figure 1.2) and $L_{e}^{\mu \nu}$ and $L_{p \mu \nu}$ are covariant tensors, given, in the approximation of a massless electron, ${ }^{8}$ by

$$
\begin{gathered}
L_{e}^{\mu \nu}=2\left(k^{\mu} k^{\prime \nu}+k^{\nu} k^{\prime \mu}-g^{\mu \nu}\left(k \cdot k^{\prime}\right)\right. \\
L_{p \mu \nu}=2\left(p_{\mu} p_{\nu}^{\prime}+p^{\nu} p^{\mu}+g^{\mu \nu}\left(M^{2}-p \cdot p^{\prime}\right)\right.
\end{gathered}
$$

Knowledge of the kinematics then allows for a complete solution of the transition amplitude. ${ }^{9}$ Substitution of the 4-vectors shown in Figure 1.2 into the above equations, and finally, into Eq. 1.30 , ultimately yields

$$
\frac{\Delta \sigma}{\Delta \Omega}=\frac{4 \alpha^{2} E^{\prime 2}}{Q^{4}} \frac{E^{\prime}}{E} \cos ^{2} \frac{\theta}{2}\left(1+\frac{2 \nu^{2}}{Q^{2}} \tan ^{2}(\theta / 2)\right) \text { elastic scattering; structureless particles }
$$

where the phase space $\Delta \Phi=\Delta \Omega \Delta E^{\prime}$ has been reduced to $\Delta \Omega$ due to the fact that, for elastic scattering from a point particle, $E^{\prime}$ is constrained by a $\delta$-function to a specifically defined function of $\theta$.

Early $e p$ scattering experiments (e.g. Ref. [10]) made it clear, however, that this result is not obeyed for the elastic scattering of electrons from protons, indicating that protons are not simple, point-like particles. To account for the hadronic structure of the proton, Eq. 1.31 must be rewritten

\footnotetext{
${ }^{6}$ See, for example Ref. [7] Section 8.3 or Ref. [1] Chapter 11.

${ }^{7}$ From this point on, "natural" $\hbar=c=1$ units are used in this thesis.

${ }^{8} m_{e}=0$ is a very good approximation for medium- and high- energy electron scattering, as the relativistic momentum is orders of magnitude greater than the rest mass. From this point onward, the very small electron mass is ignored.

${ }^{9}$ Readers unfamiliar with the covariant notation should consult any introductory field theory book, e.g. the preface to Ref. [11].
} 
as

$$
\left\langle|\mathscr{M}|^{2}\right\rangle=\frac{(4 \pi \alpha)^{2}}{q^{4}} L_{e}^{\mu \nu} W_{\mu \nu}
$$

where the new response surface tensor $W_{\mu \nu}$ replaces $L_{p \mu \nu}$. The tensor is constructed to accomodate any possible scattering response of the electron. Thus, terms representing every possible covariant 4-vector term are present in its definition [7]:

$$
W_{\mu \nu}=-W_{1} g_{\mu \nu}+W_{2} \frac{p_{\mu} p_{\nu}}{M^{2}}+W_{4} \frac{q_{\mu} q_{\nu}}{M^{2}}+W_{5}\left(p_{\mu} q_{\nu}+p_{\nu} q_{\mu}\right)+W_{6}\left(p_{\mu} q_{\nu}-p_{\nu} q_{\mu}\right)
$$

It can be easily shown [1] that current conservation implies

$$
q_{\mu} W_{\mu \nu}=W_{\mu \nu} q_{\nu}=0
$$

Applying this to the definition of $W_{\mu \nu}$, it can also be shown [1][7] that $W_{6}=0^{10}$ and that $W_{4}$ and $W_{5}$ can be expressed in terms of $W_{1}$ and $W_{2}$ :

$$
W_{4}=\frac{M}{q^{2}} W_{1}+\left(\frac{q \cdot p}{q^{2}}\right)^{2} W_{2} \quad ; \quad W_{5}=-\frac{q \cdot p}{q^{2}} W_{2}
$$

This yields

$$
W_{\mu \nu}=W_{1}\left(-g_{\mu \nu}+\frac{q_{\mu} q_{\nu}}{q^{2}}\right)+\frac{W_{2}}{M^{2}}\left(p_{\mu}+\left(\frac{q \cdot p}{q^{2}}\right) q_{\mu}\right)\left(p_{\nu}+\left(\frac{q \cdot p}{q^{2}}\right) q_{\nu}\right)
$$

Substitution into 1.31 and using the 4-vectors $p_{\mu}$ and $q_{\mu}$ in the lab frame eventually yields [3][7]

$$
\left\langle\left|\mathscr{M}_{f i}\right|^{2}\right\rangle=\frac{(4 \pi \alpha)^{2}}{4 E E^{\prime} \sin ^{4}(\theta / 2)}\left(2 W_{1} \sin ^{2} \frac{\theta}{2}+W_{2} \cos ^{2} \frac{\theta}{2}\right)
$$

Using Fermi's Golden Rule and $\Delta \Phi=E^{2} \Delta E^{\prime} \Delta \Omega$ results in

$$
\frac{\Delta \sigma}{\Delta E^{\prime} \Delta \Omega}=\frac{\alpha^{2}}{4 E^{2} \sin ^{4}(\theta / 2)}\left(2 W_{1} \sin ^{2} \frac{\theta}{2}+W_{2} \cos ^{2} \frac{\theta}{2}\right)
$$

\footnotetext{
${ }^{10}$ This can be seen by noting that $L^{\mu \nu}$ is symmetric, so that the antisymmetric $W_{6}$ term makes no contribution.
} 
It is important to note that $W_{1}$ and $W_{2}$ are kinematic functions. Changing the phase space coordinates using

$$
\Delta Q^{2} \Delta \nu=\frac{E E^{\prime}}{\pi} \Delta \Omega \Delta E^{\prime}
$$

and $Q^{2}=4 E E^{\prime} \sin ^{2}(\theta / 2)$ gives [6]

$$
\frac{\Delta \sigma}{\Delta Q^{2} \Delta \nu}=\frac{4 \pi \alpha^{2} E^{\prime} \cos ^{2}(\theta / 2)}{Q^{4} E}\left(W_{2}\left(Q^{2}, \nu\right)+2 \tan ^{2} \frac{\theta}{2} W_{1}\left(Q^{2}, \nu\right)\right) \text { generalized ep scattering }
$$

This is a central result for electron-proton scattering experiments. It shows that measurement of the inclusive cross-section in terms of the momentum and electron scattering angle provides us information about $W_{1}$ and $W_{2}$, which contain all the physics of the scattering. Two explicitly macroscopic measureable degrees of freedom, $E^{\prime}$ and $\theta$, describe $W_{1}$ and $W_{2}$, which yield microscopic details about the electromagnetic interaction between the electron and proton. As outlined in the first section of this chapter, as electron beam energy is increased, the behavior over shorter distance scales inside the proton is revealed. The quantities $W_{1}$ and $W_{2}$ are referred to as structure functions of the proton. We now concentrate on breaking down $W_{1}$ and $W_{2}$ into different terms, that is, different structure functions with specific physical interpretations.

\subsubsection{Unpolarized structure functions $\left(F_{1}, F_{2}, R\right)$}

We proceed now to the introduction of the unpolarized structure functions $F_{1}$ and $F_{2}$. Measurement of these quantities is not the purpose of this thesis. However, their understanding is an essential prerequisite to the more complex topic of polarized structure functions, introduced in the next section. Also, it will be shown later that knowledge of the unpolarized structure functions is necessary for extrication of the polarized structure functions $g_{1}$ and $g_{2}$.

\section{Form factors and structure functions}

Consider for a moment the specific case of elastic scattering of the electron from the proton, in which kinetic energy is conserved, and hence, no new particles are produced. The constraint of kinetic energy conservation on the initial and final momentum 4-vectors reduces $E^{\prime}$ to a uniquely 
determined function of the scattering angle [7]:

$$
E^{\prime}=\frac{E}{1+(2 E / M) \sin ^{2}(\theta / 2)}=E-\frac{Q^{2}}{2 M} \text { (elastic scattering) }
$$

A similar calculation to that of the previous subsection, with the added constraint of kinetic energy conservation $^{11}$, gives the analog to Equation 1.41 for elastic $e p$ scattering:

$$
\frac{\Delta \sigma}{\Delta \Omega}=\frac{\alpha^{2}}{4 E^{2} \sin ^{4}(\theta / 2)} \frac{E^{\prime}}{E}\left(2 W_{1(e l)} \sin ^{2} \frac{\theta}{2}+W_{2(e l)} \cos ^{2} \frac{\theta}{2}\right) \quad \text { (elastic scattering) }
$$

Here, the uniquely determined value of $E^{\prime}$ has been integrated, so that only $\Delta \Omega$ (and thus, only $\Delta \theta$ after a simple $\phi$-integration) is needed to completely parametrize the cross-section. The $W$ structure functions are now functions of one kinematic variable only. Because $E^{\prime}$ is no longer an independent parameter, and $\nu=E-E^{\prime}$,

$$
W_{1,2}\left(Q^{2}, \nu\right) \rightarrow W_{1,2(e l)}\left(Q^{2}\right) \quad \text { (elastic scattering) }
$$

If we define $G_{E}$ and $G_{M}$ such that

$$
G_{M}^{2}\left(Q^{2}\right) \equiv \frac{W_{1(e l)}}{\tau}
$$

and

$$
G_{E}^{2}\left(Q^{2}\right) \equiv(1+\tau) W_{2(e l)}-W_{1(e l)}
$$

where $\tau \equiv \nu^{2} / Q^{2}$, we can write the well-known Rosenbluth Formula for elastic scattering:

$$
\frac{\Delta \sigma}{\Delta \Omega}=\frac{\alpha^{2}}{4 E^{2} \sin ^{4}(\theta / 2)} \frac{E^{\prime}}{E}\left(2 \tau G_{M}^{2}\left(Q^{2}\right) \sin ^{2} \frac{\theta}{2}+\frac{G_{E}^{2}\left(Q^{2}\right)+\tau G_{M}^{2}\left(Q^{2}\right)}{1+\tau} \cos ^{2} \frac{\theta}{2}\right)
$$

$G_{M}$ and $G_{E}$ are known as the Sachs magnetic and electric form factors; in the following we show how these quantities may be interpreted as such.

The scattering matrix $\mathscr{M}$, in terms of the interaction propagator and electromagnetic current

\footnotetext{
${ }^{11}$ The quantity $\frac{q \cdot p}{q^{2}}=-\frac{1}{2}$ in this section, for the special case of elastic scattering.
} 
operators, is written as

$$
\left|\mathscr{M}_{f i}\right|^{2}=\left|\left\langle\psi_{f}\left|j_{\mu}\left(-\frac{1}{q^{2}}\right) J^{\mu}\right| \psi_{i}\right\rangle\right|^{2}
$$

where $j_{\mu}$ and $J^{\mu}$ are current operators for the electron and proton, respectively. The electron is a structureless particle, so we can write the matrix operator for the (lowest order) term $j_{\mu}$ according to standard QED formalism [3] as

$$
\left\langle\psi_{f}\left|j^{\mu}\right| \psi_{i}\right\rangle=-e \bar{u}\left(k^{\prime}\right) \gamma^{\mu} u(k)
$$

However, we do need to account for the unknown structure of the proton, so $J_{\mu}$ must be expressed as the most generalized possible (parity-conserving) 4-vector containing independent terms and Dirac $\gamma$-matrices:

$$
\left\langle\psi_{f}\left|J^{\mu}\right| \psi_{i}\right\rangle=\bar{u}\left(p^{\prime}\right)\left(\gamma^{\mu} \mathscr{F}_{1}\left(Q^{2}\right)+i \sigma^{\mu \nu} \frac{q_{\nu}}{2 M} \mathscr{F}_{2}\left(Q^{2}\right)\right) u(p)
$$

where $\mathscr{F}_{1}\left(Q^{2}\right)$ and $\mathscr{F}_{2}\left(Q^{2}\right)$ are unknown parameters, known as the Dirac and Pauli form factors, respectively. Solving for the cross-section again, inserting these current definitions into $\mathscr{M}_{f i}$, one finds ${ }^{12}$

$$
G_{E}=\mathscr{F}_{1}-\frac{Q^{2}}{4 M^{2}} \mathscr{F}_{2}
$$

and

$$
G_{M}=\mathscr{F}_{1}+\mathscr{F}_{2}
$$

We now shift to the Breit reference frame (introduced in Section 1.1.3), the reference frame in which $\nu=0$, and hence $E=E^{\prime}$. For elastic scattering, the Breit frame is the same as the $e p$

\footnotetext{
${ }^{12}$ Most texts use $F_{1}$ and $F_{2}$ to denote the Dirac and Pauli form factors in this and related equations. These are not at all the same quantities as the $F_{1}$ and $F_{2}$ structure functions. To avoid this (unfortunate) notation, $\mathscr{F}_{1}$ and $\mathscr{F}_{2}$ are used to symbolize the Dirac and Pauli form factors in this thesis. Also, some works (e.g. Ret. [3]) write the anomalous magnetic moment $\kappa$ explicitly in these equations, while others (e.g. Ref. [12]) absorb it into the form factor definitions; the latter approach is used here.
} 
center-of-mass reference frame. Evaluating Eq. 1.52 in this reference frame results in

$$
\left\langle\psi_{f}\left(-Q^{2} / 2\right)\left|J(0)^{\mu}\right| \psi_{i}\left(Q^{2} / 2\right)\right\rangle=e \bar{u}\left(p^{\prime}\right)\left(\gamma^{\mu}\left(\mathscr{F}_{1}+\mathscr{F}_{2}\right)-\frac{\left(p^{\mu}+p^{\mu}\right)}{2 M} \mathscr{F}_{2}\right) u(p)
$$

The notation $J(0)^{\mu}$ notes the current operator in the Breit frame. If the Dirac spinors are written explicitly in terms of the helicity spinors $\chi_{s}$ and $\chi_{s}^{\prime}$ and evaluated in this reference frame, one can evaluate the four components of $J^{\mu}$ as [12]

$$
\left\langle\psi_{f}\left(-Q^{2} / 2\right)\left|J^{0}(0)\right| \psi_{i}\left(Q^{2} / 2\right)\right\rangle=2 M e G_{E}\left(Q^{2}\right) \delta_{s^{\prime} s}
$$

and

$$
\left\langle\psi_{f}\left(-Q^{2} / 2\right)|\vec{J}(0)| \psi_{i}\left(Q^{2} / 2\right)\right\rangle=G_{M}\left(Q^{2}\right) \chi_{s^{\prime}}^{\dagger}(i e \vec{\sigma} \times \vec{q}) \chi_{s}
$$

This can all be simplified a bit if we define a $z$-axis along the direction of the $e p$ collision, in which case the matrix elements are diagonal. If we note that $J^{\mu}=\left(\rho, J_{x}, J_{y}, J_{z}\right)$, we can then write the nonzero matrix elements as [3]

$$
\rho=2 M e G_{E}\left(Q^{2}\right)\left(s^{\prime}=s\right)
$$

and

$$
J_{x} \pm i J_{y}=\mp 2|\vec{q}| e G_{M}\left(Q^{2}\right) \quad\left(s=-s^{\prime}=\mp \frac{1}{2}\right)
$$

Now, we have the charge $J^{0}=\rho$ as a function of $Q^{2}$, as well as $J_{x} \pm i J_{y}$, which can be interpreted as a current loop about the $z$-axis; that is, the magnetization $\mu$ of the proton as defined along our chosen axis. In this frame, then, simple 3-dimensional Fourier transforms

$$
\begin{aligned}
& \rho(\vec{r})=\int \frac{d^{3} q}{(2 \pi)^{3}} e^{-i \vec{q} \cdot \vec{r}} M G_{E}\left(Q^{2}\right) \\
& \mu_{z}(\vec{r})=\int \frac{d^{3} q}{(2 \pi)^{3}} e^{-i \vec{q} \cdot \vec{r}}|\vec{q}| G_{M}\left(Q^{2}\right)
\end{aligned}
$$

can be made to obtain the charge or magnetization density in the Breit frame.

The main point of importance to be noted here is that $G_{E}$ and $G_{M}$ can be interpreted as electric 
and magnetic form factors, respectively. In the limit $Q^{2} \ll M^{2}$, then, the charge distribution and magnetization density of the proton can then be respectively determined by the Fourier transforms of $G_{E}$ and $G_{M}$. Very precise parametrizations of $G_{E}$ and $G_{M}$ from elastic scattering experiments have been published [13][14]. These parametrizations are later used in this thesis for calculation of the beam times target polarization product (see Section 6.2).

\section{Extension of elastic formalism to inelastic scattering}

The main subject of this thesis is the study of inelastic structure functions. Why then, are we concerned with the analogous terms to $W_{1}$ and $W_{2}$ in the elastic region, as detailed in the last section? The answer becomes immediately apparent upon extension of these elastic results into the inelastic region. Looking at Equations 1.47 and 1.48, we see

$$
W_{1(e l)} \equiv \tau G_{M}^{2}\left(Q^{2}\right)
$$

and

$$
W_{2(e l)} \equiv \frac{G_{E}^{2}\left(Q^{2}\right)+\tau G_{M}^{2}\left(Q^{2}\right)}{1+\tau}
$$

If we extend the concept of $W_{1}$ and $W_{2}$ to inelastic scattering events, and we are still to believe the interpretation of the Sachs form factors in Equations 1.58 and 1.59 past this threshold, then it is clear that $W_{1}\left(Q^{2}, \nu\right)$ parametrizes the transverse part of transition matrix element $\left|\mathscr{M}_{f i}\right|^{2}$ (as represented by the magnetic dipole field in the Breit frame), but that $W_{2}\left(Q^{2}, \nu\right)$ contains both transverse and longitudinal parts of the transition matrix [6].

To separate the longitudinal part of the matrix for inelastic events, it is conventional to define $W_{L}$ as

$$
W_{L}\left(Q^{2}, \nu\right) \equiv(1+\tau) W_{2}\left(Q^{2}, \nu\right)-W_{1}\left(Q^{2}, \nu\right)
$$

(note the similarity to $\mathrm{Eq} .1 .48$ ). We can now define the ratio of unpolarized structure functions, labeled $R$ by convention:

$$
R\left(Q^{2}, \nu\right) \equiv \frac{\sigma_{L}}{\sigma_{T}}=\frac{W_{L}\left(Q^{2}, \nu\right)}{W_{1}\left(Q^{2}, \nu\right)}
$$


so that, for elastic events,

$$
R_{(e l)}=\frac{G_{E}^{2}}{\tau G_{M}^{2}}
$$

In other words, $R$ is defined as the ratio of the transverse to longitudinal transition strength [6] in the $e p$ reaction.

\section{Structure Functions and Deep Inelastic Scattering}

We now switch to a different perspective - scattering in the deep inelastic region ( $W \gtrsim 2.0 \mathrm{GeV}$ ). As explained in Section 1.1.3, if the virtual photon transfers enough energy to the proton, it will scatter elastically from the individual quarks, but only if their momentum fraction (calculated in the Breit Frame) is given by $x=Q^{2} /(2 M \nu)$. In Section 1.1.2, we considered the possibility that both valence quarks and sea quarks/antiquarks of many different flavors could "exist" inside the proton. If we then define $f(x)$ to be the probability of quark of flavor $f$ having a value between $x$ and $x+\Delta x$, then (assuming quarks have no structure of their own), that means one can simply rewrite the elastic cross-section, multiplied by $\sum_{f} f(x) \Delta x$, to express the DIS cross-section!

To do this, we use Eq. 1.34 , with a couple modifications. First, we drop the recoil factor $\frac{E^{\prime}}{E}$, and assume the target does not recoil. ${ }^{13}$ We also must consider that the coupling strength $\alpha$ is proportional to the the product of the charges of the interacting particles, so

$$
\alpha \rightarrow z_{f} \alpha
$$

for each term in the sum, where $z_{f}$ is the charge associated with the quark flavor. This yields

$$
\frac{\Delta \sigma}{\Delta \Omega}=\frac{4 \alpha^{2} E^{2} \cos \theta / 2}{Q^{4}}\left(\sum_{f} z_{f}^{2} f(x) \Delta x+\frac{2 \nu^{2}}{Q^{2}} \tan ^{2} \frac{\theta}{2} \sum_{f} z_{f}^{2} f(x) \Delta x\right)
$$

\footnotetext{
${ }^{13}$ In DIS experiments, the recoil factor in the cross-section is a very complicated quantity to calculate, due to the fact that, in this regime, some of the incident energy of the electron goes into creating different angular momentum and flavor states, while some goes into the target recoil. For this reason, DIS cross-sections are generally calculated assuming no recoil in the nucleon, and a complicated TMG (target mass correction) is factored in at the end. It is common convention in measurement of DIS quantities not to include target mass corrections until they are needed. Except for in the discussion of quark-hadron duality in Section 8.4.3, the TMC is deferred in this analysis.
} 
It is conventional to write DIS cross-sections in terms of $\Delta Q^{2}$ instead of $\Delta \Omega=\Delta \phi \Delta(\cos \theta)$. This requires integrating over $\Delta \phi$ (i.e. factor of $2 \pi$ ) and using Eq. 1.7 to show $\Delta Q^{2}=2 E E^{\prime} \sin \theta \Delta \theta$, and hence

$$
\Delta(\cos \theta)=-\sin \theta \Delta \theta=\frac{-\Delta Q^{2}}{2 E E^{\prime}}
$$

Using Eq. 1.11 to show

$$
\Delta x=\frac{-Q^{2}}{2 M \nu^{2}} \Delta \nu=\frac{-x}{\nu} \Delta \nu
$$

we can rewrite Eq. 1.68 as [6]

$$
\frac{\Delta \sigma}{\Delta Q^{2} \Delta \nu}=\frac{4 \pi \alpha^{2} E^{\prime} \cos ^{2}(\theta / 2)}{Q^{4} E}\left(\frac{x}{\nu} \sum_{f} z_{f}^{2} f(x)+\frac{1}{M} \tan ^{2} \frac{\theta}{2} \sum_{f} z_{f}^{2} f(x)\right)
$$

Finally, we are in a position to define the unpolarized structure functions $F_{1}$ and $F_{2}$ in the deep inelastic region [12]:

$$
\begin{aligned}
& F_{1}\left(x, Q^{2}\right) \equiv M W_{1}\left(\nu, Q^{2}\right) \\
& F_{2}\left(x, Q^{2}\right) \equiv \nu W_{2}\left(\nu, Q^{2}\right)
\end{aligned}
$$

It is also sometimes useful to use Eq. 1.64 to define

$$
F_{L}\left(x, Q^{2}\right) \equiv 2 M W_{L}=2 M\left[(1+\tau) W_{2}\left(Q^{2}, \nu\right)-W_{1}\left(Q^{2}, \nu\right)\right]
$$

Substituting $F_{1}$ and $F_{2}$ into Eq. 1.43 gives

$$
\frac{\Delta \sigma}{\Delta Q^{2} \Delta \nu}=\frac{4 \pi \alpha^{2} E^{\prime} \cos ^{2}(\theta / 2)}{Q^{4} E}\left(\frac{1}{\nu} F_{2}\left(x, Q^{2}\right)+\frac{1}{M} \tan ^{2} \frac{\theta}{2} F_{1}\left(x, Q^{2}\right)\right)
$$

With this definition of the $F_{1}$ and $F_{2}$ structure functions, this means, that if the quark theory holds true, then in the DIS region

$$
\begin{aligned}
& F_{1}(x)=\frac{1}{2} \sum_{f} z_{f}^{2} f(x) \quad \text { (DIS only) } \\
& F_{2}(x)=x \sum_{f} z_{f}^{2} f(x) \quad \text { (DIS only) }
\end{aligned}
$$


That is, the structure functions exhibit scaling behavior and become dependent only on $x$ at high values of $Q^{2}$. That is, in the DIS region, we can interpret $F_{1}(x)$ as representing $\frac{1}{2}$ the probability, weighted by the square of its charge, of a quark having a momentum fraction $p_{q u a r k} / p_{\text {proton }}=x$ in the Breit reference frame. It is obvious, in the scaling region, that we expect

$$
F_{1}(x)=2 x F_{2}(x) \quad(\text { DIS only })
$$

which is known as the Callan-Gross Relationship.

Also, it is obvious from the above relations that $F_{1}, F_{2}$ and $R$ are all algebraically related. A quick derivation using Eqs. 1.64, 1.65, 1.72 and 1.73 yields

$$
R=\frac{F_{2}\left(1+\frac{1}{\tau}\right)}{2 x F_{1}}+1
$$

meaning that knowledge of any two of these three quantities completely parametrizes the third.

\subsubsection{Polarized structure functions $\left(g_{1}, g_{2}\right)$}

We saw in the previous sections how structure functions for unpolarized scattering cross-sections relate information about the interior of the nucleon to measureable quantities in the laboratory. Now, we extend the discussion to polarized electron-nucleon scattering cross-sections, which provide information on distribution of the angular momentum within the proton.

\section{Generalizing the leptonic and hadronic tensors}

The leptonic and hadronic tensors $L_{\mu \nu}$ and $W_{\mu \nu}$ can provide information about the momentum and charge composition of quarks in the case of unpolarized targets. In that case, complete symmetry is required upon exchange of the indices $\mu$ and $\nu$, because spins of individual electrons and protons cannot be isolated, and the scattering configuration is identical to its reflected image in all reference frames. However, if we introduce a polarization $s_{\mu}$ to the electron and/or $S_{\mu}$ to the proton, this is definitely not the case (see Figure 1.4). Experiments that aim to extract the related spin-dependent structure functions must utilize polarized electron beams and/or proton targets. 


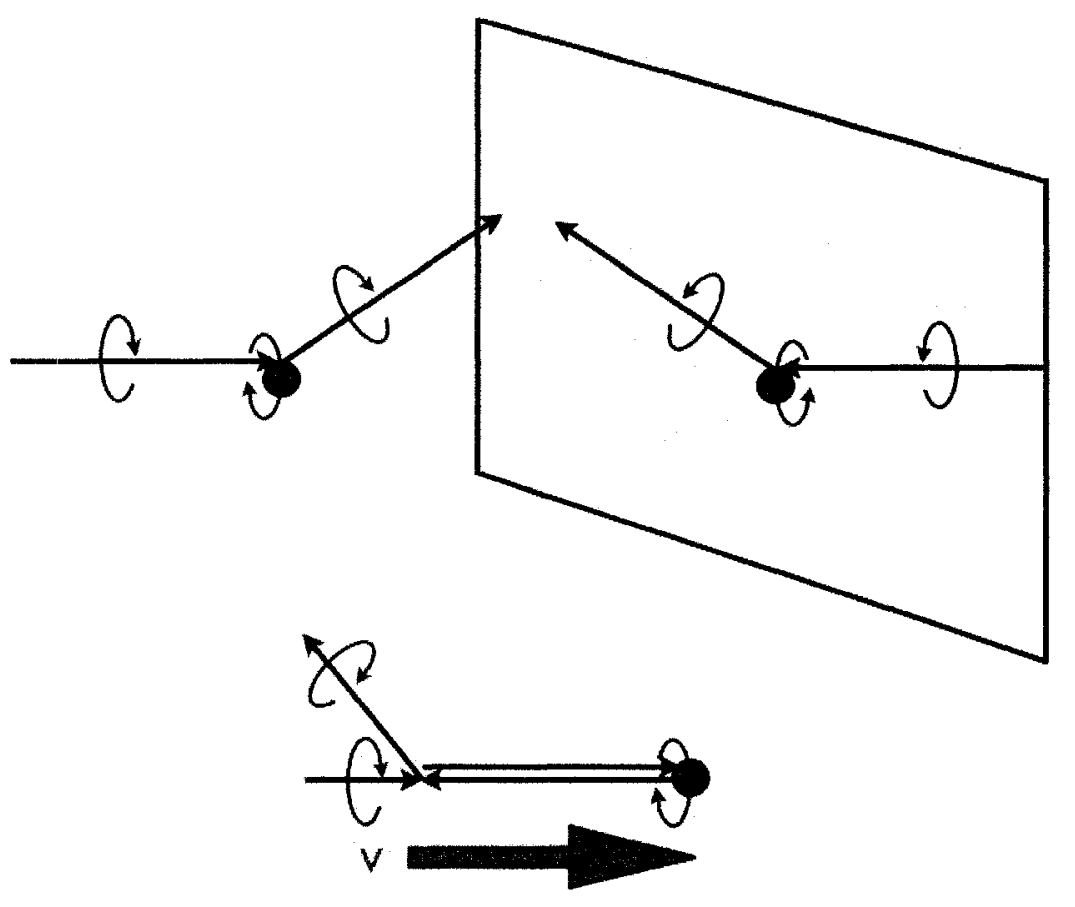

Figure 1.4: Diagram showing how the apparent polarizations of particles in $e p$ scattering change upon reflection of coordinates or change of reference frame. The top diagram shows a scattering event in the lab frame where an incident electron (black arrow) with a given spin impinges on a stationary particle, maintaining the same spin before and after the scattering event. A coordinate reflection reverses all the apparent helicities. The bottom figure shows the same event in a reference fram moving at velocity $v$. In this reference frame, the helicities of both the proton and the electron after the scattering event are opposite their direction in the lab frame. Lorentz invariance dictates that the particle interaction cannot be represented solely by matrix terms that are symmetric upon exchange of $x, y, z$ and $t$ coordinates. 
We now return to Eq. 1.35, and expand the scope of $L_{\mu \nu}$ and $W_{\mu \nu}$ to include the asymmetric space-time physics of polarized particles. Any tensor can be written as a sum of a symmetric and antisymmetric tensor, so that

$$
L^{\mu \nu}=L_{S}^{\mu \nu}+L_{A}^{\mu \nu}
$$

and

$$
W_{\mu \nu}=W_{\mu \nu}^{S}+W_{\mu \nu}^{A}
$$

The symmetric components were detailed in the previous discussion of unpolarized structure functions. The antisymmetric leptonic tensor for a structureless spin- $\frac{1}{2}$ particle is calculated as [12]

$$
L_{A \pm}^{\mu \nu}=\mp 2 i \epsilon_{\mu \nu \alpha \beta} k^{\alpha} k^{\prime \beta}
$$

where $\epsilon_{\mu \nu \alpha \beta}$ is the antisymmetric Levi-Civita tensor (defined as $\epsilon_{0123}=+1$ in this convention). Here, the \pm represents the sign of the helicity, which, for a "massless" particle (i.e. the electron), is the same as the polarization direction.

Once again, the most generalized possible tensor must be constructed to represent the hadronic contribution, with the unknown coefficients again representing the unknown response functions. First, the nuclear polarization vector $S_{\mu}$ must be introduced. We know from (nonrelativistic) quantum angular momentum theory for spin- $\frac{1}{2}$ particles [9] that

$$
\vec{S}=\chi_{S}^{\dagger} \vec{\sigma} \chi S
$$

where $\chi_{S}$ is the two-component spinor wavefunction of the proton and $\vec{\sigma}$ is the Pauli spin matrix. Relativistic generalization of this quantity to a 4-vector yields [12]

$$
S^{\mu}=\vec{u}(p) \gamma^{\mu} \gamma^{5} u(p) / 2 M
$$

where $u(p)$ is Dirac spinor representing the proton and $\gamma^{\mu} \gamma^{5}$ is the combination of Dirac matrices needed to generate an antisymmetric matrix. The most general antisymmetric matrix possible ${ }^{14}$

\footnotetext{
${ }^{14}$ Actually, it is the most general antisymmetric matrix that conserves parity. This requires the factor $\epsilon_{\mu \nu \alpha \beta}$ to cancel the parity-violating effects of the $\gamma^{5}$ matrix.
} 
that can be constructed from the vectors $p^{\mu}, q^{\mu}$ and $S^{\mu}$ (analogous to Eq. 1.39) is [12]

$$
W_{\mu \nu}^{A}=i \epsilon_{\mu \nu \alpha \beta} q^{\alpha}\left(G_{1}\left(\nu, Q^{2}\right) S^{\beta}+\frac{G_{2}\left(\nu, Q^{2}\right)}{M^{2}}\left(S^{\beta} p \cdot q-p^{\beta} S \cdot q\right)\right)
$$

Just as the symmetric term can be characterized completely by the two response functions $W_{1}$ and $W_{2}$, the antisymmetric (completely spin-dependent) term can be characterized completely by the two response functions $G_{1}$ and $G_{2}$.

In exact analogy to the case of unpolarized scattering (save a factor of $\nu$ ) we define

$$
\begin{gathered}
g_{1}\left(x, Q^{2}\right) \equiv M \nu G_{1}\left(\nu, Q^{2}\right) \\
g_{2}\left(x, Q^{2}\right) \equiv \nu^{2} G_{2}\left(\nu, Q^{2}\right)
\end{gathered}
$$

Substituting these into $W_{\mu \nu}^{A}$ gives

$$
W_{\mu \nu}^{A}=i M \frac{\epsilon_{\mu \nu \alpha \beta} q^{\alpha}}{\nu}\left[g_{1}\left(x, Q^{2}\right) S^{\beta}+g_{2}\left(x, Q^{2}\right)\left(S^{\beta}-M \frac{S \cdot q}{\nu} p^{\beta}\right)\right]
$$

Now, putting this all together, we have

$$
\left\langle\left|\mathscr{M}_{f i}\right|^{2}\right\rangle=\frac{(4 \pi \alpha)^{2}}{q^{4}}\left(L_{S}^{\mu \nu} W_{\mu \nu}^{S}+L_{A}^{\mu \nu} W_{\mu \nu}^{A}\right)
$$

where the two cross-terms $L_{A}^{\mu \nu} W_{\mu \nu}^{S}$ and $L_{S}^{\mu \nu} W_{\mu \nu}^{A}$ cancel due to the symmetry requirement of $\mathscr{M}_{f i}$.

Now, we must consider possible orientations of the electron and proton spin. The EG1 experiment utilizes a longitudinally polarized electron beam and target, so we limit our considerations to the possibilities of parallel and antiparallel orientation of the electron and proton spins.

Applying the appropriate sign to Eq. 1.82 and the appropriately polarized spinors into Eq. 1.84, one can use Fermi's Golden Rule and the $e p$ scattering 4-vectors of Figure 1.2 to evaluate the differential cross-sections in the lab frame for both the spin-parallel $(\uparrow \uparrow)$ and spin-antiparallel $(\uparrow \Downarrow)$ [12]:

$$
\frac{\Delta \sigma^{\Uparrow \Uparrow}}{\Delta \Omega \Delta E^{\prime}}=\frac{\Delta \sigma}{\Delta \Omega \Delta E^{\prime}}-\frac{2 \alpha^{2} E^{\prime}}{Q^{2} E}\left(\frac{E+E^{\prime} \cos \theta}{M \nu} g_{1}\left(x, Q^{2}\right)-\frac{1}{M \tau} g_{2}\left(x, Q^{2}\right)\right)
$$




$$
\frac{\Delta \sigma^{\dagger \Downarrow}}{\Delta \Omega \Delta E^{\prime}}=\frac{\Delta \sigma}{\Delta \Omega \Delta E^{\prime}}+\frac{2 \alpha^{2} E^{\prime}}{Q^{2} E}\left(\frac{E+E^{\prime} \cos \theta}{M \nu} g_{1}\left(x, Q^{2}\right)-\frac{1}{M \tau} g_{2}\left(x, Q^{2}\right)\right)
$$

We see the unpolarized cross-section of Eq. 1.41 is part of the total polarized cross-section. Hence, one can already see that knowledge of the unpolarized structure functions is necessary for extraction of the polarized structure functions.

It can also be seen that, by either adding/subtracting the above cross-sections, it is possible to isolate the unpolarized/polarized cross-section contributions:

$$
\begin{aligned}
& \left(\frac{\Delta \sigma}{\Delta \Omega \Delta E^{\prime}}\right)_{\text {unpolarized }}=\frac{1}{2}\left(\frac{\Delta \sigma^{\uparrow \Downarrow}}{\Delta \Omega \Delta E^{\prime}}+\frac{\Delta \sigma^{\uparrow \Uparrow}}{\Delta \Omega \Delta E^{\prime}}\right) \\
& \left(\frac{\Delta \sigma}{\Delta \Omega \Delta E^{\prime}}\right)_{\text {polarized }}=\frac{1}{2}\left(\frac{\Delta \sigma^{\dagger \Downarrow}}{\Delta \Omega \Delta E^{\prime}}-\frac{\Delta \sigma^{\uparrow \Uparrow}}{\Delta \Omega \Delta E^{\prime}}\right)
\end{aligned}
$$

This important result is paramount to the discussion of the relation between asymmetries and the spin structure functions.

\section{DIS interpretation of polarized structure functions}

Like $F_{1}$ and $F_{2}, g_{1}$ and $g_{2}$ also have an interpretation in the DIS region that implies scaling behavior, except that instead of parametrizing the linear momentum distribution of the quarks (as is the case for $F_{1}$ and $\left.F_{2}\right), g_{1}$ and $g_{2}$ parametrize the spin orientation distribution of the quarks.

It is easiest to see the scaling behavior of $g_{1}$ by introducing the double spin asymmetry

$$
A_{\|}\left(x, Q^{2}\right)=\frac{\Delta \sigma^{\dagger \Downarrow}-\Delta \sigma^{\dagger \Uparrow}}{\Delta \sigma^{\uparrow \Downarrow}+\Delta \sigma^{\uparrow \Uparrow}}=\frac{\Delta \sigma_{\text {polarized }}}{\Delta \sigma_{\text {unpolarized }}}
$$

From Eqs. 1.90 and 1.91 one can calculate

$$
\left(\frac{\Delta \sigma}{\Delta \Omega \Delta E^{\prime}}\right)_{\text {polarized }}=\frac{4 \alpha^{2} E^{\prime}}{Q^{2} M \nu}\left[\left(1+\frac{E^{\prime}}{E} \cos \theta\right) g_{1}\left(x, Q^{2}\right)-\frac{Q^{2}}{E \nu} g_{2}\left(x, Q^{2}\right)\right]
$$


Applying Eq. 1.42 to Eq. 1.75 yields

$$
\left(\frac{\Delta \sigma}{\Delta \Omega \Delta E^{\prime}}\right)_{\text {unpolarized }}=\frac{4 \alpha^{2} \cos ^{2}(\theta / 2)}{Q^{4} E^{2}}\left(\frac{1}{M} \tan ^{2} \frac{\theta}{2} F_{1}\left(x, Q^{2}\right)+\frac{1}{\nu} F_{2}\left(x, Q^{2}\right)\right)
$$

We can see from both of these equations, at large $\nu$, the contributions to the cross-sections from $F_{1}$ and $g_{1}$ are much larger than those from $F_{2}$ and $g_{2}$. ${ }^{15}$ Thus, we can see

$$
A_{\|}\left(x, Q^{2}\right) \propto \frac{g_{1}\left(x, Q^{2}\right)}{F_{1}\left(x, Q^{2}\right)}+\mathscr{O}\left(\frac{1}{\nu}\right)
$$

where $\mathscr{O}(1 / \nu)$ is a small term containing the contributions from $g_{2}$ and $F_{2,}$ which we ignore for now.

Earlier, a function $f(x)$ was defined as representing the probability of a quark with flavor $f$ containing the fraction $x$ of the total proton's momentum, when viewed in the Breit reference frame. It was seen that, at high enough $Q^{2}$, that the $F_{1}$ and $F_{2}$ structure functions become simple functions of $f(x)$, as summed over all quark flavors, assuming only that the quark model and asymptotic freedom hold true, an example of scaling behavior.

Now, consider the quark model, again assuming asymptotic freedom to be true. At high enough $Q^{2}$ (i.e. the scaling region), the absorption of the virtual photon must be by one of the individual quarks. Figure 1.5 shows the absorption of a virtual photon by the constituent quarks. When the initial spin of the electron and proton are parallel (e.g. $S=s=\frac{1}{2}$ ), not only must the quark have momentum fraction $x$ in the Breit frame (as for unpolarized DIS scattering), but angular momentum conservation dictates that the exchange must occur by the electron flipping its spin (to $s^{\prime}=-\frac{1}{2}$ ) and emitting a spin +1 virtual photon, which is in turn absorbed by the single quark with spin $-\frac{1}{2}$, which then flips its spin, resulting in a final spin of $S^{\prime}=+\frac{3}{2}$ for the nucleon. ${ }^{16}$ Conversely, when the initial electron and proton spins are antiparallel (e.g. $s=\frac{1}{2}$ and $S=-\frac{1}{2}$ ), either one of the two spin $-\frac{1}{2}$ quarks (with momentum fraction $x$ ) can absorb the spin +1 virtual photon, resulting in a final spin of $S^{\prime}=+\frac{1}{2}$. Note that the exact same situation arises with both antiparallel and parallel spins when the initial spin of the electron is $s=-\frac{1}{2}$, due to the symmetry of the configuration. ${ }^{17}$

\footnotetext{
${ }^{15}$ Because $\nu$ is large and $Q^{2} \sim E$ in the DIS region

${ }^{16}$ This changes the proton to a spin- $\frac{3}{2}$ state (e.g. $\Delta^{+}$), which in turn decays very quickly.

${ }^{17}$ This is why only the double-spin asymmetry need be considered; this is due to the cancellation of the cross-terms in Eq. 1.89 .
} 
i.

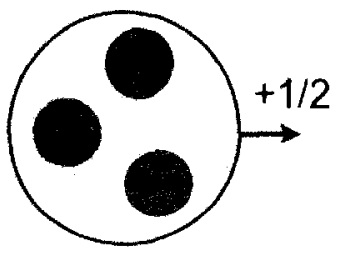

i.

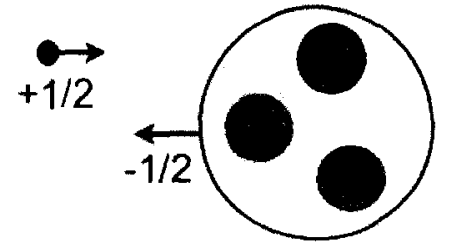

ii.

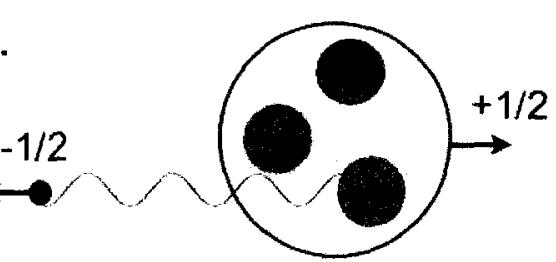

iii.

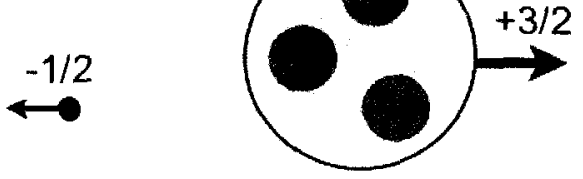

(a) Initial Spins Parallel

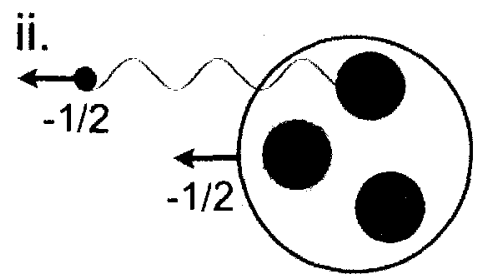

iii.
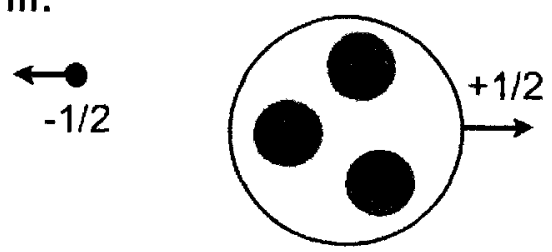

(b) Initial Spins Antiparallel

Figure 1.5: Diagrams showing the spin-transition at scaling region kinematics for polarized ep scattering, in both possible longitudinal double-spin configurations. The initial electron and proton spins are labeled, along with the quark spin directions (i). The electron (black dot) emits a virtual photon of spin 1, flipping the electron spin (ii), which is then absorbed by a quark of the same spin atignment (as it must be from angular momentum conservation), flipping its spin and changing the nucleon spin (iii). See the text for more specific details. 
We now define simple, but more specific distribution functions $f^{\dagger}(x)\left(f^{\downarrow}(x)\right)$ as the probability distribution for finding a quark of flavor $f$ and momentum fraction $x$ of the proton in the Breit frame with its spin aligned parallel (antiparalle/) to the whole proton spin. From the considerations of the preceding paragraph, then, in the scaling region only, we expect

$$
A_{||}\left(Q^{2}, x\right)=\frac{\Delta \sigma^{\dagger \Downarrow}-\Delta \sigma^{\uparrow \Uparrow}}{\Delta \sigma_{\text {unpolarized }}} \propto \frac{\sum q_{f}^{2} f^{\dagger}(x)-\sum q_{f}^{2} f^{\downarrow}(x)}{\sum q_{f}^{2} f(x)}
$$

The relation between $g_{1}$ and the polarized cross-sections now becomes clear. The constant of proportionality in the above equations is the depolarization factor of Eq. 1.19; it is dealt with later in Section 1.4. If we define

$$
\Delta f(x)=f^{\uparrow}(x)-f^{\downarrow}(x)
$$

then we can see that, in the scaling region

$$
\frac{\sum q_{f}^{2} \Delta f(x)}{\sum q_{f}^{2} f(x)}=\frac{g_{1}}{F_{1}}
$$

(where we have ignored contributions to the order $\mathscr{O}(1 / \nu)$ ). We see that, just as $F_{1}$ can be interpreted as a sum of over the distributions of the linear momentum fractions of the quarks, $g_{1}$ can be interpreted as the sum of the distribution of the linear momentum fractions of the quarks, weighted by their spin distributions. Thus, the $g_{1}$ structure function provides an indirect way of observing the angular momentum distribution of the quarks within the nucleon. Writing the quark flavors out explicitly, and extrapolating the concept to the non-scaling region, one finds

$$
g_{1}\left(x, Q^{2}\right)=\frac{1}{2} \sum q_{f}^{2} \Delta f\left(x, Q^{2}\right)=\frac{4}{18} \Delta u\left(x, Q^{2}\right)+\frac{1}{18} \Delta d\left(x, Q^{2}\right)+\frac{1}{18} \Delta s\left(x, Q^{2}\right)
$$

with the contributions from quarks and antiquarks subsumed within the $\Delta f$ functions. Note that a factor of $\frac{1}{2}$ is included by convention (just as in $F_{1}$ ) so that the ratio of Eq. 1.100 holds true.

To this point, an intuitive interpretation of the $g_{2}$ structure function has not been discussed. Unfortunately, $g_{2}$ can not be interpreted in such a (relatively) straightforward manner as $g_{1}$. We have 
seen that, in the case of parallel/anti-parallel spins at high $Q^{2}$, most of the polarized cross-section contribution comes from $g_{1}$, with $g_{2}$ only making only a lower-order contribution. This might naturally lead one to guess that the converse is true for perpendicular spin alignments; since these are spinstructure functions, electron-proton scattering for a perpendicular relative polarization difference should be dominated by $g_{2}$, with $g_{1}$ only making a minor contribution. This, however, is not true; in fact [12]

$$
\frac{\Delta \sigma \uparrow \Rightarrow}{\Delta \Omega \Delta E^{\prime}}-\frac{\Delta \sigma \uparrow \Leftarrow}{\Delta \Omega \Delta E^{\prime}}=\frac{4 \alpha^{2} E^{2}}{M \nu Q^{2} E}\left[g_{1}\left(x, Q^{2}\right)+\frac{2 E}{\nu} g_{2}\left(x, Q^{2}\right)\right] \sin \theta
$$

meaning both structure functions make a contribution of the same order. This (and the lack of any simple interpretation) occurs because of relativistic effects, relating to the fact that, in moving to the Breit (or any other reference) frame, the perpendicular spins in the lab frame do not remain perpendicular in the new reference frame [6]. (This is obviously not a problem for parallel/antiparallel spins, since the spins transform along a common axis.)

We will see, though, in the following sections that the $g_{2}$ structure function, like $g_{1}$, is also expected to obey various sum rules, and that predictions can be made as to its behavior in the DIS and resonance regions. For now, it can be seen that $g_{1}$ and $g_{2}$ are the two structure functions that completely parametrize the polarized cross-sections for the lowest order scattering diagram of Figure 1.2.

\subsection{4 $Q^{2}$ evolution and scaling violations}

In Section 1.1.2, a qualitative summary of phenomenological proton/quark models and their behavior varies with the strength of the virtual photon $Q^{2}$, was discussed. Namely, at low values of $Q^{2}$, the virtual photon interacts "coherently" with the entire nucleon, as if it was a fundamental particle, while at the highest values of $Q^{2}$, the virtual photon interacts with the (asymptotically free) individual valence quarks and sea quark-antiquark pairs in the nucleon.

This limiting behavior constrains the behavior of the structure functions by predicting behavior at these extreme kinematic values. The scaling behavior of both the polarized and unpolarized structure functions has already been discussed. The expectation of scaling behavior, though, has implicitly assumed that the entire process of the scattering of a virtual photon from the constituent 
quark can be described by a relatively simple process governed by a single Feynman diagram:

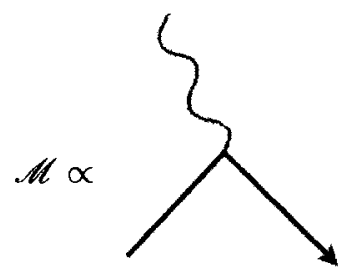

whereas, in reality, the process is better described by a sum of this and several higher order diagrams:
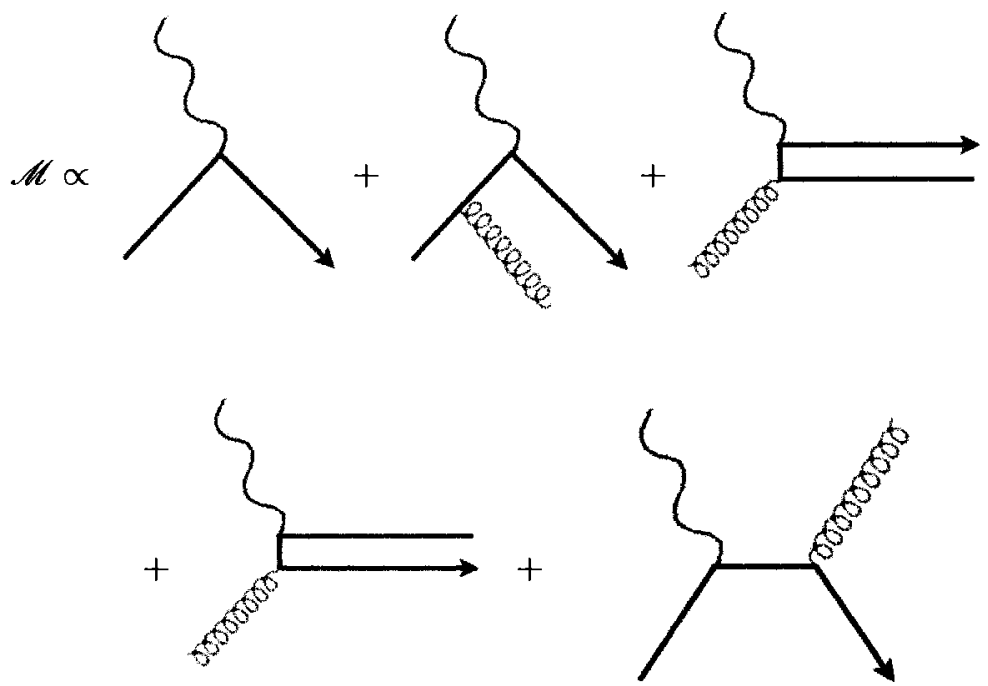

where we see additional diagrams for quark-antiquark production (from scattering off a "hard" gluon) and spontaneous radiation of gluons from the quark fermion lines. A rigorous calculation of these terms has been made by Dokshitzer, Gribov, Lipatov, Altarelli and Parisi [15] by solving for the crosssections in terms of $d \Omega$ or $d p_{T}=d\left(p^{\prime} \sin \theta\right)$, and integrating in $Q^{2}$ down to an infrared divergence limit $Q_{0}^{2}{ }^{18}$ The results for the quark and gluon spin contributions are the DGLAP equations (also

\footnotetext{
${ }^{18}$ This is necessary because, at low $Q^{2}$, due to the large size of the QCD coupling constant, simple perturbative methods (i.e. low-order approximations) can no longer be used, and more sophisticated treatments are required. These techniques are far beyond the scope of this thesis; the interested reader is directed to Ref. [16], for example, for details.
} 
called the evolution equations and the Altarelli-Parisi equations):

$$
\begin{gathered}
\frac{d}{d t} \Delta q_{f}(y, t)=\frac{\alpha_{s}(t)}{2 \pi} \int_{x}^{1} \frac{d y}{y}\left[\Delta q_{f}(y, t) \Delta P_{q q}\left(\frac{x}{y}\right)+\Delta g(y, t) \Delta P_{q g}\left(\frac{x}{y}\right)\right] \\
\frac{d}{d t} \Delta g(y, t)=\frac{\alpha_{s}(t)}{2 \pi} \int_{x}^{1} \frac{d y}{y}\left[\sum_{i=1}^{2 f} \Delta q_{f}(y, t) \Delta P_{g q}\left(\frac{x}{y}\right)+\Delta g(y, t) \Delta P_{g g}\left(\frac{x}{y}\right)\right]
\end{gathered}
$$

where

$$
t \equiv \ln \frac{Q^{2}}{Q_{0}^{2}}
$$

and $\alpha_{s}(t)$ is the strong coupling constant, which is a "running" function of $t .{ }^{19}$ The splitting functions $\triangle P_{A B}$ (where $A, B=g$ or $q$ for gluons or quarks) are defined as

$$
\Delta P_{A B} \equiv P_{A+B+}-P_{A-B+}
$$

where the "+" and "-" represent the helicities of the quarks/gluons in question, and $P$ can be interpreted as the probability for a coupling between the quarks/gluons taking place. ${ }^{20}$ These are given by

$$
\begin{gathered}
\Delta P_{q q}=C_{F} \frac{1+z^{2}}{1-z} \\
\Delta P_{g q}=C_{F} \frac{1-(1-z)^{2}}{z} \\
\Delta P_{q g}=\frac{1}{2}\left[z^{2}-(1-z)^{2}\right] \\
\Delta P_{q q}=C_{F}\left(1+z^{4}\right)\left[\left(\frac{1}{z}+\frac{1}{1-z}\right)-\frac{1-z}{z}\right]
\end{gathered}
$$

for $z<1$, where $z$ is the fraction of the given quark momentum contributed by the recoil of the emitted parton.

We can see from Eq. 1.101 that this directly affects the $g_{1}$ structure function. In fact, the main point to be made here is that QCD predicts that violations of the scaling behavior of the spinstructure functions due to changes in the spin-dependent distributions of quarks and gluons do

\footnotetext{
${ }^{19}$ The evolution of the strong coupling constant is discussed thoroughly in Ref. [16] and many other introductory texts in QCD. A relatively thorough treatise on the status of calculations of this quantity can be found in Ret. [17].

${ }^{20}$ More properly, $\left(\alpha_{s} / 2 \pi\right) P_{A B}$ is the coupling probability.
} 
indeed occur, and that the specific magnitude of the violation as a function of $Q^{2}$ and $x$ can be calculated.

Eqs. 1.105 and 1.106 are cumbersome, so, using $q$ to represent a parton (gluon or quark) distribution, these are often written in the condensed notation [18]

$$
\frac{d q(x, t)}{d t}=\frac{\alpha_{s}(t)}{2 \pi}(\Delta P \otimes q)(x, t)
$$

where the $\otimes$ symbol represents a convolution of the $\Delta P$ operators with the parton distributions.

It is important to emphasize that pure scaling behavior only manifests at high $Q^{2}$ and/or low $x$ [19], and that scaling violations can only be calculated in this manner in the kinematic region where pQCD is applicable, $Q^{2} \gtrsim 1.0 \mathrm{GeV}^{2}$ [20], in the DIS region ( $W \gtrsim 2.0 \mathrm{GeV}$ ). Outside of this range, the complications caused by the growth of the running coupling constant and the presence of resonances (see Section 1.4.3) render the methods of pQCD unusable, and other models must be used.

\subsection{Moments and sum rules}

In the resonance region $(1.08 \mathrm{GeV}<\mathrm{W}<2.0 \mathrm{GeV}$ ), we see the variation of structure functions and asymmetries due to the excitation of various hadronic states. As already mentioned, these resonances are not predictions of the standard PQCD method. At this point in time, no analytic or perturbative solution exists for the evolution of structure functions across excitations of hadronic states. Instead, we must rely upon the Operator Product Expansion (OPE) to express the moments of structure functions in terms of empirically evaluated hadronic matrix elements. The evaluation of these moments allows the testing of various physical sum rules, as well as a wide range of applications regarding other physical quantities (see Chapter 8).

\subsubsection{The Operator Product Expansion}

A basic understanding of the Operator Product Expansion is essential for proper interpretation of measured moments of the structure functions. As we will see, the measurable scattering cross- 
section is the result of the product of two electromagnetic current field operators. Unfortunately, the product of two fields is not a well-defined entity, particularly as it relates to long-range QCD effects. The OPE provides a method for separating the short distance (i.e. high-energy) correlations of the inelastic cross-section so that one can utilize perturbative expansion techniques [16].

\section{Product of two fields in ep scattering}

It has already been demonstrated, for the case of elastic ep scattering, that the square of the transition amplitude, and hence the scattering cross-section, can be expressed in terms of the electromagnetic current operator $J_{\mu}$ (Eq. 1.50), and that (in the Breit frame) this can be reduced to terms of a local current operator $J_{\mu}(0)$ (Eq. 1.55).

Now, we attempt to generalize this formalism for inelastic scattering in the lab reference frame, and show that the resulting cross-section contains an inseparable product of two field operators $J_{\mu}(r) J_{\nu}(0) .{ }^{21}$ Rewriting $W_{\mu \nu}$ (defined in Eq. 1.35) as general sum over states yields [16]

$$
W_{\mu \nu}=\frac{1}{4 \pi} \sum_{X}(2 \pi)^{4} \delta^{4}\left(p_{X}-p-q\right) \sum_{\lambda}\left\langle p \lambda\left|J_{\mu}(0)\right| X\right\rangle\left\langle X\left|J_{\nu}(0)\right| p \lambda\right\rangle
$$

The expression for $W_{\mu \nu}$ simply states that the contribution to the square of the scattering amplitude $\left\langle|\mathscr{M}|^{2}\right\rangle$ from the virtual photon-proton interaction is given by the sum of the matrix elements given by the transition amplitudes between the initial proton momentum states and eigenstates $|p \lambda\rangle$ and final state of scattered particles $|X\rangle$, as measured at the proton origin $r=0$, with the $\delta$-function enforcing momentum and energy conservation.

By simply writing the $\delta$-function in terms of its Fourier transform and using closure of states ${ }^{22}$, one finds

$$
W_{\mu \nu}=\frac{1}{4 \pi} \int d^{4} r e^{i\left(p_{X}-p-q\right) \cdot r} \sum_{\lambda}\left\langle p \lambda\left|J_{\mu}(0) J_{\nu}(0)\right| p \lambda\right\rangle
$$

By use of the translation operator $e^{i\left(\hat{P}-\hat{P}^{\prime}\right) \cdot r}$, we have

$$
\left\langle p \lambda\left|J_{\mu}(0) J_{\nu}(0)\right| p \lambda\right\rangle=e^{i\left(p_{X}-p\right) \cdot r}\left\langle p \lambda\left|J_{\mu}(x) J_{\nu}(0)\right| p \lambda\right\rangle
$$

\footnotetext{
${ }^{21}$ Here, $r$ is used as the space-time 4-vector instead of the more conventional $x$, to avoid confusion with the kinematic variable defined in Section 1.1.3.

22That is, $\sum_{X}|X\rangle\langle X|=1$.
} 
because the indices $\nu$ and $\mu$ are associated with initial $(p)$ and final $\left(p_{X}\right)$ state momenta, respectively.

Thus, we simplify $W_{\mu \nu}$ to

$$
W_{\mu \nu}=\frac{1}{4 \pi} \int d^{4} r e^{i(q) \cdot r} \sum_{\lambda}\left\langle p \lambda\left|J_{\mu}(r) J_{\nu}(0)\right| p \lambda\right\rangle
$$

Using the exact same process, we can prove

$$
\sum_{X}(2 \pi)^{4} \delta^{4}\left(q-p+p_{X}\right) \sum_{\lambda}\left\langle p \lambda\left|J_{\nu}(0)\right| X\right\rangle\left\langle X\left|J_{\mu}(0)\right| p \lambda\right\rangle=\int d^{4} r e^{i q \cdot r} \sum_{\lambda}\left(p \lambda\left|J_{\mu}(0) J_{\nu}(r)\right| p \lambda\right\rangle=0
$$

This equation equals zero because the condition $q-p+p_{X}=0$ in the $\delta$-function is kinematically forbidden, since $q=k-k^{\prime}=p_{X}-p>0$ always. ${ }^{23}$

This result can be used to rewrite Eq. 1.117 in terms of a commutator of current operators:

$$
W_{\mu \nu}=\frac{1}{4 \pi} \sum_{\lambda} \int d^{4} r e^{i q \cdot r}\left\langle p, \lambda\left|\left[J_{\mu}(r), J_{\nu}(0)\right]\right| p \lambda\right\rangle
$$

The commutator $\left[J_{\mu}(r), J_{\nu}(0)\right]$ must vanish for space-like coordinates (for which $r^{2}<0$ ). We also realize that the integrand is oscillatory, so that if the oscillations become close enough together in $r$ (i.e. if $|q \cdot r|$ becomes large enough), the integral evens out to zero. Thus, the dominant contribution to $W_{\mu \nu}$ comes from the localized region ${ }^{24}$

$$
0 \leq r^{2} \leq r_{\text {limit }}^{2}
$$

where, using $Q^{2}=-q^{2}$,

$$
r_{\text {limit }}^{2} \propto \frac{1}{Q^{2}}
$$

The main objective of this presentation is to show that the hadronic tensor $W_{\mu \nu}$ contains a field product of two current operators. While this product in itself is not an easily defined field operator, it can be shown with relative ease that the dominant contribution to the measurable cross-section comes from the localized timelike components of the product $J_{\mu}(r) J_{\nu}(0)$. This provides a motivation

\footnotetext{
${ }^{23}$ That is, the recoil particle cannot have more energy than the incident particle.

${ }^{24} \mathrm{~A}$ more rigorous proof of this relation is found in Ret. [16]; the point here is to convey the general distance scale over which the dominant contribution to the scattering cross-section is made.
} 
for extricating the dominant components of the field in terms of an operator product expansion about the point $r=0$, in the neighborhood of $r_{\text {limit }}^{2}$

\section{Summary of the OPE (Light Cone Expansion; Twist)}

The goal of the Operator Product Expansion, then, is to expand the product of two operators $\hat{A}(r)$ and $\hat{B}\left(r^{\prime}\right)$ in terms of a well defined third operator $\hat{P}(r)$ such that

$$
\hat{A}(r) \hat{B}\left(r^{\prime}\right)=\sum_{i=0}^{\infty} C_{i}\left(r-r^{\prime}\right) \hat{P}\left(\frac{r+r^{\prime}}{2}\right)
$$

A detailed proof of the O.P.E. and the specific values of all the coefficients for the hadronic tensor is beyond the scope of this thesis; the reader is hereby referred to Ref. [16] for more information. Instead, a summary providing the major points is presented, only to show the reader how the extraction of structure functions provides input into our knowledge of the terms and operators in the resultant expansion.

As already explained, the locality near the origin of the light-cone (Eq. 1.120) serves as the region of importance for the expansion of Eq. 1.122. This corresponds to the kinematic region $Q^{2} \rightarrow \infty$ with fixed $x \equiv Q^{2} /(2 p \cdot q)$. Thus, in this light-cone region [16]

$$
Q^{2}=\left|q^{2}\right| \sim|p \cdot q| \gg\left|p^{2}\right|, M^{2}
$$

The hadronic tensor $W_{\mu \nu}$ contains a product of two current operators (Eq. 1.117). In terms of the the Feynman diagram in Figure 1.2, the portion of interest to the hadronic tensor evaluation can be written mathematically equivalently in terms of an (unsquared) Compton scattering diagram as [11]

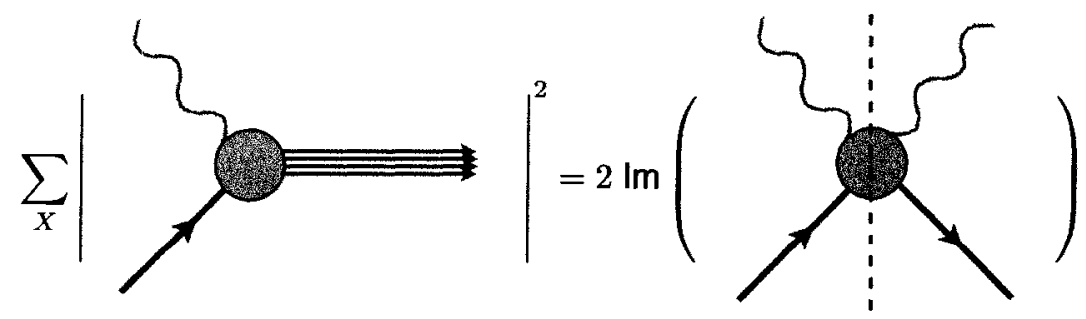


The challenge, then, is to expand the diagram term on the right in the Operator Product Expansion in the the vicinity of the light cone origin (that is, using the conditions enforced in Eq. 1.123). This diagram can be expressed perturbatively; here we consider only the highest order terms in the expansion:
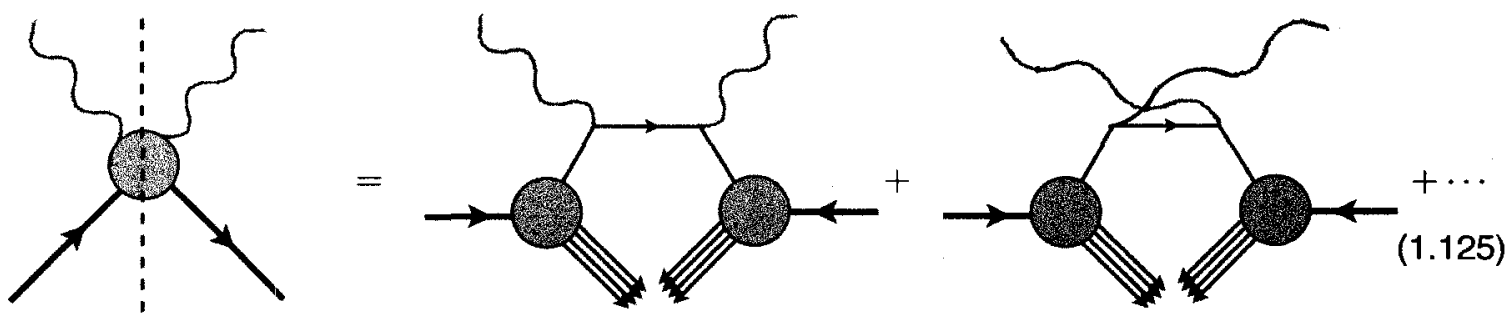

There are exactly two diagrams of order $1 / Q^{2}$ in this series, related by crossing symmetry. For now, we consider only the first diagram in this expansion. ${ }^{25}$ To show the form taken by the OPE in the light-cone expansion, we need to consider the general form of the matrix element $\mathscr{M}_{f i}$. In the DIS (scaling) region, the current product can be written

$$
J_{\mu}(r) J_{\nu}(0) \rightarrow \bar{q}(r) \gamma_{\mu} q(r) \bar{q}(0) \gamma_{\nu} q(0)=\bar{q}(r) \gamma_{\mu} \overbrace{q(r) \bar{q}(0)} \gamma_{\nu} q(0)+\cdots
$$

where $\gamma_{\mu}$ are the $4 \times 4$ Dirac matrices and $\sim$ indicates a contraction over indices in the embraced operators. Note that the operators in this equation correlate exactly to the expansion of Eq. 1.125.

According to the Feynman rules, the scattering amplitude for the proton will have the proportionality [11]

$$
\mathscr{M}_{\text {proton }} \propto \int d^{4} r e^{i q \cdot r} \bar{q}(r) \gamma_{\mu} \overbrace{q(r) \bar{q}(0)} \gamma_{\nu} q(0)=\bar{q}(r) \gamma_{\mu} \frac{i\left(\partial^{\sigma} \gamma_{\sigma}+q^{\sigma} \gamma_{\sigma}\right)}{(i \partial+q)^{2}} \gamma_{\nu} q(0)
$$

\footnotetext{
${ }^{25}$ Crossing symmetry effects on the OPE result in either even or odd moments of structure functions going to zero. This will be considered again in Section 1.3.2.
} 
Thus, we see that the OPE requires expansion of the denominator in the locality of the light cone origin. Using Eq. 1.123, a Taylor series expansion can be used to find

$$
\frac{1}{(i \partial+q)^{2}}=-\frac{1}{Q^{2}-2 i q \cdot \partial+\partial^{2}}=-\frac{1}{Q^{2}} \sum_{n=0}^{\infty}\left(\frac{2 i q \cdot \partial-\partial^{2}}{Q^{2}}\right)^{n}
$$

Terms of order $\partial^{2}$ can be ignored. By using $\gamma$-matrix identities, one can show [11] that the amplitude can be expressed as

$$
\mathscr{M}_{\text {proton }} \propto-i \bar{q}(r)\left(2 \gamma_{\mu}\left(i \partial_{\nu}\right)-g_{\mu \nu} q^{\sigma} \gamma_{\sigma}\right) \frac{1}{Q^{2}} \sum_{n=0}^{\infty}\left(\frac{2 i q \cdot \partial}{Q^{2}}\right)^{n} q(0)
$$

Note that this expression contains 2 separate terms, each summed over $n$. The $g_{\mu \nu}$ term represents a trace, ${ }^{26}$ and the $i \partial_{\nu}$ is the main part of the new combined operator in the light cone expansion. The operator corresponding to the $k$ th term in the expansion can be seen to contain the operator

$$
\bar{q}(r) \gamma_{\mu_{1}}\left(i \partial_{\mu_{2}}\right) \cdots\left(i \partial_{\mu_{k}}\right) q(0) \propto \int d^{4} r e^{i q \cdot r} \tilde{\tilde{q}}(r) \gamma_{\mu_{1}}\left(r_{\mu_{2}}\right) \cdots\left(r_{\mu_{k}}\right) \tilde{q}(0)
$$

where we have equated the momentum-space composite operator to the Fourier transform of the equivalent geometrical-space operator on the right.

Generalizing the above to any current operator in the light-cone expansion, then, the product of two current operators can be written as

$$
J(r) J(0)=\sum_{i, n} C_{n}^{(i)}\left(r^{2}\right) r^{\mu_{1}} \ldots r^{\mu_{n}} \hat{P}_{\mu_{1 \ldots \mu_{n}}}^{(i)}(0)
$$

where $\mathscr{O}_{\mu_{1} \ldots \mu_{n}}^{(i)}\left(r^{2}\right)$ is the expansion of composite operators, and $C_{n}^{(i)}\left(r^{2}\right)$ is an expansion of correlated coefficient functions. This decomposition is known as the light-cone expansion, and it is the key relation in the Operator Product Expansion for DIS electron-hadron scattering.

One last topic that must be addressed before applying the expansion is that of the analytic structure of the coefficient functions and the definition of the twist of these functions. It is simplest

\footnotetext{
${ }^{26}$ This is subtracted from the main operator to make the main current operator traceless. The subtraction of traces, while necessary, is not the primary concern here; the remainder of the explanations in this section will assume the trace terms are implicit in the OPE equations.
} 
to define twist by using dimensional analysis on the operators assuming a free-field expansion (i.e. no renormalization terms). If we define $d_{j_{0}}$ as the canonical dimension of the operator $J(r)$, and $d_{0}^{i}(n)$ as the canonical dimension of the composite operator term $\hat{P}_{\mu_{1} \ldots \mu_{n}}^{(i)}$, then clearly, we require

$$
2 d_{j_{\mathrm{o}}}=d_{0}^{i}(n)
$$

Looking at Eq. 1.131, in order to conserve dimensionality in terms of $r$, the coefficient function must be [16] of the form

$$
C_{n}^{(i)}\left(r^{2}\right) \sim\left(r^{2}\right)^{-d_{j_{0}}-n / 2+d_{0}^{i}(n) / 2}
$$

We will see (near the end of this section) that the magnitude of the operator terms is directly determined by the "strength" of the singularities in the coefficients, as determined by a contour integral in the complex plane. Looking at the form of $C_{n}^{(i)}\left(r^{2}\right)$, it can be seen that if the exponent is sufficiently large enough (that is, if $d_{0}^{i}(n)-n>2 d_{j_{0}}$ ), that the singularity structure of the $i$-th, $n$-th coefficient function disappears. Thus, the magnitude of the integral is directly determined by the value of $d_{0}^{i}(n)-n$, a quantity referred to as the twist $\tau_{n}^{i}$ of the operator term:

$$
\tau_{n}^{i} \equiv d_{0}^{i}(n)-n
$$

Generally, this naive derivation of twist through dimensional analysis does not work for the case of interacting field operators. The required renormalization of the operator terms results in dimensional regularization of the higher-order terms; the renormalization group equations [11][16] are then required in the subsequent expansion to obtain the singularity structure.

Generally, an OPE composite operator of term $n$ and dimension $d$ can be shown to make a contribution of order [11]

$$
\left(\frac{2 p \cdot q}{Q^{2}}\right)^{n}\left(\frac{1}{Q}\right)^{d-n-2}=\frac{\omega^{n}}{Q^{d-n-2}}
$$

where the value of $n$ is referred to as the "spin" of the operator [12], with the twist defined here as $\tau=d-n$. The lower the twist, the greater the contribution to the expansion from the operator. In general, twist-2 operators result from the lowest order expansion of functions, with higher twist (HT) contributions leading to (small) violations of sum rules and scaling derived using only the twist-2 
terms. Twist-1 (or lower) terms make no contributions to the expansion; in fact, the identity operator is the only possible twist-1 QCD operator [11].

\section{Application of the OPE to spin-structure functions}

In this section it is shown how the OPE can be used to expand the general hadronic tensor $W_{\mu \nu}$ for polarized scattering, and how the (polarized and unpolarized) structure functions $F_{1}, F_{2}, g_{1}$ and $g_{2}$ can be written in terms of this expansion. Once again, this section is not intended as a rigorous proof, but merely to clarify the expansion of the field operators results in a summation of moments of structure functions.

To begin with, the generalized electromagnetic current operator commutator of Eq. 1.119 is assumed to have the most generalized form possible ${ }^{27}$ under the constraints of Lorentz invariance and current conservation [16][21]:

$$
\begin{aligned}
\mathbf{T}\left[J_{\mu}(r) J_{\nu}(0)\right]= & \\
-\left(g_{\mu \nu} \square^{2}-\right. & \left.\partial_{\mu} \partial_{\nu}\right) \hat{O}_{1}(r, 0)-\left(g_{\mu \lambda} g_{\nu \sigma} \square^{2}-g_{\mu \lambda} \partial_{\nu} \partial_{\sigma}-g_{\nu \sigma} \partial_{\mu \lambda}+g_{\mu \nu} \partial_{\lambda} \partial_{\sigma}\right) \hat{O}_{2}^{\lambda \sigma}(r, 0) \\
& +i \epsilon_{\mu \nu \lambda \sigma} \partial^{\lambda} \hat{Q}_{1}^{\sigma}(r, 0)-i\left(\partial_{\nu} \epsilon_{\mu \rho \lambda \sigma} \partial^{\rho}-\partial_{\mu} \epsilon_{\nu \rho \lambda \sigma} \partial^{\rho}-\square^{2} \epsilon_{\mu \nu \lambda \sigma}\right) \hat{Q}_{2}^{\lambda \sigma}(r, 0)
\end{aligned}
$$

The expression $\mathbf{T}\left[J_{\mu}(r) J_{\nu}(0)\right]$ here refers to the time-ordered product of the currents operators [1]:

$$
\mathbf{T}\left[J_{\mu}(r) J_{\nu}(0)\right] \equiv J_{\mu}(r) J_{\nu}(0) \theta\left(r_{0}\right)+J_{\nu}(0) J_{\mu}(r) \theta\left(-r_{0}\right)
$$

where $\theta\left(r_{0}\right)$ is the step function, equal to 1 if the 0 -component of $r$ (i.e. time) is positive, and equal to 0 if this quantity is negative.

Here the (symmetric) terms containing operators $\hat{O}_{1}$ and $\hat{O}_{2}$ are relevant to the unpolarized response functions $W_{1}$ and $W_{2}$, and the (antisymmetric) terms containing operators $\hat{Q}_{1}$ and $\hat{Q}_{2}$ are relevant to the polarized response functions $G_{1}$ and $G_{2}$. Again, we use the light-cone expansion to

\footnotetext{
${ }^{27} \epsilon_{\mu \nu \lambda \sigma}$ again represents the Levi-Cevita tensor, and $\square^{2} \equiv \partial_{\mu} \partial^{\mu}$ is the D'Alembertian operator.
} 
write these bilocal operators as

$$
\begin{aligned}
& \hat{O}_{1}(r, 0)=\sum_{i, n} C_{1, n}^{(i)}\left(r^{2}\right) r^{\mu_{1}} \ldots r^{\mu_{n}} \hat{P}_{1, \mu_{1} \ldots \mu_{n}}^{(i)}\left(\frac{r}{2}\right) \\
& \hat{O}_{2}^{\lambda \sigma}(r, 0)=\sum_{i, n} C_{2, n}^{(i)}\left(r^{2}\right) r^{\mu_{1}} \ldots r^{\mu_{n}} \hat{P}_{2, \mu_{1} \ldots \mu_{n}}^{(i) \lambda \sigma}\left(\frac{r}{2}\right) \\
& \hat{Q}_{1}^{\sigma}(r, 0)=\sum_{i, n} E_{1, n}^{(i)}\left(r^{2}\right) r^{\mu_{1}} \ldots r^{\mu_{n}} \hat{R}_{1, \mu_{1} \ldots \mu_{n}}^{(i) \sigma}\left(\frac{r}{2}\right) \\
& \hat{Q}_{2}^{\lambda \sigma}(r, 0)=\sum_{i, n} E_{2, n}^{(i)}\left(r^{2}\right) r^{\mu_{1}} \ldots r^{\mu_{n}} \hat{R}_{2, \mu_{1} \ldots \mu_{n}}^{(i) \lambda \sigma}\left(\frac{r}{2}\right)
\end{aligned}
$$

The variables $C_{1,2}^{(i)}$ and $E_{1,2}^{(i)}$ are the expansion coefficient functions of the operators $O_{1,2}^{(i)}$ and $Q_{1,2}^{(i)}$, respectively.

The matrix elements of these new composite tensor operators $P_{1,2}^{(i)}$ and $R_{1,2}^{(i)}$ have the structures [16][21][22]

$$
\begin{gathered}
\left\langle p\left|P_{1 \mu_{1} \ldots \mu_{n}}^{(i)}(0)\right| p\right\rangle=A_{1, n}^{(i)} p_{\mu_{1}} \ldots p_{\mu_{n}} \\
\left\langle p\left|P_{2 \mu_{1} \ldots \mu_{n}}^{(i) \lambda \sigma}(0)\right| p\right\rangle=A_{2, n+2}^{(i)} p^{\lambda} p^{\sigma} p_{\mu_{1}} \ldots p_{\mu_{n}} \\
\left\langle p s\left|R_{1 \mu_{1} \ldots \mu_{n}}^{(i) \sigma}(0)\right| p s\right\rangle=-M_{1, n}^{(i)}\left\{S_{\sigma} p_{\mu_{1}} \ldots p_{\mu_{n}}\right\} \\
\left\langle p s\left|R_{1 \mu_{1} \ldots \mu_{n}}^{(i) \lambda \sigma}(0)\right| p s\right\rangle=-M_{2, n}^{(i)} \frac{1}{2}\left(p_{\lambda} S_{\sigma}-p_{\sigma} S_{\lambda}\right) p_{\mu_{1}} \ldots p_{\mu_{n}}
\end{gathered}
$$

where terms containing $g_{\mu \nu}$ (which reduce to traces) are simply dropped, as they do not correlate to terms into the structure function expansion, and thus have zero coefficients. The curly brackets $\{\ldots\}$ in Eq. 1.144 denote a complete symmetrization of the space-time indices $\mu$ contained within. Here the variables $A_{1}^{(i)}$ and $A_{2}^{(i)}$ are coefficients ${ }^{28}$ for the expansion of the unpolarized (symmetric) terms (correlating to $W_{1}$ and $W_{2}$ ), and $M_{1}^{(i)}$, and $M_{2}^{(i)}$ are coefficients corresponding to corresponding to the polarized (antisymmetric) terms (correlating to $G_{1}$ and $G_{2}$ ).

The other coefficient functions $C_{1}^{(i)}, C_{2}^{(i)}, E_{1}^{(i)}$ and $E_{2}^{(i)}$ are singular functions of $r$ (in the neighborhood of $r^{2}=0$ ). If we define the Fourier transforms of these functions with a tilde $(\sim)$, for

\footnotetext{
${ }^{28}$ These are not to be confused with the asymmetries $A_{1}$ and $A_{2}$, which are completely different quantities.
} 
example,

$$
\tilde{C}_{1, n}^{(i)}\left(Q^{2}\right)(-i)\left(Q^{2} / 2\right)^{-n-1} q^{\mu_{1}} \ldots q^{\mu_{n}}=\int d^{4} r e^{i q \cdot r} r^{\mu_{1}} \ldots r^{\mu_{n}} C_{1, n}^{(i)}\left(x^{2}\right)
$$

and take the Fourier transform of Eq. 1.136, then that equation can be rewritten as

$$
\begin{aligned}
& T_{\mu \nu} \equiv \int d^{4} r\left\langle p\left|\mathbf{T}\left[J_{\mu}(r) J_{\nu}(0)\right]\right| p\right\rangle= \\
& \quad 2 \sum_{i, n}\left[e_{\mu \nu} A_{1, n}^{(i)} \tilde{C}_{1, n}^{(i)}\left(Q^{2}\right)+f_{\mu \nu} A_{2, n}^{(i)} \tilde{C}_{2, n}^{(i)}\left(Q^{2}\right)+h_{\mu \nu} M_{1, n}^{(i)} \tilde{E}_{1, n}^{(i)}\left(Q^{2}\right)+j_{\mu \nu} M_{2, n}^{(i)} \tilde{E}_{2, n}^{(i)}\left(Q^{2}\right)\right] \omega^{n}
\end{aligned}
$$

where $\omega \equiv 1 / x$ (as defined in Section 1.1.3) and the tensors $e_{\mu \nu}$ through $j_{\mu \nu}$ are sums of the generalized kinematics terms in Eq. 1.136 divided by $q^{2}$; e.g.

$$
e_{\mu \nu} \equiv g_{\mu \nu}-\frac{q_{\mu} q_{\nu}}{q^{2}}
$$

This brings us to the central point in this process: The Fourier transform of the time-ordered product of current operators in the Operator Product Expansion can be expressed as a power series expansion of a kinematic variable $\omega$.

At this point, we introduce the Low equation for the scattering amplitude [1]. Inserting the completeness relation $\sum_{X}|X\rangle\langle X|=1$ between the two time-ordered currents in the left hand side of Eq. 1.147 and explicitly evaluating the integrals yields

$$
\begin{aligned}
T_{\mu \nu}=\sum_{X}(2 \pi)^{3}\left[\frac{\delta^{(3)}(\mathbf{p} \mathbf{X}-\mathbf{q}-\mathbf{p})}{p_{X 0}-q_{0}-p_{0}-i \varepsilon}\left\langle p\left|J_{\mu}(0)\right| X\right\rangle\left\langle X\left|J_{\nu}(0)\right| p\right\rangle\right. & \\
& \left.+\frac{\delta^{(3)}\left(\mathbf{p}_{\mathbf{X}}+\mathbf{q}-\mathbf{p}\right)}{p_{X 0}+q_{0}-p_{0}-i \varepsilon}\left\langle p\left|J_{\nu}(0)\right| X\right\rangle\left\langle X\left|J_{\mu}(0)\right| p\right\rangle\right]
\end{aligned}
$$

Just as in Eq. 1.118, energy conservation dictates that the second term goes to zero. Using this and the expression for $W_{\mu \nu}$ in Eq. 1.115, one finds that

$$
W_{\mu \nu}=\frac{1}{2 \pi i}\left[T_{\mu \nu}\left(q_{0}+i \varepsilon\right)-T_{\mu \nu}\left(q_{0}-i \varepsilon\right)\right] \equiv \frac{1}{\pi} \operatorname{Abs} T_{\mu \nu}
$$

Here Abs $T_{\mu \nu}$ refers to the absorptive part of $T_{\mu \nu}$ [16]. Applying a Cauchy integration along the 


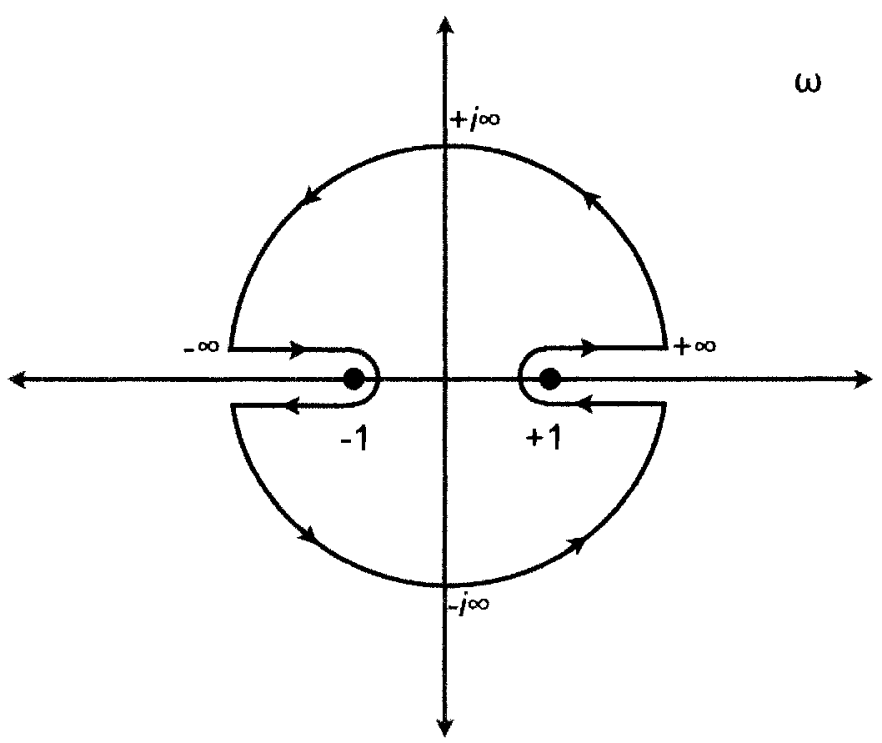

Figure 1.6: The contour $\mathscr{C}$ used to evaluate the $\omega$-integrals in Equations 1.151 and 1.152. A branch cut is made along the real axis for $|\omega|>1$. The singularity strength of the coefficient functions along this cut determine the overall magnitude of the integral. Copied from Ref. [16].

contour $\mathscr{C}$ shown in Figure 1.6 to Eq. 1.149 and Eq. 1.150, one can show [16]

$$
\frac{1}{2 \pi i} \oint_{\mathscr{C}} \frac{T_{\mu \nu}}{\omega^{n}}=\frac{2}{\pi} \int_{1}^{\infty} \frac{d \omega}{\omega^{n}} \mathrm{Abs} T_{\mu \nu}=2 \int_{0}^{1} d x x^{n-2} W_{\mu \nu}
$$

Another integration along $\mathscr{C}$ can be done to show

$$
\frac{1}{2 \pi i} \oint_{\mathscr{C}} d \omega \omega^{m-n}=\delta_{m, n-1}
$$

which, if applied to Eq. 1.147, has the effect of reducing the sum over $i$ and $n$ to a sum over $i$ only, so that

$$
\begin{aligned}
T_{\mu \nu}=2 \sum_{i}\left[e_{\mu \nu} A_{1, n-1}^{(i)} \tilde{C}_{1, n-1}^{(i)}\left(Q^{2}\right)\right. & +f_{\mu \nu} A_{2, n-1}^{(i)} \tilde{C}_{2, n-1}^{(i)}\left(Q^{2}\right) \\
& \left.+h_{\mu \nu} M_{1, n-1}^{(i)} \tilde{E}_{1, n-1}^{(i)}\left(Q^{2}\right)+j_{\mu \nu} M_{2, n-1}^{(i)} \tilde{E}_{2, n-1}^{(i)}\left(Q^{2}\right)\right]
\end{aligned}
$$

All that remains is equate the right side of Eq. 1.150 to Eq. 1.153 , to arrive at an Operator Product 
Expansion of the hadronic tensor $W_{\mu \nu}$.

At this point, we once again point out that the hadronic tensor contains separate terms (Eq. 1.81) that can be expressed algebraically in terms of the unpolarized structure functions $F_{1}$ and $F_{2}$, and the polarized structure functions $g_{1}$ and $g_{2}$. In terms of the bilinear tensors (used for abbreviation in this section), one can write the unpolarized (symmetric) contribution to $W_{\mu \nu}$ as

$$
W_{\mu \nu}^{S}=e_{\mu \nu} F_{L}+f_{\mu \nu} F_{2}
$$

where $F_{L}$ and $F_{2}$ are the structure functions defined in Section 1.2.2. Putting this into Eq. 1.150 and Eq. 1.153 yields the operator product expansion for the unpolarized structure functions:

$$
\begin{gathered}
\int_{0}^{1} d x x^{n-2} F_{L}\left(x, Q^{2}\right)=\sum_{i} A_{1, n}^{(i)} \tilde{C}_{1, n}^{(i)}\left(Q^{2}\right) \\
\int_{0}^{1} d x x^{n-2} F_{2}\left(x, Q^{2}\right)=\sum_{i} A_{2, n}^{(i)} \tilde{C}_{2, n}^{(i)}\left(Q^{2}\right)
\end{gathered}
$$

Naturally, the same process can be applied to the polarized (antisymmetric) part of $W_{\mu \nu}$, as well. Equating the antisymmetric parts of Eqs. 1.150 and 1.153, and separating the polarized structure functions $g_{1}$ and $g_{2}$ (as labeled in Eq. 1.88) ultimately yields [22]

$$
\begin{gathered}
\int_{0}^{1} d x x^{n-1} g_{1}\left(x, Q^{2}\right)=\frac{1}{2} \sum_{i} M_{1, n}^{(i)} \tilde{E}_{1, n}^{(i)}\left(Q^{2}\right) \\
\int_{0}^{1} d x x^{n-1} g_{2}\left(x, Q^{2}\right)=\frac{1}{2} \sum_{i}\left[M_{2, n}^{(i)} \tilde{E}_{2, n}^{(i)}\left(Q^{2}\right)-\frac{n-1}{n} M_{1, n}^{(i)} \tilde{E}_{1, n}^{(i)}\left(Q^{2}\right)\right]
\end{gathered}
$$

The OPE for $g_{2}$ is more complex than that for $g_{1}$ due to the prominence of transverse effects [22] that do not remain isolated in a Lorentz transformation like the longitudinal components of $g_{1}$. It is customary to define the matrix components

$$
a_{n} \equiv \sum_{i} M_{1, n}^{(i)} \tilde{E}_{1, n}^{(i)}\left(Q^{2}\right)
$$




$$
d_{n} \equiv \frac{n}{n-1} \sum_{i} M_{2, n}^{(i)} \tilde{E}_{2, n}^{(i)}\left(Q^{2}\right)
$$

so that Eqs. 1.157 and 1.158 can be rewritten more simply as

$$
\begin{gathered}
\int_{0}^{1} d x x^{n-1} g_{1}\left(x, Q^{2}\right)=\frac{1}{2} a_{n} \\
\int_{0}^{1} d x x^{n-1} g_{2}\left(x, Q^{2}\right)=\frac{1}{2} \frac{n-1}{n}\left(d_{n}-a_{n}\right)
\end{gathered}
$$

One should keep in mind that this is a greatly simplified "derivation" of these OPE relations, where the subtraction of trace elements and flavor structure of the quark fields has been suppressed [22]. The relations of Eqs. 1.161 and 1.162 are known as sum rules. The integrals on the left hand sides of these equations are referred to as the $n$th moments of $g_{1}$ and $g_{2}$.

If we look at these relations, we can begin to understand the motivation for the Operator Product Expansion. The structure functions $g_{1}$ and $g_{2}$ can be experimentally measured, as we will see in Section 1.4.2, but only with a limited amount of precision. By integrating the structure function data (in essence, just summing over the bins in $x$ ), one can greatly reduce the relative size of the error bars, providing information about the matrix coefficients, which in turn yield additional information about the $Q^{2}$ evolution of the structure functions.

The term $a_{n}$ contains the twist-2 operator sum contribution, while $d_{n}$ is the matrix element of the sum of all twist-3 operators contributing to the $n$th moment of $g_{2}$ [22]. ${ }^{29}$ It is possible to incorporate scaling violations (Section 1.2.4) and even non-perturbative phenomena into the OPE to derive additional sum rules; these sum rules involve higher twist $(\mathrm{HT})$ matrix elements, and, to leading order, can be shown to give the same results as the DGLAP equations (Section 1.2.4). Due to the enormous complexity of the involved calculations, detailed derivations of higher-twist and nonperturbative effects are not possible here; we do however, summarize some of these sum rules in the following sections.

\footnotetext{
${ }^{29}$ The expansion for $g_{1}$ and the unpolarized structure function includes only the base (twist-2) expansion terms; the twist-3 term is included in the expansion for $g_{2}$ because it is not suppressed by inverse powers of $Q^{2}$, as generalized in Eq. 1.135, due to complications introduced by transverse polarization terms and "off-shell" finteracting partons [22]. More light is shed on the behavior of $g_{2}$ in Section 1.3.2.
} 


\subsubsection{First moment of $g_{1}$ and its sum rules}

Given a configuration of a target and beam with parallel spin orientation, $g_{1}$ is the easier of the two spin structure functions to measure. It also has the simpler interpretation in the parton model, and can be expressed to leading order exclusively in terms of twist-2 operators. The first moment of $g_{1}$ for the proton

$$
\Gamma_{1}^{p}\left(Q^{2}\right)=\int_{0}^{1} d x g_{1}\left(x, Q^{2}\right)
$$

is the dominant term in the Operator Product Expansion of Eq. 1.157. In this section we discuss some of the sum rules that apply specifically to $\Gamma_{1}^{p}$, and help illuminate the expected behavior of the $g_{1}$ structure function at differing magnitudes of $Q^{2}$. The most important of these rules are the GDH Sum Rule, which constrains the behavior of $\Gamma_{1}^{p}$ as $Q^{2} \rightarrow 0$, and the Bjorken Sum Rule, which dictates the behavior of the moment as $Q^{2} \rightarrow \infty$.

\section{The Gerasimov-Drell-Hearn Sum Rule}

The Gerasimov-Drell-Hearn (GDH) Sum Rule takes advantage of the fact that the cross-section of the scattering of a photon from a nucleon can be equivalently expressed in terms of the scattering amplitude $\mathscr{M} \equiv f(\nu)$ of forward Compton scattering, as shown in Eq. 1.125. This is really a statement of the optical theorem [12],

$$
\sigma_{\gamma N}(\nu)=\frac{4 \pi}{\nu} \operatorname{Im} f(\nu)
$$

As shown in Figure 1.5, the nucleon can have a final spin of $\frac{1}{2}$ or $\frac{3}{2}$, so that we can write the optical theorem in terms of either final spin-dependent cross-section:

$$
\sigma_{\gamma N \frac{1}{2}, \frac{3}{2}}(\nu)=\frac{4 \pi}{\nu} \operatorname{Im} f_{\frac{1}{2}, \frac{3}{2}}(\nu)
$$

The total Compton amplitude transition matrix can be expressed in terms of the initial (final) photon polarization $\vec{\varepsilon}(\vec{\varepsilon})$ and initial (final) electron spinors $\chi_{i}\left(\chi_{f}\right)$ as [12]

$$
T=8 \pi M \chi_{f}^{\dagger}\left[f(\nu) \vec{\varepsilon} \cdot \vec{\varepsilon}^{*}-i g(\nu) \vec{\sigma} \cdot\left(\vec{\varepsilon} \times \vec{\varepsilon}^{* *}\right)\right] \chi_{i}
$$


where $f$ is the spin-independent amplitude, and $g$ is the spin-flip amplitude, and $\vec{\sigma}$ represents the standard $2 \times 2$ Pauli spin matrices. The amplitudes for the two spin cases can then be written

$$
f_{\frac{1}{2}}=f+g \quad ; \quad f_{\frac{3}{2}}=f-g
$$

At low photon energies (that is, $Q^{2} \rightarrow 0$ ), where the photon scatters coherently from the proton on its (zero) mass shell, the amplitudes $f$ and $g$ can be expanded in powers of $\nu$ :

$$
\begin{gathered}
f(\nu)=-\frac{e^{2}}{4 \pi M}+\left(\alpha_{E}+\beta_{M}\right) \nu^{2}+\mathscr{O}\left(\nu^{4}\right) \\
g(\nu)=-\frac{e^{2} \kappa_{P}^{2}}{8 \pi M^{2}} \nu+\gamma_{0} \nu^{3}+\mathscr{O}\left(\nu^{5}\right)
\end{gathered}
$$

Here $\kappa_{P} \approx 1.79$ is the anomalous magnetic moment of the proton. Three new terms are introduced - the electric and magnetic polarizabilities $\alpha_{E}$ and $\beta_{M}$, and $\gamma_{0}$, the forward spin polarizability. Calculation of forward spin polarizability is an important application of $g_{1}$ moments, discussed more at the end of this thesis in Section 8.4.1.

The GDH Sum Rule is a dispersion relation, which, like the derivation of the OPE expansion in terms of structure function moments (Eq. 1.153), exploits the analyticity of forward Compton scattering, utilizing Cauchy's theorem [23]

$$
F(\nu)=\int \frac{\operatorname{lm} F\left(\nu^{\prime}\right)}{\nu-\nu^{\prime}} d \nu^{\prime}
$$

along with uniformity and crossing symmetry, that is

$$
g(\nu)=-g(-\nu)
$$

By applying Cauchy's theorem to Eq. 1.169 (working to order $\nu$ only, as we assume $Q^{2} \rightarrow 0$ ), one can derive the basic form of the Gerasimov-Drell-Hearn Sum Rule:

$$
\frac{\alpha}{M^{2}} \kappa_{P}^{2}=-\frac{4}{\pi} \int_{\nu_{t h}}^{\infty} \frac{d \nu}{\nu^{2}} \operatorname{lm} g(\nu)=\frac{1}{2 \pi^{2}} \int_{\nu_{t h}}^{\infty} \frac{d \nu}{\nu}\left[\sigma_{\frac{3}{2}}(\nu)-\sigma_{\frac{1}{2}}(\nu)\right]
$$


where $\nu_{t h}$ marks the inelastic (pion production) threshold. We see from Eq. 1.100 and the caption of Figure 1.5 that the $g_{1}$ structure function can be expressed in terms of

$$
g_{1} \sim \sigma^{\dagger \Downarrow}-\sigma^{\dagger \Uparrow}=\sigma^{\frac{1}{2}}-\sigma^{\frac{3}{2}}
$$

so that we can write the GDH Sum Rule in terms of the $g_{1}$ structure function. Making a conversion of the integration variable from $\nu$ to $x$, the result ${ }^{30}$ for this substitution is [22]

$$
-\frac{\kappa_{P}^{2}}{4} \equiv I_{P}(0)=2 \frac{M^{2}}{Q^{2}} \int_{0}^{x_{t h}} g_{1}\left(x, Q^{2}\right) d x
$$

It is important to note that the expansion of Eq. 1.169 is used in deriving this sum rule, so that it only holds exactly at $Q^{2}=0$, where the virtual photon becomes a real photon. However, since the right side of Eq. 1.174 is just ( $2 \frac{M^{2}}{Q^{2}}$ times) the first moment of $g_{1}$, this relation can be used to constrain the behavior of the $\Gamma_{1}^{p}$ as $Q^{2} \rightarrow 0$. Specifically, we see

$$
\Gamma_{1}^{p}\left(Q^{2} \rightarrow 0\right)=-\frac{1}{8} \kappa_{P}^{2} \frac{Q^{2}}{M^{2}}=-\frac{0.456}{\mathrm{GeV}^{2}} Q^{2}
$$

so that we expect $\Gamma_{1}^{p}(0)=0$ with a slope of $-0.456 / \mathrm{GeV}^{2}$ as $Q^{2} \rightarrow 0$.

While this summarizes the basic application of the GDH sum rule to the low $Q^{2}$ behavior of the moment $\Gamma_{1}^{p}$, before moving on, we mention the Generalized $G D H$ integral. A generalization of the GDH Sum Rule, applicable beyond the $Q^{2} \rightarrow 0$ limit, has been suggested by Ji and Osbourne [24]. A generalized dispersion relation

$$
S_{1}\left(\nu, Q^{2}\right)=4 \int_{Q^{2} / 2 M}^{\infty} \frac{\nu^{\prime} d \nu^{\prime} G_{1}\left(\nu^{\prime}, Q^{2}\right)}{\nu^{\prime 2}-\nu^{2}}
$$

can be defined containing the $G_{1}=g_{1} /(M \nu)$ polarized response function (see Eq. 1.85). As we will see later in this thesis, $g_{1}$ (and hence $G_{1}$ ) can be measured experimentally, while $S_{1}$ can be

\footnotetext{
${ }^{30}$ Properly, a recalculation involves dividing out the contribution to $g_{1}$ from the unpolarized cross-section via recalculation of the dispersion relation with the Kramers-Kronig relation for the proper amplitude containing both $g(\nu)$ and $f(\nu)$. This is not done in detail here; the objective in this thesis is merely to demonstrate the relation between the quantities.
} 
expressed theoretically, by expanding it into moments in small $\nu$ as [23]

$$
S_{1}\left(\nu, Q^{2}\right)=\sum_{n=0,2, \mathbf{4}, \ldots} \nu^{n} S_{1}^{(n)}\left(Q^{2}\right)
$$

for which the dispersion relation can be written

$$
S_{1}^{(n)}\left(\nu, Q^{2}\right)=4 \int_{Q^{2} / 2 M}^{\infty} \frac{d \nu}{\nu^{n+1}} G_{1}\left(\nu, Q^{2}\right)
$$

The low $Q^{2}$ region is amenable to the methods of chiral perturbation theory $(\chi \mathrm{PT})$, which has been used to fourth order [24] to find

$\bar{S}_{1}\left(0, Q^{2}\right)=-\frac{\kappa_{P}^{2}}{M^{2}}+\frac{g_{A}^{2}}{12\left(4 \pi f_{\pi}\right)^{2} M m_{\pi}}\left(1+3 \kappa_{V}+2\left(1+3 \kappa_{S}\right) \tau^{3}\right) Q^{2}+\cdots \approx-\kappa_{P}^{2}+24 Q^{2}\left(\right.$ in GeV $\left.^{2}\right)+\cdots$

thus providing a method of extending the predictions of a generalized GDH integral to virtual Compton scattering for finite $Q^{2}$, for $Q^{2} \lesssim 0.1 \mathrm{GeV}^{2}$. ${ }^{31}$

\section{The Bjorken Sum Rule}

While the GDH Sum Rule governs the behavior of $\Gamma_{1}^{p}$ at low $Q^{2}$, the Bjorken Sum Rule is a relation that governs the behavior of $\Gamma_{1}^{p}$ at high $Q^{2}$. The derivation of the Bjorken Sum Rule is simple. We start with the definition of $g_{1}^{p}$ in the scaling region $\left(Q^{2} \rightarrow \infty\right)$ in Eq.1.101. If we consider the same structure function for the neutron, $g_{1}^{n}$, which, by isospin symmetry, is identical to the proton in quark structure, except that

$$
u u d \rightarrow u d d
$$

then $g_{1}^{p}$ and $g_{1}^{n}$, in terms of quark wave functions, should be identical on exchange of $\Delta u$ and $\Delta d$, such that

$$
g_{1}^{n}\left(x, Q^{2}\right)=\frac{1}{18} \Delta u\left(x, Q^{2}\right)+\frac{4}{18} \Delta d\left(x, Q^{2}\right)+\frac{1}{18} \Delta s\left(x, Q^{2}\right)
$$

\footnotetext{
${ }^{3 t}$ In this equation, $\bar{S}$ simply denotes $S$ without elastic event contributions; $g_{A}=1.26$ is the axial coupling constant, $\kappa_{V}=1.85$ and $\kappa_{A}=-0.06$ are the isovector and isoscalar anomalous magnetic moments, $f_{\pi}=92.4 \mathrm{MeV}$ is the piondecay coupling coefficient, and $m_{\pi}=137 \mathrm{MeV}$ is the pion mass.
} 
Integrating over $x$, one gets for the first moments (as $Q \rightarrow \infty$ ):

$$
\begin{aligned}
& \Gamma_{1}^{p}\left(Q^{2}\right)=\frac{4}{18} \Delta u\left(Q^{2}\right)+\frac{1}{18} \Delta d\left(Q^{2}\right)+\frac{1}{18} \Delta s\left(Q^{2}\right) \\
& \Gamma_{1}^{n}\left(Q^{2}\right)=\frac{1}{18} \Delta u\left(Q^{2}\right)+\frac{4}{18} \Delta d\left(Q^{2}\right)+\frac{1}{18} \Delta s\left(Q^{2}\right)
\end{aligned}
$$

Subtracting these two moments yields the Bjorken Sum Rule:

$$
\Gamma_{1}^{p}-\Gamma_{1}^{n}=\frac{1}{6}(\Delta u-\Delta d)=\frac{1}{6} g_{A}
$$

The quantity $g_{A}=1.26$ is the axial vector coupling constant, which is well measured from $\beta^{-}$decay, $\beta^{-}$capture, and other weak phenomena [7]. It is easy to show that $g_{A}$ is given by $\Delta u-\Delta d$ by considering, for example, the axial vector component of the $\beta^{-}$capture reaction wave function, and equating the wavefunction of the whole nucleon with the same reaction over the constituent quarks [6]:

$$
g_{A}\left\langle n, s\left|\sigma^{3} \tau^{-}\right| p, s\right\rangle=\left\langle n, s\left|\sum_{q} \tau_{q}^{-} \sigma_{q}^{3}\right| p, s\right\rangle
$$

where the nucleon spin $s=\Uparrow$ or $\Downarrow$, and $\tau^{-}$is the isospin lowering operator, which converts $|p\rangle$ to $|n\rangle$, or $u$ to $d$, in the case of the quark isospin lowering operator $\tau_{q}^{-}$on the right hand side.

Let us pause a moment to consider the meaning of this equation. The left hand side tells us nothing about the quark structure of the nucleon; it just states that the axial amplitude in the electron capture reaction converts a proton to a neutron with an unknown amplitude $g_{A}$ that must be determined experimentally. In other words, the constant $g_{A}$ "soaks up" our ignorance of the constituent quark composition. The right side expresses the same quantity by operation of the isospin lowering operator over each quark wavefunction, which must sum to the same total value as the left side, if the picture of the nucleon made of constituent quarks is indeed correct.

The SU(2) isospin symmetry result $\left\langle n\left|\tau^{-}\right| p\right\rangle=\left\langle p\left|\tau^{3}\right| p\right\rangle$ can be used to write Eq. 1.185 as [6]

$$
g_{A}\left\langle p, s\left|\sigma^{3} \tau^{3}\right| p, s\right\rangle=\left\langle p, s\left|\sum_{q} \tau_{q}^{3} \sigma_{q}^{3}\right| p, s\right\rangle
$$


Operating on the right side of the equation, summing over both possible flavor/spin wavefunctions

$$
\begin{aligned}
& \tau_{q}^{3} \sigma_{q}^{3}|u \uparrow\rangle=|u \uparrow\rangle ; \tau_{q}^{3} \sigma_{q}^{3}|u \downarrow\rangle=-|u \downarrow\rangle \\
& \tau_{q}^{3} \sigma_{q}^{3}|d \uparrow\rangle=-|d \uparrow\rangle ; \tau_{q}^{3} \sigma_{q}^{3}|d \downarrow\rangle=|d \downarrow\rangle
\end{aligned}
$$

Adding these terms together and equating both sides of Eq. 1.186 gives

$$
g_{A}=|u \uparrow\rangle-|u \downarrow\rangle-|d \uparrow\rangle+|d \downarrow\rangle=\Delta u-\Delta d
$$

hence showing that the well known constant $g_{A}$ must equal $\Delta u-\Delta d$ in the Bjorken Sum Rule. In summary, this sum rule dictates that $\Gamma_{1}^{p}-\Gamma_{1}^{n}$ approaches a constant value as $Q^{2} \rightarrow \infty$, given by a constant, $g_{A}$, which can be measured by low energy experiments such as $\beta^{-}$decay.

Much as in the case of the GDH Sum Rule, the Bjorken Sum Rule can be generalized to the case of results at different $Q^{2}$. By introducing perturbative QCD (PQCD) effects, consisting of both radiative effects (of gluons, $q \bar{q}$ production, etc. by way of the DGLAP Equations (Section 1.2.4)) and higher twist corrections in the OPE expansion, a modified virtual Compton amplitude can be derived, and a dispersion relation for the modified Bjorken Sum Rule can be extracted [12][23]:

$$
\Gamma_{1}^{p}-\Gamma_{1}^{n}=\left[1-\frac{\alpha_{s}\left(Q^{2}\right)}{\pi}-3.583\left(\frac{\alpha_{s}\left(Q^{2}\right)}{\pi}\right)^{2}-20.215\left(\frac{\alpha_{s}\left(Q^{2}\right)}{\pi}\right)^{3}+\mathscr{O}\left(\alpha_{s}\left(Q^{2}\right)^{4}\right)\right] \frac{g_{A}}{6}
$$

Here $\alpha_{s}\left(Q^{2}\right)$ is the running coupling constant of QCD. In the region of $Q^{2} \sim 2-10 \mathrm{GeV}^{2}$, the theory has been shown to match experiment within $10 \%$ accuracy, prior to the EG1b experiment [12].

\section{Behavior of $\Gamma_{1}^{p}$ at intermediate $Q^{2}$}

At $Q^{2}$ values greater than about $0.2 \mathrm{GeV}^{2}$ (below which the generalized GDH Sum Rule and $\chi \mathrm{PT}$ theory are applicable) and less than about $1 \mathrm{GeV}^{2}$ (above which pQCD and the modified Bjorken Sum Rule are applicable), predictions by theory to physical QCD systems become extremely difficult. We do know, from the constraints enforced by the aforementioned sum rules, that somewhere in the range $0.2<Q^{2}<1.0 \mathrm{GeV}$ that the (negative) moment must make an upward turn and eventually (and gradually) flatten out at high $Q^{2}$, as scaling behavior sets in (see Figure 1.7). This 


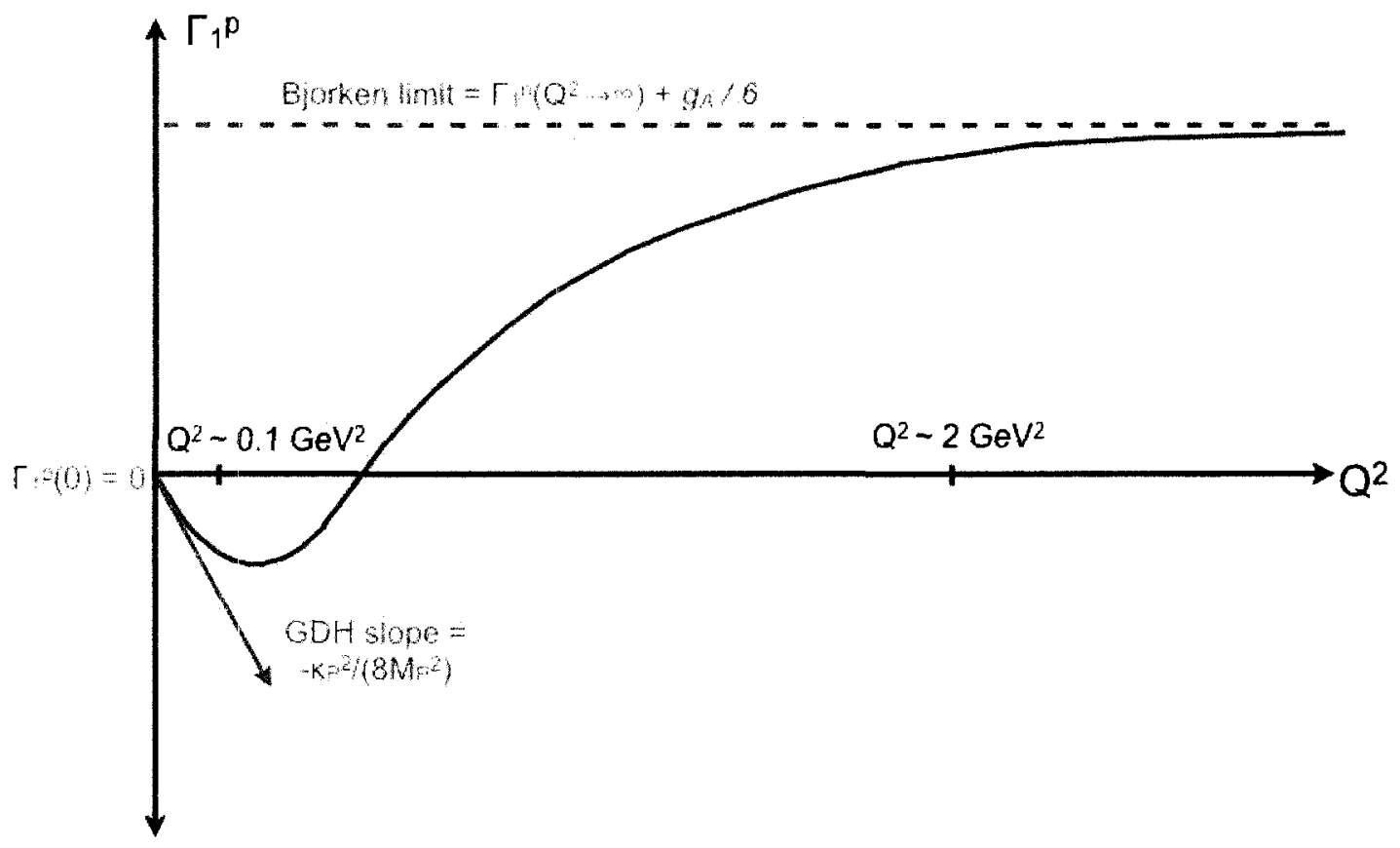

Figure 1.7: The expected evolution of the $\Gamma_{1}^{p}$ structure function moment (blue), as enforced by the GDH Sum Rule (low $Q^{2}$ ) and the Bjorken Sum Rule (high $Q^{2}$ ). See the text for details.

intermediate range is dominated by resonance behavior; aside from Lattice QCD computations, there is little that can be done at this point to directly compute the structure functions and their moments in this region, without the input of experimental data describing the structure of the resonances.

\subsubsection{Other moments of $g_{1}$}

The motivations for measuring the first moment of $g_{1}, \Gamma_{1}$, as a test of principles of QCD, including the GDH sum rule, $\chi \mathrm{PT}, \mathrm{PQCD}$ and Bjorken scaling behavior, are now clear. However, if we recall from the Operator Product Expansion (Eq. 1.157), there are higher moments of $g_{1}$ to consider, as well. In general, the $n$th moment of $g_{1}$ is given by

$$
\Gamma_{n}\left(Q^{2}\right)=\int_{0}^{1} d x x^{n-1} g_{1}\left(x, Q^{2}\right)
$$


In the light cone expansion, $\Gamma_{1}$ is guaranteed to make the dominant contribution to the OPE. $A$ complete reconstruction of the $Q^{2}$ evolution of $g_{1}$ to higher order naturally requires information regarding higher moments, as well, especially when higher twist effects are considered.

Before introducing the reconstruction of structure functions from their moments, it is important to discuss a very important consequence of the effects of crossing symmetry in the Operator Product Expansion. Namely, due to the optical theorem, the scattering of a virtual photon from a nucleon can be expressed in terms of Forward Compton Scattering (Eq. 1.124). The two leading order diagrams, shown in Eq. 1.125, are related by an interchange of photon lines, so that $q \rightarrow-q$ in the opposing diagram. ${ }^{32}$ Each moment in the OPE for the spin structure functions contains a factor of $x=Q^{2} /(2 p \cdot q)$, so that

$$
x \rightarrow-x
$$

in the OPE for the second leading order diagram. Thus, any OPE term containing a factor of an odd power of $x$ must cancel when the two diagrams are added. Thus, in Eq. 1.157,

$$
M_{1,2}^{(i)}=M_{1,4}^{(i)}=M_{1,6}^{(i)}=\ldots=0
$$

with a similar relation for even $n$ for $M_{2, n}^{(i)}$. Therefore, only odd moments of the spin structure functions need to be considered in the Operator Product Expansion. ${ }^{33}$

As previously pointed out, measurements of structure function integrals in $Q^{2}$ can be made with higher precision than their explicit dependence over both $Q^{2}$ and $x$. Of course, this integration destroys information about the shape of the resonances as a function of $x$. However, one can still reconstruct detailed information about the $Q^{2}$ evolution of $g_{1}$ through use of the inverse Mellin transform [12][25]:

$$
g_{1}\left(x, Q^{2}\right)=\int_{K-i \infty}^{K+i \infty} d n x^{1-n} \Gamma_{n}\left(Q^{2}\right)
$$

where $K$ is any real constant in the complex plane to the right of any singularities in the integrand. While this transformation preserves the analytic continuity of the structure function, naturally, it

\footnotetext{
${ }^{32}$ See also Eq. 1.171.

${ }^{33}$ This isn't explicitly shown here for higher-order diagrams, but the cancellation of even moments holds true to any order, since all possible diagrams in Forward Compton Scattering have two external photon lines that can be crossed.
} 
does not include the resonance structure in the reconstruction, because this information has been eliminated in the integration. However, this is enough information to apply the DGLAP equations, for example, to obtain information about the accuracy of pQCD regarding the $Q^{2}$ evolution of the structure functions [18][26]. In practice, the entire range $0 \leq x \leq 1$ cannot be completely known due to experimental limitations, especially as $x \rightarrow 0$ (where infinite beam energies would be needed), so truncated moments are used in the reconstruction:

$$
\Gamma_{n}\left(Q^{2}\right)=\int_{x_{0}}^{1} d x x^{n-1} g_{1}\left(x, Q^{2}\right)
$$

where $x_{0}$ is a suitable cutoff point chosen for the given $Q^{2}$ value. If we define the truncated moments of quark distribution functions as

$$
\underline{q}_{n} \equiv \int_{x_{0}}^{1} d x x^{n-1} q(x)
$$

then, using Eq. 1.182 , the moments of $g_{1}$ can be written in the parton model as

$$
\Gamma_{n}^{p}=\frac{4}{18} \underline{u}_{n}+\frac{1}{18} \underline{d}_{n}+\frac{1}{18} \underline{s}_{n}
$$

Looking now at individual quark distributions, the (abbreviated) DGLAP equations (Eq. 1.113) then imply

$$
\frac{d \underline{q}_{n}\left(x_{0}, t\right)}{d t}=\frac{\alpha_{s}(t)}{2 \pi} \int_{x_{0}}^{1} d x x^{n-1}(\Delta P \otimes q)(x, t)
$$

Using the general form of the DGLAP Equations, it can then easily be shown [18] that

$$
\int_{x_{0}}^{1} d x x^{n-1}(\Delta P \otimes q)(x)=\left(\left[z^{n} \Delta P(z)\right] \otimes \underline{q}_{n}\right)\left(x_{0}\right)
$$

so that Eq. 1.197 can be rewritten

$$
\frac{d \underline{q}_{n}\left(x_{0}, t\right)}{d t}=\frac{\alpha_{s}(t)}{2 \pi}\left(\left[z^{n} \Delta P(z)\right] \otimes \underline{q}_{n}\right)\left(x_{0}, t\right)
$$

The inverse Mellin transform of Eq. 1.193 can then be used to determine the quark distributions, including violations from scaling predicted by the evolution equations. In other words, even with a 
truncated moment, the $Q^{2}$ evolution of the DGLAP equations can be tested with an inverse Mellin transform of structure function moments.

Naturally, the more higher order moments that can be experimentally determined, the greater the precision to which PQCD theory can be verified. Due to the increasing magnitude of $x^{n-1}$ with each higher moment, higher $x$ values make up a larger proportion of the integral contribution as $n$ increases, making large-acceptance, medium-energy experiments like EG1b ideal for measuring the higher moments of $g_{1}$. The only limit imposed on the maximum value of $n$ is that of the decreasing magnitude of the higher moments, such that the proportion of systematic error (particularly due to kinematic bin smearing; see Section 7.2.6) eventually becomes too large for accurate measurement.

\subsection{4 $g_{2}$ and its Moments}

As mentioned before, a straightforward interpretation of $g_{2}$ in the parton model is problematic due to the difficulties involved in perpendicular polarization. ${ }^{34}$ We can, however, infer some behavior of this structure function by splitting it into components of differing twist. If we write

$$
g_{2}=g_{2}^{W W}+\bar{g}_{2}
$$

where $g_{2}^{W W}$, known as the Wandzura-Wilczek form of $g_{2}$, contains only leading twist (twist-2) contributions to the structure function, and $\bar{g}_{2}$ contains twist-3 (and higher) contributions, the OPE can be used to write (see Eqs. 1.161 and 1.162) [22]

$$
\int_{0}^{1} d x x^{n-1}\left[\frac{n-1}{n} g_{1}\left(x, Q^{2}\right)+g_{2}^{W W}\left(x, Q^{2}\right)\right]=0
$$

Inverting this equation [27] yields

$$
g_{2}^{W W}\left(x, Q^{2}\right)=-g_{1}\left(x, Q^{2}\right)+\int_{x}^{1} \frac{d y}{y} g_{1}\left(y, Q^{2}\right) \equiv-g_{1}\left(x, Q^{2}\right)+g_{T}^{W W}
$$

\footnotetext{
${ }^{34}$ Specifically, one can say that the perpendicular spin operator and free quark Hamiltonian operator do not commute.
} 
(with $y$ defined in Section 1.1.3), which is called the Wandzura-Wilczek relation. It is important to note that this equation considers only leading twist effects. The magnitude of the contribution of higher twist effects to $g_{2}$ is an unresolved question at this time; the matrix element $d_{n}\left(Q^{2}\right)$ in Eq. 1.162 represents the twist- 3 terms in the expansion. Thus, measurement of $g_{2}$ presents a potential probe of higher twist effects. It is customary to extract the matrix element [22]

$$
d_{n}\left(Q^{2}\right)=2 \int_{0}^{1} d x x^{n-1}\left[g_{1}\left(x, Q^{2}\right)+\frac{n}{n-1} g_{2}\left(x, Q^{2}\right)\right]=2 \frac{n}{n-1} \int_{0}^{1} d x x^{n-1} \bar{g}_{2}\left(x, Q^{2}\right)
$$

which provides a direct measurement of the deviation from the Wandzura-Wilczek relation.

Note that if higher twist effects are absent from $g_{2}$, that is, $g_{2}^{W W}=g_{2}$, then if we set $n=1$ in Eq. 1.201 , that

$$
\int_{0}^{1} d x g_{2}\left(x, Q^{2}\right)=0
$$

This is the Burkhardt-Cottingham Sum Rule. It obviously holds in the absence of twist-3 effects; though the proper derivation of this sum rule [28] requires considerably less restrictive conditions than the absence of higher-twist effects. Though it is not implied by the above (pseudo-)derivation, the only necessary conditions for this sum rule to hold true are analyticity, crossing symmetry, parity conservation and convergence of $g_{2}$ as $x \rightarrow 0,{ }^{35}$ even in the presence of higher-twist terms [29].

Measurements of $g_{2}^{p}$, at this present time, have limited accuracy for most values of $Q^{2}$, mostly due to experimental difficulties involved with large acceptance experiments involving transversely polarized targets. Reconstruction of the structure functions through an inverse Mellin transform offers potential utility in obtaining information regarding the $g_{2}$ structure function. Error bars on $g_{2}$ measurements (at most $Q^{2}$ values) are generally too large to obtain meaningful information regarding the resonance structure, but $Q^{2}$ evolution of transverse polarization elements using summed bins, as described in the previous section for $g_{1}$, can potentially be derived with this methodology. Existing measurements of $g_{2}^{p}$ are summarized in Section 1.5.

As a final note on the $g_{1}$ and $g_{2}$ structure functions, it important to point out that the measurement of $d_{n}$, as given by Eq. 1.203 , is also important for higher twist corrections to $g_{1}$ [22]. Specifically,

\footnotetext{
${ }^{35}$ The last of these assumptions is actually quite complex; a proper treatment of this issue requires an in-depth discussion of the limits of residue values in the far DIS region; see Ref. [29] for details.
} 
HT corrections to the OPE give

$$
\Gamma_{1}^{p}\left(Q^{2}\right)=\int_{0}^{1} d x g_{1}^{p}\left(x, Q^{2}\right)=\frac{1}{2} a_{1}+\frac{M^{2}}{9 Q^{2}}\left(a_{3}+4 d_{3}+4 f_{3}\right)+\mathscr{O}\left(\frac{M^{4}}{Q^{4}}\right)
$$

Here, a new matrix element $f_{n}$, which includes twist- 4 contributions, has been introduced. $f_{n}$ is defined by an explicit quark-gluon correlation operator. Thus, we see that the relation between $g_{1}$ and $g_{2}$ can not be completely separated, when all HT terms are considered. Measurement of both these structure functions is necessary for a complete QCD spin model of the nucleon.

\subsection{Asymmetries}

So far, the motivation for measuring spin-structure functions of the nucleon has been explained in some detail. However, it is yet to be explained how measurement of $g_{1}$ and $g_{2}$ can actually be extracted from experimental data. Section 1.2.1 gave an introduction to the meaning of crosssection measurements. In practice, direct measurement of cross-sections is not always the most practical approach to sensitive electroproduction measurements, because the overall acceptance ${ }^{36}$ of the spectrometer must be taken into account. Instead, asymmetries are often used instead, as they rely on a ratio of measured counts, and hence have no reliance on detector acceptance for their accuracy. ${ }^{37}$ The expression of results in terms of virtual photon asymmetries $A_{1}$ and $A_{2}$ also provides a more straightforward physical interpretation of data in terms of virtual photon exchange, and provides a link between the measured asymmetries and the extracted spin-structure functions.

\subsubsection{Measuring asymmetries}

As previously mentioned (e.g. Eq. 1.93 and 1.95), measurement of the spin-structure functions directly depends on the difference (in a given bin) between the scattering cross-sections with the

\footnotetext{
${ }^{36}$ Acceptance is defined as the ratio of measured events to physical events as a function of the free parameters (such as $E^{\prime}$ and $\theta$, or $x$ and $Q^{2}$ ).

${ }^{37}$ Measuring count ratios, however, does have the disadvantage of reducing statistical precision of measurements, since directly measuring a ratio of two statistical quantities results in a large relative statistical error [8]. For that reason, a very long running time (to acquire high statistics) was required tor the EG1 experiment.
} 
electron beam spin and target proton spin aligned parallel and anti-parallel:

$$
\Delta \sigma^{\uparrow \Downarrow}-\Delta \sigma^{\uparrow \Uparrow}
$$

This quantity can be measured directly; but, as mentioned, to avoid the need for acceptance corrections, it is easier to measure the quantity (previously defined in Eq. 1.94)

$$
A_{\| \mid}\left(x, Q^{2}\right)=\frac{\Delta \sigma^{\uparrow \Uparrow}-\Delta \sigma^{\uparrow \Downarrow}}{\Delta \sigma^{\uparrow \Uparrow}+\Delta \sigma^{\uparrow \Downarrow}}
$$

If $n$ is defined as the normalized rate of detected counts in a bin, then

$$
\Delta \sigma=\frac{n}{\text { acceptance }}
$$

making it easy to see that the acceptances cancel in Eq. $1.207^{38}$ so that

$$
A_{\|}\left(x, Q^{2}\right)=\frac{n^{-}\left(x, Q^{2}\right)-n^{+}\left(x, Q^{2}\right)}{n^{-}\left(x, Q^{2}\right)+n^{+}\left(x, Q^{2}\right)}
$$

with $n^{+}$and $n^{-}$representing the count rates for $\uparrow \uparrow$ and $\uparrow \Downarrow$ scattering events, respectively, defined as positive helicity and negative helicity events from this point forward. In practice, the counts for each helicity need to be normalized to both the amount of charge in the beam, as well as detector dead time, so that

$$
n^{ \pm}=\frac{N^{ \pm}}{F C_{g}^{ \pm}}
$$

with $N$ representing the actual number of detected particle hits and $F C_{g}$ representing the (helicitysorted) charge measured with the Faraday Cup device, gated to only include detector live time. The Faraday Cup operation and details are discussed later, in Section 2.3.4.

The quantity $A_{\|}$is referred to as the longitudinal double-spin asymmetry; it is the actual quantity directly measured in the EG1b experiment described in this thesis. As explained in Section 1.2 .1 , inclusive $e p$-scattering (at a constant beam energy) can be fully parametrized in terms of two

\footnotetext{
${ }^{38}$ This assumes, of course, that the detector has the same acceptance for both $\uparrow \Uparrow$ and $\uparrow \Downarrow$ scattering events. Acceptance is dependent only on detector geometry and efficiency, which depend only on scattered particle type, energy and location/direction, so this is a valid assumption.
} 
variables; in practice, it is actually

$$
A_{\|}\left(\Delta W, \Delta Q^{2}\right)=\frac{n^{-}\left(\Delta W, \Delta Q^{2}\right)-n^{+}\left(\Delta W, \Delta Q^{2}\right)}{n^{-}\left(\Delta W, \Delta Q^{2}\right)+n^{+}\left(\Delta W, \Delta Q^{2}\right)}
$$

that is measured for each beam energy in a two-dimensional bin array, with desired variable translations (using the equations in Section 1.1.3) being made in later analysis.

Another often-mentioned quantity is the transverse double-spin asymmetry

$$
A_{\perp}\left(x, Q^{2}\right)=\frac{\Delta \sigma^{\uparrow \Rightarrow}-\Delta \sigma^{\uparrow \Leftarrow}}{\Delta \sigma^{\uparrow \Rightarrow}+\Delta \sigma^{\uparrow \Leftarrow}}
$$

which provides much more information regarding transverse polarization effects (and thus, $g_{2}$ ) than the longitudinal double-spin asymmetry. The CLAS detector configuration is not currently suited for a perpendicularly polarized target, due to the magnetic field configuration [30], so this quantity is defined here for completeness and comparison purposes only.

In addition to double-spin asymmetries, it is obviously possible to measure single-spin asymmetries,

$$
A_{t}=\frac{\Delta \sigma^{\Uparrow}-\Delta \sigma^{\Downarrow}}{\Delta \sigma^{\Uparrow}+\Delta \sigma^{\Downarrow}}
$$

Parity-violating quantities, such as the electroweak asymmetry, take this form [31]. In order to minimize contamination effects from possible single-spin asymmetries, both the beam and target spins are periodically reversed. ${ }^{39}$ This causes a simple sign cancellation of most of the effects of the single-spin asymmetry, so that only the relative orientation of the beam and target polarization has any effect on the asymmetry measurement.

\subsubsection{Asymmetries to polarized structure functions}

\section{The virtual photon asymmetry $A_{1}$}

We now discuss how a measured double spin asymmetry $\left(A_{\|}\right.$or $\left.A_{\perp}\right)$ can be used to calculate the spin structure functions $g_{1}$ and $g_{2}$. As already shown in Figure 1.5 and Section 1.2.3, in the Breit

\footnotetext{
${ }^{39}$ In EG1, the beam polarization oscillates at $\sim 30 \mathrm{~Hz}$, while the target polanization is switched over a period of hours or days; see the next chapter for more details.
} 
frame, we can interpret the longitudinal asymmetry as the asymmetry in cross-sections between final spin- $\frac{1}{2}$ and $\frac{3}{2}$ hadronic states. As required by angular momentum conservation, this is really just an asymmetry between spin +1 and spin -1 virtual photons. Thus, we define the virtual photon spin asymmetry [6]

$$
A_{1}\left(\gamma^{*}\right) \equiv \frac{\sigma_{T}^{\frac{1}{2}}\left(\gamma^{*}\right)-\sigma_{T}^{\frac{3}{2}}\left(\gamma^{*}\right)}{\sigma_{T}^{\frac{1}{2}}\left(\gamma^{*}\right)+\sigma_{T}^{\frac{3}{2}}\left(\gamma^{*}\right)}
$$

The subscript $T$ implies that the cross-section only includes transversely polarized (i.e. spin \pm 1 ) virtual photons, because longitudinal virtual photon polarizations cannot excite the final desired states. ${ }^{40}$ Eqs. 1.97 and 1.98 imply

$$
A_{\|}\left(x, Q^{2}\right) \propto A_{1}\left(x, Q^{2}\right)+\mathscr{O}\left(\frac{1}{\nu}\right)
$$

meaning that $A_{\|} \propto A_{1}$ in the scaling region of $Q^{2} \rightarrow \infty$. Recalling from Eq. 1.94 that $A_{\|}$is ratio of polarized to unpolarized cross-sections, we refer to the equation for the DIS unpolarized crosssection, Eq. 1.43. Recalling that the transverse contribution to the cross-section is given by $W_{1}$, and that $R=W_{L} / W_{1}$ (Eq. 1.65), this unpolarized cross-section can be rewritten as [6]

$$
\frac{\Delta \sigma}{\Delta Q^{2} \Delta \nu}=\frac{4 \pi \alpha^{2} E^{\prime} \cos ^{2}(\theta / 2)}{Q^{4} E} \frac{W_{1}\left(Q^{2}, \nu\right)}{\epsilon(1+\tau)}\left[1+\epsilon R\left(Q^{2}, \nu\right)\right]
$$

The quantity in square brackets, then, is the ratio of the total cross-section to the transverse term (i.e. $W_{1}$ only) cross-section. Using the definitions of Eqs. 1.94 and 1.214, the proportionality constant in Eq. 1.215, defined $D$, is given by

$$
D=\frac{\sigma_{T}}{\sigma_{\text {unpolarized }}} \times \frac{\sigma^{\dagger \Downarrow}-\sigma^{\dagger \Uparrow}}{\sigma_{T}^{\frac{1}{2}}\left(\gamma^{*}\right)-\sigma_{T}^{\frac{3}{2}}\left(\gamma^{*}\right)}
$$

The first of these two cross-section ratios, the ratio of the total transversely polarized virtual photon cross-section to the total cross-section, is simply $(1+\epsilon R)^{-1}$, as dictated by Eq. 1.216. The second ratio can be interpreted as the inverse of the helicity fraction transferred from the electron to the virtual photon [6]; it can be calculated, in the scaling region (where the virtual photon and polarization

\footnotetext{
${ }^{40}$ Recall that virtual photons are not constrained to transverse polarizations, unlike their real counterparts.
} 
directions are parallel, as in Figure 1.5) to be $\sqrt{1-\epsilon^{2}}$, where $\epsilon$, given by Eq. 1.16, is the ratio of longitudinal to transverse polarization of the virtual photon [12]. This relation is not proven explicitly here, but this can be seen to make logical sense, in that all helicity is transferred in the limit of a purely transverse virtual photon $(\epsilon=0)$.

Therefore, Eq. 1.215 becomes [6]

$$
A_{\|}\left(x, Q^{2}\right)=\frac{\sqrt{1-\epsilon^{2}}}{1+\epsilon R}\left[A_{1}\left(x, Q^{2}\right)+\mathscr{O}\left(\frac{1}{\nu}\right)\right] \quad \text { (scaling region) }
$$

One can see that, for large $\nu$, that the longitudinal asymmetry is given completely in terms of $A_{1}$. However, at lower $Q^{2}$ (and hence lower $\nu$ ), we expect other contributions to be made.

\section{The virtual photon asymmetry $A_{2}$}

Basically, the reason the assumption that $A_{\|} \propto A_{1}$ works in the scaling region is because, in this case, the angle of the virtual photon $\theta^{*}$ (with respect to the polarizations) is always zero. That is, the direction of virtual photon exchange vector is aligned parallel to the electron and nucleon spins. At higher $x$, leaving the DIS region, this is, of course, not always the case; the virtual photon vector can have any angle $\theta^{*}$ with the polarization.

In the case of nonzero $\theta^{*}$, the $A_{1}$ contribution to $A_{\|}$naturally must be multiplied by $\cos \left(\theta^{*}\right)$. This, however, is not the end of the story, as there are still $\mathscr{O}(1 / \nu)$ terms, no longer unimportant, with which to deal. This contribution can be looked at as an "interference" between the longitudinal and transverse photon polarization cross-sections, which we can define as $\sigma_{L T}$. Simple orthogonality requires that

$$
\sigma_{L T}^{2} \leq \sigma_{L} \sigma_{T}
$$

We now define a second asymmetry

$$
A_{2}\left(\gamma^{*}\right) \equiv \frac{\sigma_{L T}}{\sigma_{T}}=\frac{2 \sigma_{L T}}{\sigma_{T}^{\frac{1}{2}}\left(\gamma^{*}\right)+\sigma_{T}^{\frac{3}{2}}\left(\gamma^{*}\right)}
$$


Eqs. 1.65 and 1.219 enforce the constraint ${ }^{41}$

$$
\left|A_{2}\right| \leq \sqrt{R}
$$

The higher order term in $A_{\|}$requires the familiar factor of $(1+\epsilon R)^{-1}$ to divide out the longitudinal cross-section contributions; the relation to $A_{2}$ depends on transverse virtual terms, so instead of $\cos \left(\theta^{*}\right), \mathrm{a} \sin \left(\theta^{*}\right)$ factor is needed, as well as a different helicity transfer factor of $\sqrt{2 \epsilon(1-\epsilon)}$. Adding in the higher order term, the complete expression for $A_{\|}$becomes [6]

$$
A_{\|}\left(E, x, Q^{2}\right)=\sqrt{1-\epsilon^{2}} \cos \left(\theta^{*}\right) \frac{A_{1}\left(\gamma^{*}\right)}{1+\epsilon R}+\sqrt{2 \epsilon(1-\epsilon)} \sin \left(\theta^{*}\right) \frac{A_{2}\left(\gamma^{*}\right)}{1+\epsilon R}
$$

It is conventional to simplify this equation to

$$
A_{\|}\left(E, x, Q^{2}, E\right)=D\left[A_{1}\left(x, Q^{2}\right)+\eta A_{2}\left(x, Q^{2}\right)\right]
$$

where $D$ is, for reasons that are now apparent, called the depolarization factor, and $\eta$ is a (beam energy-dependent) kinematic factor. Through kinematics calculations in the lab frame, these quantities can be simplified to the forms shown in Eqs. 1.17 and 1.19.

It is essential to note that the double-spin asymmetry $A_{\|}$is expressed in terms of 3 unknown parameters: $A_{1}, A_{2}$, and $R$. In practice, if $A_{||}$is the measured experimental quantity, two of these three must generally be approximated by models to extract the third quantity. For the kinematic region of interest in this experiment (that is, the resonance region), $\epsilon R$ and $\eta A_{2}$ are relatively small quantities compared to $A_{1} .{ }^{42}$ Therefore, $A_{\|}$measurements serve as a good measurement for $A_{1}$ :

$$
A_{1}=\frac{A_{\|}}{D}-\eta A_{2}=\frac{A_{\|}(1+\epsilon R)}{1-\epsilon E^{\prime} / E}-\eta A_{2}
$$

where models, evaluated in terms of $Q^{2}$ and $W$, are used to evaluate the (small) contributions from $R$ and $A_{2}$.

\footnotetext{
${ }^{41}$ In the elastic region, we have $A_{1 e l}=1$ and $A_{2 e l}=\sqrt{R_{e l}}=G_{E} /\left(\sqrt{\tau} G_{M}\right)$. These equations are useful for calculating $A_{1 \mid}$ for elastic scattering events, and are used later in Section 6.2 .

${ }^{42}$ In particular, $\epsilon \ll 1$ because $1 / \gamma^{2}+Q^{2} /(2 M x)^{2}$ is large. Then $\eta \ll 1$ because $\eta \propto \epsilon \sqrt{Q^{2}}$.
} 
However, the variables $A_{1}$ and $A_{2}$ are not entirely uncorrelated, of course, as can be inferred through the presence of the $\mathscr{O}(1 / \nu)$ term in Eq. 1.215. The correlation in the higher-order terms can be exploited to solve for both these asymmetries simultaneously (while modeling only $R$ ), but with very limited precision. The asymmetries $A_{1}$ and $A_{2}$ can be evaluated completely in terms of virtual Compton scattering, as will be be discussed momentarily. This implies that $A_{1}$ and $A_{2}$ are functions of $Q^{2}$ and $\theta^{*}$ (the virtual photon magnitude and angle) only, as these are the only free parameters involved in the $\gamma^{*} p$ scattering. Using the relations in Section 1.1.3, these asymmetries can also (equivalently) be parametrized completely by $Q^{2}$ and $x{ }^{43}$ Looking at Eq. 1.17 (as well as 1.7 and 1.16), on the other hand, we see that $\eta$, a purely kinematic parameter, requires knowledge of three independent parameters, $E, E^{\prime}$ and $\theta$, or, equivalently, $E, Q^{2}$ and $x$. This means we can write Eq. 1.224 as

$$
\frac{A_{\|}}{D}\left(E, Q^{2}, x\right)=A_{1}\left(Q^{2}, x\right)+\eta\left(E, Q^{2}, x\right) A_{2}\left(Q^{2}, x\right)
$$

Thus, inside of a particular kinematic $\Delta Q^{2}, \Delta x$ bin, one expects $A_{1}$ and $A_{2}$ to be constant, while $A_{\|} / D$ varies linearly with $\eta$, with $\eta$ depending only on beam energy. By measuring $A_{\|} / D$ at different beam energies, one can plot $A_{\|} / D$ vs. $\eta$ and solve for $A_{1}$ and $A_{2}$ for that $\Delta Q^{2}, \Delta x$ bin by linear regression:

$$
A_{1}=y \text {-intercept }\left[\frac{A_{\|}}{D}(\eta)\right]
$$

and

$$
A_{2}=\text { slope }\left[\frac{A_{\| I}}{D}(\eta)\right]
$$

Naturally, because this is a bin-by-bin measurement method, with no assumptions about the analyticity of the functions $A_{1}\left(Q^{2}, x\right)$ and $A_{2}\left(Q^{2}, x\right)$ going into the calculation, large statistical error bars are expected. The best possible measurements of $A_{1}$ and $A_{2}$ require an iterative method of using models and linear regression measurements. This issue is dealt with in Section 8.1.3. Of course, measurement of $A_{\perp}$ would provide more exact measurements of $A_{2}$. The analogous relation to Eq. 1.223 is

$$
A_{\perp}\left(E, x, Q^{2}\right)=d\left[A_{1}\left(x, Q^{2}\right)+\zeta A_{2}\left(x, Q^{2}\right)\right]
$$

\footnotetext{
${ }^{43}$ The variables $Q^{2}$ and $W$ can be used, too, as is done for most of the duration of this analysis
} 
However, as previously stated, CLAS is not configured to measure $A_{\perp}$, so the linear regression method must be used if any information regarding $A_{2}$ is to be obtained from the EG1 experiment.

\section{Extraction of spin-structure functions from $A_{1}$ and $A_{2}$}

Now, the task remains to convert the virtual photon asymmetries $A_{1}$ and $A_{2}$ into the structure functions $g_{1}$ and $g_{2}$. To do this (and to properly derive Eq. 1.222, as well), one must solve for the relation between the hadronic ep tensor $W_{\mu \nu}$ in terms of the virtual photon polarization asymmetries $A_{1}$ and $A_{2}$ to obtain explicit solutions of $\sigma_{T}^{\frac{1}{2}}, \sigma_{T}^{\frac{3}{2}}$ and $\sigma_{L T}$ in terms of $g_{1}$ and $g_{2}$. This can be done by again using the optical theorem [12] $]^{44}$

$$
2 \pi M W_{\mu \nu}\left(\nu, Q^{2}\right)=\operatorname{lm} T_{\mu \nu}\left(\nu, Q^{2}\right)
$$

where $T_{\mu \nu}$ is the amplitude tensor for virtual Compton scattering, as described previously in this thesis. The virtual photon cross-sections are calculated in terms of $g_{1}$ and $g_{2}$. The results are [12][22]

$$
\begin{gathered}
\sigma_{T}^{\frac{1}{2}}-\sigma_{T}^{\frac{3}{2}}=\frac{8 \pi^{2} \alpha}{M \nu^{*}}\left[g_{1}\left(x, Q^{2}\right)-\gamma^{2} g_{2}\left(x, Q^{2}\right)\right] \\
\sigma_{L T}=\frac{8 \pi^{2} \alpha}{M \nu^{*}} \gamma\left[g_{1}\left(x, Q^{2}\right)+g_{2}\left(x, Q^{2}\right)\right] \\
\sigma_{T}=\frac{1}{2}\left(\sigma_{T}^{\frac{1}{2}}+\sigma_{T}^{\frac{3}{2}}\right)=\frac{8 \pi^{2} \alpha}{M \nu^{*}} F_{1}\left(x, Q^{2}\right)
\end{gathered}
$$

Eq. 1.230 contains only polarized structure functions, because a subtraction of the cross-section terms cancels the symmetric (unpolarized) components of transition amplitude. Conversely, Eq. 1.232 contains only a single unpolarized structure function, because an addition of the crosssection terms cancels the antisymmetric (polarized) components of the transition amplitude. The simple form of Eq. 1.232 should come as no surprise, since $F_{1}=M W_{1}$ represents a purely transverse virtual photon amplitude (see Section 1.2.2).

The variable $\nu^{*}$ in the above equations is known as the equivalent photon energy; it is the nor-

\footnotetext{
${ }^{44}$ Two other interpretations of this theorem are applied in discussion of the OPE and GDH sum rule - graphically in Eq 1.124 and algebraically in Eq. 1.164.
} 
malization factor for the virtual photon spectrum. ${ }^{45}$ Its value, a function of kinematics, is a matter of convention. More detail on its use is given later, in the derivation of forward spin polarizability in Section 8.4.1.

Inserting these cross-sections into $A_{1}$ (Eq. 1.214) and $A_{2}$ (Eq. 1.220) yields

$$
\begin{aligned}
& A_{1}\left(x, Q^{2}\right)=\left[g_{1}\left(x, Q^{2}\right)-\gamma^{2} g_{2}\left(x, Q^{2}\right)\right] / F_{1}\left(x, Q^{2}\right) \\
& A_{2}\left(x, Q^{2}\right)=\gamma\left[g_{1}\left(x, Q^{2}\right)+g_{2}\left(x, Q^{2}\right)\right] / F_{1}\left(x, Q^{2}\right)
\end{aligned}
$$

The value of $\gamma^{2}=4 E^{\prime} E /\left(E-E^{\prime}\right)^{2} \sin ^{2}(\theta / 2)=4 M^{2} x^{2} / Q^{2}$ is small for deep inelastic kinematics, so that $g_{2}$ makes very little contribution to $A_{1}$ for DIS. Solving in terms of the structure functions yields

$$
\begin{aligned}
& g_{1}\left(x, Q^{2}\right)=\frac{1}{\gamma^{2}+1}\left[A_{1}\left(x, Q^{2}\right)+\gamma A_{2}\left(x, Q^{2}\right)\right] F_{1}\left(x, Q^{2}\right) \\
& g_{2}\left(x, Q^{2}\right)=\frac{1}{\gamma^{2}+1}\left[\frac{1}{\gamma} A_{2}\left(x, Q^{2}\right)-A_{1}\left(x, Q^{2}\right)\right] F_{1}\left(x, Q^{2}\right)
\end{aligned}
$$

which can be used to derive the polarized structure functions from the virtual photon asymmetries.

\subsubsection{Behavior of $A_{1}$ in the resonance region}

As well as providing a method of measurement for the polarized structure functions, the virtual photon asymmetries also provide direct information about the behavior of resonances within the nucleon. Asymmetry measurements in the medium to high $x$ region also provide valuable quantitative tests of QCD models.

Resonance region behavior is of great interest, because of the relative dearth of experimental data in this region (see Section 1.5), the fact that it serves as a "bridge" between the "well-behaved" realms of $\chi \mathrm{PT}$ and $\mathrm{pQCD}$ modeling where the physics behavior cannot be well-predicted, and the fact that resonances exhibit physics which cannot be accessed by DIS measurements.

To investigate resonance structure, it is best to use the Lorentz invariant missing mass $W$, in place of $x$, as it provides a clearer scale in the resonance region. In the context of ep scattering,

\footnotetext{
${ }^{45}$ Many sources (e.g. Ref. [6]) use the notation $K$ in place of $\nu^{*}$.
} 
a resonance is basically a temporary excitation, or new particle state, that arises due to the impartation of energy and angular momentum. We know that that several such excitations exist for the range $1.08 \mathrm{GeV}<W<2.0 \mathrm{GeV}$. Below $W=1.08 \mathrm{GeV}$, no such excitations are possible, because $M+m_{\pi}=1.08 \mathrm{GeV}$, and pions are the lightest particle that can be emitted from a resonance decay. Above $W=2.0 \mathrm{GeV}$, DIS dynamics dominate, as the excitations blur into a (nearly) constant cross-section, where excitations of individual quarks and gluons dominate the spectrum.

Intuitively, one might expect that, since we observe discrete mass and angular momentum states of final particles, that sharp, $\delta$-function resonances would be observed, limited only in resolution by the precision of the spectrometer. However, we know from the uncertainty principle $\Delta E \Delta t \geq \hbar$ that the energy (and hence missing mass) resolution $\Gamma$ must be inversely proportional to the decay time $\tau$ of the resonant state:

$$
\tau \propto \frac{\hbar}{\Gamma}
$$

so that structures with a finite, measureable width are expected. Only the elastic peak at $W=M=$ $0.938 \mathrm{GeV}$ is expected to be a $\delta$-function, since, for elastic scattering, there is no finite "lifetime" of any "excited" state (i.e. $\Gamma \rightarrow \infty$ ). In practice, numerous other higher-order Feynman diagrams and external radiation effects greatly broaden the elastic peak from its "exact" $\delta(M)$ form. This is explained in more detail in Section 6.4.

Experimentally, one observes several resonances, ${ }^{46}$ including the spin- $\frac{3}{2}$ hadron excitation $\Delta(1232)$, and several $\operatorname{spin} \frac{1}{2} \mathrm{~N}^{*}$ excited nucleon resonances (including the well-known Roper resonance $N^{*}(1440)$ ). Recalling Eq. 1.214, it is obvious that the $\Delta$ (spin- $\frac{3}{2}$ ) excitation implies $A_{1}<0$, while an $\mathrm{N}^{*}$ (spin- $\frac{1}{2}$ ) excitation implies $A_{1}>0$. There are several observed spin- $\frac{1}{2}$ excitations, classified by their overall angular momentum S $(L=0), \mathrm{P}\left(L=\frac{1}{2}\right), \mathrm{D}(L=1)$ or $\mathrm{F}\left(L=\frac{3}{2}\right)$, and subscripts ${ }_{m n}$

\footnotetext{
${ }^{46}$ These are typically labeled with their missing mass in MeV in parenthesis
} 
representing $L_{z}$ and total energy discrete states [5][12]:

$$
e+p \underset{s=\frac{1}{2}}{\longrightarrow}\left\{\begin{array}{c}
P_{11}(1440) \\
D_{13}(1520) \\
S_{11}(1535) \\
S_{31}(1620) \\
S_{31}(1620) \\
S_{11}(1680) \\
F_{15}(1680) \\
D_{33}(1700)
\end{array}\right.
$$

Detailed information of resonance structures is important to multipole analysis [1], a thorough study of which is far beyond the scope of this thesis. For example, the $\Delta(1232)$ resonance amplitude $A_{\frac{3}{2}}$ is known by multipole analysis to occur through a magnetic dipole (M1) and electric quadrupole (E2) transition [12]:

$$
A_{\frac{3}{2}}=-\frac{\sqrt{3}}{2}\left(M_{1+}^{(3 / 2)}-E_{1+}^{(3 / 2)}\right)
$$

The total cross-section of the resonance is then given by [12]

$$
\sigma_{\frac{3}{2}}^{T}=\frac{4 \pi^{2} \alpha}{\nu^{*}} A_{\frac{3}{2}}
$$

$A_{\frac{3}{2}}$ can then be related to the structure functions in $L_{\mu \nu} W^{\mu \nu}$ through the optical theorem, for an explicit solution of the resonance contribution. The asymmetry $A_{1}$ then arises from comparison to the contribution $A_{\frac{1}{2}}$ at that kinematic point, and application of Eq. 1.214. The $p \rightarrow \Delta$ transition can occur through the reactions

$$
e+p \underset{s=\frac{3}{2}}{\longrightarrow} \Delta(1232) \rightarrow e+N+\pi
$$

(with $N$ and $\pi$ representing final nucleon and pion states of varying isospin and charge) or, less commonly, by

$$
e+p \underset{s=\frac{3}{2}}{\longrightarrow} \Delta(1232) \rightarrow e+p+\pi^{+}+\pi^{-}
$$


For this reason, exclusive analyses, which look specifically at events containing decay products, are often done to explore the multipole structure of resonances, including the spin asymmetries of resonances such as [32]

$$
e+p \rightarrow e+n+\pi^{+}
$$

The total inclusive asymmetry contains the combination of all possible excited resonances. The inclusive cross-sections are the combination of all possible exclusive cross-sections. With all these resonances (and possibly others) combined, one expects $A_{1}$ to be composed of a blurred continuum of finite-width resonances in $W$, dipping into the negative region near $W=1232 \mathrm{MeV}$ and then rising to a positive value somewhere near the Roper resonance at $W=1440 \mathrm{MeV}$, eventually leveling off to a smooth region for DIS, at $W \gtrsim 2000 \mathrm{MeV}$ (see Figure 1.8).

It is also interesting to consider the behavior of $A_{1}$ exclusively in the DIS region. We know from Eq. 1.214 that $A_{1}=1$ for elastic scattering, because, obviously, $\sigma_{3 / 2}=0$ for these events. ${ }^{47}$ We know $x=1$ for elastic $e p$ scattering, by definition. Looking only at events where $W>2.0 \mathrm{GeV}$ (and the quark structure of the proton is probed), but as $x \rightarrow 1$, near-elastic scattering occurs from asymptotically free quarks, with few higher order corrections needed for gluons and $q \bar{q}$ pairs. This requires increasingly high values of $Q^{2}$, with $x=1$ requiring $Q^{2} \rightarrow \infty$ (i.e. scaling). The DIS region where $x \rightarrow 1$, free of the difficulties introduced by extra resonance parameters, is thus an excellent region for the testing of $\mathrm{PQCD}$ theory. The measurement of the rate that $A_{1} \rightarrow 1$ as $x \rightarrow 1$ is consequently of great interest to QCD theorists (see Section 8.5.3) [33].

\subsection{Existing Measurements}

Now that the theory, motivation and method of extraction of $g_{1}$ and $g_{2}$ from physical data have been presented, a basic summary of the published measurements of these structure functions is given.

In this section, a brief summary is given of existing spin physics experiments, outside of EG1b, that have provided measurements for $g_{1}^{p}$ and $g_{2}^{p}$, as well as a presentation of the current world data and the expectations for this analysis. Fine details about the experimental apparatus, etc., are not provided here; interested readers are directed to the included references for this information.

\footnotetext{
${ }^{47}$ In other words, if there is any spin excitation, then it is obviously not an elastic scattering event.
} 


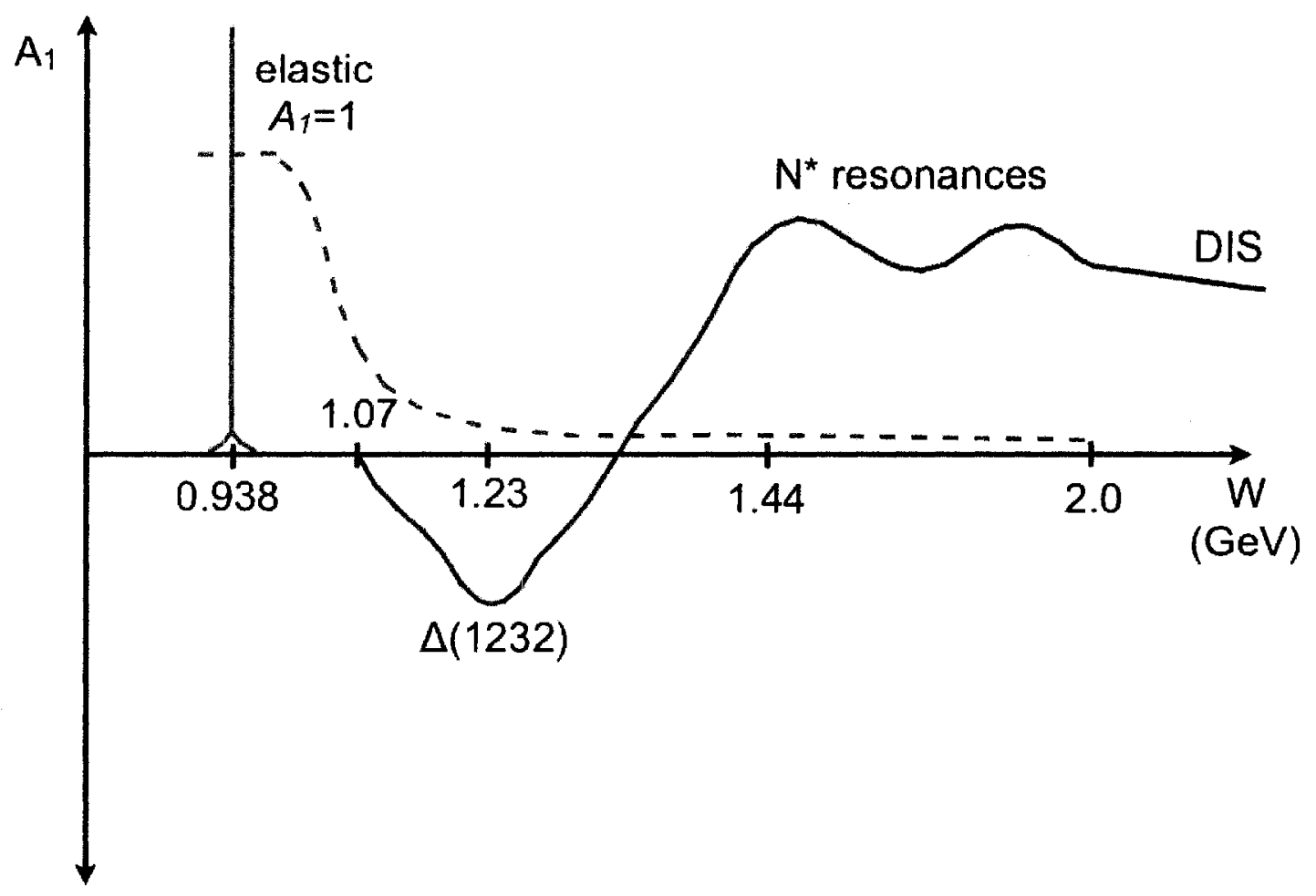

Figure 1.8: Qualitative expectation of the measured virtual photon spin asymmetry $A_{1}$. The DIS, resonance, and elastic scattering regions are highlighted in green, blue and red, respectively. Note that in practice, radiative effects greatly broaden the elastic peak from its ideal $\delta$-function shape (dotted line). See the text for more details.

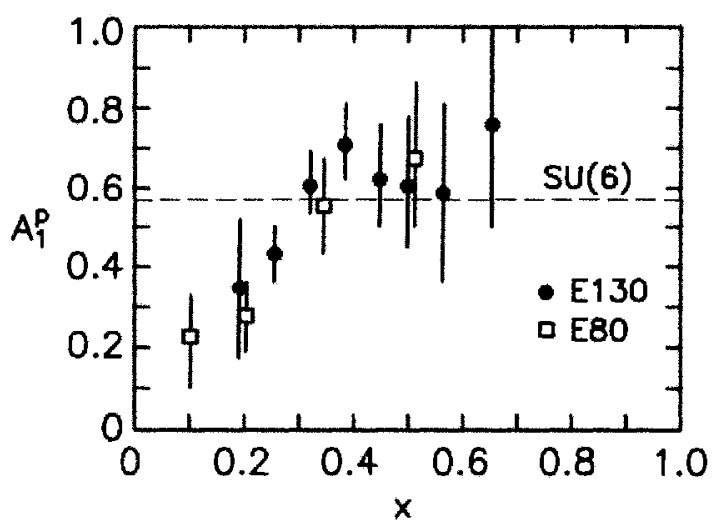

Figure 1.9: Earliest measurements of $A_{1}$ for the proton at SLAC. A value of $\frac{5}{9}$ is expected in the stationary quark SU(6) model; this value holds fairly well for high $x$. From Ref. [34]. 
It should be noted that the structure functions $g_{1}$ and $g_{2}$ for the neutron have been measured in numerous experiments, utilizing electron and muon scattering data from stationary deuteron and ${ }^{3} \mathrm{He}$ targets. The majority of experiments listed in this section (including EG1b) also incorporated measurements of $g_{1}^{n}$ and/or $g_{2}^{n}$ using one of these two targets in addition to a proton target. Additional experiments in Hall-A at Jefferson Laboratory with a stationary polarized ${ }^{3} \mathrm{He}$ target have supplied further neutron data (see, for example, Ref. [35]).

Though measurements of $g_{1}^{n}$ and $g_{2}^{n}$ are of equal importance to those of $g_{1}^{p}$ and $g_{2}^{p}$ with regard to a complete understanding of nucleon dynamics (especially where isopsin study is involved, as in the Bjorken Sum Rule in Section 1.3.2), the focus of this work is the study of the proton, so the collection of world data on $g_{1}^{p}$ and $g_{2}^{p}$ is the sole focus of this section.

\subsubsection{Early measurements of $g_{1}^{p}$}

A chronology of major experiments producing measurements for the $g_{1}$ structure function of the proton is given in Table 1.2. A very brief overview of the chronological history and summary of this measurement is presented here.

The E80 experiment at the Stanford Linear Accelerator (SLAC) in 1976 is the first notable experiment to utilize a polarized electron beam (ranging from 6-13 GeV) and a polarized (butanol) target. A low beam current limited collection to only 2 million events [36], but it enabled the first ever measurements of $A_{1}^{p}$ at $Q^{2} \sim 2 \mathrm{GeV}^{2}$ (Figure 1.9) [37]. The higher energy SLAC experiment E130 (run to reduce higher twist effects through scaling) at $23 \mathrm{GeV}$ beam energy was run in 1983, at 3.5 $\mathrm{GeV}^{2}<Q^{2}<10 \mathrm{GeV}^{2}$, collecting even fewer events, but at higher $x$ than the first experiment [38].

Polarized deep-inelastic scattering (PDIS) at CERN, in the European Muon Collaboration (EMC) experiment used a different measurement approach. Polarized muons were used in place of electrons [39]. Muons are "automatically" polarized when produced from the decay of high-energy pions

$$
\pi^{-} \rightarrow \mu^{-}+\bar{\nu}
$$

due to the weak axial-vector coupling of the decay, avoiding the difficulties involved in producing polarized electrons [22]. With the exception of a differing target mass correction (TMC) for the 


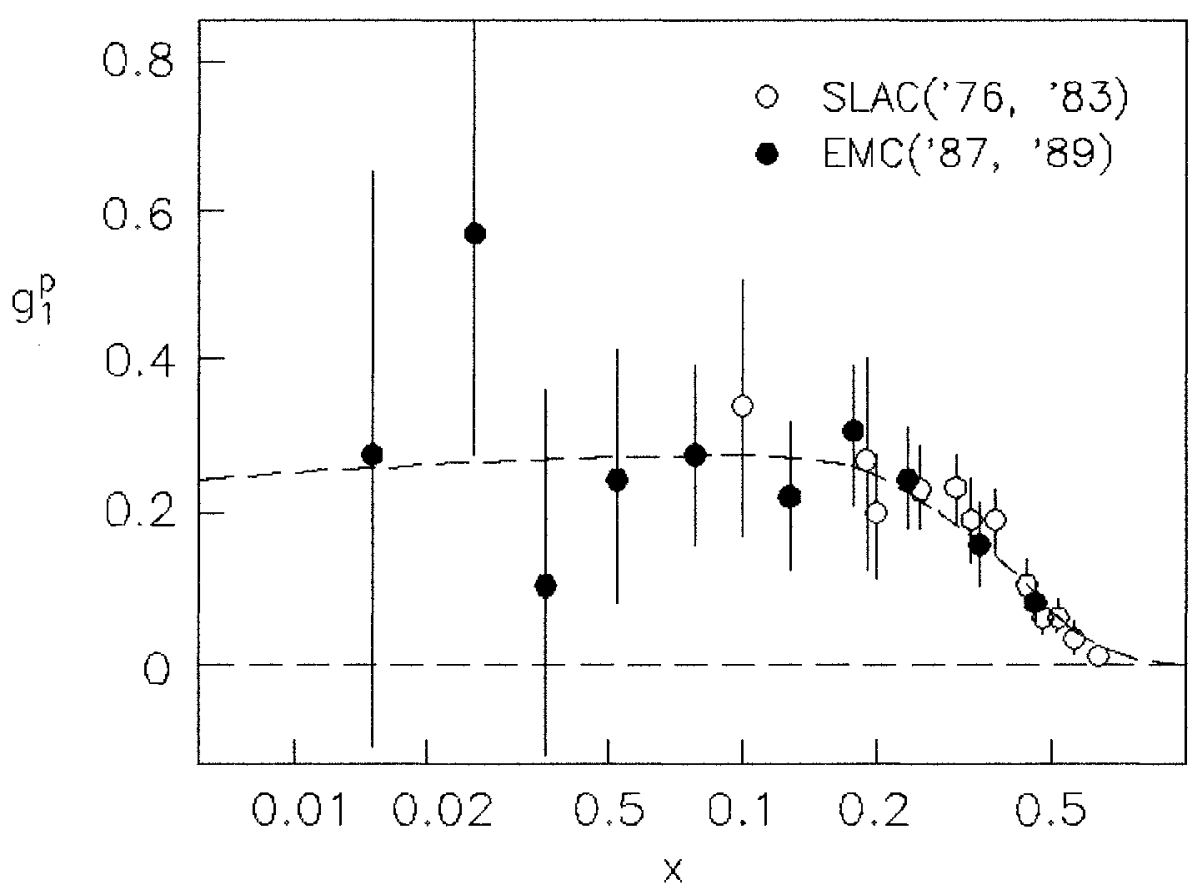

Figure 1.10: The first available data for $g_{1}^{p}$ from the SLAC E80 and E130 and the CERN EMC experiments. Resolution was not fine enough in these experiments to bin in terms of more than one kinematic variable. From Ref. [40].

recoil of the struck particle, the $\mu p$ and $e p$ reactions are identical, so that the same asymmetry and structure functions can be measured. CERN is a high-energy facility; $\mu^{-}$energies of $100-200 \mathrm{GeV}$ were produced. The EMC experiment utilized a polarized $\mathrm{NH}_{3}$ target with polarization measured by NMR. ${ }^{48}$ Muon polarization was (rather tenuously) evaluated by a Monte Carlo distribution of the scattered particles. The $g_{1}^{p}$ results from these early experiments are shown in Figure 1.10.

Obviously, these early measurements did not produce the event flux or kinematic breadth of data necessary for a fine measurement of the $Q^{2}$ or $x$ evolution of the structure function. They were, however, able to test two predictions: the stationary SU(6) quark symmetry, and the Ellis-Jaffe Sum Rule.

\footnotetext{
${ }^{48} \mathrm{~A}$ similar system is used in EG1, but used only as a secondary check for polarization. See Section 6.2 .
} 
Table 1.2: A tabular summary of experiments measuring the $g_{1}^{p}$ structure function, including the approximate $Q^{2}$ range and approximate number of triggers.

\begin{tabular}{|c|c|c|c|c|c|}
\hline Date & Experiment & Location & $Q^{2}$ range $\left(\mathrm{GeV}^{2}\right)$ & $x$ range & Approx. \# of events \\
\hline \hline 1976 & E80 & SLAC & $\sim 2$ & $0.1-0.5$ & $2 \times 10^{6}$ \\
\hline 1983 & E130 & SLAC & $3.5-10$ & $0.2-0.65$ & $1 \times 10^{6}$ \\
\hline 1987,1989 & EMC & CERN & $3.5-29.5$ & $0.01-0.70$ & $1.2 \times 10^{6}$ \\
\hline 1992 & SMC & CERN & $0.01-23.1$ & $0.00006-0.121$ & $4.5 \times 10^{6}$ \\
\hline $1993-1994$ & E143 & SLAC & $1-40$ & $0.014-0.90$ & $2.36 \times 10^{8}$ \\
\hline 1996 & HERMES & DESY & $0.18-20$ & $0.0041-0.9$ & $3.47 \times 10^{6}$ \\
\hline 1997 & E155 & SLAC & $1.22-34.72$ & $0.015-0.750$ & $1.7 \times 10^{8}$ \\
\hline 1998 & EG1a & JLAB & $0.02-5.0$ & $0.01-0.70$ & $3 \times 10^{9}$ \\
\hline $2000-2001$ & EG1b & JLAB & $\mathbf{0 . 0 2 - 5 . 0}$ & $\mathbf{0 . 0 1 - 0 . 7 0}$ & $\mathbf{2 . 3} \times 10^{10}$ \\
\hline 2002 & RSS & JLAB & $\sim 1.3$ & $0.3-0.8$ & $1.6 \times 10^{8}$ \\
\hline
\end{tabular}

\subsubsection{The spin crisis}

The stationary SU(6) quark model does not account for Fermi smearing caused by internal motion of quarks in the nucleon, nor does it account for the presence of the gluons and sea-quarks. From a basic constituent quark model, using the notation of Section 1.2.3, we can predict [6]

$$
A_{1} \sim \frac{g_{1}}{F_{1}}=\frac{q_{u}^{2} \Delta u+q_{d}^{2} \Delta d}{q_{u}^{2} u+q_{d}^{2} d}=\frac{\frac{4}{9} \cdot \frac{4}{3}+\frac{1}{9} \cdot \frac{-1}{3}}{\frac{4}{9} \cdot 2+\frac{1}{9} \cdot 1}=\frac{5}{9}
$$

which agreed reasonably with the DIS data from SLAC and CERN, at least at high $x$ (see Figure 1.9).

Another prediction of early double-spin asymmetry experiments was the Ellis-Jaffe sum rule. Using Eqs. 1.182 and 1.184, and assuming $\Delta s=0$, one can write [12]

$$
\Gamma_{\mathrm{I}}^{p}=\frac{g_{A}}{12}+\frac{5}{36}(\Delta u+\Delta d)
$$

This is a simple form of the Ellis-Jaffe Sum Rule. The sum $\Delta u+\Delta d$ can be evaluated from hyperon $\beta$-decay [12], in a manner analagous to the evaluation of $g_{A}$ presented in Section 1.3.2, assuming 
SU(3) symmetry holds for the quark triplet $u d s$. The result is

$$
\Delta u+\Delta d=0.57 \pm 0.06
$$

QCD radiative corrections [12] modify Eq. 1.246 (to first order in $\alpha_{s}$ ) to

$$
\Gamma_{1}^{p}=\frac{g_{A}}{12}\left[1-\frac{\alpha_{s}\left(Q^{2}\right)}{\pi}\right]+\frac{5}{36}(\Delta u+\Delta d)\left[1-\frac{7}{15} \frac{\alpha_{s}\left(Q^{2}\right)}{\pi}\right]
$$

Using this, along with the known value of $g_{A}=1.26$, yields

$$
\Gamma_{1}^{p}\left(Q^{2}=3 \mathrm{GeV}^{2}\right)_{E J}=0.167 \pm 0.008
$$

The EMC result of $0.114 \pm 0.012 \pm 0.026$ clearly violated this rule - meaning that very little of the proton spin resides on the consituent quarks $u$ and $d$ - an unexpected result, considering the prior success of the quark model. The discrepancy became known as the spin crisis. 49 This result made it apparent that a spin parton model of the nucleon was far from complete.

The unexpected violation of the Ellis-Jaffe Sum Rule motivated the next experiment to measure nucleon spin, the Spin Muon Collaboration (SMC) experiment at CERN, which instead used a butanol target (like the early SLAC experiments) and better beam polarization measurements using the positron spectrum from $\mu^{-}$decay [41]. The SMC data supplemented the original EMC run, and extended the kinematic reach down to even lower $x$, with a considerably higher event flux. It also provided measurements of $g_{1}^{d}$ (and hence $g_{1}^{n}$ ) from deuterated butanol, so that the Bjorken Sum Rule (Eq. 1.184) could be tested.

\subsubsection{Second generation SLAC and HERMES polarized experiments}

The next generation of PDIS experiments at SLAC utilized solid state GaAs cathodes to produce a higher flux polarized beam than was available for the E80/E130 polarized experiments. The E143 experiment, utilizing beam energies of $19.4,22.7$ and $25.5 \mathrm{GeV}$, and a polarized solid $\mathrm{NH}_{3}$ target,

\footnotetext{
${ }^{49}$ Clearly, other components $(\Delta s, \Delta g$, etc.) contribute to the total proton spin - components that are isospin invariant, assuming the Bjorken sum rule holds.
} 


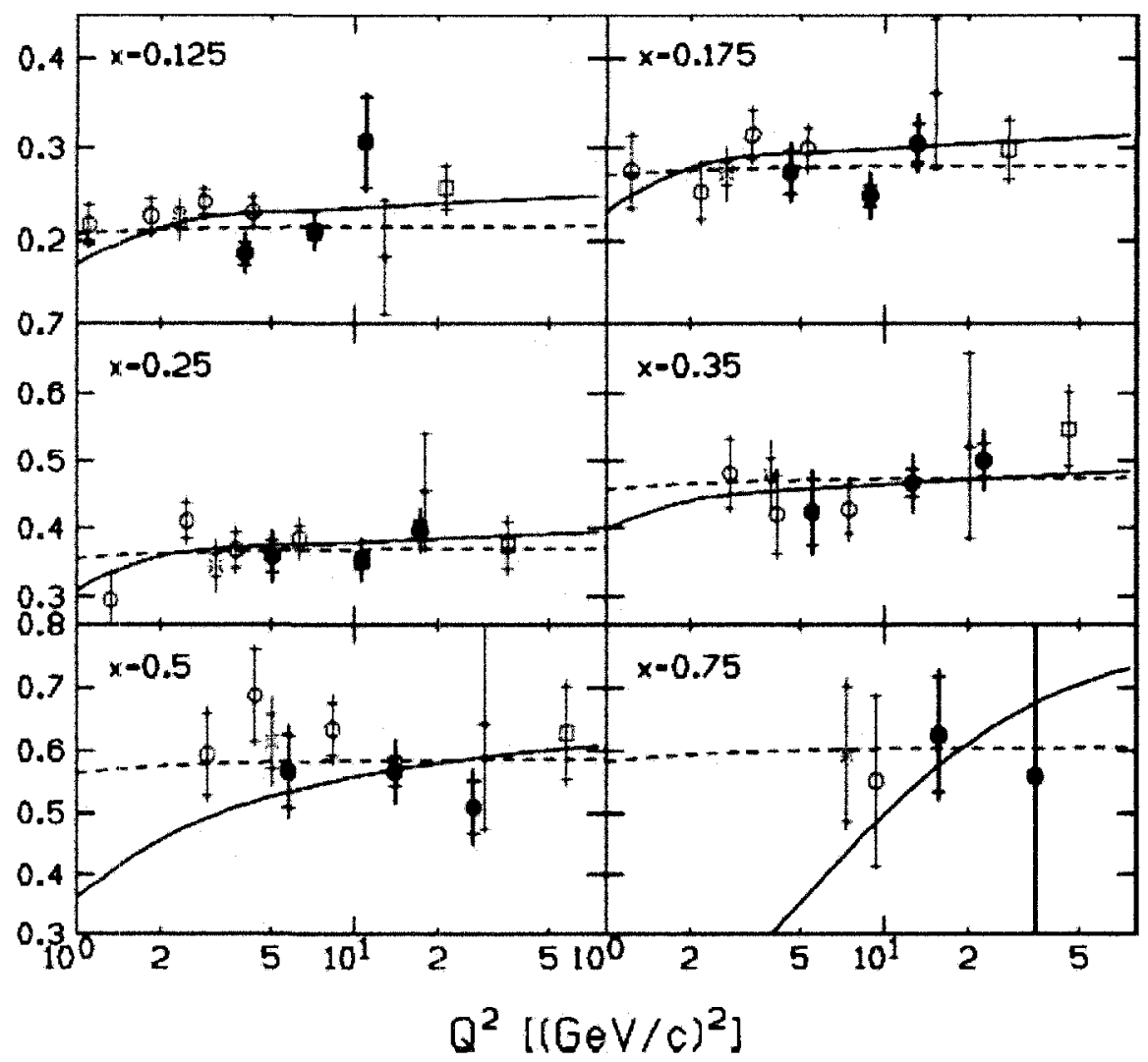

Figure 1.11: Measurements of $g_{1}^{p} / F_{1}^{p}$ in selected $x$ bins for the SLAC E155 experiment $(\bullet)$, compared to results from E143(o), HERMES $(*)$ and SMC( $\square)$. See the text for more details. The solid line is a NLO QCD model, while the dotted line is a simpler fit. From Ref. [42]. 
was thus able to generate 300 million measured events, orders of magnitude higher than available in any prior double-spin asymmetry measurement for the proton, with a more easily calculable unpolarized background subtraction [4]. The E143 target provides the basic model for the target used in the EG1 experiment (see Section 2.4). Measurements of the moment $\Gamma_{1}^{p}$ from E143

$$
\Gamma_{1}^{p}\left(Q^{2}=3 \mathrm{GeV}^{2}\right)=0.127 \pm 0.004 \pm 0.010
$$

showed once and for all that this moment lay at least two standard deviations below the Ellis-Jaffe expectation, thus showing a clear violation of this sum rule due to, presumably, higher order QCD and higher twist effects.

The most recent SLAC polarized $\mathrm{NH}_{3}$ target experiment, E155, provides the widest range of $Q^{2}$ and $x$ acceptance available prior to the EG1 experiment. E155 data covered very low $x$ values (down to 0.025 ), at a lower $Q^{2}$ range than previously available for DIS scattering in this range (see Figure 1.11) [42].

Using a very different setup, the HERMES experiment employed a unique longitudinally polarized gas target, which produced a jet of atomic hydrogen with polarized states selected by SternGerlach separation (exchanged by RF transitions) [43]. This method provides a very thin radiation length target, but also gives the advantage of scattering from a target with no large- $A$ background, and thus no dilution factor (see chapter 5 ). A high-flux electron beam ( $\sim 30 \mathrm{GeV}$ ) from the DESY HERA lepton storage ring provided a high precision measurement for very low values of $x$ (as low as 0.0041 ), at much lower $Q^{2}$ values than achieved by the E155 experiment (see Figure 1.12). With the new SLAC and HERMES data, it was finally possible to check the assumptions of QCD models and sum rules in detail, and test the behavior of models in terms of both $Q^{2}$ and $x$.

\subsubsection{The missing kinematic region: motivation for EG1}

It is apparent from a careful inspection of Figure 1.12 that a large kinematic region, namely at low $Q^{2}$ and high $x$, has still not been significantly covered by the global data set. This situation is clarified in Figure 1.13, which shows the breadth of the world data on $g_{1}^{p}$ before and after EG1. By substituting sample values (covered by the inverted red triangles) of $Q^{2}$ and $x$ into Eq. 1.9, it 


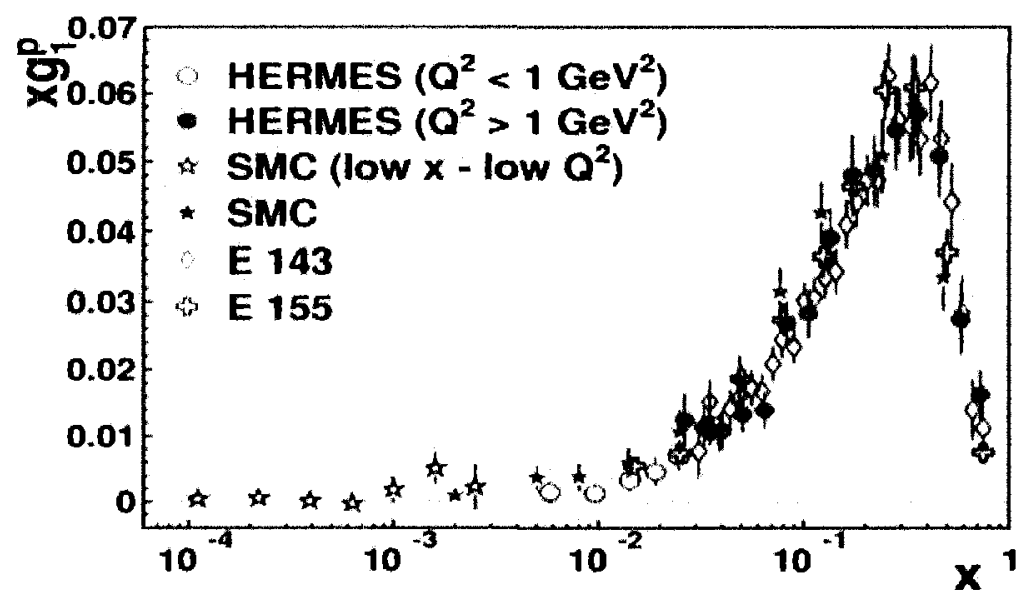

Figure 1.12: A plot of $x g_{1}^{p}$ data from HERMES, with low $Q^{2}$ data (o) and high $Q^{2}$ data (•) superimposed on the available world data. The HERMES data cover a fuller range of kinematic values than any previous data, but there is still a noticeable void for high $x$ values at low $Q^{2}$ after the completion of this experiment. From Ref. [47]; color version from Ref. [48].

is easy to see that a large portion of this region lies in the resonance region $1.08 \mathrm{GeV}<W<$ $2.0 \mathrm{GeV}$. Structure function data in this region, covering the $\Delta$ and $\mathrm{N}^{*}$ resonances, is expected to oscillate greatly, and thus the specific values of $g_{1}$ cannot be reconstructed from their OPE moments using assumptions of analyticity and continuity alone (see Section 1.3.2). As explained in Section 1.4.3, detailed data of the resonance structures must be explicitly known to account for nuclear structure in the intermediate $Q^{2}$ region to test assumptions of theories such as $\chi \mathrm{PT}$ and even Lattice QCD. Detailed interpolation from widely spread data points is not possible where the structure function value shifts rapidly in terms of $x$. Therefore, much more data are required in this region to gain the same type of precision measurements of the $g_{1}$ spectrum in this region. This requires an experiment with large acceptance and statistics, needs fulfilled by the EG1b experiment at Jefferson Laboratory.

EG1b is a follow up to the shorter EG1a experiment. EG1a was a smaller preliminary data set, intended as a "test run" of the experimental conditions employed in the later, much longer EG1b experiment. EG1a data have already been analyzed [44][45]. The kinematic coverage for EG1a is only slightly different that EG1b. However, EG1b, with its much longer running time, provides much 
more data and hence greater statistical precision than the preliminary EG1a experiment. ${ }^{50}$

\subsubsection{Current measurements of $g_{2}^{p}$ (E155x and RSS)}

Transverse polarization experiments are, in practice, more difficult to implement than longitudinal polarization experiments, and even when implemented, the resulting asymmetry provides an (approximately) equally mixed measurement of the $g_{1}$ and $g_{2}$ structure function, rather than a (nearly) pure spin-result, as in the longitudinal case. Therefore, available $g_{2}^{p}$ data are very limited in scope and detail.

At this point, only two experimental analyses contribute notably to our knowledge of $g_{2}^{p}$. E155x was an extension of the E155 experiment at SLAC, similar to its parent experiment, except that it utilized a transversely polarized target to measure $A_{\perp}$, enabling extraction of the sum $g_{1}+g_{2}$ in the DIS region [49]. The older, E143 experiment placed some constraints on the value of $g_{2}$, but these measurements are rendered obsolete by E155x (see Figure 1.14).

Measurements in the resonance region for $g_{2}^{p}$ are even rarer. The only available precision measurement comes from the recent Resonance Spin Structure (RSS) experiment in Hall-C at Jefferson Lab. RSS, like EG1, utilized a frozen ammonia target, which couid be aligned both parallel and perpendicular to the beam, with data collected by the High Momentum Spectrometer (HMS) [52]. While this was a very high precision experiment, the acceptance of the HMS was not nearly as broad as that of CLAS in Hall-B, so that only a very narrow band at $Q^{2} \sim 1.3 \mathrm{GeV}^{2}$ was covered by the data. However, the versatility of the polarized target allowed for the measurement of both $A_{\|}$and $A_{\perp}$, so that extraction of both $g_{1}$ and $g_{2}$ was possible in the resonance region (see Figure 1.15). RSS results show unambiguously that $g_{2} \neq g_{2}^{W W}$ in the measured kinematic region, meaning that higher-twist effects, and thus long-range correlations bewteen quarks and gluons, are likely a determining factor of spin-behavior at this kinematic scale (see Section 1.3.4). Thus, future measurements of $g_{2}$ in this kinematic region are of considerable physical interest.

Unfortunately, the EG1 polarized target cannot be set up in a perpendicular polarization configuration, so that $A_{\perp}$ cannot be directly measured in the experiment in this thesis. However, due to

\footnotetext{
${ }^{50}$ Prior to this thesis, a majority of the $1.6 \mathrm{GeV}$ and $5.6 \mathrm{GeV}$ data in EG1b were already analyzed in a first pass analysis [2][46]. This thesis represents a complete analysis of all inclusive proton scattering data in EG1b, with improvements upon some aspects of analysis used in the first pass.
} 


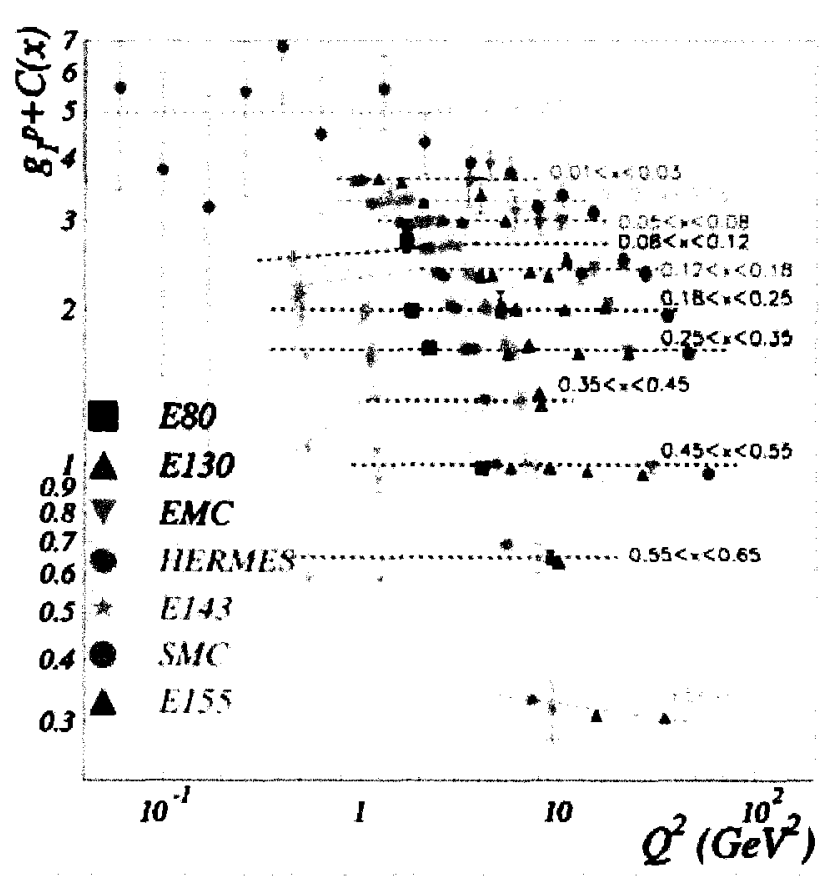

(a) Before EG1

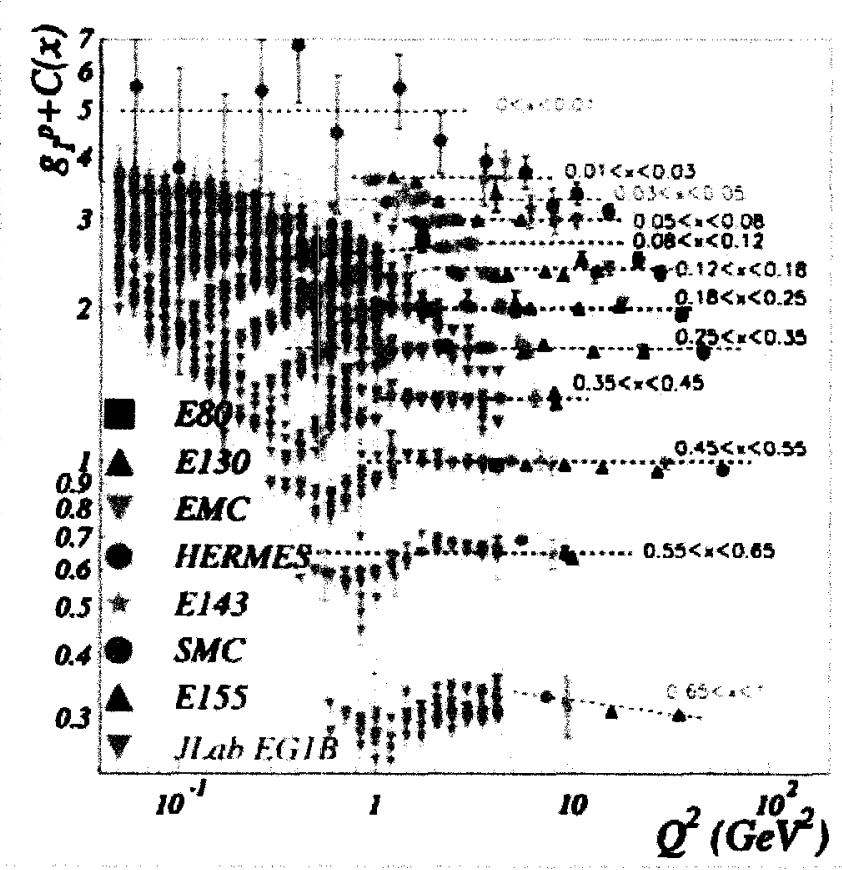

(b) After EG1

Figure 1.13: Depiction of world data set for $g_{1}^{p}$, shown before and after the EG1 experiment, logarithmically as a function of $Q^{2}$ and $x$. An offset $C(x)$ is added to $g_{1}$ to separate different values of $x$. Plots courtesy A. Deur. 


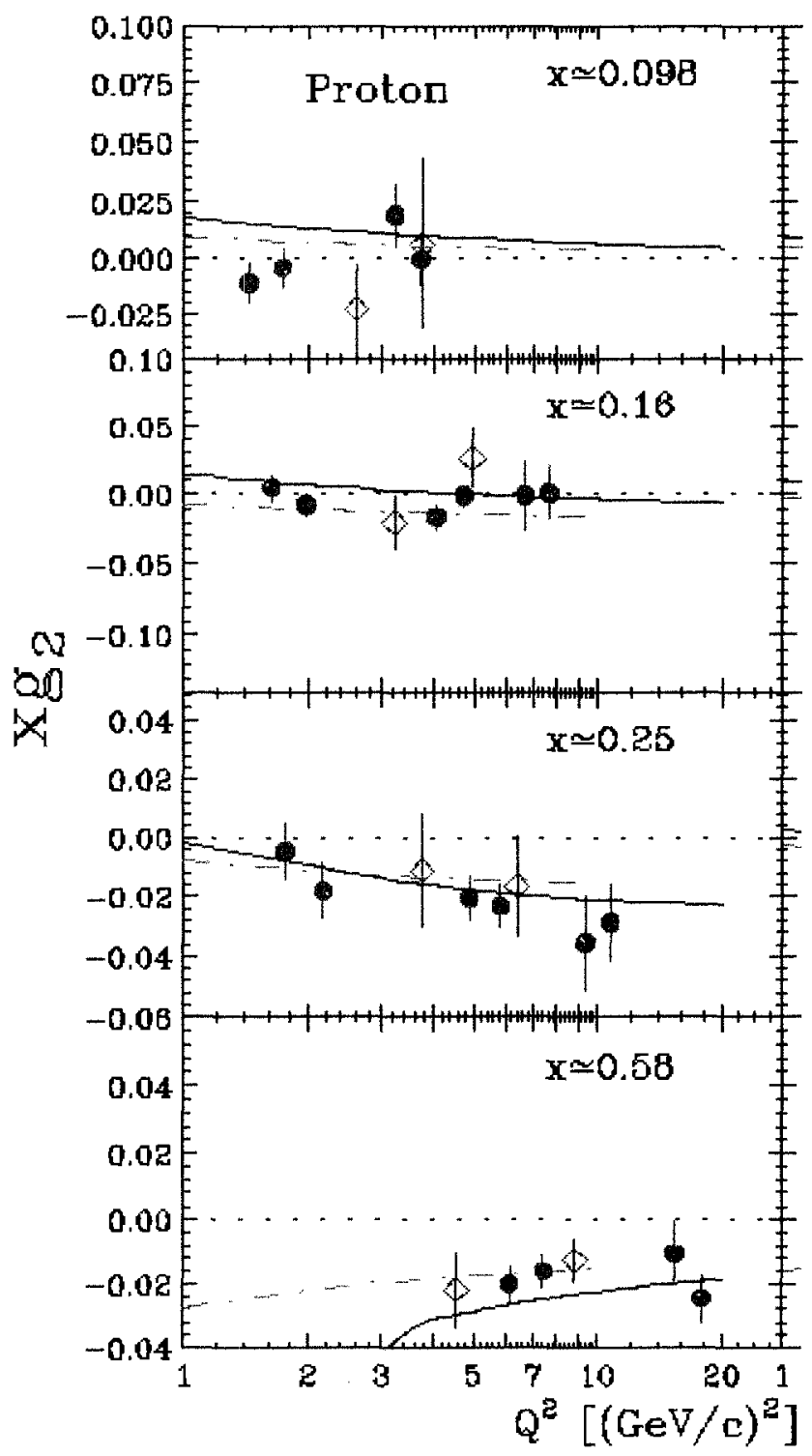

Figure 1.14: Measurements of $x g_{2}^{p}$ for the DIS region from E155x(•), compared to older constraints from E143( $\diamond)$. World data for $g_{2}^{p}$ are very limited compared to $g_{1}^{p}$. From Ref. [50]. 


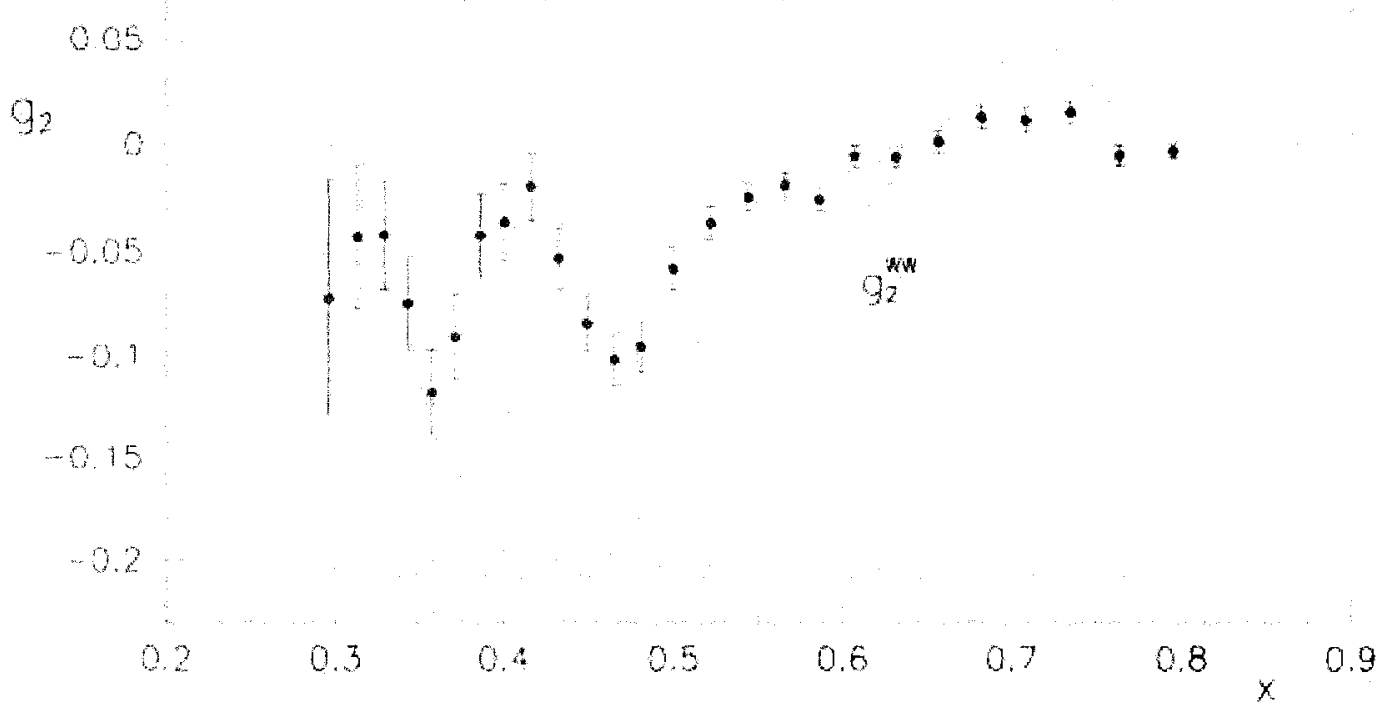

Figure 1.15: Measurements of $g_{2}^{p}$ for the resonance region at $Q^{2} \sim 1.3 \mathrm{GeV}^{2}$ for RSS in Jefferson Lab Hall-C. The leading order twist curve $g_{2}^{W W}$ is shown for comparison. Measurements for $g_{1}^{p}$ at this kinematic value were also made (not shown). From Ref. [51].

the use of multiple beam energies and the very high statistics available in EG1, the method of linear regression shown in Section 1.4 can be used to extract a lower-precision measurement of $A_{2}$, and hence $g_{2}$, for the proton, over a wider $Q^{2}$ range in the resonance region than previously available. This process is described further in Section 8.1.2, near the end of this thesis. 


\section{Chapter 2}

\section{Experimental Apparatus and Models}

\subsection{The EG1b Experiment: Introduction}

We now set the stage for analysis of the data by describing the experimental apparatus and procedure. Data were collected over approximately a 7-month period from 2000-2001, with 1-6 GeV $(\sim 70 \%)$ polarized electrons produced at a rate of approximately $20 \mathrm{nA}$ from the CEBAF electron accelerator, scattered from a ( 70\%) polarized target, ${ }^{1}$ and detected in Experimental Hall-B by the CLAS (CEBAF Large Acceptance Spectrometer) detector. Data were then archived on tapes for later analysis.

Technical details regarding the CEBAF accelerator, CLAS detector components, and EG1 polarized targets are archived in the various NIM (Nuclear Instruments and Methods) papers referenced throughout this chapter. In this thesis, only a brief description of the experimental apparatus is supplied, with appropriate references, so that the bulk of this chapter can be dedicated to detailed descriptions of this particular analysis.

In the sections that follow, all experimental components are described, beginning with the beam injector and accelerator, followed by the EG1 target, the components of the CLAS detector, and finally the storage of the data for later analysis.

\footnotetext{
${ }^{1}$ Only the $\mathrm{NH}_{3}$ target had a polarization this high; $\mathrm{ND}_{3}$ polarizations were considerably lower.
} 


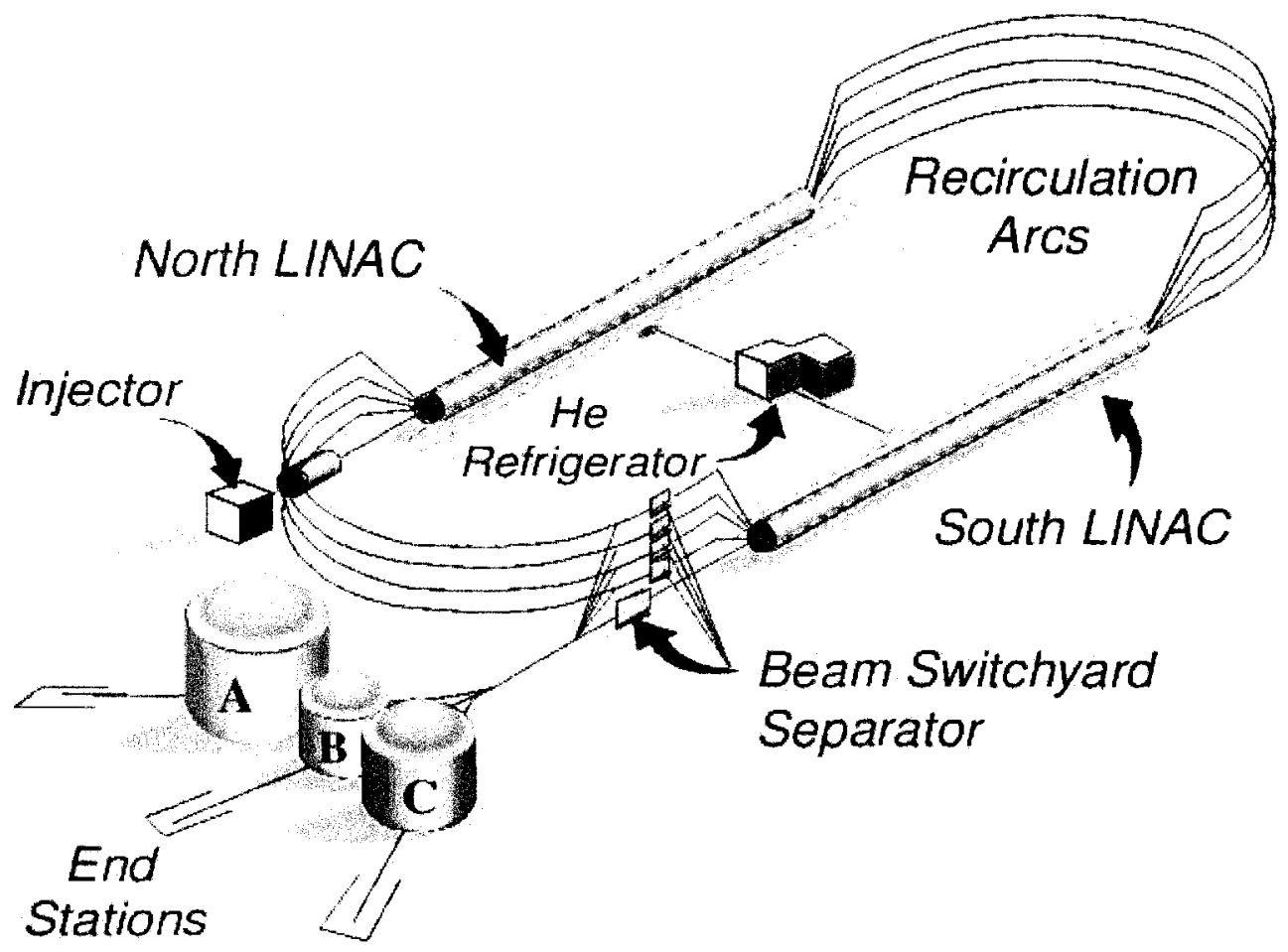

Figure 2.1: Schematic of CEBAF, the Continuous Electron Beam Accelerator Facility at Jefferson Lab. See the text for details regarding the components.

\subsection{The CEBAF Electron Accelerator}

The CEBAF electron accelerator is capable of generating beam energies of up to $5.8 \mathrm{GeV}$ (with a spread $\left.\Delta E / E \lesssim 2.5 \times 10^{-5}\right)^{2}$ at currents of up to $300 \mu \mathrm{A}$ delivered in $1497 \mathrm{MHz} \mathrm{RF}$ modulated pulses, split between three research halls. ${ }^{3}$ Electrons can be (up to $75 \%$ ) polarized in alternating bunches of up to $3 \mathrm{pC}$ of charge [53]. ${ }^{4}$

Figure 2.1 shows an overall diagram of the CEBAF accelerator. Polarized electrons at $45 \mathrm{MeV}$ are generated in a beam injector unit, then fed into a pair of $600 \mathrm{MeV}$ linear accelerators employing RF cryomodules, cooled by a central LHe refrigerator. Recirculation arcs magnetically steer the

\footnotetext{
2Plans are in place to upgrade the maximum beam energy to $\sim 12 \mathrm{GeV}$ by c.2012.

${ }^{3}$ This means an effective pulse rate of $499 \mathrm{MHz}$ is delivered to each Hall.

${ }^{4}$ Beam polarization has reached up to $85 \%$ in recent years; the $75 \%$ limit corresponds to the time of the EG1b experiment.
} 
beam through up to 5 passes (controlled by the beam switchyard operator) through the linac pair. A total maximum beam energy of $6.0 \mathrm{GeV}$ can thus be delivered to each of the 3 research halls. ${ }^{5}$ Due to spectrometer instrumentation limits (most particularly the inner layer drift chambers), only a maximum of $100 \mathrm{nA}$ can be delivered to Hall-B, with only about $20 \mathrm{nA}$ typically used for the EG1 experiment. More details (and references) regarding specific beam components are outlined later in this section.

\subsubsection{Beam Injector}

Polarized electrons used in Jefferson Lab experiments are produced initially in the Beam Injector Unit, prior to their introduction into the North Linac. First a GaAs photocathode (Figure 2.2a) is activated to a negative electron affinity by introducing $\mathrm{Cs}$ and oxidizing $\mathrm{NF}_{3}$ gas to the cathode surface [58]. Then, under ultra-high vacuum $\left(\sim 10^{-11}\right.$ Torr), polarized electrons are produced from the cathode at $100 \mathrm{keV}$ by exciting electrons from the top (spin-biased) valence state into the conduction band. The excitations are made by circularly polarized light [54] supplied by MOPA (Master-Oscillator-Power-Amplifier) or Ti:sapphire lasers configured in the ultra-high-vaccuum system (Figure 2.2b) [55]. A half-wave plate (HWP) can be inserted in the laser beam to change the polarization phase by $180^{\circ}$. The HWP is inserted and removed at semi-regular intervals throughout the experimental run to ensure that no polarity-dependent bias is manifested in the measured asymmetry.

The $100 \mathrm{kV}$ electrons are introduced into the injector line (Figure 2.3), through a prebuncher cavity and two circular apertures $\left(A_{1}\right.$ and $\left.A_{2}\right)$, which pare down the beam in length and diameter so that it can be passed to the chopper, which splits the beam into $499 \mathrm{MHz}$ bunches for delivery into each of the 3 experimental halls [55][56]. A buncher cavity, followed by a five-cell gradedelectron capture section, compresses the RF bunches and kicks the beam energy up to $\sim 500 \mathrm{keV}$. Unbunched residual electrons are steered to a beam dump. Then, a quarter-length cryomodule (containing 2 SRF (superconducting RF) cavities, explained momentarily) accelerates the experimental electrons to $5 \mathrm{MeV}$, freezing the axial beam dimension to a $90 \mu \mathrm{m}$ ( $300 \mathrm{fs}$ ) bunch. Finally, then, two complete cryomodules boost the beam energy to $45 \mathrm{MeV}$, prior to their introduction into

\footnotetext{
${ }^{5}$ However, beam quality concerns practically mandate a slightly lower maximum energy of just under $5.8 \mathrm{GeV}$.
} 


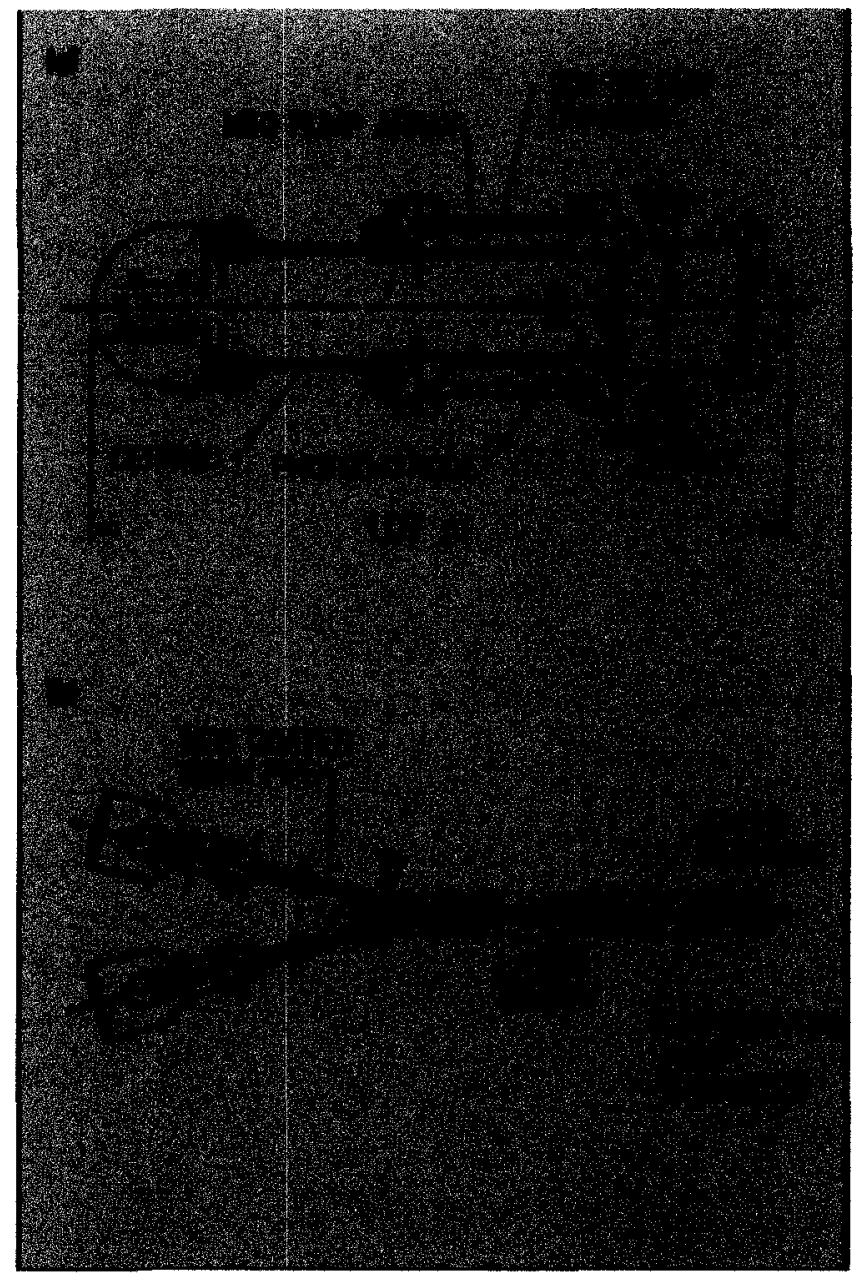

Figure 2.2: Diagram of a $100 \mathrm{kV}$ GaAs photoelectron gun, used to generate polarized electrons in CEBAF (top). Alternating pulses from two guns are fed into the photoinjector (bottom) leading to the rest of the injector unit (Figure 2.3). From Ref. [58]. 


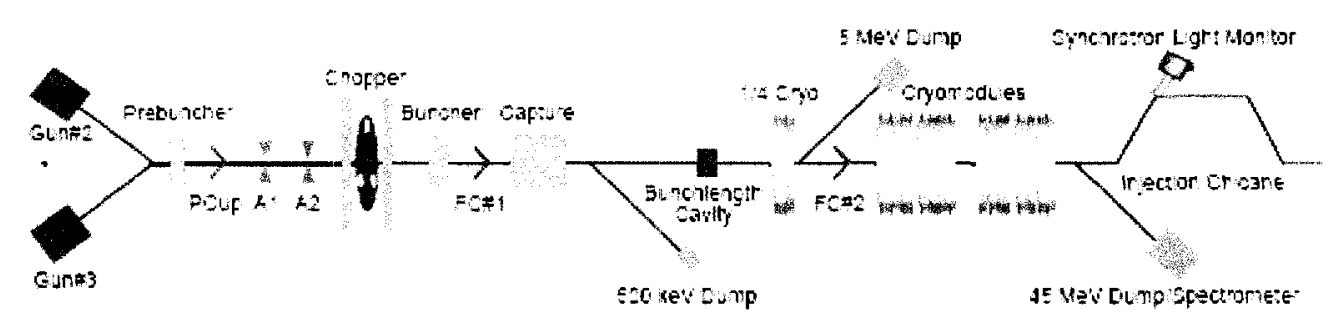

Figure 2.3: Diagram of the CEBAF Beam Injector Unit. See the text for details. From Ref. [59].

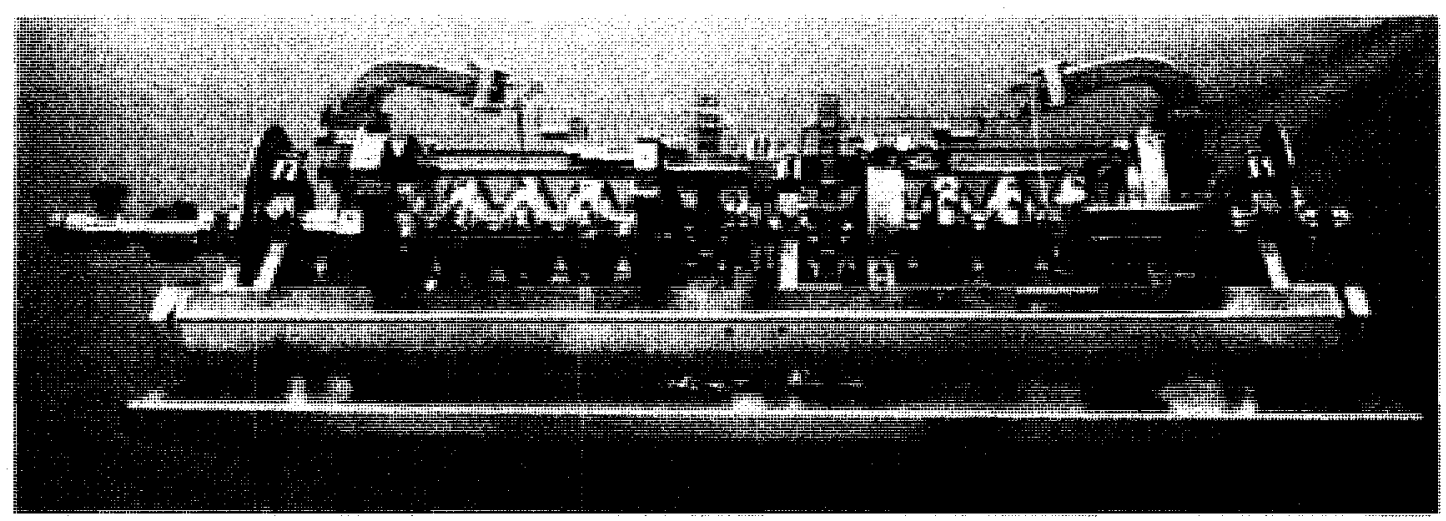

Figure 2.4: A cryounit, consisting of 2 resonant RF cavities. A cryomodule is a series of 4 cryounits (see text for details). From Ref. [53].

the North Linac of the accelerator (Figure 2.1) [56]. Electrons are bent through a chicane magnet prior to injection, producing synchrotron light. The intensity of synchrotron radiation is directly proportional to the beam current. Thus, a Synchrotron Light Monitor (SLM) measures the relative beam current at this stage [57].

\subsection{GeV Linac}

Each linear accelerator, or linac, is capable of increasing the electron energies by $\sim 600 \mathrm{MeV}$, boosting the energy by $\sim 1200 \mathrm{MeV}$ in each complete pass around the accelerator. Each linac contains a series of 160 resonant superconducting niobium RF cavities, a pair of which are shown in Figure 2.4. Eight cavities in a series comprise a "cryomodule", containing vacuum pipes/pumps, and magnetic dipoles/quadrupoles for beam steering/focusing.

Cryomodules are cooled by $2.2 \mathrm{~K}$ LHe from the central helium refrigerator, with $4.5 \mathrm{~K}$ LHe sup- 
plied from an end-station refrigerator for the radiation shields. This lowers the niobum cavities to well below the $9 \mathrm{~K}$ superconductivity point, optimized to minimize BCS energy losses. A 5-kW klystron generates RF power for each cavity, locked to the master driving RF (at the main injector cathode) to less than a $1^{\circ}$ difference in phase. This creates an oscillating phase gradient along each cavity (regulated by an RF control module to one part in $10^{4}$ ) with maxima and minima separated by a spacing equal to the distance between the nodes in the cavity. This causes a net acceleration of the electron bunches, because the klystron driving RF is in resonance with the bunch frequency [53].

Because electrons are such light particles, they effectively travel at the speed of light for energies ranging from $45 \mathrm{MeV}$ (upon leaving the injector) to $6 \mathrm{GeV}$. Thus, the same resonant cavities and driving frequencies can be used to boost the electron energies in every pass through the accelerator, which allows electrons in separate passes to be superimposed. A view of part of the linac during maintenance is shown in Figure 2.5 .

\subsubsection{Recirculation Arcs and Beam Optics}

Magnetic recirculation arcs are installed on both ends of the accelerator so that multiple passes can be made through the linacs. Four arcs are located on the west end, and five on the east side (see Figure 2.1), to accomodate up to 5 passes through both linacs. Although the spacing of all high energy electron bunches is constant (enabling them all to make multiple passes through the same linac), the separate recirculation arcs require differing magnetic field strengths for each pass. A chicane magnet at the end of each linac splits the beam into monoenergetic paths, which then pass through a series of six "periods" in each arc (Figure 2.6). Each period (nominally) contains 8 dipoles, 8 quadrupole and 4 sextupole magnets [60]. The optical configuration was designed to avoid beam degradation through dispersion and blurring, provide a path length that is an integer multiple of the RF wavelength (to avoid phase space dilution), and minimize energy spread due to the emittance of synchrotron radiation. In total, the optics design of the accelerator requires 2267 individual magnetic elements [53].

A beam switchyard separator either allows the beam to continue unhindered after one complete pass, or extracts the beam with a chicane from the appropriate recirculation arc after 2-5 passes, 


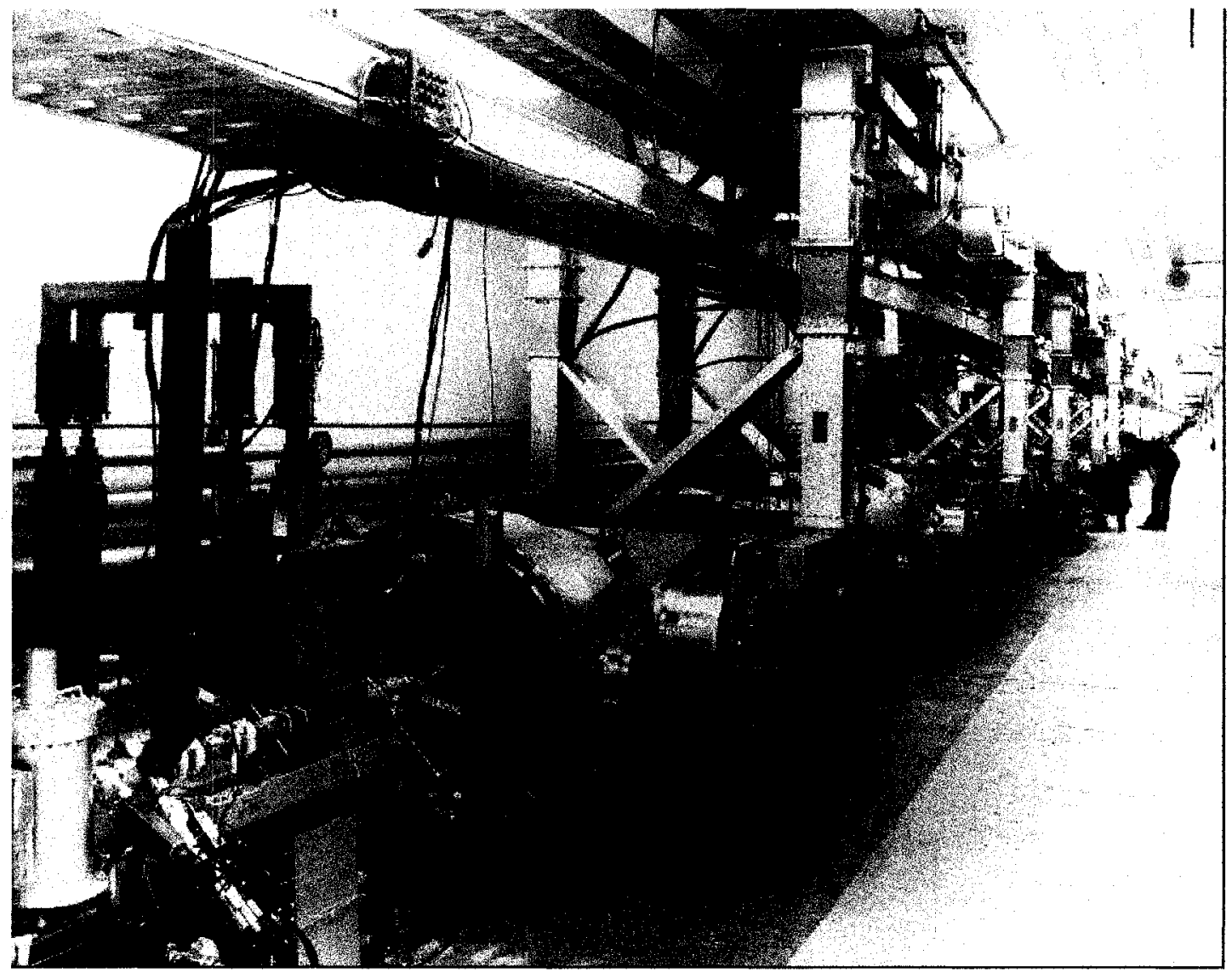

Figure 2.5: Photograph of a section of a linac during maintenance. Cryounits are concealed inside the cylindrical cryostats. From the JPIX Jefferson Lab picture exchange. 
depending on the beam energy requested. To deliver beam to all 3 of the research halls $(A, B$ and $C$ ), a $\frac{1}{3}$ harmonic RF separator system splits the interleaved bunches using an oscillating, deflecting magnetic field, steering the desired bunch toward the appropriate opening in a 3 aperture Lambertson septum, and delivers the beam to the appropriate experimental hall [53]. Different beam energies can even be delivered to different halls simultaneously by performing a similar "twobeam" split at the switchward separator. ${ }^{6}$ Electrons delivered to Halls $A$ and $C$ must be bent through arcs with steering magnets. These arcs are used to provide a precise measurement of the beam energy (see Section 4.2.4). Electrons continuing straight forward can then be delivered to the CLAS detector in Hall-B, the location of the EG1 experiment.

\subsection{Hall B Beam Line Devices}

After the Hall-B electron bunches are separated from the main beam line, they approach the entrance to Hall-B inside an evacuated beam pipe. Prior to entering (and after exiting) the EG1 polarized target and CLAS detector, the beam passes several devices, including a Meller Polarimeter, 3 Beam Position Monitors (BPMs), 3 Harp Scanners and, finally, a Faraday Cup. The uses of these beam line instruments are detailed in this section. Figure 2.7 shows a schematic diagram of Hall-B and the locations of these devices.

\subsubsection{Møller Polarimeter}

At the entrance of Hall-B, a Moller Polarimeter (Figure 2.8) is used to take measurements of the beam polarization. Møller polarimetry requires the use of a magnetized iron target, so it is an invasive measurement that cannot be done during data collection. Separate Meller data runs (taking $\sim 30$ minutes) were made periodically throughout the experiment.

The polarimeter consists of a target chamber with a $25-\mu \mathrm{m}$ thick permendur ${ }^{7}$ foil oriented at $\pm 20^{\circ}$ with respect to the beam line, longitudinally polarized to $7.5 \%$ by a 120 G Helmoltz mag-

\footnotetext{
${ }^{6}$ This is a bit more complex, though. An oscillating RF deflection field steers the selected bunch across a septum and out of the arc. The other two bunches, $120^{\circ}$ out of phase, are steered slightly in the opposite direction. Their paths are corrected by subsequent magnets before reinjection into the linac.

${ }^{7}$ Permendur is $49 \% \mathrm{Fe}, 49 \% \mathrm{Co}, 2 \% \mathrm{Va}$.
} 


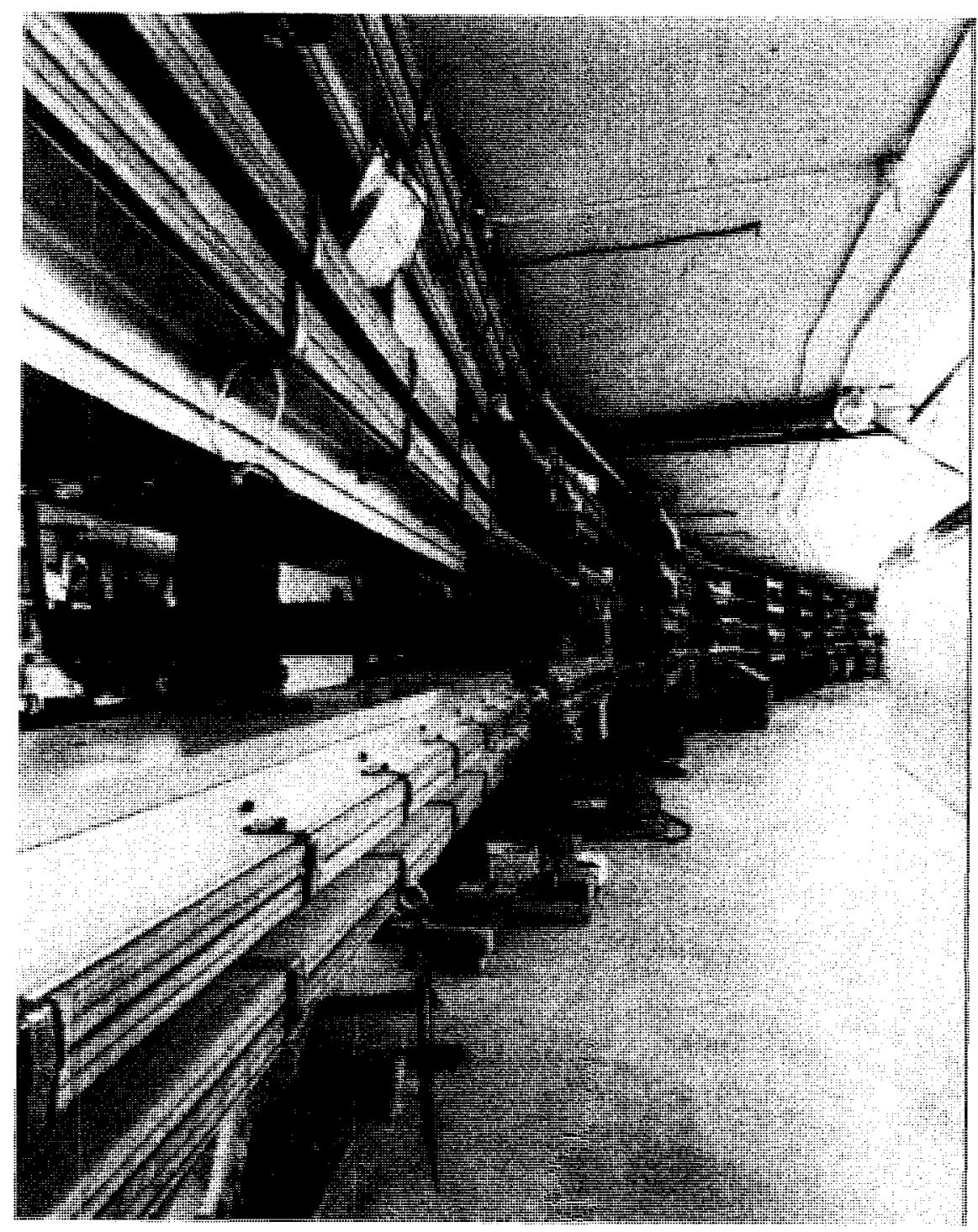

Figure 2.6: Photograph of a section of a recirculation arc. From the JPIX Jefferson Lab picture exchange. 


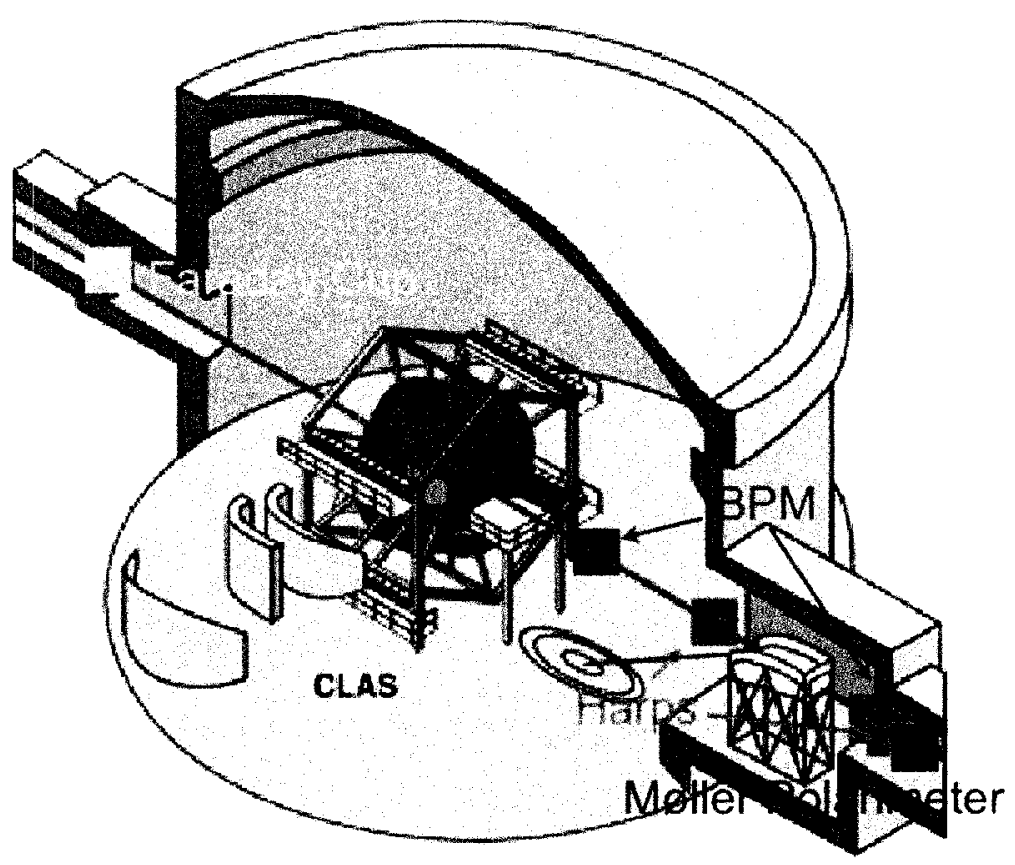

Figure 2.7: Hall-B schematic, showing the location of CLAS and the approximate locations of the beam line monitoring devices. One BPM and harp are located further up the beam line and are not shown.
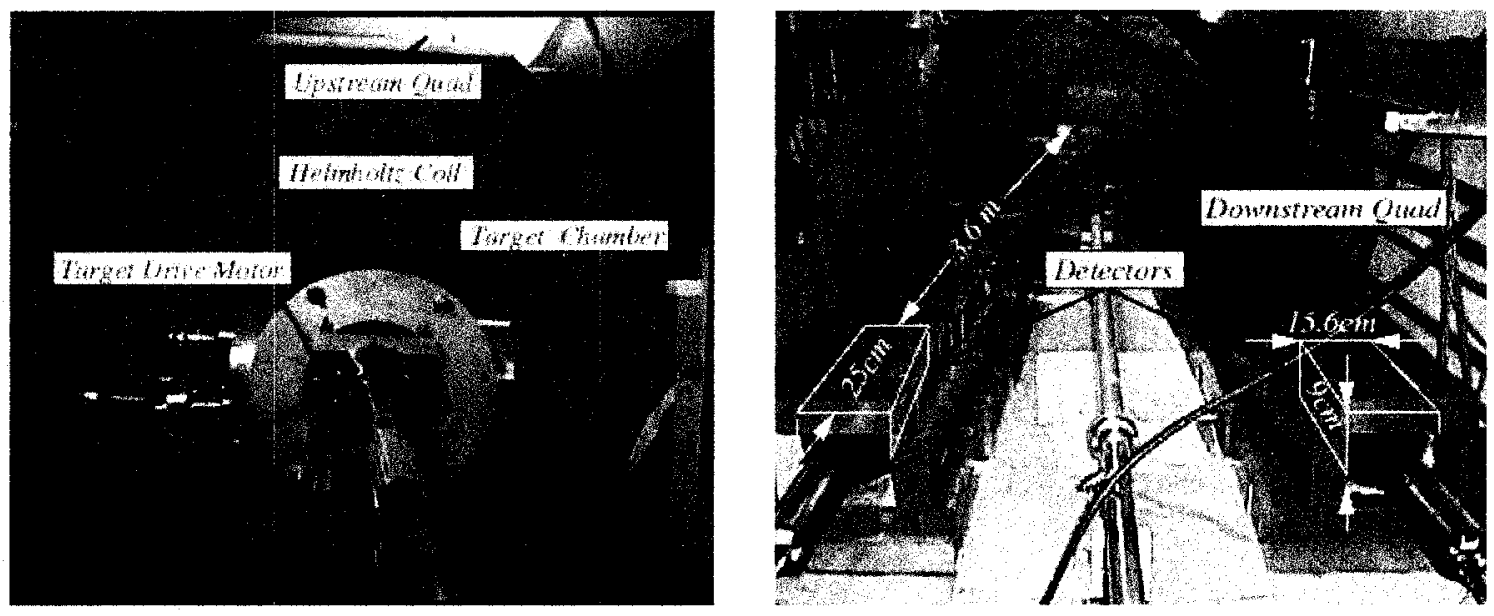

Figure 2.8: Photograph of the Moller polarimeter in Hall-B, showing upstream (left) and downstream (right) views. The electron beam travels through the thin central pipe. From Ref. [61]. 


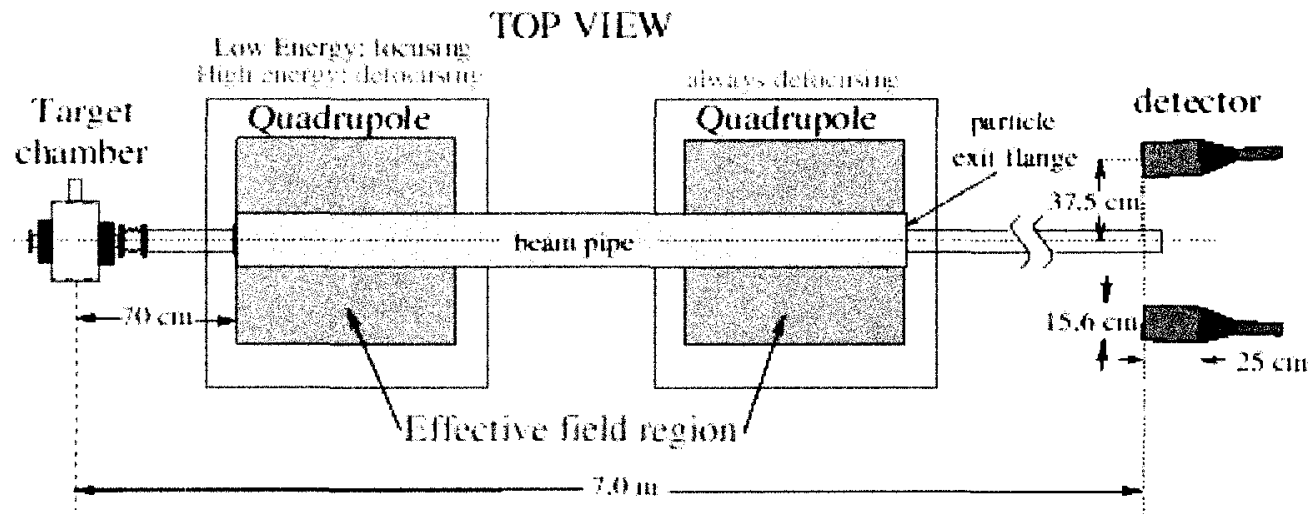

Figure 2.9: Schematic diagram of the Hall-B Møller polarimeter. From Ref. [30].

net [62]. Two quadrupoles separate the scattered electrons according to their polarizations. The electrons then enter one of two lead/scintillator/photomultiplier tube combinations for detection (see Figure 2.9) [30].

Elastic electron-electron scattering coincidences are used to determine the polarization. The differential scattering cross-section, in terms of the permendur target polarization $\left(P^{t}\right)$ and beam polarization $\left(P^{b}\right)$, is given by [2][61]

$$
\frac{d \sigma}{d \Omega} \propto\left(1+\sum_{i, j=x, y, z} P_{i}^{t} A_{i j} P_{j}^{b}\right)
$$

where

$$
\begin{gathered}
A_{y y}=-A_{x x}=\frac{\sin ^{4} \theta_{C M}}{\left(3+\cos ^{2} \theta_{C M}\right)^{2}} \\
-A_{z z}=\frac{\left(7+\cos ^{2} \theta_{C M}\right) \sin ^{2} \theta_{C M}}{\left(3+\cos ^{2} \theta_{C M}\right)^{2}} \\
A_{i j, i \neq j} \approx 0
\end{gathered}
$$

Here, $\theta_{C M}$ is the scattering angle in the CM frame, $z$ is defined as the beam axis, and the ee scattering plane is defined to be the $x z$ plane. Using knowledge of the scattering kinematics and $p^{t}$ (from the detectors and foil alignment, respectively), the beam polarization, $P_{z}^{b}$, can be determined.

The Møller measurement typically had an absolute statistical uncertainty of $1 \%$ and a system- 


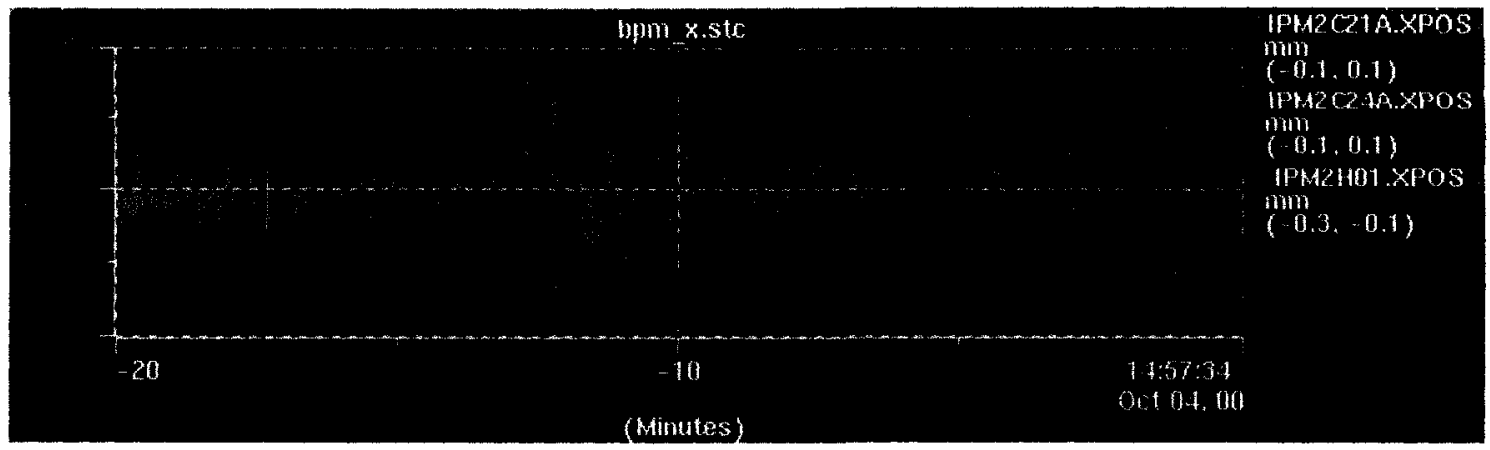

Figure 2.10: Screenshot of the online Beam Position Monitor measurement for the $x$-coordinate (in $\mathrm{mm}$ ) during part of the EG1b experiment. The three colored lines represent measurements from each of the 3 BPMs. The last several minutes of the plotted time period show marginal beam quality that required correction by the accelerator operators. From Ref. [63].

atic uncertainty of $\sim 2 \%[30]$. In practice, normalization to the elastic scattering asymmetry is used to determine the beam xtarget polarizations (see Section 6.2); the only actual uses for Moller measurements in this thesis are for determining the polarized ${ }^{15} \mathrm{~N}$ correction (Section 6.3 ) and for consistency checks on the $P_{b} P_{t}$ measurements.

\subsubsection{Beam Position Monitors (BPM)}

Three beam position monitors are located 36.0, 24.6 and $8.2 \mathrm{~m}$ upstream from the CLAS center. They measure the beam position in the $x y$ plane, as well as the (relative) beam intensity. Each BPM is composed of 3 RF cavities. The beam position is cross-calibrated using the Harp Beam Profile Monitors (described next), and the intensity is calibrated (periodically) with the Faraday Cup (Section 2.5). Measurements are taken at a rate of $1 \mathrm{~Hz}$, and these data are used in a feedback loop to keep the beam centered on the target [30]. An example of BPM measurements along one coordinate for all 3 monitors is shown in Figure 2.10.

\subsubsection{Harp Beam Profile Monitors}

The profile and diameter of the electron beam delivered to the target is measured during periodic harp scans. There are three different "harps" (at 36.7, 22.1 and $15.5 \mathrm{~m}$ upstream of the CLAS center) composed of thin, movable wires $(20 \mu \mathrm{m} \mathrm{W}, 50 \mu \mathrm{m} \mathrm{W}$ and $100 \mu \mathrm{m} \mathrm{Fe}$, respectively) in a 

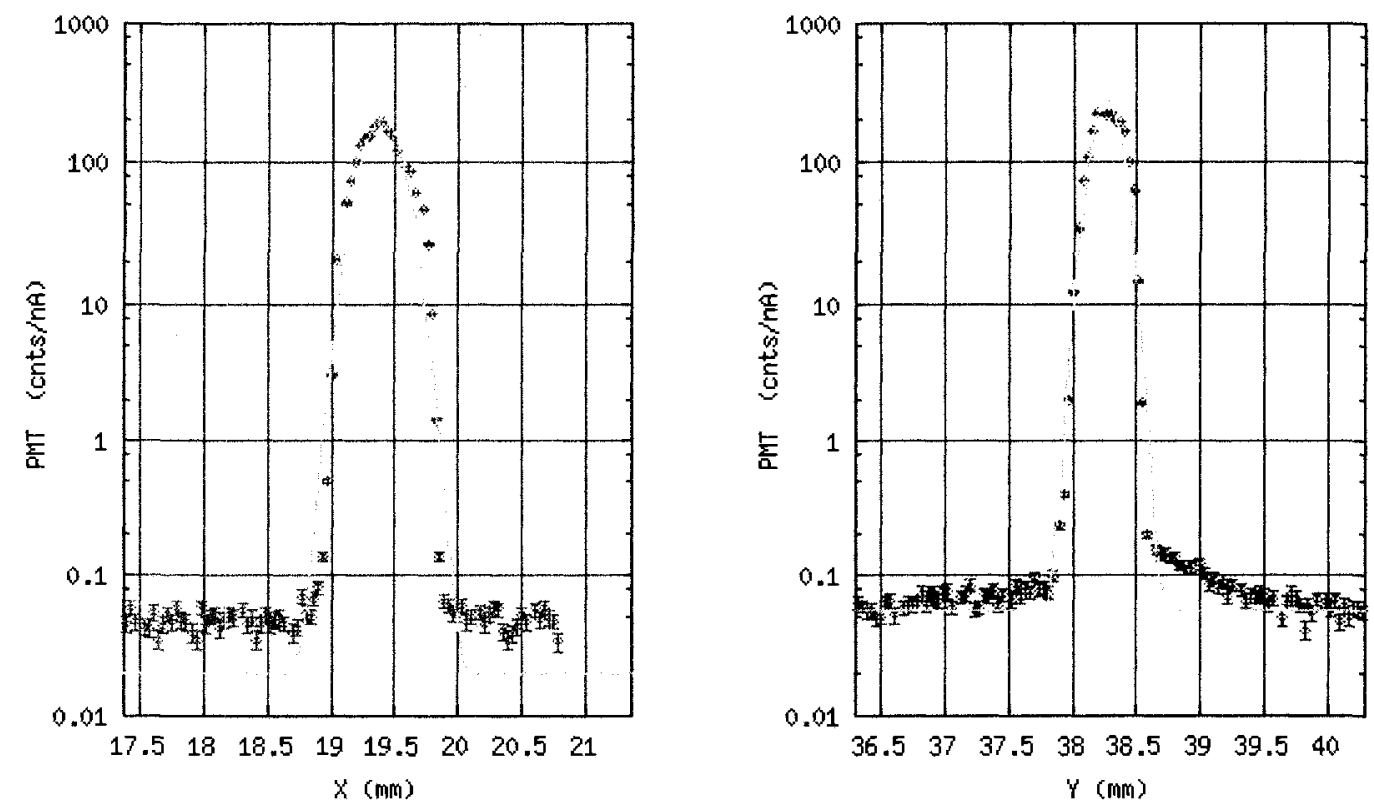

Figure 2.11: Results of a Hall-B beam harp scan during the EG1b experiment. Note that the PMT count scale is logarithmic; beam diameters smaller than $0.5 \mathrm{~mm}$ are typical. From Ref. [63].

cross-hair pattern. During a harp scan, the wires are slowly moved through the beam, resulting in scattering events whenever the wire crosses the beam. Photomultipliers (PMT) $10 \mathrm{~cm}$ from the beam line detect the scattered electrons via Cherenkov radiation in the PMT glass window. A beam profile (in $x$ and $y$ ) can then be reconstructed [30].

Figure 2.11 shows the results of a typical harp scan along both the $x$ and $y$-axes, performed during the EG1b experiment. Typical beam diameter measurements show an RMS of around 80 $\mu \mathrm{m}$, so that most of the beam is contained within a $200 \mu \mathrm{m}$ diameter. Note that, like Moller measurements, harp scans constitute an invasive measurement that cannot be completed during data collection.

\subsubsection{Faraday Cup}

The beam line ends at the Faraday Cup (FC), $29.0 \mathrm{~m}$ downstream from the CLAS center point. The Faraday Cup is used to integrate the beam current. The device is a $15 \mathrm{~cm}$ diameter long horizontal cylinder consisting of 75 radiation lengths $(4000 \mathrm{~kg})$ of lead, connected to an electric feedthrough 


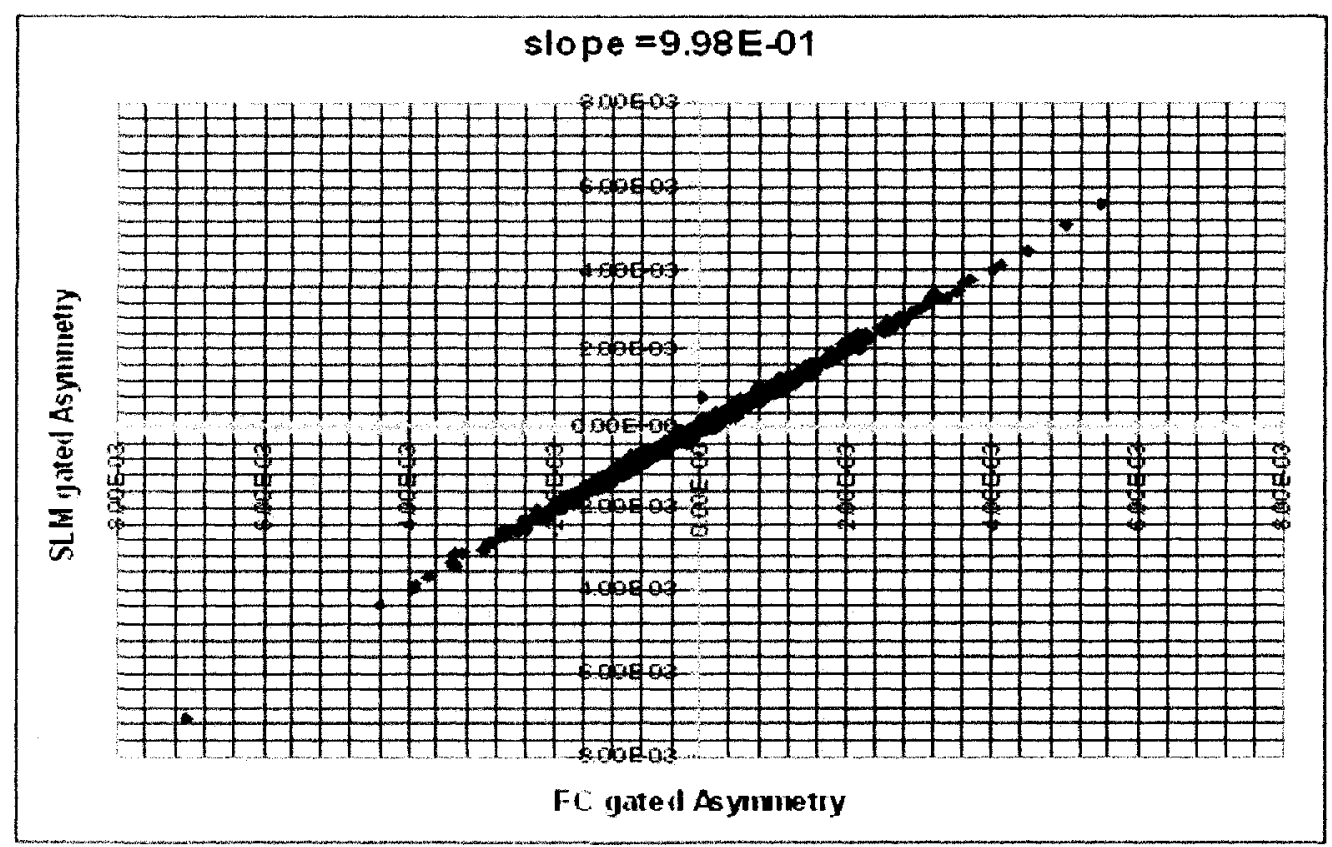

Figure 2.12: Faraday Cup charge asymmetry plotted against Synchrotron Light Monitor asymmetry for a set of EG1 runs, indicating a linear relation between measured charge and beam intensity. From Ref. [64].

to measure the collected charge [30].

It is connected through a logic gate to the CLAS data acquisition system, to record both total (ungated) and detector live-time (gated) counts. The latter omits charge collected when the readout electronics are busy. The main RF frequency is used to gate the FC so that readings for each beam helicity are recorded separately. Figure 2.12 compares Faraday Cup charge asymmetry measurements compared to asymmetry measurements from the Synchrotron Light Monitor (SLM) described earlier, from a test for helicity-related bias in the total charge measurement [64].

It is important to note that the narrow FC cylinder width $(15 \mathrm{~cm})$ means that the beam must remain tightly collimated after passing through the polarized target and CLAS detector. If multiple scattering in the target causes an angular spread in the beam, not all the incident charge will enter the Faraday Cup. This is a greater problem at lower beam energies, and is, in fact, a considerable problem in this experiment. Section 4.5 explains how this problem is handled in the analysis. 


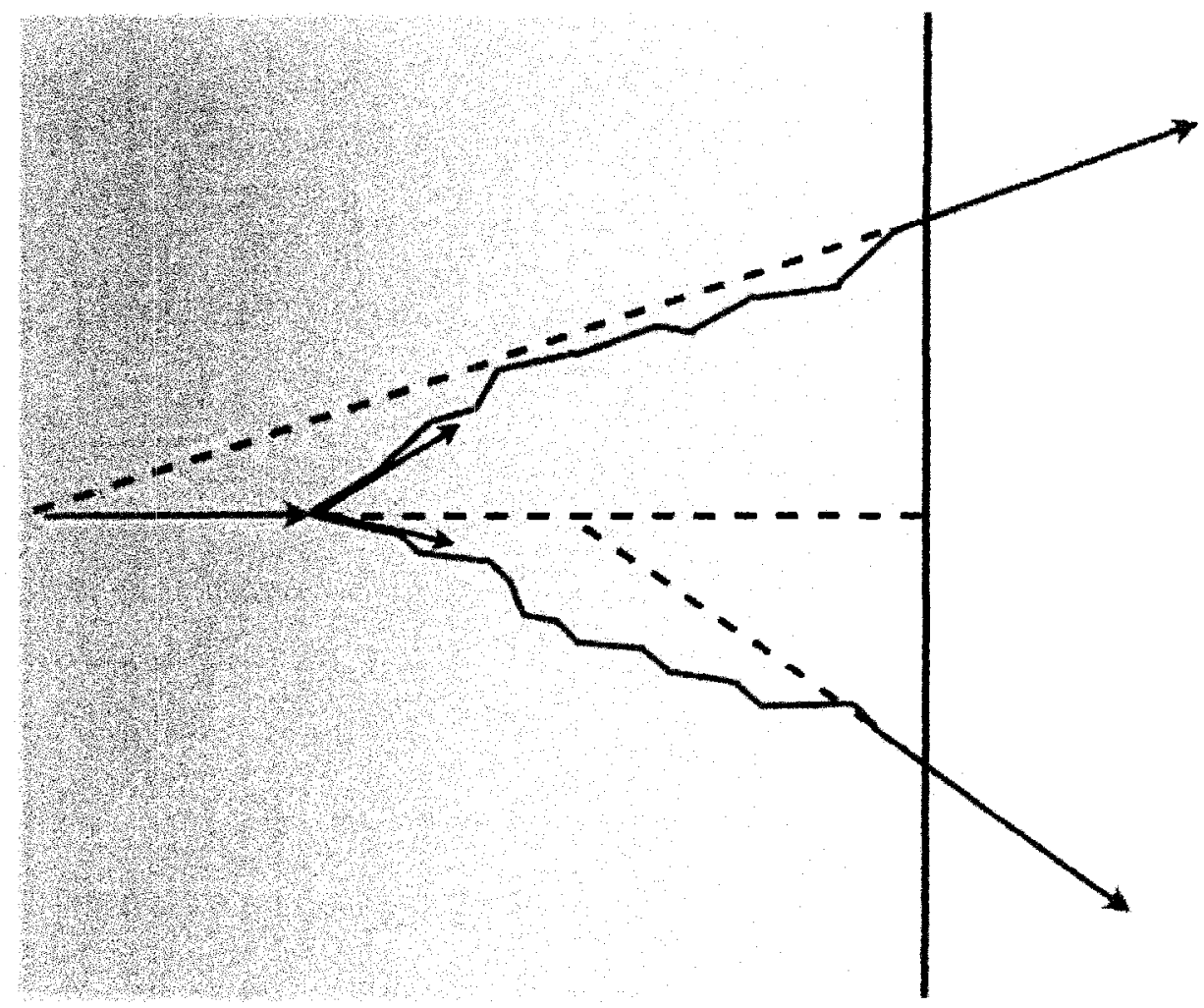

Figure 4.11: A greatly exaggerated picture of multiple scattering effects. The true vertex position and apparent angle are distorted by this effect. The black arrows show the true angles and vertex, while the blue dotted lines show the apparent angle and vertex positions of the scattered particles. Notice, in this case, that the true vertex position lies between the apparent vertex positions for each particle. While this is not necessarily the case for a specific event, using the weighted average of the vertices does, on average, increase the kinematic precison. 


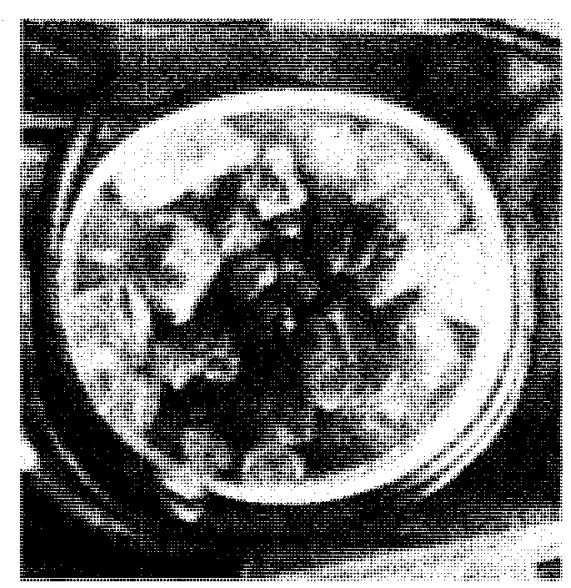

Figure 2.13: Beads of ammonia in a target cup. The purple discoloration is due to radiation damage.

the width of the target window. Helmholtz coils produce the required oscillating fields at the correct frequencies to produce the desired pattern. Raster magnet ADC amplitudes are recorded in coincidence with each scattering event, so that the raster pattern can be reconstructed (see, for example, Section 3.3.4).

\subsubsection{Dynamic Nuclear Polarization: Overview}

The method of Dynamic Nuclear Polarization (DNP) is used to polarize the ammonia target material. A complete, detailed treatise of this method is beyond the scope of this thesis; only a basic summary (specific to this experiment) and appropriate references are included here.

For spin- $\frac{1}{2}$ nuclei, which only have two possible spin orientations in an external field $\left(+\frac{1}{2}\right.$ and $-\frac{1}{2}$ ), the polarization along the magnetic field $(z)$ axis is given in terms of the spins $J$ as simply

$$
P=\left\langle J_{z}\right\rangle / J=n_{+}-n_{-}
$$

where $n_{ \pm}$represents the fraction of nuclei with each spin. Assuming internal equilibrium, the spins can then be characterized by the Boltzmann law with a characteristic temperature $T_{S}$ :

$$
\frac{n_{-}}{n_{+}}=\exp \left(-E_{m} / k T_{S}\right)
$$


where $E_{m}$ is the energy differences between the two spin states $m= \pm \frac{1}{2}$. Using $E_{m}=2 \mu H$ (for a spin of $+\frac{1}{2}$ ) where $\mu$ is the magnetic dipole moment of the nucleus and $H$ is the magnetic field magnitude, the polarization in thermal equilibrium is

$$
P=\frac{n_{+}-n_{-}}{n_{+}+n_{-}}=\frac{1-e^{2 \mu H / k T_{S}}}{1+e^{2 \mu H / k T_{S}}}=\tanh \frac{\mu H}{k T_{S}}
$$

However, in a $50 \mathrm{kG}$ magnetic field at $1 \mathrm{~K}$, this gives a polarization of $P=0.00511$, clearly too small for a polarized experiment. Dynamic polarization of the impurities must be employed to improve this value.

\subsubsection{DNP, Neglecting Spin-Spin Interactions}

Neglecting spin-exchange interactions between adjacent electrons and adjacent nuclei, the spin Hamiltonian for an electron of spin $S$ and a proton of spin $J$ can be written [67]

$$
\mathscr{H}=\mathscr{H}_{S Z}+\mathscr{H}_{J Z}+\mathscr{H}_{S J}+\mathscr{H}_{R F}
$$

where the four terms represent the Zeeman energy of the electron, Zeeman energy of the proton, the spin interaction of the electron and proton, and the externally applied microwave (RF) field, respectively. The dipole interaction term can be written [67]

$$
\mathscr{H}_{S J}=\frac{\hbar^{2} \gamma_{e} \gamma_{p}}{r^{3}}\left[\vec{S} \cdot \vec{J}-\frac{3(\vec{S} \cdot \vec{r})(\vec{J} \cdot \vec{r})}{r^{2}}\right]
$$

where $\gamma_{e}\left(\gamma_{p}\right)$ is the gyromagnetic ratio of the electron (proton). This can be calculated in terms of the products of the bilinear spin operators $S_{z,+,-}$ and $J_{z,+,-}$. First order perturbation theory yields 
the $|p e\rangle$ spin eigenfunctions

$$
\begin{aligned}
& |a\rangle=|-+\rangle+\varepsilon^{*}|--\rangle \\
& |b\rangle=|--\rangle+\varepsilon|-+\rangle \\
& |c\rangle=|++\rangle+\varepsilon^{*}|+-\rangle \\
& |d\rangle=|+-\rangle+\varepsilon|++\rangle
\end{aligned}
$$

where

$$
\varepsilon=\frac{3}{4} \frac{\hbar \gamma_{e}}{r^{3} H} \sin \theta \cos \theta e^{-i \phi}
$$

and $\varepsilon^{*}$ is the complex conjugate of $\varepsilon . \theta$ and $\phi$ represent the polar and azimuthal angles of $r$ with respect to the polarization axis.

Thus, we see that the eigenstates resulting from the e-p spin interaction are mixtures of the unperturbed free particle eigenstates. That is, the spin eigenstates $|++\rangle, 1+-\rangle, 1-+\rangle, 1--\rangle$ are 'rotated' into the eigenstates $|a\rangle,|b\rangle,|c\rangle,|d\rangle$ by the spin-spin interaction. This is essential, as now an externally applied field $\left(\mathscr{H}_{R F}\right)$ can induce transitions between the new eigenstates, flipping the spins of both the electron and proton, such as

$$
\begin{aligned}
& 1++\rangle \rightarrow|--\rangle \\
& 1+-\rangle \rightarrow|-+\rangle
\end{aligned}
$$

which are forbidden (due to dipole selection rules) if the electron-proton spin interaction is neglected (see Figure 2.14). 


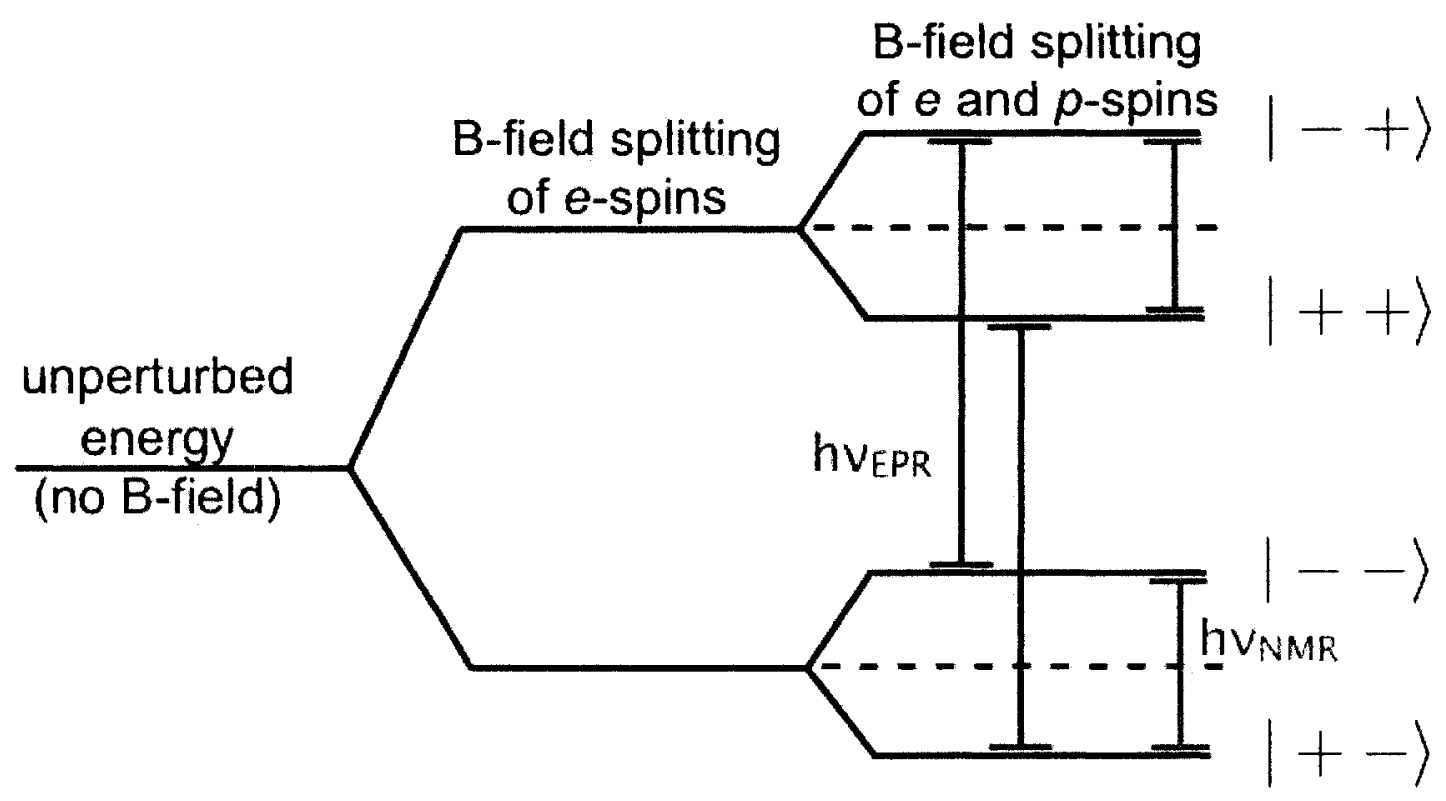

Figure 2.14: Energy levels of the $e p$ target system in an external magnetic field. The ket notation $\left|S_{P} S_{e}\right\rangle$ represents the spin states of the proton and electron. Allowed transitions $h \nu_{\text {ESR }}$ (Electron Spin Resonance) and $h \nu_{\text {NMR }}$ (Nuclear Magnetic Resonance) are shown in blue and red. Other transitions are forbidden by dipole selection rules in the absence of a changing external field. 
An external oscillating electromagnetic field with frequency

$$
\nu=\nu_{\mathrm{EPR}} \pm \nu_{\mathrm{NMR}}
$$

(where the EPR and NMR energies are shown in Figure 2.14) excites the "forbidden" transition, $W^{+}$ or $W^{-}$(shown in Figure 2.15), depending on the sign in Eq. 2.13. The probability ratio of a $W^{ \pm}$ transition to that of an "allowed" dipole transition is $4|\varepsilon|^{2}$, as calculated from the matrix elements $\mathscr{H}_{\text {RF }}$ applied to the admixed eigenstates [67].

After a simultaneous spin flip (Eq. 2.12), both the electron and proton eventually return to their ground state, as determined by their respective spin-lattice relaxation times. This relaxation time is approximately a factor of $10^{6}$ longer for the proton than the electron [65] (about $10^{-3} \mathrm{~s}$ for the electron, but $10^{3} \mathrm{~s}$ for the proton). Once the electron relaxes, it is again available to interact with another proton, and induce another "forbidden" spin flip. The process continues, albeit with a decreasing probability, as the distance $r$ increases as the protons are polarized, shrinking the magnitude of the Hamiltonian terms in Eq. 2.9. Eventually, a net polarization of the protons is induced, and an equilibrium is reached. ${ }^{10}$ When this occurs, the polarization of the target remains stable, so long as the magnetic field and microwave radiation are continuously applied.

\subsubsection{DNP in a real solid: Equal Spin Temperature (EST) Theory}

The preceding provides a relatively simple model for DNP, where the Zeeman energy levels were considered infinitely sharp, in the absence of spin-interactions within the electron lattice. However, in real solids (such as our $\mathrm{NH}_{3}$ target), the spin-spin interactions greatly complicate the system, effectively "broadening" the energy levels into bands containing many degrees of freedom. In this case, the thermodynamic approach of Equal Spin Temperature (EST) theory must be used to describe the system.

EST theory presumes that the proton Zeeman system (described above) is characterized by a temperature $T_{Z}$, and that the spin-spin Hamiltonian between electrons is characterized by a second Boltzmann distribution with temperature $T_{S S}$ [67]. If the radiating microwave energy is fixed, but the

\footnotetext{
${ }^{10}$ It is not strictly true that the value of $r$ in the Hamiltonian is the limiting factor in the inducing of polarization in the target; a mechanism known as spin diffusion is also involved in transporting the polarization to adjacent nuclei.
} 


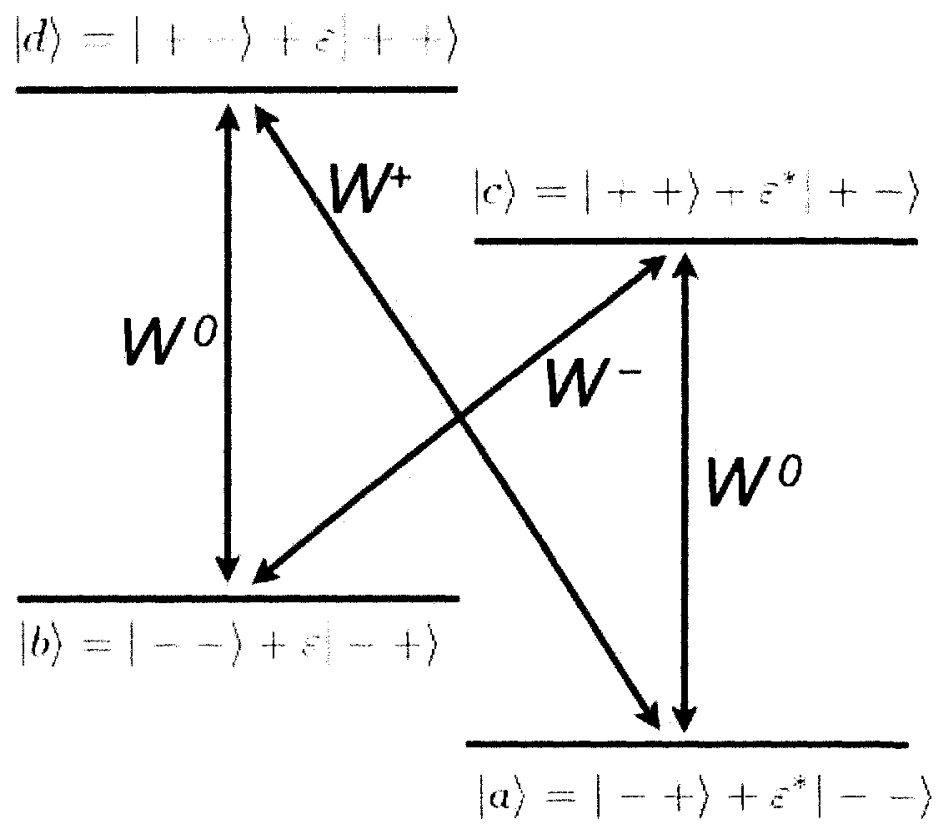

Figure 2.15: Energy levels (expressed in a new set of basis kets) after application of microwave radiation to the magnetized material. The transitions $W^{+}$or $W^{-}$enable forbidden transitions, excluded from Figure 2.14, that simultaneously flip the spins of both the electron and proton. From Ref. [67]. 


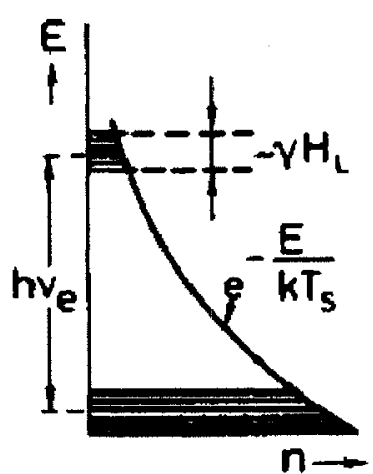

a)

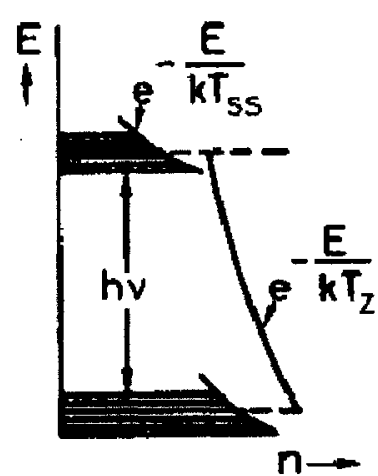

b)

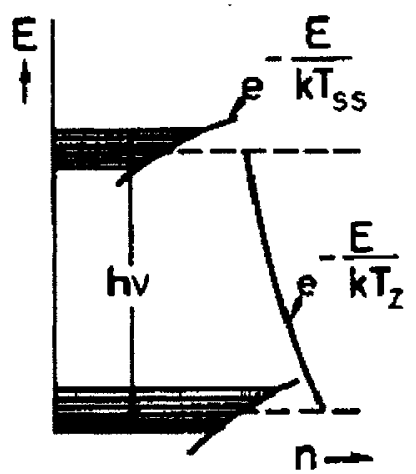

c)

Figure 2.16: Distribution of energy levels in terms of population fraction $n$. In thermal equilibrium (a), the average energy separation between the bands is $\nu_{e}=\nu_{\mathrm{ESA}}$, and a single spin temperature $T_{S}$ describes the whole system. If the energy separation is slightly perturbed (that is, the separation is $\nu=\nu_{e}-\delta$ ), then two characteristic temperatures, $T_{S S}$ and $T_{Z}$, describe the system. If $\nu<\nu_{e}$, (b) then $T_{S S}>0$. If $\nu>\nu_{e}$, (c) then $T_{S S}$ become negative, corresponding to opposite polarization. From Ref. [67].

"bands" corresponding to the polarization states (depicted in Figure 2.16) contain many (closely separated) energy levels, then the electron system absorbs an energy $h(\nu+\delta)$, with $\nu$ defined in Eq. 2.13 , with the energy $h \delta$ absorbed by the spin-spin thermodynamic system.

The Zeeman and spin-spin temperatures of the electron system move toward thermodynamic equilibrium $T_{Z}=T_{S S} \equiv T_{\ell}$ (lattice temperature). In the process of achieving this equilibrium, energy is emitted or absorbed by the spin-spin system, and the proton Zeeman system (with its own temperature, $T_{Z_{P}}$ ) either emits or absorbs energy $\nu_{\text {NMP }}$ (i.e. the proton Larmor frequency). The proton Zeeman system "cools" through an electron double-spin flip and proton single-spin flip [68]. ${ }^{11}$ The polarization then stabilizes at thermal equilibrium, with a polarization given by Eq. 2.7, with the substitution $T_{S} \rightarrow T_{Z_{P}}$.

\footnotetext{
${ }^{11}$ Note that cooling can occur through emission or absorption of energy, the latter seeming contrary to intuition. For spin- $\frac{1}{2}$ systems, however, canonical absolute temperatures can be positive or negative, with negative temperatures corresponding to spins in the negative direction. Systems with negative temperatures must absorb energy in order to cool [68].
} 


\subsection{5 ${ }^{15} \mathrm{NH}_{3}$ as a Polarized Target}

${ }^{15} \mathrm{NH}_{3}$ was selected for use as a polarized target due to several specific properties. Free protons $\left({ }^{1} \mathrm{H}\right)$ are required for $e p$ scattering experiments, and these protons must be in a polarizable (spin $\frac{1}{2}$ ) configuration. Pure $\mathrm{H}_{2}$ is (unfortunately) not a viable option, because the bonding of the two hydrogen atoms results in a non-polarizable spin-1 configuration. 12 Traditionally, butanol and ammonia have been used for polarized targets. Of these two targets, ammonia is much better at retaining its polarization under prolonged irradiation [69].

Of course, under DNP, the nitrogen nuclei are not immune to the effects of polarization, either, though the net polarization of the nitrogen is much smaller than that of the free protons. ${ }^{15} \mathrm{~N}$ is selected in lieu of the more common isotope, ${ }^{14} \mathrm{~N}$, because, viewed in the shell model, ${ }^{15} \mathrm{~N}$ has a single ( $\operatorname{spin} \frac{1}{2}$ ) unpaired neutron which carries all the polarization, whereas, ${ }^{14} \mathrm{~N}$ (a spin-1 nucleus), contains an unpaired proton and neutron, which can both be polarized [70]. EST theory predicts well the relation between the polarizations of ${ }^{15} \mathrm{~N}$ and ${ }^{1} \mathrm{H}$ in $\mathrm{NH}_{3}$; this empirical relation has been well tested in past target studies [71]. Figure 2.17 shows the polarization of ${ }^{15} \mathrm{~N}$ as a function of proton polarization in the SMC frozen $\mathrm{NH}_{3}$ target compared to the full calculation of EST theory. Note that, at low polarizations, the relation is fairly linear. At high polarizations, nonlinear saturation effects begin decreasing the efficiency of the proton polarization against that of the nitrogen. Asymmetry corrections due to ${ }^{15} \mathrm{~N}$ polarization are dealt with later, in Section 6.3.

\subsubsection{Polarized Target System}

The EG1 target system consists of the following list of elements:

1. Superconducting Helmholtz magnet (to generate the $5 \mathrm{~T}$ magnetic field)

2. Refigerator and cryostat unit (to maintain the necessary $1.5 \mathrm{~K}$ temperature)

3. Microwave system (for inducing the RF double spin-flip transitions)

4. Continuous wave NMR system (for online monitoring of the polarization)

\footnotetext{
${ }^{12}$ As noted in Section 1.5, HERMES utilized a polarized hydrogen target, but only in a gaseous form, which greatly limited the event flux.
} 


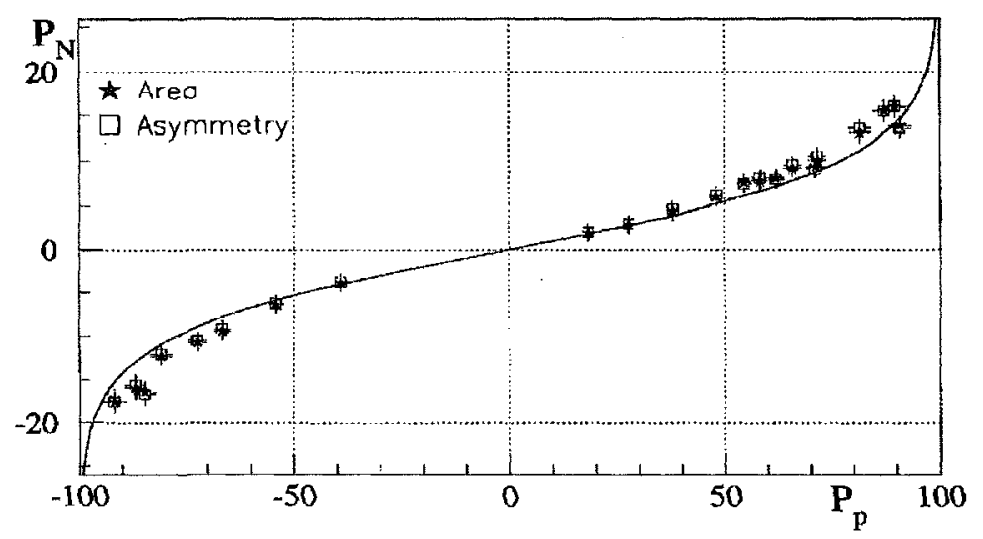

Figure 2.17: Polarization of ${ }^{15} \mathrm{~N}$ as a function of proton polarization in frozen $\mathrm{NH}_{3}$ during the SMC experiment. Actual data (obtained using two methods) are compared to the prediction of EST theory. From Ref. [71].

5. Target insert (contains the actual target material, to be changed for different runs)

The target magnet is positioned at the center of the torus magnet inside the CLAS detector (Section 2.5). It surrounds the target, producing a magnetic field coaxial with the beam line. The field is uniform (accurate within $10^{-4}$ ) over a central cylindrical volume $20 \mathrm{~mm}$ in diameter and $20 \mathrm{~mm}$ long [65]. This uniformity is necessary to ensure a narrow ESR linewidth. The axial diameter of the magnet allows for unimpeded forward scattering within $50^{\circ}$ of the beam axis. Coil superconductivity is maintained by an external liquid helium refrigerator.

The $1.5 \mathrm{~K}$ temperature necessary to maintain the polarization was achieved by pumping liquid helium at a rate of $3300 \mathrm{~m}^{3} \mathrm{mr}$ through a diagonally mounted cryostat system (Figure 2.18). The target chamber is injected with LHe from the cryostat. Evaporation occurs through hexagonal vents around the front end of the target. Temperature was monitored by a ${ }^{3} \mathrm{He}$ pressure bulb inside the evaporation chamber. Figure 2.19 shows the LHe reservoir as the target assembly is inserted into the Helmholtz cylinder.

The RF is supplied by an Extended Interaction Oscillator (EIO) that delivers about $1 \mathrm{~W}$ of microwave power with a linewidth of about $10 \mathrm{MHz}$. The $140 \mathrm{GHz}$ radiation, adjustable over a bandwidth of $2 \mathrm{GHz}$ (by varying the length of a remotely controlled resonant cavity), is generated to match the precise frequency requirements required by Eq. 2.13. The radiation is emitted from a 


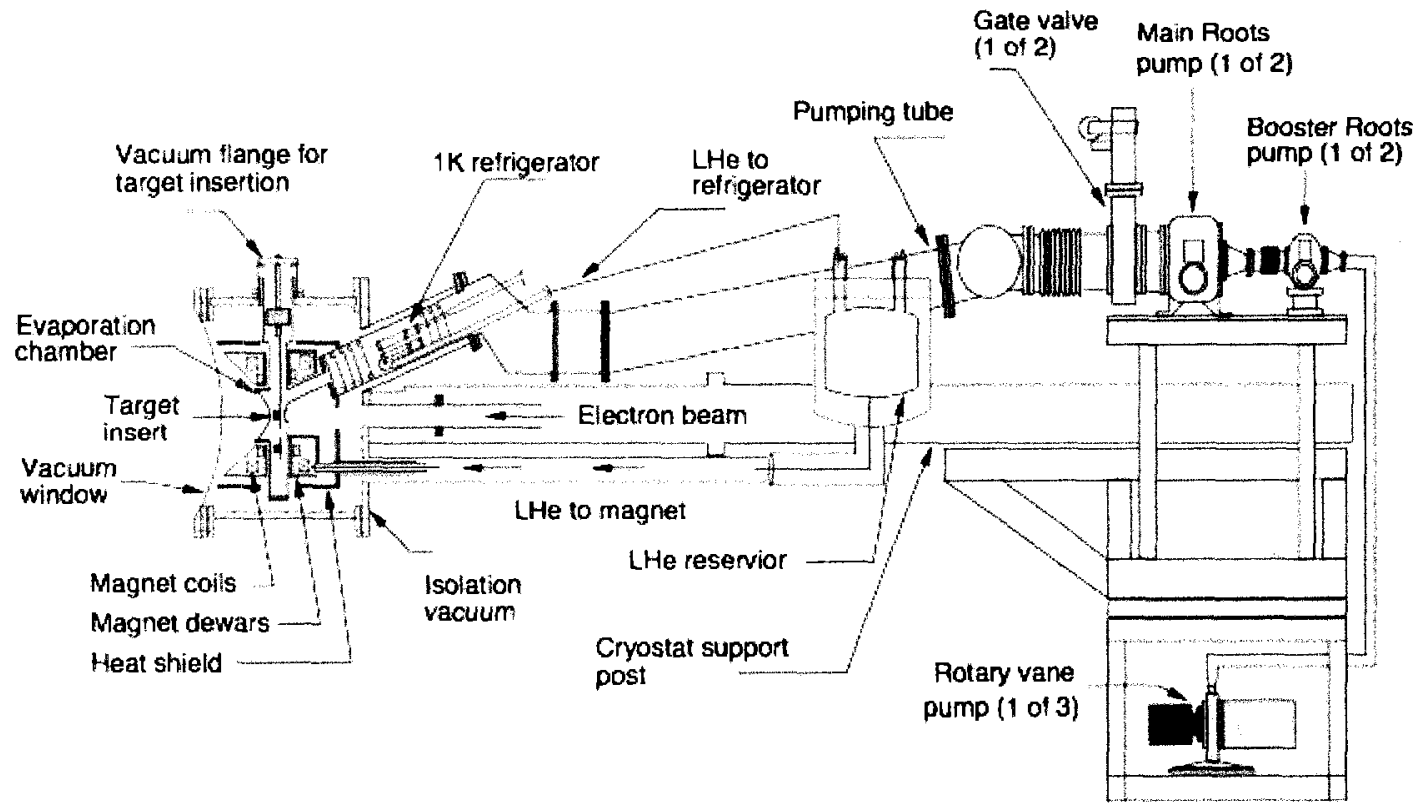

Figure 2.18: The polarized target cryostat assembly, shown in its configuration with the target chamber (banjo), insert, and magnet coil. From Ref. [65].

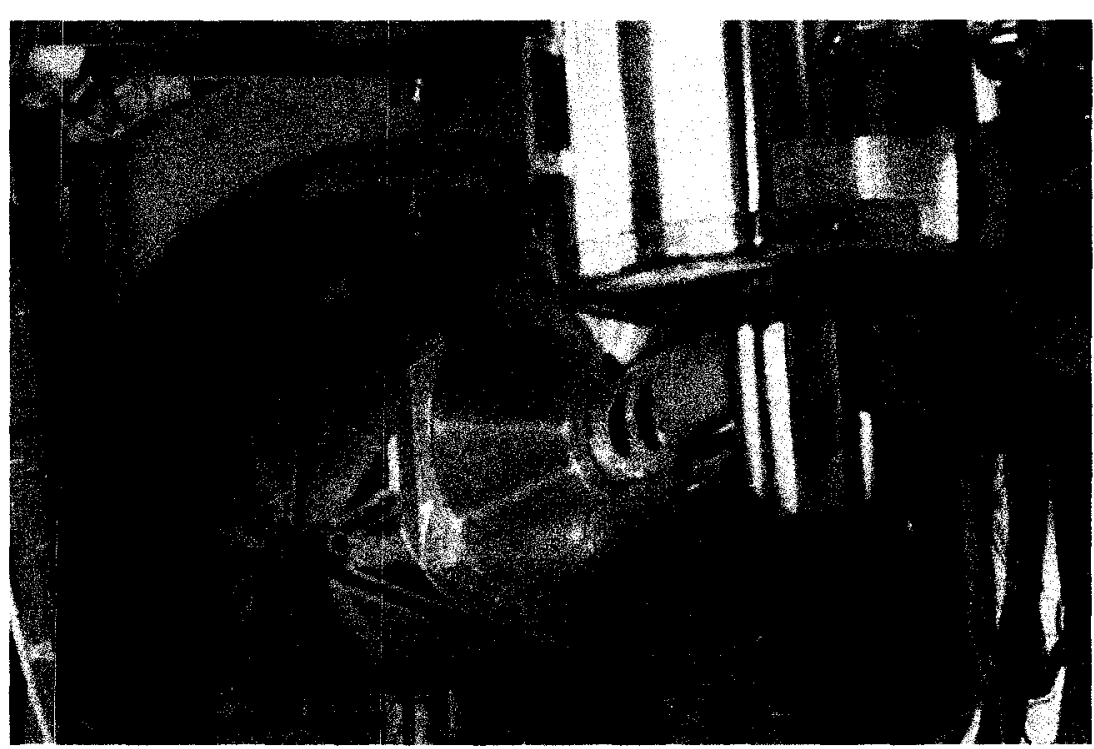

Figure 2.19: The target assembly being inserted into the superconducting Helmholtz magnet. The the reservoir is clearly visible in the foreground. 


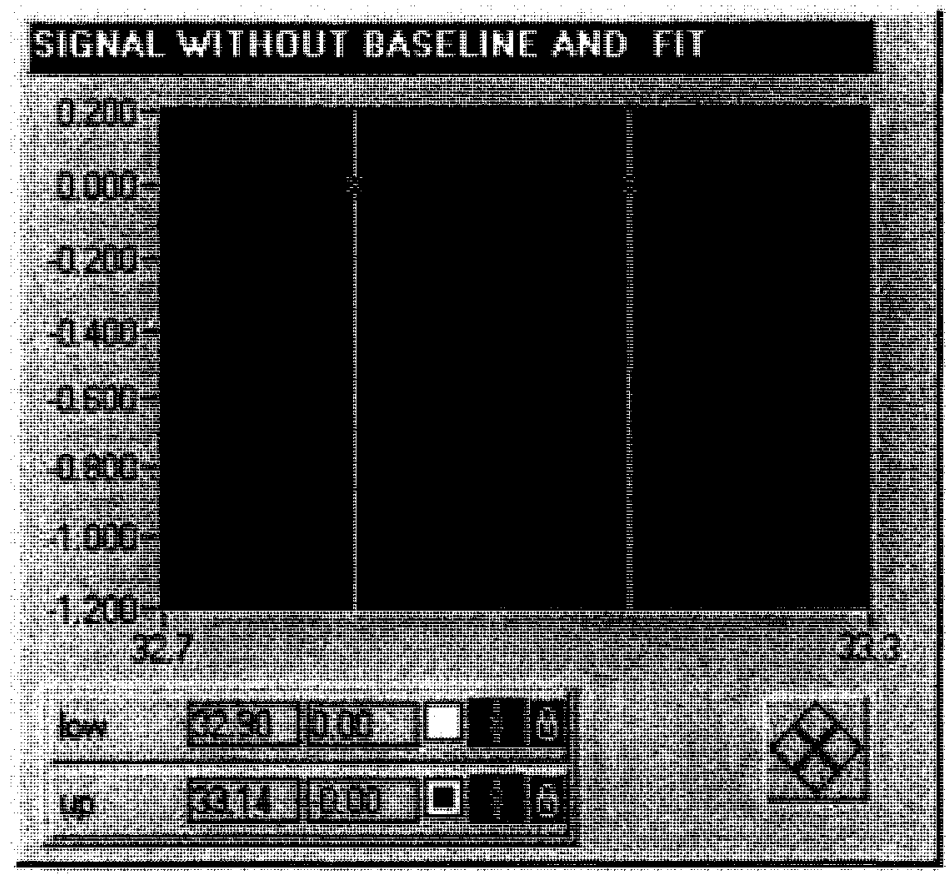

Figure 2.20: Screenshot of the online NMR polarization monitor for an $\mathrm{NH}_{3}$ target. The area under the curve gives the total polarization, measured as -0.736 in this case.

small metal "horn" (visible later in Figure 2.25) to continuously irradiate the magnetized target volume.

The NMR system, used for online monitoring of the ammonia $\left(\mathrm{NH}_{3}\right.$ and $\left.\mathrm{ND}_{3}\right)$ polarizations, consists of a coil wrapped around the polarized target material as part of a resonant RLC circuit. A varying RF frequency centered about $212.6 \mathrm{MHz}$ (the proton Larmor frequency) is swept through the circuit. ${ }^{13}$ In a plot of voltage vs. frequency, the polarization of the sample is proportional to the area under the curve. Figure 2.20 shows a screenshot of the online NMR monitor plot for the $\mathrm{NH}_{3}$ target.

Unfortunately, the proportionality constant used to calculate the polarization is difficult to determine accurately, due to thermal drifts in the NMR circuit [65], and is known to be less accurate at lower polarizations. Also, the polarization at the center of the target, where scattering actually occurs (and degradation due to radiation may be greater), may be different than that measured by

\footnotetext{
${ }^{13}$ For $\mathrm{ND}_{3}$, the Larmor frequency is $32.6 \mathrm{MHz}$. NMR analysis for the deuteron RF peak is also different, because two peaks are involved. See Ref. [65] for details.
} 


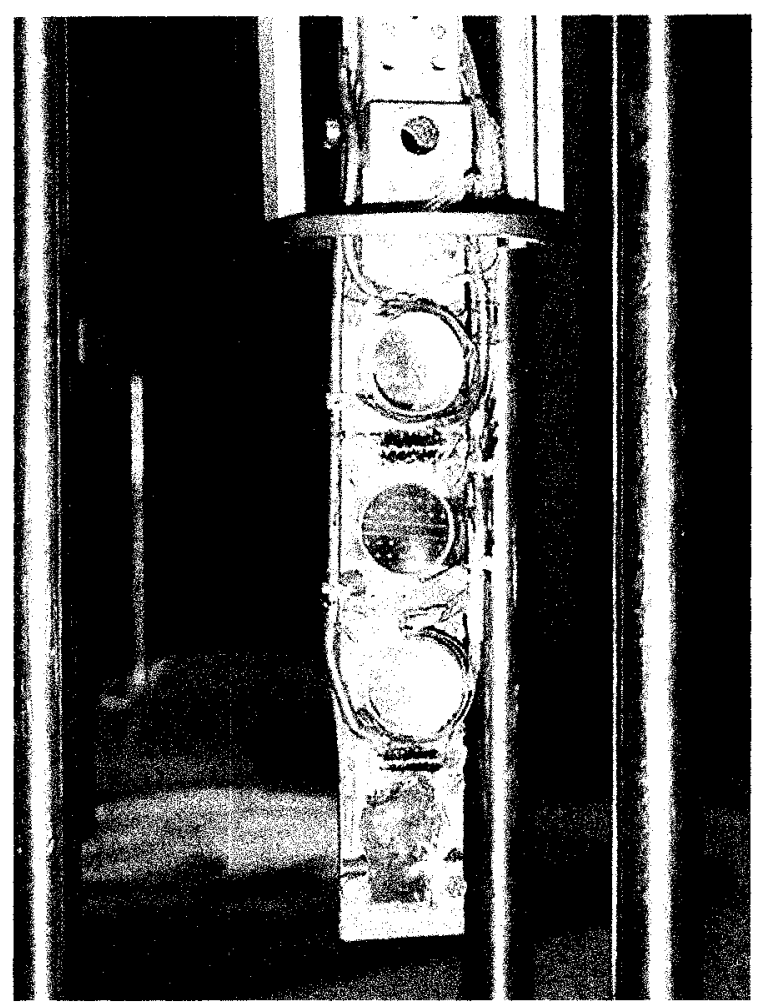

Figure 2.21: Target insert strip showing the 4 target cells used for the EG1 targets: $\mathrm{ND}_{3},{ }^{12} \mathrm{C}, \mathrm{NH}_{3}$ and empty, from the top down. Note the NMR coils that surround the $\mathrm{ND}_{3}$ and $\mathrm{NH}_{3}$ cells. Only the carbon target is full at the time of this photograph.

the NMR. Thus, although NMR is useful as an online monitor, actual analysis of polarizations relies on the $e p$ elastic peak ratio method, described in detail in Section 6.2.

The target insert is a thin aluminum strip ( $25 \mu \mathrm{m}$ thick at the target cell entrance) consisting of (nominally) $1-\mathrm{cm}$ thick cylindrical cells, shown in Figure 2.21. These cells contain the target material. Frozen $\mathrm{NH}_{3}$ and $\mathrm{ND}_{3}$ beads are placed in two of the cells (surrounded by NMR coils), and a 2.3-mm thick slab of amorphous carbon (for background subtraction) is placed in a third cell. The fourth cell is left empty, for additional background subtraction purposes. This strip is connected to a metal vacuum flange (with necessary feedthroughs) and a brass heat sink to maintain a cryogenic vacuum environment for the target (Figure 2.22). A motor shaft allows vertical motion of the target strip, so that the target cells can be alternated between experimental runs. 


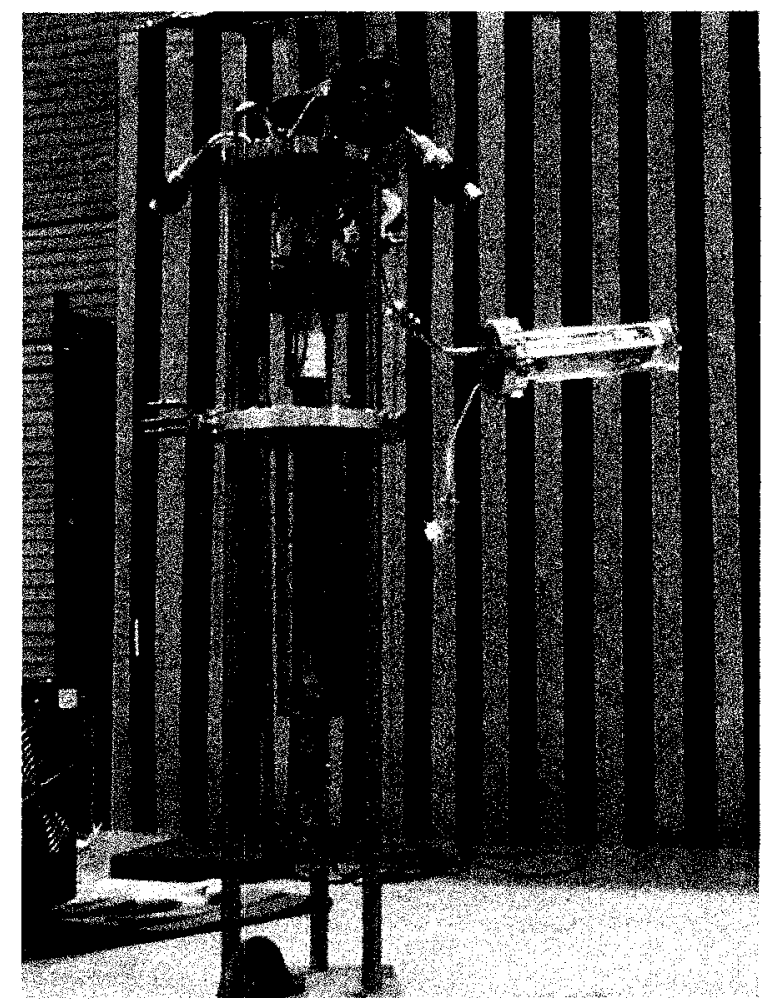

Figure 2.22: The target stick connected to a vacuum flange (with feedthroughs) for vertical insertion into the EG1 target system. 


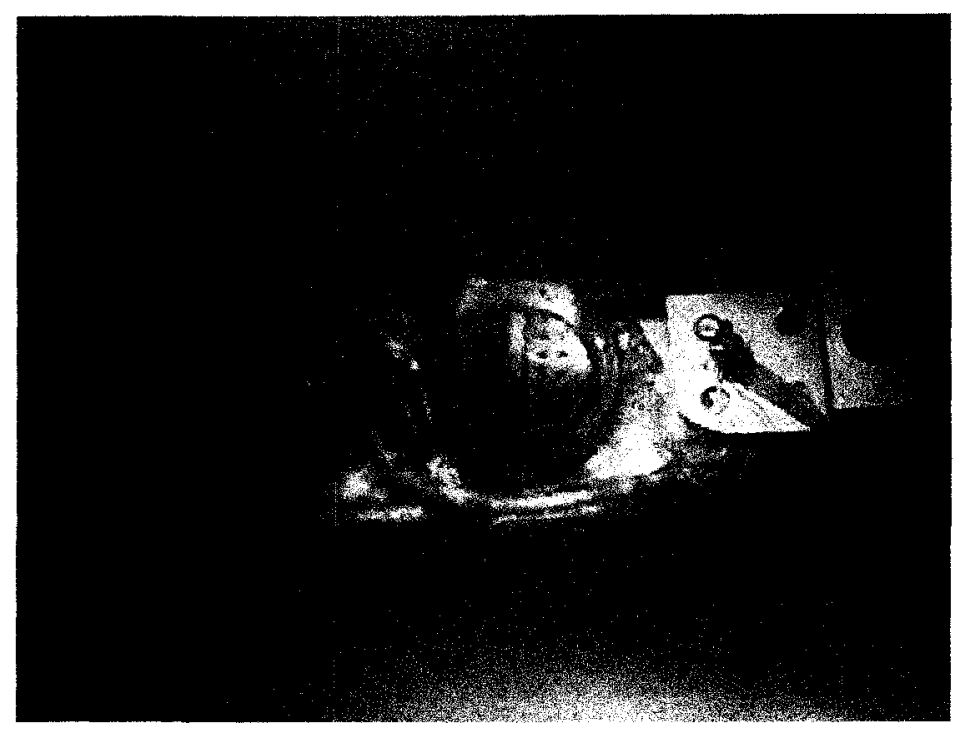

Figure 2.23: The target stick, all cells filled, immersed in a liquid nitrogen bath. The $\mathrm{NH}_{3}$ cell is shown.

The filled targets must remain immersed in liquid nitrogen when not in use (Figure 2.23), to maintain the presence of the paramagnetic free radicals. A $50 \mu \mathrm{m}$ thick Kapton foil cover (Figure 2.24) is used to keep the material in each cell in place. ${ }^{14}$ The filled target stick is inserted into a (nominally) $2.0 \mathrm{~cm}$ diameter (127 $\mu \mathrm{m}$ thick) Kapton "minicup" within a cylindrical vacuum-sealed "banjo" with thin $(71 \mu \mathrm{m})$ aluminum exit windows (Figure 2.25). The minicup is filled with liquid helium during the experimental run.

A second $\left({ }^{15} \mathrm{~N}\right)$ target stick, nearly identical to the first, except that it contained only two cells, was used for two shorter run sets during the experiment. One cell contained a $2.2 \mathrm{~mm}$ thick amorphous carbon slab, while the other was filled with isotopically enriched $(98 \%)$ solid ${ }^{15} \mathrm{~N}$. This target was used for modeling the relation between ${ }^{15} \mathrm{~N}$ and ${ }^{12} \mathrm{C}$ so that amorphous carbon data (collected throughout the experiment) could be used for accurate background subtraction.

The entire target assembly was mounted together (Figure 2.26) and inserted into the target magnet and CLAS detector, where the polarization process in the $5 \mathrm{~T}$ magnetic field could begin. More detailed descriptions of all the EG1 target components can be found in Ref. [65]. A much longer and more detailed description of the target assembly, operation and performance can be

\footnotetext{
${ }^{14}$ Kapton is added to the empty target cell, as well.
} 

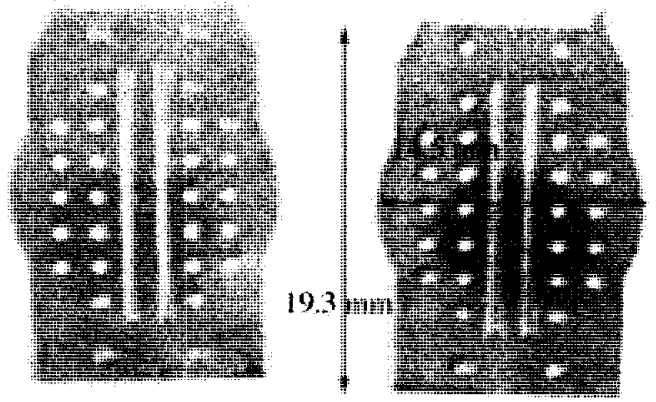

Figure 2.24: Kapton foil coverings used on the target cell. Kapton foil is used in nuclear physics experiments due to its high resistance to radiation damage.

found in Ref. [32].

\subsubsection{Modeling the Target for Background and Radiative Corrections}

The measured vertex resolution of scattered events in the EG1 experiment is good enough to subtract scattering contributions from the vacuum windows, but not nearly good enough to separate scattering events from the banjo windows, Kapton and aluminum cell windows, or minicup. Also, scattering occurs from LHe in the minicup, as well as from the ${ }^{15} \mathrm{~N}$ nuclei. Dilution factors must be determined to remove the contributions from all these elemerits, leaving only the sums over proton scattering events. This procedure is described in Chapter 5 . Making these calculations requires a precise model of the thicknesses and densities of the target through the beam path and through all possible forward scattering angles.

Table 2.1 lists the densities and thicknesses of all materials in the target(s) within $\sim 5 \mathrm{~cm}$ of the target center. ${ }^{15}$ Note that length of ammonia is only approximate, due to the unknown packing fraction of frozen granules, which can "powder" and settle within the target cell. The ${ }^{15} \mathrm{~N}$ target length is also not exactly known, since the amount of frozen material is not necessarily constant. The LHe (total cell) length is also uncertain from the target dimensions, due to possible warping of

\footnotetext{
${ }^{15}$ This includes all target materials inside of the wide event vertex cut that will be made later.
} 


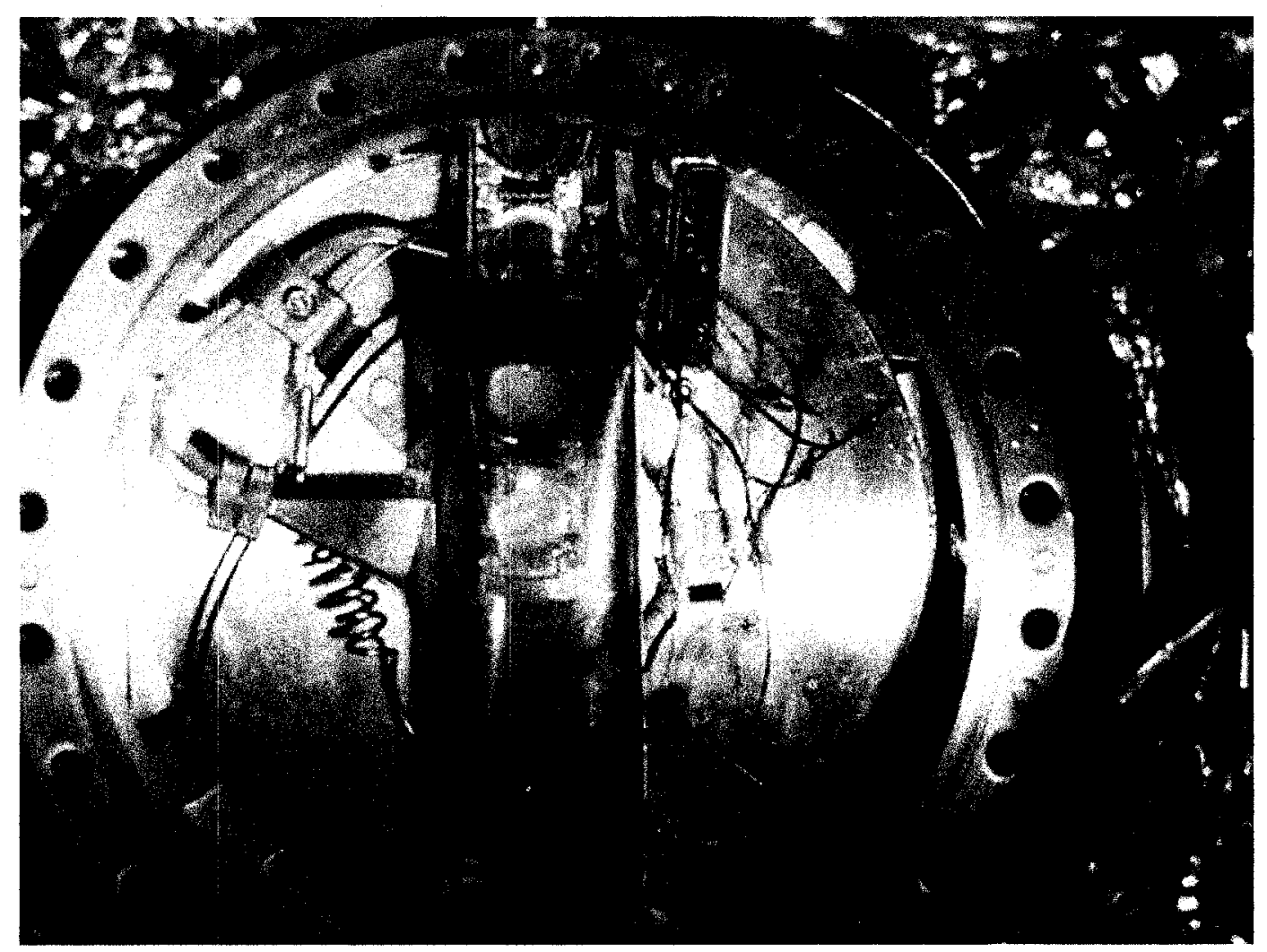

Figure 2.25: An internal view of the banjo, showing the (orange) Kapton cylindrical LHe minicup into which the target stick is inserted. Note the metal "horn", the source of microwave emission, on the left side. 


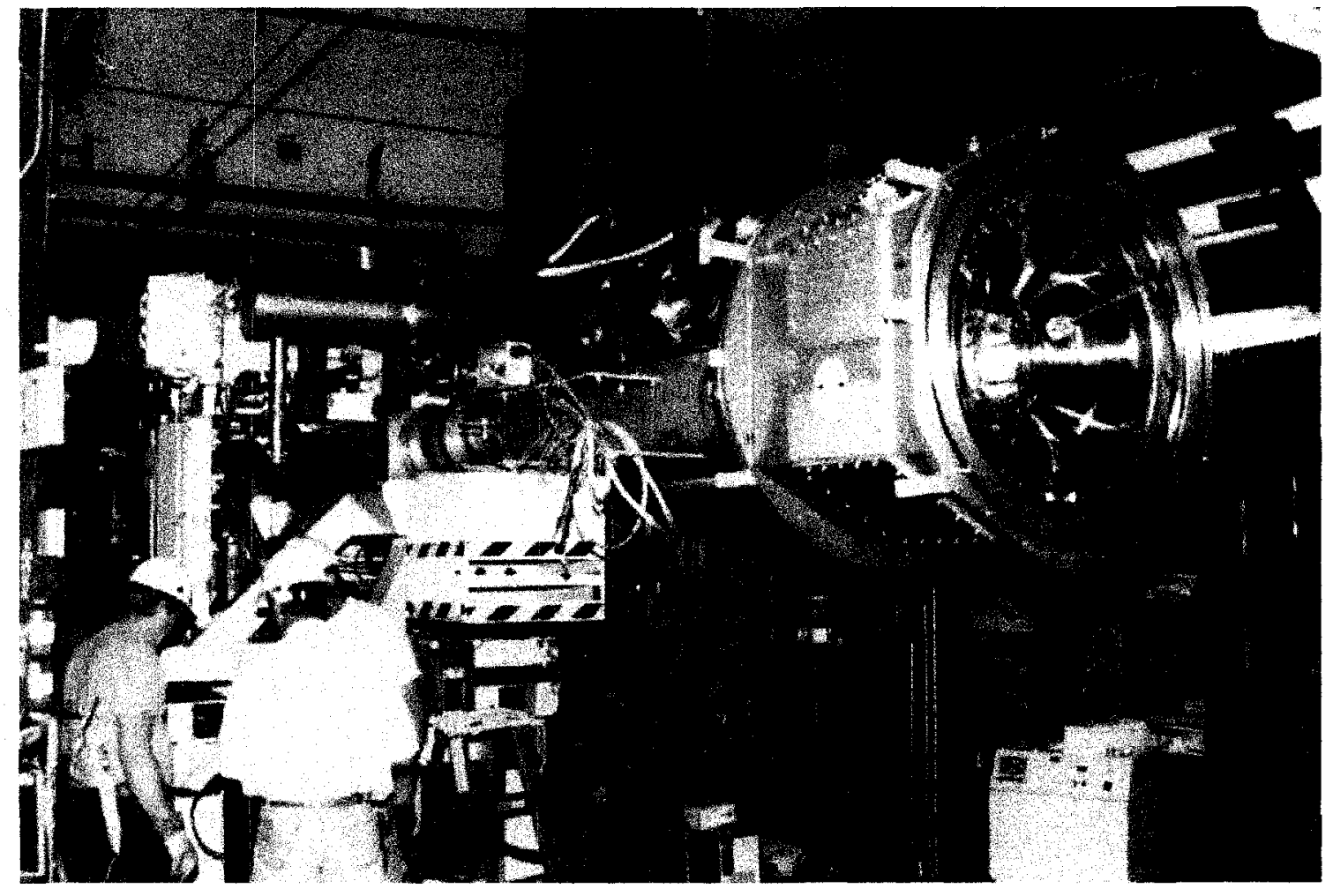

Figure 2.26: The assembled EG1 polarized target, viewed downstream, prior to insertion into CLAS. 
the Kapton minicup. These quantities are calculated from event data in a more rigorous manner in Chapter 5. Of course, there is a limit on the accuracy of length and density measurements, particularly the latter, as densities can vary with temperature. These inaccuracies are dealt with in the calculation of target model systematic errors (Section 7.2.1).

Table 2.1: Densities and lengths of materials in the EG1 target. Values are from Ref. [72] and Ref. [73]. Numbers in square brackets [ ] pertain only to the ${ }^{15} \mathrm{~N}$ target stick.

\begin{tabular}{|c|c|c|c|}
\hline Material & Density $\left(\mathrm{g} / \mathrm{cm}^{3}\right)$ & Density $\left(\mathrm{mol} / \mathrm{cm}^{3}\right)$ & Total thickness $(\mathrm{cm})$ \\
\hline ammonia $\left(\mathrm{NH}_{3}\right)$ & 0.917 & 0.0508 & $\sim 0.6$ \\
\hline ammonia $\left(\mathrm{ND}_{3}\right)$ & 1.056 & 0.0502 & $\sim 0.6$ \\
\hline carbon $\left({ }^{12} \mathrm{C}\right)$ & 2.17 & 0.180 & $0.23[0.22]$ \\
\hline nitrogen-15 $\left({ }^{15} \mathrm{~N}\right)$ & 1.1 & 0.073 & $\sim 0.5$ \\
\hline liquid helium $(\mathrm{LHe})$ & 0.145 & 0.0362 & $\sim 1.9$ minus solid target material \\
\hline Kapton $(\mathrm{K})$ & 1.42 & 0.00371 & $0.0304(0.0384$ after 27997$)[0.0354]$ \\
\hline aluminum $(\mathrm{Al})$ & 2.69 & 0.0997 & 0.0167 \\
\hline
\end{tabular}

\begin{tabular}{|c|c|c|}
\hline Material & Present in target & Comment \\
\hline ammonia $\left(\mathrm{NH}_{3}\right)$ & $\mathrm{NH}_{3}$ & length dependent on packing fraction \\
\hline ammonia $\left(\mathrm{ND}_{3}\right)$ & $\mathrm{ND}_{3}$ & length dependent on packing fraction \\
\hline carbon $\left({ }^{12} \mathrm{C}\right)$ & carbon & $\begin{array}{c}\text { carbon in }{ }^{15} \mathrm{~N} \text { target stick } \\
\text { is } 100 \mu \mathrm{m} \text { thinner }\end{array}$ \\
\hline nitrogen-15 $\left({ }^{15} \mathrm{~N}\right)$ & nitrogen-15 & length and density not well known \\
\hline liquid helium (LHe) & all & $\begin{array}{c}\text { length dependent on other thicknesses } \\
\text { \& total minicup length }\end{array}$ \\
\hline Kapton (K) & all & $\begin{array}{c}\text { extra 80 } \mu \mathrm{m} \text { added after Run 27997; } \\
\text { extra } 50 \mu \mathrm{m} \text { of Kapton on }{ }^{15} \mathrm{~N} \text { target stick }\end{array}$ \\
\hline aluminum (Al) & all & includes cell window and banjo windows \\
\hline
\end{tabular}

Note that a leak in the $\mathrm{ND}_{3}$ target during a later run set necessitated the addition of extra Kapton foil. The Kapton and target lengths also vary (slightly) between the two (ammonia and ${ }^{15} \mathrm{~N}$ ) target sticks. The $\mathrm{NH}_{3}$ and $\mathrm{ND}_{3}$ lengths are also subject to change when the target material is refilled during the experiment. Table 2.2 contains some other quantities related to the densities and lengths that are useful later in the analysis. 
Table 2.2: Some other useful quantities for calculating target parameters. Numbers in square brackets [ ] pertain to the ${ }^{15} \mathrm{~N}$ target stick.

\begin{tabular}{|c|c|c|}
\hline Quantity & Value & Description \\
\hline$\rho_{C} \ell_{C}$ & $0.498 \mathrm{~g} / \mathrm{cm}^{2}=0.0415 \mathrm{~mol} / \mathrm{cm}^{2}$ & mass thickness of carbon \\
& {$\left[0.476 \mathrm{~g} / \mathrm{cm}^{2}=0.0397 \mathrm{~mol} / \mathrm{cm}^{2}\right]$} & \\
\hline$\rho_{K} \ell_{K}$ & $\begin{array}{c}0.0432 \mathrm{~g} / \mathrm{cm}^{2}\left(0.055 \mathrm{~g} / \mathrm{cm}^{2} \text { after } 27997\right) \\
{\left[0.0503 \mathrm{~g} / \mathrm{cm}^{2}\right]}\end{array}$ & mass thickness of Kapton \\
\hline$\rho_{A l} \ell_{A l}$ & $0.045 \mathrm{~g} / \mathrm{cm}^{2}$ & \\
\hline$\rho_{F} \ell_{F}$ & $\begin{array}{c}0.0882 \mathrm{~g} / \mathrm{cm}^{2}\left(0.0996 \mathrm{~g} / \mathrm{cm}^{2} \text { after } 27997\right) \\
{\left[0.0952 \mathrm{~g} / \mathrm{cm}^{2}\right]}\end{array}$ & mass thickness of aluminum \\
\hline$f$ & $0.177(0.200$ after 27997$)[0.235]$ & $\rho_{F} \ell_{F} / \rho_{C} \ell_{C}$ \\
\hline
\end{tabular}

\subsection{The CLAS Detector}

The CEBAF Large Acceptance Spectrometer (or CLAS) detector is a large-acceptance detector capable of reconstructing multiple particle (exclusive) events, as well as providing a large acceptance ( $8^{\circ}<\theta<49^{\circ}$ in 6 azimuthal sectors) for the detection of inclusive electrons and other particles. The detector uses a toroidal magnetic field (Section 2.5.1) for momentum determination. A 3-layer drift-chamber (DC) (Section 2.5.2) is used to reconstruct charged particle tracks, with time-of-flight gauged by an extensive scintillation counter (SC) system (Section 2.5.4). Cherenkov counters (CC) (Section 2.5.3) aid in particle identification at forward angles, while electromagnetic calorimeters (EC) (Section 2.5.5) provide identification of neutral particles, additional timing information, and additional particle identification parameters [30]. ${ }^{16}$ Figure 2.27 shows a cutaway of the detector along the beam line. Figure 2.28 shows a cutaway perpendicular to the beam axis, showing the azimuthal 6-sector symmetry of the detector. In this section, a brief explanation of the functionality, capabilities and calibration of each of these detector components is given.

\subsubsection{Torus Magnet}

A toroidal superconducting magnet, approximately $5 \mathrm{~m}$ in length and diameter (Figure 2.29) is used to generate a $\vec{B}$-field along the $\phi$-direction [30]. The torus can generate up to a $2.5 \mathrm{~T} \cdot \mathrm{m}$ field

\footnotetext{
${ }^{16}$ Additional elements are also present, including a tagger for the generation of photon beams, and a large angle calorimeter (LAC) for the identification of wide-angle-scattered exclusive channels, but these are not utilized in this analysis, and hence not discussed here.
} 


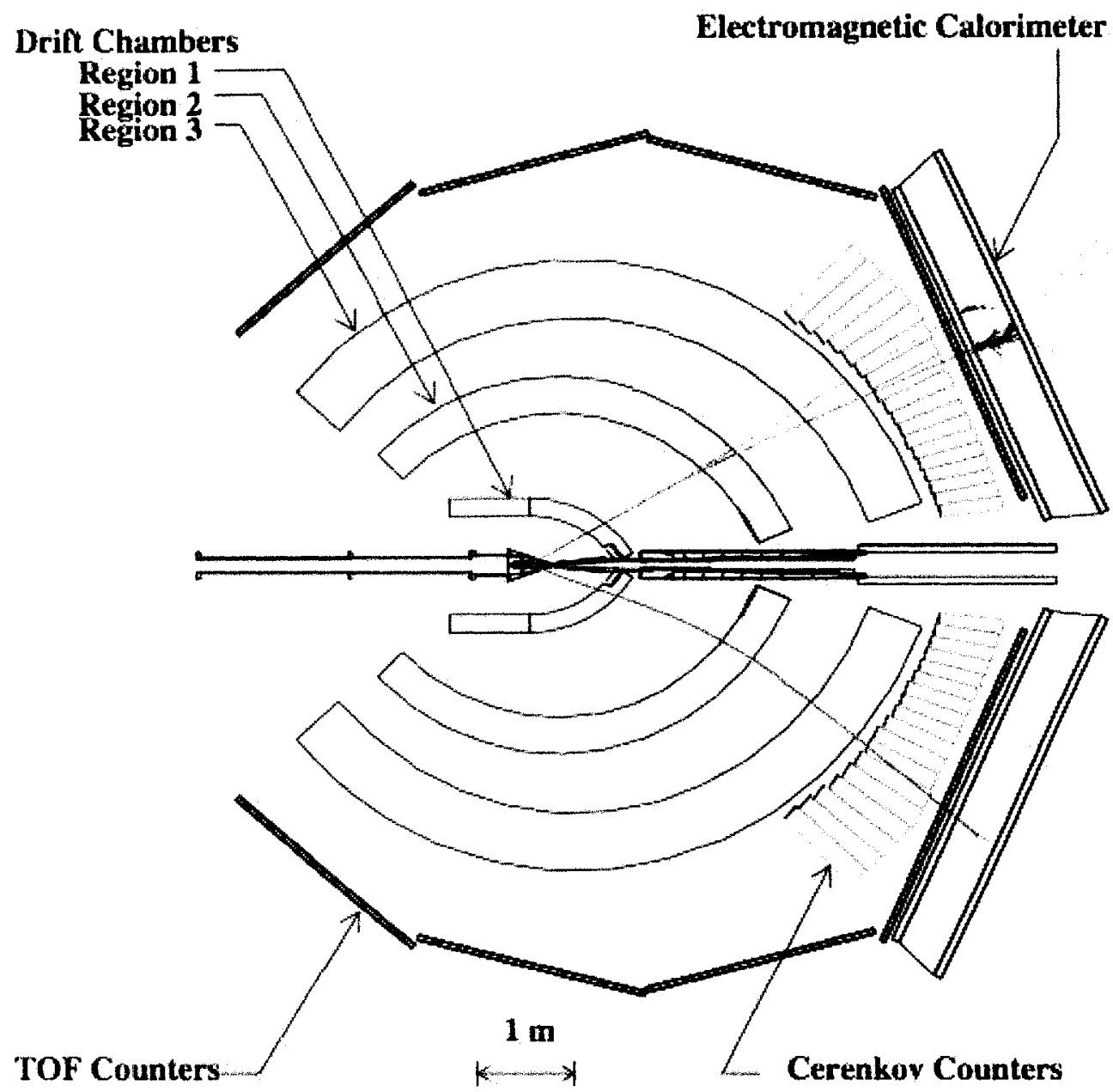

Figure 2.27: Cutaway diagram of the CLAS detector, showing components used in this analysis. A reconstructed $e p$ scattering event is superimposed; the top track shows an inbending electron, radiating a photon in the layer 2 drift chamber, and creating a particle shower in the calorimeter. The bottom track shows the correlating proton, which bends in the opposite direction in the torus field and creates little to no calorimeter shower. From Ref. [30]. 


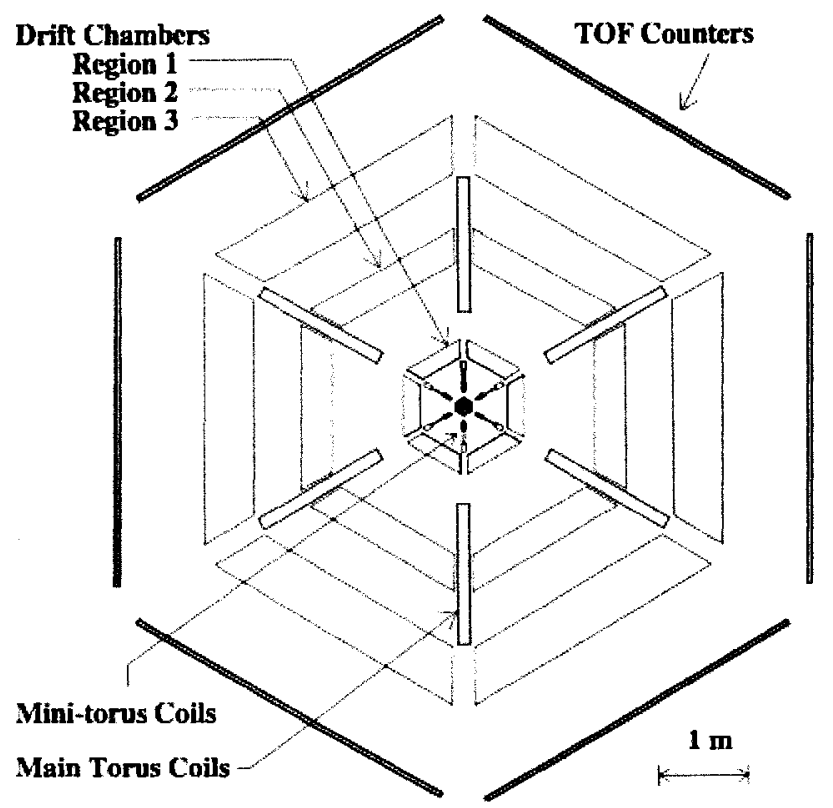

Figure 2.28: Cutaway diagram of the CLAS detector, perpendicular to the beam line. All detector components mentioned in this section are constructed in this six-fold symmetry. From Ref. [30].

integral in the forward direction, ${ }^{17}$ where the field is at its maximum intensity, to better differentiate the spectrum of high-momentum particles scattering at forward angles. Torus currents of $1500 \mathrm{~A}$ (at low beam energies) or $2250 \mathrm{~A}$ (at high beam energies) were employed in this experiment. The torus current was periodically reversed; negatively charged forward particle (i.e. electron) paths were bent toward the center for + torus current, or inbending data, while electrons were bent away from the center for - torus current, or outbending data. The former allows for better spatial detector acceptance at large scattering angles (high $\theta$ ), while the latter allows for better acceptance at small scattering angles (low $\theta$ ). Employing both polarities provides for high-precision measurements over a larger momentum range than would be possible with only a single torus current direction. The central, field-free region allows for the operation of a polarized target (Section 2.4). Coils of $\mathrm{Nb}$ Ti/Cu wire wrap around each of the six magnet spools. Superconductivity is maintained by cooling the coils to $4.5 \mathrm{~K}$ with $\mathrm{LHe}$ in cooling tubes at the edge of the wire windings.

The purpose of the torus magnet is to maintain a magnetic field, reasonably homogeneous in

\footnotetext{
${ }^{17}$ This value corresponds to the maximum achievable torus current of $3860 \mathrm{~A}$.
} 


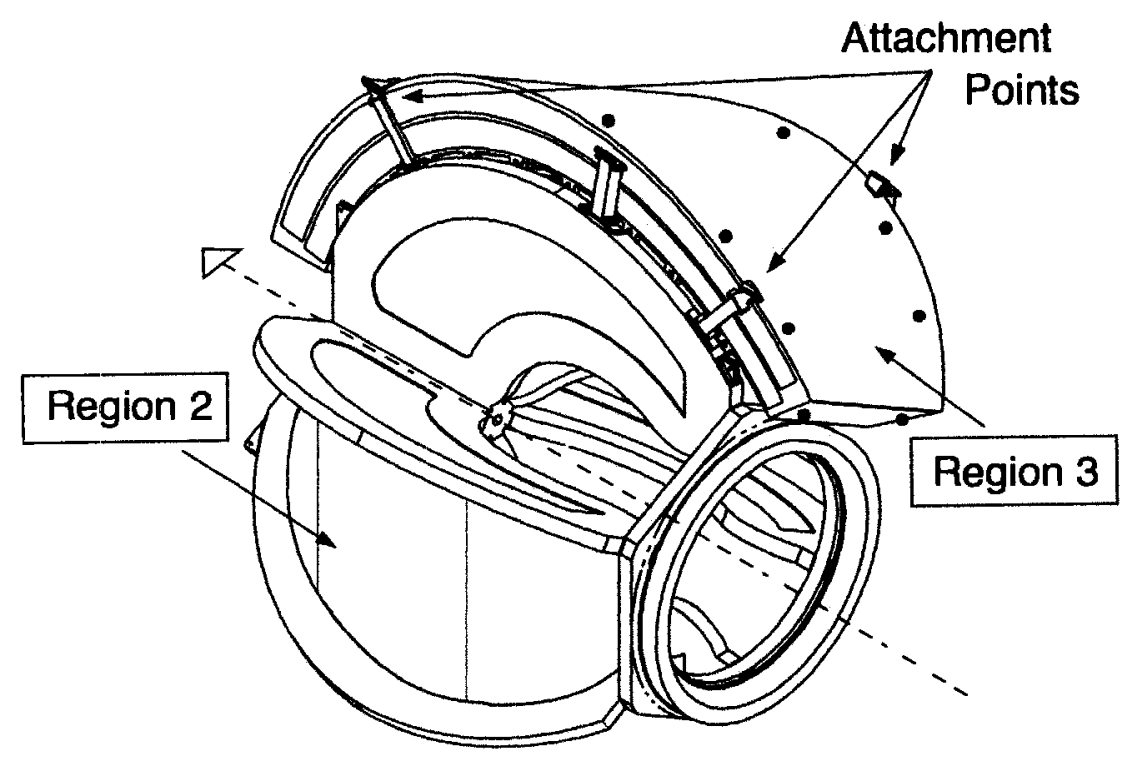

Figure 2.29: View of the torus magnet frame, showing the relative placements of the Region 2 and 3 drift chambers. The Region 1 drift chambers (not shown) are placed in the central (field-free) region of the torus. Superconducting wires are wrapped around the edges of the 6 kidney-shaped frames to generate an azimuthally-directed field. From Ref. [30].

$\phi$, for the identification of momenta based on particle path curvature. While a rudimentary map of the magnetic field exists [30], a thorough map of the CLAS torus field was never successfully completed. Calibration of the momenta using well-understood events (typically elastic $e p$ events) is therefore necessary (see Section 4.2.8).

\subsubsection{Drift Chambers}

The CLAS drift chambers consist of three "regions" (Figure 2.29), each of which contain two "superlayers" of 6 layers of multiple hexagonal cells ${ }^{18}$ (Figure 2.30). The DC chambers in each sector are installed between and around the frames of the torus magnet; one of the DC layers for a sector, prior to installation, is shown in Figure 2.31.

Each individual hexagonal cell in the drift chamber contains a voltage wire (140 $\mu \mathrm{m}$ Au-plated Al) and detection wire ( $20 \mu \mathrm{m}$ diameter Au-plated $\mathrm{W}$ ) surrounded by a $90 \% / 10 \% \mathrm{Ar} / \mathrm{CO}_{2}$ mixture [74]. This mixture of gas is optimized to produce a high ionization gain of induced charge when

\footnotetext{
${ }^{18}$ The exception is Superlayer 1 , which contains only 4 layers.
} 


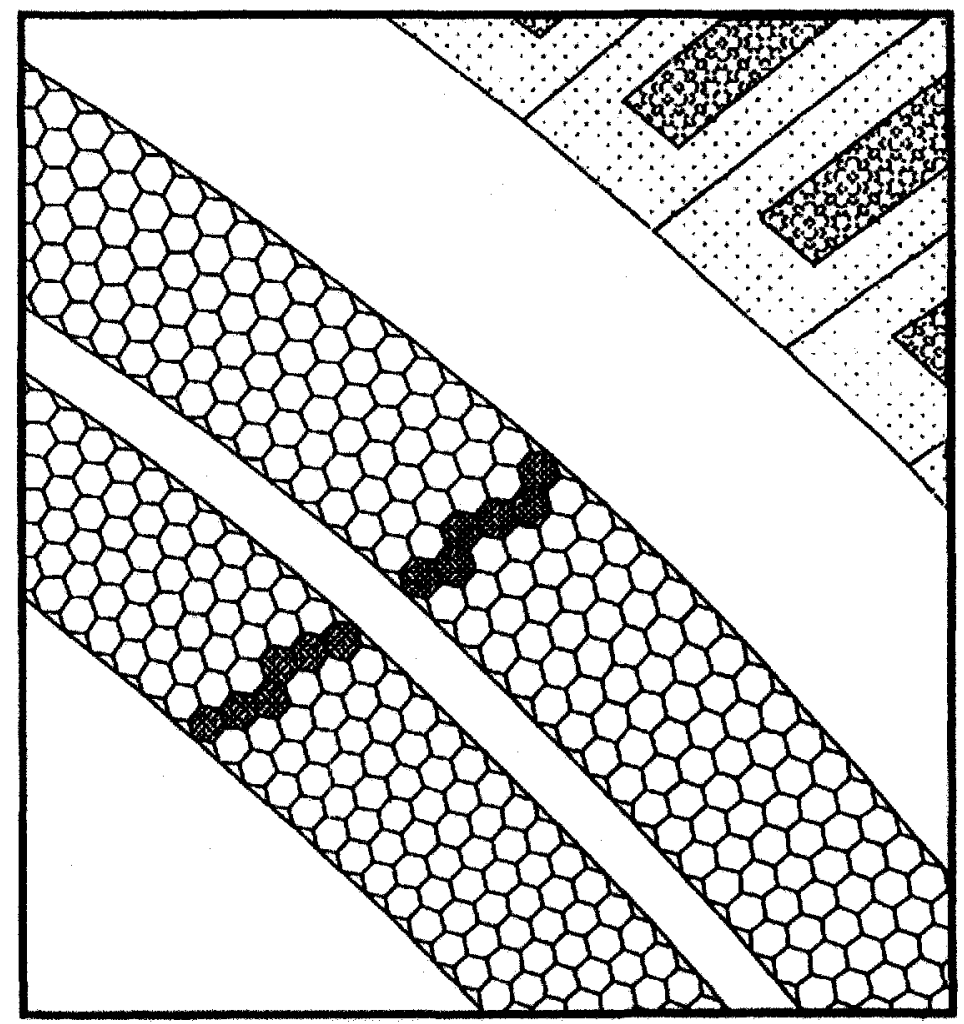

Figure 2.30: Diagram of a particle passing through the cells of the outer layer drift chamber, giving an initial estimate of the path of the particle through hit-based tracking. Each hexagonal cell contains a perpendicular sensor wire in its center. From Ref. [74]. 


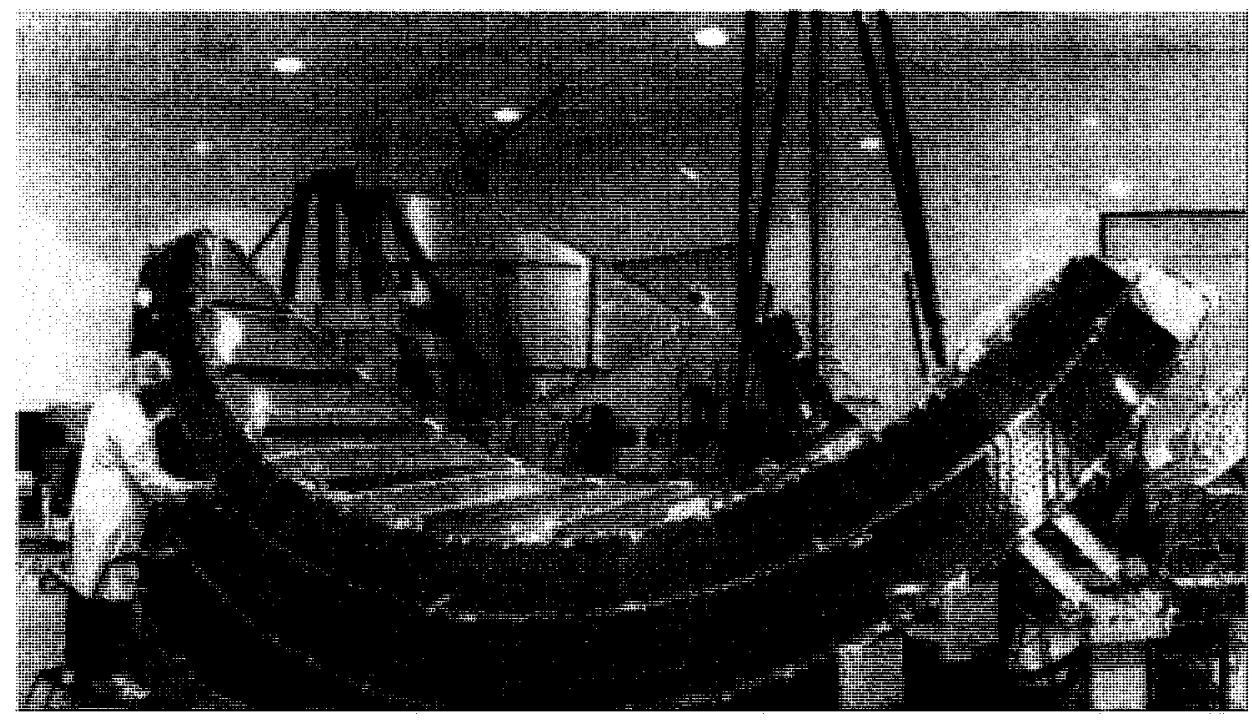

Figure 2.31: Photograph of one sector of an outer layer drift chamber section prior to installation in the CLAS detector. From the JLab JPIX picture exchange.

energetic particles pass through the cell. This induced charge drifts toward the sense wire and produces a current, thus providing information that a particle has passed through the cell. This information provides initial hit-based tracking information about the particle trajectory, reconstructing the actual particle momenta within an accuracy of $3-5 \%$.

Wires are strung perpendicular to the magnetic field in one of the two superlayers of each region, and at a $6^{\circ}$ angle around the cell radius in the other superlayer, to provide $\phi$-direction information. In total, about 130,000 wires are strung through the cells of the drift chambers. Cell material was minimized, so that only $\sim 1 \%$ of a radiation length would (on average) be encountered by a particle, to lower the incidence of multiple scattering events. There are 1296, 2262, and 2304 individual hexagonal detection cells in each sector of the Region 1, 2, and 3 drift chambers, respectively. The sizes of the individual cells range from $15 \mathrm{~mm}$ in Region 1 to $45 \mathrm{~mm}$ in Region 3 [74].

Charge induced by an ionized particle drifts toward the sensor cell at a relatively slow velocity of around $4 \mathrm{~cm} / \mu \mathrm{s}$. More accurate path information can be provided once the total time-of-flight of the particle is determined by the scintillation counters (Section 2.5.4). Then, a reference time can be used to indicate when the particle passes through each cell, and this can be compared to the TDC time of the signal generated in the sense wire. If the relation between drift time and distance within 
individual hexagonal cells is known, the distance of closest approach (DOCA) to the sensor wire in each cell can be calculated, thus greatly improving the accuracy of the path through time-based tracking (see Figure 2.32). The DOCA function is fit by a $\chi^{2}$ minimization of a polynomial function to the observed drift times. For example [74], Region 3 is fit with the function

$$
x(t)=v_{0} t+\eta\left(\frac{t}{t_{\max }}\right)^{p}+\kappa\left(\frac{t}{t_{\max }}\right)^{q}
$$

where $v_{0}$ is the saturated drift velocity (at $t=0$ ), $t_{\max }$ is the maximum drift time (at the cell edge) and $\eta, \kappa, p$ and $q$ are fit coefficients, determined by the minimization of

$$
\chi^{2}=\frac{\left|x(t)-x_{p a t h}\right|^{2}}{\sigma_{p a t h}^{2}}
$$

where $x_{p a t h}$ and $\sigma_{\text {path }}$ are the DOCA distance from the sense wire along the path and the error on this quantity, respectively. Polynomial forms are used for Regions 1 and $2 .{ }^{19}$ A sample of the parameterized DOCA vs. drift time is shown in Figure 2.33. The value $\left|x(t)-x_{p a t h}\right|$ is called the residual of the fit; the magnitude of this value is used to evaluate the quality of the drift chamber calibrations (see Figure 2.34). Time-based tracking is capable of increasing the precision of track measurement to a certainty of $<500 \mu \mathrm{m}$ (for the largest cells; i.e. Region 3 ). The radius of curvature in the magnetic field can then be used to determine the particle momentum to within a fraction of a percent accuracy.

\subsubsection{Cherenkov Counters}

Once forward angle particles pass through the regions of the drift chamber, they enter the Cherenkov counters (CC), which are used to aid in particle identification, particularly the separation of forwardscattered electrons from inelastically produced pions. The Cherenkov counters are used to detect electrons in all 6 sectors scattered at forward angles of up to $\sim 45^{\circ}$. Each sector of detectors (Figure 2.35) contains a total of 18 symmetrical mirrored chambers (segments), filled with perfluorobutane $\left(C_{4} F_{10}\right)$ gas. Individual segments are divided in half, with a photomultiplier (PMT) tube on each half

\footnotetext{
${ }^{19}$ Region 2 requires an additional correction for the fact that the main torus fields causes perturbation of the electric potential map; see Ref. [74].
} 


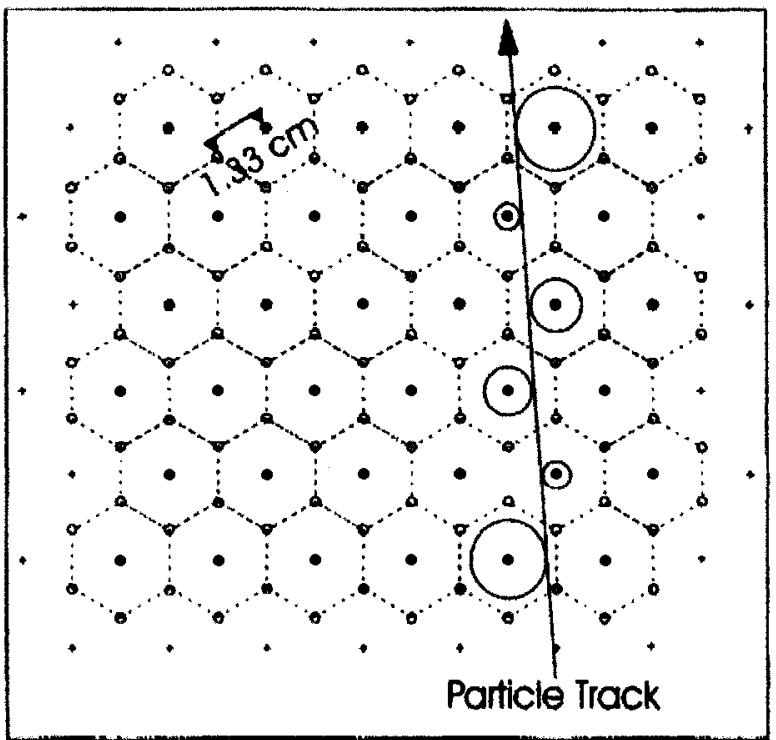

Figure 2.32: Cutaway view of a drift chamber, showing how path accuracy can be increased by the calculation of the distance of closest approach (DOCA) through time-based tracking. From Ref. [75].

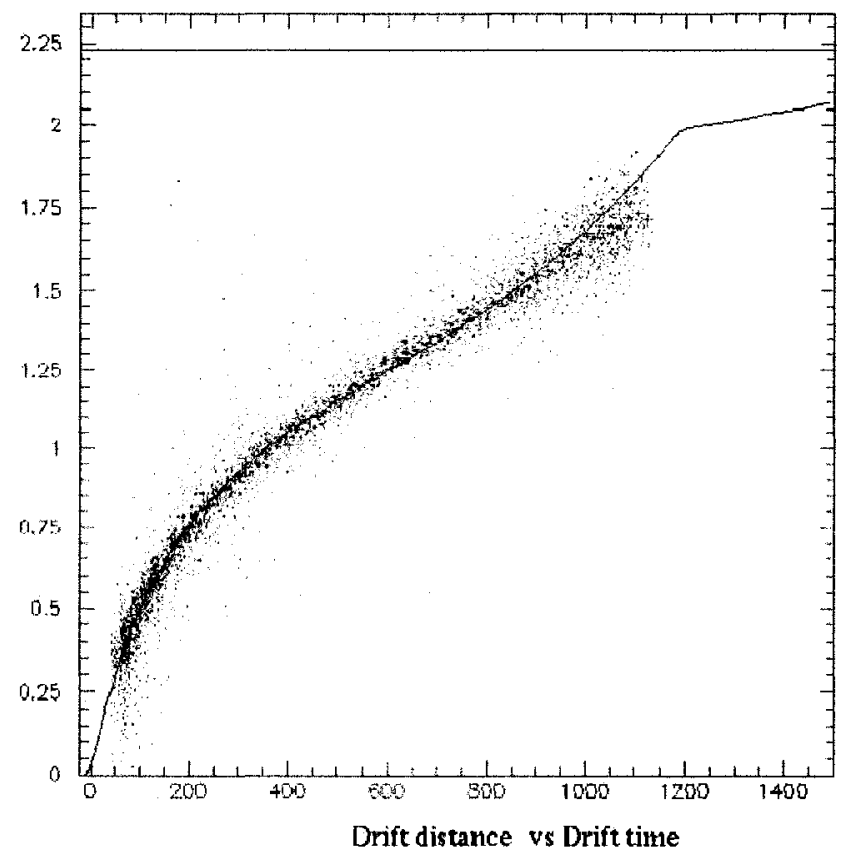

Figure 2.33: Parametrized DOCA (in $\mathrm{cm}$ ) vs. drift time (in $\mathrm{ns}$ ) for the CLAS drift chambers. From Ref. [2]. 


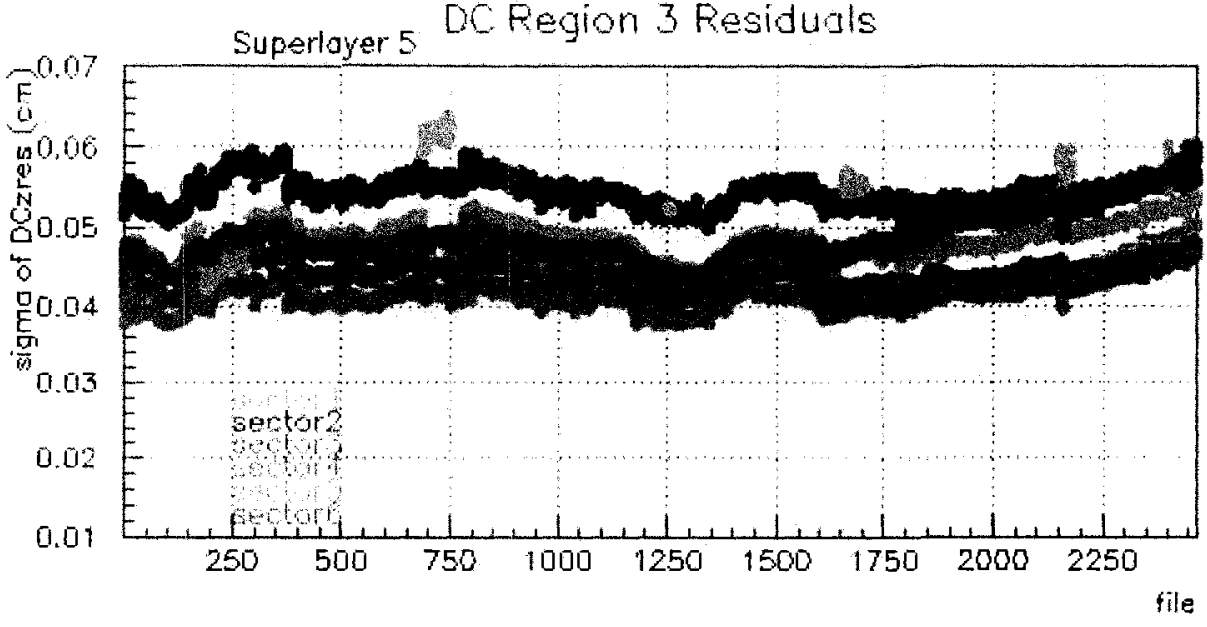

Figure 2.34: Typical residuals $\left|x(t)-x_{p a t h}\right|$ (in $\mathrm{cm}$ ) after track-fitting for the Superlayer 5 (Region 3) drift chamber, for $5.7 \mathrm{GeV}$ beam energy data in the EG1b data set. Colors show residuals for each of the 6 sectors. Accuracies of $\sim 500 \mu \mathrm{m}=0.05 \mathrm{~cm}$ (as shown here) are typical for the Region 3 DC. Regions 1 and 2 have smaller cell sizes, and, correspondingly, smaller residuals.

\section{Optical Mirror System}

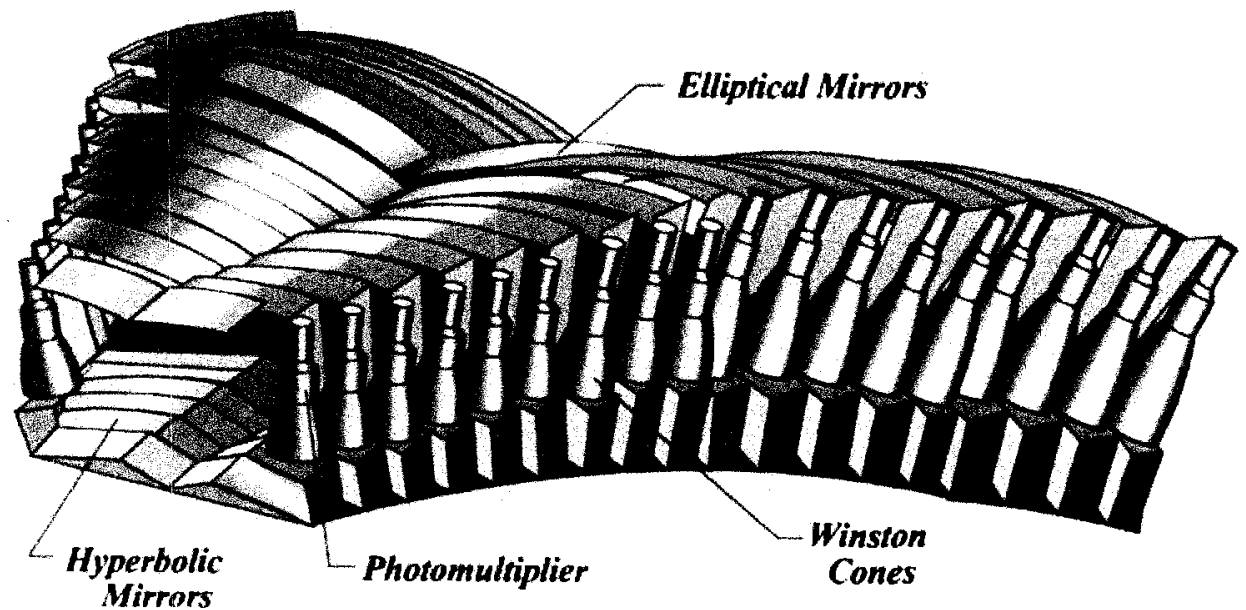

Figure 2.35: A schematic drawing of the 18 symmetrical mirrored segments in one sector of the CLAS Cherenov Counter. From Ref. [76]. 
to detect Cherenkov radiation from particles passing through the gas.

High-energy scattered particles have velocities that approach the speed of light $c$ in a vacuum, where the index of refraction $n=1$. If a high energy particle enters a medium where $n>1$, like the aforementioned $\mathrm{C}_{4} \mathrm{~F}_{10}$ gas (maintained at a positive pressure of $340 \mathrm{~Pa}$ ), such that the particle velocity $\beta \equiv v / c$ is given by

$$
\beta>\frac{1}{n(f)}
$$

where $n(f)$ is the index of refraction of light, as a function of of frequency $f$ (that is, the speed of the particle is greater than that of light in the medium), then a electromagnetic shock wave will be produced. ${ }^{20}$ The shock wave has a coherent wavefront, conical in shape, with axial angle $\theta_{C}$ defined (in a thick medium) as $[8]^{21}$

$$
\cos \theta_{C}=\frac{1}{\beta n(f)}
$$

This Cherenkov light is reflected between a (forward) elliptical mirror and (rear) hyperbolic mirror, with the optics optimized to direct reflected light from (aimost) any entry point to a parabolic reflecting "cup". The cup directs light into a photomultiplier (PMT) vacuum tube at the chamber edge (see Figure 2.36). The PMT tubes convert the UV Cherenkov light into an ADC signal corresponding to the number of photoelectrons. ${ }^{22}$ The PMT tubes are magnetically shielded from the effects of the torus field [76].

The Cherenkov counters exhibit an efficiency that falls off sharply near the PMTs and the outer edges of the chamber, as well as along the center "ridge" in the case of an outbending (negative current) torus field. This is partly due to the imperfect mirror optics and reflectivity, and partly due to the residual effects of the torus field on the PMT. Cherenkov Counter efficiency is the limiting factor in the acceptance of CLAS measurements. This is further explored and accounted for in detail in Section 4.4 .

The primary purpose of the Cherenkov counters is to distinguish light hadrons (primarily pions) from electrons, by their Cherenkov response threshold. Electrons, practically massless, radiate Cherenkov light at relatively low energies, while pions only reach a value of $\beta$ sufficient to produce

\footnotetext{
${ }^{20}$ This is analogous to the effect of a "sonic boom" for objects exceeding the speed of sound in air.

${ }^{21}$ Note that this relation holds exactly only for an infinitely thick medium. The real relation is somewhat more complex (see, for example, Ref. [8]). For the Cherenkov counters in CLAS, however, Eq. 2.17 is a good approximation.

${ }^{22}$ Calibration of this signal is performed by a single fit function detailed in Ref. [76].
} 


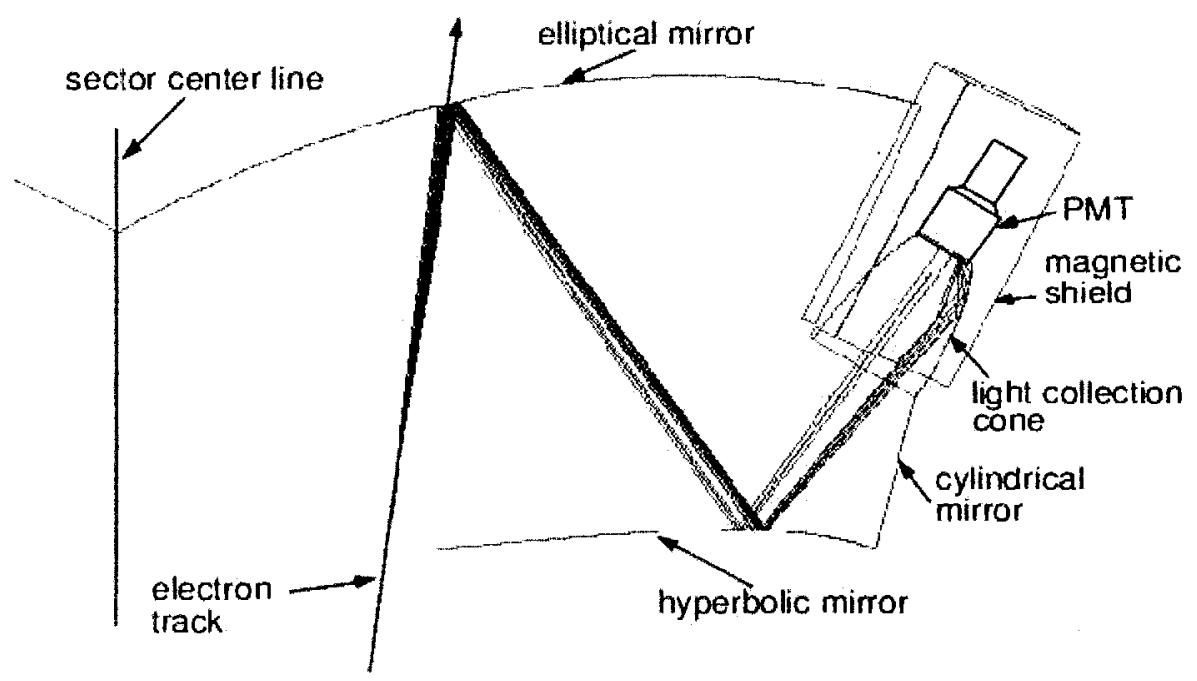

Figure 2.36: Diagram showing the reflection of Cherenkov radiation toward a PMT in half of a CC segment. From Ref. [76].

Cherenkov light at $p \approx 2.7 \mathrm{GeV}$ or higher.

Naturally, this separation works best at lower momenta, where $\beta$ differs the most between electrons and the (considerably more massive) pions. More detail on the identification of particles by their Cherenkov spectra is given in Section 3.4.1. A view of the CLAS Cherenkov counters can be seen in Figure 2.37.

\subsubsection{Scintillation Counters}

The scintillation counters (SC) surround the CLAS detector around the full solid angle of the drift chambers (DC). The scintillation counters are used to measure the time of flight (TOF) of scattered particles int order to establish time-based tracking in the drift chambers, and set a baseline time standard for event measurement. The SC system is composed of 48 paddles in each sector (see Figure 2.38). Each paddle consists of a length of scintillation material capped with photomultiplier tubes (PMTs) on either end. The scintillators are 15 or $22 \mathrm{~cm}$ wide ${ }^{23}$ bars of transparent material that produces violet $(425 \mathrm{~nm})$ light when its molecules are ionized by charged particles. Al foil is

\footnotetext{
${ }^{23}$ The scintallators are $15 \mathrm{~cm}$ at forward angles, $22 \mathrm{~cm}$ at large angles, as per Figure 2.38 .
} 


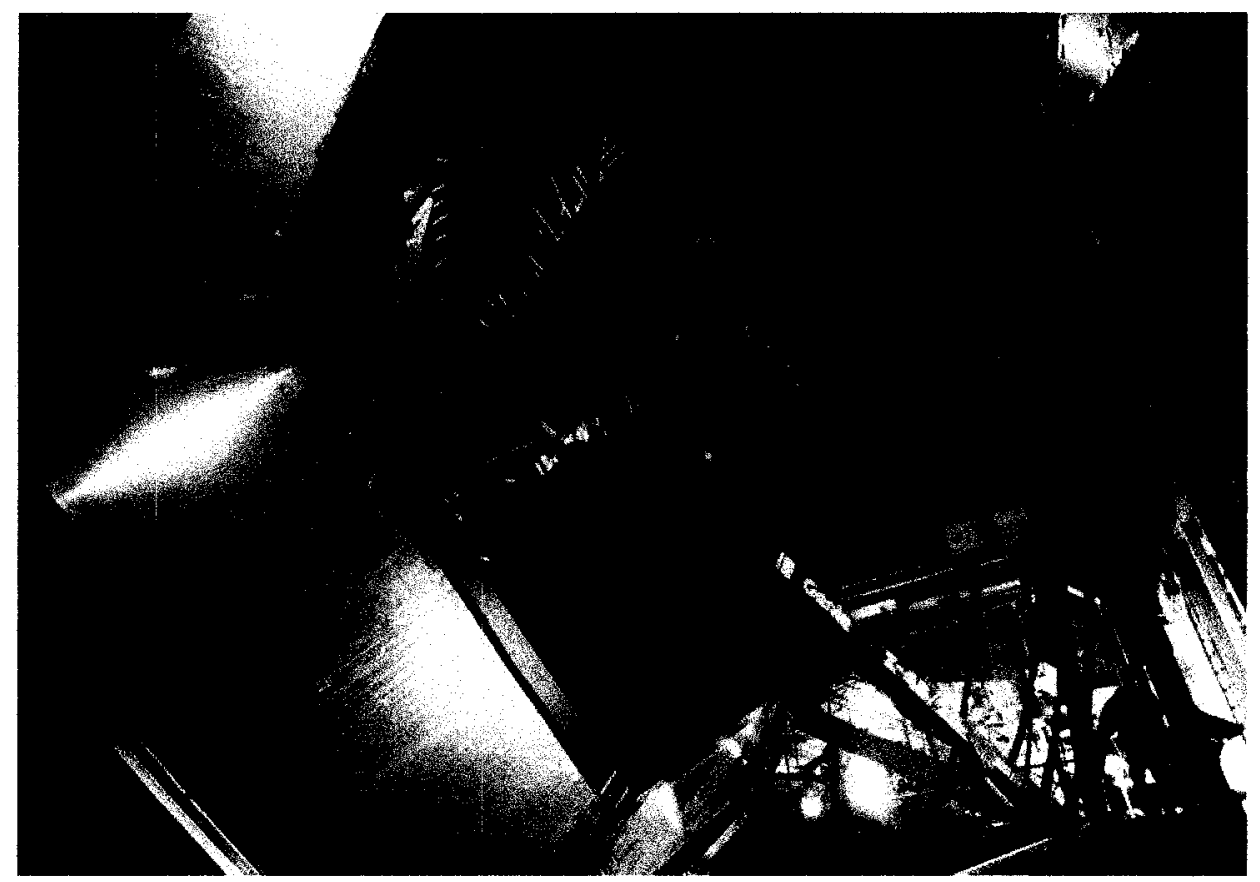

Figure 2.37: A view of the CLAS Cherenkov counter (CC), separated from the drift chambers (upper right corner) for maintenance. From the JPIX JLab picture exchange.

wrapped around the scintillator to prevent loss of light, as it reflects internally toward the PMTs. Light guides (twisted at the backward angles) are used to direct the signal to the PMT windows. Cylindrical mu-metal tubes are used to shield the PMTs from the effects of the main torus magnet [77].

There are $48 \times 6=288$ paddles, and a total of $288 \times 2=576$ PMTs in the SC. This necessitates an extensive sequence of calibrations to make the $\mathrm{SC}$ detector work as a coherent unit. Calibrations are performed in 3 stages:

1. ADC and TDC channels from each PMT are calibrated, so that a signal incident on a PMT at time $t$ records a signal correlating to this exact time. These calibrations ignore the scintillator entirely; they are performed by feeding electrical or luminous luminous LED signals directly into the PMTs. First, a pedestal value $P$ must be subtracted to provide for the "zero signal" threshold of the ADC value $A$ to get the true signal amplitude $A^{\prime}$ :

$$
A^{\prime}=A-P
$$




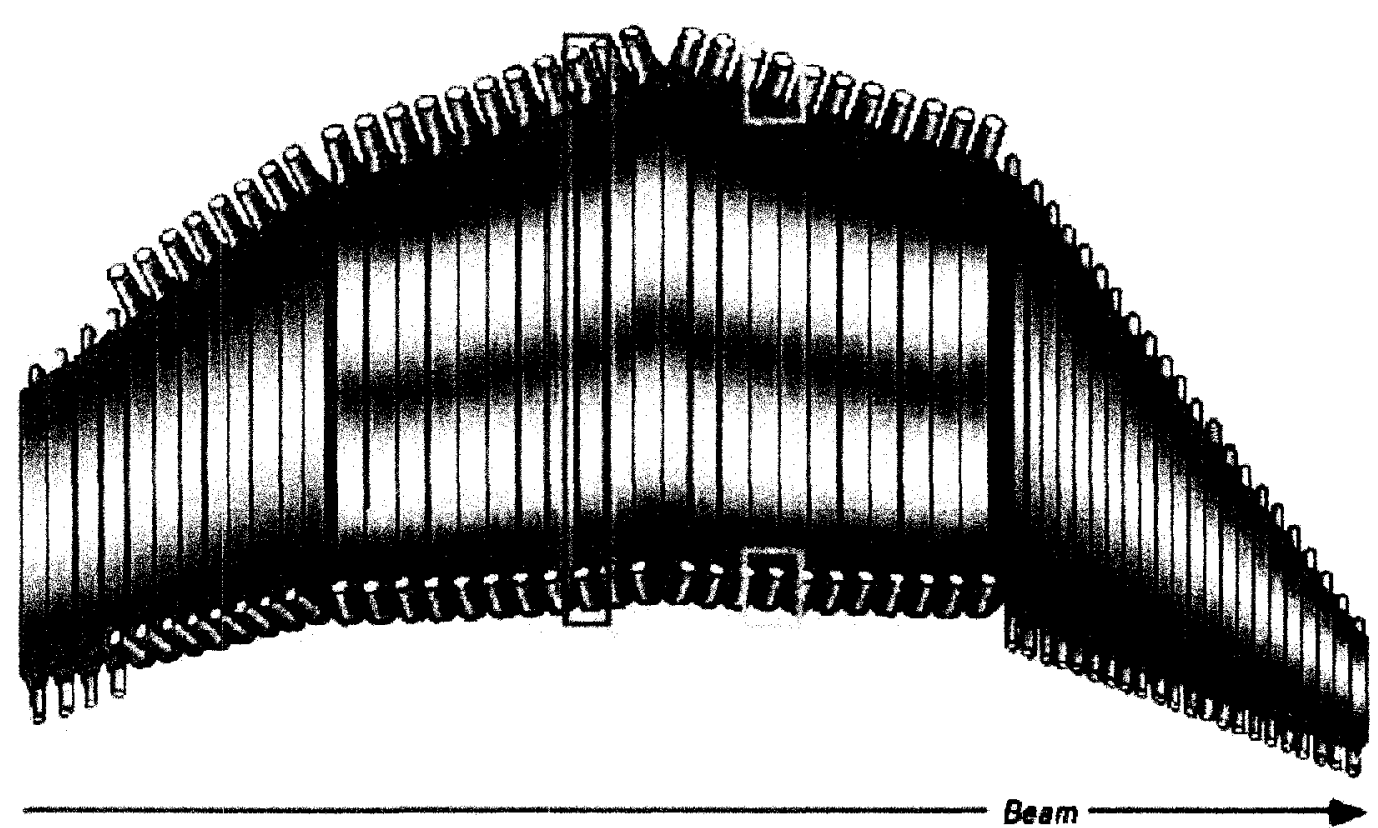

Figure 2.38: Diagram of the CLAS scintillation counter (SC) is one sector. The large red box contains a whole paddle, while the smaller yellow boxes surround PMTs. Modified from Ref. [77].

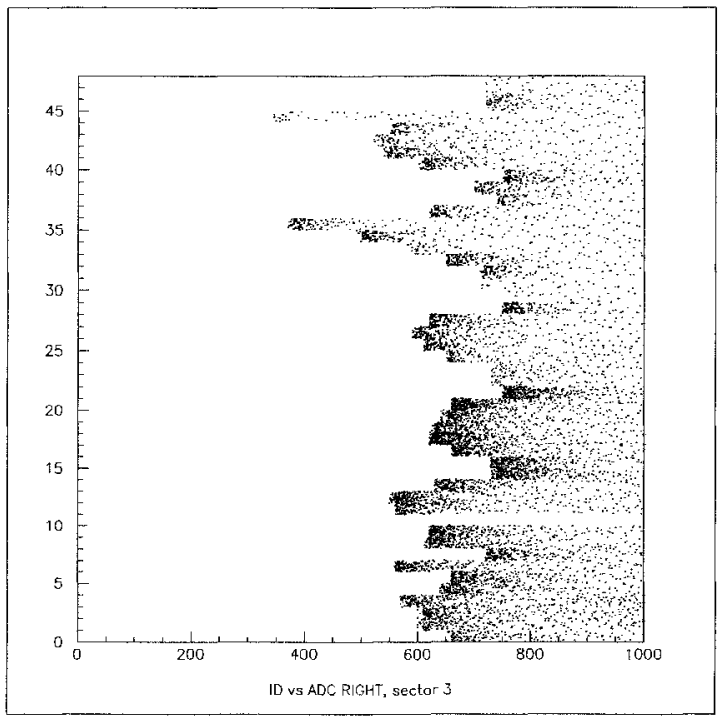

(a)

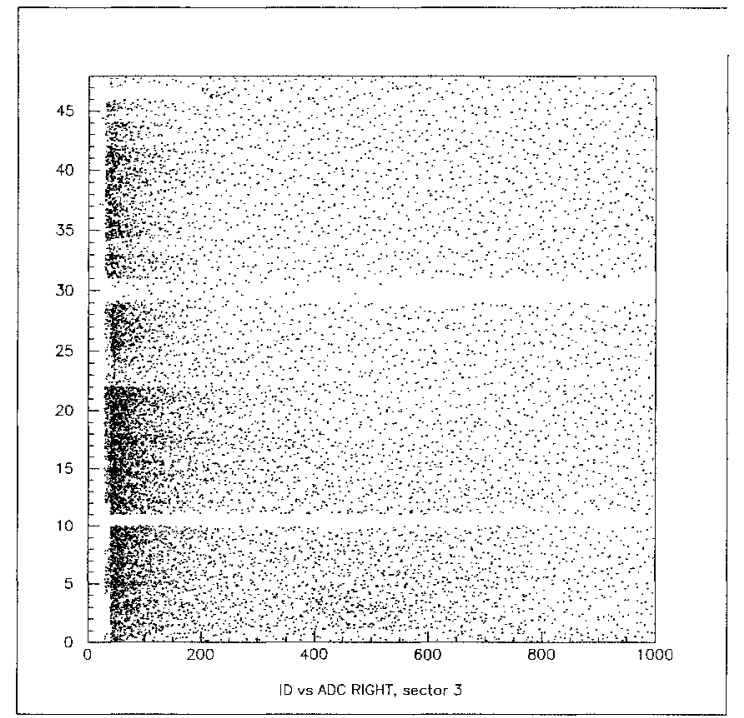

(b)

Figure 2.39: ADC channels in SC PMTs as read from electronic pulser trigger data, shown before and after the addition of pedestals. CLAS detector components require the addition of pedestals to define the ADC readout for a null signal. 


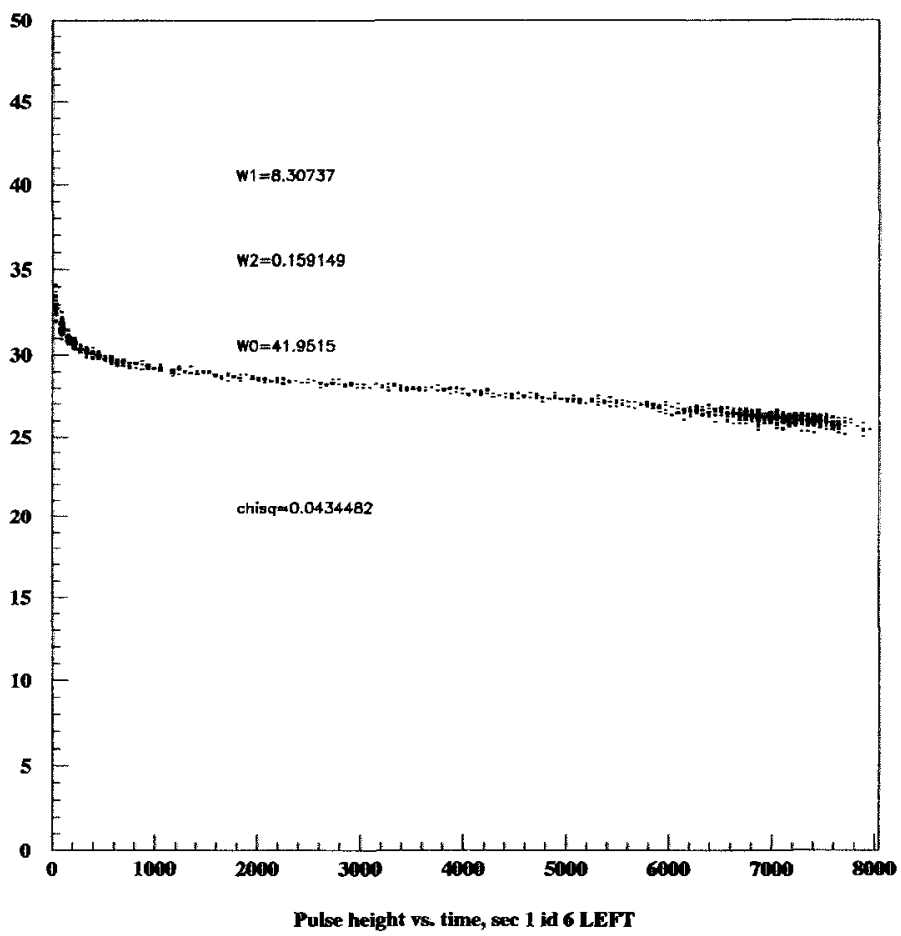

Figure 2.40: Time-walk evolution of pulse height (ADC value) vs. time (TDC value) for the left PMT on Paddle 6, Sector 1 . The values of the three fit constants $w_{0}, w_{1}$ and $w_{2}$ are shown. 
(see Figure 2.39). Then, input from a pulse generator is used to calibrate the TDC signal $T$ to the true signal time $t$ with a quadratic fit:

$$
t=c_{0}+c_{1} T+c_{2} T^{2}
$$

Then, laser light generated by photodiodes on each PMT is used to calibrate against the dependence of the TDC signal on the ADC amplitude (a phenomenon known as time-walk). This fit is considerably more complex [78]; the corrected time $t_{w}$ is given by

$$
t_{w}=t-f_{w}\left(\frac{A^{\prime}}{T h}\right)+f_{w}\left(\frac{600}{T h .}\right)
$$

where $T h$. is the TDC channel number corresponding to the leading pulse edge ${ }^{24}$ and

$$
f_{w}(x)= \begin{cases}\frac{w_{1}}{x^{w_{2}}} & \left(x<w_{0}\right) \\ \frac{w_{1}}{w_{0}^{w_{2}}}\left(1+w_{2}\right)-\frac{w_{1} w_{2}}{w_{0}^{w_{2}+1}} & \left(x>w_{0}\right)\end{cases}
$$

where $w_{0}, w_{1}$ and $w_{2}$ are fit constants (see Figure 2.40).

2. Once a consistent time and amplitude response is obtained from the individual PMTs, each paddle, composed of 2 PMTs and a length of scintillator, must be calibrated so that it provides a consistent TOF response from particle hits along any point of the scintillator length. Cosmic rays can be used for these calibrations, as can actual scattering data. ${ }^{25}$ Light from ionization requires finite times $t_{L}$ and $t_{R}$ to travel to the left and right PMTs, respectively. $A$ hit at the exact center of the paddle (i.e. the center of the hit distribution) should record a simultaneous signal on both PMTs. To enforce this, it is necessary to add a left-right calibration offset [78]

$$
\delta t_{L R}=\left(e d g e_{L}+e d g e_{R}\right) / v_{e f f}
$$

to the TDC output (see Figure 2.41), where $e d g e_{L / R}$ is the coordinate distance from the central point to the appropriate edge of the of the scintillator, and $v_{\text {ef } f}$ is the effective velocity of light

${ }^{24} 600$ is the ADC channel corresponding to an MIP (minimum ionizing particle) response.

${ }^{25} \mathrm{Cosmic}$ ray runs are the only way to ensure accurate calibration over the full detector acceptance. 


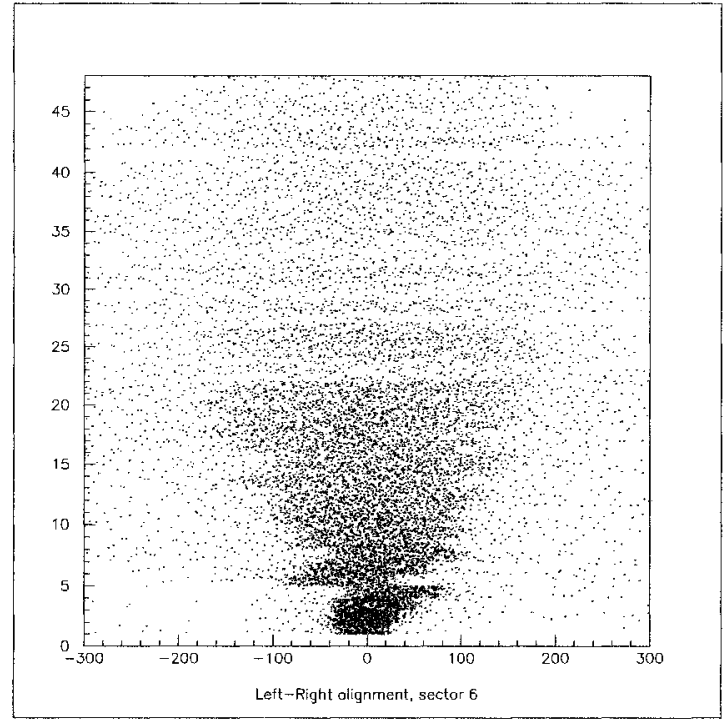

(a)

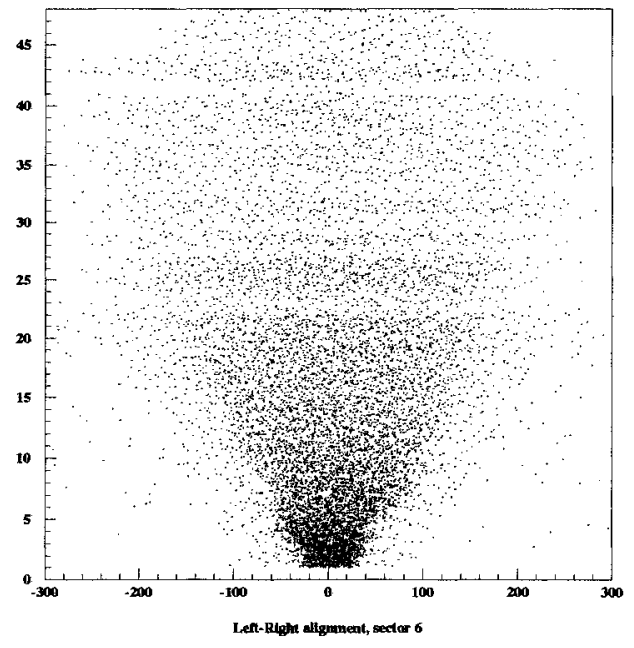

(b)

Figure 2.41: Left-right alignment plots for Sector 6, shown before and after calibration. The axes represent paddle number vs. distance from the scintillator center, in $\mathrm{cm}$.

along the scintillator toward the PMTs. The effective velocity is less than the speed of light in the scintillator, due to oblique internal reflections of light within the scintillator. Effective velocity is determined by using [78]

$$
t_{L / R}=t_{0} \pm \frac{y}{v_{e f f}}
$$

where $t_{0}$ is the measured time of an event at the geometric scintillator center, and $y$ is the position along the scintillator. The value of $v_{\text {eff }}$ is approximately $16 \mathrm{~cm} / \mathrm{ns}$, and in fact, this value can be assumed with little reduction in timing resolution. ${ }^{26}$ In addition, the ADC channels are calibrated for energy loss and attenuation (i.e. dispersion of the light signal) along the scintillator. The attenuation is exponential; the fit to the ADC channels (see Figure 2.42) is

$$
A_{L / R}^{\prime}=\frac{M_{0 L / R}}{10 \mathrm{MeV}} E e^{-y / \lambda}
$$

where $E$ is particle energy deposited in the scintillator, $M_{0 L / R} / 10 \mathrm{MeV}$ is a normalization

\footnotetext{
${ }^{26}$ This is indeed the case for this analysis.
} 


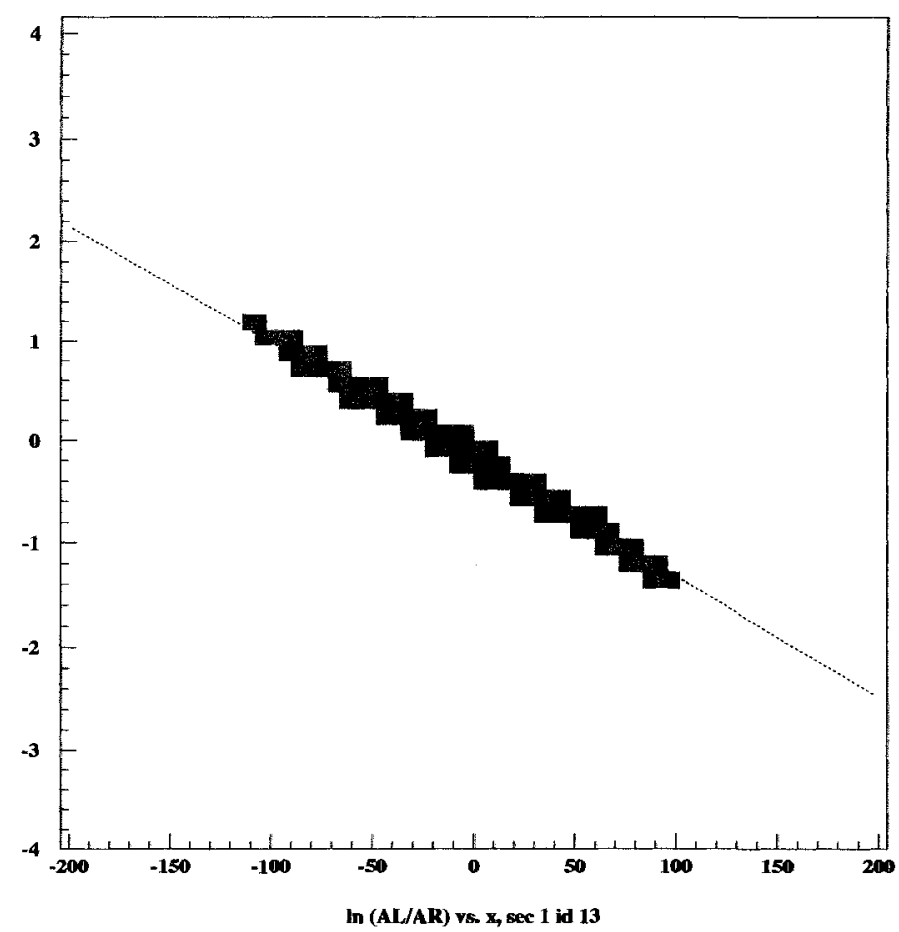

Figure 2.42: Plot of the log of the ratio of ADC values vs. scintillator position (in $\mathrm{cm}$ ) for Paddle 13, Sector 1, after the attenuation length calibration of Eq. 2.24.

factor (determined in the fit), $\lambda$ is the fit attentuation length and $y$ is again the hit position, defined by

$$
y=\frac{v_{e f f}}{2}\left(t_{L}-t_{R}-y_{\text {offset }}\right)
$$

where $y_{\text {offset }}$ is another fit variable. Note that due to the codependence of the variables, the calibration steps for the paddle must be performed iteratively, with reasonable initializations of the fit parameters [78].

3. When individual paddles are properly calibrated, the final step is to ensure that all the active paddles in every sector act as a coherent, single detector, tracing the start time of an (approximately zero-mass) electron scattered through any angle properly back to the vertex point. Then, the TOF of slower, heavier particles, and hence their mass, can be calculated. 


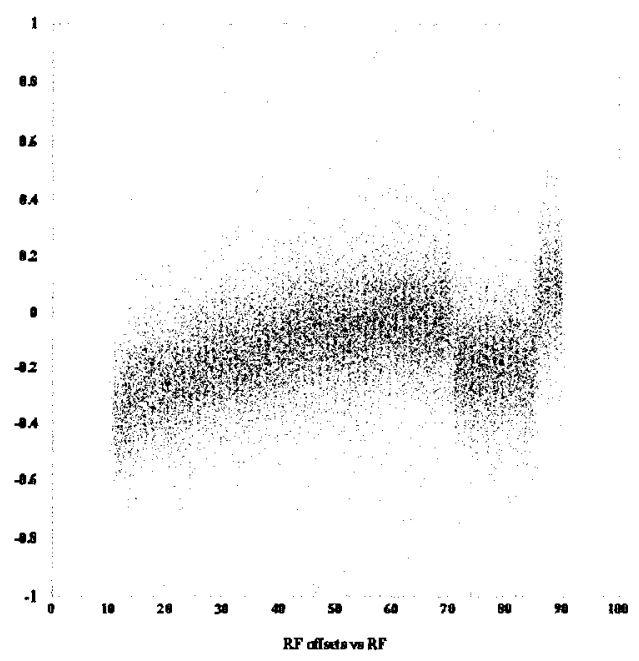

(a)

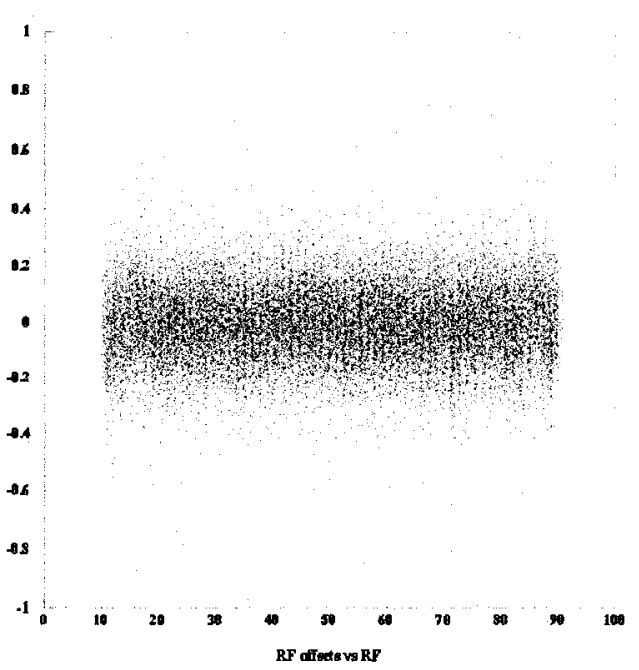

(b)

Figure 2.43: RF bunch timing offsets, before and after calibration of the RF start time. The points represent the reconstructed start time of electron events vs. the position in the RF bunch. A polynomial offset function is fitted in segments to center the offset at zero.

First, the start time must be determined. As detailed in Section 2.2, the electron beam is delivered to Hall-B in RF-pulsed bunches. The phase of these bunches is subject to periodic changes. Event start timing is offset by a function of its phase within the RF signal. To adjust the start time, a third-degree polynomial and an overall offset constant are fit as a function of offset time within the pulse distribution ${ }^{27}$ (see Figure 2.43).

Finally, then paddle-to-paddle or counter-to-counter delay offsets $c_{c 2 c}$ are determined for each paddle from a fit of scattered electron and/or pion data [78]:28

$$
t_{L / R}=t_{w} \pm \frac{\delta t_{L R}}{2}+c_{c 2 c}
$$

Plots of reconstructed hadron mass before and after this calibration are shown in Figure 2.44.

\footnotetext{
${ }^{27}$ In event that the RF pulse information is unavailable, in fact a problem for large sections of the EG1b run set, electron TOF was used to normalize the start time of the heavier hadrons.

${ }^{28}$ This equation is slightly different than the one shown in Ref. [78], as unused calibration constants are omitted here.
} 

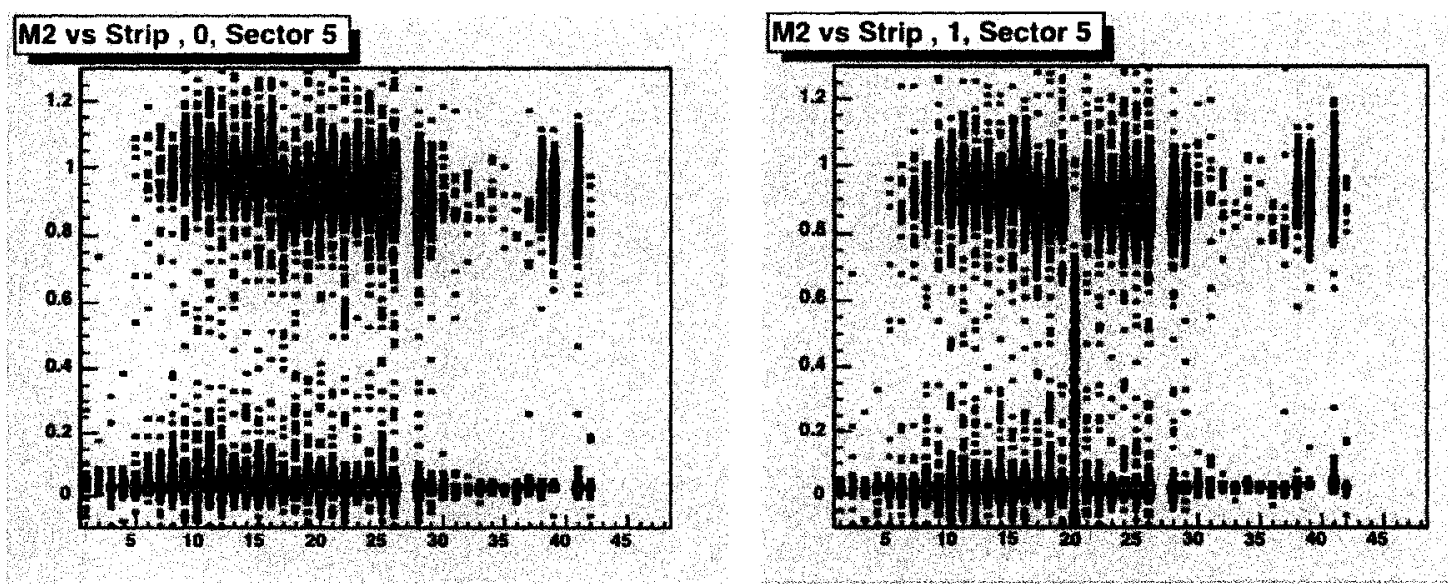

Figure 2.44: TOF-reconstructed mass squared (in $\mathrm{GeV}^{2}$ ) vs. paddle number for sector 5 events, before and after paddle-to-paddle delay calibrations. The wide band represents protons $\left(M^{2}=\right.$ $\left.0.879 \mathrm{GeV}^{2}\right)$ and the bottom band represents pions $\left(M^{2}=0.019 \mathrm{GeV}^{2}\right)$. (Paddle 20 failed to calibrate properly with the automated software (right plot), and required manual recalibration.)

Assuming these offsets are chosen properly, the time-of-flight $\bar{t}$ for any SC hit can be found as

$$
\bar{t}=\frac{t_{L}+t_{R}}{2}
$$

The time of flight not only establishes time-based tracking in the DC, it normalizes timing for the EC and CC. Timing resolution within $0.2-0.3 \mathrm{~ns}$ can usually be achieved with proper calibration of the SC constants.

\subsubsection{Electromagnetic Calorimeters}

After passing through the various (DC, CC, SC) detector components, forward-scattered particles enter the outermost detector portion in CLAS, the electromagnetic calorimeters (EC). The EC absorbs energy from the scattered particles in 15 radiation lengths of lead, interleaved with scintillation detectors that provide energy and timing information for the scattered particle.

The calorimeters in each sector are composed of 39 dual alternating layers of ( $2.2 \mathrm{~mm}$ thick) $\mathrm{Pb}$ and (10 $\mathrm{mm}$ thick) scintillator, the lengths optimized for maximal energy resolution and minimal transverse light attentuation [79]. The calorimeter in a given sector is triangular in shape. The scintillators are cut into 36 parallel strips in each layer. The scintillator direction alternates to provide 


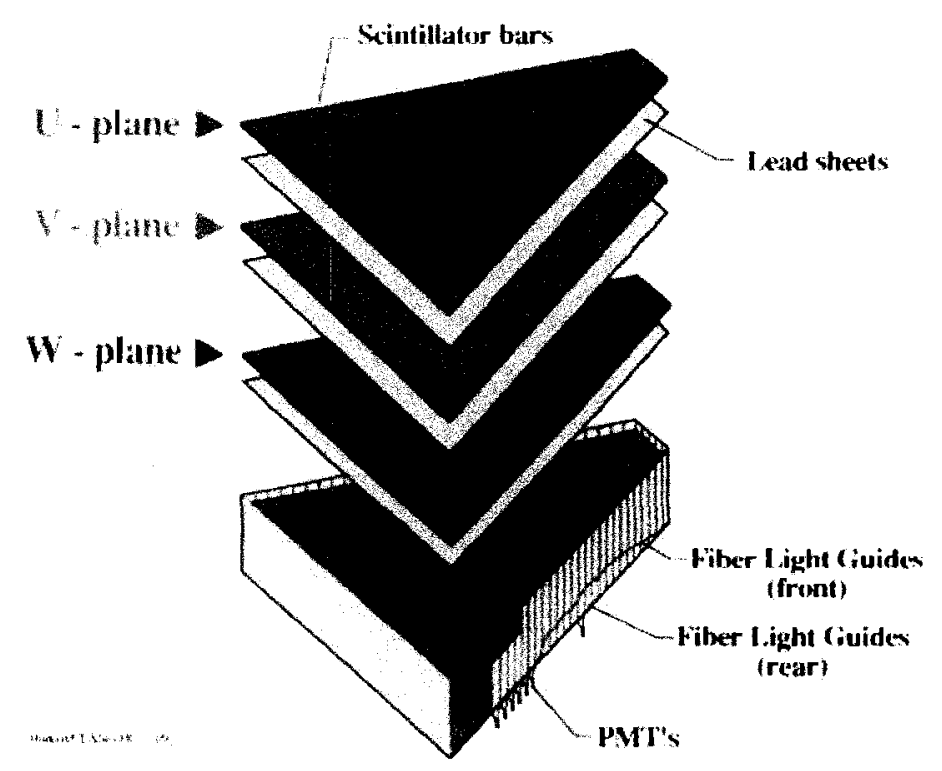

Figure 2.45: Diagram of one sector of the CLAS electromagnetic calorimeter, showing the stacks of scintillator strips aligned along 3 different orientations, alternating with lead sheets. Fiber light guides send light from the scintillator planes to the PMTs. From Ref. [79].

spatial resolution along 3 orientations, labeled as $U, V$, and $W$ (see Figure 2.45). Each orientation thus has $36 \div 3=13 \mathrm{~Pb} / \mathrm{scintillator} \mathrm{layers.} \mathrm{Light} \mathrm{from} \mathrm{the} \mathrm{first} 5$ scintillators along a given orientation $\left(E C_{\text {in }}\right.$ ) leads through light guides to one PMT, while light from the remaining 8 scintillators along a given orientation ( $\left(\mathrm{CC}_{\text {out }}\right.$ ) leads to a second $\mathrm{PMT}$. This arrangement is capped with steel/foam plates. A diagram of this configuration is shown in Figure 2.46. Each "column" of 13 strips has its own pair of PMTs, for a total of $13 \times 3 \times 36=216$ PMTs in each sector [79].

Incident charged particles in the EC (above a minimum energy threshold of $\sim 0.5 \mathrm{GeV}$ ) produce either ionization reactions (in the case of incident hadrons) or showers of $e^{+} e^{-}$pairs and Bremsstrahlung photons (in the case of incident electrons or positrons). ${ }^{29}$ lonizing tracks are the easier case to localize; the hit location along the intersection of the $U, V$, and $W$ orientations gives the location of the particle. Showering events, which produce several signals over the scintillators in each layer, require more sophisticated reconstruction. First, adjacent strips along each orientation meeting a certain energy threshold are grouped, and peaks, in the form of a centroid and RMS of

\footnotetext{
${ }^{29}$ Neutrons and photons can also be detected in the $\mathrm{EC}$, but this is of little relevance to inclusive analysis, and is not dealt with in this thesis.
} 


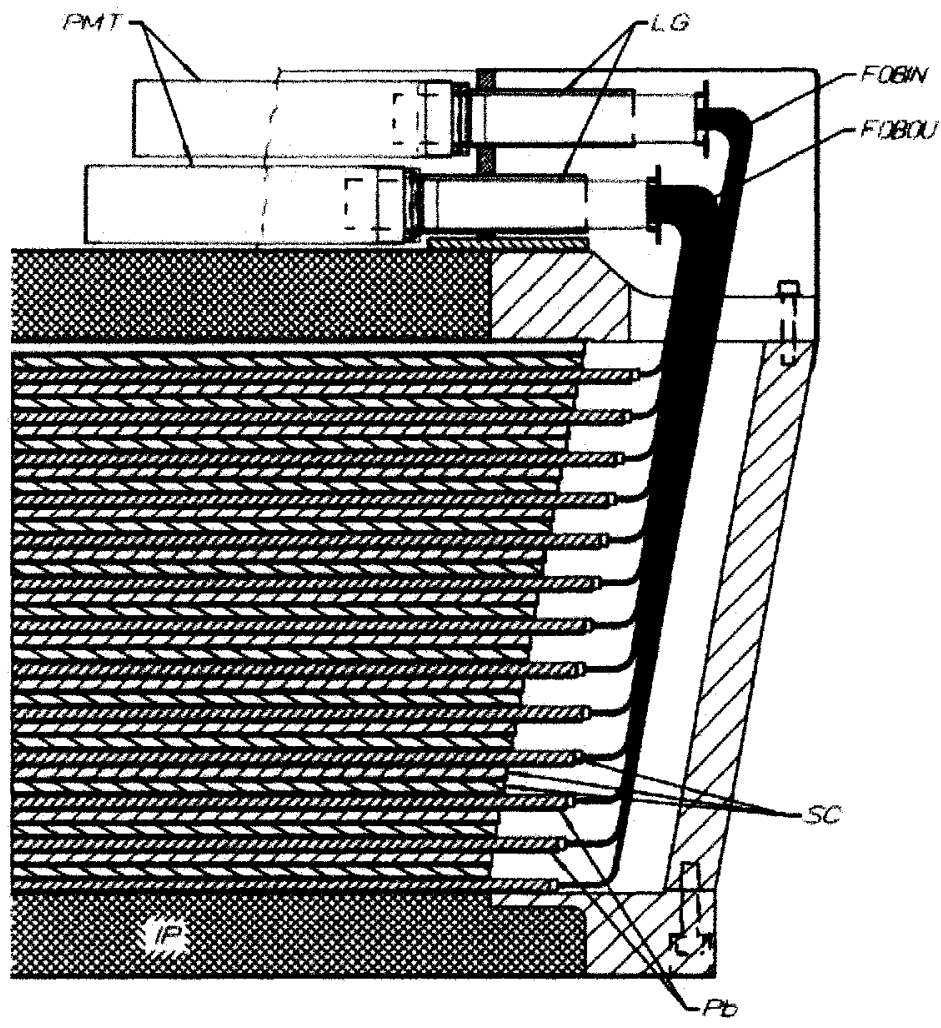

Figure 2.46: Vertical cross section of one edge of the EC in one sector, showing lead (Pb) plates and scintillators (SC), light guides (LG), fiber optic bundles for the inner and outer calorimeter layers (FOBIN and FOBOU) and the inner plate (IP) of steel face sheets and foam core, needed for structural support. From Ref. [79].

each group, are calculated. Then, the peaks above another threshold are matched for geometrical compatibility in a three-iteration loop over the $\mathrm{U}, \mathrm{V}$ and $\mathrm{W}$ coordinates to produce a hit location.

Once the hit is identified, energy measurements for $\mathrm{EC}_{\text {in }}, \mathrm{EC}_{\text {out }}$, and the signal sum $\mathrm{EC}_{\text {tot }}$ are recorded for all PMTs in the peak. The total energy deposited in the EC is equal to the total particle energy multiplied by a sampling fraction $f_{s}$. The EC energy resolution is then

$$
\frac{\sigma}{E} \propto \sqrt{\frac{t_{s}}{f_{s}}}
$$

where $t_{s}$ is the calorimeter thickness in radiation lengths. A fairly constant sampling fraction of $f_{s}=0.27 \pm 0.02$ is characteristic at electron energies higher than $1.5 \mathrm{GeV}$, with lower (and less ac- 


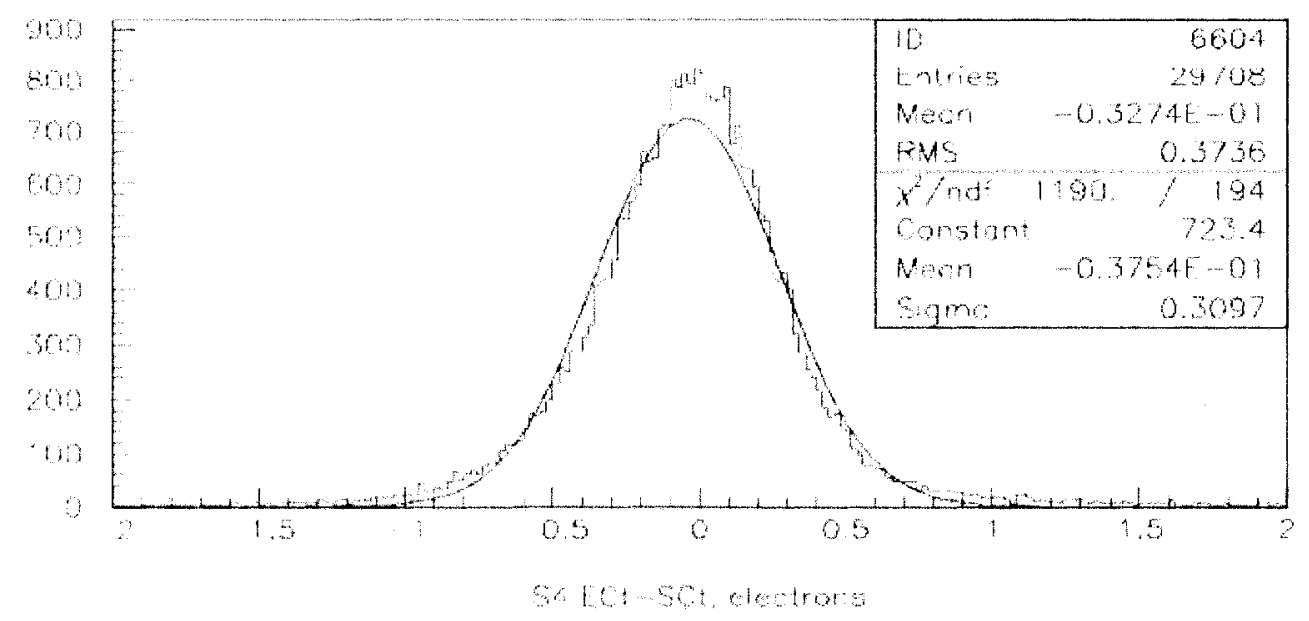

Figure 2.47: Difference between EC and SC times (in ns) for reconstructed electron events in sector 4, after EC timing calibrations.

curate) sampling fractions for lower energies [79]. Thus, to translate EC energies to actual particle energies, the EC energies must be divided by 0.27 .

Much like the TOF (SC) detectors, the scintillators and PMTs in the EC require calibrations. As for the SC, PMTs require internal calibrations (for ADC pedestals), as well as corrections due to the scintillator properties (i.e. exponential attentuation). Gain matching is also required, such that the sum of channel energies totals $E \times f_{s} .{ }^{30}$ After the experimental run, during pre-analysis, the EC timing signal is calibrated to the SC signal, by using a 5-parameter model to minimize the average difference between the SC and EC timing. A sample plot of the overall resulting time resolution is shown in Figure 2.47.

The main purpose of the EC in the EG1b experiment is to help aid in particle identification. Due to the $e^{+} e^{-}$showers produced by incident electrons at high energies, the EC is most effective at discriminating between electrons and hadrons with higher momenta (unlike the $\mathrm{CC}$, which discriminates most efficiently between low-momentum particles). The use of the EC in particle identification is dealt with in detail in Section 3.4.2. The secondary purpose of the EC is to supply additional timing information to aid in event reconstruction (see Section 2.6.2).

\footnotetext{
${ }^{30}$ This is the ADC analogy to paddle-to-paddle calibration of TDC values described in Section 2.5.4.
} 


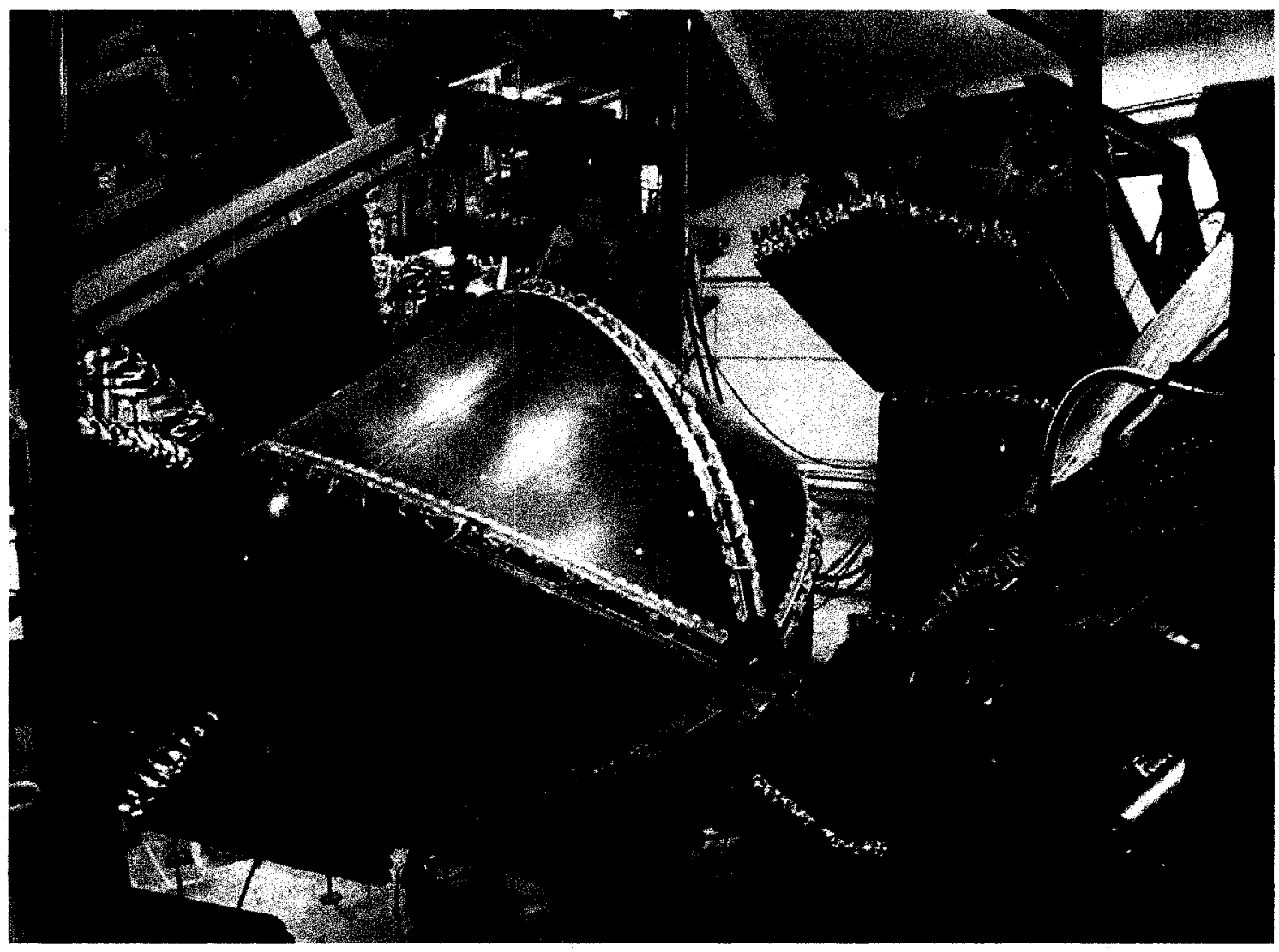

Figure 2.48: A photograph of the CLAS detector, partially assembled. The spherical drift chambers (DC) are in the center. The foil-wrapped, hexagonal scintillation counters (SC) surround the DC on all sides when enclosed. On the far right, gas feedthroughs into the Cherenkov counters (CC) and PMT feedthroughs into the electromagnetic calorimeters (EC) can be seen.

\subsection{Data Collection}

The individual CLAS components, shown together in Figure 2.48, must be made to operate together during the data collection process. A brief description of the detector electronics, data acquision (DAQ) system, and simple event builder (SEB) used for event reconstruction follows, along with a brief summary of the EG1b data set. Again, the goal is not to provide a complete blueprint of the data collection and event reconstruction procedure, but merely to provide an outline and direction to relevant references regarding the hardware and software methods. 


\subsubsection{Data Acquisition}

All analog signals from the CLAS detector are digitized by FASTBUS and VME modules in 24 crates. CLAS has two different trigger levels that can be used as conditions for event recording. For this experiment, Level 1 triggers, which require minimum thresholds in both the inner layer EC and in the CC [2] were employed. All PMT signals (i.e. SC, EC, and CC) within 90 ns of the trigger are processed, as well as drift chamber TDC signals [30]. The trigger supervisor (TS) electronics board then takes the Level 1 trigger inputs and generates all signals, busy gates and resets required to generate event parameters. These are then fed into the data aquisition, or DAQ system.

The CLAS DAQ system was designed to handle event rates of up to $2 \mathrm{kHz}$ and data rates of 25 $\mathrm{MB} / \mathrm{s}$ [30]. ${ }^{31}$ CLAS data flow is shown in Figure 2.49, and is described in this brief outline:

1. Digital output from the 24 crates is read into 24 Readout Controllers (ROCs), then through fast ethernet lines into the central DAQ.

2. The Event Builder (EB) software assembles the digital signals into complete particle events. Each event is labeled with a number and the trigger bits (see Section 3.6.2).

3. Assembled event data is passed through shared online memory managed by the Event Transport (ET) system, so that online reconstruction and monitoring can take place. ET1 manages the memory, sending data to ET2 for online monitoring, and ET3 for online reconstruction and analysis (see Figure 2.50).

4. ET1 sends data to the Event Recorder (ER), which in turn temporarily stores the data on local RAID (Redundant Arrays of Inexpensive Disks) disks.

5. Data is transferred (in parallel) to be written on magnetic tapes in a remote recording and retrieval silo. The data can then be retrieved as needed for analysis.

Data collection is managed by CODA (CEBAF Online Data Acquisition) software [80], which provides the configuration of the DAQ components outlined above. The CODA software writes the data separated into 2 GB file blocks. Typically, 15-30 of these file blocks constitutes a complete

\footnotetext{
${ }^{3}$ These figures correspond to 2000-01, when these data were taken. The DAQ has received substantial upgrades since that time.
} 


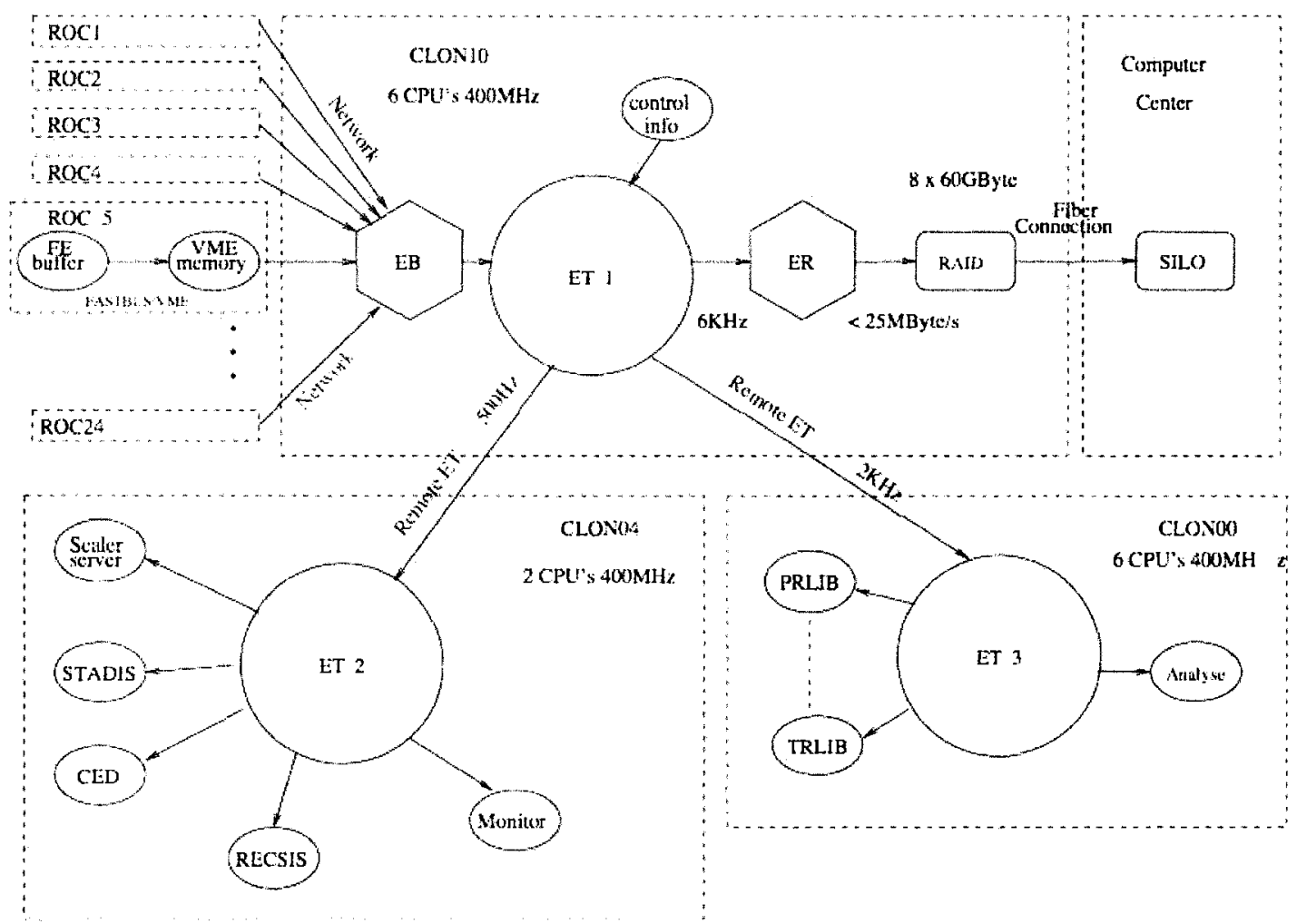

Figure 2.49: A flowchart showing data transfer in the CLAS DAQ system. See the text for details. From Ref. [30].
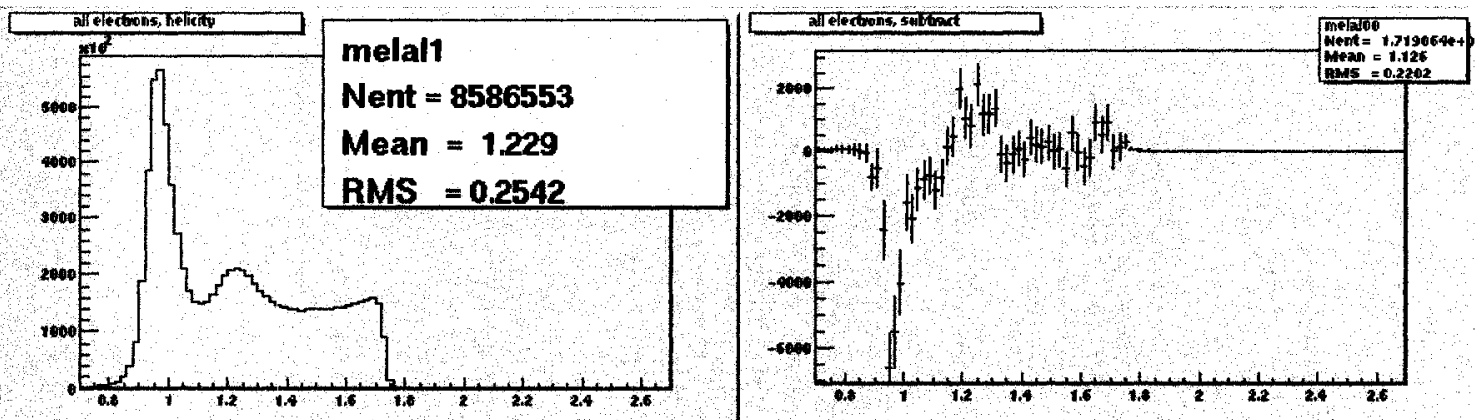

Figure 2.50: A screenshot of online event reconstruction at $1.7 \mathrm{GeV}$ beam energy during the EG1 experiment. The left plot shows total reconstructed event counts in terms of missing mass $W$ (in $\mathrm{GeV}$ ). The right plot shows the raw double-spin asymmetry (Eq. 1.209). In both cases, the elastic peak $(W=0.938 \mathrm{GeV})$ and the $\Delta$-resonance $(W=1.23 \mathrm{GeV})$ are clearly visible. The absolute sign of the asymmetry is inverted due to a negative beam-target polarization product. From Ref. [63]. 
data run. There were approximately 2000 runs in the EG1 data set, meaning at least 40 terabytes of data were written to the silo data tapes. Because this is an unwieldy quantity of data for analysis, several stages of data compression were employed.

\subsubsection{The Simple Event Builder (SEB)}

The simple event builder (SEB) is used during a process known as "cooking" to convert the raw TDC and ADC channel data into kinematic and particle identification data by incorporating geometric parameters and calibration constants. Calibration and mapping constants are read from the CalDB (calibration database). The CalDB is a MySQL database with a user interface that contains all $A D C$ and TDC offsets and corrections, geometric constants, status flags and run information for all Hall-B experiments [81].

Once particle track momentum, geometry and crucial EC and CC information has been established, the superfluous digital channel data can be excluded from the new, compressed files. The RECSIS (Reconstruction and Analysis) package uses a Tcl initialization file to instruct the SEB to produce smaller "cooked" data files from the "raw" data files, writing data only into specified banks to minimize the required disk space [82].

The SEB works by matching tracks/hits in the CLAS coordinate system [83], which is defined geometrically in terms of $x$ (horizontally), $y$ (vertically) and $z$ (along the beam axis) for the DC, EC, and SC, and along polar coordinates $\phi$ and $\theta$ for the CC. First, the geometric DC cell tracks for each particle are assumed to be master tracks, then hits in the CC, SC and EC $\left(a_{i}\right)$ are matched to the track in the detector plane $\left(a_{p l}\right)$ by the minimizing

$$
\chi^{2}=\sum_{i} \frac{a_{i}^{2}-a_{p l}^{2}}{\sigma_{a_{i}}^{2}}
$$

Neutral particles, of course, have no DC track, so straight lines from their EC hit points traced to the target vertex (at $z=-55.1 \mathrm{~cm}$ ) are used as the master tracks. This process is known as hit-based tracking.

The SEB then cycles through particles in the event to search for a single trigger electron ${ }^{32}$ as

\footnotetext{
${ }^{32}$ That is, it looks for an electron unpaired (by momentum conservation) with a positon, as that would indicate a $e^{+} e^{-}$ pair production, not a scattered electron.
} 
the trigger particle, requiring that it has a negative charge and produces an EC shower. If more than one electron candidate is found, the one with the highest momentum is selected. Tracing this particle back to the vertex along its geometric path (assuming $v=c$ ) determines the trigger start time. The time-of-flight of other particles can then be determined relative to the start time using SC TDC values. EC TDC values are used if SC values are not available for the particle. The mass $m$ of any charged particle in the event can then be determined from the relation

$$
t_{S C}-t_{\text {start }} \pm \delta=\frac{\ell_{\mathrm{path}}}{\beta}=\frac{\ell_{\mathrm{path}}}{c} \sqrt{\frac{p^{2}+m^{2}}{p^{2}}}
$$

where $t_{S C}$ and $t_{\text {start }}$ are the particle SC time and trigger start time, $\ell_{\text {path }}$ is the particle path length (determined by hit-based tracking), $c$ is the speed of light, $p$ is the particle momentum, and $\beta \equiv v / c$. The uncertainty $\delta$ is the inherent Gaussian detector error in determining the TOF; it is usually in the neighborhood of a few to several hundred picoseconds after TOF calibration. A (preliminary) particle type ID can then be assigned to each particle based on its position in the TOF spectrum (see Figure 2.51). This is known as time-based tracking, and it provides the minimal present criteria for an event to be considered for further analysis.

\subsubsection{The EG1b Data Set: Preparation for Analysis}

The EG1b experiment collected electron data at 4 approximate beam energies (1.6, 2.5, 4.2, and $5.7 \mathrm{GeV}$ ). In reality, beam energies could not be kept at precisely these values for the whole 7 month EG1b run, so several different beam energies (1.606, 1.723, 2.286, 2.561, 4.238, 5.615, 5.725 and $5.743 \mathrm{GeV}$ ) were used, with two possible (inbending and outbending) main torus currents. Calibration constants were determined for a total of 12 different data sets (or brackets) for each combination of beam energy and torus current. CLAS runs $25488-28570$ comprise the EG1b data set. Kinematic coverage of the EG1 data for the 4 nominal beam energies is shown in Figure 2.52. Comparing to Figure 1.13 , one can indeed see that the new data provides coverage in the low $x$ and $Q^{2}$ region.

Each bracket contains runs using each of the available targets on the main insert: $\mathrm{NH}_{3}, \mathrm{ND}_{3}$, 


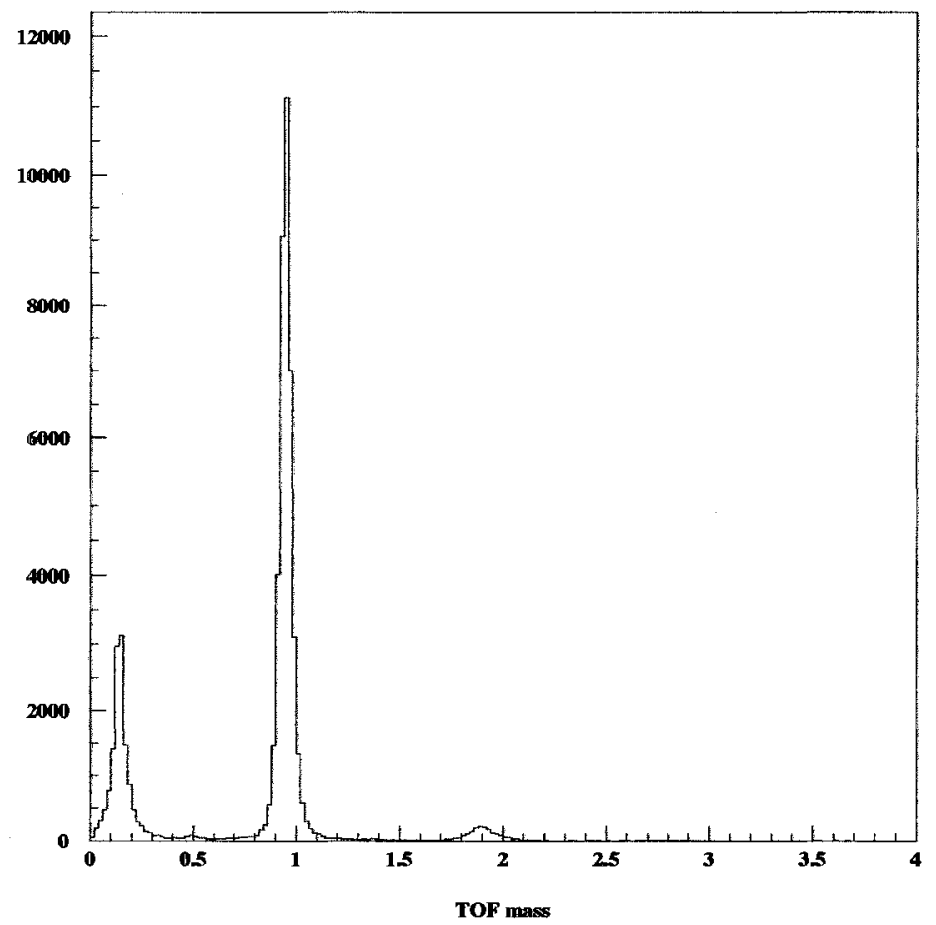

Figure 2.51: TOF mass (in GeV) of secondary (non-trigger) particles for an EG1b data run, shown after TOF calibration. Mass is given in GeV. The pion and proton peaks can clearly be seen at 140 and $938 \mathrm{MeV}$. Much smaller kaon and deuteron peaks can also be resolved at 494 and $1876 \mathrm{MeV}$. 


\section{EG1b kinematic range}

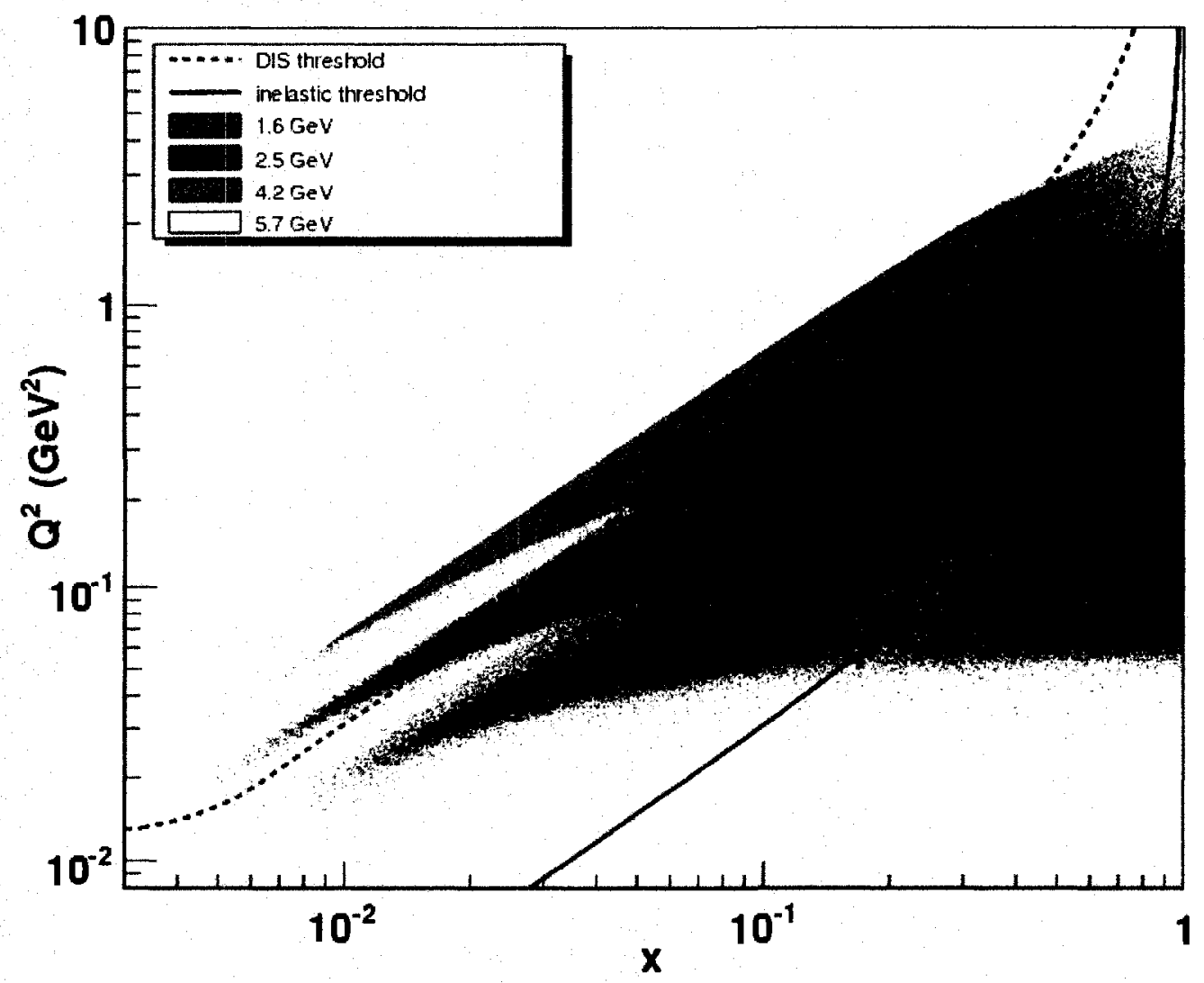

Figure 2.52: Kinematic coverage of data (by energy) in terms of $Q^{2}$ and $x$ in the EG1b data set. This plot was constructed by analysis of the events of an inbending and outbending run at each of the 4 energies. The solid and dotted lines mark missing mass $W=1.08 \mathrm{GeV}$ and $2.0 \mathrm{GeV}$, respectively. 
${ }^{12} \mathrm{C}$, and empty (LHe and windows only). Shorter, special run sets were taken with the ${ }^{15} \mathrm{~N}$ target insert, as well. In addition to these production runs, numerous other calibration and test runs were made, including runs for DAQ tests, Møller runs, cosmic ray calibration, TDC calibration, laser calibrations and pedestal calibrations [84]. These runs were separated from the production runs.

After calibrations were completed as outlined in Section 2.5, quality checks of the calibration of each data set were made. The RECSIS code was applied to the whole run set, first for only the first file in each data set, a procedure referred to as passO cooking, to test overall TOF quality, EC timing quality, and residuals from DC calibrations. Necessary adjustments to subsets of runs (sometimes required for a change in $\mathrm{RF}$ offset, for example) were then made. Figure 2.53 shows mean electron RF timing offsets after passo cooking for the $2.286 \mathrm{GeV}$ data. Assuming the calibration quality of the run set is acceptable, then, pass1 cooking is done, processing all production data into timebased tracking event data for physics analysis. Analysis of these "cooked" data is the subject of the remainder of this thesis.

\subsection{Structure function and asymmetry models}

Section 1.4 indicated that quantitative models parametrizing the contributions from physical quantities are required to extract structure functions from the data. This section provides a brief description of the models package (designed by S. Kuhn et al.) used to evaluate contributions from unpolarized structure functions and virtual photon asymmetries. The 'models' are parametrized by FORTRAN code originally included in the RCSLACPOL code used at SLAC, modified for the lower beam energies found at $\mathrm{JLab}$, especially in the resonance region.

\subsubsection{Model of unpolarized structure functions $F_{1}^{p}$ and $R^{p}$}

The $F_{1}$ and $F_{2}$ structure functions for the proton have long been well-parametrized by data in the DIS region. The ratio of longitudinal to transverse cross-sections for unpolarized scattering, $R$ (Eq. 1.65), is related to $F_{2}$ and $F_{1}$ by Eq. 1.79. The fit for $R$ uses JLab Hall-C High Momentum Spectrometer (HMS) measurements of LT separated virtual photoabsorption cross-sections [85] combined with an older SLAC parametrization of DIS world data [86]. Specifically, Hall-C measurements of 


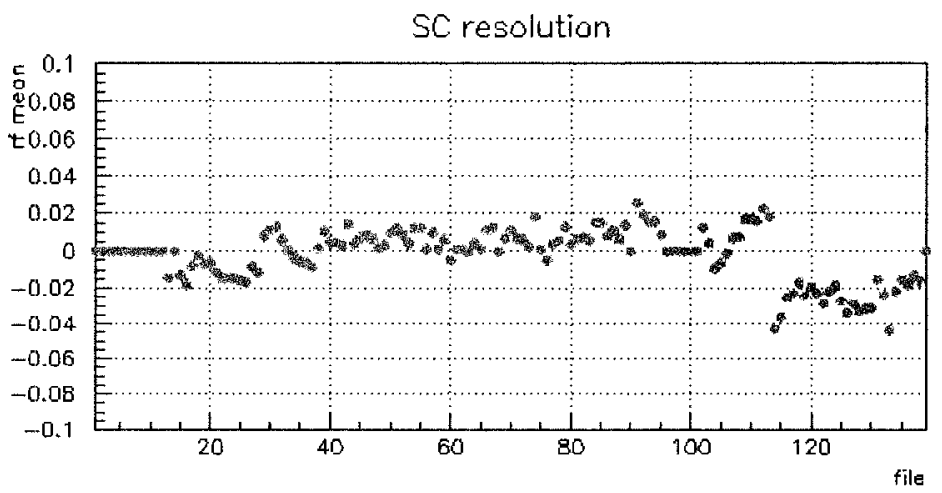

(a)

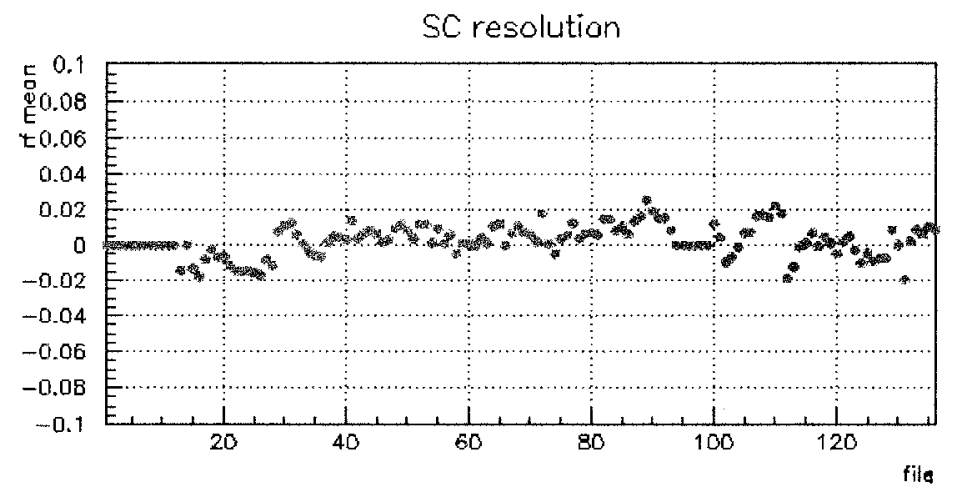

(b)

Figure 2.53: Quality check plots for event timing of the $2.286 \mathrm{GeV}$ data. a) shows the mean difference in electron trigger start and RF bunch time for the first file in each data set. This plot shows an improper RF offset for the last several runs, which is shown corrected in b). All times are in ns. 
the reduced cross-section [87]

$$
\sigma_{r}=\frac{1}{\Gamma} \frac{d \sigma}{d \Omega d E^{\prime}}=\sigma_{T}\left(W^{2}, Q^{2}\right)+\epsilon \sigma_{L}\left(W^{2}, Q^{2}\right)
$$

were used, where the virtual photon flux $\Gamma$ is given by

$$
\Gamma=\frac{\alpha E^{\prime}\left(W^{2}-M^{2}\right)}{(2 \pi)^{2} Q^{2} M E(1-\epsilon)}
$$

Values of $\sigma_{L}$ and $\sigma_{T}$ were extracted from $\sigma_{r}$ using the older DIS fit to $R$. These extracted data were then fit independently, and the newly determined $R$ value was used to iteratively extract new values of $\sigma_{L}$ and $\sigma_{T}$ until convergence was reached. With $R$ known, fits to the total unpolarized cross-sections, together with Eq. 1.79 , were used to extract $F_{1}$ [87]. Values of $F_{1}^{p}$ are known to a precision of better than $3 \% ; R^{p}$ errors are accurate to approximately $3 \%$ over the kinematic range covered by EG1b.

The total cross-section is fit by contributions from resonant production (described by thresholddependent Breit-Wigner forms and $Q^{2}$-dependent amplitudes) superimposed on a smooth nonresonant background. Data from JLab and SLAC are used for the resonance and DIS regions, while DA $\Phi$ HNE and other older photoproduction data constrain the $Q^{2} \rightarrow 0$ limit. Exact details of the fit equations, data sets, and a full description of the fit procedure by $\mathrm{E}$. Christy and P. Bosted can be found in Ref. [87]. Plots of the unpolarized structure function models of $F_{1}^{p}$ and $R^{p}$ for various $Q^{2}$ bins are shown in Figures 2.54 and 2.55. In this thesis, the $F_{1}$ model provides the basic unpolarized ratio for the extraction of $g_{1}$ and $g_{2}$ from virtual photon asymmetries, while $R$ is used exclusively for calculation of the depolarization factor $D$ (Eq. 1.19).

\subsubsection{Model of virtual photon asymmetries $A_{1}^{p}$ and $A_{2}^{p}$}

Fits for modeling the virtual photon asymmetries $A_{1}$ and $A_{2}$ utilized all the pre-EG1b data described in Section 1.5, i.e. E80, E130, EMC, SMC, E143, HERMES, E155 and EG1a for $A_{1}$ and $g_{1}$; and E155x for $A_{2}$ and $g_{2}$. ${ }^{33}$ The $A_{1}$ model was generally used only for comparison and general

\footnotetext{
${ }^{33}$ RSS data was not yet included in the model fit at the time of the writing of this thesis.
} 

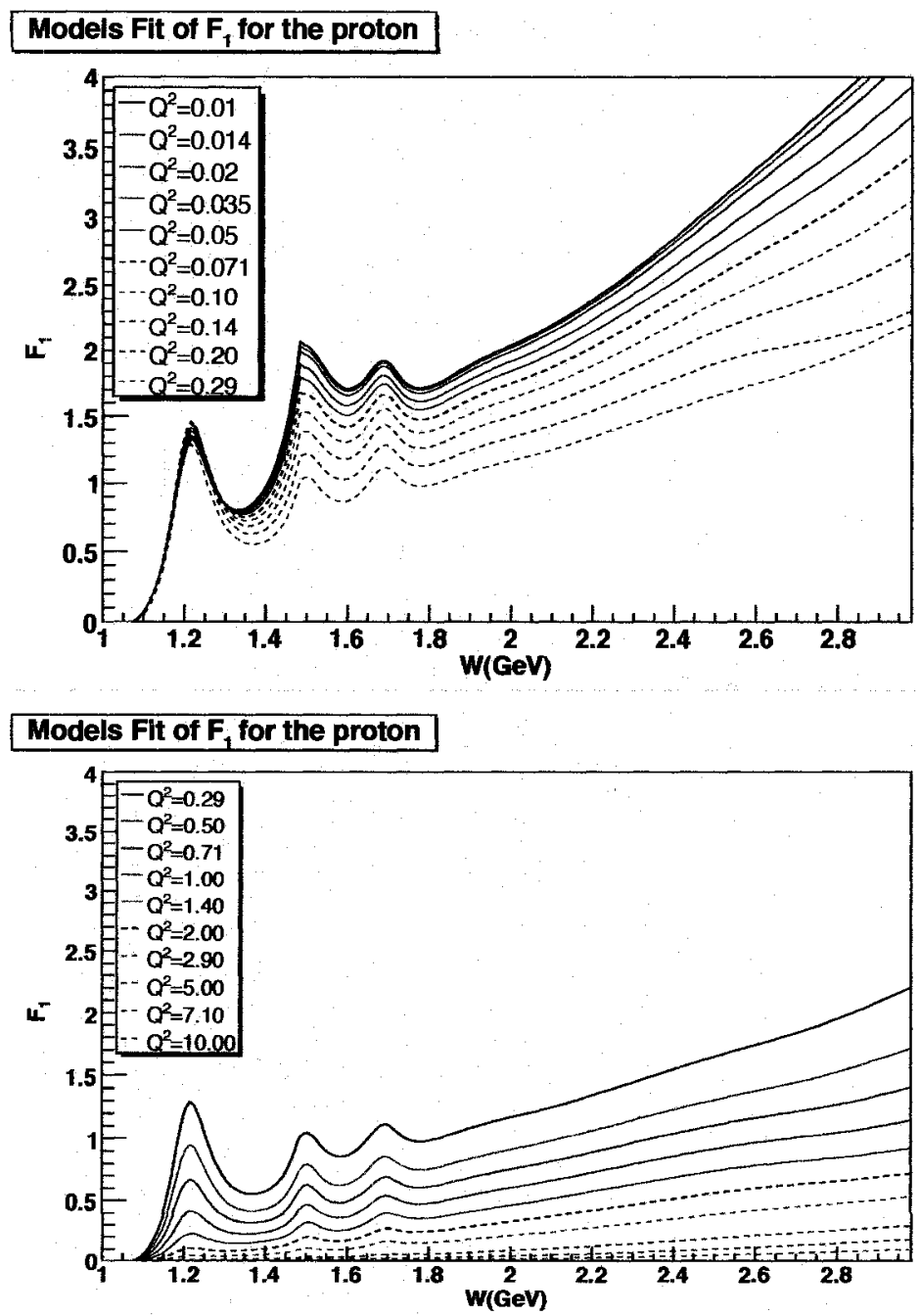

Figure 2.54: Model values of $F_{1}$ [87] for the proton used to extract the $g_{1}$ and $g_{2}$ structure functions in this thesis, shown in terms of missing mass $W$. Each curve corresponds to a different $Q^{2}$ bin. The highest $Q^{2}$ value shown in the top plot is the same as the lowest $Q^{2}$ value shown in the bottom plot. 

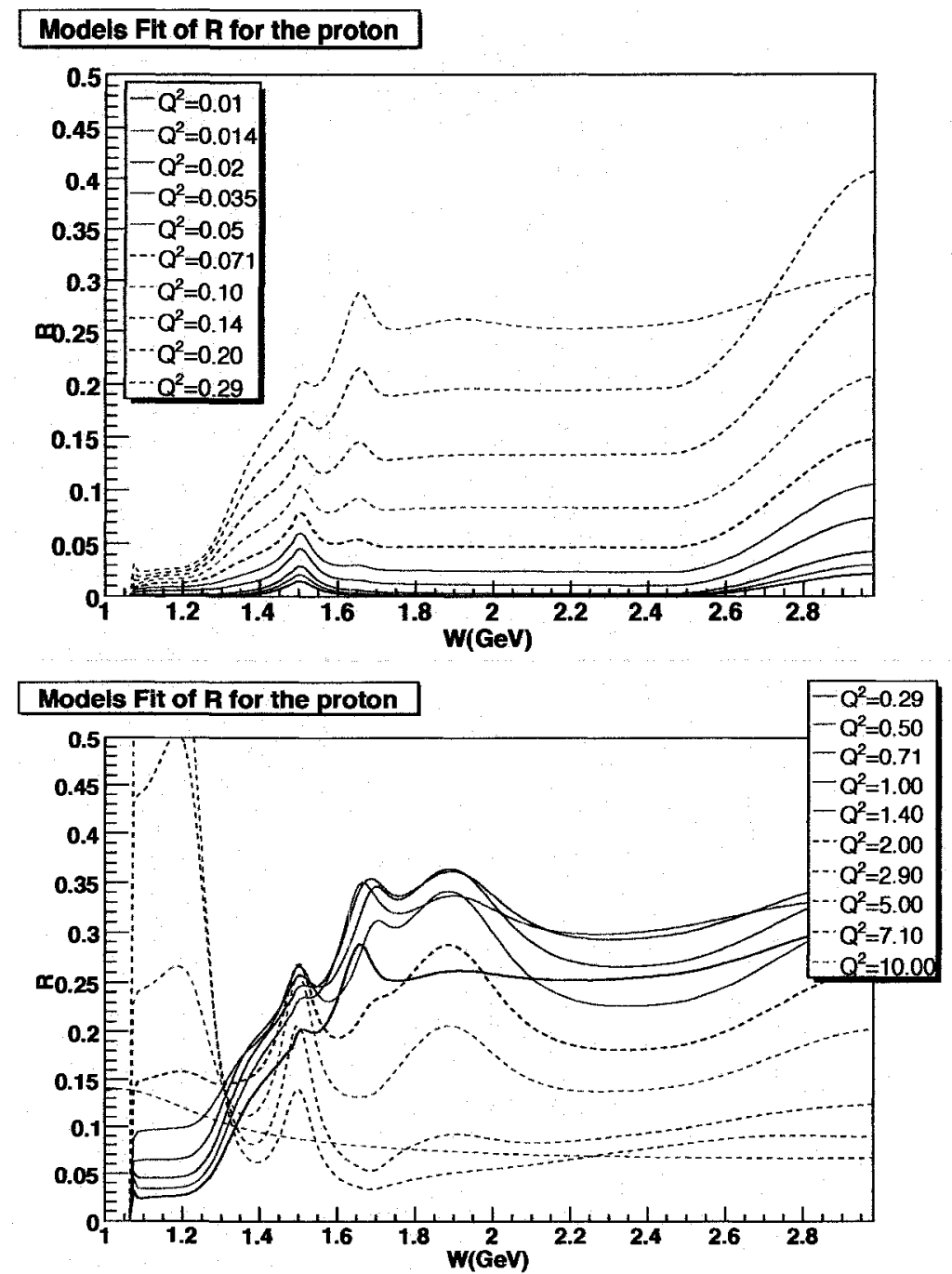

Figure 2.55: Model values of $R=\sigma_{L} / \sigma_{T}$ [87] for the proton used to extract the $g_{1}$ and $g_{2}$ structure functions in this thesis, shown in terms of missing mass $W$. Each curve represents a different $Q^{2}$ bin. The highest $Q^{2}$ value shown in the top plot is the same as the lowest $Q^{2}$ value shown in the bottom plot. 
weighting between target polarizations, while the $A_{2}$ model was essential for extracting the $A_{1}$ asymmetry. In fact, the $A_{2}$ model incurs the single largest systematic error on the extraction of $A_{1}$; see Section 8.1.1.

The parametrization by N. Bianchi [89] uses Regge theory to constrain the $A_{1}$ fit at $Q^{2}=0$, describing high-energy cross-sections as

$$
\sigma^{\frac{1}{2}}-\sigma^{\frac{3}{2}} \sim s^{\alpha_{0}-1}
$$

(with $s$ defined in Section 1.1.3) and

$$
\alpha_{0}=J-\alpha^{\prime} m_{t}^{2}
$$

is given in terms of a spin $J$ and mass $m_{t}$ of an exchanged meson, assumed to be the $a_{1}(1260)$ or $f_{1}(1285)$ for the isovector and isoscalar contributions to the cross-section, respectively [89], and $\alpha^{\prime} \sim 0.85$. A global fit in terms of $W$ was applied using this general form at the real photon point. The parametrization in the resonance region uses an extrapolation from the DIS fit to the world data, with resonance data added as a separately parametrized contribution, fit with a total of 9 parameters.

Very little data exists to parametrize $A_{2}$, but some constraints can be applied to develop a rudimentary model of this quantity. The first constraint, which can be derived from Eqs. 1.214, 1.220 and 1.219 , is the Soffer bound [90]

$$
\left|A_{2}\left(x, Q^{2}\right)\right| \leq \sqrt{\frac{A_{1}+1}{2}} R\left(x, Q^{2}\right)
$$

The Wandzura-Wilczek relation (Eq. 1.202) and Burkhardt-Cottingham Sum Rule (Eq. 1.204) were used as further constraints to the model, although it is not yet known at this point whether they hold exactly. ${ }^{34}$ The model also makes the assumption that all higher twist effects are contributed by the resonance region, with a smooth transition to the DIS region, ${ }^{35}$ where $A_{2} \rightarrow 0$ in the scaling region [90]. Model values of $A_{2}$ used in this thesis are shown in Figure 2.56.

\footnotetext{
${ }^{34}$ In fact, we know from RSS data that it is very unlikely at least one these rules holds exactly, but RSS was not incorporated into the described version of the model, and, in any case, these rules serve as a good first-order approximation.

${ }^{35}$ not necessarily true, but compatible with a fit to E155x data
} 


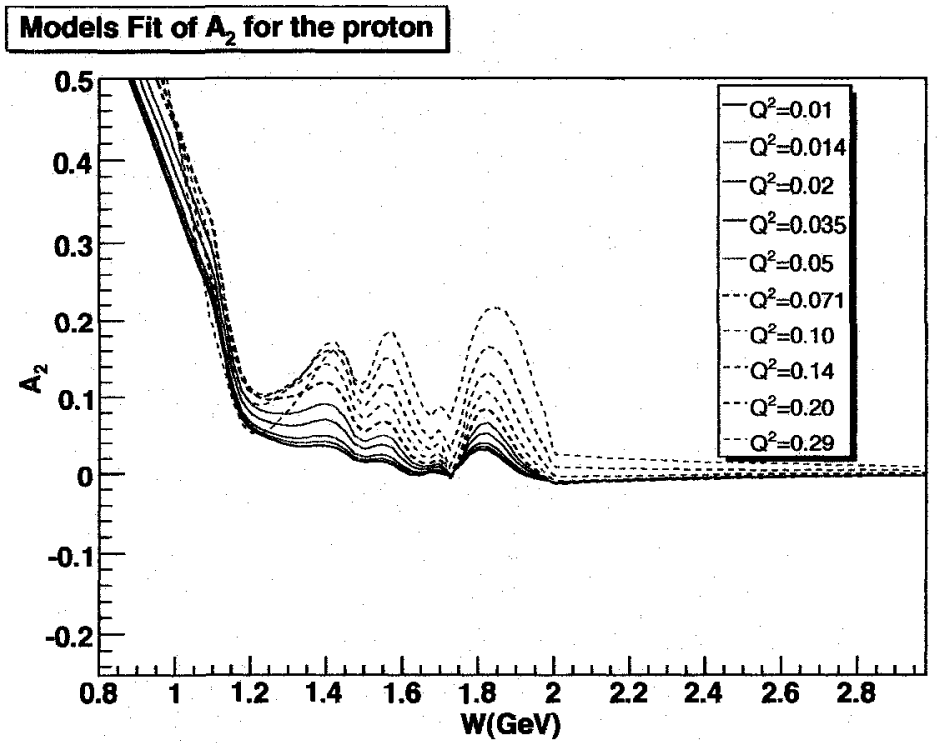

Models Fit of $\mathrm{A}_{\mathbf{2}}$ for the proton

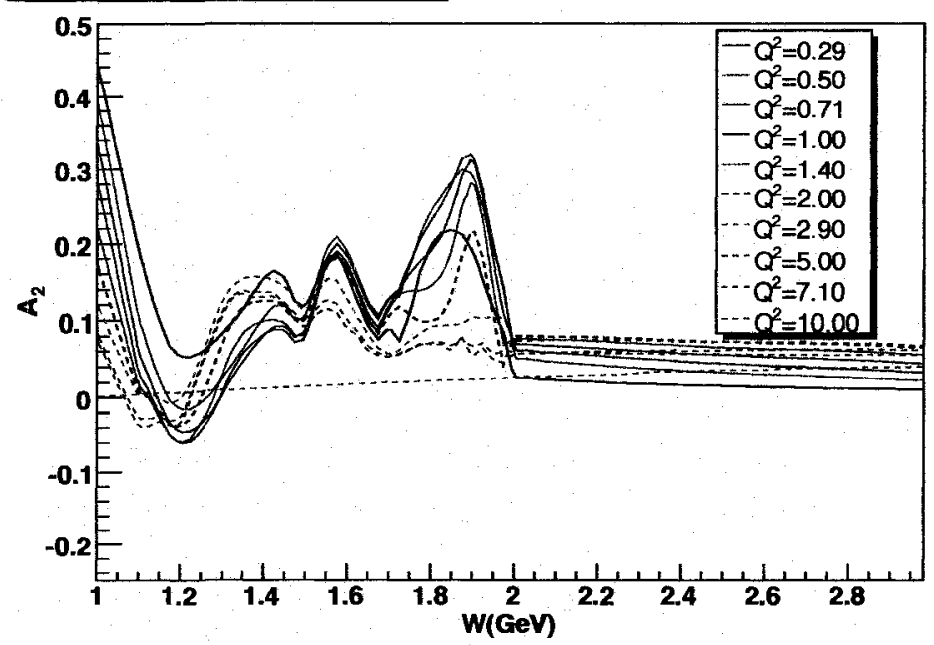

Figure 2.56: Model values of $A_{2}$ [90] for the proton used to extract the $g_{1}$ and $g_{2}$ structure functions in this thesis, shown in terms of missing mass $W$. Each curve represents a different $Q^{2}$ bin. The highest $Q^{2}$ value shown in the top plot is the same as the lowest $Q^{2}$ value shown in the bottom plot. 


\subsubsection{Model for $\sigma_{n} / \sigma_{d}$}

Derivations of the dilution factor in the elastic region rely partially upon the assumption that electron scattering cross-sections of the various target materials are proportional to the number of $n-p$ pairs in the nucleus. Neglecting the small nuclear EMC effect (see Section 2.8 ), this is generally a valid assumption. However, when using ${ }^{12} \mathrm{C}$ to model the ${ }^{15} \mathrm{~N}$ (for background subtraction), we must account for the effects of the unpaired neutron in ${ }^{15} \mathrm{~N}$. This requires knowledge of the ratio of the neutron cross-section $\sigma_{n}$ to that of the deuteron $\sigma_{d}$. For inelastic scattering, Equation 1.75 gives, for the neutron $(n)$ or proton $(p)$,

$$
\sigma_{(n, p)} \propto 2 \sin ^{2}\left(\frac{\theta}{2}\right) F_{1(n, p)}+\frac{M}{\nu} \cos ^{2}\left(\frac{\theta}{2}\right) F_{2(n, p)}
$$

in the inelastic region ( $W \geq 1.07 \mathrm{GeV}$ ). The Rosenbluth formula (Eq. 1.49) gives, for elastic scattering,

$$
\sigma_{n, p} \propto \frac{G_{E(n, p)}^{2}+\tau G_{M(n, p)}^{2}}{1+\tau} \cos ^{2}\left(\frac{\theta}{2}\right)+2 \tau G_{M(n, p)}^{2} \sin ^{2}\left(\frac{\theta}{2}\right)
$$

In both cases, the proportionality constants for the proton and neutron are equal. Neglecting Fermi smearing in the deuteron, we assume

$$
\frac{\sigma_{n}}{\sigma_{d}}=\frac{\sigma_{n}}{\sigma_{n}+\sigma_{p}}
$$

For elastic scattering events, values of $G_{M}$ and $G_{E}$ for the neutron and proton are derived from fits to the world elastic scattering data [14]. For inelastic scattering, the $F_{1}$ and $F_{2}$ models explained in the previous section are used for the proton. The structure function $F_{1}$ for the deuteron is fit to the world data in a similar method as described in the previous section, with $F_{1}$ for the neutron in the DIS region extracted using a specific free nucleon fit form, also developed by $E$. Christy and $P$. Bosted as detailed in Ref. [88].

If we use Eq. 1.79 to rewrite Eq. 2.36 as

$$
\sigma_{(n, p)} \propto\left[2 \sin ^{2}\left(\frac{\theta}{2}\right)-\frac{M}{\nu} \frac{2 x}{1+1 / \tau} \cos ^{2}\left(\frac{\theta}{2}\right)\left(R_{(n, p)}-1\right)\right] F_{1(n, p)}
$$


and make the assumption that the longitudinal to transverse virtual cross-section ratio is approximately equal for all nucleons, that is

$$
R_{(p)} \approx R_{(n)}
$$

then we can see that its contribution to the cross-section ratio is negligible, ${ }^{36}$ so that

$$
\frac{\sigma_{n}}{\sigma_{d}} \approx \frac{F_{1(n)}}{F_{1(n)}+F_{1(p)}}
$$

and $F_{2}$ can be neglected within the systematic bounds of accuracy for this model (see Section 7.2).

\subsection{Radiated nuclear cross-section models}

In order to properly calculate a model for dilution factors (Chapter 5) to remove scattering contributions from background material in the target, it is essential to have good models of the unpolarized cross-sections of the various materials in the target. As shown in Section 2.4, the materials present in the targets that must be modeled are free protons $(p)$ and nitrogen-15 $\left({ }^{15} \mathrm{~N}\right)$ in the ammonia, aluminum (Al) and Kapton $\left(\mathrm{C}_{22} \mathrm{~N}_{2} \mathrm{O}_{5} \mathrm{H}_{10}\right)$ in the target entrance and exit windows, helium-4 $\left({ }^{4} \mathrm{He}\right)$ used to freeze the ammonia, and carbon-12 $\left({ }^{12} \mathrm{C}\right)$, used in a separate cell to determine the total length $L$ of the target + helium and the effective length $\ell_{A}$ of the frozen $\mathrm{NH}_{3}$ target material.

The unpolarized cross-section models used in this thesis are generated by a code developed by P. Bosted, and include radiative corrections using the (now standard) treatment of Mo and Tsai [91]. Modeling radiative effects requires both internal and external corrections; detailed explanation of these radiative corrections is deferred until Section 6.4. A cross-section of the form

$$
\sigma_{\mathrm{TOTAL}}=\sigma_{\mathrm{Bom}}+\sigma_{R C}
$$

is derived for each material compared. Ratios of all the cross-sections involved are required for determination of the material lengths and dilution factors (see Chapter 5).

The Born cross-section $\sigma_{\text {Born }}$ is the basic (tree-level Feynman diagram) scattering cross-section

\footnotetext{
${ }^{36}$ At the large energies involved in inelastic scattering, electric and magnetic modes of the virtual photon are comparable, so that the quantity $(R-1)$ is small, allowing this approximation.
} 

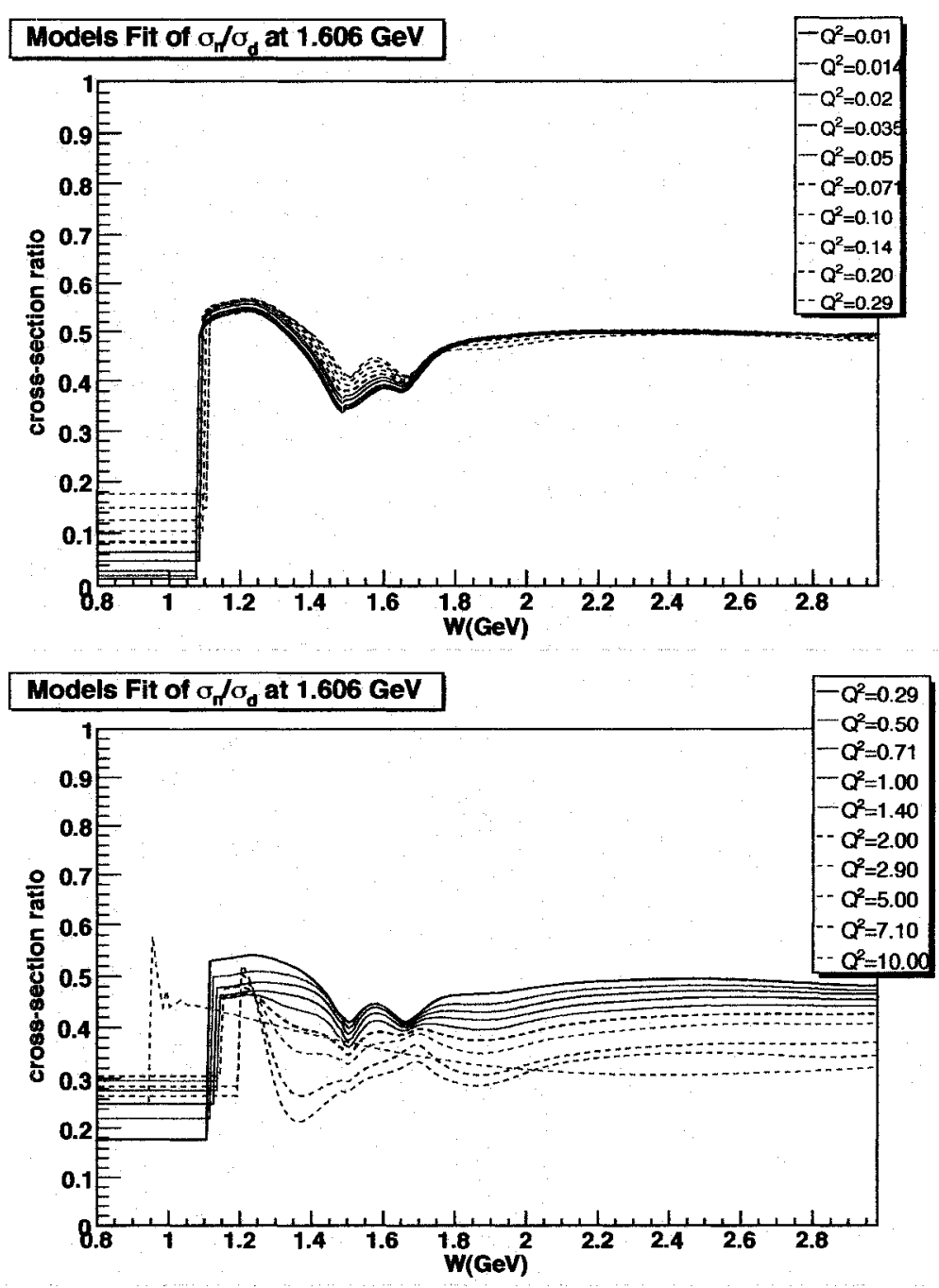

Figure 2.57: Model values of $\sigma_{n} / \sigma_{d}$ [87][88] used in the statistical (bin-by-bin) method of unpolarized background calculation later in this thesis. Each curve represents a different $Q^{2}$ bin. The highest $Q^{2}$ value shown in the top plot are the same as the lowest $Q^{2}$ value shown in the bottom plot. The flat region for $W<1.08 \mathrm{GeV}$ shows the elastic cross-section ratio. All other values are $W$-dependent inelastic ratios. The values depend slightly on beam energy $(E=1.606 \mathrm{GeV}$ in this figure). 
from the nucleon; its value is dependent only on the 3 free inclusive parameters $E, Q^{2}$ and $W$. For the nuclear cross-sections $\left({ }^{4} \mathrm{He},{ }^{12} \mathrm{C},{ }^{15} \mathrm{~N}, \mathrm{Al}\right)$ the effective charge radii of the nuclei was taken from Ref. [92]. To parametrize the quasi-elastic peaks ${ }^{37}$, the formalism of superscaling by Donnelly and Sick [93] is used, in which cross-sections are determined in terms of a kinematic function $\psi$ which gives results independent of $A$ (atomic mass) and the momentum transfer $q$. Specifically, a polynomial fit to the superscaling function of Ref. [94] was used, with binding energies per nucleon $E_{s}$ ranging from 15-20 MeV, and the effective Fermi width parameter $K_{F}$ used in Fermi smearing ranging from $180-260 \mathrm{MeV}$, depending on the nucleon species [95]. This function was fit to both longitudinal and transverse cross-sections in the quasi-elastic region, with values for the $G_{E}$ and $G_{M}$ form factors parametrized in Ref. [13].

For inelastic scattering, a Fermi-convolution of the smearing of free nucleon cross-sections was fit to cross-section data. The fit equation employed a total of 15 free parameters $F_{i}$ and 15 Fermismearing parameters $\eta_{i}$ in the form [95]

$$
\sigma_{A}\left(W, Q^{2}\right)=\sum_{i=1}^{15}\left[Z \sigma_{p}\left(W_{i}^{\prime}, Q^{2}\right)+(A-Z) \sigma_{n}\left(W_{i}^{\prime}, Q^{2}\right)\right] F_{i}
$$

where

$$
\left(W_{i}^{\prime}\right)^{2}=W^{2}+\eta_{i} K_{F}|\bar{q}|-2 E_{s}(\nu+M)
$$

Specific values for all the parameters $\eta_{i}, F_{1}, K_{F}$ and $E_{s}$ can be found in Ref. [95]. Cross-sections for the free proton and deuteron (neutron) were calculated from a fit to the world data of $F_{1}^{p}$ and $F_{2}^{p}$ [87], or $F_{1}^{n}$ and $F_{2}^{n}$ [88], respectively (see Eq. 1.75).

The last factor that needs to be employed in any $A$-dependent nuclear scattering model is the well-known nuclear EMC effect [97], which is, in effect, a deviation from the linear scaling of inelastic scattering cross-section magnitude as a function of $A$. This effect was parametrized using SLAC data [98], ${ }^{38}$ and was assumed to be only $x$-dependent, with no dependence on $Q^{2}$ or beam energy, and assumed constant for $x>0.7$.

\footnotetext{
${ }^{37}$ The term quasi-elastic is used because scattering from events in a bound nucleus at $W \sim 0.938$ GeV can include correlations between nucleons, and thus aren't elastic in the true sense.

${ }^{38}$ This reference contains an empirical fit to scattering data from nuclei in various targets ranging from $A=4$ to $A=197$, specifically measuring the $A$-dependence of cross-sections at SLAC energies.
} 
The radiative correction cross-section $\sigma_{R C}$, unlike $\sigma_{\mathrm{Born}}$, directly depends on the thickness of the material, due to external radiative correction and range staggling effects. In practice, the radiated cross-section for $1 \%$ and $2 \%$ of a radiation length ${ }^{39} X_{0}$ is calculated. The target mass thickness is approximately $1.5 \%$ of a radiation length. The total cross-section $\sigma_{\text {TOTAL }}$ is given simply by interpolating to the radiation length fraction $\rho \ell / X_{0}$ of the material. Note that the radiation length of ${ }^{15} \mathrm{~N}$ is not well known, so it is assumed that $X_{0}\left({ }^{15} \mathrm{~N}\right)=\frac{15}{14} X_{0}\left({ }^{14} \mathrm{~N}\right)$. Radiation lengths of the various target materials are listed in Table 2.3.

Using the formalism for unpolarized cross-sections outlined in Section 6.4, the radiative corrections to each Born cross-section can be found, and using

$$
n \propto \rho \ell \sigma_{\text {TOTAL }}
$$

the model can be used to determine the ratio of counts attributable to unpolarized backgrounds. The probability of external Bremsstrahlung goes as $t d \nu / \nu$, where $\nu$ is the photon energy, and $t$ is the thickness in terms of $X_{0}$. External radiative corrections must take into account the total value of $t$, so precise knowledge of mass thicknesses (in $\mathrm{g} / \mathrm{cm}^{2}$ ) is needed for accurate radiative corrections to unpolarized cross-sections. The following materials are accounted for:

1. Materials along the beam line from the back edge of the target to the scattering center $\left(t_{t a r g}\right)$

2. Materials from the scattering center to the edge of the target along the scattering path $\left(t_{\text {targ }} / \cos \theta\right)$

3. Combined thickness of banjo windows and target cell exit windows (see Section 2.4).

4. All elements external to the target traversed by the scattered electron.

The last of these is an explicit function of $\theta$, and accounts for aluminum in the (inward bowed) target vacuum shield exit window, aluminum in the thermal shields (which increases in thickness at wider angles), layers of superinsulation (for the target solenoid; see Section 2.4), air (outside the vacuum shielding), and the inner layer drift chambers (see Section 2.5). 40 The total mass thickness of

\footnotetext{
${ }^{39} \mathrm{~A}$ radiation length is defined as the amount of a material, usually measured in $\mathrm{g} / \mathrm{cm}^{2}$, necessary to cause a high-energy electron to lose all but 1/e of its original energy; it is characteristic to a given material in terms of $Z$ and $A$.

${ }^{40}$ Of the 3 layers of drift chambers, only the first is considered for radiative corrections. The complex magnetic torus field dominates the second layer (so it is neglected). By the time the third layer is reached, any radiated photons will be emitted in the particle direction, and hence absorbed by the calorimeters, so they are not considered.
} 
these external elements as a function of $\theta$ is shown in Figure 2.58. As one can see, the radiation lengths of these external elements (ranging from $1.1 \%$ to $1.5 \%$ of $X_{0}$ ) are comparable in magnitude to that of the actual target, and therefore need to be accounted for if accurate modeling of the crosssections is to be made.

Table 2.3: Radiation lengths of irradiated materials in the EG1 experiment. Kapton has a very similar cross-section and radiation length to ${ }^{12} \mathrm{C}$, and the latter is used for its cross-section calculations.

\begin{tabular}{|c|c|}
\hline Material & Radiation Length $\left(X_{0}\right)\left(\mathrm{g} / \mathrm{cm}^{2}\right)$ \\
\hline $\mathrm{p}$ (free proton) & 61.28 \\
\hline $\mathrm{d}$ (free deuteron) & 122.4 \\
\hline${ }^{4} \mathrm{He}$ (liquid helium) & 94.32 \\
\hline${ }^{12} \mathrm{C}$ (amorphous carbon) & 42.7 \\
\hline${ }^{15} \mathrm{~N}$ (nitrogen-15) & $37.99 \times \frac{15}{14}=40.70$ \\
\hline $\mathrm{Al}$ (aluminum) & 24.01 \\
\hline
\end{tabular}




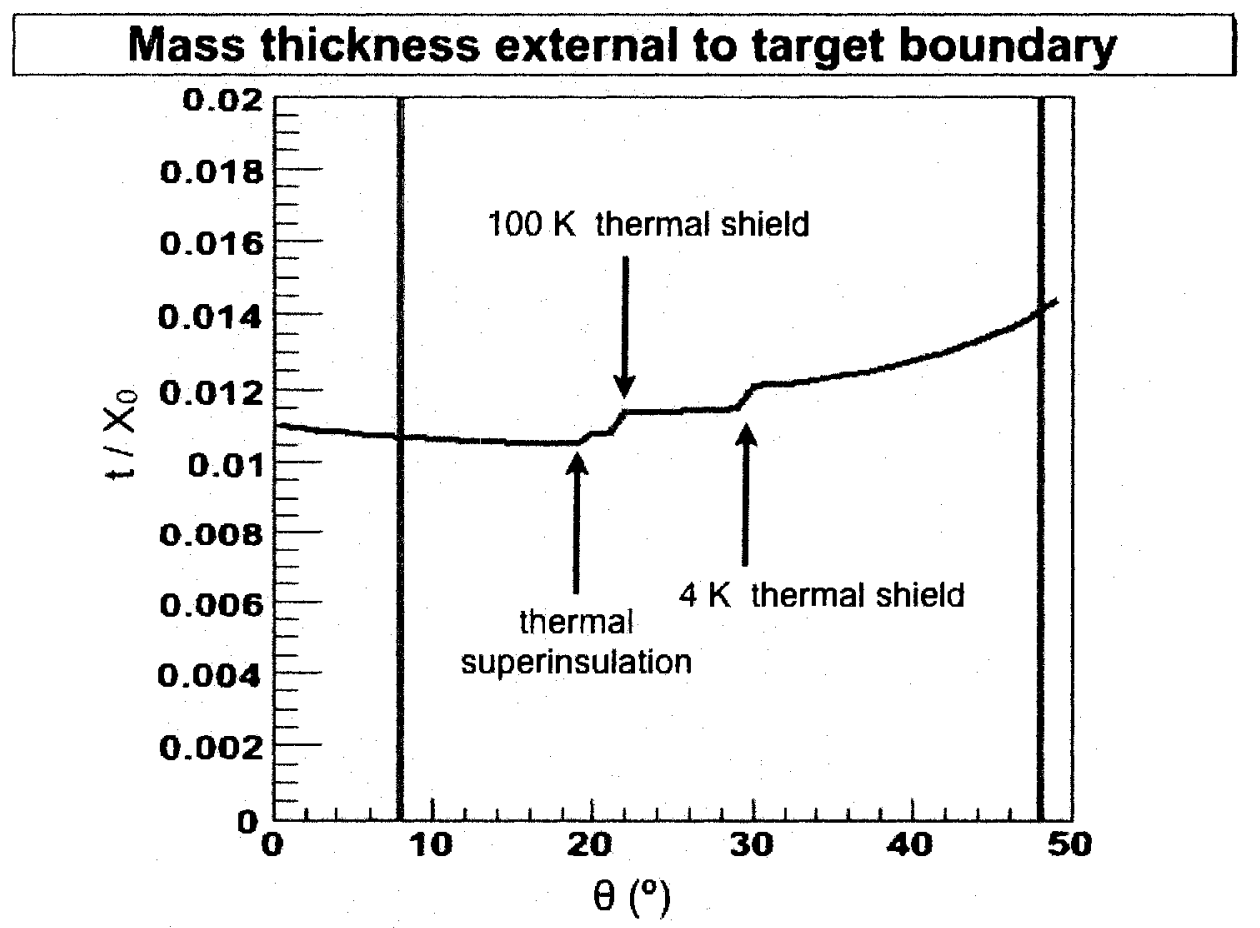

Figure 2.58: Mass thicknesses, expressed as a dimensionless fraction of radiation length $X_{0}$, attributable to materials outside the target (e.g. thermal shields, insulation, air, inner layer DC; that is, item 4 in the text) as a function of $\theta$. Arrows point out reasons for sudden increases in the mass thickness. The approximate limits of acceptance for this experiment are bounded by the vertical red lines. 


\section{Chapter 3}

\section{Helicity and Particle Identification}

\subsection{Reading the Data Summary Tapes (DSTs)}

\subsubsection{DST organization}

All crucial information regarding reconstructed events (produced by the SEB) and their constituent particles is written to Data Summary Tape (DST) files and stored in the permanent storage silos with the other important (raw data, n-Tuple, etc.) files from the EG1 data set. Only information relevant to the analysis of reconstructed events (i.e. kinematics, event- and particle-correlated ADC measurements required for particle identification, Faraday Cup counts, etc.) is recorded in the the DST files, to conserve both analysis time and disk space.

When all calibration and raw data processing were completed, the final versions of the DST files were uploaded from the silo to a semi-permanent 3 terabyte cache disk for easy reading. DST files from viable runs ${ }^{1}$ were then organized in directories by beam energy and torus current (see Table 3.1).

\footnotetext{
1 This includes all sets utilizing a normal electron beam, consistent main torus current and one of the main EG1b targets (either $\mathrm{NH}_{3}, \mathrm{ND}_{3},{ }^{12} \mathrm{C}$, empty (LHe), or frozen ${ }^{15} \mathrm{~N}$ ).
} 
Table 3.1: All EG1b run sets with usable electron beam data, organized by beam energy and torus polarity. These set labels are used throughout this and later chapters.

\begin{tabular}{|c|c|c|c|}
\hline Set Label & Run Number Range(s) & Beam Energy(GeV) & Torus Current(A) \\
\hline \hline $1.6+$ & $25488-25559 ; 25669-26221$ & 1.606 & +1500 \\
\hline $1.6-$ & $26222-26359$ & 1.606 & -1500 \\
\hline $1.7+$ & $28512-28526$ & 1.724 & +1500 \\
\hline $1.7-$ & $27644-27798 ; 28527-28532$ & 1.724 & -1500 \\
\hline $2.3+$ & $27205-27351$ & 2.288 & +1500 \\
\hline $2.5+$ & $28001-28069$ & 2.562 & +1500 \\
\hline $2.5-$ & $27799-27924 ; 27942-27995$ & 2.562 & -1500 \\
\hline $2.8-$ & $27936-27941$ & 2.792 & -1500 \\
\hline $4.2+$ & $28074-28277 ; 28482-28494 ; 28506-28510$ & 4.239 & +2250 \\
\hline $4.2-$ & $28280-28479 ; 28500-28505$ & 4.239 & -2250 \\
\hline $5.6+$ & $27356-27364 ; 27386-27499$ & 5.627 & +2250 \\
\hline $5.6-$ & $27366-27380$ & 5.627 & -2250 \\
\hline $5.7+$ & $27069-27198$ & 5.735 & +2250 \\
\hline $5.73-$ & $26874-27068$ & 5.735 & -2250 \\
\hline $5.76-$ & $26468-26722 ; 26776-26851$ & 5.764 & -2250 \\
\hline
\end{tabular}

\subsubsection{Reading particle data from the DST}

The data in the DST were written in a compressed format using a a FORTRAN-based bit-packing algorithm [99]. Events were written to sequential "buckets" containing a single beam helicity. A C++ program, constructed around a previously used DST bank opening prototype [100], unpacked the compressed data banks and converted the data to numerical arrays. After unpacking and accessing the event-by-event DST data, it was then possible to make kinematic corrections and cuts not included in the SEB or calibration processes. In practice, it was easiest to compress the data to successively more compact forms (beginning with ROOT trees [101]) for quick, successive reading. The basic particle identification cuts and kinematic corrections were implemented in the DST reader, and the output was written to ROOT tree files for temporary storage. More specific cuts and corrections could then be added later, so that various cut options could be applied and tested as needed with relative ease.

The basic purpose of the DST reader was to:

1. Read run, helicity, event and particle information from the DST file. 
2. Truncate unwanted helicity buckets that could potentially lead to biased or erroneous asymmetry measurements (see Section 3.2).

3. Apply basic particle identification (PID) cuts on the scattered electron candidate for each event (see Section 3.4), and cut out pion background (see Section 4.1).

4. Correct momentum and other kinematic quantities for all particles (see Section 4.2).

5. Write the needed electron event data to a ROOT tree file, along with data pertaining to any additional particles in each event. Secondary charged particles in a 2-charged particle event are candidates for an elastically scattered proton, needed for determination of beam $\times$ target polarization $\left(P_{b} P_{t}\right)$ (see Section 6.2.2).

\subsubsection{Tree File Structure}

A ROOT Tree is an array, or n-Tuple, with elements that need not be of the same data type [101]. 2 Each event constitutes an entry in the tree. Each entry has members divided into branches for organization. Separate branches are defined to hold crucial run information (e.g. beam energy, torus current, etc.), event-specific information (e.g. beam raster position, number of particles, helicity information etc.), and additional branches pertain to crucial information for each particle in the event (e.g. $x, y$ and $z$ position and momentum, EC and CC channel data, etc.) Text log files, containing scalars, including total gated and ungated Faraday cup charges, were also produced.

Tree files provide a legible repository of event-by-event data, are much faster to read than the compact DST files, and are useful for the application of cuts requiring refined detail (such as fiducial cuts (Section 4.4) and elastic proton kinematic cuts (Section 3.5)). However, there are still millions of electron events in each run, leading to a tree file size exceeding 2 GB for some runs. ${ }^{3}$

It became clear that more data compression was required for an efficient, repeatable analysis in kinematic bins of $Q^{2}$ vs. $W$. This process is outlined at the end of this chapter in Section 4.6. First, however, details must be provided regarding the basic cuts and corrections made prior to the further compression of data.

\footnotetext{
${ }^{2}$ In fact, they need not even be numbers. $\mathrm{C}++$ and HOOT data objects can also be members of a ROOT tree.

'Low energy outbending runs, in particular, included up to ten times as many reconstructed events as a higher energy inbending run.
} 


\subsection{Removal of Problematic Helicity Buckets}

\subsubsection{Identifying helicity buckets for removal}

The electron beam is longitudinally polarized, with the polarization direction alternating pseudorandomly at a rate of $30 \mathrm{~Hz}$. The events that occur within the $\sim 0.03$ second window are referred to as a helicity bucket. The raw asymmetry is a function of the kinematics, defined as

$$
A=\frac{n^{-}-n^{+}}{n^{-}+n^{+}}
$$

where $n^{+}$and $n^{-}$are the count rates of scattered events in the + and - helicity buckets, respectively. Measurement of asymmetries is the goal of this analysis. Thus, it is very important that the helicity buckets are properly identified. Also, one must be careful not to remove problematic helicity buckets (those containing unreadable data, for example) in a way that biases one of the two helicities; this can also result in a non-physical asymmetry. For this reason, whenever a helicity bucket is labeled for removal, the opposite member of its pair, or complement, is also removed to prevent possible bias in a measured asymmetry.

Helicity buckets alternated pseudorandomly at $30 \mathrm{~Hz}$, and the helicity state information was recorded in two separate data outbank files. ${ }^{4}$ Occasionally, due to detector dead time or other errors, mistakes occured in the recording of helicity buckets. To correct these errors, a synchronization clock signal with exactly double the frequency of the polarization switch was used to identify skipped helicity buckets (see Figures 3.1 and 3.2), and reorder helicity buckets by pair. They could then be labeled according to Table 3.2. Helicity buckets without a complement were deleted from the data.

This makes the identification of unpaired helicity buckets (unpaired because their "partners" failed to write due to dead time or other reasons) very easy, as a bucket labeled 1 should always be followed by a 4 , and a 2 always followed by a 3 . Buckets that do not properly fit into this scheme were flagged with a negative number label for removal.

To ensure that the helicity bucket fittering worked as expected, HeLP (Helicity Pairing) tables

\footnotetext{
${ }^{4}$ These are labeled TGBI and HLS, with the latter taking precedence where the information differs - see Ref. [46], pages $121-123$
} 


\section{Clock Sync Bit}

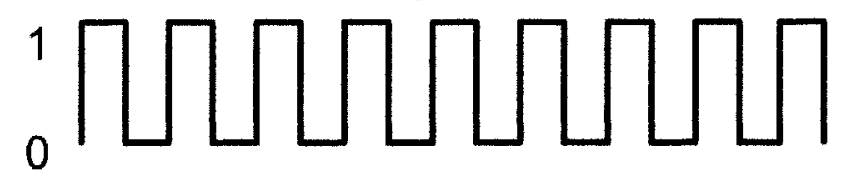

Helicity Bit
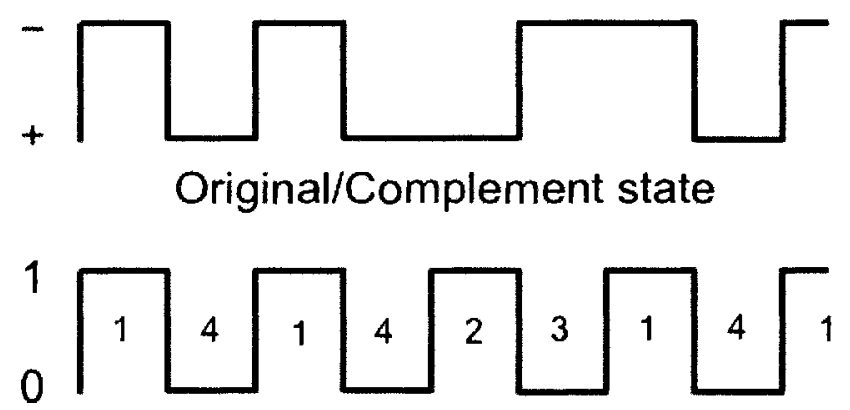

Figure 3.1: The clock sync bit is used to detect a missing helicity bit and arrange the helicity buckets into original/complement pairs labeled 1 through 4 , according to helicity and position in the pair. This aids in identifying bad helicity buckets. (See also Ref. [2], p.97-98.)

Table 3.2: Helicity label definitions.

\begin{tabular}{|c|c|c|}
\hline Label & Polarization & Position in Pair \\
\hline \hline 1 & - & original \\
\hline 2 & + & original \\
\hline 3 & - & complement \\
\hline 4 & + & complement \\
\hline
\end{tabular}




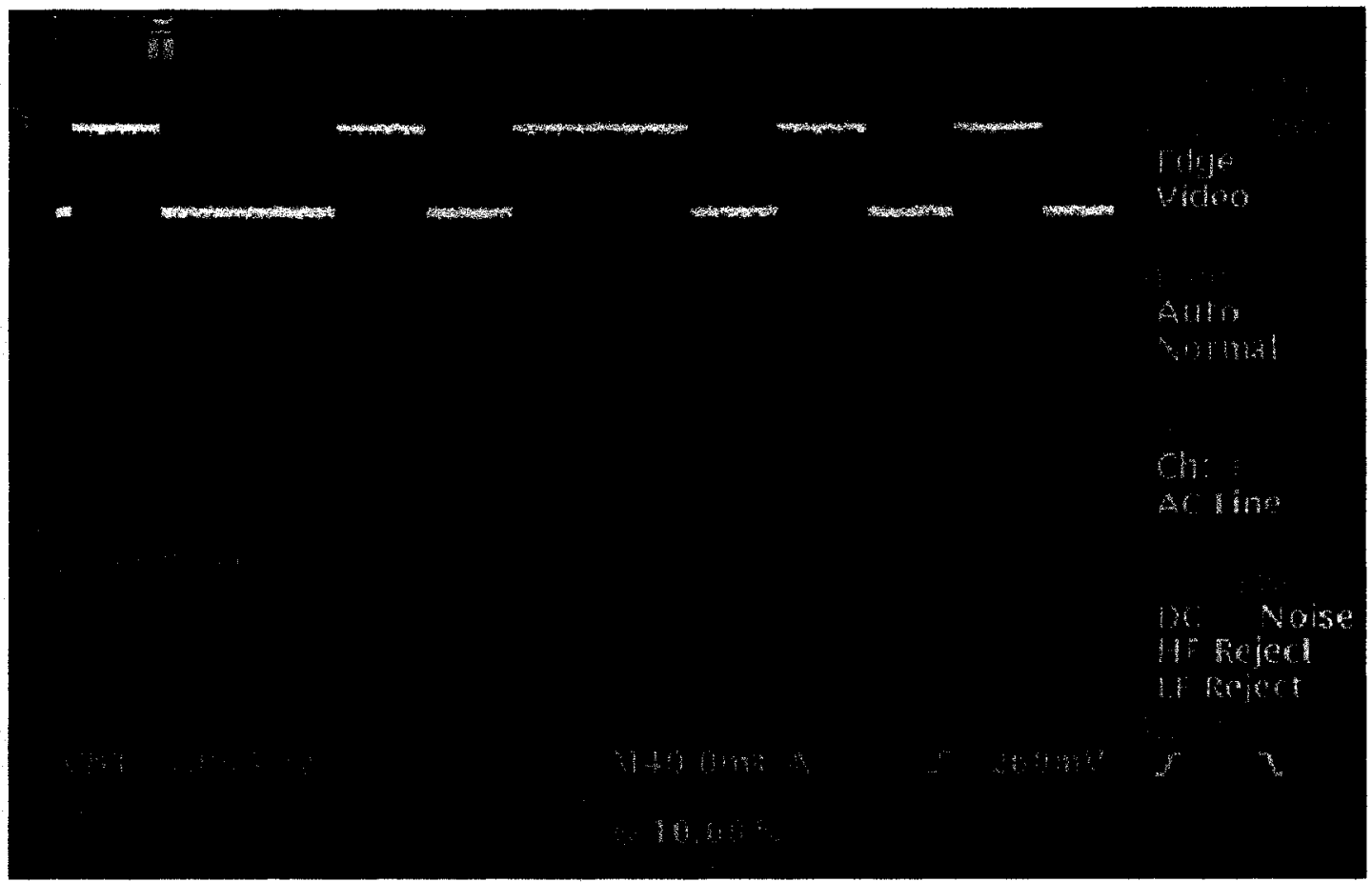

Figure 3.2: Helicity bits monitored online during the EG1b experiment, as explained by Figure 3.1. Helicity bits are shown in yellow (Ch1); and the original/complement states in cyan (Ch2). The magenta (Ch3) shows the triggering of the helicity flips by the clock sync bit. From Ref. [63].

were generated, listing the sequence of helicity labels, polarization states and applied flags (see following subsection).

\subsubsection{Further helicity bucket problems}

In spite of the above precautions, review of the HeLP tables showed that the algorithm used to eliminate unpaired helicity buckets when generating the DSTs did not work perfectly. Namely, the following problems were found:

- Not all unpaired helicity buckets were properly flagged.

- Not all event numbers were properly matched between the two helicity data banks and HeLP tables.

- A possible file closing error generated suspicious-looking helicity labels at the end of every 
Table 3.3: Short excerpt from a HeLP (Helicity Pairing) table. The three (normally) single digit numbers are, from left to right, the helicity label, polarization and status flag, respectively. A status flag of 1 represents a good bin, while negative flags mark helicity buckets that are removed from the

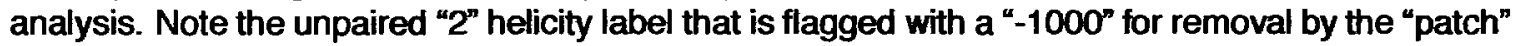
program. This is done to complement the " -500 " (an unreadable helicity), previously flagged for removal in the data banks. The other (6-digit) numbers represent the ranges of reconstructed scattering event numbers (which number around 100-200 for a given bucket in this particular sample). Events numbered in the specified range are assigned the nominal polarization if the flag is equal to 1.

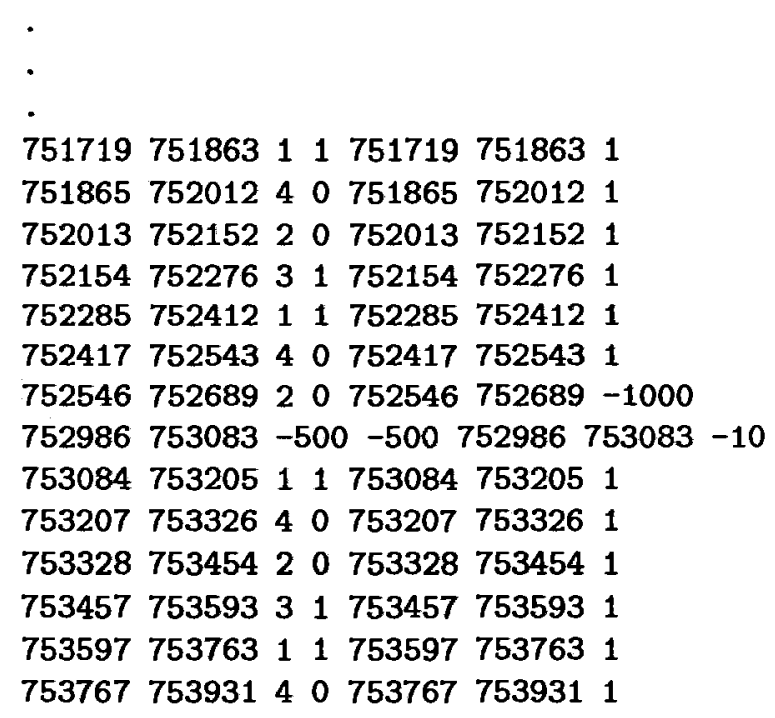

file, possibly corresponding to repeated writing of the same events.

A C++ program and PERL script were written [102] as a patch for the DST reader, which reads the HeLP tables, looks for the above discrepencies, adds appropriate flags ${ }^{5}$ and rewrites the table. This new table is then used as a reference for labeling the helicity states of particles when the DST reader loops through the particle events (see Table 3.3).

Note that these tables determine the relative helicities between events in adjacent helicity buckets, since insertion of the half-wave-plate and energy-dependent spin precession can reverse the meaning of the recorded labels. To determine the absolute helicities (i.e., whether 0,1 represents

\footnotetext{
${ }^{5}$ The last of these three problems is solved simply by flagging all helicity buckets a fixed distance from the end of the file for removal.
} 
$+,-$ or,-+$)$ are determined by checking that the sign of the elastic asymmetry is always positive (see Section 3.3.3).

\subsection{Quality Checks}

There are over 2000 runs in the EG1b data set, but not all of them contain usable data. Runs are categorized by target (either $\mathrm{NH}_{3}, \mathrm{ND}_{3},{ }^{12} \mathrm{C}$, empty, or ${ }^{15} \mathrm{~N}$ ), and removed from the data pool if unusable. Runs used for diagnostic purposes (Møller runs, DAQ tests, cosmic ray runs, etc.) were excluded, as well as runs with problems encountered during data collection (bad beam charge asymmetry, massive DC high voltage trips, loss of target polarization, etc.). In cases where problems only affected part of a run, individual DST data files in the run were systematically checked and kept if possible.

\subsubsection{Beam charge asymmetry check}

The Faraday Cup records the cumulative beam charge incident on the target (Section 2.3.4). It is desirable to have the same amount of beam charge corresponding to both of the beam helicity states, in order to avoid a false asymmetry that might correlate with unknown deviations in the behavior of the Faraday Cup over time. Thus, the total beam charge asymmetry was measured for each file in every run:

$$
A_{\text {beam }}=\frac{F C^{+}-F C^{-}}{F C^{+}+F C^{-}}
$$

where $F C^{ \pm}$is the ungated Faraday Cup counts for $\mathrm{a}+$ or - helicity state. ${ }^{6}$ A rigorous study of the beam charge asymmetry was made [64], finding the expected null result. The study showed that a cut of \pm 0.005 on the beam charge asymmetry removed only obviously anomalous files. Figure 3.3 shows this cut applied to two different runs. Because a beam charge asymmetry affects only the polarized data, this cut was not enforced on unpolarized background subtraction runs (carbon, frozen nitrogen and empty).

\footnotetext{
${ }^{6}$ The ungated charge is desired because the task at hand is the search for biases in the physical beam line and detector, independent of DAQ performance.
} 

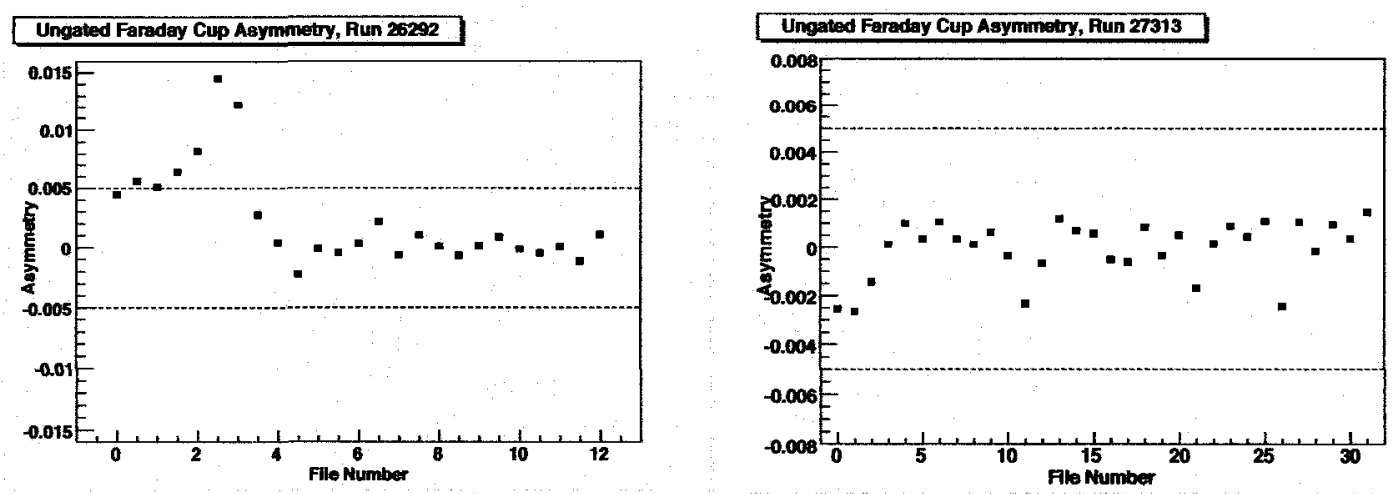

Figure 3.3: Beam charge asymmetries for two two representative runs. Files with asymmetries of greater magnitude than 0.005 were cut, including several of the early files in Run 26292 (left). This is an unusual case; less than $1 \%$ of the total data were excluded by this cut. Run 27313 (right) was a more typical case. (Half-integer file numbers represent data overflow from the previous file exceeding the $2 \mathrm{~GB}$ file size limit.)

\subsubsection{Event rate check}

Measurements of the count rates, normalized by the (livetime gated) Faraday Cup, are useful for checking the quality of the detector data. A sudden change in the count rate of inclusive electrons in an isolated sector can indicate a high voltage trip or detector problem (usually in the DC or EC channels). Uniform drops in count rate across all sectors indicate a change in the target, DAQ or general electronics (e.g. a leak in the target, an unrecorded change in EC thresholds, etc.). Also, because the count rates varies with target type, it is possible to identify an improperly labeled target or Faraday Cup bit (see Section 4.5). Figure 3.4 shows an example of this method of target identification. $^{7}$

Count rates were studied individually for all DST files in every run containing viable data. A change in count rate likely indicates a change in detector acceptance, which does not in any way alter an asymmetry measurement (see Section 1.4). Therefore, no $\mathrm{NH}_{3}$ files were cut from the asymmetry measurement based on count rate. However, for the purposes of determining dilution factors (and other background subtraction), it is important for the acceptance of the $\mathrm{NH}_{3}$ runs to match that of the ${ }^{12} \mathrm{C}$ and $\mathrm{LHe}$ runs.

\footnotetext{
${ }^{7}$ Unfortunately, the software configuration at the time of this experiment necessitated the manual entry of the target label by shift-takers for each run, resulting in many emoneously labeled runs.
} 


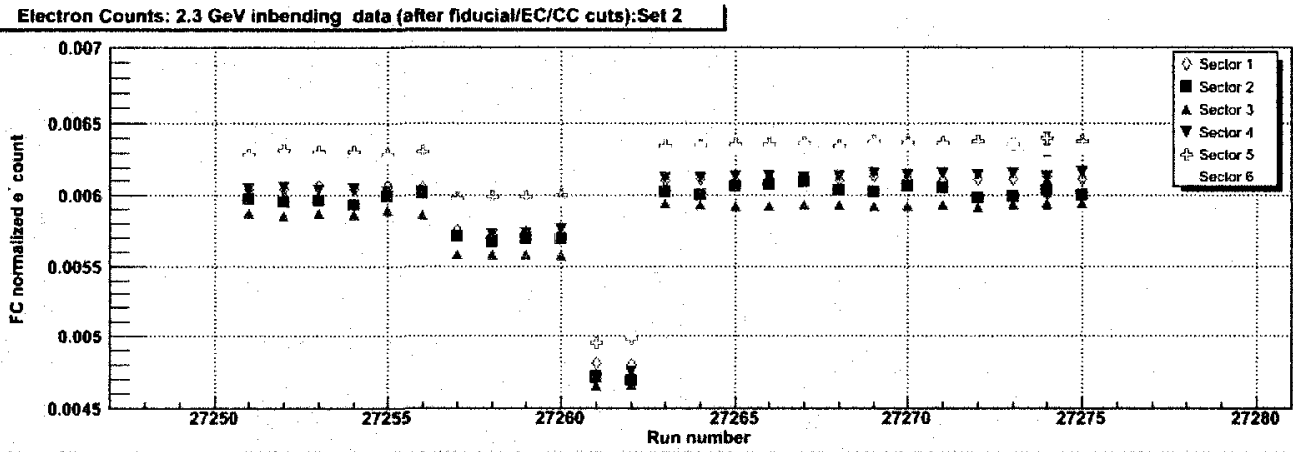

Figure 3.4: Charge-weighted count rates in all 6 sectors for a subset of the $2.3 \mathrm{GeV}$ inbending data. The change in count rates corresponds to differing targets. Most runs are ammonia $\left(\mathrm{NH}_{3}\right)$ with 4 carbon $\left({ }^{12} \mathrm{C}\right)$ runs and 2 empty target $(\mathrm{LHe})$ runs corresponding to the points with obviously lower rates. Plots like this were useful for verifying the target labels. (The LHe target count rates here are plotted before the Faraday Cup bit correction (Section 4.5.2), so these two runs show count rates that are too high by a factor of 2.)

The unpolarized target runs are interspersed throughout the experiment, so that long-term changes in the behavior of the detector affect all data equally (see Section 2.4). However, shortterm changes (due to HV trips, for example) can lead to a change in the count ratio bewteen targets, causing an error in the dilution factor. For this reason, the count rates of every file in every run were measured for each individual sector. Files with a count rate (in any one or more of the six sectors) outside $a \pm 5 \%$ limit relative to the count rate average for a given target in a given set were cut from the files used to calculate dilution factors and other background. This limit was kept in place for the lower energy (1.x and 2.x GeV) runs. The higher energy runs, however, showed greater statistical fluctuations in their count rate, ${ }^{8}$ so the threshold for the higher energies (4.x and 5.x GeV) was increased to $\pm 8 \%$ (see Figure 3.5 ).

Whenever there was a prolonged change in the file count rate, an effort was made to scrutinize the online electronic logbook [63] for the cause. Drops in count rate caused by acceptance changes could then be removed with confidence. Changing rates caused by target refills (a particularly prevalent issue with the $2.5 \mathrm{GeV}$ outbending data) did not warrant removal of data. ${ }^{9}$ In virtually

\footnotetext{
${ }^{8}$ This is due to a greater spread in the momentum of inclusive electrons from scattering processes.

9 Instead, the averaged value of the count rate and resulting ammonia target length was incorporated into the dilution factor tor this case. Because this change in count rate actually conresponds to a change in the physical composition of the target, the change needs to be incorporated into the overall dilution factor. In the case where target refills caused a count rate change, the average rate was calculated for each segment of nuns between the target refills, and the $\pm 5 \%$ limit was entorced for each of these subsets individually.
} 


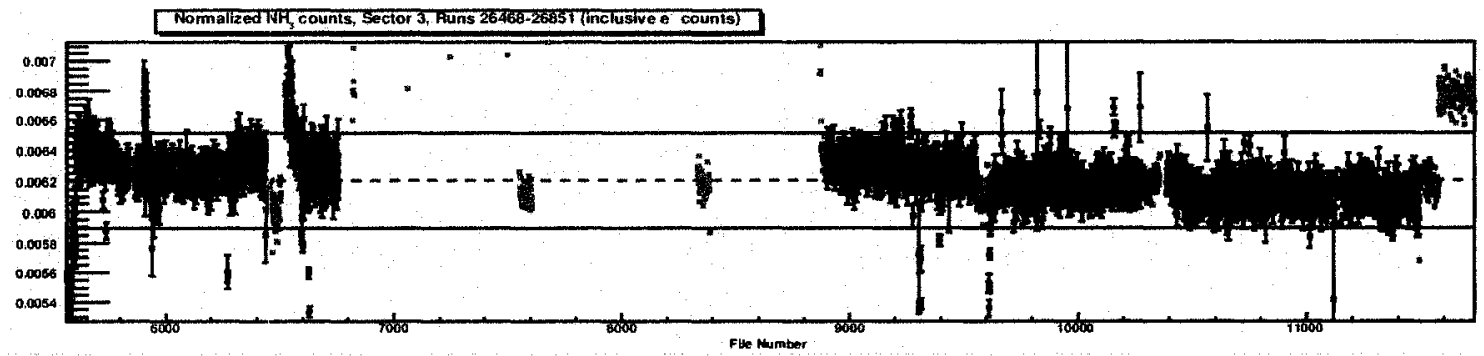

Figure 3.5: A sample count rate check in Sector 3 for $5.76 \mathrm{GeV}$ outbending $\mathrm{NH}_{3}$ runs (highlighted in blue). The dotted line is the average count rate; files outside the range of the solid lines (denoting the $\pm 8 \%$ cut limit) are removed from the dilution factor and background calculation data (but still kept for asymmetry measurement). Gray points represent files for other targets (analyzed separately). Note that the scale is selected to show details in the fluctuations.

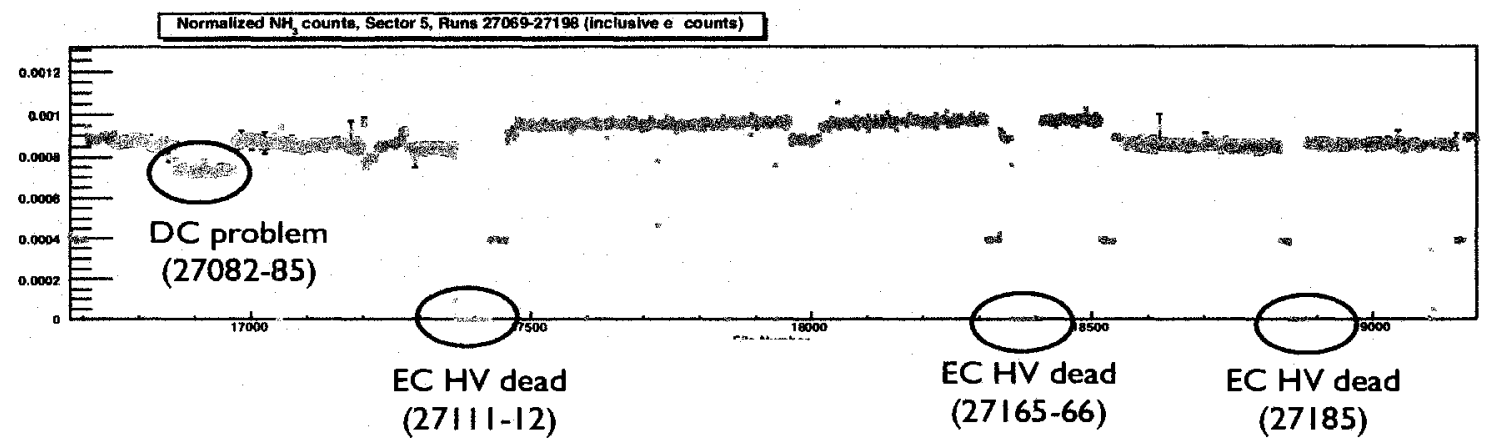

Figure 3.6: A example of the identification of data collection problems for $5.7 \mathrm{GeV}$ inbending data in sector 5 . The online logbook was used to correlate the problems with recorded issues during data collection. FC-weighted count rates are shown. Magenta-highlighted data are $\mathrm{NH}_{3}$ runs.

all cases where the count rate dropped suddenly in a sector for prolonged time periods, the reason was clearly identified, and the proper course of action was taken (Figure 3.6).

\subsubsection{Target polarization and half-wave plate check}

Measurement of the double-spin asymmetry is dependent on the relative longitudinal polarization direction between the beam and the target. The beam polarization flips approximately every 30 ms (Section 2.2); great care has already been taken that "buckets" of alternating beam helicity are symmetrically labeled (Section 3.2). The constant alternating of this helicity prevents any bias in the double-spin asymmetry resulting from acceptance changes over time. The half-wave plate (which reverses the beam helicity states) is also periodically inserted into the beam injection line (Section 
2.2.1) to rule out any possible false asymmetries dependent the absolute polarity. The target polarization remains constant throughout periods lasting several runs (see Section 2.4), but is also periodically reversed to minimize systematic errors from single-spin scattering or inherent detector asymmetries.

Unfortunately, the half-wave-plate (HWP) status and target polarization were not always recorded correctly in the DST. ${ }^{10}$ To further complicate matters, electron spin precession in the recirculation arcs causes multiple reversals of the beam polarity within the accelerator. This phenomenon is beam-energy dependent, meaning some energy sets require a factor of -1 on the double-spin asymmetry, in addition to the HWP and target polarization sign corrections.

Fortunately, the elastic double-spin asymmetry is known to be positive by definition (Section 1.4). This fact, along with the information recorded in the online logbook [63], was used to resolve ambiguities in the HWP status, target polarization and spin precession factor. For each individual DST file, the inclusive double-spin asymmetry (Eq. 3.1) was measured in the elastic region only. This region was defined by a cut on $W$ depending on the elastic peak resolution for the given beam energy (found later in Table 6.4). If all three of the above Boolean $(+/-)$ variables are properly defined, the asymmetry should be positive (or, equivalentty, the raw elastic assymetry (corrected only for spin precession) multiplied by the HWP status should have the same sign as the target polarization - see Figure 3.7). If the asymmetry is not positive, one or more of these variables must be fixed in the database, usually based on a careful reading of the online logbook.

Once these factors are correctly determined, the corrected value of the raw asymmetry evaluated henceforth is given by

$$
A_{\text {raw (corr) }}=A_{\text {raw }} \times f_{\text {targ }} \times f_{\mathrm{HWP}} \times f_{\text {linac }}
$$

where the factors $f$ are the corrective values $( \pm 1)$ for the target polarization, HWP status and electron spin precession.

Ultimately, a reference table was compiled containing correct target labels, HWP status, target polarization, spin precession factor, and Faraday cup charge corrections after a check of all runs.

\footnotetext{
${ }^{10}$ Typically, the polarization reading was only faulty at the experiment start (1.6+ data) and during an NMR readout failure in the 2.3+ set, but the recorded hali-wave-plate status was often incorrect throughout the whole experiment.
} 

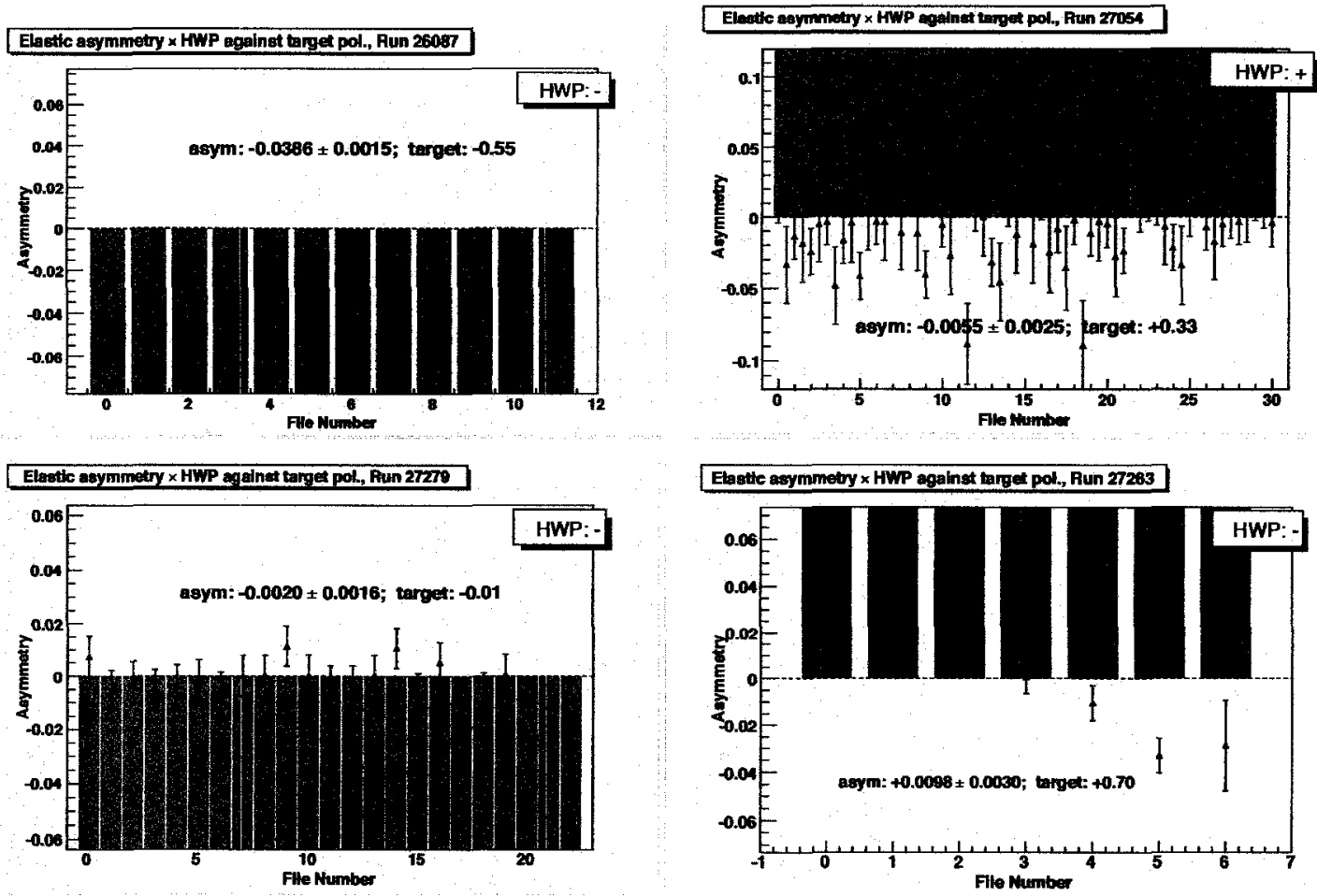

Figure 3.7: Plots showing the raw elastic asymmetry times HWP, against the target polarization sign (colored background). Also shown are the averaged asymmetry value and NMR target polarization readout. Each color represents a different target. The first plot shows the correct HWP and target sign for an $\mathrm{NH}_{3}$ run (indicated by cyan); note that the HWP corrected asymmetry matches the target sign (negative). The second plot shows an $\mathrm{ND}_{3}$ run (magenta) for $5.7 \mathrm{GeV}$ with the wrong HWP sign (note the HWP-corrected asymmetry does not match the target polarization). Asymmetry signs for $\mathrm{ND}_{3}$, especially at high energy, were the most difficult case to discern, but were necessary to know to put together a complete experimental history. The third plot is a carbon target (red) with no discernable elastic asymmetry. The recorded target polarization for these (and other unpolarized) runs was changed to zero. The last plot shows an $\mathrm{NH}_{3}$ run that reversed target polarization during the middle of a run due to a polarized target malfunction; this run was removed from the analysis. 


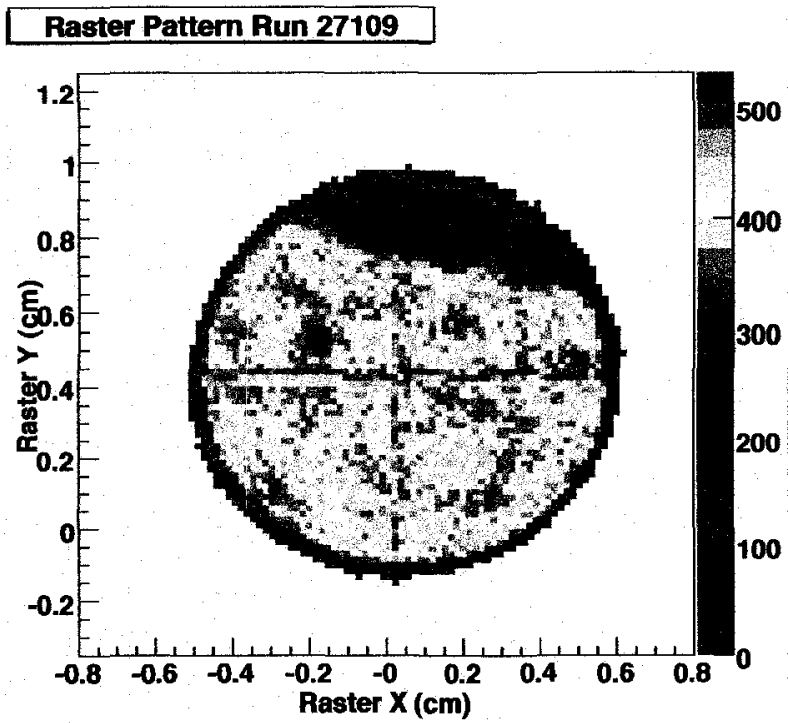

Figure 3.8: Histogram of event rate density over different regions of the beam raster. The blue area at the top is an area of lower event density, most likely caused by settling of the frozen $\mathrm{NH}_{3}$ suspension in the surrounding LHe. The 'crosshair' pattern is an anomaly caused by a bug in the null ADC channels, and has no physical meaning.

\subsubsection{Raster pattern check}

Another useful test for checking file quality is the plotting of the number of events as a function of raster coordinate position, as is shown in Figure 3.8. Inhomogeneities in these plots were then carefully scrutinized to determine if they were inconsequential to the analysis (like the area of lower rate seen in Figure 3.8), or whether they indicated a possible problem with the run. In general, a lower count rate area (particularly near the top of the pattern) indicates "settling" of the target material, and does not affect overall packing fraction or consequent dilution factors/asymmetry, while an area of elevated count rate (particularly a crescent-shaped "hot spot") indicates the electron beam scraping the target edge or other interfering material in the target. These runs must be checked for further problems, then either be removed or corrected as necessary. Few runs exhibited problems requiring further investigation, but two specific recurring cases warrant mentioning here.

Two separate target inserts were used in EG1b (Section 2.4), one containing the main $\left(\mathrm{NH}_{3}\right.$ and $\mathrm{ND}_{3}$ ) targets, and the other the frozen ${ }^{15} \mathrm{~N}$ target. Both inserts contained a ${ }^{12} \mathrm{C}$ target. All carbon run raster patterns in the first (of two) ${ }^{15} \mathrm{~N} /{ }^{12} \mathrm{C}$ "mini-experiments" (runs 27326-27380) showed a 
strange 'crescent' shape of unknown origin (Figure 3.9). A comparison was made between the count rates in the top half vs. the bottom half of the target. By application of the same model used for packing fraction calculation (Section 5.5.2, applied to the carbon instead of ammonia target), it was determined that the rate difference corresponded to no more than a $2 \%$ error in the overall effective carbon target length, so the runs were kept, accounting for this systematic error [95].

Of greater concern was a raster pattern anomaly that appears in all empty target (LHe only) runs, beginning at Run 27899, persisting through the end of the EG1b experiment. The start of the problem correlates to the identification of a leak in the $\mathrm{ND}_{3}$ target during the $2.5 \mathrm{GeV}$ outbending set. The crescent-shaped "hot spot" that persists in the empty target thereafter (see Figure 3.10) likely results from a wire or other debris in the window path after the disturbing of the target. 11 The empty ( $\mathrm{LHe}$ ) target is of crucial importance to the measurement of the total target length $L$, needed for unpolarized background subtraction. The special correction required to this target data is discussed later, in Chapter 5.

\subsubsection{Inclusive $W$-spectrum check}

Due to the beam energy dependence of the elastic peak prominence, it was sometimes difficult to identify the difference between ammonia and carbon targets based on an overall inclusive count rate. Also, there were occasionally slight changes in count rate within a particular target type (due to target leakage or EC threshold change, for example) that warranted closer inspection.

As a final check on overall run quality, the spectrum of each run, in terms of invariant mass $W$, was checked for the presence of any obvious problems (see Figure 3.11). Where integrated inclusive count rates between differing ammonia and/or carbon targets are similar, the identification of an elastic peak at $W=0.938 \mathrm{GeV}$ easily distinguishes these cases. In the case of a varying $\mathrm{EC}$ threshold, a slight shift in the high- $W$ limit was noticed. Runs with the differing count rate ${ }^{12}$ were excluded from dilution factor analysis (but still kept for asymmetry measurements).

\footnotetext{
${ }^{11}$ An analysis of the "hot spot" indicated no sign of an elastic proton peak, so it is not frozen ammonia or trozen $\mathrm{H}_{2} \mathrm{O}$ contamination.

${ }^{12}$ This indicates a change in the detection threshold at low momenta, occurring in the early part of the 2.3+ data. The effect diminished when a reasonable low-momentum cut was implemented.
} 

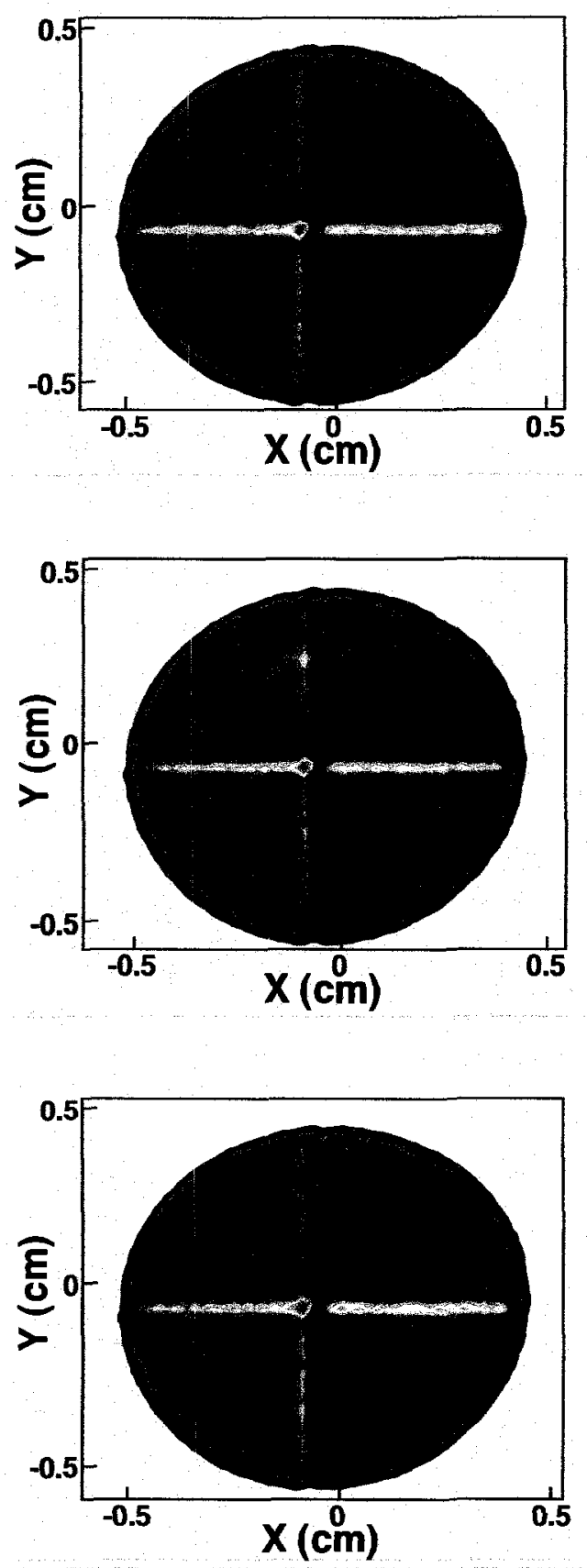

Figure 3.9: Raster pattern quality checks for three different runs. The top pattern shows the (good) raster pattern from a ${ }^{12} \mathrm{C}$ run on the main (ammonia) target insert (Run 27313). The middle pattern shows the problematic raster pattern common to ${ }^{12} \mathrm{C}$ runs on the nitrogen/carbon target insert, in the first of these special run sets (Run 27340 shown). The third pattern shows a nearby ${ }^{15} \mathrm{~N}$ raster pattern (Run 27342), indicating this problem affected only the carbon runs, not the whole target insert. This figure is also found in Ref. [95]. 


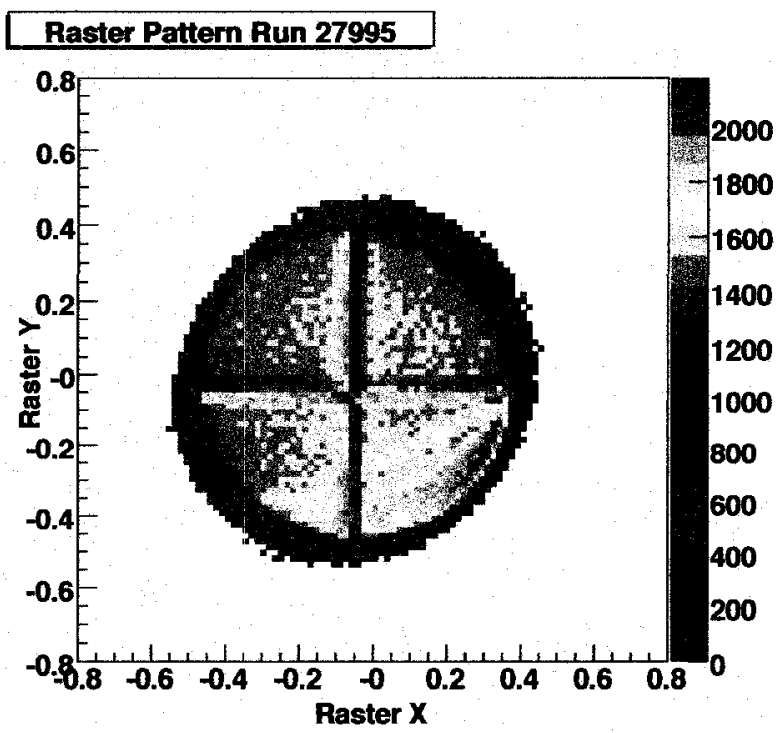

Figure 3.10: Raster pattern for an empty (LHe) target run in the latter part of the EG1b run set. The elevated count rate in the lower right indicates a problem of unknown origin.

\subsubsection{Miscellaneous issues}

Naturally, given the large number of variations that occur during an experiment of this duration, many issues arise that cannot be encompassed by a simple set of well-defined rules for inclusion and exclusion of data. Where cases involving selection based on quantifiable measurements occurred (i.e. beam charge asymmetry, count rates, etc.), data were cut according to strict criteria unrelated to the douple-spin asymmetry, so as to avoid introducing any unintentional bias into the analysis.

Scrutiny of the online logbooks revealed many underlying problems with certain runs that usually, but not always, correlated to one or more of the previously described quality check violations. Obviously problematic runs were removed from all analysis. Problems explicitly labeled in the logbook included ${ }^{13}$

- Experiment not ready (all runs prior to 25747)

- DAQ problems $(26275,26230,28388)$

\footnotetext{
${ }^{13}$ This list is not by any means comprehensive, and is intended only to serve as an example of problems encountered during data collection.
} 

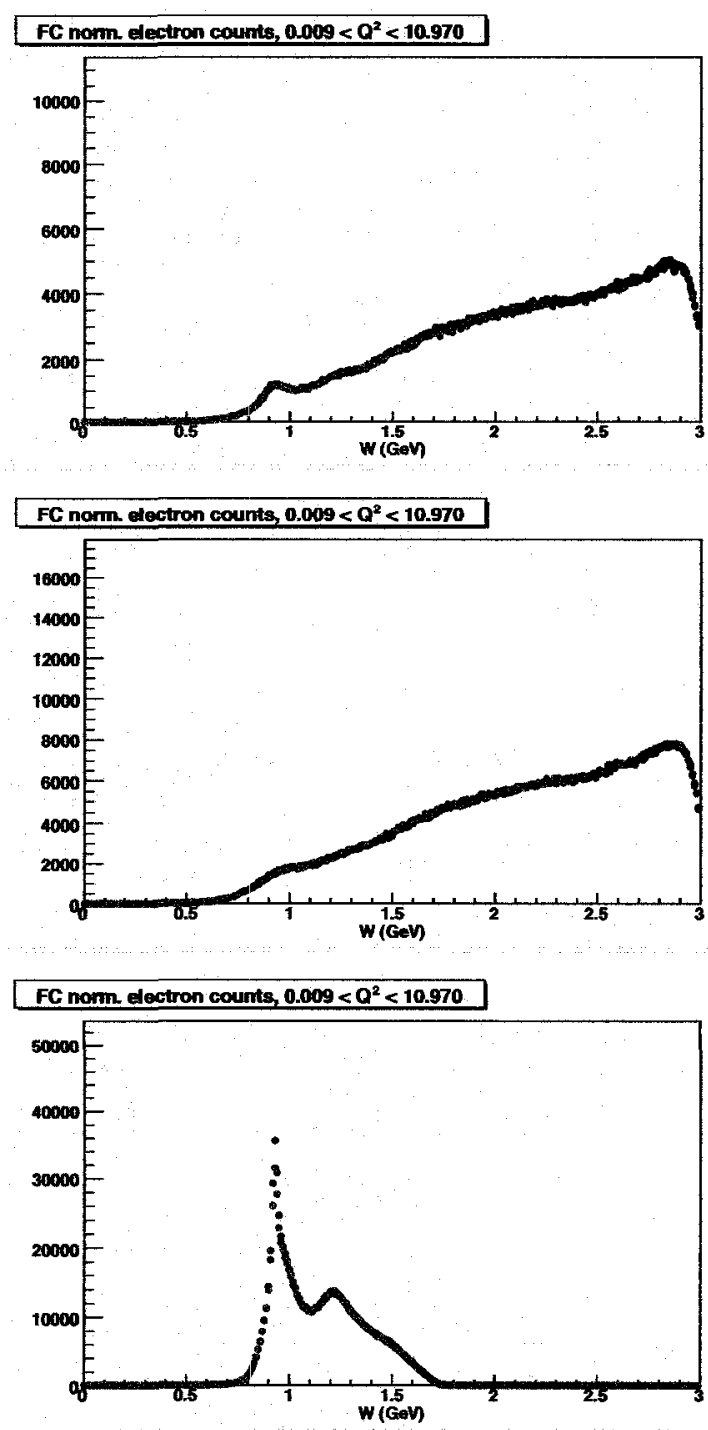

Figure 3.11: $W$-spectra check for three EG1b runs. The blue and red represent positive and negative relative polarization inclusive counts, respectively; these generally overlap, in the shown plots. The top two diagrams show the spectra of two consecutive runs $(26720,26721)$, the top $\mathrm{NH}_{3}$ and the middle ${ }^{12} \mathrm{C}$. Note the difference in the (quasi)elastic peak $(W \sim 0.938 \mathrm{GeV})$ region. These two runs are high-energy $(5.76 \mathrm{GeV})$ runs. Low energy runs have a much more prominent elastic peak (bottom diagram, run $25790,1.6 \mathrm{GeV}$ ). Note the obvious asymmetry between the polarization states in the elastic region at the lower beam energy. 
- Torus current malfunction (26723-26775)

- Large number of HV trips (26506)

- Beam off target (26558-26559, 27835, 26903-26924)

- Target irradiation/annealing not completed (26842)

- Bad EC thresholds (27490)

- Bad CC threshold (28310)

- Incorrect pedestal values (28343-28349)

- Thresholds set for wrong energy (28513-28521)

- Sudden target polarization loss/change (25802,27263)

- Beam helicity labels changed during run (25888)

- Wrong DAQ configuration file (26510-26522,27123)

- Target not yet polarized (26036)

- Changed target during run (26178)

- Wrong size raster and thresholds (26468-26480)

- Time-of-flight malfunction in 3 sectors (27399)

- Missing EC, CC channels (27445)

- Target only half full $(27803-27819 ; 27821)$

- Beam energy $200 \mathrm{MeV}$ higher than rest of set (27933-27941)

- HWP inserted during run (28324)

- Wrong beam energy used in SEB reconstruction $(28415,28444)$ 
In addition, all runs containing 2 or fewer raw data files ${ }^{14}$ were also removed, regardless of documentation. These runs compose only a very small minority of the data, and runs were usually stopped this quickly only if a problem occurred.

\subsubsection{Data Organization and Tests of Quality Checks}

After comprehensive lists of good files for the cases of asymmetry measurement and background removal were organized, directories containing soft links to the cached files were created. One was (labeled RATE) for files of all targets used for background removal (including cuts on the inclusive count rates), and another (labeled ASYM/ linked only to frozen ammonia target runs considered good for asymmetry measurements (no count rate cuts included).

As a final check, tests on the overall asymmetry sign (Figure 3.7) were rerun using corrected polarizations. Also, count rate checks for complete runs (Figure 3.4) were redone to check for correct target labeling and rate cuts.

With confidence that only high-quality data are referenced in the database, and all preliminary particle identification cuts and kinematic corrections in place, analysis then proceeded to the next steps.

\subsection{Electron Identification}

The SEB labels particles by their time-of-flight, charge and momentum, mainly as determined by the scintillation counter (SC) and drift chamber (DC) detectors (see Section 2.6.2). However, it is difficult to distinguish light hadrons (especially $\pi^{-}$) from electrons simply by their trajectory and flight time, as, at GeV energies, their time-of-flight distributions overlap, especially at forward scattering angles [30]. Fortunately, the interaction of hadrons with matter differs significantly from that of electrons. This fact is exploited by the Cherenkov counters (CC) and electromagnetic calorimeters (EC) to make a basic preliminary particle identification (PID) for the DST reader to utilize as a starting point in identifying the electron and its associated particles. In addition to the requirement that the charge $q=-1$ and $\beta \equiv v / c=1$ (a good approximation at GeV beam energies), limits

\footnotetext{
${ }^{14}$ Complete runs typically contained around 20 raw data files.
} 
are set on the values of the ADC signals associated with the $E C$ and $C C$ for the particle to be considered an electron candidate.

\subsubsection{Cherenkov Counter Cuts}

As previously noted, the Cherenkov Counter is used to distinguish electrons from hadrons by counting the radiated photons as a superluminal particle travels through a gas (see Section 2.5.3). The rate of photon production (with respect to distance traveled, $x$ ) of a negatively charged particle due to Cherenkov radiation can be calculated to be [8]

$$
\frac{d^{2} N}{d \lambda d x}=\frac{2 \pi \alpha}{\lambda^{2}}\left(1-\frac{1}{\beta^{2} n^{2}(\lambda)}\right)
$$

where $\alpha=\frac{1}{137}$ is the fine structure constant, $\lambda$ is the wavelength of the emitted light, and $n(\lambda)$ is the refractive index of the medium.

At the 1-6 GeV energies of Jefferson Lab, electrons can safely be considered ultrarelativistic $(\beta \approx 1)$. Pions, however, have velocities considerably smaller than $c$. Therefore, one expects a smaller number of Cherenkov photoelectrons for pions than for electrons. Recall from Section 2.5.3 that particles do not emit Cherenkov light unless $\beta>c / n(\lambda)$; this condition is not met for pions below a momentum of $2.7 \mathrm{GeV}$, making the $\mathrm{CC}$ the ideal tool for separating low momentum pions from electrons. Statistical (Poisson) distributions of measured photoelectrons are expected. ${ }^{15}$ Specifically, we observe two approximately overlapping Poisson distributions with different peaks corresponding to pion and electron events, with pions having a sharper, lower peak, consisting mostly of sub-threshold noise. Figure 3.12 shows these distributions.

A minimum of a 2.0 photoelectron signal was required for an electron candidate at momenta of less than $3.0 \mathrm{GeV}$. This removes a large number of pions from the inclusive electron candidates, but not all of them, of course, as the upper tail of the Poisson distribution trails under the electron distribution. At higher momenta, where $\beta \rightarrow 1$ for both pions and electrons, it is not possible to segregate the electrons and pions in this manner without incurring a very high electron loss, as they mix heavily (see lower figure). Therefore, at momenta greater than $3.0 \mathrm{GeV}$, a very low cut of

\footnotetext{
${ }^{15}$ Poisson distributions are generally expected whenever a phenomenon with a constant expected number of events per unit time is measured during a sufficiently high number of observations [103].
} 
0.5 photoelectrons is used, ${ }^{16}$ and other methods, outlined in the following sections, are relied upon to prevent pion contamination.

\subsubsection{Electromagnetic Calorimeter Cuts}

The Electromagnetic Calorimeter (EC) separates electrons from pions by the differentiation of minimum ionizing particles (MIPs), which include high-energy pions (and other hadrons), from electrons, through their differing interactions with matter.

Pions, with a mass of $\sim 140 \mathrm{MeV}$, are considerably more massive than electrons ( $\sim 0.5 \mathrm{MeV})$. The primary mechanism of energy loss for pions is through collisions with heavy nuclei. Highenergy pions obey the Bethe-Bloch formula of energy loss (see Eq. 4.25). According to this formula, the energy loss rate $d E / d x$ of a heavy charged particle decreases with increasing energy and reaches a minimum at $\beta \approx 0.96$. At speeds higher than this, it begins to increase again, but only very gradually, never again achieving the energy loss rate exhibited at lower energies [8]. ${ }^{17}$ Past this threshold of minimum energy loss, particles are referred to as minimum ionizing.

At energies in the GeV range, pions can safely be considered to be minimum ionizing. Electrons, on the other hand, cannot be considered MIPs, due to the fact that the energy loss mechanism governing electrons is very different than that for heavier particles, due to their much higher charge-to-mass ratio. High-energy electrons, even at energies as low as $100 \mathrm{MeV}$, lose energy primarily through Bremsstrahlung radiation (i.e. photon radiation emitted due to negative acceleration of the charge) resulting in subsequent electron/positron pair production. The photon emission probability varies as the inverse square of the particle mass [8]

$$
\sigma \propto \frac{e^{2}}{\left(m c^{2}\right)^{2}}
$$

meaning photon radiation loss by electrons is almost $\mathbf{8 0 0 0 0}$ times as intense as from pions. The energy loss rate $d E / d x$ varies considerably with the energy of the electron, in contrast to the nearly constant energy loss rate of MIPs [8].

\footnotetext{
${ }^{16}$ Below this value, PMT and other internal noise dominates the $\mathrm{CC}$ response.

${ }^{17}$ When energies near $100 \mathrm{GeV}$ are reached, Bremsstrahlung radiation becomes significant; but this is far beyond the energy range encountered at Jefferson Lab
} 


\section{Photoelectron Signal $(p<3 \mathrm{GeV})$}

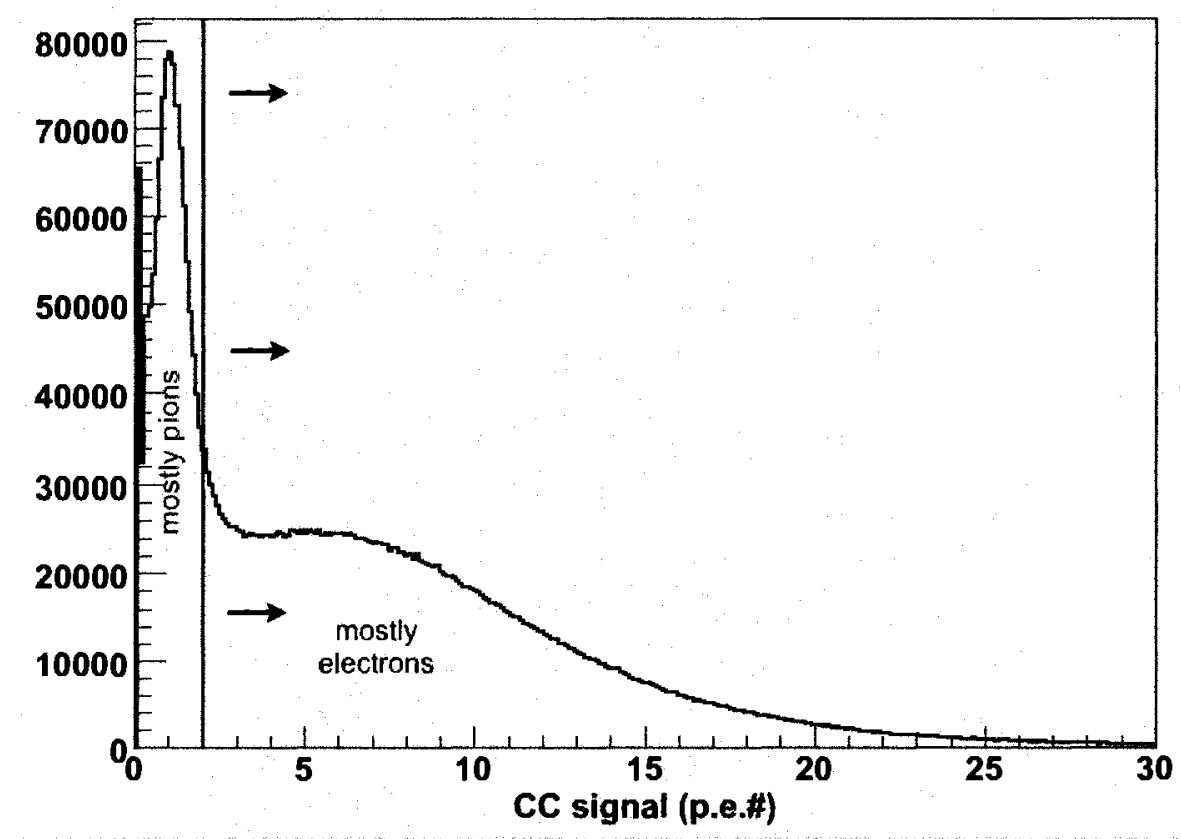

CC Photoelectron Signal ( $p>3 \mathrm{GeV}$ )

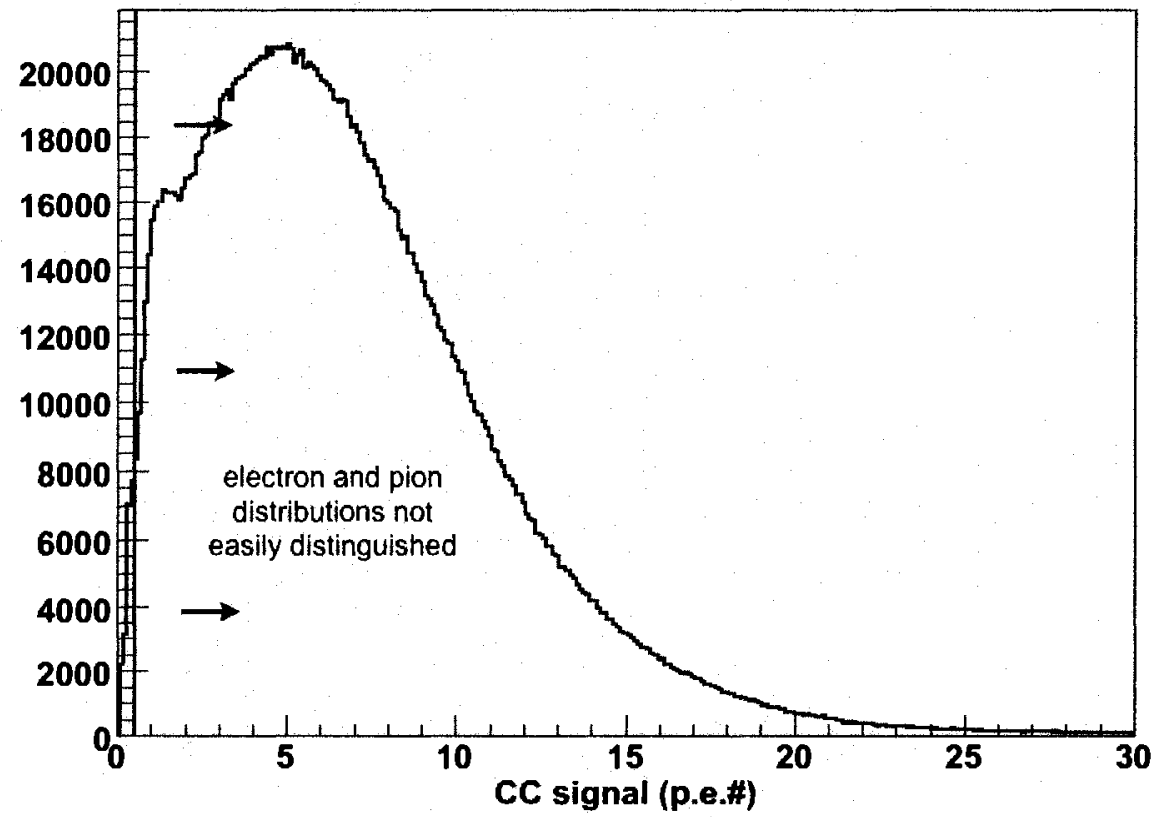

Figure 3.12: Spectrum of photoelectrons (p.e.) corresponding to photons detected by the Cherenkov counters for the leading particle in each event. There are two superimposed (approximately Poisson) distributions in each plot. The high peak at $<2$ p.e. represents primarily $\pi^{-}$ particles, while electrons dominate the wider, higher distribution. The line represents the minimum cut requirement imposed on the data. At high momenta $(>3 \mathrm{GeV}$ ), a minimal cut of 0.5 p.e. (to block out sub-threshold noise) is used. Data shown are from the $5.76 \mathrm{GeV}$ beam energy set. 


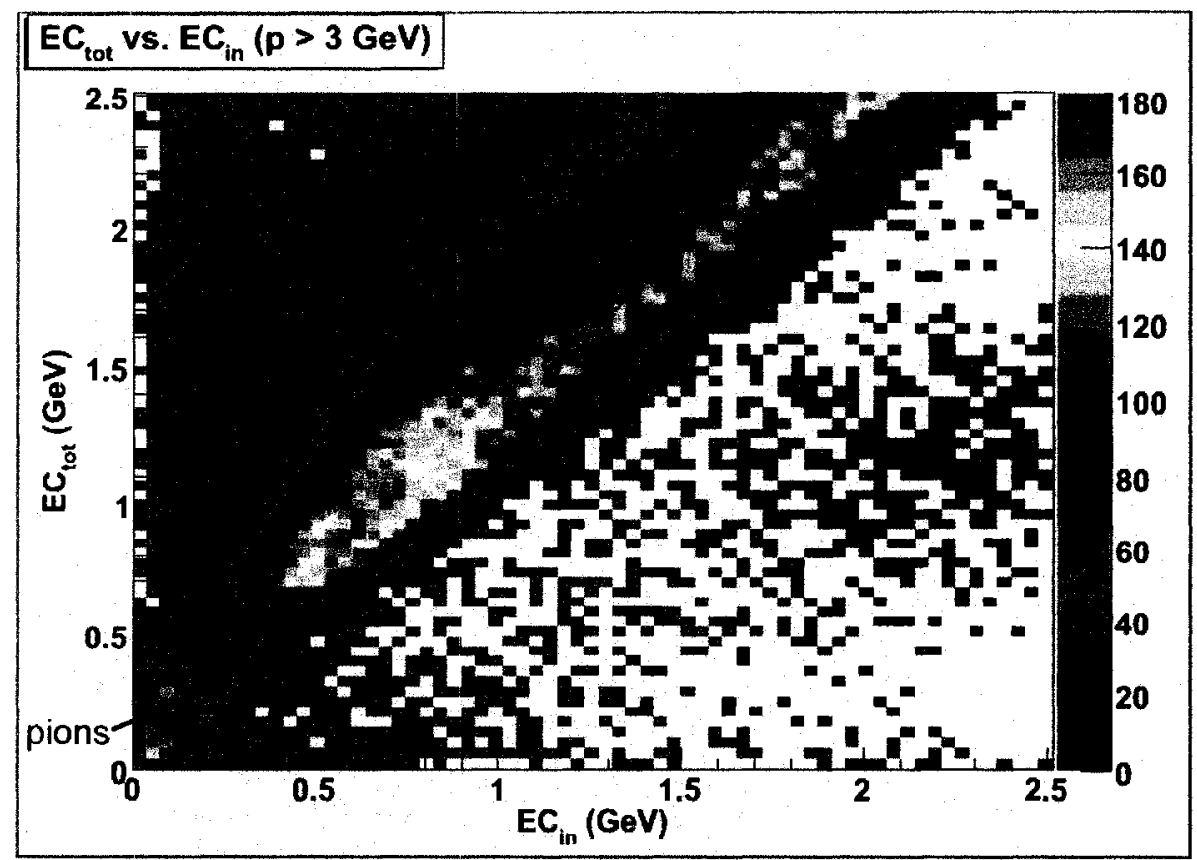

Figure 3.13: Total energy deposited in the calorimeter vs. energy deposited in the inner calorimeter layer only, for high ( $>3 \mathrm{GeV}$ ) outgoing momenta in $5.7 \mathrm{GeV}$ data. Pions appear as a small spot in the lower left, while electrons fan out over a wide range of deposited energies. The enforced cut is shown by the black line and arrows.

To differentiate between electrons and pions in the electromagnetic calorimeter (EC), plots of $E_{\text {EClotal }}$ vs. $E_{\text {ECinner }}$ were generated. This shows the total energy deposited in the calorimeter vs. the energy deposited in the inner layers of the calorimeter only (see Section 2.5 .5 for more details on the calorimeter layer structure). The differences exhibited between pions and electrons are dramatic, especially at higher momenta, as shown in Figure 3.13. The minimum ionizing nature of the pions, resulting from their nearly constant energy loss rate, confines them to a very small region on the plot, as opposed to the wide range of energy loss spanned by the photon radiation and subsequent $e^{+} e^{-}$pair-production loss characteristic of electrons in matter. A lower minimum of $0.22 \mathrm{GeV}$ energy loss in the inner layer of the calorimeter ${ }^{18}$ was used to define an inclusive electron.

Because the energy loss of electrons and pions is statistical, inserting a simple cut on the EC

\footnotetext{
${ }^{18}$ This corresponds to an actual reading of 0.06 in the inner EC channel ADC. EC values must be divided by 0.27 to convert to actual energy lost. See Section 2.5.5.
} 

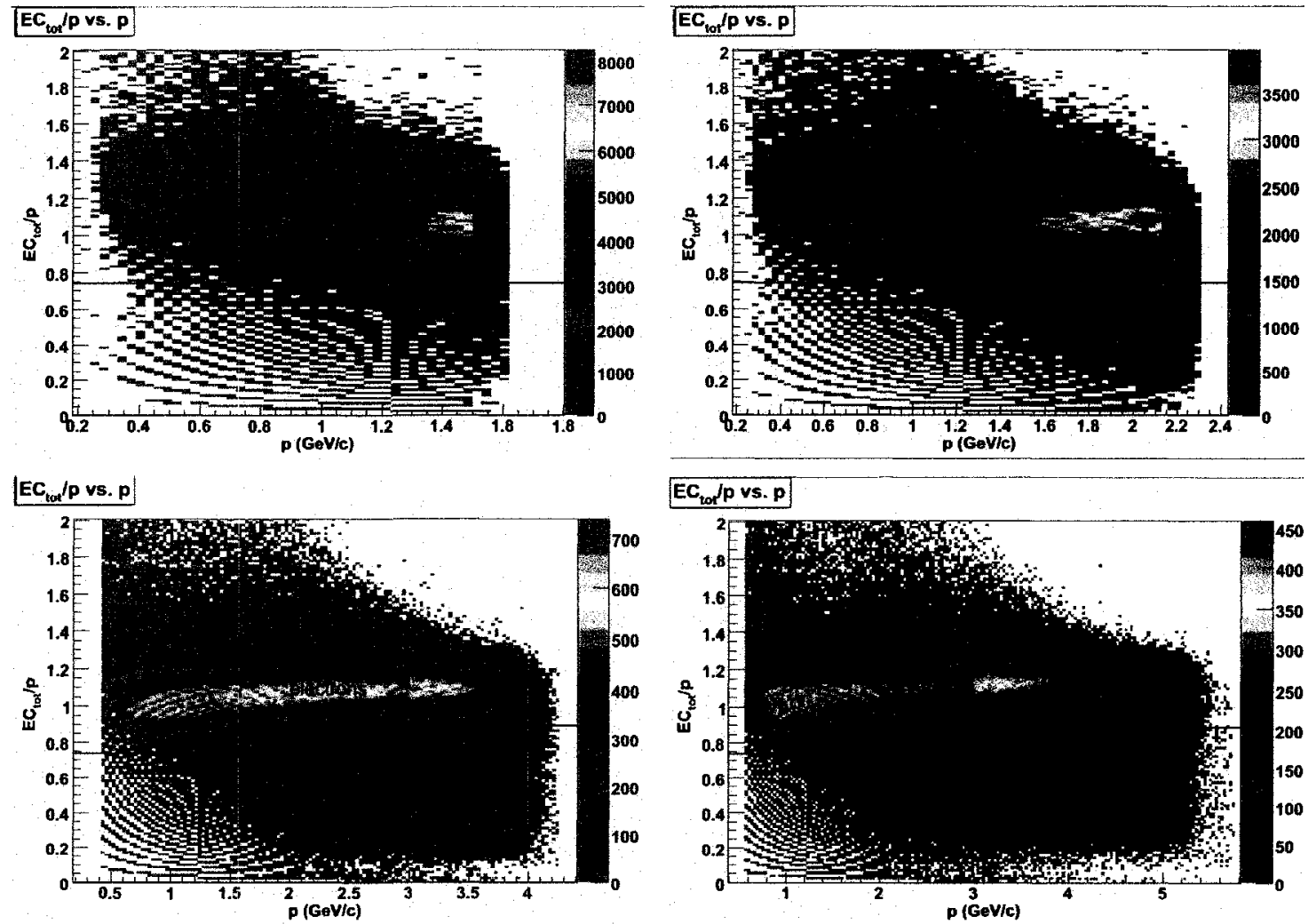

Figure 3.14: A final cut on the total EC energy per unit momentum restricts accepted particles to the region of electrons. Plots for all 4 beam energies are shown. The other $\mathrm{CC}$ and EC parameter cuts, as well as fiducial cuts (see Section 4.4) have already been implemented in these plots. Note the resulting discontinuity in the histogram totals at $p=3.0 \mathrm{GeV}$ in the latter two figures.

energy does not completely separate the two particles. To improve the situation, cuts are also made on the ratio of the deposited energy to particle momentum. With all the previous (CC and EC) cuts in place, plots of $\mathrm{EC}_{\text {tot }} / p$ vs. $p$ (where $p$ is particle momentum) were generated. Additional cuts were then implemented to remove the bulk of any remaining pion contamination. More stringent cuts were used at $p>3.0 \mathrm{GeV}$ to remove the greater $\pi^{-}$background (see Figure 3.14). Based on these plots, a minimum EC energy absorption to momentum ratio of 0.74 for $p<3.0 \mathrm{GeV}$ and 0.89 $\mathrm{GeV}$ for $p>3.0 \mathrm{GeV}$ were used for identifying electrons. ${ }^{19}$

${ }^{19}$ These correspond to values of the EC ADC channel $p$ (with $p$ in $\mathrm{GeV}$ ) of 0.20 and 0.24 , respectively. 


\subsubsection{Particle identification parameters for elastic $e p$ events}

Exclusive analysis of elastic electron-proton scattering events is also required to measure the beam $x$ target polarization (Section 6.2.2). Momentum and kinetic energy conservation at the interaction vertex is applied to 2-particle events for identification. ${ }^{20}$ Because of the strict constraint of 4momentum conservation, we can afford to loosen the $\mathrm{CC}$ and EC cut criteria considerably without contaminating the sample with non-electron triggered events. Instead, we rely on the restriction of the kinematic conservation requirements to remove pions and other inelastically generated particles. Very loose cuts are used for both the electron and proton. The cut requirements used for all 4 cases in this analysis (inclusive electron ( $p<3 \mathrm{GeV}$ ), inclusive electron ( $p>3 \mathrm{GeV}$ ), exclusive $e p$ electron $(p<3 \mathrm{GeV})$, and exclusive ep electron $(p>3 \mathrm{GeV})$ and exclusive $e p$ proton are recorded in Table 3.4.

Table 3.4: PID cuts for electrons in inclusive and exclusive elastic $e p$ analysis. EC values are given here as EC ADC parameters for easy reference. All EC values must be divided by the sampling fraction of 0.27 to get the actual absorbed energy in $\mathrm{GeV}$.

\begin{tabular}{|c|c|c|}
\hline Quantity & Inclusive $(p<3.0)$ & Inclusive $(p>3.0)$ \\
\hline \hline CC photoelectrons & $>2.0$ & $>0.5$ \\
\hline$E_{\text {ECinner }}$ & $>0.06$ & $>0.06$ \\
\hline$E_{\text {ECtotal }} / p$ & $>0.20$ & $>0.24$ \\
\hline
\end{tabular}

\begin{tabular}{|c|c|c|}
\hline Quantity & Exclusive $\left.e p\left(p_{e}<3.0\right)\right)$ & Exclusive $e p\left(p_{e}>3.0\right)$ \\
\hline \hline CC photoelectrons & $>0.5$ & $>0.5$ \\
\hline$E_{\text {ECtotal }} / p$ & $>0.15$ & $>0.20$ \\
\hline
\end{tabular}

\footnotetext{
${ }^{20}$ Actually, all events with 2 charged particles are looked at. Neutral particles, which could just be Brehmsstrahlung photons, are ignored.
} 


\subsection{Elastic $e p$ Event Selection}

In addition to inclusive electrons, which must be very strictly identified by the cuts explained in this chapter, exclusive $e p$ events, scattering elastically as

$$
e p \rightarrow e p
$$

are required for this analysis, not only for momentum corrections (Section 4.2.8), but for asymmetry measurement for $P_{b} P_{t}$ determination (Section 6.2.2).

For the identification of these protons, only events with 2 detected charged particles (including the electron) are considered. Cuts used to make a preliminary identification of the electron are identified in Section 3.4 and Table 3.4. Protons were identified by their time-of-flight using Equation 2.30 , with the condition $|\delta| \leq 0.9 \mathrm{~ns}$. Cuts exploiting 4-momentum conservation are placed on the proton, dependent on the kinematics of the electron, to identify elastic ep events.

The energy of elastically scattered electrons can be calculated by setting $W=M$ in Eq. 1.9 as [7]

$$
E_{e}^{\prime}=\frac{E_{b e a m}}{1+2 E_{b e a m} \sin ^{2}\left(\theta_{e} / 2\right) / M}
$$

where $M$ is the proton mass and $\theta_{e}$ is the polar electron scattering angle. If we apply energy conservation

$$
E_{b e a m}+M=E_{e}^{\prime}+E_{p}^{\prime}
$$

and use $\sin ^{2}\left(\theta_{e} / 2\right)=\frac{1}{2}\left(1-\cos \left(\theta_{e}\right)\right)$, this yields

$$
E_{p}^{\prime}=E_{\text {beam }}+M-\frac{M E_{\text {beam }}}{M+E_{\text {beam }}\left(1-\cos \theta_{e}\right)}
$$

By noting $E=\sqrt{p^{2}+M^{2}}$, the "missing energy" of the proton can then be defined as

$$
\Delta E=E_{\text {beam }}+M-\frac{M E_{\text {beam }}}{M+E_{\text {beam }}\left(1-p_{z_{e}} / p_{e}\right)}-\sqrt{p_{p}^{2}+M^{2}}
$$


This quantity is used to help define an elastic $e p$ event. Constraints can also be placed on the polar angle $\theta_{p}$ by using momentum conservation on the $z$-axis:

$$
E_{b e a m}=E_{e}^{\prime} \cos \theta_{e}+p_{p} \cos \theta_{p}
$$

Solving for $\cos \theta_{p}$ and applying relativistic momentum, this gives

$$
\cos \theta_{p}=\frac{E_{b e a m}-E_{e}^{\prime} \cos \theta_{e}}{\sqrt{E_{p}^{\prime 2}-M^{2}}}
$$

so that the "missing theta" is given by

$$
\Delta \theta=\arccos \left[\frac{E_{\text {beam }}-E_{e}^{\prime} \cos \theta_{e}}{\sqrt{E_{p}^{\prime 2}-M^{2}}}\right]-\arccos \left(p_{z_{p}} / p_{p}\right)
$$

Momentum conservation in the radial direction must also be considered. Since the initial momentum in this direction is zero, the particles should travel in directly opposite radial directions, so that the "missing phi" (in radians) is defined by

$$
\Delta \phi=\left|\phi_{p}-\phi_{e}\right|-\pi
$$

or, in terms of momenta,

$$
\Delta \phi=\left|\arctan \left(p_{y_{p}} / p_{x_{p}}\right)-\arctan \left(p_{y_{e}} / p_{x_{e}}\right)\right|-\pi
$$

If the event is an elastic ep event, the $\Delta E, \Delta \theta$ and $\Delta \phi$ should all be close to zero, within a margin of error allowing for CLAS resolution. Deteriming optimal cuts was an iterative process. Histograms of the "missing" quantities were made for all charged 2-particle candidates meeting the electron ep cut criteria in Table 3.4 and proton TOF cut. Very wide elastic cuts were initially applied. The histograms were then regenerated, with all these cuts in place (except the cut on the plotted quantity), and the cut was tightened to border the (now narrower) elastic peak. This was continued until peak 

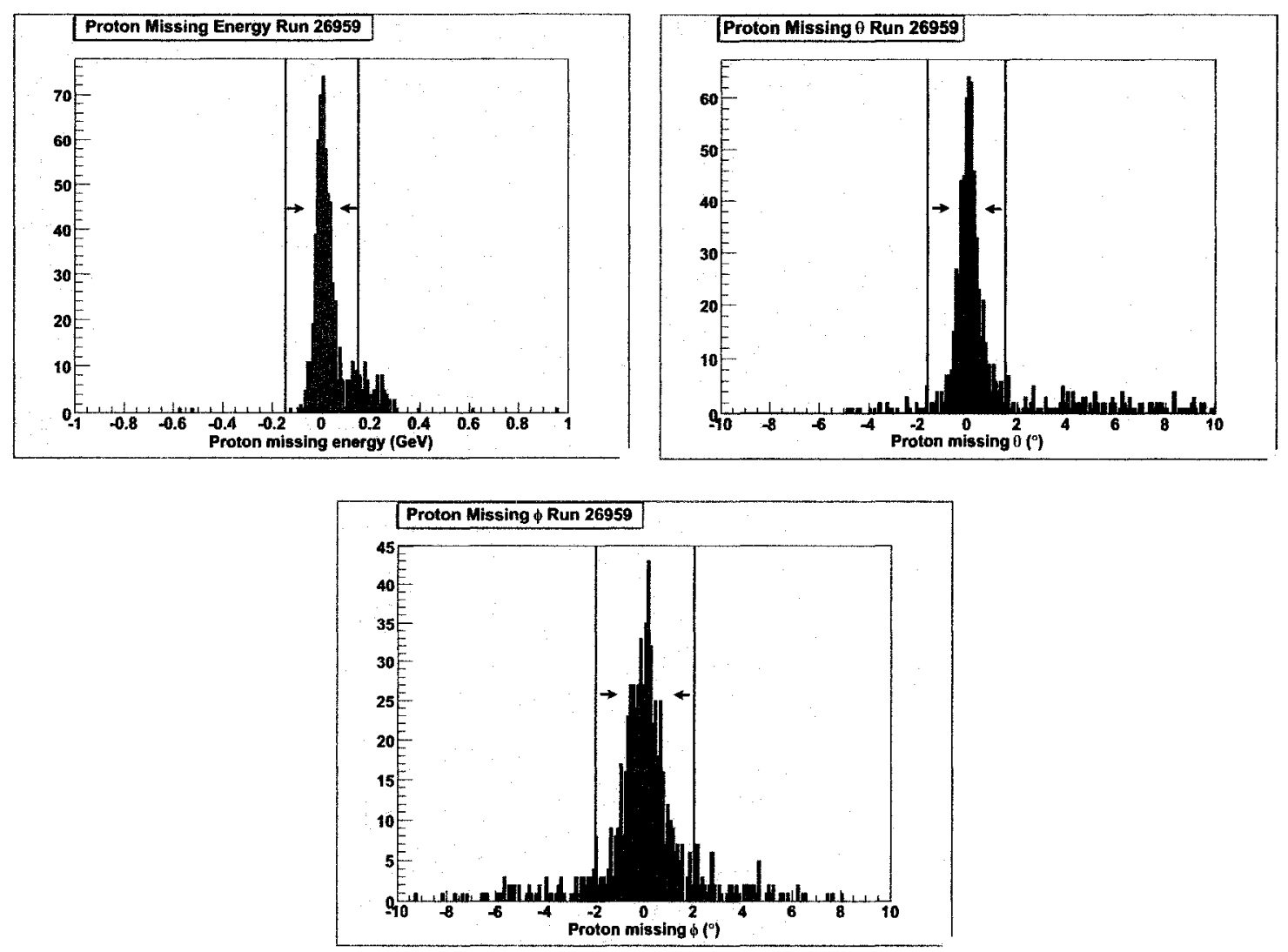

Figure 3.15: Cuts on $\Delta E, \Delta \theta$, and $\Delta \phi$ for elastic ep events in a $5.7 \mathrm{GeV}$ outbending data run. Each plot has the cuts on the other two quantities already applied. Also, a cut of $0.88 \mathrm{GeV}<W<0.99$ $\mathrm{GeV}$ was used prior to plotting.

resolution reached its limit. Final plots of $\Delta E, \Delta \theta$ and $\Delta \phi$ for a $5.7 \mathrm{GeV} \mathrm{NH}$ run are shown in Figure 3.15 .

In addition to these cuts, a cut was placed on the missing mass $W$. Depending on the resolution of the elastic peak (which worsens as beam energy increases), a cut of $30-60 \mathrm{MeV}$ from $W=M=$ $0.938 \mathrm{GeV}$ is used (these are listed explicitly in Section 6.2). Because the data skim files preserve the $W$ and $Q^{2}$ values of the event (Section 4.6), it is not necessary to implement a fixed $W$ cut in this analysis stage. The $W$ cut is instead optimized according to the resolution for each data set just prior to the measurement of $P_{b} P_{t}$. The other final cuts used for elastic $e p$ events are listed in Table 3.5.

Of course, a small percentage of the isolated events are not actual ep events scattered from the 
free protons in the $\mathrm{NH}_{3}$, but are instead quasielastic background events scattered from ${ }^{15} \mathrm{~N}$, LHe and the target windows. Removal of the background is done by scaling of the same data from ${ }^{12} \mathrm{C}$ in the "wings" of the $\phi$ distribution; this procedure is also outlined in Section 6.2.

Table 3.5: Kinematic cuts used to select elastic $e p$ events. See the text for details on these variables. $W$-cuts are energy-dependent and not implemented until after binning of the data takes place.

\begin{tabular}{|c|c|}
\hline Quantity & cut \\
\hline \hline$\Delta E$ & $\pm 0.15 \mathrm{GeV}$ \\
\hline$\Delta \theta$ & $\pm 1.5^{\circ}$ \\
\hline$\Delta \phi$ & $\pm 2.0^{\circ}$ \\
\hline$W$ & See Table 6.6 \\
\hline
\end{tabular}

\subsection{Miscellaneous Minor Cuts}

At this point, all preliminary PID cuts have been developed for application to the data. Now, some minor cuts are added addressing various issues, to prevent spurious events from surfacing in the data.

\subsubsection{Vertex cut}

To help ensure scattering from target materials only, a longitudinal cut on the reconstructed $z$-vertex position $v_{z}$ must be considered. Unfortunately, the target configuration makes it impossible to cut out the target window material with a vertex cut, as the resolution of the event vertex reconstruction is not fine enough to resolve distinct scattering peaks within the $\sim 2.3 \mathrm{~cm}$ distance of the target banjo length. Thus, other techniques (i.e. dilution factor calculation) must be used to eliminate contributions from the aluminum, Kapton and liquid helium on either end of the frozen ammonia target material.

This considered, a vertex cut was put in place narrow enough to exclude events that scatter off the LHe refrigerator ends ${ }^{21}$ but still include all properly scattered events within $3 \sigma$ of the target

\footnotetext{
${ }^{21}$ This can be seen by the secondary purple "stripe" on the right of Figure 4.9, later in the text.
} 


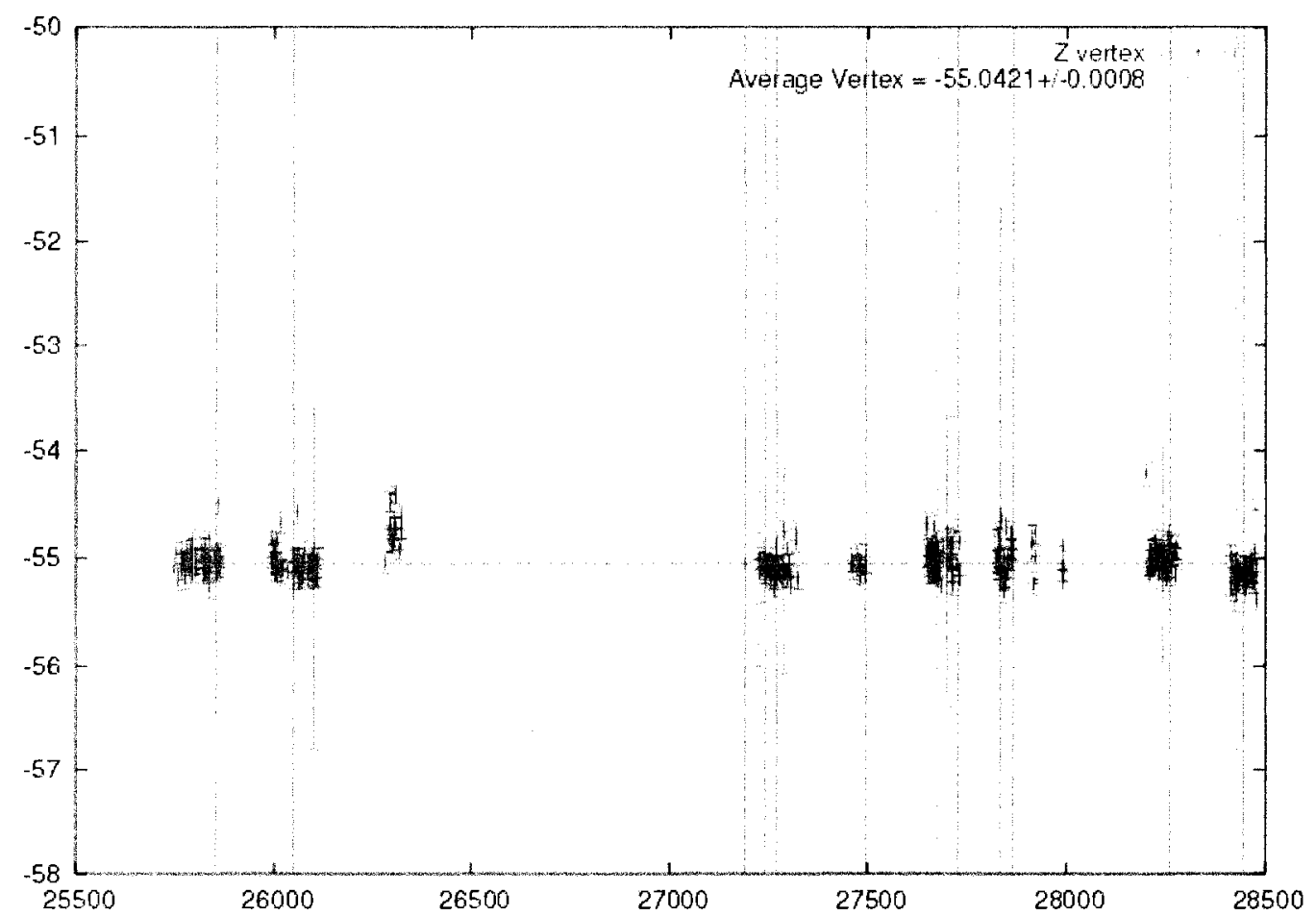

Figure 3.16: Average $z$-vertex position (in $\mathrm{cm}$ ) as a function of run number. The error bars on each run correspond to the standard deviations of the vertex position distributions of each run. The weighted average over all runs agrees quite well with the nominal value of $-55.1 \mathrm{~cm}$. Plot courtesy J. Pierce.

center (nominally located at $-55.1 \mathrm{~cm}$ in CLAS coordinate space). The loose cut

$$
-58 \mathrm{~cm}<v_{z}<-52 \mathrm{~cm}
$$

was therefore placed on the data. Figure 3.16 shows the average $z$-vertex position as a function of run number for the EG1b data.

Cuts on the $x$ and $y$ positions of the vertex were not made. However, raster patterns were inspected for each run to ensure that the beam impacted the target and not the surrounding cell (see Section 3.3.4). 


\subsubsection{Trigger bits}

Every reconstructed event in the DST contains a trigger bits variable.This integer represents a 16-bit binary number. An N-bit binary number can be represented in the form

$$
a_{1} 2^{0}+a_{2} 2^{1}+a_{3} 2^{2}+\ldots+a_{N} 2^{N-1}
$$

where the coefficients $a_{n}$ are either 1 or 0 (i.e. the bit is present or not present). A trigger bit of 49313, for example, can be written as

$$
49313=2^{0}+2^{5}+2^{7}+2^{14}+2^{15}
$$

meaning that bits $1,6,8,15$, and 16 are present, in this example. Trigger bits 1-6 represent a proper event trigger $(C C+E C)$ in respective sectors 1-6. Trigger bits 7 and 8 represent triggers in the EC only, with 8 representing a lower EC threshold than that normally used for event triggers. Trigger bits 9-14 are unused, and trigger bits 15-16 (redundantly) record the value of the helicity bucket. ${ }^{22}$

Only events that contain valid hits in at least one sector are desired for inclusive analysis, so only events containing trigger bits 1-6 were included in the data. Events with only bits 7 and higher were cut. For pion background analysis, it was important to consider the behavior of the EC background triggers, so trigger bits 7 and 8 were used for these studies (see Section 4.1).

\subsubsection{Status flags}

Each individual particle in the DST was correlated to a one or two-digit status flag variable indicating the nature of the detector signal corresponding to the particle. A flag least digit value greater than 5 corresponds to trajectories reconstructed from hit-based tracking only (Section 2.6.2). Only accurately reconstructed events using time-based tracking are desired; these correspond to flag least digit values in the range 0-5.

In the event that a particle is detected in all 3 superlayers of the DC (see Section 2.5.2), resulting

\footnotetext{
${ }^{22}$ Bits 15-16 are overriden by the modified variable read from the fixed helicity tables (see Section 3.2) and are not used.
} 


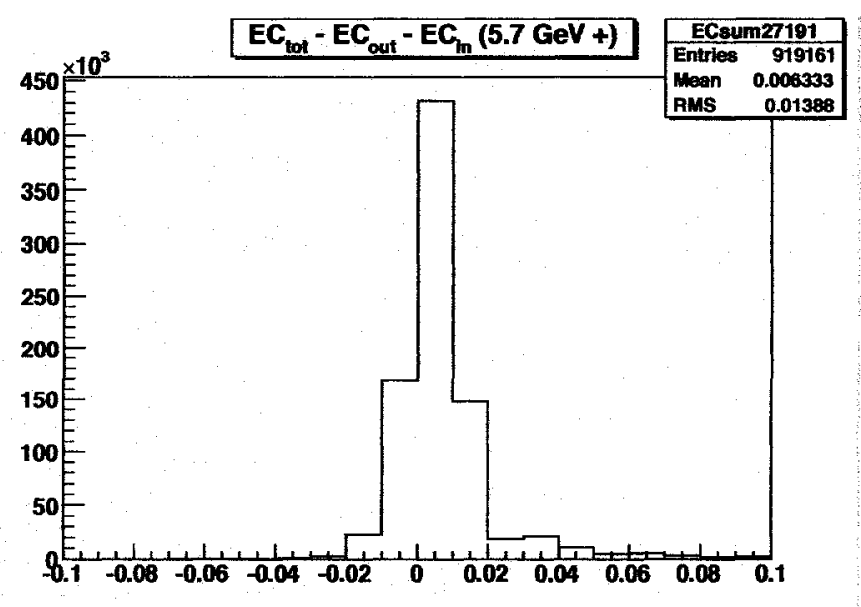

Figure 3.17: Difference in deposited EC energy between the total signal and sum of both layers of the calorimeter. Events far from zero indicate an inconsistency in the internal signals of the EC.

in a very accurately reconstructed trajectory, $a+10$ is added to the flag variable. These are definitely events that should be kept. Therefore, particles corresponding to $0 \leq$ flag $\leq 5$ or $10 \leq$ flag $\leq 15$ are kept, while all other particles are discarded.

\subsubsection{EC energy sum correction}

The Electromagnetic Calorimeter records 3 ADC signals for the energy deposited by an incident particle, denoting the inner calorimeter layer $\left(E C_{\text {in }}\right)$, outer calorimeter layer $\left(E C_{\text {out }}\right)$ and total energy deposited $\left(E C_{\text {tot }}\right)$ (see Section 2.5.5). Occasionally, the readings in these channels did not match. That is, $E_{\text {tot }} \neq E C_{\text {in }}+E C_{\text {out }}$ (see Figure 3.17). This occurred because, occasionally, part of the energy was not recorded in one or more of the channels, even though these represented perfectly good events in every other respect. Thus, the correction

$$
E C_{\text {tot }}=\max \left(E C_{\text {tot }}, E C_{\text {in }}+E C_{\text {out }}\right)
$$

was enforced to ensure that the full measured EC energy was employed in the PID cuts. 


\subsubsection{Minor kinematic and geometric cuts}

Analysis of CLAS data becomes problematic when scattered particles get too close to the edge of the geometric acceptance of the detector, or when momentum becomes so low that the efficiency of the detectors and momentum measurement is unpredictable. Also, certain events (such as those with $E^{\prime}>E$ ) are obviously non-physical particles. For that reason, the following cuts are added for inclusive electrons:

$$
\begin{gathered}
\nu \equiv E-E^{\prime}>0 \\
y \equiv 1-\frac{E^{\prime}}{E}<0.80 \\
8.5^{\circ}<\theta_{D C}<49^{\circ}
\end{gathered}
$$

Here $\theta_{D C}$ is the polar angle measured at the inner layer drift chamber. The low angle here marks the innermost forward angle definitively covered by CLAS acceptance, and the upper angle is limited by physical interference from the target magnet coils.

Another cut necessitated by closer inspection of the data in each sector is

$$
\theta_{D C}<18^{\circ} \text { and } \theta_{D C}>22^{\circ} \text { (Sector } 5 \text { only) }
$$

A comparison of plots of $\theta_{D C}$ between sectors showed a discrepancy in the angle reconstruction of inclusive electrons in sector 5 . This was confirmed by plotting the reconstructed $z$-vertex position vs. $\theta_{D C}$ (see Figure 3.18). Data in the excluded range were obviously reconstructed incorrectly by the SEB, possibly due to mislabelled channels in the drift chamber wire map. 


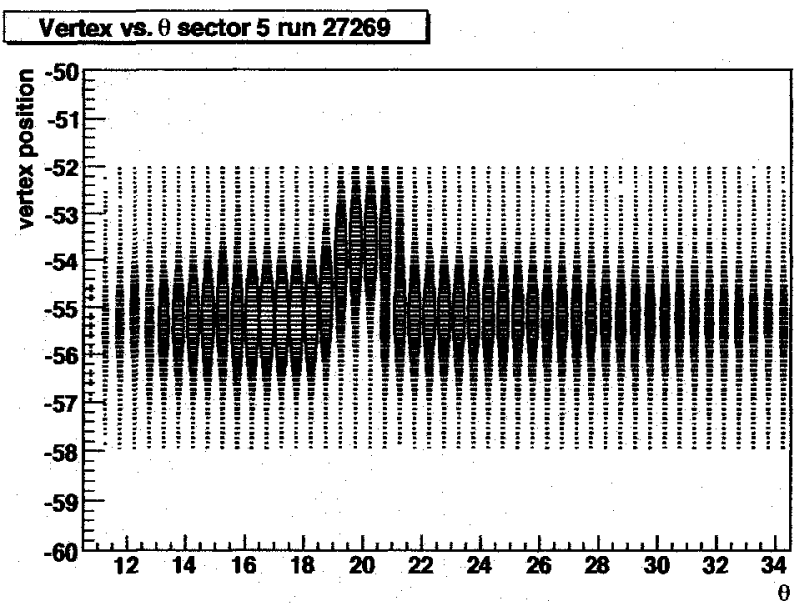

Figure 3.18: Reconstructed event vertex position (in $\mathrm{cm}$ ) along the longitudinal axis vs. $\theta$ (in degrees), as reconstructed from the drift chamber data, for sector 5 data only. Note the obviously incorrect vertex position between 18 and 22 degrees. 


\section{Chapter 4}

\section{Precision Cuts and Corrections}

\subsection{Pion Background Removal}

\subsubsection{Remaining pion background}

The detector cuts explained in the previous chapter remove a large percentage of remaining pions among the inclusive electrons. However, due to the statistical nature of the pion and electron distributions in terms of these cut parameters, it is not possible to remove all pions from the electron data sample using such basic cut parameters. A small but significant background of pions still remains in the data.

By inverting the electromagnetic calorimeter cuts in the previous sections, and selecting trigger bits corresponding only to particles not matched to Cherenkov triggers, ${ }^{1}$ the shape of the pion distribution in the Cherenkov counter photoelectron spectrum (Figure 3.12) can be estimated [104]. Figure 4.1 shows a comparison of photoelectrons remaining after all the applied PID cuts (except, of course, the CC cut) and the approximate photoelectron spectrum of pions. Of course, due to the unknown relative cross-sections of the cut regions, the relative height of these spectra is of little relevance. However, a look at the $\pi^{-}$spectrum shows a statistical tail that overflows into

\footnotetext{
'See Section 3.6 .2 for definition and selection of trigger bits. For the plots shown in this section, "pions' were identified by cuts of $E_{\mathrm{ECOt}} / p<0.24$ and $E_{\mathrm{EChot}} / p<0.28$ were used for $p<3.0$ and $p>3.0 \mathrm{GeV}$, respectively, atong with a general cut of $E_{E C \text { in }}<0.01$ (all values given in ADC channels, not GeV). Events containing trigger bits 1-6 were excluded from pion spectra.
} 


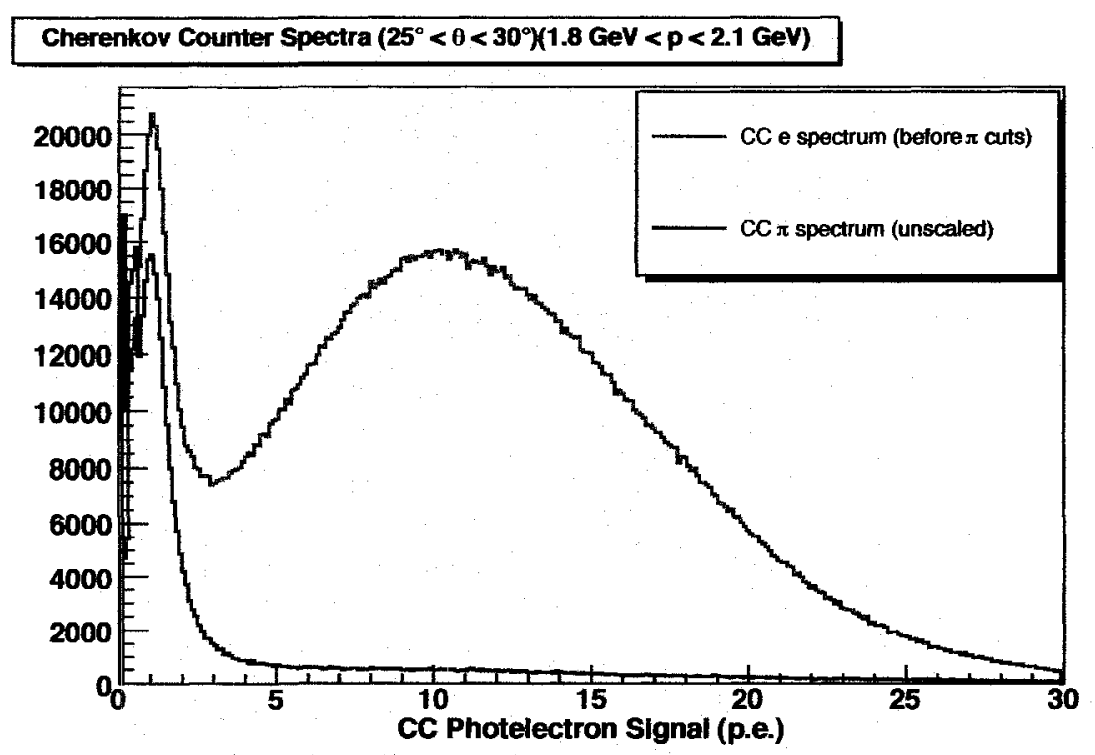

Figure 4.1: The Cherenkov photoelectron distribution for a given bin in $p$ and $\theta$. Distribution shapes for both negative pions and electrons, as determined by calorimeter and trigger bit cuts, are shown. Pion tracking and timing cuts (Sections 4.1 .2 - 4.1.4) are not yet used in this plot. Data shown are from a combination of $5 . x \mathrm{GeV} \mathrm{NH}_{3}$ runs.

the electron distribution. Part of this tail is, no doubt, electrons, but at least a portion of it is part of the pion distribution. Apparently, $\pi^{-}$contamination is still an issue above the threshold of the Cherenkov photoelectron cuts (Table 3.4).

Older analyses of CLAS inclusive electron data utilized a model to estimate the magnitude of remaining pion contamination as a function of $p$ and $\theta$, and a function was fit to the data. This function was subtracted as background from the inclusive spectrum, and counts were rebinned the in terms of $Q^{2}$ and $W$ [2][46]. ${ }^{2}$ Unfortunately, this method of background subtraction is prone to high systematic error.

M. Osipenko et al. have determined that most of the pion background results from indeterminate track matching of the Cherenkov counter photomultipliers to the DC and SC-determined track path and timing, allowing internal PMT noise to correlate with the particle [105]. Applying restrictions to the geometry and trigger timing in the $\mathrm{CC}$, the overwhelming majority of remaining $\pi^{-}$background can be removed, and the (very low) remaining residue can be considered a systematic error (see

\footnotetext{
${ }^{2} \mathrm{~A}$ (more accurate) variant of this method is still used for removal of the $e^{+} e^{-}$pair production background removal. See Section 6.1.
} 
Section 7.2.5).

\subsubsection{Geometric cuts on accepted CC triggers}

For a hit triggering a given $\mathrm{CC}$ segment $\mathrm{t}^{3}$, a geometric cut of the form

$$
\left|\theta_{p}-\theta_{p}^{\text {center }}-\theta_{p}^{o f f s e t}\right|<3 \sigma_{p}
$$

is used, where $\theta_{p}$ is the polar angle of the particle angle in the CC projective plane, calculated using the SC-plane trajectory angle. A detailed depiction of this projective plane is shown in Figure 4.2.

The quantity $\theta_{p}^{\text {center }}$ is the polar angle of the CC segment center image in the CC projective

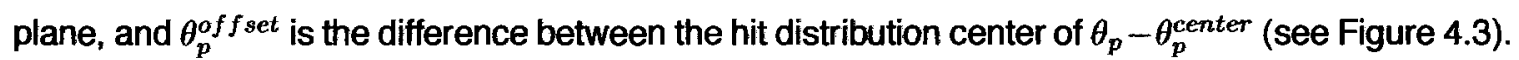
The CC projective plane is the plane through all points extrapolated from the original Cherenkov ray direction through the equivalent distance traveled to the CC PMT [105]. In the CLAS coordinate system, this plane is given in $x_{S}$ and $z$ by

$$
1-a x_{S}-b z=0
$$

where $a=0.0007841 \mathrm{~cm}^{-1}, b=0.001681 \mathrm{~cm}^{-1}$, and $x_{S}$ is the radial particle distance along the sector center line. ${ }^{4}$ The distribution width, $\sigma_{p}$, is the width of the $\theta_{p}$ distribution for the electron events. Figure 4.3 demonstrates the efficacy of this geometric cut.

\subsubsection{PMT cuts on accepted CC triggers}

Each segment of the Cherenkov Counter contains two different photomultiplier (PMT) tubes for the detection of Cherenkov light. Once $\theta_{p}$ and $\phi_{p}$ (the polar and azimuthal angles of the track with respect to the projective plane) are known for an electron track, the PMT corresponding to the half

\footnotetext{
${ }^{3} 18$ segments per sector $\times 6$ sectors $=108$ segments. See Section 2.5.3.

${ }^{4}$ This is $x$ in CLAS sector coordinates.
} 


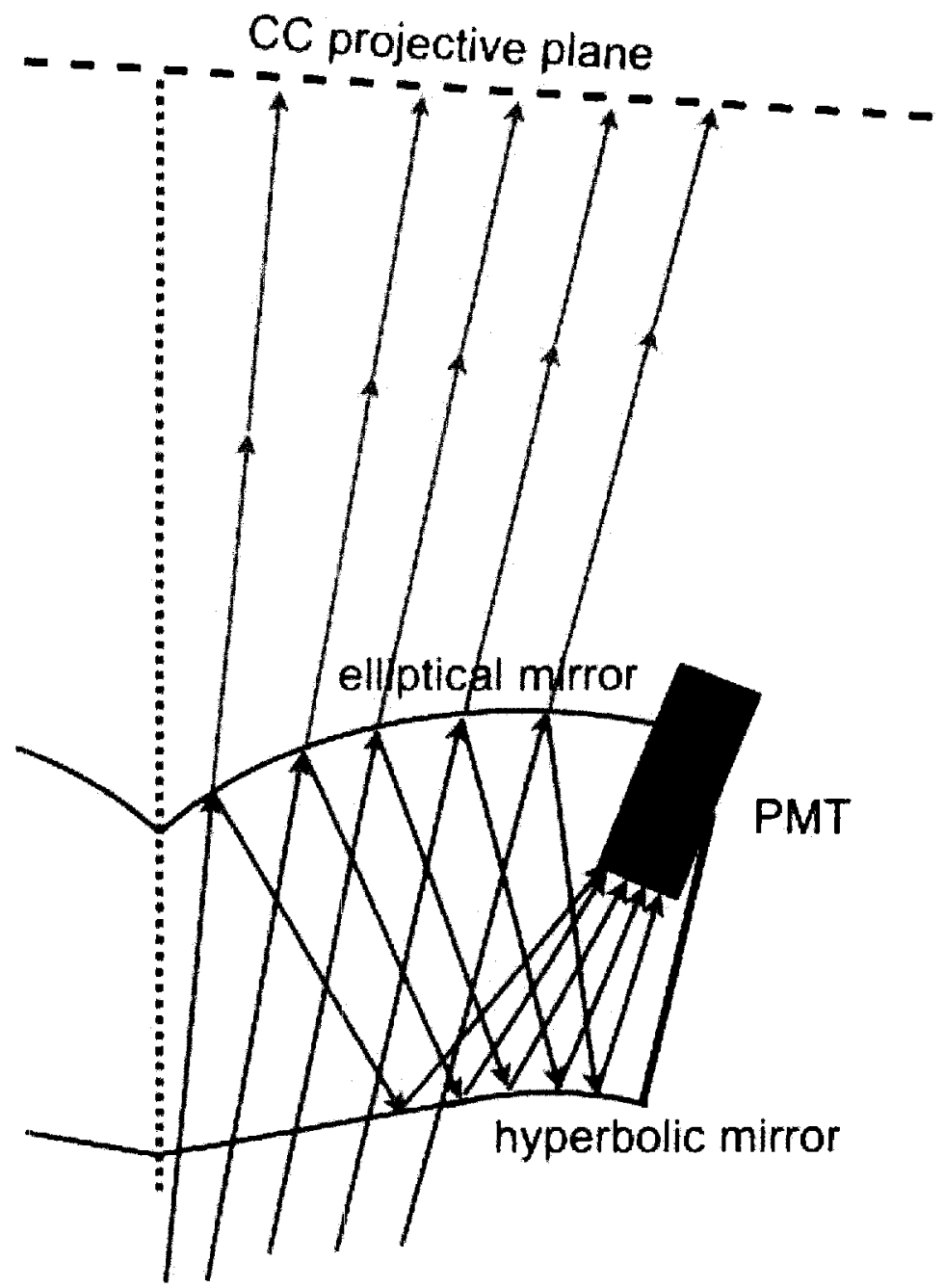

Figure 4.2: A profile of the location of the Cherenkov counter projection plane for a particular CC segment. The blue arrows represent the paths taken by incoming particles, and the reflection paths of their subsequent Cherenkov light between the mirrors to the PMT. To form the projective plane, the equivalent distance to the PMT is traced along the initial direction of the particle (red arrows); the resultant plane formed by the projection of possible paths is the projective plane. The angle between an extended red arrow and the normal to the projective plane is $\theta_{p}$, and the angle made with the center projection (vertical dotted line) and the plane normal is $\theta_{p}^{\text {center. }}$. 


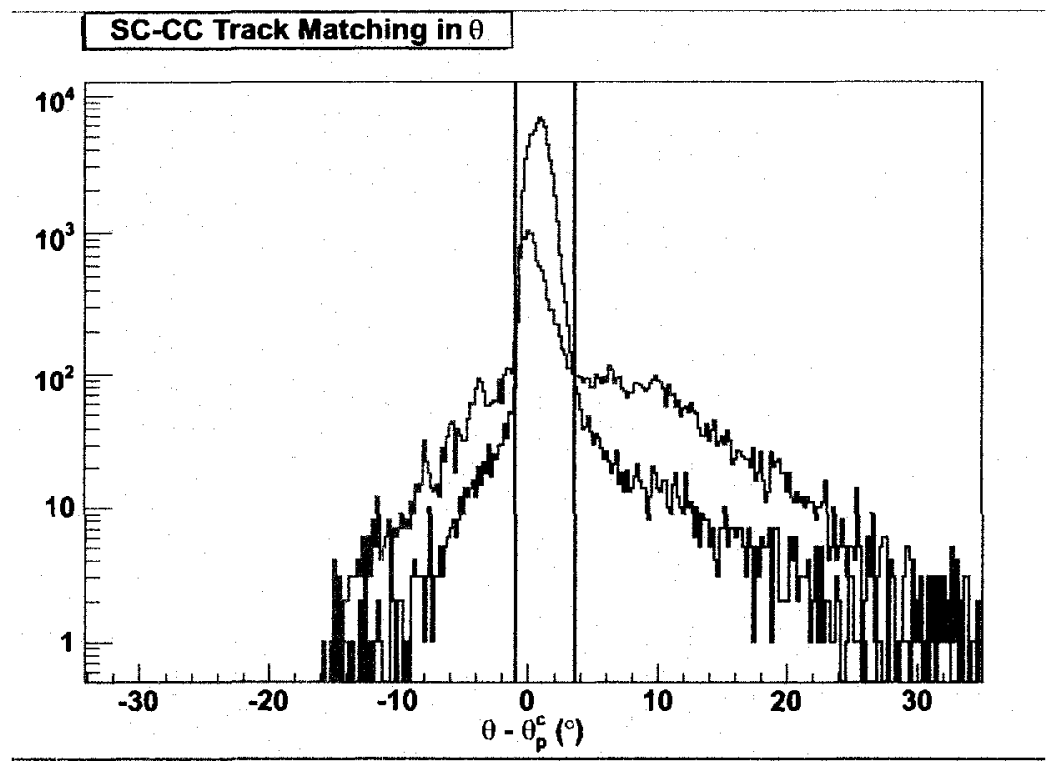

Figure 4.3: The difference $\theta-\theta_{p}$ for low photoelectron peak events (red) and events with $>2.5$ photoelectrons (blue). Actual electrons exhibit a sharp peak, easily distinguishable from the background from a simple cut (black lines). Note the small offset $\left(\theta_{p}^{\text {off } f \text { set }}\right)$ from zero. Data shown are from 4.2 GeV outbending data specific to sector 4, CC segment 9. (Compare to Ref. [105], Fig. 5.)

of the counter segment containing the track can easily be identified. If the event-triggered PMT does not correspond to the electron track identified as the event trigger, then the event is cut from the data, as it is most likely a pion with a PMT triggered by background particles and/or noise. In simpler terms, a good event is identified by $\phi_{S}<0$ for a left PMT trigger, and by $\phi_{S}>0$ for a right PMT trigger (with $\phi_{S}$ being the azimuthal angle with respect to the sector center).

\subsubsection{Timing cuts on accepted $\mathrm{CC}$ triggers}

Assuming that the SC and $\mathrm{CC}$ are triggered by the same particle, the expected time difference between the two signals is obviously given by dividing the distance difference by the electron speed. That is, 5

$$
\Delta t_{\text {coincidence }}=-\frac{r^{S C}-r^{C C}}{c \beta}
$$

\footnotetext{
${ }^{5}$ This must be done after time-of-flight calibration, of course, so that effective velocity of light in the scintillator need not be considered. See Section 2.5 .4
} 


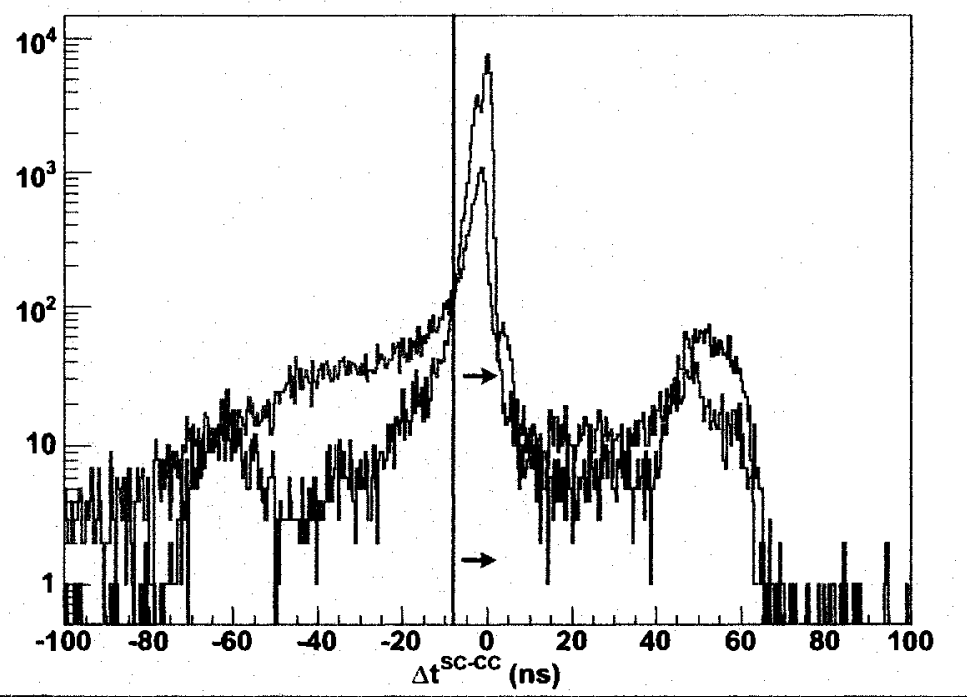

Figure 4.4: This is the same data shown in Figure 4.3, except in terms of $\Delta t^{S C-C C}$. Due to the multiple peak structure exhibited by good electrons at higher $\Delta t^{S C-C C}$, only a lower cut (black line) is used. (The narrow twin peaks are caused by timing differences in the CC PMTs, while the wider peak at $\sim 55 \mathrm{~ns}$ is of undetermined origin but definitely internal to the CC.) (Compare to Ref. [105], Fig. 6.) 
where $r^{S C}-r^{C C}$ is the track distance between the SC paddles and CC projection plane. The observed time difference from that expected is thus given by

$$
\Delta t^{S C-C C}=t^{S C}-t^{C C}-\frac{r^{S C}-r^{C C}}{c \beta}
$$

As with the geometric cuts, the electron peak (now measured in terms of this time difference), rises above the pion background. The edge of this peak is again used to determine the edge of the cut. A plot of the event rates vs. $\triangle t^{S C-C C}$ is shown in Figure 4.4. The timing cut is decided based on this and similar figures for each $\mathrm{CC}$ segment. Due to a strange prominence at the upper end of the spectrum, related to timing discrepancies internal to the CC [105], only a lower edge cut is used on $\Delta t^{S C-C C}$, to avoid cutting electrons that may be present in the structures at higher $\Delta t^{S C-C C}$ values. ${ }^{6}$

\subsubsection{Results of Osipenko's cuts and Implementation}

The effectiveness of these cuts can be investigated by looking at the Cherenkov photoelectron spectrum in various $p$ and $\theta$ bins, with and without the cuts, demonstrated in Figure 4.5. Figure 4.6 shows that very little pion contamination remains after these cuts are implemented. The shape of the photoelectron distribution is not well understood [105], but a function of $x^{2}$ or $x^{3 / 2}$ times a Gaussian worked well for fitting the post-cut photoelectron distribution. This curve is extrapolated to the low photoelectron region and subtracted to approximate the remaining pion contamination at the low end of the spectrum. The contamination beyond the range of the CC PID cut can then be estimated by scaling the pion distribution (shown in blue in Figure 4.1) to the height of the difference, and extrapolating the fit into the high-photoelectron region. ${ }^{7}$ The small remaining $\pi^{-}$ contribution can then be treated as a systematic error on the asymmetry (Section 7.2.5).

To implement these cuts, a function is referenced with the necessary input parameters. Values for $\theta_{p}^{\text {center }}, \theta_{p}^{\text {offset }}, \sigma_{p}$ and the lower cut on $\Delta t^{S C-C C}$ were uniquely determined for each CC seg-

\footnotetext{
${ }^{6}$ The origin of this extra "hump" in Figure 4.4 is not well understood, but is definitely internal to the $C_{\text {, }}$ as can be determined by replacing the SC timing signal with the EC timing signal (see Ref. [105]). It may represent electron signals undergoing extra reflections in the $\mathrm{CC}$, and cannot be assumed to be pion background.

${ }^{7}$ This may seem like a rather slipshod method of calculating the background, but it is only an estimation of what turns out to be a very small remaining contamination. Due to the presence of electrons in the "pion" distribution, it is guaranteed to at least slightly overestimate the remaining contamination, so the error is on the side of caution.
} 

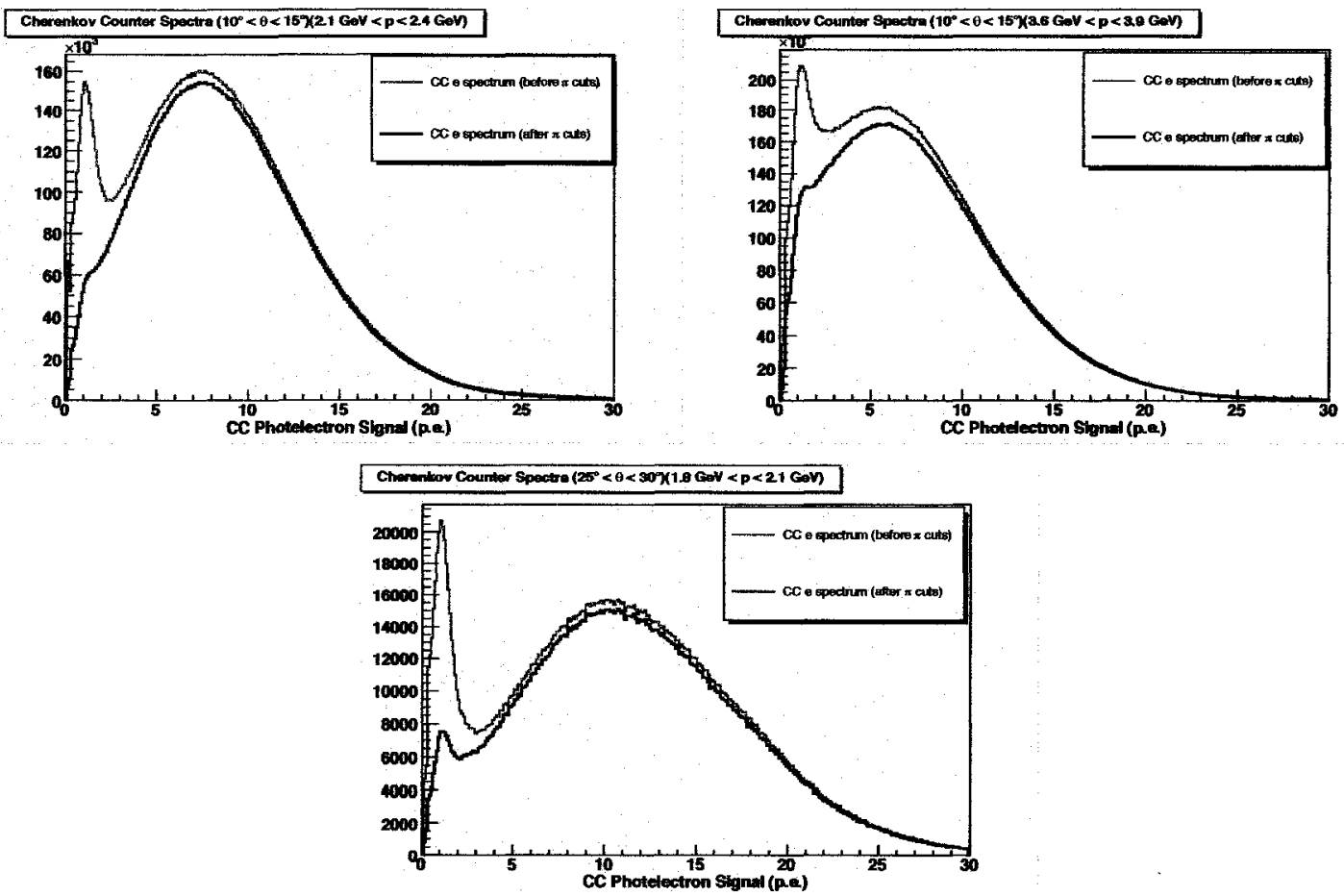

Figure 4.5: Photoelectron spectra for $5.7 \mathrm{GeV}$ electrons in three different $p$ and $\theta$ bins, before (red) and after (black) M. Osipenko's Cherenkov tracking geometry and timing cuts. Note the nearly complete diminishing of the low photoelectron peak. The contamination problem becomes somewhat worse near the edge of geometric acceptance (bottom diagram). 

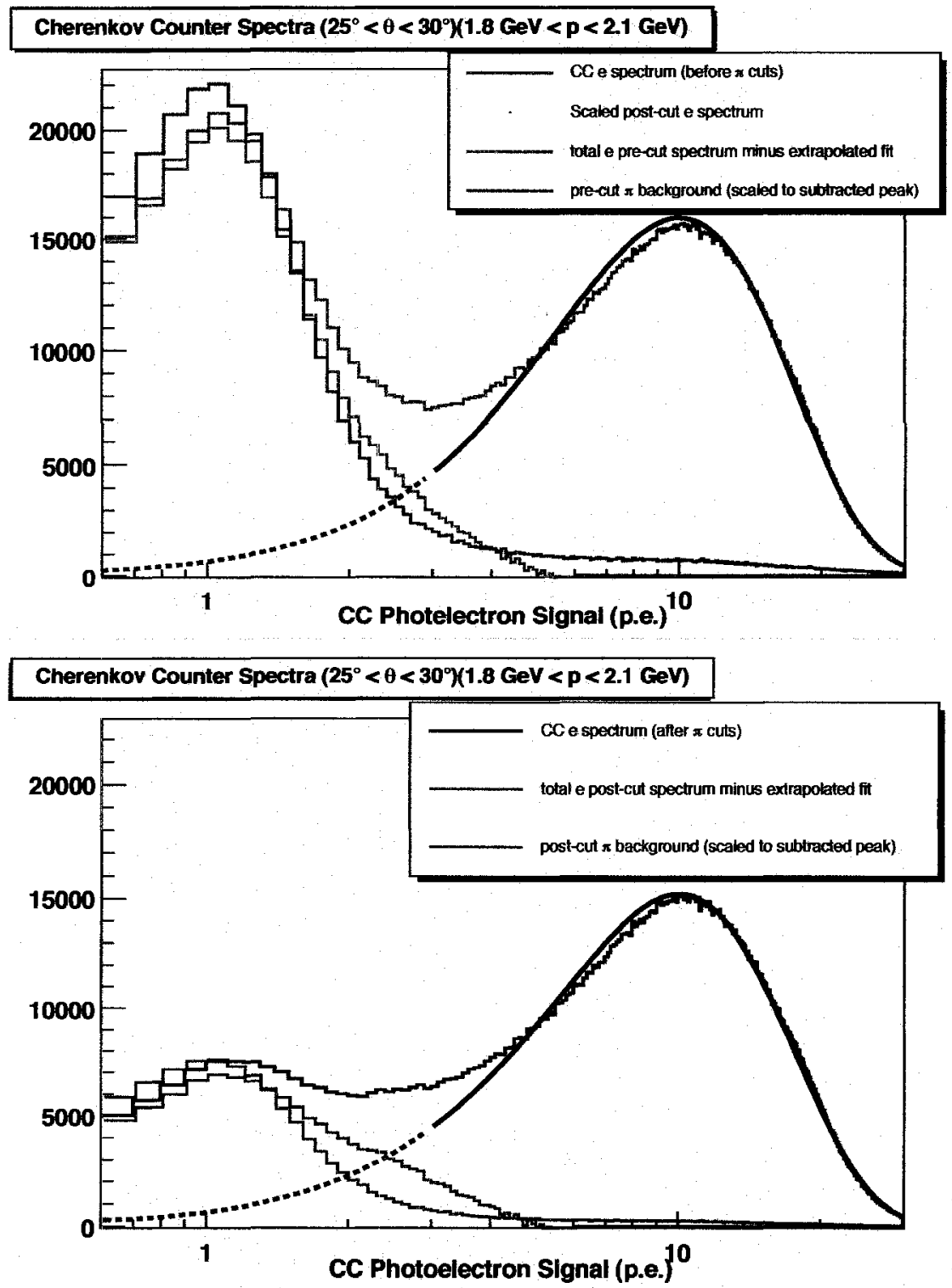

Figure 4.6: Photoelectron spectra for $5.7 \mathrm{GeV}$ electrons (on a logarithmic scale), showing low photoelectron contamination before (top) and after (bottom) Osipenko's cuts. In the top diagram, the post-cut electron spectrum is shown scaled (yellow) to the total pre-cut electron spectrum (red). A fit function to this curve in the region p.e. $>3$ was extrapolated downward; this was subtracted from the electron curves to show the remaining pions in the low p.e. region (magenta). The pion curve (blue in Figure 4.1) is scaled to this difference and extrapolated upward (green) to show an estimate of the $\pi^{-}$contamination before the cuts described in this section. The bottom fig shows the same principle after Osipenko's cuts, with the post-cut electrons (black histogram), the remains after subtracting an extrapolated fit (gray) and the extrapolated scaled pions (cyan), which, compared to the green curve above, shows a significant reduction in the remaining pions. 
ment and sector by N. Guler. Because of the differing path track geometries and timing, separate cut parameters were used for inbending and outbending data. A complete record of these variables for reference purposes is included in Table B.3.

\subsection{Kinematics Corrections}

\subsubsection{The kinematics correction package}

Necessary kinematic corrections were integrated directly into the DST reading program. Adjustments were made to the momentum components $p_{x}, p_{y}, p_{z}$ and the vertex position $v_{z}$ to compensate for both known inaccuracies in the CLAS detector and lack of precision in the event reconstruction code. The following corrections were made for each particle:

1. Raster correction (to correct for vertex position given by the beam raster point on the target at the time of the event)

2. Torus scaling correction (to correct for discrepancies between the physical torus current and the current used for event reconstruction)

3. Beam energy correction (to make sure the exact beam energy is used, corrected for energy loss of the beam within target materials prior to the event vertex)

4. Multiple scattering correction (to correct for the angle and vertex displacement caused by multiple scattering of all particles before they exit the target)

5. Stray magnetic field correction (correction for angular deflections in particle path caused by the target solenoid field)

6. Energy loss correction ( $d E / d x$ corrections for each scattered particle to account for energy loss in the target after scattering)

7. Momentum correction (final sector-by-sector calibrations due to inaccuracies in magnetic field mapping and drift chamber geometry).

Each correction is described individually in detail in this section. 


\subsubsection{Raster correction}

\section{Translation of raster ADC coordinates}

As explained in Section 2.4.1, the electron beam is continuously rastered in a spiraling, circular pattern. The $x$ and $y$ rastering amplitude ADC values were recorded for each reconstructed event time in the DST. Assuming a linear relation between the magnet ADC values and the raster displacement, ${ }^{8}$ the ADC amplitudes can be converted to centimeters as

$$
\begin{gathered}
x_{c m}=\left(x_{A D C}-x_{0}\right) x_{s c a l e} \\
y_{c m}=\left(y_{A D C}-y_{0}\right) y_{s c a l e}
\end{gathered}
$$

The translation coefficient values are shown in Table 4.1.9

Table 4.1: Raster ADC to $\mathrm{cm}$ translation parameters for EG1b, for use with Equations 4.5

\begin{tabular}{|c|c|c|c|c|}
\hline Run Set & $x_{0}$ & $x_{\text {scale }}$ & $y_{0}$ & $y_{\text {scale }}$ \\
\hline \hline $1.6+; 1.6-$ & 3800 & -0.000175 & 5600 & $-0,000180$ \\
\hline $5.76-; 5.73-; 5.7+$ & 4250 & -0.000195 & 6360 & -0.000190 \\
\hline $2.3+$ & 3900 & -0.00048 & 4000 & -0.00048 \\
\hline $5.6+; 5.6-$ & 3900 & -0.00019 & 4000 & -0.00019 \\
\hline $1.7-$ (main set) & 3900 & -0.00060 & 4000 & -0.00060 \\
\hline $2.5-; 2.5+$ & 3900 & -0.00041 & 4000 & -0.00041 \\
\hline $4.2+; 4.2-$ & 3900 & -0.00026 & 4000 & -0.00026 \\
\hline $1.7+; 1.7-\left(^{12} \mathbf{C}^{15} \mathrm{~N}\right)$ & 3900 & -0.00060 & 4000 & -0.00060 \\
\hline
\end{tabular}

Using this coordinate system, it is possible to create a histogram image of the number of events in the physical raster coordinate space, as was done in Figure 3.8.

Due to the varying entry point of the beam into the target, a geometric correction was made to the vertex position. The basic correction method is outlined in [106], and the correction geometry is elucidated here.

\footnotetext{
${ }^{8}$ Given the very small displacements, this is a reasonable assertion.

${ }^{9}$ These were determined as fit parameters for each set by actualiy applying the geometric corrections described in this subsection to the $z$-vertex position $\left(v_{z}\right)$ and using MINUIT to minimize $\chi^{2}=\sum\left(v_{z(\operatorname{cor} r)}-v_{z 0}\right)^{2}$. See Ret. [106] tor more details.
} 


\section{Correction of vertex coordinate $v_{z}$}

If one takes into account that the beam entry point into the target cell is actually along a path parallel to the "center" assumed by the SEB for kinematic reconstruction ${ }^{10}$, then it becomes obvious that the actual location of the scattering event vertex along the $z$ (beamline) axis is slightly displaced from the scattering point, had the event actually occurred at the target center. To correct the position $v_{z}$, first the sector angle is defined as

$$
\phi_{s}=(\text { sector } \#-1) \times 60^{\circ}
$$

and the azimuthal scattering angle $\left(\phi=\arctan \left(p_{y} / p_{x}\right)\right)$ is calculated.

Then, the, projection of the raster coordinates $r_{x}$ and $r_{y}$ (in $\mathrm{cm}$ ) on to the radius sector ray at $\phi_{s}$ is calculated as

$$
s=r_{x} \cos \phi_{s}+r_{y} \sin \phi_{s}
$$

as shown in Figure 4.7. Then, this segment is projected on to the $\phi$ component of the SEB reconstructed track direction to get the displacement of the particle along the the $r$-direction of the track:

$$
x^{\prime}=s / \cos \left(\phi-\phi_{s}\right)=\left(r_{x} \cos \phi_{s}+r_{y} \sin \phi_{s}\right) / \cos \left(\phi-\phi_{s}\right)
$$

Then, finally, the corrected vertex position along the $z$-direction $v_{z c}$ can be calculated from the uncorrected position $v_{z_{0}}$ by backtracking:

$$
v_{z(c o r r)}=v_{z 0}+x^{\prime} / \tan (\theta)
$$

as shown in Figure 4.8.

\footnotetext{
${ }^{10}$ Strictly speaking, of course, the beam path isn't exactly parallel tor every point on the raster, but the raster magnet is distant enough relative to the target dimensions that the approximation of parallel beam paths at every raster point is adequate.
} 


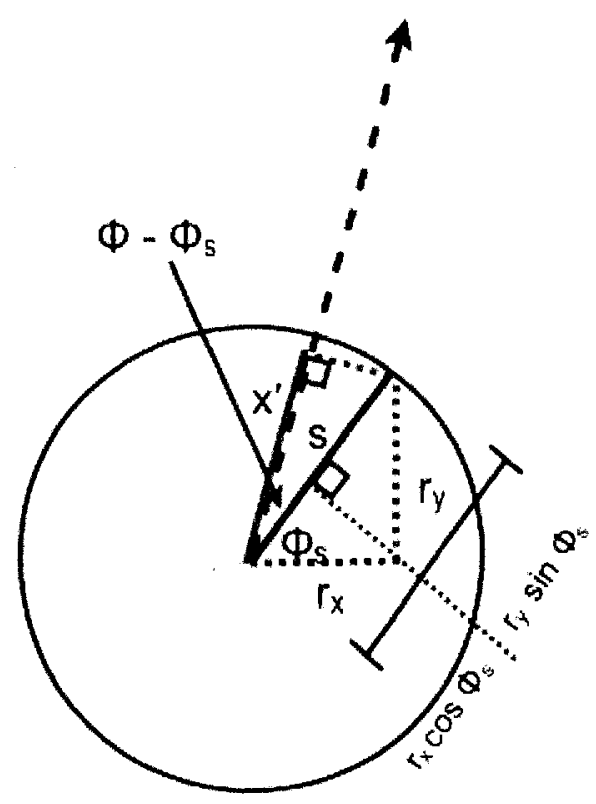

Figure 4.7: Raster correction geometry, viewed from the front of the target. The black dotted line represents the (uncorrected) particle trajectory; while the solid black line $(s)$ is the reference line of the triggered sector. The projection of the raster coordinates (green) on the trajectory is defined as $x^{\prime}$ (blue).

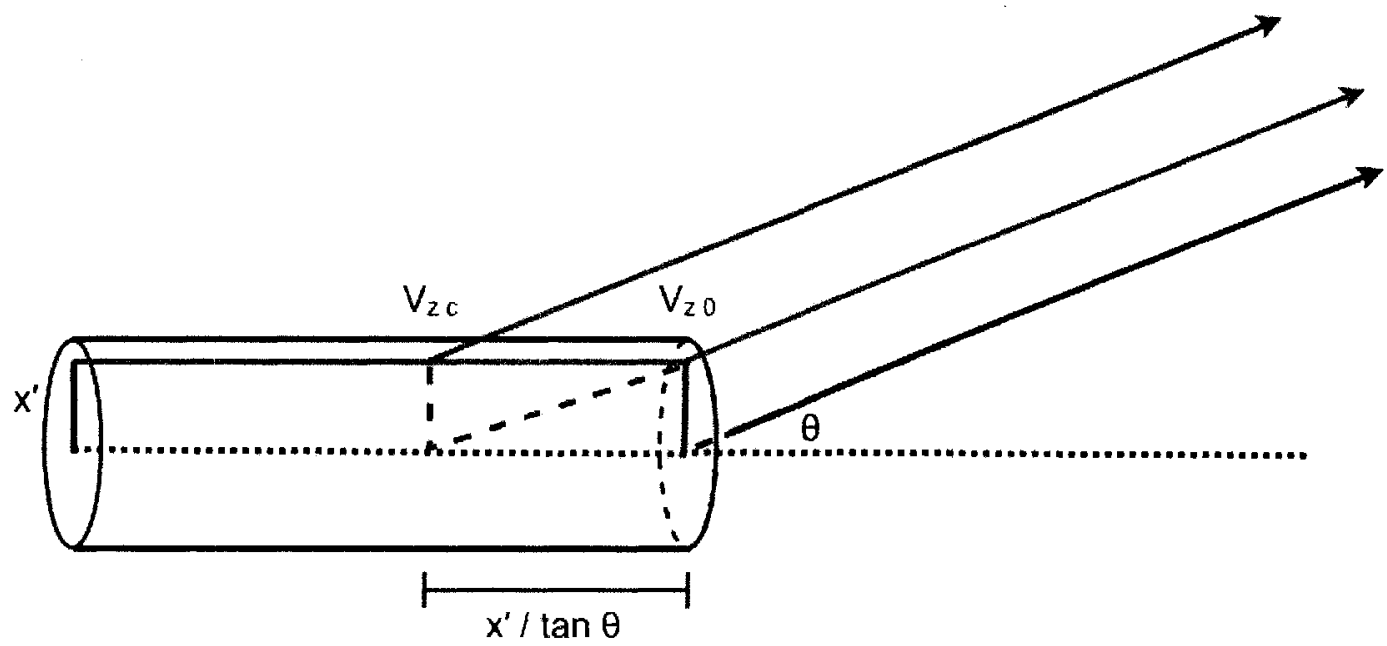

Figure 4.8: Raster correction geometry, viewed from the side. The corrected $z$-vertex position $v_{z(\text { corr })}$ is calculated from the uncorrected position $v_{z 0}$ by backtracking through the end of $x^{\prime}$ (drawn in Figure 4.7). Here, the black ray is the uncorrected particle path, the red ray is the "backtracked" ray, and the blue path is the final, raster corrected path through the true vertex. 
$\phi$ correction due to $v_{z}$ shift

The SEB code automatically scales the azimuthal deflection through $\phi$ to compensate for the 5 Tesla magnetic field in the vicinity of the target (see Section 2.4). However, due to the raster correction to the position of the $z$-vertex, the amount of time the particle spends in the target magnetic field is different than that assumed by the SEB, so the $\phi$ deflection must be corrected.

The correction required exactly compensates for the extra distance traveled, just as the SEB reconstruction code:

$$
\phi_{c}=\phi_{0}-\left(q \times 50 \times x^{\prime} / 100 \times 0.02998 / p_{t}\right)
$$

where the charge $q= \pm 1,50 \mathrm{kG}=5 \mathrm{~T}$ is the field strength, $0.02998=c$ (in $\mathrm{cm} / \mu \mathrm{s}$ ) and $p_{t}=p \sin \theta$ is the transverse momentum (in GeV) [106].

For inclusive electron analysis, however, the $\phi$-component is not a concern. Even for exclusive $e p$ analysis, which is used in this procedure, ${ }^{11}$ the signs of both $x^{\prime}$ and the charge $q$ reverse sign under an exchange of $e$ and $p$, causing an identical $\phi$ shift for both (oppositely directed) particles, so the analysis is unaffected. Only in multiple-particle exclusive events (such as $e p \pi^{+} \pi^{-}$) does the $\phi$ correction become relevant, but it is mentioned here for completeness. The improvements made to inclusive events in terms of $\phi$ and $v_{z}$ due to raster corrections can be seen in Figure 4.9.

\subsubsection{Torus scaling correction}

During the pass1 SEB and DST generation process (see Section 2.6.3), there was a coding error in some of the Tcl files omitting the fixed torus current. Instead, the value for read from a current monitoring transducer was used in track reconstruction. This torus current value produced values fluctuating by up to \pm 8 A between runs, in contrast to the fixed, regular values consistent with a superconducting electromagnet. The SEB tracking code allows for a margin of error of $0.2 \%$ within this fluctuation range for the commonly used settings $[107]^{12}$, so that, in most cases, despite the coding error, the correct torus current was used in particle reconstruction, and no correction was required.

\footnotetext{
${ }^{11}$ Elastic exclusive events are needed for $P b P t$ calculations. See Section 6.2.2.

${ }^{12}$ These "common" settings include the \pm 1500 and $\pm 2250 \mathrm{~A}$ used in this experiment.
} 


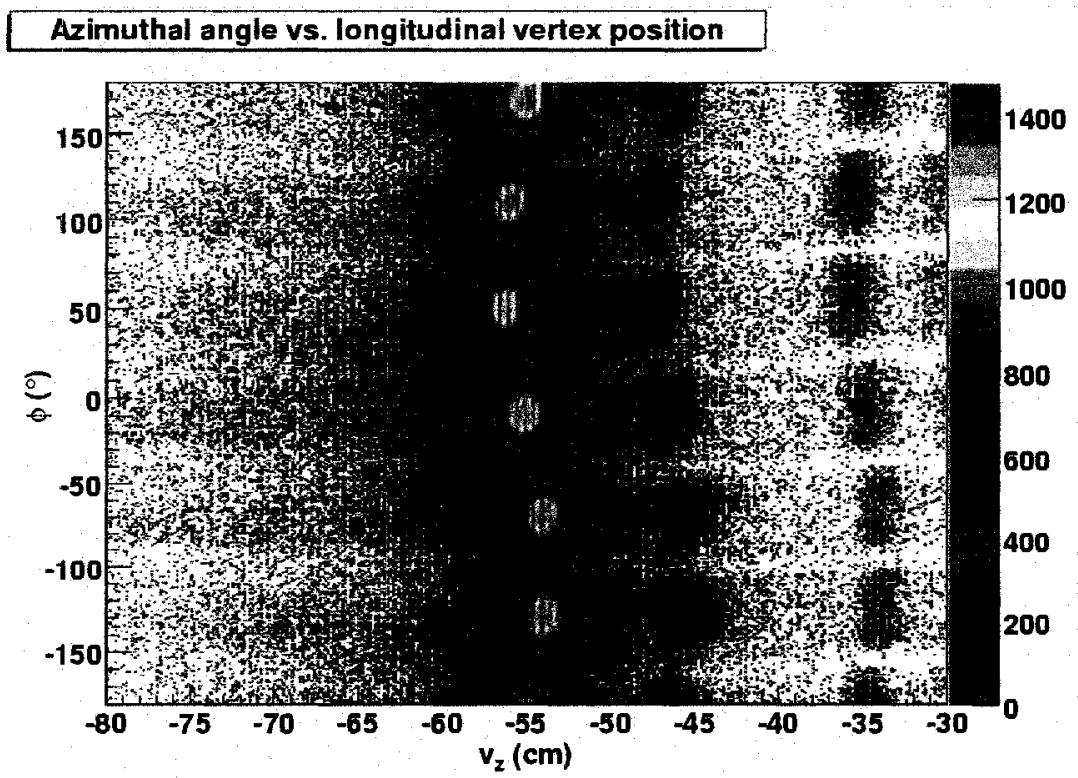

Azimuthal angle vs. Iongitudinal vertex position

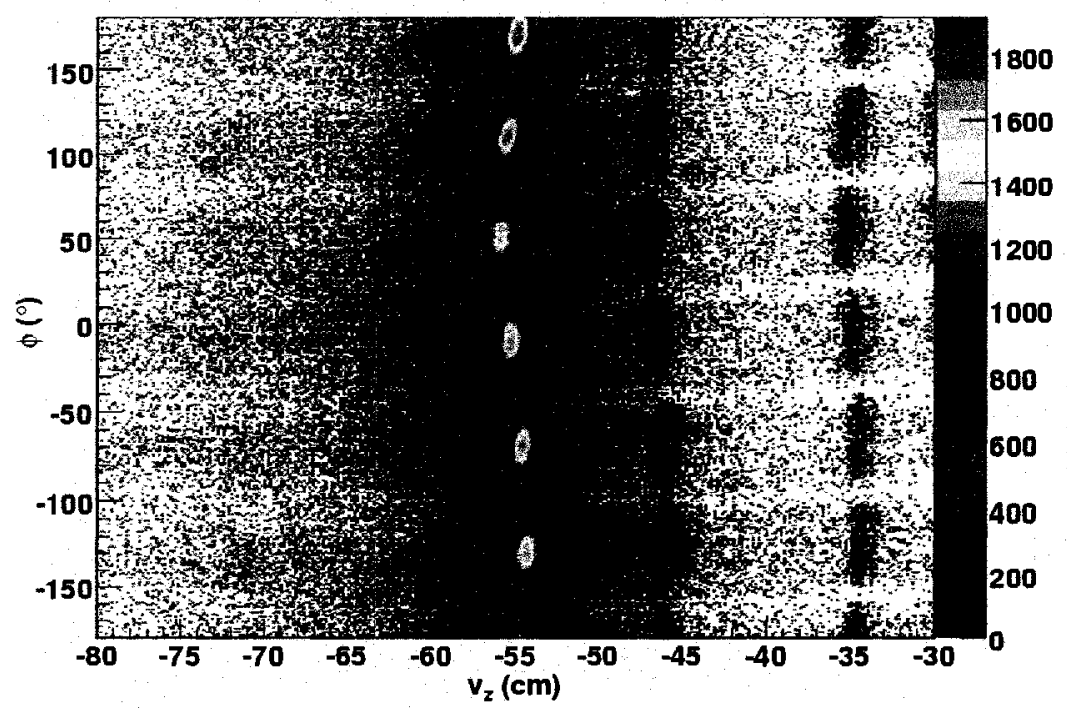

Figure 4.9: Plots of $\phi$ vs. $z$-vertex position before and after raster corrections, shown for $1.6 \mathrm{GeV}$ inbending data (no other kinematic corrections added). Note the significant increase in vertex resolution after the correction. The purple "strip" on the far right represents scattering from the vacuum shield/refrigerator end. These events are removed with a wide vertex cut (Section 3.6.1). 
However, for some runs, the torus current transducer reading occasionally fluctuated to a value that did not fall within the $\pm 0.2 \%$ limit of self-correction. Therefore, the wrong torus current was used in the event reconstruction. Also, in one case (the $4.2 \mathrm{GeV}$ outbending data), a fixed torus current was used, but the value was $10 \mathrm{~A}$ too high (i.e. $2260 \mathrm{~A}$ instead of $2250 \mathrm{~A}$ ). In these cases, the reconstructed particle momentum required correction to reflect the actual magnetic field value. Since the magnetic field $B$ is proportional to the torus current, and the measured momentum of the particle is proportional to the field, it is easy to see that the first-order correction to the initial (incorrect) momentum $p_{i}$ is given by

$$
p_{\text {true }}=\frac{B_{\text {true }}}{B_{i}} p_{i}=\frac{I_{\text {true }}}{I_{i}} p_{i}
$$

This leading-order correction suffices for the $0.4 \%$ torus current error in the $4.2 \mathrm{GeV}$ outbending data, as seen in Figure 4.10. Other run sets requiring the correction fluctuate within an even smaller limit, making this crude correction adequate. The torus scaling was applied to all $4.2 \mathrm{GeV}$ runs with negative torus current, ${ }^{13}$ as well as a few other data sets in which the used torus current incorrectly fluctuated (specifically, 2.3+, 2.5+, 2.5-, and 4.2+).

\subsubsection{Beam energy correction}

High precision knowledge of the beam energy prior to the electron-nucleon interaction is crucial for accurate kinematics determination. Therefore, precise determination of the beam energy electrons prior to interaction with the target and degradation due to the beam energy loss $d E / d x$ within the target are implemented into the kinematics correction package.

\section{Accurate determination of real beam energy}

Nominal beam energy measurements are supplied from the MCC (Machine Control Center) based on the number of passes through the accelerator and spin precession, measured by beam polarimeters [62]. This nominal number is limited in accuracy, however, and may not accurately reflect

\footnotetext{
${ }^{13}$ For the specific case of ${ }^{15} \mathrm{~N}^{12} \mathrm{C}$ analysis, the DSTs for involved nuns were regenerated with $I=2250 \mathrm{~A}$ tor precision measurements of the cross-section ratios [96]. For general calculation of the ditution factor, though, any accuracy gained thereby is overshadowed by other systematic errors, so the scaling method is deemed satisfactory for this analysis.
} 


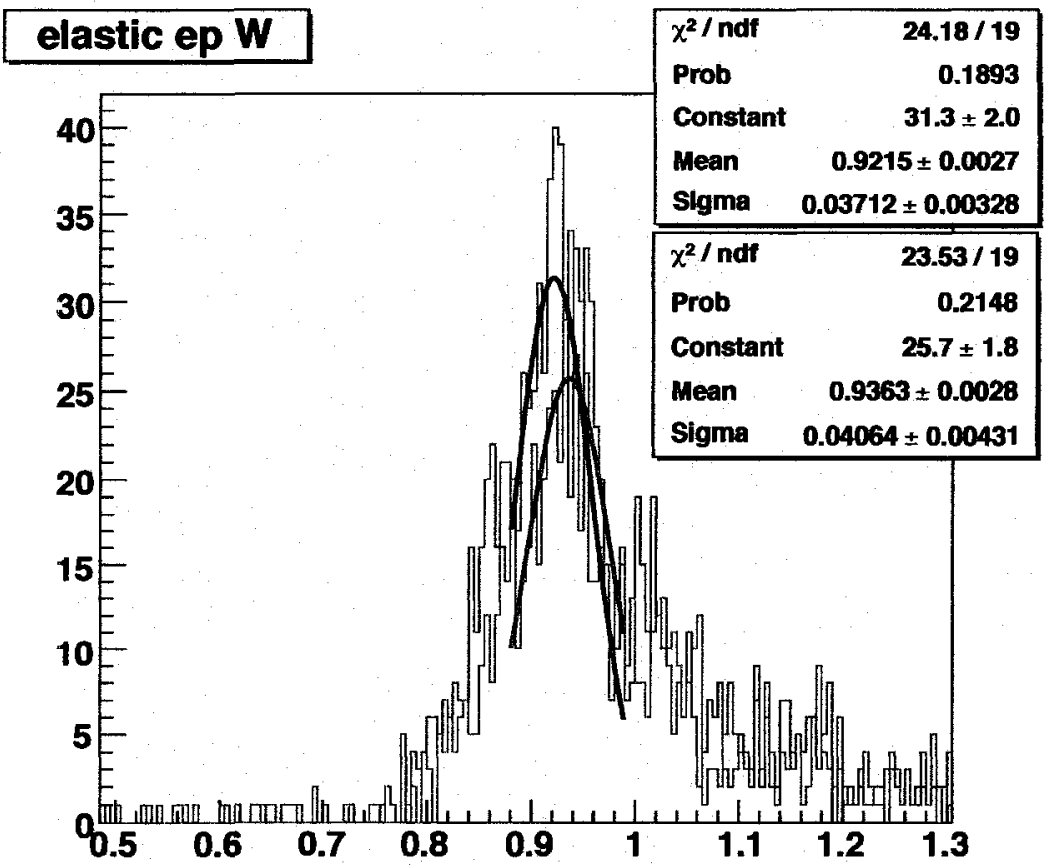

Figure 4.10: $4.2 \mathrm{GeV}$ inbending data before (blue, top stats box) and after (red, bottom stats box) torus current corrections. (Both plots are raster corrected, but no other kinematic corrections are applied.) The distribution width (sigma) is not changed much (and is actually not improved until later corrections), but the peak location is improved significantly due to the momentum scaling, moved closer to the true elastic value of $W=0.938 \mathrm{GeV}$. 
the true Hall-B beam energy. To better determine the delivered electron energy, accurate energy measurements made during the same time period by Hall-A were scaled by the relative number of passes of the beam through the accelerator to get the Hall-B energy.

Two methods were used by Hall-A to determine the beam energy, accurate within a factor of $\sim 2$ $\times 10^{-4}[108]$. The first method is a direct measurement of the beam energy as it passes through a series of magnets in the $40 \mathrm{~m}$ arc section between the accelerator and experimental Hall-A (see Figure 2.1), in terms of the magnetic field integral $\int \mathbf{B} \cdot \mathrm{dl}$ and the bend angle $\phi_{b}$, using the equation

$$
E=\frac{c}{\phi_{b}} \int \mathbf{B} \cdot \mathbf{d l}
$$

summed over the eight bend magnets. 14 The second method [109] utilizes a more complex iterative procedure utilizing Hall-A BPMs (calibrated against magnetic measurements from the 9th dipole of the arc magnets) combined with the field integral of Eq. 4.12.

Both methods gave very close results for the Hall-A beam energy. The average was assumed to be the correct Hall-A energy. Then, the average energy $E_{\ell}$ produced in each linac can be found from the Hall-A energy $E_{A}$ by

$$
E_{\ell}=\left(E_{A}-45 \mathrm{MeV}\right) / 2 N_{A}
$$

where $N_{A}$ is the number of accelerator passes made for the Hall-A beam, and $45 \mathrm{MeV}$ is the injector energy (see Section 2.2.1). The Hall-B energies are then found simply, depending on the number of accelerator passes delivered $\left(N_{B}\right)$, as

$$
E_{B}=2 N_{B} E_{\ell}+45 \mathrm{MeV}
$$

The validity of this relationship within a value of $2 \mathrm{MeV}$ can be verified by comparing Hall-A and Hall-C direct measurements from the same time period [110].

If the magnetic field map in Hall-B was precisely known, the exact beam energy could be verified by selecting elastic $e p$ events and measuring the scattering angle of each particle. The energy

\footnotetext{
${ }^{14}$ The magnetic field must be measured indirectly through comparison to a reterence magnet connected in series to the other magnets, since the arc is inaccessible. See Ret. [108] for more information.
} 
would then be given by [111]

$$
E \approx M_{p} \frac{\cos \theta_{e}+\left(\sin \theta_{e} / \tan \theta_{p}\right)-1}{1-\cos \theta_{e}}
$$

However, because of lack of precise knowledge of the magnetic field, the exact beam energy is required for performing momentum corrections (Section 4.2.8), to center the elastic peak at its correct kinematic location. Therefore, elastic ep events cannot also be used to calculate the Hall-B beam energy, and external sources must be relied upon instead. The scaled (Hall-A determined) vs. nominal (MCC) beam energies for EG1b are listed in Table 4.2.

Table 4.2: Nominal vs. actual beam energies for EG1b

\begin{tabular}{|c|c|c|}
\hline Set Label & Nominal Beam Energy(GeV) & Actual Beam Energy(GeV) \\
\hline \hline $1.6 \pm$ & 1.606 & 1.606 \\
\hline $1.7 \pm$ & 1.724 & 1.723 \\
\hline $2.3+$ & 2.288 & 2.286 \\
\hline $2.5 \pm$ & 2.562 & 2.561 \\
\hline $4.2 \pm$ & 4.239 & 4.238 \\
\hline $5.6 \pm$ & 5.627 & 5.615 \\
\hline $5.7+; 5.73-$ & 5.735 & 5.723 \\
\hline $5.76-$ & 5.764 & 5.743 \\
\hline
\end{tabular}

\section{Effective beam energy due to $d E / d x$ in matter}

At energies in the GeV range, energy loss due to Bremsstrahlung radiation outweighs the energy loss by atomic collisons by an order of magnitude or more [112]. However, the effects of Bremsstrahlung radiation are accounted for by external radiative corrections (Section 6.4), so they should not be accounted for prior to that point. Energy loss due to atomic collisions $\left(d E / d x_{\text {coll }}\right)$ prior to the interaction vertex, however, must be subtracted to accurately determine the initial electron energy, $E$. Since this, like all other corrections in this package, is a leading order correction, only a good estimate, not an exact value, for $d E / d x_{\text {coll }}$ is needed. Assuming a ${ }^{12} \mathrm{C}$ target ${ }^{15}$ and $\beta \gamma=p /(M c) \sim 2000$, the energy loss rate $d E / d x_{\text {coll }}$ measures as approximately $2.8 \mathrm{MeV} /\left(\mathrm{gm} / \mathrm{cm}^{2}\right)$

\footnotetext{
${ }^{15}$ This is a good approximation, since the value of $d E / d x$ is proportional to $Z / A$, which is roughly the same tor all the EG1 targets
} 
[5]; this value was used for the energy loss of electrons. The energy loss due to collisions remains fairly constant as a function of electron momentum in this energy range, so using a constant value is an adequate approximation. Using the modified vertex position $v_{z}$, the amount of energy loss can easily be calculated from the densities and lengths listed in Table 2.1 as

$$
\Delta E[\mathrm{MeV}]=2.8 \rho \Delta x
$$

with mass thickness $\rho \Delta x$ given by

$$
\rho \Delta x\left[\frac{\mathrm{g}}{\mathrm{cm}^{2}}\right]=0.4+0.6(0.5-\delta z)
$$

where $\delta z$ is the distance between $v_{z}$ and the target center $\left(\right.$ i.e. $\left.(-55.1 \mathrm{~cm})-v_{z}\right)$. The other numbers correspond to the mass thicknesses of target window materials plus $\mathrm{LHe}$ and $\mathrm{NH}_{3}(0.4)$, packing fraction (0.6), and target half-thickness (0.5). See Section 4.2.7 for details on these numbers. ${ }^{16}$ The average energy loss is typically $\sim 2.0 \mathrm{MeV}$. The effective energy $E_{b e a m}$ is adjusted accordingly downward to account for this loss.

\subsubsection{Multiple scattering correction}

After an $e N$ event occurs, the scattered particles usually still have a short distance left to continue traveling through the target material before continuing on to the detector. During this interval, the particle(s) undergo multiple scattering within the target material. Also, unwanted scattering can occur between the electron and detector components (especially the drift chambers). These multiple scattering collisions cause both a net angular deflection and displacement of the apparent vertex position (see Figure 4.11).

The GEANT Hall B simulation package GSIM [113] was used to study the effects of multiple scattering in the EG1 configuration [114]. The angular effects of multiple scattering produce an (approximately) Gaussian distribution about the mean angle, and are accounted for as part of the systematic error on the kinematic precision (see Section 7.2.6). The GSIM study showed, though,

\footnotetext{
${ }^{16}$ Specifically, use the same equations as in Section 4.2.7, excepting that the particle is entering, not leaving the target $(\delta z \rightarrow-\delta z)$, and $\theta=0$.
} 


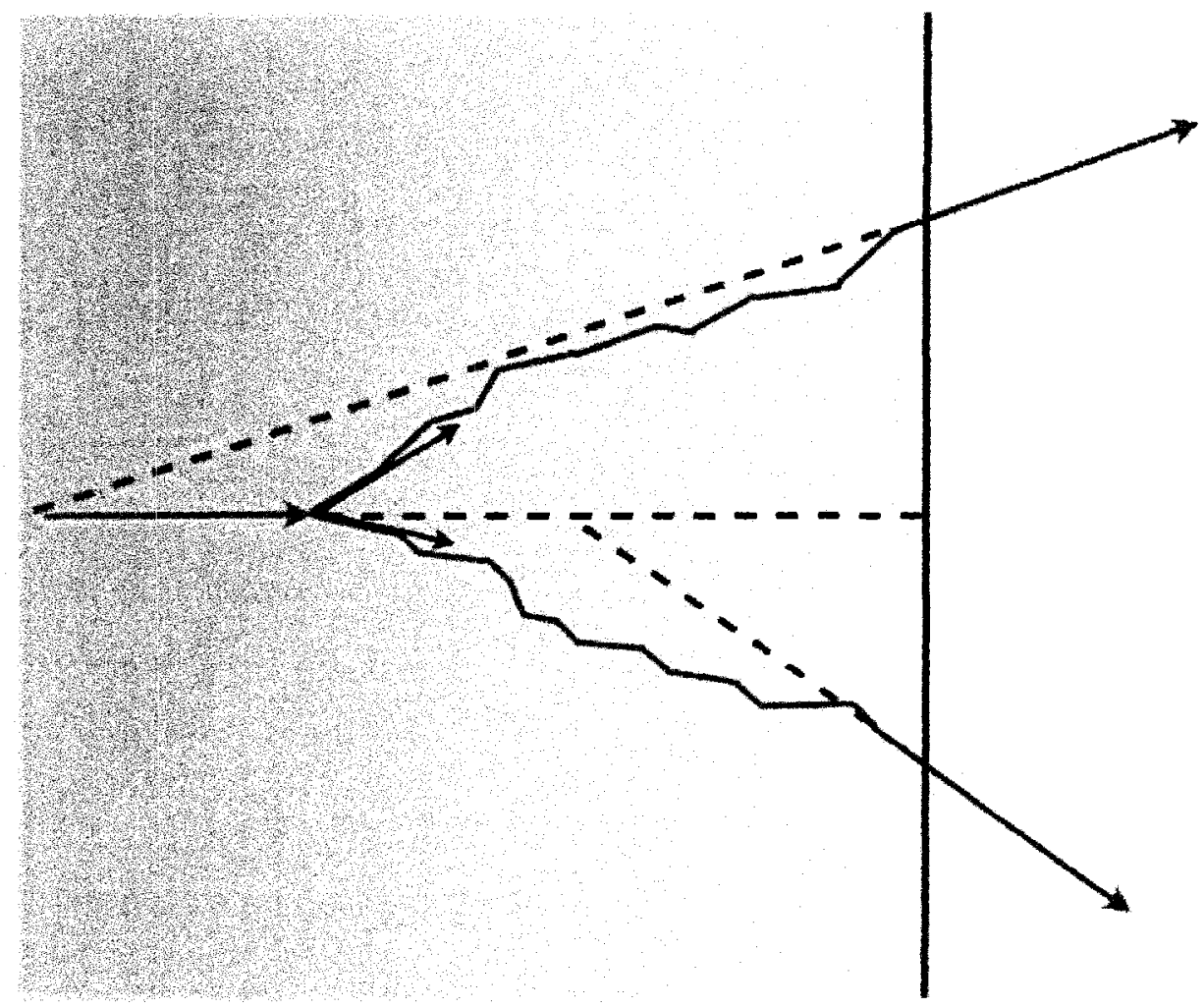

Figure 4.11: A greatly exaggerated picture of multiple scattering effects. The true vertex position and apparent angle are distorted by this effect. The black arrows show the true angles and vertex, while the blue dotted lines show the apparent angle and vertex positions of the scattered particles. Notice, in this case, that the true vertex position lies between the apparent vertex positions for each particle. While this is not necessarily the case for a specific event, using the weighted average of the vertices does, on average, increase the kinematic precison. 
that using the weighted average of all vertex positions

$$
\left\langle v_{z}\right\rangle=\frac{\sum v_{z i} / \sigma_{i}^{2}}{\sum 1 / \sigma_{i}^{2}}
$$

as the particle vertex (in place of the $v_{z}$ value for the individual particle) increased the accuracy of the determination of $\phi$ and $\theta$, where the weighting factor $\sigma$ is 17

$$
\sigma \equiv 0.10 /\left(\beta \sqrt{p_{x}^{2}+p_{y}^{2}}\right)
$$

The (raster-corrected) vertex positions for all particles in each event were used to calculate this average. This derived vertex position is then used as the "real" vertex position for all other corrections.

The GSIM study also showed a necessary correction to the polar angle $\theta$ correlating to multiple scattering within the Region 1 and Region 2 drift chambers (Section 2.5.2):

$$
d \theta=-\delta z(0.018 \theta+0.002 / p)
$$

where the particle momentum $p$ is measured in $\mathrm{GeV}$, and $\delta z \equiv v_{z}-\left\langle v_{z}\right\rangle$.

As in the case of raster corrections, a correction must also be made to the azimuthal scattering angle $\phi$, due to effects of the target magnet and the changed location of $v_{z}$. The GSIM parametrized correction needed is ${ }^{18}$

$$
d \phi=0.015 q \delta z / p
$$

where the charge $q= \pm 1$ as appropriate.

\subsubsection{Stray magnetic field correction}

The SEB track reconstruction software accounts for the target solenoid field in the vicinity of the target. However, it is unable to account for the effect of the field in the region of overlap with the first layer of drift chambers and outward (see Section 2.5.1). Along with the multiple scattering effects

\footnotetext{
${ }^{17}$ See the note in Ref. [114], Section 5 and Ref. [8] Eq. 2.86 (for example) for a justification of this weighting scheme.

${ }^{18}$ Unlike the case for the $\phi$-correction in $\mathrm{Eq}$. 4.10, this $\phi$-correction can affect elastic ep analysis, because the value of $\delta z$ can be different for both the electron and proton.
} 
(previous section), the GSIM software was used to parametrize the necessary corrections due to extension of the magnetic field into the inner detector components.

$$
d \theta=0.005(\theta-0.26) / p
$$

and

$$
d \phi=-0.0015 q \sqrt{2.2(\theta)} / p
$$

are the corrections, ${ }^{19}$ fit from a parametrization of the GSIM data, with $\theta$ and $\phi$ expressed in radians. A reevaluation of the beam energy using elastic $e p$ data and $\mathrm{Eq} .4 .15$ showed beam energies in much closer agreement with the the Hall-A scaled beam energies than previous studies after this correction was applied [114].

\subsubsection{Energy loss $(d E / d x)$ correction}

As explained in Section 4.2.4, particle energy loss through collisions within the target must be taken into account for accurate energy/momentum determination. The situation is slightly more complex for particles emerging from the target after the scattering event.

The correction is identical, in principle, to the beam energy loss correction, except that there is a polar scattering angle through the remainder of the target thickness, meaning the total energy loss of the scattered particle must be divided by $\cos \theta$. Also, while the collisional energy loss of 2.8 $\mathrm{MeV} / \mathrm{g}-\mathrm{cm}^{2}$ (see Section 4.2.4) can be used again for electrons, for which $\beta \approx 1$, collisional energy loss for hadrons is $\beta$-dependent, and can vary considerably.

Collisional energy loss in heavy ionized particles is governed by the Bethe-Bloch formula [8]:20

$$
-\frac{1}{\rho} \frac{d E}{d x}=4 \pi N_{a} r_{e}^{2} m_{e} c^{2} \frac{Z}{A} \frac{1}{\beta^{2}}\left[\ln \left(\frac{2 m_{e} c^{2} \gamma^{2} \beta^{2}}{I}\right)-\beta^{2}\right]
$$

\footnotetext{
${ }^{19}$ These equations are similar to, but not exactly the same as those listed in Ref. [114]. This is due to an update in the parametrization since Ref. [114] was written.

${ }^{20}$ We have used $W_{\max }=2 m c^{2} \beta^{2} \gamma^{2}$ (a valid highenergy assumption) and $\beta=v / c$ to get this form from that shown in Ref. [8]. The constant $r_{e}$ is the "classical electron radius", and $N_{a}$ is Avogadro's number.
} 
Using the approximations $Z / A \approx 0.5$, and $I \approx 10 \mathrm{eV} \times Z \approx 90 \mathrm{eV}$ (where $I$ is the mean excitation potential), this yields

$$
-\frac{1}{\rho} \frac{d E}{d x}\left[\frac{\mathrm{MeV}}{\mathrm{g} \mathrm{cm}}\right]=0.306 \times \frac{0.5}{\beta^{2}} \times\left[\ln \left(2 \times 511 \beta^{2} \gamma^{2} / 0.090\right)-\beta^{2}\right]
$$

which is the energy correction for scattered hadrons.

To calculate the total energy loss, $\Delta E$, the above quantity must be multiplied by $\rho \Delta x,{ }^{21}$ with

$$
\rho \Delta x\left[\mathrm{~g} / \mathrm{cm}^{2}\right]=\left[(\delta z+0.5 \mathrm{~cm}) \rho_{\mathrm{NH}_{3}}+\rho_{\mathrm{Al}} \ell_{\mathrm{Al}}+\rho_{\mathrm{K}} \ell_{\mathrm{K}}+\rho_{\mathrm{He}} \ell_{\mathrm{He}}\right] / \cos \theta
$$

where $\delta z$ is the distance between the target center $(-55.1 \mathrm{~cm})$ and the average vertex position (given by Eq. 4.18), $\theta$ is the polar scattering angle of the particle and $0.5 \mathrm{~cm}$ is the (approximate) distance from the center of the target material to the window. The remaining densities and lengths are given in Table 2.1. Using these values (for ammonia, aluminum, Kapton and liquid helium) ${ }^{22}$ we find

$$
\rho_{\mathrm{Al}} \ell_{\mathrm{Al}}+\rho_{\mathrm{K}} \ell_{\mathrm{K}} \approx 0.1 \mathrm{~g} / \mathrm{cm}^{2}
$$

and

$$
\rho_{\mathrm{He}} \ell_{\mathrm{He}} \approx 0.3 \mathrm{~g} / \mathrm{cm}^{2}
$$

We also have $\rho_{\mathrm{NH}_{3}} \approx 1 \mathrm{~g} / \mathrm{cm}^{3}$, but this must be multiplied by the packing fraction, that is, the fraction of the target volume actually occupied by solid ammonia. This quantity is derived formally in Section 5.5.1, and equals approximately 0.6 . Thus, we have

$$
\rho \Delta x\left[\mathrm{~g} / \mathrm{cm}^{2}\right]=[0.6(\delta z+0.5)+0.4] / \cos \theta
$$

${ }^{21} \frac{\Delta E}{\Delta x}=\frac{d E}{d x}$ can salely be assumed for a thin target and high energies.

22 Naturally, this equation holds "strictly" only for the ammonia target runs. However, the carbon target was chosen so that it has the same approximate radiation length $X_{0}$ as the ammonia target, so that the same correction is usable as an approximation. The ${ }^{15} \mathrm{~N}$ target is also approximately the same in dimensions and density as the ammonia target. The empty LHe target differs considerably in radiation length, but the emply target is only used as a small correction to the inclusive dilution factors, utilizing only high energy electrons, which are only minimally affected by $d E / d x$ corrections. Measurements of the total cell length $L$ also use the UHe target, but these again rely on an average across all DIS electrons compared to carbon, and are raciation length corrected. The $d E / d x$ corrections tor the ammonia target thus sufficed generally as a leading-order correction. 
for the desired quantity. This is multiplied by Eq. 4.25 (for hadrons) or $2.8 \mathrm{MeV} /\left(\mathrm{g} / \mathrm{cm}^{2}\right.$ ) (for electrons) for the appropriate correction to the outgoing particle energy. This value must be added to the measured energy, as it reflects energy lost in the target and missed by the detectors. ${ }^{23}$

Note that by no means is this an exact correction, due to all the approximations involved, and the margin of error involved in the calculation of the vertex position. The total internal target energy loss is generally less than a few $\mathrm{MeV}$ for inclusive electrons, ${ }^{24}$ though, making this approximation generally adequate for energy scales on the order of $0.1-5.0 \mathrm{GeV}$. Like the other kinematic corrections detailed in this section, it is only a first-order correction meant to improve the general precision and accuracy of particle momentum measurements, and provide as reliable as possible a starting point for the momenturn corrections, the most important and final part of the base correction package.

\subsubsection{Momentum corrections}

The kinematics corrections previously described account for the physical effects of known, directly quantifiable phenomena. These corrections serve to reduce the systematic biases in the measurement of particle momenta and angles (a necessary precursor to a complete momentum correction scheme [115]). However, the task still remains to account for the effect of "unknowns" on the particle kinematics, that is, effects that cannot, due to practical considerations, be accounted for by direct physical measurements or calculations. Such effects include (but are not limited to)

- Slight misalignment of the drift chambers from their nominally determined geometric positions

- Errors in the calculation of the location of drift chamber wire feedthrough holes

- Effects of gravitational sag and thermal expansion on the drift chamber wires

- Inexact knowledge of both the main torus and target solenoid magnetic fields

- Possible (but indeterminable) errors and/or miscalculations regarding the physical effects accounted for in previous stages of the kinematic corrections (e.g. raster ADC timing lag, unknown torus current offset, systematic timing errors missed during calibrations, etc.)

\footnotetext{
${ }^{23}$ The resolution limits of the $z$-vertex occasionally resulted in a particle with a reconstructed vertex outside the target window. In that case, the vertex was assumed to be on the target edge for purposes of the $d E / d x$ calculations.

${ }^{24}$ Multi-particle exclusive event data have have lower momentum and higher $d E / d x$. Low-momentum protons, for example, can lose up to $20 \mathrm{MeV}$ in this manner.
} 
These miniscule but cumulatively significant effects can definitely not be accounted for by specific physical corrections. Obviously, other, more indirect means must be used to correct for these effects.

\section{Momentum corrections: initial strategy}

The process used to precisely correct the EG1b particle momenta utilizes the fact that 4-momentum conservation is required for specific dynamic scattering processes (i.e. resonances). The particle momenta are corrected for these miscellaneous effects by the fitting of an expression, containing 14 different correction coefficients, corresponding to the most pertinent of the expected physical effects mentioned above. The expression is fit (separately, in each of the 6 CLAS sectors) to the momentum and scattering angles corresponding to these resonances, such that 4-momentum is conserved to the best possible precision [115].

Eight fit parameters $(A-H)$ were used to parametrize the effect of drift chimber dislocations (relative to Region 1) on $p$ and $\theta$ in terms of $p, \theta$ and $\phi$ (with $\phi$ written in sector coordinates, that is, $\left(\phi-\phi_{S}\right) \rightarrow \phi$, where $\phi_{S}$ marks the center "baseline"of the sector).25

$$
\begin{gathered}
\Delta \theta=(A+B \phi) \frac{\cos \theta}{\cos \phi}+(C+D \phi) \sin \theta \\
\frac{\Delta p}{p}=\left((E+F \phi) \frac{\cos \theta}{\cos \phi}+(G+H \phi) \sin \theta\right) \frac{p}{q B_{\text {torus }}}
\end{gathered}
$$

Terms are included to correct for offsets in the radial position (factor $\cos \theta / \cos \phi)^{26}, \phi$-dependent radial displacements (i.e. rotations around the $z$-axis; factor $\phi \cos \theta / \cos \phi$ terms), displacements along the $z$-axis (factor $\sin \theta)^{27}$, and rotations about the $\phi$-direction (factor $\phi \sin \theta$ ). The quantity $B_{\text {torus }} \equiv \int B_{\perp} d l$ along the track path is given by [116]

$$
B_{\text {torus }}=0.76 \frac{I_{\text {torus }} \sin ^{2}(4 \theta)}{3375 \cdot \theta} \quad(\theta<\pi / 8)
$$

\footnotetext{
${ }^{25}$ Note no $\phi$-correction terms are included. This is because $\phi$-coordinates are calculated with a larger intrinsic uncertainty, so that the precision corrections in this phase would have little, if no noticeable effect on the final resolution [115].

${ }^{26}$ The factor of $\cos \theta$ arises from a vertical offset $\Delta y$ because the offset in $p$ and $\theta$ becomes largest at forward (smaller) angles. The $1 / \cos \phi$ factor arises because of the fiatness of the drift chambers and because the particle track in $\phi$ is only perpendicular to the DC surface at $\phi=0$, the sector center.

${ }^{27}$ The factor of $\sin \theta$ arises from a horizontal offset because the effect of the offset is greatest at larger potar angles.
} 


$$
B_{\text {torus }}=0.76 \frac{I_{\text {torus }}}{3375 \cdot \theta} \quad(\theta \geq \pi / 8)
$$

with $\theta$ given in radians.

As well as mechanical displacements of the drift chambers, differences between the real magnetic field and the field map used in event reconstruction must be taken into account. As these corrections are a function only of the field geometry, no $p$-dependence is expected. To account for this effect, the terms

$$
J \cos \theta+K \sin \theta+L \sin (2 \theta)+(M \cos \theta+N \sin \theta+O \sin (2 \theta)) \phi
$$

were added to Eq. 4.31. ${ }^{28}$ Note that each variable $A-O$ represents six actual variables, one for each sector. From this point on, it is implicitly assumed that the fit variables $A, B, C, \ldots, O$ are actually $A_{s}, B_{s}, C_{s}, \ldots O_{s}$ where $s=1 \ldots 6$ corresponds to the sector number of the particle.

Drift chamber dislocation and magnetic field uncertainty are the two greatest impediments to accurate momentum determination, and are the effects directly accounted for by the terms in the momentum corrections. However, other, smaller effects on the momentum not accounted for earlier are also absorbed into the $14 \times 6=84$ coefficients used in the momentum corrections, as the missing momenta in exclusive reactions are centered exactly at zero by the correct choice of coefficients. For this reason, care was taken that prior corrections were not be altered in any way once the coefficients $A-O$ were determined.

To determine the coefficients, the transverse momenta $\left(p_{x}, p_{y}, p_{z}\right)$ of elastic ep scattering events were analyzed. For each event, the missing momenta and energy were calculated:

$$
\begin{gathered}
p_{x_{m i s s}}=p_{x_{e}}+p_{x_{p}}=p_{e} \sin \theta_{e} \cos \phi_{e}+p_{p} \sin \theta_{p} \cos \phi_{p} \\
p_{y_{m i s s}}=p_{y_{e}}+p_{y_{p}}=p_{e} \sin \theta_{e} \sin \phi_{e}+p_{p} \sin \theta_{p} \sin \phi_{p} \\
p_{z_{m i s s}}=p_{x_{e}}+p_{z_{p}}-E_{b e a m}=p_{p} \cos \theta_{e}+p_{p} \cos \theta_{p}-E_{b e a m} \\
E_{m i s s}=p_{e}+\sqrt{p_{p}^{2}+M^{2}}-M-E_{b e a m}
\end{gathered}
$$

\footnotetext{
${ }^{28}$ Unlike the drift chamber position terms, the field correction terms were arrived at by trial-and-error [115], and are not easily respresented term-by-term as dependent on phenemonological corrections.
} 
Then, the functions for $\Delta p$ and $\Delta \theta$ were added to the values of $p$ and $\theta$ in the missing momentum, to find values of the coefficients $A-O$ that brought the $\chi^{2}$ of these values closest to zero. Eqs. 4.30 - 4.33 were applied (starting with all coefficients $A-O$ at zero), the substitution

$$
\theta \rightarrow \theta+\Delta \theta ; p \rightarrow p\left(1+\frac{\Delta p}{p}\right)
$$

was made, and the missing 4-momenta (Eq. 4.34-4.37) were recalculated.

The sum

$$
\Delta \chi^{2}=\sum_{\text {events }}\left(\frac{E_{m i s s}^{2}}{\sigma_{E}^{2}}+\frac{p_{x_{m i s s}}^{2}}{\sigma_{p_{x}}^{2}}+\frac{p_{y_{m i s s}}^{2}}{\sigma_{p_{y}}^{2}}+\frac{p_{z_{m i s s}}^{2}}{\sigma_{p_{z}}^{2}}\right)+\sum_{\text {sector }=1}^{6} \sum_{a=1}^{14} \frac{X_{a}^{2}}{\sigma_{X_{a}}^{2}}
$$

was then minimized (through iterative reapplication of the correction equations), resulting in the best values for the parameters $A-O$ in each sector using the MINUIT minimization package. ${ }^{29}$ The intrinsic measurement uncertainties for the 4-momenta are set to $\sigma_{E}=\sigma_{p_{x}}=0.020 \mathrm{GeV}$ and $\sigma_{p_{x}}=\sigma_{p_{y}}=0.014 \mathrm{GeV}$.

The sum over coefficients at the end of Eq. 4.39 is added in order to prevent the problem of "runaway solutions" for the fit variables (a common problem in fitting large numbers of variables in any fitting algorithm). Here the $X_{a}$ are the 14 fit variables $A-O$ specific to each sector. An intrinsic uncertainty of 0.001 was used for $\sigma_{X_{a}}$, except for the $\phi$-dependent displacement terms ( $F$ and $H$ ), which have a larger intrinsic uncertainty $\left(\sigma_{F}=\sigma_{H}=0.01\right)$.

Once MINUIT optimized the fit variables, the correction Eqs. $4.30-4.33$ were applied to $p_{x}=$ $p \sin \theta \cos \phi, p_{y}=p \sin \theta \sin \phi$ and $p_{z}=p \cos \theta$ as part of the kinematics correction package, prior to writing these quantities to the ROOT tree files. This method makes a significant improvement to both the precision and accuracy of the elastic peak location, as the cumulative effect of the corrections shows (Figure 4.12).

\footnotetext{
${ }^{29}$ Minimization of 84 variables might appear to be formidable task for MINUIT. However, many of the constants are eftectively decoupled trom one another (e.g. variables in different sectors; magnetic field from DC displacement constants (if inbending and outbending are both included), etc.), and the stable equilibrium diplacement is close enough to the initialization point that the values rapidly converge when Eq. 4.39 is applied [117].
} 

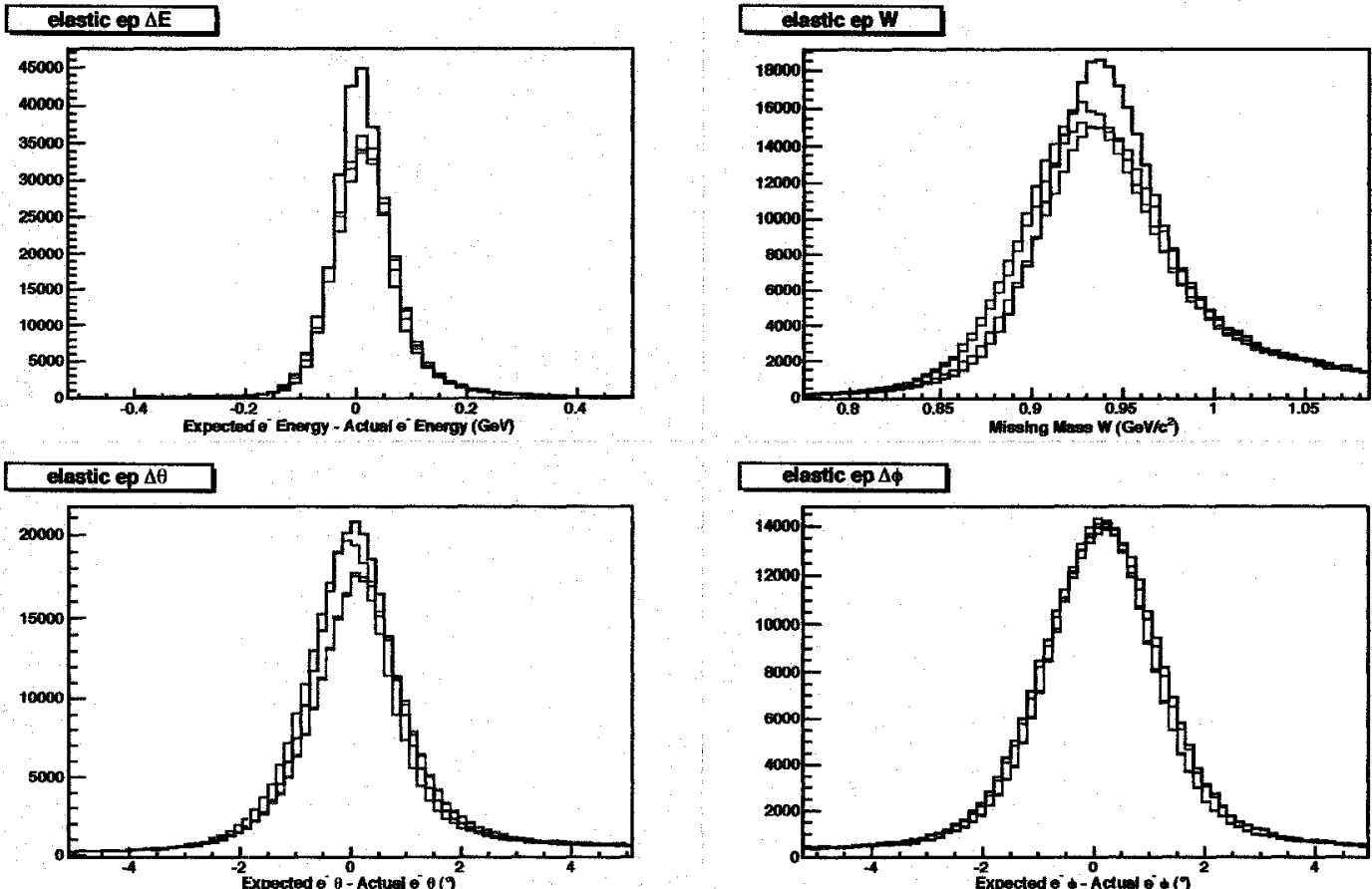

Figure 4.12: Deviations of elastic ep scattering kinematics (of the electron) from the values expected for elastic events. Missing energy, theta, and phi values are expected to be centered at zero, while invariant mass $(W)$ should equal the proton mass $(0.938 \mathrm{GeV})$. (See Section 3.5 for calculation of these quantities.) The blue histograms represent data with raster and torus scaling corrections only; the red histrogram adds energy loss and beam energy corrections; green adds stray target field and multiple scattering corrections; and black marks the addition of final sector-dependent momentum corrections. Combined $e p$ data from all 2.x $\mathrm{GeV} \mathrm{NH}_{3}$ runs are shown. 


\section{Additional corrective terms}

After the completion of momentum corrections, there were still a couple minor problems with the proper centering of the elastic $W$-peak between inbending and outbending sets, when using the same correction constants for every set. It was determined that the resolution of the elastic missing mass peak could be improved if the total momentum was slightly shifted for the outbending (i.e. negative torus current) sets:

$$
\left(\frac{\Delta p}{p}\right)_{\text {final }}=\frac{\Delta p}{p}+T_{s e t}
$$

This minor correction was necessary to preserve the ease of drawing from a single set of constants with uniform utility. As one can see from a quick look at the $T_{\text {set }}$ values in Table 4.4, they are very small corrections indeed. In the largest case, less than half a percent of an adjustment is made to the total momentum.

It was very difficult to get a correct fit of the parameters to low momentum particles, due to their sensitivity to changes in the coefficients. Since these particles do make some contribution to the elastic peak, ${ }^{30}$ it is possible to include correction terms that give a slightly better improvement to elastic events if a correction is also applied directly to $p$ instead of just to $\Delta p / p$. In practice, two terms are added to the total momentum, one of them a factor of $\phi^{31}$

$$
p_{\text {final }}=p+Q+R \phi
$$

where $Q$ and $R$ are sector-dependent constants of opposite signs. While these are negligible corrections for high momentum particles, they contribute a comparably larger percentage to the correction as momentum decreases, since the terms are added directly to the momentum, not the ratio $\Delta p / p$.

After adding these additional torus polarity-dependent and low-momentum corrective terms, the fit was redone, and found to give a better distribution for the elastic peak region than Eqs. 4.30 4.33 alone.

\footnotetext{
${ }^{30}$ One can calculate $M y=2 E \sin ^{2}(\theta / 2)$, for elastic events. Thus, these events are at the largest measurable scattered angles in CLAS

${ }^{31}$ The angle $\phi$ is defined, as before, in the sector coordinate system.
} 


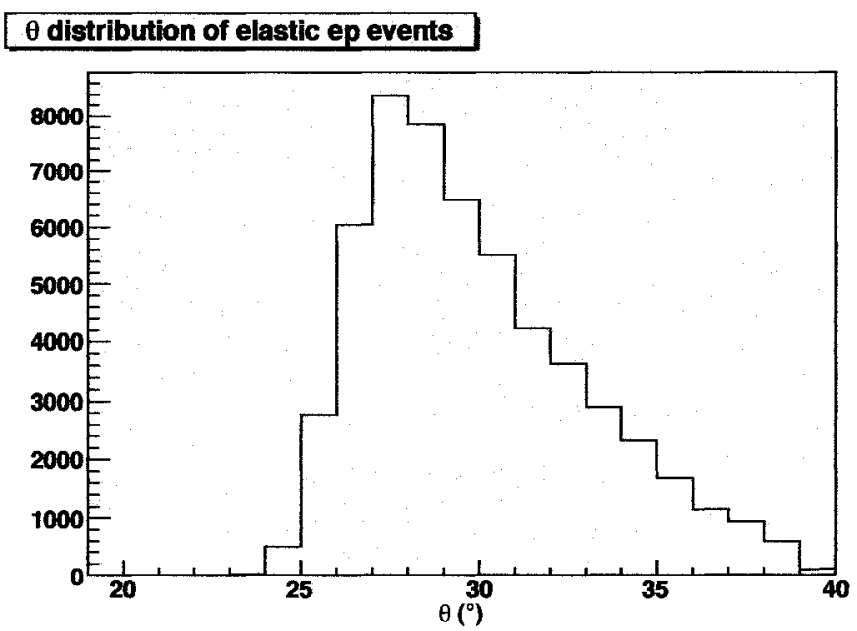

Figure 4.13: Distribution in $\theta$ of elastic $e p$ events for the $2.5 \mathrm{GeV}$ outbending data. Approximately equal numbers of events were sampled from each shown bin for momentum correction. Bins with too little data for a significant sample were not used in the fit at all.

\section{Proper weighting of the correction function in $\theta$}

Because the distribution of scattering events is a statistical function, the correction factor must be weighted properly as a function of the magnetic field and detector geometry. The scattering is homogeneous in $\phi$, so this coordinate constitutes no problem. However, the distribution is definitely not homogeneous in $\theta$ (see Figure 4.13), leading to a heavier weighting of the function in certain regions (peaking at $\theta_{e}=27^{\circ}$ in the shown set). The correction function should not be given preferential weighting for events in any specific part of the detector geometry.

To correct for the possibility of improper weighting, elastic ep events were divided into $1^{\circ} \theta$-bins, and only a certain percentage of events in each bin (selected randomly) were used in making the MINUIT fit. The percentage used for each bin was chosen so that the distribution of the elastic $e p$ events used for the momentum correction was constant in $\theta$, eliminating any possibility of a weighting bias introduced by the strong polar angle dependence of the detected elastic events.

In spite of this precaution, extension of the fit to far forward scattering angles $\left(\theta_{e}<12^{\circ}\right)$ still proved to be problematic, as there simply were not enough exclusive scattering events in this kinematic range for a reliable fit. This problem is addressed in Section 4.3. 


\section{Extending the fit to the resonance and DIS region}

Although this fit gives satisfactory results for elastic ep events at $W=M=0.938 \mathrm{GeV}$, the question remains open as to whether extrapolation of the fit to the inelastic region $(W>1.077)$ gives equally valid results. To ensure a reasonable fit to the data at all kinematics, a scattering reaction was chosen for the fit in the inelastic region, in addition to the elastic ep events. The exclusive reaction

$$
e p \rightarrow e p \pi^{+} \pi^{-}
$$

is a dominant decay channel, just far enough in the inelastic region $(W \approx 1.23 \mathrm{GeV}$ ) to serve this purpose, while still providing enough statistics to contribute significantly to the fit, assuming an appropriate weighting scheme is used.

The process for incorporating these events is virtually identical to that used for elastic $e p$ events. Events where all 4 outgoing particles can be identified (by time-of-flight) are used to reconstruct the given events. Then, 4-momentum conservation is enforced for the sum over all particles at the vertex (resulting in twice as many terms for the fit equation parameters in the equations analogous to Eq. $4.34-4.39$, of course). For each configuration of beam energy and torus current, the ratio of $e p \pi^{+} \pi^{-}$to $e p$ events used for the corrections was approximately $1: 5 .{ }^{32}$

Study of the distributions of missing transverse and longitudinal and momenta for exclusive $e p \pi^{+} \pi^{-}$reactions before and after corrections [114] show that the momentum corrections improve the kinematic fit in the inelastic as well as elastic region. It is important to keep in mind that these fits are iterative in nature. After a fit is done with the initialization of $A-R$ and $T_{\text {set }}$ all to zero initially, it is redone with improved values of these coefficients until stable values are reached. Final values of the correction coefficients are listed in Table 4.3. If done correctly, only one set of fit parameters is needed, regardless of the beam energies and torus currents used. ${ }^{33}$

With these final corrections applied, the most accurate and precise momentum corrections possible with the available data can be ensured, as the procedure utilizes well-known reactions and the actual data, as opposed to externally measured parameters. Two differing scattering processes

\footnotetext{
${ }^{32} 10000 \mathrm{ep \pi ^{+ }} \pi^{-}$and $50000 \mathrm{ep}$ events per set were used, specifically. If 10000 inelastic events were unavailable, then the fit was simply done with a lower ratio of inelastic to elastic events. Also, no theta-dependence scaling was employed for the $e p \pi^{+} \pi^{-}$events.

33The sole exception is the single $T_{\text {set }}$ correction variable.
} 
(one elastic and one inelastic) are used, removing the inherent uncertainty of extrapolating from a single value of invariant mass $W$. Figure 4.14 demonstrates the net improvement of missing momentum as function of $\phi$ as a result of this correction.

Table 4.3: Sector-dependent Momentum Correction Parameters for EGtb.

\begin{tabular}{|c|c|c|c|c|c|c|}
\hline Parameter & Sector 1 & Sector 2 & Sector 3 & Sector 4 & Sector 5 & Sector 6 \\
\hline \hline$A$ & 0.00091 & 0.00085 & -0.00005 & -0.00084 & -0.00152 & -0.00162 \\
\hline$B$ & -0.00265 & -0.00112 & -0.00425 & 0.00269 & -0.00052 & 0.000145 \\
\hline$C$ & -0.00369 & -0.00465 & -0.00130 & 0.00103 & 0.00147 & 0.00091 \\
\hline$D$ & 0.00236 & 0.00266 & -0.00156 & -0.00363 & -0.00355 & -0.00534 \\
\hline$E$ & 0.00003 & -0.00063 & -0.00423 & 0.00239 & 0.00041 & 0.00072 \\
\hline$F$ & 0.02302 & 0.01214 & 0.01677 & -0.01380 & 0.00404 & -0.02218 \\
\hline$G$ & 0.00261 & 0.00715 & 0.00510 & -0.00439 & -0.00065 & -0.00552 \\
\hline$H$ & -0.03800 & -0.01755 & -0.01946 & 0.02098 & -0.00409 & 0.04574 \\
\hline$J$ & 0.00117 & -0.00593 & -0.00277 & 0.00258 & 0.00273 & 0.000992 \\
\hline$K$ & -0.00348 & 0.00304 & -0.01295 & -0.01154 & -0.780 & -0.00584 \\
\hline$L$ & -0.00000976 & -0.00000899 & 0.00000164 & -0.0000162 & -0.0000183 & -0.00000878 \\
\hline$M$ & -0.00200 & -0.00393 & 0.00 & -0.00400 & -0.00678 & 0.00319 \\
\hline$N$ & -0.00778 & -0.01507 & -0.01295 & -0.01491 & -0.00755 & -0.00623 \\
\hline$O$ & -0.0001340 & -0.0000603 & 0.0000082 & 0.0000144 & -0.0000485 & 0.0000755 \\
\hline$Q$ & 0.00196 & 0.00183 & 0.00120 & 0.00117 & 0.00080 & 0.00139 \\
\hline$R$ & -0.00094 & -0.00463 & -0.00486 & -0.00523 & -0.00120 & -0.00437 \\
\hline
\end{tabular}

Table 4.4: Torus current-dependent parameter $T_{\text {set }}$ for outbending sets.

\begin{tabular}{|c|c|}
\hline Set & $T_{\text {set }}$ \\
\hline \hline $1.6-$ & -0.000159 \\
\hline $1.7-$ & 0.000705 \\
\hline $2.5-$ & 0.000308 \\
\hline $4.2-$ & 0.003203 \\
\hline $5.6-$ & $-1.64 \times 10^{-12}$ \\
\hline $5.73-$ & 0.000854 \\
\hline $5.76-$ & -0.000589 \\
\hline
\end{tabular}

\subsection{Momentum Correction at Low $\theta$}

The momentum corrections described in the previous section are satisfactory for the correction of data in kinematic regions where exclusive $e p$ and $e p \pi^{+} \pi^{-}$events are plentiful, which is over most of the CLAS acceptance. However, at low values of $\theta$ (the electron scattering angle) there are no 

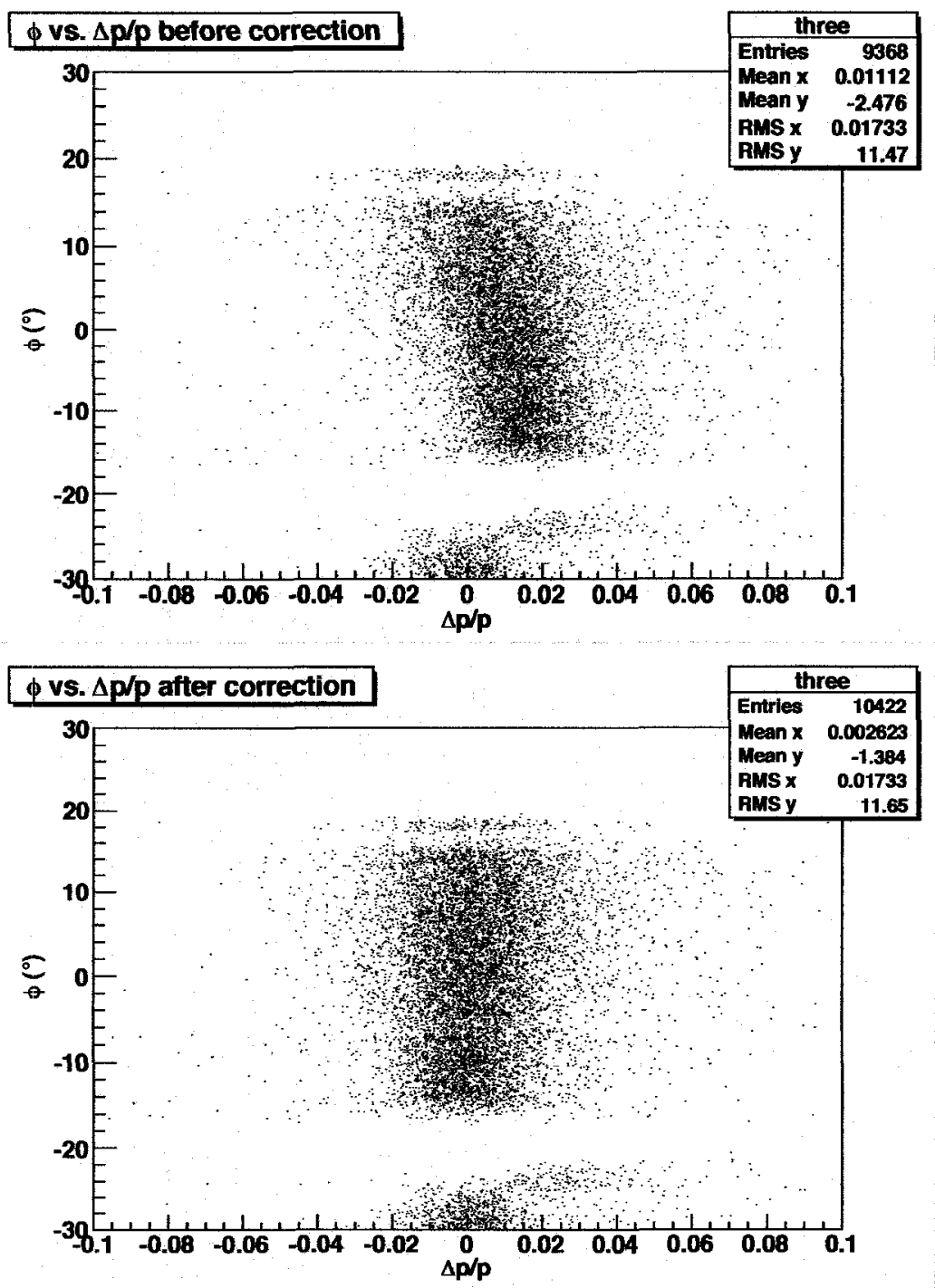

Figure 4.14: Plots of $\phi$ vs. $\Delta p / p$ for elastic $e p$ events before and after sector-dependent momentum corrections, shown for sector 3 for $2.5 \mathrm{GeV}$ outbending data. The mean value of $\Delta p / p$ is greatly improved after application of the correction. 
elastic $e p$ events ${ }^{34}$ and very few $e p \pi^{+} \pi^{-}$events. Therefore, in the region $\theta \lesssim 12^{\circ}$, the momentum corrections of Section 4.2 .8 are using an unreliable extrapolation of the momentum correction functions into the forward angle region. A large proportion of the inclusive data (especially at low $Q^{2}$ ) lies in this low angle range, so that accurate momentum corrections are essential for a complete analysis.

Study of inclusive scattering events at low angles, after momentum corrections were complete, indeed showed that the elastic peak location was not consistent as a function of $\phi$. Only inclusive scattering events consistently cover this kinematic region, meaning that momentum conservation at the vertex cannot be employed to correct the kinematics. Instead, a correction function in terms of $\phi$ and $\theta$ is fit to the momentum $p$ such that the offset of the elastic missing mass peak value $W_{\text {peak }}-M$ is minimized.

P. Bosted and N. Guler developed, through trial and error, a fit function that changed rapidly enough in $\theta$ to accomodate the complex magnetic field in the forward region:

$$
E_{f}^{\prime}=E_{i}^{\prime}\left\{1+0.02\left[U+\left(V+W \frac{1}{30^{\circ}}\left(\phi-30^{\circ}\right)\right)\left(\frac{10^{\circ}}{\theta}\right)^{3}\right]\right\}
$$

where $U, V$ and $X$ are fit coefficients, determined independently in each of the 6 sectors. ${ }^{35}$ Separate parameters are required for inbending and outbending torus currents, but there is no dependence on the torus current magnitude or beam energy. Note that the listed values (in Table 4.5) correspond to negatively charged particles. Particles with positive charge require use of the coefficients corresponding to the opposite polarity.

Because inclusive electrons are scattered from $\mathrm{NH}_{3}$ molecules, not just free protons, the $\mathrm{W}$ peak is not just an elastic peak, but rather an elastic peak superimposed on a broader quasi-elastic background, contributed by scattering from the ${ }^{15} \mathrm{~N}$ nucleus. To better locate the true $W$-position of the elastic peak (rather than the position of the peak plus background), it is useful to divide the normalized count rate of $\mathrm{NH}_{3}$ events by that of the $\mathrm{ND}_{3}$ target scattering events. ${ }^{36}$ Then, the ${ }^{15} \mathrm{~N}$

\footnotetext{
${ }^{34}$ This is due to an inability to detect back-scattered protons, at large angles where the target solenoid and support frame cut off the acceptance.

${ }^{35} \mathrm{Here}, \theta$ and $\phi$ are determined at the inner layer drift chamber, not the event vertex.

${ }^{36}$ In $2.3 \mathrm{GeV}$ inbending, where $\mathrm{ND}_{3}$ is unavailable, ${ }^{12} \mathrm{C}$ was substituted. This gives similar results, though with less statistics.
} 


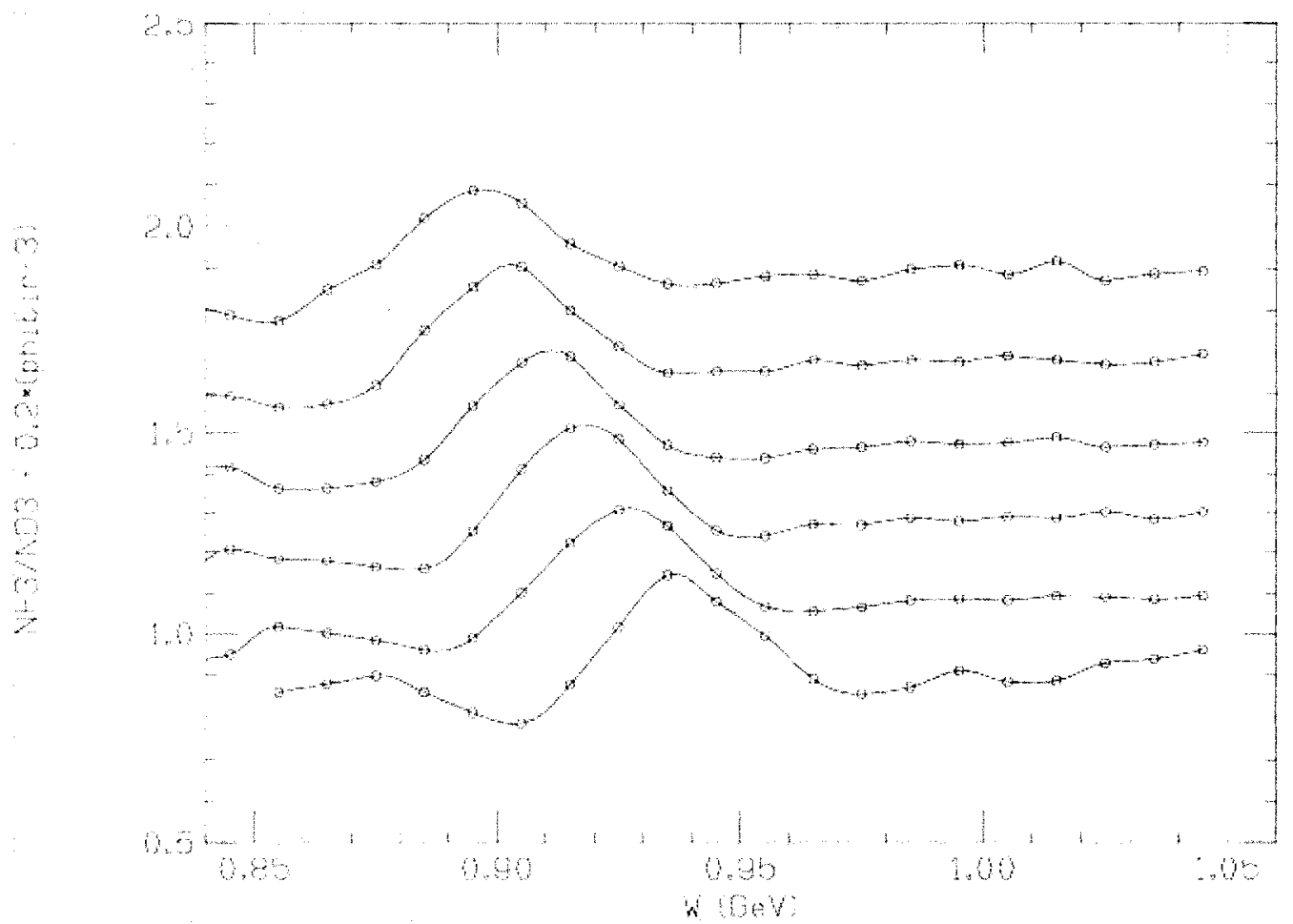

Figure 4.15: Ratios of $\mathrm{NH}_{3} / \mathrm{ND}_{3}$ spectra for six different $\phi$-values in Sector 1 , at $\theta<13^{\circ}$, separated by an arbitrary offset for visibility. Corrections are obviously needed to center the peaks at the proper elastic value of $W=0.938 \mathrm{GeV}$. Plot courtesy P. Bosted.

background cancels in the ratio, leaving the ratio of the free proton elastic peak to the deuteron quasi-elastic peak. The deuteron peak is considerably more narrow than the ${ }^{15} \mathrm{~N}$ peak, resulting in considerable narrowing and more precise location of the exact elastic peak position. This ratio was calculated in $10^{\circ} \phi$ and $1^{\circ} \theta$ bins, and the peak location $W_{\text {peak }}$ was then simply the maximum point of this ratio. ${ }^{37}$ Figure 4.15 shows a (pre-correction) example of this peak ratio in each of the 6 sectors.

The coefficients $U-X$ were then fit using MINUIT such that

$$
\sum_{\theta, \phi} W_{p e a k}(\theta, \phi)-M \rightarrow 0
$$

An example of the fit, made individually for each sector, is shown in Figure 4.16. Sector 6 did not

\footnotetext{
${ }^{37}$ This assumes a reasonable $W$-range of course. At extreme low $W$, where statistics are low, the count ratio can trail spuriously up to extreme values that are obviously not associated with elastic events.
} 


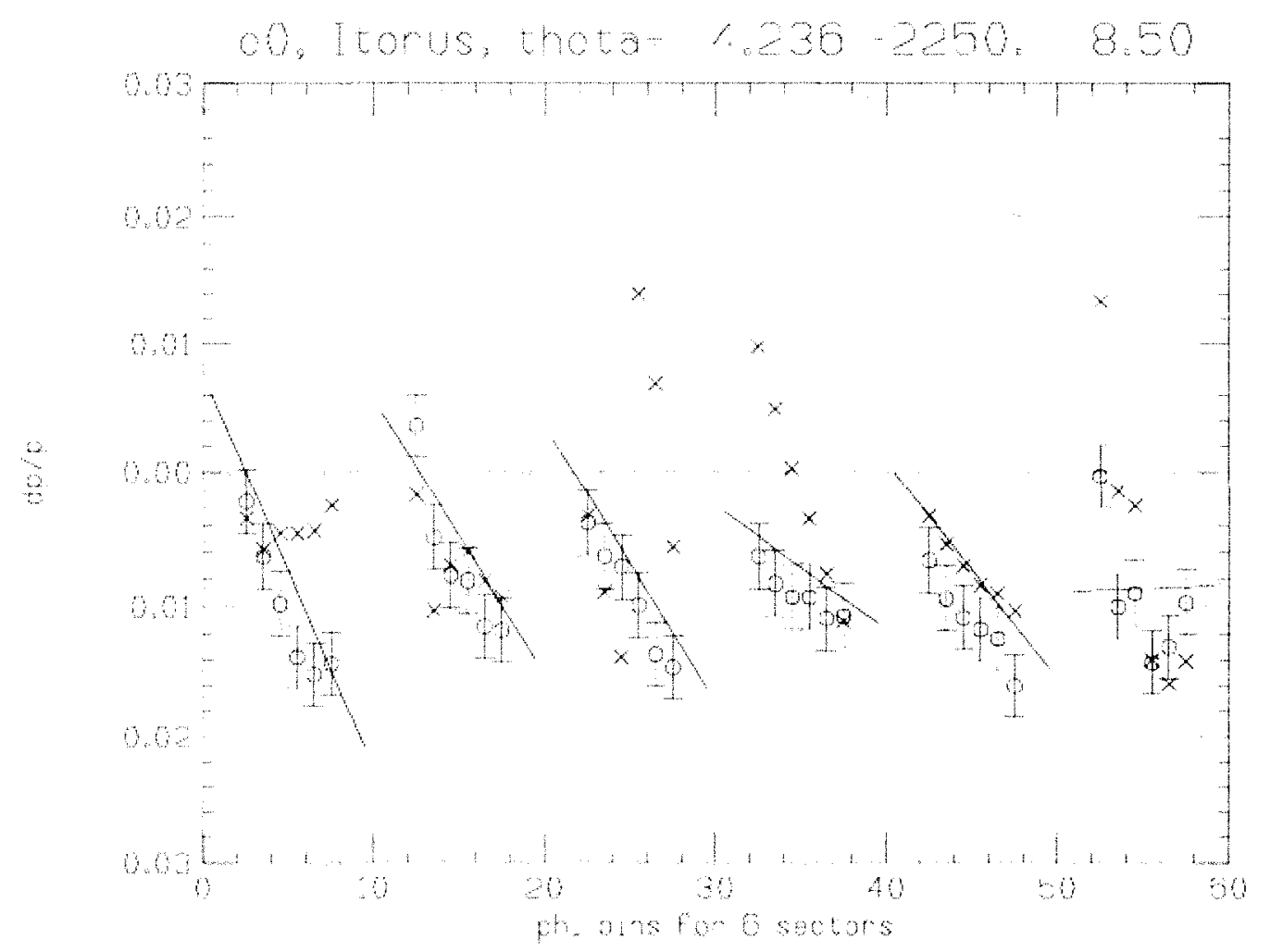

Figure 4.16: A fit of the low angle momentum corrections for $8^{\circ}<\theta<9^{\circ}$ for $4.2 \mathrm{GeV}$ outbending data, shown for missing momentum $\Delta p / p$. Lines represent a fit to the data points after the corrections of Section 4.2.8. Error bars are estimated, and depend only on beam energy. The $x$ points represent the data before the momentum corrections of Section 4.2.8. Plot courtesy P. Bosted.

behave according to any easily fit function; the improvement in this sector is only slight compared to the other five.

Fit coefficients in each of the 6 sectors are listed in Table 4.5. The fit function is designed to maximize influence at forward angles (manifested by the inclusion of the $10^{\circ} / \theta$ term). It has little effect in higher $\theta$ regions, where the momentum corrections of Section 4.2 .8 have already adequately calibrated the momentum. At small $\theta$, however, the corrective effect is quite significant (see Figure 4.17), and indeed necessary for consistent kinematic determination between energy sets.

In practice, the correction was most effective for data with low beam energies and outbending torus current, where low $Q^{2}$ data are most abundant. This "patch" correction was thus applied only to the data sets where improvement in the elastic peak resulted, namely all outbending data where $E_{\text {beam }} \leq 4.2 \mathrm{GeV}$, as well as $1.6 \mathrm{GeV}$ inbending data. Note these coefficients were determined 

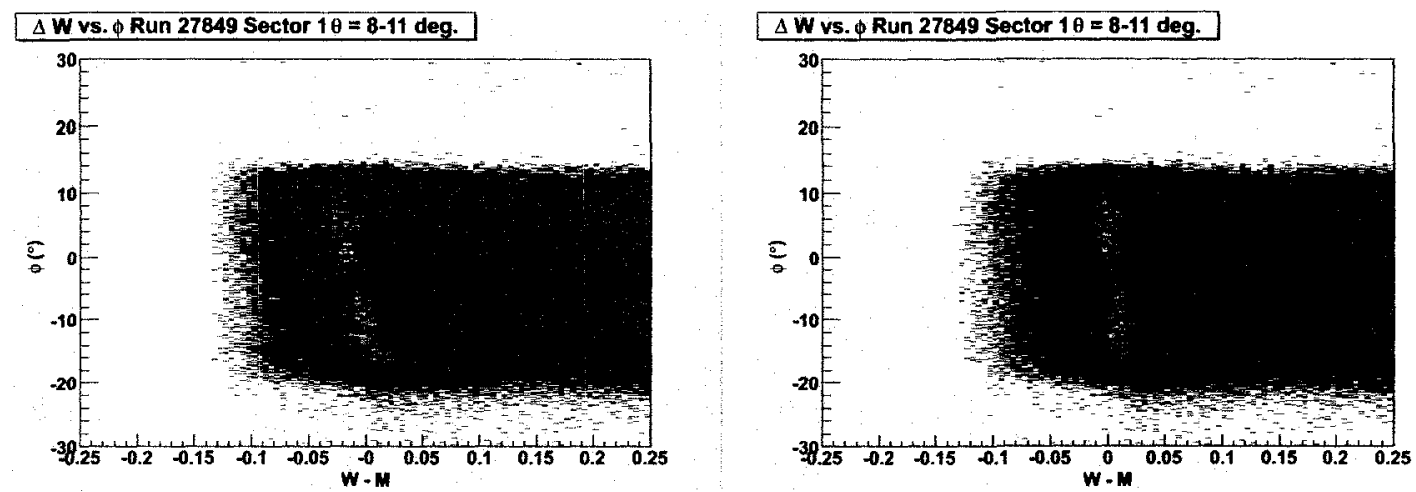

Figure 4.17: Inclusive electons for $\theta<11^{\circ}$ shown for Sector 1 , from one run of $2.5 \mathrm{GeV}$ outbending data. The histograms represent events in $\phi$ vs. $W-M$ before (left) and after (right) the special low$\theta$ momentum corrections. In both cases, the standard corrections of Section 4.2 have already been applied. The corrections are not perfect, but do show a marked improvement, without sacrificing the smoothness ensured by a global correction.

independently of the momentum corrections of the previous section, only after the previous corrections were completed. Figure 4.18 shows the net effects of all the kinematic corrections on some sampled data.

Table 4.5: Sector-dependent forward-angle correction coefficients for EG1b, for inbending and outbending electrons. For positively charged particles, coefficients corresponding to the opposite torus polarity are used.

\begin{tabular}{|c|c|c|c|c|c|c|}
\hline Parameter & Sector 1 & Sector 2 & Sector 3 & Sector 4 & Sector 5 & Sector 6 \\
\hline \hline Outbending & \multicolumn{7}{|c|}{} \\
\hline$U$ & -0.0472 & -0.0378 & -0.2485 & -0.0066 & 0.0257 & -0.5182 \\
\hline$V$ & -0.2235 & -0.1650 & -0.0532 & -0.2370 & -0.2588 & 0.1406 \\
\hline$X$ & -0.2730 & -0.1789 & -0.4687 & -0.1929 & -0.1733 & -0.2743 \\
\hline Inbending & -0.2238 & -0.2621 & -0.0024 & -0.1918 & -0.1217 & -0.1203 \\
\hline$U$ & -0.2786 & 0.3348 & -0.4065 & 0.3624 & 0.2378 & 0.1846 \\
\hline$V$ & -0.963 & -0.748 & 0.713 & 0.591 & -0.032 & -1.070 \\
\hline$X$ &
\end{tabular}

\subsection{Fiducial Cuts}

As already noted, the calculation of asymmetries does not require knowledge of the acceptance of different detector regions. However, the calculation of dilution factors (see Chapter 5) requires 

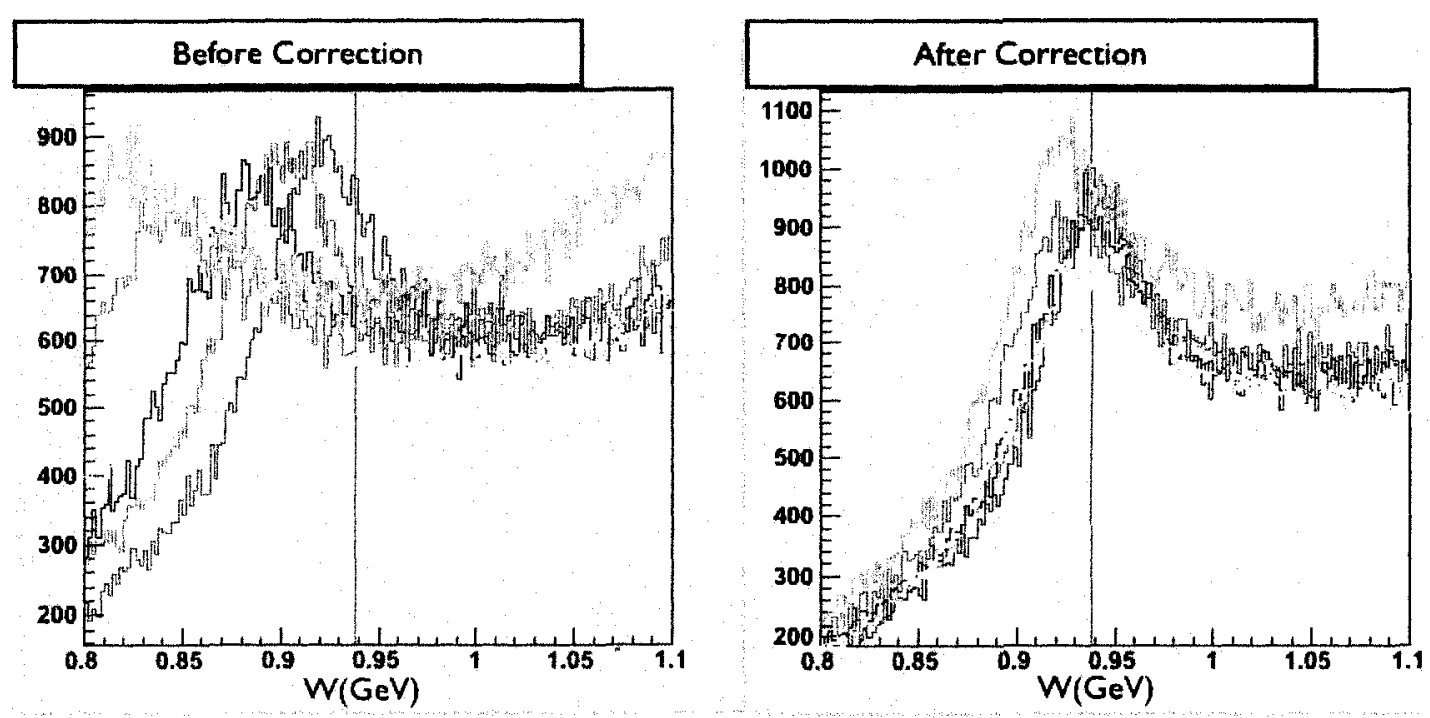

Figure 4.18: Net effect of kinematic corrections at $\theta=13^{\circ}$ for $4.2 \mathrm{GeV}$ outbending data on the inclusive event spectrum. Various colors represent differing $\phi$-bins, all in Sector 3 . Plots courtesy N. Guler.

that the acceptances of the ${ }^{12} \mathrm{C}$ and $\mathrm{LHe}$ runs are the same as the acceptance of the $\mathrm{NH}_{3}$ runs, as dilution factors are directly dependent on the ratios of counts from these differing targets. The kinematic acceptance of a detector is a direct function of the geometry of the apparatus. Therefore, specific regions of the detector where the acceptance is not well-understood must be removed from the analysis.

Drift wire chamber and scintillator components of the CLAS detector (i.e. the DC, SC, and EC) can be considered as "perfectly" efficient devices, in that they respond to practically $100 \%$ of inclusively scattered electrons. ${ }^{38}$ Due both to limitations incurred by properties of the Cherenkov gas, and reflective loss within the mirror geometry, however, the Cherenkov Counter (CC) exhibits definite inefficiencies in the identification of electron triggers.

The purpose of fiducial cuts, then, is to remove electron hits in inefficient regions of the Cherenkov Counter from the data used in any acceptance-dependent calculations. In an inefficient region of the $\mathrm{CC}$, statistical fluctuation in the number of observed photoelectrons is too great to determine an accurate measurement of electrons, due to the narrow Poisson distribution of the photoelectrons.

\footnotetext{
${ }^{38}$ This is true during gated live time, assuming the momentum is not too low. This is part of the reason a low-momentum cut is used on inclusive data. The drift chamber is $98+\%$ efficient (assuming occupancies are not too high) [74], and scintillator devices (like the EC and SC) are generally nearly $100 \%$ efficient to high-energy electrons [8].
} 


\subsubsection{Determination of Inefficient CC Regions}

To implement the fiducial cuts, a criterion for determining the expected number of photoelectrons in each region of the Cherenkov Counter was established. A thorough study of the behavior of the $\mathrm{CC}$, using $1.6 \mathrm{GeV}$ data taken before the the $\mathrm{EG1b}$ data, ${ }^{39}$ has already been done by $\mathrm{A}$. Vlassov [120].

This study entailed measuring the average number of photoelectrons generated by hits from elastically scattered electrons as a function of $\theta$ and $\phi$ (measured at the EC/CC). Preliminary cuts were made in $W$, the fraction of particle energy deposited in the $\mathrm{EC}$, and the vertex reconstruction point in order to enforce a rudimentary exclusion of inelastic hits and pions. A limit on the deviation from the matching point between the EC and $\mathrm{CC}$ was also made to exclude poorly matched tracks. The average number of photoelectrons produced for these events was then recorded over the full area of the detector, as a function of $\theta$ and $\phi$, measured at the SC/EC plane. Once the geometric dependence of the expected number of photoelectrons was empirically determined, an applicable function was developed to determine the expected number of photoelectrons in the $\mathrm{CC}$ as a function of particle track coordinates.

To calculate the actual efficiency as a function of $\theta$ and $\phi$ in each sector, it was assumed that the number of photoelectrons generated by a hit in the detector region obeys a Poisson distribution. 40 For purposes of cutting down pion contamination, a lower limit of 2.0 photoelectrons was used for electron definition. The efficiency at any particular point in the $\mathrm{CC}$ can then be determined by finding the percentage of events in the Poisson distribution (with a mean value determined by $A$. Vlassov's function) that remain after all the events with less than 2.0 photoelectrons are removed from the distribution. Thus, the efficiency of a detector location can be expressed in terms of the expected photoelectrons $(\mu)$ and minimum photoelectron cutoff $(c)$ as

$$
\text { efficiency }=\sum_{n>c} \frac{\mu^{n} e^{-\mu}}{n !}
$$

\footnotetext{
${ }^{39}$ The E1b experiment, completed in 1999, was used.

${ }^{40}$ See Footnote 15 in Chapter 3.
} 
$\phi$ vs. $\theta$ (reconstructed $\phi$ )

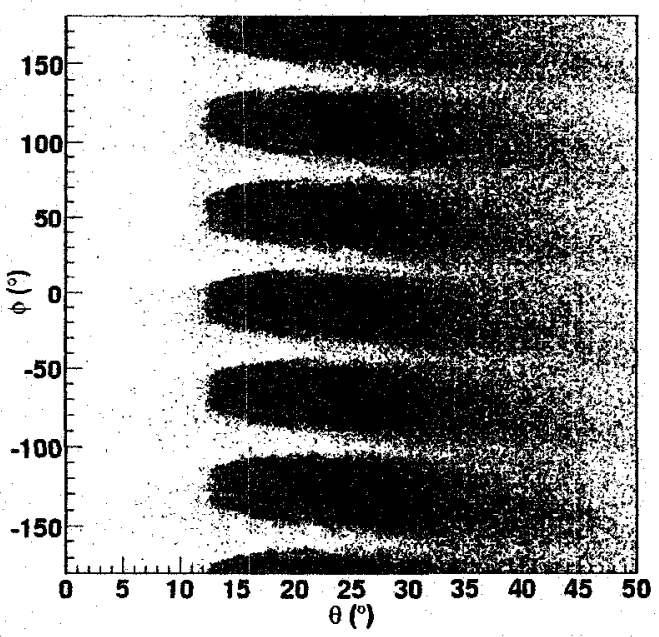

$\phi$ vs. $\theta$ ( $\phi$ measured at inner DC)

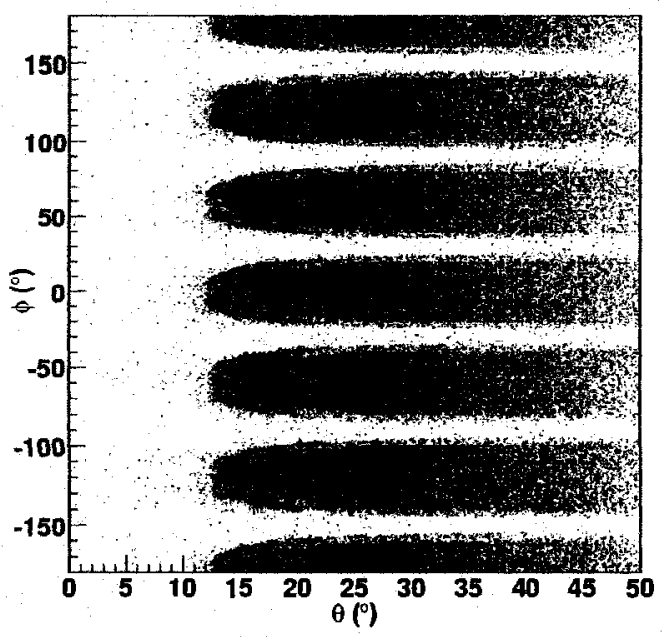

Figure 4.19: Plots of detected electrons in $\theta$ vs. $\phi$ for a quarter million $2.3 \mathrm{GeV}$ EG1b events, contrasting the measurement of $\phi$ as reconstructed at the vertex (left) against actual Region 1 DC coordinates (right). The inner drift chamber $\phi$ measurement (right) shows less distortion with respect to $\theta$, and is the value used for both determining and applying fiducial cuts.

An efficiency of $80 \%$ was a used as a cutoff for exclusion of inefficient CC detector regions, ${ }^{41}$ which corresponds to an approximate value of 4.3 expected photoelectrons in a Poisson distribution.

Plots in $\theta$ vs. $\phi$ were made for each sector, showing only events that registered above the calculated photoelectron threshold. Geometric cuts were then made on these plots to exclude regions that did not contain a significant density of events meeting this minimum requirement. The polar angle $\theta$ was measured as $\arctan \left(p_{z} / p_{r}\right)$ (reconstructed from the DC and SC tracking), but, due to the axial target polarization field, the azimuthal angle $\phi$ was measured at a point independent of the vertex. The innermost layer drift chamber provided a more geometrically stable determination of the $\phi$-coordinate (see Figure 4.19). ${ }^{42}$

\footnotetext{
${ }^{41}$ This is a somewhat arbitrary number. However, it was noted from the fiducial cut histograms that the dropoff in expected photoelectron rate occurs at such a steep slope with respect to location that even large variations in this value do not change the location of the cut boundary in any significant way.

${ }^{42}$ The sector-by-sector $\phi_{D C}$ coordinates translate to the equivalent reconstructed $\phi_{e}$ as $\phi_{e}\left(^{\circ}\right)=\phi_{D C}+60 \times$ (sector1) -30 , minus 360 if $\phi_{e}>180^{\circ}$.
} 


\subsubsection{Determining the Fiducial Cut Boundaries}

Different cuts were used for inbending and outbending electrons, due to the differing trajectories through the Cherenkov Counters corresponding to a given scattering angle. In both cases, a function symmetrical in $\phi$ that suitably evolved with the electron momentum was used. Inbending electrons were the simpler case, as only one cut curve was required, and each sector has a similar enough geometry that the same cut could be used for all six sectors. Outbending electrons showed greater variation in photoelectron efficiency from sector to sector, and required a more complex cut.

\section{Inbending Cuts}

For inbending electrons, the fiducial cut limits for $\phi$ and $\theta$ are given by

$$
30^{\circ}-\Delta \phi<\phi<30^{\circ}+\Delta \phi
$$

and

$$
\theta>\theta_{\text {cut }}
$$

where the cut limits $\Delta \phi$ and $\theta_{c u t}$ are defined by

$$
\Delta \phi=A \cdot\left(\sin \left(\theta-\theta_{\text {cut }}\right)\right)^{e x p}
$$

with

$$
\operatorname{exp.}=B \cdot\left(p_{e} \cdot \frac{3375 \text { amp. }}{I_{\text {torus }}}\right)^{C}
$$

and

$$
\theta_{\text {cut }}=D+\frac{E}{\left(p_{e}+F\right) \frac{3375 \text { amp. }}{I_{\text {torus }}}}
$$

In these equations, $p_{e}$ represents the electron momentum, $I_{\text {torus }}$ the torus current, and $A, B$, $C, D, E$, and $F$ are empirically determined constants. Roughly speaking, $A, B$ and $C$ control the width and curvature of the cut, while $D$ determines the minimum $\theta$-value of the cut curve, and $E$ 
and $F$ control the rate of evolution of the curve with momentum. ${ }^{43}$

To determine the values used for these empirical constants, a curve drawn using these equations was superimposed on sector-dependent $\phi$ vs. $\theta$ plots of particle events meeting the threshold of required expected number of photoelectrons, as explained in the previous section. Bins in $p_{e}$ of $0.15 \mathrm{GeV}$ were used, ranging from $10 \%$ to $100 \%$ of the beam energy (in GeV). Previous determinations of fiducial cuts in Hall B used fixed values of the empirical constants over large regions of the entire beam energy range, with no more than two sets of constants used for any set [2]. In order to better circumscribe the efficiency region, with no sharp "edges" cutting into the data between kinematic bins, values for the six empirical constants were slightly varied for each 0.15 $\mathrm{GeV}$ momentum bin, and the values were stored in an array. At $p_{e}>4.0 \mathrm{GeV}$, variables were no longer varied with momentum, as the pattern remained fairly stable. In addition, a parameter $\theta_{\max }$ was added to determine the location of the "corner" of the cut (i.e. the maximum $\theta$-value before $\phi$ becomes constant). A maximum polar angle ( $\theta$ ) limit of $49.5^{\circ}$ was also added to cut electrons scattering from the target solenoid. Inbending fiducial cuts for a couple of different momentum bins are shown in Figures 4.20 and 4.21.

The fiducial cuts are specifically dependent on the electron path geometry as a function of momentum. Thus, different fit coefficients must be assigned for each torus current value. The values of the coefficients outlined above, for both $+1500 \mathrm{~A}$ and $+2250 \mathrm{~A}$ main torus current, are summarized in Table B.3.

\section{Outbending Cuts}

Outbending electrons exhibited a more complex efficiency pattern than inbending electrons. Variation between sectors was too pronounced to use the same cut for every sector, so different cut coefficients were determined for every sector.

A slightly different outer curve was used for outbending electrons:

$$
30^{\circ}-\Delta \phi<\phi<30^{\circ}+\Delta \phi
$$

\footnotetext{
${ }^{43}$ In practice, $A, B$ and $D$ were adjusted, while the other three variables were left at nearly static values. Due to the narrow momentum binning and eventual interpolation of the variables, adjustment of the other parameters was seldom necessary.
} 

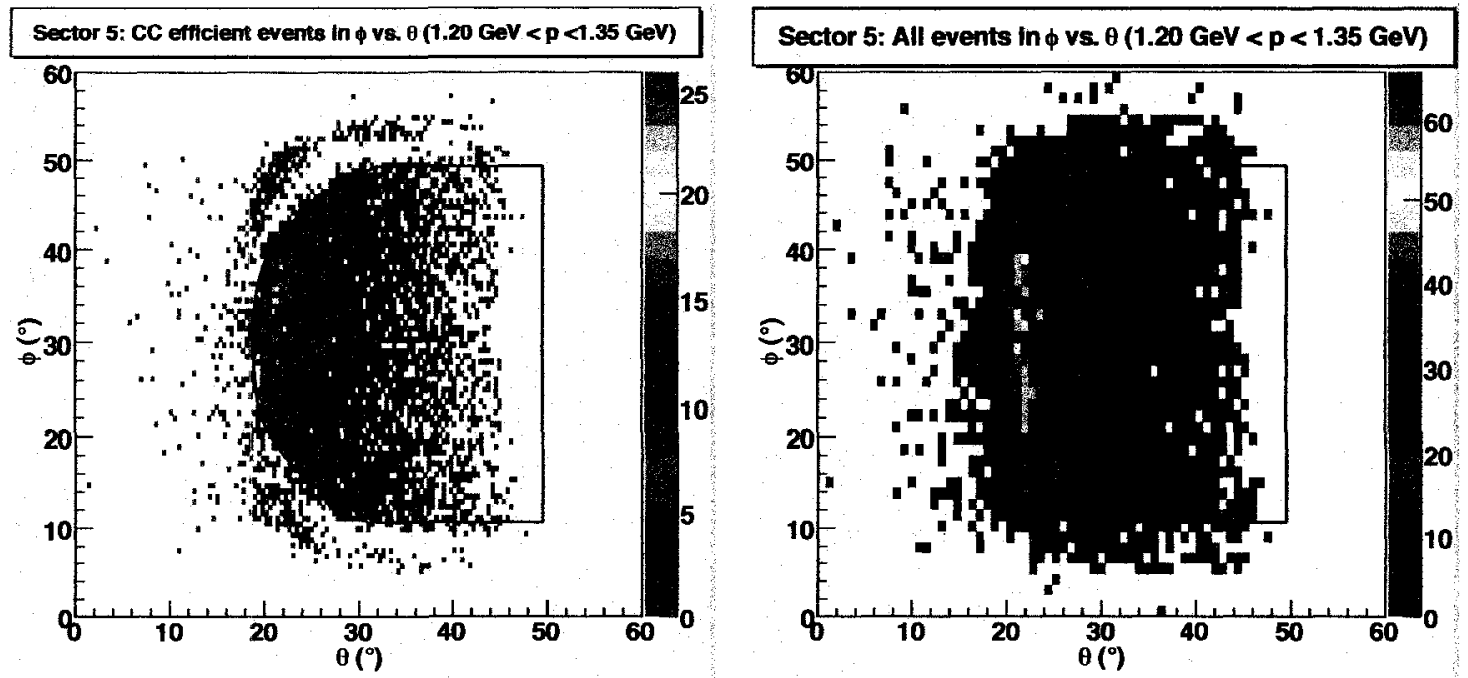

Figure 4.20: Inbending fiducial cuts for one momentum bin and sector for a torus current of 2250 A. The first figure shows only electron events meeting the $80 \%$ efficiency criterion as defined in the text. The second figure shows all potential electron events, demonstrating that these cuts remove a significant number of particles from the data. The excluded "eyebrow" structures that wrap around the first plot are direct particle impacts on the CC PMT.
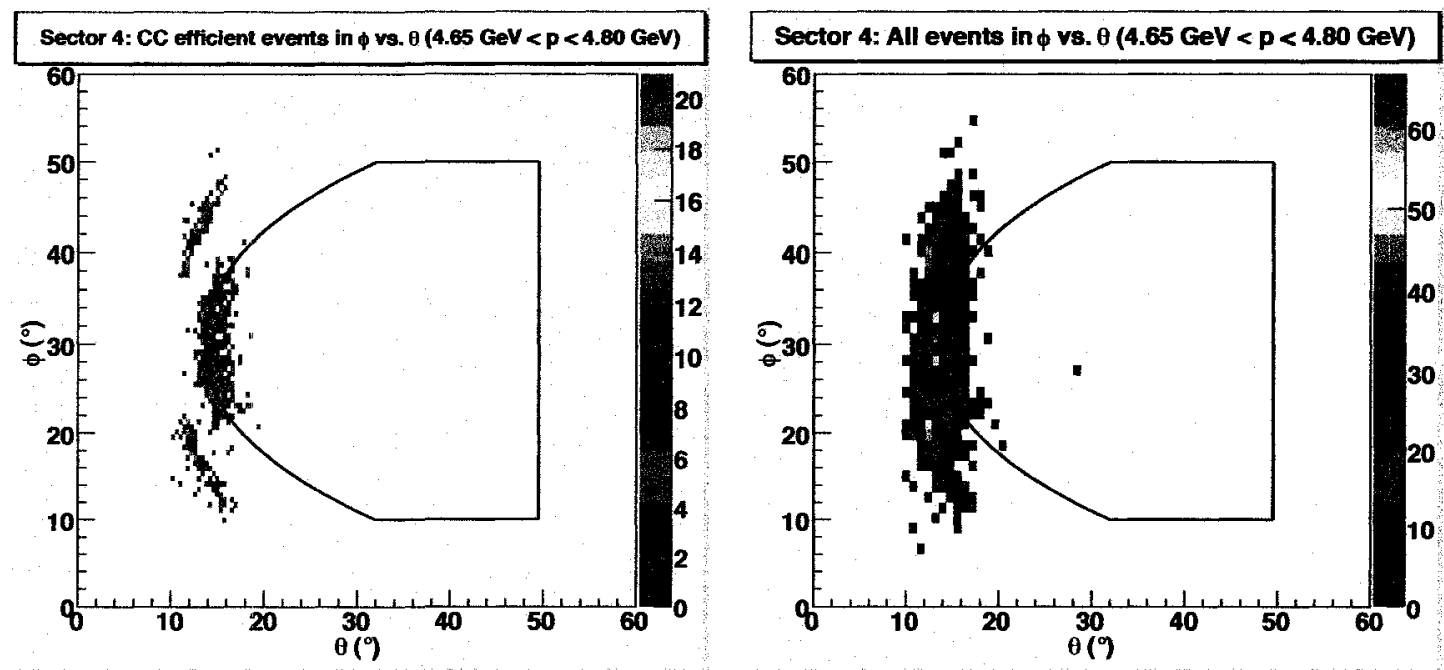

Figure 4.21: Same as Figure 4.20, except for a different (higher) momentum bin and different sector. 
and

$$
\theta_{\text {cut }}<\theta<\theta_{\text {high }}
$$

where

$$
\begin{gathered}
\Delta \phi=A \cdot\left(\sin \left(\theta-6.5^{\circ}\right)\right)^{e x p .} \\
\exp .=B \cdot\left(\frac{1}{4} p_{e}\right)^{C} \\
\theta_{\text {cut }}=D+E \cdot\left(1-\frac{1}{4} p_{\text {scale }}\right)^{F} \\
\theta_{\text {high }}=\min \left(40^{\circ}, \theta_{\text {nom }}\right) \\
\theta_{\text {nom }}=\frac{35^{\circ}}{(\mathrm{GeV} / \mathrm{c})^{1 / 3}} \cdot\left[\frac{1}{5}\left(p_{e^{-}} \cdot \frac{3375 \mathrm{amp} .}{\left|I_{\text {torus }}\right|}+2.5 \mathrm{GeV} / \mathrm{c}\right)\right]^{\frac{1}{3}} \\
p_{\text {scale }}=p_{e} \cdot \frac{1500 \mathrm{amp} .}{\left|I_{\text {torus }}\right|}
\end{gathered}
$$

Here, $A$ through $F$ are empirically determined constants, just as in the case of inbending cuts. Note that a maximum value in $\theta$ is used in order to exclude events that might miss the EC due to a large outbending angle. Additionally, the maximum $\phi$-dependent $\theta$ value of the cut boundary was parametrized, just as in the inbending case. However, due to asymmetry in $\phi$, different values of this angle were used for the upper $\phi$ and lower $\phi$ "corners" $\left(\theta_{\text {upper }}\right.$ and $\left.\theta_{\text {lower }}\right)$.

In many cases, the center strip along $\phi$ in each $\mathrm{CC}$ sector (corresponding to the mirror joint at the center "ridge" of the $\mathrm{CC}$ ) showed regions of low efficiency. To remove this center strip from the data, the following curve was used

$$
30^{\circ}+\phi_{c e n t e r}<\phi<30^{\circ}-\phi_{c e n t e r}
$$

where

$$
\phi_{\text {center }}=\frac{G}{\sin (\theta+H)}
$$

Due to pervasive asymmetric efficiency patterns with respect to $\phi$, separate parameters were determined for the upper and lower bounds of the curve, resulting in 4 (instead of 2) extra parameters $\left(G_{\text {upper }}, G_{\text {lower }}, H_{\text {upper }}, H_{\text {lower }}\right)$. In addition, two extra overall additive offsets in $\phi$ were included for 
both the inner and outer boundaries (inneroff $f$ set and outeroff $f$ set).

As in the case of inbending fiducial cuts, values of all these parameters were determined for each $0.15 \mathrm{GeV}$ momentum bin ranging between $10 \%$ and $100 \%$ of the beam energy (again varied only up to $4.0 \mathrm{GeV}$, and their values were recorded in a variable array. Some examples of outbending fiducial cuts for various momentum bins are included in Figure 4.22. Unlike the inbending cuts, outbending fiducial cuts differed for each sector. This resulted in a very large number of parameters. However, many of the parameters are similar in value, requiring only minor adjustments from sector-to-sector and bin-to-bin. The outbending fiducial cut parameters, for torus currents of both -1500 and $-2250 \mathrm{~A}$, are listed in Table B.5.

\subsubsection{Interpolation of Cut Parameters Between Momentum Bins}

To avoid sharp "edges" that can appear between bins when cuts changed suddenly (potentially with unpredictable effects on the measurement of count ratios near such an "edge"), the parameters were smoothly interpolated between momentum bins, and the fiducial parameters were made into a variable function of the particle momentum. The cut function was modified to make a uniquely determined fiducial cut boundary on each electron based on its individual momentum by interpolating the values of each parameter with a curve from bin to bin. A third-degree polynomial was fit in up to 4 (but usually only 2 or 3) momentum regions for each parameter to generate a variable cut function in terms of $\theta$ and $\phi$. All variable parameters were treated this way for inbending cuts (see Figure 4.23 for two examples). For outbending cuts, only the outer cut boundary was treated in this manner; the center strip was left determined by a discrete function in $p .44$

The interpolated values ${ }^{45}$ were then referenced (along with the discrete center-strip outbending parameters) in a $\mathrm{C}++$ function which returned an acceptable hit value only for electron events with $\theta$ and $\phi$ values within the designated cut boundaries.

\footnotetext{
${ }^{44}$ Evolution of the center strip cut parameters $G$ and $H$ varied too unpredictably between momentum bins to be reasonably interpolated by a polynomial fit.

${ }^{45}$ These are $A, B, C, D, E, F$ for both inbending and outbending cuts, plus $\theta_{\max }$ for inbending cuts or $\theta_{\text {upper }}, \theta_{\text {lower }}$ and outerof $f$ set tor outbending cuts.
} 

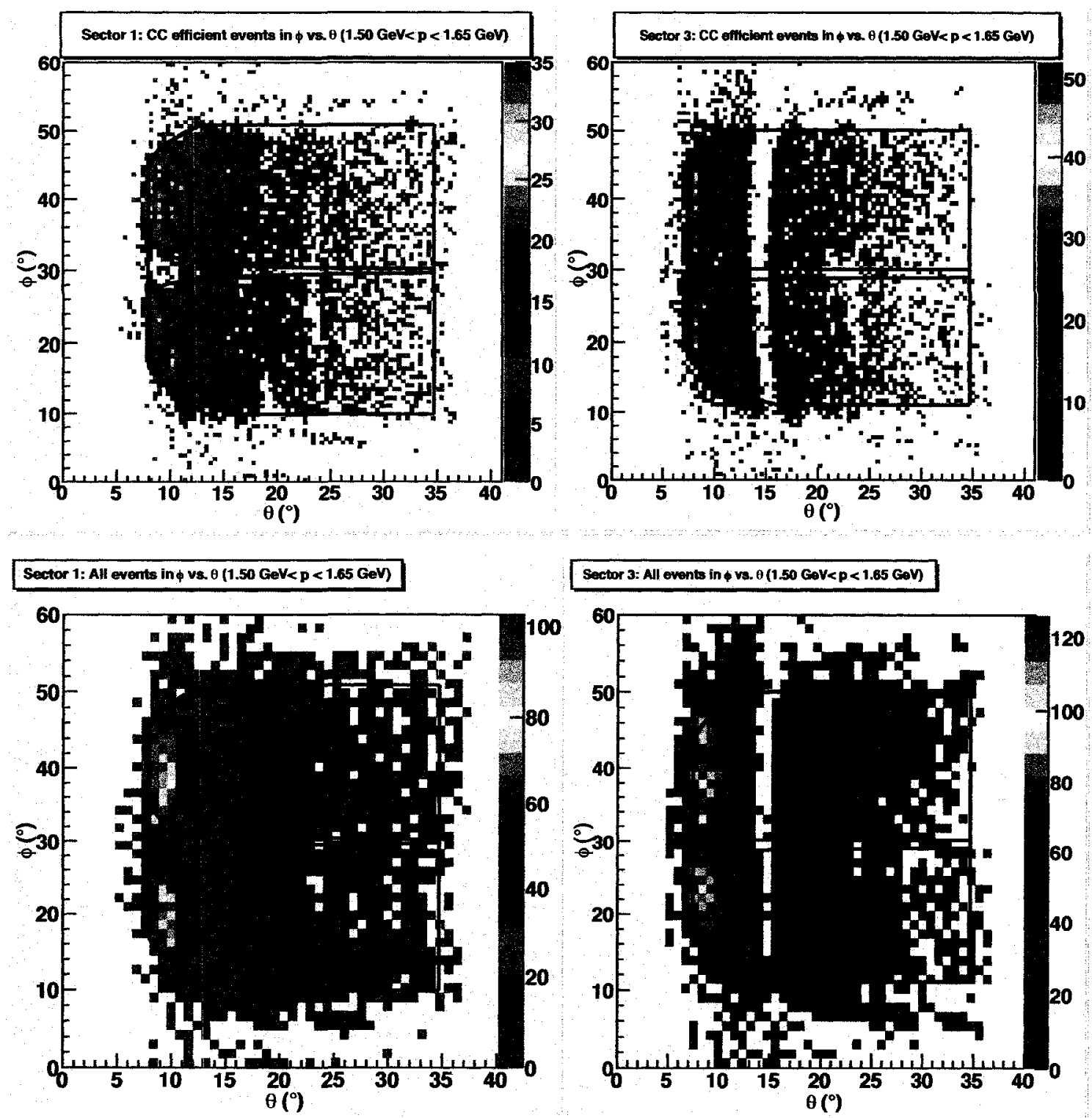

Figure 4.22: Outbending fiducial cuts, shown for two different sectors in the same momentum bin. Notice the differences between the two cuts (especially in the center strip). The top image shows electrons meeting the $80 \%$ efficiency requirement, and the bottom shows the same data set, showing all events in both sectors. (The empty vertical "strip" on the right hand plots is due to an inactive SC paddle.) 

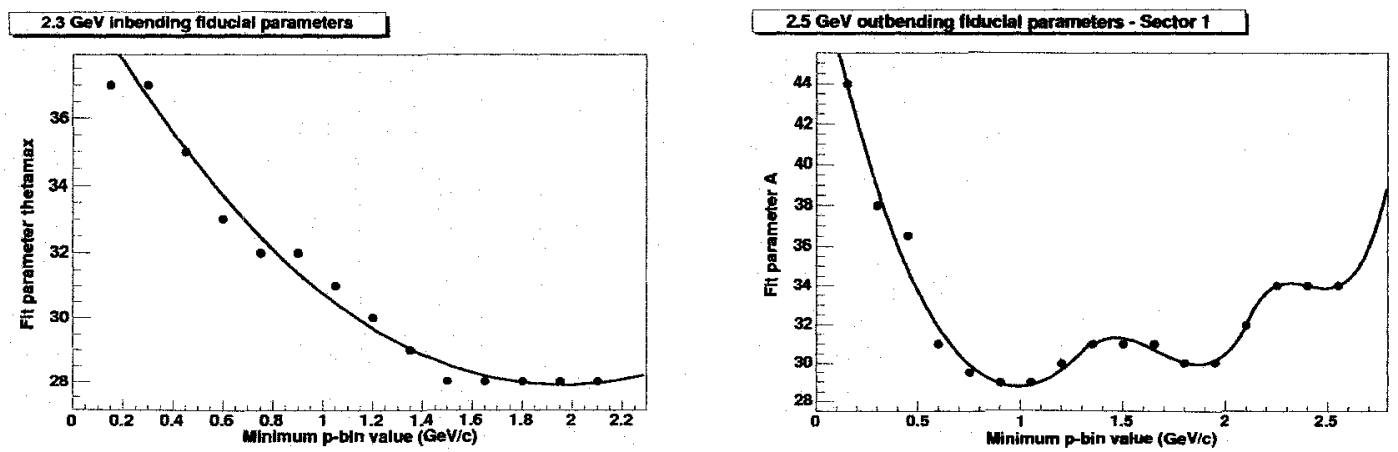

Figure 4.23: Graphs of the interpolated values between momentum bins of fiducial cut constants. Shown are the $\theta_{\max }$ values for torus current $+1500 \mathrm{~A}$ and $A$ in sector 1 for $-1500 \mathrm{~A}$, as two examples. The polynomial fit parameters were then used in the fiducial cut function in place of the discrete fit parameters.

\subsubsection{Application and Effects of Fiducial Cuts}

The fiducial cuts outlined in this section are necessary for removing regions of the Cherenkov detectors where the response and acceptance are not well understood. This is crucial when direct count rates are used in analysis, as in the case of dilution factor (Section 5.6) measurement. However, asymmetry measurements are not dependent on the detector efficiency or response.

Fiducial cuts are a very restrictive set of cuts, especially for high momentum inbending data (as can be seen in Figure 4.21). It is desirable, therefore, to use the fiducial cuts only when needed. Therefore, fiducial cuts are only used for measurements where clean count measurements are needed (e.g. dilution factors, pion background calculation, etc.). The asymmetry $A_{\| \mid}(\mathrm{Eq}$. 1.209) is then measured without the fiducial cuts.

The case of inbending data, however, still presents a concern, due to the electrons directly impacting the Cherenkov photomultiplier tubes in this configuration (depicted in Figure 4.20). The high photoelectron counts in this region are not necessarily a Cherenkov response, and thus this data cannot be relied upon for a good PID. Therefore, a second set of "loose" fiducial cuts was made for the inbending data that only cuts out the region of direct PMT hits. ${ }^{46}$ This cut, shown in Figure 4.24, uses only 2 sets of parameters (for $p<3 \mathrm{GeV}$ and $p>3 \mathrm{GeV}$ ) and uses no parameter inter-

\footnotetext{
${ }^{46}$ The high angle limit is also left in place to prevent scattering from the target solenoid.
} 


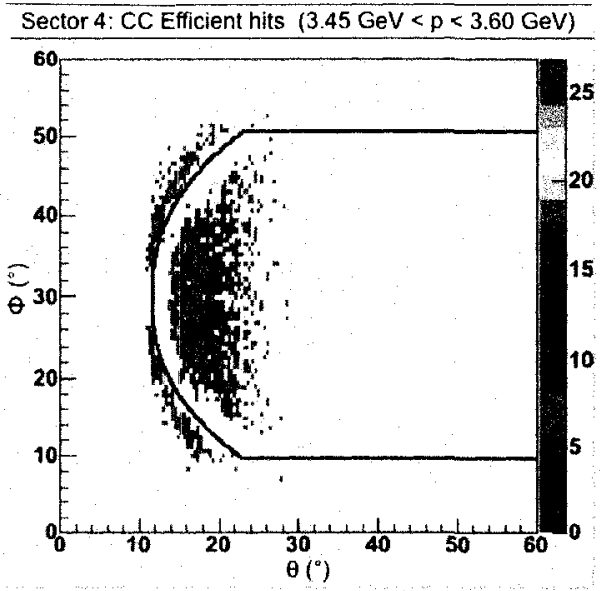

Figure 4.24: "Loose" fiducial cut for one momentum bin and sector for inbending data. The bands of direct PMT hits in the CC were specifically removed by these cuts.

polation. ${ }^{47}$ This loose cut was applied to inbending $A_{\|}$data, while no fiducial cuts were used on the outbending $A_{\|}$data. Table 4.6 summarizes the parameters used for these less restrictive cuts for inbending data. Unlike the the other fiducial cuts, the parameter $\theta_{\max }$ is replaced with $\phi_{\text {limit }}$. That is, the constant $\phi$-edge of the cut is explicitly defined, instead of determined in terms of $\theta$.

For convenient reference, a simple set of "starting parameters" are also included for the normal (tighter) inbending and outbending fiducial cuts in Tables 4.7 and 4.8, respectively. These parameters do not provide the smooth transition between bins like the refined cut parameters in the longer parameter tables, but provide a reasonable starting point for the development of future fiducial cuts.

The decision to use different cuts for asymmetries and absolute count rates arose partially from an investigation of the $Q^{2}$ and $W$ dependence of the measured rates of data with and without fiducial cuts. Omitting fiducial cuts from asymmetries allows for an expansion of the measured range of $A_{\|}$slightly beyond the viable range of the measured dilution factor. The dilution factor can then be applied to the asymmetry in all kinematic regions by the extrapolation of a smooth model (see

\footnotetext{
${ }^{47}$ This less intricate method resulted in limited accidental regions where the "tighter" cuts actually kept dala excluded by the supposedly "looser" cuts. Thus, the cut subroutine was modified in the "loose" case to keep events that pass either cut, so that the "tight" cuts always retained a subset of the "loose" cut events.
} 
Table 4.6: "Loose" inbending fiducial cut parameters. These cuts are intended for removal of direct Cherenkov PMT hits only, and should not be applied to acceptance-dependent measurements. These numbers correspond to $p$ in $\mathrm{GeV}$ and all angles are in degrees.

\begin{tabular}{|c|c|c|}
\hline Parameter & $p<3 \mathrm{GeV}$ & $p>3 \mathrm{GeV}$ \\
\hline \hline$A$ & 41 & 41 \\
\hline$B$ & 0.26 & 0.26 \\
\hline$C$ & 0.30 & 0.30 \\
\hline$D$ & 9 & 8 \\
\hline$E$ & 16.72 & 16.72 \\
\hline$F$ & 0.06 & 0.06 \\
\hline$\phi_{\text {limit }}$ & 21.5 & 21.5 \\
\hline
\end{tabular}

Table 4.7: "Tight" inbending fiducial cut parameters. These parameters are not used in this analysis, and are merely included as a reference for a starting point for future fiducial cuts. These numbers correspond to $p$ in $\mathrm{GeV}$ and all angles are in degrees.

\begin{tabular}{|c|c|c|}
\hline Parameter & $p<3 \mathrm{GeV}$ & $p>\mathbf{3 ~ G e V}$ \\
\hline \hline$A$ & 36 & 36 \\
\hline$B$ & 0.28 & 0.25 \\
\hline$C$ & 0.30 & 0.30 \\
\hline$D$ & 10 & 10 \\
\hline$E$ & 16.72 & 16.72 \\
\hline$F$ & 0.06 & 0.06 \\
\hline$\phi_{\text {limit }}$ & 20 & 20 \\
\hline
\end{tabular}


Table 4.8: "Tight" outbending fiducial cut parameters. These parameters are not used in this analysis, and, like the previous table, they are merely included as a reference for a starting point for future fiducial cuts. These numbers correspond to $p$ in $\mathrm{GeV}$ and all angles are in degrees.

\begin{tabular}{|c|c|c|c|}
\hline Parameter & $p<3 \mathrm{GeV}(-2250 \mathrm{~A})$ & $p>3 \mathrm{GeV} c(-2250 \mathrm{~A})$ & $-1500 \mathrm{~A}$ \\
\hline \hline$A$ & 34 & 45 & 34 \\
\hline$B$ & 0.28 & 0.54 & 0.33 \\
\hline$C$ & 0.22 & 0.21 & 0.22 \\
\hline$D$ & 5 & 9.5 & 6.2 \\
\hline$E$ & 3 & -4 & 3 \\
\hline$F$ & 1.46 & 1.2 & 1.46 \\
\hline$G_{\text {upper }}$ & 0.15 & 0.3 & 0.15 \\
\hline$H_{\text {upper }}$ & -0.09 & 0.1 & -0.09 \\
\hline$G_{\text {lower }}$ & 0.15 & 0.3 & 0.15 \\
\hline$H_{\text {lower }}$ & -0.09 & 0.1 & -0.09 \\
\hline$\phi_{\text {limit }}$ hi & 21 & 21 & 21 \\
\hline$\phi_{\text {limit }_{l o}}$ & 22 & 22 & 22 \\
\hline outeroffset $_{\text {in }}$ & 1.2 & -0.6 & 1.2 \\
\hline inneroffset & 0 & 0 & 0 \\
\hline
\end{tabular}

Section 5.6 .2 for details).

The effectiveness of fiducial cuts can be seen by viewing the number of pion events (characterized by a low CC photoelectron peak) removed by the cuts, in the absence of the pion track matching cuts (Section 4.1). Figure 4.25 shows the basic inclusive PID cuts of Chapter 3 applied to data with and without fiducial cuts, for comparison.

\subsection{Faraday Cup Corrections}

\subsubsection{Correction for multiple scattering divergence}

The Faraday Cup lies about $29.5 \mathrm{~m}$ downstream from the CLAS target [30] and has a diameter of $15 \mathrm{~cm}$. As mentioned in Section 4.2.5, multiple scattering effects within the target cause the exit angle of electrons from solid materials to change (Figure 4.11). This creates an overall statistical divergence, or "spread" in the beam as it leaves the target.

Given these dimensions, the entire beam will not enter the Faraday Cup unless the spread angle 


\section{Particle Identification Parameters}
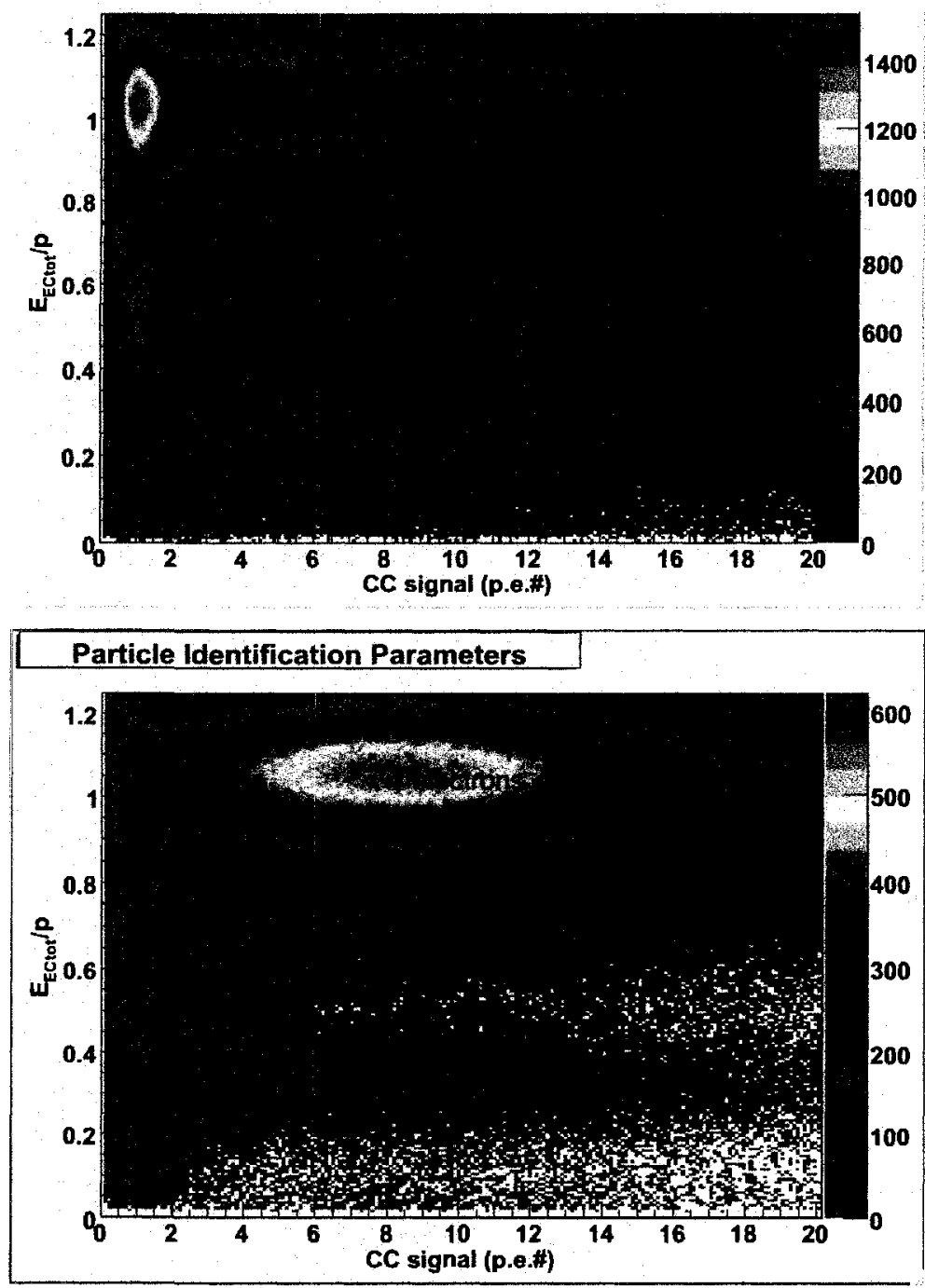

Figure 4.25: Basic electron PID cuts (black lines) for $2.3 \mathrm{GeV}$ inbending data. Electrons are separated from pions (red "hot spot") by application of these cuts. The top and bottom plots show the same data and cuts, except that fiducial cuts (Section 4.4) are added in the bottom plot, greatly reducing the pion peak in the upper left corner. Note that no pion track matching cuts have been applied in either case. 
of the scattered beam is less than $\arctan (15 / 2 / 2950)=0.146^{\circ}$. The divergence caused by multiple scattering is a given by a statistical (Mollière) distribution [5], for which the root mean square is given by

$$
\theta_{r m s}=\sqrt{2} \frac{13.6 \mathrm{MeV}}{\beta c p} \sqrt{x / X_{0}}\left[1+0.038 \ln \left(x / X_{0}\right)\right]
$$

To estimate the effects on the worst case (i.e. lowest energy) EG1b data, we use $\beta \approx 1$ and $p \approx$ $1600 \mathrm{MeV}$. The value of $t / X_{0}$ (radiation length fraction of target material) for the ${ }^{12} \mathrm{C}$ target $t^{48}$ is given by

$$
\begin{gathered}
\frac{t}{X_{0}}=\frac{\rho_{\mathrm{C}} \ell_{\mathrm{C}}}{X_{0 \mathrm{C}}}+\frac{\rho_{\mathrm{Al}} \ell_{\mathrm{Al}}}{X_{0 \mathrm{Al}}}+\frac{\rho_{\mathrm{K}} \ell_{\mathrm{K}}}{X_{0 \mathrm{~K}}}+\frac{\rho_{\mathrm{He}} \ell_{\mathrm{He}}}{X_{0 \mathrm{He}}} \\
=\frac{0.498}{42.7}+\frac{0.045}{24.01}+\frac{0.0432}{42.7}+\frac{0.145(1.90-0.23)}{94.32} \approx 0.017
\end{gathered}
$$

All numbers in the above can be found on Tables 2.1 and 2.3. This yields a value of $\theta_{r m s}=$ $0.0017 \mathrm{rad}=0.097^{\circ}$, which translates to a projected width on the Faraday Cup of $(29.5 \mathrm{~m}) \times \tan \theta_{r m s}=$ $5.0 \mathrm{~cm}$.

Clearly, this is approaching the Faraday Cup radius of $7.5 \mathrm{~cm}$. Within the inner $98 \%$ of the distribution, a Mollière and Gaussian distribution are similar [5]. This means the Faraday Cup can only detect (approximately) 1.5 standard deviations, or $87 \%$ of the electron beam. ${ }^{49}$ On the other hand, if the same calculation is repeated for an empty (LHe only) target, ${ }^{50} t / X_{0}=0.005$ and the corresponding $\theta_{r m s}$ (projected spread) is given by $0.029^{\circ}(1.4 \mathrm{~cm})$, meaning almost no charge is missed by the Faraday Cup, in this case.

In theory, these calculations could be used to calculate a target and beam energy-dependent multiplicative number for the Faraday Cup charge, but the situation is complicated by the possibility of "overfocussing" by the Helmholtz magnet [117], where the beam focal point falls short of the Faraday Cup, causing an additional divergence. ${ }^{51}$ Thus, empirical methods must be relied upon to renormalize the Faraday Cup values to account for this issue.

\footnotetext{
${ }^{48}$ The ${ }^{12} \mathrm{C}$ and $\mathrm{NH}_{3}$ targets have approximately the same radiation lengths, so we use the simpler case here.

${ }^{49}$ This assumes the Gaussian approximation translates to the projection, a valid assumption for angles as small as these.

${ }^{50}$ This is an identical calculation, except that $\ell_{\mathrm{C}}=0$ and $\ell_{\mathrm{He}}=1.90$.

${ }^{51}$ This "spread" is proportional to the inverse square of the beam energy, so it is also worse at lower beam energies.
} 
A study was made by R. Minehart et al. measuring the current at the upstream BPMs (Beam Position Monitors) for different targets. The assumption was made that no correction was needed at $5.7 \mathrm{GeV}^{52}$ and this was established as a "baseline" for a 1:1 correspondence between the BPM and Faraday Cup (i.e., an appropriate "weighting factor" for translation between the two was determined). Then, the ratio of the (weighted) BPM value to the ungated Faraday Cup measurement was recorded for each beam energy and each target. The average of the ratios for the 3 BPMs (see Section 2.2) determined a "normalization factor" for the total charge delivered to a given target. No corrections were used for 4.x and 5.x GeV data, since the scattering angle is too small to be noticed at those energies. Corrective factors for the lower beam energies for each target are listed in Table 4.9. Note that it is the ratios between differing targets that is important here, not the absolute correction on any particular target. ${ }^{53}$

Table 4.9: Faraday Cup normalization factors correcting for angular spread caused by multiple scattering effects. The recorded Faraday Cup value must be divided by the appropriate number to get the "true" weighting value. The $1.723 \mathrm{GeV}$ values were determined by linear interpolation from $1.606 \mathrm{GeV}$ values.

\begin{tabular}{|c|c|c|c|c|}
\hline Beam Energy (GeV) & $\mathrm{NH}_{3}$ & $\mathrm{ND}_{3}$ & ${ }^{12} \mathrm{C}$ & empty(LHe) \\
\hline \hline 1.606 & 0.846 & 0.828 & 0.850 & 0.965 \\
\hline 1.723 & 0.856 & 0.840 & 0.860 & 0.967 \\
\hline 2.286 & 0.951 & 0.951 & 0.962 & 1.000 \\
\hline 2.561 & 0.986 & 0.986 & 0.986 & 1.000 \\
\hline
\end{tabular}

\subsubsection{Bit factor correction}

Empty ( $\mathrm{LHe}$ ) target runs utilized a higher beam current than the other target, because of the much shorter radiation length $t / X_{0}$ of the empty target, meaning a much higher beam current could be tolerated with the same dead time. To accomodate this change, one bit was removed from the Faraday Cup count response rate. In other words, the FC recorded "clicks" at half the rate it did for other runs. Thus, to get an accurate count rate for the empty target runs, the FC counts for these runs had to be multiplied by 2 .

\footnotetext{
${ }^{52}$ This is a valid assumption, as $\theta_{r m s}$ is less than $1 / 3$ that for $1.6 \mathrm{GeV}$, and thus the distribution is almost $100 \%$ contained in the Faraday Cup opening.

${ }^{53} \mathrm{For}{ }^{12} \mathrm{C} /{ }^{15} \mathrm{~N}$ analysis, the radiation tengths of the only two targets used were believed to be similar, so no corrective factors for the FC were used, at least initially (see Section 5.4).
} 
There were also a few exceptions of other target runs erroneously missing an FC bit in the DST. ${ }^{54}$ Runs 26055, 26491, 27294, 26683, and runs 27982-27986 all required a factor of 2 on the FC charge to get the proper value. Conversely, one LHe run (28201) was not missing the FC bit as it should have been; the FC charge for this run was doubled.

To ease the confusion caused by the Faraday Cup issues, the Faraday Cup charge for every run read both the multiple scattering factor (Table 4.9) and a bit factor correction from a reference table developed after quality checks were complete.

\subsection{Generation of Skims}

At this point, the DST files have been converted to an easily readable (ROOT tree) format, and all essential preliminary cuts and corrections are in place. The next necessary step is to convert the data into compact skims retaining only the essential information needed for physics analysis. Gated Faraday cup charges, target and polarization information, beam energy and torus current were written to short text files for each run. Remaining analysis steps were mainly completed in terms of $Q^{2}$ and $W$, so cumulative counts for each run, divided by helicity, were written to designated $Q^{2}$ and $W$ bins for various combinations of PID and sector cuts. This simplified checks and changes in later analysis steps. Counts for both inclusive electrons and exclusive ep events in terms of $Q^{2}$ and $W$ were recorded to the skims.

$300 \times 40$ arrays were used in $W$ and $Q^{2}$. $W$ bins were constant in size, exactly $0.01 \mathrm{GeV}$ in width, ranging from a bin minimum of 0.0 to $2.99 \mathrm{GeV}$. A logarithmic scale was used for the designation of $Q^{2}$ bins, ${ }^{55}$ ranging from 0.01 to $10.0 \mathrm{GeV}^{2}$. Labels for the standard $Q^{2}$ bins are listed in Table B.1.

Also, kinematic values, averaged over the number of events, were recorded in each $Q^{2}, W$ bin, for extraction in future asymmetry calculations. Averaged values of $W, Q^{2}, s, x, \nu, D, \eta, \epsilon, \gamma, \theta$ and $E^{\prime}$ (defined in Section 1.1.3) were also calculated for each bin and recorded in $300 \times 40$ arrays, to avoid later difficulties of relative weighting within bins. The $Q^{2}$ values, weighted by the elastic

\footnotetext{
${ }^{54}$ This is evidenced by exactly double an inclusive count rate, and target confirmation by $W$-spectrum analysis (see Section 3.3).

${ }^{55}$ This is done to obtain finer resolution at lower $Q^{2}$ values.
} 
double-spin asymmetry, were also recorded for inclusive and exclusive events (see Section 6.2 for details).

Some other miscellaneous information was also kept in the skims, as required. Elastic ep events in terms of $\phi$ (in $0.2^{\circ}$ bins) were kept for future exclusive background subtraction (Section 6.2.2), for example. 1-D histograms in terms of various kinematic parameters were kept for reference, as well as 2-D raster pattern histograms, but these had little future use in analysis aside from diagnostics. For analysis of this data, two complete passes were made through each run set (outlined in Table 3.1). In the first pass, only runs and files passing all quality checks (that is, the RATE/files described in Section 3.3.7) were analyzed. These runs were needed for the calculation of the target length, ${ }^{12} \mathrm{C} \rightarrow{ }^{15} \mathrm{~N}$ modeling, packing fraction, dilution factors, and other (unpolarized) background-related calculations. The strict fiducial cuts (Section 4.4) were applied to all the inclusive data analyzed in the first pass, and the resulting skims were stored in a permanent disk space.

In the second pass, only ammonia files were analyzed, specifically for the measurement of asymmetries. These files were subject to less stringent cut criteria than those analyzed in the first pass. Namely, no cuts were made on fluctuations in the count rate ${ }^{56}$ (that is, these files are those in the ASYM directory described in Section 3.3.7). Strict fiducial cuts were not applied to these files. No fiducial cuts were used at all for outbending (negative torus current) data, and only the "loose" fiducial cuts were applied to inbending (positive torus current) data (see Section 4.4.4).

Data from these two passes are stored separately. The analysis steps in the following chapters are then applied to the appropriate data set(s). As a general rule, any analysis step requiring different target types requires the first pass (RATE/) files, while any involving only asymmetries uses the the second pass (ASYM) files.

Skim files for each run containing the crucial physics information were thus stored to disk and used for the remaining analysis. The following chapters explain in detail how diluted double-spin asymmetries were extracted from the essential physics data stored in the skims.

\footnotetext{
${ }^{56}$ As previously noted, asymmetry measurements are not dependent on detector acceptance, so this is acceptable for asymmetries only.
} 


\title{
Measurement of Inclusive Proton Double-Spin Asymmetries
} and Polarized Structure Functions

Robert G. Fersch, Jr.

Annapolis, MD

B.A. Physics Education, University of Delaware, 1996 M.S. Physics, College of William and Mary, 2003

\begin{abstract}
A Dissertation presented to the Graduate Faculty of the College of William and Mary in Candicacy for the Degree of Doctor of Philosophy
\end{abstract}

Physics

The College of William and Mary

August, 2008 


\section{Part 2}




\section{Chapter 5}

\section{Dilution Factors: Removal of}

\section{Unpolarized Background}

\subsection{Calculation of Background-subtracted Asymmetries}

At this point in the analysis, all kinematic corrections and PID cuts are in place, and counts (for each beam helicity) and average kinematic values have been written in $300 \times 40$ arrays (representing bins in $W$ and $Q^{2}$ ) to skim files for each run (see Section 4.6). All components are now in place for the calculation of the double-spin asymmetry (Eq. 3.1).

The inclusive asymmetry is still heavily contaminated with background. Only a small percentage of the asymmetry represents the scattering of electrons from the free polarized protons in the target $\mathrm{NH}_{3}$. To find the actual physics asymmetry $A_{\| \mid}$, one must divide out the contributions from unpolarized target materials (i.e. ${ }^{15} \mathrm{~N}$, LHe, and target windows) in the form of an overall kinematicsdependent dilution factor $\left(F_{D F}\right)$. Also, since the polarizations of both the beam electrons are free protons is incomplete, one must also divide out the total beam polarization $\left(P_{b}\right)$ and target polarization $\left(P_{t}\right)$ from the asymmetry. This yieids an actual double-spin asymmetry of

$$
A_{\|}=\frac{1}{P_{b} P_{t} F_{D F}} \frac{n^{-}-n^{+}}{n^{-}+n^{+}}
$$


Here, $n^{+}$and $n^{-}$are counts for each helicity normatized by the (livetime-gated) cumulative Faraday Cup charge for the given helicity bucket. Here, and in the rest of this thesis, lowercase $n$ is used to denote Faraday charge-normalized counts, while capital $N$ represents actual (unormalized) numbers of detected events. The procedure for removing unpolarized background is demonstrated in this chapter.

\subsection{Combining Data from Different Runs}

The next analysis steps require the combination of run data by target type, separated into subsets containing runs with similar characteristics (specifically beam energy, torus current, target polarization sign and half-wave plate status). For $\mathrm{NH}_{3},{ }^{12} \mathrm{C}$ and $\mathrm{LHe}$ runs, cumulative counts in each bin were summed for each $Q^{2}$ and $W$ bin in each of the 12 data "brackets" listed in Table 5.1. In addition, combined count skims were also written for ${ }^{12} \mathrm{C}$ and ${ }^{15} \mathrm{~N}$ target runs in each of the 7 special frozen nitrogen-carbon "brackets" listed in Table 5.2.

Table 5.1: "Brackets" of EG1 ammonia/carbon/empty target data, ordered in the sequence in which runs were taken. The combinations of HWP/target polarization sign for $\mathrm{NH}_{3}$ data present for each bracket are listed. See Table 3.1 for run, beam energy and torus current information on these sets.

\begin{tabular}{|c|c|c|}
\hline Set Label & Bracket & HWP/target pol. present \\
\hline \hline $1.6+$ & 1 &,,,+++--+-- \\
\hline $1.6-$ & 2 & ++ \\
\hline $5.76-$ & 3 &,,,+++--+-- \\
\hline $5.73-$ & 4 &,,,++--+-- \\
\hline $5.7+$ & 5 &,,,+++--+-- \\
\hline $2.3+$ & 6 &,,+++-- \\
\hline $5.6+$ & 7 &,,++-+-- \\
\hline $1.7-$ & 8 &,,+++--- \\
\hline $2.5-$ & 9 &,,,+++--+- \\
\hline $2.5+$ & 10 & none $\left(\mathrm{ND}_{3,},{ }^{12} \mathrm{C}\right.$, LHe only $)$ \\
\hline $4.2+$ & 11 &,,,+++--+-- \\
\hline $4.2-$ & 12 &,,,+++--+-- \\
\hline
\end{tabular}

For $\mathrm{NH}_{3}$ runs only, separate counts, as well as cumulative raw asymmetries and their errors (in each bin), were written for each individual half-wave-plate (HWP) and target polarization sign 
Table 5.2: "Brackets" of EG1 carbon/frozen nitrogen target data, ordered in the sequence in which runs were taken. See Table 3.1 for run, beam energy and torus current information on these sets. These brackets are marked with an apostrophe (') to avoid confusion with the main data brackets.

\begin{tabular}{|c|c|}
\hline Set Label & Bracket \\
\hline \hline $2.3+$ & $1^{\prime}$ \\
\hline $5.6+$ & $2^{\prime}$ \\
\hline $5.6-$ & $3^{\prime}$ \\
\hline $4.2-$ & $4^{\prime}$ \\
\hline $4.2+$ & $5^{\prime}$ \\
\hline $1.7+$ & $6^{\prime}$ \\
\hline $1.7-$ & $7^{\prime}$ \\
\hline
\end{tabular}

combination $(+t,+-,-+,--)$. Raw asymmetries were calculated as

$$
A_{\text {raw }}\left(W, Q^{2}\right)=\frac{\left(\frac{N^{-}\left(W, Q^{2}\right)}{F C^{-}}\right)-\left(\frac{N^{+}\left(W, Q^{2}\right)}{F C^{+}}\right)}{\left(\frac{N^{-}\left(W, Q^{2}\right)}{F C^{-}}\right)+\left(\frac{N+\left(W, Q^{2}\right)}{F C^{+}}\right)}
$$

where $N^{ \pm}$represents the total count of each helicity per bin and $F C^{ \pm}$represents the total helicity and livetime-gated Faraday Cup charge for the run. The statistical error bar can be calculated using quadrature [103] as

$$
\sigma_{A}\left(W, Q^{2}\right)=\sqrt{\left(\frac{\partial A}{\partial N^{-}}\right)^{2} \sigma_{N^{-}}^{2}+\left(\frac{\partial A}{\partial N^{+}}\right)^{2} \sigma_{N^{+}}^{2}+\left(\frac{\partial A}{\partial F C^{-}}\right)^{2} \sigma_{F C^{-}}^{2}+\left(\frac{\partial A}{\partial F C^{+}}\right)^{2} \sigma_{F C^{+}}^{2}}
$$

The error on the Faraday Cup counts is small compared to that of the total (Poisson distributed) detector counts ${ }^{1}$, so the last two terms can be neglected. The positive and negative helicity cumulative charges are approximately equal, so we can assume $\mathrm{FC}^{+} \approx F C^{-}$, yielding

$$
\frac{\partial A_{\text {raw }}}{\partial N^{+}}=\frac{1}{2 N^{-}} ; \frac{\partial A_{\text {raw }}}{\partial N^{-}}=\frac{1}{2 N^{+}}
$$

\footnotetext{
${ }^{1}$ The Faraday Cup, once normalized for multiple scattering spread (Section 4.5 ), is nearly $100 \%$ efficient.
} 
If $\sigma_{N^{+}}=\sqrt{N^{+}}$(characteristic of a Poisson distribution), the error becomes

$$
\sigma_{A}\left(W, Q^{2}\right)=\sqrt{\frac{1}{4} \frac{N^{-}+N^{+}}{N^{-} N^{+}}}
$$

The raw asymmetries are typically on the order of $10^{-2}$, so generally we can assume, for the purposes of error calculation, $N\left(W, Q^{2}\right) \approx 2 N^{+} \approx 2 N^{-}$, which yields

$$
\sigma_{\Lambda}\left(W, Q^{2}\right)=\frac{1}{\sqrt{N\left(W, Q^{2}\right)}}
$$

for the error bar on the asymmetry, calculated independently for each bin in the ammonia runs.

The cumulative asymmetry and error for several combined runs is added in the standard manner of combining statistical errors:

$$
\begin{gathered}
A_{\text {raw }}\left(W, Q^{2}\right)=\frac{\sum_{\text {rand }} A_{\text {raw }}\left(W, Q^{2}\right) / \sigma_{A}\left(W, Q^{2}\right)^{2}}{\sum_{\text {runs }} 1 / \sigma_{A}\left(W, Q^{2}\right)^{2}} \\
\sigma_{A_{\text {total }}}\left(W, Q^{2}\right)=\frac{1}{\sqrt{\sum_{\text {runs }} 1 / \sigma_{A}\left(W, Q^{2}\right)^{2}}}
\end{gathered}
$$

In addition to the total summed bin-by-bin counts and the cumulative asymmetries for each "bracket", the averaged kinematic values of $Q^{2}, W, x, s, \nu, D, E^{\prime}, \theta, \epsilon, \eta$, and $\gamma$ are tracked for each run set, weighted by the total counts in each bin. (See Section 1.1.3 for definitions of these variables.) That is,

$$
v a l=\frac{\sum_{\text {rans }} v a l \times N\left(W, Q^{2}\right)}{\sum_{\text {runs }} N\left(W, Q^{2}\right)}
$$

is simply recorded in each bin for each of these 11 kinematic quantities val. Tracking of the averages allows for easier calculations later, without having to worry about the relative weighting within the bin.

For the purposes of accurate evaluation of the elastic asymmetry for beam $\times$ target polarization determination (Section 6.2), the average $Q^{2}$, weighted by the calculated elastic asymmetry

\footnotetext{
${ }^{2}$ For bins with very small cumulative counts, this approximation breaks down. However, the net effect, summing over many runs, is for these statistical effects in these bins to average out and cancel. To make sure this was the case, analysis was done with both cumulative asymmetries and cumulative counts, with no significant discrepancies found between the two methods.
} 
$A_{e l}\left(Q^{2}, E\right)$ given by equation 6.22 , is averaged over the runs, for both inclusive and exclusive $e p$ events:

$$
Q_{\text {weighted } \text { totad }^{2}}^{2}=\frac{\sum_{\text {runs }} Q_{\text {weighted }}^{2} A_{e l}\left(Q^{2}, E\right) N\left(W, Q^{2}\right)}{\sum_{\text {runs }} A_{e l}\left(Q^{2}, E\right) N\left(W, Q^{2}\right)}
$$

where $Q_{w e i g h t e d}^{2}$ is the similarly weighted $Q^{2}$ from each individual run.

For ammonia and carbon runs, the summed counts in $\Delta \phi$ particular to each target are also recorded for elastic ep events only ${ }^{3}$ for exclusive event background subtraction (see Section 6.2.2).

Finally, the cumulative Faraday Cup counts were recorded for each target label (in the case of ammonia targets, for each HWP and target polarization combination). The checking of FaradayCup normalized count rates is a valuable diagnostic tool. Comparative count rates for each target, sampled at each beam energy, are shown in Figure 5.1.

\subsubsection{Corrections to empty (LHe) target counts}

Empty (LHe) target runs presented a special case, due to both the significant difference in mass thickness compared to the other targets (and thus the need for differing external radiative corrections), and the raster-related problems present in empty targets in Brackets 9-12 of the EG1 run set (see also Section 3.3.4). Because of these factors, data in the empty (LHe only) target runs presented additional complications when summing over the runs for use in statistical models.

Before explaining the corrections made to the LHe runs, it is useful to consider the purpose of collecting the empty target data. Subsequent sections of this chapter explain two methods for the calculation of the target length $(L)$, ammonia packing fraction $\left(\ell_{A}\right)$, and dilution factors $\left(F_{D F}\right)$. One involves bin-by-bin statistical averaging of the actual data, and the other uses a radiated crosssection model to provide a definition of these quantities free from domination by statistical deviations. (The latter, of course, is the favored method, where its applicability is valid. See subsequent sections for a more complete explanation.)

\footnotetext{
${ }^{3}$ These were divided into $0.2^{\circ}$ bins for all bins within $30^{\circ}$ of $\Delta \phi=0$.
} 

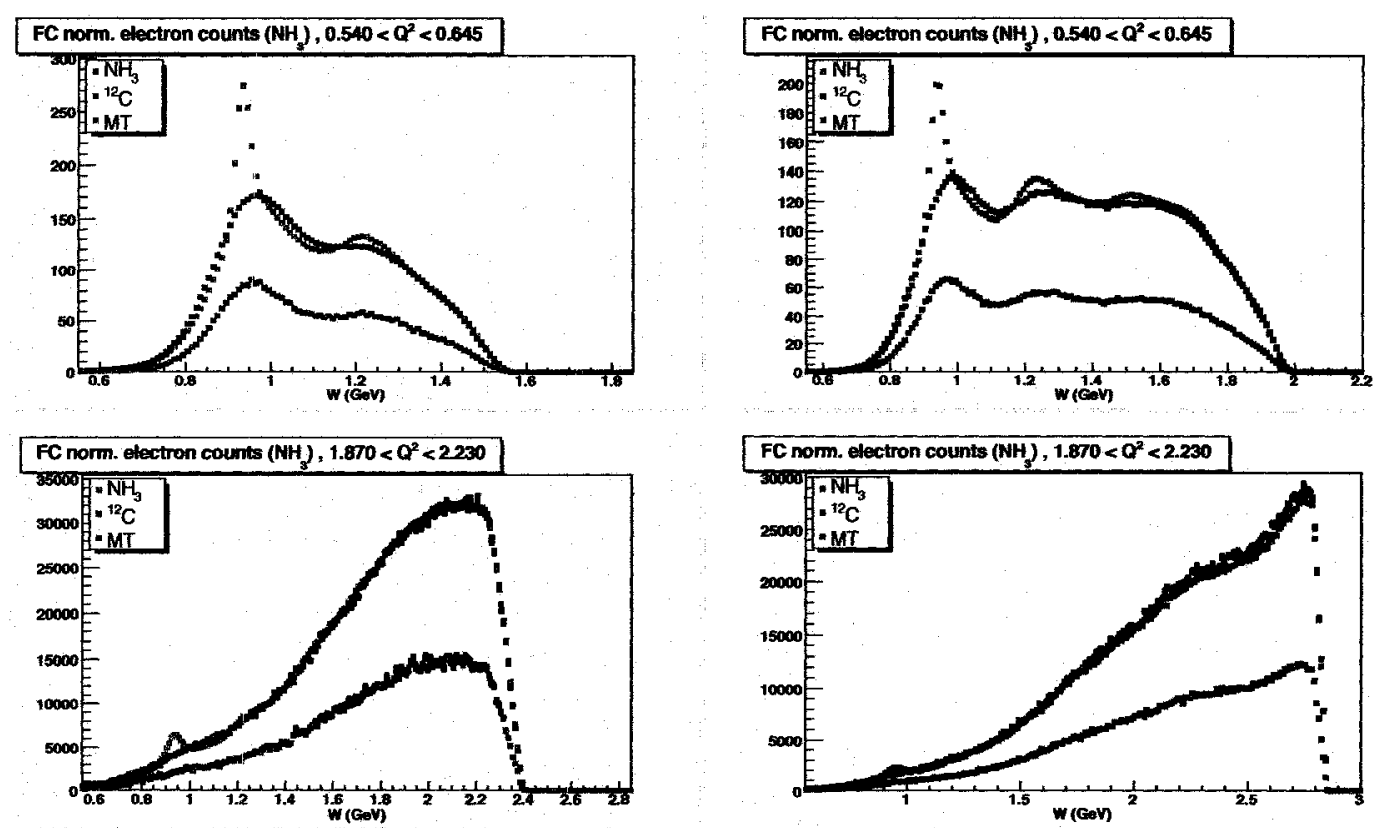

Figure 5.1: Inclusive count rates in $W$ shown for $\mathrm{NH}_{3}$ (red), ${ }^{12} \mathrm{C}$ (blue) and empty LHe (green) targets, for inbending data. The top two plots show the same $Q^{2}$ bin for 1.6 and $2.3 \mathrm{GeV}$ data, while the bottom plots show a higher $Q^{2}$ bin at 4.2 and $5.7 \mathrm{GeV}$ beam energy, respectively. $\mathrm{NH}_{3}$ counts are actual counts, while the other two targets are Faraday charge-normalized relative to the ammonia target. Note the presence of the elastic peak (more prominent at lower energies) for $\mathrm{NH}_{3}$ counts, while empty (MT) target counts are much lower, corresponding to the much smaller radiation length fraction $t / X_{0}$ of this target. Error bars, calculated as $\sqrt{N} / F C$ (Poisson statistics normalized by the Faraday Cup charge), are generally too small to see. 


\section{Radiative corrections for empty target counts}

The empty target is of considerably smaller thickness in terms of radiation length $\left(t / X_{0}\right)$ [8] than the carbon or ammonia targets, which were both designed with approximately the same mass thickness [65]. The quantities mentioned in the preceding paragraph are dependent on count ratios between the three targets $\left(\mathrm{NH}_{3},{ }^{12} \mathrm{C}, \mathrm{LHe}\right)$. Because $t / X_{0}$ is similar for the first two of these, no correction need be considered for any ratio between these two targets. However, empty target counts require a count rate correction to account for external radiative effects. The corrections thus generated should be applied to any model using the bin-by-bin averaging of statistical data (but should not be applied to models using radiated cross-sections, as such models already have the radiative differences inherently built-in). Therefore, for the empty target, two $300 \times 40$ arrays of summed counts were produced for each bracket, one representing uncorrected counts, and the second incorporating (unpolarized) radiative corrections.

The model outlined in Section 2.8 was used to generate a $300 \times 40$ array of multiplicative factors for each $Q^{2}$ and $W$ bin used at each beam energy in the experiment. ${ }^{4}$ To calculate the ratio, first, the total target length $L$ is calculated as according to the radiated cross-section model (see Section 5.3.2 for details on this procedure). Then, a length $L_{\text {sim }}$ is calculated, representing what the total length of the empty target would be if it had the same radiation length $X_{0}$ as the ${ }^{12} \mathrm{C}$ target. This length is calculated by subtracting the length of the carbon material $\left(\ell_{C}\right)$ and adding back in a length of liquid helium with the same radiation length as the carbon. In other words, we replace the fraction of a radiation length of carbon with the same fraction of a radiation length of helium, using the relation

$$
\frac{\rho_{H e} \ell_{H e}}{X_{0(H e)}}=\frac{\rho_{C} \ell_{C}}{X_{0(C)}}
$$

This results in

$$
L_{s i m}=L-\ell_{C}+\frac{X_{0(H e)}}{X_{0(C)}} \frac{\rho_{C}}{\rho_{H e}} \ell_{C}
$$

Then, the total radiated cross-section (accounting for all contributions from LHe, Kapton, and aluminum listed in Table 2.1) is calculated for each material in the empty target configuration, the first

\footnotetext{
${ }^{4}$ This is indeed the exact same model used to calculate the target lengths and dilution factors in the inelastic regions explained in later sections.
} 
time using $L$ for the total target length, and the second time using $L_{s i m}$ for the target length. In both cases, the total target radiated cross-section

$$
\sigma_{M T}=\rho_{A l} \ell_{A l} \sigma_{A l}+\rho_{K} \ell_{K} \sigma_{K}+\rho_{H e} \ell_{H e} \sigma_{H e}
$$

is calculated. ${ }^{5}$ The ratio $\sigma_{M T(s i m)} / \sigma_{M T(t r u e)}$ (using values of $\ell_{H e}$ equal to $L_{\text {sim }}$ and $L$, minus the foil thickness, in the numerator and denominator, respectively) was then written to an array for each bin, to be multiplied by the raw empty target counts. This gives the corrected count appropriate for unradiated model comparisons to carbon and ammonia targets. 6

\section{Raster pattern cuts for contaminated empty target runs}

The second correction to empty target runs applly only to the last three data brackets. All the empty runs in these brackets required a raster pattern cut to remove a bad part of the target (see Section 3.3.4). To evaluate the effect of the contamination in the lower half of the target, $L$ was measured, by both methods detailed in Section 5.3.1, for each of the last 4 data sets, using different cuts on the raster pattern (shown in Figure 5.2). The results of the study are organized in Table 5.2.1. As the table shows, the problem is more extensive than the whole pattern plot suggests. As pieces of the raster are removed, the count ratio (and hence $L$ ) decreases, but it does not stabilize until events correlating to the bottom half of the pattern are removed all together. Thus, for all empty target runs from 27899 onward, we cut events scattered from the bottom target haif, and double the remaining (upper half) event count rate. Precise determination of the ratio between whole- and half-targets resulted in a factor slightly different than 2.00 , however.

To calculate the appropriate multiplicative factor for the empty target runs after the raster cut, the same raster cut was employed on the (perfectly good) $\mathrm{NH}_{3}, \mathrm{ND}_{3}$ and ${ }^{12} \mathrm{C}$ targets in these run sets, and the remaining total counts were divided into the total (uncut) inclusive counts. The derived carbon target factor was the corrective factor actually used, since the ${ }^{12} \mathrm{C}$ target was closest on the target stick to the empty target, minimizing the effects of any difference in position due to motion of

\footnotetext{
${ }^{5}$ The cross-section of Kapton is considered to be equal to that of carbon tor the purpose of this analysis.

${ }^{6}$ The correction was typically small, ranging fom $\sim 0.97$ below the elastic region to $\sim 1.07$ at high $W$, rising more quickly in $W$ for lower beam energies.
} 

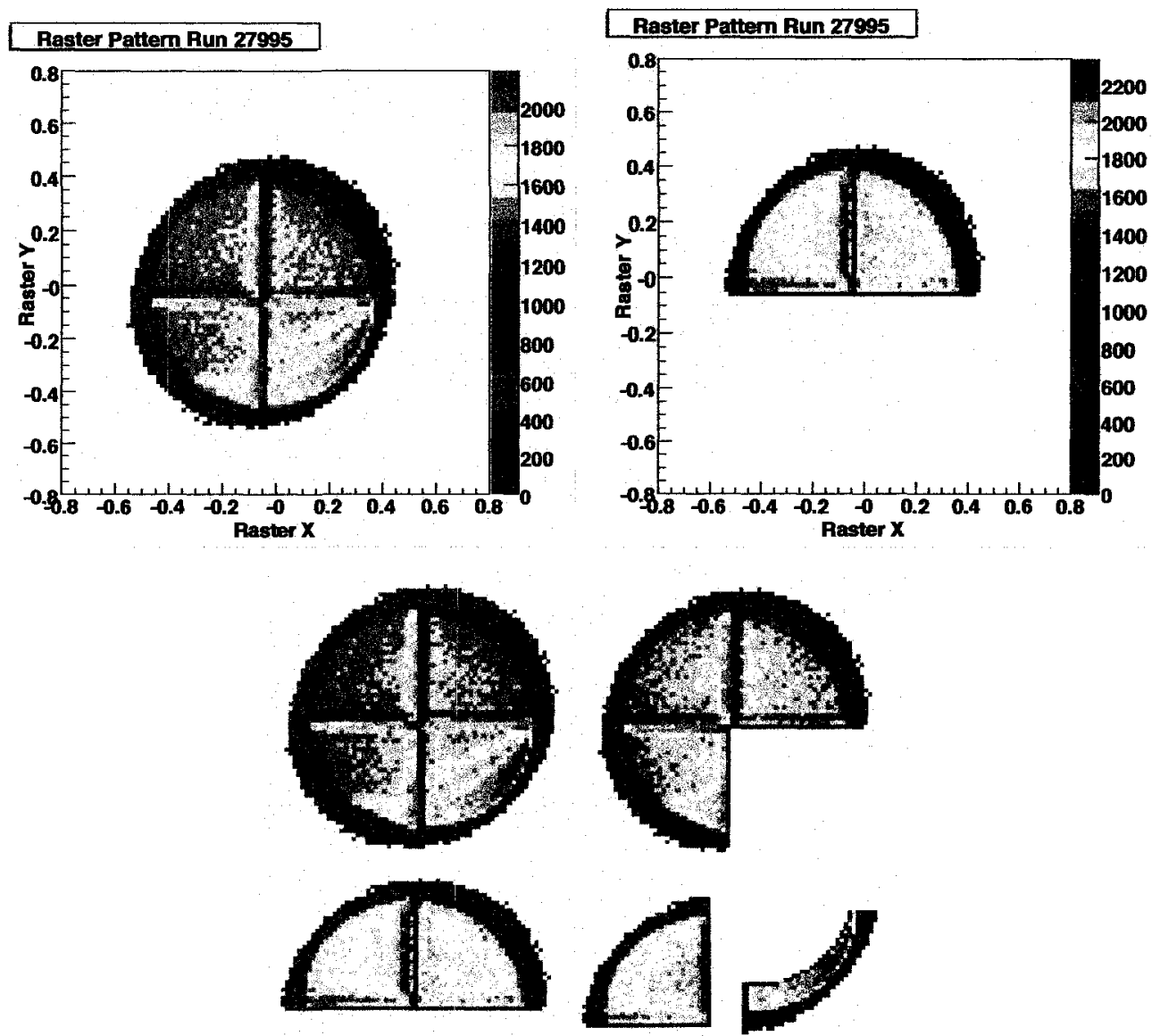

Figure 5.2: Raster pattern for an empty (LHe) target run in the latter part of the EG1b run set (shown also in Figure 3.10). A half-pattern (second figure) was decided as a final, stable cut on the raster pattern to remove the anomaly in the bottom half. The 5 cut configurations analyzed in the study of the counts are shown in the bottom figure. 
Table 5.3: Measured values of total target length $L$ using the two different methods outlined in Sections 5.3.1 and 5.3.2, labeled $L_{\text {rate }}$ and $L_{\text {model }}$, respectively. Different raster pattern cuts (shown in Figure 5.2) were used. Measurements from the last 4 data sets, plus "good" 2.5- runs prior to Run 27899 (for control purposes) are shown. Note the columns labeled "good only" remain constant, while the others fluctuate, only stabilizing when half or less of the target remains. All lengths are given in $\mathrm{cm}$.

\begin{tabular}{|c|c|c|c|c|}
\hline Raster Fraction & $L_{\text {rate }}(2.5-$, good only) & $L_{\text {model }}(2.5-$, good only) & $L_{\text {rate }}(2.5-$,all $)$ & $L_{\text {model }}(2.5-$,all) \\
\hline \hline whole & 1.95 & 1.86 & 2.61 & 2.49 \\
\hline $3 / 4$ & 1.95 & 1.86 & 2.51 & 2.39 \\
\hline top half & 1.94 & 1.85 & 2.50 & 2.37 \\
\hline $1 / 4$ & 1.95 & 1.85 & 2.47 & 2.35 \\
\hline \hline bad crescent & 1.93 & 1.81 & 3.35 & 3.13 \\
\hline
\end{tabular}

\begin{tabular}{|c|c|c|c|c|c|c|}
\hline Raster Fraction & $L_{\text {rate }}(2.5+)$ & $L_{\text {model }}(2.5+)$ & $L_{\text {rate }}(4.2+)$ & $L_{\text {model }}(4.2+)$ & $L_{\text {rate }}(4.2-)$ & $L_{\text {model }}(4.2-)$ \\
\hline \hline whole & 2.24 & 2.17 & 2.22 & 2.16 & 2.34 & 2.28 \\
\hline $3 / 4$ & 2.06 & 1.98 & 2.07 & 2.00 & 2.17 & 2.11 \\
\hline top half & 1.99 & 1.92 & 2.04 & 1.97 & 2.17 & 2.11 \\
\hline $1 / 4$ & 1.99 & 1.90 & 2.02 & 1.92 & 2.09 & 2.02 \\
\hline bad crescent & 3.45 & 3.20 & 3.44 & 3.05 & 4.08 & 3.86 \\
\hline
\end{tabular}

the target stick. The $\mathrm{NH}_{3}$ values were used for a determination of the systematic errors due to this problem (Section 7.2). These factors, which are close to the estimate of 2, are recorded in Table 5.4 .

Table 5.4: Ratio for total vs. half-raster cut counts for three different targets in the last 3 brackets of the EG1 data. The ${ }^{12} \mathrm{C}$ ratio was used as the multiplicative factor for (raster-cut) empty target counts in each given bracket. Note that some empty runs in Bracket 9 (2.5-) were unaffected by the anomaly, so bad runs in this set were simply removed, and no corrective factor was required.

\begin{tabular}{|c|c|c|c|}
\hline Set Label/Bracket & $\mathrm{NH}_{3}$ ratio & $\mathrm{ND}_{3}$ ratio & ${ }^{12} \mathrm{C}$ ratio \\
\hline \hline $2.5+/ 10$ & - & 1.989 & 1.957 \\
\hline $4.2+/ 11$ & 1.904 & 1.896 & 1.866 \\
\hline $4.2-/ 12$ & 1.90 & 1.904 & 1.871 \\
\hline
\end{tabular}

\subsection{Calculation of Target Length $L$}

The total length of the target (i.e. the length of the whole LHe mini-cup, including all LHe and aluminum, as well as the Kapton and target material in the target cell itself) was nominally 1.90 
$\mathrm{cm}$ (see Table 2.1). However, this cannot be taken as an exact measurement, due to variations in target length due to unobservable factors that occur during the experiment. These factors include

- Concavity of the aluminum target ends due to the negative pressure within the cryotarget

- Possible overflow of LHe from the target minicup (increasing the total length by up tp $0.2 \mathrm{~cm}$ )

- Slight variation vs. beam position due to curvature of the entrance and exit windows.

Accurate knowledge of the target length $L$ is essential for the accurate calculation of dilution factors (Section 5.6) for unpolarized background subtraction. It is desirable to have a method for measuring $L$ that accounts for minute variations in the total target length.

Two separate methods were developed for calculation of $L$, both of which require use of inclusive empty (LHe) and ${ }^{12} \mathrm{C}$ target counts. The first (Section 5.3.1) utilizes only real EG1 data and a bin-bybin model in the high resonance and deep-inelastic regions to calculate $L$. The radiative correction factor outlined in the previous section must be used for the empty target data in this method. The second method (Section 5.3.2) uses a radiated cross-section model to calculate $L$, and thus can use raw carbon and empty inclusive counts across both resonance and DIS regions. In practice, both methods were compared for validity, and the radiated cross-section model method was used for the actual measurement of $L$.

Because of possible variations over time, the value of $L$ used for dilution factor calculations was specific to each data bracket. For the purposes of ${ }^{12} \mathrm{C} /{ }^{15} \mathrm{~N}$ data analysis, which used a completely different target insert, similar measurements of $L$ were not possible for each individual set, because there were no empty target runs during the carbon/nitrogen analysis phases. Instead, the errorweighted average

$$
L_{\text {avg }}=\frac{\sum_{\text {brackets }} L / \sigma_{L}^{2}}{\sum_{\text {brackets }} 1 / \sigma_{L}^{2}}
$$

was used as an estimate for the total target length in the carbon/nitrogen target runs, with an appropriate systematic error included on any results from this target insert. 


\subsubsection{Calculation of $L$ from data}

The normalized count rates for the empty and carbon targets can be expressed in terms of $\sigma_{C}$ $\left({ }^{12} \mathrm{C}\right.$ scattering cross-section), $\sigma_{H e}\left({ }^{4} \mathrm{He}\right.$ scattering cross-section) and $\sigma_{F}$ (the averaged scattering cross-section of the foil Kapton and aluminum) as

$$
\begin{gathered}
n_{C} \propto \rho_{C} \ell_{C} \sigma_{C}+\rho_{F} \ell_{F} \sigma_{F}+\rho_{H e}\left(L-\ell_{C}\right) \sigma_{H e} \\
n_{M T} \propto \rho_{F} \ell_{F} \sigma_{F}+\rho_{H e} L \sigma_{H e}
\end{gathered}
$$

where the constant of proportionality is directly dependent on the acceptance and is assumed to be the same for both targets. If we assume $\sigma_{F} / \sigma_{C}=\rho_{F} \ell_{F} / \rho_{C} \ell_{C} \equiv f$, then ${ }^{7}$ these reduce to

$$
\begin{gathered}
n_{C} \propto(1+f) \rho_{C} \ell_{C} \sigma_{C}+\rho_{H e}\left(L-\ell_{C}\right) \sigma_{H e} \\
n_{M T} \propto f \rho_{C} \ell_{C} \sigma_{C}+\rho_{H e} L \sigma_{H e}
\end{gathered}
$$

Dividing the first relation into the second and assuming $\sigma_{C}=3 \sigma_{H e},{ }^{8}$ we get

$$
r \equiv \frac{n_{M T}}{n_{C}}=\frac{3(1+f) \rho_{C} \ell_{C}+\rho_{H e}\left(L-\ell_{C}\right)}{3 f \rho_{C} \ell_{C}+\rho_{H e} L}
$$

Solving for $L$ then yields

$$
L=\left(\frac{3 \rho_{C} e l l_{C}[(1+f) r-f]}{\rho_{H e}}-r \ell_{C}\right) /(1-r)
$$

$L$ was calculated each bin, with statistical error bars calculated for only one variable $(r)[103]$ as

$$
\sigma_{L}=\frac{\partial L}{\partial r} \sigma_{r}=\left[\frac{N(1+f)-\ell_{C}}{1-r}+\frac{N[(1+f) r-f]-r \ell_{C}}{(1-r)^{2}}\right] \sigma_{r}
$$

\footnotetext{
${ }^{7}$ This is not strictly true, of course, but foil contributions are generally small compared to that of the carbon target, Kapton approximates carbon in its cross-section, and the per nucleon cross-section difference between aluminum and carbon (i.e. the nuclear EMC effect) is small. The cross-sectional model does not have the drawback of this assumption, but the differences are minor, as the final plots of $L, \ell_{A}$ and $F_{D F}$ show.

${ }^{8}$ This is also not strictly true, due to the nuclear EMC effect, etc.; see previous footnote.
} 
where $N \equiv 3 \rho_{C} \ell_{C} / \rho_{H e}$. The error on $r, \sigma_{r}$, is given in quadrature by

$$
\sigma_{r}=\sqrt{\left(\frac{\partial r}{\partial n_{C}}\right)^{2} n_{C}+\left(\frac{\partial r}{\partial n_{M T}}\right)^{2} n_{M T}}
$$

which yields

$$
\frac{\sigma_{r}}{r}=\sqrt{n_{C}^{-1}+n_{M T}^{-1}}
$$

Plots of $L$ as a function of both $W$ and $Q^{2}$, with the error-weighted average taken over the opposing variable, are shown in Figure 5.3.

The determination of $L$ for each run set was then determined by the error-weighted average over the bins,

$$
L=\frac{\sum_{Q^{2}} \sum_{W} L / \sigma_{L}^{2}}{\sum_{Q^{2}} \sum_{W} 1 / \sigma_{L}^{2}}
$$

with error ${ }^{9}$

$$
\sigma_{L}=\frac{1}{\sqrt{\sum_{Q^{2}} \sum_{W} 1 / \sigma_{L}^{2}}}
$$

Only ranges of $W$ and $Q^{2}$ were used where the model was valid. Because of the nuclear EMC effect (i.e. the breakdown of assumptions like $N \equiv 3 \rho_{C} \ell_{C} / \rho_{H e}$ ), the $\Delta(1232)$ region did not present a completely "flat" measurement of $L$, so a lower cut of $W=1.40 \mathrm{GeV}$ was used. The higher cut on $W$ and selection of $Q^{2}$ bins was beam energy dependent; the same boundaries used for dilution factor modeling (Table 5.10) were employed here. Results for this method of determination of $L$ are listed in Table 5.5 under the "Method 1" heading.

\subsubsection{Calculation of $L$ from radiated cross-sections}

$L$ was also calculated using the radiated cross-sections contributed by each material in the ${ }^{12} \mathrm{C}$ and LHe targets. The model described in Section 2.8 was used. Because this model calculates radiated cross-sections, which are dependent on the amount of material in each target, the cross-sections for like materials must be calculated independently for each target. To resolve the ambiguity, a

\footnotetext{
${ }^{9}$ It is important to note that this error is just the statistical error on $L$ assuming statistical compatibility of the data, so that it does not account for systematic variations of $L\left(W, Q^{2}\right)$, and therefore grossly underestimates the actual error on $L$. Its only use in this analysis is as a weighting factor for calculating $L_{a v g}$ in ${ }^{12} \mathrm{C} / 15 \mathrm{~N}$ analysis.
} 

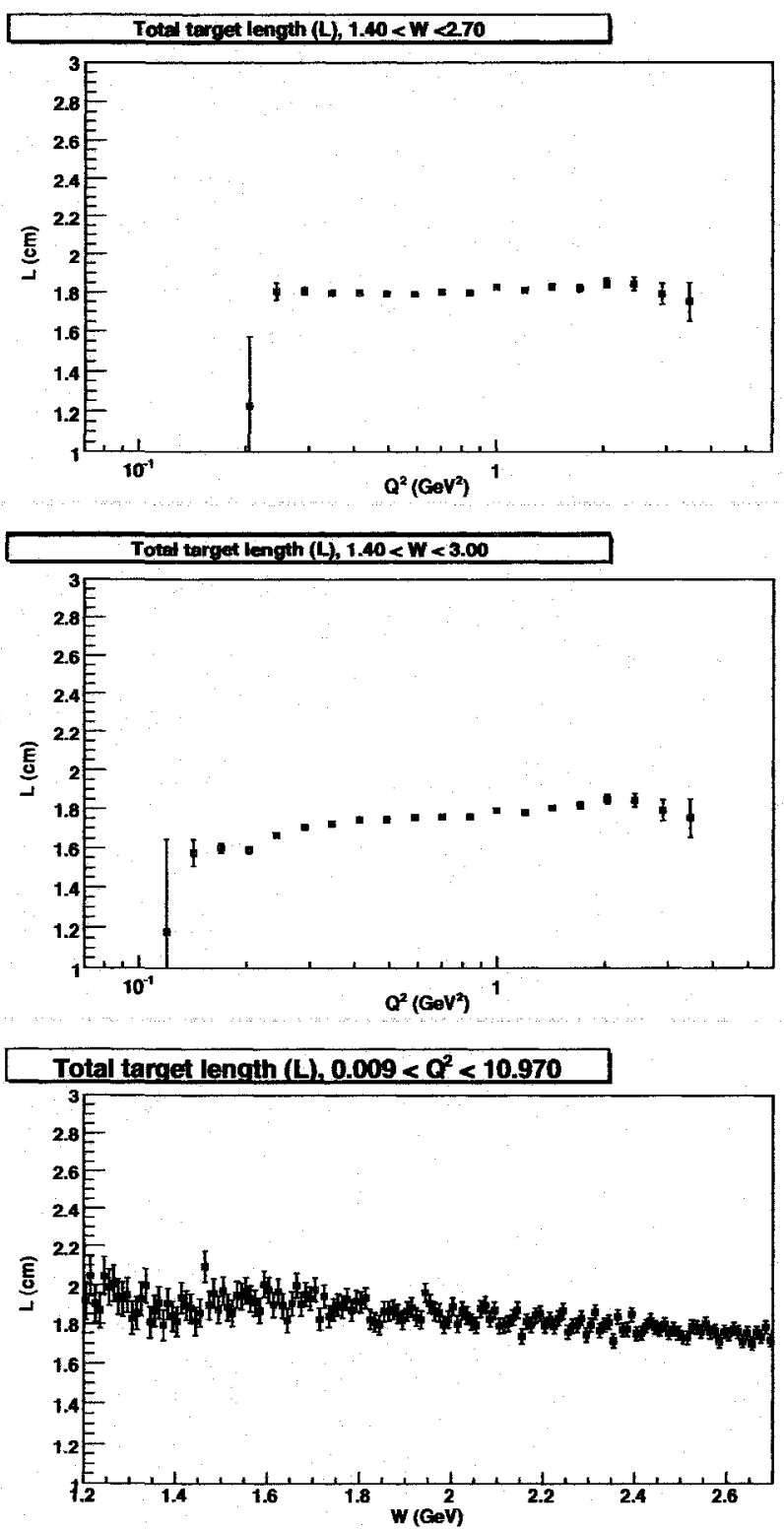

Figure 5.3: Total target length, $L$, calculated using EG1 data, shown as a function of $Q^{2}$ (top two figures) and $W$ (bottom figure). Note that below $W \sim 1.4 \mathrm{GeV}$, the $\Delta$-resonance affects the measurement (bottom), so $W$ values below this threshold are not used in calculating the average. High- $W$ values are avoided, as well, due to the extreme sensitivity of radiative corrections between the two targets, as can be seen in a comparison of the top two plots. Shown are the $5.76 \mathrm{GeV}$ outbending data. 

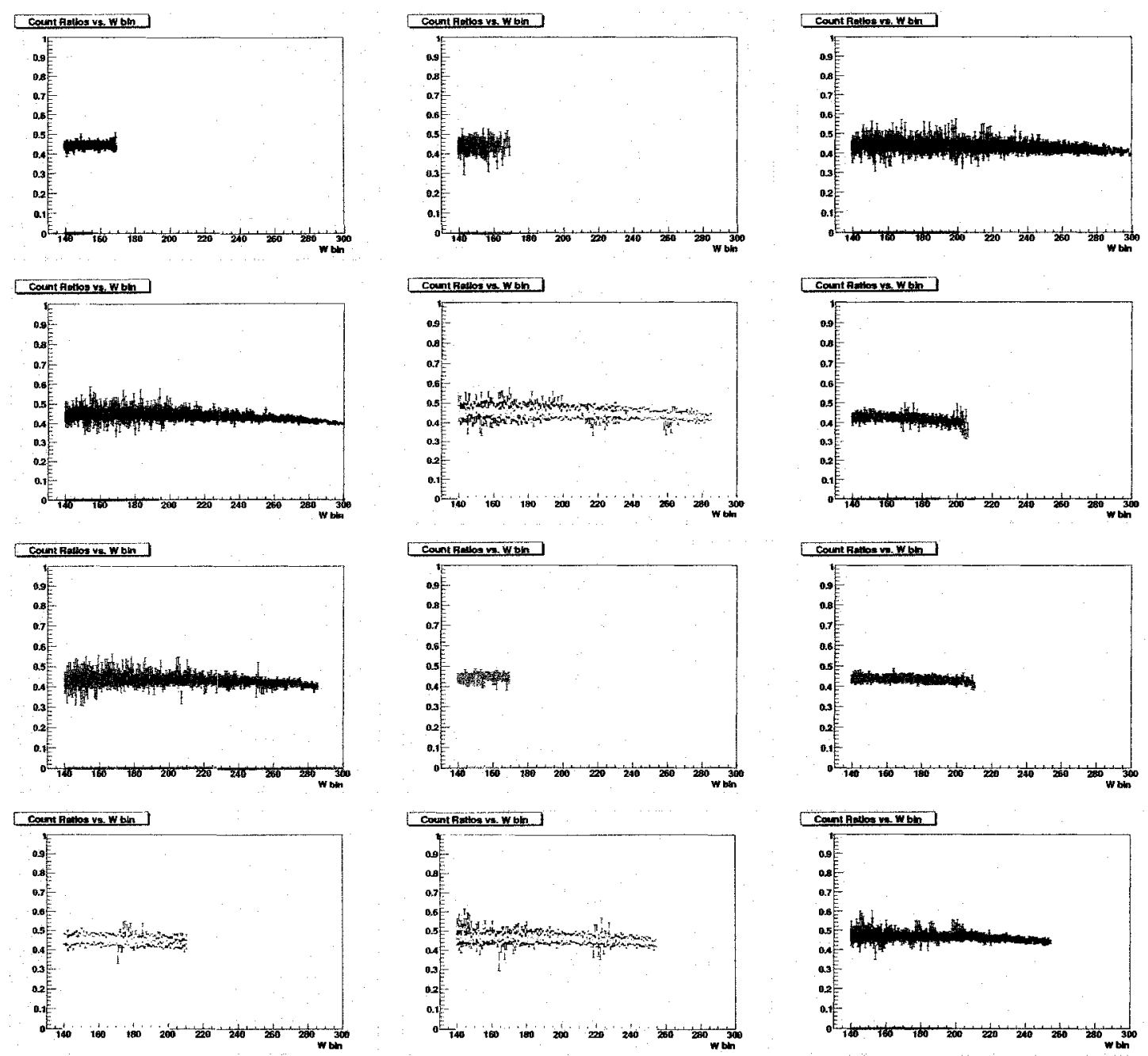

Figure 5.4: The count ratio $n_{M T} / n_{C}$, shown as a function of $W$, including the correction for differing radiation lengths, for all 12 data brackets in sequence. Points of constant $W$ across varying $Q^{2}$ were superimposed. The ratio measured $\sim 0.44$ throughout data collection. This value was used to determine $L$. Points with an error bar larger than 0.1 were excluded for clarity. 
subscript in brackets [ ] is used to label which target configuration was used to calculate the given cross-section.

Beginning with Eqs. 5.15-5.16, with the foil terms expanded into Kapton and aluminum crosssections:

$$
\begin{gathered}
n_{C} \propto \rho_{A l} \ell_{A l} \sigma_{A l[C]}+\rho_{K} \ell_{K} \sigma_{C[C]}+\rho_{C} \ell_{C} \sigma_{C[C]}+\rho_{H e}\left(L-\ell_{C}\right) \sigma_{H e[C]} \\
n_{M T} \propto \rho_{A l} \ell_{A l} \sigma_{A l[M T]}+\rho_{K} \ell_{K} \sigma_{C[M T]}+\rho_{H e} L \sigma_{H e[M T]}
\end{gathered}
$$

(where it is approximated $\sigma_{C} \approx \sigma_{K}$, and the constants of proportionality are equal for both targets), defining $W \equiv \rho_{A l} \ell_{A l} \sigma_{A l}+\rho_{K} \ell_{K} \sigma_{C}$ and $r \equiv n_{M T} / n_{C}$, and dividing, we find

$$
r=\frac{\mathrm{W}_{[M T]}+\rho_{H e} L \sigma_{H e[M T]}}{\mathrm{W}_{[C]}+\rho_{C} \ell_{C} \sigma_{C[C]}+\rho_{H e}\left(L-\ell_{C}\right) \sigma_{H e[C]}}
$$

Solving for $L$ yields

$$
L=\frac{r \mathrm{~W}_{[C]}-\mathrm{W}_{[M T]}+r \ell_{C}\left(\rho_{C} \sigma_{C[C]}-\rho_{H e} \sigma_{H e[C]}\right)}{\rho_{H e}\left(\sigma_{H e[M T]}-r \sigma_{H e[C]}\right)}
$$

To calculate a statistical error bar, it was assumed that the foil contributions were small (so that W could be neglected) and that $\sigma_{\mathrm{He}[\mathrm{MT}]} \approx \sigma_{\mathrm{He}[\mathrm{C}]}$, so that

$$
\frac{\sigma_{L}}{\sigma_{r}}=\frac{\partial L}{\partial r}=\frac{\ell_{C}\left(\rho_{C} \sigma_{C[C]}-\rho_{H e} \sigma_{H e[C]}\right)}{\rho_{H e} \sigma_{H e[C]}(1-r)^{2}}
$$

where the error $\sigma_{r}$ is calculated using equation 5.23.

An apparent catch in the use of this method is that the amounts of all materials must be known in order to calculate the radiated cross-sections (see Section 2.8), which means $L$ must be kriown to calculate the cross-sections! Fortunately, the radiative corrections are generally small compared to the Born (unradiated) cross-section, so that only a very rough approximation of $L$ is needed for the radiative correction. In practice, an initial value of $L=1.90 \mathrm{~cm}$ is used, and when $L$ is calculated (and averaged over all valid bins), the new value of $L$ is used to calculate the cross-sections again, which are inserted back into the model. This process is repeated until $L$ stabilizes. In practice, this never required more than 3 iterations. Using different initial values of $L$ ranging from 1.60 to 2.40 $\mathrm{cm}$ showed no change in the final measurement when the iterative method was used. 
Values of $L$ were then combined for each bin just as in the first method (see Footnote 9), but because phenemona like the nuclear EMC effect were included in the model, the $\Delta(1232)$ resonance could be included, so a lower limit of $W=1.10 \mathrm{GeV}$ was used instead, in this case. Unfortunately, the heavy dependence on the model caused a large dip in the measurement of $L$ when higher $W$ values were approached (see Figure 5.5), causing a systematic lowering in the averaged value of $L$. For this reason, a more stringent cut on the high value of $W$ was used. Specifically,

$$
W<1.10 \mathrm{GeV}+\frac{2}{3}\left(W_{\text {limit }}-1.10 \mathrm{GeV}\right)
$$

was used, that is, $2 / 3$ of the inelastic region used for dilution factor calculation. The value of $W_{\text {limit }}$ is defined as the appropriate value listed in Table 5.10.

The two methods yield approximately the same values for $L$, as can be seen in Table 5.5 .

Table 5.5: Measured target length $L$ using both methods outtined in the text. Errors shown are statistical (i.e. for weighting purposes) only, and do not reflect systematical variations in terms of $W$ and $Q^{2}$. Method 2 was used for the value of $L$ in actual analysis. $L_{\text {avg }}$ is used only for ${ }^{12} \mathrm{C} /{ }^{15} \mathrm{~N}$ analysis. Lengths are in $\mathrm{cm}$.

\begin{tabular}{|c|c|c|}
\hline Set Label/Bracket & $L$ (Method 1) & $L$ (Method 2) \\
\hline \hline $1.6+/ 1$ & $1.93 \pm 0.0056$ & $1.90 \pm 0.0038$ \\
\hline $1.6-/ 2$ & $1.82 \pm 0.0109$ & $1.85 \pm 0.0054$ \\
\hline $5.76-/ 3$ & $1.79 \pm 0.0036$ & $1.83 \pm 0.0045$ \\
\hline $5.73-/ 4$ & $1.82 \pm 0.0023$ & $1.87 \pm 0.0029$ \\
\hline $5.7+/ 5$ & $1.93 \pm 0.0044$ & $1.95 \pm 0.0065$ \\
\hline $2.3+/ 6$ & $1.76 \pm 0.0038$ & $1.77 \pm 0.0035$ \\
\hline $5.6+/ 7$ & $1.77 \pm 0.0044$ & $1.78 \pm 0.0063$ \\
\hline $1.7-/ 8$ & $1.87 \pm 0.0036$ & $1.87 \pm 0.0019$ \\
\hline $2.5-/ 9$ & $1.84 \pm 0.0028$ & $1.86 \pm 0.0022$ \\
\hline $2.5+/ 10$ & $1.93 \pm 0.0072$ & $1.92 \pm 0.0070$ \\
\hline $4.2+/ 11$ & $2.01 \pm 0.0060$ & $2.00 \pm 0.0076$ \\
\hline $4.2-/ 12$ & $2.04 \pm 0.0021$ & $2.05 \pm 0.0022$ \\
\hline \hline$L_{\text {avg }}$ & $1.89 \pm 0.0010$ & $1.90 \pm 0.00095$ \\
\hline & &
\end{tabular}




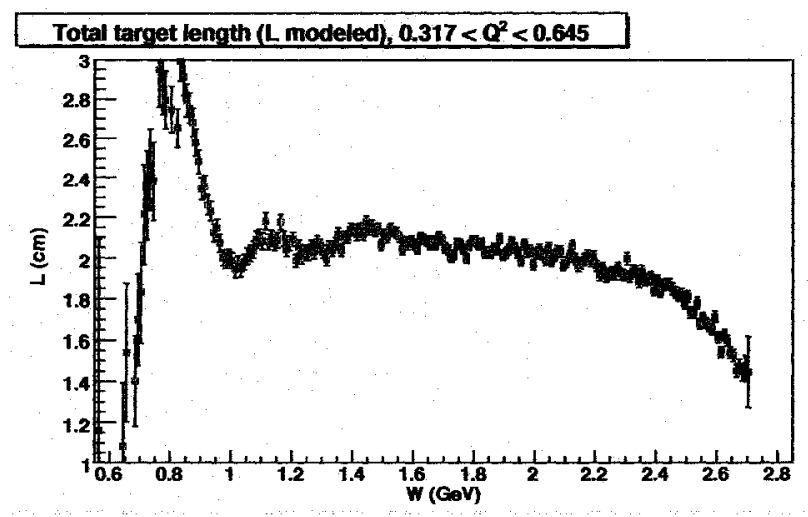

Total target length ( $L$ modeled), $0.317<Q^{2}<0.645$

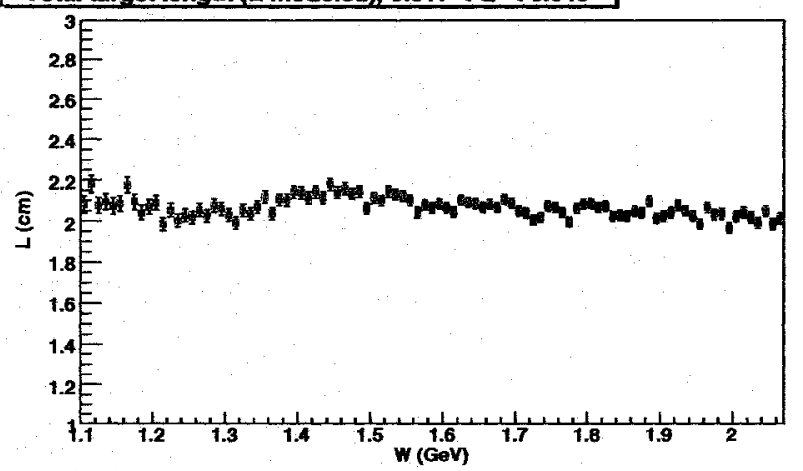

Figure 5.5: Total target length, $L$, calculated using a radiated cross-section model, as a function of $W$, averaged over $4 Q^{2}$ bins. The top figure clearly demonstrates the inadequacy of the model in the elastic region, as well as its failure, likely due to $e^{+} e^{-}$pair production, at high $W$. The bottom figure is the same data, showing only the region used to make the actual calculation of $L$. Data shown are for the last bracket, the 4.2- data. 

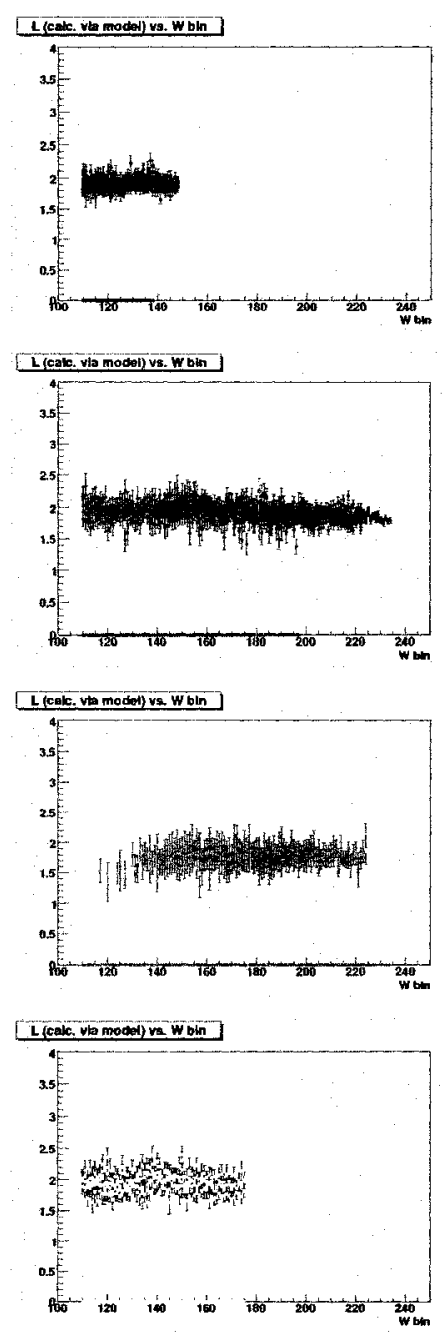
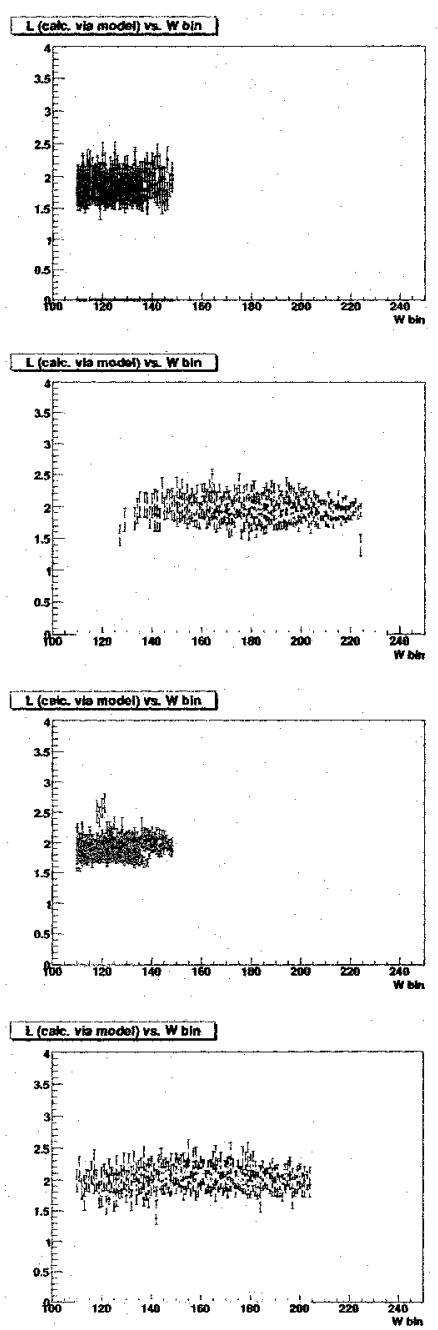
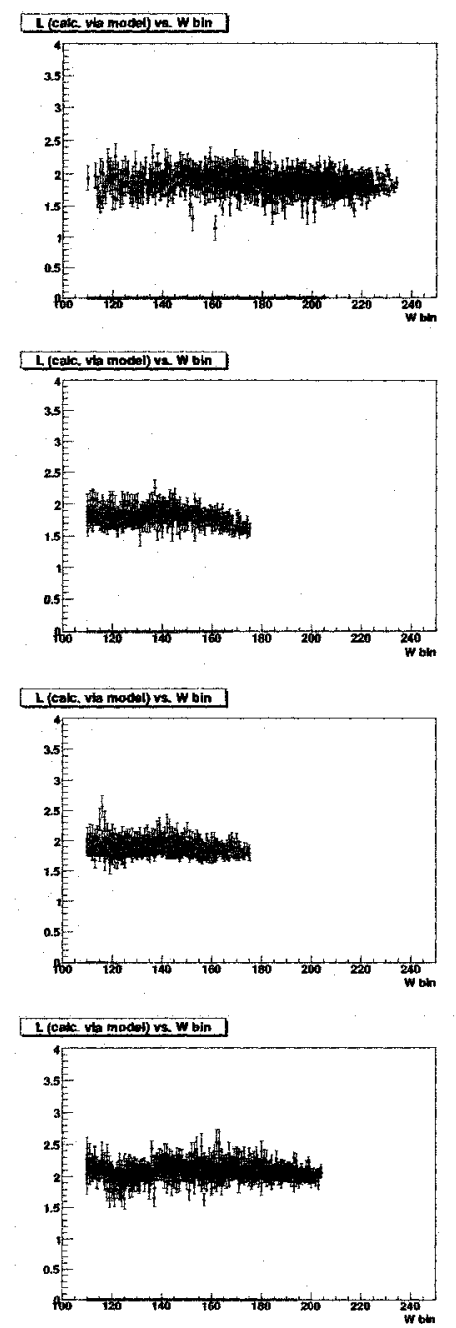

Figure 5.6: $L$ shown as function of $W$ bin for all 12 data brackets, in chronological sequence. As in Figure 5.4, points of different $Q^{2}$ are superimposed. Only points with an error of less than $0.2 \mathrm{~cm}$ are included for clarity. 


\subsection{Modeling ${ }^{12} \mathrm{C}$ to ${ }^{15} \mathrm{~N}$ Data}

Solid ${ }^{12} \mathrm{C}$ was used in EG1 as an approximation to the ${ }^{15} \mathrm{~N}$ in ${ }^{15} \mathrm{NH}_{3}$, scaled to the proper nuclear mass per scattering center (see below), for purposes of non-polarized background removal. This works well as a first-order approximation, but due to the likelihood of additional effects arising from the unpaired neutron in the ${ }^{15} \mathrm{~N}$ nucleus, it is desirable to have a model relating the cross-section ratios of these two elements.

Fortunately, a limited number of solid ${ }^{15} \mathrm{~N}$ runs, using a separate target insert (Section 2.4), were interspersed with ${ }^{12} \mathrm{C}$ runs for comparison. The original method of comparison of these data, seen in Refs. [2] and [46], assumed that the frozen target length $\ell_{N}$ was well-known, and that the crosssections could be scaled as a function of the neutron-deuteron cross-section ratio $\left(\sigma_{n} / \sigma_{d}\right)$ and two constant coefficients (labeled $a$ and $b$ ). Unfortunately, this method does not accord precision measurements to the relationship, as the frozen nitrogen length $\ell_{N}$ is not known to better than $\sim 0.1$ $\mathrm{cm}$, leading to large uncertainties in the relation coefficients. The development of a physical model to relate the ratio of cross-sections of ${ }^{15} \mathrm{~N}$ and ${ }^{12} \mathrm{C}$ was needed in this experiment.

\subsubsection{Development of the model}

An in-depth analysis of the carbon-nitrogen subsets (contained in 7 beam energy/torus current brackets, see Table 5.2), was performed during the most recent EG1 analysis. Of these 7 data sets, only the first ( $2.3 \mathrm{GeV}$ inbending) contained enough data to be used to develop a precision model of ${ }^{15} \mathrm{~N}$ cross-sections relative to ${ }^{12} \mathrm{C}$. The model outlined in Section 2.8 was used for the cross-sectional data of ${ }^{12} \mathrm{C}$, LHe and Al (with Kapton approximated as ${ }^{12} \mathrm{C}$, as usual). A best fit of the model parameters to the ${ }^{15} \mathrm{~N}$ data generated a model that could reproduce the observed ratio of nitrogen/carbon counts as a function of $W$ and $Q^{2}$. The other 6 brackets could then be used as a test of the model. Ref. [95] contains a thorough description of the process used to develop the model; only the basics are outlined in this section. To generate the model, the ratio of nitrogencarbon target inclusive electron counts was recorded as a function of $Q^{2}$ and $W$ in the standard manner. ${ }^{10}$ Because a different target insert was used for these runs, it was not possible to directly

\footnotetext{
${ }^{10} \mathrm{PID}$ cuts were somewhat different than those specifically outlined in the previous chapter, but comparison between the ratios using varying PIDs showed no substantial discrepancies. See Ref. [95] for more details.
} 


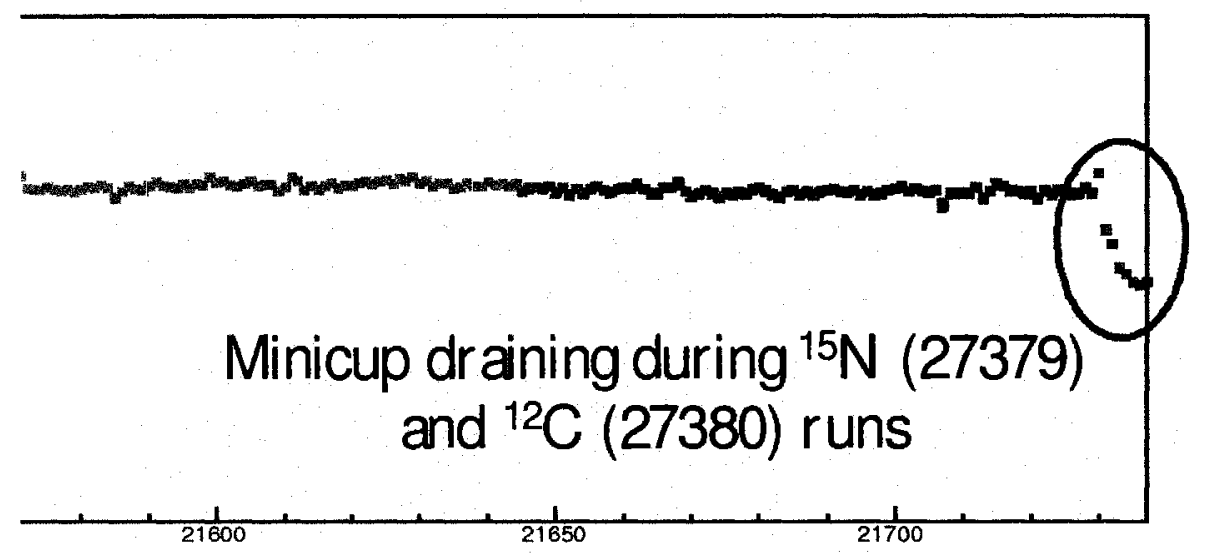

Figure 5.7: An enlargement of a quality check plot of the 5.6 GeV outbending carbon-nitrogen run set, showing FC-normalized count rate as a function of DST file number. (Normal ${ }^{12} \mathrm{C}$ run files are highlighted in green.) Note the decrease in count rate near the end of the set. Closer scrutiny of the rate counts and log book entries showed that the LHe began draining during a frozen ${ }^{15} \mathrm{~N}$ run (Run 27379) and had nearly completed draining by the beginning of the short (carbon) Run 27380. This run was useful for comparison to find out the total contribution to the cross-section from LHe scattering, and hence an approximation of the total target length $L$.

measure $L$. However, it was known from physical measurement of the assembled target that the total path length through the LHe in the target was $\sim 1.80 \mathrm{~cm}$, with the possibility of overflow of LHe in the target minicup, which could potentially increase the effective target length to up to $2.10 \mathrm{~cm}$.

To detect the presence of overflow, we analyzed a single data run containing carbon, throughout which the LHe was draining (Run 27380). Observation of the file-by-file count rate of this run shows that the target was (nearly) drained by the end of the run (see Figure 5.7). Comparing a "full" carbon target run to the files near the end of Run 27380 , and using a method very similar to that outlined in Section 5.3.2, ${ }^{11}$ an estimate of the total LHe length could be derived. It was indicated that the minicup had not overflowed, so $L=1.80 \mathrm{~cm}$ was used in the analysis. ${ }^{12}$ The possibility of $\mathrm{H}_{2} \mathrm{O}$ contamination was also investigated by a careful inspection of the elastic peak region of the count ratio [95]. No tangible evidence of an elastic peak resulting from free hydrogen was found, so we concluded that water vapor contamination was not an issue.

\footnotetext{
"The only difference is that the term $\rho_{H e}\left(L-\ell_{C}\right) \sigma_{H e}$ for the "empty" target is replaced by $\rho_{C} \ell_{C} \sigma_{C}$ before solving for $L$.

${ }^{12}$ The value of $2.10 \mathrm{~cm}$ was used to estimate the systematic error. The remaining analysis in this thesis uses $L_{\text {avg }} \approx 1.88$ cm, well within the systematic error.
} 
The count ratio could then be modeled as ${ }^{13}$

$$
r_{N C} \equiv \frac{n_{N}}{n_{C}}=\frac{\rho_{A l} \ell_{A l} \sigma_{A l[N]}+\rho_{K} \ell_{K} \sigma_{C[N]}+\rho_{N} \ell_{N} \sigma_{N[N]}+\rho_{H e}\left(L-\ell_{N}\right) \rho_{H e[N]}}{\rho_{A l} \ell_{A l} \sigma_{A l[C]}+\rho_{K} \ell_{K} \sigma_{C[C]}+\rho_{C} \ell_{C} \sigma_{C[C]}+\rho_{H e}\left(L-\ell_{C}\right) \rho_{H e[C]}}
$$

with $\ell_{N}$ determined by a minimization of

$$
\chi^{2}=\sum_{W, Q^{2}}\left(r_{N C}-r_{N C(\text { model })}\right)^{2} / \sigma_{r_{N C}}^{2}
$$

As in the case of using a model to determine $L$ in the previous section, this must be done iteratively, as initial knowledge of the nitrogen/carbon model is not precise. For that reason, only values of $W>1.2 \mathrm{GeV}$ were used in the fit, to avoid sensitivity to the peak structure in the inelastic range. The model parameters (described in Section 2.8) used to determine the unknown $\sigma_{N}$ were then optimized with MINUIT to generate the new model.

Though there was not enough data to fit the model at the 1.7, 4.2 and 5.6 GeV beam energies, the model could be extrapolated to these data sets to test the viability of the fit. Ratios of counts between frozen nitrogen and carbon using the finished model and Eq. 5.32 are shown in Figure 5.8. The model fits the data well, except for the quasielastic region when $Q^{2}$ becomes too low (bottom figures).

The most obvious major systematic errors in this mini-analysis are the target material lengths $L$ and $\ell_{N}$. However, there were a couple more significant discrepancies in the data which required correction before an accurate model could be derived.

\section{Scaling of ${ }^{12} \mathrm{C}$ runs from different target inserts}

This correction applies only to the $4.2 \mathrm{GeV}$ outbending data. Most nitrogen-carbon data sets contained an equal quantity of ${ }^{12} \mathrm{C}$ and ${ }^{15} \mathrm{~N}$ data. The 4.2 - data (bracket $4^{2}$ ), however, contained only one (very brief) carbon run (Run 28500), which suffered a DC HV trip in Sector 3, making overall count comparisons involving the (already meager amount of) data exceedingly difficult.

\footnotetext{
${ }^{13}$ All cross-sections and count rates here are implicitly assumed to be functions of $W$ and $Q^{2}$.
} 

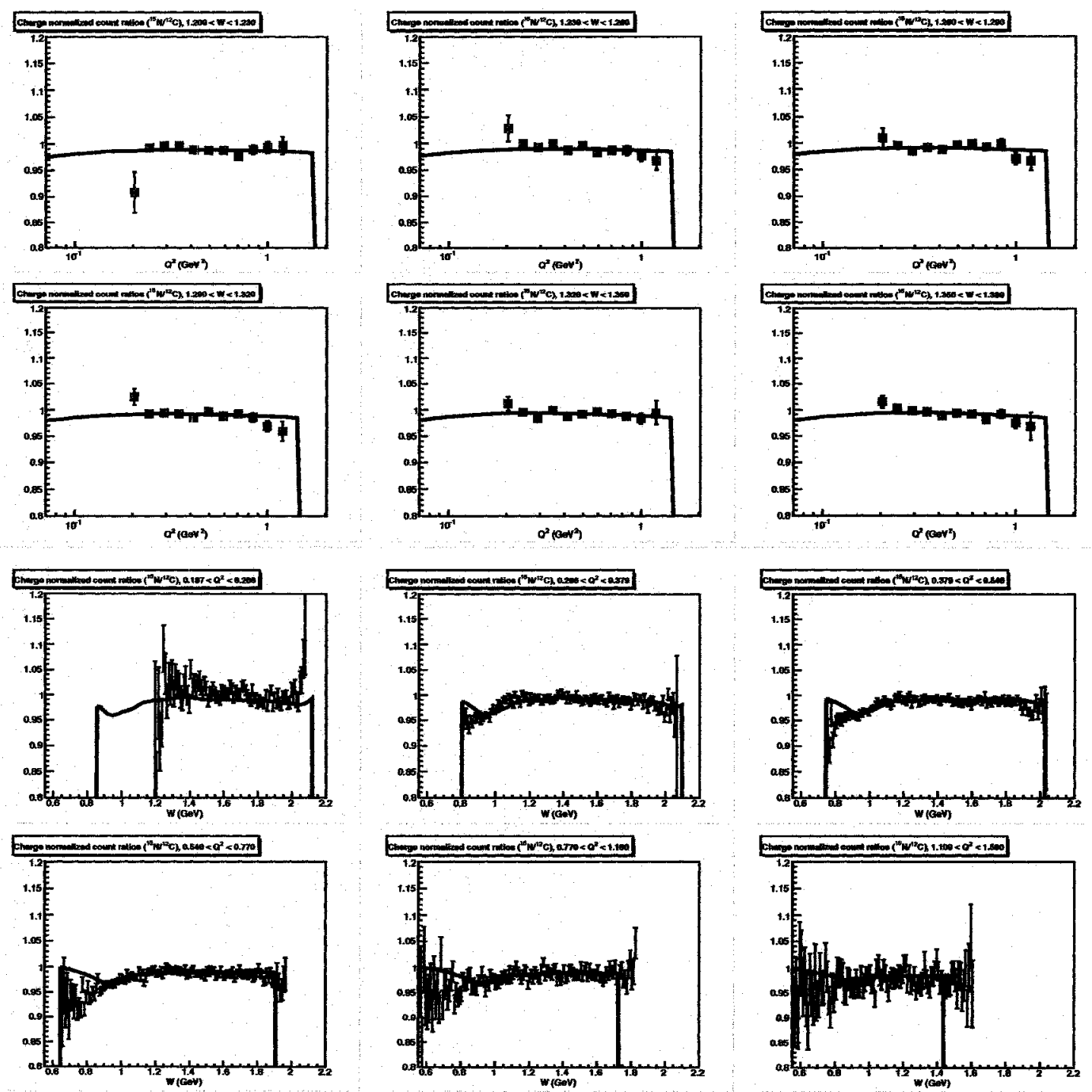

Figure 5.8: The count ratio $r_{N C}=\frac{n_{N}}{n_{C}}$ compared to the finished model calculation of the same quantity, showing only the $2.3 \mathrm{GeV}$ data used in construction of the model. Extended $W$ and $Q^{2}$ ranges are shown. More information on the model can be found in Ref. [95]. 
It was thus desirable to take advantage of the large number of ${ }^{12} \mathrm{C}$ runs taken during the main (ammonia) data collection of 4.2- runs (Bracket 11), taken not too long before the runs in bracket 4'. These carbon runs, however, used a different insert stick, and thus could not be trusted for comparison unless some sort of normalization was made. For this purpose, the inclusive (charge normalized) count rate in all $\mathbf{5}$ good sectors of Run 28500 was divided by the inclusive count rate in the same 5 sectors for the Bracket 11 carbon runs.

An average of the ratio between the bracket $11 /$ bracket $4^{\prime}$ counts yielded a value of 1.047 . The carbon insert used in the nitrogen-15 target stick was measured as $4.5 \%$ thicker than that used in the regular target stick [65], so this is an expected result.

Thus, for the purposes of testing the fit, and further carbon-nitrogen analysis, the bracket 11 carbon runs were used for bracket 4' analysis in place of Run 28500, and inclusive counts in every bin were multiplied by 1.047 .

\section{Adjustment of Faraday Cup charges due to $X_{0}$ discrepancies}

Initial plots of the ratios of ${ }^{15} \mathrm{~N} /{ }^{12} \mathrm{C}$ target counts showed overall offsets of indeterminate physical origin. Though knowledge of the intracacies of the resonance structures in the two targets are not obvious (and is, in fact, the goal of creating this model), we know the ratio in the deep inelastic (DIS) region should be 1.00 , assuming equal density $\times$ length $/ X_{0}$ for both targets, as the internal 3-quark point structure is the same for all matter.

This was definitely not the case, however, for raw normalized count ratios between the two targets. In fact, there was an energy-dependent offset in the DIS ratio, highest at low (1.7 GeV) beam energy, diminishing to nil at the high ( $5.6 \mathrm{GeV}$ ) beam energy. This phenomenon is consistent with the failure to account for multiple scattering spread at the Faraday Cup aperture between targets of differing radiation length (see Section 4.5$)$. The ratio offsets $(20.8 \%, 6.5 \%, 2.6 \%$, and $0 \%$ for 1.7 , 2.3, 4.2, and 5.6 GeV, respectively) are indicative of a larger frozen nitrogen target mass thickness $t / X_{0}$ than for the carbon target. ${ }^{14}$

The source of the extra radiation length is unknown; visual investigation of the target found

\footnotetext{
${ }^{14}$ The ratios were determined by the error-weighted average over the DIS region.
} 
no discernable difference between the nitrogen and carbon target cells. To account for the extra radiation length, normalization factors were multiplied by the overall ${ }^{12} \mathrm{C}$ target counts. These normalization factors are listed in Table 5.6.

Table 5.6: Required normalization factors for nitrogen-carbon data to account for the differing radiation lengths of the ${ }^{12} \mathrm{C}$ and ${ }^{15} \mathrm{~N}$ targets. The factor was applied to the carbon inclusive electron counts.

\begin{tabular}{|c|c|}
\hline Set Label/Bracket & Normalization factor \\
\hline \hline $2.3+11^{\prime}$ & 1.065 \\
\hline $5.6+/ 2^{\prime}$ & 1.000 \\
\hline $5.6-13^{\prime}$ & 1.000 \\
\hline $4.2-14^{\prime}$ & $1.026 \times 1.047=1.074$ \\
\hline $4.2+/ 5^{\prime}$ & 1.026 \\
\hline $1.7+/ 6^{\prime}$ & 1.208 \\
\hline $1.7-/ 7^{\prime}$ & 1.208 \\
\hline
\end{tabular}

This may appear to be (and indeed is, to an extent) a "messy cleanup" of the target data. However, application of the normalized count rates to calculation of the target length $\ell_{N}$ (as described in the remainder of this section) reveals a consistent value. This consistency is not present without the proper normalization factors on the data. Without the normalizations, an obviously improper correlation between between $\ell_{N}$ and the beam energy appears. Therefore, the normalization factors are testable for internal consistency, and are, in fact, a viable method for salvaging the validity of the data as a tool to test the model. Sample plots of the $W$-dependence of the model compared to real data are shown for the other 6 data sets in Figure 5.9.

\subsubsection{Comparison to the older fit method}

To check the internal consistency of the model, it is useful to check it against the older fit method cited in the introduction to this section. We begin by defining $n_{C}^{\prime} \propto \rho_{C} \ell_{C} \sigma_{C}$ and $n_{H e}^{\prime} \propto \rho_{H e} \sigma_{H e}$ where the proportionality constant is the same as in Eqs. 5.17 and $5.18,{ }^{15}$ and insert these values into these equations, to get

$$
n_{C}=(1+f) n_{C}^{\prime}+\left(L-\ell_{C}\right) n_{H e}^{\prime}
$$

\footnotetext{
${ }^{15}$ This gives the interpretation of $n_{C}^{\prime}$ as the charge normalized counts scattered from the ${ }^{12} \mathrm{C}$ stab only, and $n_{H e}^{\prime}$ as the normalized count rate scattered per $1 \mathrm{~cm}$ of LHe.
} 

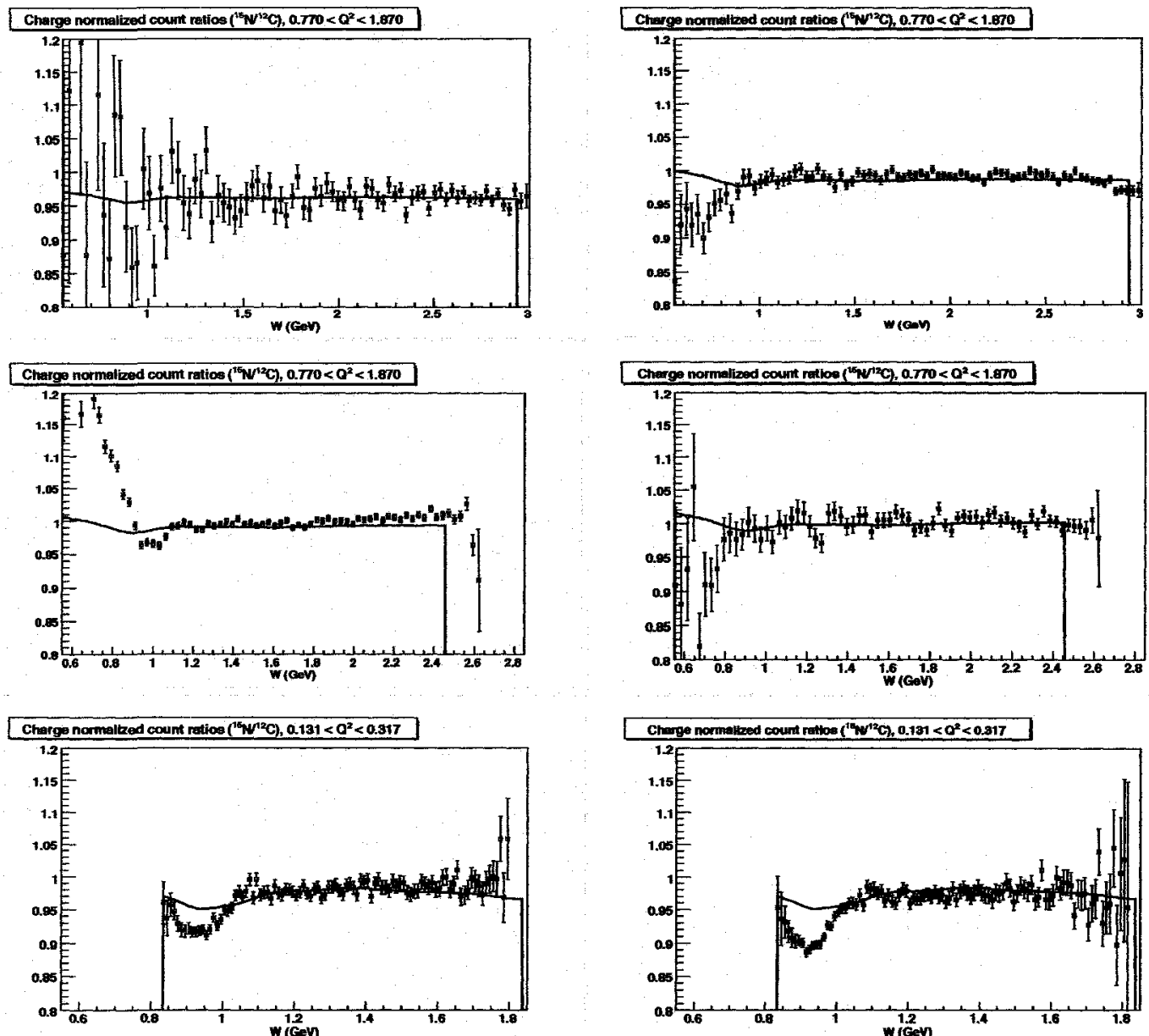

Figure 5.9: Extrapolations of the ${ }^{15} \mathrm{~N} /{ }^{12} \mathrm{C}$ model from the $2.3 \mathrm{GeV}$ data set to data sets at other beam energies. The six plots correspond to brackets 2'-7', in sequence. The three rows represent $5.6 \mathrm{GeV}, 4.2 \mathrm{GeV}$ and $1.7 \mathrm{GeV}$ data, in descending order. Extension of the fit to the elastic region at lower beam energies proved troublesome, due to the rapid change as a function of $W$ and the presence of the resolution-dependent elastic radiative tail. 


$$
n_{M T}=f n_{C}^{\prime}+L n_{H e}^{\prime}
$$

Solving for $n_{C}^{\prime}$ and $n_{H e}^{\prime}$ yields

$$
n_{C}^{\prime}=A n_{C}+B n_{M T}
$$

and

$$
n_{H e}^{\prime}=C n_{M T}+D n_{C}
$$

where

$$
\begin{array}{ll}
A=\frac{L}{L+f \ell_{C}} & B=-\frac{L-\ell_{C}}{L+f \ell_{C}} \\
C=\frac{1+f}{L+f \ell_{C}} & D=-\frac{f}{L+f \ell_{C}}
\end{array}
$$

The inclusive count rate from a ${ }^{15} \mathrm{~N}$ target (using the notation of Eqs. 5.15 and 5.16) is given by

$$
n_{N} \propto \rho_{F} \ell_{F} \sigma_{F}+\rho_{H e}\left(L-\ell_{C}\right) \sigma_{H e}+\rho_{N} \ell_{N} \sigma_{N}
$$

Using the definition of $f$, this becomes

$$
n_{N} \propto f \rho_{C} \ell_{C} \sigma_{C}+\rho_{H e}\left(L-\ell_{C}\right) \sigma_{H e}+\rho_{N} \ell_{N} \sigma_{N}
$$

Using the above, we find

$$
n_{N}=f n_{C}^{\prime}+\left(L-\ell_{C}\right) n_{H e}^{\prime}+n_{N}^{\prime}
$$

where

$$
n_{N}^{\prime} \propto \rho_{N} \ell_{N} \sigma_{N}
$$

(same proportionality constant again assumed) is the only remaining term with unknowns, namely $\ell_{N}$ and $\sigma_{N}$. The value $\ell_{N}$ is just a number, but $\sigma_{N}$ is a modeled function that varies with kinematics. The simple model assumed here is

$$
\sigma_{N}=\left(a+b \frac{\sigma_{n}}{\sigma_{d}}\right) \sigma_{C}
$$


where $\sigma_{n} / \sigma_{d}$ is the cross-section ratio of the neutron to the deuteron. Naively, $a=7 / 6$ and $b=1 / 6$, with only very slight deviations. However, in reality, other inaccuracies in the model are "soaked up" into the constants, as we will see. Inserting this into Eq. 5.41 and using the definition of $n_{C}^{\prime}$ yields

$$
n_{N}=f n_{C}^{\prime}+\left(L-\ell_{C}\right) n_{H e}^{\prime}+\frac{\rho_{N} \ell_{N}}{\rho_{C} \ell_{C}}\left(a+b \frac{\sigma_{n}}{\sigma_{d}}\right) n_{C}^{\prime}
$$

or, using Eq. 5.35,

$$
n_{N}=n_{M T}-\ell_{C} n_{H e}^{\prime}+\frac{\rho_{N} \ell_{N}}{\rho_{C} \ell_{C}}\left(a+b \frac{\sigma_{n}}{\sigma_{d}}\right) n_{C}^{\prime}
$$

In principle, then, using empty target, carbon and frozen nitrogen runs, one can make a best fit of $\ell_{N}, a$ and $b$ to the data. Because empty target runs were actually taken in separate sets from the carbon/nitrogen data, the count ratios to carbon were actually used: ${ }^{16}$

$$
\frac{n_{N}}{n_{C}}=\frac{n_{M T}}{n_{C}}-\ell_{C} n_{H e}^{\prime \prime}+\frac{\rho_{N} \ell_{N}}{\rho_{C} \ell_{C}}\left(a+b \frac{\sigma_{n}}{\sigma_{d}}\right) n_{C}^{\prime \prime}
$$

with

$$
n_{C}^{\prime \prime}=A+B \frac{n_{M T}}{n_{C}}
$$

and

$$
n_{H e}^{\prime \prime}=C \frac{n_{M T}}{n_{C}}+D
$$

This way, carbon runs from the same bracket could be used in comparison to the other target, ${ }^{17}$ ensuring proper normalization between run sets separated across time.

In practice, MINUIT had difficulty fitting all 3 unknown parameters $\ell_{N}, a$ and $b$ at once; it was just not possible to find a stable equilibrium in the fit without some more constraints on the data. In practice, the model outlined in Section 5.4.1 was used, so that

$$
a+b \frac{\sigma_{n}}{\sigma_{d}} \rightarrow \frac{\sigma_{N}}{\sigma_{C}}
$$

\footnotetext{
${ }^{16}$ Because of the $0.1 \mathrm{~mm}$ difference in thicknesses of the 2 carbon targets used, a multiplicative factor of 1.047 was used on the $n_{M T} / n_{C}$ count ratio. See Section 5.4 and Table 5.6.

${ }^{17}$ Pairings between carbon-nitrogen brackets to normal data brackets (for empty target data) were as follows: $1^{\prime} \leftrightarrow 6$; $2^{\prime}, 3^{\prime} \leftrightarrow 3 ; 4^{\prime}, 5^{\prime} \leftrightarrow 12 ; 6^{\prime}, 7^{\prime} \leftrightarrow 8$. Outbending data sets were preferrable to inbending due to wider overall kinematic coverage.
} 
in Eq. 5.46, then MINUIT was used to find a fit for $\ell_{N}$. In other words, the histogram of $\mathrm{LHe} /{ }^{12} \mathrm{C}$ counts (in terms of $W$ and $Q^{2}$ ) was fit to the histogram of ${ }^{15} \mathrm{~N}^{12} \mathrm{C}$ counts, using the function in Eq. 5.46. The parameter $\ell_{N}$ was varied (with $\sigma_{N} / \sigma_{C}$ read from an array determined by the model), until the best fit, which minimized the value of

$$
\chi^{2}=\sum_{W, Q^{2}}\left(\left(\frac{n_{N}}{n_{C}}\right)^{\text {true }}-\left(\frac{n_{N}}{n_{C}}\right)^{t h}\right)^{2}
$$

was found, where $\left(\frac{n_{N}}{n_{C}}\right)^{t h}$ is the result of Eq. 5.46. Values of $\ell_{N}$ determined for each set (after the charge normalization process described in the previous section) can be found in Table 5.8, under the heading "Method 1 ".

To test the consistency of the old model, the same fit was repeated, except this time using the $\ell_{N}$ just derived in the (unmodified) Eq. 5.46, to find $a$ and $b$. Final values of $a$ and $b$ are printed, along with their statistically weighted average, in Table 5.7. The precision of the fit can be seen in Figure 5.10, in which the scaled ${ }^{12} \mathrm{C}$ counts are compared to the (unmodified) ${ }^{15} \mathrm{~N}$ counts. Average values of $a$ and $b$ approximate $7 / 6$ and $1 / 6$, respectively, and the fit is good, showing a good degree of internal consistency between the old fit model and the new.

Table 5.7: Values of the fit coefficients $a$ and $b$ relating the cross-sections of ${ }^{12} \mathrm{C}$ to ${ }^{15} \mathrm{~N}$. The weighted averages are also shown. These data are used for comparative purposes, and have no direct bearing on the final derived value of $A_{||}$in this analysis.

\begin{tabular}{|c|c|c|}
\hline Set Label/Bracket & $a$ & $b$ \\
\hline \hline $2.3+/ 1^{\prime}$ & $1.18 \pm 0.0015$ & $0.12 \pm 0.0036$ \\
\hline $5.6+/ 2^{\prime}$ & $1.04 \pm 0.0186$ & $0.47 \pm 0.0461$ \\
\hline $5.6-/ 3^{\prime}$ & $1.24 \pm 0.0070$ & $-0.01 \pm 0.0155$ \\
\hline $4.2-/ 4^{\prime}$ & $1.20 \pm 0.0014$ & $0.07 \pm 0.0031$ \\
\hline $4.2+/ 5^{\prime}$ & $1.12 \pm 0.0187$ & $0.28 \pm 0.0452$ \\
\hline $1.7+/ 6^{\prime}$ & $1.12 \pm 0.0030$ & $0.27 \pm 0.0073$ \\
\hline $1.7-/ 7^{\prime}$ & $1.08 \pm 0.0019$ & $0.37 \pm 0.0047$ \\
\hline \hline average & $1.16 \pm 0.0008$ & $0.15 \pm 0.0019$ \\
\hline
\end{tabular}



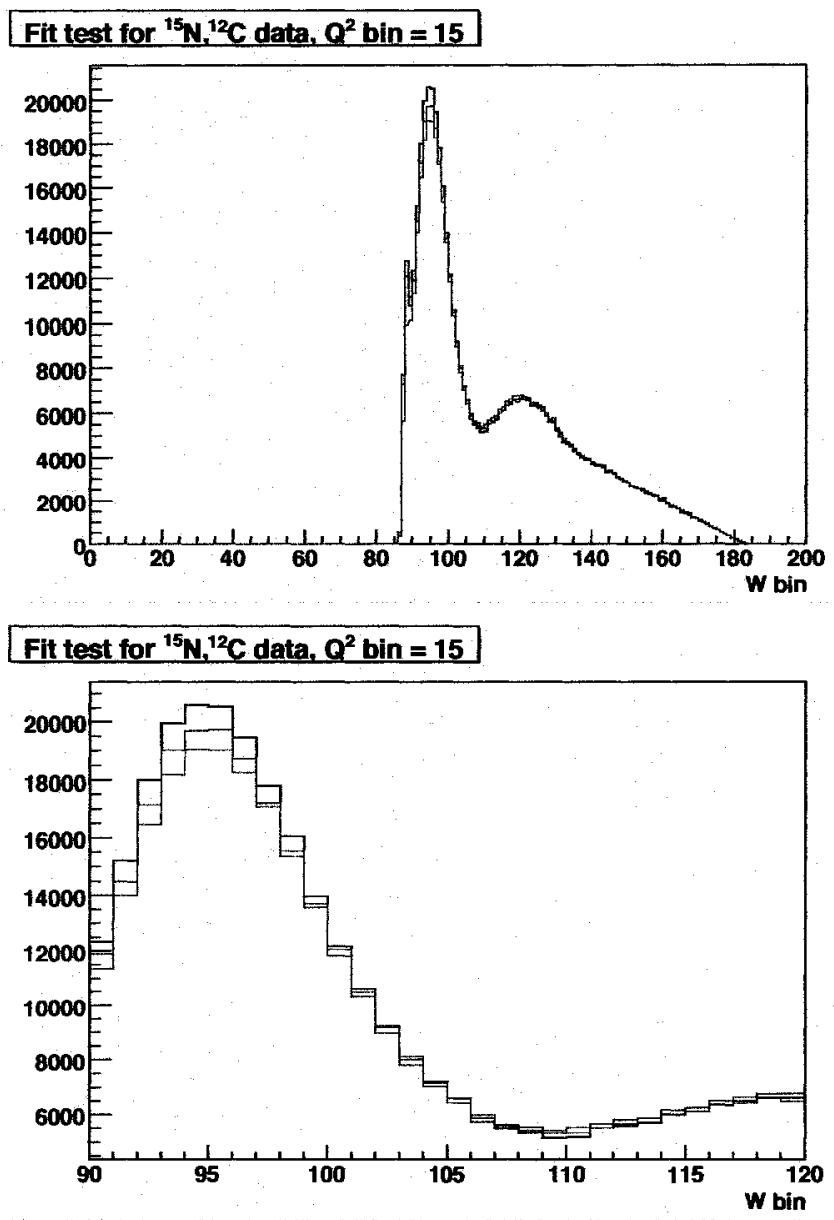

Figure 5.10: Fit of modeled ${ }^{12} \mathrm{C}$ counts (i.e. , Eq. 5.45) to ${ }^{15} \mathrm{~N}$ inclusive counts. The raw nitrogen data are shown in red, the raw carbon data are shown in black, and the fit of the carbon to nitrogen is shown in blue. The improvement of the fit over raw data is only seen when closer detail is revealed (bottom). 1.7 GeV outbending data (Bracket 7') is shown here. 


\subsubsection{Direct determination of $\ell_{N}$ with the model}

The previous subsection outlined the methodology of determining $\ell_{N}$ in terms of a best fit to the cross-sectional model given by Eq. 5.46. As a test of internal consistency, it is useful to apply the new ${ }^{15} \mathrm{~N}$ cross-section model directly to derive $\ell_{N}$. We start with Eq. 5.15 for the carbon target and the equivalent expression for the frozen nitrogen target:

$$
n_{N} \propto \rho_{A l} \ell_{A l} \sigma_{A l[N]}+\rho_{K} \ell_{K} \sigma_{C[N]}+\rho_{N} \ell_{N} \sigma_{N[N]}+\rho_{H e}\left(L-\ell_{N}\right) \sigma_{H e[N]}
$$

Again, the proportionality constant is assumed to be the same for both equations, so that we can divide them to get

$$
r_{N C} \equiv \frac{n_{N}}{n_{C}}=\frac{\mathrm{W}_{[N]}+\rho_{N} \ell_{N} \sigma_{N[N]}+\rho_{H e}\left(L-\ell_{N}\right) \sigma_{H e[N]}}{\mathrm{W}_{[C]}+\rho_{C} \ell_{C} \sigma_{C[C]}+\rho_{H e}\left(L-\ell_{C}\right) \sigma_{H e[C]}}
$$

with $\mathrm{W} \equiv \rho_{A l} \ell_{A l} \sigma_{A l}+\rho_{K} \ell_{K} \sigma_{C}$ as before. Solving for $\ell_{N}$ then yields

$$
\ell_{N}=\frac{r \mathrm{~W}_{[C]}-\mathrm{W}_{[N]}+r \rho_{C} \ell_{C} \sigma_{C[C]}-\rho_{H e} L \sigma_{H e[N]}+r \rho_{H e}\left(L-\ell_{C}\right) \sigma_{H e[C]}}{\rho_{N} \sigma_{N[N]}-\rho_{H e} \sigma_{H e[N]}}
$$

As was done for $L$, we calculate the error bar (for relative weighting only - see Footnote 9 ) by assuming the foil contributions are small $(W \rightarrow 0)$ and $\sigma_{H e[N]} \approx \sigma_{H e[C]}$, yielding

$$
\sigma_{\ell_{N}}=\frac{\partial \ell_{N}}{\partial r} \sigma_{r_{N C}}=\frac{\rho_{C} \ell_{C} \sigma_{C[C]}+\rho_{H e}\left(L-\ell_{C}\right) \sigma_{H e[C]}}{\rho_{N} \sigma_{N[N]}-\rho_{H e} \sigma_{H e[N]}} \sigma_{r_{N C}}
$$

with

$$
\frac{\sigma_{r_{N C}}}{r_{N C}}=\sqrt{n_{C}^{-1}+n_{N}^{-1}}
$$

analogous to Eq. 5.23. These calculations were made, using the model for the cross-sections, for all $W$ and $Q^{2}$ bins containing data. The error weighted mean and error

$$
\ell_{N_{a v g}}=\frac{\sum_{Q^{2}} \sum_{W} \ell_{N} / \sigma_{\ell_{N}}^{2}}{\sum_{Q^{2}} \sum_{W} 1 / \sigma_{\ell_{N}}^{2}}
$$



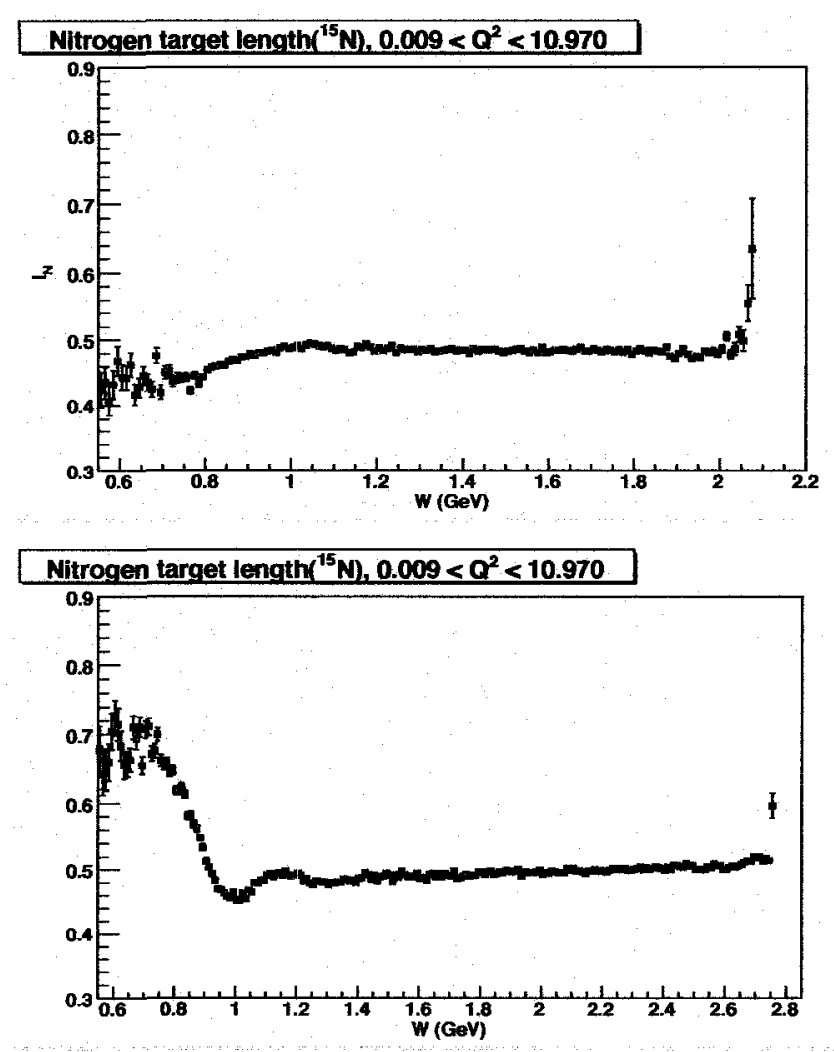

Figure 5.11: Solid ${ }^{15} \mathrm{~N}$ target length, averaged over $Q^{2}$, shown as a function of $W$, calculated using the radiated cross-section model and ${ }^{15} \mathrm{~N} /{ }^{12} \mathrm{C}$ inclusive count ratios. The average remains fairly constant so long as the elastic region $(W<1.10)$ is avoided. The two plots show $2.3 \mathrm{GeV}$ inbending (Bracket 1') and 4.2 GeV outbending (Bracket 4') data, respectively.

$$
\sigma_{\ell_{N a v g}}=\frac{1}{\sqrt{\sum_{Q^{2}} \sum_{W} 1 / \sigma_{\ell_{N}}^{2}}}
$$

were calculated, using all inelastic ( $W>1.10)$ bins, with an upper $W$ bound again used from Table 5.10. Plots of $\ell_{N}$ as function of $W$ are shown in Figure 5.11. Values calculated using the model matched well with those made in the fit, and are listed for comparison in Table 5.8.

\subsection{Calculation of Ammonia Target Length $\ell_{A}$}

Before calculating dilution factors, one more critical piece of information is required - the effective length of the frozen ammonia target material $\ell_{A}$. As detailed in Section 2.4 , the actual target 
Table 5.8: Values of the nitrogen target length $\left(\ell_{N}\right)$, using the best fit of the radiated model to different count ratios (Method 1) and a direct fit to cross-sections generated from the (Method 2). All lengths are given in $\mathrm{cm}$.

\begin{tabular}{|c|c|c|}
\hline Set Label/Bracket & $\ell_{N}($ Method 1) & $\ell_{N}$ (Method 2) \\
\hline \hline $2.3+/ /^{\prime}$ & $0.45 \pm 0.00015$ & $0.46 \pm 0.00023$ \\
\hline $5.6+/ 2^{\prime}$ & $0.43 \pm 0.00066$ & $0.44 \pm 0.00119$ \\
\hline $5.6-/ 3^{\prime}$ & $0.45 \pm 0.00028$ & $0.46 \pm 0.00043$ \\
\hline $4.2-/ 4^{\prime}$ & $0.47 \pm 0.00008$ & $0.47 \pm 0.00022$ \\
\hline $4.2+/ 5^{\prime}$ & $0.47 \pm 0.00086$ & $0.48 \pm 0.00103$ \\
\hline $1.7+/ 6^{\prime}$ & $0.44 \pm 0.00039$ & $0.45 \pm 0.00057$ \\
\hline $1.7-/ 7^{\prime}$ & $0.45 \pm 0.00033$ & $0.45 \pm 0.00056$ \\
\hline \hline average & $0.46 \pm 0.00007$ & $0.46 \pm 0.00014$ \\
\hline
\end{tabular}

material consists of frozen $\mathrm{NH}_{3}$ granules immersed in a LHe bath. Only a particular fraction (known as the packing fraction) of the cell length actually consists of frozen target material at any given time. The consistency of frozen material may even vary between locations in the cell (see Figure 3.8). We are interested in the effective overall target length, averaged over all the data. To first order, the approximation $\ell_{A}=0.6 \mathrm{~cm}$ can be used for some calculations (see, for example, Section 4.2.4). However, for accurate background removal, a more precise figure is desired. Again, two methods are accorded for deriving this quantity, one utilizing actual statistical data, and another (the preferred method) using radiated cross-section data and the count ratio $n_{N H_{3}} / n_{C}$. Both methods are outlined in this section.

\subsubsection{Calculation of $\ell_{A}$ from data}

The same logic used to derive $\ell_{N}$ (the frozen nitrogen target length) can be applied to find $\ell_{A}$ (the frozen ammonia target length), since both targets are constucted similarly. Thus, we start with Eq. 5.45, using the simplification of Eq. 5.49 and changing the target label from $\mathrm{N}$ (nitrogen) to $\mathrm{A}$ (ammonia):

$$
n_{A}=n_{M T}-\ell_{C} n_{H e}^{\prime}+\frac{\rho_{A} \ell_{A}}{\rho_{C} \ell_{C}} \frac{\sigma_{A}}{\sigma_{C}} n_{C}^{\prime}
$$



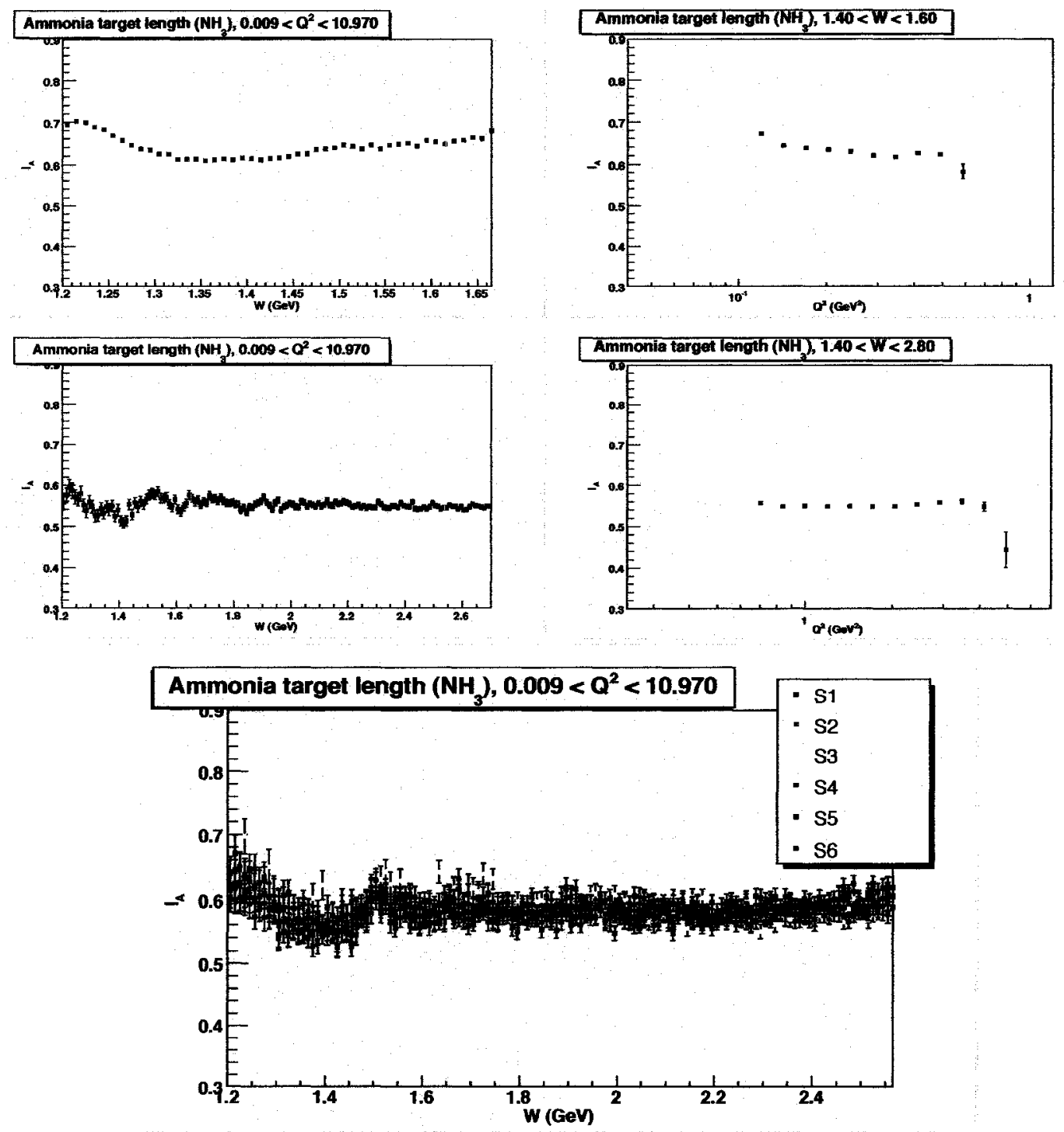

Figure 5.12: $\mathrm{NH}_{3}$ target length, shown as both a function of $W$ and $Q^{2}$, as calculated using the method in Section 5.5.1. The top row shows 1.6 GeV inbending (Bracket 1) data. Low energies were the worst-case scenario for this method, due to the narrow coverage in $W$. The center row shows $5.6 \mathrm{GeV}$ inbending (Bracket 7), showing the broad, flat region characteristic of higher beam energies. The bottom figure shows the sector-by-sector dependence of $\ell_{A}$ for $4.2 \mathrm{GeV}$ inbending (Bracket 11) data. When correct, the dependence should only show statistical fluctuations from sector to sector, as shown. 
with all other notation the same as in Section 5.4.2. Assuming $\sigma_{A}=\sigma_{N}+3 \sigma_{p}{ }^{18}$ this yields

$$
n_{A}=n_{M T}-\ell_{C} n_{H e}^{\prime}+\frac{\rho_{A} \ell_{A}}{\rho_{C} \ell_{C}}\left(\frac{\sigma_{N}}{\sigma_{C}}+3 \frac{\sigma_{p}}{\sigma_{C}}\right) n_{C}^{\prime}
$$

Now, making the assumption that the carbon nucleus is merely an assembly of 6 deuterons ${ }^{19}$

$$
\frac{3 \sigma_{p}}{\sigma_{C}}=\frac{3\left(\sigma_{d}-\sigma_{n}\right)}{\sigma_{C}}=\frac{3}{6}-\frac{3 \sigma_{n}}{6 \sigma_{d}}=\frac{1}{2}\left(1-\frac{\sigma_{n}}{\sigma_{d}}\right)
$$

we get

$$
n_{A}=n_{M T}-\ell_{C} n_{H e}^{\prime}+\frac{\rho_{A} \ell_{A}}{\rho_{C} \ell_{C}}\left[\frac{\sigma_{N}}{\sigma_{C}}+\frac{1}{2}\left(1-\frac{\sigma_{n}}{\sigma_{d}}\right)\right] n_{C}^{\prime}
$$

where $\sigma_{n} / \sigma_{d}$ and $\sigma_{N} / \sigma_{C}$ are functions of beam energy, $Q^{2}$ and $W$ determined by the models in this chapter and Section 2.8. ${ }^{20}$

Solving for $\ell_{A}$ then yields

$$
\ell_{A}=\left(n_{A}-n_{M T}\right) /\left(\frac{\rho_{A}}{\rho_{C} \ell_{C}}\left[a+b \frac{\sigma_{n}}{\sigma_{d}}+0.5\left(1-\frac{\sigma_{n}}{\sigma_{d}}\right)\right] n_{C}^{\prime}-n_{H \mathbf{e}}^{\prime}\right)
$$

This quantity can thus be calculated in all bins where the assumptions hold (i.e. higher $W$, away from the realm of the EMC effect), just as $L$ and $\ell_{N}$ were in previous sections of this chapter.

The error can be calculated in quadrature [103] (assuming $\sigma_{n}=\sqrt{n}$ ) as

$$
\sigma_{\ell_{A}}=\sqrt{\left(\frac{\partial \ell_{A}}{\partial n_{A}}\right)^{2} n_{A}+\left(\frac{\partial \ell_{A}}{\partial n_{C}}\right)^{2} n_{C}+\left(\frac{\partial \ell_{A}}{\partial n_{M T}}\right)^{2} n_{M T}}
$$

Calculation of the partial derivatives yields

$$
\begin{gathered}
\frac{\partial \ell_{A}}{\partial n_{A}}=1 / \mathscr{D} \\
\frac{\partial \ell_{C}}{\partial n_{C}}=-\frac{\left(n_{A}-n_{M T}\right)\left(\frac{\sigma_{A}}{\sigma_{C}} A-D\right)}{\mathscr{D}^{2}}
\end{gathered}
$$

\footnotetext{
${ }^{18}$ This is a good assumption, considering the negligibly low energy bonding the free protons to the nitrogen atom in $\mathrm{NH}_{3}$. ${ }^{19}$ This is a more tenuous assumption, neglecting the nuclear EMC effect, avoided by the alternate (model) method.

${ }^{20}$ For a deuteron target, the calculation only differs in that the factor of $\frac{1}{2}\left(1-\frac{\sigma_{n}}{\sigma_{d}}\right)$ is instead simply $\frac{1}{2}$, as substituting $\sigma_{p} \rightarrow \sigma_{d}$ in Eq.5.60 shows.
} 


$$
\frac{\partial \ell_{M T}}{\partial n_{M T}}=-\frac{\left(\mathscr{D}+\left(n_{A}-n_{M T}\right)\left(\frac{\sigma_{A}}{\sigma_{C}} B+C\right)\right)}{\mathscr{D}^{2}}
$$

where

$$
\mathscr{D} \equiv \frac{\rho_{A}}{\rho_{C} \ell_{C}} \frac{\sigma_{A}}{\sigma_{C}} n_{C}^{\prime}-n_{H e}^{\prime}
$$

and $\sigma_{A} / \sigma_{C}$ is defined by the quantity in square brackets in Eq. 5.61 .

The fit region ranged from $W=1.4$ (safely above the $\Delta(1232)$ resonance) up to the maximum value listed in Table 5.10, to avoid the high $W$-region where systematic errors (i.e. pion contamination, radiative corrections) dominate. ${ }^{21}$ The weighted average and error

$$
\begin{gathered}
\ell_{A a v g}=\frac{\sum_{Q^{2}} \sum_{W} \ell_{A} / \sigma_{\ell_{A}}^{2}}{\sum_{Q^{2}} \sum_{W} 1 / \sigma_{\ell_{A}}^{2}} \\
\sigma_{\ell_{A} \text { avg }}=\frac{1}{\sqrt{\sum_{Q^{2}} \sum_{W} 1 / \sigma_{\ell_{A}}^{2}}}
\end{gathered}
$$

were again used over this region to calculate the average $\ell_{A}$ for each data set. Plots of $\ell_{A}$ derived with this method as a function of $W$ are shown in Figure 5.12.

\subsubsection{Calculation of $\ell_{A}$ from radiated cross-sections}

The exact same formalism used to extract $\ell_{N}$ in Section 5.4.3 using radiated cross-section models can be used to determine $\ell_{A}$. The only difference is that $\mathbf{N} \rightarrow A$ in every equation in this section, and we use

$$
\sigma_{A}=\frac{15}{18} \sigma_{N}+\frac{3}{18} \sigma_{p}
$$

to account for the molar masses of the constituents of $\mathrm{NH}_{3} .{ }^{22}$ This substitution yields

$$
\begin{gathered}
\ell_{A}=\frac{r_{A C} \mathrm{~W}_{[C]}-\mathrm{W}_{[A]}+r_{A C} \rho_{C} \ell_{C} \sigma_{C[C]}-\rho_{H e} L \sigma_{H e[A]}+r_{A C} \rho_{H e}\left(L-\ell_{C}\right) \sigma_{H e[C]}}{\rho_{A}\left(\frac{15}{18} \sigma_{N[A]}+\frac{3}{18} \sigma_{p[A]}\right)-\rho_{H e} \sigma_{H e[A]}} \\
\sigma_{\ell_{A}}=\frac{\partial \ell_{A}}{\partial r_{A C}} \sigma_{r_{A C}}=\frac{\rho_{C} \ell_{C} \sigma_{C[C]}+\rho_{H e}\left(L-\ell_{C}\right) \sigma_{H e[C]}}{\rho_{A}\left(\frac{15}{18} \sigma_{N[A]}+\frac{3}{18} \sigma_{p[A]}\right)-\rho_{H e} \sigma_{H e[A]}} \sqrt{n_{C}^{-1}+n_{A}^{-1}} r_{A C}
\end{gathered}
$$

\footnotetext{
${ }^{21}$ Unfortunately, this leaves a narrow region tor measurement at beam energies below $2 \mathrm{GeV}$. The second (radiated cross-section) method is clearly superior in this case, as it allows inclusion of lower $W$ bins.

${ }^{22}$ For $\mathrm{ND}_{3}$, the respective fractions are $\frac{15}{21}$ and $\frac{6}{21}$, of course.
} 

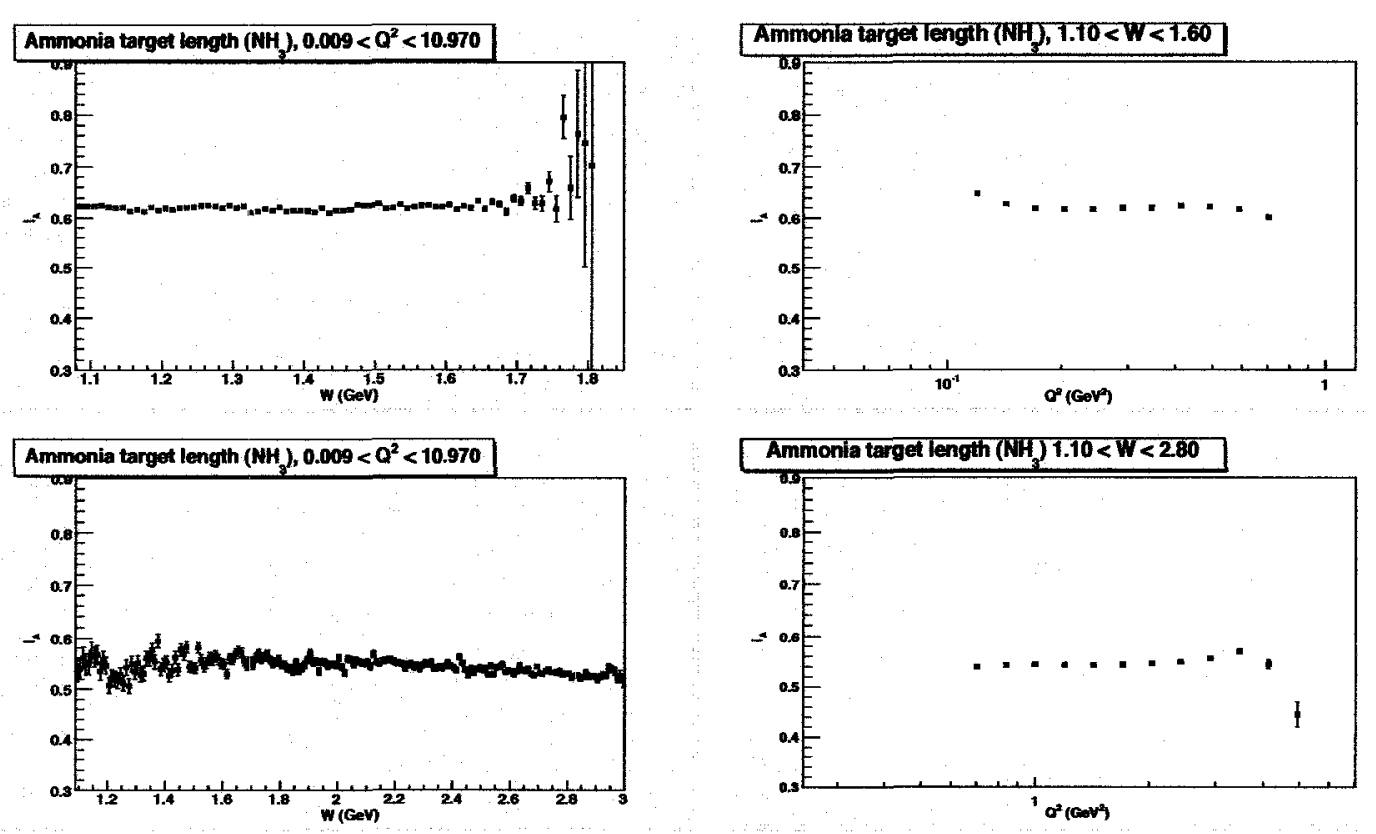

Figure 5.13: $\mathrm{NH}_{3}$ target length, shown as both a funcition of $W$ and $Q^{2}$, calculated using radiated cross-section models. The two data sets shown are the same as those shown in the top two rows of Figure 5.12. Note the significant improvement in stability for the $1.6 \mathrm{GeV}$ data.

with $r_{A C} \equiv n_{A} / n_{C}$. Combination of data points proceeds as in the preceding method for $\ell_{A}$, except that the lower limit in $W$ is lowered to $W=1.10 \mathrm{GeV}$. Because the model accounts for the nuclear EMC effect, there are no assumptions relating the consistency of cross-section ratios going into this calculation, making for a much smoother average over the resonance region (see Figure 5.13). Calculations of the average value of $\ell_{A}$ for each data set, using both the previous method (Method 1) and the radiated cross-section method (Method 2) are listed in Table 5.9.

\subsection{Dilution Factors}

With adequate knowledge of the total target length $L$, ammonia packing fraction $\ell_{A}$, and with a functional model for the scaling of the ${ }^{12} \mathrm{C}$ cross-sections to ${ }^{15} \mathrm{~N}$ in place, we can proceed to calculate the dilution factor, $F_{D F}\left(W, Q^{2}\right)$. As usual, both the statistical, data-derived methodology (S. Kuhn) and the radiated cross-section model (P. Bosted) were used to determine dilution factors. In the end, it was determined that both methods have a kinematic region of applicability in the EG1b 
Table 5.9: Values of the ammonia target length $\left(\ell_{A}\right)$, using the best fit of the radiated model to different count ratios (Method 1) and a direct fit to cross-sections generated from the model (Method 2). Once again, the error bar reflects only the error on the statistical fit, not the true uncertainty on the value. Target refills occured before Brackets 3 and 6 and during Bracket 9 [63], explaining the sudden changes in $\ell_{A}$ at these points. All lengths are given in $\mathrm{cm}$.

\begin{tabular}{|c|c|c|}
\hline Set Label/Bracket & $\ell_{A}$ (Method 1) & $\ell_{A}$ (Method 2) \\
\hline \hline $1.6+/ 1$ & $0.62 \pm 0.00054$ & $0.63 \pm 0.00019$ \\
\hline $1.6-/ 2$ & $0.64 \pm 0.0019$ & $0.65 \pm 0.00044$ \\
\hline $5.76-/ 3$ & $0.60 \pm 0.00055$ & $0.61 \pm 0.00026$ \\
\hline $5.73-/ 4$ & $0.57 \pm 0.00047$ & $0.57 \pm 0.00024$ \\
\hline $5.7+/ 5$ & $0.55 \pm 0.00012$ & $0.56 \pm 0.00056$ \\
\hline $2.3+/ 6$ & $0.62 \pm 0.00060$ & $0.63 \pm 0.00026$ \\
\hline $5.6+/ 7$ & $0.55 \pm 0.0010$ & $0.56 \pm 0.00048$ \\
\hline $1.7-/ 8$ & $0.54 \pm 0.00072$ & $0.55 \pm 0.00019$ \\
\hline $2.5-/ 9$ & $0.56 \pm 0.00033$ & $0.57 \pm 0.00017$ \\
\hline $4.2+/ 11$ & $0.59 \pm 0.00093$ & $0.59 \pm 0.00042$ \\
\hline $4.2-/ 12$ & $0.59 \pm 0.00026$ & $0.60 \pm 0.00014$ \\
\hline
\end{tabular}

analysis. To ensure internal consistency, the methods used to calculate $L$ and $\ell_{A}$ for a given $F_{D F}$ were always kept the same. That is, if the former (latter) method was used to calculate $F_{D F}$, then the former (latter) method was used to derive $L$ and $\ell_{A}$.

To derive the dilution factor $F_{D F}$, one begins with the regular formula for $A_{\|}$:

$$
A_{\|}=\frac{n^{-}-n^{+}}{n^{-}+n^{+}}
$$

where it is assumed the counts $n^{ \pm}$are FC normalized. We then assume that each count $n^{ \pm}$ contains a background $\frac{1}{2} n_{b a c k}{ }^{23}$ which must be subtracted to get the true asymmetry:

$$
A_{\|}=\frac{\left(n^{-}-\frac{1}{2} n_{b a c k}\right)-\left(n^{+}-\frac{1}{2} n_{b a c k}\right)}{\left(n^{-}-\frac{1}{2} n_{b a c k}\right)+\left(n^{+}-\frac{1}{2} n_{b a c k}\right)}=\frac{n^{-}-n^{+}}{n^{-}+n^{+}-n_{b a c k}}
$$

If $F_{D F}$ is defined as the ratio of the target proton scattered events to total events

$$
F_{D F} \equiv \frac{n_{\text {total }}-n_{\text {back }}}{n_{\text {total }}}
$$

\footnotetext{
${ }^{23}$ That is, we assume that the asymmetry is relatively small, so that the contribution from each polarization state to the background is approximately equal.
} 
(where of course, $n_{\text {total }}=n^{-}+n^{+}$), then

$$
n_{b a c k}=\left(1-F_{D F}\right)\left(n^{-}+n^{+}\right)
$$

Thus,

$$
A_{\|}=\frac{n^{-}-n^{+}}{n^{-}+n^{+}-\left(1-F_{D F}\right)\left(n^{-}+n^{+}\right)}=\frac{n^{-}-n^{+}}{n^{-}+n^{+}-n^{-}-n^{+}+F_{D F} n^{-}+F_{D F} n^{+}}
$$

which reduces to

$$
A_{\|}=\frac{1}{F_{D F}} \frac{n^{-}-n^{+}}{n^{-}+n^{+}}
$$

Aside from the factor $P_{b} P_{t}$, this is the necessary equation for background removal from the doublespin asymmetry. $F_{D F}$ is a function of $Q^{2}$ and $W$, as it is a description of the relative count rates for the free protons and background. The effective response of the background varies as a function of these parameters, particularly near the elastic peak, and to a lesser (but significant) degree, near the resonances, as well.

\subsubsection{Calculation of $F_{D F}$ from data}

To find $n_{\text {back }}$, one simply reworks Eqs. 5.58 to 5.61 , but this time omitting the contribution from the free protons. That is, we omit the $3 \sigma_{p}$ term, as we are interested only the background cross-section. The resulting replacement for Eq. $\mathbf{5 . 5 8}$ is

$$
n_{\text {back }}=n_{M T}-\ell_{A} n_{H e}^{\prime}+\frac{\rho_{A} \ell_{A}}{\rho_{C} \ell_{C}} \frac{\sigma_{N}}{\sigma_{C}} n_{C}^{\prime}
$$

The dilution factor is thus given by Eq. 5.75 :

$$
F_{D F}=1-\frac{1}{n_{A}}\left(n_{M T}-\ell_{A} n_{H e}^{\prime}+\frac{\rho_{A} \ell_{A}}{\rho_{C} \ell_{C}} \frac{\sigma_{N}}{\sigma_{C}} n_{C}^{\prime}\right)
$$


Table 5.10: Upper $W$ limits (in $\mathrm{GeV}$ ) on the data used for dilution factors (as calculated from data), also used for limits on measurements of $L, \ell_{A}$, and $\ell_{N}$. Dilution factors generated from the crosssectional model (Section 5.6.2) do not need an upper limit, since they can be smoothly extrapolated to higher $W$. Bins not listed do not contain enough data to be included.

\begin{tabular}{|c|c|c|c|c|c|}
\hline$Q^{2}$ bin & $Q^{2}$ & $1 . x \mathrm{GeV}$ & $2 . x \mathrm{GeV}$ & $4 . x \mathrm{GeV}$ & $5 . \times \mathrm{GeV}$ \\
\hline \hline 10 & 0.050 & 1.55 & - & - & - \\
\hline 11 & 0.059 & 1.55 & - & - & - \\
\hline 12 & 0.071 & 1.50 & - & - & - \\
\hline 13 & 0.084 & 1.50 & - & - & - \\
\hline 14 & 0.10 & 1.50 & - & - & - \\
\hline 15 & 0.12 & 1.50 & 1.90 & - & - \\
\hline 16 & 0.14 & 1.50 & 1.90 & - & - \\
\hline 17 & 0.17 & 1.70 & 1.90 & - & - \\
\hline 18 & 0.20 & 1.70 & 2.00 & 2.40 & - \\
\hline 19 & 0.24 & 1.70 & 2.10 & 2.40 & - \\
\hline 20 & 0.29 & 1.70 & 2.10 & 2.40 & - \\
\hline 21 & 0.35 & 1.65 & 2.10 & 2.40 & - \\
\hline 22 & 0.42 & 1.60 & 2.05 & 2.50 & - \\
\hline 23 & 0.50 & 1.55 & 2.00 & 2.55 & 3.00 \\
\hline 24 & 0.59 & 1.45 & 1.90 & 2.55 & 2.95 \\
\hline 25 & 0.71 & 1.35 & 1.80 & 2.55 & 2.85 \\
\hline 26 & 0.84 & 1.20 & 1.65 & 2.55 & 2.85 \\
\hline 27 & 1.0 & 1.00 & 1.50 & 2.40 & 2.85 \\
\hline 28 & 1.2 & - & 1.30 & 2.30 & 2.85 \\
\hline 29 & 1.4 & - & 1.00 & 2.20 & 2.80 \\
\hline 30 & 1.7 & - & - & 2.05 & 2.75 \\
\hline 31 & 2.0 & - & - & 1.90 & 2.60 \\
\hline 32 & 2.4 & - & - & 1.60 & 2.50 \\
\hline 33 & 2.9 & - & - & 1.00 & 2.40 \\
\hline 34 & 3.5 & - & - & - & 2.20 \\
\hline 35 & 4.2 & - & - & - & 2.00 \\
\hline 36 & 5.0 & - & - & - & 1.50 \\
\hline 37 & 5.9 & - & - & - & 1.00 \\
\hline & & & & & \\
\hline
\end{tabular}


The error on $F_{D F}$ is again calculated by quadrature:

$$
\sigma_{F_{D F}}=\sqrt{\left(\frac{\partial F_{D F}}{\partial n_{A}}\right)^{2} n_{A}+\left(\frac{\partial F_{D F}}{\partial n_{C}}\right)^{2} n_{C}+\left(\frac{\partial F_{D F}}{\partial n_{M T}}\right)^{2} n_{M T}}
$$

The partial derivatives can be calculated as

$$
\begin{gathered}
\frac{\partial F_{D F}}{\partial n_{A}}=n_{b a c k} / n_{A}^{2} \\
\frac{\partial F_{D F}}{\partial n_{C}}=-\frac{1}{n_{A}} \ell_{A}(\mathscr{B} A-D) \\
\frac{\partial F_{D F}}{\partial n_{M T}}=\frac{1}{n_{A}}\left[1-\ell_{A}(\mathscr{B} B-C)\right]
\end{gathered}
$$

with

$$
\mathscr{B} \equiv \frac{\rho_{A}}{\rho_{C} \ell_{C}} \frac{\sigma_{N}}{\sigma_{C}}
$$

Inclusive scattering dilution factors derived using this method, and the effects of background removal, are shown in Figures 5.14, 5.15, 5.16, and 5.17. It is important to notice the dependence as a function of $W$. This distribution makes sense, considering that the scattering response in the elastic region ( $W \sim 0.938 \mathrm{GeV}$ ) is predominantly from the free protons, while in the the DIS region, the effective response of all unpolarized matter is equivalent.

\section{Dilution factors for elastic ep events}

For use in $P_{b} P_{t}$ derivation (Section 6.2), it is desirable to have dilution factors for both inclusive electron scattering and elastic ep exclusive events, as both of these are used in calculation of beam $\times$ target polarization. A much lower background is expected for $e p$ events, as kinematic cuts are relied upon for the separation of these events from background (see Section 3.5).

The principle for generating dilution factors $F_{D F}$ for exclusive events is exactly the same as that outlined for inclusive events, except that the inclusive counts $n_{A}, n_{C}$ and $n_{M T}$ are replaced by exclusive $e p$ counts in the same $W$ and $Q^{2}$ bins. The target length $\ell_{A}$ derived from inclusive scattering is used. For a model of $\sigma_{C} \rightarrow \sigma_{N}$, Eq. 5.43 was used ${ }^{24}$, but with $b=0$, as only elastic

\footnotetext{
${ }^{24}$ No exclusive model of the cross-section ratios for ${ }^{15} \mathrm{~N}$ to ${ }^{12} \mathrm{C}$ was developed.
} 

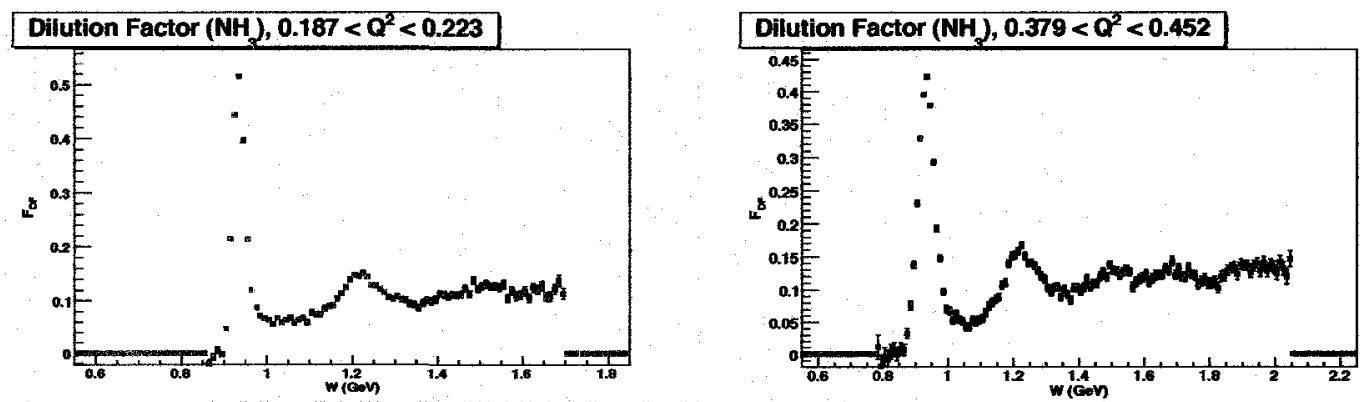

Dilution Factor $\left(\mathrm{NH}_{3}\right), 0.919<\mathrm{Q}^{2}<1.100$
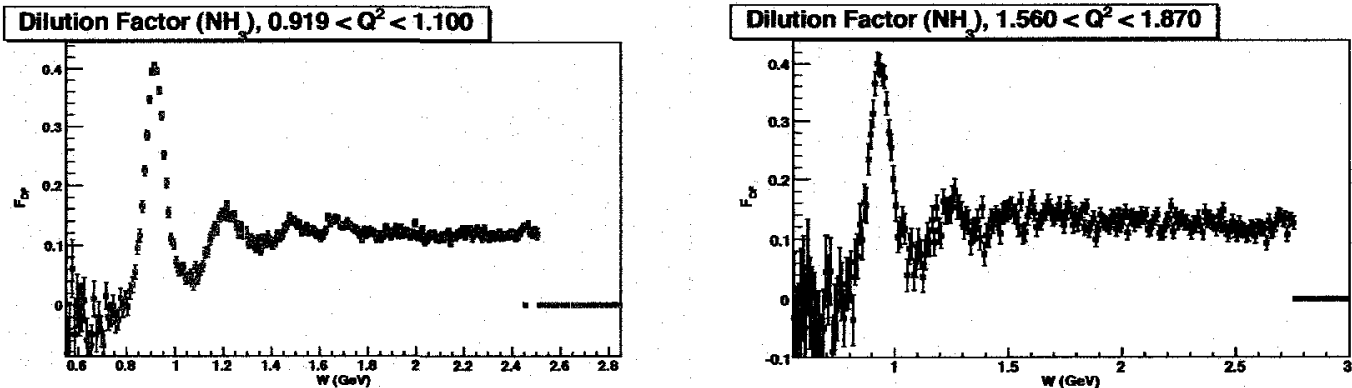

Figure 5.14: Dilution factors as a function of $W$, shown at 4 different beam energies. These $F_{D F}$ values were generated bin-by-bin using data. Note the prominence of the elastic peak, followed by fluctuations corresponding to the resonances, before a general flattening occurs in the $W>\mathbf{2 . 0}$ GeV DIS region. Shown are the data from 4 outbending data sets (1.7- (top left), 2.5- (top right), 4.2- (bottom left) and 5.73- (bottom right)). 

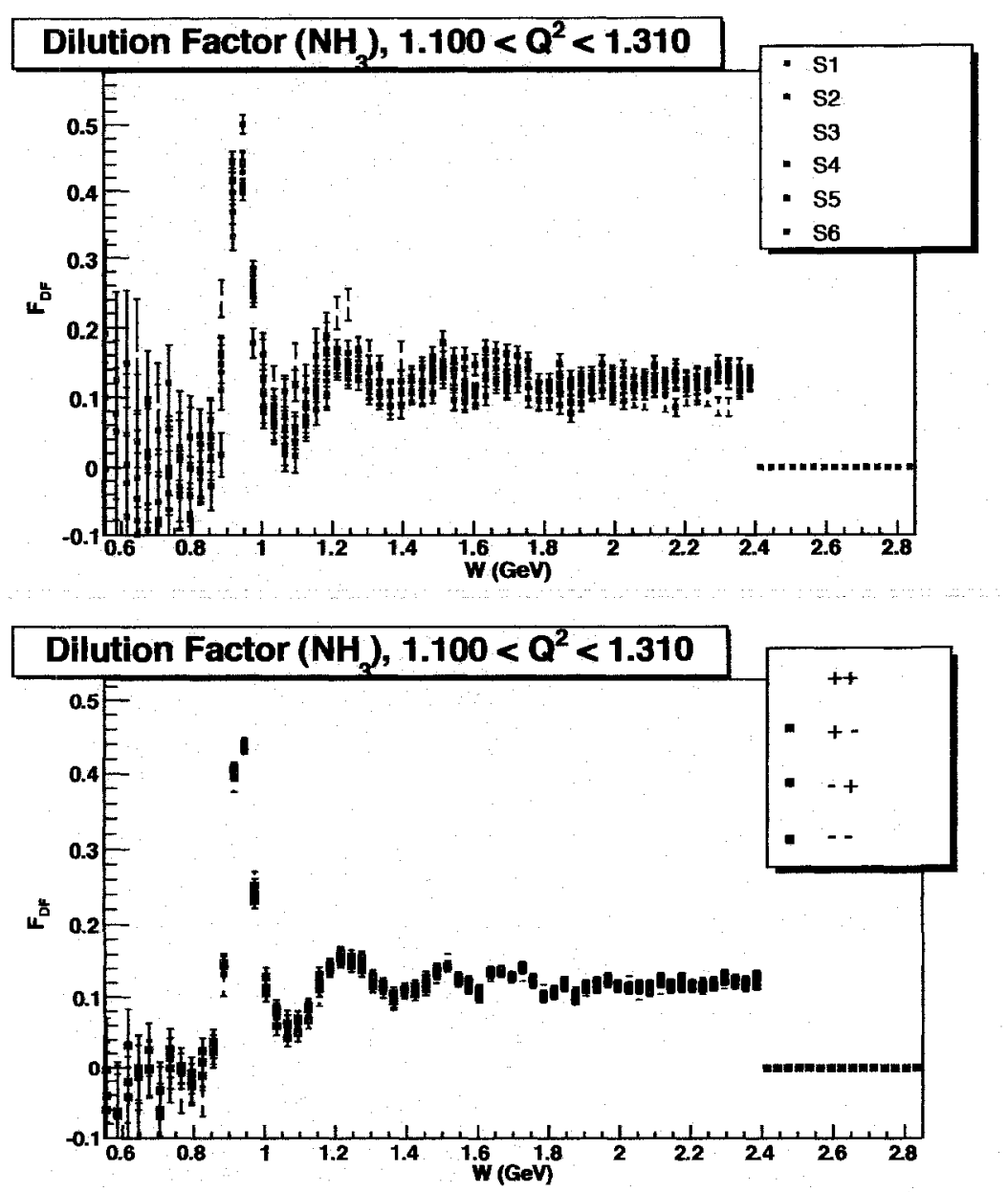

Figure 5.15: Dilution factors as a function of $W$ for $4.2+$ data (Bracket 11), calculated individualiy by sector (top) and by HWP/target polarization (bottom). Separating the dilution factors by sectors helped to identify problematic detector regions, while separating by polarizations helped isolate acceptance changes over time. 

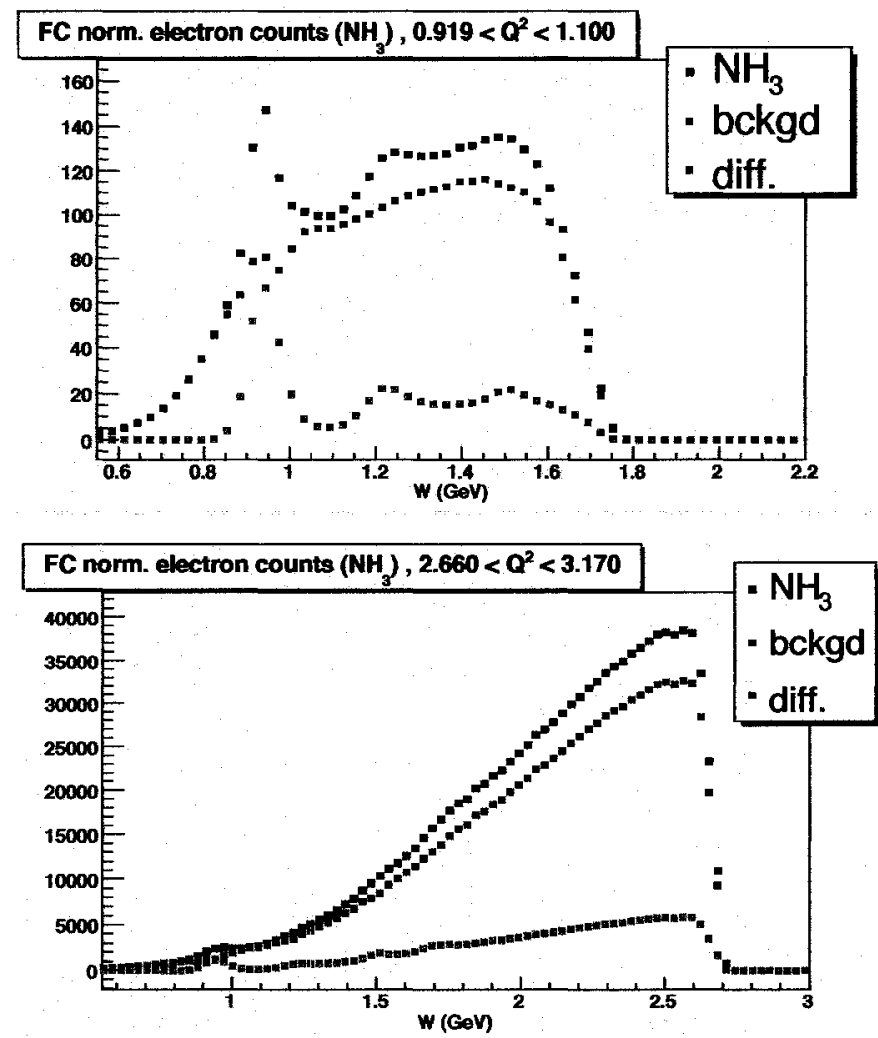

Figure 5.16: $\mathrm{NH}_{3}$ data (black) showing the subtracted background (defined by $\left.\left(1-F_{D F}\right) n_{A}\right)$ (blue) and the resulting free proton scattering spectrum (red). Shown are 2.3+ (Bracket 6) data (top) and 5.7+ (Bracket 5) data (bottom). 

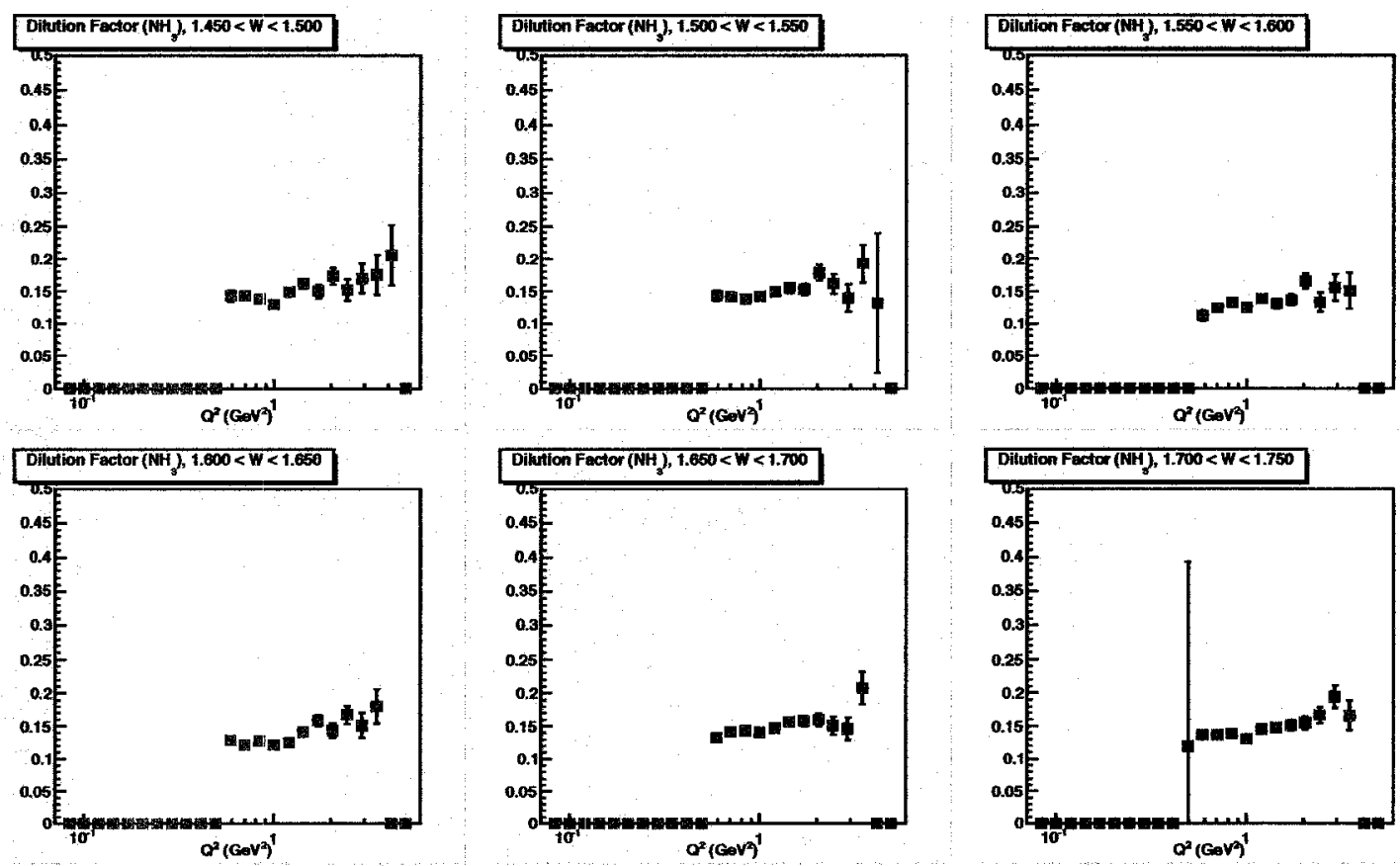

Figure 5.17: $F_{D F}$ as a function of $Q^{2}$, shown for several different $W$ bins, for the 5.76- set (Bracket $3)$. There is a slight but significant $Q^{2}$ dependence of the dilution factor which can easily be seen here. 
scattering events from free protons were in present in the data.

Elastic $e p$ events compared to their subtracted background are shown later, in Figure 6.6. In the course of this analysis, it was seen that the background was low enough that a simpler model using carbon-background subtracted events in $\phi$ was sufficient for removal of unwanted $e p$ events (Section 6.2.2), so this method was, in the end, employed only as a method of comparison for the derivation of $P_{b} P_{t}$.

\subsubsection{Calculation of $F_{D F}$ from radiated cross-sections}

Calculation of the dilution factors from radiated cross-sections is simple once all the target material lengths and densities are known. According to Eq. 5.75, one simply divides the sum of total counts from the protons only by the sum of all counts in the target:

$$
F_{D F}=\frac{n_{p}}{n_{A}}=\frac{\frac{3}{18} \rho_{A} \ell_{A} \sigma_{p}}{\mathrm{~W}+\rho_{A} \ell_{A}\left(\frac{3}{18} \sigma_{p}+\frac{15}{18} \sigma_{N}\right)+\rho_{H e}\left(L-\ell_{A}\right) \sigma_{H e}}
$$

where, as usual, W represents the foil (Al and Kapton) contributions. All cross-sections here pertain to the ammonia target, so no disambiguation is needed. Because this is a smooth-fitting model, no statistical errors are calculated, though systematic errors need to be handled properly (see Section 7.2).

Using a model for the dilution factor has two advantages over the statistical method:

1. It exploits the continuity in the dilution factor from bin to bin, so that artificially large statistical fluctuations do not dominate the calculated asymmetry. ${ }^{25}$

2. Extrapolation of the model can be made into kinematic regions where adequate data inside fiducial regions is not available.

A comparison of the modeled dilution factors to the dilution factors calculated from the first method are shown in Figure 5.18.

\footnotetext{
${ }^{25}$ In other words, there are correlations between bins that the statistical errors in the first method do not use advantageously, thus having the net effect of underrepresenting our precision
} 

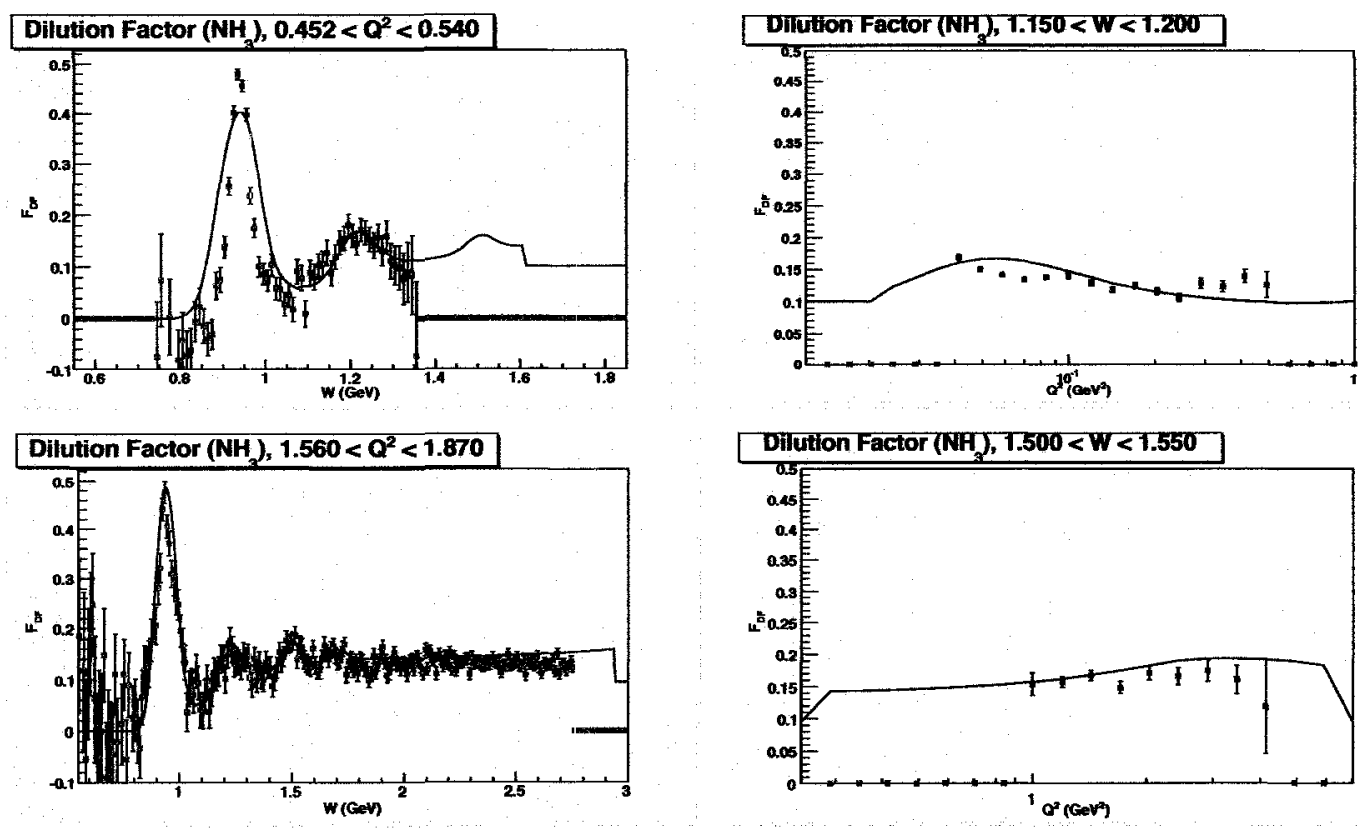

Figure 5.18: Dilution factors (statistical and modeled) as a function of $W$ (left) and $Q^{2}$ (right) for selected bins of the 1.6- (Bracket 2) and 5.6+ (Bracket 7) data. It is important to note that this is not a direct fit to the data, but rather an independent model of cross-section data employing only information about the target material quantities. Note that the elastic peak is not very well fit for the lower beam energy, a point of concern addressed in the text. 


\subsubsection{Application of dilution factors}

Two methods have been demonstrated for calculating the inclusive dilution factors for the EG1 data. As already explained, the model method is preferrable to use, assuming that its accuracy is verified, because it is not governed by the statistical error of the data. ${ }^{26}$ However, as seen in Figure 5.18, and explained in Section 2.8, the model does not provide a very good fit to the data for the elastic region, due to the dependency on detector resolution, which must be externally determined and inserted into the model.

The model appears to fit quite well for the inelastic region, where the analysis of $A_{1}$ and $A_{2}$ is of interest. In the elastic region, where the model is less accurate, there is plenty of data available, with less statistical fluctuation than in the DIS region, even for high-energy data. Also, the only use in this analysis for the elastic region dilution factors is for the determination of $\boldsymbol{P}_{b} \boldsymbol{P}_{t}$, where all elastic data are averaged into a single bin, so statistical fluctuations are not a considerable problem therein.

Therefore, the modeled dilution factors were used at $W \geq 1.08 \mathrm{GeV}$, above the inelastic threshold, while the older (statistical) method was used at $W<1.08 \mathrm{GeV}$. There was no guarantee of perfect continuity of $F_{D F}$ across this boundary, so values on the opposing sides of the boundary were never used in the same integration or average. Finished dilution factor arrays were determined for each data bracket and every bin for the removal of non-polarized background.

\footnotetext{
${ }^{26}$ In previous analyses ([2] and [46]), a statistical averaging technique was used to "smooth over" the sparsely populated bins at higher energies. Since the model is now used, this is no longer necessary.
} 


\section{Chapter 6}

\section{Removal of Polarized and Other Target Background Events}

\section{1 $e^{+} e^{-}$Background Correction}

Analysis so far has assumed that detected electrons, after $\pi^{-}$removal, are indeed exclusively scattered electrons $e^{\prime}$. However, it is possible that the detected electron originated from other processes.

Inclusive $e p$ scattering can produce $\pi^{0}$ mesons at the event vertex. These mesons subsequently decay, must commonly through Dalitz decay [118]:

$$
\pi^{0} \rightarrow e^{+} e^{-} \gamma
$$

where the pair-symmetric decay of the neutral pion has a branching ratio of $1.2 \%$ [5]. Because the $\pi^{0}$ decays electromagnetically and thus has an extremely short lifespan, the $e^{+} e^{-}$pair is effectively produced at the event vertex. If the electron from the decay is accidentally detected as the event trigger particle instead of the inclusive $\left(e^{\prime}\right)$ electron, the inclusive cross-section will be contaminated with invalid data.

While Eq. 6.1 is the most common decay mode that produces superfluous electrons, other 
decays are also possible, including the Bethe-Heitler process [119]

$$
\gamma N \rightarrow e^{+} e^{-} N
$$

which is more prominent at forward angles [118], and

$$
\pi^{0} \rightarrow \gamma \gamma \rightarrow e^{+} e^{-} e^{+} e^{-}
$$

though contribution from the latter is minimal. Similar decays of of the (pseudoscalar) $\eta$ mesons also contribute to the pair symmetric background. Very small $e^{+} e^{-}$contributions from $\rho, \omega$ and $\phi$ (vector) meson decays are also made [118]. 1

To correct for contamination from these events, we take advantage of the fact that a positron is always produced with every electron (as required by charge and lepton number conservation) and make the following assumptions:

1. The event reconstruction for positrons in outbending runs is identical to that of electrons for inbending runs, and vice versa.

2. The detection efficiency of positrons is similar to that of electrons.

3. The overall contamination is small, so that slight differences in beam energy or acceptance have little effect on the first two assumptions.

With this in mind, we realize that the rate of $e^{-}$contamination for an inbending run should be identical to the rate of $e^{+}$triggers for an outbending run of the same beam energy (and vice versa, of course). Using subsequently calculated $e^{+} / e^{-}$ratios and $e^{+}$double-spin asymmetries, the effect on the asymmetry can be calculated.

We look for a coefficient $C_{b a c k}$ that can be multiplied by the uncorrected asymmetry $A_{\|}$, to yield the $e^{+} e^{-}$background-corrected asymmetry. This changes Eq. 5.1 to

$$
A_{\|}=\frac{C_{b a c k}}{P_{b} P_{t} F_{D F}} \frac{n^{-}-n^{+}}{n^{-}+n^{+}}
$$

\footnotetext{
${ }^{1}$ Non-pair-symmetric production can also occur from the decay of $K_{L}^{-} \rightarrow \pi^{0} e^{-} \nu_{e}$. These electrons much more difficult to account for, since there is no $e^{+}$produced in the reaction. Fortunately, the $K$ lifetime is long enough (and the $K / \pi^{0}$ ratio is small enough) that the vertex contamination is negligible [118].
} 
We assume that the dilution factors are approximately equal for each data set (a valid assumption for small contaminations. One can see from Figure 5.14 that these numbers are approximately equal in the inelastic range). We also neglect $P_{b} P_{t}$, for now, as this factor is of the same magnitude between data sets, and this is a small correction. We can thus apply the correction directly to the raw asymmetry:

$$
A_{\text {corr }}=C_{\text {back }} \frac{n^{-}-n^{+}}{n^{-}+n^{+}}
$$

where $n^{+}$and $n^{-}$are the raw FC-normalized count rates for each helicity state. To find $C_{b a c k}$, we consider that the uncontaminated asymmetry can be found from the raw asymmetry by subtracting the (as of now, unknown) pair production electron rates $\left(n_{p}^{+}\right.$and $n_{p}^{-}$) from the sample:

$$
A_{\text {corr }}=\frac{\left(n^{-}-n_{p}^{-}\right)-\left(n^{+}-n_{p}^{+}\right)}{\left(n^{-}-n_{p}^{-}\right)+\left(n^{+}-n_{p}^{+}\right)}
$$

If we take $n \equiv n^{+}+n^{-}$and $n_{p} \equiv n_{p}^{+}+n_{p}^{-}$, and divide both the numerator and denominator by $n$, we find

$$
A_{\text {corr }}=\frac{\frac{n^{-}-n^{+}}{n}-\frac{n_{p}^{-}-n_{p}^{+}}{n}}{1-n_{p} / n}
$$

If $R \equiv n_{p} / n$, this can be written

$$
A_{\text {corr }}=\frac{A_{r a w}-\frac{n_{p}^{-}-n_{p}^{+}}{n_{p} / R}}{1-R}
$$

Defining $A_{p} \equiv\left(n_{p}^{-}-n_{p}^{+}\right) / n_{p}$ yields

$$
A_{\text {corr }}=\frac{A_{\text {raw }}-R A_{p}}{1-R}
$$

so that

$$
C_{\text {back }}=\frac{1-R A_{p} / A_{\text {raw }}}{1-R}
$$

Therefore, the asymmetry can be corrected if the contamination rate $(R)$ and the pair-production electron asymmetry $\left(A_{p}\right)$ are known. The following sections provide information on the evaluation of these quantities. 


\subsubsection{Measurement of pair-production rates and asymmetries}

The rate of contamination of pair-produced electrons in the inclusive electron data should be approximately equal to the rate of positrons detected at the same beam energy and opposite main torus current. Events triggered by positrons, instead of electrons, were written to separate DST files. To calculate the number of positrons, these files were analyzed in exactly the same manner as the regular (electron-triggered) files. Similarly to the oppositely charged electrons, $\pi^{+}$contamination was a possible issue. Therefore, the respective pion contamination and fiducial cuts were applied, except that the parameters for the opposing torus current were used (see Sections 4.1 and 4.4), due to the reflected geometry of the positively charged particle tracks.

In a given bracket, charge-normalized counts were recorded for total inclusive $\left(e^{\prime}\right)$ counts, along with positron $\left(e^{+}\right)$counts for an equal-energy bracket with opposing torus current. The counts were rebinned in terms of $\theta$ and momentum $p$. This binning scheme is outlined in Table B.2. Plots were generated of the resulting $e^{+} / e^{-}$ratio in terms of $\theta$ and $p .{ }^{2}$ Ratios were observed to diverge at both low $p$ and high $\theta$, because a more homogeneous spread of the (randomly directed) pair symmetric decays is expected than of the forward-scattered inclusive electrons.

In terms of $p$, the background ratio $R \equiv e^{+} / e^{-}$could be smoothly fit with

$$
R(p)=e^{A+B p}
$$

while, in terms of $\theta$, the fit

$$
R(\theta)=e^{C+D \theta}
$$

was employed.

Some examples of isometric fits in terms of both $p$ and $\theta$ are shown in Figures 6.1 and 6.2, respectively. Ultimately, a fit of $R$ to both $p$ and $\theta$ simultaneously, for each beam energy, was required, so that the contamination could be calculated for any kinematic value, including values extrapolated outside the range of accurate ratio data. Because of the success of the above exponential forms in

\footnotetext{
${ }^{2}$ Error bars, generally too small to be noticed, but important for fit purposes, can be calculated by the suitable analog to Eq. 5.23.
} 
parametrizing the contamination, a fit of the equation

$$
R(\theta, p)=e^{a+b \theta+c p+d \theta p}
$$

was used for each beam energy. Given a value of $\theta_{\text {avg }}$ and $p_{\text {avg }}$ in each $W$ and $Q^{2}$ bin, the value of $R$, to be applied to Eq. 6.10 , can be calculated to appropriately modify the raw asymmetry.

The only piece of information still needed for this correction is the value of $A_{p} / A_{\text {raw }}$. This quantity, given simply by

$$
A_{p} / A_{\text {raw }}=\frac{\left(n_{p}^{-}-n_{p}^{+}\right)\left(n^{-}+n^{+}\right)}{\left(n_{p}^{-}+n_{p}^{+}\right)\left(n^{-}-n^{+}\right)}
$$

can be calculated by separately summing over electron and positron events in the DST . The double-spin asymmetry for the contaminating electrons is, by definition, identical to that for the positrons (for the opposite torus current). Plots of $A_{p}$ as a function of momentum are shown in Figure 6.3. As one can see from these plots, the pair-production asymmetry is very small, so that its effects on $A_{\|}$are negligible. Thus, the approximation

$$
A_{p} / A_{\text {raw }} \approx 0
$$

is used in this analysis.

\subsubsection{Application of pair symmetric correction}

The pair symmetric correction is applied to raw asymmetries for each data set in the analysis. The raw asymmetry is multiplied times a factor $C_{b a c k}$ determined using the smooth paramatrization of $R$ given by Eq. 6.13, with $A_{p} / A_{\text {raw }}=0$.

Fit values for $a, b, c$ and $d$, as well as the fit errors on each of these parameters, are listed in Table 6.1. Note that this correction to the asymmetry is applied before the calculation of $P_{b} P_{t}$, so that more accurate values of the raw elasitc asymmetry can be determined before evaluating the polarization product. 

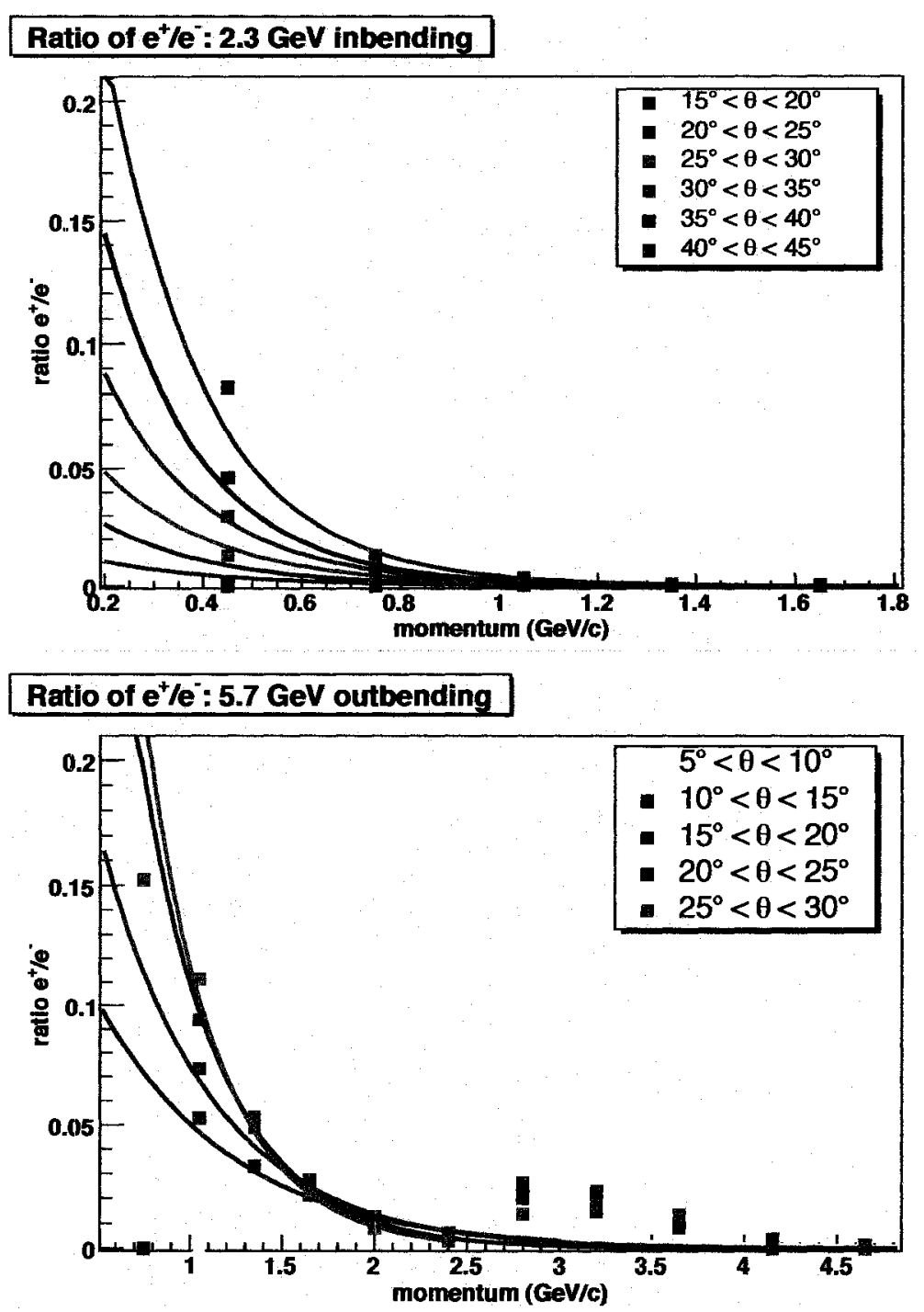

Figure 6.1: Ratios of $e^{+} / e^{-}$as a function of $p$, shown for various $\theta$ values, fit with the exponential curve of Eq. 6.11. 2.5 GeV outbending positrons were used for the top plot, while 5.7 GeV inbending positrons were used for the bottom plot. The normalized counts were divided by the inclusive electron counts. Note that the contamination is highest at low $p$ and high $\theta$. The "bump in the bottom plot near $3 \mathrm{GeV}$ is pion contamination, above the Cherenkov detection threshold of $p \approx 2.7$ GeV. 

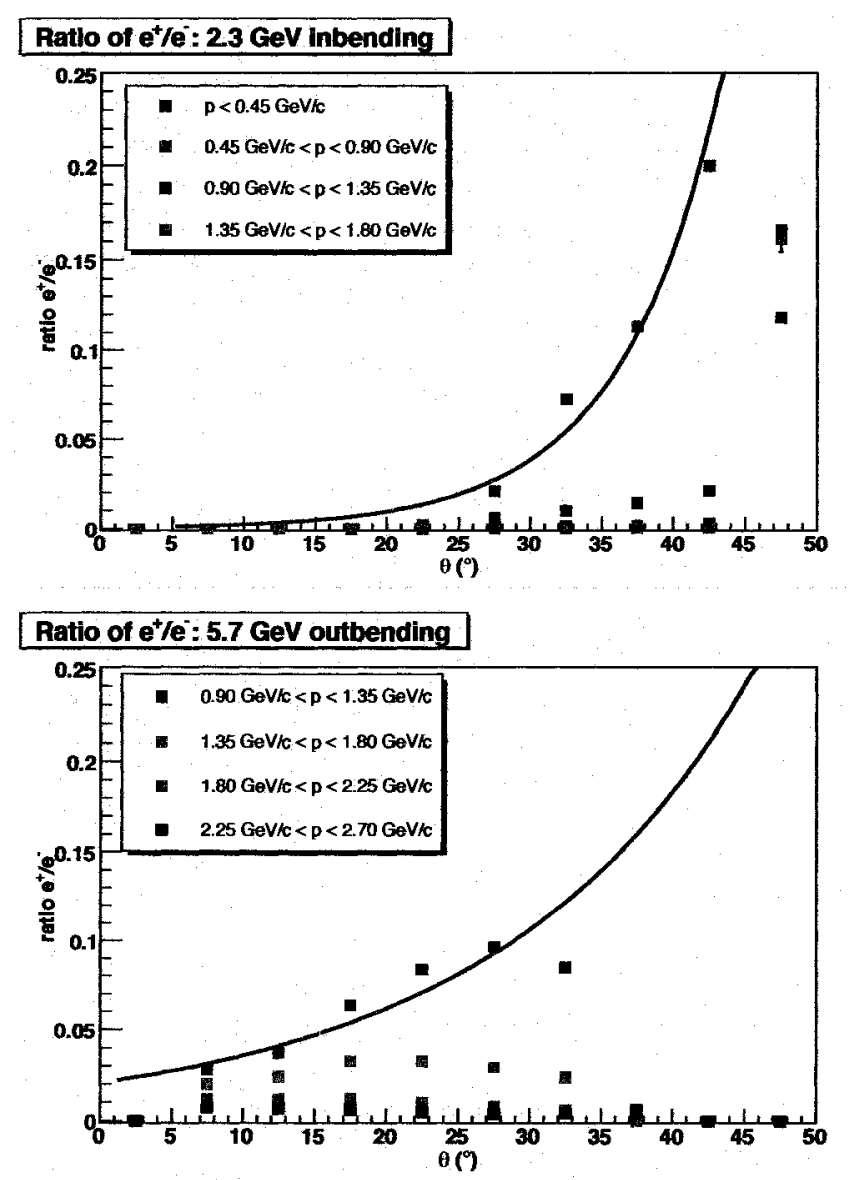

Figure 6.2: Ratios of $e^{+} / e^{-}$as a function of $\theta$, this time shown for various $p$ values. The lowest $p$-bin, where pair-production contamination dominates, is fit with the exponential curve of Eq. 6.12. Note that the contamination rapidly diminishes at higher momenta. 
Table 6.1: Values for the parametrization of $R \equiv e^{+} / e^{-}$, as given by Eq. 6.13, shown for each data bracket. These units apply to $\theta$ in degrees and $p$ in $\mathrm{GeV}$.

\begin{tabular}{|c|c|c|}
\hline Set/Bracket & $a$ & $b$ \\
\hline \hline $1.6+/ 1$ & $-5.96 \pm 0.067$ & $0.131 \pm 0.0021$ \\
\hline $1.6-/ 2$ & $-1.54 \pm 0.016$ & $0.0141 \pm 0.00071$ \\
\hline $5.76-/ 3$ & $-2.29 \pm 0.018$ & $0.129 \pm 0.00087$ \\
\hline $5.73-/ 4$ & $-2.31 \pm 0.017$ & $0.123 \pm 0.00084$ \\
\hline $5.7+/ 5$ & $-1.07 \pm 0.039$ & $0.0799 \pm 0.0015$ \\
\hline $2.3+/ 6$ & $-2.13 \pm 0.030$ & $0.0442 \pm 0.00099$ \\
\hline $5.6+/ 7$ & $-1.23 \pm 0.040$ & $0.0877 \pm 0.0016$ \\
\hline $1.7-/ 8$ & $0.0798 \pm 0.015$ & $-0.0151 \pm 0.00068$ \\
\hline $2.5-/ 9$ & $-1.98 \pm 0.0097$ & $0.0442 \pm 0.00044$ \\
\hline $4.2+/ 11$ & $-1.64 \pm 0.058$ & $0.0868 \pm 0.0022$ \\
\hline $4.2-/ 12$ & $-2.45 \pm 0.024$ & $0.118 \pm 0.0012$ \\
\hline
\end{tabular}

\begin{tabular}{|c|c|c|}
\hline Set/Bracket & $c$ & $d$ \\
\hline \hline $1.6+/ 1$ & $-4.22 \pm 0.10$ & $-0.0469 \pm 0.0033$ \\
\hline $1.6-/ 2$ & $-6.40 \pm 0.028$ & $0.00492 \pm 0.0012$ \\
\hline $5.76-/ 3$ & $-0.438 \pm 0.015$ & $-0.0932 \pm 0.00070$ \\
\hline $5.73-/ 4$ & $-0.434 \pm 0.014$ & $-0.0896 \pm 0.00067$ \\
\hline $5.7+/ 5$ & $-1.10 \pm 0.029$ & $-0.0635 \pm 0.0011$ \\
\hline $2.3+/ 6$ & $-3.95 \pm 0.042$ & $-0.00336 \pm 0.0014$ \\
\hline $5.6+/ 7$ & $-1.02 \pm 0.030$ & $-0.0681 \pm 0.0012$ \\
\hline $1.7-/ 8$ & $-6.56 \pm 0.028$ & $0.0216 \pm 0.0012$ \\
\hline $2.5-/ 9$ & $-3.91 \pm 0.016$ & $-0.0137 \pm 0.00068$ \\
\hline $4.2+/ 11$ & $-1.88 \pm 0.046$ & $-0.0538 \pm 0.0017$ \\
\hline $4.2-/ 12$ & $-1.21 \pm 0.021$ & $-0.0837 \pm 0.00098$ \\
\hline
\end{tabular}



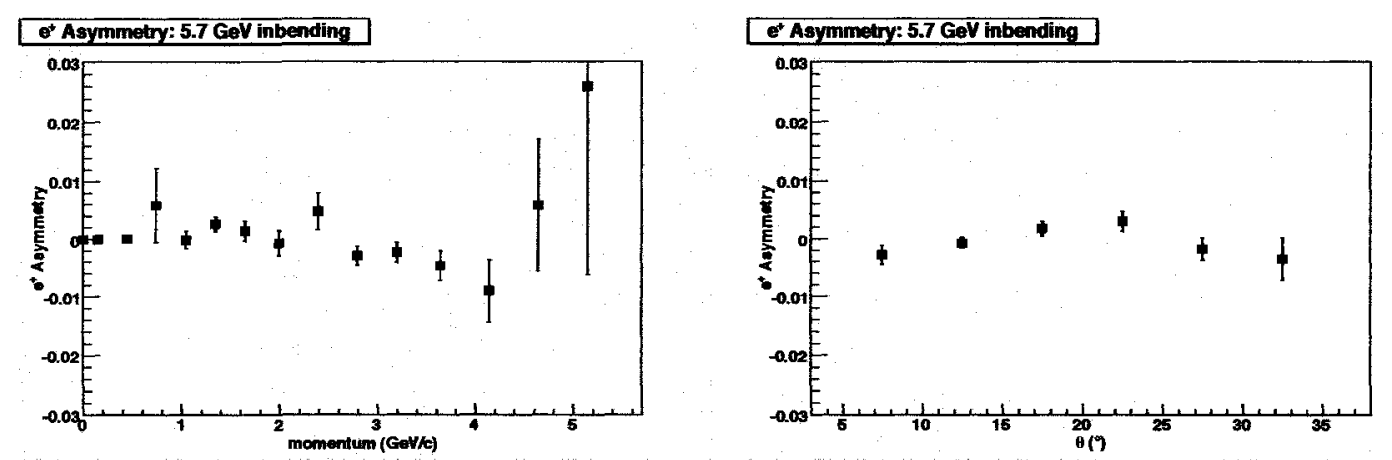

Figure 6.3: Total integrated positron asymmetries for $5.7+($ Bracket 5) data, shown as both a function of $p$ (left) and $\theta$ (right). Magnitudes are very small compared compared to the raw inclusive electron asymmetries, showing that pair-production makes only a very tiny contribution to the measured asymmetry.

\subsection{Beam $\times$ Target Polarizations}

Dilution factors quantify non-polarizable target background, leaving only events scattered from the free protons in the $\mathrm{NH}_{3}$. However, since we are interested only in measuring the double-spin asymmetry, it remains to remove scattering data for which either the incoming beam electron or target proton was not polarized. While the CEBAF electron beam maintains a fairly constant polarization of approximately $70 \%$, the polarization of the target protons can vary considerably. While the target NMR (see Section 2.4) makes a measurement of the polarization of the target, it is not well understood how the polarization varies throughout the volume (both in thickness and radius) of the target, nor is a precise manner of determining the error on the target NMR reading readily known. To further complicate metters, there are large stretches of target runs (particularly in the 1.6+ and 2.3+ data sets) with no NMR measurements.

To provide this polarization, we instead take advantage of the fact that the double-spin asymmetry $A_{\|}$can be easily calculated from the electric and magnetic form factors $G_{E}$ and $G_{M}$, and is well-understood for the proton in the elastic region, lower in $W$ (i.e. higher in $x$ ) than the region of interest of the measurement of $A_{1}$ and $A_{2}$. Elastic scattering is a purely spin- $\frac{1}{2}$ phenomenon, so $A_{1}=1$ and $A_{2}$ is purely transverse [6], so that

$$
A_{1}=1 \quad \text { (elastic) }
$$


and

$$
A_{2}=\sqrt{R_{(e l)}}=\frac{G_{E}}{\sqrt{\tau} G_{M}} \text { (elastic) }
$$

where $R_{(e l)}$ is the value analogous to the structure function $R$ in the elastic region (Eq. 1.66). 3 Substitution of these into Eq. 1.223, after applying Eqs. 1.19 and 1.66 yields

$$
A_{\|}=\frac{\left(1-E^{\prime} \epsilon / E\right)\left(1+\eta /\left(r_{G} \sqrt{\tau}\right)\right)}{1+\epsilon /\left(\tau r_{G}^{2}\right)}
$$

The number $r_{G}$ is the ratio of electric and magnetic form factors $r_{G} \equiv G_{M} / G_{E}$ for the proton. With some algebra, this can be written

$$
A_{\|}=\frac{\tau\left(1 / \epsilon-E^{\prime} / E\right)\left(r_{G}^{2}+r_{G} \eta / \sqrt{\tau}\right)}{1+r_{G}^{2} \tau / \epsilon}
$$

The numerator now contains terms proportional to $r_{G}^{2}$ and $r_{G}$. Eqs. 1.7, 1.15 and 1.44 can be used to show

$$
\begin{gathered}
Q^{2}=2 M\left(E-E^{\prime}\right)=2 M \nu \quad \text { (elastic) } \\
\tau=\frac{Q^{2}}{4 M^{2}} \quad \text { (elastic) }
\end{gathered}
$$

Using these relations, application of Eq. 1.16 to the $r_{G}^{2}$ terms, and application of Eq. 1.17 (with the numerator and denominator divided by $\epsilon E$ ) to the $r_{G}$ terms yields a more mundane equation for $A_{\|}$ in the elastic region [46]:

$$
A_{\|}=\frac{2 \tau r_{G}\left[\frac{M}{E}+r_{G}\left(\tau \frac{M}{E}+(1+\tau) \tan ^{2}\left(\frac{\theta}{2}\right)\right)\right]}{1+r_{G}^{2} \tau / \epsilon}
$$

The form factors $G_{M}$ and $G_{E}$ can be parametrized from world data [13] as

$$
\begin{gathered}
G_{E}\left(Q^{2}\right)=\frac{1}{1+0.62 Q+0.68 Q^{2}+2.80 Q^{3}+0.83 Q^{4}} \\
G_{M}\left(Q^{2}\right)=\frac{\mu_{p}}{1+0.35 Q+2.44 Q^{2}+0.50 Q^{3}+1.04 Q^{4}+0.34 Q^{5}}
\end{gathered}
$$

${ }^{3}$ Refer back to Sections 1.2 .2 and 1.4 for more information. 
where the proton magnetic moment is $\mu_{p}=2.79$.

A more recent parametrization of the form factors for the proton is given in Ref. [14]:

$$
\begin{gathered}
G_{E}\left(Q^{2}\right)=\left[1+p_{2} Q^{2}+p_{4} Q^{4}+\ldots+p_{12} Q^{12}\right]^{-1} \\
G_{M}\left(Q^{2}\right)=\mu_{p}\left[1+p_{2} Q^{2}+p_{4} Q^{4}+\ldots+p_{12} Q^{12}\right]^{-1}
\end{gathered}
$$

where the coefficients $p_{2}-p_{12}$ are replicated in Table 6.2. Later in analysis, this parametrization was used in place of the parametrization of Eqs. 6.23 and 6.24. The change in the resulting asymmetries was small, showing that knowledge of these form factors is not a limiting factor in the accuracy of our measurement. ${ }^{4}$

Table 6.2: Fit parameters for the Rosenbluth form factors $G_{E}$ and $G_{M}$ by J. Arrington. From Ref. [14].

\begin{tabular}{|c|c|c|}
\hline Parameter & $G_{E}$ & $G_{M}$ \\
\hline \hline$p_{2}$ & 3.226 & 3.19 \\
\hline$p_{4}$ & 1.508 & 1.355 \\
\hline$p_{6}$ & -0.3773 & 0.151 \\
\hline$p_{8}$ & 0.611 & -0.0114 \\
\hline$p_{10}$ & -0.1853 & $5.33 \times 10^{-4}$ \\
\hline$p_{12}$ & 0.01596 & $-9.00 \times 10^{-6}$ \\
\hline
\end{tabular}

Knowing the proper value of the elastic asymmetry, one can then measure the actual backgroundsubtracted asymmetry of the real data, integrated over the elastic region. This asymmetry always turns out smaller than the pure double-spin asymmetry calculated in $\mathrm{Eq} .6 .22$, because, though the non-proton background is already removed, not all the target protons and beam electrons are polarized. Using Eq. 5.1, the product $P_{b} P_{t}$ (beam $\times$ target polarizations) is

$$
P_{b} P_{t}=\frac{A_{\text {measured }}}{A_{\|}}
$$

where, in this case, $A_{\|}$is defined by the above equations and $A_{\text {measured }}$ is the diluted experimental asymmetry.

\footnotetext{
${ }^{4}$ This is considered in determination of the systematic errors. See Section 7.2.3.
} 
Knowledge of both detector behavior and the accuracy of the (dilution factor) is dubious at low values of $Q^{2}$. Therefore, a lower limit of $Q^{2}=0.2 \mathrm{GeV}^{2}$ was used in all measurements of $P_{b} P_{t}$. Both background subtracted inclusive data and exclusive ep events were used for the measurement of $A_{\text {measured }}$. These two methods are explained individually in this section.

It is important to note that measurement of $P_{b} P_{t}$ (on average) incurs the largest systematic errors of any of the individual contributions to the asymmetry (see Section 7.2.3), as the number of elastic $e p$ events is statistically limited. Also, as the final answer is a single number for each data set (unlike the contnuous spectrum of dilution factors), great care was taken to cross-check the final values using variations on the methodology, detailed in the following subsections.

\subsubsection{Inclusive method}

The first method of determining $P_{b} P_{t}$ uses a cut on $W$ to isolate events from the inclusive electron scattering spectrum in the elastic region, and then uses their background-subtracted asymmetry to evaluate Eq. 6.27 for each $Q^{2}$ bin. The error-weighted average is then used for the final value of $P_{b} P_{t}$. Using inclusive events is advantageous because it offers the most possible statistics (i.e. it includes elastic events even when the recoil proton was not detected), but it has the disadvantage of requiring a very large removal of background (see Figure 6.4), potentially leading to large systematic errors.

\section{Isolation of true elastic events}

First, the width of the elastic peak must be defined. Including the entire elastic peak is desirable to minimize the statistical error. However, widening the cut by too much risks the inclusion of nonelastic electrons. This can happen both at the high $W$ end (if $W \rightarrow M+m_{\pi}$, allowing for radiative inelastic tails) and at the low $W$ end (where coherent nuclear scattering creates a tail at low $W$ ). For lower beam energies, where elastic scattering events dominate over the inelastic scattering events, the elastic peak is clearly discernable from the background. As beam energy increases, though, it becomes more challenging to isolate the less visible elastic peak (see Figure 5.1, for example). 
The simplest way to determine the width of the elastic peak was to subtract away the background. Removal of the non-proton scattering events excises multiple-nucleon correlated events, and the width of the elastic peak can then be more easily distinguished. The background removal can be done one of two ways. The more exact method ${ }^{5}$ is to use the dilution factors $F_{D F}$ and Eq. 5.76. The other, simpler method, takes advantage of the fact that, in the low $W$ tail, scattering events involving "long-distance" $(x>1)$ correlations between nucleons dominate the event spectrum. At the relatively high beam energies of EG1, these event cross-sections are roughly equal for ${ }^{12} \mathrm{C}$ and ${ }^{15} \mathrm{~N}$, so long as $W$ is not too low (where $x \rightarrow Z$, and unequal effects for nitrogen and carbon are expected).

Given that the spectra of ${ }^{12} \mathrm{C}$ and $\mathrm{NH}_{3}$ should have the same shape in the low $W$ region, apart from an overall multiplicative "scaling factor" $S$ accounting for slight differences in mass thickness, ${ }^{6}$ the total background can be found by scaling the counts in the low- $W$ tail of the carbon target events to the same magnitude as the $\mathrm{NH}_{3}$ events. That is, one finds the factor $S$ such that

$$
\sum_{0.40 \mathrm{GeV}<W<W_{l i m i t}}\left(n_{N H_{3}}-S n_{C}\right) \approx 0
$$

by looping over small increments in $S$ until the above quantity is minimized. $W_{\text {limit }}$ was set low enough to be safely far away enough from the elastic peak, as including the peak risks 'underscaling' of the carbon events. The quantity $n_{\mathrm{NH}_{3}}-S n_{C}$ then yields the approximate backgroundsubtracted spectrum, when extended into the elastic region.

Accuracy can be improved if the subtracted spectrum of ${ }^{12} \mathrm{C}$ is related to the predicted ${ }^{15} \mathrm{~N}$ spectrum by a model. Once again, the model of Section 2.8 was used, specifically the newly-modeled ${ }^{15} \mathrm{~N} /{ }^{12} \mathrm{C}$ data, to predict the count ratio between the carbon target and the ammonia target, minus the free proton contribution. The model is used with Eq. 5.32 , with $\ell_{N} \rightarrow \ell_{A} \approx 0.60 \mathrm{~cm}$ and $\rho_{N} \rightarrow \frac{15}{18} \rho_{A}$, to account for the length and density of ${ }^{15} \mathrm{~N}$ in the ammonia target, respectively. ${ }^{7}$ The array of numbers $r_{N C}\left(W, Q^{2}\right)$ was then used as multiplicative factor on the carbon counts. The resulting numbers ( $\sim 0.87$ in the elastic region) are very close to the scaling factors required when

\footnotetext{
${ }^{5}$ This assumes models for $\sigma_{n} / \sigma_{d}$ and $\sigma_{N} / \sigma_{C}$ are precise, which was not the case during the early phase of the analysis, hence previously necessitating another method.

${ }^{6}$ This factor should be fairly constant, with no more variations than those inherent in the ammonia target length $\ell_{A}$.

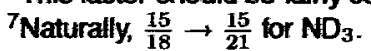


no model is used. ${ }^{8}$ This lends further credence to the utility of the model. A summary of the scaling integration limits and derived scaling factors $S$ is recorded in Table 6.3.

Plots of background-subtracted spectra in the elastic region for inclusive events are shown in Figure 6.4. Using these plots, one can easily find the boundaries of the elastic peak. However, in no case was an upper limit of $W=1.00 \mathrm{GeV}$ in the elastic peak range exceeded, in order to avoid inelastic tail contamination (plainly visible in the lower energy background-subtracted $W$ spectra). Boundaries for inclusive elastic peaks (along with the upper limits used for the case of carbon background subtraction) are listed in Table 6.4 .

Table 6.3: Upper limit of integration for ${ }^{12} \mathrm{C}$ scaling, as well as the weighted average of the scaling factor $S$ for carbon counts, with and without the use of the nitrogen/carbon scaling model. Two examples of the model, one using a fixed ammonia target length $\ell_{A}$, and another using the precise value of $\ell_{A}$ for each set (from Table 5.9), are shown. See the text for more details.

\begin{tabular}{|c|c|c|c|c|}
\hline Set/Bracket & $W_{\text {limit }}$ & $S$ (no model) & $S$ (N/C model, $\left.\ell_{A}=0.6\right)$ & $S$ (N/C model, exact $\ell_{A}$ ) \\
\hline \hline $1.6+/ 1$ & 0.86 & 0.86 & 1.06 & 1.04 \\
\hline $1.6-/ 2$ & 0.86 & 0.88 & 1.09 & 1.05 \\
\hline $5.76-/ 3$ & 0.70 & 0.87 & 1.01 & 1.01 \\
\hline $5.73-/ 4$ & 0.70 & 0.85 & 0.98 & 1.01 \\
\hline $5.7+/ 5$ & 0.74 & 0.82 & 0.94 & 0.98 \\
\hline $2.3+/ 6$ & 0.82 & 0.90 & 1.07 & 1.04 \\
\hline $5.6+/ 7$ & 0.74 & 0.83 & 0.96 & 1.00 \\
\hline $1.7-/ 8$ & 0.86 & 0.82 & 1.01 & 1.06 \\
\hline $2.5-/ 9$ & 0.82 & 0.83 & 1.01 & 1.04 \\
\hline $4.2+/ 11$ & 0.80 & 0.84 & 0.97 & 0.98 \\
\hline $4.2-/ 12$ & 0.78 & 0.86 & 1.00 & 1.00 \\
\hline
\end{tabular}

Table 6.4: Lower and upper limits of elastic $W$-bounds used for measurement of $P_{b} P_{t}$, for the inclusive method. All values are in GeV.

\begin{tabular}{|c|c|c|}
\hline Beam Energy (GeV) & Elastic Bound (lo) & Elastic Bound (hi) \\
\hline \hline $1 . x$ & 0.90 & 0.96 \\
\hline $2 . x$ & 0.89 & 0.97 \\
\hline $4 . x$ & 0.88 & 0.98 \\
\hline $5 . x$ & 0.86 & 1.00 \\
\hline
\end{tabular}

\footnotetext{
${ }^{8}$ This means that when the model is incorporated, the resultant scaling factor becomes $1.00 \pm 0.06$.
} 

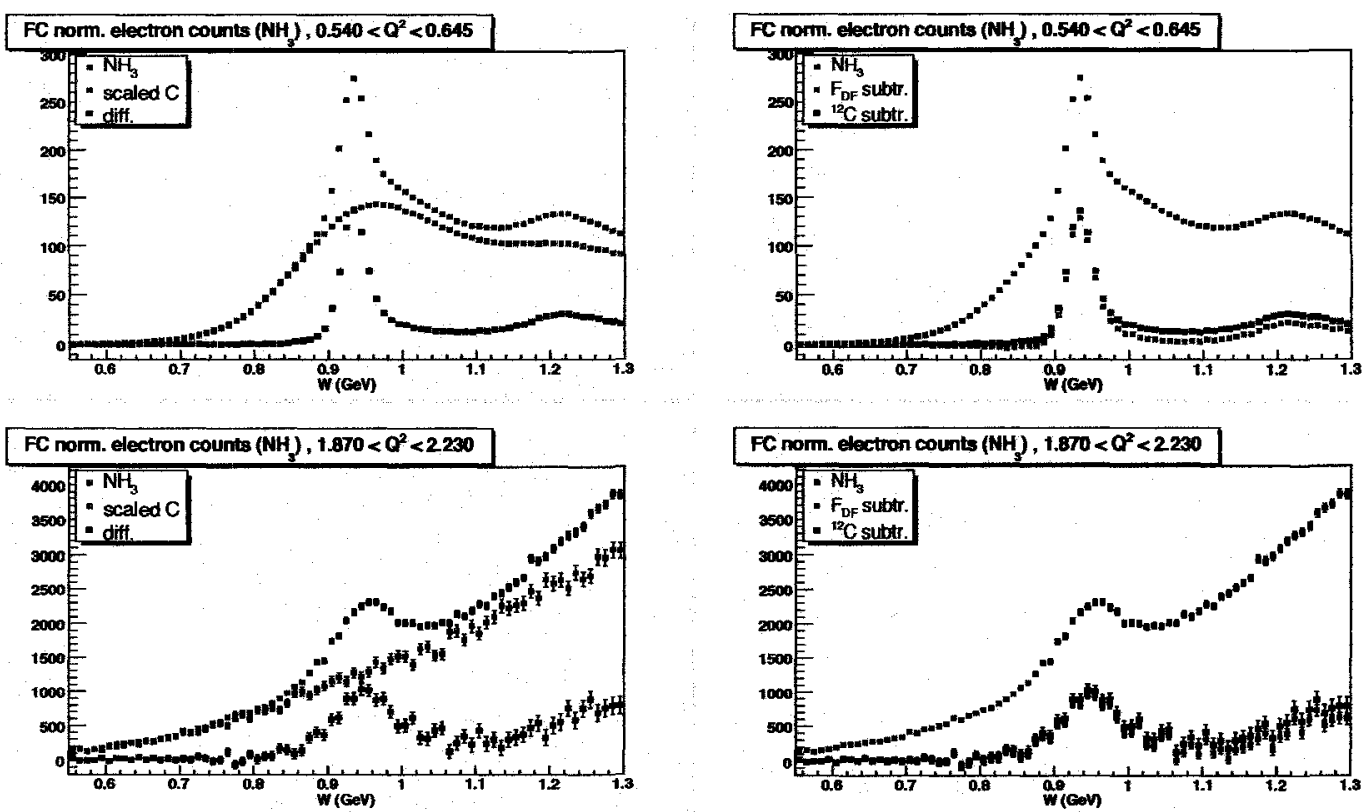

Figure 6.4: Background subtracted inclusive spectra, near the elastic region in $W$, for $1.6 \mathrm{GeV}$ (top) and $5.7 \mathrm{GeV}$ (bottom) data. The left and right plots show the same data, and are only separated for clarity. Shown are the total $\mathrm{NH}_{3}$ counts (blue), the ${ }^{12} \mathrm{C}$ counts scaled to the ammonia spectrum (green), the remaining difference of these two (i.e. the elastic events)(black). The same subtracted spectrum using the dilution factor $F_{D F}$ to determine background is also shown (red). 


\section{Integration over the elastic peak}

The background subtraction must not be improperly weighted by the relative counts in each bin. There are two approaches one can take to handle this issue. The simplest method is to derive the number of background counts bin-by-bin and integrate the remaining counts directly. The value of $n_{\text {back }}$ is calculated using either Eq. 5.76 (for proper ditution factors) or $n_{N_{3}}-S n_{C}$ (for the carbon scaling method). For the case of dilution factors, the error on $n_{b a c k}$ is given by

$$
\sigma_{n_{b a c k}}=\sqrt{\left(\frac{\partial n_{b a c k}}{\partial n_{N H_{3}}}\right)^{2} \sigma_{n_{N H_{3}}}^{2}+\left(\frac{\partial n_{b a c k}}{\partial F_{D F}}\right)^{2} \sigma_{F_{D F}}^{2}}
$$

This calculation yields

$$
\sigma_{n_{b a c k}}=\sqrt{\left(1-F_{D F}\right)^{2} n_{N H_{3}}+n_{N H_{3}}^{2} \sigma_{F_{D F}}^{2}}
$$

For the carbon-subtraction method, the error is simply given by

$$
\sigma_{n_{b a c k}}=\sqrt{S n_{C(a d j .)}}
$$

where $n_{C(a d j .)}$ is the carbon count with the minor carbon/nitrogen-model adjustment mentioned above.

To apply these equations, the sums of the $+/$ - helicity $\mathrm{NH}_{3}$ counts between the elastic $W$ limits were calculated for each $Q^{2}$ bin. This integrated sum was then treated as a single bin. An overall dilution factor, weighted by the error on the raw asymmetry in each $W$-bin, is calculated as

$$
\begin{gathered}
F_{D F_{\text {total }}}=\frac{\sum_{W} N F_{D F}}{\sum_{W} N} \\
\sigma_{F_{D F_{\text {total }}}}=\frac{1}{\sqrt{\sum_{W} N}}
\end{gathered}
$$

This dilution factor is then applied to the sum of all the counts over the elastic region in Eq. 5.76 to get $n_{\text {back }}$ and Eq. 6.30 to get $\sigma_{n_{\text {back }}}$.

After $n_{b a c k}$ and its error are calculated (using either dilution factors or scaled carbon counts), the background-subtracted asymmetry for the given $Q^{2}$ bin is then given by Eq. 5.74 , with the change 
$n_{\text {back }} \rightarrow 2 n_{\text {back. }}{ }^{9}$ Thus we have (with the $n$ 's this time representing the summed counts over $W$ ):

$$
A_{\|}=\frac{n^{-}-n^{+}}{n^{-}+n^{+}-2 n_{\text {back }}}
$$

Using the usual method for statistical errors, one gets

$$
\sigma_{A_{\|}}=\sqrt{\left(\frac{\partial A_{\|}}{\partial n_{b a c k}}\right)^{2} \sigma_{n_{b a c k}}^{2}+\left(\frac{\partial A_{\|}}{\partial n^{-}}\right)^{2} n^{-}+\left(\frac{\partial A_{\|}}{\partial n^{+}}\right)^{2} n^{+}}
$$

where (approximately)

$$
\begin{aligned}
& \frac{\partial A_{\|}}{\partial n_{\text {back }}}=\frac{2\left(n^{-}-n^{+}\right)}{\mathscr{R}^{2}} \\
& \frac{\partial A_{\|}}{\partial n^{-}}=\frac{2\left(n^{+}-n_{\text {back }}\right)}{\mathscr{R}^{2}} \\
& \frac{\partial A_{\|}}{\partial n^{+}}=\frac{2\left(n^{-}-n_{\text {back }}\right)}{\mathscr{R}^{2}}
\end{aligned}
$$

with

$$
\mathscr{R} \equiv n^{-}+n^{+}-2 n_{b a c k}
$$

With the background-subtracted asymmetry and its error now calculated for every populated $Q^{2}$ bin, Eq. 6.27 can then be applied in each bin. The weighted average over these bins is then the actual $P_{b} P_{t}$ value.

As a check on this method, a second method of background subtraction was applied, in which the integrated dilution factor (Eq. 6.32) was applied to the averaged asymmetry, summed over the elastic $W$ bins, as according to Eqs. 5.7 and 5.8 (but summed only over elastic $W$ and not $Q^{2}$ ). The diluted asymmetry was then found in the standard manner, that is

$$
A_{\|_{\text {diluted }}}=\frac{1}{F_{D F_{\text {total }}}} A_{\|_{\text {raw }}}
$$

\footnotetext{
${ }^{9}$ This is done because the Faraday Cup charge sum of both the plus and minus helicities is used to define the count rate $n_{\text {back }}$ in this section, whereas the Faraday Cup of either $+/$ - helicity was used in Section 5.6. This is a subtle and often confusing point that must be remembered when doing charge-weighted background subtraction. Generally, appropriate Faraday Cup charge weights are not explicitly stated in this text, due to the heaviness of notation required to do so. Care must be taken to include them when working out exact calculations for use in coding.
} 
Calculating the standard error yields

$$
\sigma_{A_{\|}}=\sqrt{\frac{F_{D F}^{2}}{N}+\frac{A_{\|\|_{\text {raw }}}^{2}}{F_{D F_{t o t a l}}^{4}} \sigma_{F_{D F_{t o t a l}}^{2}}^{2}}
$$

This method can also be used if carbon scaling is used for the background subtracting, by defining the dilution factor according to Eq. 5.76, using the calculated scaled background. In this case, the error on $F_{D F_{\text {total }}}$ is

$$
\sigma_{F_{D F_{t o t a l}}}=\sqrt{\frac{n_{b a c k}}{\left(n^{-}+n^{+}\right)^{2}}+\frac{n_{b a c k}^{3}}{\left(n^{-}+n^{+}\right)^{4}}}
$$

with the remaining calculation of $A_{\|}$going just as for regular dilution factors. In summary, there are 4 (non-independent) methods for subtracting the background presented here:

1. Using an averaged dilution factor to subtract backgrounds from the summed counts over $W$, then calculating the total asymmetry

2. Using scaled carbon to subtract the summed background, then calculating the total asymmetry

3. Using an error-weighted dilution factor, averaged over the peak, to dilute the average asymmetry

4. Calculating a primitive dilution factor from the scaled carbon counts, then error-weighting and averaging it to dilute the average asymmetry.

In the end, all 4 methods were found to produce asymmetries with approximately equal error bars for all data sets, with resulting $P_{b} P_{t}$ values generally compatible within the resulting statistical error bar. In the end, it was Method 3. that was used to do background subtraction for the inclusive case, because dilution factors were developed to make the most accurate subtraction of background possible. 


\section{Averaging $P_{b} P_{t}$ over $Q^{2}$}

The asymmetry calculated in Eq. 6.22 varies as a function of $Q^{2}$. The asymmetry-weighted average of $Q^{2}$ in each of the $40 Q^{2}$ bins over all elastic events was calculated as

$$
\left\langle Q^{2}\right\rangle=\frac{\sum_{\text {events }} Q^{2} A_{\| !}\left(Q^{2}\right)}{\sum_{\text {events }} A_{\|}\left(Q^{2}\right)}
$$

with $A_{\|}$calculated in Eq. 6.22. Then, Eq. 6.27 was applied in each $Q^{2}$ bin, yielding a separate measurement of $P_{b} P_{t}$ in each $Q^{2}$ bin, with statistical error given simply by

$$
\sigma_{P_{b} P_{t}}=\sigma_{A_{\text {measured }}} / A_{\|}
$$

This value should be constant for all $Q^{2}$ (within a statistical error).

The inclusive method of calculating $P_{b} P_{t}$ works well at low energies, where elastic peak statistics are plentiful and background is (relatively) low, but it leaves much to be desired at higher energies. For this reason, a second method was devised.

\subsubsection{Exclusive $e p$ method}

Beam $\times$ target polarizations can also be calculated from exclusive elastic ep events (Eq. 3.6). Due to the strict kinematic cuts used to identify these events, very little background remains to be subtracted. The kinematic cuts on missing energy, $\theta$ and $\phi$ used to identify these events are described in detail in Section 3.5, while the PID cuts used to identify potential ep events are explained and summarized in Section 3.4. Only a final cut on $W$ remains to be defined in this section. The basic strategy for finding a good $W$-cut is essentially the same as for inclusive electrons; only the method of background subtraction differs.

\section{Removal of remaining background}

With only a slight difference in method as used for inclusive electrons (see Section 5.6.1), dilution factors can also be calculated for elastic ep events. Scaling of ${ }^{12} \mathrm{C}$ events can also be used, but since (practically) all coherent scattering events in the low- $W$ tail are already removed from the 
spectrum by the kinematic cuts, a different strategy must be employed here.

A spectrum of counts in $\phi$ (in $0.1^{\circ}$ bins) is produced for elastic $e p$ events, with all the cuts in Section 3.5 in place, except for the $\Delta \phi$ cut. This is done for both $\mathrm{NH}_{3}$ and ${ }^{12} \mathrm{C}$ runs. Using the method of minimizing Eq. 6.28, except over bins in $\phi$, a carbon scaling factor $S$ can be found, using the same reasoning as in the preceding section. A cut of

$$
2^{\circ}<|\Delta \phi|<6^{\circ}
$$

(with $\Delta \phi$ defined in Eq. 3.14) was used for the scaling region, so that

$$
\sum_{2^{\circ}<|\Delta \phi|<6^{\circ}}\left(n_{N H_{3}}-S n_{C}\right)
$$

was minimized. Figure 6.5 shows the scaled carbon counts in $\phi$, showing just how small the remaining background actually is. Scaling factors for exclusive carbon events are listed in Table 6.5. Using the carbon scaling factor $S$ and the exclusive $e p$ dilution factors, plots analogous to those in Figure 6.4, showing the background subtraction using each method, can be drawn (Figure 6.6). W-cut boundaries for elastic $e p$ events are considerably wider than for inclusive events, as listed in Table 6.6.

Table 6.5: Carbon scaling factors required for elastic ep events for each data set in the region of $2^{\circ}<|\Delta \phi|<6^{\circ}$. Details are given in the text.

\begin{tabular}{|c|c|}
\hline Set/Bracket & $S$ \\
\hline \hline $1.6+/ 1$ & 1.16 \\
\hline $1.6-/ 2$ & 1.52 \\
\hline $5.76-/ 3$ & 1.25 \\
\hline $5.73-/ 4$ & 1.11 \\
\hline $5.7+/ 5$ & 0.99 \\
\hline $2.3+/ 6$ & 1.06 \\
\hline $5.6+/ 7$ & 1.31 \\
\hline $1.7-/ 8$ & 1.53 \\
\hline $2.5-/ 9$ & 1.13 \\
\hline $4.2+/ 11$ & 1.23 \\
\hline $4.2-/ 12$ & 1.34 \\
\hline
\end{tabular}




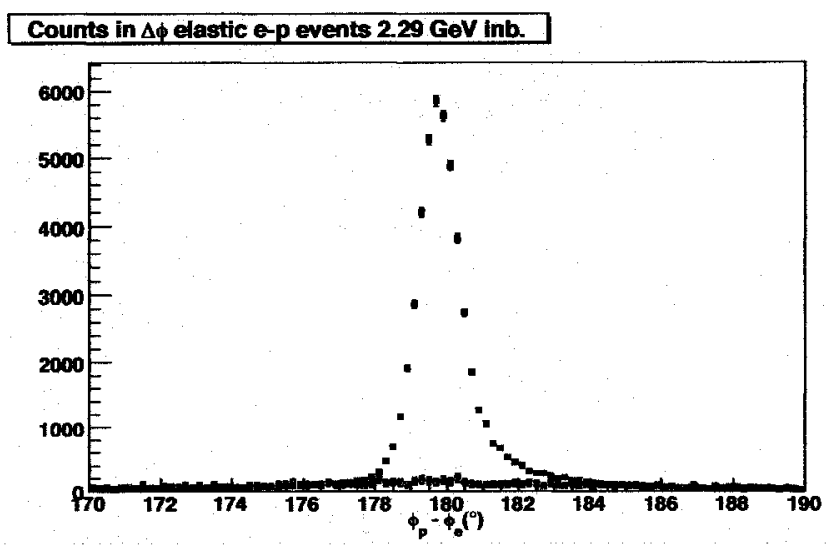

Counts in $\Delta \phi$ elastic e-p events $5.74 \mathrm{GoV}$ outb.

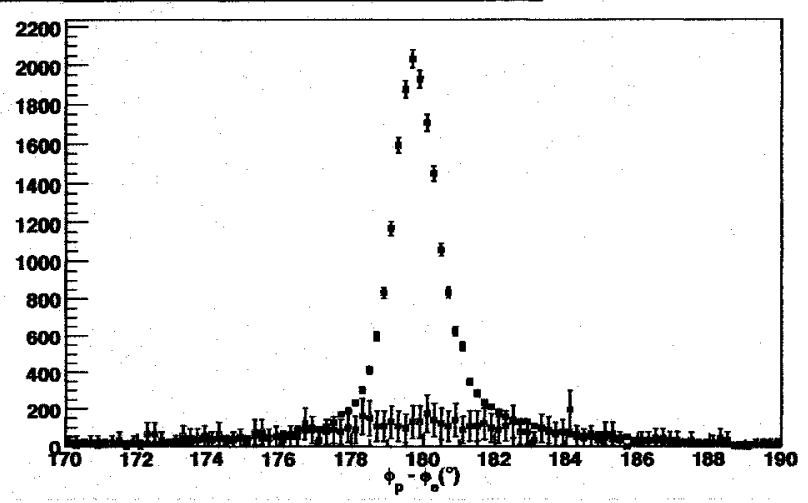

Figure 6.5: Elastic $e p$ events in terms of $\phi_{p}-\phi_{e}$ (blue) showing scaled carbon background (red). Remaining background from exclusive events is very low if good kinematic cuts are used. Data from Brackets $6(2.3+)$ and $3(5.76-)$ are shown.

Table 6.6: Lower and upper limits of elastic $W$-bounds used for measurement of $P_{b} P_{t}$, for the exclusive method. All values are in GeV.

\begin{tabular}{|c|c|c|}
\hline Beam Energy (GeV) & Elastic Bound (lo) & Elastic Bound (hi) \\
\hline \hline $1 . x$ & 0.88 & 0.98 \\
\hline $2 . x$ & 0.87 & 0.99 \\
\hline $4 . x$ & 0.86 & 1.00 \\
\hline $5 . x$ & 0.84 & 1.02 \\
\hline
\end{tabular}



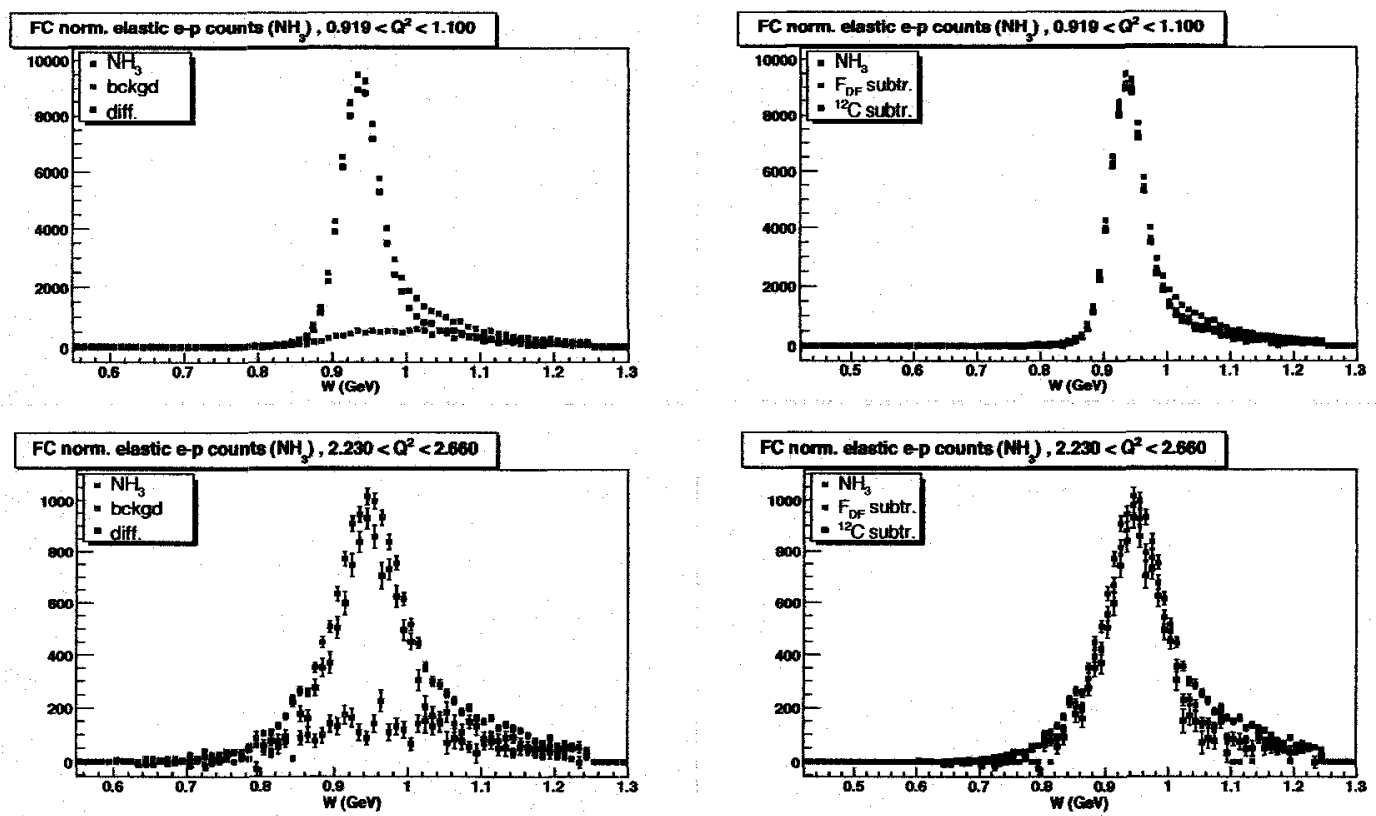

Figure 6.6: Background subtracted exclusive ep spectra, near the elastic region in $W$, prior to a $W$-cut. The left and right plots show the same data, and are only separated for clarity. Shown are the total elastic $\mathrm{NH}_{3}$ counts (blue), the ${ }^{12} \mathrm{C}$ counts scaled to the ammonia spectrum (green), and the remaining difference of these two (i.e. the elastic events) (black). The same subtracted spectrum using an elastic dilution factor $F_{D F}$ to determine background is also shown (red, barely visible behind the black on the right-hand plots). Brackets 6 and 3 are again shown. 


\section{Applying the exclusive method}

Application of the exclusive $e p$ procedure of deriving $P_{b} P_{t}$ can also proceed as according to the four methods listed in the description of the inclusive method. In each case, the mathematical formalism, including error bars, is exactly the same as for the inclusive method, only the exclusive $e p$ counts, backgrounds and dilution factors are used.

Just as in the inclusive case, the four methods of deriving the exclusive $P_{b} P_{t}$ yielded consistent results. However, in this case, the carbon scaling method was applied to cumulative asymmetries (Method 4) to generate $P_{b} P_{t}$. For exclusive events, the background subtraction was very small, so there was little to gain in using a dilution factor in place of background subtraction, and the carbonscaling method provided greater overall kinematic coverage. ${ }^{10}$

Again, $P_{b} P_{t}$ was evaluated in every $Q^{2}$ bin. These can be shown compared to the inclusive values in the figures on the following pages. Just as in the inclusive method, the $A_{\|}$-weighted $Q^{2}$ was used to calculate the theoretical asymmetry. ${ }^{11}$ Because of the small background subtraction, the exclusive method gave slightly smaller error bars than the inclusive at the highest beam energies, but was less successful at lower beam energies, where the less energetic proton recoil was not as often detected.

However, even though the statistical error bar was larger in the exclusive case for low energies, there are far fewer sources of systematic errors in the exclusive evaluation than in the inclusive case, due to very small background subtraction in the former. Therefore, the exclusive $P_{b} P_{t}$ values were employed as the "official" measurements for the proton analysis.

\subsubsection{Final determination of $P_{b} P_{t}$ values}

By this point, inclusive and exclusive $P_{b} P_{t}$ values were calculated for each $Q^{2}$ bin. Values were checked for constancy for each HWP and target polarization sign combination (Figure 6.7), for separate target polarizations only (Figure 6.8) and whole sets (Figure 6.9), for diagnostic purposes. In every case, $Q^{2}$ bins where the asymmetry error was larger than 1.5 was excluded, to prevent the

\footnotetext{
${ }^{10}$ Calculation of dilution factors in the elastic region relied upon empty LHe count data being present in every bin. Populated bins sometimes were sparse for the limited ep events in the limited numbers of these runs.

${ }^{11}$ This is actually more crucial in the exclusive case, where weaker statistics are more fikely to result in an average $Q^{2}$ away from the bin center.
} 
Table 6.7: $P_{b} P_{t}$ values, inclusive and exclusive, for each half-wave-plate state and target polarization combination. Error bars on high-energy values are quite large. For this reason, asymmetry data for separate HWP states and target polarizations are combined before finally evaluating $P_{b} P_{t}$, and these values are for diagnostics and reference only. The signs represent HWP/targetpol; e.g. " ++ " is HWP out, positive target polarization.

\begin{tabular}{|c|c|c|c|c|}
\hline Set/Bracket & ++ (inclusive) & ++ (exclusive) & -+ (inclusive) & -+ (exclusive) \\
\hline \hline $1.6+/ 1$ & $0.551 \pm 0.0041$ & $0.550 \pm 0.0063$ & $0.634 \pm 0.0063$ & $0.645 \pm 0.010$ \\
\hline $1.6-/ 2$ & $0.561 \pm 0.012$ & $0.567 \pm 0.041$ & - & - \\
\hline $5.76-/ 3$ & $0.443 \pm 0.023$ & $0.449 \pm 0.023$ & $0.415 \pm 0.027$ & $0.502 \pm 0.028$ \\
\hline $5.73-/ 4$ & $0.500 \pm 0.029$ & $0.572 \pm 0.031$ & $0.459 \pm 0.032$ & $0.502 \pm 0.035$ \\
\hline $5.7+/ 5$ & $0.582 \pm 0.056$ & $0.473 \pm 0.030$ & $0.468 \pm 0.070$ & $0.489 \pm 0.038$ \\
\hline $2.3+/ 6$ & $0.531 \pm 0.0056$ & $0.556 \pm 0.0079$ & - & - \\
\hline $5.6+/ 7$ & $0.534 \pm 0.062$ & $0.612 \pm 0.036$ & $0.520 \pm 0.066$ & $0.584 \pm 0.039$ \\
\hline $1.7-/ 8$ & $0.586 \pm 0.0082$ & $0.656 \pm 0.019$ & - & - \\
\hline $2.5-/ 9$ & $0.511 \pm 0.088$ & $0.611 \pm 0.157$ & $0.606 \pm 0.0080$ & $0.630 \pm 0.014$ \\
\hline $4.2+/ 11$ & $0.584 \pm 0.027$ & $0.609 \pm 0.019$ & $0.562 \pm 0.026$ & $0.538 \pm 0.018$ \\
\hline $4.2-/ 12$ & $0.538 \pm 0.034$ & $0.600 \pm 0.042$ & $0.563 \pm 0.015$ & $0.606 \pm 0.019$ \\
\hline
\end{tabular}

\begin{tabular}{|c|c|c|c|c|}
\hline Set/Bracket & +- (inclusive) & +- (exclusive) & - - (inclusive) & - -(exclusive) \\
\hline \hline $1.6+/ 1$ & $0.530 \pm 0.0035$ & $0.545 \pm 0.0054$ & $0.515 \pm 0.0054$ & $0.518 \pm 0.0086$ \\
\hline $1.6-/ 2$ & - & - & - & - \\
\hline $5.76-13$ & $0.462 \pm 0.031$ & $0.506 \pm 0.032$ & $0.392 \pm 0.028$ & $0.439 \pm 0.029$ \\
\hline $5.73-/ 4$ & $0.470 \pm 0.030$ & $0.571 \pm 0.032$ & $0.465 \pm 0.033$ & $0.505 \pm 0.036$ \\
\hline $5.7+/ 5$ & $0.25 \pm 0.12$ & $0.465 \pm 0.062$ & $0.472 \pm 0.036$ & $0.488 \pm 0.020$ \\
\hline $2.3+/ 6$ & $0.460 \pm 0.0078$ & $0.476 \pm 0.010$ & $0.421 \pm 0.0051$ & $0.451 \pm 0.0069$ \\
\hline $5.6+/ 7$ & - & - & $0.452 \pm 0.046$ & $0.519 \pm 0.027$ \\
\hline $1.7-/ 8$ & $0.564 \pm 0.043$ & $0.423 \pm 0.096$ & $0.489 \pm 0.0088$ & $0.526 \pm 0.019$ \\
\hline $2.5-/ 9$ & $0.559 \pm 0.0089$ & $0.605 \pm 0.016$ & $0.543 \pm 0.041$ & $0.680 \pm 0.069$ \\
\hline $4.2+/ 11$ & $0.459 \pm 0.025$ & $0.498 \pm 0.019$ & $0.572 \pm 0.027$ & $0.541 \pm 0.019$ \\
\hline $4.2-/ 12$ & $0.556 \pm 0.016$ & $0.560 \pm 0.021$ & $0.492 \pm 0.019$ & $0.511 \pm 0.023$ \\
\hline
\end{tabular}


incorporation of non-Gaussian measurements. Final $P_{b} P_{t}$ were calculated using exclusive asymmetries for whole run sets, after asymmetries for opposing target polarizations were combined with the appropriate weights (see Section 7.1). These final $P_{b} P_{t}$ calculations cut out all $Q^{2}$ bins containing less than 10 events. ${ }^{12}$

Table 6.8: $P_{b} P_{t}$ values, inclusive and exclusive, for each target polarization combination.

\begin{tabular}{|c|c|c|}
\hline Set/Bracket & + (inclusive) & + (exclusive) \\
\hline \hline $1.6+/ 1$ & $0.574 \pm 0.0034$ & $0.577 \pm 0.0053$ \\
\hline $1.6-/ 2$ & $0.559 \pm 0.012$ & $0.592 \pm 0.043$ \\
\hline $5.76-/ 3$ & $0.431 \pm 0.017$ & $0.471 \pm 0.018$ \\
\hline $5.73-/ 4$ & $0.481 \pm 0.021$ & $0.542 \pm 0.023$ \\
\hline $5.7+/ 5$ & $0.539 \pm 0.044$ & $0.480 \pm 0.023$ \\
\hline $2.3+/ 6$ & $0.530 \pm 0.0056$ & $0.556 \pm 0.0079$ \\
\hline $5.6+/ 7$ & $0.528 \pm 0.045$ & $0.600 \pm 0.026$ \\
\hline $1.7-/ 8$ & $0.582 \pm 0.0082$ & $0.661 \pm 0.019$ \\
\hline $2.5-/ 9$ & $0.603 \pm 0.0080$ & $0.633 \pm 0.015$ \\
\hline $4.2+/ 11$ & $0.572 \pm 0.019$ & $0.573 \pm 0.013$ \\
\hline $4.2-/ 12$ & $0.556 \pm 0.014$ & $0.606 \pm 0.017$ \\
\hline
\end{tabular}

\begin{tabular}{|c|c|c|}
\hline Set/Bracket & - (inclusive) & - (exclusive) \\
\hline \hline $1.6+/ 1$ & $0.524 \pm 0.0029$ & $0.536 \pm 0.0046$ \\
\hline $1.6-/ 2$ & - & - \\
\hline $5.76-/ 3$ & $0.424 \pm 0.021$ & $0.470 \pm 0.022$ \\
\hline $5.73-/ 4$ & $0.467 \pm 0.022$ & $0.542 \pm 0.024$ \\
\hline $5.7+/ 5$ & $0.452 \pm 0.034$ & $0.486 \pm 0.019$ \\
\hline $2.3+/ 6$ & $0.432 \pm 0.0042$ & $0.458 \pm 0.0058$ \\
\hline $5.6+/ 7$ & $0.452 \pm 0.046$ & $0.520 \pm 0.027$ \\
\hline $1.7-/ 8$ & $0.489 \pm 0.0086$ & $0.526 \pm 0.020$ \\
\hline $2.5-/ 9$ & $0.557 \pm 0.0087$ & $0.612 \pm 0.016$ \\
\hline $4.2+/ 11$ & $0.531 \pm 0.013$ & $0.531 \pm 0.013$ \\
\hline $4.2-/ 12$ & $0.528 \pm 0.012$ & $0.555 \pm 0.015$ \\
\hline
\end{tabular}

Final $P_{b} P_{t}$ values as a function of $Q^{2}$ bin for each data bracket are shown in Figure 6.9. A tabulation of the weighted averages, for each combination of HWP and target polarization, are listed in Tables 6.7 - 6.9.

\footnotetext{
${ }^{12}$ Specifically, any $Q^{2}$ bins with $\sigma /\left(P_{b} P_{t}\right)<1 /\left(3 A_{\| \mid}\right)$were cut, where $A_{\| \mid}$was modeled. This approximately corresponds to 9 events or less. (Gaussian error combination is spurious for distributions containing less than $\sim 10$ events.) The differing error bar cuts explains the difference in error bar sizes between the diagnostic and final exclusive $P_{b} P_{t}$ values in Table 6.9.
} 

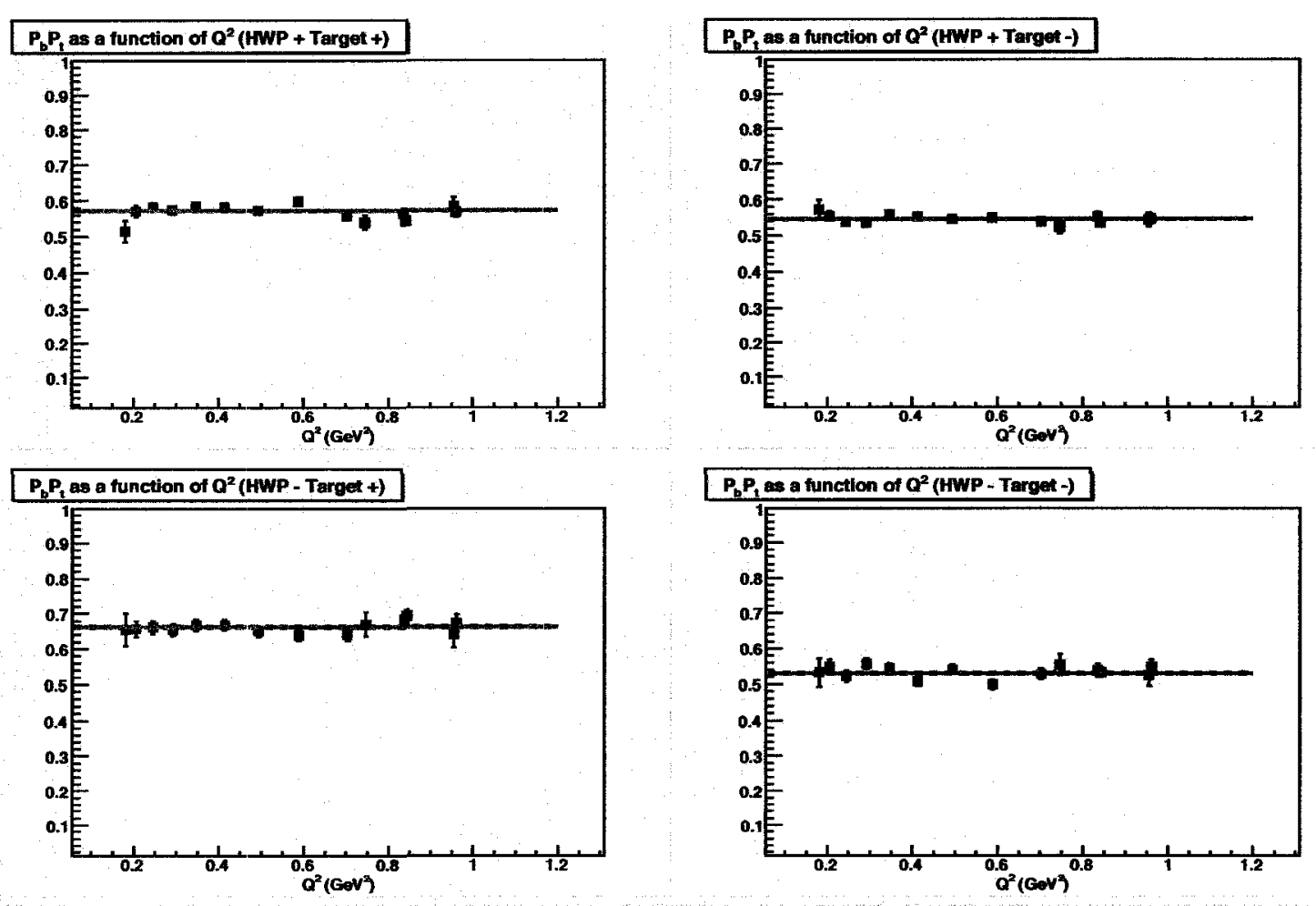

Figure 6.7: $P_{b} P_{t}$ values as a function of the asymmetry-weighted average $Q^{2}$, shown for the first data bracket (1.6+). Both inclusive (blue) and exclusive (black) data points are shown. Data are divided into 4 sets according the HWP status and target polarization direction. The red bar is the average for the given HWP and target polarization.

Table 6.9: $P_{b} P_{t}$ values for each whole data set, calculated using the properly weighted asymmetries (see Section 7.1). The final value is the exclusive value, recalculated with the asymmetries weighted for target polarization (see Section 6.2.5).

\begin{tabular}{|c|c|c|c|}
\hline Set/Bracket & inclusive & exclusive & final \\
\hline \hline $1.6+/ 1$ & $0.547 \pm 0.0022$ & $0.554 \pm 0.0035$ & $\mathbf{0 . 5 5 6 \pm \mathbf { 0 . 0 0 2 5 }}$ \\
\hline $1.6-/ 2$ & $0.561 \pm 0.012$ & $0.567 \pm 0.041$ & $\mathbf{0 . 5 6 7} \pm \mathbf{0 . 0 2 9}$ \\
\hline $5.76-/ 3$ & $0.428 \pm 0.013$ & $0.471 \pm 0.014$ & $\mathbf{0 . 4 7 1} \pm \mathbf{0 . 0 0 9 8}$ \\
\hline $5.73-/ 4$ & $0.475 \pm 0.015$ & $0.541 \pm 0.017$ & $\mathbf{0 . 5 4 3} \pm \mathbf{0 . 0 1 2}$ \\
\hline $5.7+/ 5$ & $0.487 \pm 0.027$ & $0.483 \pm 0.015$ & $\mathbf{0 . 4 8 4} \pm \mathbf{0 . 0 1 0}$ \\
\hline $2.3+/ 6$ & $0.468 \pm 0.0034$ & $0.493 \pm 0.0047$ & $\mathbf{0 . 5 0 4} \pm \mathbf{0 . 0 0 3 4}$ \\
\hline $5.6+/ 7$ & $0.493 \pm 0.032$ & $0.560 \pm 0.019$ & $\mathbf{0 . 5 6 9} \pm \mathbf{0 . 0 1 4}$ \\
\hline $1.7-/ 8$ & $0.541 \pm 0.0051$ & $0.593 \pm 0.013$ & $\mathbf{0 . 6 0 4} \pm \mathbf{0 . 0 0 9 7}$ \\
\hline $2.5-/ 9$ & $0.584 \pm 0.0059$ & $0.620 \pm 0.011$ & $\mathbf{0 . 6 2 1} \pm \mathbf{0 . 0 0 7 6}$ \\
\hline $4.2+/ 11$ & $0.543 \pm 0.013$ & $0.552 \pm 0.0093$ & $\mathbf{0 . 5 5 3} \pm \mathbf{0 . 0 0 6 5}$ \\
\hline $4.2-/ 12$ & $0.542 \pm 0.0090$ & $0.577 \pm 0.011$ & $\mathbf{0 . 5 7 9} \pm \mathbf{0 . 0 0 8 0}$ \\
\hline
\end{tabular}



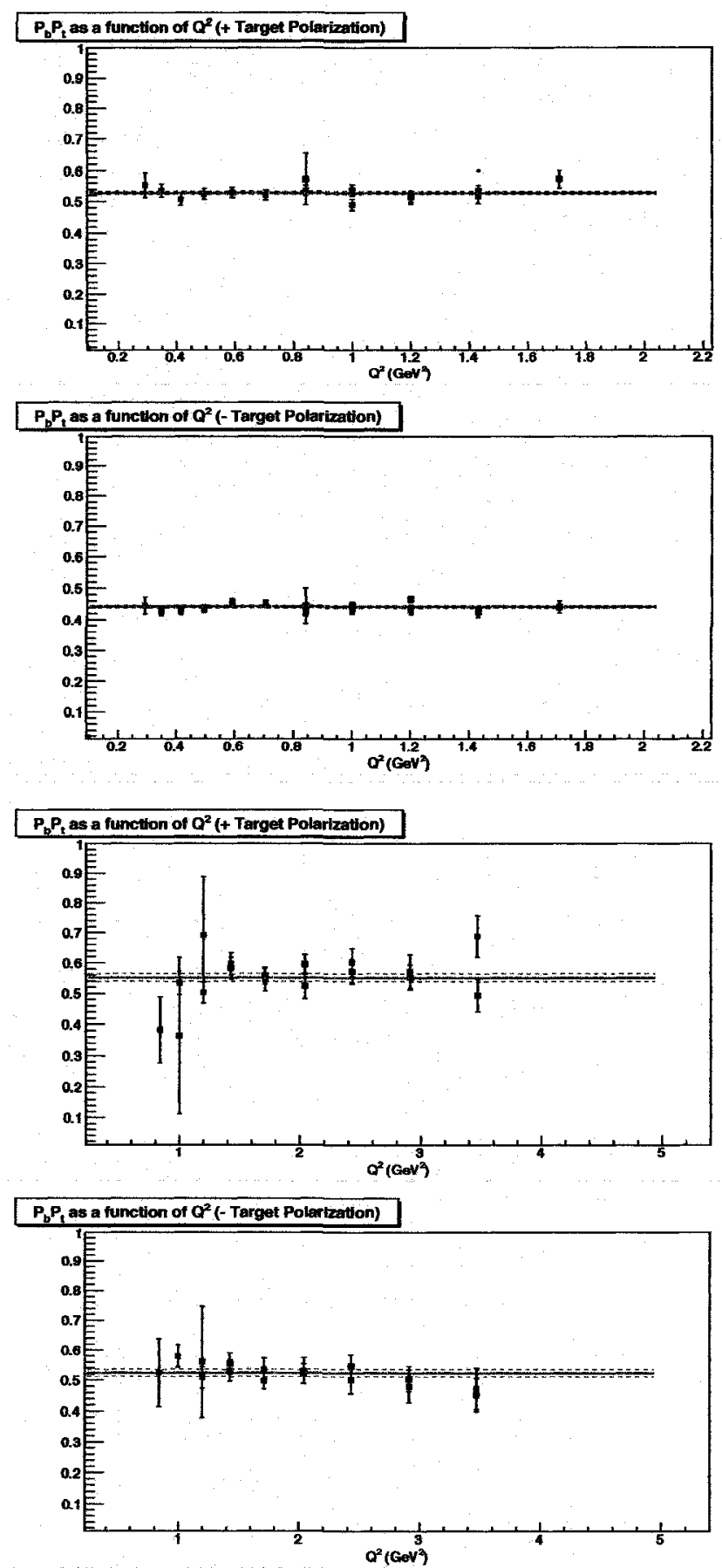

Figure 6.8: $P_{b} P_{t}$ values for two data sets (2.3+ (top two plots) and 4.2+ (bottom two plots)), split up by target polarization direction. Opposing HWP data are combined, here. Note that statistics for the elastic peak worsen as energy increases, but that the exclusive data (black) begins to span a wider range in $Q^{2}$ with increasing energy. 

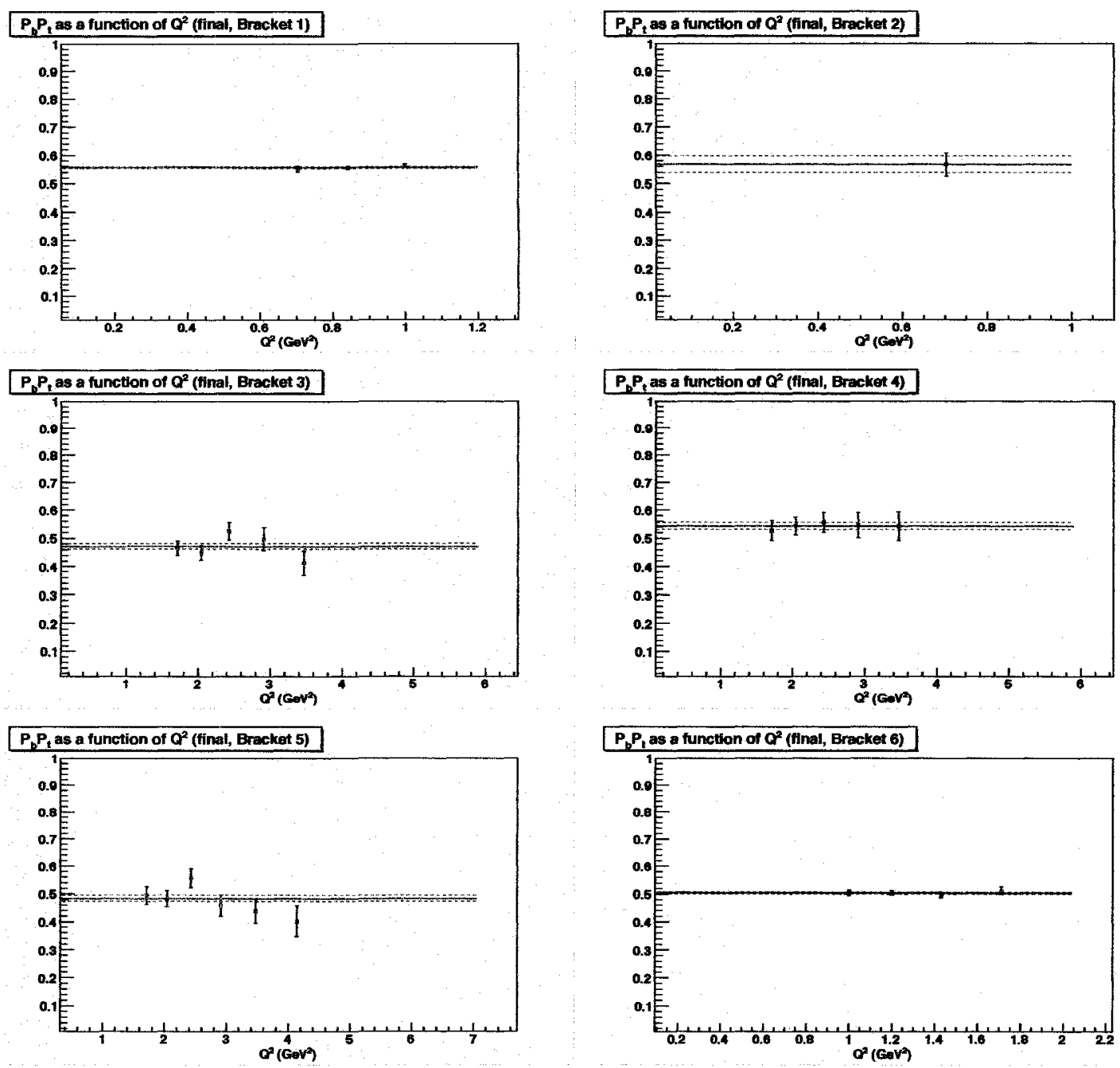

Figure 6.9: Tiles showing the final $P_{b} P_{t}$ values for the first $6 \mathrm{NH}_{3}$ data brackets as a function of $Q^{2}$ (numbered left to right, starting in the top row). The red line shows the final weighted average of the data. 

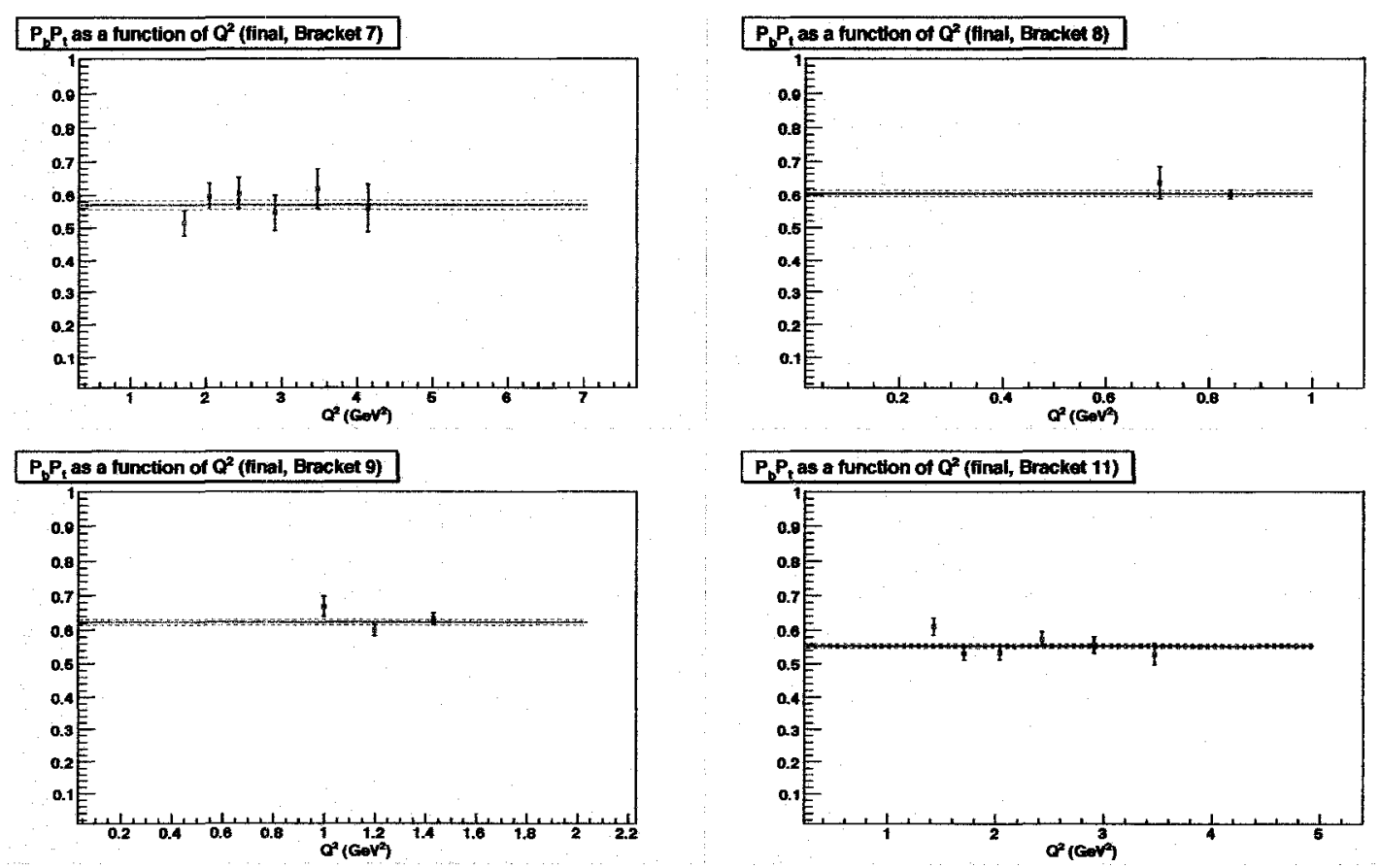

\section{$P_{b} P_{t}$ as a function of $Q^{2}$ (final, Bracket 11)}
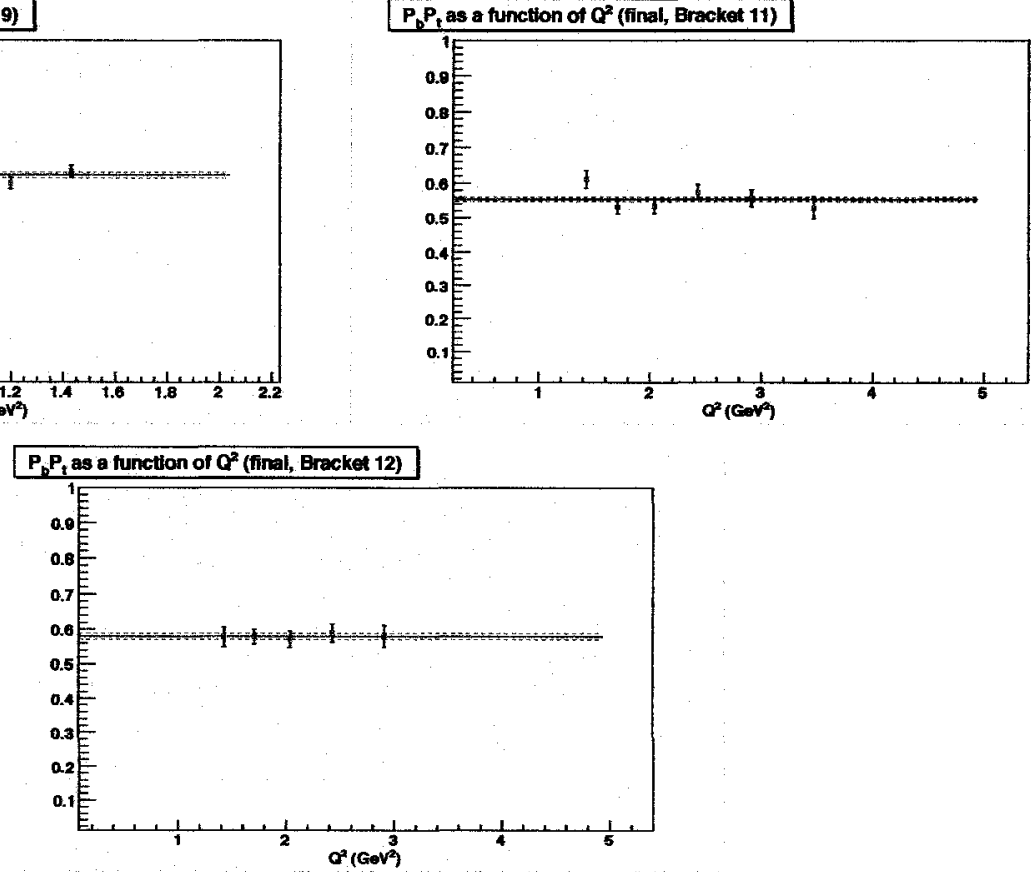

Figure 6.10: Tiles showing the final $P_{b} P_{t}$ values for the last $6 \mathrm{NH}_{3}$ data brackets as a function of $Q^{2}$ (continued from the previous figure. Bracket 10 is skipped because it contains no $\mathrm{NH}_{3}$ data). 


\subsubsection{Comparison to target NMR}

Having evaluated the values of $P_{b} P_{t}$ for each set and target polarization, it is useful to make at least a qualitative check on the accuracy of this measurement by comparing it to another, more external measurement. The continuous wave NMR (see Section 2.4) provides an approximate reading of the target polarization. This measurement cannot, however, be relied upon for an accurate determination of $P_{t}$ because the error is unknown, as is the consistency of the measurement throughout the target volume. The beam polarization measurement $P_{b}$ is consistently around $70 \%$, and can be accurately determined by Møller runs (see Section 2.3.1). It is the target polarization that is the limiting factor on the accuracy of $P_{b} P_{t}$ measurement.

Both the Møller beam polarization and NMR target polarization readout were recorded for each run in the DST files. While these are not employable for a primary measurement of $P_{b} P_{t}$, it is informative to compare the elastic peak ratio method with the product of these two values in the database. Unfortunately, there were large sections of runs where the NMR was definitely not working. Many early (1.6+) runs, for example, did not properly record the value at all, while the logbook noted that the NMR transducer broke down later, during part of the 2.3+ data set. Runs without good NMR and Møller information were excluded from this study.

Figure 6.11 shows the elastic peak ratio method of $P_{b} P_{t}$ determination for each run set (explained in the previous sections), separated according to target polarization direction, compared to the DST Møller $\times$ NMR product for the entire EG1 data set. There is a definite correlation with the database values and ratio-determined $P_{b} P_{t}$ products. The accuracy of the comparison appears to break down when the target polarization becomes too low, as the case with the third (5.76-) data bracket, not all together an unexpected result (see Section 2.4). However, this quick study (and a similar study completed for $\mathrm{ND}_{3}$ data, not shown) lends more confidence to the $P_{b} P_{t}$ measurements, and is useful as a general overall quality check.

\subsection{5 $P_{b} P_{t}$ for weighting purposes}

Eventually, the point is reached where the asymmetries from various data sets must be combined (Section 7.1). When the target polarization reverses directions (as it does over the course of several 


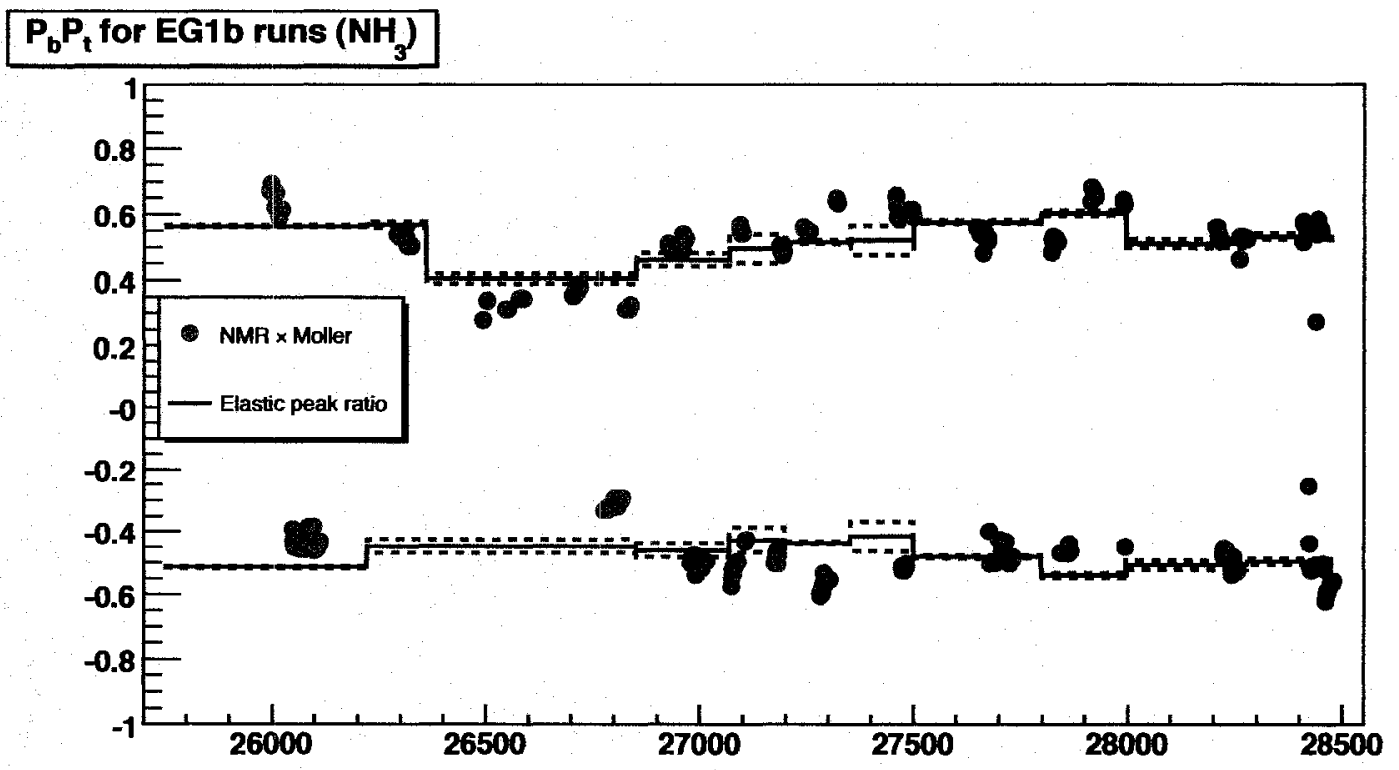

Figure 6.11: $P_{b} P_{t}$ values, calculated as described in the text (shown by the lines with errors for each bracket) compared to the product of the NMR and Moller values in the database. Values are separated by positive (top) and negative (bottom) target polarization. Though a precise evaluation using the target NMR was not completed, a general correlation between the two measurements can be seen. 
runs), the polarization magnitude changes 'suddenly' to a new value, with minimal correlation to the previous value. Raw asymmetries vary directly in magnitude with target polarization. For this reason, asymmetries of opposite target polarizations were evaluated separately, and when combined, required weighting by the target polarization value. An accurate target polarization is not needed for this purpose; what is important is that the relative measurements (i.e. $P_{t}^{+} / P_{t}^{-}$) have as much precision as possible.

For this purpose, yet another $P_{b} P_{t}$ measurement was made. This measurement uses the same principle as the inclusive elastic peak ratio method, but instead of using the elastic data, a model for the whole range of data in $W$, including the DIS region, was used to compare $A_{\text {measured }}$ and $A_{\|}$. Models values (see Section 2.7) in the inelastic region ( $W>1.07 \mathrm{GeV}$ ) for $A_{1}$ and $A_{2}$, along with the tracked average for $D$ and $\eta$ in each bin, were used in Eq. 1.223 to calculate $A_{\|}$, and the ratio was taken to generate a $P_{b} P_{t}$ value in each bin:

$$
\begin{gathered}
P_{b} P_{t \text { inel }}\left(W, Q^{2}\right)=\frac{A_{\text {raw }} / F_{D F}}{A_{\|}} \\
\sigma_{\text {inel }}\left(W, Q^{2}\right)=\frac{\sigma_{A_{\text {raw }}} / F_{D F}}{A_{\|}}
\end{gathered}
$$

The error-weighted average of all these was then taken to find the total $P_{b} P_{t}$ :

$$
\left\langle P_{b} P_{t \text { inel }}\right\rangle=\frac{\sum_{Q^{2}} \sum_{W} P_{b} P_{t \text { inel }} / \sigma^{2}}{\sum_{Q^{2}} \sum_{W} 1 / \sigma^{2}}
$$

with

$$
\sigma_{\text {inel }}=\frac{1}{\sqrt{\sum_{Q^{2}} \sum_{W} 1 / \sigma^{2}}}
$$

The values of $P_{b} P_{t}$ inel were then statistically combined with the true (elastic) value of $P_{b} P_{t}$ for each target polarization, to yield a $P_{b} P_{t}$ value for weighting purposes only:

$$
\begin{gathered}
P_{b} P_{t w g t}=\frac{P_{b} P_{t \text { inel }} / \sigma_{\text {inel }}^{2}+P_{b} P_{t} / \sigma^{2}}{1 / \sigma_{\text {inel }}^{2}+1 / \sigma^{2}} \\
\sigma_{w g t}=1 / \sqrt{1 / \sigma_{\text {inel }}^{2}+1 / \sigma^{2}}
\end{gathered}
$$


Plots of $P_{b} P_{t}$ inel as a function of $Q^{2}$ are shown in Figure 6.12. Note that $P_{b} P_{t}$ generated in this manner is not nearly as constant in $Q^{2}$ as that generated only from the elastic peak, mainly because of our ignorance regarding the accuracy of the model, specifically at low $Q^{2}$. The statistical error (for larger energies) is small, due to the larger amount of data incorporated into the calculation, but the systematic errors imposed are very large. Simply put, it is a precise model, but not an accurate model of $P_{b} P_{t}$. However, it is good for comparing the relative weights accorded to data of the same beam energy/torus current but opposing target polarizations. The application of these $P_{b} P_{t}$ values to weighting of the data is described in Section 7.1. Values of $P_{b} P_{t}$ inel and the final $P_{b} P_{t}$ wgt for each data set are listed in Table 6.10.

Table 6.10: Values of $P_{b} P_{t}$ wgt used for the weighting of asymmetries from opposing target polarizations. The contribution from the inelastic region only $\left(P_{b} P_{t \text { inel }}\right)$ is also shown.

\begin{tabular}{|c|c|c|c|c|c|}
\hline Set/Bracket & Target Pol. & $P_{b} P_{t \text { inel }}$ & $\sigma_{\text {inel }}$ & $P_{b} P_{t \text { wgt }}$ & $\sigma_{\text {wgt }}$ \\
\hline \hline $1.6+/ 1$ & + & 0.562 & 0.0078 & 0.574 & 0.0029 \\
\hline $1.6+/ 1$ & - & 0.516 & 0.0092 & 0.528 & 0.0025 \\
\hline $1.6-/ 2$ & + & & & not needed & \\
\hline $5.76-/ 3$ & + & 0.429 & 0.0074 & 0.435 & 0.0066 \\
\hline $5.76-/ 3$ & - & 0.470 & 0.0061 & 0.468 & 0.0058 \\
\hline $5.73-/ 4$ & + & 0.509 & 0.0081 & 0.509 & 0.0074 \\
\hline $5.73-/ 4$ & - & 0.473 & 0.0076 & 0.479 & 0.0070 \\
\hline $5.7+/ 5$ & + & 0.486 & 0.008 & 0.486 & 0.0073 \\
\hline $5.7+/ 5$ & - & 0.486 & 0.010 & 0.486 & 0.0086 \\
\hline $2.3+/ 6$ & + & 0.620 & 0.007 & 0.568 & 0.0042 \\
\hline $2.3+/ 6$ & - & 0.489 & 0.0095 & 0.451 & 0.0035 \\
\hline $5.6+/ 7$ & + & 0.562 & 0.011 & 0.567 & 0.010 \\
\hline $5.6+/ 7$ & - & 0.438 & 0.011 & 0.449 & 0.010 \\
\hline $1.7-/ 8$ & + & 0.570 & 0.028 & 0.595 & 0.0075 \\
\hline $1.7-/ 8$ & - & 0.535 & 0.027 & 0.502 & 0.0078 \\
\hline $2.5-/ 9$ & + & 0.666 & 0.015 & 0.620 & 0.0066 \\
\hline $2.5-/ 9$ & - & 0.602 & 0.013 & 0.578 & 0.0068 \\
\hline $4.2+/ 11$ & + & 0.575 & 0.0076 & 0.574 & 0.0065 \\
\hline $4.2+/ 11$ & - & 0.538 & 0.0076 & 0.536 & 0.0066 \\
\hline $4.2-/ 12$ & + & 0.652 & 0.0076 & 0.634 & 0.0064 \\
\hline $4.2-/ 12$ & - & 0.597 & 0.0086 & 0.574 & 0.0067 \\
\hline
\end{tabular}



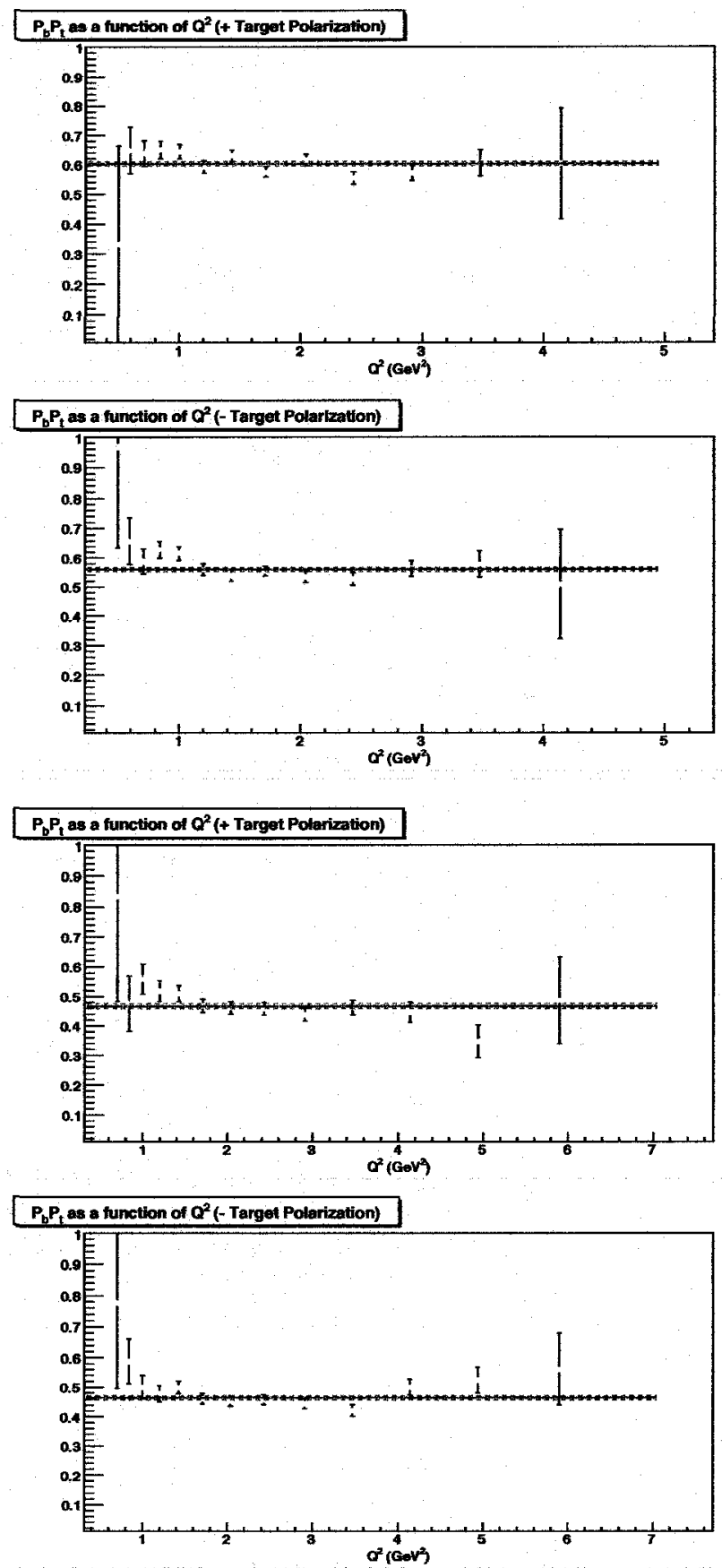

Figure 6.12: $P_{b} P_{t \text { inel }}$ values for use in relative weighting of opposing target polarizations, shown for 4.2 GeV inbending (top two plots) and $5.7 \mathrm{GeV}$ inbending (bottom two plots) data. Overall statistics are much better here than for the standard elastic peak ratio method, but systematic errors (not shown) are much larger. 


\subsection{Polarized Nitrogen Correction}

At this point, most significant backgrounds (remaining after PID cuts) have been removed from the asymmetry. Non-polarized background with $A>1$ is removed ${ }^{13}$ by dilution factors $\left(F_{D F}\right)$; unpolarized protons and electrons are removed by dividing out beam and target polarization $\left(P_{b} P_{t}\right)$; and pair-production electrons are accounted for by calculating their contamination rates $\left(C_{b a c k}\right)$. The calculations so far, however, neglect the nonzero polarizations of $A>1$ backgrounds. In particular, ${ }^{15} \mathrm{~N}$ also becomes partially polarized by DNP. While this particular isotope was chosen for its minimal polarization relative to the free protons, the effects of this factor on the double-spin asymmetry must be considered for an accurate calculation.

An in-depth treatment of the problem of the spin-polarization of nuclei is beyond the scope of this thesis. We instead take advantage of the fact that this is a small correction, and use a simple (nuclear shell) model application, along with empirical information regarding the relative polarization $\left(P_{15} / P_{p}\right)$ of the target elements.

According to EST (Equal Spin Temperature) theory [121], when the target polarization is low enough, the relative polarizations of two spin-interacting atoms in the same medium is given (to lowest order) simply by the ratio of their magnetic moments:

$$
\frac{P_{15} N}{P_{p}}=\frac{\mu_{15} N}{\mu_{p}}=\frac{-0.264 \mu_{n}}{2.793 \mu_{n}}=-0.0945
$$

As the polarization grows larger, however, dipole interactions of the electron spins become a nonnegligible factor in the polarization ratio, and the simple model shows deviations from the experimental ratio [121]. To account for this, rather than using the complex formalism of higher-order EST theory, we use an empirical relation based on a fit of NMR polarization values of the free protons and ${ }^{15} \mathrm{~N}$ for ${ }^{15} \mathrm{NH}_{3}$ in the E143 experiment at SLAC [4]:

$$
P_{15}=0.136 P_{p}-0.183 P_{p}^{2}+0.335 P_{p}^{3}
$$

A comparison of these parametrizations is shown in Figure 6.13.

\footnotetext{
${ }^{13} A$ represents atomic mass, here.
} 


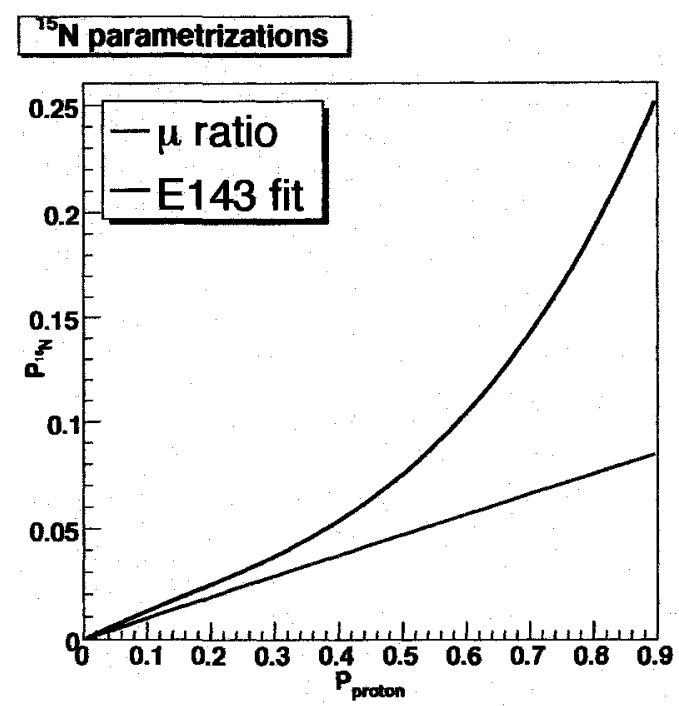

Figure 6.13: The E143 parametrization of nitrogen vs. proton polarizations in frozen ${ }^{15} \mathrm{NH}_{3}$ (Eq. 6.54) compared to the simple ratio of magnetic dipole moments (Eq. 6.53).

According to the nuclear shell model, ${ }_{2}^{14}$ the 8 neutrons in ${ }^{15} \mathrm{~N}$ pair in the $s_{1 / 2}$ and $p_{3 / 2}$ shells with opposing spins, as do 3 pairs among the 7 protons, leaving a single unpaired proton the $p_{1 / 2}$ shell to carry the spin of the nucleus [70].

To obtain the polarization contribution of this free proton, we must calculate the polarization on the "free" nuclear proton relative to the (known) ${ }^{15} \mathrm{~N}$ polarization. This can be done through a separation of the $p_{1 / 2}$ "free" nuclear proton state ${ }^{15}$ into substates of the intrinsic fermion spin $\left(m_{s}= \pm 1 / 2\right)$ and the remaining orbital angular momentum $\left(m_{l}= \pm 1\right)$ using the standard method of decomposition with Clebsch-Gordan coefficients:

$$
\left|1, \pm \frac{1}{2}\right\rangle=\sqrt{\frac{2}{3}}\left| \pm 1, \mp \frac{1}{2} ; \pm \frac{1}{2}\right\rangle+\sqrt{\frac{1}{3}}\left|0, \pm \frac{1}{2} ; \pm \frac{1}{2}\right\rangle
$$

We see here that the second term is antialigned with the (spin-1) orbital spin. Squaring the amplitude yields a $\frac{1}{3}$ probability of the "free" proton being antialigned with the net nuclear spin.

Thus, for an equal number of protons and ${ }^{15} \mathrm{~N}$ nuclei, the expected correction is approximately $-\frac{1}{3} P_{15} / P_{p}$. Of course, there are 3 free protons for every nitrogen nucleus, so another factor of $\frac{1}{3}$

\footnotetext{
${ }^{14}$ The nuclear shell model requires that $s, p, d$, eic. orbitals are filled by nucleons as per the Pauli Exclusion Principle, with protons and neutrons capable of pairing in the same orbitals (as they have opposite isospin).

${ }^{15}$ This is the $j=1, j_{z}=1 / 2$ state.
} 
is needed. Putting all this together, the effective nitrogen polarization correction is given by

$$
P_{15}^{*}=-\frac{1}{3} \cdot \frac{1}{3}\left(0.136 P_{t}-0.183 P_{t}^{2}+0.335 P_{t}^{3}\right)
$$

where $P_{t}=P_{p}$ is the target polarization. ${ }^{16}$ This factor should be added to the target polarization when calculating $A_{\| 1}$. (That is, to be specific, adding the negative value of $P_{15}^{*}$ lowers the effective target polarization). That is,

$$
P_{b} P_{t} \rightarrow P_{b}\left(P_{t}+P_{15}^{*}\right)
$$

in the final asymmetry measurement. All measured asymmetries that include nitrogen background should have this correction - this includes inclusive elastic asymmetries used to measure $P_{b} P_{t}$. In this case, since the asymmetry is used to scale $P_{b} P_{t}$, it is assumed $P_{b}=P_{t}=1$ so that the raw asymmetry is simply divided by $\left(1+P_{{ }_{15} N}^{*}\right)$ (see Section 6.2.3). Exclusive ep events do not require this correction, as nearly all nitrogen background is removed by the kinematic cuts.

This is only an approximate correction. However, a typical $P_{t}$ value of 0.75 yields a correction of $P_{15}^{*} \approx-0.016$, making it a very small correction, for which the minor details of our approximations have little bearing on the final asymmetry. To implement this correction, we use Eq. 6.56 and assume $P_{t}=P_{b} P_{t} / P_{b}$, where $P_{b} P_{t}$ values are derived using the elastic peak method in Section 6.2 and $P_{t}$ is the average Møller polarization given for each run, weighted by the gated FC charge for each run:

$$
P_{b} \approx \frac{\sum_{\text {run }} F C \times\left|P_{b(\text { Meller })}\right|}{\sum_{\text {run }} F C}
$$

Resultant $P_{b}$ values for each bracket, along with the final target polarization corrections $P_{15}^{*}$, are listed in Table 6.11.

\footnotetext{
${ }^{16}$ To be precise, this equation should be multiplied by an $x$-dependent factor to account for the nuclear EMC effect [97]. However, the effect for ${ }^{1.5} \mathrm{~N}$ would result in a coefficiem within several percent of 1.00 tor the kinematic regions of concern in this analysis. Because the nitrogen polarization has such a small total effect, we neglect the EMC factor in this analysis.
} 
Table 6.11: $P_{b}$ estimates for each data set, using the Moller polarization weighted by the $\mathrm{NH}_{3}$ Faraday cup counts. Also shown are the resulting target polarizations $\left(P_{t}\right)$ using the peak ratio method (Section 6.2). The resulting polarized nitrogen correction $P_{{ }_{15}}^{*}$ is also shown.

\begin{tabular}{|c|c|c|c|}
\hline Set/Bracket & $P_{b}$ & $P_{t}$ & $P_{15}^{*}$ \\
\hline \hline $1.6+/ 1$ & 0.72 & 0.78 & -0.017 \\
\hline $1.6-/ 2$ & 0.69 & 0.82 & -0.019 \\
\hline $5.76-/ 3$ & 0.64 & 0.73 & -0.015 \\
\hline $5.73-/ 4$ & 0.71 & 0.76 & -0.016 \\
\hline $5.7+/ 5$ & 0.69 & 0.70 & -0.013 \\
\hline $2.3+/ 6$ & 0.72 & 0.70 & -0.013 \\
\hline $5.6+/ 7$ & 0.72 & 0.79 & -0.017 \\
\hline $1.7-/ 8$ & 0.70 & 0.86 & -0.022 \\
\hline $2.5-/ 9$ & 0.75 & 0.82 & -0.019 \\
\hline $4.2+/ 11$ & 0.73 & 0.76 & -0.016 \\
\hline $4.2-/ 12$ & 0.78 & 0.74 & -0.015 \\
\hline
\end{tabular}

\subsection{Radiative corrections}

The basic goal of this analysis is the extraction of $A_{1}$ and $A_{2}$, which can be expressed in terms of the spin- $\frac{1}{2}$ and spin- $\frac{3}{2}$ final state virtual photon cross-sections $\sigma_{1 / 2}$ and $\sigma_{3 / 2}$, and the longitudinal single-photon interference term $\sigma_{L T}$ (see Section 1.4). These quantities result from the crosssection calculations of the simplest possible Feynman diagram, involving a single-photon exchange between the proton and electron. 17 The sum of all the constituent cross-sections should then be given by

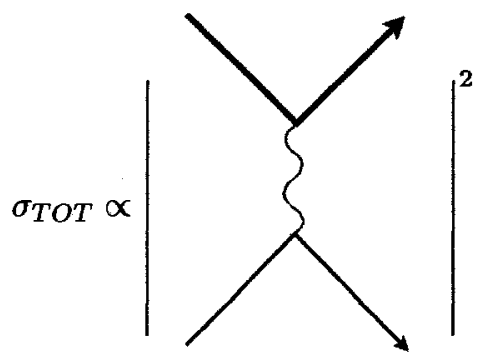

\footnotetext{
${ }^{17}$ In reality, the outgoing bold arrow represents the variety of scattering products $X$, not just a single proton, except in the special case of elastic scattering.
} 
At high values of $Q^{2}$, the studied reaction $e p \rightarrow e X$ approximates this expression. However, as $Q^{2}$ lowers, the validity of this approximation breaks down, and higher-order Feynman diagrams contribute to the measured experimental cross-section:
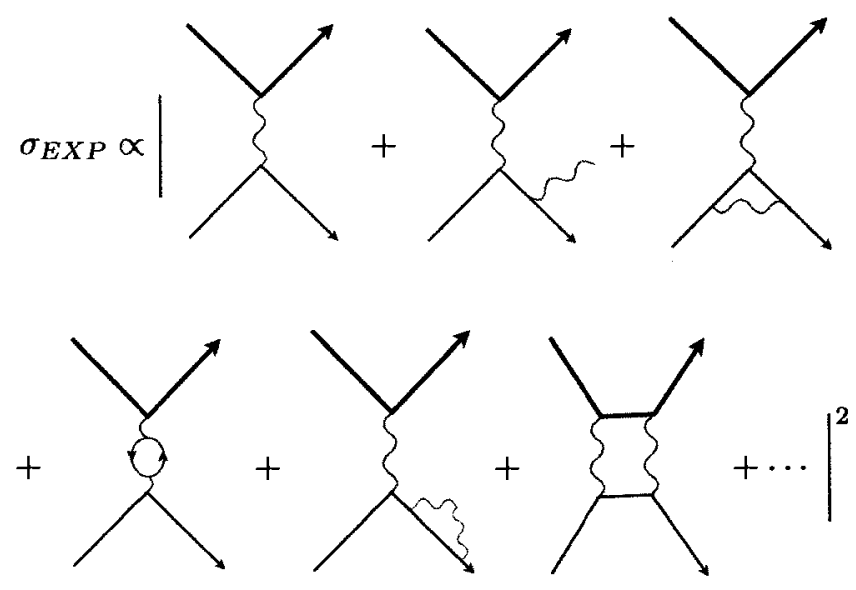

These second-order corrections occur at the interaction vertex and are known as internal radiative effects. Basically, given the measured asymmetries in terms of the experimental cross-sections $\sigma_{E X P},{ }^{18}$ the contributions from the second-order terms must be removed as a function of $W$ and $Q^{2}$ to extract $\sigma_{T O T}$. For example, the last shown term, which involves a 2-photon exchange, can result in the production of spin- $\frac{5}{2}$ resonances, which must be removed from the inclusive spectrum if $A_{1}$ (which is dependent only on spin- $\frac{1}{2}$ and spin- $\frac{3}{2}$ states) is to be derived from the data. ${ }^{19}$

In addition to the internal radiative effects from the addition of higher-order Feynman diagrams, electrons accelerating through the target and main torus fields, as well as those interacting with target and detector materials, can undergo Bremsstrahlung production of photons, which can slightly alter the measured kinematics of the detected electron. Range straggling, the statistical effect of a spread in the possible range of the target [8], is also an effect not yet accounted for, which must be incorporated into the radiative corrections. ${ }^{20}$ These effects, which occur after the measured

\footnotetext{
${ }^{18}$ See the definitions of $A_{1}$ and $A_{2}$, in Section 1.4 to see the asymmetries written explicitly in terms of cross-sections.

${ }^{19}$ The 2-photon exchange term, in reality, makes only a very small contribution, because of the overwhelming likelihood, in such a case, of one of the two photons transferring most of the energy (see Ref. [122], page 4, tor example). This example is used here just to make a discernible point about the need for radiative corrections.

${ }^{20}$ The multiple scattering of electrons in matter is also a concern, but this issue (with the exception of the range straggling) is handled separately in the kinematics correction package (see Sections 4.2.4 and 4.2.5). Range-straggling effects are most efficiently handled at the same time as radiative corrections, because the probability of external Brehmsstrahlung is directly accorded to the penetration range in matter.
} 
reaction, and are dependent on the experimental configuration, are known as external radiative effects, and must be accounted for as well.

This is clearly a complicated scenario. Fortunately, we can take advantage of the fact that both internal and external radiative effects on $A_{\|}$are small compared to the measured asymmetry. In the case of internal corrections, this can be assumed due the small size of the QED coupling constant $\alpha$, because higher-order diagrams are proportional to higher powers of this small constant. In the case of external corrections, a thin target and a large-scale homogeneous magnetic field ensure that these radiative effects are small. ${ }^{21}$ Thus, we can use older (less accurate) models of $A_{\| \mid}$to calculate the higher order and external radiative terms, and subtract their contribution from the overall asymmetry. In kinematic regions where the proportion of the radiative corrections to the actual asymmetry is too high (that is, near the elastic peak and at high $W$ ), we can assume the EG1 experiment provides little to no new information about the asymmetry; these points are then excluded from the analysis. ${ }^{22}$

\subsubsection{Basic methodology of radiative corrections}

The complete, detailed treatment of radiative corrections in ep scattering is a problem clearly beyond the scope of this thesis. In this and the following subsections, a description of the iterative nature of the radiative corrections is supplied, followed by a summary of internal and external radiative corrections and references to the appropriate source materials.

To calculate the radiative corrections, the RCSLACPOL code developed by L. Stuart et al. [4] is applied iteratively to calculate the radiative contribution to the polarized and unpolarized Born crosssections as a function $W, Q^{2}$, and beam energy $E$. The total experimentally measured asymmetry can be written

$$
A_{\|\|_{e x}}=\frac{n^{-}-n^{+}}{n^{-}+n^{+}}=\frac{\sigma^{P}}{\sigma^{a}}
$$

where $\sigma^{P}$ and $\sigma^{a}$ are the (radiated) polarization-dependent and polarization-independent crosssections, respectively. The goal is to calculate the corrective terms necessary to find the $\alpha^{2}$ (lowest)

\footnotetext{
${ }^{21}$ Still, the effects of external Bremstrahlung at momenta of several hundred MeV or higher are several times stronger than the $d E / d x$ correction for nuclear collisions [5], making this an important correction.

${ }^{22}$ This is done by according a reasonable systematic error proportional to the correction. This error then "blows up" tor large corrections, defining a de facto boundary on the data (see Section 7.2.5).
} 
order Born asymmetry, that is, the solutions $A_{R C}$ and $f_{R C}$ of

$$
A_{\|_{B o r n}}=\frac{1}{f_{R C}} \frac{\sigma^{P}}{\sigma^{\alpha}}+A_{R C}
$$

The variable $f_{R C}\left(E, W, Q^{2}\right)$ includes all the unpolarized corrections and can be interpreted as a "radiative dilution factor", while $A_{R C}\left(E, W, Q^{2}\right)$ accounts for (polarization-dependent) terms that cannot be easily accounted for in $f_{R C}$. Corrections could, technically, be absorbed in a single coefficient $A_{R C}$. However, using a second correction $f_{R C}$ allows for simple rescaling of the asymmetry error bars

$$
\sigma_{A_{B o r n}}=\frac{\sigma_{A_{\text {exp }}}}{f_{R C}}
$$

to the bulk of the correction.

The RCSLACPOL code works by an iterative procedure. The elastic Born cross-section is described by a Dirac $\delta$-function at $W=M$. The entire contribution of the long radiative elastic tail, which contaminates the entire resonance region to some extent [91][122], must first be removed from the cross-sectional spectra $\sigma^{P}$ and $\sigma^{a}$. Radiative corrections to the inelastic resonances are more complex, since, unlike the elastic peak, the resonances are characterized by a distinct structure across $W$, with peaks at 1232, 1525, and $1700 \mathrm{MeV}$ dominated by many different resonances. Fortunately, the radiative tails of the resonances are much smaller than the elastic tail, so that only their effects on nearby resonances in $W$ need be considered [122]. The peaking approximation, that is, the assumption that the radiative tail is proportional to the non-radiative cross-section, is used to iteratively derive the contributions of these peaks.

After the elastic tail, the next-largest resonance ${ }^{23}$ is isolated from its radiative tail via calculations of its internal and external radiative corrections (summarized in the following subsections). Then, a neighboring resonance ${ }^{24}$ is given the same treatment, and so on, until all known resonance contributions are analyzed. Then, the results are iterated over again. The contributions of the largest $(\Delta)$ resonance are adjusted, again using the peaking approximation to calculate internal and external radiative contributions, to account for the subtraction of the radiative tails of its nearby (i.e. $P_{11}$, et

${ }^{24} P_{11}(1440)$ is the closest resonance in the inclusive spectrum. 
al.) resonances. This iterative procedure is repeated for all excited states up to the threshold of the DIS region, until stable values for the magnitudes of the radiative contributions to $\sigma^{P}$ and $\sigma^{a}$ are reached.

After the contributions of radiative tails for the elastic and resonance regions are "untangled", the radiative contributions from the continuum (DIS) region can be calculated. Using the peaking approximation, it can be shown (Ref. [122], Appendix $\mathrm{C}$ ) that the radiative contribution in the region of a continuum of states can be calculated by an integration over the region bounded in $W,{ }^{25}$ if only the Born cross-sections at the boundaries are known. Therefore, the calculation of radiative cross-sections in the DIS (high- $W$ ) region is much less complicated than in the resonance region.

Once all the calculations are completed, the various contributions from the elastic and inelastic radiative tails are absorbed into the corrective terms $f_{R C}$ and $A_{R C}$. The bulk of the contribution comes from the elastic radiative tail, so we must ensure the error on $A_{\|}$is properly scaled by this contribution, as well. Therefore, we are motivated to include this contribution in $f_{R C}$. However, the polarization-dependent contribution to this term cannot be included in an overall "radiative dilution factor", so this (small) part of the correction is absorbed into $A_{R C}$. Thus, we have

$$
f_{R C} \equiv \frac{\sigma_{\text {total }}-\left(\sigma_{e l}^{a}-\sigma_{0 e l}^{a}\right)}{\sigma_{\text {total }}}
$$

where $\sigma_{e l}^{a}-\sigma_{0 e l}^{a}$ is the polarization-independent contribution of the elastic radiative tail and $\sigma_{t o t a l}$ is the radiated (i.e. experimentally measured) cross-section. (Compare to Eq. 5.75 to see the analogy to the dilution factor.) The remaining term $A_{R C}$ is then defined as the correction term for the polarized elastic tail contribution, as well as all inelastic contributions to the total asymmetry.

\subsubsection{Internal radiative corrections}

Internal radiative corrections, that is, corrections based on the addition of higher-order Feynman diagrams (as shown in Eq. 6.60), are based on the formalism of Kuchto and Schumeiko as outlined in Ref. [123]. Of the various higher-order terms that must be calculated, the most important are

\footnotetext{
${ }^{25}$ Ref. [122] uses the kinematic values $E$ and $E^{\prime}$ instead of $E$ and $W$, but at a given $Q^{2}$, the kinematics of inclusive scattering can be expressed completely using any two kinematic variables, so the descriptions are equivalent.
} 
internal Brehmsstrahlung ${ }^{26}$

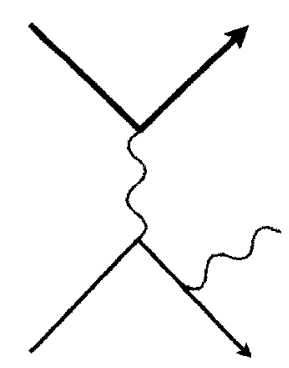

and the vertex correction term

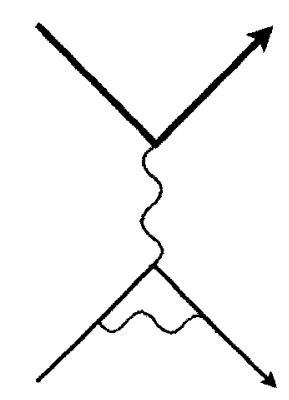

applied to the electron vertex. ${ }^{27}$ The Bremsstrahlung contribution $\sigma_{R}$ is calculated as the sum of polarization-dependent and independent cross-sections:

$$
\sigma_{R}\left(E, W, Q^{2}\right)=\sigma_{R}^{a}\left(E, W, Q^{2}\right)+\sigma_{R}^{P}\left(E, W, Q^{2}\right)
$$

These cross-sections are solved explicitly in terms of the kinematics in Ref. [123]. For the inclusive scattering case, the whole phase space of the radiated photon is integrated over. This requires the separation of the respective cross-sections into the sum of a finite and a (renormalizable) infrareddivergent term. The contribution of these terms, relative to the Born cross-section, can be used together with the aforementioned peaking approximation to specify the magnitude of this specific radiative effect.

The vertex correction contribution is calculated in terms of a vertex correction factor $\delta_{V}$ to the

\footnotetext{
${ }^{26}$ Here the radiated photon can be emitted from either the incoming or outgoing electron line, though the latter is shown. ${ }^{27}$ The much larger mass $M$ of the proton makes the same effect, reflected to the hadronic line, negligibly small compared to the like diagrams on the lepton line.
} 
Born cross-section $\sigma_{0}$ and the anomalous magnetic moment contribution $\sigma^{A M M}$ :

$$
\sigma_{V}\left(E, W, Q^{2}\right)=\sigma_{0}\left(E, W, Q^{2}\right) \frac{\alpha}{\pi} \delta_{V}+\sigma^{A M M}\left(E, W, Q^{2}\right)
$$

Again, these are calculated explicitly and are detailed in Ref. [123]. Polarization-dependent terms are all contained in $\sigma_{0}$. The correction term $\delta_{V}$ includes a contribution from leptonic vacuum polarization in the photon line.

The radiative tail from the elastic peak due to internal contributions $\sigma_{R}^{N}$ is also explicitly calculated as

$$
\sigma_{R}^{N}\left(E, W, Q^{2}\right)=\frac{2 \alpha^{2}}{\sqrt{s^{2}-4 m^{2} M^{2}}} \frac{\alpha}{\pi} \int_{T_{m i n}^{N}}^{T_{m a x}^{N}} \frac{d T}{T^{2}} R(T)
$$

where $s$ is the Mandelstam variable (Eq. 1.10) and $T \equiv q^{2}=-Q^{2} ; R(T)$ and the integration limits are functions of the leptonic response functions, explicitly calculated in Ref. [123].

Other contributions due to internal corrections are also considered. Vacuum polarization of the virtual photon, for example

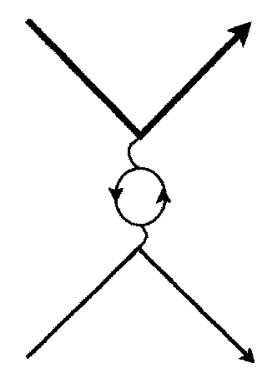

as well as emission of Bremsstrahlung photons by the hadron line, emission of multiple Bremsstrahlung photons, and multiple-photon exchange

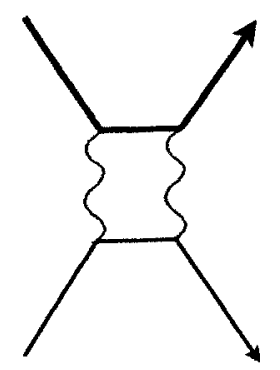


have only very small effects on the asymmetry $A_{\|}$, except at $x \rightarrow 0$ and $y \rightarrow 1$ [123]. Values of systematic errors attributable to radiative corrections become prohibitively large for evaluation near these limits.

The internally corrected cross-section can now be expressed in terms of the Born cross-section and correction terms as

$$
\sigma_{i n t}=\sigma_{0} \cdot\left(1+\delta_{V R}\right)+\sigma_{R}^{F}+\sigma_{R}^{N}
$$

where $\sigma_{R}^{F}$ is the finite contribution of the Brehmsstrahlung cross-section and $\delta_{V R}$ is defined to absorb the vertex correction and aforementioned infrared divergence terms (denoted by $\sigma_{R}^{I R}$ ):28

$$
\sigma_{0} \delta_{V R} \approx \sigma_{R}^{I R}+\sigma_{V}
$$

These corrections are done separately for the polarization-dependent and independent crosssections. The internally-corrected asymmetry can then be written

$$
A_{\|}^{i n t}=\frac{\sigma_{i n t}^{P}}{\sigma_{i n t}^{a}}=\frac{\sigma_{0}^{P} \cdot\left(1+\delta_{V R}\right)+\sigma_{R}^{F P}+\sigma_{R}^{N P}}{\sigma_{0}^{a} \cdot\left(1+\delta_{V R}\right)+\sigma_{R}^{F a}+\sigma_{R}^{N a}}
$$

with the $P$ and $a$ superscripts noting the polarization-dependent and independent contributions, as before.

\subsubsection{External radiative corrections}

External radiative corrections depend specifically on the experimental configuration, and manifest themselves in the form of external Bremsstrahlung radiation and range straggling due to ionization. Both effects are results of the interaction of the scattered electrons with matter in the target material and shielding. The methods pioneered by Mo, Tsai et al. [91][122] are used. The corrected

\footnotetext{
${ }^{28}$ This is little more than a manner of convenient notation involved in adoption of the peaking approximation, and contains no polarization-dependent terms. See Ret. [123] for more details.
} 
polarization-independent cross-section can be expressed, if the probability of an electron having final energy $E_{f}$ after passing through $t$ radiation lengths of material (written $I_{b}\left(E, E_{f}, t\right)$ ) is known, by integration over the electron momenta:

$$
\sigma_{\text {total }}^{a}\left(E, E^{\prime}, \theta\right)=\int_{E_{\min }^{\prime}}^{E} \int_{E_{F}^{\prime}}^{E_{m a x}^{\prime}} I_{b}\left(E, E_{f}, t\right) \sigma_{i n t}^{a}\left(E, E^{\prime}, \theta\right) I_{b}\left(E^{\prime}, E_{f}^{\prime}, t^{\prime}\right) d E_{f} d E^{\prime}
$$

Here, $E_{m i n}^{\prime}$ and $E_{m a x}^{\prime}$ are limits on $E^{\prime}$ for elastic scattering, $\sigma_{i n t}^{a}$ is the cross-section after internal radiative corrections, and $t\left(t^{\prime}\right)$ is the fraction of the radiation length $X_{0}$ passed through by the incoming (outgoing) electron.

The calculation for the polarization-dependent cross-section is nearly identical, except that a contribution due to the depolarization of the electron from Bremsstrahlung $D\left(E, E^{\prime}, Z\right)$ [124] must be factored into the equation:

$$
\sigma_{\text {total }}^{P}\left(E, E^{\prime}, \theta\right)=\int_{E_{\min }^{\prime}}^{E} \int_{E_{F}^{\prime}}^{E_{\max }^{\prime}} I_{b}\left(E, E_{f}, t\right) \sigma_{\text {int }}^{P}\left(E, E^{\prime}, \theta\right) I_{b}\left(E^{\prime}, E_{f}^{\prime}, t^{\prime}\right)\left[1-D\left(E, E^{\prime}, Z\right)\right] d E_{f} d E^{\prime}
$$

The calculation of the energy loss probability function $I_{b}\left(E, E_{f}, t\right)$ is complicated, due to the statistical nature of external Brehmsstrahlung radiation. Range straggling effects due to both Brehmsstrahlung and ionization of matter must be taken into account. The statistical nature of energy loss phenomena results not in a fixed range for the electron, but rather a variable range with an upper tail described by a Landau distribution [5].

A full treatment of this problem requires solving integral equations of $I_{b}\left(E, E_{f}, t\right)$ with boundary conditions and is not treated here; the reader is hereby referred to Appendix B of Ref. [122] for an in-depth look at the problem. Basically, straggling due to ionization affects the shape of discrete peaks in the resonance distribution, while Brehmsstrahlung affects the subtraction of wider tails trom nearby resonances.

The result (from Ref. [122]) is ${ }^{29}$

$$
I_{b}\left(E, E_{f}, t\right)=\frac{b t}{\left(E-E_{F}\right) \Gamma(1+b t)}\left(\frac{E-E_{f}}{E}\right)^{b t} \phi\left(\frac{E-E_{f}}{E}\right)
$$

\footnotetext{
${ }^{29}$ Equations B.3 and B.43 are combined from Ret. [122] to get this result. Here $\Gamma(x)$ is the mathematical Gamma function.
} 
where

$$
\begin{gathered}
b=\frac{4}{3}\left[1+\frac{1}{9}\left(\frac{Z+1}{Z+\eta}\right)\left(\frac{1}{\ln \left(183 Z^{-1 / 3}\right)}\right)\right] \\
\eta=\frac{\ln \left(1440 Z^{-2 / 3}\right)}{\ln \left(183 Z^{-1 / 3}\right)}
\end{gathered}
$$

and $\phi(v)$ is the normalized distribution of the Brehmsstrahlung function. For a small energy loss, as is the case in this experiment,

$$
\phi(v) \approx 1-v+\frac{3}{4} v^{2}
$$

These results are known to be good within $0.5 \%$ of the total cross-section, assuming a target of thickness less than $0.1 X_{0}$ (definitely the case in EG1b).

Combining all the above, the external radiative corrections can then be calculated, with the only necessary remaining input being the thickness, in radiation lengths, traversed by the incoming and outgoing electrons. ${ }^{30}$ The variable $t$ is just a number (the incoming electron travels straight along the beam line), while $t^{\prime} \equiv t^{\prime}(\theta, \phi)$ is a function of the scattering angle. The radiation length fractions of target materials, including the solid angle distributions of the radiation thickness, are detailed in the target description of Section 2.4 and in the unpolarized model description in Section 2.8. These thicknesses are used for a given scattering angle in the above equations, filling in the last needed parameters for the external corrections.

\subsubsection{Application of radiative corrections}

In practice, the external radiative corrections must be done first. The aforementioned iterative procedure is used to numerically solve for $\sigma_{i n t}$ in Eqs. 6.75 and 6.76 from the experimental crosssection. Then, these results are substituted into Eq. 6.74 to get the Born cross-sections $\sigma_{0}^{P}$ and $\sigma_{0}^{a}$, the ratio of which yields the Born asymmetry $A_{\| 1}$.

As already explained, all correction coefficients are absorbed into a radiative dilution factor $\left(f_{R C}\right)$ and additive constant $\left(A_{R C}\right)$ to correct the asymmetry. These corrections are written to a $300 \times 40$ array for the mean $Q^{2}$ and $W$ values for each bin, at each of the 4 major beam energies. ${ }^{31}$ After the

\footnotetext{
${ }^{30}$ It is important to note that $d E / d x$ due to ionization energy loss is not included in these radiative corrections. See Section 4.2.4.

${ }^{31}$ At one point, it was considered whether it was necessary to generate asymmetry corrections for specific beam energies. That is, for example, whether different corrections were required for $1.606 \mathrm{GeV}$ and $1.723 \mathrm{GeV}$ energies. Because the
} 
asymmetry is calculated at each individual beam energy, and before division by the depolarization factor $D$, Eq. 6.62 is applied to each $A_{\|}\left(W, Q^{2}\right)$ to derive the proper Born asymmetry from the data.

Plots of the final $A_{\| \mid}$for each beam energy are shown in the next chapter in Figure 7.5, with the contribution specifically from radiative corrections shown shaded. As noted, in the inelastic region, as long as $W$ is not too large, or too close to the elastic region, the contribution from the corrections is small compared to the total asymmetry.

corrections are so small, the difference turned out to be less the incurred systematic error due to the corrections themselves, so only the nominal energies $1.6,2.5,4.2$ and $5.7 \mathrm{GeV}$ were used for correcting $A_{1 \mid}$. This was desirable because of the long amount of computing time required to run the code for polarization-dependent cross-section corrections. 


\section{Chapter 7}

\section{Combination of Data Sets and \\ Calculation of Systematic Errors}

\subsection{Evaluation and Combination of $A_{\|}$Values}

We are now the position to accurately calculate the total proton double-spin asymmetry $A_{\|}$. Using Eq. 5.1, and explicitly writing factors for $e^{+} e^{-}$pair production, polarized nitrogen corrections, and radiative corrections we have

$$
A_{\|}=\frac{C_{b a c k}}{F_{D F} P_{b}\left(P_{t}+P_{15}^{*}\right) f_{R C}} \frac{n^{-}-n^{+}}{n^{-}+n^{+}}+A_{R C}
$$

The statistical error is given by the properly scaled version of Eq. 5.6:

$$
\sigma_{A}=\frac{C_{b a c k}}{F_{D F} P_{b}\left(P_{t}+P_{15}^{*}\right) f_{R C}} \frac{1}{\sqrt{N}}
$$

The errors on all the correction factors are accounted for in the systematic error analysis of this chapter (Section 7.2).

The raw asymmetry (Eq. 3.1) is first measured individually for every run, and then combined together for a total raw asymmetry, for each combination of target polarization sign and HWP sign 

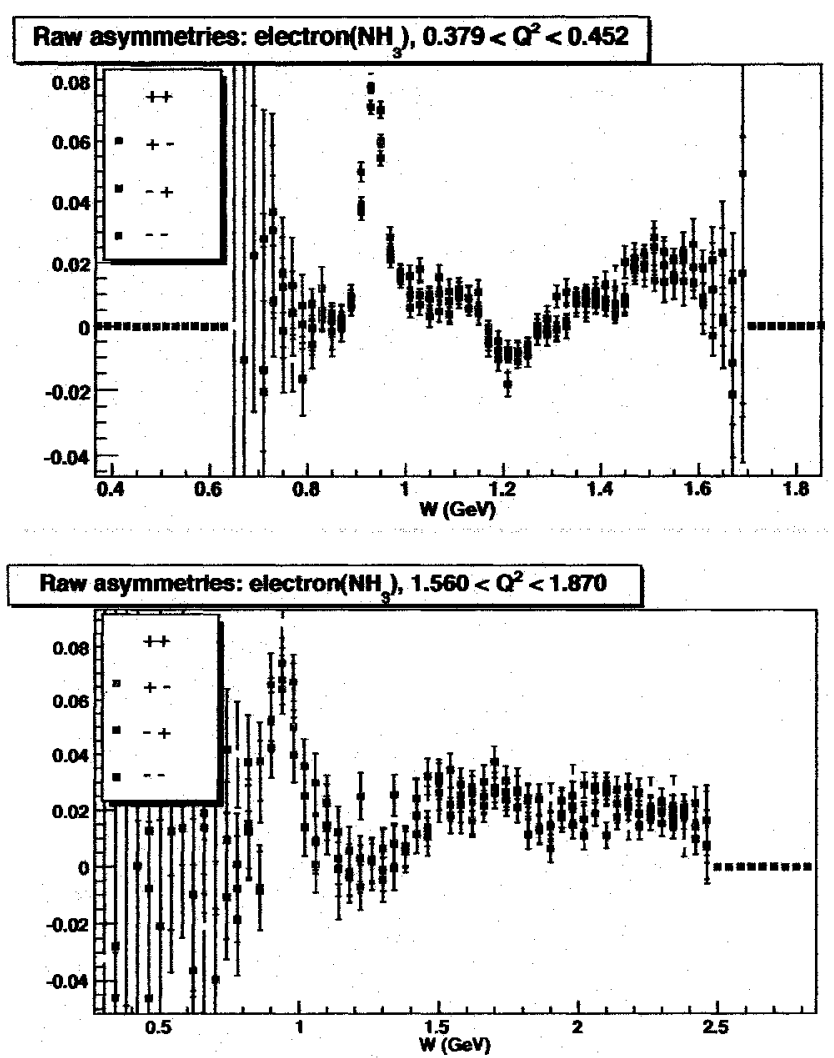

Figure 7.1: Raw asymmetries superimposed for each combination of HWP status/target polarization as a function of $W$, shown in a selected $Q^{2}$ bin in two different data brackets. The $1.6 \mathrm{GeV}$ inbending (Bracket 1,top) and $4.2 \mathrm{GeV}$ inbending (Bracket 11, bottom) data are shown, with 20 $\mathrm{MeV}$ and $40 \mathrm{MeV} W$-bins, respectively.

(see Section 3.3). Raw asymmetries for each HWP and target polarization state are shown for two sets in Figure 7.1.

The goal of this section is to find a total value of $A_{\| \mid}$for each one of the 4 main beam energies, so that $A_{1}$ and $A_{2}$ can be extracted (Section 1.4). Proper weighting of data and statistical compatibility are important issues to consider when combining subsets of data. These issues are addressed at each stage of data combination, later in this section.

Beginning with a total of 38 raw asymmetries for the present HWP/target polarization states (explicated in Table 5.1), the data were combined in the following sequence:

- Raw asymmetries from run sets of differing HWP status (but same bracket and target polar- 
ization) were combined, reducing the number of independent asymmetries from 38 to 21 .

- Raw asymmetries of opposite target polarization sign were combined, weighted by relative beam and target polarizations (Section 6.2.5), resulting in 11 raw asymmetries (one per bracket).

- $A_{\|}$and its error was calculated using Eqs. 7.1 and 7.2 (excluding radiative terms $A_{R C}$ and $f_{R C}$ ) for each bracket.

- $A_{||}$values were combined for brackets with exactly the same beam energy, but opposite torus polarity, resulting in 8 sets of $A_{\|}$values.

- Radiative corrective terms $A_{R C}$ and $f_{R C}$ were factored in, and $A_{\|} / D$ was calculated. ${ }^{1}$

- $A_{\|} / D$ values for similar beam energies were combined, resulting in 4 arrays in $Q^{2}$ and $W$ for each of the 4 main beam energies, the desired result.

Each of these stages is described in some detail in the first part of this section. Then, the development of systematic error estimates is presented in detail.

\subsubsection{Combining asymmetries from opposite HWP status}

Combination of cumulative asymmetries from different half-wave-plate status proceeds first. No relative weighting is needed, since the beam polarization magnitude does not change upon insertion/removal of the HWP. So, raw asymmetries from each HWP state $\left(A_{I N}\right.$ and $\left.A_{O U T}\right)$ are combined by the standard method of weighting by their statistical errors [103]:

$$
\begin{gathered}
A=\frac{A_{I N} / \sigma_{A_{I N}}^{2}+A_{O U T} / \sigma_{A_{O U T}}^{2}}{1 / \sigma_{A_{I N}}^{2}+1 / \sigma_{A_{O U T}}^{2}} \\
\sigma_{A}=\frac{1}{\sqrt{1 / \sigma_{A_{I N}}^{2}+1 / \sigma_{A_{O U T}}^{2}}}
\end{gathered}
$$

\footnotetext{
${ }^{1}$ The quantity $D$ is the depolarization tactor, which is required for the calculation of $A_{1}$ and $A_{2}$. It is defined in Section 1.1.3.
} 
The various average kinematic values that are tracked for each bin $\left(Q^{2}, W, x, s, D, E^{\prime}, \theta, \epsilon, \eta, \gamma\right.$ and $\nu$ ) are also combined as weighted averages

$$
v a l=\frac{v a l_{I N} / \sigma_{A_{I N}}^{2}+v a l_{O U T} / \sigma_{A_{O U T}}^{2}}{1 / \sigma_{A_{I N}}^{2}+1 / \sigma_{A_{O U T}}^{2}}
$$

In order to test that our assumption of statistical compatibility is correct, a mathematical Student's $t$-test is run on the data [125][126]. To run the compatibility test, we calculate

$$
t\left(W, Q^{2}\right)=\frac{A_{O U T}-A_{I N}}{\sqrt{\sigma_{A_{O U T}}^{2}+\sigma_{A_{I N}}^{2}}}
$$

for each populated bin. We then find the mean and standard deviation of $t$ :

$$
\bar{t}=\sum_{N} t / N \quad \sigma_{t}=\sqrt{\sum_{N} t^{2} / N}
$$

where $N$ is the number of populated bins in the sample. Good statistical compatibility is indicated by $\bar{t} \approx 0$ and $\sigma_{t} \approx 1.00$.

After the combination of opposing HWP states, two sets of data remain for each bracket, one per target polarization sign. ${ }^{2}$ Results of the $t$-test for each target polarization and data bracket are listed in Table 7.1. Statistical compatibility was not an issue for opposing HWP states. (A similar combination of data was made for exclusive $e p$ asymmetries, for use in evaluating the $P_{b} P_{t}$ values for each total bracket.) An example of raw asymmetries combined between opposing HWP states is shown in Figure 7.2.

\subsubsection{Combining asymmetries from opposite target polarizations}

Combining data from subsets with opposing target polarizations is similar to the case of HWP set combination. However, now we must consider that the magnitudes of the target polarizations of opposite signs can differ considerably. The relative weighting of the raw asymmetries is an important procedure, in such a case.

\footnotetext{
${ }^{2}$ The exception is $1.6 \mathrm{GeV}$ outbending, for which there is only data for + target polarization and HWP status.
} 

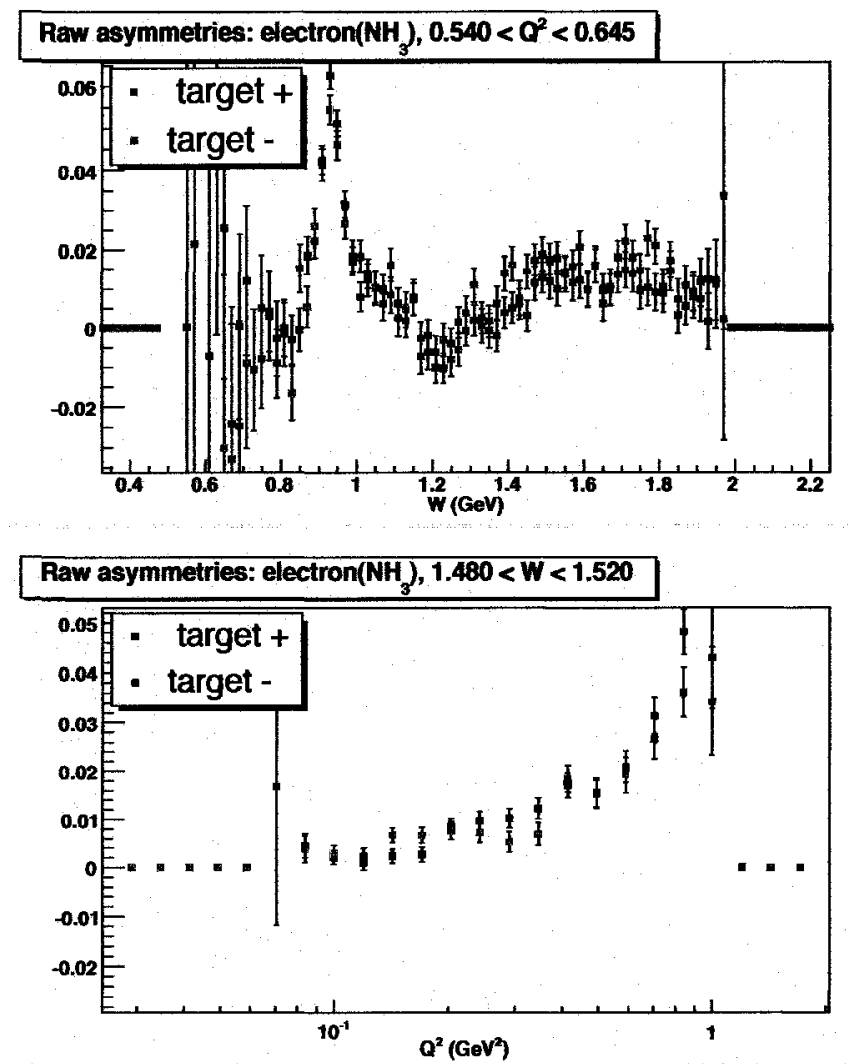

Figure 7.2: Raw asymmetries superimposed for each target polarization sign, after combination of asymmetries from opposing HWP sets. The $2.5 \mathrm{GeV}$ outbending data (Bracket 9) are shown, for a chosen $Q^{2}$ bin as a function of $W$ (top), and averaged over a small range in $W$ as a function of $Q^{2}$ (bottom). 
Table 7.1: Students $t$-test results for the combination of opposing HWP states over each target polarization. Sets with only one HWP state available are listed as "n/a".

\begin{tabular}{|c|c|c|c|}
\hline Set/Bracket & Target Pol. & $\bar{t}$ & $\sigma_{t}$ \\
\hline \hline $1.6+/ 1$ & + & 0.15 & 1.10 \\
\hline $1.6+/ 1$ & - & -0.03 & 1.01 \\
\hline $1.6-/ 2$ & + & $\mathrm{n} / \mathrm{a}$ & $\mathrm{n} / \mathrm{a}$ \\
\hline $5.76-/ 3$ & + & 0.00 & 1.00 \\
\hline $5.76-/ 3$ & - & -0.05 & 1.01 \\
\hline $5.73-/ 4$ & + & -0.05 & 1.00 \\
\hline $5.73-/ 4$ & - & -0.01 & 1.01 \\
\hline $5.7+/ 5$ & + & 0.03 & 1.00 \\
\hline $5.7+/ 5$ & - & 0.03 & 1.01 \\
\hline $2.3+/ 6$ & + & $\mathrm{n} / \mathrm{a}$ & $\mathrm{n} / \mathrm{a}$ \\
\hline $2.3+/ 6$ & - & -0.02 & 1.00 \\
\hline $5.6+/ 7$ & + & 0.00 & 0.99 \\
\hline $5.6+/ 7$ & - & $\mathrm{n} / \mathrm{a}$ & $\mathrm{n} / \mathrm{a}$ \\
\hline $1.7-/ 8$ & + & $\mathrm{n} / \mathrm{a}$ & $\mathrm{n} / \mathrm{a}$ \\
\hline $1.7-/ 8$ & - & 0.03 & 1.00 \\
\hline $2.5-/ 19$ & + & 0.02 & 1.00 \\
\hline $2.5-/ 9$ & - & -0.02 & 1.00 \\
\hline $4.2+/ 11$ & + & -0.04 & 1.02 \\
\hline $4.2+/ 11$ & - & 0.08 & 1.02 \\
\hline $4.2-/ 12$ & + & 0.00 & 1.01 \\
\hline $4.2-/ 12$ & - & -0.04 & 1.02 \\
\hline \multicolumn{5}{|r}{} \\
\end{tabular}


The calculated values of $P_{b} P_{t}$ are used to weight the results. However, the $P_{b} P_{t}$ values for some of the (higher energy) data sets have particularly large statistical errors (see Table 6.8), and thus run the risk of improperly scaling the relative weights. Therefore, we use a second, more precise calculation of the beam and target polarizations, using a model of $A_{\|}$in the inelastic $W$-region. The process for deriving this factor $\left(P_{b} P_{t} w g t\right)$ was detailed explicitly in Section 6.2.5. Values of $P_{b} P_{t} w g t$ for each target polarization are listed in Table 6.10.

To combine the data from opposite target polarizations, the $P_{b} P_{t}$-weighted average is taken:

$$
A=\frac{\left(P_{b} P_{t w g t+}\right)^{2} A_{+} / \sigma_{A+}^{2}+\left(P_{b} P_{t w g t-}\right)^{2} A_{-} / \sigma_{A-}^{2}}{\left(P_{b} P_{t w g t+}\right)^{2} / \sigma_{A+}^{2}+\left(P_{b} P_{t w g t-}\right)^{2} / \sigma_{A-}^{2}}
$$

and the error is

$$
\sigma_{A}=\sqrt{\frac{\left(P_{b} P_{t w g t+}\right)^{2}+\left(P_{b} P_{t w g t-}\right)^{2}}{\left(P_{b} P_{t w g t+}\right)^{2} / \sigma_{A+}^{2}+\left(P_{b} P_{t w g t-}\right)^{2} / \sigma_{A-}^{2}}}
$$

where the + and - subscripts denote the values from the respective target polarizations. Average values of kinematics quantities are also combined as

$$
v a l=\frac{\left(P_{b} P_{t w g t+}\right)^{2} v a l_{+} / \sigma_{A+}^{2}+\left(P_{b} P_{t w g t-}\right)^{2} v a l_{-} / \sigma_{A-}^{2}}{\left(P_{b} P_{t w g t+}\right)^{2} / \sigma_{A+}^{2}+\left(P_{b} P_{t w g t-}\right)^{2} / \sigma_{A-}^{2}}
$$

Just as in the case with opposing half-wave-plate status (previous subsection), a students $t$-test is again run on the data to ensure statistical compatibility, where $t$ is given by

$$
t\left(W, Q^{2}\right)=\frac{A_{+} / P_{b} P_{t w g t+}-A_{-} P_{b} P_{t w g t-}}{\sqrt{\sigma_{A_{+}}^{2} /\left(P_{b} P_{t w g t+}\right)^{2}+\sigma_{A_{-}}^{2} /\left(P_{b} P_{t} w g t-\right)^{2}}}
$$

and $\bar{t}$ and $\sigma_{t}$ are again given by Eq. 7.7. Again, if there is no systematic bias between the two data sets, we expect $\bar{t} \approx 0$ and $\sigma_{t} \approx 1$. A list of these values for raw asymmetries can be found in Table 7.2. (Again, a similar combination of data was made for exclusive ep asymmetries, for use in evaluating the $P_{b} P_{t}$ values for each total bracket.) Data combined using this method for a sample bracket are shown in Figure 7.3. 


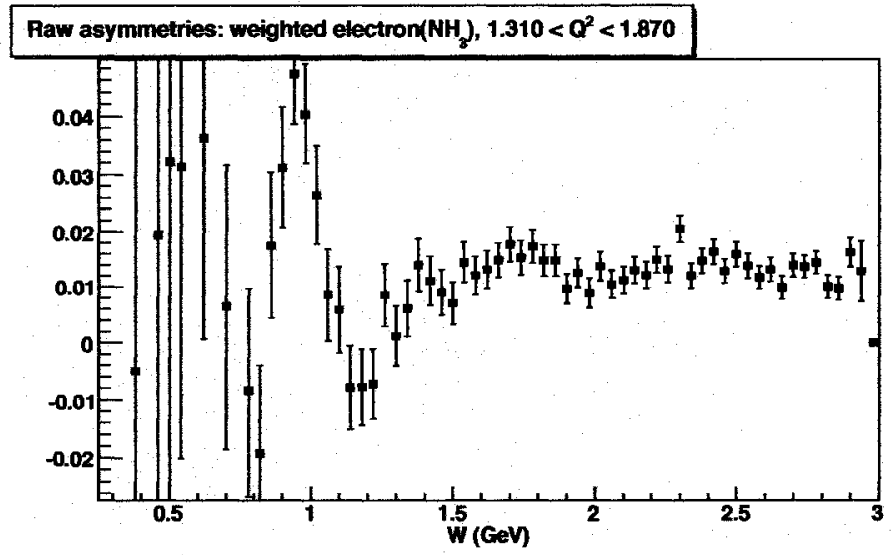

Figure 7.3: Raw asymmetry as a function of $W$ (using $40 \mathrm{MeV}$ bins) for a chosen $Q^{2}$ range (two standard bins combined), for the $5.7 \mathrm{GeV}$ inbending data set (Bracket 5). The method of weighting between opposing target polarizations, as described in the text, was used to generate the data.

Table 7.2: Students $t$-test results for the combination of opposing target polarization states. The set with only one polarization state available is listed as " $n / a$ ".

\begin{tabular}{|c|c|c|}
\hline Set/Bracket & $\bar{t}$ & $\sigma_{t}$ \\
\hline \hline $1.6+/ 1$ & 0.05 & 1.02 \\
\hline $1.6-/ 2$ & n/a & n/a \\
\hline $5.76-/ 3$ & -0.01 & 1.01 \\
\hline $5.73-/ 4$ & 0.02 & 1.00 \\
\hline $5.7+/ 5$ & 0.01 & 1.01 \\
\hline $2.3+/ 6$ & 0.00 & 0.99 \\
\hline $5.6+/ 7$ & 0.00 & 1.00 \\
\hline $1.7-/ 8$ & -0.02 & 1.00 \\
\hline $2.5-/ 9$ & -0.02 & 0.98 \\
\hline $4.2+/ 11$ & 0.00 & 0.98 \\
\hline $4.2-/ 12$ & 0.01 & 1.01 \\
\hline
\end{tabular}



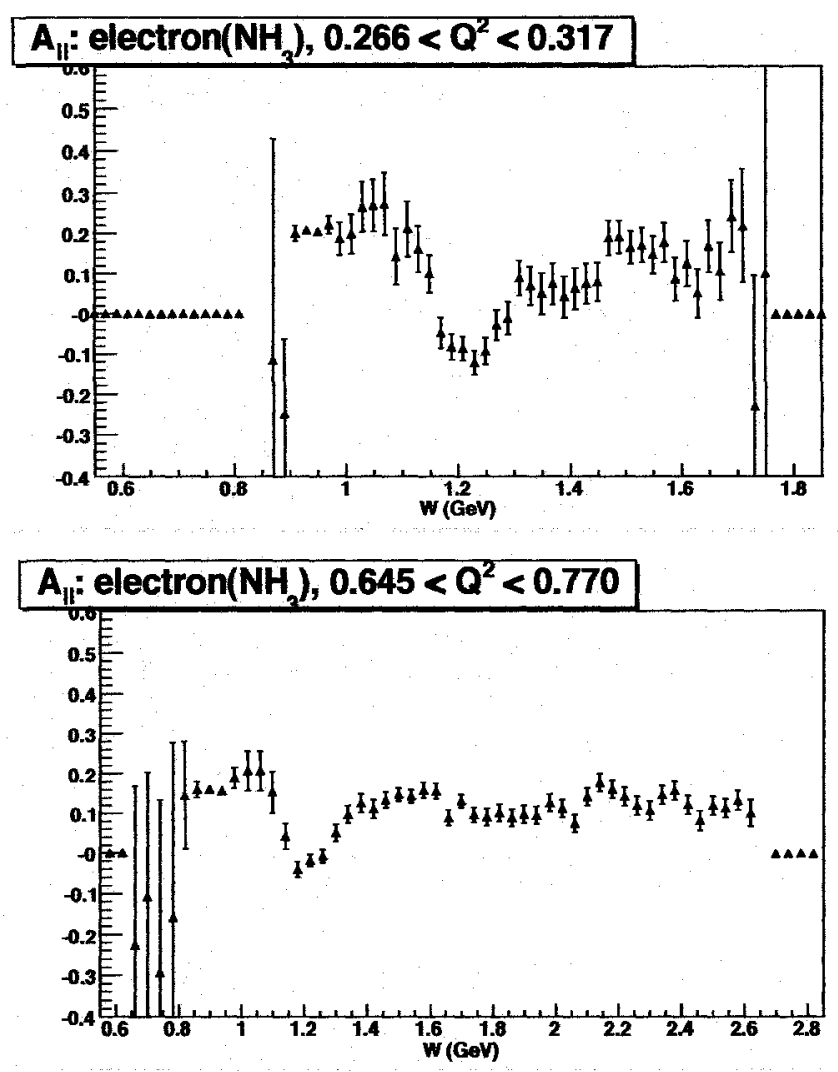

Figure 7.4: Values of $A_{\|}$calculated from the background-corrected double spin asymmetry, as a function of $W$, in a $Q^{2}$ bin for the $1.7 \mathrm{GeV}$ outbending (Bracket 8 , top) and $4.2 \mathrm{GeV}$ outbending (Bracket 12, bottom) data. Radiative corrections have not yet been added.

\subsubsection{Combining asymmetries from opposite torus currents}

At this point, the 11 raw asymmetries (from each data bracket) are converted to $A_{\|}$values using Eq. 7.1 (without radiative corrections, so that $A_{R C}=0$ and $f_{R C}=1$ at this point). The backgrounds $P_{b} P_{t}, F_{D F}, C_{b a c k}$ and $P_{15}^{*}$ for the whole bracket are used. Sample results of the value $A_{\|}$for two brackets are shown in Figure 7.4.

Once the values are weighted by the backgrounds, there is no reason to expect that the magnitude of the physics quantity $A_{\|}\left(W, Q^{2}\right)$ should have any dependence on torus current. ${ }^{3}$ Therefore, we can now combine values of $A_{\| \mid}$(and the average kinematics per bin) between inbending and

\footnotetext{
${ }^{3}$ The size of the error bar in a given bin, of course, will vary between opposite torus currents, as inbending and outbending torus currents alter the range of kinematic acceptance. This is why all combinations are weighted by their statistical errors.
} 
outbending torus currents, where the energies remain unchanged. Specifically, we combine Brackets 1 and $2(1.606 \mathrm{GeV})$, Brackets 4 and $5(5.725 \mathrm{GeV})$, and Brackets 11 and $12(4.238 \mathrm{GeV})$.

The equations for combining the data are exactly the same as in Section 7.1.1, except that the subscripts $I N$ and $O U T$ denote inbending and outbending torus currents instead of HWP status. Again, a student's $t$-test is run for statistical compatibility; the results are listed in Table 7.3.

Once all data from identical beam energies are combined, radiative correction terms $\left(A_{R C}\right.$ and $f_{R C}$; see Eq. 6.62) are factored in. Radiative corrections are explained in detail in Section 6.4. Combined data by energy, showing the effects of radiative corrections, can be seen in Figure 7.5.

Table 7.3: Students $t$-test results for the combination of $A_{\|}$values between opposing torus currents.

\begin{tabular}{|c|c|c|}
\hline Beam Energy (GeV) & $\bar{t}$ & $\sigma_{t}$ \\
\hline \hline 1.606 & -0.02 & 1.06 \\
\hline 4.238 & 0.06 & 1.00 \\
\hline 5.725 & 0.12 & 1.07 \\
\hline
\end{tabular}

\subsubsection{Combining asymmetries from slightly differing beam energies}

To calculate the desired asymmetries $\left(A_{1}\right.$ and $A_{2}$ ), we need values of $A_{\|} / D$ for each of the 4 main beam energies (see Section 1.4), where $D$ is the depolarization factor, defined in Section 1.1.3. The average running value of $D$ in each $W, Q^{2}$ bin has been tracked up to this point. The radiative corrected value of $A_{\|}$(see previous subsection) is simply divided by the tracked average of $D$ in each $W, Q^{2}$ bin.

$A_{\| /} / D$, its error $\left(\sigma_{A_{\| \mid}} / D\right)$, and the averages of all the kinematics values are then combined using the exact same method used in the previous section. It is important to divide by $D$ before combining similar beam energies, because its value is dependent on $E$. In the particular case (5.x GeV) where 3 beam energies were combined, the two $5.7 \times \mathrm{GeV}$ sets were combined first, and the resulting set was combined with the $5.6 \mathrm{GeV}$ data.

Again, to make sure that the imparted difference due to the beam energy discrepancy does not cause a weighting issue, a student's $t$-test is again used on the data. The resultant compatibility 

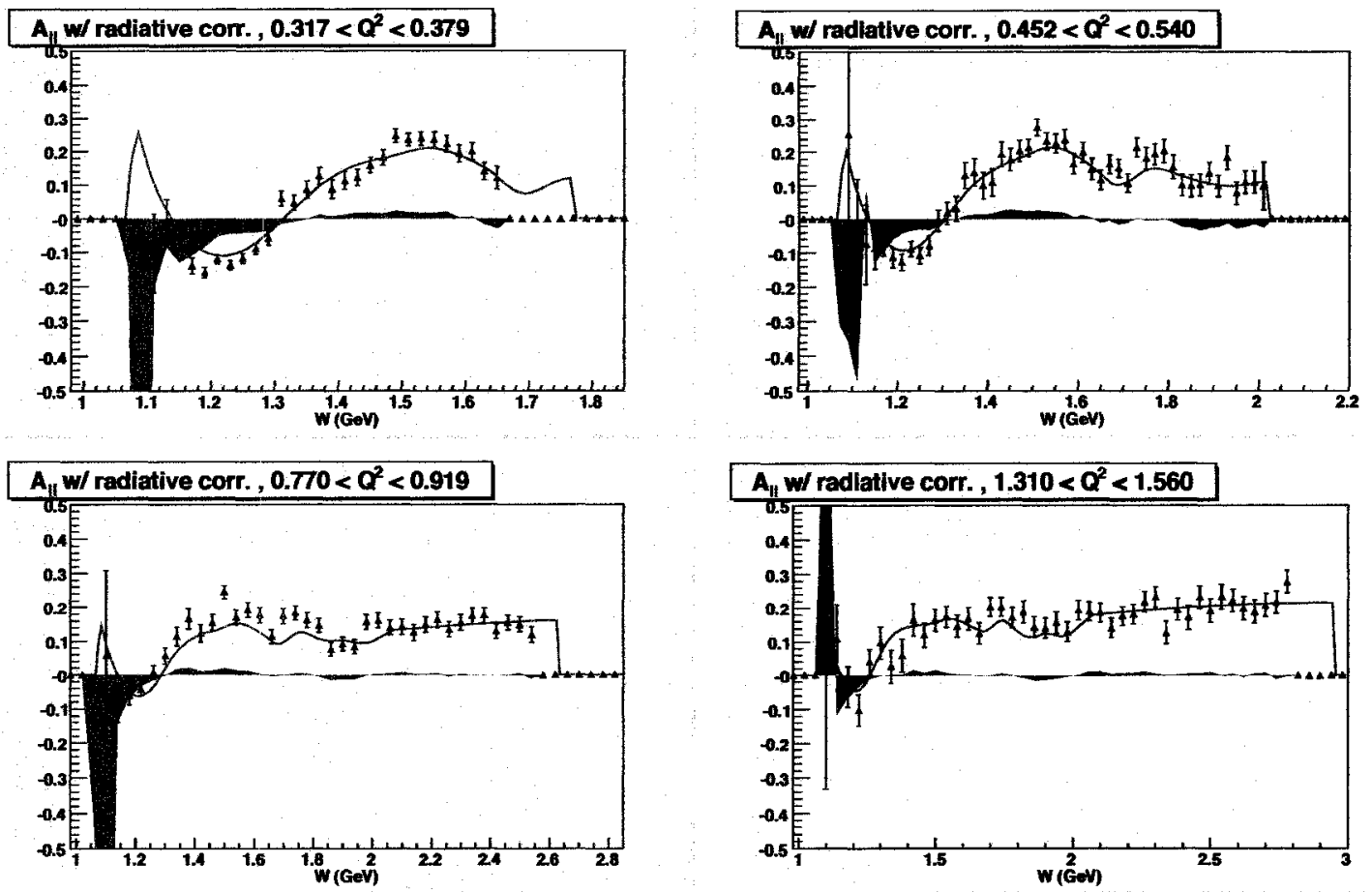

Figure 7.5: Values of $A_{\|}$shown at $1.606 \mathrm{GeV}, 2.286 \mathrm{GeV}, 4.238 \mathrm{GeV}$, and $5.725 \mathrm{GeV}$ respectively, in selected $Q^{2}$ bins, opposite torus current data combined. Radiative corrections have been added. The radiative contribution (that is, $A_{\| \text {uncorrected }}-A_{\| \text {corrected }}$ ) is shown in the cyan shading. Note that radiative contributions blow up near the elastic region. The red line is the models comparison of $A_{||}=D\left(A_{1}+\eta A_{2}\right)$. 
test results are shown in Table 7.4.

Table 7.4: Students $t$-test results for the combination of $A_{\|} / D$ values for slightly differing beam energies. After this combination process, only 4 asymmetries for each $\left(W, Q^{2}\right)$ bin remain, one for each major beam energy. The $5.73 \mathrm{GeV}$ set is a temporary combination that is immediately afterward combined with the $5.6 \mathrm{GeV}$ data.

\begin{tabular}{|c|c|c|c|c|}
\hline Approx. Combined Beam Energy & set A (GeV) & set B (GeV) & $\bar{t}$ & $\sigma_{t}$ \\
\hline \hline $1.6 \mathrm{GeV}$ & 1.606 & 1.723 & 0.16 & 0.96 \\
\hline $2.5 \mathrm{GeV}$ & 2.286 & 2.561 & 0.05 & 0.97 \\
\hline $4.2 \mathrm{GeV}$ & 4.238 & - & n/a & n/a \\
\hline $5.73 \mathrm{GeV}$ (temporary) & 5.725 & 5.743 & 0.00 & 1.01 \\
\hline $5.7 \mathrm{GeV}$ & 5.615 & 5.73 & 0.00 & 0.98 \\
\hline
\end{tabular}

After this combination of data, up to 4 values of $A_{\|} / D$ (and their statistical errors) remain per $W, Q^{2}$ bin. These values can be used to derive values of $A_{1}$, and in some cases, $A_{2}$, as explained in Section 1.4. Values of $A_{\|} / D$, for all 4 beam energies, are shown in Figure 7.6.

\subsection{Systematic error calculation}

In addition to the statistical errors in the analysis, which are rigorously calculated as according to the formalism in the preceding chapters and sections, there are systematic errors resulting from uncertainties in measured quantities. These must be determined in order make an appropriate assessment of the accuracy of the measured asymmetries.

The basic strategy used to calculate systematic errors required identifying potential sources of uncertainty (in physical quantities, models, etc.). We then altered the numerical value of the given error source to the extent of the uncertainty, and reran the entire analysis from the start, keeping all other parameters the same. The systematic error due to the particular cause could then be estimated as the measured difference between the two analyses. The systematic errors on any given quantity due to a particular source of uncertainty (models, kinematics, backgrounds, etc.) on any quantity $\left(A_{\| \mid} / D, A_{1}, g_{1}\right.$, etc.) can be calculated in this manner.

Systematic errors were divided into 8 total categories:

1. Target model errors (i.e. material thicknesses and densities) 

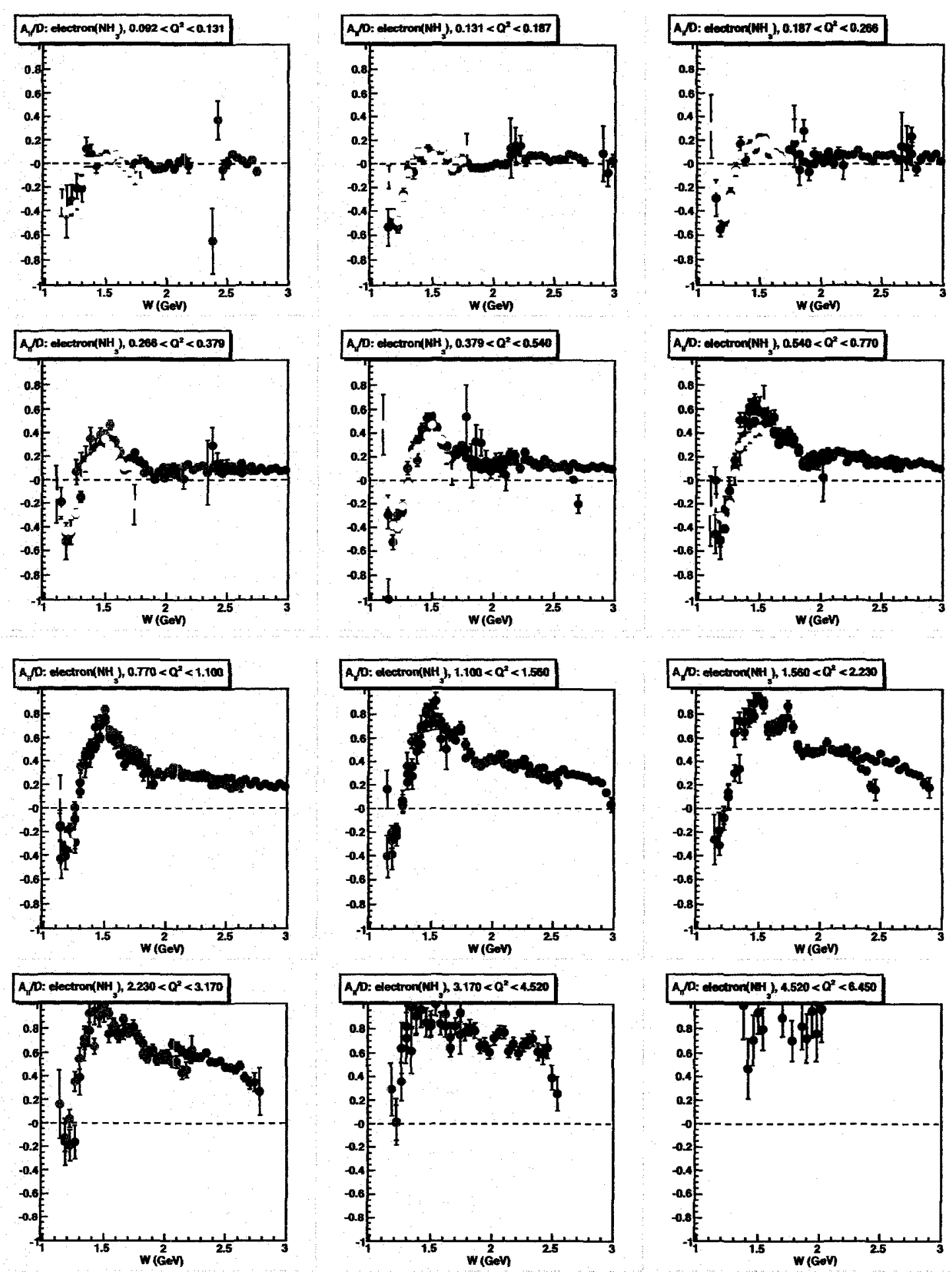

Figure 7.6: Combined values of $A_{||} / D$ over the full range of $Q^{2}$, showing the full kinematic expanse of the EG1 data. Data for each beam energy are represented by different colors (yellow $=1.6 \mathrm{GeV}$, green $=2.5 \mathrm{GeV}$, red $=4.2 \mathrm{GeV}$, blue $=5.7 \mathrm{GeV}$ ). Points with the largest error bars are removed for clarity. Regions where points overlap can be used to determine $A_{2}$ by linear regression (see Section 1.4). 
2. Dilution factor model errors (i.e. unpolarized cross-section error)

3. Beam $\times$ target polarization errors (systematic)

4. Miscellaneous background errors

5. Kinematics errors

6. Charge normalization and false asymmetry errors (i.e. Faraday cup issues)

7. Errors on modeled asymmetries and structure functions

8. Beam $\times$ target polarization errors (statistical)

The total effects of these errors (for selected $Q^{2}$ ranges at 2 different beam energies) on the measurement of $A_{1}+\eta A_{2}$ are shown in Figure 7.7.

The sources and magnitudes of these individual errors are individually described in detail in the following subsections. The analysis was rerun for each source of error (listed in Table 7.5), and the various errors were combined in quadrature, as described in Section 7.2.10. Throughout this section, parenthesized ( ) numbers are periodically inserted to correlate the described errors with their error index label in Table 7.5.

\subsubsection{Target model errors (1-9)}

Accurate modeling of the lengths and densities of the materials in the target is essential for the subtraction of unpolarized backgrounds (i.e. the dilution factors). There were, of course, physical uncertainties in the measurement of the dimensions of the various materials in the target. Lengths of the carbon, Kapton and aluminum, in particular, were used to derive the total target length $L$ and the frozen ammonia target length $\ell_{A}$. Precise knowledge of the densities of all target materials were needed to derive these quantities, and hence, the dilution factors $F_{D F}$.

\section{Densities and lengths used in calculations}

Reasonable uncertainties were included on each of these lengths and densities (1-8), recorded in Table 2.1, and the analysis was rerun for each possible error source, to determine the systematic 

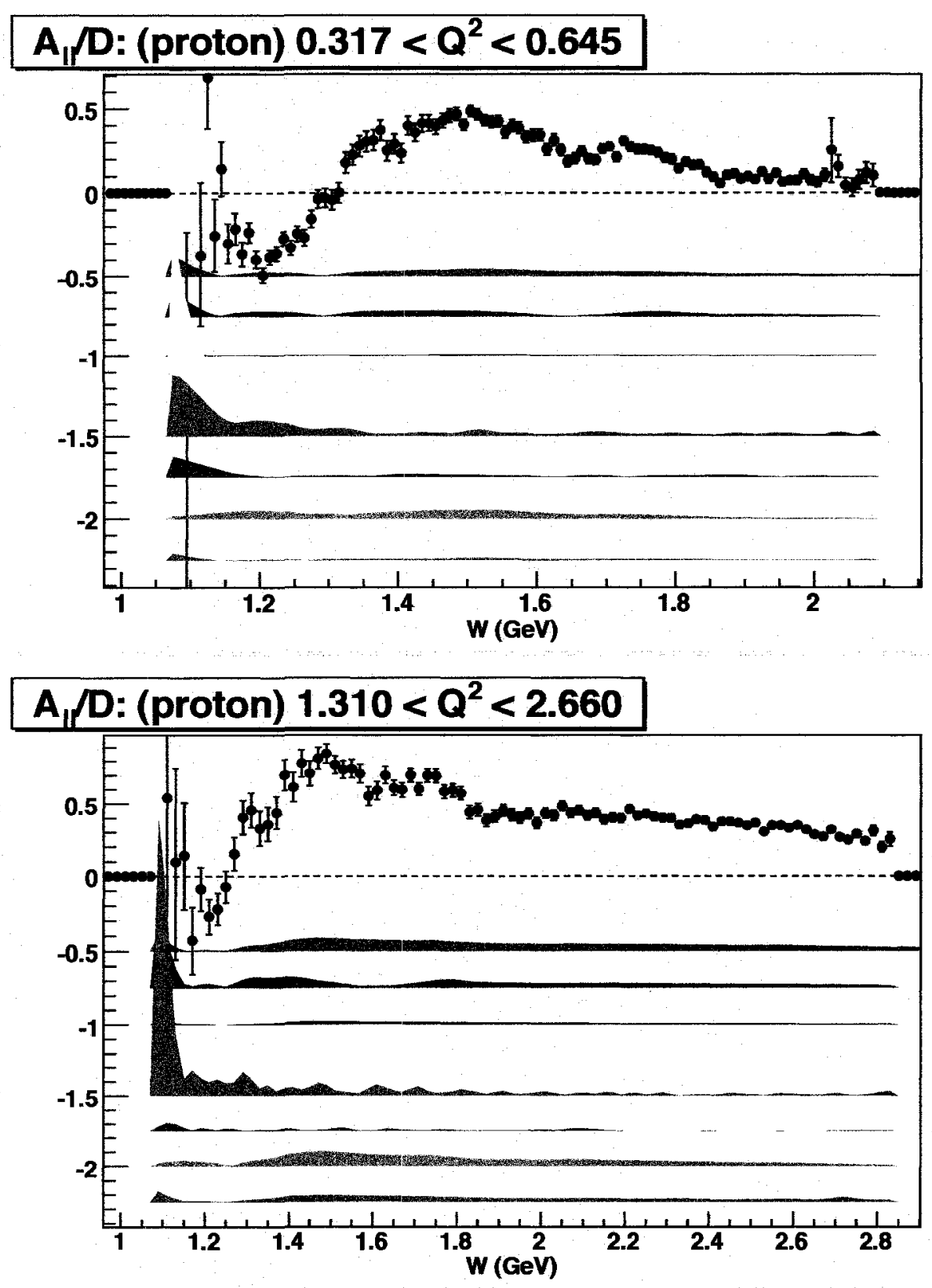

Figure 7.7: Systematic errors on $A_{1}+\eta A_{2}$ for the 7 categories of error described in the beginning of Section 7.2 , shown for $2.5 \mathrm{GeV}$ (top) and $4.2 \mathrm{GeV}$ (bottom) data. Errors are multiplied by a factor of 5 for visibility. Shown are the total errors for all 8 sources listed in the text, specifically, from top to bottom, errors due to the target model (red), dilution factor models (light green), systematic errors on $P_{b} P_{t}$ (blue), miscellaneous backgrounds (yellow), kinematics (magenta), false asymmetries (cyan), model errors (dark green), and $P_{b} P_{t}$ statistical errors (purple). 
Table 7.5: Index key used for systematic error determination, with a brief summary of what parameters were varied in order to determine each error. Boldfaced numbers note major (average $>2 \%$ ) contributions to the total asymmetry error. Italicized numbers did not (and were not expected to) contribute to the error on $A_{\|}$at all, and were used only in ${ }^{15} \mathrm{~N}$ analysis or development versions of analysis software.

\begin{tabular}{|c|c|c|}
\hline Error Index & Alteration & Quantities Affected \\
\hline 1 & LHe multiplicative factor $\left({ }^{12} \mathrm{C}\right.$ factor $\rightarrow \mathrm{NH}_{3}$ factor), brackets $10-12$ & $\overline{F_{D F}}$ \\
\hline 2 & $\rho_{C} \ell_{C}\left({ }^{12} \mathrm{C}\right.$ density $(+0.01 \mathrm{~cm}) \times$ thickness $\left.(+2 \%)\right)$ & $F_{D F}$ \\
\hline 3 & $\ell_{K}$ (Kapton thickness $(+10 \%),{ }^{12} \mathrm{C}$ target only) & $F_{D F}$ \\
\hline 4 & $\rho_{A}\left(\mathrm{NH}_{3}\right.$ density $\left.(+1 \%)\right)$ & $F_{D F}$ \\
\hline 5 & $\rho_{\text {He }}($ LHe density $(-4 \%))$ & $F_{D F}$ \\
\hline 6 & $\ell_{K}$ (Kapton thickness $\left.(+10 \%)\right)$ & $F_{D F}$ \\
\hline 7 & $\ell_{A l}$ (Al foil thickness $\left.(+10 \%)\right)$ & $F_{D F}$ \\
\hline 8 & $L$ averaging range in $W$ (increased to $\ell_{A}$ range) & $F_{D F}$ \\
\hline 9 & $\ell_{A}$ averaging range in $W$ (decreased to $L$ range) & $F_{D F}$ \\
\hline 10 & $\sigma_{N} / \sigma_{C}\left({ }^{15} \mathrm{~N}\right.$ cross-section model; changed to simple function of $\left.\sigma_{n} / \sigma_{D}\right)$ & $P_{b} P_{t_{1}} \ell_{N}, a, b$ \\
\hline 11 & normalization offset for ${ }^{15} \mathrm{~N}$ target $(-10 \%)$ & $\ell_{N}, a, b$ \\
\hline 12 & ${ }^{15} \mathrm{~N}$ density $(-15 \%)$ & $\ell_{N}, a, b$ \\
\hline 13 & $\sigma_{n} / \sigma_{D}$ cross-section ratio model (used $\sigma_{n} / \sigma_{p}=1-0.8 x$ ) & $P_{b} P_{t} \ell_{N}, a, b$ \\
\hline 14 & $L$ (total target length) used in ${ }^{15} \mathrm{~N} / 12 \mathrm{C}$ analysis $(+0.3 \mathrm{~cm})$ & $\ell_{N}, a, b$ \\
\hline 15 & $\ell_{N}$ averaging range in $W\left(\frac{1}{3}\right.$ of inelastic range removed) & $\ell_{N}, a, b$ \\
\hline 16 & $a+b \sigma_{n} / \sigma_{D} \rightarrow \sigma_{N} / \sigma_{C}$ modeling accuracy (combine 10,13 for ${ }^{15} N^{12} \mathrm{C}$ ) & $\ell_{N}, a, b$ \\
\hline 17 & $Q_{m i n}^{2}$ for $P_{b} P_{t}$ ang. (decreased by $0.1 \mathrm{GeV}^{2}$ ) & $P_{b} P_{t}$ \\
\hline 18 & exclusive ep ${ }^{12} \mathrm{C}$ scaling region in $\Delta \phi$ (shifted by $2^{\circ}$ ) & $\overline{P_{b} P_{t}}$ \\
\hline 19 & elastic peak width in $W$ (10 MeV cut on each side) & $\boldsymbol{P}_{b} P_{t}$ \\
\hline 20 & $P_{b} P_{t}$ statistical error (not used; replaced by $41-52$ ) & $\boldsymbol{P}_{\mathbf{b}} \boldsymbol{P}_{t}$ \\
\hline 21 & $A_{\text {|lth }}$ elastic asymmetry value (older $G_{M}, G_{E}$ fits used) & $P_{b} P_{t}$ \\
\hline 22 & $\pi^{-}$contamination (modeled - see Section 4.1) & $C_{b c k g d}$ \\
\hline 23 & $e^{+} e^{-}$contamination (one std. dev. added to fit) & $C_{b c k g d}$ \\
\hline 24 & radiative corrections ( $5 \%$ variation assumed) & $A_{R C}, f_{R C}$ \\
\hline 25 & ${ }^{15} \mathrm{~N}$ polarization (leading order EST theory used) & $P_{N} *$ \\
\hline 26 & ${ }^{14} \mathrm{~N}$ contamination ( $2 \%$ contamination assumed) & $P_{N^{*}}$ \\
\hline 27 & deuteron contamination ( $0.5 \%$ contamination assumed) & $A_{\|}$ \\
\hline 28 & $F_{D F}$ models accuracy (determined by polynomial fit) & $F_{D F}$ \\
\hline 29 & beam energy $(E)(+2 \mathrm{MeV})$ & all \\
\hline 30 & radial momentum $\left(p_{r}\right)(+1 \mathrm{MeV})$ & all \\
\hline 31 & longitudinal momentum $\left(p_{z}\right)(+1 \mathrm{MeV})$ & all \\
\hline 32 & bin smearing effects (momenta $p_{r} / p_{z}$ randomly varied $\pm 17 / 20 \mathrm{MeV}$ ) & all \\
\hline 33 & accounting of possible false asymmetries ( $\triangle A=10^{-4}$ added) & $A_{\| 1}$ \\
\hline 34 & NOT USED (reserved for single-spin asymmetry tests) & - \\
\hline 35 & dead time error in count ratios $\left(10^{-7} n\right.$ added to counts) & all \\
\hline 36 & DIS fit of $A_{1}$ model (1 std. dev. added) & $\boldsymbol{A}_{\|}$ \\
\hline 37 & $A_{2}$ model (increased to Sofler limit (Eq. 2.35)) & $A_{11}, A_{1}, g_{1}$ \\
\hline 38 & resonance region fit of $A_{1}$ model (alternate fit used) & $A_{1 L}$ \\
\hline 39 & $F_{2}$ model (1 std. dev. added) & $A_{11}, g_{1}$ \\
\hline 40 & $R$ model (1 std dev added) & $A_{11}, g_{1}$ \\
\hline $41-52$ & $\begin{array}{l}\qquad P_{b} \bar{P}_{t} \text { statistical error; evaluated } \\
\text { independently for each bracket } 1-12 \text { (stat. err. added to } P_{b} P_{t} \text { ) }\end{array}$ & $\boldsymbol{P}_{b} P_{t}$ \\
\hline
\end{tabular}


error as a result from variations in these measurements. Alternate measurements of the ${ }^{12} \mathrm{C}$ target thickness $\ell_{C}$ yielded $0.225 \mathrm{~cm}$ and $0.24 \mathrm{~cm}$ [72], so the original value of $0.23 \mathrm{~cm}$ was increased by $0.01 \mathrm{~cm}$ to calculate the error. The carbon density $\rho_{C}$ was checked against a SLAC measurement of $2.2 \mathrm{~g} / \mathrm{cm}^{3}$ [72]. The value of $\rho_{C}$ was thus increased by roughly $2 \%$ to determine the error. The quantities $\rho_{C}$ and $\ell_{C}$ always appear multiplied together as $\rho_{C} \ell_{C}$ in dilution factor analysis, so this systematic error was analyzed in a single pass of the data by increasing this quantity (2).

The total Kapton material length $\left(\ell_{K}\right)$ had an approximate uncertainty of $10 \%$, due to perforation in the surface of the material [72], so the thickness was increased accordingly in reanalysis. Perforations in the foil could affect either a single target (3) or all targets (6); both cases were considered. Varying the thicknesses $\ell_{K}$ for the radiated cross-section method of analysis was simple. However, for the older (statistical) method of dilution factor analysis, the values of $f$ and $A, B, C$ and $D$ (defined in Sections 5.3.1 and 5.4.2) required appropriate alteration. Changing all foil thicknesses simultaneously was simple, and required only changing the overall value of $f \equiv \rho_{F} \ell_{F} / \rho_{C} \ell_{C}$ accordingly. To change the foil thickness exclusively on the carbon target required reworking the equations for $A$ through $D$ in Sections 5.4.2, 5.3.1 and 5.5.1, using a separately calculated value $f_{C}$ for the carbon target. This results in the substitutions

$$
1+f \rightarrow 1+f_{C}
$$

in all equations for $L, \ell_{A}$ and $F_{D F}$, and the substitution

$$
L+f \ell_{C} \rightarrow L+f \ell_{C}+L\left(f_{C}-f\right)
$$

in all the denominators of Eq. 5.38. While varying the thickness of the foils simultaneously (6) had little effect on the count ratios (and hence the dilution factor), the possibility of a perforation in a single foil (3) only was the largest single systematic error contribution resulting from target model uncertainties.

The aluminum shielding thickness was also assumed to have an equivalent uncertainty (7) in the (separate) determination of its related systematic error. This contributed very little to the overall 
error. ${ }^{4}$

Due to possible expansion as a function of temperature, the LHe density was given a wider margin of error. The density of LHe at $1.0 \mathrm{~K}$ is $0.145 \mathrm{~g} / \mathrm{cm}^{3}$, and at its boiling point of $4.2 \mathrm{~K}$, it rises to $0.125 \mathrm{~g} / \mathrm{cm}^{3}$. To approximate the density error for $\mathrm{LHe}$, a linear relation between density and temperature was assumed, along with a maximum temperature under $2.0 \mathrm{~K}$, giving approximately a $4 \%$ variance in the LHe density (5); this decrease was used in the error analysis. A similar rough calculation of the density of solid $\mathrm{NH}_{3}$ yields a density change of slightly less than $1 \%$. The density $\rho_{A}$ was thus given a variation of $1 \%$ to reflect the uncertainty (4).

\section{Derivation of $L$ and $\ell_{A}$}

The lengths of the $\mathrm{NH}_{3}$ and $\mathrm{LHe}$ are derived quantities. Their systematic errors are directly correlated functions of the other lengths and densities, so they are not varied independently to produce systematic errors. Rather, they are recalculated with each iteration of the analysis. However, any other factors of uncertainty that may affect the evaluation of $L$ and $\ell_{A}$ must also be considered. The values of $L$ and $\ell_{A}$ are evaluated by averaging the results determined by the ratios of $\mathrm{LHe}^{12} \mathrm{C}$ and $\mathrm{NH}_{3} /{ }^{12} \mathrm{C}$ target counts, respectively, and the input from a radiated cross-section model (see Sections 5.3.1 and 5.5.1), averaged over the inelastic kinematic region. The extent of the kinematic region is chosen, somewhat arbitrarily, as the part of the inelastic region where the measured value appears constant. Thus, we consider variations of the $W$-ranges over which $L$ and $\ell_{A}$ are measured.

The range given for the evaluation of $L$ in the main analysis is $\frac{2}{3}$ of the inelastic range between $W=1.08 \mathrm{GeV}$ and the upper limit chosen for the statistical evaluation of $F_{D F}$. For the evaluation of systematic errors, the whole inelastic region, up to the $F_{D F}$ upper limit, was used instead (8). For the regular evaluation of $\ell_{A}$, the entire inelastic range (from $\mathrm{W}=1.08$ to the upper limit) was used in the average. This was shortened to $\frac{2}{3}$ of this range for systematic error determination (9).

\footnotetext{
${ }^{4}$ The densities of solid Kapton and aluminum are very precisely known, so their uncertainty is not considered here.
} 


\section{LHe correction error for Brackets 10-12}

The last 3 brackets of data $(2.5+, 4.2+$ and $4.2-)$ required a raster-based correction to empty (LHe only) runs due to the presence of unknown material contaminating the lower half of the target (see Section 5.2.1). Accuracy in measurement of $L$ critically depends on the ratio of $\mathrm{LHe}$ to ${ }^{12} \mathrm{C}$ target counts, so it is important to consider the error on the multiplicative factor used to correct the empty target counts. To estimate this error, the multiplicative factor based on the ${ }^{12} \mathrm{C}$ target cell raster was replaced with the factor based on the ammonia $\left(\mathrm{NH}_{3}\right.$ and $\left.\mathrm{ND}_{3}\right)$ targets listed in Table 5.4 (1). Note that this systematic error applies only to these last 3 brackets.

The systematic errors outlined above affect dilution factors. Both the modeled dilution factor quantity (used for unpolarized background subtraction in $A_{\|}$) and the statistical model used in the elastic region (for inclusive method $P_{b} P_{t}$ calculation) are affected. The various errors affect the two regions differently, but the total ratio of the target model effects is approximately equal $(\sim 2.5 \%)$ for both methods of dilution factor calculation (see Figure 7.8).

\subsubsection{Errors in dilution factor model (28)}

The dilution factor used for subtraction of unpolarized background material was generated using a world data model of unpolarized cross-sections (see Section 2.8). Unfortunately, the systematic errors on this model, as a function of $Q^{2}$ and $W$, are not well-defined, so an external method is required to estimate the veracity of this model.

\section{Contrasting dilution factor models}

Fortunately, a statistical construction of the dilution factors, using the actual data from the empty, carbon and ammonia targets (with the more spurious assumption that $\sigma_{C}=3 \sigma_{\mathrm{He}}$ ), was made using an older method (see Section 5.6). A comparison between two viable models is a good way to estimate the magnitude of their systematic error. However, the older method of calculating $F_{D F}$ resulted in large statistical fluctuations from bin to bin. A mere subtraction of results using either dilution factor model would result in an error dominated by these fluctuations. This behavior is not characteristic of a proper systematic error. Also, since the modeled dilution factor was extrapolated 


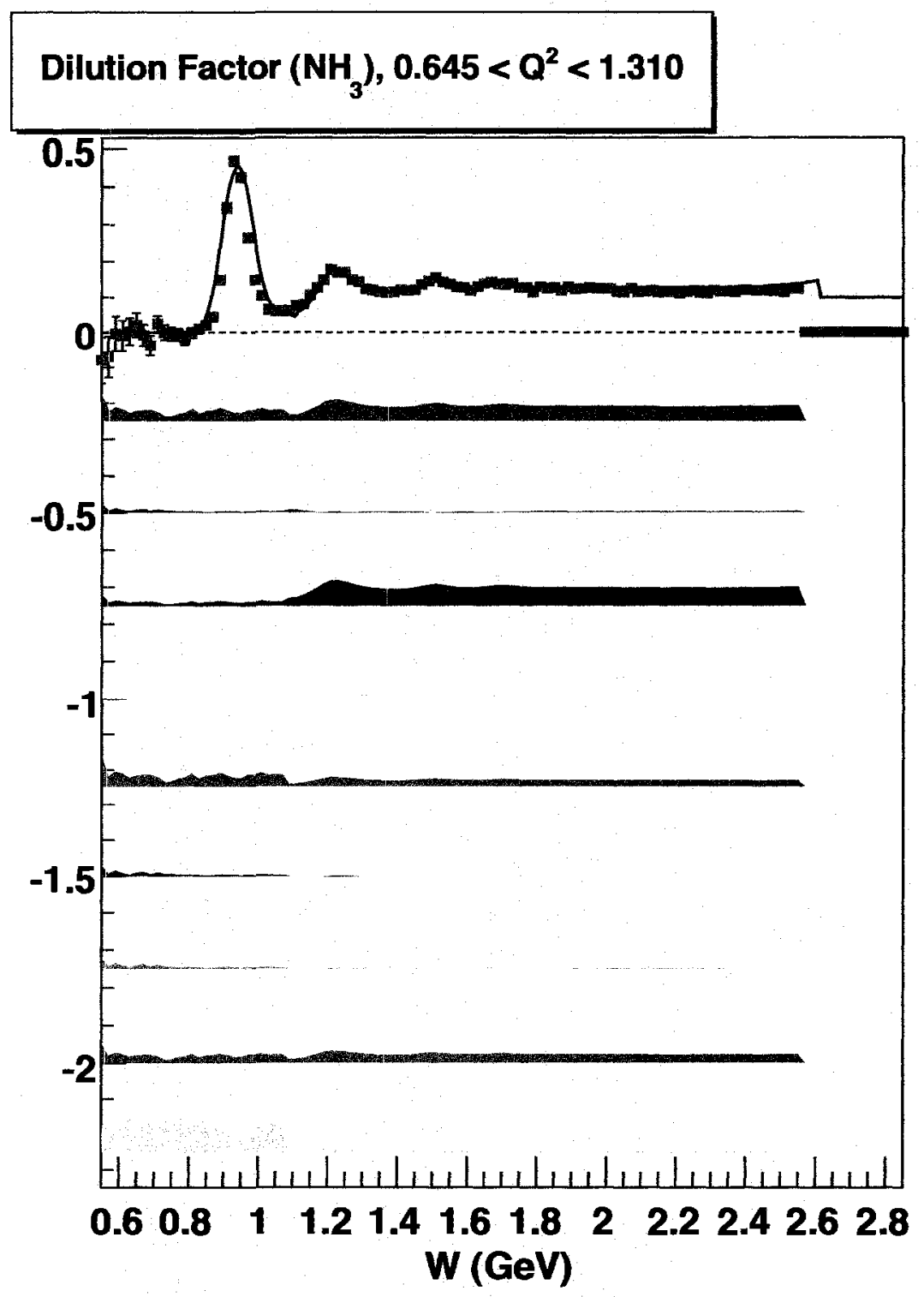

Figure 7.8: Dilution factor for a selected $Q^{2}$ range for $4.2 \mathrm{GeV}$ outbending data, showing the relative size of systematic errors related specifically to the target model. All shown error values bars are multiplied by a factor of 25 for visibility. Different methods for calculating the dilution factor were used below and above $W=1.08 \mathrm{GeV}$; the sizes of the errors change accordingly at this boundary. From top to bottom are errors due to the empty target correction factor (red), carbon length/density (light green), ${ }^{12} \mathrm{C}$ target Kapton foil thickness (blue), $\mathrm{NH}_{3}$ density (yellow), LHe density (magenta), average Kapton foil thickness (cyan), Al foil thickness (dark green), $L$ averaging range (purple) and $\ell_{A}$ averaging range (gray). 
to bins outside the fiducial region of the statistical dilution factor, another means of estimating the error in these extended kinematic regions was required.

To remedy these issues, the "smooth" cross-section derived $F_{D F}$ model used in analysis was adjusted to the average levels of the older, statistically derived dilution factor by means of a fit by a multiplicative factor. Fitting all inelastic regions simultaneously proved difficult, especially as the fit function approached the elastic region. Through trial and error, a polynomial (nonphenomenological) fit was found that realized the differences between the two models, but remained "well-behaved" in the extrapolated regions. The difference between the fit function and the modeled dilution factor defined the systematic error.

First, for each of the 11 data brackets, an overall multiplicative constant $r$ was used to fit the modeled $F_{D F}$ to the statistically derived $F_{D F}$, from the range $W=1.15 \mathrm{GeV}$ to the end of the DIS region. Data for $Q^{2}<0.077 \mathrm{GeV}^{2}$ were excluded from the whole fit, due to unpredictable (and obviously non-physical) behavior of the statistical dilution factor.

With this overall factor $(r)$ held constant, a second fit of the form

$$
F_{D F s y s} \equiv\left(r+s W+t W^{2}+u W^{3}\right) F_{D F}
$$

was used in the region $1.15 \mathrm{GeV}<W<1.80 \mathrm{GeV}$, where $r$ - $u$ were determined by a $\chi^{2}$ minimization fit of $F_{D F s y s}$ to the statistically derived dilution factor for each data bracket. Then, the extrapolated fit function

$$
\begin{gathered}
F_{D F s y s} \equiv\left(r+s W+t W^{2}+u W^{3}\right) F_{D F} \quad(W<1.80 \mathrm{GeV}) \\
F_{D F s y s} \equiv r F_{D F} \quad(W \geq 1.80 \mathrm{GeV})
\end{gathered}
$$

was used in place of $F_{D F}$ for the remainder of the analysis iteration to determine the systematic error (28). Values of $\chi^{2}$ and fit coefficients for each bracket are shown in Table 7.6. Figure 7.9 shows sample bins comparing the (regular) systematic error to the (systematic error) fit dilution factors for two different data sets.

Values of the error varied with the beam energy, with the highest errors in the resonance region at the lowest beam energies, as one would expect from knowledge of the unpolarized cross- 

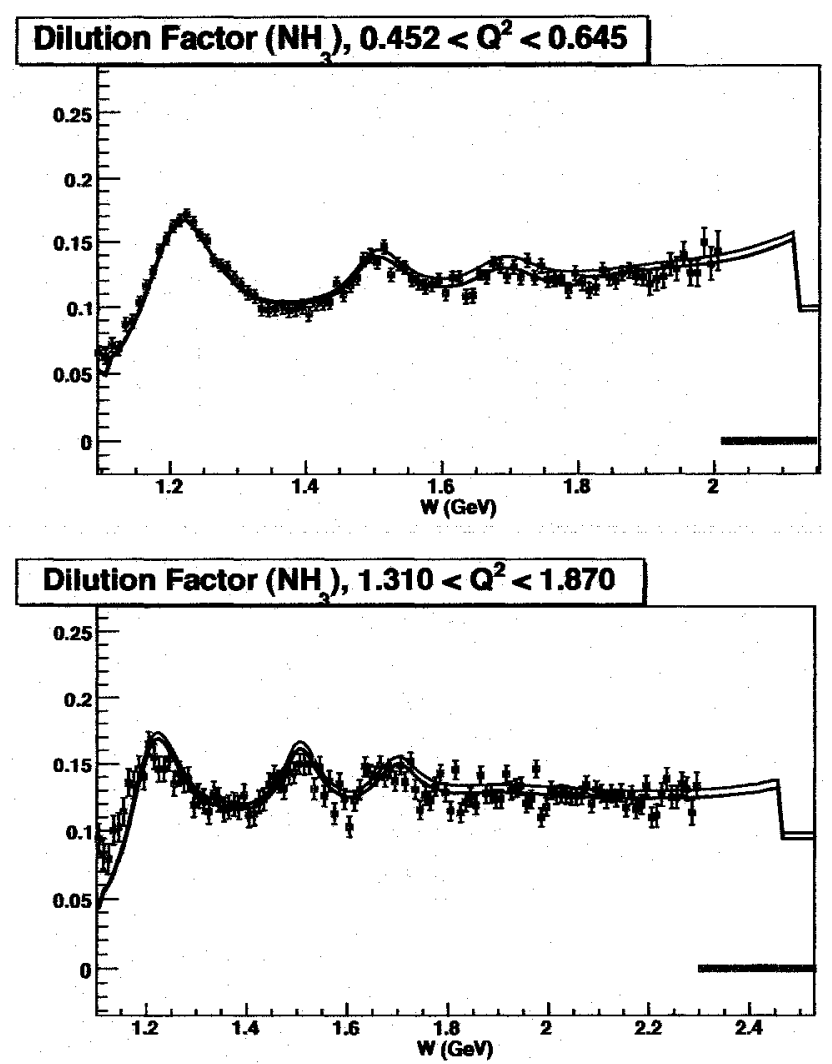

Figure 7.9: Dilution factors fit to the data for $2.5 \mathrm{GeV}$ outbending (top) and $4.2 \mathrm{GeV}$ outbending (bottom) data. The modeled dilution factor (blue line) is compared to the parametrized dilution factor (black line), which is fit to the statistically-determined dilution factor (red) for an approximate determination of the magnitude of the systematic error. 
sections. Typically, the total error amounted to $\sim 3-4 \%$ of the dilution factor, and hence the measured asymmetry. The magnitude of this error $(\times 5)$ can be seen as the light green error bar in Figure 7.7.

Table 7.6: Fit parameters and $\chi^{2}$ values (per degree of freedom) for each data bracket for determining the (approximate) dilution factor cross-section model errors. See the text for more details. To leading order, the value of $r$ gives the ratio of agreement between the two models.

\begin{tabular}{|c|c|c|c|c|c|c|}
\hline Bracket & Set & $r$ & $s$ & $t$ & $u$ & $\chi^{2} /$ ndf \\
\hline 1 & $1.6+$ & 0.948 & -0.060 & 0.091 & -0.034 & $1704 / 435$ \\
\hline 2 & $1.6-$ & 0.957 & 0.318 & -0.240 & 0.001 & $678 / 445$ \\
\hline 3 & $5.76-$ & 0.970 & -0.926 & 1.168 & -0.368 & $826 / 761$ \\
\hline 4 & $5.73-$ & 0.986 & -0.992 & 1.380 & -0.460 & $1370 / 765$ \\
\hline 5 & $5.7+$ & 0.984 & -0.711 & 0.861 & -0.262 & $494 / 529$ \\
\hline 6 & $2.3+$ & 0.973 & -0.516 & 0.784 & -0.284 & $692 / 571$ \\
\hline 7 & $5.6+$ & 0.983 & -0.693 & 0.858 & -0.263 & $552 / 542$ \\
\hline 8 & $1.7-$ & 0.971 & 0.224 & -0.056 & -0.082 & $2338 / 508$ \\
\hline 9 & $2.5-$ & 0.962 & 0.362 & -0.342 & 0.070 & $1986 / 822$ \\
\hline 11 & $4.2+$ & 0.957 & -0.594 & 0.781 & -0.253 & $643 / 527$ \\
\hline 12 & $4.2-$ & 0.955 & -0.109 & 0.167 & -0.059 & $2510 / 890$ \\
\hline
\end{tabular}

\section{Correlation with other systematic errors}

The most major drawback to this method of determining the systematic error is the possibility of correlations to other systematic errors (specifically, those errors resulting from the target model, outlined in the previous subsection). Should an error or shift in one of the target modeling parameters cause unequal shifts between the values of the dilution factors in the statistical and modeled methods, then inclusion of this model error can cause a significant overcalculation of the systematic error.

To rectify this issue, the fit factor $r$, which, to leading order, represents the overall ratio of the two methods of finding the dilution factor, was recalculated for each of the first 9 error indices relating to the target model (i.e. 1-9 in Table 7.5). In most cases, absolutely no significant shift could be seen in the fit value of $r$ when the parameters would change, indicating little to no correlation to these errors. However, varying the $W$-range of the averaging of the ammonia target length $\ell_{A}(9)$ had a very significant effect on the difference between the two models. This occurs mostly due to the $W$-range sensitivity of the $\ell_{A}$ measurement in the older (statistical) model, a problem not seen in 
the smoother radiated cross-section model. In fact, varying the fit range for $\ell_{A}$ between the decided systematic error range could change the value of $r$ by a few percent, in the most extreme cases. Clearly, a large portion of the model error was correlated with the uncertainty in ammonia target length, meaning that this significant source of error was propagated into the total error twice.

To remedy the situation, it was assumed that the variation of the overall differential multiplicative factor $r$ can be attributed to this uncertainty in $\ell_{A}$.This error is already accounted for (9), so the averaging range of $\ell_{A}$ in $W$ (for the statistical method only) was varied between $33 \%$ and $100 \%$ of the inelastic region ${ }^{5}$, and optimized for each set so that $r=1.00$ (or as close to possible to 1.00) when the fit is made. (This is done for systematic error index (28) only - the normal range for $\ell_{A}$ is still used in the main analysis and in determination of other errors.)

Adding this constraint into the fit reduced the systematic error by an approximate factor of 2 , presumably by removing any correlation between this error calculation and that caused by errors in the calculation of $\ell_{A}$. The highest remaining errors are in the resonances at lower beam energies, an observation consistent with the expected errors on the cross-sectional models.

\subsubsection{Uncertainties in determination of beam $\times$ target polarization $(10,13,17-$} 21)

The systematic errors due to uncertainties in the beam and target polarization products must also be included in the final error estimate. By far, the largest contribution to the uncertainty of $P_{b} P_{t}$ is the statistical error on this quantity. To account for the statistical uncertainty, the final statistical error is merely added to the value of $P_{b} P_{t}$ for each data bracket, and the asymmetries are reevaluated with the adjusted $P_{b} P_{t}(20)$. This error is considered in more detail later. Though the statistical uncertainty is the dominant contribution to the error on $P_{b} P_{t}$, other sources of systematic error were also considered.

For inclusive data, the background subtraction in the elastic peak used the older (statistical) $F_{D F}$ model, which relied upon the ${ }^{15} \mathrm{~N} /{ }^{12} \mathrm{C}$ cross-section model ratio for its calculation. To estimate the systematic error due to the model used in background subtraction for the elastic peak, cross-section

\footnotetext{
${ }^{5}$ Recall that the range used for $\ell_{A}$ calculation in this model was $66 \%$ of the inelastic region, with $100 \%$ of the range used for systematic error estimation. An additional $33 \%$ allows for a variation (roughly) consistent with the assumed systematic error range.
} 
ratio substitution

$$
\frac{\sigma_{N}}{\sigma_{C}} \rightarrow \frac{7}{6}+\frac{1}{6} \frac{\sigma_{n}}{\sigma_{D}}
$$

was used to give a rough estimate on the background error (10). Apart from this, the $\sigma_{n} / \sigma_{D}$ crosssection made a minimal contribution of in determination the ammonia target length $\ell_{A}$ used to generate the elastic region dilution factor (see Eq. 5.62). As an estimate of the systematic error on this modeled quantity, measured in the inelastic region only, the substitution

$$
\sigma_{n} / \sigma_{p} \rightarrow 1-0.8 x
$$

(with $x=Q^{2} / 2 M \nu$ ) served as a viable substitution for the neutron/proton cross-section ratio [127]. Assuming that the deuteron is just an $n+p$ combination, this gives the approximation (13) ${ }^{6}$

$$
\sigma_{n} / \sigma_{D} \rightarrow(1+1 /(1-0.8 x))^{-1}
$$

Both cross-section ratio models, which affect the background removal of inclusive events through the elastic region dilution factor, made only a small contribution to the systematic errors $(10,13)$.

For exclusive data, the background subtraction was calculated by the scaling of exclusive ep carbon to ammonia data in a $\Delta \phi$ region safely away from the elastic peak (18). For systematic error calculation, this region was shifted $2^{\circ}$ to the outside of the peak, so that Eq. 6.45 was changed to

$$
4^{\circ}<|\Delta \phi|<8^{\circ}
$$

This was shown to have small effects on $P_{b} P_{t}$, due to the already small background for exclusively selected $e p$ events.

Other variations to parameters used to determine $P_{b} P_{t}$ were tried, as well. Narrowing the width of the $W$-cut on the elastic peak by $10 \mathrm{MeV}$ on each side resulted in a measurable error at larger beam energies (19). Other adjustments were considered, as well. Lowering of the minimum $Q^{2}$ bin used in evaluating the elastic asymmetry (from 0.2 to $0.1 \mathrm{GeV}^{2}$ ) (17), and changing the theoretical

\footnotetext{
${ }^{6}$ This approximation serves well for inelastic events only. Elastic events, (which, for the $\ell_{A}$ calculation, are inconsequential to the final results) do not fit this approximation, due to Fermi smearing in the elastic peak.
} 
asymmetry within the (very narrow) uncertainty given by our knowledge of $G_{M}$ and $G_{E}$ and the kinematic accuracy of $Q^{2}(21)^{7}$ produced negligible errors.

The comparative effects of the $P_{b} P_{t}$ systematic errors (except for the statistical uncertainty) on the measurement of $A_{\|}$are shown in Figure 7.10. Because only exclusive $P_{b} P_{t}$ values were used in this analysis, only the $\phi$-scaling factor change (18) and $W$-peak width change (19) have any significant effects in these plots.

\subsubsection{Statistical uncertainty on $P_{b} P_{t}(41-52)$}

Simply adding the statistical error to each $P_{b} P_{t}$ product (20) produced only a rough estimate of the effects of this uncertainty. This method was later replaced with a more thorough method of evaluating this error. Because this error is purely statistical in nature, simply adding the upper bound to all the $P_{b} P_{t}$ values simultaneously creates a false additive correlation between these errors, likely overestimating the error measurement on $A_{1}+\eta A_{2}$. On the contrary, errors on measurements between the data, such as linear regression for the determination of $A_{2}$, can be underestimated. ${ }^{8}$ Therefore, this crude estimate was replaced with a lengthier but more cautious evaluation.

Twelve new additional error indices (41-52) were defined, representing perturbations of $P_{b} P_{t}$ by a standard deviation for each individual bracket prior to combination. The analysis was then run 12 more times. Errors were added in quadrature to find the total systematic error due to this statistical effect. Figure 7.11 shows the addition of statistical errors for $5.7 \mathrm{GeV}$ data, and a comparison between this and the more crude method $(20)$ of evaluating the error. It is important to note that a more exact evaluation of this particular error is possible because it the only source of systematic uncertainty in this analysis than can be evaluated precisely, because of its purely statistical origin.

\subsubsection{Miscellaneous background subtraction errors (22-27)}

Effects due to the presence of other backgrounds also required consideration, including errors due to the effects of $\pi^{-}$and $e^{+} e^{-}$contamination, radiative corrections, and errors due to polarized

\footnotetext{
${ }^{7}$ Specifically, an older empirical fit of $G_{M}$ and $G_{E}[13]$ was used and the bin median value of $Q^{2}$ was substituted for the asymmetry-weighted average of $Q^{2}$.

${ }^{8}$ The latter underestimation can occur because the assumed correlation perturbs all asymmetries in the same direction for the error evaluation, whereas the statistical $P_{b} P_{t}$ errors can move the true values in different directions.
} 

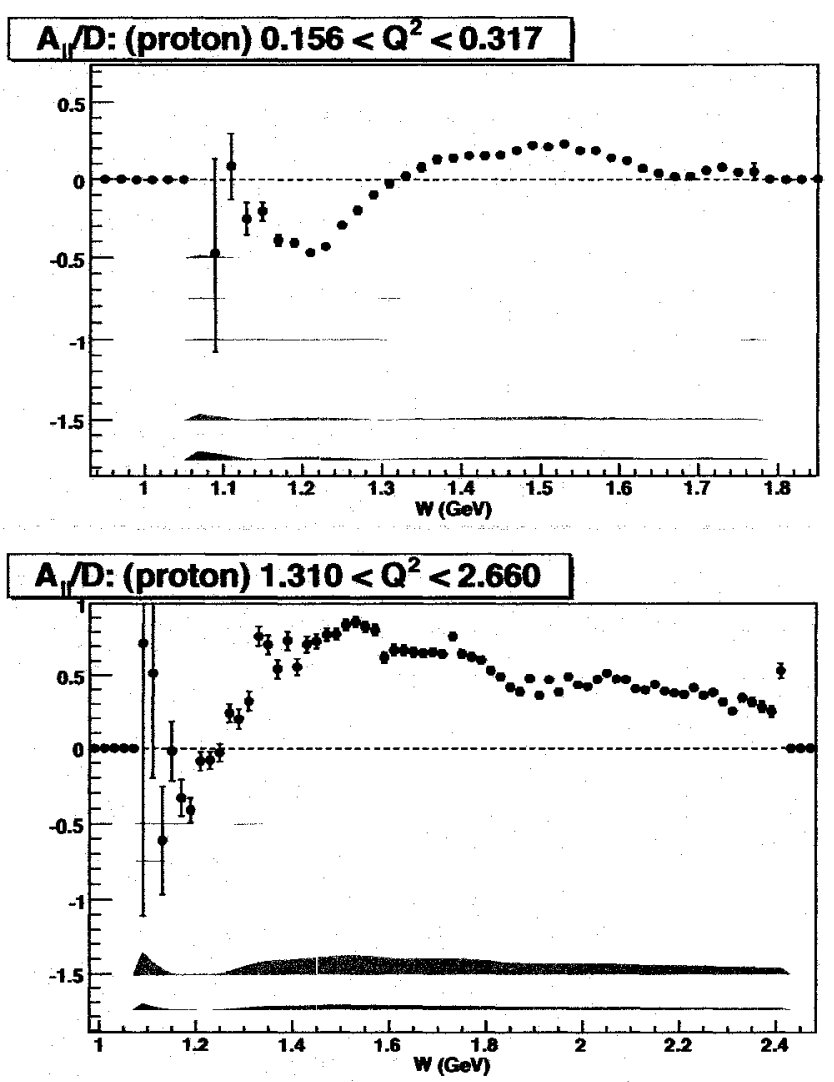

Figure 7.10: $A_{1}+\eta A_{2}$ showing the effects of systematic errors on $P_{b} P_{t}$ measurements, for 1.6 $\mathrm{GeV}$ (top) and $4.2 \mathrm{GeV}$ (bottom) data. Systematic errors are multiplied by a factor of 25 for visibility. Shown, from the top down, are the errors due to the ${ }^{15} \mathrm{~N} /{ }^{12} \mathrm{C}$ cross-section model (red), the $\mathrm{n} / \mathrm{D}$ cross-section model (light green), low $Q^{2}$ limit (blue), $\Delta \phi$ exclusive background subtraction range (yellow), elastic peak width (magenta), and the elastic asymmetry model (cyan). The $P_{b} P_{t}$ statistical errors, the largest individual systematic effect, are not shown here; see Figure 7.11 for these. 

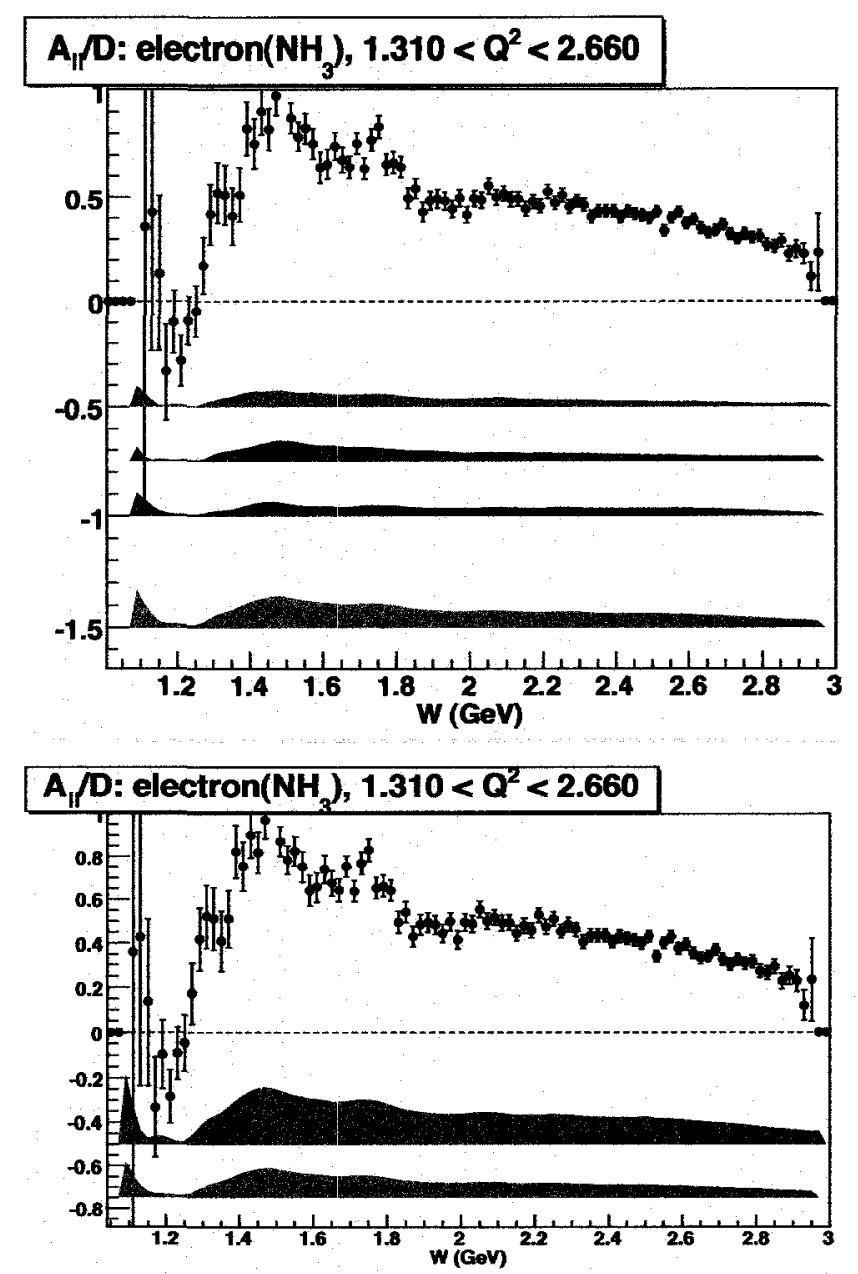

Figure 7.11: $A_{1}+\eta A_{2}$ showing the effects of systematic errors due to statistical errors in $P_{b} P_{t}$ for the $5.7 \mathrm{GeV}$ data. Systematic errors are multiplied by a factor of $\mathbf{2 5}$ for easy visibility. The top plot shows, from top to bottom, the individual effects of the 5.76-(red), 5.73- (green), 5.7+ (blue) and 5.6+ (yellow) set $P_{b} P_{t}$ errors, with the quadrature-added sum shown (at the bottom) in magenta. The bottom plot shows a comparison bewteen the crude (red, top) and proper (magenta, bottom) methods of error evaluation, demonstrating that the crude method (index 20) substantially overestimates the systematic error. 
background and isotopic contaminations. The relative effects of these errors on the measurement of $A_{1}+\eta A_{2}$ can be seen in Figure 7.12.

$\pi^{-}$and $e^{+} e^{-}$correction errors

The majority of $\pi^{-}$contamination was removed through the use of SC/CC track matching cuts (see Section 4.1). The approximate remaining background was calculated in bins of $\theta$ and $p$ as according to Section 4.1.5, and a curve of the exact same form used for $e^{+} e^{-}$background removal (Eq. 6.13) was fit to the remaining background to determine $R_{\pi}$. The variation in the the asymmetry due to this approximate background could then then be calculated as

$$
A_{\text {corr }}=\frac{A_{r a w}-R_{\pi} A_{\pi}}{1-R_{\pi}}
$$

just as for the $e^{+} e^{-}$correction, with $A_{\pi} \approx 0$. This asymmetry, now including a (very approximate) "correction" for $\pi^{-}$contamination, was subtracted from the normally-determined asymmetry to yield the pion contamination error (22). ${ }^{9}$

To calculate the error for the pair-symmetric correction, the same correction described in Section 6.1 was applied, except that one standard deviation of a flat (slope $=0$ ) linear fit to the contamination across $\theta$ (Figure 6.2), determined individually in each $p$ bin, was added to the overall contamination at all $\theta$. The resulting difference in the final asymmetries determined the systematic error (23).

Systematic error contributions from both of these sources were generally small. The total error from both these sources was generally less than $1 \%$ of the total asymmetry. ${ }^{10}$

\section{Radlative correction errors}

Radiative corrections (Section 6.4) on the asymmetry $A_{||}$remove higher-order radiative terms from the desired Born asymmetry. A rigorous treatment of the systematic errors due to radiative correc-

\footnotetext{
${ }^{9}$ This correction is applied only as as a systematic error because there is no secure method of parametrizing the exact number of remaining pions. This equation serves only as a rough approximation.

${ }^{10}$ Values for pion contamination error are somewhat higher in the resonance region at high $Q^{2}$ - see Figure 7.12.
} 
tions requires rerunning of the RCSLACPOL software using differing target model dimensions and alternate parametrizations of structure function models, just as is described in this section for the main analysis. The resulting differences to the terms $A_{R C}$ and $f_{R C}$ can then be added in quadrature to determine the relative systematic errors on these corrective quantities.

At the time of the writing of this thesis, calculation of the systematic errors in the latest version of the ACSLACPOL code was not yet complete, due to ongoing modernization of the models. Past analyses ([2] and [46], for example) realized no more than about a $5 \%$ error in the total radiative corrections. So, as a temporary solution, a factor of 1.05 was multiplied by $A_{R C}$ and $\left(1-f_{R C}\right)$ to approximate the systematic error (24), until such time that upgraded models with more thorough corrections can be included. It can be seen, with this estimate, that radiative corrections present the largest error due to miscellaneous backgrounds, and, in fact, become the limiting factor in measurement of the asymmetries near the inelastic threshold $(W=1.077 \mathrm{GeV})$.

\section{Contamination and background polarization errors}

A polarization correction to $A_{\| \mid}$accounts for polarized ${ }^{15} \mathrm{~N}$ in the background material (Section 6.3). To estimate the magnitude of the error on this small correction, it was considered that the SLAC parametrization might not accurately describe the polarization response in this experimental configuration. The SLAC parametrization of the ${ }^{15} \mathrm{~N}$ polarization $(\mathrm{Eq}$. 6.54) was replaced by the simpler model of leading order EST theory (Eq. 6.53) (25). The resultant change in the correction was small, but significant enough to warrant inclusion ( $\sim 1 \%$ of the asymmetry).

The effects of impurities in the ${ }^{15} \mathrm{NH}_{3}$ material (specifically ${ }^{14} \mathrm{~N}$ and deuterium) were also taken into account. The target material contamination was limited to $<2 \%{ }^{14} \mathrm{~N} /{ }^{15} \mathrm{~N}$ [65]. The residual polarization of ${ }^{14} \mathrm{~N}$ cannot be measured as accurately as that of ${ }^{15} \mathrm{~N}$. Its polarization response is roughly $2 \%$, give or take about a $10 \%$ error [70]. To account for this, we calculate

$$
P_{N^{*} \text { sys }} \equiv P_{N^{*}}(1.00-\ell)+\iota\left(P_{\mathbf{1 4} N}+\sigma_{14}\right)
$$


where $\iota=0.02$ is the contamination, $P_{14}=0.02$ is the ${ }^{14} \mathrm{~N}$ polarization, and $\sigma_{14}=0.10 \times 0.02$ is the uncertainty in the ${ }^{14} \mathrm{~N}$ polarization. This value of $P_{N^{*} \text { sys }}$ is substituted in place of $P_{N^{*}}$ to evaluate the systematic error, which turns out to be a negligible contribution to the asymmetry.

The isotopic contamination of deuterium in the $\mathrm{NH}_{3}$ was known to be considerably less than $1 \%$ of the hydrogen content. To estimate the maximal effects of polarized deuterium, a contamination of $0.5 \% \mathrm{p} \rightarrow \mathrm{d}$ was assumed. The final value of $A_{\| \mid}$was then adjusted for this by assuming the deuteron double-spin asymmetry was given by

$$
A_{\| D} \approx A_{1(D)} D
$$

where $D$ is the depolarization (Section 1.1.3) and $A_{1}$ for the deuteron is modeled. The necessary adjustment is then easily calculated by diluting the proper asymmetry by the contamination contribution and subtracting a term representing the contribution of the polarized deuterium: ${ }^{11}$

$$
A_{\| s y s}=\frac{A_{\|}}{1-\lambda}-\frac{\lambda}{(1-\lambda)} \frac{P_{D}}{P_{t}} A_{\| D}
$$

where the deuteron polarization $P_{D} \approx 0.35, \lambda=0.005$ is the contamination, and $P_{t}$ is the target polarization, calculated in Section 6.3. Again, the total error contribution is quite small (less than $1 \%)$.

\subsubsection{Kinematics resolution errors (29-32)}

Another source of error not yet considered is the error on the kinematics of identified particles. This includes both errors on the accuracy of the electron energy and momenta, as well as "bin smearing" effects, resulting from statistical uncertainties in measurement. To measure the approximate effects of these errors (29-32), the entire analysis was rerun from the start for each of these error index options, with the specified inaccuracies/uncertainties added to the quantity in question. The resulting asymmetry differences naturally showed statistical variations, requiring averaging over bins (see

\footnotetext{
${ }^{11}$ The ratio of polarizations in the latter arises due to the fact that the proton and deuteron have a differing polarization response.
} 

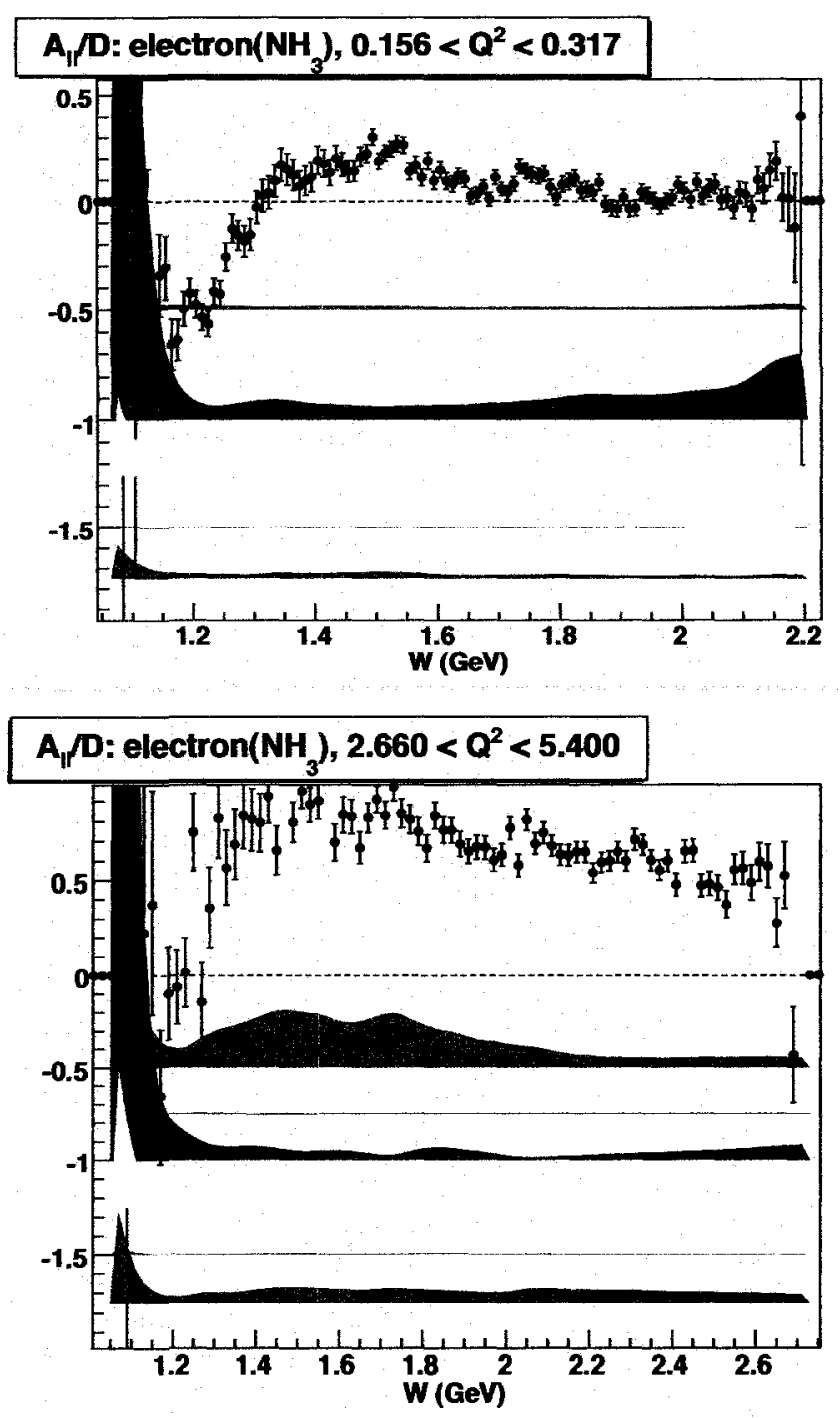

Figure 7.12: $A_{1}+\eta A_{2}$ showing the effects of miscellaneous background errors for $2.5 \mathrm{GeV}$ (top) and $5.7 \mathrm{GeV}$ (bottom) data. Systematic errors are multiplied by a factor of 25 for visibility. Shown, from top to bottom, are the errors due to $\pi^{-}$contamination (red), $e^{+} e^{-}$contamination (green), radiative corrections (blue), ${ }^{15} \mathrm{~N}$ polarization (yellow), ${ }^{14} \mathrm{~N}$ contamination (magenta) and deuteron contamination (cyan). Radiative correction uncertainties tend to dominate at lower beam energies, while remaining pion contamination dominates at higher energies. 
Section 7.2.10) to obtain average systematic errors. ${ }^{12}$

\section{Errors on momenta and beam energy}

As mentioned in Section 4.2.4, Hall A beam energy measurements provide an accuracy $\Delta E / E$ of about $2 \times 10^{-4}$ at $5 . \times \mathrm{GeV}$ energies, implying an uncertainty of less than $2 \mathrm{MeV}$. ${ }^{13}$ Thus, to determine the maximal uncertainty due to this factor, $2 \mathrm{MeV}$ was added to the incident energy of the electrons (29) (after all kinematic corrections).

The momenta $p_{x}, p_{y}$ and $p_{z}$ were corrected with the kinematic corrections package (see Section 4.2.8) to, in most cases, an accuracy of $\sim 1 \mathrm{MeV}$. To estimate the error caused by the remaining (very small) inacurracy, $1.0 \mathrm{MeV}$ was added to the momenta. Because of the symmetry in $\phi$ for inclusive scattering, the $x$ and $y$ components of $p$ could be tested together by adding $1.0 \mathrm{MeV}$ to $p_{r}=\sqrt{p_{x}^{2}+p_{y}^{2}}(30)$, while the $1.0 \mathrm{MeV}$ inaccuracy was added to $p_{z}$ in a separate run of the analysis (31). These tests attribute a small collective error due to inaccuracies in the measurement of the electron kinematics.

\section{Bin smearing error}

With the approximate inaccuracies in kinematic measurement propagated through analysis to determine systematic errors, it remains to determine the effects of inprecision in the momentum determination, or bin smearing effects, caused by the fact that the measured momentum of a particle, in actuality, forms a finite uncertainty distribution about the true momentum (32).

Proper analysis of bin smearing effects is a complicated process. For this analysis, where the expected uncertainty from the effect is small compared to the sum of other errors, it suffices to make an approximation resulting in an effect of similar magnitude. To start with, the standard deviations on (corrected) measurements of $p_{x}, p_{y}$ and $p_{z}$ were determined, by a standard Gaussian fit

\footnotetext{
${ }^{12}$ Only events used to construct asymmetries (those in the ASYM/ file list) were tested for errors. Events used to determine $F_{D F}$ (those in the RATE/file list) were either fit or averaged over large numbers of bins, thus minimizing the effects of shifted kinematics and/or bin smearing. The miniscule error on $F_{D F}$ due to kinematic effects was not considered here.

${ }^{13}$ This factor becomes even smaller for lower beam energies.
} 
to elastic ep events, to be $\Delta_{p_{x}} \approx \Delta_{p_{y}} \approx 17 \mathrm{MeV}$ and $\Delta_{p_{z}} \approx 30 \mathrm{MeV} .{ }^{14}$

To estimate the effects of the error, the data were reanalyzed, but this time, for each event, different randomly generated values ranging between $-17 \mathrm{MeV}$ and $+17 \mathrm{MeV}$ were added to each $p_{x}$ and $p_{y}$, while a value between $-20 \mathrm{MeV}$ and $+20 \mathrm{MeV}$ was added to $p_{z}$. This effectively "smears" the data a second time by an uncertainty similar to the natural smearing of the CLAS detector. The asymmetry $A_{\|}$is then recalculated, with the difference producing the approximate error due to bin smearing.

Note that this is, of course, not technically a proper way to calculate the exact magnitude of the effect, but if one makes the first-order assumption that the magnitude of the bin smearing effects is linear with respect to the magnitude of the uncertainty, it provides a reasonable approximation of this effect.

Because of the statistical nature of the binning process in $W$ and $Q^{2}$, these systematic errors (29-32), more than any other, require "smoothing" over local bins (see Section 7.2.10), so that the systematic effects of these errors can be separated from the statistical "forest" that results from rebinning. The total effects of the kinematic uncertainty errors were small, but worth considering, amounting $2-3 \%$ of the measured asymmetry. Errors due to these effects, with and without "smoothing" over the bins, are shown in Figure 7.13.

\subsubsection{False asymmetry and Faraday cup errors $(33,35)$}

A careful study of Faraday cup asymmetries (by S. Kuhn and N. Guler) ensured that there were no correlations between the spin charge asymmetry (Eq. 3.2) and the measured double-spin asymmetry (Eq. 3.1). Such would imply a false single-spin asymmetry due to the Faraday charge measurement. Final results of the study showed no correlation to a certainty $|\delta A / A|<10^{-4}[64]$.

There are other possible sources of false asymmetries, such as the parity-violating electroweak asymmetry in ep-scattering, though its magnitude $\left(\sim 10^{-5}\right)[31]$ is considerably smaller than the asymmetry uncertainty attributable to Faraday charge measurement. To estimate the maximum possible error resulting from false (single-spin) asymmetries, we assume a raw asymmetry of $10^{-4}$,

\footnotetext{
${ }^{14}$ These results are from an analysis of 4.2 GeV EG1 data by N. Guler. Optimal results cited in Ref. [115] are only slightly smaller in magnitude (14 MeV and $20 \mathrm{MeV}$, specifically).
} 

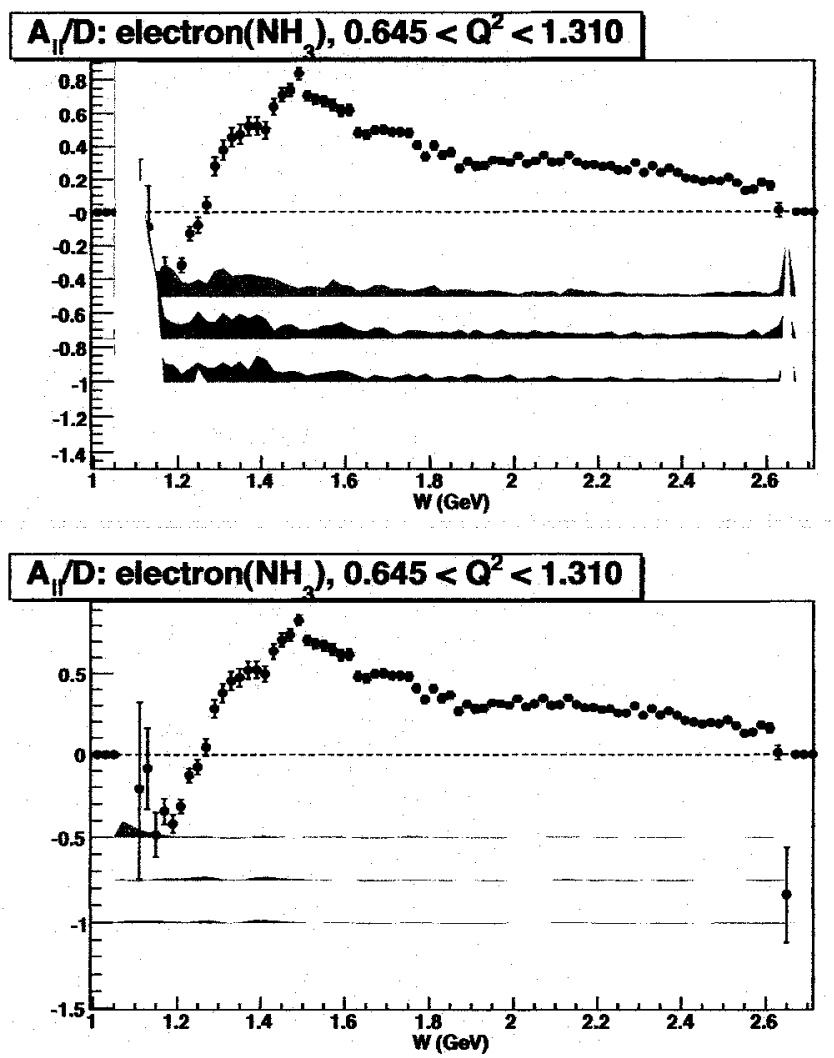

Figure 7.13: $A_{1}+\eta A_{2}$ showing the magnitudes of kinematic errors for $4.2 \mathrm{GeV}$ data, before (top) and after (bottom) bin "smoothing" by iteratively averaging over adjacent bins. Systematic errors are shown actual size. Removing statistical fluctuations is essential before averaging errors of this type. Shown, from top to bottom, are the errors due to beam energy determination (red), $p_{r}$ determination (green), $p_{z}$ determination (blue), and bin smearing (yellow). Note the error is largest where the asymmetry slope is steepest, as would be expected from a shifting of bin contents. 
divided by the dilution factor $F_{D F}$ and $P_{b} P_{t}$ (estimated as 0.4 ), and add it to the measured asymmetry $A_{\|}$, yielding what would initially appear to be a 1-2\% error on $A_{\|}$.

However, this is not the end of the story, as this is a single-spin asymmetry, meaning that states of opposing HWP and target polarization will act to cancel the asymmetry. Thus, to estimate the false asymmetry contamination, we rerun the analysis, but assume all target polarizations to be positive, and the half-wave-plate to be permanently removed (33). ${ }^{15}$ This reduces resulting asymmetry to considerably less than $1 \%$ of $A_{\|}$, due to the cancellation of opposing polarizations.

Another considered error source is the mathematical error that results from assuming the sum of the ratios is equal to the ratio of the sums, when calculating helicity-independent count rates. In other words, the analysis, particularly the calculation of backgrounds (see Eq. 5.74) has assumed that

$$
n=2 \frac{N_{b}^{+}+N_{b}^{-}}{2 F C^{-}+\Delta} \doteq \frac{N_{b}^{+}}{F C^{-}+\Delta}+\frac{N_{b}^{-}}{F C^{-}}
$$

where $N_{b}$ symbolizes background counts (of each helicity) and $\Delta$ is the difference between the positive and negative helicity-gated Faraday cup counts. This tentative relation ( $\doteq$ ) is an equality so long as no asymmetry exists in the background counts. If there is an asymmetry in the background, then

$$
N_{b}^{+}=N_{b}^{-}+\delta N_{b}
$$

Thus we have

$$
n=\frac{2\left(2 N_{b}^{-}+\delta N_{b}\right)}{2 F C^{-}+\Delta} \doteq \frac{N_{b}^{-}+\delta N_{b}}{F C^{-}+\Delta}+\frac{N_{b}^{-}}{F C^{-}}
$$

This difference $\Delta$ is small, so by working out a binomial expansion of the denominators of both sides, one can easily calculate a difference

$$
\delta n \approx-\frac{1}{2} \frac{\delta N_{b}}{F C^{-}}\left(\frac{\Delta}{F C^{-}}\right)
$$

\footnotetext{
${ }^{15}$ In other words, the sign of the asymmetry for runs with +- or -+ HWPhargetpol status is reversed.
} 
Defining $\delta n_{b} \equiv \delta N_{b} / F C^{-}$and realizing our false asymmetry is given by $A_{\text {false }} \approx \delta n_{b} / n_{b}$, and approximating $\mathrm{FC}^{\text {total }}=2 \mathrm{FC}^{-}$, the difference in the tentative relation can be written

$$
-n_{b} A_{\text {false }}\left(\frac{\Delta}{F C^{\text {total }}}\right)
$$

A study of the Faraday counts between helicities of the various run sets yields a maximal value of $\Delta / F C^{\text {total }} \leq 0.001 .{ }^{16}$ Given our maximal false asymmetry of $10^{-4}$ and Eq. 5.74, accounting for the background effects of the false asymmetry requires multiplying the raw asymmetry by a factor of $1 /\left(1-n_{b} A_{\text {false }}\left(\frac{\Delta}{F C^{\text {total }}}\right)\right) \approx 1+10^{-7} n_{b}$, making this source of error (35) completely negligible.

The effects of false asymmetries, in the end, account for a very small $(<1 \%)$ fraction of the asymmetry, as can be seen from the cyan error in Figure 7.7.

\subsubsection{Models errors (36-40)}

Finally, we consider errors due to the use of modeled asymmetries and structure functions. A description of the fits used to determine the models for $F_{1}, F_{2}, R, A_{1}$ and $A_{2}$ is described in Section 2.7. Most of the errors on these quantities are determined by varying the fit parameters to the world data by a standard deviation. The relative error effects due to uncertainties on these models is shown in Figure 7.14.

In the derivation of $A_{\|} / D=A_{1}+\eta A_{2}$, the most significant model errors appear due to the $R$ structure function ratio, which propagate into the depolarization $D$ via Eq. 1.19. To find the error due to the model of $R$, one standard deviation is added to the fit parameters for $R(40)$ and the data are reanalyzed.

The $F_{1}$ unpolarized structure function is required for calculation of the spin structure functions (Eqs. 1.235 and 1.236). The value of $F_{1}$ can be expressed in terms of $R$ and $F_{2}$ by Eq. 1.79. To calculate the systematic error on $F_{1}$, then, a standard deviation is added to the fits of $F_{2}(39)$ and $R(40)$ in separate systematic error tests. We then recalculate $F_{1}$ in terms of the modified model of $F_{2}$ or $R$.

The models of $A_{1}$ and $A_{2}$ also make a very minor contribution to the derivation of $A_{\| \mid} / D$, in that

\footnotetext{
${ }^{16}$ This figure represents the early $(1.6 \mathrm{GeV})$ data, where the beam charge asymmetry was at its worst.
} 


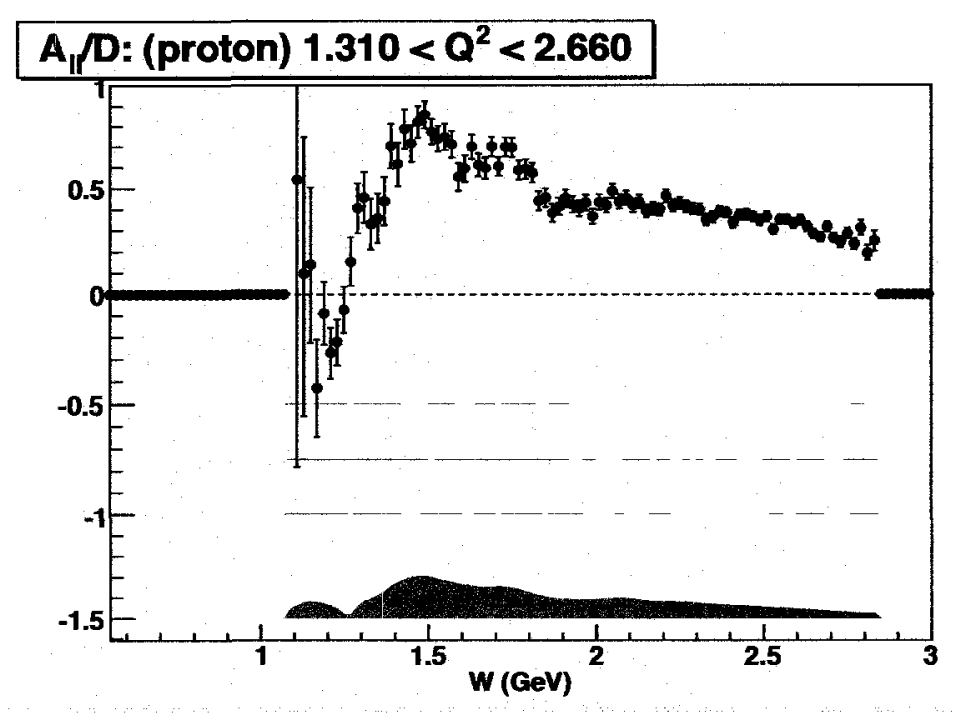

Figure 7.14: $A_{1}+\eta A_{2}$ showing the effects of models errors for $5.7 \mathrm{GeV}$ data. Systematic errors are multiplied by a factor of $\mathbf{1 0}$ for visibility. From top to bottom are shown the errors due to the $A_{1}$ DIS fit (red), $A_{2}$ (green), $A_{1}$ resonance region fit (blue), $F_{2}$ (yellow) and $R$ (magenta). Only the last error has any noticeable effects on the measurement of $A_{1}+\eta A_{2}$. Other errors, negligible in this plot, become important later, in the evaluation of virtual photon asymmetries and polarized structure functions.

they are used to model $A_{\| \mid}$for the derivation of $P_{b} P_{t}$ wgt used only for the relative weighting of data between target polarizations (see Section 7.1.2). It is important to show that our choice of models for $A_{1}$ and $A_{2}$ has no significant effect on the final outcome of our measured $A_{\|} / D$ values, so these quantities are varied, as well, in separate error analyses. Specifically, the DIS fit to $A_{1}$ is varied by one standard deviation (36), and an alternate fit of $A_{1}$ in the resonance region is also separately applied (38). $A_{2}$, the most poorly known modeled quantity in this analysis, is varied by increasing its value to the Soffer limit (Eq. 2.35) (37). It can be seen that varying the asymmetry models has a negligible effect on the outcome of the $A_{\|}$measurement.

It is essential to note, though, that while the asymmetry models (as expected) have negligible effects on the value of $A_{||} / D=A_{1}+\eta A_{2}$, that the $A_{2}$ model, will, of course, have a huge effect on the extraction of $A_{1}$ from $A_{\|} / D$, and that our knowledge of this asymmetry is the limiting factor in the accuracy of the measurement of $A_{1}$. This topic is addressed in more detail in Section 8.1.1. 


\subsubsection{Uncertainties with no effects on asymmetry analysis $(11-12,14-16,20,34)$}

Several of the (italicized) indices in Table 7.5 have no effect at all on the derivation of the doublespin asymmetry. Most of these were used specifically for systematic error determination in ${ }^{15} \mathrm{~N} /{ }^{12} \mathrm{C}$ analysis. These error tests included altering the density of frozen ${ }^{15} \mathrm{~N}(12)$, varying the target length $L$ for the (separate) ${ }^{15} \mathrm{~N}$ target (14), changing the $W$-range used to determine $\ell_{N}$ (15), varying the cross-section model used to fit the target length (16), and altering the overall radiative multiplicative offset on the ${ }^{15} \mathrm{~N}$ target counts (11). The effects of systematic errors in this mini-analysis are summarized in Ref. [95], and are not expounded in detail here.

It was useful to keep these error indices as a debugging check, to confirm null results for variation of parameters unrelated to the double-spin asymmetry analysis. In all the aforementioned cases, an error of exactly zero was measured on $A_{\|} / D$, demonstrating that the analysis code produced consistent measurements, eliminating many possible sources of technical errors.

\subsubsection{Combination of systematic errors}

All these errors must be combined to produce a total estimate of the systematic error. In doing so, it is assumed that, on average, the various systematic errors do not correlate with one another. ${ }^{17}$ Therefore, we add the errors in quadrature:

$$
\sigma_{\mathrm{SYS}}\left(W, Q^{2}\right)=\sqrt{\sigma_{1}\left(W, Q^{2}\right)^{2}+\sigma_{2}\left(W, Q^{2}\right)^{2}+\ldots}
$$

Before combination, however, statistical fluctuations must be eliminated, lest statistical errors propagate into the systematic errors. Systematic errors, unlike statistical errors, do not decrease in magnitude when bins are combined and averaged. Thus, "freezing" statistical errors in place in a systematic error can produce a huge overestimation in the total error. This is an especially important concern in the case of kinematic error evalution (29-32).

This problem can be solved by averaging over neigboring bins, in effect "smoothing" over the

\footnotetext{
${ }^{17}$ This is not, of course, a guaranteed assumption, given the complexities of systematic error effects. For a large number of error sources, however, our only viable option is to assume that the correlations, constructive and destructive, approximately cancel when all is done.
} 


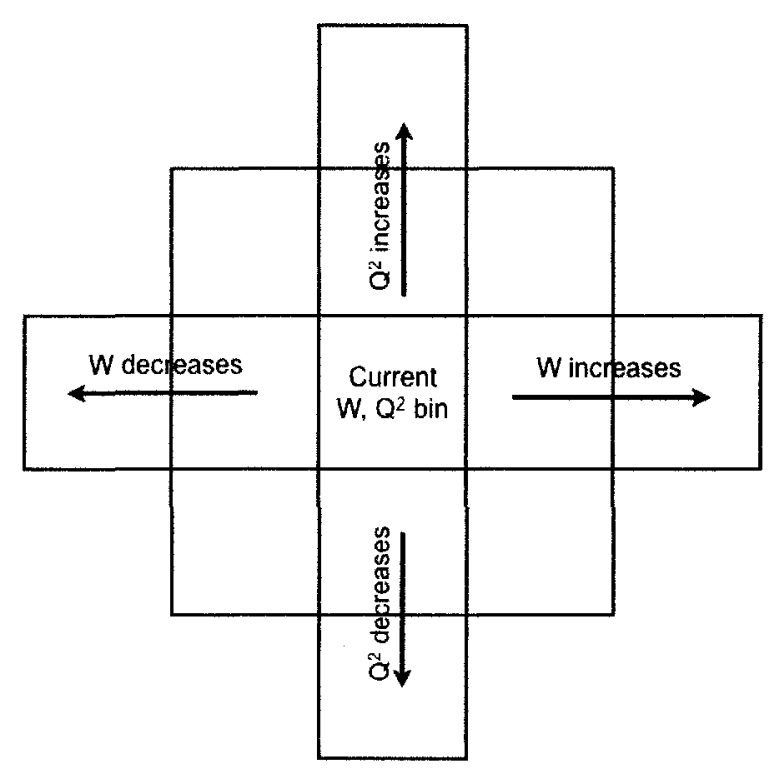

Figure 7.15: Diagram showing local bins used for the smoothing process. All bins containing data located within 2 perpendicular "steps" of the current bins are statistically averaged using Eq. 7.30, and the current bin value is replaced with the new average.

error distribution until statistical fluctuations diminish. We are then left with the relatively flat, underlying average of the error. This is done for each $W$ and $Q^{2}$ bin by averaging the systematic errors in all bins located within two "steps" (see Figure 7.15) of the current bin, weighted by the asymmetry statistical errors:

$$
\sigma_{\text {new }}\left(W, Q^{2}\right)=\frac{\sum_{\text {bins }} \sigma_{\text {sys }} / \sigma_{\text {stat }}^{2}}{\sum_{\text {bins }} 1 / \sigma_{\text {stat }}^{2}}
$$

This procedure is iterated for all data a total of 6 times, to ensure removal of the most egregious statistical fluctuations. After the smoothing process is completed for each individual error, Eq. 7.29 is applied to calculate the total systematic error.

Typically, in the resonance region (away from the elastic peak) and DIS region, a total systematic error in the range of 6-8\% was found for $A_{1}+\eta A_{2}$. To provide reasonable statistical error bars, the standard bins were combined into larger bins using the standard method of combining values with statistical errors [103]:

$$
A_{\|} / D_{\text {avg }}\left(W, Q^{2}\right)=\frac{\sum_{\text {bins }}\left(A_{\| \mid} / D\right) / \sigma_{\text {stat }}^{2}}{\sum_{\text {bins }} 1 / \sigma_{\text {stat }}^{2}}
$$




$$
\sigma_{\text {avg }}\left(W, Q^{2}\right)=\frac{1}{\sqrt{\sum_{\text {bins }} 1 / \sigma_{\text {stat }}^{2}}}
$$

The systematic errors must also be combined into larger bins, using Eq. 7.31, with the systematic error in place of $A_{\|} / D$. After bins are combined, the statistical and systematic errors can be combined to find the total error on the measurement at a given kinematic value:

$$
\sigma_{\mathrm{TOTAL}}\left(W, Q^{2}\right)=\sqrt{\sigma_{\mathrm{SYS}}\left(W, Q^{2}\right)^{2}+\sigma_{\mathrm{STAT}}\left(W, Q^{2}\right)^{2}}
$$

Final values (and errors) for the asymmetry $A_{\|} / D=A_{1}+\eta A_{2}$ at each of the four nominal beam energies can be found in Appendix Section B.4. Also shown are the average values of all tracked kinematic values over events in each bin. In order that the tables could be reasonably contained in a document of this size, the standard $Q^{2}$ bins were quadrupled in size, and the $W$-bins size was increased by a factor of 6 (to $60 \mathrm{MeV} / \mathrm{bin}$ ). Bins with statistical errors of greater than 1.0 were excluded from the tables. Values of $A_{\|} / D=A_{1}+\eta A_{2}$ for all 4 beam energies are plotted in Figures 7.16 and 7.17.

At this point, extraction of double-spin asymmetries and kinematic values is complete. Final values of physical importance can now be extracted from the data: the asymmetries $A_{1}$ and $A_{2}$, structure functions $g_{1}$ and $g_{2}$, and various moments of these structure functions. The final chapter continues the propagation of errors, as described in this section, to final measured results for these physical quantities. 

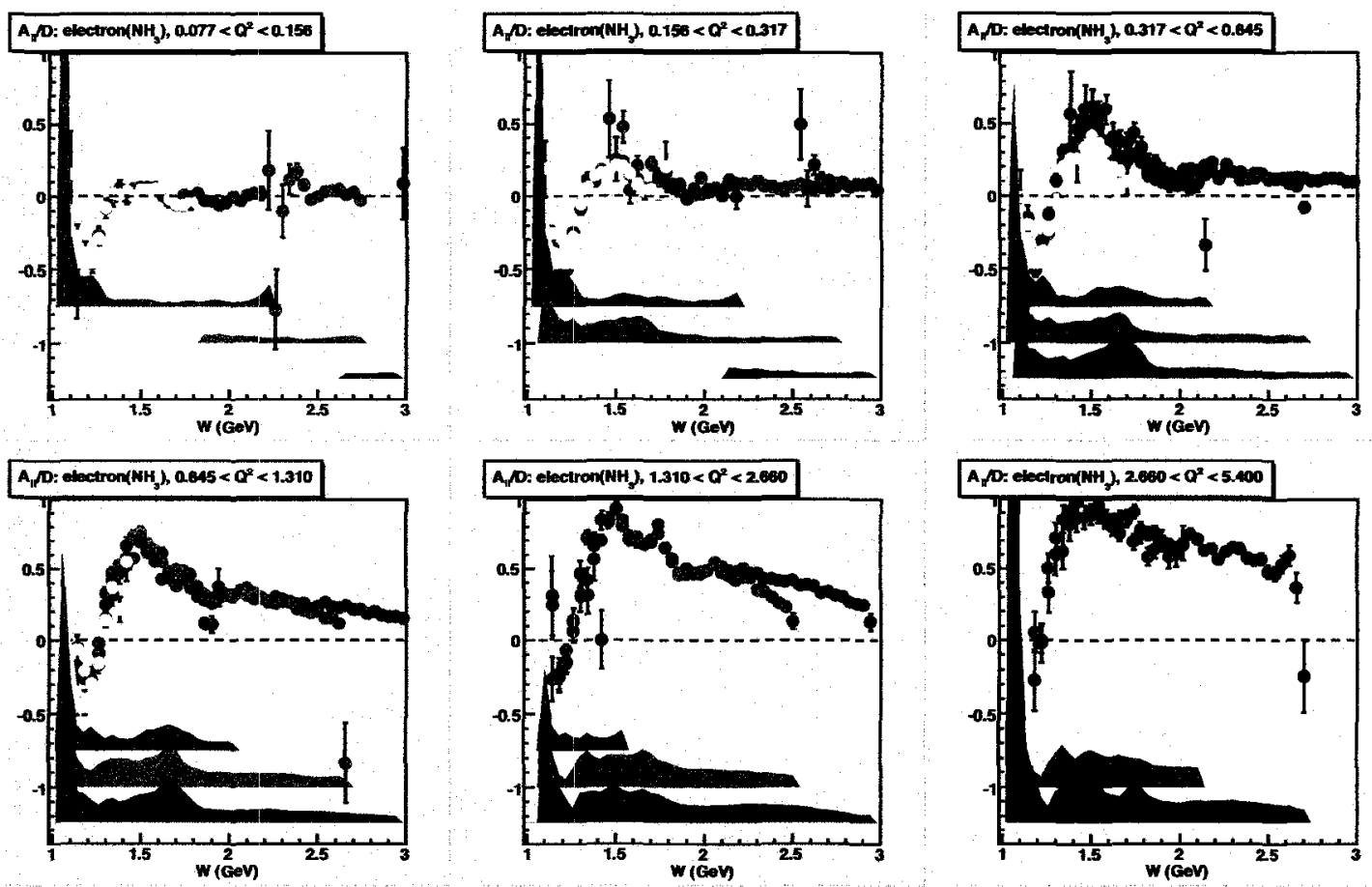

Figure 7.16: $A_{1}+\eta A_{2}$ showing total systematic errors for all 4 beam energies. Systematic errors are multiplied by 5 for visibility. Note that since larger $Q^{2}$ bins are used here, the average $Q^{2}$ in a given plot can vary between beam energies, causing wider apparent variations between two energies than really exist at a given $Q^{2}$ value. (This is especially apparent in the bottom middle plot at high $W$.) The yellow, green, red and blue represent $1.6,2.5,4.2$ and $5.7 \mathrm{GeV}$, respectively. 

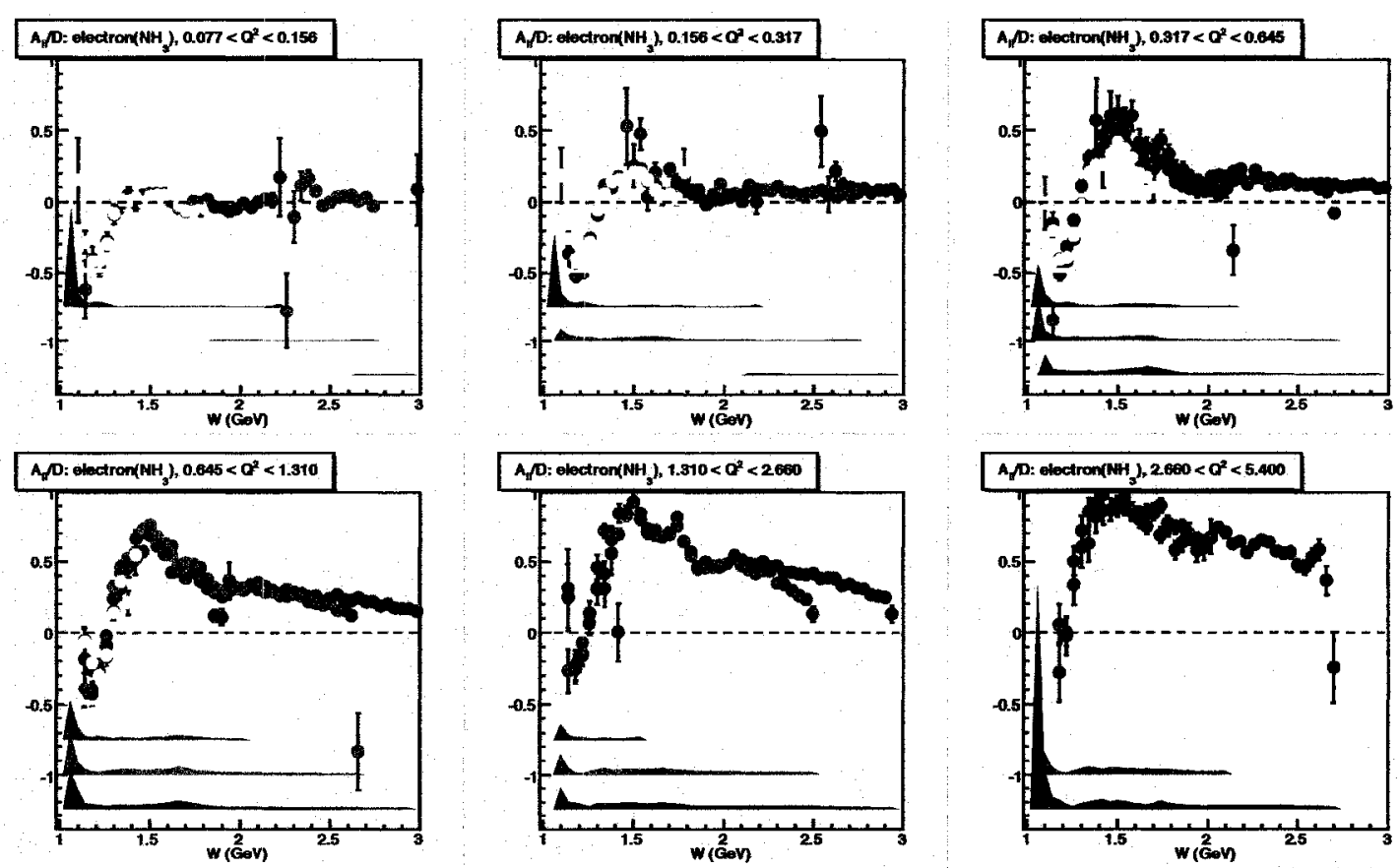

Figure 7.17: Same plot shown in Figure 7.16, but with systematic errors shown at their actual size. 


\section{Chapter 8}

\section{Final Results}

In the final chapter of this thesis, the physical results (that is, the asymmetries $A_{1}$ and $A_{2}$, as well as the structure functions $g_{1}$ and $g_{2}$ ) are extracted and presented in detail, along with their moments and several applications of the data. Extensive numerical data are printed in the tables in the Appendix Sections B.4-B.7. All data listed in the tables will also be listed in the CLAS Collaboration Database [128]. In the case of structure functions and asymmetries, where bins have been combined in the printed tables, the original, uncombined data will be provided in the online database.

Where possible, world data from Jefferson Lab, SLAC, DESY and CERN for the structure functions and asymmetries (described in Section 1.5) are included in the plots of the final results. Unless explicitly stated otherwise, symbols used to represent data from differing experiments correspond to the key shown in Figure 8.1, for easy reference. In some cases where data points from the same experiment were too crowded together in the same plot, the statistical average of the local point was plotted instead, for clarity. Note that world data points include only statistical errors. A table of specific references for the world data is given in Table 8.1.

Note that, by the completion of this thesis, there are a few unresolved issues with the data analysis requiring future attention. These are explained in detail near the end of this thesis, in Section 8.5.1. 


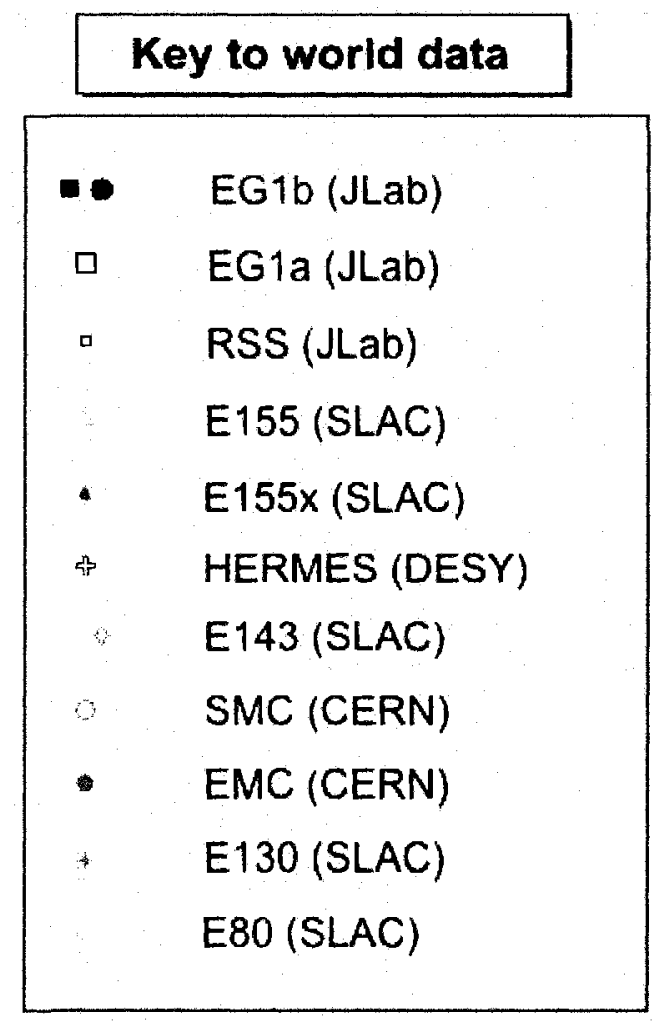

Figure 8.1: Key to the world data plots spread throughout this chapter. Details regarding specific experiments can be found in Section 1.5. 
Table 8.1: Quick reference table of sources of world data for the proton double-spin asymmetries and structure functions presented in this chapter, as given in the Bibliography. In the case of RSS data, at the time of this thesis, only plots, not numeric data, are publicly available. The plotted RSS values in this chapter are close estimates graphically interpreted from these plots, not exact data. (The exception is the $d_{2}$ integral value (Section 8.3.3), which is published in Ref. [51].)

\begin{tabular}{|c|c|}
\hline Experiment & Source \\
\hline \hline E80 & {$[36]$} \\
\hline E130 & {$[129]$} \\
\hline EMC & {$[40]$} \\
\hline SMC & {$[130]$} \\
\hline E143 & {$[131]$} \\
\hline HERMES & {$[132]$} \\
\hline E155/E155x & {$[133]$} \\
\hline RSS & {$[51]$} \\
\hline EG1a & {$[134]$} \\
\hline
\end{tabular}

\subsection{Virtual Photon Asymmetry Results}

\subsubsection{Extraction of $A_{1}$}

At this point, the quantity $A_{\|} / D=A_{1}+\eta A_{2}$ has been carefully extracted from the data, subtracting contributions from all backgrounds, for the 4 different beam energies in the EG1 experiment. Now, we extract the physics quantities defined in Chapter 1 , namely, the virtual photon asymmetries $A_{1}$ and $A_{2}$ defined in Section 1.4. The most precise way to measure $A_{1}$ from $A_{\|\|} / D$ is to use Eq. 1.224, with a reasonable model (Section 2.7.2) employed for the relatively small contribution from $A_{2}$. As Section 1.4 points out, measurements of $A_{2}$ can be extracted from these data, but the measurements are of poor precision. Though they can be used to constrain future versions of the model, a smooth-fitting (i.e. analytical, not statistical) curve is required for this subtraction to obtain accurate results. The subtraction of $A_{2}$ imparts a substantial systematic error, due to its inaccuracy. (see Figure 8.2).

To solve for $A_{1}$, Eq. 1.224 was applied in each $W$ and $Q^{2}$ bin, for the final 4 sets of $A_{||} / D$ corresponding to each $(1.6,2.5,4.2$ and $5.7 \mathrm{GeV})$ beam energy. The statistically averaged values of $\eta$ in each bin were used to prevent weighting errors. Systematic errors were calculated as described in in the previous chapter, and combined in quadrature, as usual. Values and errors 

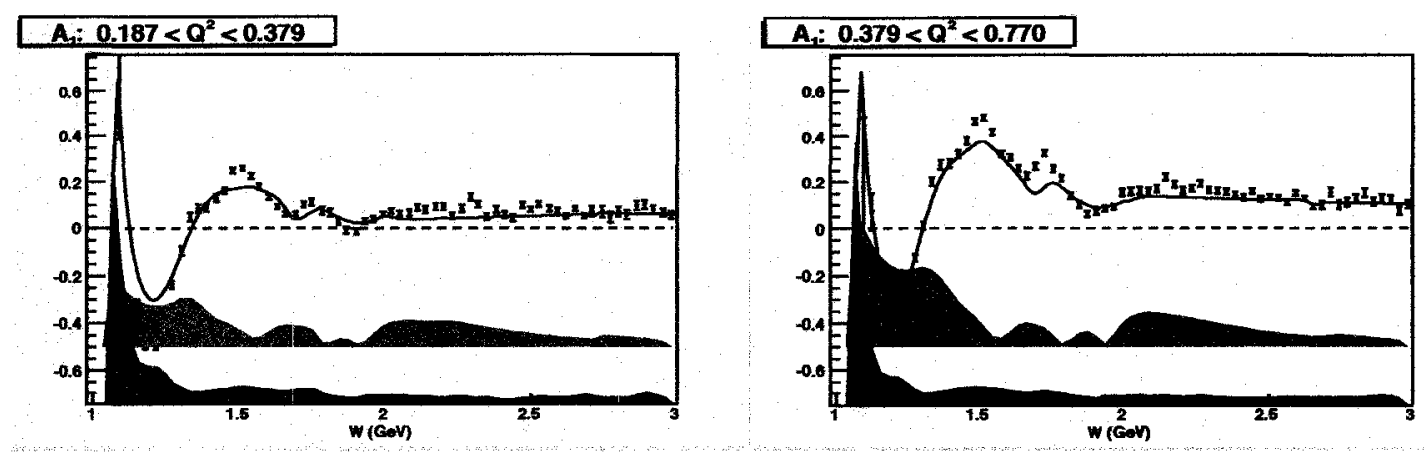

Figure 8.2: Results for $A_{1}$ in terms of $W$ for two neighboring $Q^{2}$ bins, comparing the size of the estimated systematic error from $A_{2}$ (red, top) to the effects of all other systematic errors (blue, bottom). Error magnitudes are multiplied by 5 for visibility. Obviously, the error on $A_{2}$ is the largest uncertainty in the extraction of $A_{1}$.

were then combined for each beam energy as

$$
\begin{gathered}
A_{1}=\frac{\sum_{\text {energies }} A_{1} / \sigma^{2}}{\sum_{\text {energies }} 1 / \sigma^{2}} \\
\sigma=\frac{1}{\sqrt{\sum_{\text {energies }} 1 / \sigma^{2}}}
\end{gathered}
$$

A student's $t$-test was again run on the combined data, though this time, with more spurious results (see Table 8.2). This most likely results from systematic errors between data sets, including inaccurate modeling of the poorly known $A_{2}$ value. The discrepancy should improve with newer models, constrained by experimental knowledge of $A_{2}$ from this and future data sets.

Plots of the virtual photon asymmetry $A_{1}$ as a function of $W$, across the $Q^{2}$ range for EG1b, are shown in Figures 8.3 and 8.4. Comparing these plots to Figure 1.8, one sees the negative (spin- $\frac{3}{2}$ ) $\Delta$ resonance at $W=1.23 \mathrm{GeV}$, with a transition to a positive value in the higher resonance region, which is dominated by the (spin- $\frac{1}{2}$ ) $\mathrm{N}^{*}$ transitions (Eq. 1.238). The same quantity in terms of $Q^{2}$, for different $W$ bins, is shown in Figure 8.4. It can be seen here that $A_{1}$ nearly uniformly increases with $Q^{2}$. This can be understood in terms of Figure 1.1, where we expect the virtual photon asymmetry $A_{1}$ to be zero for DIS scattering at $Q^{2} \rightarrow 0$ (where scattering from the whole proton occurs), and increasing at higher $Q^{2}$, where the model of Figure 1.5 becomes valid. 
Numeric data for these values are listed in Table B.10. ${ }^{1}$ Results for $A_{1}$ were also plotted in terms of Bjorken $x$ (Figure 8.5). When compared to a large $Q^{2}\left(10 \mathrm{GeV}^{2}\right)$ model of $A_{1}$ DIS results, one notices that the resonances (with the exception of the large, negative $\Delta$ resonance at the higher $x$ end) "oscillate" around the DIS curve, approximately conserving the area under the curve, when compared to the (blue) DIS model. This phenomenon, shown qualitatively in this figure, is known as quark-hadron duality, and is explained in greater detail in Section 8.4.3.

Table 8.2: Students $t$-test results for combinations of the $A_{1}$ data between beam energies. Results are more suspect than in previous $t$-tests in this thesis, most likely due to varying systematic errors between data sets, which are not included in the $t$-test error bar. The $4.2 \mathrm{GeV}$ data show the worst result.

\begin{tabular}{|c|c|c|c|}
\hline Set A & Set B & $t$ & $\sigma_{t}$ \\
\hline \hline $1.6 \mathrm{GeV}$ & $2.5 \mathrm{GeV}$ & -0.004 & 1.01 \\
\hline $1.6 \mathrm{GeV}$ & $4.2 \mathrm{GeV}$ & -0.135 & 1.39 \\
\hline $1.6 \mathrm{GeV}$ & $5.7 \mathrm{GeV}$ & 0.126 & 1.04 \\
\hline $2.5 \mathrm{GeV}$ & $4.2 \mathrm{GeV}$ & -0.090 & 1.31 \\
\hline $2.5 \mathrm{GeV}$ & $5.7 \mathrm{GeV}$ & 0.138 & 1.02 \\
\hline $4.2 \mathrm{GeV}$ & $5.7 \mathrm{GeV}$ & 0.039 & 1.18 \\
\hline
\end{tabular}

\subsubsection{Extraction of $A_{2}$}

A rudimentary measurement of $A_{2}$ can be made by using the linear regression method described in detail in Section 1.4.2, explicitly defined for each bin by Eqs. 1.226 and 1.227. Using MINUIT to fit a straight line to the plot of $A_{\|} / D$ vs. $\eta$ in each bin, the value of $A_{2}\left(W, Q^{2}\right)$ is given by the resultant slope.

Since two or more data points at differing beam energies are required to make the linear fit, kinematic coverage of this measurement is limited to the space where data from different beam energies overlap (refer to Figure 2.52). Figure 8.6 shows the approximate kinematic coverage of the linear fit between energies, in terms of $W$ and $Q^{2}$, showing sample linear fits of $A_{\|} / D$ vs. $\eta$ in the bins.

Before trusting the resulting values of $A_{2}$ in each bin, a consistency check was made of the

\footnotetext{
${ }^{1}$ Again, these tables combine $W(\times 6)$ and $Q^{2}(\times 4)$ bins, so that results can fit into printed tables. More finely binned results will be compiled in Ret. [128].
} 

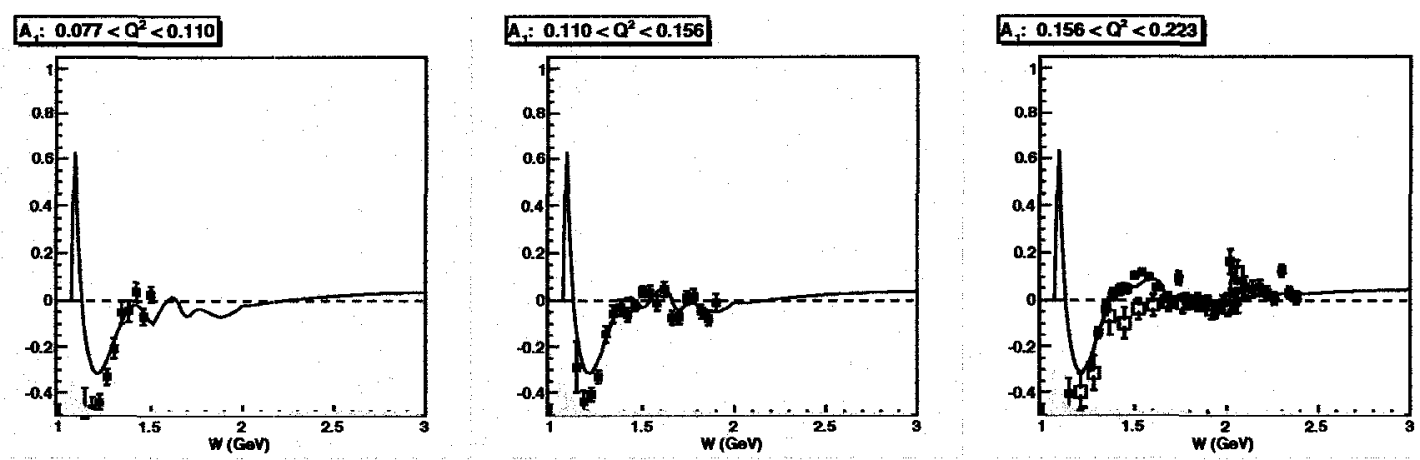

$A_{1}: 0.223<Q^{2}<0.317$
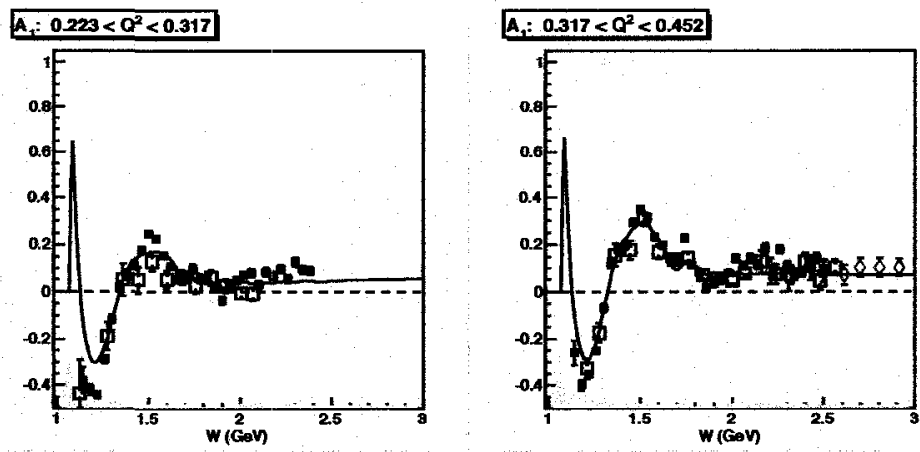

\section{$A_{1}: 0.452<Q^{2}<0.645$}

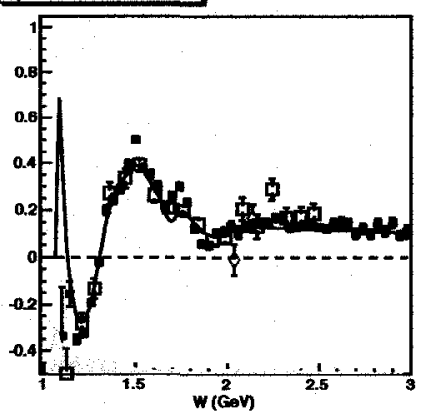

$A_{1}: 0.645<0^{2}<0.919$

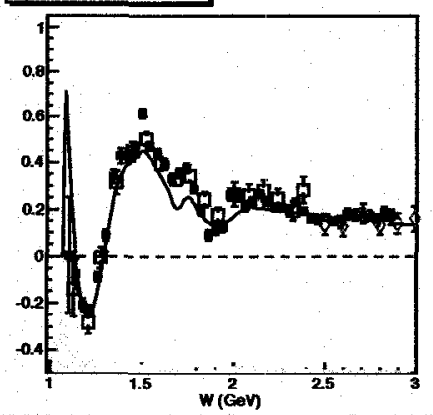

$A_{0}: 0.819<0^{2}<1.310$

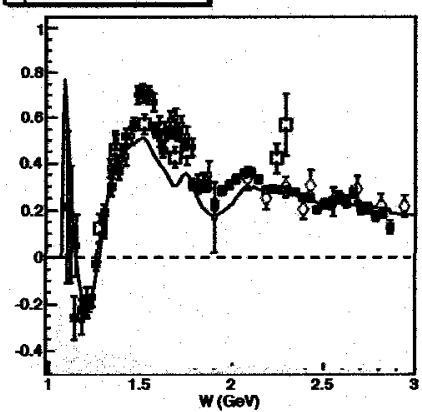

$A_{1}: 1.310<Q^{2}<1.870$

$A_{i}: 1.870<Q^{2}<2.660$
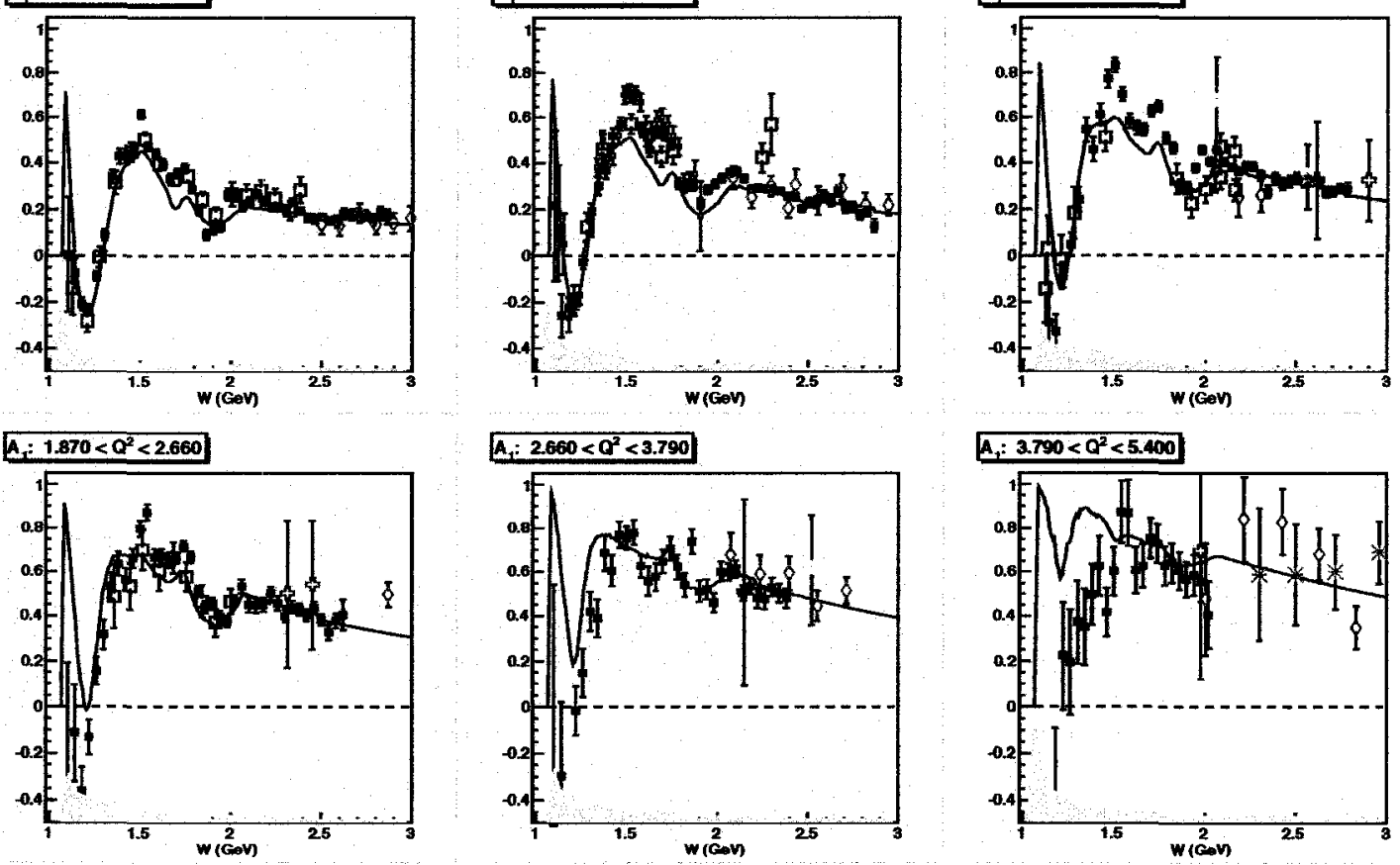

$A_{1}: 3.790<Q^{2}<5.400$

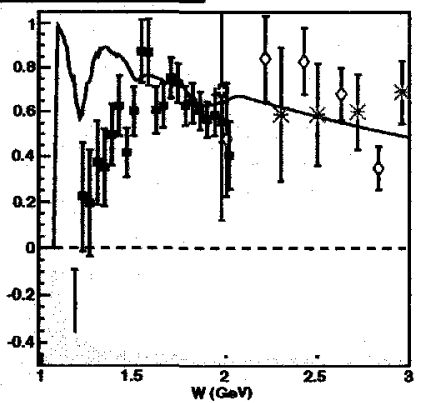

Figure 8.3: Final results for $A_{1}$ vs. $W$ across the whole $Q^{2}$ range covered by the EG1b data. Data from other experiments, summarized in Figure 8.1, are also shown. Gray background represents total systematic errors, shown actual size. 
$A_{1}: 1.060<W<1.200$

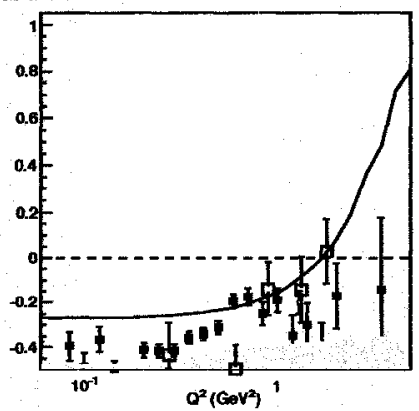

$A_{1}: 1.440<W<1.500$

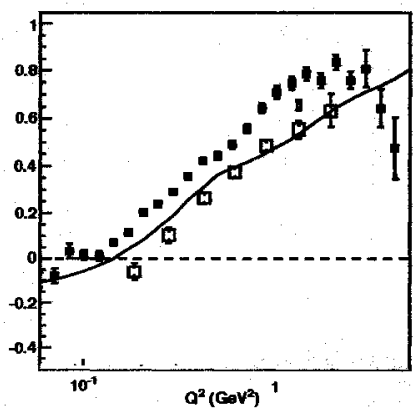

$A_{1}=1.000<W<1.920$

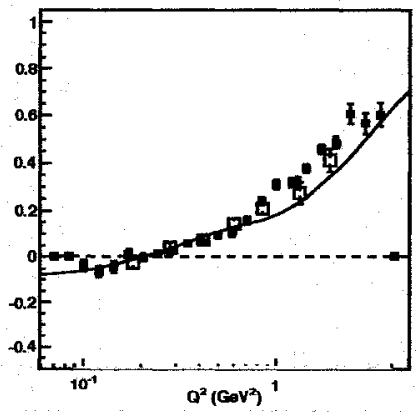

$A_{1}: 2.160<W<2200$

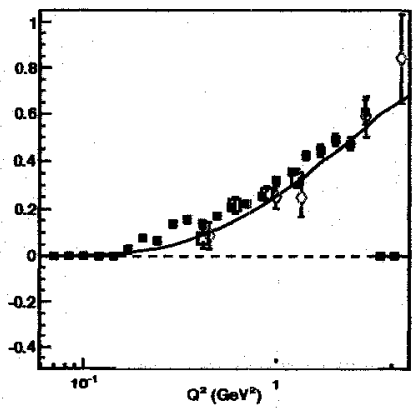

$A_{i}: 1200<W<1320$

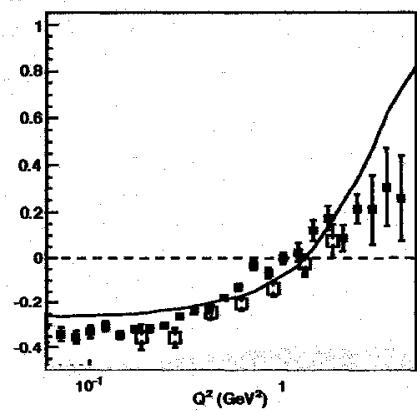

$A_{1}: 1.500<W<1.600$

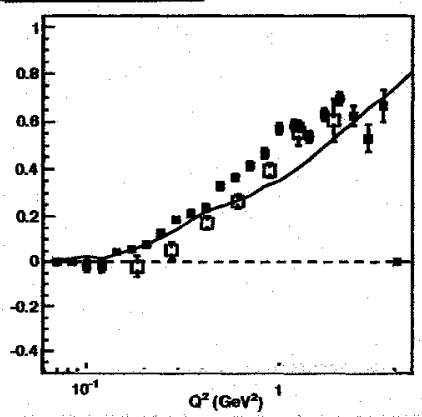

$A_{1}: 1.020<W<2.040$

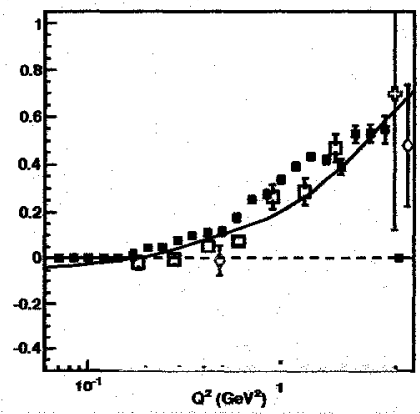

$A_{1}: 2.260<W<2400$

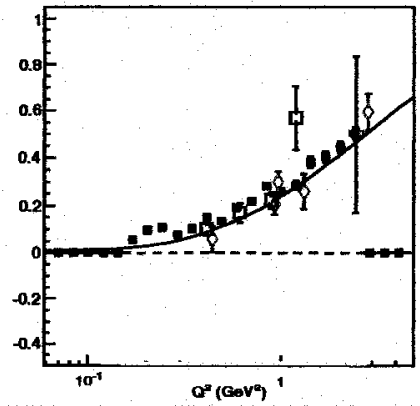

$R_{1}: 1.320<W<1.40$

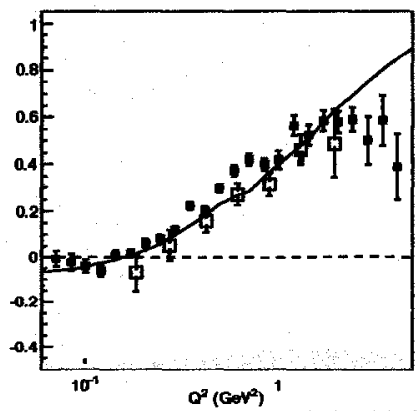

$A_{1} ; 1.600<W<1.800$

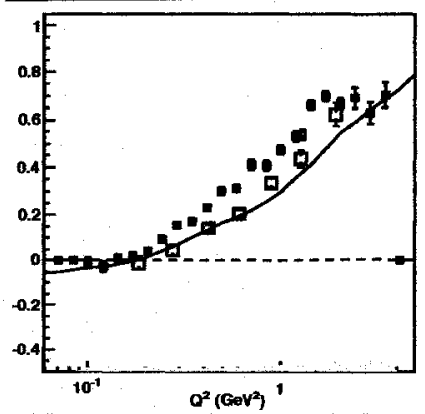

$A_{1}: 2040<W<2.160$

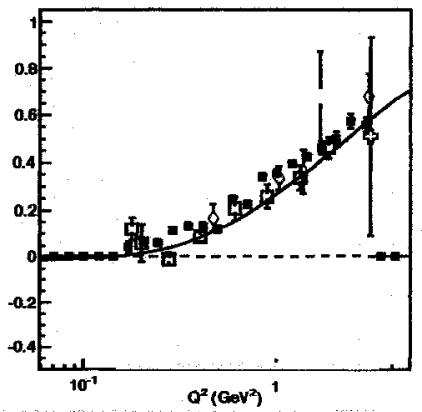

$A_{1}: 2400<W<2520$

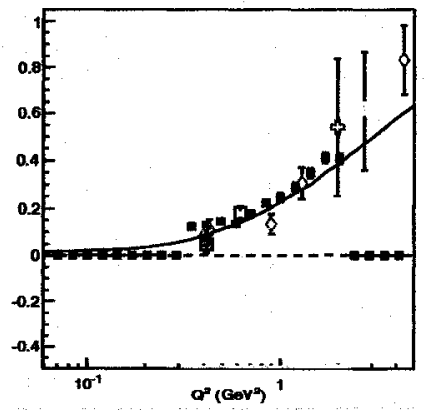

Figure 8.4: Final results for $A_{1}$ vs. $Q^{2}$ across part of the inelastic $W$ range covered by the EG1b data, compared to other experimental data. Gray background represents total systematic errors. 


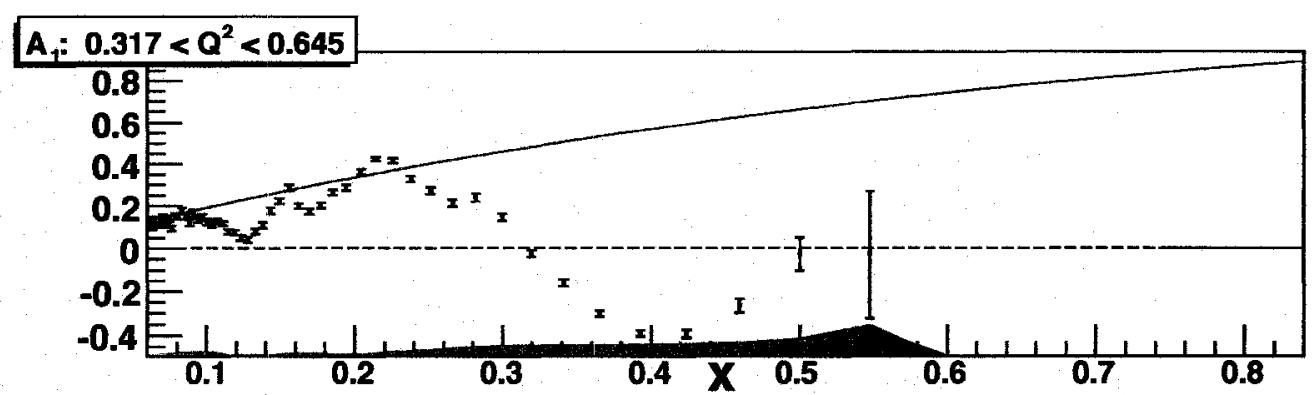

$A_{1}: 0.645<Q^{2}<1.310$
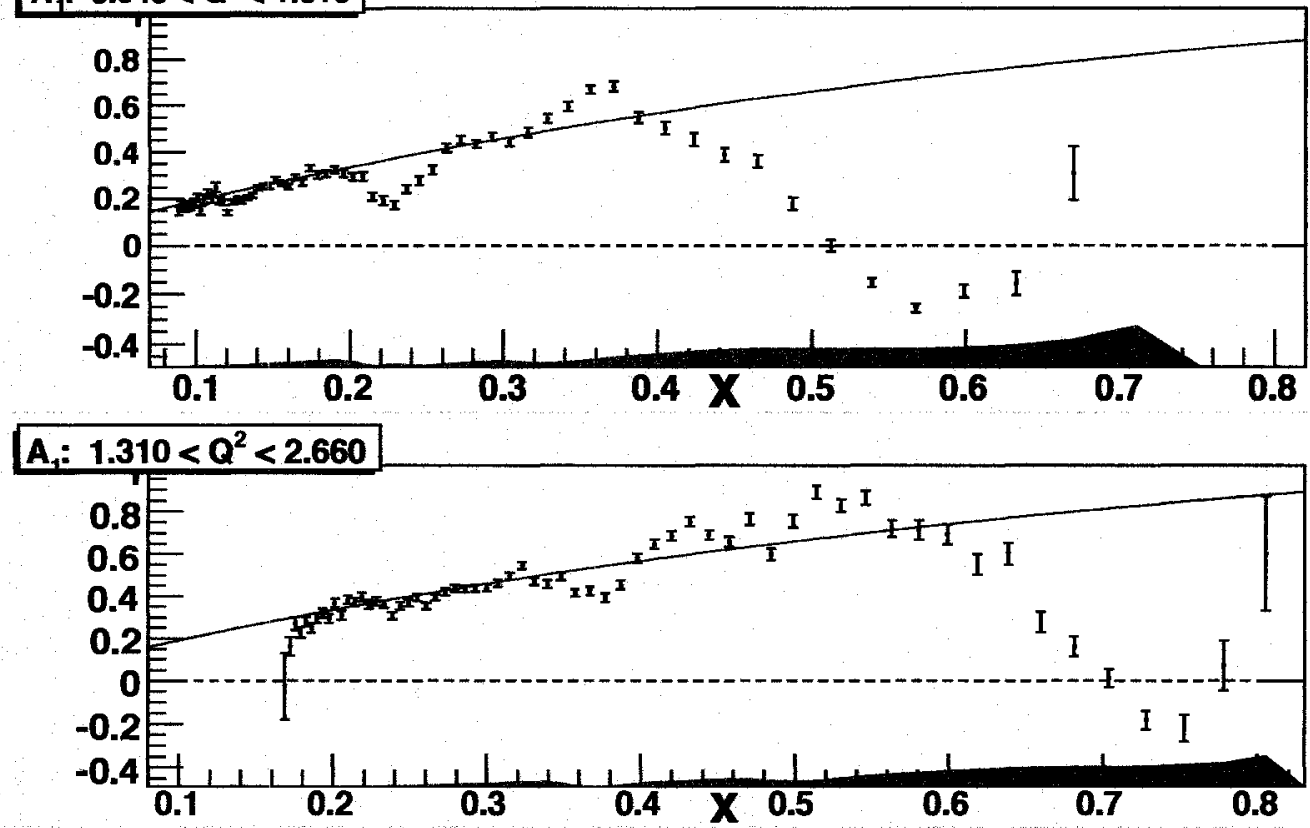

Figure 8.5: EG1b results for $A_{1}$ vs. $x$ for selected $Q^{2}$ bins. The blue line is a superimposed DIS $Q^{2}=10 \mathrm{GeV}^{2}$ model of $A_{1}$. Note the oscillations of the resonances about the DIS model; this is a manifestation of quark-hadron duality (Section 8.4.3). 

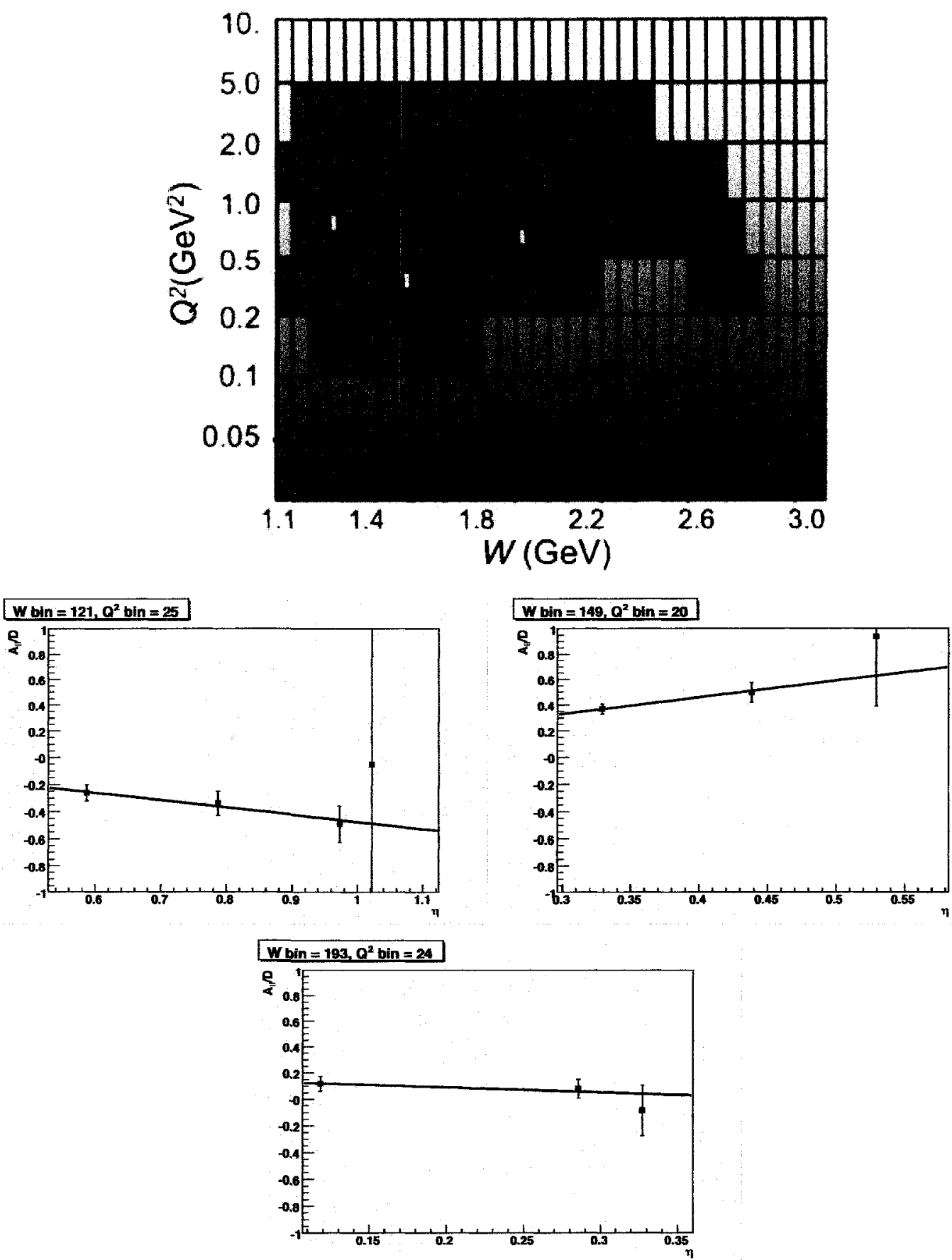

Figure 8.6: Approximate kinematic coverage of the linear regression method (top). The other plots show the linear fit in terms of $\eta$ for the highlighted (yellow) bins in the top figure, in order of increasing $W$. Note that even though they are marked as valid bins, here, DIS $(W>2.0 \mathrm{GeV})$ values were ultimately excluded from the regression analysis. 

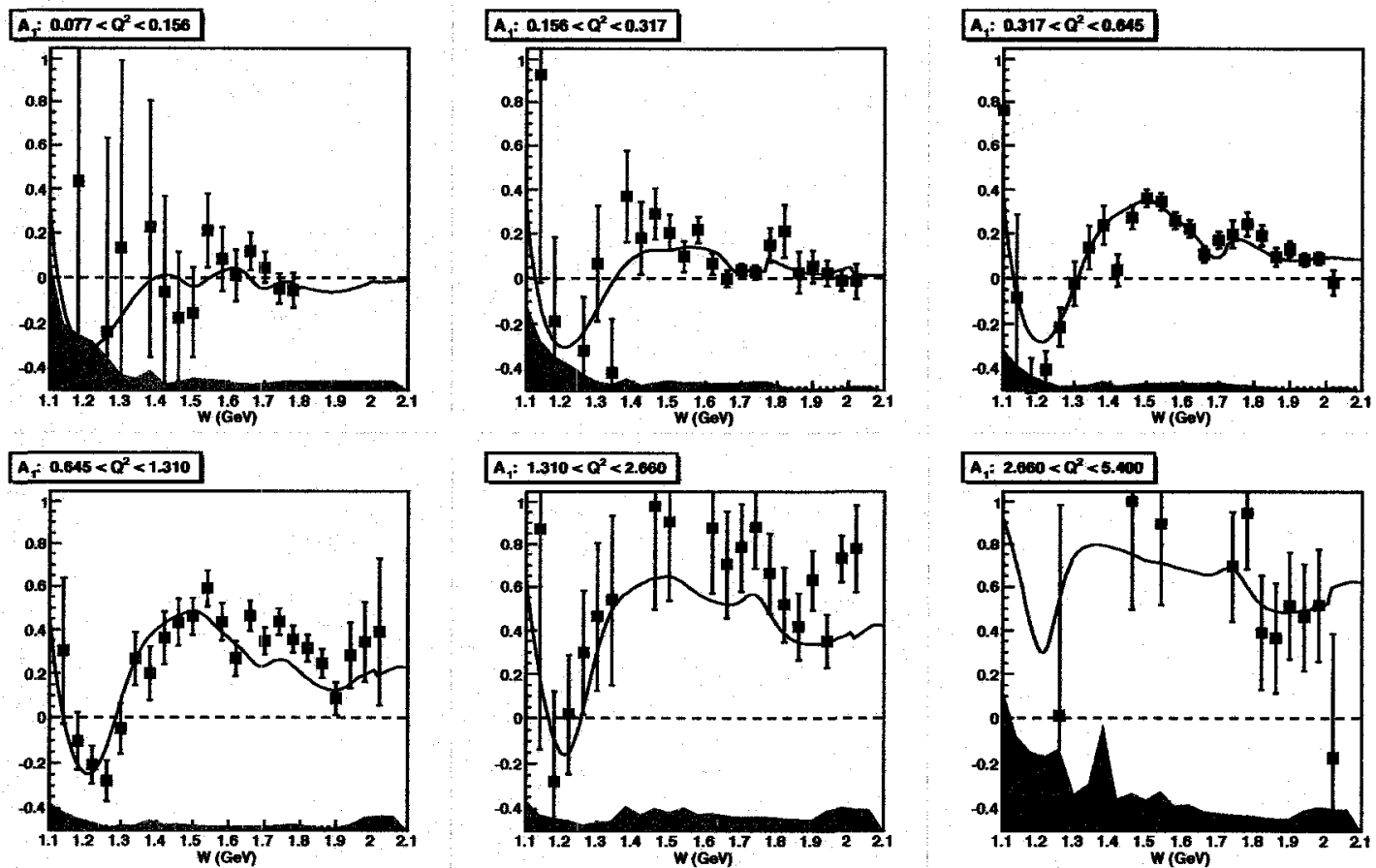

Figure 8.7: $A_{1}$ vs. $W$, derived without using an $A_{2}$ model, as the $y$-intercept of $A_{\|} / D$ vs. $\eta$. Because the analytic structure of $A_{2}$ is not assumed in creating this plot, statistical error bars are considerably larger than in Figure 8.3. 
resultant values of $A_{1}$, determined by the $y$-intercept, that they match the expected values of $A_{1}$ when a model is used for $A_{2}$. Figure 8.7 shows some sample plots of $A_{1}\left(W, Q^{2}\right)$, derived by the linear regression method. The error bars are considerably larger than those in the plots shown for the standard method of using the $A_{2}$ model (Figure 8.3), because the linear regression method makes no assumptions regarding the nature of $A_{2}$, not even its analyticity (i.e. "smoothness" across bins). Therefore, statistical fluctuations in this plot are substantially larger than those in Figure 8.3. However, the plotted values are statistically compatible, at least partially validating the method.

Corresponding values of the slope $\left(A_{2}\right)$ are shown in Figures 8.8 and 8.9. Bins have been combined to improve the statistical resolution. The results show slightly higher values than those predicted by the existing model in the resonance region, a result compatible with that found by the Jefferson Lab Hall-C RSS experiment at $Q^{2}=1.3 \mathrm{GeV}^{2}$ [51].

Caution must be used in deriving $A_{1}$ and $A_{2}$ using this method. The results are very sensitive to statistical differences in normalization factors between data sets of differing beam energy, particularly $P_{b} P_{t}$ statistical errors. At values of $W>2.0 \mathrm{GeV}$, in the DIS region, derived values of $A_{1}$ were definitely not compatible with those in Figure 8.3, and derived values of $A_{2}$ exceeded the Soffer bound (Eq. 2.35), a physical impossibility. Values derived from linear regression in this region (correlated to large angle scattering, where results become more tenuous) are unreliable. Thus, a cut of $W<2.0 \mathrm{GeV}$ was placed on the $A_{2}$ data, and results were limited to the resonance region. Current measurements of $A_{2}$ from the EG1b data can be found in Table B.5.

\subsubsection{Refining Accuracy of $A_{1}$ and $A_{2}$ Measurements}

The accuracy of the $A_{1}$ measurement is limited by our knowledge of the asymmetry $A_{2}$. What little is known about $A_{2}$, namely the Soffer bound, Burkhardt-Cottingham Sum Rule, and the more tentative Wandzura-Wilczek relation, along with DIS constraints supplied by the SLAC E155x data, ${ }^{2}$ is used as the basis for the existing $A_{2}$ model. Optimally, a new model must be developed, extending the fit into the resonance region using both the EG1 data for $A_{2}$ and the precise data at $Q^{2}=1.3 \mathrm{GeV}^{2}$ from RSS [51]. Then, the new $A_{2}$ model can be used to more accurately calculate $A_{1}$, as per Section 8.1.1.

\footnotetext{
${ }^{2}$ Chapter 1 and Section 2.7 explain these constraints in more detail.
} 

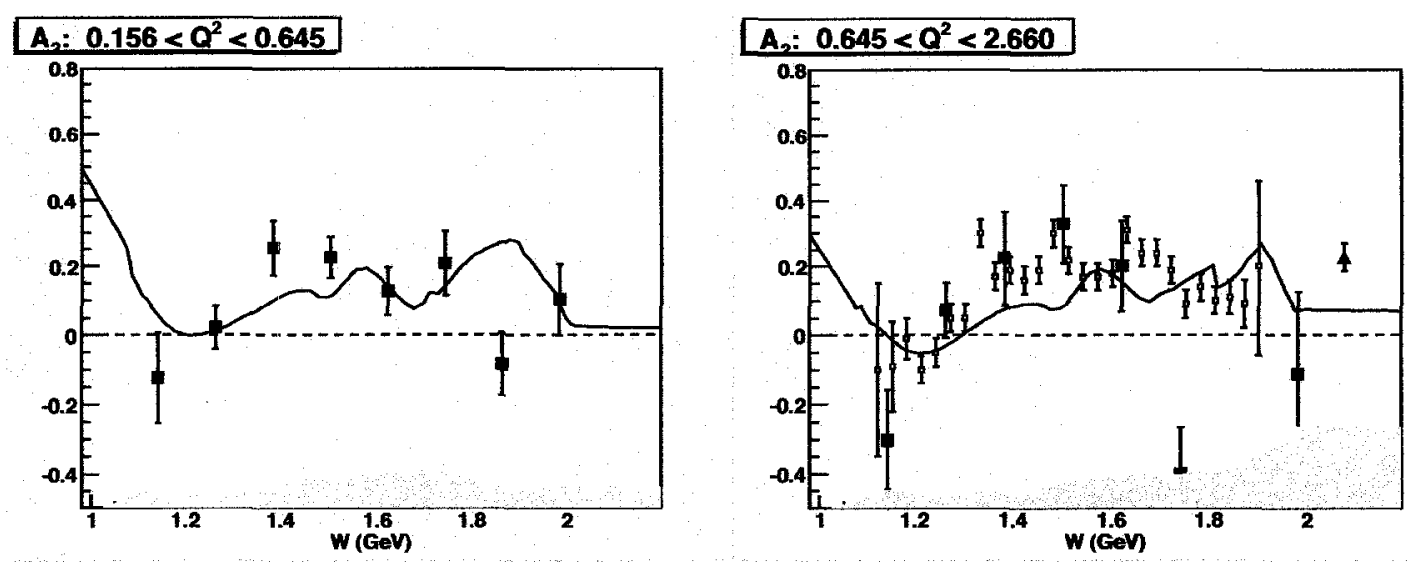

Figure 8.8: $A_{2}$ vs. $W$, extracted from the EG1b data. While not as precise as RSS data (small blue squares), EG1b extends the measurement to a wider $Q^{2}$ range.

The present limit in accuracy arises from the fact that, while the statistical information regarding $A_{2}$ is as good as it can get using the present data, the analytic information, that is, the continuity of $A_{2}$ from bin to bin, is a necessary physical constraint unrevealed by the statistically independent measurements in each bin. Fitting a new model to the $A_{2}$ data can help improve our picture of $A_{2}$, as well as provide a more accurate measurement of its contribution to $A_{\|} / D$ (and thus decrease the large systematic error illustrated in Figure 8.2). At the time of completion of this thesis, a new fit of the EG1 $A_{2}$ model is not yet completed, and attempts to exploit the analyticity of the model while factoring in the new $A_{2}$ data yielded inconclusive results. ${ }^{3}$ A rigorous extension of the model fit into the resonance region is necessary to improve the measurement in this way, a task that remains outstanding (see Section 8.5.1).

\subsection{Spin Structure Function Results}

\subsubsection{Results for $g_{1}$}

it is useful to point out that, due to the model uncertainty imparted by our lack of knowledge regarding $A_{2}$, the structure function ratio $g_{1} / F_{1}$ is more precisely measured by $A_{\|}$data than $A_{1}$ itself

\footnotetext{
${ }^{3}$ Tried methods included feeding $A_{1}$ with the modeled $A_{2}$ contribution back into the linear fit at $\eta=0$, as well as averaging $A_{1}+\eta A_{2}$ with the $A_{\|} / D$ results before the fit, and reweighting errors. Any increases in resolution thereby gained were coupled to equivalentty ambiguous systematic oftsets.
} 

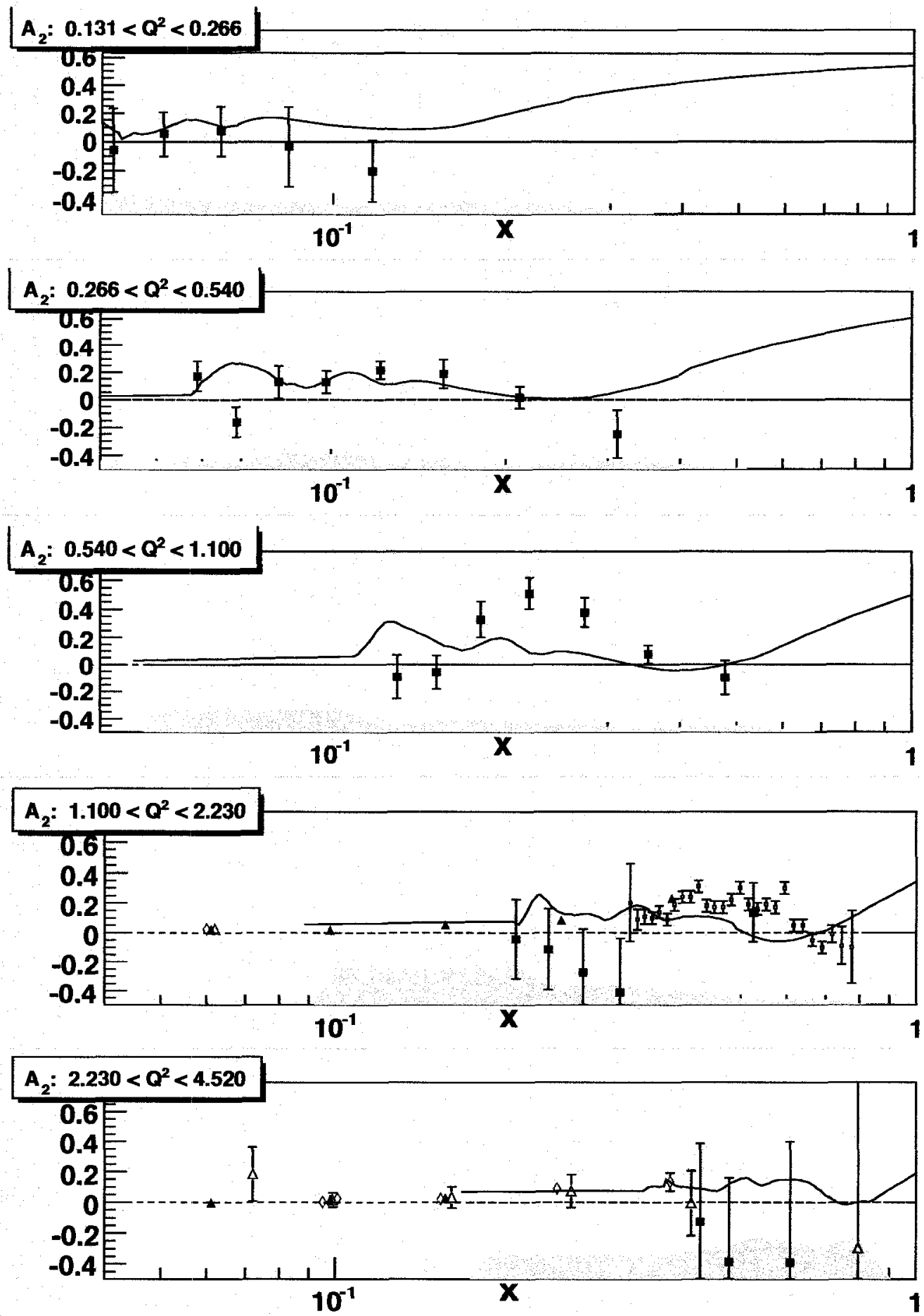

Figure 8.9: $A_{2}$ vs. $x$, extracted from the EG1b data. The EG1b model, terminating at $W=3.0 \mathrm{GeV}$, is shown for comparison (solid line). 

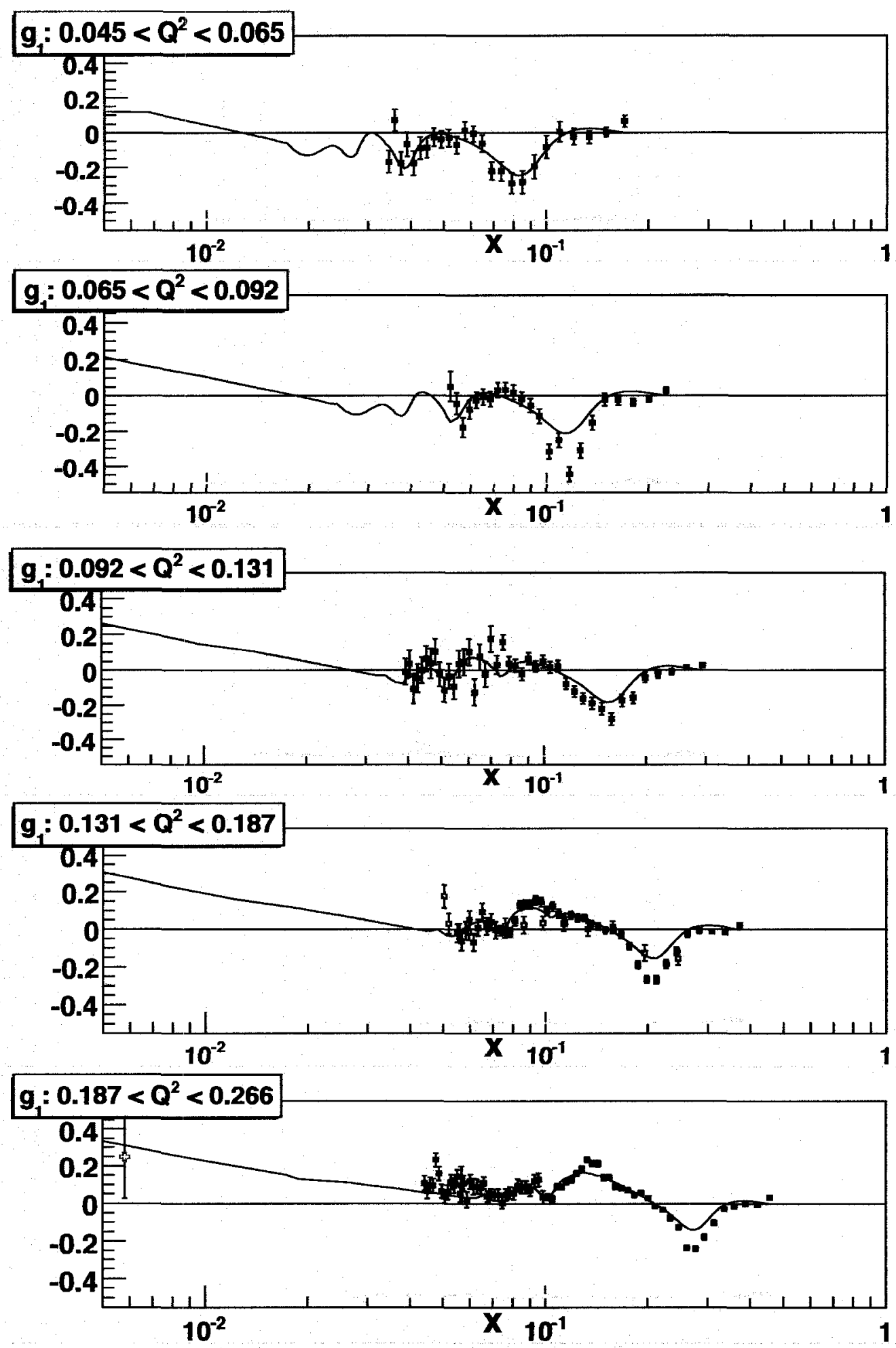

Figure 8.10: The spin-structure function $g_{1}$ vs. Bjorken $x$, in low $Q^{2}$ bins, extracted from the EG1b data. 

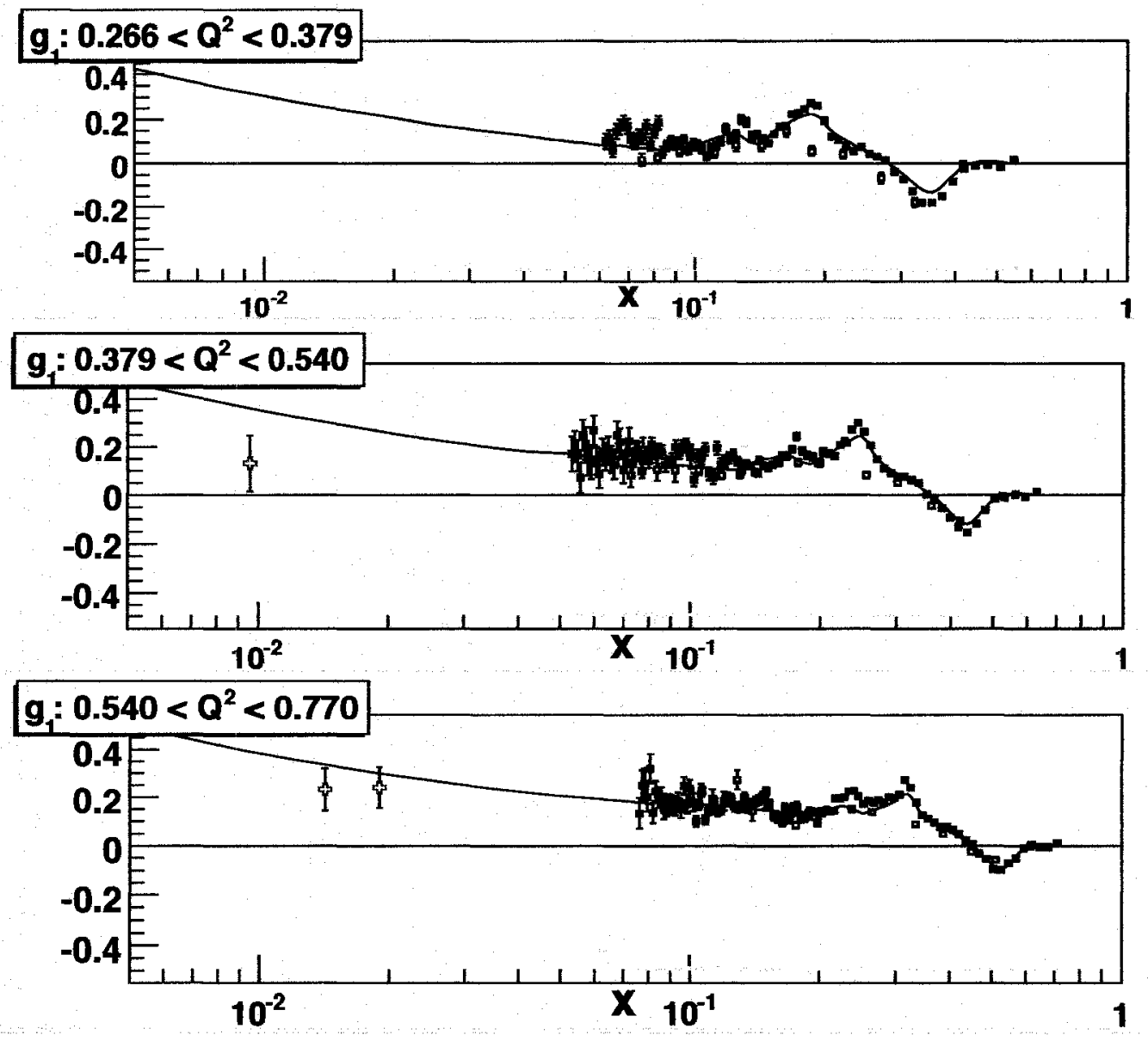

Figure 8.11: The spin-structure function $g_{1}$ vs. Bjorken $x$, in low-intermediate $Q^{2}$ bins, extracted from the EG1b data. 

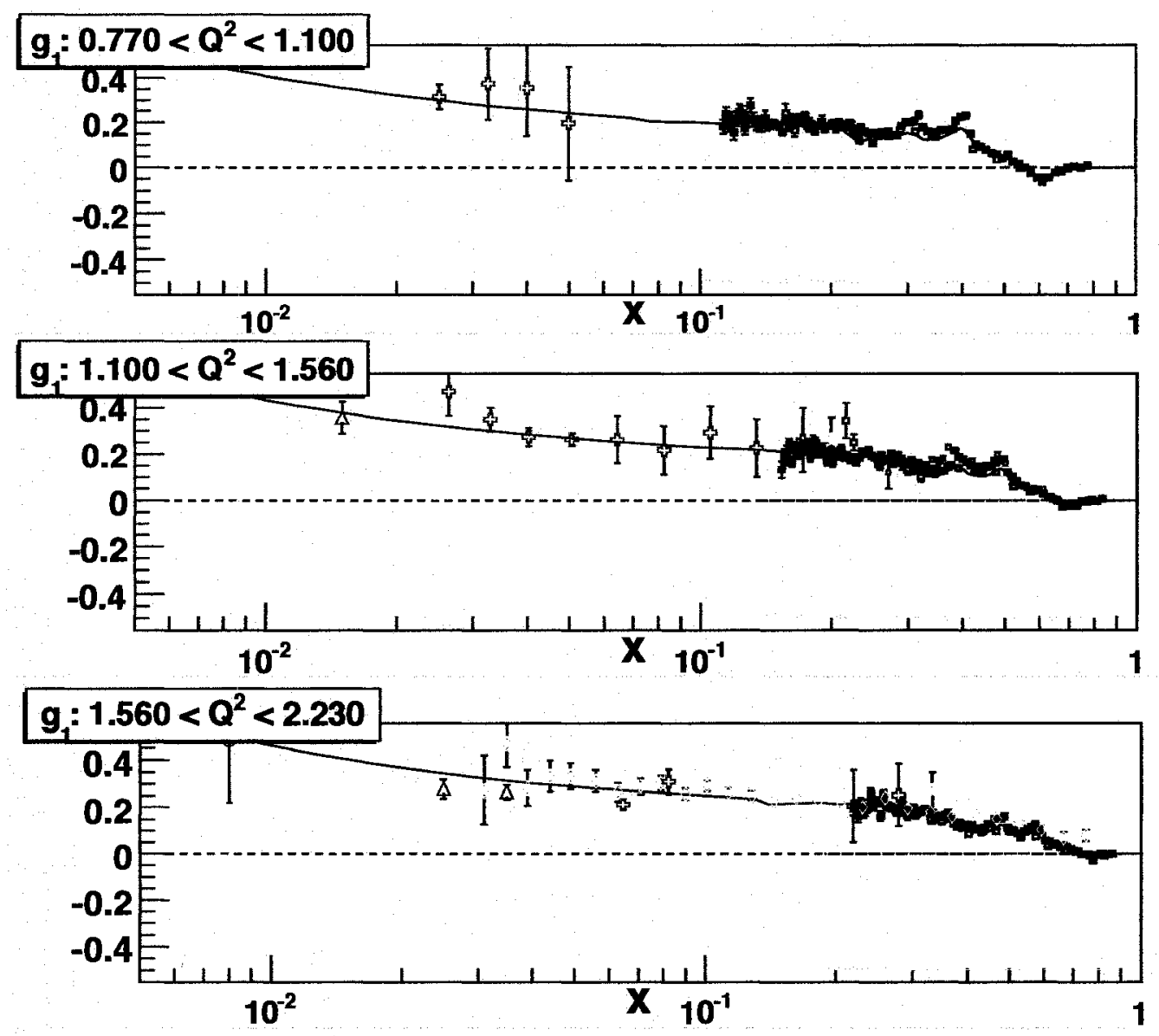

Figure 8.12: The spin-structure function $g_{1}$ vs. Bjorken $x$, in high-intermediate $Q^{2}$ bins, extracted from the EG1b data. 

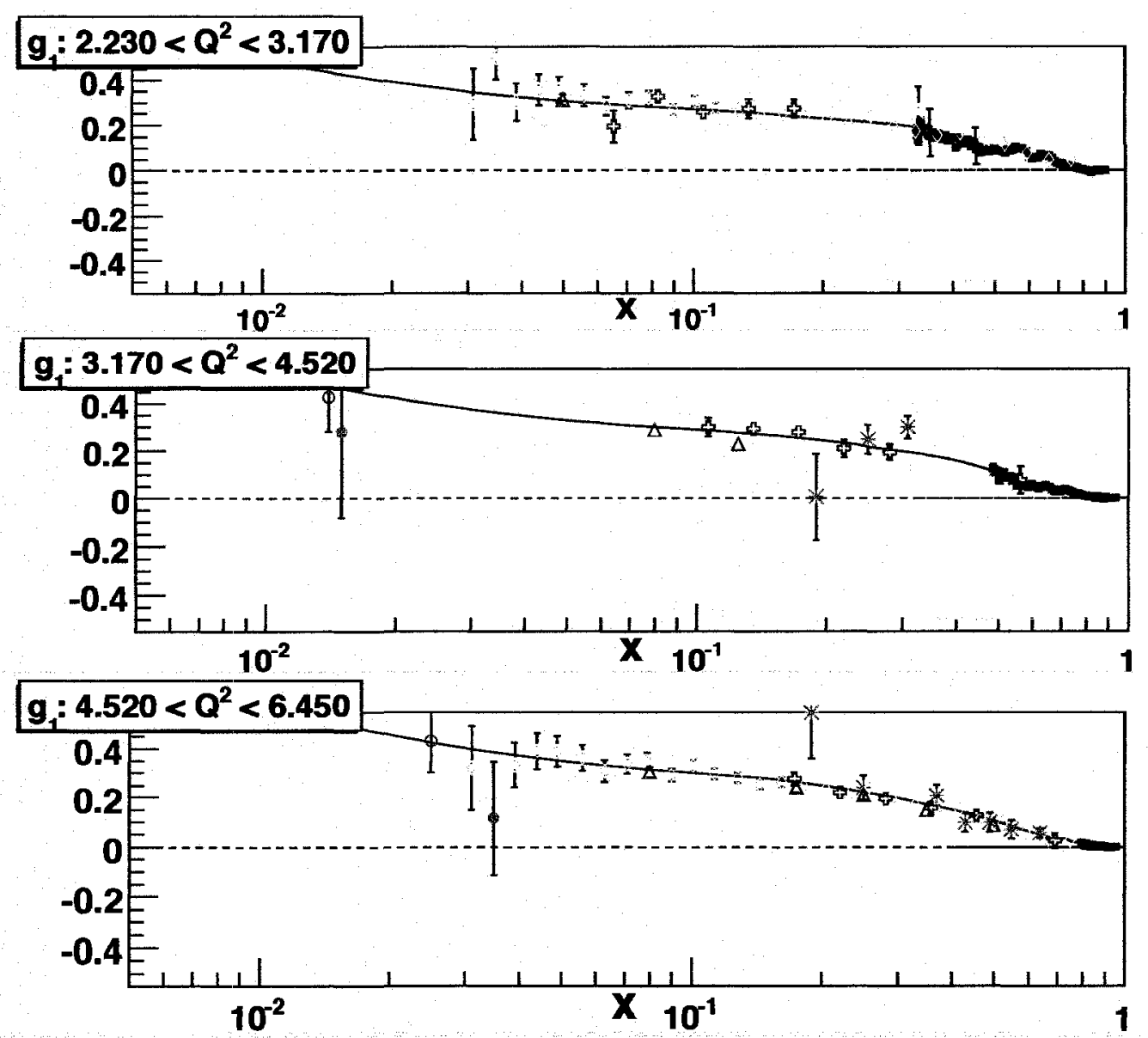

Figure 8.13: The spin-structure function $g_{1}$ vs. Bjorken $x$, in the highest $Q^{2}$ bins, extracted from the EG1b data. 

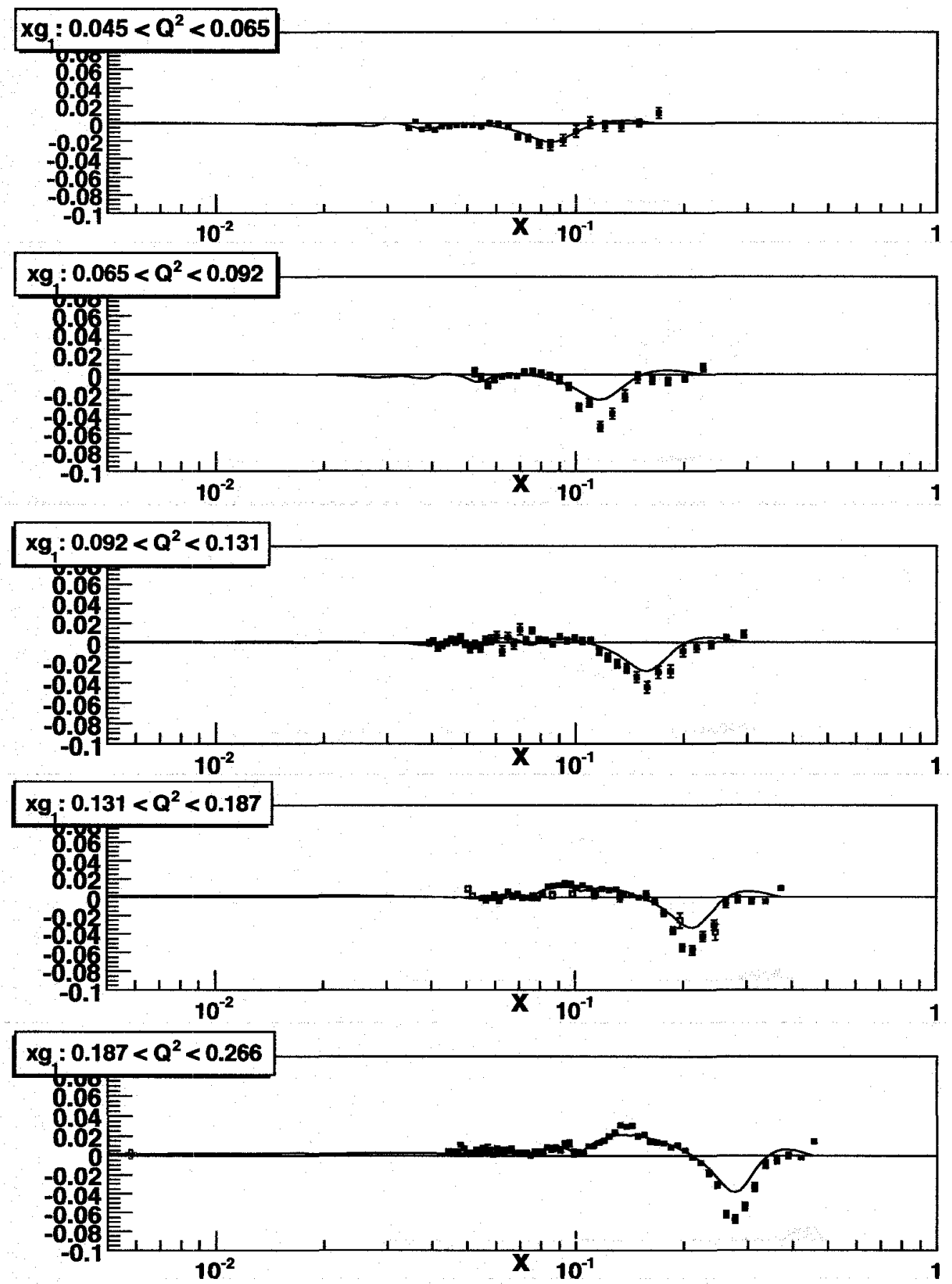

Figure 8.14: The quantity $x g_{1}$ vs. Bjorken $x$, in low $Q^{2}$ bins, extracted from the EG1b data. 

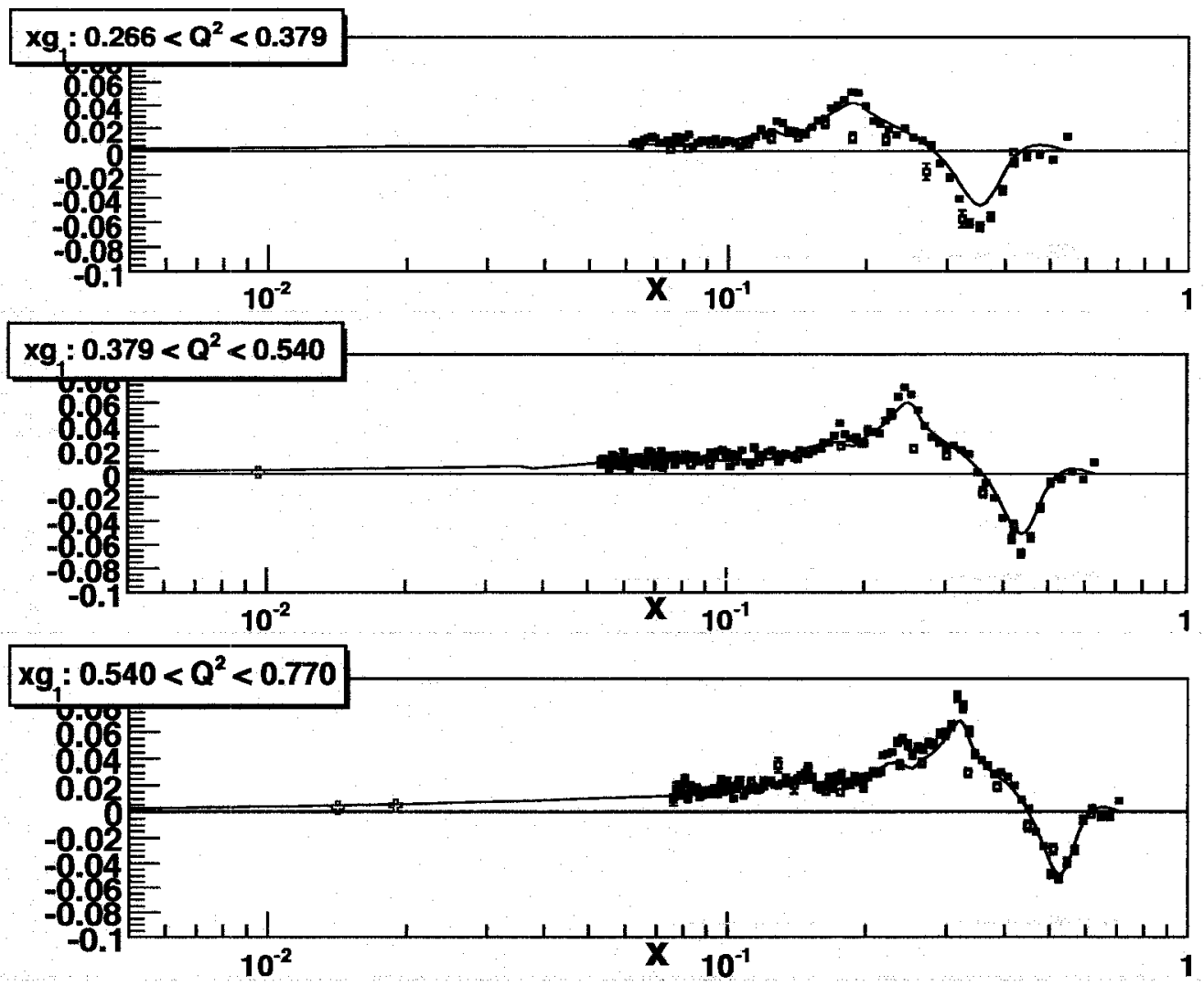

Figure 8.15: The quantity $x g_{1}$ vs. Bjorken $x$, in low-intermediate $Q^{2}$ bins, extracted from the EG1b data. 

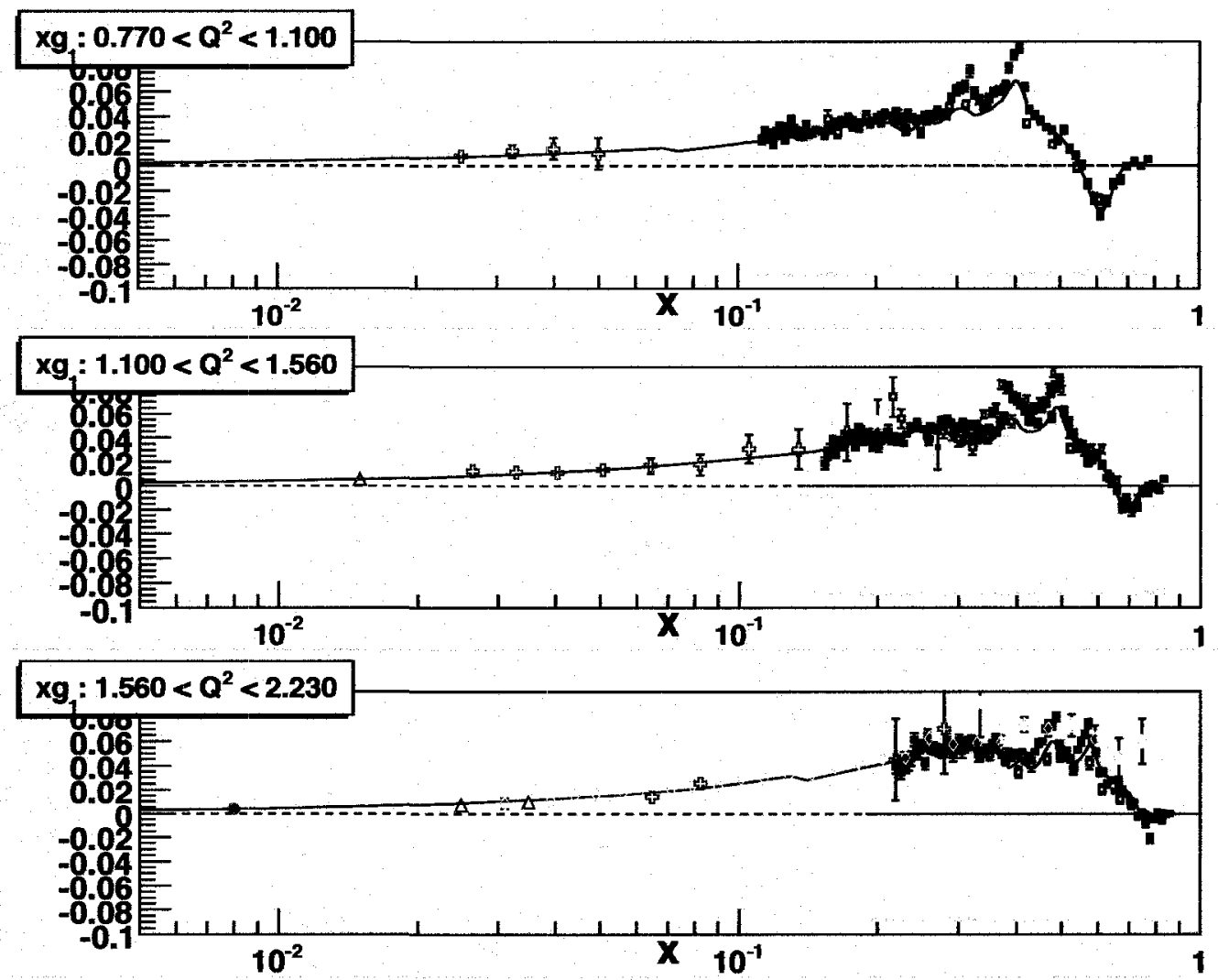

Figure 8.16: The quantity $x g_{1}$ vs. Bjorken $x$, in high-intermediate $Q^{2}$ bins, extracted from the EG1b data. 

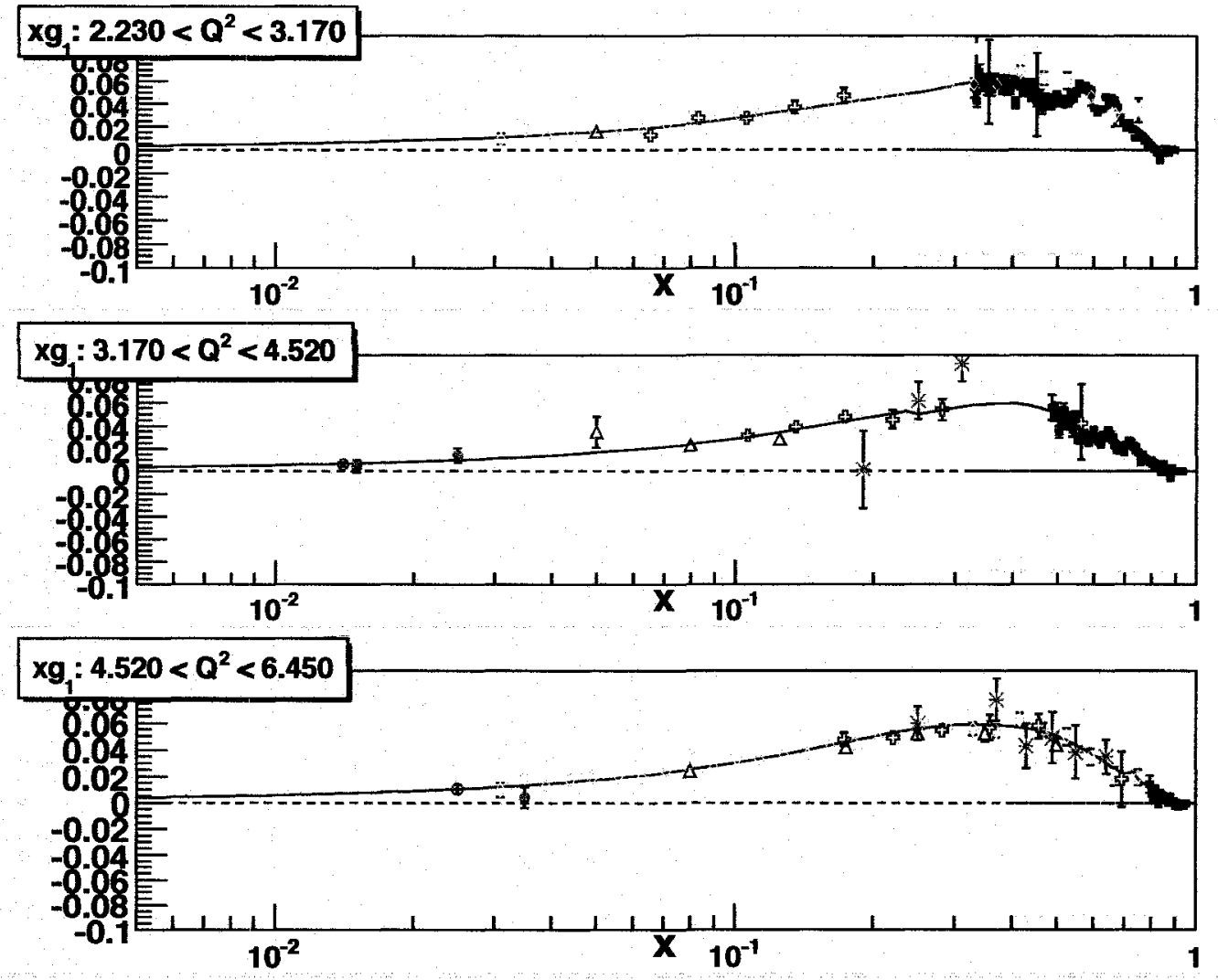

Figure 8.17: The quantity $x g_{1}$ vs. Bjorken $x$, in the highest $Q^{2}$ bins, extracted from the EG1b data. 
[155]. Using Eqs. 1.223 and 1.235, one can easily derive

$$
g_{1}\left(x, Q^{2}\right)=\frac{1}{\gamma^{2}+1}\left(\frac{A_{\|}}{D}+(\gamma-\eta) A_{2}\right) F_{1}\left(x, Q^{2}\right)
$$

Combining Eqs. 1.8, 1.17 and 1.14 yields

$$
\eta=\frac{\epsilon \gamma\left(E-E^{\prime}\right)}{E-E^{\prime} \epsilon}
$$

At small scattering angles, Eq. 1.16 implies $\epsilon \rightarrow 1$ and thus $\eta \rightarrow \gamma$. Even at the widest scattering angles (i.e. high $W$ ) in EG1, $\epsilon$ is around 0.3 ; so that $\eta \sim \gamma .{ }^{4}$ Therefore,

$$
|\gamma-\eta| \ll|\eta| \quad(\text { EG1 data) }
$$

so that the systematic error caused by $A_{2}$ is much smaller for an extraction of $g_{1} / F_{1}$ than for $A_{1}$. The statistical error on $A_{||} / D$ is propagated so that

$$
\sigma_{g_{1}}=\frac{1}{\gamma^{2}+1} \sigma_{\frac{A_{1}}{D}} F_{1}
$$

with the error from the $A_{2}$ contribution assumed as a systematic error, as usual. Values are calculated for each beam energy and statistically averaged. Final results for $g_{1}$ in terms of $x$, for various $Q^{2}$ bins, are shown in Figures 8.10 through 8.13. Also shown are plots of $x g_{1}$ vs. $x$ (Figures 8.14 through 8.17), which more prominently feature the high $x$ data measured in this experiment. Numerical results in terms of $Q^{2}$ and $W$ are listed in Table B.6.

Because the actual experimental measurement must be multiplied by a model of $F_{1}$ to derive $g_{1}$, it is customary to plot/publish results of the structure function ratio $g_{1} / F_{1}$ in terms of $x$ and $Q^{2}$. Plots of $g_{1} / F_{1}$ are good for showing the relative contribution of polarization-dependent components to the overall scattering cross-section. A combined plot of $g_{1} / F_{1}$, statistically averaged over all $Q^{2}$, also provides a great visual medium for resolving the fine structure of the resonances provided by the EG1 experiment, minimizing the effect of energy-dependent $A_{2}$ model effects. A plot of this

\footnotetext{
${ }^{4}$ That is, they are positive numbers of the same order of magnitude.
} 


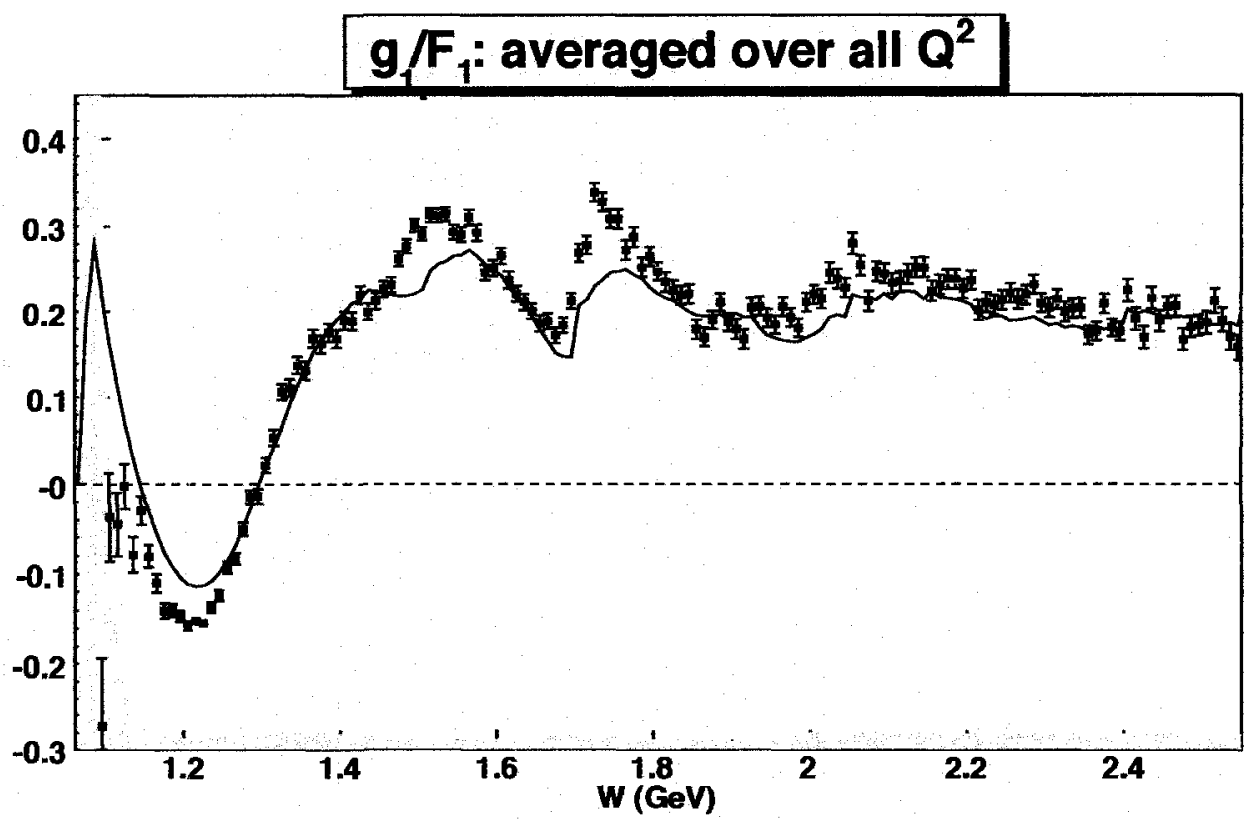

Figure 8.18: $g_{1} / F_{1} \sim A_{1}$ vs. $W$, averaged over all $Q^{2}$. Detailed resonance structure can be seen when all data are combined. The solid line is the average of the model weighted by the statistical error on the bins.

type, of $g_{1} / F_{1}$ vs. $W$, is shown in Figure 8.18 .

Plots of $g_{1} / F_{1}$ vs. $Q^{2}$ in various $x$ bins are shown in Figure 8.19. These plots serve as an illustrative example of the level of experimental precision now available in spin-physics experiments (compare to Figure 1.11). As another consistency check, measurements of $g_{1} / F_{1}$ for each beam energy were compared for compatibility in similar plots (Figure 8.20). A plot of $x g_{1}$ vs. $x$, emphasizing high $Q^{2}$ results, is shown in Figure 8.21, as an update to the HERMES data comparison shown in Figure 1.12. Tables of $g_{1} / F_{1}$ for the EG1b data are listed in Table B.6.

\subsubsection{Results for $g_{2}$}

Unlike $g_{1}$, the $g_{2}$ structure function contains a very large contribution from the $A_{2}$ virtual photon asymmetry, a quantity poorly measured by the current experiment. However, because there is also a sizeable $A_{1}$ contribution (Eq. 1.236), a reasonable reconstruction of $g_{2}$ in the resonance region can be made from the EG1 data. As with the measurement of the $g_{1}$ structure function (Eq. 8.3), 

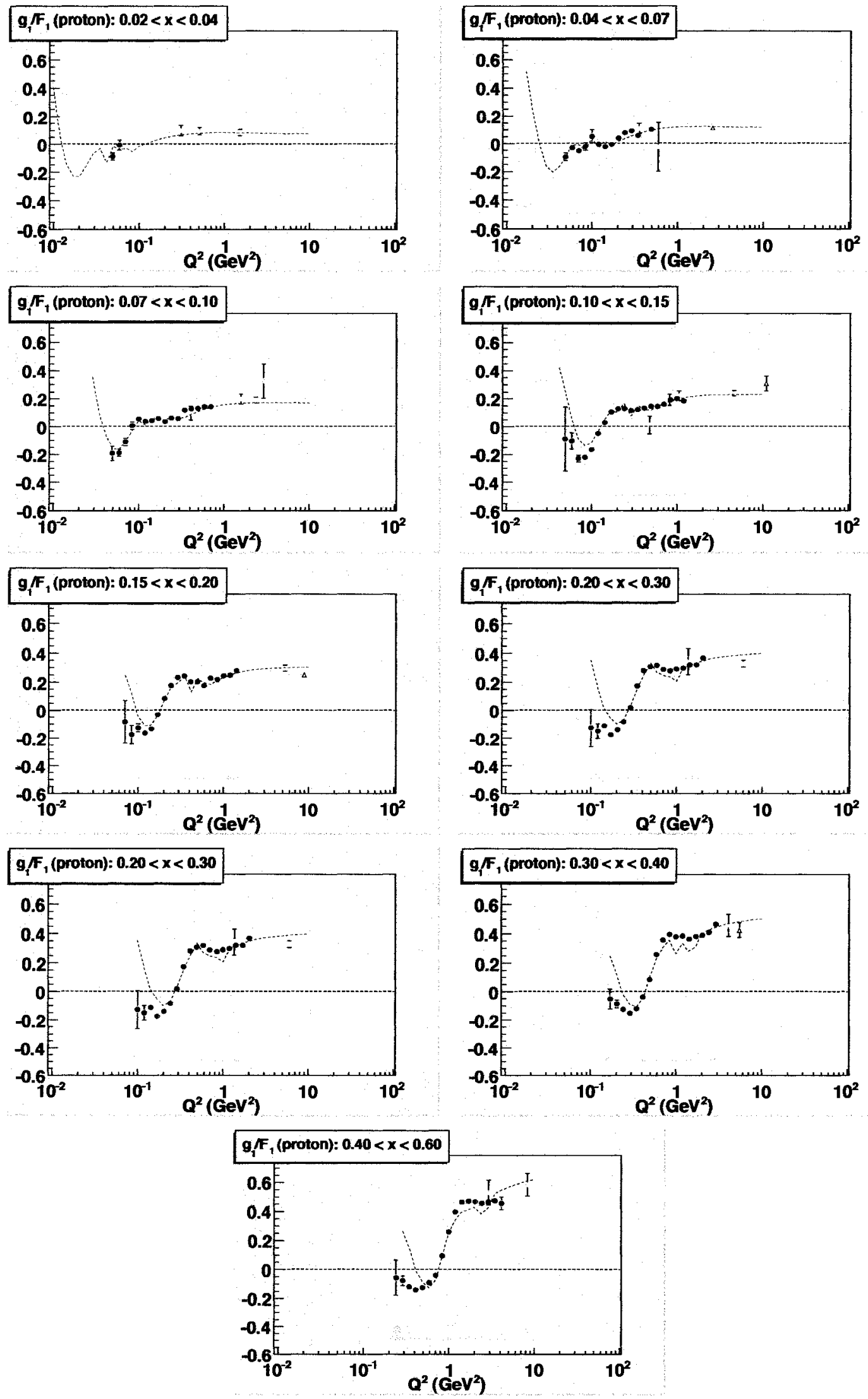

Fiaure 8.19: Plots of $a_{1} / F_{1}$ for different $x$ values for combined EG1b data. also showing SLAC F143 

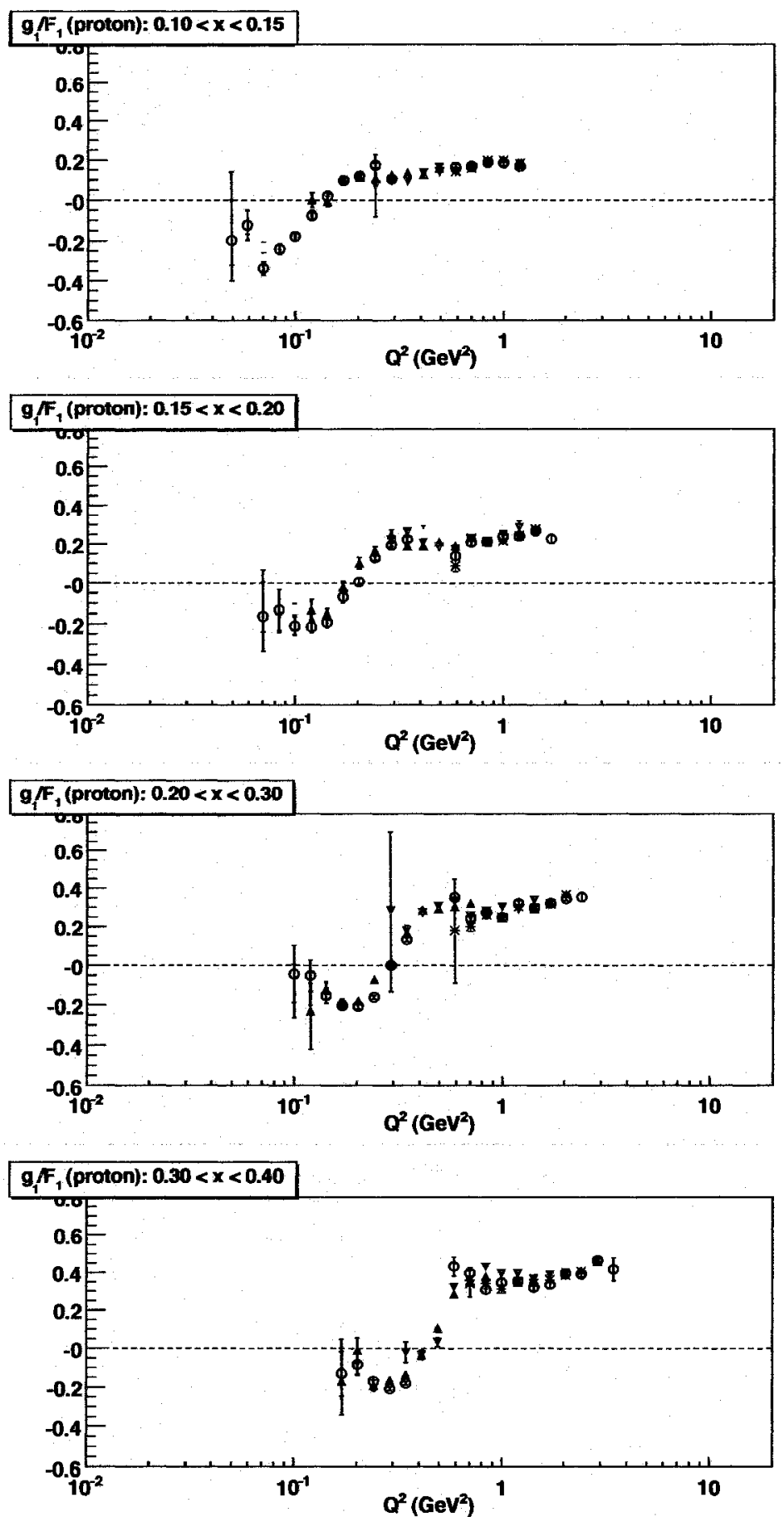

Figure 8.20: Plots of $g_{1} / F_{1}$ for different $x$ values by nominal beam energy. Shown are $1.6 \mathrm{GeV}$ data (yellow circles), $2.5 \mathrm{GeV}$ data (green triangles), $4.2 \mathrm{GeV}$ data (red inverted triangles) and $5.7 \mathrm{GeV}$ data (blue asterisks) compared to an older analysis of $1.6 \mathrm{GeV}$ and $5.7 \mathrm{GeV}$ data by Yelena Prok (black open circles) [46]. These plots were useful for demonstrating compatibility between differing analyses and beam energies. 


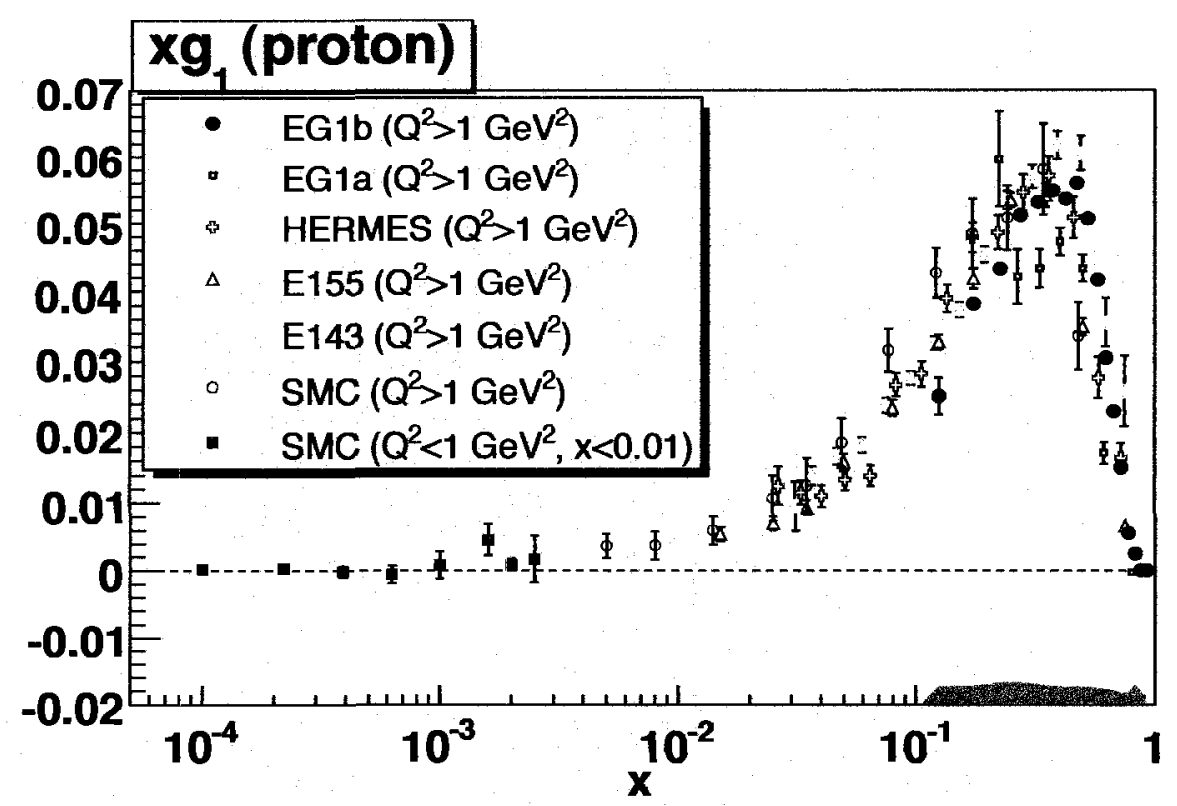

Figure 8.21: Measurements of $x g_{1}$ for $Q^{2}>1 \mathrm{GeV}^{2}$, extracted from the EG1b data, as compared to other world data. Compare to Figure 1.12.

$g_{2}$ can be written directly in terms of $A_{\|} / D$ and $A_{2}$ :

$$
g_{2}\left(x, Q^{2}\right)=\frac{1}{\gamma^{2}+1}\left[\left(\frac{1}{\gamma}+\eta\right) A_{2}-\frac{A_{\|}}{D}\right] F_{1}\left(x, Q^{2}\right)
$$

as easily calculated from Eqs.1.223 and 1.236. It is assumed that the statistical error from $A_{2}$ dominates over the much smaller error from $A_{\|} / D$, so that

$$
\sigma_{g_{2}}=\frac{1}{\gamma^{2}+1}\left(\frac{1}{\gamma}+\eta\right) \sigma_{A_{2}} F_{1}
$$

The structure function $g_{2}$ and its error were measured for each beam energy, and statistically combined in the usual manner. Numeric results for $g_{2}$ in combined bins are listed in Table B.6. Plots of $g_{2}$ vs. $x$ averaged over 4 different $Q^{2}$ ranges are shown in Figure 8.22. Similar plots of $x g_{2}$ vs. $x$ are shown in Figure 8.14. While precision is poor compared to the $g_{1}$ data, it does provide information for the constraint of future models of $g_{2}$, particularly near $Q^{2} \sim 0.5 \mathrm{GeV}^{2}$ in the middle resonance region, where the EG1 data are most plentiful. Averaging the data together over $x$ reveals a definite 
trend. Figure 8.24 shows $g_{2}^{p}$ averaged over all available $x$ in the resonance region (RR), as well as a plot of $x g_{2}^{p}$ vs. $Q^{2}$. While these plots do not preserve the resonance shape, graphs of this style are useful for viewing overall changes in $g_{2}$ with respect to virtual photon coupling strength, and comparison to extrapolated DIS functions (a basic concept behind quark-hadron duality, Section 8.4.3). The same plot, split into different $W$-bins, thus showing localized regions of resonances, is shown in Figure 8.25 .

\subsection{Spin Structure Function Moments}

Now that the spin structure functions are evaluated, the moments of $g_{1}$ and $g_{2}$ in the Operator Product Expansion can be calculated. We recall from Section 1.3.1 that the $n$th moment of a structure function $\mathscr{S}\left(x, Q^{2}\right)$ is defined as

$$
[n \text {th moment of } \mathscr{S}]\left(Q^{2}\right)=\int_{0}^{1} x^{n-1} \mathscr{S}\left(x, Q^{2}\right) d x
$$

Recall also that the even moments in the OPE are not physically significant, due to symmetry considerations; they are thus not considered in these results (see Section 1.3.3).

Naturally, the finite detector acceptance and beam energy range in EG1b does not allow for complete integration of physical data for $x=0$ to 1 . Therefore, where physical data from this experiment are not available, contributions from the $g_{1}$ and $g_{2}$ models are used instead. Confidence in the world data model behavior below $x=0.001$ is not good, so all moments are truncated on the lower end at this value. This truncation is expected to cause little to no change in the total integral value (see Section 1.3.3).

\subsubsection{Procedure for calculating moments}

In practice, of course, completely smooth integrals over data are not possible. Instead, a sum over bins is taken. Basically,

$$
[n \text {th moment of } \mathscr{S}]\left(Q^{2}\right)=I_{\text {model }\left(x_{\text {low }}\right)}\left(Q^{2}\right)+I_{\text {data }}\left(Q^{2}\right)+I_{\text {model }\left(x_{\text {high }}\right)}\left(Q^{2}\right)
$$



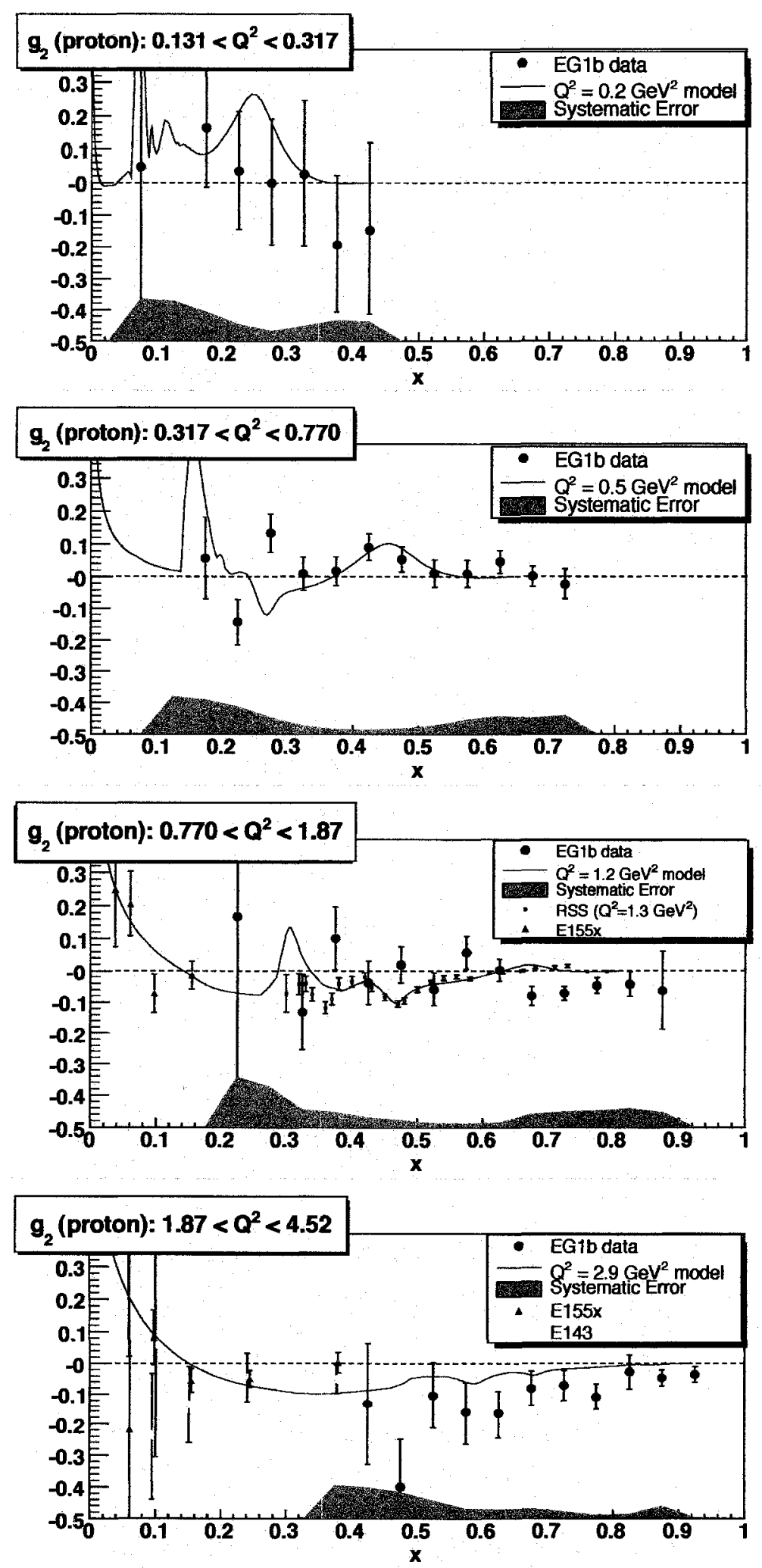

Figure 8.22: $g_{2}$ vs. Bjorken $x$ for the proton, extracted from the EG1b data. A $g_{2}$ model (blue line), based on the $A_{1}$ and $A_{2}$ models described in this thesis, is shown for comparison. (The "spike" in the model at low $x$ in the low $Q^{2}$ plots is an erroneous relic of fitting the resonance $\rightarrow$ DIS transition, and is not physical.) 

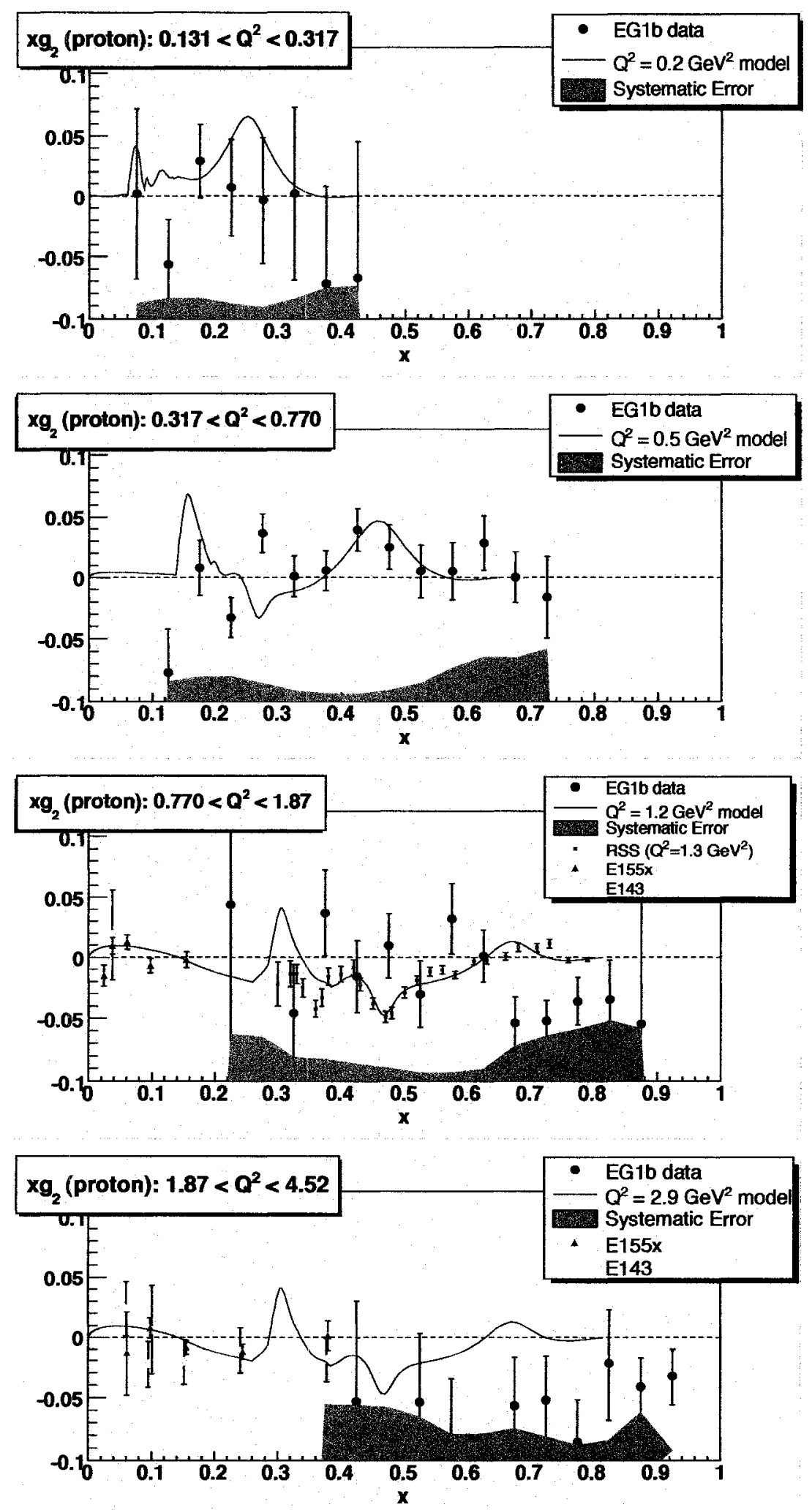

Figure 8.23: Similar plots as shown in Figure 8.22, showing $x g_{2}$ vs. Bjorken $x$ for the proton, extracted from the EG1b data. 

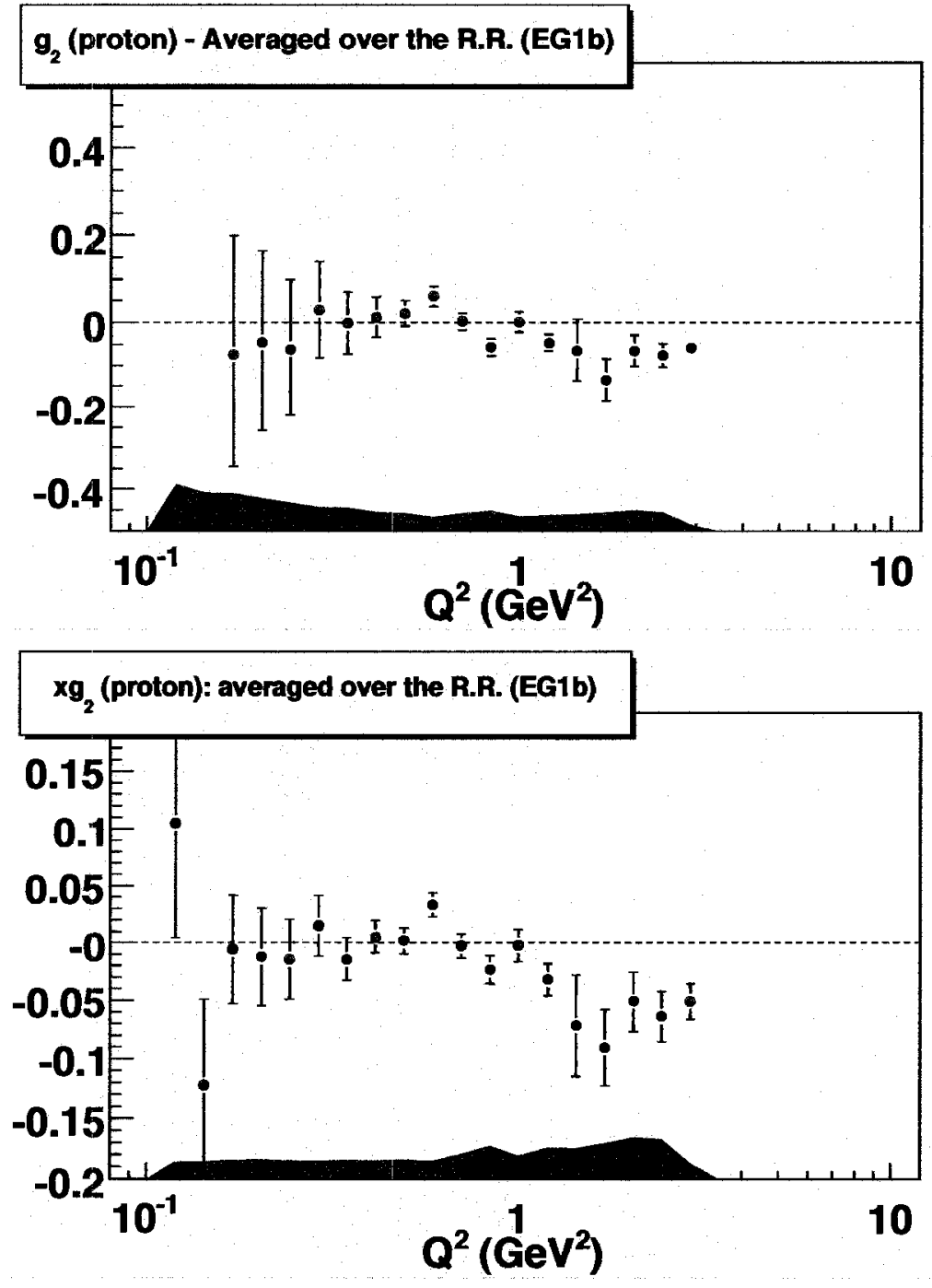

Figure 8.24: Plots of $g_{2}^{p}$ vs. $Q^{2}$ (top) and $x g_{2}^{p}$ vs $Q^{2}$ (bottom) averaged over all available $x$ in the EG1b experiment. Note that only results in the resonance region (RR) are plotted. 

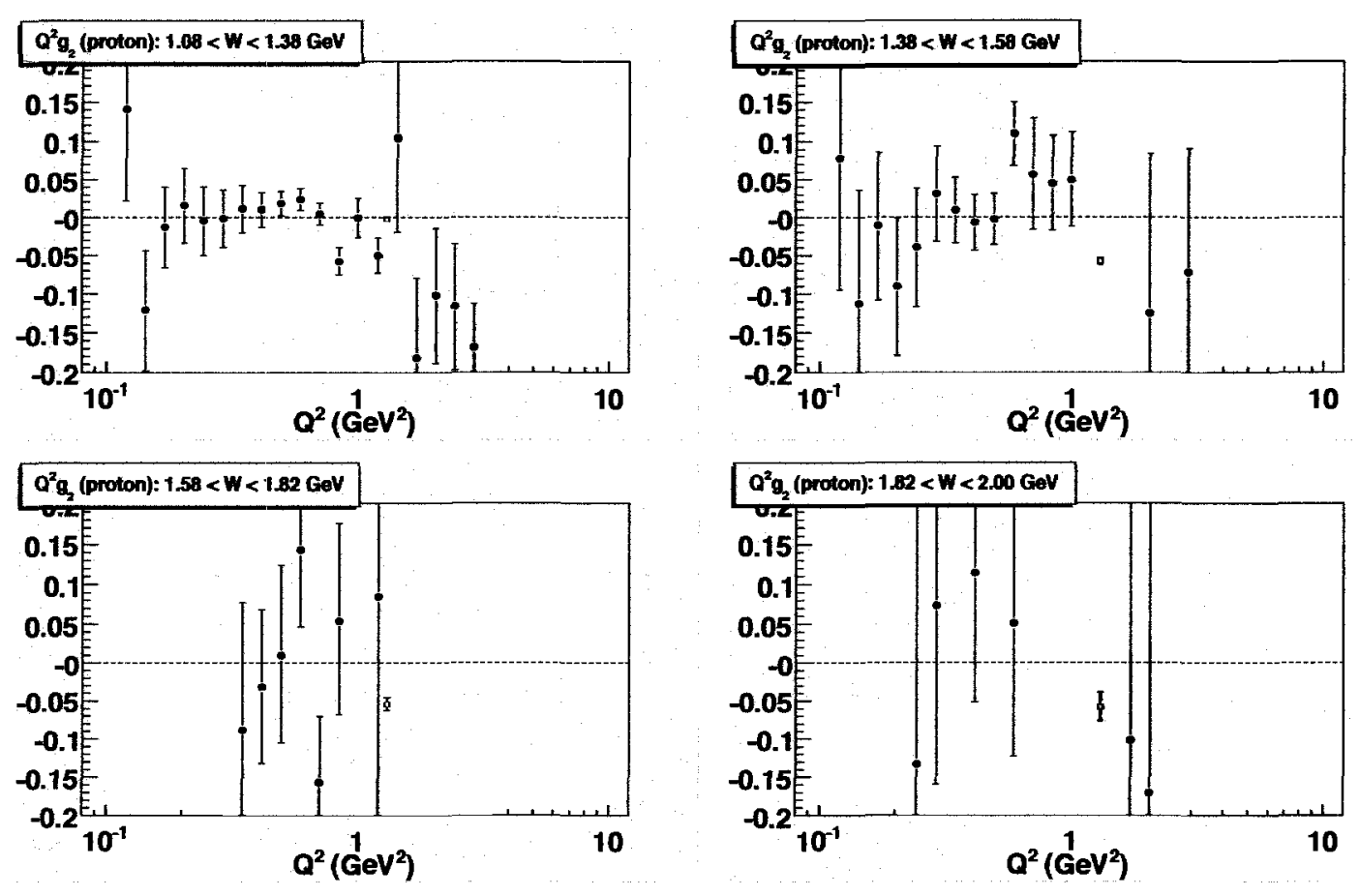

Figure 8.25: $x g_{2}^{p}$ vs $Q^{2}$ (bottom) for various $x$ ranges. Only resonance region data are plotted; this should be kept in mind before comparisons are made to Figure 1.14 (which shows DIS data only). 
The contribution from the experimental data is given by

$$
I_{d a t a}\left(Q^{2}\right)=\sum_{\left(W, Q^{2}\right)} x_{a v g}^{n-1} \mathscr{S}\left(W, Q^{2}\right)\left[x_{a}-x_{b}\right]
$$

Here, $x_{a v g}$ is the average value of $x$ in each $W$ and $Q^{2}$ bin (tracked throughout the experiment) and $x_{a}$ and $x_{b}$ are the $x$-values corresponding to the $W$-bin boundaries. Statistical errors are combined in quadrature as

$$
\sigma_{I}=\sqrt{\sum_{\left(W, Q^{2}\right)}\left(x_{a v g}^{n-1}\right)^{2} \sigma_{\mathscr{S}}^{2}\left[x_{a}-x_{b}\right]^{2}}
$$

Thus, bins with the largest statistical error bars were excluded, so as not to incur too large of a statistical error on the final result (avoiding the possibility of a single bin with a huge statistical error from dominating the uncertainty). Generally, only bins where the error on $A_{\|}$was less than 0.6 (corresponding to a minimum of 400-500 events) were used in the integration.

After the calculation of $I_{\text {data }}$, the lowest and highest bin edges used in the summation for each $Q^{2}$ bin were recorded, so that the remaining contributions, given by the models, could be added. Reference tables were printed containing the modeled $g_{1}, g_{2}$ and median $x$ values for every standard $Q^{2}$ bin (from $Q^{2}=0.01 \mathrm{GeV}^{2}$ to $10 \mathrm{GeV}^{2}$ ). The standard $W$-bin size (10 MeV) was used. All bins with a median $x$ of 0.001 or greater were printed to the reference table, from just below the inelastic threshold $(W=1.07 \mathrm{GeV}$ ) up to

$$
W=\sqrt{M^{2}+Q^{2} / x-Q^{2}}=\sqrt{0.938^{2}+10 / 0.001-10}=99.5 \mathrm{GeV} \approx 100 \mathrm{GeV}
$$

The low and high end $x$-contributions were then summed over all bins not used in the experimental sum in the same way:

$$
I_{\text {model }}\left(Q^{2}\right)=\sum_{\left(W, Q^{2} ; x>0.001\right)} x_{a v g}^{n-1} \mathscr{S}\left(W, Q^{2}\right)\left[x_{a}-x_{b}\right]
$$

All plots of integrals in this thesis chapter show two results - the experimental integral $I_{\text {data }}$ (shown in red with its systematic error, connected with a black line showing the expectations from the model) and the total integral (Eq. 8.10, shown in cyan, with its systematic error, connected with a 
blue line showing the model expectation). Systematic errors were calculated as usual; that is, each of the systematic error indices defined in the previous chapter were enforced, and the experiment was rerun from start to conclusion. The resulting differences were added in quadrature.

As a final note before moving on to the results, it should be clarified that conventions regarding inclusion of elastic peak contributions in the moments vary in the literature. To avoid confusion, all plots and tables of moments in this thesis include inelastic contributions only. Elastic values are often plotted for comparison, but are not included in the plotted moments. Where necessary, it is simple to calculate the elastic contribution. As noted in Figure 1.8, the (unradiated) asymmetry contribution from the elastic peak is represented by a $\delta$-function. Therefore, integrating over this point requires simply adding the contribution(s) from $g_{1}$ and/or $g_{2}$ defined by Eqs. 1.235 and 1.236, where $A_{1}=1$ and $A_{2}=G_{E} /\left(\sqrt{\tau} G_{M}\right)$ for elastic scattering (see Section 6.2). The results for the elastic contribution (where $x=1$ ) are

$$
\int_{1}^{1} x^{n-1} g_{1} d x=g_{1 e l}=\frac{Q^{4}}{4 M\left(4 M^{2}+Q^{2}\right)}\left[1+\frac{Q^{2}}{4 M^{2}} \frac{G_{E}}{G_{M}}\right] G_{M}^{2}
$$

and

$$
\int_{1}^{1} x^{n-1} g_{2} d x=g_{2 e l}=\frac{Q^{4}}{4 M\left(4 M^{2}+Q^{2}\right)}\left[\frac{G_{E}}{G_{M}}-1\right] G_{M}^{2}
$$

where $F_{1 e l}=M G_{M}^{2} / \gamma^{2}$ (from Eqs. 1.62 and 1.72) and $\gamma=2 M / \sqrt{Q^{2}}$ (for elastic scattering kinematics) are also employed. These terms, with $G_{M}$ and $G_{E}$ parametrized as functions of $Q^{2}$ in Section $6.2,{ }^{5}$ can be added as necessary to the total integral when needed. Cases requiring the elastic contribution ${ }^{6}$ are treated as they individually arise.

\subsubsection{Moments of $g_{1}$}

The $n$th moment of $g_{1}$ is denoted by the shorthand $\Gamma_{n}$. The first several moments of $g_{1}$ were calculated for the EG1b data. Because $x<1$, successive moments are progressively smaller in magnitude (see Eq. 8.9), so that one eventually reaches a point at high enough $n$ where the systematic error magnitude grows large compared to the moment. The first three physically significant

\footnotetext{
${ }^{5}$ The Bosted parametrization [13] is used in the plots in this thesis.

${ }^{6}$ Testing of the Burkhardt-Cottingham Sum Rule requires the elastic term.
} 
(i.e. odd) moments of $g_{1}\left(\Gamma_{1}^{p}, \Gamma_{3}^{p}\right.$, and $\Gamma_{5}^{p}$, in Figures 8.26, 8.27, and 8.28) can be calculated with some degree of accuracy from this data. Data for these moments are listed in Tables B.16, B.17 and B.18. As noted in Section 1.3.3, the analytic structure of the $g_{1}$ structure function can be reconstructed through an inverse Mellin transform, at the cost of the resonance information, in terms of $x$. The reconstructed structure function serves as a baseline for the generation of parton distribution functions (PDFs) and the testing of quark-hadron duality (Section 8.4.3).

The first moment $\Gamma_{1}^{p}$, in particular, provides a mechanism for the testing of various sum rules. At the $Q^{2} \rightarrow 0$ and $Q^{2} \rightarrow \infty$ limits, physical consequences of real photon QED and isospin conservation, respectively, can be tested, by virtue of the GDH and Bjorken Sum Rules (see Section 1.3.2). The $\Gamma_{1}^{p}$ vs. $Q^{2}$ results, over two different $Q^{2}$ ranges, are shown in Figure 8.26. The larger scale plot shows the expected Bjorken limit $\Gamma_{1}^{n}+\frac{1}{6} g_{A}=0.143$, for testing comparison. ${ }^{7}$ It can be seen that the integral approaches this limit at large $Q^{2}$, a partial vindication of the data + model results. In the low $Q^{2}$ limit (lower plot), the GDH sum rule line ${ }^{8}$ is plotted against the data. This is the line that $\Gamma_{1}^{p}$ is expected to follow as $Q^{2} \rightarrow 0$. However, both the value and the slope of the moment change very rapidly in this region. EG1b data do not extend to low enough values of $Q^{2}$ to serve as a concrete test of the fundamental GDH sum rule. We can qualitatively see that the values of $\Gamma_{1}^{p}$ fall within the valid physical limit constrained by the GDH sum rule. It remains for analysis of future spin-physics data at lower $Q^{2}$ to better confront the $Q^{2} \rightarrow 0$ limit with data. ${ }^{9}$

Testing of the simplest forms of the Bjorken and GDH sum rules is not possible in the intermediate $Q^{2}$ range. However, the data analyzed in this experiment provide a rich environment for the testing of the generalized GDH integral (incorporating $\chi \mathrm{PT}$ corrective terms, Eq. 1.179) and the modified Bjorken Sum Rule (incorporating pQCD corrective terms, Eq. 1.189). Application of these extended sum rules is an involved process, requiring the analytic solution of dispersion integrals [23] and knowledge of the $Q^{2}$ evolution of the strong coupling constant $\alpha_{s}$ [20], respectively. This subject is not investigated any further in this thesis, but data are provided in the applicable $Q^{2}$ ranges $^{10}$ for future application of these theories.

\footnotetext{
${ }^{7}$ The value of $\Gamma_{1}^{n} \approx-0.064$ comes from SMC results [22].

${ }^{8}$ This line is defined by a stope of $-0.456 / \mathrm{GeV}^{2}$ intersecting zero at $Q^{2}=0$

${ }^{9}$ The newer EG4 experiment in Hall-B serves exactly this purpose. See Section 8.5.2.

${ }^{10}$ These ranges cover up to $\sim 0.1 \mathrm{GeV}^{2}$ for the generalized GDH integral [23], and greater than $\sim 1.0 \mathrm{GeV}^{2}$ for the modified Bjorken sum rule [12].
} 


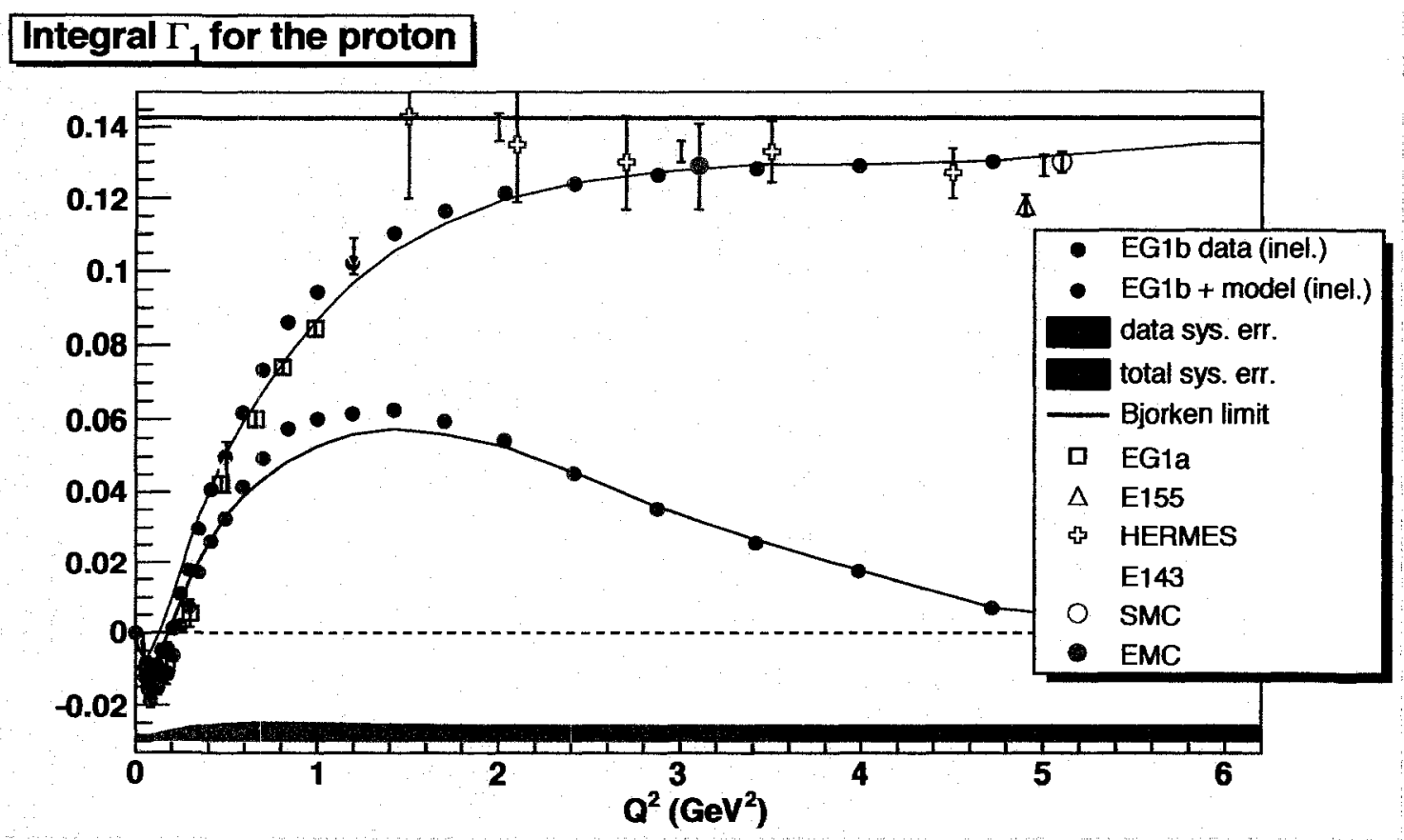

\section{Integral $\Gamma_{1}$ for the proton}

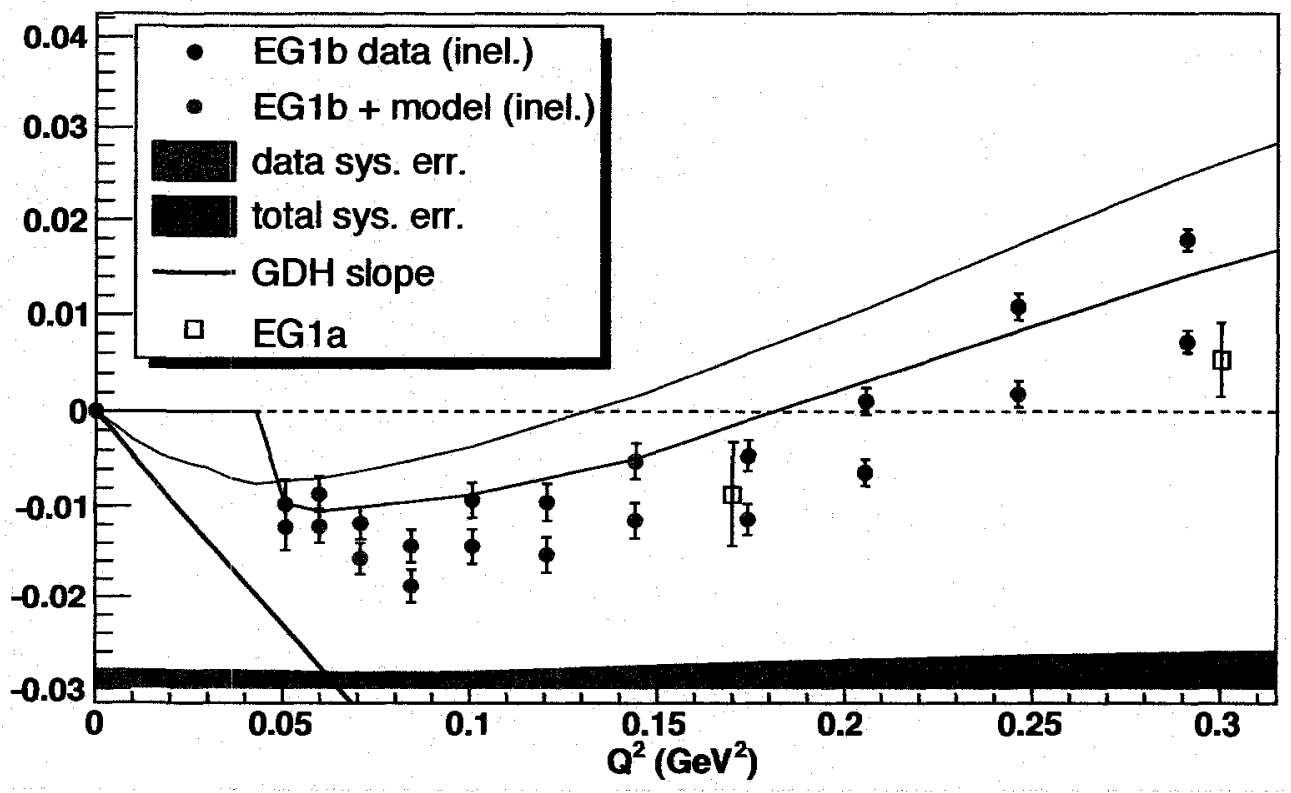

Figure 8.26: $\Gamma_{1}$ vs. $Q^{2}$ for EG1b data. The high $Q^{2}$ limit (top plot) can be used (along with neutron data) to test the modified Bjorken sum rule (Eq. 1.189). The low $Q^{2}$ limit (bottom plot) can be used to test generalized GDH integrals (Eq. 1.179). See the text for details. 


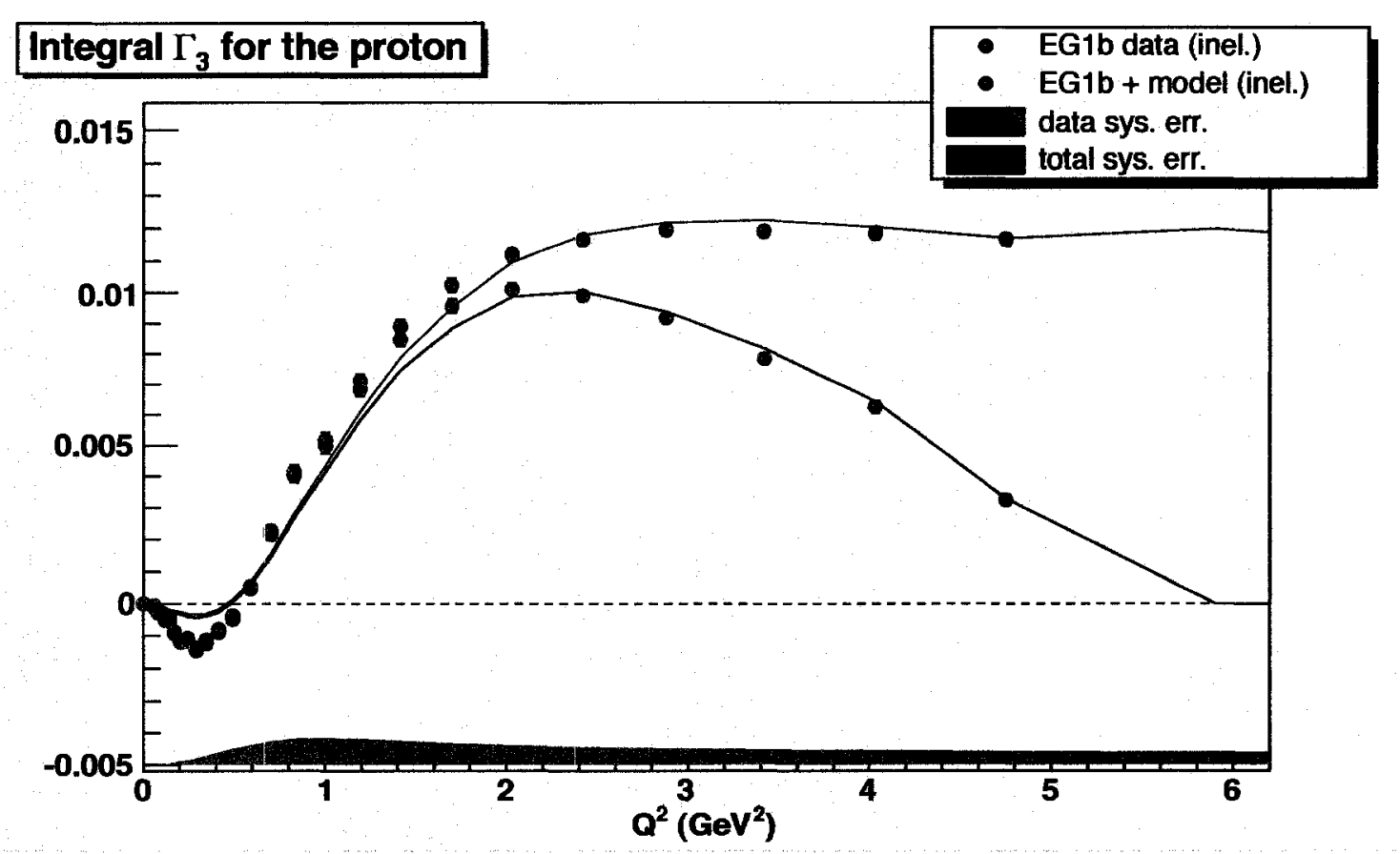

Figure 8.27: The third moment of $g_{1}$ in the Operator Product Expansion, extracted from EG1b data.

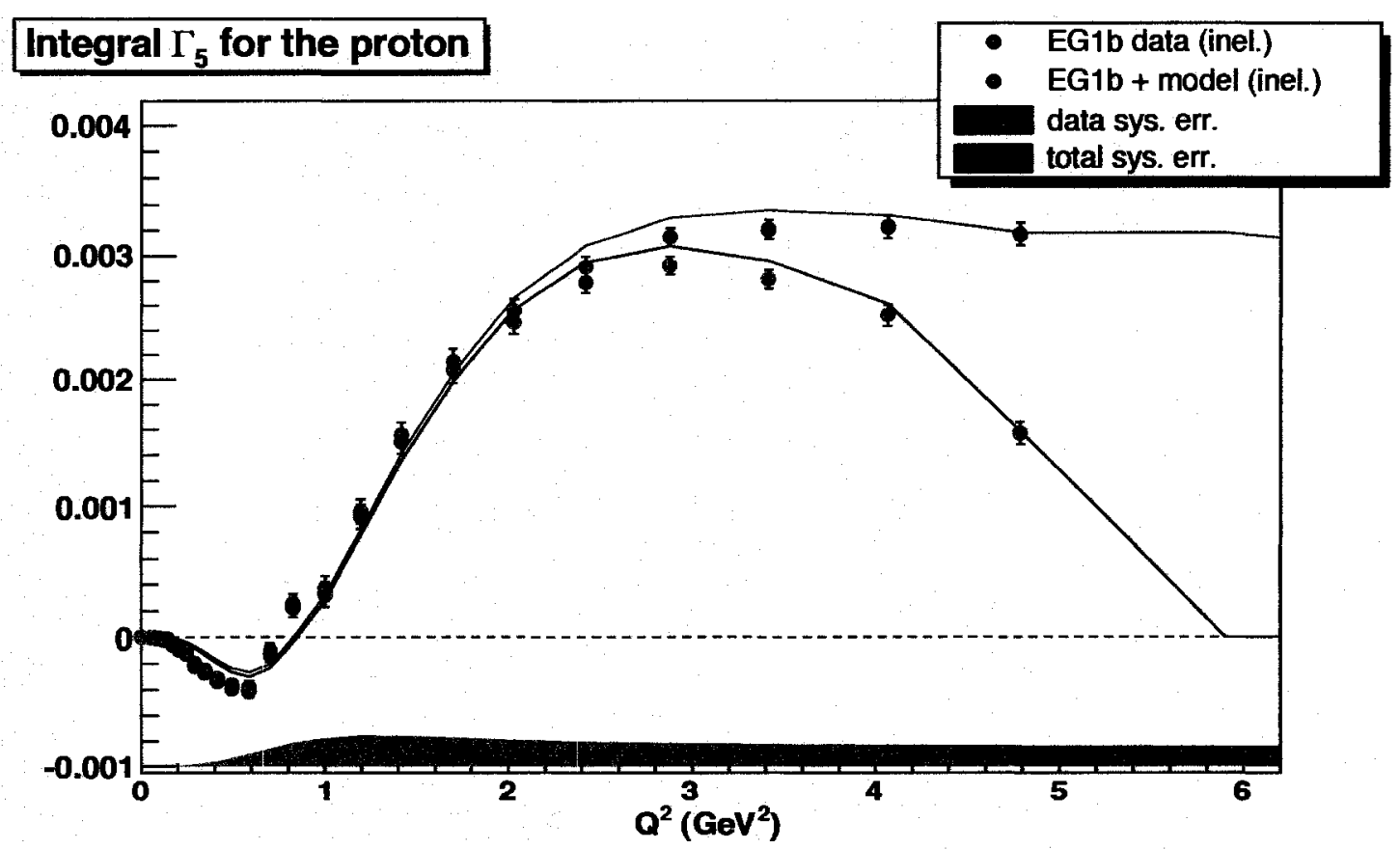

Figure 8.28: The fifth moment of $g_{1}$ in the Operator Product Expansion, extracted from EG1b data. 


\subsubsection{Moments of $g_{2}$}

Moments of the $g_{2}$ structure function can also be measured for the EG1 experiment, albeit to much less precision than those for $g_{1}$. The first and third moments of $g_{2}$ (that is, $\int g_{2} d x$ and $\int x^{2} g_{2} d x$ ) were calculated for the EG1b data. Results are shown for these integrals in Figures 8.29 and 8.30; data are given in Tables B.19 and B.20. $A_{2}$ errors were not included in the systematic error estimate for the model, and were truncated at $x=0.1$ for estimation of the overall systematic error, due to a non-physical divergence of the error at low $x .{ }^{11}$ Measurement of the first moment of $g_{2}$, in theory, allows for an approximate test of the Burkhardt-Cottingham sum rule (Eq. 1.204). To test this sum rule, the elastic contribution (Eq. 8.16) must be added to the total integral. This term is also shown in Figure 8.29. Though error bars are large, it can be seen that the net contribution due to the inelastic integral is slightly positive, and that the elastic contribution is slightly negative. Within the limited accuracy of the data, this gives a net result compatible with zero at lower $Q^{2}$. The data are not good enough for a conclusive test of the Burkardt-Cottingham sum rule, though we can say these results raise no immediate contradictions with its validity. ${ }^{12}$

Though its application will not be explored in-depth in this thesis, the $d_{n}$ matrix element integrals (Eq. 1.203) serve as a useful probe of higher-twist ${ }^{13}$ effects, particularly the second order term

$$
d_{3}\left(Q^{2}\right)=2 \int_{0}^{1} x\left[g_{1}\left(x, Q^{2}\right)+\frac{3}{2} g_{2}\left(x, Q^{2}\right)\right] d x=3 \int_{0}^{1} x^{2} \bar{g}_{2}\left(x, Q^{2}\right)
$$

At this time, we pause to take note of a conflict between common notation in the source literature. The $d_{3}$ term, as defined by Eq. 1.203, and used in much of the literature [22], is now more commonly referred to as the $d_{2}$ term. In other words, there is a difference of 1 in the counting of the index $n$ in Eq. 1.203 and more recent references [136], so caution must be used:

$$
d_{2} \rightleftharpoons d_{3} \quad \text { (notation ambiguity!) }
$$

\footnotetext{
${ }^{11}$ This turther necessitates work on a better $A_{2}$ model and a more accurate estimate of its error.

12 The two higher $Q^{2}$ points present more of a quandary, but systematic errors are large here, and may not all be properly accounted for (especially the error on the $g_{2}$ model, which is not added in, and known to be problematic at low $x$ ) and the bins are very wide here, so we cannot to put too much stock into the net negative result at these values. Better experiments in the future, such as SANE (see Section 8.5.2), will provide more precise results.

${ }^{13}$ Specifically, they quantify twist-3 contributions [22].
} 


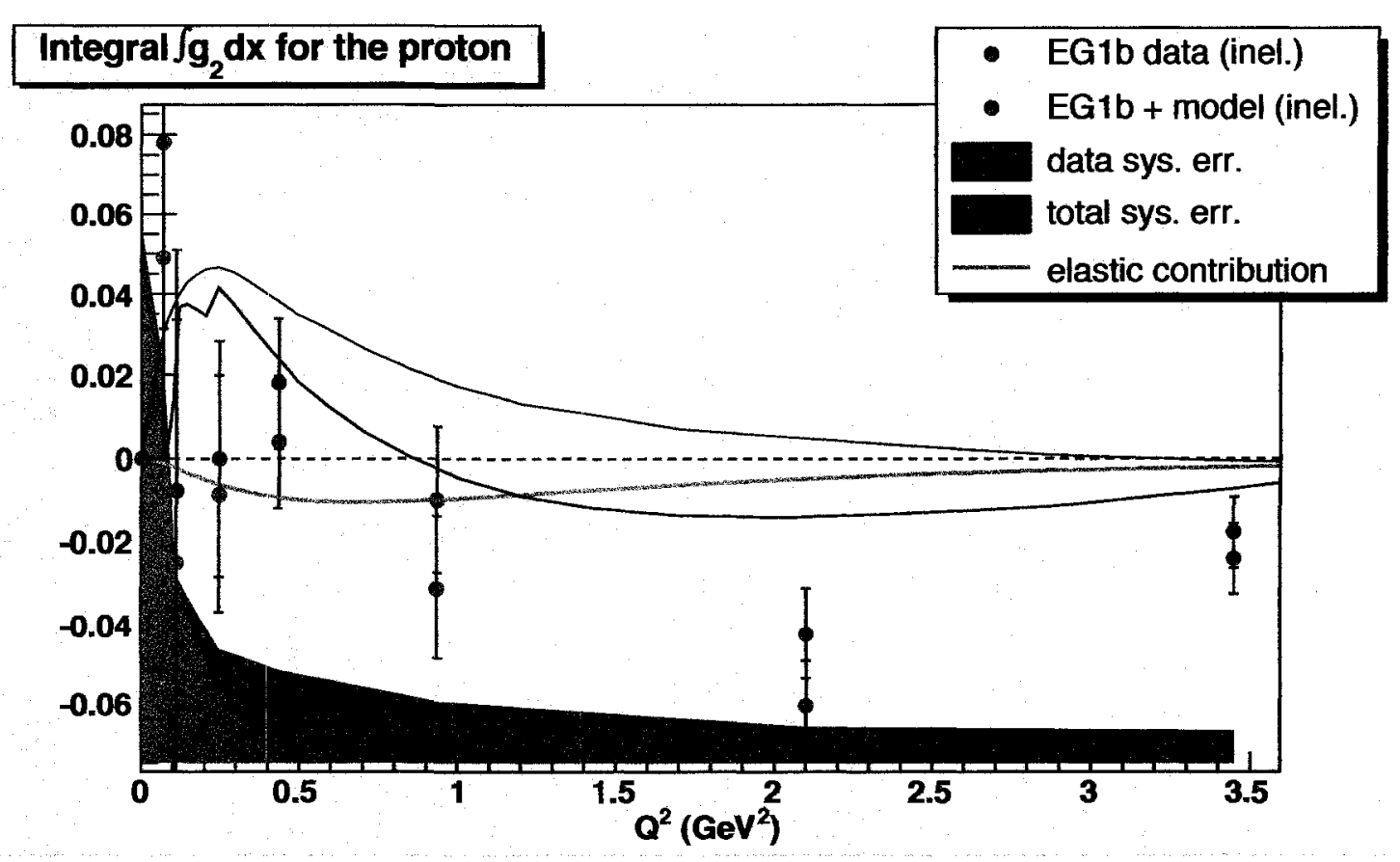

Figure 8.29: The first moment of $g_{2}$ for the proton in the Operator Product Expansion, extracted from EG1b data. Addition of the elastic (green) curve to the inelastic integral should total zero if the Burkhardt-Cottingham sum rule is correct.

The remainder of this thesis uses the (now more common) $d_{2}$ definition for this quantity. Inelastic values for this integral are listed in Table B.21; a plot is shown in Figure 8.31. ${ }^{14}$ The elastic contribution, which needs to be added to get the total integral, is also shown.

It is useful to note that [136]

$$
\int_{0}^{1} x^{2} \bar{g}_{2}\left(x, Q^{2}\right)=\frac{1}{3} d_{2}\left(Q^{2}\right)
$$

so that the $d_{2} / 3$ gives the higher twist contribution to the integral plotted in Figure 8.30 .

\subsection{Applications}

At this point, the measurements made from the EG1b data have been described in detail. Before concluding the thesis, we explore some of the applications of $g_{1}$ and $g_{2}$ and their moments.

Keeping in mind that $e p$ scattering probes the electromagnetic structure of the proton, we can

\footnotetext{
${ }^{14}$ Note the concern in Footnote 12.
} 


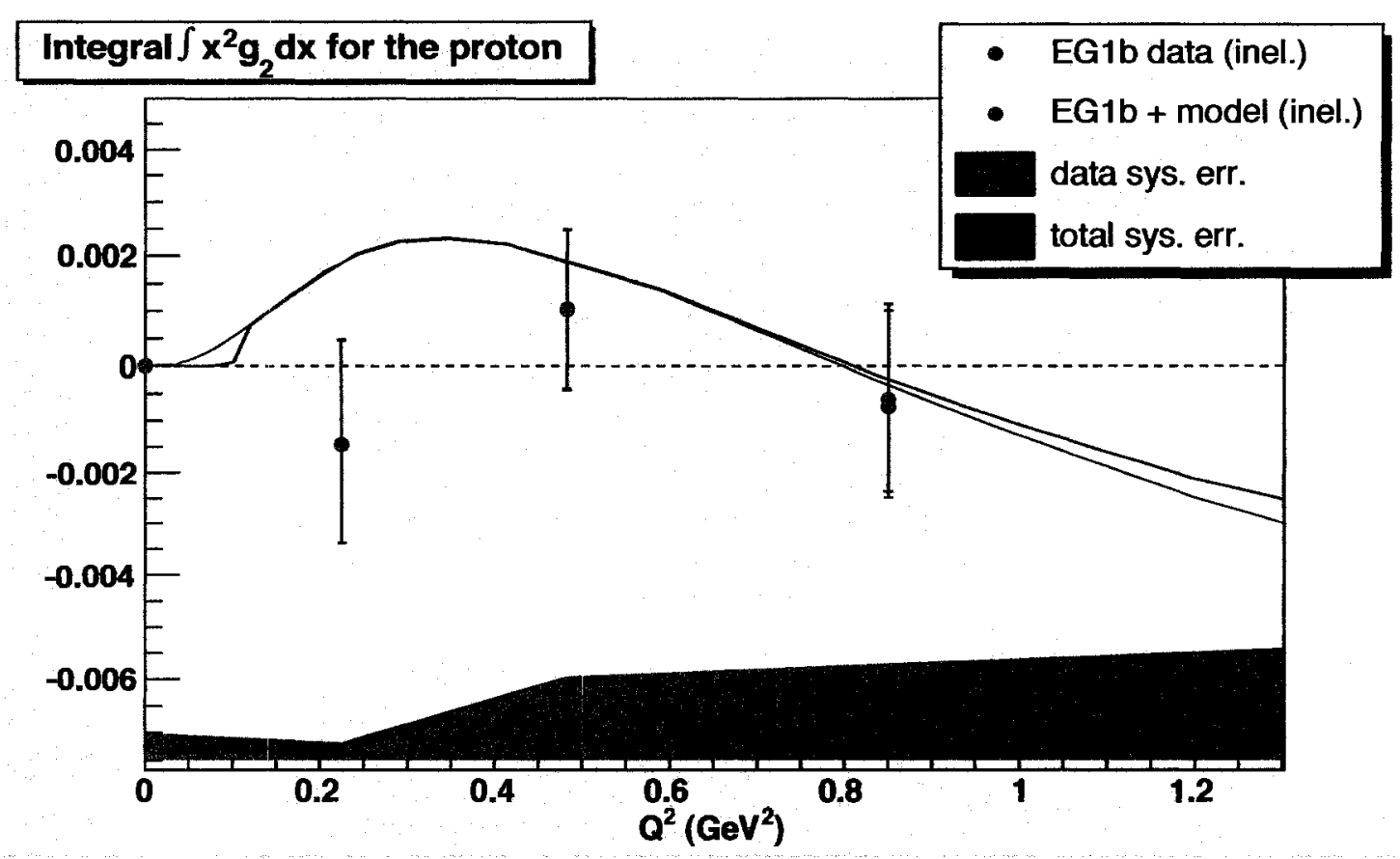

Figure 8.30: The third moment of $g_{2}$ for the proton in the Operator Product Expansion, extracted from EG1b data. Very limited information is available for higher $g_{2}$ moments in the EG1b data set.

use this structure information to calculate the characteristic response of the proton to external electromagnetic fields at different distance scales by measuring polarizabilities. We also look at a totally different kind of application of the moment data in the realm of atomic physics, in the calculation of the energy of hydrogen hyperfine splitting. Then, finally, we investigate the analytic behavior of the resonances with respect to the extrapolated DIS structure through the study of quark-hadron duality.

\subsubsection{Forward Spin Polarizability $\left(\gamma_{0}\right)$}

To introduce the concept of forward spin polarizability, we first refer back to Eqs. 1.168 and 1.169. Here, $f(\nu)$ and $g(\nu)$ represent the spin-independent and spin-flip forward Compton scattering amplitudes as $Q^{2} \rightarrow 0$. We recall that the optical theorem allows expression of the $e p$ scattering crosssection in terms of these amplitudes, and consider the terms $\alpha_{E}, \beta_{M}$ and $\gamma_{0}$ in these equations. These variables are called polarizabilities. 

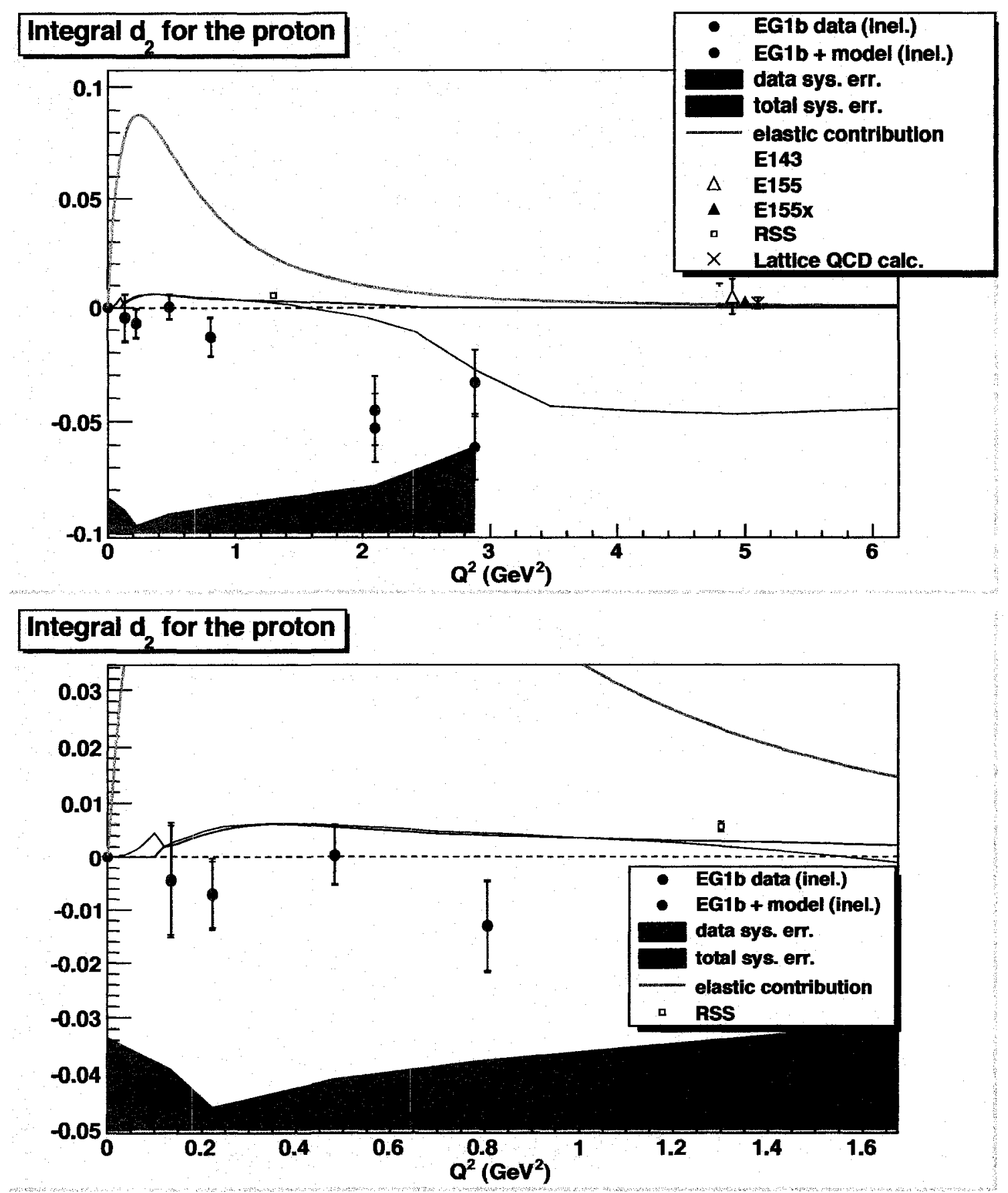

Figure 8.31: The matrix element $d_{2}$ (excluding elastic contributions) for the EG1b data, showing two different $Q^{2}$ ranges. The function $d_{2}\left(Q^{2}\right)$, a matrix component in the OPE, is a useful probe of higher-twist effects. (Note the notational ambiguity $d_{2} \rightleftharpoons d_{3}$ explained in the text.) The elastic value of $d_{2}$ (not included in the plotted sum of the data) is shown for comparison (green line). 
In Eq. 1.168 , in $f(\nu)$, the leading term $-e^{2} /(4 \pi M)$ is just a Thomson scattering term, which appears in non-relativistic scattering [137]. Thus, the $\nu^{2}$ term contains all the internal information (i.e. excitation spectrum) for unpolarized scattering, in the form of electric ( $\left.\alpha_{E}\right)$ and magnetic $\left(\beta_{M}\right)$ dipole polarizabilities. These variables characterize the "response" of the proton to an externally applied electric or magnetic field. Note that this picture specifically applies to the $Q^{2} \rightarrow 0$ limit. Analogous views of these quantities can be extended to shorter distance scales within the nucleon at higher $Q^{2}$.

Likewise, in Eq. 1.169, the terms in $g(\nu)$ can be viewed in a similar manner. The first term $-e^{2} \kappa_{P}^{2} /\left(8 \pi M^{2}\right) \nu$ is the leading-order term for polarized scattering; it is, in fact, exploited in the derivation of the GDH Sum Rule. The next term, which contains $\gamma_{0}$ (the quantity of interest here), can be interpreted (at $Q^{2}=0$ ) as the "forward spin-dependent electromagnetic response" of the proton, or more conventionally, the forward spin polarizability. The GDH Sum Rule was derived by applying Cauchy's Theorem to the $\mathscr{O}(\nu)$ term in $g(\nu)$. By applying the same process to the $\mathscr{O}\left(\nu^{3}\right)$ term containing $\gamma_{0}$, one obtains a similar sum rule [12] for the forward spin polarizability: ${ }^{15}$

$$
\gamma_{0}=\frac{1}{4 \pi^{2}} \int_{\nu_{t h}}^{\infty} \frac{\sigma_{3 / 2}\left(\nu^{\prime}\right)-\sigma_{1 / 2}\left(\nu^{\prime}\right)}{\nu^{3}} d \nu^{\prime}
$$

(compare to Eq. 1.172). We now refer to Eq. 1.230, which contains the equivalent photon energy, $\nu^{*}$. The definition of this value normalizes the total virtual photon cross-section to the lab frame kinematics. The value of $\nu^{*}$ depends on convention. Most commonly, we see Hand's Definition

$$
\nu^{*} \equiv \nu-\frac{Q^{2}}{2 M}=\nu(1-x)
$$

or the simpler Gilman's Definition

$$
\nu^{*} \equiv \nu
$$

\footnotetext{
${ }^{15}$ Once again, a contusion in conventions arises, here. Some sources include a factor of -1 in this equation, and define the polarizability (sometimes written $\gamma$, sometimes $\gamma_{0}$ ) as positive (e.g. Ref. [137]), while others leave the polarizability as a negative number (e.g. Rets. [12] and [135]). The latter approach is adopted here.
} 
The latter is used in this section. Converting the integration variable in Eq. 8.20 from $\nu$ to $x$ (by using Eq. 1.11 and calculating $\left.d \nu=-Q^{2} /\left(2 M x^{2}\right) d x\right)$, one finds

$$
\gamma_{0}=\frac{16 M^{2} \alpha}{Q^{6}} \int_{0}^{x_{t h}} x^{2}\left(g_{1}\left(x, Q^{2}\right)-\gamma^{2} g_{2}\left(x, Q^{2}\right)\right) d x
$$

Using Eq. 1.233, this can be more conveniently written

$$
\gamma_{0}=\frac{16 M^{2} \alpha}{Q^{6}} \int_{0}^{x_{t h}} x^{2} A_{1}\left(x, Q^{2}\right) F_{1}\left(x, Q^{2}\right) d x
$$

Results for the integral only of this equation (that is, without the factor of $16 M^{2} \alpha / Q^{6}$ ) are shown in Figure 8.32. Like the other integrals in this chapter, both the experimental contribution (red) and total integral (cyan) are shown, though, in this case, both are nearly equal for $Q^{2} \lesssim 1 \mathrm{GeV}^{2}$, showing that this result is very accurately measured almost completely by the experimental data, with little magnitude contributed by unmeasured regions (models). We can see that $\gamma_{0}$ is an ideal quantity for measurement in the CLAS EG1b experiment.

Using a conversion factor of $1 \mathrm{fm}^{-1}=5.07 \mathrm{GeV}^{-1}$ [3], $\gamma_{0}$ itself was found by multiplying the integral by $16 M^{2} \alpha / Q^{6}$ (all units in factors of $\mathrm{GeV}$ ) and multiplying the final value by

$$
\frac{1 \mathrm{fm}^{-4}}{1 \mathrm{GeV}^{-4}} \times 10^{-4}=\frac{10^{-4}}{5.07^{-4}}[\mathrm{fm} / 10 \mathrm{GeV}]^{4}=\frac{1}{0.06607}[\mathrm{fm} / 10 \mathrm{GeV}]^{4}=15.134[\mathrm{fm} / 10 \mathrm{GeV}]^{4}
$$

to convert to units of $10^{-4} \mathrm{fm}$. This value of $\gamma_{0}$ is plotted in Figure 8.33. Due to the factor of $Q^{-6}$, this quantity, unlike the other moments and integrals in this chapter, does not diminish to zero at small $Q^{2}$. This means the forward spin polarizability integral is an excellent testing ground for $\chi$ PT theories, which are only valid at $Q^{2}$ values around $0.1 \mathrm{GeV}^{2}$ and lower. So far, heavy baryon and relativistic $\chi$ PT theories have had little success at fitting the available data for the forward spin polarizability at low $Q^{2}[135][138]$.

Results for $\gamma_{0}$ at $Q^{2} \rightarrow 0$ have been measured in the MAMI GDH experiment as [137]

$$
\gamma_{0}^{p}=[-1.01 \pm 0.08 \pm 0.10] \cdot 10^{-4} \mathrm{fm}^{4}
$$




\section{Forward Spin Polarizability Integral for the proton}

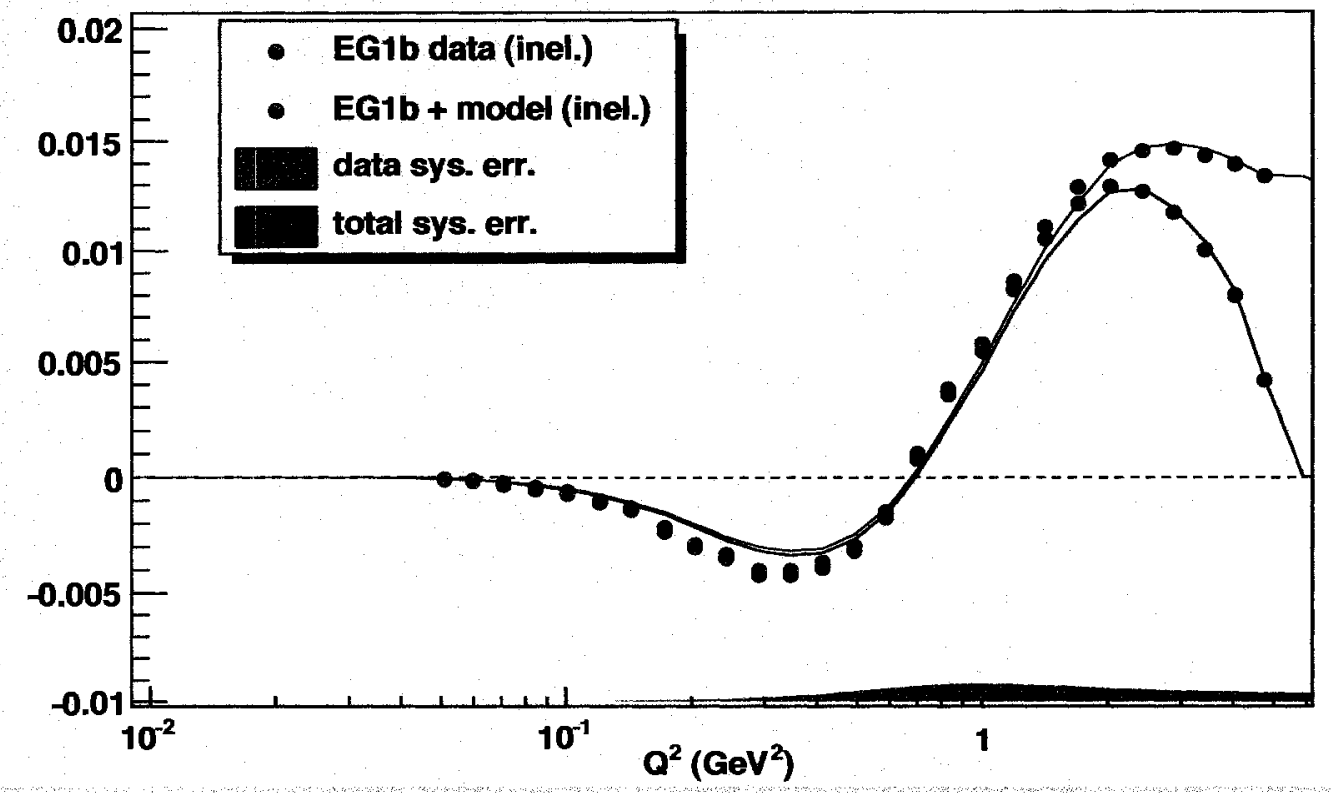

Figure 8.32: The forward spin polarizability integral, extracted from EG1b data. EG1b provides an ideal measurement of this quantity, as very little contribution from models terms is required.

EG1b data are unreliable for this integral below $Q^{2} \sim 0.08 \mathrm{GeV}^{2}$, though no substantial incompatibility with this $Q^{2} \rightarrow 0$ result is seen, within the bounds of statistical and systematic errors. (Extrapolation of the experimental curve intersects the axis at this point, within the limits of our errors).

Prior to this point, analysis of global ep scattering data has provided ample opportunity for measurement of the unpolarized dipole polarizabilities $\alpha_{E}$ and $\beta_{M}$, but not before the advent of adequate spin structure function data in the resonance region arose in the form of EG1b, were measurements of $\gamma_{0}$ possible for virtual photons [135]. Tables of both the forward spin polarization integral $\int x^{2} A_{1} F_{1} d x$ and $\gamma_{0}$ are listed in Tables B.22 and B.23.

\subsection{2 ${ }^{1}$ H Atomic Hyperfine Splitting}

Traditionally, the realms of nuclear physics and atomic physics are dealt with in completely separate experimental and theoretical applications, as the characteristic excitation spectra of nuclei and atomic electron orbitals are separated by several orders of magnitude ( $\mathrm{MeV}$ vs. eV, typically). How- 


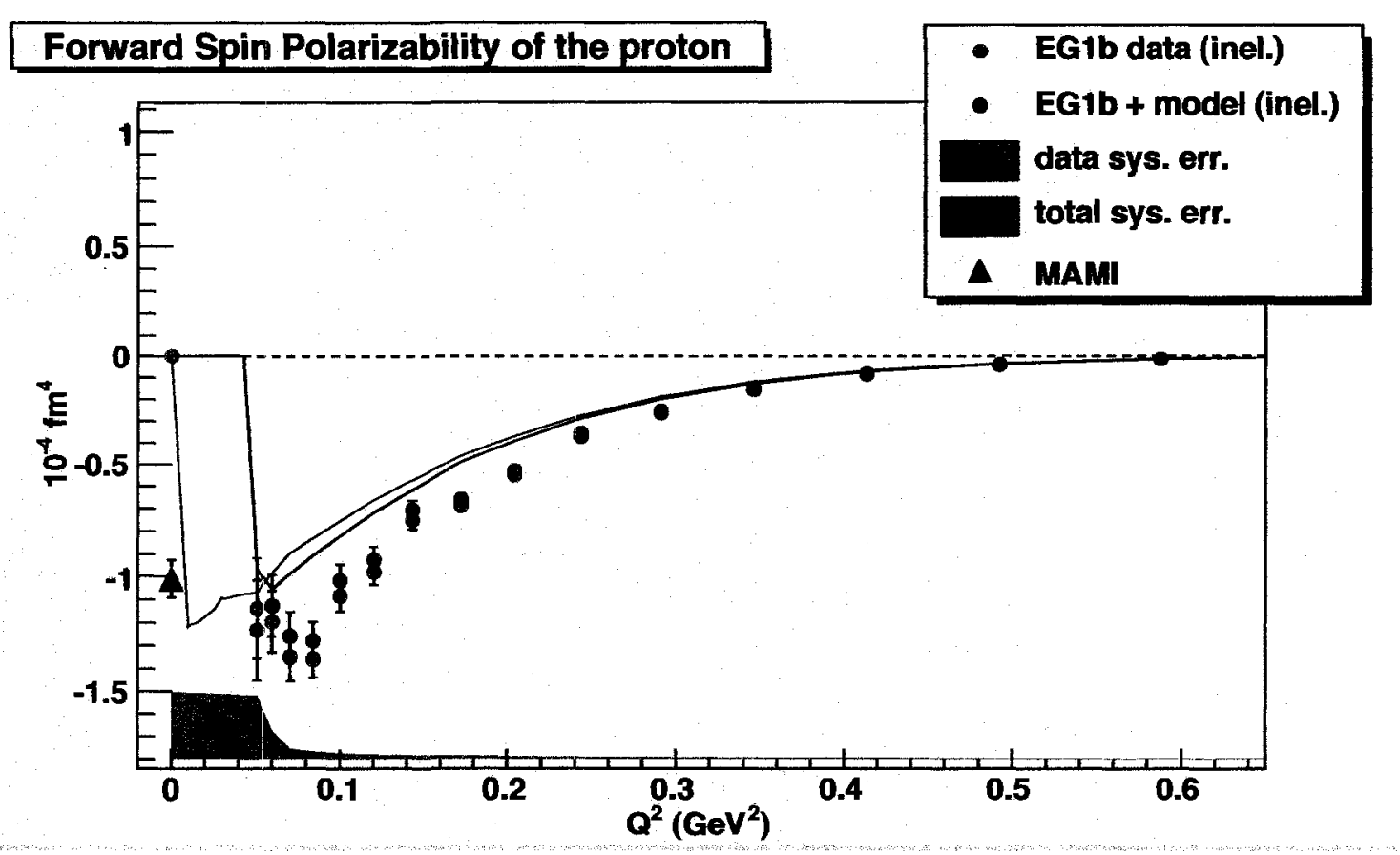

Figure 8.33: Forward spin polarizability $\gamma_{0}$, calculated from the polarizability integral. New data analysis in intermediate $Q^{2}$ regions (provided by 2.5 and $4.2 \mathrm{GeV}$ analyses) yields new information in the sensitive region around $Q^{2} \sim 0.1-0.2 \mathrm{GeV}^{2}$. 
ever, this treatment is not exactly correct, of course. If one makes sensitive enough measurements in either realm, the excited states within nuclei and electron orbitals are found to couple to one another, albeit only very weakly. One can picture the ${ }^{1} \mathrm{H}$ atom as the world's smallest electron accelerator, ${ }^{16}$ operating at an energy of $13.6 \mathrm{eV}$. Like any electron in an accelerator, the orbital energy is determined by the electromagnetic field acting on the particle. This coupling is primarily electrical in nature; the assumption of an electric field from a point-like proton results in the base $13.6 \mathrm{eV}$ energy of the ground-state orbital. However, the proton and electron have spin, of course, too (a point central to this thesis!) - and thus act as tiny magnets, which also couple to one another through their magnetic fields. Depending on whether the resultant dipoles are aligned or antialigned, this coupling strength will perturb the total energy. Thus, the observed spectrum exhibits hyperfine splitting of the $13.6 \mathrm{eV}$ ground state between these two spin-induced states, with an energy difference $\Delta E_{h f}$ given by $[7]$

$$
\Delta E_{h f}=\frac{8}{3} \alpha^{4} \frac{m_{e}}{M} m_{e}\left(1+\kappa_{P}\right)
$$

where $1+\kappa_{P}=\mu_{p}=2.79$ is the proton magnetic moment. This simple calculation is just an approximation which assumes $m_{e} \ll M$. Removing this assumption, this expands to

$$
\Delta E_{h f}=\frac{8}{3} \alpha^{4} \frac{M^{2} m_{e}^{2}}{\left(M+m_{e}\right)^{3}}\left(1+\kappa_{P}\right)
$$

Evaluation of the magnitude of this hyperfine splitting difference is one of the great achievements of modern experimental precision measurement techniques. Its value is known to 13 significant figures, more precision than any other measured quantity [139]:

$$
\Delta E_{h f}=1420.4057517667(9) \mathrm{MHz}
$$

This level of precision necessitates a better model than that used to derive Eq. 8.28 , which treats the proton as a point particle, and does not account for the structure of the proton, higher order radiative effects, vacuum polarization, proton recoil, or the effects of the weak interaction (i.e. $Z^{0}$

\footnotetext{
${ }^{16}$ This analogy should not be taken too far - the electron in an atom is in a quantum stationary state (and does not emit synchrotron radiation, for example), so it isn't really "accelerated", per se, unless an external perturbation is applied.
} 
boson exchange). All these must be taken into account at this level of precision. A more exact relation is given by [139][140]

$$
\Delta E_{h f}=\frac{8 \alpha^{4} M^{2} m_{e}^{2}}{3\left(M+m_{e}\right)^{3}}\left(1+\kappa_{P}\right)\left(1+\Delta_{Q E D}+\Delta_{R}+\Delta_{h v p}+\Delta_{\mu v p}+\Delta_{\text {weak }}+\Delta_{S}\right)
$$

where the various corrective terms are related to the following respective physical phenomena:

$$
\left\{\begin{array}{cl}
\Delta_{Q E D} & \text { QED radiative corrections } \\
\Delta_{R} & \text { recoil corrections } \\
\Delta_{h v p} & \text { hadronic vacuum polarization } \\
\Delta_{\mu v p} & \text { muonic vacuum polarization } \\
\Delta_{w e a k} & \text { weak } Z^{0} \text { virtual boson coupling } \\
\Delta_{S} & \text { proton structure corrections }
\end{array}\right.
$$

The last of these terms has relevance to the quantities measured in this thesis. Subtracting out the other terms, calculated theoretically, from the experimental value (Eq. 8.29) yields [140]

$$
\Delta_{S}=\Delta_{z}+\Delta_{p o l}=-38.58(16) \mathrm{ppm}
$$

where the uncertainty is split into a term calculated by A.C. Zemach [141] in 1956, and a much smaller polarization-dependent term. The Zemach term is given in terms of the proton form factors as

$$
\Delta_{Z}=-\frac{8 \alpha m_{e}}{\pi}\left(1+\delta_{Z}^{r a d}\right) \int_{0}^{\infty} \frac{d Q}{Q^{2}}\left[G_{E}\left(Q^{2}\right) \frac{G_{M}\left(Q^{2}\right)}{1+\kappa_{P}}-1\right]
$$

where $\delta_{Z}^{\text {rad }}=0.015(0)$ is an anomalous calculated higher-order contribution to the form factors [140].

It is the other term in $\Delta_{S}$ that is of interest in this thesis; it is given by [140]

$$
\Delta_{p o l}=\frac{\alpha^{3 / 2} \sqrt{\pi} m_{e}}{2 M^{2} \mu_{p}}\left(\Delta_{1}+\Delta_{2}\right)
$$


where

$$
\Delta_{1}=\frac{9}{4} \int_{0}^{\infty} \frac{d Q^{2}}{Q^{2}}\left\{\mathscr{F}_{2}^{2}\left(Q^{2}\right)+\frac{8 M^{2}}{Q^{2}} B_{1}\left(Q^{2}\right)\right\}
$$

and

$$
\Delta_{2}=-24 M^{2} \int_{0}^{\infty} \frac{d Q^{2}}{Q^{2}} B_{2}\left(Q^{2}\right)
$$

Here, $\mathscr{F}_{2}$ is the Pauli form factor (defined in Section 1.2.2) and $B_{1}$ and $B_{2}$ are spin-structure function integrals, specifically

$$
\begin{gathered}
B_{1}\left(Q^{2}\right)=\frac{4}{9} \int_{0}^{x_{t h}}\left(-3 \tau+2 \tau^{2}+2(2-\tau) \sqrt{\tau(\tau+1)}\right) g_{1}\left(x, Q^{2}\right) d x \\
B_{2}\left(Q^{2}\right)=\int_{0}^{x_{\text {th }}}(1+2 \tau-2 \sqrt{\tau(\tau+1)}) g_{2}\left(x, Q^{2}\right) d x
\end{gathered}
$$

where $x_{t h}$ is the threshold corresponding to inelastic pion production $(W=1.077 \mathrm{GeV})$ and $\tau$ is the kinematic factor defined in Eq. 1.15.

With these calculations, one can see the immediate application of the $g_{1}$ and $g_{2}$ structure function results to a physically measurable quantity in atomic physics. Low $Q^{2}$ kinematics contribute the bulk of the above integrals [140]. Evaluation of these structure functions in the resonance region at low $Q^{2}$ thus greatly increases the known accuracy of $\Delta_{\text {pol }}$. The EG1b analysis supplies an excellent resource for the $g_{1}$ structure function. The $g_{2}$ contribution presents a more difficult case, and is the limiting factor in our knowledge of $\Delta E_{h f}$ [142]. Plots of these integrals are shown in Figures 8.34 and 8.35.

More accuracy can be obtained if we neglect the effect of $H T$ corrections to $g_{2}$. That is, we assume $g_{2}=g_{2}^{W W}$, as defined in Eq. 1.202. Thus, $B_{2}$ is decomposed into a leading order $\left(B_{2}^{W W}\right)$ and higher-twist $\left(\bar{B}_{2}\right)$ term:

$$
B_{2}=B_{2}^{W W}+\bar{B}_{2}
$$


Making the change $g_{2} \rightarrow g_{2}^{W W}$ in Eq. 8.38 , it is possible to apply Eq. 1.202 , and with some manipulation [142], derive

$$
B_{2}^{W W}\left(Q^{2}\right)=\int_{0}^{x_{t h}}\left[4 \sqrt{\tau(\tau+1)}-4 \tau-2 \sqrt{\tau} \ln \left(\frac{\sqrt{\tau(\tau+1)}+\sqrt{\tau}}{\tau}\right)\right] g_{1}\left(x, Q^{2}\right) d x
$$

Information regarding $H T$ effects, given by $\bar{B}_{2}$, would complete this picture. As explained in Section 8.3.3, the integral $d_{2}\left(Q^{2}\right)$ (Eq. 8.17; note Eq. 8.18) quantifies the magnitude of higher twist effects on $g_{2}$ (see Eq. 8.19). Assuming only that $d_{2}$ is small at low $Q^{2}$ and high $Q^{2},{ }^{17}$ it is possible to place a limit on the magnitude of the higher twist effects [142]:

$$
\bar{B}_{2}<\frac{M^{2}}{Q^{2}} \frac{d_{2}\left(Q^{2}\right)}{3}
$$

A plot of $B_{2}^{W W}$ is shown in Figure 8.36. Values for all 3 of these integrals $\left(B_{1}, B_{2}\right.$ and $\left.B_{2}^{W W}\right)$ are given in Tables B.24-B.26. Insertion of EG1 analysis values into the above sequence of equations has yielded a value of [142]

$$
\Delta_{\text {pot }}=1.8 \pm 0.7 \mathrm{ppm}
$$

A recent computation [143] gives

$$
\Delta_{Z}=-41.01(49) \mathrm{ppm}
$$

Adding these numbers yields a value obviously compatible with the experimental value of $\Delta_{S}$ in Eq. 8.32 , within the statistical precision of the data and calculation. The largest remaining uncertainty is the constraint given by the value of $d_{2}$, offering an immediate future application for more accurate $g_{2}$ structure function data in upcoming experiments (see Section 8.5.2).

As a concluding remark to this section, we note that the forward spin polarizability of the preceding section can be directly related to the required corrections for HF splitting at low $Q^{2}$. In fact, to generate the value of $\mathrm{Eq}$. 8.42, the contribution of the lowest $Q^{2}$ value (where EG1b data were

\footnotetext{
${ }^{17}$ There is every indication that this is true, assuming only very basic constraints on the model for $A_{2}$. See Section 2.7 .2 for information on these constraints.
} 
unavailable) used the value of $\gamma_{0}$ in Eq. 8.26 and

$$
\Delta_{1}\left[0, Q_{1}^{2}\right]=\left[-\frac{3}{4} r_{P}^{2} \kappa_{P}^{2}+18 M^{2} c_{1}-\frac{5 M^{2}}{4 \alpha} \gamma_{0}\right] Q_{1}^{2}
$$

and

$$
\Delta_{2}\left[0, Q_{1}^{2}\right]=\frac{3 M^{2} Q_{1}^{2}\left(\gamma_{0}-\delta_{L T}\right)}{2 \alpha}
$$

where $c_{1}=2.95 \mathrm{GeV}^{-4}, \delta_{L T}=1.35 \times 10^{-4} \mathrm{fm}^{-4}, r_{P}^{2} \sim 0.81 \mathrm{fm}$ is the Pauli proton radius squared [3] and $Q_{1}$ is the cutoff value of integration at low $Q^{2}$. Use of this estimate, which exploits necessary physical constraints as $Q^{2} \rightarrow 0$, is necessary to bridge the gap in available data at the lowest $Q^{2}$ values.

\subsubsection{Bloom-Gilman Duality}

As noted in Chapter 1, theoretical descriptions of particle interactions differ at low and high $Q^{2}$, with quark-gluon degrees of freedom (e.g. the DGLAP equations and pQCD) used at high $Q^{2}$, and hadronic/mesonic degrees of freedom (e.g. $\chi$ PT and multipole expansions) used at low $Q^{2}$. The question ensues, then, as to whether these two approaches are equivalent. Bloom-Gilman duality, a phenomenon first observed in 1970 in the unpolarized $F_{2}$ structure function, provides an experimental test of this question [144]. The theoretical concerns that underpin duality are very involved, and clearly beyond the scope of this thesis. Instead, a qualitative picture with some important definitions is presented here to convey the overall concept.

If duality between the quark-hadron pictures holds true, then there must be an equivalence between the resonance region structure functions, and an extrapolation of the structure functions from the DIS region into the resonance region, described by functions generated solely by QCD (quarkgluon) degrees of freedom. In this picture, the resonances can be viewed as parity-dependent excitations superimposed on the basic (extrapolated) scaling function. The average of the structure function over the resonances, then, should match up with the extrapolated function. The areas under both the true (resonant) structure function and extrapolated DIS function should be equal.

Figure 8.5 shows a simple (and very qualitative) manifestation of duality in the asymmetry $A_{1}$. 

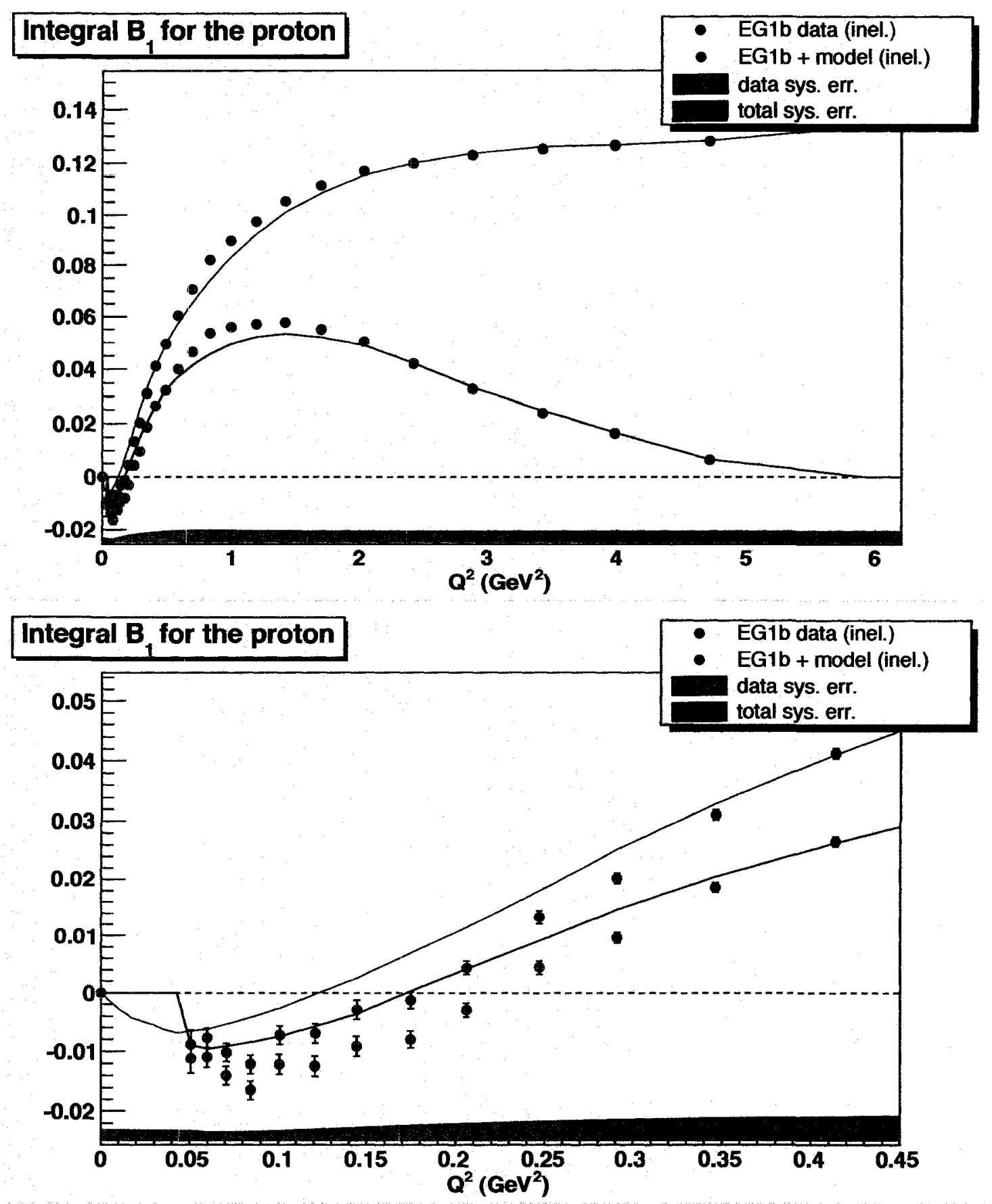

Figure 8.34: The integral $B_{1}$, shown at high (top) and low (bottom) $Q^{2}$. This integral is a required calculation in the determination of higher order correction in ${ }^{1} \mathrm{H}$ hyperfine splitting, and is directly dependent on the $g_{1}$ structure function. 


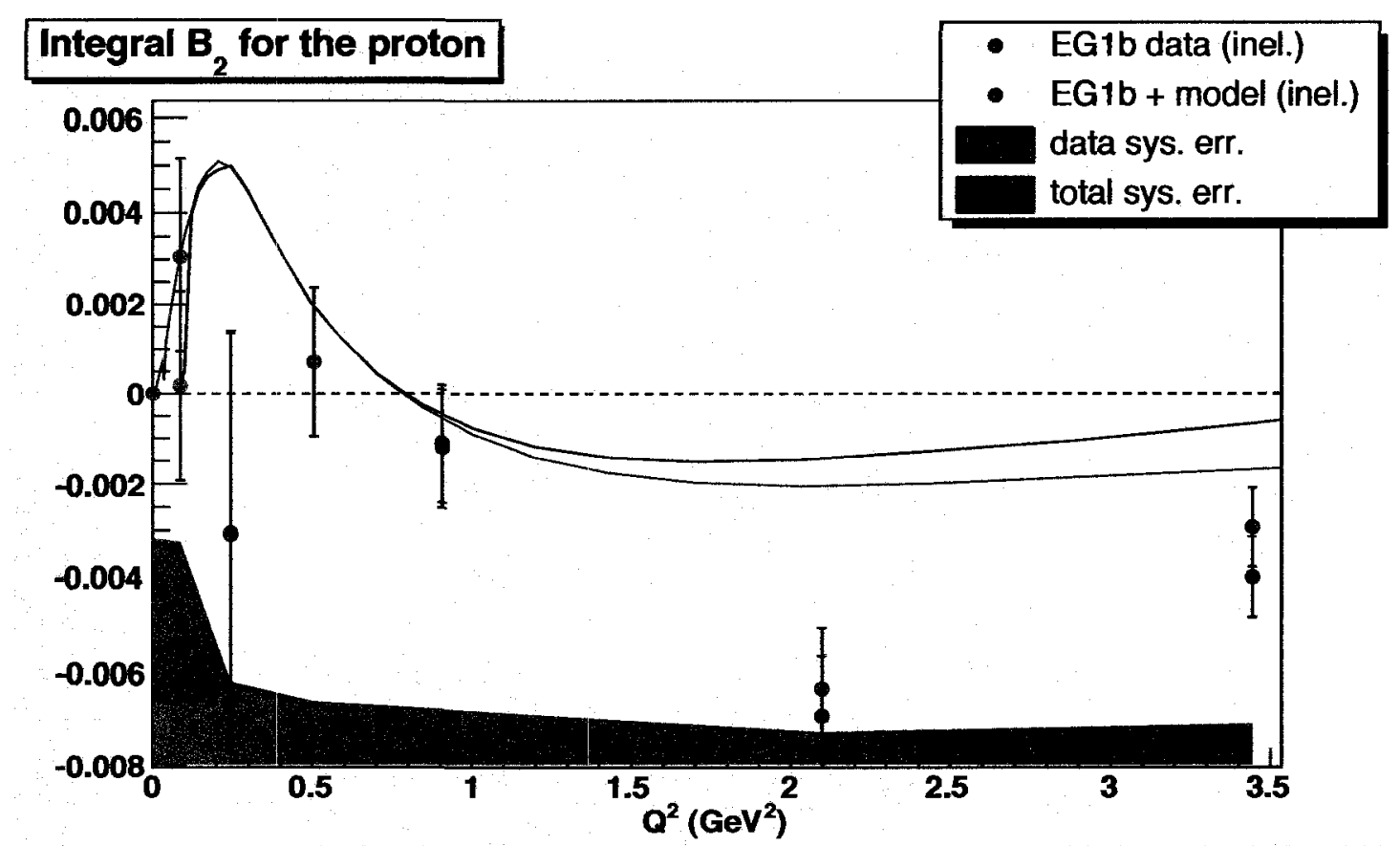

Figure 8.35: The integral $B_{2}$, derived from the EG1b data. Though the EG1b experiment provides little information on this quantity, it may help constrain the largest uncertainty in ${ }^{1} \mathrm{H}$ hyperfine splitting measurement. 

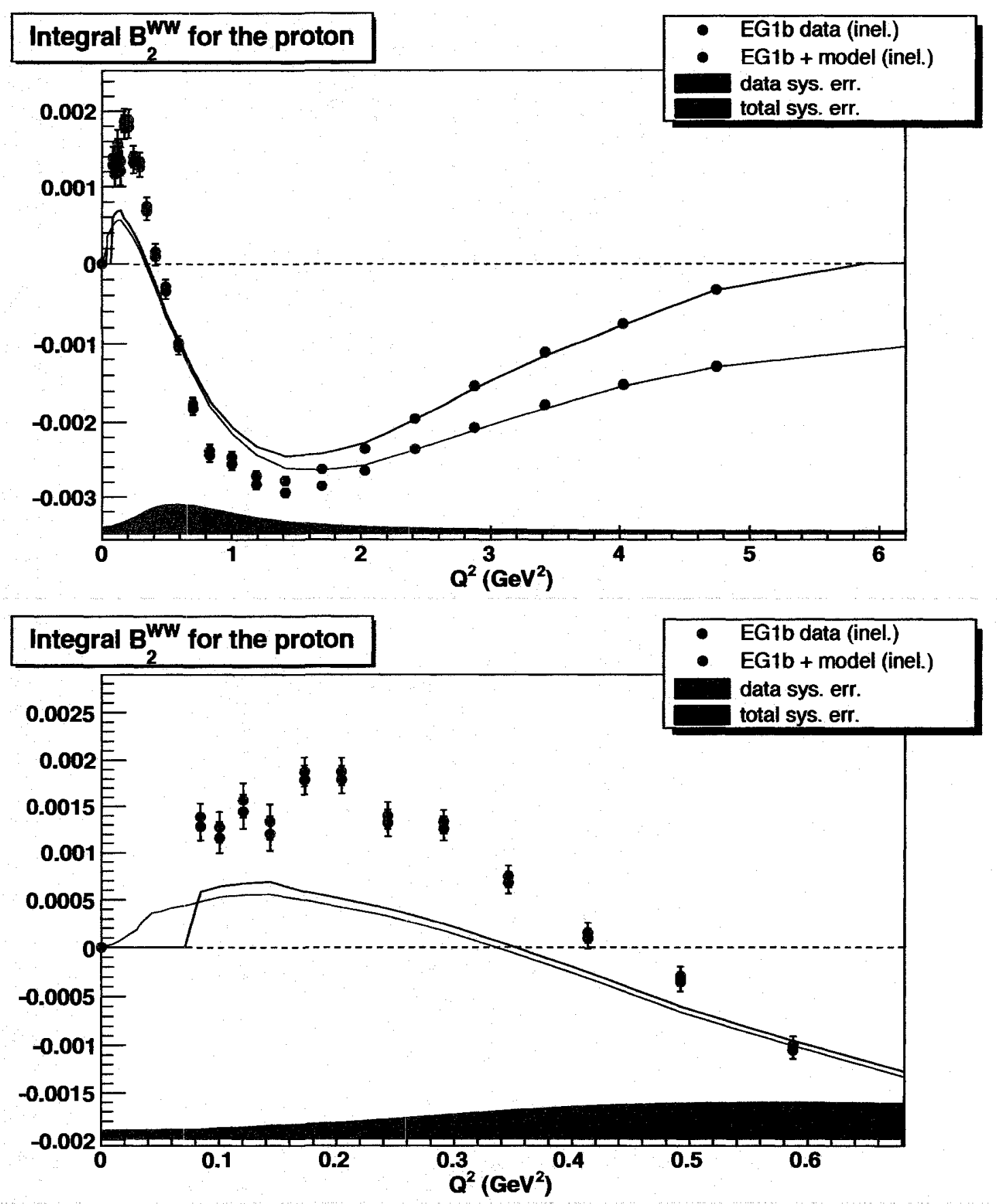

Figure 8.36: $B_{2}^{W W}$ from the EG1b data; that is, $B_{2}$ to leading-order twist only. Un upper bound of higher twist effects can be derived from the $d_{2}$ integral (Eq. 8.17), making it a useful estimate in the total value of $B_{2}=B_{2}^{W W}+\bar{B}_{2}$. 
18 Note that in the two higher $Q^{2}$ bins, if one ignores the large, negative $\Delta$-resonance at high- $x$, that $A_{1}$ appears to "oscillate" around the blue line (which represents the high- $Q^{2}$ extrapolation of $A_{1}$ into the plotted region). The area under the $Q^{2}=10 \mathrm{GeV}^{2}$ line and the resonant structure function are approximately equal, until one approaches the neighborhood of the $\Delta$-resonance. In addition to not holding in the $\Delta$ region, the phenomenon begins to break down below $Q^{2}<1 \mathrm{GeV}^{2}$ (i.e. the top plot), as well. Keep in mind that the compared function is not a "properly" extrapolated QCD function, as will be momentarily described, though it does, in this case, approximate an extrapolated function enough to visually introduce the basic concept of duality.

Duality has, in fact, been shown to break down for the unpolarized structure functions at $Q^{2}<1$ $\mathrm{GeV}^{2}$ [145]. Above this value, resonances are dominated by magnetic multipole transitions. Below this value, a more complex combination of electric and magnetic transitions complicates the correlations of resonant states to quark-gluon parton degrees of freedom, and duality is not expected to hold [146]. Duality is related to the cancelation of higher-twist effects in the summation of moments in the OPE [146]. The constancy observed at higher $Q^{2}$ in leading order moments (such as $\Gamma_{1}\left(Q^{2}\right)$ at high $Q^{2}$ ), which are primarily composed of lower-twist terms, implies a cancellation of the highertwist effects in summing over the resonances. Recent theoretical QCD studies predict that duality should hold for the structure function $g_{1}^{p}$, though not as precisely as for the unpolarized structure functions, due to sizeable negative contributions in the polarized structure functions. ${ }^{19}$

Before the analysis of EG1b data, no comprehensive test of quark-hadron duality for polarized structure functions existed, as their resolution in the resonance region was too poor to discern their structure in functions of both $W$ and $Q^{2}$, a necessary precursor to any tests of duality. The detailed EG1b resonance-region data on $g_{1}$ fulfill the first requirement for the testing of duality. The other requirement is a properly scaled and extrapolated DIS QCD function in the resonance region. An exact extrapolation for the comparison of polarized structure functions is considerably more complex than the qualitative introduction given by Figure 8.5 .

To show that duality is indeed a test of internal nucleon physics, and not kinematic phenemena, we must account for the "recoil" of the target proton in the lab frame, which varies with $x$ and $Q^{2}$.

\footnotetext{
${ }^{10}$ This is not a structure function, but recall that $A_{1}$ can be expressed in terms of severat structure functions, and is dominated by the behavior of $g_{1}$ and $F_{1}$, so it manifests aspects of duality.

${ }^{19}$ Spin- $\frac{3}{2}$ suppression is related to the cancellation of higher-twist eflects [146]; this suppression is definitely violated tor the $\Delta$-resonance.
} 
Recall (see Footnote 13 in Chapter 1) that the conventional definition of the structure functions does not account for the target recoil. This correction is given by a simple factor of $E^{\prime} / E$ for elastic scattering cross-sections (see Eq. 1.34). However, for inelastic scattering, the corrections are considerably more complicated. Detailed target mass corrections (TMC) are required to eliminate the functional dependence on reference frames.

These effects can be approximated by replacing the Bjorken scaling variable $x$ with a variable that approximates the purely kinematic corrections in higher twist terms [147], known as the Nachtmann scaling variable:

$$
\xi=\frac{2 x}{1+\sqrt{1+4 M^{2} x^{2} / Q^{2}}}
$$

A more exact TMC can be made using the prescription of Blümlein and Tkabladze [145][148]:

$$
\begin{aligned}
g_{1}^{T M C}\left(x, Q^{2}\right)=\frac{x}{\xi(1+\gamma)^{3 / 2}} g_{1}^{Q C D}\left(\xi, Q^{2}\right)+\frac{(x+\xi) \gamma}{\xi(1+\gamma)^{2}} \int_{\xi}^{1} \frac{d u}{u} g_{1}^{Q C D}\left(u, Q^{2}\right) \\
\quad-\frac{\gamma(2-\gamma)}{2(1+\gamma)^{5 / 2}} \int_{\xi}^{1} \frac{d u}{u} \int_{u}^{1} \frac{d v}{v} g_{1}^{Q C D}\left(v, Q^{2}\right)
\end{aligned}
$$

The extrapolated structure function $g_{1}^{Q C D}$ is fit to next-to-leading order (NLO) QCD fits of parton distribution functions (PDFs) above the resonance region. ${ }^{20}$ Correcting for divergences in perturbative expansions at high $x$ (where soft gluon production introduces complications) presents a more complex challenge [149]. This resummation at high $x$ is most easily estimated instead of calculated, with a suitable systematic error included in the PDF extension.

Using the NLO PDFs and the EG1b resonance data, the validity of duality can be tested. Figure 8.37 shows $x g_{1}$ vs. $x$ against the extrapolated PDFs. Note that, with the exception of the $\Delta$-resonance, the $g_{1}$ data "oscillate" around the the extended DIS function. To make a final, comprehensive test of duality, both the extrapolated PDF and the data need to be averaged over the evaluated range in $x$ :

$$
\left\langle g_{1}\left(Q^{2}\right)\right\rangle=\frac{\int_{x_{l o}}^{x_{h i}} g_{1}\left(x, Q^{2}\right) d x}{x_{h i}-x_{l o}}
$$

\footnotetext{
${ }^{20}$ That is, one determines $\Delta u, \Delta d$ and $\Delta s$ based on the available world data (see Section 8.5.2). The functional form of $g_{1}$ can then interpreted in the form of Eq. 1.101.
} 


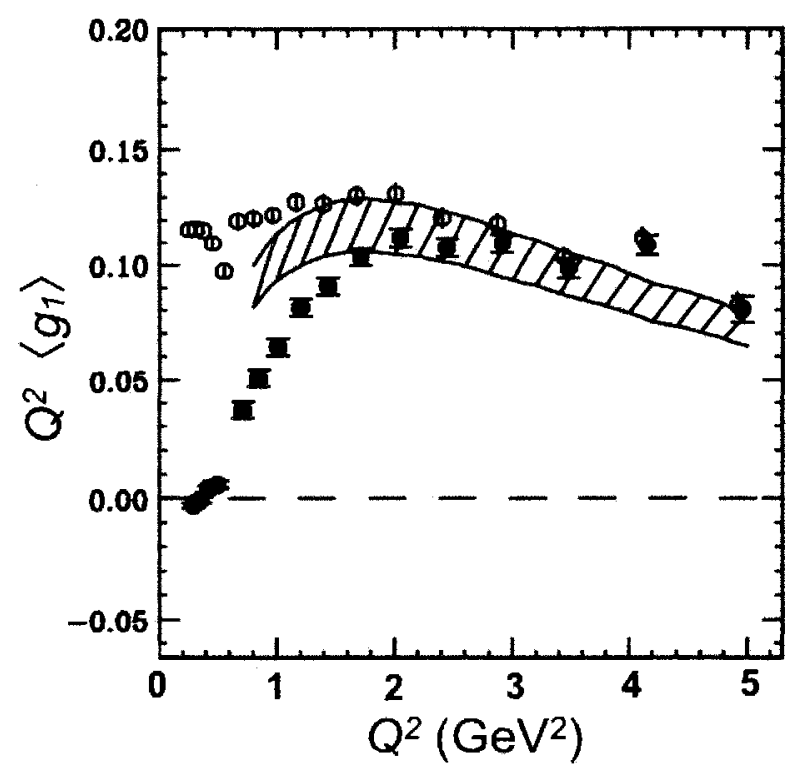

Figure 8.37: Values of $x g_{1}$ vs. $x$ for $g_{1}^{p}$, plotted against the extrapolated NLO PDFs, as described in the text. The structure function data oscillate about the mean, except near the $\Delta$ resonance, an effect of quark-hadron duality. This plot represents a full EG1b analysis extension of the similar plot published in Ref. [145] (which included only 1.6 and 5.7 GeV data), courtesy of P. Bosted.

This average can be taken over the full range of resonance region data, or over a smaller range in $W$ for local tests of duality. If duality holds, both the averaged extrapolated function and data should be equal. Figure 8.38 shows a test of global duality over the whole resonance region, ${ }^{21}$ showing the averaged $g_{1}$ structure function data plotted against the NLO PDF extension. Note that the elastic peak contribution (given by Eq. 8.15) must be added in for duality to be observed at the low $Q^{2}$ values, showing that this state must be added in to counteract the effects of the negative $\Delta$ resonance for duality to be complete. With the exception of the $\Delta$ region (at $W<1.38 \mathrm{GeV}$ ), local duality, where a sum is taken over only a limited range in $W$, appears to hold as well [145], showing that higher twist effects and parity separation of resonances cancel locally in the $g_{1}$ spectrum.

Testing of quark-hadron duality is, unfortunately, not possible for $g_{2}$ from EG1b, due to the poor resolution of this data. Whether or not this structure function exhibits similar behavior remains an open question. 22

\footnotetext{
${ }^{21}$ The term global does not truly imply "global" in the sense that it includes a complete set of basis states, but "global" in the sense that it covers the whole resonance region $(1.08<W<2 \mathrm{GeV})$.

${ }^{22}$ It is also a pertinent question, due to the presumed dominance of HT effects in the $g_{2}$ structure function.
} 


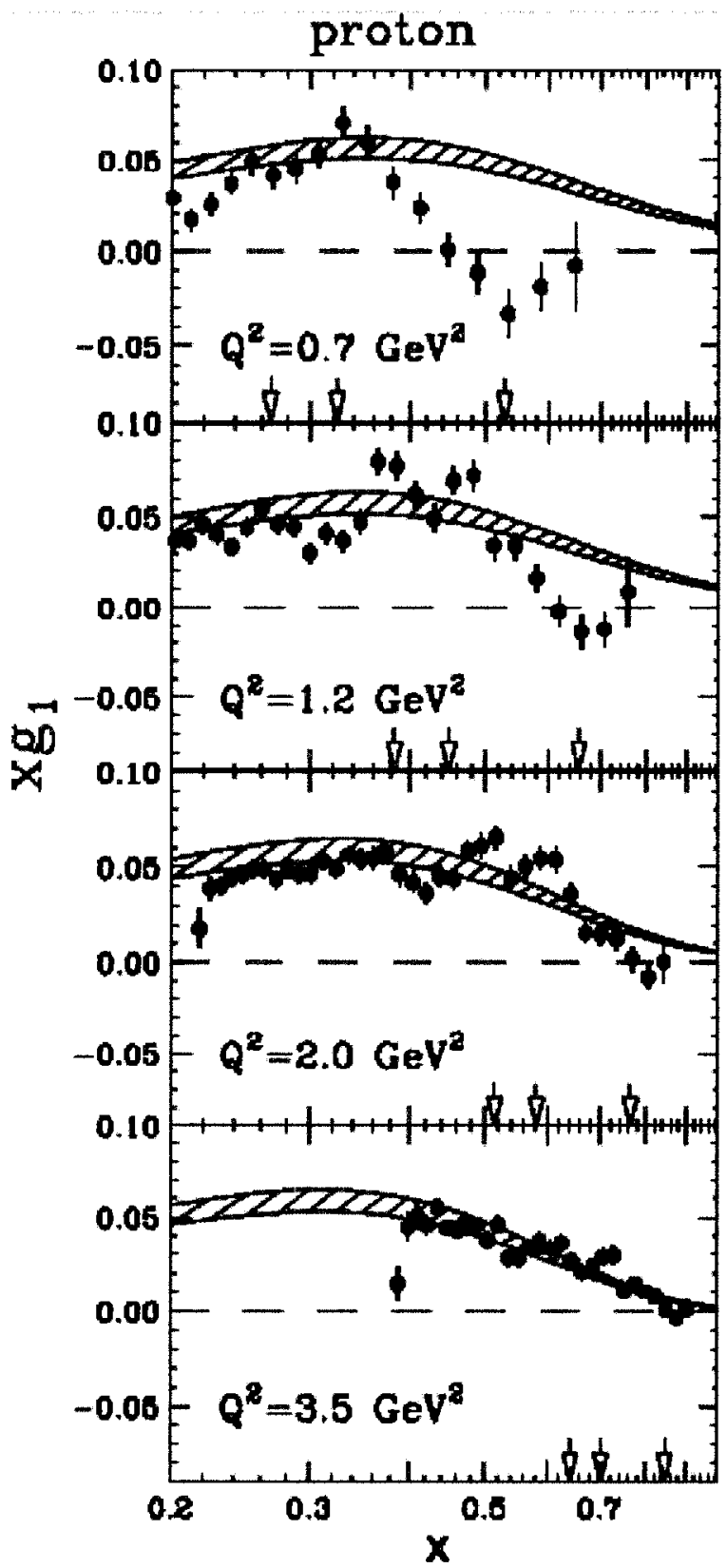

Figure 8.38: Statistically averaged values of $Q^{2} g_{1}$ (dark circles) vs. $Q^{2}$ for the proton, plotted against the extrapolated NLO PDF. When elastic contributions are added in (hollow circles), duality appears to hold. Again, these plots are an extension of those in Ref. [145] for the newer, full analysis of EG1b. The duality plots (and NLO PDFs) were generated by P. Bosted. 


\subsection{Future Directions}

\subsubsection{Outstanding Details}

As pointed out throughout this chapter, inclusive proton analysis for EG1b data is not yet $100 \%$ complete. There are a few remaining issues with the polarization-dependent models that must be resolved before "final" values of $g_{1}$ and $g_{2}$ for the proton are obtained.

Radiative corrections to $A_{\|}$(Section 6.4) require updating and genuine error calculation. The corrective terms $A_{R C}$ and $f_{R C}$ used to determine the Born asymmetry do not include corrections fit to the most recently available data, and while large changes are not expected, they should be updated. Also, recalling Section 7.2.5, systematic errors due to radiative corrections were not rigorously calculated (and were instead merely estimated). A more technical approach toward determining these errors must be completed.

Also, as mentioned in Section 8.1.3, the $A_{2}$ model should be updated to include the final EG1b and RSS results, so that $A_{1}$ (and $g_{1}$ ) can be more accurately determined. This could reduce the effects of the single largest systematic error in the extraction of the spin-structure functions, and provide a means to incorporate both the statistical $A_{2}$ values derived from EG1b data and the physical/anaiytic constraints of the $A_{2}$ virtual photon asymmetry into the extraction of $A_{1}$. Note that, while these details require attention before the analysis can be declared "finished", that even in the state presented in this thesis, this analysis presents the most precise measurements available of spin structure functions of the proton in and above the resonance region.

\subsubsection{Upcoming Experiments and Data}

The EG1b data provide a comprehensive set of polarized structure function measurements in and near the resonance region at $0.05<Q^{2}<5 \mathrm{GeV}^{2}$. However, in conclusion, we note that there are still voids of physical interest for spin structure function measurements. Though EG1b data extend to lower $Q^{2}$ and $x$ than any other (analyzed) wide acceptance spin experiment to date, the $Q^{2}$ values are still not low enough to accurately test the GDH sum rule and other leading order $Q^{2} \rightarrow 0$ dispersion relations. Also, measurements of $A_{2}$ and $g_{2}$ are meager compared to those of $A_{1}$ and $g_{1}$. 
Clearly, an experiment using a perpendicularly polarized target (to measure $A_{\perp}$ ) would be desirable (though a practical impossibility for the current configuration of CLAS). In this light, brief attention is given to two other experiments, one already complete (and in early analysis phase), and the other upcoming: the CLAS EG4 experiment [150] and the JLab Hall-C SANE experiment [136].

The EG4 experiment, which also utilized the CLAS detector, completed in 2006, used a polarized beam (from 1.0 to $3.0 \mathrm{GeV}$ ) and a longitudinally polarized target (nearly identical to that used for EG1), collecting approximately 27 billion trigger events for $\mathrm{NH}_{3}$ and $\mathrm{ND}_{3}$ targets [151]. Experimental objectives were similar to EG1. However, the $Q^{2}$ range was considerably lower, ranging from $0.015<Q^{2}<1 \mathrm{GeV}^{2}$ in a lower $x$ region than that covered by EG1b resonance region data (see the empty space in the lower left corner of Figure 1.13). To facilitate better efficiency for measuring the outbending data required to make these low $Q^{2}$ measurements, a new Cherenkov Counter [150] was constructed in one sector. One of the main objectives of the EG4 experimental analysis is the extension of inclusive $A_{\|}$measurements made in EG1 to this new low $Q^{2}$ kinematic range. Combined together, EG1b and EG4 will provide a more global data set for the reconstruction of structure function moments like $\Gamma_{1}^{p}$, and allow for a true test of the GDH sum rule, better measurements of forward spin polarizability $\gamma_{0}$, and ${ }^{1} \mathrm{H}$ hyperfine splitting. (All these measurements are heavily weighted by low $Q^{2}$ data.) EG4 will also provide a more comprehensive data set for the testing of generalized dispersion relations based on $\chi^{\mathrm{PT}}$, and detailed resonance information near the $Q^{2} \rightarrow 0$ limit that is not currently available. Figure 8.39 shows a projection of expected kinematic coverage for $\Gamma_{1}^{p}$, based on preliminary simulations and recent structure function models.

While EG4 provides valuable information regarding the $g_{1}$ structure function, it does little to elucidate the poor data available for the $g_{2}$ structure function, which requires $A_{\perp}$ data to precisely measure. As stated, the RSS experiment [51] completed this measurement for a very small $Q^{2}$ range. Widening the kinematic acceptance is necessary to provide more detailed intormation over a wide range of $Q^{2}$ and $W$. Unfortunately, the magnetic field configuration in CLAS impedes the use of a perpendicularly polarized target, so one must look elsewhere for opportunities to make this set of measurements.

Hall-C at Jefferson Lab, unlike Hall-B, supports a target configuration versatile enough to allow $A_{\perp}$ measurements. In late 2008, the SANE (Spin Asymmetries on the Nucleon Experiment) will run 


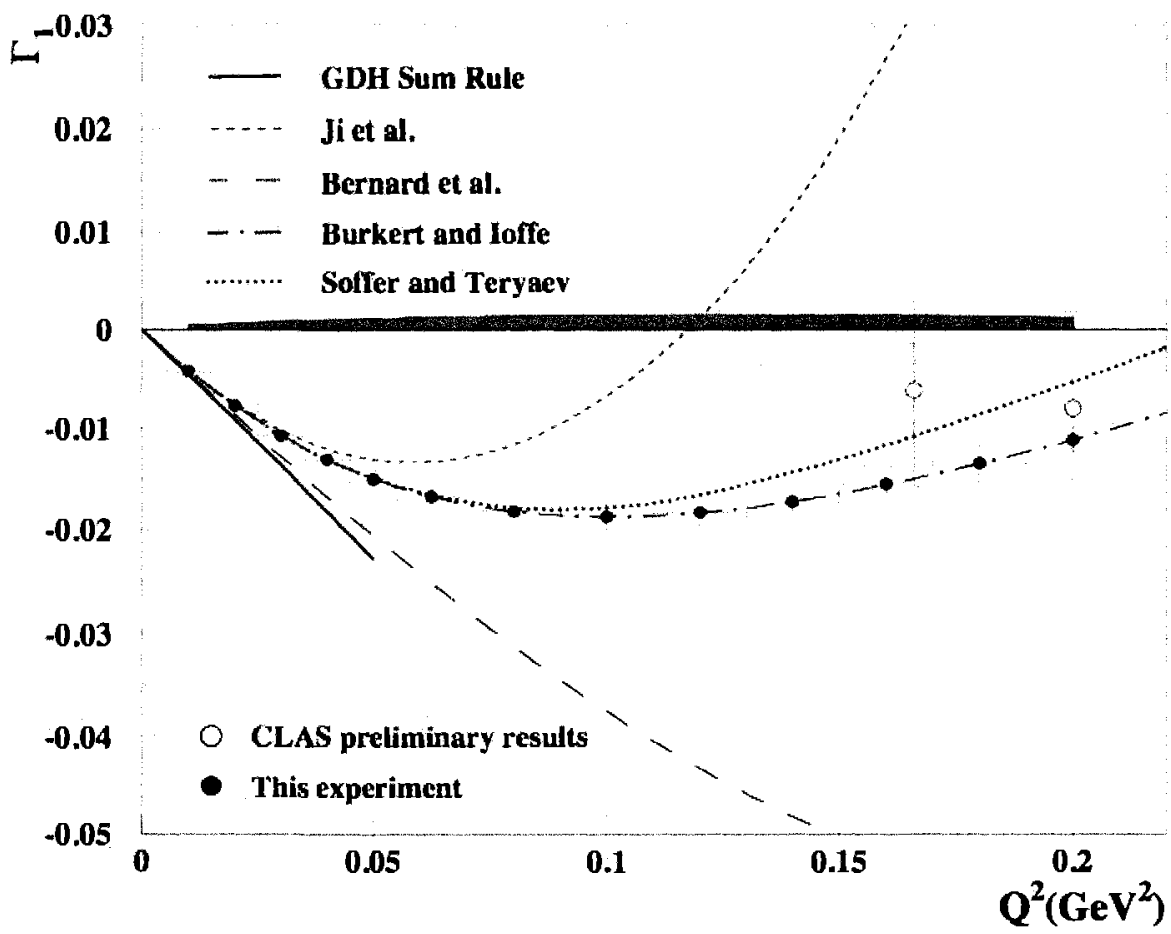

Figure 8.39: Projected experimental results for EG4 analysis (solid circles) of the first moment of $g_{1}^{p}, \Gamma_{1}^{p}$, plotted at low $Q^{2}$ values. (Here, "This experiment" refers to EG4, not EG1.) The hollow circles show (approximate) limits of EG1b analysis for relative comparison. From Ref. [150]. 


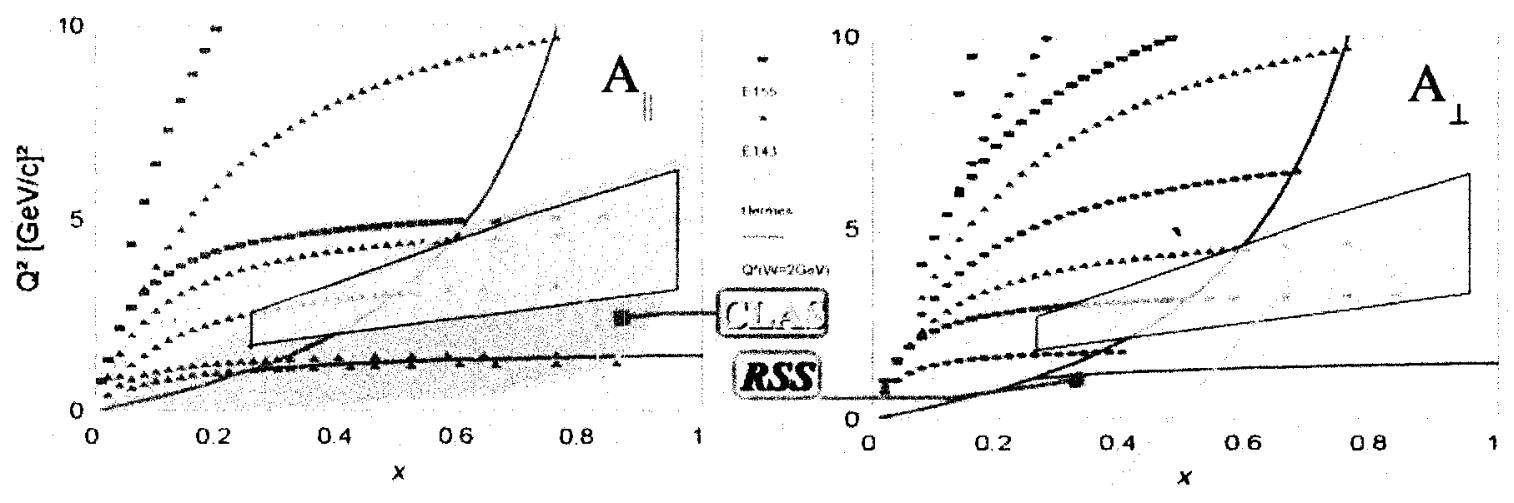

Figure 8.40: Region of kinematic coverage for the approved SANE experiment at Jefferson Lab, compared to coverage of world data. Regions to be covered by SANE are pointed out by the arrows. From Ref. [153]

in Hall-C, utilizing the University of Virginia polarized ammonia target system [152] $]^{23}$ and the BETA (Big Electron Telescope Array) detector [153], consisting of the BigCal lead glass calorimeter, a gas Cherenkov detector and lucite hodoscopes. Operating at the higher end of CEBAF beam energies ( $\sim 4.6$ and $\sim 5.7 \mathrm{GeV}$ ), this experiment will measure both $A_{\|}$and $A_{\perp}$ for $2.5<Q^{2}<6.5 \mathrm{GeV}^{2}$ and $0.3<x<0.8$ [136], providing the most precise measurements of both these quantities available in the specified kinematic region (see Figure 8.40). Not only will SANE provide higher precision measurements of $g_{1}$ in the intermediate $Q^{2}$ range, it will perform the first precision measurements of $g_{2}$ over an extended acceptance, allowing for much better measurements of the $d_{2}$ integral and probes of higher twist effects (see Figure 8.41).

\subsubsection{Testing Quark Models}

Future measurements of double-spin asymmetries stand to greatly improve our understanding of QCD models and generalized parton distributions. Data from this experiment are merely a small part of the larger set of world data that can be used in a global fit of parton distributions [154]. A global fit of the quark distribution functions $\Delta u, \Delta d$ and $\Delta s$ (see $\mathrm{Eq}$. 1.99) requires not only measurements of $g_{1}$ for the proton, but neutron measurements (so that isospin conservation can be exploited) and SIDIS (semi-inclusive DIS) data. Also, NN collision data from RHIC (Relativistic

\footnotetext{
${ }^{23}$ This target was also used in the RSS experiment.
} 


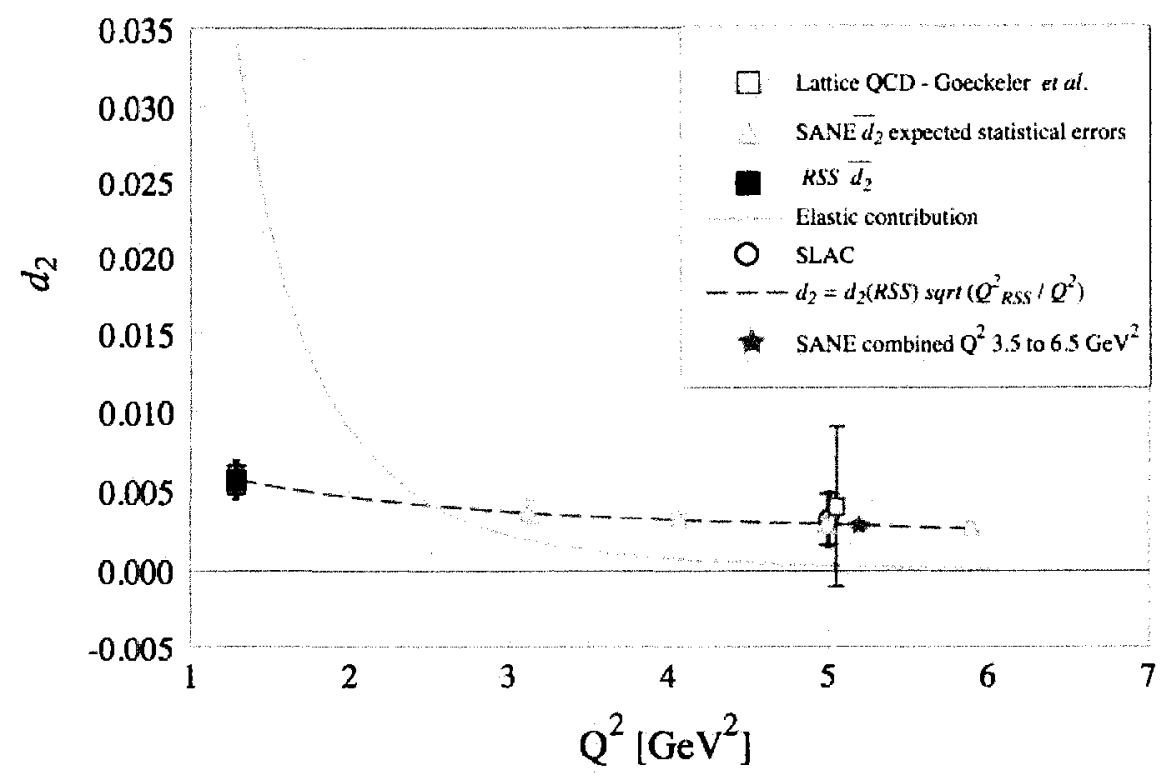

Figure 8.41: Projected SANE results for the $d_{2}$ integral. Compare to Figure 8.31 for this experiment. The RSS result is also shown. From Ref. [136].

Heavy lon Collider at Brookhaven) ${ }^{24}$ and $\mu N$ scattering experiments from COMPASS (at CERN) are needed to provide a means of extracting the gluon contribution $\Delta g$. The integral

$$
\Delta f^{1}\left(Q^{2}\right) \equiv \int_{0}^{1} \Delta f\left(x, Q^{2}\right) d x
$$

measures the spin contribution any given parton $f$ to the proton, making parton distributions and their moments of high physical importance in QCD spin physics. The most up-to-date information available (at the time of this thesis) on global analysis of these parton distributions can be found in Ref. [154].

Even within Jefferson Lab, there are great strides to be made in the study of QCD models. DIS measurements at high $x$ provide valuable ground for the testing of pQCD models. The virtual photon asymmetry $A_{1}$ must equal 1 at $x=1$, as this is the elastic scattering limit. The pure SU(6) (non-relativistic) constituent quark model gives a prediction of $A_{1}=\frac{5}{9}$ (Eq. 1.245). Resonance data involves complexities that cannot be fit by pure pQCD models, but the smooth DIS data can be used

\footnotetext{
${ }^{24}$ This is measured in both the STAR and PHENIX detectors, collecting jet and $x^{0}$ data, respectively.
} 
to test the limit as $x \rightarrow 1$, where the asymmetry must smoothly transition from the (average) SU(6) neighborhood to $A_{1}=1$, as dictated by symmetry-breaking processes. These processes include one-gluon exchange hyperfine interactions between quarks, spin- $\frac{3}{2}$ suppression due to helicity conservation, and/or connections to duality-based models [155]. These different symmetry-breaking scenarios lead to differing predictions of the value of DIS $A_{1}$ values in the high $x$ limit.

Using DIS $(W>2.0 \mathrm{GeV})$ data, from EG1b runs and world experimental measurements, a plot of $A_{1}$ vs. $x$ can be generated (Figure 8.42). ${ }^{25}$ At energies lower than $6 \mathrm{GeV}$, the current limit at JLab, maximum values of $x \sim 0.55$ can be reached. Previously published EG1b data for $A_{1}$ at 5.7 GeV have already helped provide precise physical constraints on QCD models at this kinematic limit [155]. Clearly, better constraints could be yielded if precise higher $x$ data were available. This thesis concludes with a brief look of what the future of JLab Hall-B has to offer in this uncharted realm of inclusive double-spin asymmetry measurement.

Currently, upgrades are being planned to double the maximum beam energy at CEBAF to 12 GeV by c. 2014, with corresponding upgrades to the Hall-B detector, concentrating on the forward (i.e. small- $\theta$ ) angle detection required in a large acceptance spectrometer at these higher beam energies [156]. The new CLAS12 detector (which, incidentally, will also accomodate measurements of $A_{£}$ ) is designed, in part, with the very purpose of obtaining high- $x$ data for polarized structure functions. A plot of expected $A_{1}^{p}$ measurements, after $\mathbf{4 0}$ simulated days of beam time at $11 \mathrm{GeV}$ beam energy in CLAS12, is shown in Figure 8.43. Comparing to Figure 8.42, one can see that future data collection at higher energies will allow for higher- $x$ extension of $A_{1}$ DIS measurements. Combining this information with large acceptance measurements of $A_{\perp}$ at the same energy will provide further versatility and precision in future asymmetry measurements in CLAS12. Future expansions of JLab facilities will clearly enable more precise and expansive measurements of nucleon spin phenomena, and lead to fuller descriptions of QCD physics through medium energy accelerator experiments.

${ }^{25}$ EG1b numerical data in this plot are listed in Table B.12. 


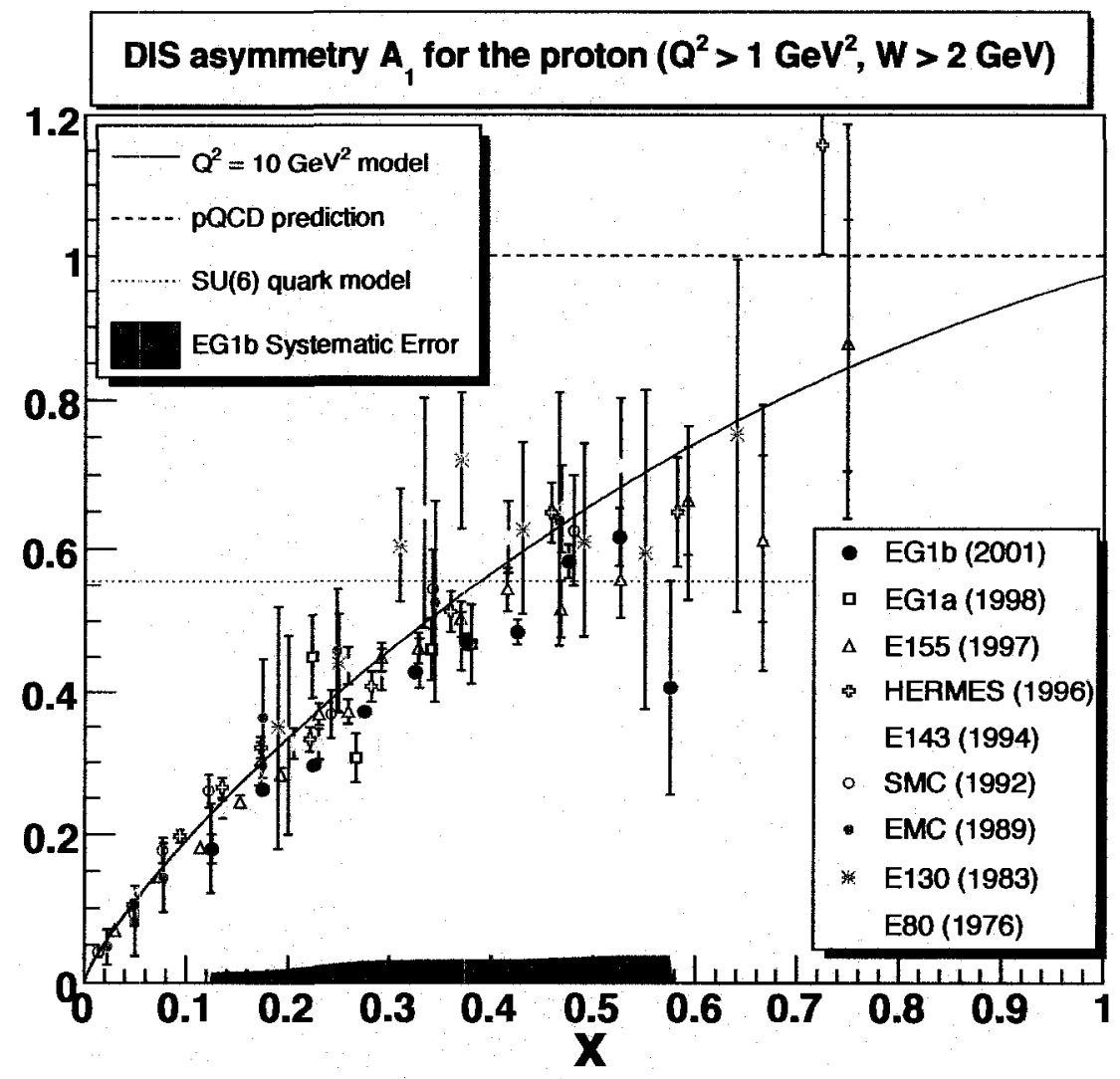

Figure 8.42: World data of $A_{1 p}$, showing $W>2 \mathrm{GeV}$ and $Q^{2}>1 \mathrm{GeV}^{2}$ values only. DIS measurements of $A_{1}$ at high Bjorken $x$ provide an ideal test of pQCD models, which constrain the behavior as $x \rightarrow 1$. 


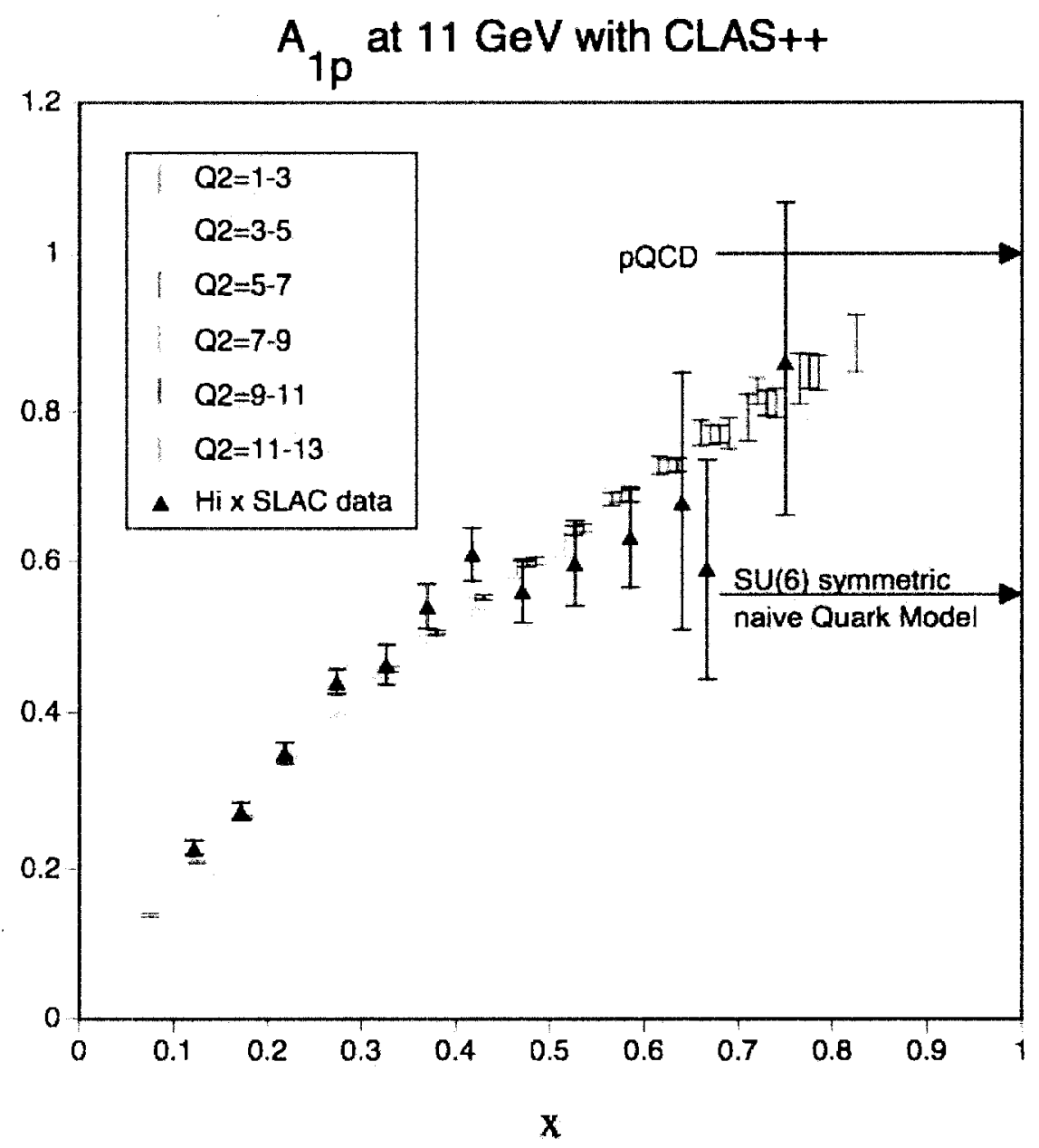

Figure 8.43: Projected precision of averaged $A_{1}$ DIS measurements for the proton at $11 \mathrm{GeV}$ beam energy, using CLAS12 simulation software. Operation at higher beam energies will enable the collection of precision DIS asymmetry data at higher $x$ values than currently possible below $6 \mathrm{GeV}$. From Ref. [156]. 


\title{
Appendix A
}

\section{Glossary of Acronyms}

\author{
ADC Amplitude $\rightarrow$ Digital Converter \\ BCDMS Bologna-CERN-Dubna-Münich-Saclay (experiment) \\ BCS Bardeen-Cooper-Schieffer (superconductivity theory) \\ BETA Big Electron Telescope Array \\ BPM Beam Position Monitor \\ CaIDB (CLAS) Calibration Database \\ cc Cherenkov Counters \\ CEBAF Continuous Electron Beam Accelerator Facility
}

CERN Conseil Européen pour la Recherche Nucléaire (European Council for Nuclear Research)

$\chi$ PT Chiral Perturbation Theory

CLAS CEBAF Large Acceptance Spectrometer

CLAS12 CLAS at $12 \mathrm{GeV}$

CM Center-of-mass

CODA CEBAF Online Data Acquisition

COMPASS COmmon Muon and Proton Apparatus for Structure and Spectroscopy

DA $\Phi$ NE An $e \bar{e}$ collider in Frascati, Italy 
DAQ Data Acquisition (System)

DC Drift Chambers

DESY Deutsches Elektronen Synchrotron (German Electron Synchrotron)

DGLAP Dokshitzer-Gribov-Lipatov-Altarelli-Parisi (pQCD evolution equations)

DIS Deep Inelastic Scattering

DNP Dynamic Nuclear Polarization

DOCA Distance of Closest Approach

DST Data Summary Tape

E80 Early polarized SLAC experiment

E130 Another early polarized SLAC experiment (after E80)

E143 Later, more comprehensive polarized SLAC experiment

E155 Yet another polarized SLAC experiment

E155x Subdivision of E155 experiment with perpendicularly polarized target

E665 Unpolarized scattering experiment at CERN

EB Event Builder

EC Electromagnetic Calorimeters

EG1 JLab Hall-B Experiment using electron (E) and photon (G) beam data. (Photon runs are not studied in this thesis.)

EG1a First (preliminary) part of EG1 experiment; test run of the experiment

EG1b Second (main) part of EG1 experiment; electron beam/proton target data is the subject of this thesis

EG4 Experiment similar to the previous EG1, but at lower $Q^{2}$ values

ElO Extended Interaction Oscillator

EMC European Muon Collaboration

EPR Electron Paramagnetic Resonance

ER Event Recorder

ESR Electron Spin Resonance

EST Equal Spin Temperature

ET Event Transport 
FASTBUS A standard electronics for particle physics applications (successor to CAMAC and NIM electronics)

FC Faraday Cup

FEL Free Electron Laser

FORTRAN Formula Translating System (progamming language)

FPACK FORTRAN package for input/output

GEANT Geometry and Tracking (Detector Description and Simulation Toof; describes passage of elementary particles through matter)

GDH Gerasimov-Drell-Hearn (usually in reference to the sum rule)

GSIM GEANT Simulation package for CLAS

H1 Particle detector in operation at HERA (at DESY)

HeLP Helicity Pairing

HERA Hadron Elektron Ring Anlange (Hadron-Electron Ring Accelerator) at DESY

HERMES A particle detector in use at HERA (at DESY)

HMS High Momentum Spectrometer

HWP Half Wave Plate

HF Hyperfine

HT Higher Twist

HV High Voltage

LAC Large Angle Calorimeter

MAMI Mainz Microtron

MCC Machine Control Center (accelerator)

MINUIT Program for function minimization and error analysis

MIP Minimum lonizing Particle

MOPA Master-Oscillator-Power-Amplifier

MT empty (target cell)

MySQL Multi-user Structured Query Language (Swedish)

NIM Nuclear Instruments and Methods

NLO Next-to-leading Order 
NMC New Muon Collaboration

NMR Nuclear Magnetic Resonance

OPE Operator Product Expansion

PID Particle Identification

PDF Parton Distribution Function

PDIS Polarized Deep Inelastic Scattering

PERL Practical Extraction and Report Language (dynamic programming language)

PHENIX Pioneering High Energy Nuclear Interactions eXperiment (at RHIC)

PMT Photomultiplier Tube

pQCD Perturbative QCD (Quantum Chromodynamics)

QCD Quantum Chromodynamics

QED Quantum Electrodynamics

RAID Redundant Arrays of Inexpensive Disks

RCSLACPOL SLAC Radiative Correction for Polarized Scattering

RECSIS Reconstruction and Analysis

RF Radiofrequency

RHIC Relativistic Heavy Ion Collider

RLC Resistor-Inductor-Capacitor

RMS Root Mean Square

ROC Readout Controller

ROOT An object-oriented data analysis framework

RR Resonance Region

RSS Resonance Spin Structure

SANE Spin Asymmetries on the Nucleon Experiment

SC Scintillation Counters

SEB Simple Event Builder

SIDIS Semi-inclusive Deep Inelastic Scattering

SLAC Stanford Linear Accelerator

SLM Synchrotron Light Monitor 
SMC Spin Muon Collaboration

SRF Superconducting Radiofrequency

STAR Solenoidal Tracker at RHIC

TOF Time-of-Flight

TDC Time $\rightarrow$ Digital Converter

TMC Target Mass Correction

TS Trigger Supervisor

VME Virtual Machine Environment

ZEUS A particle detector in use at HERA (at DESY) 
Appendix B

\title{
Additional Tables
}

\author{
B.1 Kinematic Bin Tables
}


Table B.1: Standard $Q^{2}$ bins for EGtb. Both the geometric (integrated by relative weight) and arithmetic averages are shown.

\begin{tabular}{|c|c|c|c|c|}
\hline Bin & $Q_{\min }^{2}$ & $Q_{\max }^{2}$ & $Q^{2}$ (geometric ave.) & $Q^{2}$ (arithmetic ave.) \\
\hline 0 & 0 & 0.00919 & - & - \\
\hline 1 & 0.00919 & 0.0110 & 0.0100 & 0.010 \\
\hline 2 & 0.0110 & 0.0131 & 0.0120 & 0.012 \\
\hline 3 & 0.0131 & 0.0156 & 0.0143 & 0.014 \\
\hline 4 & 0.0156 & 0.0187 & 0.0171 & 0.017 \\
\hline 5 & 0.0187 & 0.0223 & 0.0204 & 0.020 \\
\hline 6 & 0.0223 & 0.0266 & 0.0243 & 0.024 \\
\hline 7 & 0.0266 & 0.0317 & 0.0291 & 0.029 \\
\hline 8 & 0.0317 & 0.0379 & 0.0347 & 0.035 \\
\hline 9 & 0.0379 & 0.0452 & 0.0414 & 0.042 \\
\hline 10 & 0.0452 & 0.0540 & 0.0494 & 0.050 \\
\hline 11 & 0.0540 & 0.0645 & 0.0590 & 0.059 \\
\hline 12 & 0.0645 & 0.0770 & 0.0704 & 0.071 \\
\hline 13 & 0.0770 & 0.0919 & 0.0841 & 0.084 \\
\hline 14 & 0.0919 & 0.110 & 0.100 & 0.10 \\
\hline 15 & 0.110 & 0.131 & 0.120 & 0.12 \\
\hline 16 & 0.131 & 0.156 & 0.143 & 0.14 \\
\hline 17 & 0.156 & 0.187 & 0.171 & 0.17 \\
\hline 18 & 0.187 & 0.223 & 0.204 & 0.20 \\
\hline 19 & 0.223 & 0.266 & 0.243 & 0.24 \\
\hline 20 & 0.266 & 0.317 & 0.291 & 0.29 \\
\hline 21 & 0.317 & 0.379 & 0.347 & 0.35 \\
\hline 22 & 0.379 & 0.452 & 0.414 & 0.42 \\
\hline 23 & 0.452 & 0.540 & 0.494 & 0.50 \\
\hline 24 & 0.540 & 0.645 & 0.590 & 0.59 \\
\hline 25 & 0.645 & 0.770 & 0.704 & 0.71 \\
\hline 26 & 0.770 & 0.919 & 0.841 & 0.84 \\
\hline 27 & 0.919 & 1.10 & 1.00 & 1.0 \\
\hline 28 & 1.10 & 1.31 & 1.20 & 1.2 \\
\hline 29 & 1.31 & 1.56 & $\overline{1.43}$ & 1.4 \\
\hline 30 & 1.56 & 1.87 & 1.71 & 1.7 \\
\hline 31 & 1.87 & 2.23 & 2.04 & 2.0 \\
\hline 32 & 2.23 & 2.66 & 2.43 & 2.4 \\
\hline 33 & 2.66 & 3.17 & 2.91 & 2.9 \\
\hline 34 & 3.17 & 3.79 & $\mathbf{3 . 4 7}$ & 3.5 \\
\hline 35 & 3.79 & 4.52 & 4.14 & 4.2 \\
\hline 36 & 4.52 & 5.40 & 4.94 & 5.0 \\
\hline 37 & 5.40 & 6.45 & 5.90 & 5.9 \\
\hline 38 & 6.45 & 7.70 & 7.04 & 7.1 \\
\hline 39 & 7.70 & 9.19 & 8.41 & 8.4 \\
\hline 40 & 9.19 & 10.97 & 10.0 & 10 \\
\hline
\end{tabular}


Table B.2: $\theta$ and momentum $(p)$ bins for $e^{+} e^{-}$background removal.

\begin{tabular}{|c|c|c|}
\hline Bin & $\theta_{\min }$ & $\theta_{\max }$ \\
\hline \hline 1 & 2.0 & 5.0 \\
\hline 2 & 5.0 & 10.0 \\
\hline 3 & 10.0 & 15.0 \\
\hline 4 & 15.0 & 20.0 \\
\hline 5 & 20.0 & 25.0 \\
\hline 6 & 25.0 & 30.0 \\
\hline 7 & 30.0 & 35.0 \\
\hline 8 & 35.0 & 40.0 \\
\hline 9 & 40.0 & 45.0 \\
\hline 10 & 45.0 & 49.0 \\
\hline
\end{tabular}

\begin{tabular}{|c|c|c|}
\hline Bin & $p_{\min }$ & $p_{\max }$ \\
\hline \hline 1 & 0.03 & 0.30 \\
\hline 2 & 0.30 & 0.60 \\
\hline 3 & 0.60 & 0.90 \\
\hline 4 & 0.90 & 1.20 \\
\hline 5 & 1.20 & 1.50 \\
\hline 6 & 1.50 & 1.80 \\
\hline 7 & 1.80 & 2.20 \\
\hline 8 & 2.20 & 2.60 \\
\hline 9 & 2.60 & 3.00 \\
\hline 10 & 3.00 & 3.40 \\
\hline 11 & 3.40 & 3.90 \\
\hline 12 & 3.90 & 4.40 \\
\hline 13 & 4.40 & 4.90 \\
\hline 14 & 4.90 & 5.40 \\
\hline
\end{tabular}




\section{B.2 Pion Background Cut Parameters}


Table B.3: Pion background cut parameters for use in Eq. 4.1 - 4.4, Each sector and CC segment has its own parameters.

Sectors 1 and 2 , inbending only

\begin{tabular}{|c|c|c|c|c|c|}
\hline sector & segment & $\theta_{p}^{\text {center }}$ & $\theta_{p}^{\text {offset }}$ & $\sigma_{p}$ & $\Delta t^{S C-C C}$ \\
\hline 1 & 1 & 7.715 & 0 & 1.25 & -10.06 \\
\hline 1 & 2 & 9.435 & 1.61667 & 0.558811 & -10.06 \\
\hline 1 & 3 & 11.17 & 1.17703 & 0.710307 & -11.42 \\
\hline 1 & 4 & 12.925 & 1.25518 & 0.761159 & -10.88 \\
\hline 1 & 5 & 14.69 & 1.29892 & 0.83303 & -11.15 \\
\hline 1 & 6 & 16.47 & 1.47686 & 0.799495 & -10.6 \\
\hline 1 & 7 & 18.29 & 1.67202 & 0.889586 & -11.42 \\
\hline 1 & 8 & 20.15 & 1.8122 & 0.79602 & -11.15 \\
\hline 1 & 9 & 22.04 & 1.79007 & 0.765841 & -11.42 \\
\hline 1 & 10 & 24.01 & 1.75959 & 0.922235 & -10.88 \\
\hline 1 & 11 & 26.05 & 1.70721 & 1.12133 & -10.88 \\
\hline 1 & 12 & 28.17 & 2.19133 & 1.09796 & -10.88 \\
\hline 1 & 13 & 30.475 & 2.28175 & 1.36657 & -28.53 \\
\hline 1 & 14 & 32.935 & 2.97774 & 1.12487 & -9.246 \\
\hline 1 & 15 & 35.445 & 3.10636 & 1.02596 & -6.531 \\
\hline 1 & 16 & 37.97 & 3.03297 & 1.11458 & -5.988 \\
\hline 1 & 17 & 40.515 & 2.4122 & 0.836312 & -8.67905 \\
\hline$T$ & 18 & 43.12 & $\overline{0}$ & 0 & 100 \\
\hline 2 & 1 & 7.715 & 0 & 1.25 & -9.518 \\
\hline 2 & 2 & 9.435 & 1.57819 & 0.511605 & -8.975 \\
\hline 2 & 3 & 11.17 & 1.11335 & 0.679164 & -10.06 \\
\hline 2 & 4 & 12.925 & 0.978316 & 0.689107 & -9.518 \\
\hline 2 & 5 & 14.69 & 0.949305 & 0.827985 & -9.789 \\
\hline 2 & 6 & 16.47 & 1.13836 & 0.877119 & -10.06 \\
\hline 2 & 7 & 18.29 & 1.3449 & 0.742062 & -10.6 \\
\hline 2 & 8 & 20.15 & 1.54754 & 1.1438 & -80 \\
\hline 2 & 9 & 22.04 & 1.79137 & 0.758517 & -11.96 \\
\hline 2 & 10 & 24.01 & 1.73608 & 0.912447 & -9.789 \\
\hline 2 & 11 & 26.05 & 1.74283 & 1.11211 & -10.88 \\
\hline 2 & 12 & 28.17 & 2.11168 & 1.03115 & -11.69 \\
\hline 2 & 13 & 30.475 & 2.34247 & 1.34492 & -10.6 \\
\hline 2 & 14 & 32.935 & 2.89912 & 1.09128 & -9.246 \\
\hline 2 & 15 & 35.445 & 2.89664 & 1.08348 & -7.617 \\
\hline 2 & 16 & 37.97 & 2.83338 & 1.08412 & -4.358 \\
\hline 2 & 17 & 40.515 & 2.19907 & 0.880703 & -5.72487 \\
\hline 2 & 18 & 43.12 & 0 & 0 & 100 \\
\hline
\end{tabular}


Sectors 3 and 4 , inbending only

\begin{tabular}{|c|c|c|c|c|c|}
\hline sector & segment & $\theta_{p}^{\text {center }}$ & $\theta_{p}^{\text {offset }}$ & $\sigma_{p}$ & $\Delta t^{S C-C C}$ \\
\hline 3 & $\overline{1}$ & 7.715 & 0 & 1.25 & -10.06 \\
\hline 3 & 2 & 9.435 & 1.59668 & 0.531775 & -10.88 \\
\hline 3 & $\overline{3}$ & 11.17 & 1.09262 & 0.685662 & -11.96 \\
\hline 3 & 4 & 12.925 & 1.12444 & 0.740032 & -15.49 \\
\hline 3 & 5 & 14.69 & 1.10406 & 0.765128 & -16.31 \\
\hline 3 & 6 & 16.47 & 1.1937 & 0.770151 & -15.49 \\
\hline 3 & 7 & 18.29 & 1.40898 & 0.791015 & -11.96 \\
\hline 3 & 8 & 20.15 & 1.63203 & 0.88898 & -11.96 \\
\hline 3 & 9 & 22.04 & 1.68464 & 0.688376 & -7.346 \\
\hline 3 & 10 & 24.01 & 2.04233 & 1.05516 & -80 \\
\hline 3 & 11 & 26.05 & 1.8272 & 1.05423 & -23.91 \\
\hline 3 & 12 & 28.17 & 2.04524 & 1.08444 & -20.92 \\
\hline 3 & 13 & 30.475 & 2.3309 & 1.41215 & -20.65 \\
\hline 3 & 14 & 32.935 & 3.09273 & 1.0571 & -9.789 \\
\hline 3 & 15 & 35.445 & 3.19737 & 1.01388 & -7.889 \\
\hline$\overline{3}$ & 16 & 37.97 & 2.97263 & 1.04351 & -3.815 \\
\hline 3 & 17 & 40.515 & 2.24073 & 0.841379 & -5.52649 \\
\hline 3 & 18 & 43.12 & 0 & 0 & 100 \\
\hline 4 & 1 & 7.715 & 0 & 1.25 & -8.432 \\
\hline 4 & 2 & 9.435 & 1.68515 & 0.571923 & -8.703 \\
\hline 4 & 3 & 11.17 & 1.27287 & 0.702654 & -9.518 \\
\hline 4 & 4 & 12.925 & 1.25499 & 0.725934 & -13.32 \\
\hline 4 & 5 & 14.69 & 1.21428 & 0.780379 & -10.6 \\
\hline 4 & 6 & 16.47 & 1.39868 & 0.847354 & -11.15 \\
\hline 4 & 7 & 18.29 & 1.60879 & 0.880012 & -10.33 \\
\hline 4 & 8 & 20.15 & 1.83014 & 0.798267 & -12.23 \\
\hline 4 & 9 & 22.04 & 1.80748 & 0.827193 & -12.78 \\
\hline 4 & 10 & 24.01 & 1.76519 & 0.887549 & -11.15 \\
\hline 4 & 11 & 26.05 & 1.63676 & 1.04132 & -11.96 \\
\hline 4 & 12 & 28.17 & 2.0624 & 1.16812 & -10.88 \\
\hline 4 & 13 & 30.475 & 2.04637 & 1.42806 & -12.23 \\
\hline 4 & 14 & 32.935 & 2.8001 & 1.09894 & -9.246 \\
\hline 4 & 15 & 35.445 & 3.00113 & 1.1128 & -6.802 \\
\hline 4 & 16 & 37.97 & 3.05102 & 1.08308 & -5.173 \\
\hline 4 & 17 & 40.515 & 2.37987 & 0.866787 & -7.08273 \\
\hline 4 & 18 & 43.12 & 0 & 0 & 100 \\
\hline
\end{tabular}


Sectors 5 and 6, inbending only

\begin{tabular}{|c|c|c|c|c|c|}
\hline sector & segment & $\theta_{p}^{\text {center }}$ & $\theta_{p}^{\text {offset }}$ & $\sigma_{p}$ & $\Delta t^{S C-C C}$ \\
\hline 5 & 1 & 7.715 & 0 & 1.25 & -7.074 \\
\hline 5 & 2 & 9.435 & 1.77528 & 0.531322 & -8.703 \\
\hline 5 & 3 & 11.17 & 1.65381 & 0.889448 & -13.86 \\
\hline 5 & 4 & 12.925 & 1.52975 & 0.829391 & -16.03 \\
\hline 5 & 5 & 14.69 & 1.36857 & 0.795689 & -10.33 \\
\hline 5 & 6 & 16.47 & 1.46561 & 0.80882 & -10.6 \\
\hline 5 & 7 & 18.29 & 1.65247 & 0.89631 & -9.518 \\
\hline 5 & 8 & 20.15 & 1.90336 & 0.861988 & -11.42 \\
\hline 5 & 9 & 22.04 & 2.25316 & 0.968319 & -11.96 \\
\hline 5 & 10 & 24.01 & 2.30237 & 1.01662 & -10.6 \\
\hline 5 & 11 & 26.05 & 2.73447 & 1.00332 & -10.6 \\
\hline 5 & 12 & 28.17 & 2.95056 & 1.14894 & -13.86 \\
\hline 5 & 13 & 30.475 & 3.05246 & 1.00229 & -11.15 \\
\hline 5 & 14 & 32.935 & 3.06401 & 1.03006 & -8.703 \\
\hline 5 & 15 & 35.445 & 3.12805 & 1.12716 & -8.16 \\
\hline 5 & 16 & 37.97 & 3.1899 & 1.08879 & -5.445 \\
\hline 5 & 17 & 40.515 & 2.53884 & 0.838787 & -8.62813 \\
\hline 5 & 18 & 43.12 & 0 & 0 & 100 \\
\hline$\overline{6}$ & 1 & 7.715 & 0 & 1.25 & -7.346 \\
\hline 6 & 2 & 9.435 & 1.64922 & 0.531754 & -10.6 \\
\hline 6 & 3 & 11.17 & 1.18211 & 0.715696 & -10.33 \\
\hline 6 & 4 & 12.925 & 1.22371 & 0.749207 & -9.789 \\
\hline 6 & 5 & 14.69 & 1.17344 & 0.738545 & -13.59 \\
\hline 6 & $\overline{6}$ & 16.47 & 1.28719 & 0.743982 & -11.15 \\
\hline$\overline{6}$ & 7 & 18.29 & 1.30081 & 0.898898 & -11.96 \\
\hline 6 & 8 & 20.15 & 1.6235 & 0.778548 & -14.95 \\
\hline$\overline{6}$ & 9 & 22.04 & 1.65107 & 0.788323 & -11.42 \\
\hline 6 & 10 & 24.01 & 1.78634 & 0.95529 & -10.6 \\
\hline 6 & 11 & 26.05 & 1.74261 & 1.11471 & -10.6 \\
\hline 6 & 12 & 28.17 & 2.13715 & 1.06596 & -11.15 \\
\hline 6 & $\overline{13}$ & 30.475 & 2.41261 & 1.33136 & -10.88 \\
\hline 6 & 14 & 32.935 & 2.94335 & 1.18341 & -8.16 \\
\hline 6 & 15 & 35.445 & 3.31166 & 1.15398 & -7.074 \\
\hline$\overline{6}$ & 16 & 37.97 & 3.37013 & 1.0801 & -3.272 \\
\hline 6 & 17 & 40.515 & 2.74791 & 0.744176 & -7.22474 \\
\hline 6 & 18 & 43.12 & 0 & 0 & 100 \\
\hline
\end{tabular}


Sectors 1 and 2, outbending only

\begin{tabular}{|c|c|c|c|c|c|}
\hline sector & segment & $\theta_{p}^{\text {center }}$ & $\theta_{p}^{\text {offset }}$ & $\sigma_{p}$ & $\Delta t^{S C-C C}$ \\
\hline \hline 1 & 1 & 7.715 & 9.44136 & 0.882966 & -18.3827 \\
\hline 1 & 2 & 9.435 & 9.78426 & 2.2021 & -18.3168 \\
\hline 1 & 3 & 11.17 & 5.11882 & 1.12383 & -16.3986 \\
\hline 1 & 4 & 12.925 & 2.38628 & 0.587813 & -10.88 \\
\hline 1 & 5 & 14.69 & 1.01974 & 0.419864 & -11.15 \\
\hline 1 & 6 & 16.47 & 0.928088 & 0.718078 & -10.6 \\
\hline 1 & 7 & 18.29 & 0.897623 & 0.728103 & -11.42 \\
\hline 1 & 8 & 20.15 & 0.85664 & 0.803771 & -11.15 \\
\hline 1 & 9 & 22.04 & 0.81016 & 0.814512 & -18.957 \\
\hline 1 & 10 & 24.01 & 0.534905 & 0.808717 & -16.8534 \\
\hline 1 & 11 & 26.05 & -0.0567701 & 0.830669 & -12.0472 \\
\hline 1 & 12 & 28.17 & -0.0894648 & 1.20216 & -10.88 \\
\hline 1 & 13 & 30.475 & -0.476507 & 1.43163 & -28.53 \\
\hline 1 & 14 & 32.935 & -0.512086 & 1.79071 & -9.246 \\
\hline 1 & 15 & 35.445 & -0.564243 & 1.88346 & -7.64864 \\
\hline 1 & 16 & 37.97 & -1.52835 & 2.55906 & -8.26228 \\
\hline 1 & 17 & 40.515 & 0.930158 & 1.11536 & -6.32124 \\
\hline 1 & 18 & 43.12 & 0.0818546 & 0.856084 & -3.74568 \\
\hline 2 & 1 & 7.715 & 9.70018 & 0.725683 & -21.08 \\
\hline 2 & 2 & 9.435 & 9.30676 & 1.61795 & -22.6378 \\
\hline 2 & 3 & 11.17 & 5.62216 & 0.945182 & -19.3352 \\
\hline 2 & 4 & 12.925 & 3.8231 & 1.32925 & -9.518 \\
\hline 2 & 5 & 14.69 & 1.75844 & 0.358314 & -9.789 \\
\hline 2 & 6 & 16.47 & 0.727494 & 0.753471 & -10.06 \\
\hline 2 & 7 & 18.29 & 0.73484 & 0.71621 & -10.6 \\
\hline 2 & 8 & 20.15 & 0.591899 & 0.684617 & -12.23 \\
\hline 2 & 9 & 22.04 & 0.81292 & 1.04731 & -80 \\
\hline 2 & 10 & 24.01 & 0.565134 & 0.672787 & -9.789 \\
\hline 2 & 11 & 26.05 & -0.0962878 & 0.849402 & -10.88 \\
\hline 2 & 12 & 28.17 & -0.314749 & 1.21073 & -11.69 \\
\hline 2 & 13 & 30.475 & -0.411599 & 1.41265 & -10.6 \\
\hline 2 & 14 & 32.935 & -0.73257 & 1.7549 & -9.246 \\
\hline 2 & 15 & 35.445 & -0.728269 & 1.917 & -7.617 \\
\hline 2 & 16 & 37.97 & -1.90306 & 2.58271 & -6.31366 \\
\hline 2 & 17 & 40.515 & 0.873927 & 1.13948 & -3.92716 \\
\hline 2 & 18 & 43.12 & -0.112584 & 0.947823 & -1.94926 \\
\hline & & & & & \\
\hline 1 & & & & \\
\hline 1 & 1 & & & & \\
\hline & 1 & & & &
\end{tabular}


Sectors 3 and 4, outbending only

\begin{tabular}{|c|c|c|c|c|c|}
\hline sector & segment & $\theta_{p}^{\text {center }}$ & $\theta_{p}^{\text {off set }}$ & $\sigma_{p}$ & $\Delta t^{S C-C C}$ \\
\hline 3 & 1 & 7.715 & 9.34863 & 1.25515 & -19.3524 \\
\hline 3 & 2 & 9.435 & 9.34325 & 2.17895 & -21.1789 \\
\hline 3 & 3 & 11.17 & 4.72228 & 0.797899 & -14.7606 \\
\hline 3 & 4 & 12.925 & 1.41506 & 0.875569 & -15.49 \\
\hline 3 & 5 & 14.69 & 0.85164 & 0.687879 & -16.31 \\
\hline 3 & 6 & 16.47 & 0.807327 & 0.698243 & -15.49 \\
\hline$\overline{3}$ & 7 & 18.29 & 0.765179 & 0.726155 & -11.96 \\
\hline 3 & 8 & 20.15 & 0.662392 & 0.704451 & -20.9295 \\
\hline 3 & 9 & 22.04 & 0.771579 & 0.80965 & -8.80298 \\
\hline 3 & 10 & 24.01 & 0.235635 & 0.765732 & -25.54 \\
\hline 3 & 11 & 26.05 & -0.605803 & 0.755947 & -80 \\
\hline 3 & 12 & 28.17 & 0.0507434 & 1.14428 & -80 \\
\hline 3 & 13 & 30.475 & -0.0564292 & 1.41699 & -20.65 \\
\hline 3 & 14 & 32.935 & -0.490215 & 1.78919 & -9.789 \\
\hline 3 & 15 & 35.445 & -0.454119 & 2.15891 & -21.4961 \\
\hline 3 & 16 & 37.97 & -2.02456 & 3.03551 & -9.94321 \\
\hline 3 & 17 & 40.515 & 1.12373 & 1.03818 & -4.15478 \\
\hline 3 & 18 & 43.12 & 0.116346 & 0.842354 & -2.4432 \\
\hline 4 & 1 & 7.715 & 9.44417 & 1.71035 & -16.8448 \\
\hline 4 & 2 & 9.435 & 9.50459 & 2.76454 & -17.4693 \\
\hline 4 & 3 & 11.17 & 4.61428 & 0.780718 & -9.518 \\
\hline 4 & 4 & 12.925 & 1.53946 & 1.02494 & -13.32 \\
\hline 4 & 5 & 14.69 & 0.974502 & 0.794278 & -10.6 \\
\hline 4 & 6 & 16.47 & 0.817862 & 0.724484 & -11.15 \\
\hline 4 & 7 & 18.29 & 0.89449 & 0.762863 & -10.33 \\
\hline 4 & 8 & 20.15 & 0.835993 & 0.8058 & -12.23 \\
\hline 4 & 9 & 22.04 & 0.877967 & 0.785631 & -12.78 \\
\hline 4 & 10 & 24.01 & 0.534746 & 0.846716 & -11.15 \\
\hline 4 & 11 & 26.05 & -0.0503515 & 0.805624 & -11.96 \\
\hline 4 & 12 & 28.17 & -0.17271 & 1.20144 & -10.88 \\
\hline 4 & 13 & 30.475 & -0.598793 & 1.39201 & -12.23 \\
\hline 4 & 14 & 32.935 & -0.732761 & 1.87927 & -9.246 \\
\hline 4 & 15 & 35.445 & -0.596728 & 1.89874 & -12.319 \\
\hline 4 & 16 & 37.97 & -1.98561 & 2.74207 & -9.8736 \\
\hline 4 & 17 & 40.515 & 0.897553 & 1.0876 & -22.7609 \\
\hline 4 & 18 & 43.12 & -0.0168865 & 0.884155 & -32.4434 \\
\hline
\end{tabular}


Sectors 5 and 6 , outbending only

\begin{tabular}{|c|c|c|c|c|c|}
\hline sector & segment & $\theta_{p}^{\text {center }}$ & $\theta_{p}^{\text {offset }}$ & $\sigma_{p}$ & $\Delta t^{S C-C C}$ \\
\hline 5 & 1 & 7.715 & 9.43056 & 0.902969 & -16.5621 \\
\hline 5 & 2 & 9.435 & 9.66316 & 2.46374 & -19.5467 \\
\hline 5 & 3 & 11.17 & 4.48558 & 1.06914 & -15.9008 \\
\hline 5 & 4 & 12.925 & 2.35348 & 0.574084 & -16.03 \\
\hline 5 & 5 & 14.69 & 1.06895 & 0.463329 & -10.33 \\
\hline 5 & 6 & 16.47 & 0.957842 & 0.682332 & -10.6 \\
\hline 5 & 7 & 18.29 & 0.873888 & 0.707929 & -9.518 \\
\hline 5 & 8 & 20.15 & 0.843671 & 0.842153 & -11.42 \\
\hline 5 & 9 & 22.04 & 0.898008 & 0.864229 & -11.96 \\
\hline 5 & 10 & 24.01 & 0.758749 & 0.94682 & -10.6 \\
\hline 5 & 11 & 26.05 & 0.46833 & 1.14142 & -10.6 \\
\hline 5 & 12 & 28.17 & 0.359037 & 1.24246 & -13.86 \\
\hline 5 & 13 & 30.475 & 0.114843 & 1.513 & -18.7278 \\
\hline 5 & 14 & 32.935 & 0.248881 & 1.52748 & -20.6778 \\
\hline 5 & 15 & 35.445 & -0.461898 & 1.7839 & -8.16 \\
\hline 5 & 16 & 37.97 & -0.556424 & 2.38824 & -5.87629 \\
\hline 5 & 17 & 40.515 & 1.21186 & 1.12693 & -5.06078 \\
\hline 5 & 18 & 43.12 & 0.236514 & 0.871704 & -4.01868 \\
\hline 6 & 1 & 7.715 & 9.55355 & 0.810562 & -17.9514 \\
\hline 6 & 2 & 9.435 & 9.72445 & 1.98536 & -18.7144 \\
\hline 6 & $\overline{3}$ & 11.17 & 5.3406 & 1.07633 & -20.7031 \\
\hline 6 & 4 & 12.925 & 2.52474 & 0.511665 & -9.789 \\
\hline 6 & 5 & 14.69 & 1.48256 & 0.154967 & -13.59 \\
\hline 6 & 6 & 16.47 & 0.842704 & 0.694377 & -11.15 \\
\hline 6 & 7 & 18.29 & 0.823057 & 0.718335 & -11.96 \\
\hline 6 & 8 & 20.15 & 0.764655 & 0.748196 & -14.95 \\
\hline 6 & 9 & 22.04 & 0.749473 & 0.815529 & -11.42 \\
\hline 6 & 10 & 24.01 & 0.509091 & 0.739087 & -13.8422 \\
\hline 6 & 11 & 26.05 & -0.0659918 & 0.846994 & -10.6 \\
\hline 6 & 12 & 28.17 & -0.105601 & 1.19791 & -11.15 \\
\hline 6 & 13 & 30.475 & -0.354063 & 1.45182 & -10.88 \\
\hline 6 & 14 & 32.935 & -0.283529 & 1.80117 & -8.16 \\
\hline 6 & 15 & 35.445 & -0.48233 & 2.08146 & -7.59534 \\
\hline 6 & 16 & 37.97 & -1.04792 & 2.74191 & -6.12133 \\
\hline 6 & 17 & 40.515 & 1.12619 & 1.15726 & -4.37422 \\
\hline 6 & 18 & 43.12 & -0.0166473 & 0.95124 & -2.87116 \\
\hline
\end{tabular}




\section{B.3 Fiducial Cut Parameters}

Table B.4: Inbending fiducial cut parameters, designated by the lower value on each momentum bin. At all momenta higher than $4.2 \mathrm{GeV} / c$, the highest bin values are used, as there is little evolution of the event geometry after this point.

Torus Current $=+1500 \mathrm{~A}$

\begin{tabular}{|c|c|c|c|c|c|c|c|}
\hline $\operatorname{Bin} p_{\min }$ & $A$ & $B$ & $C$ & $D$ & $E$ & $F$ & $\theta_{\max }$ \\
\hline \hline 0.15 & 36 & 0.28 & 0.30 & 5 & 16.72 & 0.06 & 37 \\
\hline 0.30 & 32 & 0.28 & 0.30 & 8.5 & 16.72 & 0.06 & 37 \\
\hline 0.45 & 32.5 & 0.28 & 0.30 & 10 & 16.72 & 0.06 & 35 \\
\hline 0.60 & 33 & 0.28 & 0.30 & 10.5 & 16.72 & 0.06 & 33 \\
\hline 0.75 & 33 & 0.28 & 0.30 & 10.5 & 16.72 & 0.06 & 32 \\
\hline 0.90 & 33 & 0.28 & 0.30 & 10.5 & 16.72 & 0.06 & 32 \\
\hline 1.05 & 33 & 0.28 & 0.30 & 10.5 & 16.72 & 0.06 & 31 \\
\hline 1.20 & 34 & 0.28 & 0.30 & 10.5 & 16.72 & 0.06 & 30 \\
\hline 1.35 & 35 & 0.28 & 0.30 & 10.5 & 16.72 & 0.06 & 29 \\
\hline 1.50 & 35 & 0.28 & 0.30 & 10.5 & 16.72 & 0.06 & 28 \\
\hline 1.65 & 35 & 0.28 & 0.30 & 10.5 & 16.72 & 0.06 & 28 \\
\hline 1.80 & 35.5 & 0.28 & 0.30 & 10.5 & 16.72 & 0.06 & 28 \\
\hline 1.95 & 36.5 & 0.28 & 0.30 & 10.5 & 16.72 & 0.06 & 28 \\
\hline 2.10 & 36.5 & 0.28 & 0.30 & 10.5 & 16.72 & 0.06 & 28 \\
\hline 2.25 & 36 & 0.28 & 0.30 & 10.5 & 16.72 & 0.06 & 28 \\
\hline
\end{tabular}


Torus Current $=+2250 \mathrm{~A}$

\begin{tabular}{|c|c|c|c|c|c|c|c|}
\hline Bin $p_{\min }$ & $A$ & $B$ & $C$ & $D$ & $E$ & $F$ & $\theta_{\max }$ \\
\hline \hline 0.15 & 36 & 0.28 & 0.30 & 0 & 16.72 & 0.06 & 41 \\
\hline 0.30 & 36 & 0.28 & 0.30 & 6 & 16.72 & 0.06 & 40 \\
\hline 0.45 & 32 & 0.28 & 0.30 & 8 & 16.72 & 0.06 & 39 \\
\hline 0.60 & 32 & 0.28 & 0.30 & 9.5 & 16.72 & 0.06 & 34 \\
\hline 0.75 & 32 & 0.28 & 0.30 & 10.5 & 16.72 & 0.06 & 32 \\
\hline 0.90 & 32 & 0.28 & 0.30 & 10.5 & 16.72 & 0.06 & 32 \\
\hline 1.05 & 32 & 0.28 & 0.30 & 10.5 & 16.72 & 0.06 & 32 \\
\hline 1.20 & 32 & 0.28 & 0.30 & 10.5 & 16.72 & 0.06 & 32 \\
\hline 1.35 & 32 & 0.28 & 0.30 & 10.5 & 16.72 & 0.06 & 32 \\
\hline 1.50 & 32 & 0.28 & 0.30 & 10.5 & 16.72 & 0.06 & 32 \\
\hline 1.65 & 32 & 0.28 & 0.30 & 10.5 & 16.72 & 0.06 & 32 \\
\hline 1.80 & 32.5 & 0.28 & 0.30 & 10.5 & 16.72 & 0.06 & 32 \\
\hline 1.95 & 32.5 & 0.28 & 0.30 & 10.5 & 16.72 & 0.06 & 32 \\
\hline 2.10 & 33 & 0.28 & 0.30 & 10.5 & 16.72 & 0.06 & 32 \\
\hline 2.25 & 33.5 & 0.28 & 0.30 & 10.5 & 16.72 & 0.06 & 32 \\
\hline 2.40 & 34 & 0.28 & 0.30 & 10.5 & 16.72 & 0.06 & 32 \\
\hline 2.55 & 34 & 0.28 & 0.30 & 10.5 & 16.72 & 0.06 & 32 \\
\hline 2.70 & 34 & 0.28 & 0.30 & 10.5 & 16.72 & 0.06 & 32 \\
\hline 2.85 & 34 & 0.28 & 0.30 & 10.5 & 16.72 & 0.06 & 32 \\
\hline 3.00 & 34 & 0.28 & 0.30 & 10.5 & 16.72 & 0.06 & 32 \\
\hline 3.15 & 34 & 0.28 & 0.30 & 10.5 & 16.72 & 0.06 & 32 \\
\hline 3.30 & 34 & 0.28 & 0.30 & 10.5 & 16.72 & 0.06 & 32 \\
\hline 3.45 & 34 & 0.28 & 0.30 & 10.5 & 16.72 & 0.06 & 32 \\
\hline 3.60 & 34 & 0.28 & 0.30 & 10.5 & 16.72 & 0.06 & 32 \\
\hline 3.75 & 34 & 0.28 & 0.30 & 10.5 & 16.72 & 0.06 & 32 \\
\hline 3.90 & 34 & 0.28 & 0.30 & 10.5 & 16.72 & 0.06 & 32 \\
\hline 4.05 & 34 & 0.28 & 0.30 & 10.5 & 16.72 & 0.06 & 32 \\
\hline 4.20 & 34 & 0.28 & 0.30 & 10.5 & 16.72 & 0.06 & 32 \\
\hline & & & & & & & \\
\hline
\end{tabular}


Table B.5: Outbending fiducial cut parameters, designated by the lower value on each momentum bin.As for inbending cuts, all momenta greater than $4.2 \mathrm{GeV} / c$ use the highest bin values. Torus Current $=-1500 \mathrm{~A}$, Sector 1

\begin{tabular}{|c|c|c|c|c|c|c|c|c|}
\hline Bin $p_{\min }$ & $A$ & $B$ & $C$ & $D$ & $E$ & $F$ & $G_{\text {upper }}$ & $H_{\text {upper }}$ \\
\hline \hline 0.15 & 44 & 0.77 & 0.17 & 12 & 3 & 1.46 & 1 & 1 \\
\hline 0.30 & 38 & 0.55 & 0.27 & 5 & 3 & 1.46 & 0 & 0 \\
\hline 0.45 & 36.5 & 0.44 & 0.22 & 5.5 & 3 & 1.46 & 0.1 & -0.11 \\
\hline 0.60 & 31 & 0.33 & 0.22 & 5.5 & 3 & 1.46 & 0.1 & 0.1 \\
\hline 0.75 & 29.5 & 0.28 & 0.22 & 5.5 & 3 & 1.46 & 0.2 & 0.5 \\
\hline 0.90 & 29 & 0.28 & 0.22 & 5.8 & 3 & 1.46 & 0.1 & -.12 \\
\hline 1.05 & 29 & 0.28 & 0.22 & 5.8 & 3 & 1.46 & 0.3 & 0 \\
\hline 1.20 & 30 & 0.28 & 0.22 & 6.2 & 3 & 1.46 & 0.2 & 0 \\
\hline 1.35 & 31 & 0.28 & 0.22 & 6.3 & 3 & 1.46 & 0.06 & -0.12 \\
\hline 1.50 & 31 & 0.28 & 0.22 & 6.5 & 3 & 1.46 & 0.3 & -0.02 \\
\hline 1.65 & 31 & 0.28 & 0.22 & 7.2 & 3 & 1.46 & 0.2 & -0.04 \\
\hline 1.80 & 30 & 0.28 & 0.22 & 7.2 & 3 & 1.46 & 0.22 & -0.06 \\
\hline 1.95 & 28 & 0.28 & 0.22 & 7.2 & 3 & 1.46 & 0.22 & -0.06 \\
\hline 2.10 & 32 & 0.37 & 0.22 & 7.5 & 3 & 1.46 & 0.18 & -0.06 \\
\hline 2.25 & 34 & 0.43 & 0.22 & 8 & 3 & 1.46 & 0.18 & -0.06 \\
\hline 2.40 & 34 & 0.43 & 0.22 & 8.3 & 3 & 1.46 & 0.18 & -0.06 \\
\hline 2.55 & 34 & 0.43 & 0.22 & 8.6 & 3 & 1.46 & 0.18 & -0.06 \\
\hline Bin $p_{\min }$ & $G_{\text {lower }}$ & $H_{\text {lower }}$ & $\theta_{\text {upper }}$ & $\theta_{\text {lower }}$ & inneroffset & outeroffset \\
\hline \hline 0.15 & 1. & 1 & 23 & 23 & \multicolumn{2}{|c|}{2} & 0 \\
\hline 0.30 & 0.15 & -0.1 & 13 & 18 & 2.0 & 1.5 \\
\hline 0.45 & 0.1 & -0.11 & 13 & 19 & 1.7 & 0.4 \\
\hline 0.60 & 1. & 0.5 & 14 & 19 & 1.5 & 1 \\
\hline 0.75 & 0.6 & 0.5 & 15 & 20 & 1.4 & 0.8 \\
\hline 0.90 & 0.1 & -.12 & 15 & 19 & 1.2 & 0 \\
\hline 1.05 & 0.3 & 0. & 14 & 18 & 1.2 & 0 \\
\hline 1.20 & 0.2 & 0 & 13.5 & 17 & 1.2 & 0 \\
\hline 1.35 & 0.12 & -0.09 & 13 & 16 & 1.2 & 0 \\
\hline 1.50 & 0.1 & -0.01 & 12.5 & 15 & 1.3 & 0 \\
\hline 1.65 & 0.2 & -0.04 & 12 & 14 & 1.2 & 0 \\
\hline 1.80 & 0.22 & -0.04 & 14 & 13 & 0. & 0 \\
\hline 1.95 & 0.22 & -0.04 & 17 & 13 & -0.4 & 0 \\
\hline 2.10 & 0.18 & -0.04 & 18 & 14 & -0.4 & 0 \\
\hline 2.25 & 0.18 & -0.04 & 19 & 15 & -0.4 & 0 \\
\hline 2.40 & 0.18 & -0.04 & 19 & 15 & -0.4 & 0 \\
\hline 2.55 & 0.18 & -0.04 & 19 & 15 & -0.4 & 0 \\
\hline
\end{tabular}


Torus Current $=-1500 \mathrm{~A}$, Sector 2

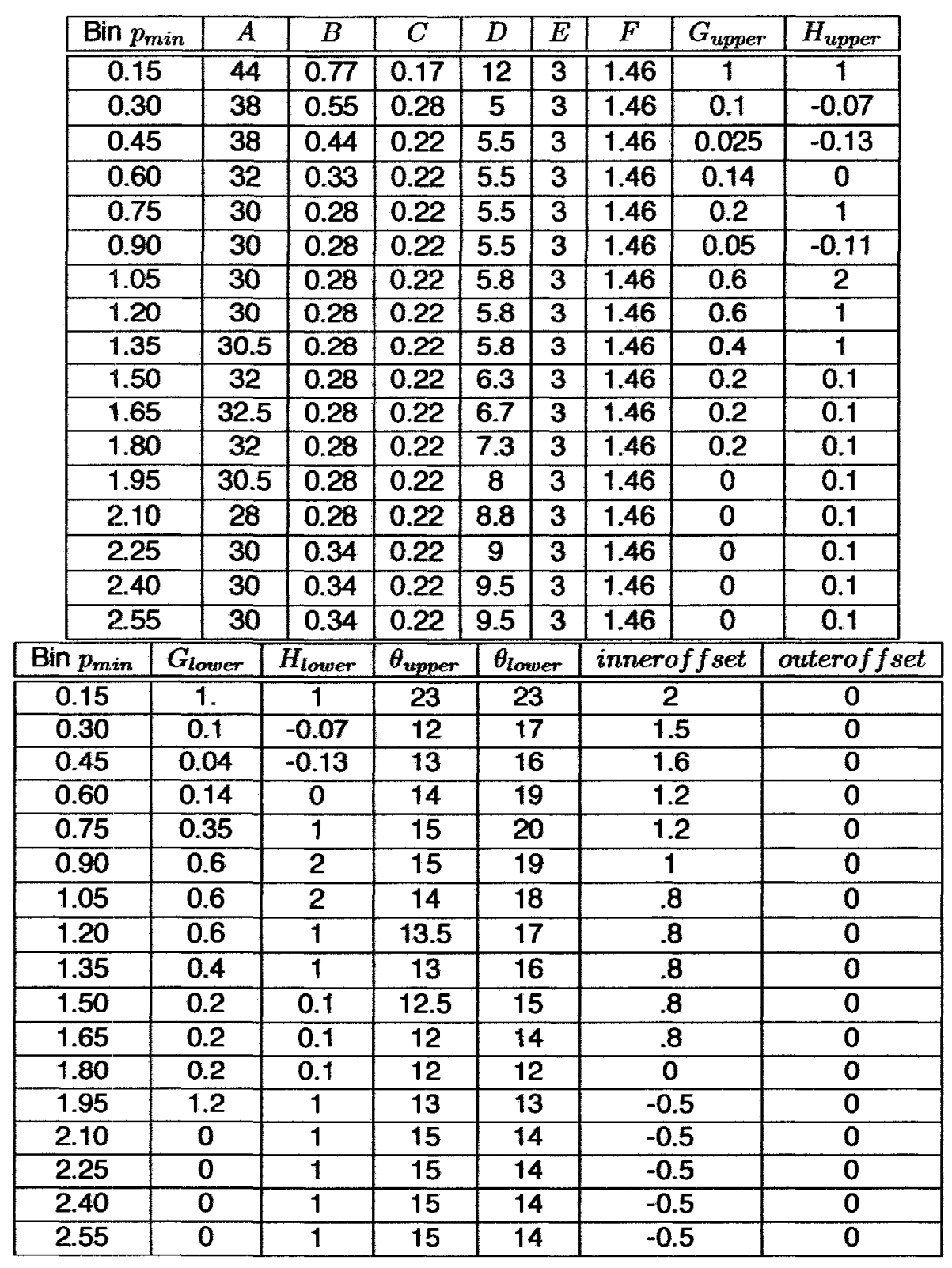


Torus Current $=-1500 \mathrm{~A}$, Sector 3

\begin{tabular}{|c|c|c|c|c|c|c|c|c|}
\hline Bin $p_{\min }$ & $A$ & $B$ & $C$ & $D$ & $E$ & $F$ & $G_{\text {upper }}$ & $H_{\text {upper }}$ \\
\hline \hline 0.15 & 44 & 0.77 & 0.17 & 12 & 3 & 1.46 & 1 & 1 \\
\hline 0.30 & 39 & 0.55 & 0.28 & 5 & 3 & 1.46 & 0 & 0 \\
\hline 0.45 & 39 & 0.44 & 0.22 & 5 & 3 & 1.46 & 1 & 1 \\
\hline 0.60 & 34 & 0.36 & 0.22 & 5 & 3 & 1.46 & 0.15 & 0.1 \\
\hline 0.75 & 31 & 0.3 & 0.22 & 5.5 & 3 & 1.46 & 0 & 0.1 \\
\hline 0.90 & 30 & 0.28 & 0.22 & 5.5 & 3 & 1.46 & 1 & 1 \\
\hline 1.05 & 30.5 & 0.28 & 0.22 & 5.5 & 3 & 1.46 & 0 & 0.1 \\
\hline 1.20 & 31 & 0.28 & 0.22 & 5.7 & 3 & 1.46 & 0 & 1 \\
\hline 1.35 & 31.5 & 0.28 & 0.22 & 5.7 & 3 & 1.46 & 0 & 1 \\
\hline 1.50 & 32 & 0.28 & 0.22 & 6 & 3 & 1.46 & 0 & 1 \\
\hline 1.65 & 32 & 0.28 & 0.22 & 6 & 3 & 1.46 & 0. & 1 \\
\hline 1.80 & 31 & 0.28 & 0.22 & 6.3 & 3 & 1.46 & 0.5 & 1 \\
\hline 1.95 & 29 & 0.28 & 0.22 & 6.6 & 3 & 1.46 & 0.5 & 1 \\
\hline 2.10 & 31 & 0.35 & 0.22 & 7 & 3 & 1.46 & 0.2 & 1 \\
\hline 2.25 & 32 & 0.35 & 0.22 & 7.4 & 3 & 1.46 & 0 & 1 \\
\hline 2.40 & 32 & 0.4 & 0.22 & 7.8 & 3 & 1.46 & 0 & 1 \\
\hline 2.55 & 32 & 0.4 & 0.22 & 8 & 3 & 1.46 & 0 & 1 \\
\hline Bin $p_{\text {min }}$ & $G_{\text {lower }}$ & $H_{\text {lower }}$ & $\theta_{\text {upper }}$ & $\theta_{\text {lower }}$ & inneroffset & outeroff fet \\
\hline \hline 0.15 & 1. & 1 & 23 & 23 & \multicolumn{2}{|c|}{2} & 0 \\
\hline 0.30 & 0.5 & 0.1 & 12 & 17 & 1.8 & 1 \\
\hline 0.45 & 1.5 & 1. & 12 & 17.5 & 1.8 & 1 \\
\hline 0.60 & 0.15 & 0.1 & 12 & 18 & 1.4 & 0 \\
\hline 0.75 & 0 & 1 & 13 & 20 & 1.2 & 0 \\
\hline 0.90 & 1 & 1 & 13 & 19 & 1.2 & -1 \\
\hline 1.05 & 1 & 1 & 13 & 18 & 1.3 & 0 \\
\hline 1.20 & 0 & 1 & 13.5 & 17 & 1 & 0 \\
\hline 1.35 & 0. & -0.10 & 12.5 & 16 & 0.7 & -0.9 \\
\hline 1.50 & 0. & 1 & 12. & 15 & 0.7 & -0.9 \\
\hline 1.65 & 0. & 1 & 12 & 14 & 0.7 & -0.9 \\
\hline 1.80 & 0.5 & 1 & 13 & 13 & -1 & -0.9 \\
\hline 1.95 & 0.5 & 1 & 14 & 13 & -1.5 & -0.9 \\
\hline 2.10 & 0.2 & 1 & 15.5 & 13 & -1.5 & -0.9 \\
\hline 2.25 & 0 & 1 & 17 & 14 & -1.5 & -0.9 \\
\hline 2.40 & 0 & 1 & 18 & 15 & -1.5 & -0.9 \\
\hline 2.55 & 0 & 1 & 18 & 15 & -1.5 & -0.9 \\
\hline
\end{tabular}


Torus Current $=-1500 \mathrm{~A}$, Sector 4

\begin{tabular}{|c|c|c|c|c|c|c|c|c|}
\hline Bin $p_{\min }$ & $A$ & $B$ & $C$ & $D$ & $E$ & $F$ & $G_{\text {upper }}$ & $H_{\text {upper }}$ \\
\hline \hline 0.15 & 44 & 0.77 & 0.17 & 12 & 3 & 1.46 & 1 & 1 \\
\hline 0.30 & 40 & 0.55 & 0.29 & 4 & 3 & 1.46 & 0.5 & 1 \\
\hline 0.45 & 35 & 0.44 & 0.28 & 4.5 & 3 & 1.46 & 0.2 & -0.08 \\
\hline 0.60 & 33.5 & 0.35 & 0.23 & 4.9 & 3 & 1.46 & 1.7 & 1 \\
\hline 0.75 & 29 & 0.28 & 0.22 & 5.1 & 3 & 1.46 & 0.07 & -0.11 \\
\hline 0.90 & 31 & 0.28 & 0.22 & 5.1 & 3 & 1.46 & 2 & 1 \\
\hline 1.05 & 31 & 0.28 & 0.22 & 5.3 & 3 & 1.46 & 0.5 & 1 \\
\hline 1.20 & 32 & 0.28 & 0.22 & 6.2 & 3 & 1.46 & 0.4 & 1 \\
\hline 1.35 & 32 & 0.28 & 0.22 & 6 & 3 & 1.46 & 0.25 & 0 \\
\hline 1.50 & 32 & 0.28 & 0.22 & 5.8 & 3 & 1.46 & 0.2 & -0.02 \\
\hline 1.65 & 31 & 0.28 & 0.22 & 5.8 & 3 & 1.46 & 0.22 & -0.04 \\
\hline 1.80 & 31 & 0.28 & 0.22 & 6.3 & 3 & 1.46 & 0.22 & -0.06 \\
\hline 1.95 & 27.5 & 0.28 & 0.22 & 6.5 & 3 & 1.46 & 0.22 & -0.06 \\
\hline 2.10 & 29.5 & 0.34 & 0.22 & 7 & 3 & 1.46 & 0.22 & -0.06 \\
\hline 2.25 & 34 & 0.43 & 0.22 & 7.5 & 3 & 1.46 & 0.22 & -0.06 \\
\hline 2.40 & 38.5 & 0.5 & 0.22 & 7.9 & 3 & 1.46 & 0.22 & -0.06 \\
\hline 2.55 & 38.5 & 0.5 & 0.22 & 8.3 & 3 & 1.46 & 0.22 & -0.06 \\
\hline Bin $p_{\text {min }}$ & $G_{\text {lower }}$ & $H_{\text {lower }}$ & $\theta_{\text {upper }}$ & $\theta_{\text {lower }}$ & inneroffset & outeroffset \\
\hline \hline 0.15 & 1. & 1 & 23 & 23 & \multicolumn{2}{|c|}{2} & 0 \\
\hline 0.30 & 0.5 & 1 & 10.5 & 16.5 & 2.5 & -0.5 \\
\hline 0.45 & 0.08 & -0.12 & 12 & 19 & 1.8 & 0 \\
\hline 0.60 & 0.03 & -0.11 & 11 & 17 & 1.8 & -0.5 \\
\hline 0.75 & 0.07 & -0.11 & 15 & 20 & 1.2 & 0 \\
\hline 0.90 & 0.2 & -0.08 & 15 & 19 & 1.2 & 0 \\
\hline 1.05 & 0.25 & -0.06 & 14 & 18 & 1.2 & -0.40 \\
\hline 1.20 & 0.1 & -0.11 & 13 & 16 & 1.0 & -0.4 \\
\hline 1.35 & 0.35 & 0 & 13 & 16 & \multicolumn{2}{|c|}{1.0} & -0.4 \\
\hline 1.50 & 0.25 & -0.02 & 11.5 & 14.5 & 1.0 & -0.6 \\
\hline 1.65 & 0.1 & 0 & 12 & 14 & 1.0 & -0.6 \\
\hline 1.80 & 0.1 & 0 & 12 & 15 & 1.0 & -0.6 \\
\hline 1.95 & 0.1 & 0. & 13 & 15 & 1.0 & -0.4 \\
\hline 2.10 & 0.1 & 0 & 14 & 16 & 0.6 & -0.4 \\
\hline 2.25 & 0.1 & 0 & 15 & 17 & 0.6 & -0.4 \\
\hline 2.40 & 0.1 & 0 & 15 & 17 & 0.6 & -0.4 \\
\hline 2.55 & 0.1 & 0 & 15 & 17 & 0.6 & -0.4 \\
\hline
\end{tabular}


Torus Current $=-1500 \mathrm{~A}$, Sector 5

\begin{tabular}{|c|c|c|c|c|c|c|c|c|}
\hline Bin $p_{\min }$ & $A$ & $B$ & $C$ & $D$ & $E$ & $F$ & $G_{\text {upper }}$ & $H_{\text {upper }}$ \\
\hline \hline 0.15 & 44 & 0.77 & 0.17 & 12 & 3 & 1.46 & 1 & 1 \\
\hline 0.30 & 39 & 0.55 & 0.29 & 4 & 3 & 1.46 & 0 & 0 \\
\hline 0.45 & 34 & 0.38 & 0.22 & 4.6 & 3 & 1.46 & 0 & 0 \\
\hline 0.60 & 32 & 0.34 & 0.22 & 5 & 3 & 1.46 & 0 & 0 \\
\hline 0.75 & 30 & 0.28 & 0.22 & 5 & 3 & 1.46 & 0 & 0 \\
\hline 0.90 & 29.5 & 0.28 & 0.22 & 5.3 & 3 & 1.46 & 0.1 & -0.1 \\
\hline 1.05 & 29.5 & 0.28 & 0.22 & 5.5 & 3 & 1.46 & 0.1 & -0.1 \\
\hline 1.20 & 30.5 & 0.28 & 0.22 & 5.7 & 3 & 1.46 & 0.1 & -0.1 \\
\hline 1.35 & 31 & 0.28 & 0.22 & 5.7 & 3 & 1.46 & 0.09 & -0.1 \\
\hline 1.50 & 31 & 0.28 & 0.22 & 6. & 3 & 1.46 & 0.09 & -0.1 \\
\hline 1.65 & 32 & 0.28 & 0.22 & 6. & 3 & 1.46 & 0.1 & -0.09 \\
\hline 1.80 & 29 & 0.28 & 0.22 & 6.5 & 3 & 1.46 & 0.18 & -0.08 \\
\hline 1.95 & 27.5 & 0.28 & 0.22 & 6.9 & 3 & 1.46 & 0.19 & -0.06 \\
\hline 2.10 & 29.5 & 0.34 & 0.22 & 7.6 & 3 & 1.46 & 0.19 & -0.06 \\
\hline 2.25 & 34 & 0.43 & 0.22 & 7.8 & 3 & 1.46 & 0.19 & -0.06 \\
\hline 2.40 & 38.5 & 0.5 & 0.22 & 8.3 & 3 & 1.46 & 0.19 & -0.06 \\
\hline 2.55 & 38.5 & 0.5 & 0.22 & 8.7 & 3 & 1.46 & 0.19 & -0.06 \\
\hline Bin $p_{\text {min }}$ & $G_{\text {lower }}$ & $H_{\text {lower }}$ & $\theta_{\text {upper }}$ & $\theta_{\text {lower }}$ & inneroff set & outeroffset \\
\hline \hline 0.15 & 1. & 1 & 23 & 23 & \multicolumn{2}{|c|}{2} & 0 \\
\hline 0.30 & 0 & 0 & 11 & 18 & 2.7 & 1 \\
\hline 0.45 & 0 & 0 & 13 & 19 & 2.5 & 2.5 \\
\hline 0.60 & 0 & 0 & 14 & 19.5 & 2.1 & 0.8 \\
\hline 0.75 & 0 & 0 & 14.5 & 19.5 & 1.8 & 0.8 \\
\hline 0.90 & 0.05 & -0.1 & 15 & 19 & 1.5 & 0 \\
\hline 1.05 & 0.05 & -0.1 & 14 & 18 & 1.2 & 0 \\
\hline 1.20 & 0.15 & -0.1 & 13.5 & 17 & 1.2 & 0 \\
\hline 1.35 & 0.15 & -0.09 & 13 & 16 & 1.2 & 0 \\
\hline 1.50 & 0.15 & -0.09 & 12.5 & 15 & 1.2 & 0 \\
\hline 1.65 & 0.13 & -0.09 & 13 & 16 & 1. & 0 \\
\hline 1.80 & 0.18 & -0.08 & 15 & 13 & -0.5 & 0 \\
\hline 1.95 & 0.22 & -0.04 & 18 & 14 & -0.5 & 0 \\
\hline 2.10 & 0.22 & -0.04 & 20 & 18 & -0.5 & 0 \\
\hline 2.25 & 0.22 & -0.04 & 20 & 18 & -0.5 & 0 \\
\hline 2.40 & 0.22 & -0.04 & 20 & 18 & -0.5 & 0 \\
\hline 2.55 & 0.22 & -0.04 & 20 & 18 & -0.5 & 0 \\
\hline
\end{tabular}


Torus Current $=-1500 \mathrm{~A}$, Sector 6

\begin{tabular}{|c|c|c|c|c|c|c|c|c|}
\hline Bin $p_{\min }$ & $A$ & $B$ & $C$ & $D$ & $E$ & $F$ & $G_{\text {upper }}$ & $H_{\text {upper }}$ \\
\hline \hline 0.15 & 44 & 0.77 & 0.17 & 12 & 3 & 1.46 & 1 & 1 \\
\hline 0.30 & 39.5 & 0.55 & 0.27 & 5 & 3 & 1.46 & 0. & 0 \\
\hline 0.45 & 36 & 0.40 & 0.22 & 5 & 3 & 1.46 & 0.17 & 0 \\
\hline 0.60 & 32 & 0.33 & 0.22 & 5.3 & 3 & 1.46 & 0.2 & 0 \\
\hline 0.75 & 30 & 0.28 & 0.22 & 5.7 & 3 & 1.46 & 0. & 0 \\
\hline 0.90 & 31.5 & 0.28 & 0.22 & 5.7 & 3 & 1.46 & 0 & 0 \\
\hline 1.05 & 31.5 & 0.28 & 0.22 & 5.7 & 3 & 1.46 & 0.3 & 1 \\
\hline 1.20 & 33 & 0.28 & 0.22 & 6.1 & 3 & 1.46 & 0.2 & 0. \\
\hline 1.35 & 33 & 0.28 & 0.22 & 6.1 & 3 & 1.46 & 0.2 & -0.06 \\
\hline 1.50 & 33 & 0.28 & 0.22 & 6.5 & 3 & 1.46 & 0.3 & -0.06 \\
\hline 1.65 & 33 & 0.28 & 0.22 & 6.5 & 3 & 1.46 & 0.3 & -0.03 \\
\hline 1.80 & 31. & 0.28 & 0.22 & 6.9 & 3 & 1.46 & 0.22 & -0.08 \\
\hline 1.95 & 29 & 0.28 & 0.22 & 7.3 & 3 & 1.46 & 0.22 & -0.06 \\
\hline 2.10 & 32 & 0.36 & 0.22 & 7.9 & 3 & 1.46 & 0.22 & -0.06 \\
\hline 2.25 & 36 & 0.43 & 0.22 & 8.3 & 3 & 1.46 & 0.22 & -0.06 \\
\hline 2.40 & 38 & 0.47 & 0.22 & 8.6 & 3 & 1.46 & 0.22 & -0.06 \\
\hline 2.55 & 38 & 0.47 & 0.22 & 8.9 & 3 & 1.46 & 0.22 & -0.06 \\
\hline Bin $p_{\text {min }}$ & $G_{\text {lower }}$ & $H_{\text {lower }}$ & $\theta_{\text {upper }}$ & $\theta_{\text {lower }}$ & inneroff fet & outeroff set \\
\hline \hline 0.15 & 1. & 1 & 23 & 23 & \multicolumn{2}{|c|}{2} & 1.5 \\
\hline 0.30 & 0. & 0 & 13 & 20 & 3.8 & 2 \\
\hline 0.45 & 0.17 & 0 & 13 & 20 & 3.4 & 0.5 \\
\hline 0.60 & 0.1 & 0 & 14 & 21 & 2.8 & 0.2 \\
\hline 0.75 & 0. & 0 & 14 & 20 & 2.4 & 0.2 \\
\hline 0.90 & 0 & 0 & 15 & 19 & 2.2 & 0 \\
\hline 1.05 & 0.3 & 1 & 14 & 18 & 2.2 & 1.9 \\
\hline 1.20 & 0.2 & 0 & 13.5 & 17 & 2.2 & 1.9 \\
\hline 1.35 & 0.2 & -0.06 & 13 & 16 & 2.2 & 1. \\
\hline 1.50 & 0.2 & 0 & 12.5 & 15 & 2.3 & 0.3 \\
\hline 1.65 & 0.3 & -0.03 & 12 & 14 & 1.2 & 0.6 \\
\hline 1.80 & 0.20 & -0.08 & 14 & 13 & 0.4 & 0.6 \\
\hline 1.95 & 0.22 & -0.07 & 18 & 14 & 0. & 0.6 \\
\hline 2.10 & 0.22 & -0.04 & 19 & 15 & 0 & 0.6 \\
\hline 2.25 & 0.22 & -0.04 & 19 & 15 & 0 & 0.6 \\
\hline 2.40 & 0.22 & -0.04 & 19 & 15 & \multicolumn{2}{|c|}{0} & 0.6 \\
\hline 2.55 & 0.22 & -0.04 & 19 & 15 & 0 & 0.6 \\
\hline
\end{tabular}


Torus Current $=-2250$ A, Sector $1, p<2.55 \mathrm{GeV}$

\begin{tabular}{|c|c|c|c|c|c|c|c|c|}
\hline Bin $p_{\min }$ & $A$ & $B$ & $C$ & $D$ & $E$ & $F$ & $G_{\text {upper }}$ & $H_{\text {upper }}$ \\
\hline \hline 0.15 & 24 & 0.28 & 0.30 & 5 & 3 & 1.46 & 0 & 0 \\
\hline 0.30 & 24 & 0.28 & 0.30 & 5 & 3 & 1.46 & 0 & 0 \\
\hline 0.45 & 35 & 0.55 & 0.30 & 5 & 3 & 1.46 & 0 & 0 \\
\hline 0.60 & 36.5 & 0.44 & 0.22 & 5.5 & 3 & 1.46 & 0 & 0 \\
\hline 0.75 & 31 & 0.33 & 0.22 & 5.5 & 3 & 1.46 & 0.5 & 0.1 \\
\hline 0.90 & 28 & 0.28 & 0.22 & 5.5 & 3 & 1.46 & 0.5 & 0.5 \\
\hline 1.05 & 29 & 0.28 & 0.22 & 5.5 & 3 & 1.46 & 0.4 & 1 \\
\hline 1.20 & 29 & 0.28 & 0.22 & 5.8 & 3 & 1.46 & 0.03 & -0.135 \\
\hline 1.35 & 29 & 0.28 & 0.22 & 6 & 3 & 1.46 & 0.08 & -0.13 \\
\hline 1.50 & 31 & 0.28 & 0.22 & 6 & 3 & 1.46 & 0.11 & -0.12 \\
\hline 1.65 & 31 & 0.28 & 0.22 & 6 & 3 & 1.46 & 0.33 & -0.02 \\
\hline 1.80 & 31 & 0.28 & 0.22 & 6.3 & 3 & 1.46 & 0.22 & -0.04 \\
\hline 1.95 & 31 & 0.28 & 0.22 & 6.3 & 3 & 1.46 & 0.22 & -0.06 \\
\hline 2.10 & 31 & 0.28 & 0.22 & 6.5 & 3 & 1.46 & 0.22 & -0.06 \\
\hline 2.25 & 31 & 0.28 & 0.22 & 6.5 & 3 & 1.46 & 0.22 & -0.06 \\
\hline 2.40 & 31 & 0.28 & 0.22 & 6.7 & 3 & 1.46 & 0.22 & -0.06 \\
\hline Bin $p_{\min }$ & $G_{\text {lower }}$ & $H_{\text {lower }}$ & $\theta_{\text {upper }}$ & $\theta_{\text {lower }}$ & inneroffset & outeroffset \\
\hline 0.15 & 1.0 & 0.1 & 25 & 25 & \multicolumn{2}{|c|}{1.8} & 1 \\
\hline 0.30 & 1.0 & 0.1 & 24 & 17 & 1.8 & 1 \\
\hline 0.45 & 1.0 & 0.1 & 13 & 18 & 1.8 & 1 \\
\hline 0.60 & 0.7 & 0. & 13 & 19 & 2.5 & 2.5 \\
\hline 0.75 & 1.3 & 0.5 & 14 & 19 & 1.2 & 0.8 \\
\hline 0.90 & 0.6 & 0.5 & 15 & 20 & 1.2 & 0.8 \\
\hline 1.05 & 0.4 & 1 & 15 & 19 & 1.2 & 0 \\
\hline 1.20 & 0.025 & -0.135 & 14 & 18 & 1.2 & 0 \\
\hline 1.35 & 0.07 & -0.13 & 13.5 & 17 & 1.2 & 0 \\
\hline 1.50 & 0.15 & -0.09 & 13 & 16 & 1.2 & 0 \\
\hline 1.65 & 0.30 & -0.01 & 12.5 & 15 & 1.3 & 0 \\
\hline 1.80 & 0.22 & -0.04 & 12 & 14 & 1.2 & 0 \\
\hline 1.95 & 0.22 & -0.04 & 12 & 13 & 1.2 & 0 \\
\hline 2.10 & 0.22 & -0.04 & 11.5 & 13 & 1.2 & 0 \\
\hline 2.25 & 0.22 & -0.04 & 11 & 13 & 1.2 & 0 \\
\hline 2.40 & 0.22 & -0.04 & 11.5 & 13 & 1.2 & 0 \\
\hline
\end{tabular}


Torus Current $=-2250 \mathrm{~A}$, Sector $1, \mathrm{p}>2.55 \mathrm{GeV}$

\begin{tabular}{|c|c|c|c|c|c|c|c|c|c|}
\hline Bir $p_{\min }$ & $A$ & $B$ & $C$ & $D$ & $E$ & $F$ & $G_{\text {upper }}$ & $H_{\text {upper }}$ \\
\hline \hline 2.55 & 31 & 0.28 & 0.22 & 6.8 & 3 & 1.46 & 0.22 & -0.06 \\
\hline 2.70 & 29.5 & 0.28 & 0.22 & 6.9 & 3 & 1.46 & 0.22 & -0.06 \\
\hline 2.85 & 28 & 0.28 & 0.22 & 7 & 3 & 1.46 & 0.22 & -0.04 \\
\hline 3.00 & 53 & 0.6 & 0.22 & 7.3 & 3 & 1.46 & 0.22 & -0.04 \\
\hline 3.15 & 53 & 0.60 & 0.22 & 7.3 & 3 & 1.46 & 0.22 & -0.04 \\
\hline 3.30 & 53 & 0.65 & 0.22 & 7.7 & 3 & 1.46 & 0.18 & -0.04 \\
\hline 3.45 & 53 & 0.63 & 0.22 & 8 & 3 & 1.46 & 0.18 & -0.04 \\
\hline 3.60 & 53 & 0.66 & 0.22 & 8.3 & 3 & 1.46 & 0.3 & 0.1 \\
\hline 3.75 & 53 & 0.66 & 0.22 & 8.5 & 3 & 1.46 & 0.3 & 0.1 \\
\hline 3.90 & 53 & 0.69 & 0.22 & 8.8 & 3 & 1.46 & 0.25 & 0.1 \\
\hline 4.05 & 53 & 0.73 & 0.22 & 9 & 3 & 1.46 & 0.25 & 0.1 \\
\hline 4.20 & 53 & 0.73 & 0.22 & 9.2 & 3 & 1.46 & 0.25 & 0.1 \\
\hline Bin $p_{\min }$ & $G_{\text {lower }}$ & $H_{\text {lower }}$ & $\theta_{\text {upper }}$ & $\theta_{\text {lower }}$ & inneroff fset & outeroffset \\
\hline \hline 2.55 & 0.22 & -0.04 & 12 & 13 & \multicolumn{2}{|c|}{0.8} & 0 \\
\hline 2.70 & 0.22 & -0.04 & 13.5 & 13 & -0.5 & 0 \\
\hline 2.85 & 0.22 & -0.04 & 14.5 & 13 & -0.5 & 0 \\
\hline 3.00 & 0.22 & -0.04 & 13.5 & 13 & -0.3 & 0 \\
\hline 3.15 & 0.22 & -0.04 & 13.5 & 13 & -0.3 & 0 \\
\hline 3.30 & 0.18 & -0.04 & 14 & 14 & -0.6 & 0 \\
\hline 3.45 & 0.18 & -0.04 & 14.5 & 14 & -0.6 & 0 \\
\hline 3.60 & 0.3 & 0.1 & 15 & 15 & -0.6 & 0 \\
\hline 3.75 & 0.3 & 0.1 & 15 & 15 & -0.6 & 0 \\
\hline 3.90 & 0.25 & 0.1 & 15 & 15 & -0.6 & -0.1 \\
\hline 4.05 & 0.25 & 0.1 & 15 & 15 & -0.6 & -0.1 \\
\hline 4.20 & 0.25 & 0.1 & 15 & 15 & -0.6 & 0 \\
\hline
\end{tabular}


Torus Current $=-2250 \mathrm{~A}$, Sector $2, \mathrm{p}<2.55 \mathrm{GeV}$

\begin{tabular}{|c|c|c|c|c|c|c|c|c|}
\hline Bin $p_{\min }$ & $A$ & $B$ & $C$ & $D$ & $E$ & $F$ & $G_{\text {upper }}$ & $H_{\text {upper }}$ \\
\hline \hline 0.15 & 25 & 0.28 & 0.30 & 5 & 3 & 1.46 & 0.05 & -0.13 \\
\hline 0.30 & 25 & 0.28 & 0.30 & 5 & 3 & 1.46 & 0.05 & -0.13 \\
\hline 0.45 & 37 & 0.55 & 0.30 & 5 & 3 & 1.46 & 0.05 & -0.13 \\
\hline 0.60 & 38 & 0.44 & 0.22 & 5.5 & 3 & 1.46 & 0.025 & -0.13 \\
\hline 0.75 & 30.5 & 0.33 & 0.22 & 5.5 & 3 & 1.46 & 0.08 & -0.12 \\
\hline 0.90 & 28.5 & 0.28 & 0.22 & 5.5 & 3 & 1.46 & 0.2 & 1 \\
\hline 1.05 & 28.5 & 0.28 & 0.22 & 5.5 & 3 & 1.46 & 0.6 & 2 \\
\hline 1.20 & 29.5 & 0.28 & 0.22 & 5.5 & 3 & 1.46 & 0.6 & 2 \\
\hline 1.35 & 29.5 & 0.28 & 0.22 & 5.8 & 3 & 1.46 & 0.2 & -0.05 \\
\hline 1.50 & 29.5 & 0.28 & 0.22 & 5.8 & 3 & 1.46 & 0.2 & -0.05 \\
\hline 1.65 & 30.5 & 0.28 & 0.22 & 5.8 & 3 & 1.46 & 0.2 & 0.1 \\
\hline 1.80 & 30.5 & 0.28 & 0.22 & 5.8 & 3 & 1.46 & 0.2 & 0.1 \\
\hline 1.95 & 30.5 & 0.28 & 0.22 & 5.8 & 3 & 1.46 & 0.2 & 0.1 \\
\hline 2.10 & 32.5 & 0.28 & 0.22 & 6 & 3 & 1.46 & 0 & 0.1 \\
\hline 2.25 & 32.5 & 0.28 & 0.22 & 6.3 & 3 & 1.46 & 0 & 0.1 \\
\hline 2.40 & 32.5 & 0.28 & 0.22 & 6.5 & 3 & 1.46 & 0 & 0.1 \\
\hline Bin $p_{\min }$ & $G_{\text {lower }}$ & $H_{\text {lower }}$ & $\theta_{\text {upper }}$ & $\theta_{\text {lower }}$ & inneroffset & outeroffset \\
\hline \hline 0.15 & 0.04 & -0.13 & 25 & 25 & \multicolumn{2}{|c|}{0.5} & 0 \\
\hline 0.30 & 0.04 & -0.13 & 24 & 24 & 0.5 & 0 \\
\hline 0.45 & 0.04 & -0.13 & 12 & 17 & 1.5 & 0 \\
\hline 0.60 & 0.04 & -0.13 & 13 & 16 & 1.6 & 0 \\
\hline 0.75 & 0.14 & -0.11 & 14 & 19 & 1.2 & 0.8 \\
\hline 0.90 & 0.35 & 0 & 15 & 20 & 1.2 & 0.8 \\
\hline 1.05 & 0.6 & 2 & 15 & 19 & 1.2 & 0 \\
\hline 1.20 & 0.6 & 2 & 14 & 18 & 0.5 & 0 \\
\hline 1.35 & 0.2 & -0.05 & 13.5 & 17 & 0.8 & 0 \\
\hline 1.50 & 0.2 & -0.05 & 13 & 16 & 1.1 & 0.6 \\
\hline 1.65 & 0.2 & 0.1 & 12.5 & 15 & 1.1 & 0 \\
\hline 1.80 & 0.2 & 0.1 & 12 & 14 & 1.1 & 0 \\
\hline 1.95 & 0.2 & 0.1 & 12 & 13 & 1.1 & 0 \\
\hline 2.10 & 1.2 & 1 & 11.5 & 13 & 1.1 & 0 \\
\hline 2.25 & 0 & 1 & 11 & 13 & 1.1 & 0 \\
\hline 2.40 & 0 & 1 & 11 & 13 & 1.1 & 0 \\
\hline
\end{tabular}


Torus Current $=-2250 \mathrm{~A}$, Sector $2, \mathrm{p}>2.55 \mathrm{GeV}$

\begin{tabular}{|}
\begin{tabular}{|c|c|c|c|c|c|c|c|c|}
\hline Bin $p_{\min }$ & $A$ & $B$ & $C$ & $D$ & $E$ & $F$ & $G_{\text {upper }}$ & $H_{\text {upper }}$ \\
\hline \hline 2.55 & 33 & 0.28 & 0.22 & 7 & 3 & 1.46 & 0 & 0.1 \\
\hline 2.70 & 31 & 0.28 & 0.22 & 7.3 & 3 & 1.46 & 0 & 0.1 \\
\hline 2.85 & 31 & 0.28 & 0.22 & 7.8 & 3 & 1.46 & 0.005 & -0.152 \\
\hline 3.00 & 29 & 0.28 & 0.22 & 8.2 & 3 & 1.46 & 0.005 & -0.152 \\
\hline 3.15 & 27 & 0.28 & 0.22 & 8.6 & 3 & 1.46 & 0.005 & -0.16 \\
\hline 3.30 & 26 & 0.28 & 0.22 & 9 & 3 & 1.46 & 0 & -0.16 \\
\hline 3.45 & 45 & 0.53 & 0.22 & 9 & 3 & 1.46 & 0 & -0.16 \\
\hline 3.60 & 45 & 0.56 & 0.22 & 9.3 & 3 & 1.46 & 0.005 & -0.16 \\
\hline 3.75 & 45 & 0.56 & 0.22 & 9.7 & 3 & 1.46 & 0.005 & -0.16 \\
\hline 3.90 & 45 & 0.56 & 0.22 & 10.1 & 3 & 1.46 & 0 & -0.16 \\
\hline 4.05 & 45 & 0.58 & 0.22 & 10.1 & 3 & 1.46 & 0 & -0.16 \\
\hline 4.20 & 45 & 0.58 & 0.22 & 10.1 & 3 & 1.46 & 0 & -0.16 \\
\hline Bin $p_{\min }$ & $G_{\text {lower }}$ & $H_{\text {lower }}$ & $\theta_{\text {upper }}$ & $\theta_{\text {lower }}$ & inneroffset & outeroffset \\
\hline \hline 2.55 & 0 & 1 & 11 & 12 & \multicolumn{2}{|c|}{0.6} & 0 \\
\hline 2.70 & 0 & 1 & 13 & 12.5 & \multicolumn{2}{|c|}{0} & 0 \\
\hline 2.85 & 0.005 & -0.152 & 14 & 12.5 & -0.5 & 0 \\
\hline 3.00 & 0.005 & -0.152 & 13.5 & 13 & -0.5 & 0 \\
\hline 3.15 & 0.005 & -0.16 & 13.5 & 13 & \multicolumn{2}{|c|}{-0.5} & 0 \\
\hline 3.30 & 0 & -0.16 & 14 & 14 & -0.5 & 0 \\
\hline 3.45 & 0 & -0.16 & 14.5 & 14 & -0.5 & 0 \\
\hline 3.60 & 0.005 & -0.16 & 15 & 15 & -0.5 & 0 \\
\hline 3.75 & 0.005 & -0.16 & 15 & 15 & -0.5 & 0 \\
\hline 3.90 & 0 & -0.16 & 15 & 15 & -0.5 & 0 \\
\hline 4.05 & 0 & -0.16 & 15 & 15 & -0.5 & 0 \\
\hline 4.20 & 0 & -0.16 & 15 & 15 & -0.5 & 0 \\
\hline
\end{tabular}
\end{tabular}


Torus Current $=-2250 \mathrm{~A}$, Sector $3, \mathrm{p}<2.55 \mathrm{GeV}$

\begin{tabular}{|c|c|c|c|c|c|c|c|c|}
\hline Birl $p_{\min }$ & iin & $B$ & $C$ & $D$ & $E$ & $F$ & $G_{\text {upper }}$ & $H_{\text {upper }}$ \\
\hline $\begin{array}{c}.15 \\
0.30\end{array}$ & 24 & 0.28 & 0.30 & 5 & $\overline{3}$ & 1.46 & 0 & 0 \\
\hline 0.30 & 24 & 0.28 & 0.30 & 5 & 3 & 1.46 & 0 & 0 \\
\hline 0.45 & 37 & 0.55 & 0.30 & 5 & 3 & 1.46 & 0 & 0 \\
\hline 0.60 & 38 & 0.44 & 0.22 & 5 & 3 & 1.46 & 1 & 1 \\
\hline 0.75 & 34 & 0.36 & 0.22 & 5.5 & 3 & 1.46 & 0.65 & 0.1 \\
\hline 0.90 & 29 & 0.28 & 0.22 & 5.5 & 3 & 1.46 & 0 & 0.1 \\
\hline 1.05 & 29 & 0.28 & 0.22 & 5.5 & 3 & 1.46 & 0 & 0.1 \\
\hline 1.20 & 29 & 0.28 & 0.22 & 5.5 & 3 & 1.46 & 0 & 0.1 \\
\hline 1.35 & 29. & 0.28 & 0.22 & 5.5 & 3 & 1.46 & 1 & 1 \\
\hline 1.50 & 29. & 0.28 & 0.22 & 5.5 & 3 & 1.46 & 1 & 1 \\
\hline 1.65 & 29. & 0.28 & 0.22 & 5.5 & 3 & 1.46 & 1 & 1 \\
\hline 1.80 & 30. & 0.28 & 0.22 & 5.7 & 3 & 1.46 & 0.3 & 1 \\
\hline 1.95 & 30. & 0.28 & 0.22 & 6 & 3 & 1.46 & 0 & 1 \\
\hline 2.10 & 32 & 0.28 & 0.22 & 6 & 3 & 1.46 & 0 & 1 \\
\hline \multirow{2}{*}{$\begin{array}{l}2.25 \\
2.40\end{array}$} & 33 & 0.28 & 0.22 & 6.3 & 3 & 1.46 & 0 & 1 \\
\hline & \begin{tabular}{l|l}
33. \\
\end{tabular} & 0.28 & 0.22 & 6.3 & 3 & 1.46 & 0 & 1 \\
\hline \multirow{2}{*}{$\frac{n p_{\text {min }}}{0.15}$} & \begin{tabular}{|l|}
$G_{\text {lower }}$ \\
\end{tabular} & $\mathrm{H}_{\text {lower }}$ & $\theta_{\text {upper }}$ & \multicolumn{2}{|c|}{$\theta_{\text {lower }}$} & \multicolumn{2}{|c|}{ inneroffset } & outeroff $f$ set \\
\hline & 1.0 & 0.1 & 25 & \multicolumn{2}{|c|}{25} & \multicolumn{2}{|c|}{1.8} & 0 \\
\hline 0.30 & 1.0 & 0.1 & 24 & \multicolumn{2}{|c|}{24} & \multicolumn{2}{|c|}{1.8} & 0 \\
\hline 0.45 & 1.0 & 0.1 & 12 & \multicolumn{2}{|c|}{17} & \multicolumn{2}{|c|}{1.8} & 0 \\
\hline 0.60 & 1 & 1. & 11.5 & 17 & & & 8 & 1 \\
\hline 0.75 & 0.65 & 0.1 & 11 & 17 & & & 8 & 0 \\
\hline 0.90 & 2 & 1 & 15 & 20 & & & 2 & 0.8 \\
\hline 1.05 & 1.5 & 1 & 15 & 19 & & & 2 & 0.8 \\
\hline 1.20 & 1.5 & 1 & 14 & 18 & & & 3 & 0.8 \\
\hline 1.35 & 0.08 & -0.12 & 13.5 & 17 & & & 3 & 0 \\
\hline 1.50 & 0.06 & -0.10 & 13 & 16 & & & 3 & -0.9 \\
\hline 1.65 & 0.3 & 1 & 12.5 & 15 & & & 3 & -0.9 \\
\hline 1.80 & 0.3 & 1 & 12 & 14 & & & 3 & -0.9 \\
\hline 1.95 & 0 & 1 & 12 & 13 & & 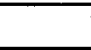 & 1 & -0.9 \\
\hline 2.10 & 0 & 1 & 11.5 & 13 & & 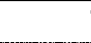 & 1 & -0.9 \\
\hline 2.25 & 0 & 1 & 11 & 13 & & . & 1 & -0.9 \\
\hline 2.40 & 0 & 1 & 11 & 13 & & 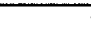 & & -0.9 \\
\hline
\end{tabular}


Torus Current $=-2250 \mathrm{~A}$, Sector $3, p>2.55 \mathrm{GeV}$

\begin{tabular}{|c|c|c|c|c|c|c|c|c|}
\hline $\operatorname{Bin} p_{\min }$ & $\bar{A}$ & $B$ & $C$ & $\bar{D}$ & $E$ & $\bar{F}$ & $G_{\text {upper }}$ & $H_{\text {upper }}$ \\
\hline 2.55 & 32.5 & 0.28 & 0.22 & 6.3 & 3 & 1.46 & 0 & 1 \\
\hline 2.70 & 31.5 & 0.28 & 0.22 & 6.3 & 3 & 1.46 & 0 & 7 \\
\hline 2.85 & 30 & 0.28 & 0.22 & 6.7 & 3 & 1.46 & 0 & 1 \\
\hline 3.00 & 28 & 0.28 & 0.22 & 6.7 & 3 & 1.46 & 0 & $T$ \\
\hline 3.15 & 26 & 0.28 & 0.22 & 7 & 3 & 1.46 & 0 & 1 \\
\hline 3.30 & 40 & 0.48 & 0.22 & 7.4 & 3 & 1.46 & 0 & 1 \\
\hline 3.45 & 37 & 0.48 & 0.22 & 7.7 & 3 & 1.46 & 0 & 1 \\
\hline 3.60 & 37 & 0.48 & 0.22 & 8 & 3 & 1.46 & 0 & 1 \\
\hline 3.75 & 42 & 0.55 & 0.22 & 8 & 3 & 1.46 & 0 & 1 \\
\hline 3.90 & 48 & 0.62 & 0.22 & 8.5 & 3 & 1.46 & 0 & 1 \\
\hline 4.05 & 48 & 0.62 & 0.22 & 8.5 & 3 & 1.46 & 0 & 1 \\
\hline 4.20 & 48 & 0.62 & 0.22 & 8.5 & 3 & 1.46 & 0 & 1 \\
\hline $\operatorname{Bin} p_{\min }$ & $G_{\text {lower }}$ & $H_{\text {lower }}$ & $\theta_{\text {upper }}$ & \multicolumn{2}{|c|}{ | $\theta_{\text {lower }}$} & \multicolumn{2}{|c|}{ inneroff set } & outerof f set \\
\hline 2.55 & 0 & 1 & 11 & 1 & & 0 & & -0.9 \\
\hline 2.70 & 0 & 1 & 13 & 12 & & & 5 & -0.9 \\
\hline 2.85 & 0 & 1 & 14 & 12 & & & .3 & -0.9 \\
\hline 3.00 & 0 & 1 & 13.5 & 1 & & & 6 & -0.9 \\
\hline 3.15 & 0 & 1 & 13.5 & 1 & & & 6 & -0.9 \\
\hline 3.30 & 0 & 1 & 14 & 1 & & & .2 & -0.9 \\
\hline 3.45 & 0 & 1 & 14.5 & 1 & & & .2 & -0.9 \\
\hline 3.60 & 0 & 1 & 15 & 1 & & & .2 & -0.9 \\
\hline 3.75 & 0 & 1 & 15 & 1 & & -1 & .2 & -0.9 \\
\hline 3.90 & 0 & 1 & 15 & 1 & & -1 & .2 & -0.9 \\
\hline 4.05 & 0 & 1 & 15 & 1 & & -1 & .2 & -0.9 \\
\hline 4.20 & 0 & 1 & 15 & 1 & & & .2 & -0.9 \\
\hline
\end{tabular}


Torus Current $=-2250$ A, Sector $4, p<2.55 \mathrm{GeV}$

\begin{tabular}{|c|c|c|c|c|c|c|c|c|}
\hline Bin $p_{\min }$ & $A$ & $B$ & $C$ & $D$ & $E$ & $F$ & $G_{\text {upper }}$ & $H_{\text {upper }}$ \\
\hline \hline 0.15 & 24 & 0.28 & 0.30 & 5 & 3 & 1.46 & 0 & 0 \\
\hline 0.30 & 24 & 0.28 & 0.30 & 5 & 3 & 1.46 & 0 & 0 \\
\hline 0.45 & 30 & 0.40 & 0.30 & 5 & 3 & 1.46 & 0 & 0 \\
\hline 0.60 & 33 & 0.44 & 0.30 & 5.3 & 3 & 1.46 & 0.2 & -0.08 \\
\hline 0.75 & 32 & 0.35 & 0.22 & 5.3 & 3 & 1.46 & 0.5 & 0 \\
\hline 0.90 & 29 & 0.28 & 0.22 & 5.3 & 3 & 1.46 & 0.6 & 0.5 \\
\hline 1.05 & 29 & 0.28 & 0.22 & 5.5 & 3 & 1.46 & 0.4 & 1 \\
\hline 1.20 & 29 & 0.28 & 0.22 & 5.5 & 3 & 1.46 & 0.03 & -0.13 \\
\hline 1.35 & 29 & 0.28 & 0.22 & 5.5 & 3 & 1.46 & 0.12 & -0.11 \\
\hline 1.50 & 30 & 0.28 & 0.22 & 5.5 & 3 & 1.46 & 0.3 & 0 \\
\hline 1.65 & 31 & 0.28 & 0.22 & 6 & 3 & 1.46 & 0.33 & -0.02 \\
\hline 1.80 & 31 & 0.28 & 0.22 & 6.3 & 3 & 1.46 & 0.22 & -0.04 \\
\hline 1.95 & 31 & 0.28 & 0.22 & 6.3 & 3 & 1.46 & 0.22 & -0.06 \\
\hline 2.10 & 31 & 0.28 & 0.22 & 6.3 & 3 & 1.46 & 0.22 & -0.06 \\
\hline 2.25 & 31 & 0.28 & 0.22 & 6.3 & 3 & 1.46 & 0.22 & -0.06 \\
\hline 2.40 & 31 & 0.28 & 0.22 & 6.3 & 3 & 1.46 & 0.22 & -0.06 \\
\hline Bin $p_{\text {min }}$ & $G_{\text {lower }}$ & $H_{\text {lower }}$ & $\theta_{\text {upper }}$ & $\theta_{\text {lower }}$ & inneroffset & outeroffset \\
\hline \hline .15 & 1.0 & 0.1 & 25 & 25 & 2.5 & 1 \\
\hline 0.30 & 1.0 & 0.1 & 24 & 24 & 2.5 & 1 \\
\hline 0.45 & 1.0 & 0.1 & 12.5 & 24 & 2.5 & 1 \\
\hline 0.60 & 0.08 & -0.12 & 12 & 18 & \multicolumn{2}{|c|}{1.8} & 0 \\
\hline 0.75 & 0.5 & 0 & 13 & 21 & 1.8 & 0 \\
\hline 0.90 & 0.6 & 0.4 & 15 & 20 & 1.2 & 0 \\
\hline 1.05 & 0.4 & 1 & 15 & 19 & 1.2 & 0 \\
\hline 1.20 & 0.025 & -0.13 & 14 & 18 & \multicolumn{2}{|c|}{1.2} & 0 \\
\hline 1.35 & 0.12 & -0.11 & 13.5 & 17 & 1.0 & -0.4 \\
\hline 1.50 & 0.45 & 0 & 13 & 16 & \multicolumn{2}{|c|}{1.0} & -0.4 \\
\hline 1.65 & 0.33 & -0.02 & 12.5 & 15 & 1.0 & -0.6 \\
\hline 1.80 & 0.22 & -0.04 & 12 & 14 & 1.0 & -0.6 \\
\hline 1.95 & 0.22 & -0.04 & 12 & 13 & 1.0 & -0.6 \\
\hline 2.10 & 0.22 & -0.04 & 11.5 & 13 & 1.0 & -0.6 \\
\hline 2.25 & 0.22 & -0.04 & 11 & 13 & 0.6 & -0.6 \\
\hline 2.40 & 0.22 & -0.04 & 11 & 13 & 0.6 & -0.6 \\
\hline
\end{tabular}


Torus Current $=-2250 \mathrm{~A}$, Sector $4, p>2.55 \mathrm{GeV}$

\begin{tabular}{|c|c|c|c|c|c|c|c|c|c|}
\hline \multicolumn{2}{|c|}{ Bin $p_{\text {min }}$} & $A$ & $B$ & $C$ & $D$ & $E$ & $F$ & $G_{\text {upper }}$ & $H_{\text {upper }}$ \\
\hline \multicolumn{2}{|c|}{2.55} & 31 & 0.28 & 0.22 & 6.3 & $\overline{3}$ & 1.46 & 0.22 & -0.06 \\
\hline \multicolumn{2}{|c|}{2.70} & 29.5 & 0.28 & 0.22 & 6.3 & 3 & 1.46 & 0.22 & -0.06 \\
\hline \multicolumn{2}{|c|}{2.85} & 28 & 0.28 & 0.22 & 6.3 & 3 & 1.46 & 0.22 & -0.06 \\
\hline \multicolumn{2}{|c|}{3.00} & 57 & 0.6 & 0.22 & 7 & 3 & 1.46 & 0.22 & -0.04 \\
\hline \multicolumn{2}{|c|}{3.15} & 57 & 0.60 & 0.22 & 7 & 3 & 1.46 & 0.22 & -0.04 \\
\hline \multicolumn{2}{|c|}{3.30} & 56 & 0.67 & 0.22 & 7.4 & 3 & 1.46 & 0.18 & -0.04 \\
\hline \multicolumn{2}{|c|}{3.45} & 54 & 0.63 & 0.22 & 7.8 & 3 & 1.46 & 0.18 & -0.04 \\
\hline \multicolumn{2}{|c|}{3.60} & 53 & 0.66 & 0.22 & 7.8 & 3 & 1.46 & 0.3 & 0.1 \\
\hline \multicolumn{2}{|c|}{3.75} & 53 & 0.66 & 0.22 & 8. & 3 & 1.46 & 0.3 & 0.1 \\
\hline \multicolumn{2}{|c|}{3.90} & 53 & 0.69 & 0.22 & 8.3 & 3 & 1.46 & 0.25 & 0.1 \\
\hline \multicolumn{2}{|c|}{4.05} & 53 & 0.73 & 0.22 & 8.5 & 3 & 1.46 & 0.25 & 0.1 \\
\hline \multicolumn{2}{|c|}{4.20} & 53 & 0.73 & 0.22 & 8.7 & 3 & 1.46 & 0.25 & 0.1 \\
\hline Bin $p_{\min }$ & \multicolumn{2}{|c|}{$G_{\text {lower }}$} & $H_{\text {lower }}$ & $\theta_{\text {upper }}$ & \multicolumn{2}{|c|}{$\theta_{\text {lower }}$} & \multicolumn{2}{|c|}{ innerof f set } & outeroff set \\
\hline \multirow{2}{*}{$\begin{array}{l}2.55 \\
2.70\end{array}$} & \multicolumn{2}{|c|}{0.22} & 1 & 11 & \multicolumn{2}{|c|}{12} & \multicolumn{2}{|c|}{0.6} & -0.6 \\
\hline & \multicolumn{2}{|c|}{0.22} & 1 & 13 & \multicolumn{2}{|c|}{12.5} & \multicolumn{2}{|c|}{0.3} & -0.6 \\
\hline 2.85 & 0.2 & & 1 & 14 & \multicolumn{2}{|c|}{12.5} & \multicolumn{2}{|c|}{0.3} & -0.6 \\
\hline \multicolumn{3}{|l|}{3.00} & -0.04 & 13.5 & 1 & & & & 0.5 \\
\hline 3.15 & \multicolumn{2}{|c|}{0.22} & -0.04 & 13.5 & 1 & & & & 0.5 \\
\hline 3.30 & 0.18 & & -0.04 & 14 & 1 & & & & 0.5 \\
\hline 3.45 & 0.18 & & -0.04 & 14.5 & 1 & & & & 0.5 \\
\hline 3.60 & 0.3 & & 0.1 & 15 & 1 & & & & 0.5 \\
\hline 3.75 & 0.3 & & 0.1 & 15 & 1 & & & .6 & 0.5 \\
\hline 3.90 & 0.2 & & 0.1 & 15 & 1 & & & 6 & 0.5 \\
\hline 4.05 & 0.2 & & 0.1 & 15 & 1 & & & .6 & 0.5 \\
\hline 4.20 & 0.2 & & 0.1 & 15 & 1 & & & 6 & 0.5 \\
\hline
\end{tabular}


Torus Current $=-2250 \mathrm{~A}$, Sector $5, p<2.55 \mathrm{GeV}$

\begin{tabular}{|c|c|c|c|c|c|c|c|c|}
\hline Bin $p_{\min }$ & $A$ & $B$ & $C$ & $D$ & $E$ & $F$ & $G_{\text {upper }}$ & $H_{\text {upper }}$ \\
\hline \hline 0.15 & 24 & 0.28 & 0.30 & 5 & 3 & 1.46 & 0 & 0 \\
\hline 0.30 & 24 & 0.28 & 0.30 & 5 & 3 & 1.46 & 0 & 0 \\
\hline 0.45 & 25 & 0.28 & 0.30 & 5 & 3 & 1.46 & 0 & 0 \\
\hline 0.60 & 34 & 0.38 & 0.22 & 5 & 3 & 1.46 & 0 & 0 \\
\hline 0.75 & 30 & 0.34 & 0.22 & 5 & 3 & 1.46 & 0 & 0 \\
\hline 0.90 & 28.5 & 0.28 & 0.22 & 5 & 3 & 1.46 & 0 & 0 \\
\hline 1.05 & 28 & 0.28 & 0.22 & 5.3 & 3 & 1.46 & 0 & 0 \\
\hline 1.20 & 29 & 0.28 & 0.22 & 5.5 & 3 & 1.46 & 0.02 & -0.1 \\
\hline 1.35 & 29 & 0.28 & 0.22 & 5.7 & 3 & 1.46 & 0.25 & -0.06 \\
\hline 1.50 & 31 & 0.28 & 0.22 & 5.7 & 3 & 1.46 & 0.09 & -0.1 \\
\hline 1.65 & 31 & 0.28 & 0.22 & 5.7 & 3 & 1.46 & 0.2 & 0 \\
\hline 1.80 & 31 & 0.28 & 0.22 & 5.9 & 3 & 1.46 & 0.03 & -0.13 \\
\hline 1.95 & 31 & 0.28 & 0.22 & 6.0 & 3 & 1.46 & 0.22 & -0.08 \\
\hline 2.10 & 31 & 0.28 & 0.22 & 6.2 & 3 & 1.46 & 0.22 & -0.06 \\
\hline 2.25 & 31 & 0.28 & 0.22 & 6.3 & 3 & 1.46 & 0.22 & -0.06 \\
\hline 2.40 & 31 & 0.28 & 0.22 & 6.3 & 3 & 1.46 & 0.22 & -0.06 \\
\hline Bin $p_{\min }$ & $G_{\text {lower }}$ & $H_{\text {lower }}$ & $\theta_{\text {upper }}$ & $\theta_{\text {lower }}$ & inneroffset & outeroffset \\
\hline 0.15 & 0 & 0 & 25 & 25 & \multicolumn{2}{|c|}{1.8} & 1 \\
\hline 0.30 & 0 & 0 & 24 & 24 & 1.8 & 1 \\
\hline 0.45 & 0 & 0 & 14 & 24 & 1.2 & 1 \\
\hline 0.60 & 0 & 0 & 12 & 22 & 2 & 2.5 \\
\hline 0.75 & 0 & 0 & 15 & 21 & 1.8 & 0.8 \\
\hline 0.90 & 0 & 0 & 15 & 20 & 1.4 & 0.8 \\
\hline 1.05 & 0 & 0 & 15 & 19 & 1.2 & 0 \\
\hline 1.20 & 0.015 & -0.1 & 14 & 18 & 1.2 & 0 \\
\hline 1.35 & 0.15 & 0 & 13.5 & 17 & 1.2 & 0 \\
\hline 1.50 & 0.08 & -0.09 & 13 & 16 & 1.2 & 0 \\
\hline 1.65 & 0.2 & 0 & 12.5 & 15 & 1.3 & 0 \\
\hline 1.80 & 0.03 & -0.13 & 12 & 14 & 1.2 & 0 \\
\hline 1.95 & 0.20 & -0.08 & 12 & 13 & 1.2 & 0 \\
\hline 2.10 & 0.22 & -0.04 & 11.5 & 13 & 1.2 & 0 \\
\hline 2.25 & 0.22 & -0.04 & 11 & 13 & 1.2 & 0 \\
\hline 2.40 & 0.1 & -0.12 & 11 & 13 & 1 & 0 \\
\hline
\end{tabular}


Torus Current $=-2250 \mathrm{~A}$, Sector $5, \mathrm{p}>2.55 \mathrm{GeV}$

\begin{tabular}{|}
\begin{tabular}{|c|c|c|c|c|c|c|c|c|}
\hline Bin $p_{\min }$ & $A$ & $B$ & $C$ & $D$ & $E$ & $F$ & $G_{\text {upper }}$ & $H_{\text {upper }}$ \\
\hline \hline 2.55 & 31 & 0.28 & 0.22 & 6.3 & 3 & 1.46 & 0.22 & -0.06 \\
\hline 2.70 & 29.5 & 0.28 & 0.22 & 6.3 & 3 & 1.46 & 0.22 & -0.06 \\
\hline 2.85 & 28 & 0.28 & 0.22 & 6.8 & 3 & 1.46 & 0.22 & -0.04 \\
\hline 3.00 & 55 & 0.6 & 0.22 & 7.3 & 3 & 1.46 & 0.22 & -0.04 \\
\hline 3.15 & 54 & 0.60 & 0.22 & 7.3 & 3 & 1.46 & 0.22 & -0.04 \\
\hline 3.30 & 56 & 0.67 & 0.22 & 7.7 & 3 & 1.46 & 0.18 & -0.04 \\
\hline 3.45 & 52.5 & 0.63 & 0.22 & 8 & 3 & 1.46 & 0.18 & -0.04 \\
\hline 3.60 & 53 & 0.66 & 0.22 & 8.3 & 3 & 1.46 & 0.3 & 0.1 \\
\hline 3.75 & 52 & 0.66 & 0.22 & 8.8 & 3 & 1.46 & 0.3 & 0.1 \\
\hline 3.90 & 53 & 0.69 & 0.22 & 9 & 3 & 1.46 & 0.25 & 0.1 \\
\hline 4.05 & 53 & 0.73 & 0.22 & 9 & 3 & 1.46 & 0.25 & 0.1 \\
\hline 4.20 & 53 & 0.73 & 0.22 & 9 & 3 & 1.46 & 0.25 & 0.1 \\
\hline Bin $p_{\min }$ & $G_{\text {lower }}$ & $H_{\text {lower }}$ & $\theta_{\text {upper }}$ & $\theta_{\text {lower }}$ & inneroffset & outeroffset \\
\hline \hline 2.55 & 0.36 & -0.04 & 11 & 12 & 0.5 & 0 \\
\hline 2.70 & 0.32 & -0.04 & 13 & 12.5 & -0.5 & 0 \\
\hline 2.85 & 0.22 & -0.04 & 14 & 12.5 & -0.8 & 0 \\
\hline 3.00 & 0.22 & -0.04 & 13.5 & 13 & -0.8 & 0 \\
\hline 3.15 & 0.26 & -0.04 & 13.5 & 13 & -0.3 & 0 \\
\hline 3.30 & 0.18 & -0.04 & 14 & 14 & -0.6 & 0 \\
\hline 3.45 & 0.18 & -0.04 & 14.5 & 14 & -0.6 & 0 \\
\hline 3.60 & 0.39 & 0.1 & 15 & 15 & -0.6 & 0 \\
\hline 3.75 & 0.45 & 0.1 & 15 & 15 & -0.6 & 0 \\
\hline 3.90 & 0.45 & 0.1 & 15 & 15 & -0.6 & -0.1 \\
\hline 4.05 & 0.45 & 0.1 & 15 & 15 & -0.6 & -0.1 \\
\hline 4.20 & 0.45 & 0.1 & 15 & 15 & -0.6 & 0 \\
\hline
\end{tabular}
\end{tabular}


Torus Current $=-2250 \mathrm{~A}$, Sector $6, p<2.55 \mathrm{GeV}$

\begin{tabular}{|c|c|c|c|c|c|c|c|c|}
\hline Bin $p_{\min }$ & $A$ & $B$ & $C$ & $D$ & $E$ & $F$ & $G_{\text {upper }}$ & $H_{\text {upper }}$ \\
\hline \hline 0.15 & 24 & 0.28 & 0.30 & 5 & 3 & 1.46 & 0 & 0 \\
\hline 0.30 & 24 & 0.28 & 0.30 & 5 & 3 & 1.46 & 0 & 0 \\
\hline 0.45 & 31 & 0.42 & 0.30 & 5 & 3 & 1.46 & 0.2 & 0 \\
\hline 0.60 & 36.5 & 0.40 & 0.22 & 5 & 3 & 1.46 & 0.2 & 0 \\
\hline 0.75 & 32 & 0.33 & 0.22 & 5.3 & 3 & 1.46 & 0.2 & 0 \\
\hline 0.90 & 30 & 0.28 & 0.22 & 5.3 & 3 & 1.46 & 0.2 & 0 \\
\hline 1.05 & 30 & 0.28 & 0.22 & 5.3 & 3 & 1.46 & 0 & 0 \\
\hline 1.20 & 29 & 0.28 & 0.22 & 5.7 & 3 & 1.46 & 0 & -0.1 \\
\hline 1.35 & 32 & 0.28 & 0.22 & 5.7 & 3 & 1.46 & 0 & -0.06 \\
\hline 1.50 & 32 & 0.28 & 0.22 & 5.7 & 3 & 1.46 & 0.04 & -0.13 \\
\hline 1.65 & 32 & 0.28 & 0.22 & 5.7 & 3 & 1.46 & 0.15 & 0 \\
\hline 1.80 & 32 & 0.28 & 0.22 & 5.9 & 3 & 1.46 & 0.02 & -0.13 \\
\hline 1.95 & 32 & 0.28 & 0.22 & 6.0 & 3 & 1.46 & 0.22 & -0.08 \\
\hline 2.10 & 33 & 0.28 & 0.22 & 6.2 & 3 & 1.46 & 0.22 & -0.06 \\
\hline 2.25 & 34 & 0.28 & 0.22 & 6.3 & 3 & 1.46 & 0.22 & -0.06 \\
\hline 2.40 & 34 & 0.28 & 0.22 & 6.3 & 3 & 1.46 & 0.26 & -0.06 \\
\hline Bin $p_{\text {min }}$ & $G_{\text {lower }}$ & $H_{\text {lower }}$ & $\theta_{\text {upper }}$ & $\theta_{\text {lower }}$ & inneroffset & outeroffset \\
\hline \hline 0.15 & 0 & 0 & 25 & 25 & \multicolumn{2}{|c|}{2.8} & 2 \\
\hline 0.30 & 0 & 0 & 24 & 24 & 2.8 & 2 \\
\hline 0.45 & 0.2 & 0 & 13 & 24 & 2.8 & 2 \\
\hline 0.60 & 0.2 & 0 & 14 & 18 & \multicolumn{2}{|c|}{2} & 0 \\
\hline 0.75 & 0.1 & 0 & 14 & 21 & 2.8 & 0 \\
\hline 0.90 & 0.1 & 0 & 14 & 20 & 2.4 & 0 \\
\hline 1.05 & 0 & 0 & 15 & 19 & 2.2 & 1 \\
\hline 1.20 & 0 & -0.1 & 14 & 18 & 2.2 & 1 \\
\hline 1.35 & 0 & 0 & 13.5 & 17 & 2.2 & 1 \\
\hline 1.50 & 0.04 & -0.11 & 13 & 16 & 2.2 & 1.5 \\
\hline 1.65 & 0.15 & 0 & 12.5 & 15 & 2.3 & 1 \\
\hline 1.80 & 0.02 & -0.13 & 12 & 14 & 2.2 & 1 \\
\hline 1.95 & 0.20 & -0.08 & 12 & 13 & 2.2 & 1 \\
\hline 2.10 & 0.22 & -0.07 & 11.5 & 13 & 2.2 & 1 \\
\hline 2.25 & 0.22 & -0.04 & 11 & 13 & 2.2 & 0 \\
\hline 2.40 & 0.1 & -0.12 & 11 & 13 & & 2 & 0 \\
\hline
\end{tabular}


Torus Current $=-2250 \mathrm{~A}$, Sector $6, \mathrm{p}>2.55 \mathrm{GeV}$

\begin{tabular}{|c|c|c|c|c|c|c|c|c|}
\hline Bin $p_{\min }$ & $A$ & $B$ & $C$ & $D$ & $E$ & $F$ & $G_{\text {upper }}$ & $H_{\text {upper }}$ \\
\hline \hline 2.55 & 34 & 0.28 & 0.22 & 6.3 & 3 & 1.46 & 0.22 & -0.06 \\
\hline 2.70 & 32 & 0.28 & 0.22 & 6.7 & 3 & 1.46 & 0.22 & -0.06 \\
\hline 2.85 & 29 & 0.28 & 0.22 & 7 & 3 & 1.46 & 0.22 & -0.04 \\
\hline 3.00 & 58 & 0.6 & 0.22 & 7.3 & 3 & 1.46 & 0.22 & -0.04 \\
\hline 3.15 & 56 & 0.60 & 0.22 & 7.7 & 3 & 1.46 & 0.22 & -0.04 \\
\hline 3.30 & 60 & 0.67 & 0.22 & 8 & 3 & 1.46 & 0.18 & -0.04 \\
\hline 3.45 & 55 & 0.63 & 0.22 & 8.2 & 3 & 1.46 & 0.18 & -0.04 \\
\hline 3.60 & 55 & 0.66 & 0.22 & 8.5 & 3 & 1.46 & 0.3 & 0.1 \\
\hline 3.75 & 53 & 0.66 & 0.22 & 8.8 & 3 & 1.46 & 0.3 & 0.1 \\
\hline 3.90 & 54 & 0.69 & 0.22 & 9 & 3 & 1.46 & 0.25 & 0.1 \\
\hline 4.05 & 53 & 0.73 & 0.22 & 9 & 3 & 1.46 & 0.25 & 0.1 \\
\hline 4.20 & 53 & 0.73 & 0.22 & 9 & 3 & 1.46 & 0.25 & 0.1 \\
\hline
\end{tabular}

\begin{tabular}{|c|c|c|c|c|c|c|}
\hline Bin $p_{\min }$ & $G_{\text {lower }}$ & $H_{\text {lower }}$ & $\theta_{\text {upper }}$ & $\theta_{\text {lower }}$ & innerof f set & outerof f set \\
\hline \hline 2.55 & 0.36 & -0.04 & 11 & 12 & .5 & 0 \\
\hline 2.70 & 0.32 & -0.04 & 13 & 12.5 & 0.5 & 1 \\
\hline 2.85 & 0.32 & -0.04 & 14 & 12.5 & -0.3 & 1 \\
\hline 3.00 & 0.36 & -0.04 & 13.5 & 13 & 0.2 & 1 \\
\hline 3.15 & 0.36 & -0.04 & 13.5 & 13 & 0.3 & 1 \\
\hline 3.30 & 0.36 & -0.04 & 14 & 14 & 0 & 1 \\
\hline 3.45 & 0.36 & -0.04 & 14.5 & 14 & 0 & 1 \\
\hline 3.60 & 0.45 & 0.1 & 15 & 15 & 0 & 1 \\
\hline 3.75 & 0.45 & 0.1 & 15 & 15 & 0 & 1 \\
\hline 3.90 & 0.45 & 0.1 & 15 & 15 & 0 & 0.9 \\
\hline 4.05 & 0.45 & 0.1 & 15 & 15 & 0 & 0.9 \\
\hline 4.20 & 0.45 & 0.1 & 15 & 15 & 0 & 0.9 \\
\hline
\end{tabular}




\section{B.4 $A_{1}+\eta A_{2}$ Results}

Table B.6: Values of $A_{1}+\eta A_{2}$ for $E=1.6 \mathrm{GeV}$ data, with statistical and systematic errors. Average kinematic values in each bin are also shown.

\begin{tabular}{|c|c|c|c|c|c|c|c|}
\hline$Q^{2}$ bin & $W$ bin & $A_{1}+\eta A_{2}$ & $\sigma_{\text {stat }}$ & $\sigma_{\text {sys }}$ & $x_{\text {avg }}$ & $Q_{\text {avg }}^{2}$ & $W_{\text {avg }}$ \\
\hline 3 & 19 & -0.346 & 0.223 & 0.06 & 0.14 & 0.06 & 1.13 \\
\hline 3 & 20 & -0.286 & 0.051 & 0.021 & 0.11 & 0.06 & 1.18 \\
\hline 3 & 21 & -0.333 & 0.027 & 0.018 & 0.09 & 0.06 & 1.23 \\
\hline 3 & 22 & -0.145 & 0.031 & 0.017 & 0.07 & 0.06 & 1.29 \\
\hline 3 & 23 & -0.016 & 0.035 & 0.007 & 0.06 & 0.06 & 1.35 \\
\hline 3 & 24 & -0.091 & 0.03 & 0.008 & 0.05 & 0.06 & 1.41 \\
\hline 3 & 25 & -0.089 & 0.023 & 0.006 & 0.04 & 0.06 & 1.47 \\
\hline 3 & 26 & -0.058 & 0.023 & 0.005 & 0.04 & 0.06 & 1.53 \\
\hline
\end{tabular}

\begin{tabular}{|c|c|c|c|c|c|c|c|c|}
\hline$Q^{2}$ bin & $W$ bin & $s_{\text {avg }}$ & $D_{\text {avg }}$ & $\eta_{\text {avg }}$ & $\epsilon_{\text {avg }}$ & $\gamma_{\text {avg }}$ & $E_{\text {avg }}^{\prime}$ & $\theta_{\text {avg }}$ \\
\hline 3 & 19 & 4.04 & 0.16 & 0.9 & 0.98 & 1.06 & 1.44 & 0.9 \\
\hline 3 & 20 & 4.04 & 0.21 & 0.69 & 0.97 & 0.82 & 1.38 & 0.69 \\
\hline 3 & 21 & 4.04 & 0.25 & 0.56 & 0.96 & 0.68 & 1.31 & 0.56 \\
\hline 3 & 22 & 4.04 & 0.3 & 0.45 & 0.94 & 0.55 & 1.24 & 0.45 \\
\hline 3 & 23 & 4.05 & 0.37 & 0.36 & 0.92 & 0.46 & 1.15 & 0.36 \\
\hline 3 & 24 & 4.05 & 0.43 & 0.29 & 0.89 & 0.39 & 1.06 & 0.29 \\
\hline 3 & 25 & 4.05 & 0.49 & 0.24 & 0.85 & 0.34 & 0.97 & 0.24 \\
\hline 3 & 26 & 4.05 & 0.55 & 0.2 & 0.81 & 0.29 & 0.89 & 0.2 \\
\hline
\end{tabular}




\begin{tabular}{|c|c|c|c|c|c|c|c|}
\hline$Q^{2}$ bin & $W$ bin & $A_{1}+\eta A_{2}$ & $\sigma_{\text {stat }}$ & $\sigma_{\text {sys }}$ & $x_{\text {avg }}$ & $Q_{\text {avg }}^{2}$ & $W_{\text {avg }}$ \\
\hline 4 & 19 & -0.228 & 0.144 & 0.063 & 0.22 & 0.11 & 1.13 \\
\hline 4 & 20 & -0.334 & 0.033 & 0.023 & 0.18 & 0.11 & 1.18 \\
\hline 4 & 21 & -0.346 & 0.019 & 0.019 & 0.15 & 0.11 & 1.23 \\
\hline 4 & 22 & -0.148 & 0.023 & 0.013 & 0.13 & 0.11 & 1.29 \\
\hline 4 & 23 & 0.019 & 0.027 & 0.007 & 0.11 & 0.11 & 1.35 \\
\hline 4 & 24 & 0.033 & 0.023 & 0.008 & 0.09 & 0.11 & 1.41 \\
\hline 4 & 25 & 0.024 & 0.017 & 0.007 & 0.08 & 0.12 & 1.47 \\
\hline 4 & 26 & 0.015 & 0.037 & 0.007 & 0.08 & 0.12 & 1.5 \\
\hline
\end{tabular}

\begin{tabular}{|c|c|c|c|c|c|c|c|c|}
\hline$Q^{2}$ bin & $W$ bin & $s_{\text {avg }}$ & $D_{\text {avg }}$ & $\eta_{\text {avg }}$ & $\epsilon_{\text {avg }}$ & $\gamma_{\text {avg }}$ & $E_{\text {avg }}^{\prime}$ & $\theta_{\text {avg }}$ \\
\hline 4 & 19 & 4.05 & 0.19 & 1 & 0.96 & 1.25 & 1.42 & 1 \\
\hline 4 & 20 & 4.05 & 0.23 & 0.8 & 0.95 & 1 & 1.36 & 0.8 \\
\hline 4 & 21 & 4.06 & 0.27 & 0.66 & 0.94 & 0.84 & 1.3 & 0.66 \\
\hline 4 & 22 & 4.05 & 0.33 & 0.54 & 0.92 & 0.71 & 1.22 & 0.54 \\
\hline 4 & 23 & 4.05 & 0.38 & 0.44 & 0.9 & 0.6 & 1.13 & 0.44 \\
\hline 4 & 24 & 4.05 & 0.44 & 0.36 & 0.86 & 0.51 & 1.03 & 0.36 \\
\hline 4 & 25 & 4.04 & 0.5 & 0.3 & 0.82 & 0.45 & 0.94 & 0.3 \\
\hline 4 & 26 & 4.04 & 0.54 & 0.27 & 0.79 & 0.42 & 0.88 & 0.27 \\
\hline
\end{tabular}




\begin{tabular}{|c|c|c|c|c|c|c|c|}
\hline$Q^{2}$ bin & $W$ bin & $A_{1}+\eta A_{2}$ & $\sigma_{\text {stat }}$ & $\sigma_{\text {sys }}$ & $x_{\text {avg }}$ & $Q_{\text {avg }}^{2}$ & $W_{\text {avg }}$ \\
\hline 5 & 19 & -0.319 & 0.084 & 0.058 & 0.38 & 0.24 & 1.13 \\
\hline 5 & 20 & -0.364 & 0.018 & 0.023 & 0.31 & 0.24 & 1.18 \\
\hline 5 & 21 & -0.353 & 0.01 & 0.019 & 0.27 & 0.24 & 1.23 \\
\hline 5 & 22 & -0.111 & 0.012 & 0.011 & 0.23 & 0.24 & 1.29 \\
\hline 5 & 23 & 0.071 & 0.014 & 0.01 & 0.2 & 0.24 & 1.35 \\
\hline 5 & 24 & 0.146 & 0.013 & 0.01 & 0.17 & 0.23 & 1.41 \\
\hline 5 & 25 & 0.195 & 0.01 & 0.011 & 0.15 & 0.23 & 1.47 \\
\hline 5 & 26 & 0.21 & 0.008 & 0.012 & 0.14 & 0.23 & 1.53 \\
\hline 5 & 27 & 0.156 & 0.009 & 0.013 & 0.12 & 0.23 & 1.59 \\
\hline 5 & 28 & 0.048 & 0.008 & 0.014 & 0.11 & 0.23 & 1.65 \\
\hline 5 & 29 & 0.026 & 0.013 & 0.016 & 0.1 & 0.22 & 1.69 \\
\hline
\end{tabular}

\begin{tabular}{|c|c|c|c|c|c|c|c|c|}
\hline$Q^{2}$ bin & $W$ bin & $s_{\text {avg }}$ & $D_{\text {avg }}$ & $\eta_{\text {avg }}$ & $\epsilon_{\text {avg }}$ & $\gamma_{\text {avg }}$ & $E_{\text {avg }}^{\prime}$ & $\theta_{\text {avg }}$ \\
\hline 5 & 19 & 3.95 & 0.26 & 1.03 & 0.92 & 1.45 & 1.3 & 1.03 \\
\hline 5 & 20 & 3.95 & 0.31 & 0.85 & 0.91 & 1.21 & 1.23 & 0.85 \\
\hline 5 & 21 & 3.94 & 0.35 & 0.73 & 0.89 & 1.04 & 1.17 & 0.73 \\
\hline 5 & 22 & 3.94 & 0.4 & 0.61 & 0.87 & 0.89 & 1.09 & 0.61 \\
\hline 5 & 23 & 3.93 & 0.45 & 0.5 & 0.83 & 0.77 & 1 & 0.5 \\
\hline 5 & 24 & 3.93 & 0.51 & 0.42 & 0.79 & 0.67 & 0.91 & 0.42 \\
\hline 5 & 25 & 3.93 & 0.56 & 0.34 & 0.74 & 0.59 & 0.81 & 0.34 \\
\hline 5 & 26 & 3.93 & 0.62 & 0.29 & 0.68 & 0.53 & 0.72 & 0.29 \\
\hline 5 & 27 & 3.93 & 0.69 & 0.23 & 0.61 & 0.48 & 0.62 & 0.23 \\
\hline 5 & 28 & 3.92 & 0.75 & 0.18 & 0.52 & 0.43 & 0.52 & 0.18 \\
\hline 5 & 29 & 3.92 & 0.8 & 0.15 & 0.45 & 0.4 & 0.45 & 0.15 \\
\hline
\end{tabular}




\begin{tabular}{|c|c|c|c|c|c|c|c|}
\hline$Q^{2}$ bin & $W$ bin & $A_{1}+\eta A_{2}$ & $\sigma_{\text {stat }}$ & $\sigma_{\text {sys }}$ & $x_{\text {avg }}$ & $Q_{\text {avg }}^{2}$ & $W_{\text {avg }}$ \\
\hline 6 & 19 & -0.184 & 0.079 & 0.056 & 0.53 & 0.45 & 1.13 \\
\hline 6 & 20 & -0.359 & 0.016 & 0.023 & 0.46 & 0.45 & 1.18 \\
\hline 6 & 21 & -0.311 & 0.009 & 0.018 & 0.41 & 0.45 & 1.23 \\
\hline 6 & 22 & -0.085 & 0.012 & 0.01 & 0.36 & 0.45 & 1.29 \\
\hline 6 & 23 & 0.203 & 0.014 & 0.012 & 0.32 & 0.44 & 1.35 \\
\hline 6 & 24 & 0.255 & 0.014 & 0.011 & 0.28 & 0.44 & 1.41 \\
\hline 6 & 25 & 0.378 & 0.012 & 0.013 & 0.24 & 0.41 & 1.47 \\
\hline 6 & 26 & 0.397 & 0.01 & 0.014 & 0.22 & 0.41 & 1.53 \\
\hline 6 & 27 & 0.277 & 0.015 & 0.015 & 0.18 & 0.37 & 1.59 \\
\hline 6 & 28 & 0.173 & 0.026 & 0.015 & 0.16 & 0.35 & 1.64 \\
\hline
\end{tabular}

\begin{tabular}{|c|c|c|c|c|c|c|c|c|}
\hline$Q^{2}$ bin & $W$ bin & $s_{\text {avg }}$ & $D_{\text {avg }}$ & $\eta_{\text {avg }}$ & $\epsilon_{\text {avg }}$ & $\gamma_{\text {avg }}$ & $E_{\text {avg }}^{\prime}$ & $\theta_{\text {avg }}$ \\
\hline 6 & 19 & 3.92 & 0.38 & 0.9 & 0.84 & 1.49 & 1.17 & 0.9 \\
\hline 6 & 20 & 3.92 & 0.43 & 0.78 & 0.82 & 1.3 & 1.11 & 0.78 \\
\hline 6 & 21 & 3.92 & 0.47 & 0.68 & 0.8 & 1.16 & 1.04 & 0.68 \\
\hline 6 & 22 & 3.92 & 0.51 & 0.58 & 0.77 & 1.02 & 0.97 & 0.58 \\
\hline 6 & 23 & 3.92 & 0.55 & 0.49 & 0.72 & 0.9 & 0.88 & 0.49 \\
\hline 6 & 24 & 3.92 & 0.6 & 0.41 & 0.67 & 0.8 & 0.79 & 0.41 \\
\hline 6 & 25 & 3.91 & 0.64 & 0.34 & 0.63 & 0.7 & 0.71 & 0.34 \\
\hline 6 & 26 & 3.91 & 0.7 & 0.28 & 0.56 & 0.64 & 0.62 & 0.28 \\
\hline 6 & 27 & 3.9 & 0.74 & 0.23 & 0.5 & 0.57 & 0.54 & 0.23 \\
\hline 6 & 28 & 3.89 & 0.77 & 0.19 & 0.44 & 0.52 & 0.46 & 0.19 \\
\hline
\end{tabular}




\begin{tabular}{|c|c|c|c|c|c|c|c|}
\hline$Q^{2}$ bin & $W$ bin & $A_{1}+\eta A_{2}$ & $\sigma_{\text {stat }}$ & $\sigma_{\text {sys }}$ & $x_{\text {avg }}$ & $Q_{\text {avg }}^{2}$ & $W_{\text {avg }}$ \\
\hline 7 & 19 & 0.188 & 0.161 & 0.057 & 0.66 & 0.74 & 1.13 \\
\hline 7 & 20 & -0.203 & 0.037 & 0.023 & 0.59 & 0.73 & 1.18 \\
\hline 7 & 21 & -0.233 & 0.025 & 0.016 & 0.53 & 0.7 & 1.23 \\
\hline 7 & 22 & -0.032 & 0.032 & 0.01 & 0.47 & 0.7 & 1.29 \\
\hline 7 & 23 & 0.339 & 0.054 & 0.01 & 0.43 & 0.69 & 1.34 \\
\hline
\end{tabular}

\begin{tabular}{|c|c|c|c|c|c|c|c|c|}
\hline$Q^{2}$ bin & $W$ bin & $s_{\text {avg }}$ & $D_{\text {avg }}$ & $\eta_{\text {avg }}$ & $\epsilon_{\text {avg }}$ & $\gamma_{\text {avg }}$ & $E_{\text {avg }}^{\prime}$ & $\theta_{\text {avg }}$ \\
\hline 7 & 19 & 3.9 & 0.53 & 0.71 & 0.72 & 1.43 & 1.01 & 0.71 \\
\hline 7 & 20 & 3.9 & 0.57 & 0.63 & 0.7 & 1.29 & 0.95 & 0.63 \\
\hline 7 & 21 & 3.89 & 0.6 & 0.57 & 0.68 & 1.18 & 0.89 & 0.57 \\
\hline 7 & 22 & 3.89 & 0.64 & 0.49 & 0.63 & 1.07 & 0.82 & 0.49 \\
\hline 7 & 23 & 3.89 & 0.67 & 0.42 & 0.59 & 0.97 & 0.75 & 0.42 \\
\hline
\end{tabular}


Table B.7: Values of $A_{1}+\eta A_{2}$ for $E=2.5 \mathrm{GeV}$ data, with statistical and systematic errors. Average kinematic values in each bin are also shown.

\begin{tabular}{|c|c|c|c|c|c|c|c|}
\hline$Q^{2}$ bin & $W$ bin & $A_{1}+\eta A_{2}$ & $\sigma_{\text {stat }}$ & $\sigma_{\text {sys }}$ & $x_{\text {avg }}$ & $Q_{\text {avg }}^{2}$ & $W_{\text {avg }}$ \\
\hline 4 & 19 & -0.195 & 0.471 & 0.064 & 0.27 & 0.14 & 1.13 \\
\hline 4 & 20 & -0.389 & 0.098 & 0.027 & 0.21 & 0.14 & 1.18 \\
\hline 4 & 21 & -0.277 & 0.047 & 0.023 & 0.18 & 0.14 & 1.23 \\
\hline 4 & 22 & -0.162 & 0.05 & 0.017 & 0.15 & 0.14 & 1.29 \\
\hline 4 & 23 & -0.036 & 0.052 & 0.013 & 0.13 & 0.14 & 1.35 \\
\hline 4 & 24 & 0.012 & 0.042 & 0.008 & 0.11 & 0.13 & 1.41 \\
\hline 4 & 25 & 0.042 & 0.031 & 0.007 & 0.09 & 0.13 & 1.47 \\
\hline 4 & 26 & 0.068 & 0.026 & 0.007 & 0.08 & 0.13 & 1.53 \\
\hline 4 & 27 & 0.055 & 0.027 & 0.005 & 0.07 & 0.13 & 1.59 \\
\hline 4 & 28 & -0.046 & 0.024 & 0.004 & 0.07 & 0.13 & 1.65 \\
\hline 4 & 29 & -0.045 & 0.022 & 0.005 & 0.06 & 0.13 & 1.71 \\
\hline 4 & 30 & 0.033 & 0.024 & 0.007 & 0.06 & 0.13 & 1.77 \\
\hline 4 & 31 & -0.036 & 0.024 & 0.006 & 0.05 & 0.13 & 1.83 \\
\hline 4 & 32 & -0.035 & 0.028 & 0.007 & 0.05 & 0.13 & 1.88 \\
\hline
\end{tabular}




\begin{tabular}{|c|c|c|c|c|c|c|c|c|}
\hline$Q^{2}$ bin & $W$ bin & $s_{\text {avg }}$ & $D_{\text {avg }}$ & $\eta_{\text {avg }}$ & $\epsilon_{\text {avg }}$ & $\gamma_{\text {avg }}$ & $E_{\text {avg }}^{\prime}$ & $\theta_{\text {avg }}$ \\
\hline 4 & 19 & 5.68 & 0.13 & 1.13 & 0.98 & 1.33 & 2.27 & 1.13 \\
\hline 4 & 20 & 5.68 & 0.15 & 0.91 & 0.98 & 1.07 & 2.21 & 0.91 \\
\hline 4 & 21 & 5.68 & 0.18 & 0.77 & 0.97 & 0.9 & 2.15 & 0.77 \\
\hline 4 & 22 & 5.68 & 0.21 & 0.64 & 0.97 & 0.76 & 2.07 & 0.64 \\
\hline 4 & 23 & 5.68 & 0.24 & 0.53 & 0.96 & 0.64 & 1.98 & 0.53 \\
\hline 4 & 24 & 5.68 & 0.28 & 0.45 & 0.94 & 0.55 & 1.89 & 0.45 \\
\hline 4 & 25 & 5.68 & 0.31 & 0.38 & 0.93 & 0.48 & 1.8 & 0.38 \\
\hline 4 & 26 & 5.68 & 0.35 & 0.33 & 0.91 & 0.43 & 1.71 & 0.33 \\
\hline 4 & 27 & 5.68 & 0.4 & 0.29 & 0.89 & 0.38 & 1.61 & 0.29 \\
\hline 4 & 28 & 5.68 & 0.44 & 0.25 & 0.86 & 0.34 & 1.5 & 0.25 \\
\hline 4 & 29 & 5.68 & 0.5 & 0.21 & 0.83 & 0.31 & 1.4 & 0.21 \\
\hline 4 & 30 & 5.68 & 0.55 & 0.19 & 0.79 & 0.29 & 1.29 & 0.19 \\
\hline 4 & 31 & 5.68 & 0.6 & 0.16 & 0.74 & 0.26 & 1.17 & 0.16 \\
\hline 4 & 32 & 5.68 & 0.65 & 0.14 & 0.7 & 0.24 & 1.07 & 0.14 \\
\hline
\end{tabular}




\begin{tabular}{|c|c|c|c|c|c|c|c|}
\hline$Q^{2}$ bin & $W$ bin & $A_{1}+\eta A_{2}$ & $\sigma_{\text {stat }}$ & $\sigma_{\text {sys }}$ & $x_{\text {avg }}$ & $Q_{\text {avg }}^{2}$ & $W_{\text {avg }}$ \\
\hline 5 & 19 & -0.412 & 0.192 & 0.066 & 0.37 & 0.24 & 1.13 \\
\hline 5 & 20 & -0.426 & 0.04 & 0.027 & 0.31 & 0.24 & 1.18 \\
\hline 5 & 21 & -0.385 & 0.022 & 0.022 & 0.27 & 0.24 & 1.23 \\
\hline 5 & 22 & -0.109 & 0.026 & 0.014 & 0.23 & 0.24 & 1.29 \\
\hline 5 & 23 & 0.123 & 0.03 & 0.012 & 0.2 & 0.24 & 1.35 \\
\hline 5 & 24 & 0.136 & 0.027 & 0.01 & 0.17 & 0.23 & 1.41 \\
\hline 5 & 25 & 0.201 & 0.02 & 0.01 & 0.15 & 0.23 & 1.47 \\
\hline 5 & 26 & 0.246 & 0.016 & 0.01 & 0.14 & 0.23 & 1.53 \\
\hline 5 & 27 & 0.152 & 0.017 & 0.008 & 0.12 & 0.23 & 1.59 \\
\hline 5 & 28 & 0.077 & 0.015 & 0.006 & 0.11 & 0.24 & 1.65 \\
\hline 5 & 29 & 0.07 & 0.014 & 0.007 & 0.1 & 0.24 & 1.71 \\
\hline 5 & 30 & 0.094 & 0.014 & 0.008 & 0.09 & 0.24 & 1.77 \\
\hline 5 & 31 & 0.058 & 0.014 & 0.007 & 0.09 & 0.24 & 1.83 \\
\hline 5 & 32 & 0.012 & 0.013 & 0.007 & 0.08 & 0.25 & 1.89 \\
\hline 5 & 33 & 0.024 & 0.013 & 0.006 & 0.08 & 0.25 & 1.95 \\
\hline 5 & 34 & 0.072 & 0.014 & 0.007 & 0.08 & 0.26 & 2.01 \\
\hline 5 & 35 & 0.072 & 0.023 & 0.008 & 0.07 & 0.27 & 2.07 \\
\hline 5 & 36 & -0.046 & 0.075 & 0.009 & 0.06 & 0.24 & 2.11 \\
\hline
\end{tabular}




\begin{tabular}{|c|c|c|c|c|c|c|c|c|}
\hline$Q^{2}$ bin & $W$ bin & $s_{\text {avg }}$ & $D_{\text {avg }}$ & $\eta_{\text {avg }}$ & $\epsilon_{\text {avg }}$ & $\gamma_{\text {avg }}$ & $E_{\text {avg }}^{\prime}$ & $\theta_{\text {avg }}$ \\
\hline 5 & 19 & 5.62 & 0.16 & 1.17 & 0.97 & 1.45 & 2.19 & 1.17 \\
\hline 5 & 20 & 5.61 & 0.19 & 0.97 & 0.96 & 1.21 & 2.12 & 0.97 \\
\hline 5 & 21 & 5.61 & 0.21 & 0.84 & 0.96 & 1.04 & 2.06 & 0.84 \\
\hline 5 & 22 & 5.6 & 0.24 & 0.71 & 0.95 & 0.89 & 1.98 & 0.71 \\
\hline 5 & 23 & 5.58 & 0.27 & 0.6 & 0.94 & 0.77 & 1.88 & 0.6 \\
\hline 5 & 24 & 5.59 & 0.31 & 0.51 & 0.92 & 0.67 & 1.79 & 0.51 \\
\hline 5 & 25 & 5.58 & 0.34 & 0.44 & 0.9 & 0.59 & 1.69 & 0.44 \\
\hline 5 & 26 & 5.57 & 0.38 & 0.39 & 0.88 & 0.53 & 1.6 & 0.39 \\
\hline 5 & 27 & 5.56 & 0.42 & 0.34 & 0.85 & 0.48 & 1.49 & 0.34 \\
\hline 5 & 28 & 5.55 & 0.46 & 0.29 & 0.82 & 0.43 & 1.38 & 0.29 \\
\hline 5 & 29 & 5.54 & 0.51 & 0.25 & 0.78 & 0.4 & 1.27 & 0.25 \\
\hline 5 & 30 & 5.52 & 0.57 & 0.22 & 0.73 & 0.36 & 1.15 & 0.22 \\
\hline 5 & 31 & 5.49 & 0.63 & 0.18 & 0.67 & 0.34 & 1.01 & 0.18 \\
\hline 5 & 32 & 5.44 & 0.69 & 0.15 & 0.6 & 0.32 & 0.87 & 0.15 \\
\hline 5 & 33 & 5.39 & 0.76 & 0.12 & 0.5 & 0.3 & 0.71 & 0.12 \\
\hline 5 & 34 & 5.37 & 0.82 & 0.1 & 0.42 & 0.28 & 0.58 & 0.1 \\
\hline 5 & 35 & 5.55 & 0.84 & 0.09 & 0.38 & 0.27 & 0.53 & 0.09 \\
\hline 5 & 36 & 5.68 & 0.85 & 0.08 & 0.38 & 0.24 & 0.54 & 0.08 \\
\hline
\end{tabular}




\begin{tabular}{|c|c|c|c|c|c|c|c|}
\hline$Q^{2}$ bin & $W$ bin & $A_{1}+\eta A_{2}$ & $\sigma_{\text {stat }}$ & $\sigma_{\text {sys }}$ & $x_{\text {avg }}$ & $Q_{\text {avg }}^{2}$ & $W_{\text {avg }}$ \\
\hline 6 & 19 & -0.117 & 0.146 & 0.06 & 0.54 & 0.47 & 1.13 \\
\hline 6 & 20 & -0.3 & 0.03 & 0.024 & 0.47 & 0.46 & 1.18 \\
\hline 6 & 21 & -0.327 & 0.017 & 0.02 & 0.42 & 0.46 & 1.23 \\
\hline 6 & 22 & -0.099 & 0.02 & 0.011 & 0.37 & 0.47 & 1.29 \\
\hline 6 & 23 & 0.265 & 0.024 & 0.012 & 0.33 & 0.47 & 1.35 \\
\hline 6 & 24 & 0.323 & 0.022 & 0.014 & 0.29 & 0.47 & 1.41 \\
\hline 6 & 25 & 0.433 & 0.017 & 0.017 & 0.26 & 0.47 & 1.47 \\
\hline 6 & 26 & 0.432 & 0.014 & 0.017 & 0.24 & 0.47 & 1.53 \\
\hline 6 & 27 & 0.346 & 0.015 & 0.013 & 0.22 & 0.47 & 1.59 \\
\hline 6 & 28 & 0.234 & 0.013 & 0.01 & 0.2 & 0.46 & 1.65 \\
\hline 6 & 29 & 0.257 & 0.011 & 0.011 & 0.18 & 0.47 & 1.71 \\
\hline 6 & 30 & 0.24 & 0.011 & 0.011 & 0.17 & 0.46 & 1.77 \\
\hline 6 & 31 & 0.151 & 0.011 & 0.008 & 0.16 & 0.46 & 1.83 \\
\hline 6 & 32 & 0.091 & 0.011 & 0.008 & 0.14 & 0.45 & 1.89 \\
\hline 6 & 33 & 0.099 & 0.011 & 0.007 & 0.12 & 0.42 & 1.95 \\
\hline 6 & 34 & 0.11 & 0.017 & 0.007 & 0.11 & 0.38 & 2 \\
\hline 6 & 35 & 0.087 & 0.03 & 0.008 & 0.09 & 0.35 & 2.06 \\
\hline
\end{tabular}




\begin{tabular}{|c|c|c|c|c|c|c|c|c|}
\hline$Q^{2}$ bin & $W$ bin & $s_{\text {avg }}$ & $D_{\text {avg }}$ & $\eta_{\text {avg }}$ & $\epsilon_{\text {avg }}$ & $\gamma_{\text {avg }}$ & $E_{\text {avg }}^{\prime}$ & $\theta_{\text {avg }}$ \\
\hline 6 & 19 & 5.37 & 0.24 & 1.07 & 0.93 & 1.49 & 1.93 & 1.07 \\
\hline 6 & 20 & 5.36 & 0.27 & 0.94 & 0.92 & 1.3 & 1.87 & 0.94 \\
\hline 6 & 21 & 5.37 & 0.3 & 0.83 & 0.91 & 1.16 & 1.81 & 0.83 \\
\hline 6 & 22 & 5.37 & 0.33 & 0.73 & 0.9 & 1.02 & 1.73 & 0.73 \\
\hline 6 & 23 & 5.37 & 0.36 & 0.63 & 0.88 & 0.9 & 1.64 & 0.63 \\
\hline 6 & 24 & 5.36 & 0.39 & 0.55 & 0.86 & 0.81 & 1.55 & 0.55 \\
\hline 6 & 25 & 5.35 & 0.42 & 0.48 & 0.83 & 0.72 & 1.45 & 0.48 \\
\hline 6 & 26 & 5.35 & 0.46 & 0.42 & 0.8 & 0.66 & 1.35 & 0.42 \\
\hline 6 & 27 & 5.34 & 0.5 & 0.36 & 0.76 & 0.6 & 1.25 & 0.36 \\
\hline 6 & 28 & 5.33 & 0.53 & 0.31 & 0.72 & 0.55 & 1.14 & 0.31 \\
\hline 6 & 29 & 5.33 & 0.58 & 0.27 & 0.67 & 0.51 & 1.03 & 0.27 \\
\hline 6 & 30 & 5.33 & 0.64 & 0.23 & 0.61 & 0.47 & 0.92 & 0.23 \\
\hline 6 & 31 & 5.33 & 0.69 & 0.19 & 0.55 & 0.43 & 0.81 & 0.19 \\
\hline 6 & 32 & 5.32 & 0.74 & 0.16 & 0.48 & 0.4 & 0.69 & 0.16 \\
\hline 6 & 33 & 5.33 & 0.79 & 0.13 & 0.41 & 0.36 & 0.59 & 0.13 \\
\hline 6 & 34 & 5.35 & 0.83 & 0.1 & 0.37 & 0.33 & 0.52 & 0.1 \\
\hline 6 & 35 & 5.62 & 0.83 & 0.1 & 0.38 & 0.3 & 0.55 & 0.1 \\
\hline
\end{tabular}




\begin{tabular}{|c|c|c|c|c|c|c|c|}
\hline$Q^{2}$ bin & $W$ bin & $A_{1}+\eta A_{2}$ & $\sigma_{\text {stat }}$ & $\sigma_{\text {sys }}$ & $x_{\text {avg }}$ & $Q_{\text {avg }}^{2}$ & $W_{\text {avg }}$ \\
\hline 7 & 19 & 0.136 & 0.145 & 0.057 & 0.69 & 0.9 & 1.13 \\
\hline 7 & 20 & -0.232 & 0.033 & 0.018 & 0.63 & 0.9 & 1.18 \\
\hline 7 & 21 & -0.221 & 0.019 & 0.017 & 0.58 & 0.9 & 1.23 \\
\hline 7 & 22 & 0.063 & 0.022 & 0.011 & 0.53 & 0.9 & 1.29 \\
\hline 7 & 23 & 0.366 & 0.027 & 0.014 & 0.47 & 0.84 & 1.35 \\
\hline 7 & 24 & 0.443 & 0.026 & 0.018 & 0.43 & 0.84 & 1.41 \\
\hline 7 & 25 & 0.556 & 0.02 & 0.021 & 0.39 & 0.84 & 1.47 \\
\hline 7 & 26 & 0.564 & 0.019 & 0.02 & 0.35 & 0.78 & 1.53 \\
\hline 7 & 27 & 0.476 & 0.022 & 0.015 & 0.32 & 0.77 & 1.59 \\
\hline 7 & 28 & 0.378 & 0.022 & 0.012 & 0.29 & 0.74 & 1.65 \\
\hline 7 & 29 & 0.382 & 0.024 & 0.013 & 0.26 & 0.7 & 1.71 \\
\hline 7 & 30 & 0.384 & 0.024 & 0.012 & 0.24 & 0.7 & 1.77 \\
\hline 7 & 31 & 0.336 & 0.06 & 0.011 & 0.23 & 0.7 & 1.8 \\
\hline
\end{tabular}

\begin{tabular}{|c|c|c|c|c|c|c|c|c|}
\hline$Q^{2}$ bin & $W$ bin & $s_{\text {avg }}$ & $D_{\text {avg }}$ & $\eta_{\text {avg }}$ & $\epsilon_{\text {avg }}$ & $\gamma_{\text {avg }}$ & $E_{\text {avg }}^{\prime}$ & $\theta_{\text {avg }}$ \\
\hline 7 & 19 & 5.28 & 0.39 & 0.85 & 0.84 & 1.38 & 1.66 & 0.85 \\
\hline 7 & 20 & 5.29 & 0.42 & 0.77 & 0.83 & 1.26 & 1.6 & 0.77 \\
\hline 7 & 21 & 5.32 & 0.44 & 0.7 & 0.81 & 1.16 & 1.55 & 0.7 \\
\hline 7 & 22 & 5.33 & 0.47 & 0.63 & 0.79 & 1.06 & 1.48 & 0.63 \\
\hline 7 & 23 & 5.33 & 0.47 & 0.57 & 0.78 & 0.96 & 1.42 & 0.57 \\
\hline 7 & 24 & 5.32 & 0.5 & 0.5 & 0.75 & 0.88 & 1.33 & 0.5 \\
\hline 7 & 25 & 5.32 & 0.54 & 0.44 & 0.72 & 0.8 & 1.24 & 0.44 \\
\hline 7 & 26 & 5.32 & 0.56 & 0.4 & 0.7 & 0.74 & 1.18 & 0.4 \\
\hline 7 & 27 & 5.32 & 0.6 & 0.35 & 0.66 & 0.68 & 1.08 & 0.35 \\
\hline 7 & 28 & 5.32 & 0.62 & 0.3 & 0.62 & 0.63 & 1 & 0.3 \\
\hline 7 & 29 & 5.33 & 0.65 & 0.26 & 0.58 & 0.57 & 0.91 & 0.26 \\
\hline 7 & 30 & 5.31 & 0.71 & 0.22 & 0.51 & 0.53 & 0.79 & 0.22 \\
\hline 7 & 31 & 5.31 & 0.74 & 0.19 & 0.47 & 0.51 & 0.72 & 0.19 \\
\hline
\end{tabular}


Table B.8: Values of $A_{1}+\eta A_{2}$ for $E=4.2 \mathrm{GeV}$ data, with statistical and systematic errors. Average kinematic values in each bin are also shown.

\begin{tabular}{|c|c|c|c|c|c|c|c|}
\hline$Q^{2}$ bin & $W$ bin & $A_{1}+\eta A_{2}$ & $\sigma_{\text {stat }}$ & $\sigma_{\text {sys }}$ & $x_{\text {avg }}$ & $Q_{\text {avg }}^{2}$ & $W_{\text {avg }}$ \\
\hline 5 & 24 & -0.077 & 0.476 & 0.023 & 0.21 & 0.31 & 1.43 \\
\hline 5 & 25 & -0.207 & 0.172 & 0.027 & 0.19 & 0.31 & 1.48 \\
\hline 5 & 26 & 0.256 & 0.105 & 0.024 & 0.17 & 0.31 & 1.54 \\
\hline 5 & 27 & 0.29 & 0.074 & 0.019 & 0.16 & 0.3 & 1.59 \\
\hline 5 & 28 & 0.166 & 0.054 & 0.014 & 0.14 & 0.3 & 1.65 \\
\hline 5 & 29 & 0.155 & 0.039 & 0.016 & 0.13 & 0.3 & 1.71 \\
\hline 5 & 30 & 0.066 & 0.034 & 0.011 & 0.11 & 0.29 & 1.77 \\
\hline 5 & 31 & 0.08 & 0.029 & 0.007 & 0.1 & 0.29 & 1.83 \\
\hline 5 & 32 & 0.012 & 0.023 & 0.006 & 0.09 & 0.28 & 1.89 \\
\hline 5 & 33 & 0.054 & 0.02 & 0.006 & 0.09 & 0.28 & 1.95 \\
\hline 5 & 34 & 0.073 & 0.018 & 0.006 & 0.08 & 0.27 & 2.01 \\
\hline 5 & 35 & 0.058 & 0.016 & 0.005 & 0.07 & 0.26 & 2.07 \\
\hline 5 & 36 & 0.066 & 0.014 & 0.005 & 0.07 & 0.26 & 2.13 \\
\hline 5 & 37 & 0.077 & 0.013 & 0.005 & 0.06 & 0.25 & 2.19 \\
\hline 5 & 38 & 0.048 & 0.012 & 0.006 & 0.06 & 0.25 & 2.25 \\
\hline 5 & 39 & 0.11 & 0.012 & 0.006 & 0.05 & 0.25 & 2.31 \\
\hline 5 & 40 & 0.069 & 0.011 & 0.005 & 0.05 & 0.25 & 2.37 \\
\hline
\end{tabular}




\begin{tabular}{|c|c|c|c|c|c|c|c|c|}
\hline$Q^{2}$ bin & $W$ bin & $s_{\text {avg }}$ & $D_{\text {avg }}$ & $\eta_{\text {avg }}$ & $\epsilon_{\text {avg }}$ & $\gamma_{\text {avg }}$ & $E_{\text {avg }}^{\prime}$ & $\theta_{\text {avg }}$ \\
\hline 5 & 24 & 8.83 & 0.18 & 0.61 & 0.97 & 0.72 & 3.45 & 0.61 \\
\hline 5 & 25 & 8.83 & 0.2 & 0.55 & 0.96 & 0.65 & 3.38 & 0.55 \\
\hline 5 & 26 & 8.83 & 0.22 & 0.49 & 0.96 & 0.58 & 3.28 & 0.49 \\
\hline 5 & 27 & 8.83 & 0.23 & 0.44 & 0.95 & 0.53 & 3.19 & 0.44 \\
\hline 5 & 28 & 8.83 & 0.25 & 0.39 & 0.94 & 0.48 & 3.09 & 0.39 \\
\hline 5 & 29 & 8.83 & 0.28 & 0.35 & 0.93 & 0.44 & 2.98 & 0.35 \\
\hline 5 & 30 & 8.83 & 0.31 & 0.31 & 0.92 & 0.4 & 2.88 & 0.31 \\
\hline 5 & 31 & 8.83 & 0.33 & 0.28 & 0.9 & 0.36 & 2.76 & 0.28 \\
\hline 5 & 32 & 8.83 & 0.36 & 0.25 & 0.89 & 0.33 & 2.65 & 0.25 \\
\hline 5 & 33 & 8.83 & 0.39 & 0.22 & 0.87 & 0.31 & 2.53 & 0.22 \\
\hline 5 & 34 & 8.83 & 0.43 & 0.2 & 0.85 & 0.28 & 2.41 & 0.2 \\
\hline 5 & 35 & 8.83 & 0.47 & 0.18 & 0.82 & 0.26 & 2.28 & 0.18 \\
\hline 5 & 36 & 8.83 & 0.5 & 0.16 & 0.8 & 0.24 & 2.15 & 0.16 \\
\hline 5 & 37 & 8.83 & 0.54 & 0.14 & 0.76 & 0.23 & 2.01 & 0.14 \\
\hline 5 & 38 & 8.83 & 0.58 & 0.13 & 0.73 & 0.21 & 1.87 & 0.13 \\
\hline 5 & 39 & 8.83 & 0.62 & 0.11 & 0.69 & 0.2 & 1.73 & 0.11 \\
\hline 5 & 40 & 8.83 & 0.66 & 0.1 & 0.64 & 0.19 & 1.58 & 0.1 \\
\hline
\end{tabular}




\begin{tabular}{|c|c|c|c|c|c|c|c|}
\hline$Q^{2}$ bin & $W$ bin & $A_{1}+\eta A_{2}$ & $\sigma_{\text {stat }}$ & $\sigma_{\text {sys }}$ & $x_{\text {avg }}$ & $Q_{\text {avg }}^{2}$ & $W_{\text {avg }}$ \\
\hline 6 & 19 & -0.308 & 0.293 & 0.055 & 0.57 & 0.52 & 1.13 \\
\hline 6 & 20 & -0.459 & 0.055 & 0.032 & 0.5 & 0.52 & 1.18 \\
\hline 6 & 21 & -0.227 & 0.03 & 0.016 & 0.44 & 0.51 & 1.23 \\
\hline 6 & 22 & -0.075 & 0.035 & 0.014 & 0.39 & 0.5 & 1.29 \\
\hline 6 & 23 & 0.245 & 0.039 & 0.018 & 0.34 & 0.49 & 1.35 \\
\hline 6 & 24 & 0.37 & 0.034 & 0.022 & 0.3 & 0.48 & 1.41 \\
\hline 6 & 25 & 0.479 & 0.024 & 0.027 & 0.27 & 0.48 & 1.47 \\
\hline 6 & 26 & 0.502 & 0.02 & 0.026 & 0.24 & 0.47 & 1.53 \\
\hline 6 & 27 & 0.38 & 0.021 & 0.021 & 0.22 & 0.47 & 1.59 \\
\hline 6 & 28 & 0.255 & 0.018 & 0.015 & 0.2 & 0.46 & 1.65 \\
\hline 6 & 29 & 0.303 & 0.015 & 0.017 & 0.18 & 0.46 & 1.71 \\
\hline 6 & 30 & 0.255 & 0.016 & 0.012 & 0.17 & 0.46 & 1.77 \\
\hline 6 & 31 & 0.125 & 0.015 & 0.008 & 0.16 & 0.46 & 1.83 \\
\hline 6 & 32 & 0.099 & 0.014 & 0.008 & 0.14 & 0.46 & 1.89 \\
\hline 6 & 33 & 0.123 & 0.013 & 0.007 & 0.13 & 0.45 & 1.95 \\
\hline 6 & 34 & 0.161 & 0.013 & 0.007 & 0.12 & 0.45 & 2.01 \\
\hline 6 & 35 & 0.138 & 0.012 & 0.006 & 0.12 & 0.44 & 2.07 \\
\hline 6 & 36 & 0.13 & 0.011 & 0.006 & 0.11 & 0.45 & 2.13 \\
\hline 6 & 37 & 0.144 & 0.011 & 0.006 & 0.1 & 0.45 & 2.19 \\
\hline 6 & 38 & 0.159 & 0.011 & 0.007 & 0.1 & 0.46 & 2.25 \\
\hline 6 & 39 & 0.141 & 0.011 & 0.006 & 0.09 & 0.45 & 2.31 \\
\hline 6 & 40 & 0.101 & 0.01 & 0.006 & 0.09 & 0.45 & 2.37 \\
\hline 6 & 41 & 0.149 & 0.012 & 0.006 & 0.09 & 0.5 & 2.43 \\
\hline 6 & 42 & 0.13 & 0.012 & 0.007 & 0.09 & 0.5 & 2.49 \\
\hline 6 & 43 & 0.139 & 0.019 & 0.008 & 0.09 & 0.55 & 2.54 \\
\hline & & & & & & & \\
\hline
\end{tabular}




\begin{tabular}{|c|c|c|c|c|c|c|c|c|}
\hline$Q^{2}$ bin & $W$ bin & $s_{\text {avg }}$ & $D_{\text {avg }}$ & $\eta_{\text {avg }}$ & $\epsilon_{\text {avg }}$ & $\gamma_{\text {avg }}$ & $E_{\text {avg }}^{\prime}$ & $\theta_{\text {avg }}$ \\
\hline 6 & 19 & 8.83 & 0.13 & 1.22 & 0.98 & 1.48 & 3.75 & 1.22 \\
\hline 6 & 20 & 8.83 & 0.15 & 1.08 & 0.97 & 1.3 & 3.68 & 1.08 \\
\hline 6 & 21 & 8.83 & 0.16 & 0.97 & 0.97 & 1.17 & 3.63 & 0.97 \\
\hline 6 & 22 & 8.83 & 0.17 & 0.86 & 0.97 & 1.03 & 3.56 & 0.86 \\
\hline 6 & 23 & 8.83 & 0.19 & 0.76 & 0.96 & 0.91 & 3.47 & 0.76 \\
\hline 6 & 24 & 8.83 & 0.2 & 0.67 & 0.96 & 0.81 & 3.38 & 0.67 \\
\hline 6 & 25 & 8.83 & 0.22 & 0.59 & 0.95 & 0.73 & 3.29 & 0.59 \\
\hline 6 & 26 & 8.83 & 0.23 & 0.54 & 0.95 & 0.66 & 3.21 & 0.54 \\
\hline 6 & 27 & 8.83 & 0.25 & 0.48 & 0.94 & 0.6 & 3.11 & 0.48 \\
\hline 6 & 28 & 8.83 & 0.26 & 0.43 & 0.93 & 0.55 & 3 & 0.43 \\
\hline 6 & 29 & 8.83 & 0.29 & 0.39 & 0.92 & 0.5 & 2.9 & 0.39 \\
\hline 6 & 30 & 8.83 & 0.32 & 0.35 & 0.9 & 0.47 & 2.79 & 0.35 \\
\hline 6 & 31 & 8.83 & 0.34 & 0.32 & 0.89 & 0.43 & 2.67 & 0.32 \\
\hline 6 & 32 & 8.83 & 0.37 & 0.29 & 0.87 & 0.4 & 2.55 & 0.29 \\
\hline 6 & 33 & 8.83 & 0.4 & 0.26 & 0.85 & 0.37 & 2.44 & 0.26 \\
\hline 6 & 34 & 8.83 & 0.44 & 0.23 & 0.82 & 0.35 & 2.31 & 0.23 \\
\hline 6 & 35 & 8.83 & 0.48 & 0.21 & 0.8 & 0.32 & 2.18 & 0.21 \\
\hline 6 & 36 & 8.83 & 0.51 & 0.19 & 0.77 & 0.3 & 2.05 & 0.19 \\
\hline 6 & 37 & 8.83 & 0.55 & 0.17 & 0.73 & 0.29 & 1.91 & 0.17 \\
\hline 6 & 38 & 8.83 & 0.59 & 0.15 & 0.69 & 0.27 & 1.76 & 0.15 \\
\hline 6 & 39 & 8.83 & 0.63 & 0.14 & 0.65 & 0.26 & 1.62 & 0.14 \\
\hline 6 & 40 & 8.83 & 0.67 & 0.12 & 0.6 & 0.24 & 1.47 & 0.12 \\
\hline 6 & 41 & 8.83 & 0.72 & 0.11 & 0.54 & 0.24 & 1.29 & 0.11 \\
\hline 6 & 42 & 8.83 & 0.76 & 0.09 & 0.48 & 0.23 & 1.14 & 0.09 \\
\hline 6 & 43 & 8.83 & 0.8 & 0.08 & 0.42 & 0.23 & 0.98 & 0.08 \\
\hline & & & & & & & \\
\hline
\end{tabular}




\begin{tabular}{|c|c|c|c|c|c|c|c|}
\hline$Q^{2}$ bin & $W$ bin & $A_{1}+\eta A_{2}$ & $\sigma_{\text {stat }}$ & $\sigma_{\text {sys }}$ & $x_{\text {avg }}$ & $Q_{\text {avg }}^{2}$ & $W_{\text {avg }}$ \\
\hline 7 & 19 & -0.314 & 0.201 & 0.054 & 0.7 & 0.96 & 1.13 \\
\hline 7 & 20 & -0.281 & 0.044 & 0.027 & 0.64 & 0.93 & 1.18 \\
\hline 7 & 21 & -0.195 & 0.026 & 0.014 & 0.59 & 0.93 & 1.23 \\
\hline 7 & 22 & 0.159 & 0.03 & 0.015 & 0.54 & 0.93 & 1.29 \\
\hline 7 & 23 & 0.419 & 0.034 & 0.019 & 0.5 & 0.94 & 1.35 \\
\hline 7 & 24 & 0.557 & 0.031 & 0.023 & 0.45 & 0.94 & 1.41 \\
\hline 7 & 25 & 0.685 & 0.023 & 0.029 & 0.42 & 0.94 & 1.47 \\
\hline 7 & 26 & 0.726 & 0.02 & 0.03 & 0.39 & 0.94 & 1.53 \\
\hline 7 & 27 & 0.61 & 0.021 & 0.025 & 0.36 & 0.93 & 1.59 \\
\hline 7 & 28 & 0.473 & 0.018 & 0.021 & 0.34 & 0.95 & 1.65 \\
\hline 7 & 29 & 0.528 & 0.016 & 0.02 & 0.31 & 0.94 & 1.71 \\
\hline 7 & 30 & 0.4 & 0.016 & 0.017 & 0.29 & 0.94 & 1.77 \\
\hline 7 & 31 & 0.334 & 0.016 & 0.014 & 0.27 & 0.93 & 1.83 \\
\hline 7 & 32 & 0.284 & 0.015 & 0.013 & 0.26 & 0.94 & 1.89 \\
\hline 7 & 33 & 0.29 & 0.014 & 0.013 & 0.24 & 0.95 & 1.95 \\
\hline 7 & 34 & 0.318 & 0.013 & 0.012 & 0.23 & 0.95 & 2.01 \\
\hline 7 & 35 & 0.31 & 0.012 & 0.012 & 0.22 & 0.95 & 2.07 \\
\hline 7 & 36 & 0.32 & 0.011 & 0.011 & 0.2 & 0.95 & 2.13 \\
\hline 7 & 37 & 0.275 & 0.01 & 0.011 & 0.2 & 0.96 & 2.19 \\
\hline 7 & 38 & 0.264 & 0.01 & 0.01 & 0.19 & 0.96 & 2.25 \\
\hline 7 & 39 & 0.261 & 0.009 & 0.01 & 0.18 & 0.96 & 2.31 \\
\hline 7 & 40 & 0.252 & 0.009 & 0.009 & 0.17 & 0.95 & 2.37 \\
\hline 7 & 41 & 0.183 & 0.009 & 0.008 & 0.15 & 0.87 & 2.43 \\
\hline 7 & 42 & 0.179 & 0.009 & 0.007 & 0.14 & 0.83 & 2.49 \\
\hline 7 & 43 & 0.123 & 0.018 & 0.008 & 0.12 & 0.74 & 2.53 \\
\hline & & & & & & & \\
\hline
\end{tabular}




\begin{tabular}{|c|c|c|c|c|c|c|c|c|}
\hline$Q^{2}$ bin & $W$ bin & $s_{\text {avg }}$ & $D_{\text {avg }}$ & $\eta_{\text {avg }}$ & $\epsilon_{\text {avg }}$ & $\gamma_{\text {avg }}$ & $E_{\text {avg }}^{\prime}$ & $\theta_{\text {avg }}$ \\
\hline 7 & 19 & 8.83 & 0.2 & 1.05 & 0.95 & 1.36 & 3.52 & 1.05 \\
\hline 7 & 20 & 8.83 & 0.21 & 0.97 & 0.95 & 1.25 & 3.46 & 0.97 \\
\hline 7 & 21 & 8.83 & 0.23 & 0.9 & 0.95 & 1.15 & 3.4 & 0.9 \\
\hline 7 & 22 & 8.83 & 0.24 & 0.81 & 0.94 & 1.05 & 3.32 & 0.81 \\
\hline 7 & 23 & 8.83 & 0.25 & 0.74 & 0.93 & 0.96 & 3.23 & 0.74 \\
\hline 7 & 24 & 8.83 & 0.27 & 0.67 & 0.92 & 0.88 & 3.14 & 0.67 \\
\hline 7 & 25 & 8.83 & 0.28 & 0.61 & 0.91 & 0.81 & 3.05 & 0.61 \\
\hline 7 & 26 & 8.83 & 0.3 & 0.56 & 0.9 & 0.75 & 2.96 & 0.56 \\
\hline 7 & 27 & 8.83 & 0.32 & 0.51 & 0.89 & 0.7 & 2.86 & 0.51 \\
\hline 7 & 28 & 8.83 & 0.34 & 0.46 & 0.88 & 0.65 & 2.74 & 0.46 \\
\hline 7 & 29 & 8.83 & 0.36 & 0.42 & 0.86 & 0.6 & 2.64 & 0.42 \\
\hline 7 & 30 & 8.83 & 0.39 & 0.39 & 0.84 & 0.57 & 2.53 & 0.39 \\
\hline 7 & 31 & 8.83 & 0.41 & 0.35 & 0.82 & 0.53 & 2.42 & 0.35 \\
\hline 7 & 32 & 8.83 & 0.44 & 0.32 & 0.8 & 0.5 & 2.3 & 0.32 \\
\hline 7 & 33 & 8.83 & 0.48 & 0.29 & 0.77 & 0.47 & 2.17 & 0.29 \\
\hline 7 & 34 & 8.83 & 0.52 & 0.27 & 0.74 & 0.44 & 2.04 & 0.27 \\
\hline 7 & 35 & 8.83 & 0.56 & 0.24 & 0.71 & 0.42 & 1.91 & 0.24 \\
\hline 7 & 36 & 8.83 & 0.6 & 0.22 & 0.68 & 0.39 & 1.78 & 0.22 \\
\hline 7 & 37 & 8.83 & 0.64 & 0.19 & 0.63 & 0.37 & 1.64 & 0.19 \\
\hline 7 & 38 & 8.83 & 0.68 & 0.17 & 0.59 & 0.35 & 1.5 & 0.17 \\
\hline 7 & 39 & 8.83 & 0.72 & 0.15 & 0.54 & 0.34 & 1.35 & 0.15 \\
\hline 7 & 40 & 8.83 & 0.76 & 0.13 & 0.49 & 0.32 & 1.2 & 0.13 \\
\hline 7 & 41 & 8.83 & 0.78 & 0.11 & 0.45 & 0.3 & 1.09 & 0.11 \\
\hline 7 & 42 & 8.83 & 0.81 & 0.09 & 0.4 & 0.28 & 0.96 & 0.09 \\
\hline 7 & 43 & 8.83 & 0.83 & 0.08 & 0.38 & 0.26 & 0.89 & 0.08 \\
\hline & & & & & & & \\
\hline
\end{tabular}




\begin{tabular}{|c|c|c|c|c|c|c|c|}
\hline$Q^{2}$ bin & $W$ bin & $A_{1}+\eta A_{2}$ & $\sigma_{\text {stat }}$ & $\sigma_{\text {sys }}$ & $x_{\text {avg }}$ & $Q_{\text {avg }}^{2}$ & $W_{\text {avg }}$ \\
\hline 8 & 19 & -0.717 & 0.265 & 0.071 & 0.82 & 1.79 & 1.12 \\
\hline 8 & 20 & -0.382 & 0.063 & 0.029 & 0.77 & 1.77 & 1.18 \\
\hline 8 & 21 & -0.062 & 0.04 & 0.019 & 0.73 & 1.79 & 1.23 \\
\hline 8 & 22 & 0.251 & 0.038 & 0.017 & 0.69 & 1.82 & 1.29 \\
\hline 8 & 23 & 0.64 & 0.037 & 0.026 & 0.66 & 1.85 & 1.35 \\
\hline 8 & 24 & 0.668 & 0.034 & 0.026 & 0.62 & 1.88 & 1.41 \\
\hline 8 & 25 & 0.813 & 0.026 & 0.029 & 0.58 & 1.84 & 1.47 \\
\hline 8 & 26 & 0.896 & 0.022 & 0.033 & 0.55 & 1.86 & 1.53 \\
\hline 8 & 27 & 0.721 & 0.022 & 0.029 & 0.53 & 1.87 & 1.59 \\
\hline 8 & 28 & 0.647 & 0.018 & 0.024 & 0.5 & 1.88 & 1.65 \\
\hline 8 & 29 & 0.712 & 0.016 & 0.024 & 0.47 & 1.88 & 1.71 \\
\hline 8 & 30 & 0.654 & 0.016 & 0.022 & 0.45 & 1.86 & 1.77 \\
\hline 8 & 31 & 0.506 & 0.016 & 0.019 & 0.42 & 1.83 & 1.83 \\
\hline 8 & 32 & 0.41 & 0.015 & 0.017 & 0.4 & 1.78 & 1.89 \\
\hline 8 & 33 & 0.455 & 0.015 & 0.017 & 0.37 & 1.73 & 1.95 \\
\hline 8 & 34 & 0.444 & 0.015 & 0.016 & 0.35 & 1.7 & 2.01 \\
\hline 8 & 35 & 0.486 & 0.015 & 0.016 & 0.32 & 1.59 & 2.07 \\
\hline 8 & 36 & 0.405 & 0.015 & 0.013 & 0.3 & 1.57 & 2.13 \\
\hline 8 & 37 & 0.393 & 0.016 & 0.013 & 0.28 & 1.53 & 2.19 \\
\hline 8 & 38 & 0.399 & 0.019 & 0.012 & 0.25 & 1.43 & 2.25 \\
\hline 8 & 39 & 0.299 & 0.032 & 0.012 & 0.24 & 1.41 & 2.29 \\
\hline
\end{tabular}




\begin{tabular}{|c|c|c|c|c|c|c|c|c|}
\hline$Q^{2}$ bin & $W$ bin & $s_{\text {avg }}$ & $D_{\text {avg }}$ & $\eta_{\text {avg }}$ & $\epsilon_{\text {avg }}$ & $\gamma_{\text {avg }}$ & $E_{\text {avg }}^{\prime}$ & $\theta_{\text {avg }}$ \\
\hline 8 & 19 & 8.83 & 0.32 & 0.8 & 0.89 & 1.16 & 3.08 & 0.8 \\
\hline 8 & 20 & 8.83 & 0.33 & 0.75 & 0.88 & 1.1 & 3.02 & 0.75 \\
\hline 8 & 21 & 8.83 & 0.35 & 0.71 & 0.87 & 1.04 & 2.94 & 0.71 \\
\hline 8 & 22 & 8.83 & 0.37 & 0.66 & 0.86 & 0.97 & 2.85 & 0.66 \\
\hline 8 & 23 & 8.83 & 0.4 & 0.6 & 0.84 & 0.91 & 2.75 & 0.6 \\
\hline 8 & 24 & 8.83 & 0.42 & 0.55 & 0.83 & 0.86 & 2.64 & 0.55 \\
\hline 8 & 25 & 8.83 & 0.42 & 0.52 & 0.82 & 0.81 & 2.57 & 0.52 \\
\hline 8 & 26 & 8.83 & 0.45 & 0.48 & 0.8 & 0.77 & 2.47 & 0.48 \\
\hline 8 & 27 & 8.83 & 0.49 & 0.44 & 0.78 & 0.73 & 2.36 & 0.44 \\
\hline 8 & 28 & 8.83 & 0.51 & 0.41 & 0.75 & 0.69 & 2.25 & 0.41 \\
\hline 8 & 29 & 8.83 & 0.53 & 0.37 & 0.73 & 0.65 & 2.14 & 0.37 \\
\hline 8 & 30 & 8.83 & 0.55 & 0.35 & 0.71 & 0.62 & 2.04 & 0.35 \\
\hline 8 & 31 & 8.83 & 0.57 & 0.32 & 0.68 & 0.59 & 1.94 & 0.32 \\
\hline 8 & 32 & 8.83 & 0.59 & 0.29 & 0.66 & 0.56 & 1.85 & 0.29 \\
\hline 8 & 33 & 8.83 & 0.62 & 0.27 & 0.64 & 0.53 & 1.76 & 0.27 \\
\hline 8 & 34 & 8.83 & 0.66 & 0.24 & 0.61 & 0.5 & 1.64 & 0.24 \\
\hline 8 & 35 & 8.83 & 0.68 & 0.22 & 0.59 & 0.47 & 1.57 & 0.22 \\
\hline 8 & 36 & 8.83 & 0.72 & 0.2 & 0.55 & 0.45 & 1.45 & 0.2 \\
\hline 8 & 37 & 8.83 & 0.75 & 0.18 & 0.52 & 0.43 & 1.34 & 0.18 \\
\hline 8 & 38 & 8.83 & 0.77 & 0.16 & 0.49 & 0.4 & 1.24 & 0.16 \\
\hline 8 & 39 & 8.83 & 0.79 & 0.15 & 0.45 & 0.39 & 1.15 & 0.15 \\
\hline
\end{tabular}




\begin{tabular}{|c|c|c|c|c|c|c|c|}
\hline$Q^{2}$ bin & $W$ bin & $A_{1}+\eta A_{2}$ & $\sigma_{\text {stat }}$ & $\sigma_{\text {sys }}$ & $x_{\text {avg }}$ & $Q_{\text {avg }}^{2}$ & $W_{\text {avg }}$ \\
\hline 9 & 19 & -0.493 & 0.495 & 0.08 & 0.88 & 2.88 & 1.13 \\
\hline 9 & 20 & -0.512 & 0.218 & 0.042 & 0.85 & 2.88 & 1.18 \\
\hline 9 & 21 & 0.046 & 0.1 & 0.029 & 0.82 & 2.88 & 1.23 \\
\hline 9 & 22 & 0.497 & 0.095 & 0.02 & 0.79 & 2.88 & 1.29 \\
\hline 9 & 23 & 0.638 & 0.084 & 0.029 & 0.75 & 2.88 & 1.35 \\
\hline 9 & 24 & 0.716 & 0.078 & 0.027 & 0.72 & 2.88 & 1.41 \\
\hline 9 & 25 & 0.823 & 0.06 & 0.03 & 0.69 & 2.87 & 1.47 \\
\hline 9 & 26 & 0.849 & 0.058 & 0.034 & 0.66 & 2.86 & 1.52 \\
\hline 9 & 27 & 0.661 & 0.063 & 0.03 & 0.64 & 2.86 & 1.58 \\
\hline
\end{tabular}

\begin{tabular}{|c|c|c|c|c|c|c|c|c|}
\hline$Q^{2}$ bin & $W$ bin & $s_{\text {avg }}$ & $D_{\text {avg }}$ & $\eta_{\text {avg }}$ & $\epsilon_{\text {avg }}$ & $\gamma_{\text {avg }}$ & $E_{\text {avg }}^{\prime}$ & $\theta_{\text {avg }}$ \\
\hline 9 & 19 & 8.83 & 0.46 & 0.56 & 0.77 & 0.97 & 2.49 & 0.56 \\
\hline 9 & 20 & 8.83 & 0.47 & 0.54 & 0.76 & 0.94 & 2.43 & 0.54 \\
\hline 9 & 21 & 8.83 & 0.5 & 0.51 & 0.74 & 0.91 & 2.36 & 0.51 \\
\hline 9 & 22 & 8.83 & 0.54 & 0.48 & 0.73 & 0.87 & 2.28 & 0.48 \\
\hline 9 & 23 & 8.83 & 0.58 & 0.45 & 0.71 & 0.83 & 2.2 & 0.45 \\
\hline 9 & 24 & 8.83 & 0.6 & 0.42 & 0.69 & 0.8 & 2.1 & 0.42 \\
\hline 9 & 25 & 8.83 & 0.6 & 0.39 & 0.67 & 0.77 & 2.02 & 0.39 \\
\hline 9 & 26 & 8.83 & 0.61 & 0.37 & 0.65 & 0.74 & 1.94 & 0.37 \\
\hline 9 & 27 & 8.83 & 0.66 & 0.34 & 0.62 & 0.71 & 1.85 & 0.34 \\
\hline
\end{tabular}


Table B.9: Values of $A_{1}+\eta A_{2}$ for $E=5.7 \mathrm{GeV}$ data, with statistical and systematic errors. Average kinematic values in each bin are also shown.

\begin{tabular}{|c|c|c|c|c|c|c|c|}
\hline$Q^{2}$ bin & $W$ bin & $A_{1}+\eta A_{2}$ & $\sigma_{\text {stat }}$ & $\sigma_{\text {sys }}$ & $x_{\text {avg }}$ & $Q_{\text {avg }}^{2}$ & $W_{\text {avg }}$ \\
\hline 6 & 30 & 0.2 & 0.343 & 0.022 & 0.22 & 0.63 & 1.78 \\
\hline 6 & 31 & 0.133 & 0.152 & 0.02 & 0.2 & 0.62 & 1.84 \\
\hline 6 & 32 & 0.122 & 0.092 & 0.018 & 0.18 & 0.61 & 1.9 \\
\hline 6 & 33 & -0.03 & 0.062 & 0.015 & 0.17 & 0.6 & 1.95 \\
\hline 6 & 34 & 0.088 & 0.05 & 0.014 & 0.16 & 0.58 & 2.01 \\
\hline 6 & 35 & 0.11 & 0.038 & 0.014 & 0.14 & 0.57 & 2.07 \\
\hline 6 & 36 & 0.18 & 0.032 & 0.014 & 0.13 & 0.56 & 2.13 \\
\hline 6 & 37 & 0.156 & 0.028 & 0.013 & 0.12 & 0.55 & 2.19 \\
\hline 6 & 38 & 0.206 & 0.025 & 0.012 & 0.12 & 0.55 & 2.25 \\
\hline 6 & 39 & 0.163 & 0.022 & 0.01 & 0.11 & 0.55 & 2.31 \\
\hline 6 & 40 & 0.127 & 0.02 & 0.008 & 0.1 & 0.54 & 2.37 \\
\hline 6 & 41 & 0.145 & 0.02 & 0.007 & 0.1 & 0.54 & 2.43 \\
\hline 6 & 42 & 0.119 & 0.019 & 0.008 & 0.09 & 0.54 & 2.49 \\
\hline 6 & 43 & 0.115 & 0.018 & 0.008 & 0.09 & 0.54 & 2.55 \\
\hline 6 & 44 & 0.156 & 0.018 & 0.007 & 0.08 & 0.54 & 2.61 \\
\hline 6 & 45 & 0.133 & 0.017 & 0.007 & 0.08 & 0.54 & 2.67 \\
\hline 6 & 46 & 0.118 & 0.017 & 0.006 & 0.08 & 0.54 & 2.73 \\
\hline 6 & 47 & 0.107 & 0.017 & 0.007 & 0.07 & 0.54 & 2.79 \\
\hline 6 & 48 & 0.136 & 0.017 & 0.007 & 0.07 & 0.54 & 2.85 \\
\hline 6 & 49 & 0.132 & 0.017 & 0.007 & 0.07 & 0.54 & 2.91 \\
\hline 6 & 50 & 0.101 & 0.021 & 0.004 & 0.06 & 0.52 & 2.96 \\
\hline & & & & & & & \\
\hline
\end{tabular}




\begin{tabular}{|c|c|c|c|c|c|c|c|c|}
\hline$Q^{2}$ bin & $W$ bin & $s_{\text {avg }}$ & $D_{\text {avg }}$ & $\eta_{\text {avg }}$ & $\epsilon_{\text {avg }}$ & $\gamma_{\text {avg }}$ & $E_{\text {avg }}^{\prime}$ & $\theta_{\text {avg }}$ \\
\hline 6 & 30 & 11.62 & 0.24 & 0.41 & 0.94 & 0.51 & 4.16 & 0.41 \\
\hline 6 & 31 & 11.63 & 0.26 & 0.38 & 0.93 & 0.47 & 4.07 & 0.38 \\
\hline 6 & 32 & 11.63 & 0.27 & 0.35 & 0.92 & 0.44 & 3.96 & 0.35 \\
\hline 6 & 33 & 11.63 & 0.29 & 0.32 & 0.91 & 0.41 & 3.85 & 0.32 \\
\hline 6 & 34 & 11.63 & 0.32 & 0.29 & 0.9 & 0.38 & 3.73 & 0.29 \\
\hline 6 & 35 & 11.63 & 0.34 & 0.27 & 0.89 & 0.35 & 3.61 & 0.27 \\
\hline 6 & 36 & 11.63 & 0.37 & 0.24 & 0.88 & 0.33 & 3.48 & 0.24 \\
\hline 6 & 37 & 11.63 & 0.4 & 0.22 & 0.86 & 0.31 & 3.35 & 0.22 \\
\hline 6 & 38 & 11.63 & 0.42 & 0.2 & 0.84 & 0.29 & 3.21 & 0.2 \\
\hline 6 & 39 & 11.63 & 0.45 & 0.19 & 0.82 & 0.28 & 3.06 & 0.19 \\
\hline 6 & 40 & 11.63 & 0.48 & 0.17 & 0.8 & 0.26 & 2.91 & 0.17 \\
\hline 6 & 41 & 11.63 & 0.51 & 0.16 & 0.77 & 0.25 & 2.76 & 0.16 \\
\hline 6 & 42 & 11.63 & 0.54 & 0.14 & 0.74 & 0.24 & 2.6 & 0.14 \\
\hline 6 & 43 & 11.63 & 0.57 & 0.13 & 0.71 & 0.22 & 2.44 & 0.13 \\
\hline 6 & 44 & 11.63 & 0.6 & 0.12 & 0.68 & 0.21 & 2.28 & 0.12 \\
\hline 6 & 45 & 11.63 & 0.64 & 0.11 & 0.64 & 0.2 & 2.11 & 0.11 \\
\hline 6 & 46 & 11.63 & 0.67 & 0.1 & 0.6 & 0.19 & 1.94 & 0.1 \\
\hline 6 & 47 & 11.63 & 0.7 & 0.08 & 0.55 & 0.18 & 1.76 & 0.08 \\
\hline 6 & 48 & 11.63 & 0.74 & 0.07 & 0.5 & 0.18 & 1.58 & 0.07 \\
\hline 6 & 49 & 11.63 & 0.77 & 0.06 & 0.45 & 0.17 & 1.4 & 0.06 \\
\hline 6 & 50 & 11.63 & 0.8 & 0.06 & 0.4 & 0.16 & 1.24 & 0.06 \\
\hline
\end{tabular}




\begin{tabular}{|c|c|c|c|c|c|c|c|}
\hline$Q^{2}$ bin & $W$ bin & $A_{1}+\eta A_{2}$ & $\sigma_{\text {stat }}$ & $\sigma_{\text {sys }}$ & $x_{\text {avg }}$ & $Q_{\text {avg }}^{2}$ & $W_{\text {avg }}$ \\
\hline 7 & 19 & -1.483 & 0.468 & 0.099 & 0.73 & 1.06 & 1.13 \\
\hline 7 & 20 & -0.397 & 0.122 & 0.021 & 0.67 & 1.04 & 1.18 \\
\hline 7 & 21 & -0.264 & 0.071 & 0.026 & 0.62 & 1.03 & 1.23 \\
\hline 7 & 22 & 0.154 & 0.076 & 0.026 & 0.57 & 1.03 & 1.29 \\
\hline 7 & 23 & 0.327 & 0.084 & 0.024 & 0.52 & 1.02 & 1.35 \\
\hline 7 & 24 & 0.566 & 0.071 & 0.034 & 0.48 & 1.04 & 1.41 \\
\hline 7 & 25 & 0.743 & 0.055 & 0.039 & 0.44 & 1.01 & 1.47 \\
\hline 7 & 26 & 0.574 & 0.045 & 0.029 & 0.41 & 1.01 & 1.53 \\
\hline 7 & 27 & 0.589 & 0.044 & 0.029 & 0.38 & 1.01 & 1.59 \\
\hline 7 & 28 & 0.529 & 0.039 & 0.026 & 0.35 & 1.01 & 1.65 \\
\hline 7 & 29 & 0.469 & 0.033 & 0.025 & 0.32 & 0.99 & 1.71 \\
\hline 7 & 30 & 0.378 & 0.031 & 0.024 & 0.3 & 0.99 & 1.77 \\
\hline 7 & 31 & 0.293 & 0.029 & 0.02 & 0.28 & 0.97 & 1.83 \\
\hline 7 & 32 & 0.216 & 0.027 & 0.018 & 0.26 & 0.96 & 1.89 \\
\hline 7 & 33 & 0.309 & 0.023 & 0.016 & 0.25 & 0.96 & 1.95 \\
\hline 7 & 34 & 0.281 & 0.022 & 0.016 & 0.23 & 0.95 & 2.01 \\
\hline 7 & 35 & 0.317 & 0.019 & 0.016 & 0.22 & 0.94 & 2.07 \\
\hline 7 & 36 & 0.291 & 0.018 & 0.015 & 0.2 & 0.94 & 2.13 \\
\hline 7 & 37 & 0.274 & 0.017 & 0.013 & 0.19 & 0.93 & 2.19 \\
\hline 7 & 38 & 0.245 & 0.016 & 0.013 & 0.18 & 0.94 & 2.25 \\
\hline 7 & 39 & 0.255 & 0.015 & 0.012 & 0.17 & 0.94 & 2.31 \\
\hline 7 & 40 & 0.208 & 0.015 & 0.01 & 0.16 & 0.93 & 2.37 \\
\hline 7 & 41 & 0.225 & 0.014 & 0.009 & 0.16 & 0.94 & 2.43 \\
\hline 7 & 42 & 0.2 & 0.014 & 0.009 & 0.15 & 0.94 & 2.49 \\
\hline 7 & 43 & 0.214 & 0.014 & 0.009 & 0.14 & 0.94 & 2.55 \\
\hline 7 & 44 & 0.223 & 0.013 & 0.009 & 0.14 & 0.95 & 2.61 \\
\hline 7 & 45 & 0.218 & 0.013 & 0.009 & 0.13 & 0.94 & 2.67 \\
\hline 7 & 46 & 0.2 & 0.013 & 0.009 & 0.13 & 0.95 & 2.73 \\
\hline 7 & 47 & 0.174 & 0.012 & 0.009 & 0.12 & 0.96 & 2.79 \\
\hline 7 & 48 & 0.175 & 0.015 & 0.009 & 0.12 & 0.96 & 2.84 \\
\hline & & & & & & & \\
\hline
\end{tabular}




\begin{tabular}{|c|c|c|c|c|c|c|c|c|}
\hline$Q^{2}$ bin & $W$ bin & $s_{\text {avg }}$ & $D_{\text {avg }}$ & $\eta_{\text {avg }}$ & $\epsilon_{\text {avg }}$ & $\gamma_{\text {avg }}$ & $E_{\text {avg }}^{\prime}$ & $\theta_{\text {avg }}$ \\
\hline 7 & 19 & 11.64 & 0.15 & 1.1 & 0.97 & 1.33 & 4.96 & 1.1 \\
\hline 7 & 20 & 11.63 & 0.16 & 1.01 & 0.97 & 1.23 & 4.9 & 1.01 \\
\hline 7 & 21 & 11.63 & 0.17 & 0.94 & 0.97 & 1.14 & 4.84 & 0.94 \\
\hline 7 & 22 & 11.63 & 0.18 & 0.86 & 0.96 & 1.05 & 4.77 & 0.86 \\
\hline 7 & 23 & 11.63 & 0.18 & 0.79 & 0.96 & 0.96 & 4.68 & 0.79 \\
\hline 7 & 24 & 11.63 & 0.2 & 0.72 & 0.96 & 0.88 & 4.58 & 0.72 \\
\hline 7 & 25 & 11.63 & 0.2 & 0.66 & 0.95 & 0.82 & 4.5 & 0.66 \\
\hline 7 & 26 & 11.63 & 0.22 & 0.61 & 0.95 & 0.76 & 4.41 & 0.61 \\
\hline 7 & 27 & 11.63 & 0.23 & 0.56 & 0.94 & 0.71 & 4.31 & 0.56 \\
\hline 7 & 28 & 11.63 & 0.24 & 0.52 & 0.93 & 0.66 & 4.21 & 0.52 \\
\hline 7 & 29 & 11.63 & 0.25 & 0.48 & 0.93 & 0.61 & 4.11 & 0.48 \\
\hline 7 & 30 & 11.63 & 0.27 & 0.44 & 0.92 & 0.57 & 4 & 0.44 \\
\hline 7 & 31 & 11.63 & 0.29 & 0.41 & 0.91 & 0.53 & 3.89 & 0.41 \\
\hline 7 & 32 & 11.63 & 0.31 & 0.38 & 0.9 & 0.5 & 3.78 & 0.38 \\
\hline 7 & 33 & 11.62 & 0.33 & 0.35 & 0.89 & 0.47 & 3.66 & 0.35 \\
\hline 7 & 34 & 11.62 & 0.36 & 0.32 & 0.87 & 0.44 & 3.53 & 0.32 \\
\hline 7 & 35 & 11.62 & 0.39 & 0.3 & 0.86 & 0.42 & 3.41 & 0.3 \\
\hline 7 & 36 & 11.62 & 0.42 & 0.27 & 0.84 & 0.39 & 3.27 & 0.27 \\
\hline 7 & 37 & 11.62 & 0.45 & 0.25 & 0.82 & 0.37 & 3.14 & 0.25 \\
\hline 7 & 38 & 11.62 & 0.48 & 0.23 & 0.8 & 0.35 & 2.99 & 0.23 \\
\hline 7 & 39 & 11.62 & 0.51 & 0.21 & 0.78 & 0.33 & 2.85 & 0.21 \\
\hline 7 & 40 & 11.62 & 0.54 & 0.19 & 0.75 & 0.32 & 2.7 & 0.19 \\
\hline 7 & 41 & 11.62 & 0.57 & 0.18 & 0.72 & 0.3 & 2.54 & 0.18 \\
\hline 7 & 42 & 11.62 & 0.6 & 0.16 & 0.69 & 0.29 & 2.38 & 0.16 \\
\hline 7 & 43 & 11.61 & 0.63 & 0.15 & 0.65 & 0.28 & 2.22 & 0.15 \\
\hline 7 & 44 & 11.61 & 0.66 & 0.13 & 0.61 & 0.26 & 2.05 & 0.13 \\
\hline 7 & 45 & 11.61 & 0.69 & 0.12 & 0.57 & 0.25 & 1.89 & 0.12 \\
\hline 7 & 46 & 11.61 & 0.72 & 0.11 & 0.53 & 0.24 & 1.71 & 0.11 \\
\hline 7 & 47 & 11.61 & 0.76 & 0.09 & 0.48 & 0.23 & 1.53 & 0.09 \\
\hline 7 & 48 & 11.6 & 0.78 & 0.08 & 0.43 & 0.22 & 1.37 & 0.08 \\
\hline
\end{tabular}




\begin{tabular}{|c|c|c|c|c|c|c|c|}
\hline$Q^{2}$ bin & $W$ bin & $A_{1}+\eta A_{2}$ & $\sigma_{\text {stat }}$ & $\sigma_{\text {sys }}$ & $x_{\text {avg }}$ & $Q_{\text {avg }}^{2}$ & $W_{\text {avg }}$ \\
\hline 8 & 19 & -0.438 & 0.415 & 0.175 & 0.83 & 1.91 & 1.12 \\
\hline 8 & 20 & -0.16 & 0.119 & 0.033 & 0.77 & 1.77 & 1.18 \\
\hline 8 & 21 & -0.172 & 0.068 & 0.022 & 0.73 & 1.79 & 1.23 \\
\hline 8 & 22 & 0.317 & 0.067 & 0.03 & 0.69 & 1.82 & 1.29 \\
\hline 8 & 23 & 0.36 & 0.064 & 0.027 & 0.66 & 1.88 & 1.35 \\
\hline 8 & 24 & 0.692 & 0.059 & 0.034 & 0.62 & 1.87 & 1.41 \\
\hline 8 & 25 & 0.8 & 0.044 & 0.037 & 0.58 & 1.86 & 1.47 \\
\hline 8 & 26 & 0.768 & 0.038 & 0.031 & 0.55 & 1.84 & 1.53 \\
\hline 8 & 27 & 0.601 & 0.037 & 0.03 & 0.52 & 1.83 & 1.59 \\
\hline 8 & 28 & 0.631 & 0.032 & 0.028 & 0.5 & 1.86 & 1.65 \\
\hline 8 & 29 & 0.696 & 0.028 & 0.029 & 0.47 & 1.85 & 1.71 \\
\hline 8 & 30 & 0.633 & 0.028 & 0.028 & 0.45 & 1.86 & 1.77 \\
\hline 8 & 31 & 0.499 & 0.027 & 0.024 & 0.43 & 1.87 & 1.83 \\
\hline 8 & 32 & 0.436 & 0.025 & 0.02 & 0.4 & 1.86 & 1.89 \\
\hline 8 & 33 & 0.438 & 0.023 & 0.019 & 0.39 & 1.87 & 1.95 \\
\hline 8 & 34 & 0.409 & 0.021 & 0.019 & 0.37 & 1.87 & 2.01 \\
\hline 8 & 35 & 0.456 & 0.019 & 0.019 & 0.35 & 1.88 & 2.07 \\
\hline 8 & 36 & 0.425 & 0.019 & 0.018 & 0.33 & 1.86 & 2.13 \\
\hline 8 & 37 & 0.425 & 0.017 & 0.016 & 0.32 & 1.87 & 2.19 \\
\hline 8 & 38 & 0.439 & 0.017 & 0.016 & 0.31 & 1.88 & 2.25 \\
\hline 8 & 39 & 0.393 & 0.016 & 0.015 & 0.29 & 1.88 & 2.31 \\
\hline 8 & 40 & 0.388 & 0.015 & 0.014 & 0.28 & 1.89 & 2.37 \\
\hline 8 & 41 & 0.375 & 0.015 & 0.013 & 0.27 & 1.9 & 2.43 \\
\hline 8 & 42 & 0.374 & 0.015 & 0.013 & 0.26 & 1.89 & 2.49 \\
\hline 8 & 43 & 0.341 & 0.016 & 0.012 & 0.23 & 1.73 & 2.55 \\
\hline 8 & 44 & 0.333 & 0.017 & 0.012 & 0.22 & 1.66 & 2.61 \\
\hline 8 & 45 & 0.287 & 0.017 & 0.011 & 0.2 & 1.58 & 2.67 \\
\hline 8 & 46 & 0.284 & 0.017 & 0.011 & 0.19 & 1.57 & 2.73 \\
\hline 8 & 47 & 0.291 & 0.029 & 0.009 & 0.17 & 1.43 & 2.78 \\
\hline & & & & & & & \\
\hline
\end{tabular}




\begin{tabular}{|c|c|c|c|c|c|c|c|c|}
\hline$Q^{2}$ bin & $W$ bin & $s_{\text {avg }}$ & $D_{\text {avg }}$ & $\eta_{\text {avg }}$ & $\epsilon_{\text {avg }}$ & $\gamma_{\text {avg }}$ & $E_{\text {avg }}^{\prime}$ & $\theta_{\text {avg }}$ \\
\hline 8 & 19 & 11.6 & 0.23 & 0.87 & 0.94 & 1.14 & 4.49 & 0.87 \\
\hline 8 & 20 & 11.6 & 0.23 & 0.85 & 0.94 & 1.1 & 4.5 & 0.85 \\
\hline 8 & 21 & 11.6 & 0.25 & 0.79 & 0.93 & 1.03 & 4.42 & 0.79 \\
\hline 8 & 22 & 11.6 & 0.26 & 0.74 & 0.93 & 0.97 & 4.33 & 0.74 \\
\hline 8 & 23 & 11.6 & 0.28 & 0.68 & 0.92 & 0.91 & 4.21 & 0.68 \\
\hline 8 & 24 & 11.6 & 0.29 & 0.64 & 0.91 & 0.86 & 4.12 & 0.64 \\
\hline 8 & 25 & 11.6 & 0.3 & 0.6 & 0.9 & 0.81 & 4.04 & 0.6 \\
\hline 8 & 26 & 11.59 & 0.31 & 0.56 & 0.9 & 0.77 & 3.95 & 0.56 \\
\hline 8 & 27 & 11.6 & 0.34 & 0.53 & 0.89 & 0.73 & 3.86 & 0.53 \\
\hline 8 & 28 & 11.59 & 0.36 & 0.49 & 0.88 & 0.69 & 3.73 & 0.49 \\
\hline 8 & 29 & 11.59 & 0.37 & 0.46 & 0.87 & 0.65 & 3.64 & 0.46 \\
\hline 8 & 30 & 11.59 & 0.39 & 0.43 & 0.85 & 0.62 & 3.52 & 0.43 \\
\hline 8 & 31 & 11.59 & 0.41 & 0.4 & 0.84 & 0.59 & 3.4 & 0.4 \\
\hline 8 & 32 & 11.59 & 0.42 & 0.37 & 0.82 & 0.56 & 3.28 & 0.37 \\
\hline 8 & 33 & 11.59 & 0.45 & 0.35 & 0.8 & 0.53 & 3.15 & 0.35 \\
\hline 8 & 34 & 11.59 & 0.49 & 0.32 & 0.79 & 0.51 & 3.03 & 0.32 \\
\hline 8 & 35 & 11.59 & 0.52 & 0.3 & 0.76 & 0.48 & 2.89 & 0.3 \\
\hline 8 & 36 & 11.59 & 0.55 & 0.28 & 0.74 & 0.46 & 2.77 & 0.28 \\
\hline 8 & 37 & 11.59 & 0.59 & 0.25 & 0.72 & 0.44 & 2.62 & 0.25 \\
\hline 8 & 38 & 11.59 & 0.62 & 0.23 & 0.69 & 0.42 & 2.48 & 0.23 \\
\hline 8 & 39 & 11.59 & 0.65 & 0.21 & 0.66 & 0.4 & 2.33 & 0.21 \\
\hline 8 & 40 & 11.59 & 0.68 & 0.19 & 0.62 & 0.39 & 2.17 & 0.19 \\
\hline 8 & 41 & 11.59 & 0.71 & 0.18 & 0.59 & 0.37 & 2.02 & 0.18 \\
\hline 8 & 42 & 11.59 & 0.74 & 0.16 & 0.55 & 0.36 & 1.87 & 0.16 \\
\hline 8 & 43 & 11.59 & 0.75 & 0.15 & 0.53 & 0.33 & 1.79 & 0.15 \\
\hline 8 & 44 & 11.59 & 0.77 & 0.13 & 0.5 & 0.32 & 1.66 & 0.13 \\
\hline 8 & 45 & 11.59 & 0.78 & 0.12 & 0.47 & 0.3 & 1.53 & 0.12 \\
\hline 8 & 46 & 11.59 & 0.81 & 0.1 & 0.42 & 0.29 & 1.36 & 0.1 \\
\hline 8 & 47 & 11.59 & 0.82 & 0.09 & 0.4 & 0.27 & 1.3 & 0.09 \\
\hline & & & & & & & & \\
\hline
\end{tabular}




\begin{tabular}{|c|c|c|c|c|c|c|c|}
\hline$Q^{2}$ bin & $W$ bin & $A_{1}+\eta A_{2}$ & $\sigma_{\text {stat }}$ & $\sigma_{\text {sys }}$ & $x_{\text {avg }}$ & $Q_{\text {avg }}^{2}$ & $W_{\text {avg }}$ \\
\hline 9 & 19 & -1.762 & 0.448 & 0.185 & 0.91 & 4.14 & 1.12 \\
\hline 9 & 20 & -0.404 & 0.193 & 0.171 & 0.89 & 3.94 & 1.17 \\
\hline 9 & 21 & 0.298 & 0.125 & 0.039 & 0.85 & 3.74 & 1.23 \\
\hline 9 & 22 & 0.194 & 0.1 & 0.043 & 0.82 & 3.63 & 1.29 \\
\hline 9 & 23 & 0.411 & 0.084 & 0.055 & 0.79 & 3.76 & 1.35 \\
\hline 9 & 24 & 0.691 & 0.074 & 0.038 & 0.76 & 3.67 & 1.41 \\
\hline 9 & 25 & 0.667 & 0.06 & 0.036 & 0.74 & 3.72 & 1.47 \\
\hline 9 & 26 & 0.804 & 0.056 & 0.033 & 0.7 & 3.42 & 1.53 \\
\hline 9 & 27 & 0.729 & 0.056 & 0.031 & 0.67 & 3.38 & 1.59 \\
\hline 9 & 28 & 0.596 & 0.043 & 0.029 & 0.65 & 3.43 & 1.65 \\
\hline 9 & 29 & 0.729 & 0.038 & 0.034 & 0.62 & 3.41 & 1.71 \\
\hline 9 & 30 & 0.658 & 0.038 & 0.03 & 0.6 & 3.41 & 1.77 \\
\hline 9 & 31 & 0.651 & 0.038 & 0.027 & 0.58 & 3.4 & 1.83 \\
\hline 9 & 32 & 0.588 & 0.036 & 0.023 & 0.56 & 3.43 & 1.89 \\
\hline 9 & 33 & 0.535 & 0.034 & 0.023 & 0.53 & 3.31 & 1.95 \\
\hline 9 & 34 & 0.57 & 0.033 & 0.02 & 0.51 & 3.28 & 2.01 \\
\hline 9 & 35 & 0.626 & 0.032 & 0.022 & 0.48 & 3.17 & 2.07 \\
\hline 9 & 36 & 0.549 & 0.029 & 0.02 & 0.46 & 3.15 & 2.13 \\
\hline 9 & 37 & 0.549 & 0.031 & 0.018 & 0.44 & 3.09 & 2.19 \\
\hline 9 & 38 & 0.476 & 0.038 & 0.018 & 0.41 & 2.89 & 2.25 \\
\hline 9 & 39 & 0.524 & 0.034 & 0.017 & 0.39 & 2.89 & 2.31 \\
\hline 9 & 40 & 0.511 & 0.036 & 0.015 & 0.38 & 2.88 & 2.37 \\
\hline
\end{tabular}




\begin{tabular}{|c|c|c|c|c|c|c|c|c|}
\hline$Q^{2}$ bin & $W$ bin & $s_{\text {avg }}$ & $D_{\text {avg }}$ & $\eta_{\text {avg }}$ & $\epsilon_{\text {avg }}$ & $\gamma_{\text {avg }}$ & $E_{\text {avg }}^{\prime}$ & $\theta_{\text {avg }}$ \\
\hline 9 & 19 & 11.58 & 0.42 & 0.52 & 0.77 & 0.85 & 3.3 & 0.52 \\
\hline 9 & 20 & 11.59 & 0.41 & 0.52 & 0.79 & 0.85 & 3.35 & 0.52 \\
\hline 9 & 21 & 11.58 & 0.42 & 0.52 & 0.79 & 0.83 & 3.37 & 0.52 \\
\hline 9 & 22 & 11.6 & 0.47 & 0.51 & 0.79 & 0.81 & 3.36 & 0.51 \\
\hline 9 & 23 & 11.58 & 0.52 & 0.47 & 0.77 & 0.78 & 3.19 & 0.47 \\
\hline 9 & 24 & 11.59 & 0.53 & 0.45 & 0.77 & 0.75 & 3.16 & 0.45 \\
\hline 9 & 25 & 11.57 & 0.52 & 0.43 & 0.74 & 0.72 & 3.03 & 0.43 \\
\hline 9 & 26 & 11.58 & 0.5 & 0.43 & 0.76 & 0.71 & 3.1 & 0.43 \\
\hline 9 & 27 & 11.58 & 0.55 & 0.41 & 0.75 & 0.69 & 3.02 & 0.41 \\
\hline 9 & 28 & 11.58 & 0.58 & 0.38 & 0.73 & 0.66 & 2.89 & 0.38 \\
\hline 9 & 29 & 11.58 & 0.6 & 0.36 & 0.72 & 0.63 & 2.8 & 0.36 \\
\hline 9 & 30 & 11.58 & 0.61 & 0.34 & 0.69 & 0.61 & 2.69 & 0.34 \\
\hline 9 & 31 & 11.58 & 0.63 & 0.31 & 0.67 & 0.59 & 2.57 & 0.31 \\
\hline 9 & 32 & 11.58 & 0.65 & 0.29 & 0.65 & 0.57 & 2.44 & 0.29 \\
\hline 9 & 33 & 11.58 & 0.66 & 0.28 & 0.64 & 0.55 & 2.38 & 0.28 \\
\hline 9 & 34 & 11.58 & 0.69 & 0.26 & 0.62 & 0.53 & 2.28 & 0.26 \\
\hline 9 & 35 & 11.58 & 0.71 & 0.24 & 0.6 & 0.51 & 2.2 & 0.24 \\
\hline 9 & 36 & 11.58 & 0.73 & 0.23 & 0.57 & 0.49 & 2.07 & 0.23 \\
\hline 9 & 37 & 11.59 & 0.75 & 0.21 & 0.55 & 0.47 & 1.98 & 0.21 \\
\hline 9 & 38 & 11.57 & 0.76 & 0.2 & 0.55 & 0.45 & 1.93 & 0.2 \\
\hline 9 & 39 & 11.59 & 0.78 & 0.18 & 0.51 & 0.43 & 1.79 & 0.18 \\
\hline 9 & 40 & 11.59 & 0.81 & 0.16 & 0.47 & 0.42 & 1.65 & 0.16 \\
\hline
\end{tabular}




\section{B.5 Virtual Photon Asymmetry Results}

Table B.10: Values of $A_{1}$ for the proton from the EG1b data, showing errors (statistical and systematic) and averaged kinematics in each bin.

\begin{tabular}{|c|c|c|c|c|c|c|c|}
\hline$Q^{2}$ bin & $W$ bin & $A_{1}$ & $\sigma_{\text {stat }}$ & $\sigma_{\text {sys }}$ & $x_{\text {avg }}$ & $Q_{\text {avg }}^{2}$ & $W_{\text {avg }}$ \\
\hline 3 & 23 & -0.051 & 0.035 & 0.052 & 0.06 & 0.06 & 1.35 \\
\hline 3 & 24 & -0.12 & 0.03 & 0.046 & 0.05 & 0.06 & 1.41 \\
\hline 3 & 25 & -0.105 & 0.023 & 0.047 & 0.04 & 0.06 & 1.47 \\
\hline 3 & 26 & -0.068 & 0.023 & 0.009 & 0.04 & 0.06 & 1.53 \\
\hline
\end{tabular}

\begin{tabular}{|c|c|c|c|c|c|c|c|}
\hline$Q^{2}$ bin & $W$ bin & $A_{1}$ & $\sigma_{\text {stat }}$ & $\sigma_{\text {sys }}$ & $x_{\text {avg }}$ & $Q_{\text {avg }}^{2}$ & $W_{\text {avg }}$ \\
\hline 4 & 19 & -0.471 & 0.137 & 0.389 & 0.23 & 0.11 & 1.13 \\
\hline 4 & 20 & -0.445 & 0.032 & 0.12 & 0.18 & 0.12 & 1.18 \\
\hline 4 & 21 & -0.407 & 0.017 & 0.09 & 0.15 & 0.12 & 1.23 \\
\hline 4 & 22 & -0.212 & 0.021 & 0.09 & 0.13 & 0.12 & 1.29 \\
\hline 4 & 23 & -0.053 & 0.024 & 0.116 & 0.11 & 0.12 & 1.35 \\
\hline 4 & 24 & -0.028 & 0.02 & 0.116 & 0.1 & 0.12 & 1.41 \\
\hline 4 & 25 & -0.004 & 0.015 & 0.112 & 0.08 & 0.12 & 1.47 \\
\hline 4 & 26 & 0.022 & 0.021 & 0.097 & 0.08 & 0.13 & 1.52 \\
\hline 4 & 27 & 0.029 & 0.027 & 0.113 & 0.07 & 0.13 & 1.59 \\
\hline 4 & 28 & -0.055 & 0.024 & 0.103 & 0.07 & 0.13 & 1.65 \\
\hline 4 & 29 & -0.05 & 0.022 & 0.139 & 0.06 & 0.13 & 1.71 \\
\hline 4 & 30 & 0.019 & 0.024 & 0.212 & 0.06 & 0.13 & 1.77 \\
\hline 4 & 31 & -0.056 & 0.024 & 0.023 & 0.05 & 0.13 & 1.83 \\
\hline
\end{tabular}




\begin{tabular}{|c|c|c|c|c|c|c|c|}
\hline$Q^{2}$ bin & $W$ bin & $A_{1}$ & $\sigma_{\text {stat }}$ & $\sigma_{\text {sys }}$ & $x_{\text {avg }}$ & $Q_{\text {avg }}^{2}$ & $W_{\text {avg }}$ \\
\hline 5 & 19 & -0.516 & 0.077 & 0.286 & 0.38 & 0.24 & 1.13 \\
\hline 5 & 20 & -0.454 & 0.017 & 0.078 & 0.31 & 0.24 & 1.18 \\
\hline 5 & 21 & -0.416 & 0.009 & 0.04 & 0.27 & 0.24 & 1.23 \\
\hline 5 & 22 & -0.169 & 0.011 & 0.047 & 0.23 & 0.24 & 1.29 \\
\hline 5 & 23 & 0.014 & 0.013 & 0.078 & 0.2 & 0.24 & 1.35 \\
\hline 5 & 24 & 0.073 & 0.012 & 0.088 & 0.17 & 0.23 & 1.41 \\
\hline 5 & 25 & 0.149 & 0.009 & 0.08 & 0.15 & 0.23 & 1.47 \\
\hline 5 & 26 & 0.176 & 0.007 & 0.095 & 0.14 & 0.23 & 1.53 \\
\hline 5 & 27 & 0.12 & 0.008 & 0.12 & 0.12 & 0.23 & 1.59 \\
\hline 5 & 28 & 0.041 & 0.007 & 0.109 & 0.11 & 0.23 & 1.65 \\
\hline 5 & 29 & 0.04 & 0.009 & 0.151 & 0.1 & 0.23 & 1.7 \\
\hline 5 & 30 & 0.061 & 0.013 & 0.118 & 0.1 & 0.25 & 1.77 \\
\hline 5 & 31 & 0.024 & 0.012 & 0.063 & 0.09 & 0.25 & 1.83 \\
\hline 5 & 32 & -0.018 & 0.012 & 0.046 & 0.09 & 0.25 & 1.89 \\
\hline 5 & 33 & 0.018 & 0.011 & 0.044 & 0.08 & 0.26 & 1.95 \\
\hline 5 & 34 & 0.069 & 0.011 & 0.115 & 0.08 & 0.26 & 2.01 \\
\hline
\end{tabular}




\begin{tabular}{|c|c|c|c|c|c|c|c|}
\hline$Q^{2}$ bin & $W$ bin & $A_{1}$ & $\sigma_{\text {stat }}$ & $\sigma_{\text {sys }}$ & $x_{\text {avg }}$ & $Q_{\text {avg }}^{2}$ & $W_{\text {avg }}$ \\
\hline 6 & 19 & -0.249 & 0.068 & 0.171 & 0.54 & 0.46 & 1.13 \\
\hline 6 & 20 & -0.361 & 0.014 & 0.054 & 0.47 & 0.46 & 1.18 \\
\hline 6 & 21 & -0.311 & 0.008 & 0.022 & 0.41 & 0.46 & 1.23 \\
\hline 6 & 22 & -0.105 & 0.01 & 0.033 & 0.37 & 0.46 & 1.29 \\
\hline 6 & 23 & 0.181 & 0.012 & 0.054 & 0.32 & 0.45 & 1.35 \\
\hline 6 & 24 & 0.228 & 0.011 & 0.064 & 0.29 & 0.45 & 1.41 \\
\hline 6 & 25 & 0.36 & 0.009 & 0.08 & 0.25 & 0.44 & 1.47 \\
\hline 6 & 26 & 0.368 & 0.008 & 0.096 & 0.23 & 0.43 & 1.53 \\
\hline 6 & 27 & 0.262 & 0.009 & 0.114 & 0.21 & 0.43 & 1.59 \\
\hline 6 & 28 & 0.191 & 0.01 & 0.104 & 0.19 & 0.45 & 1.65 \\
\hline 6 & 29 & 0.236 & 0.009 & 0.121 & 0.18 & 0.46 & 1.71 \\
\hline 6 & 30 & 0.193 & 0.009 & 0.083 & 0.17 & 0.46 & 1.77 \\
\hline 6 & 31 & 0.081 & 0.009 & 0.053 & 0.16 & 0.46 & 1.83 \\
\hline 6 & 32 & 0.038 & 0.008 & 0.042 & 0.14 & 0.45 & 1.89 \\
\hline 6 & 33 & 0.074 & 0.008 & 0.046 & 0.13 & 0.43 & 1.95 \\
\hline 6 & 34 & 0.13 & 0.01 & 0.099 & 0.12 & 0.43 & 2.01 \\
\hline
\end{tabular}




\begin{tabular}{|c|c|c|c|c|c|c|c|}
\hline$Q^{2}$ bin & $W$ bin & $A_{1}$ & $\sigma_{\text {stat }}$ & $\sigma_{\text {sys }}$ & $x_{\text {avg }}$ & $Q_{\text {avg }}^{2}$ & $W_{\text {avg }}$ \\
\hline 7 & 19 & -0.019 & 0.093 & 0.105 & 0.68 & 0.86 & 1.13 \\
\hline 7 & 20 & -0.207 & 0.021 & 0.051 & 0.62 & 0.86 & 1.18 \\
\hline 7 & 21 & -0.186 & 0.013 & 0.026 & 0.57 & 0.86 & 1.23 \\
\hline 7 & 22 & 0.074 & 0.015 & 0.031 & 0.52 & 0.87 & 1.29 \\
\hline 7 & 23 & 0.347 & 0.019 & 0.051 & 0.47 & 0.86 & 1.35 \\
\hline 7 & 24 & 0.447 & 0.019 & 0.056 & 0.44 & 0.89 & 1.41 \\
\hline 7 & 25 & 0.579 & 0.015 & 0.065 & 0.41 & 0.89 & 1.47 \\
\hline 7 & 26 & 0.572 & 0.013 & 0.068 & 0.37 & 0.87 & 1.53 \\
\hline 7 & 27 & 0.47 & 0.014 & 0.091 & 0.34 & 0.87 & 1.59 \\
\hline 7 & 28 & 0.398 & 0.013 & 0.081 & 0.32 & 0.88 & 1.65 \\
\hline 7 & 29 & 0.429 & 0.012 & 0.076 & 0.3 & 0.88 & 1.71 \\
\hline 7 & 30 & 0.329 & 0.012 & 0.079 & 0.28 & 0.88 & 1.77 \\
\hline 7 & 31 & 0.244 & 0.014 & 0.056 & 0.27 & 0.93 & 1.83 \\
\hline 7 & 32 & 0.169 & 0.013 & 0.059 & 0.26 & 0.95 & 1.89 \\
\hline 7 & 33 & 0.239 & 0.012 & 0.147 & 0.24 & 0.96 & 1.95 \\
\hline 7 & 34 & 0.288 & 0.011 & 0.232 & 0.23 & 0.95 & 2.01 \\
\hline
\end{tabular}




\begin{tabular}{|c|c|c|c|c|c|c|c|}
\hline$Q^{2}$ bin & $W$ bin & $A_{1}$ & $\sigma_{\text {stat }}$ & $\sigma_{\text {sys }}$ & $x_{\text {avg }}$ & $Q_{\text {avg }}^{2}$ & $W_{\text {avg }}$ \\
\hline 8 & 19 & -0.636 & 0.224 & 0.135 & 0.82 & 1.82 & 1.12 \\
\hline 8 & 20 & -0.303 & 0.055 & 0.07 & 0.77 & 1.77 & 1.18 \\
\hline 8 & 21 & -0.068 & 0.034 & 0.055 & 0.73 & 1.79 & 1.23 \\
\hline 8 & 22 & 0.241 & 0.033 & 0.065 & 0.69 & 1.82 & 1.29 \\
\hline 8 & 23 & 0.506 & 0.032 & 0.128 & 0.66 & 1.86 & 1.35 \\
\hline 8 & 24 & 0.606 & 0.029 & 0.109 & 0.62 & 1.88 & 1.41 \\
\hline 8 & 25 & 0.756 & 0.023 & 0.123 & 0.58 & 1.85 & 1.47 \\
\hline 8 & 26 & 0.794 & 0.019 & 0.146 & 0.55 & 1.85 & 1.53 \\
\hline 8 & 27 & 0.612 & 0.019 & 0.135 & 0.53 & 1.86 & 1.59 \\
\hline 8 & 28 & 0.6 & 0.016 & 0.18 & 0.5 & 1.87 & 1.65 \\
\hline 8 & 29 & 0.665 & 0.014 & 0.143 & 0.47 & 1.87 & 1.71 \\
\hline 8 & 30 & 0.606 & 0.014 & 0.164 & 0.45 & 1.86 & 1.77 \\
\hline 8 & 31 & 0.458 & 0.014 & 0.151 & 0.42 & 1.84 & 1.83 \\
\hline 8 & 32 & 0.348 & 0.013 & 0.148 & 0.4 & 1.81 & 1.89 \\
\hline 8 & 33 & 0.414 & 0.013 & 0.248 & 0.37 & 1.77 & 1.95 \\
\hline 8 & 34 & 0.412 & 0.012 & 0.229 & 0.35 & 1.75 & 2.01 \\
\hline
\end{tabular}




\begin{tabular}{|c|c|c|c|c|c|c|c|}
\hline$Q^{2}$ bin & $W$ bin & $A_{1}$ & $\sigma_{\text {stat }}$ & $\sigma_{\text {sys }}$ & $x_{\text {avg }}$ & $Q_{\text {avg }}^{2}$ & $W_{\text {avg }}$ \\
\hline 9 & 19 & -1.187 & 0.332 & 0.817 & 0.9 & 3.57 & 1.13 \\
\hline 9 & 20 & -0.445 & 0.144 & 0.612 & 0.87 & 3.48 & 1.17 \\
\hline 9 & 21 & 0.129 & 0.078 & 0.327 & 0.83 & 3.21 & 1.23 \\
\hline 9 & 22 & 0.299 & 0.069 & 0.274 & 0.8 & 3.24 & 1.29 \\
\hline 9 & 23 & 0.456 & 0.059 & 0.358 & 0.77 & 3.33 & 1.35 \\
\hline 9 & 24 & 0.638 & 0.054 & 0.281 & 0.74 & 3.29 & 1.41 \\
\hline 9 & 25 & 0.694 & 0.043 & 0.282 & 0.71 & 3.3 & 1.47 \\
\hline 9 & 26 & 0.773 & 0.04 & 0.332 & 0.68 & 3.15 & 1.53 \\
\hline 9 & 27 & 0.648 & 0.042 & 0.224 & 0.66 & 3.15 & 1.59 \\
\hline 9 & 28 & 0.566 & 0.043 & 0.259 & 0.65 & 3.43 & 1.65 \\
\hline 9 & 29 & 0.708 & 0.038 & 0.211 & 0.62 & 3.41 & 1.71 \\
\hline 9 & 30 & 0.633 & 0.038 & 0.213 & 0.6 & 3.41 & 1.77 \\
\hline 9 & 31 & 0.624 & 0.038 & 0.172 & 0.58 & 3.4 & 1.83 \\
\hline 9 & 32 & 0.562 & 0.036 & 0.153 & 0.56 & 3.43 & 1.89 \\
\hline 9 & 33 & 0.51 & 0.034 & 0.24 & 0.53 & 3.31 & 1.95 \\
\hline 9 & 34 & 0.551 & 0.033 & 0.192 & 0.51 & 3.28 & 2.01 \\
\hline
\end{tabular}


Table B.11: Values of $A_{2}$ for the proton from the EG1b data, showing errors (systematic and statistical) and averaged kinematics for each bin.

\begin{tabular}{|c|c|c|c|c|c|c|c|}
\hline$Q^{2}$ bin & $W$ bin & $A_{2}$ & $\sigma_{\text {stat }}$ & $\sigma_{\text {sys }}$ & $x_{\text {avg }}$ & $Q_{\text {avg }}^{2}$ & $W_{\text {avg }}$ \\
\hline 4 & 21 & 0.756 & 0.735 & 0.081 & 0.18 & 0.14 & 1.23 \\
\hline 4 & 22 & -0.68 & 0.884 & 0.08 & 0.15 & 0.14 & 1.29 \\
\hline 4 & 23 & -0.972 & 0.996 & 0.106 & 0.13 & 0.14 & 1.35 \\
\hline 4 & 24 & -0.177 & 0.823 & 0.115 & 0.11 & 0.13 & 1.41 \\
\hline 4 & 25 & -0.173 & 0.608 & 0.107 & 0.09 & 0.13 & 1.47 \\
\hline
\end{tabular}

\begin{tabular}{|c|c|c|c|c|c|c|c|}
\hline$Q^{2}$ bin & $W$ bin & $A_{2}$ & $\sigma_{\text {stat }}$ & $\sigma_{\text {sys }}$ & $x_{\text {avg }}$ & $Q_{\text {avg }}^{2}$ & $W_{\text {avg }}$ \\
\hline 5 & 20 & -0.529 & 0.36 & 0.078 & 0.32 & 0.25 & 1.18 \\
\hline 5 & 21 & -0.249 & 0.213 & 0.037 & 0.28 & 0.25 & 1.23 \\
\hline 5 & 22 & 0.017 & 0.276 & 0.044 & 0.24 & 0.25 & 1.29 \\
\hline 5 & 23 & 0.537 & 0.336 & 0.075 & 0.21 & 0.25 & 1.35 \\
\hline 5 & 24 & -0.098 & 0.305 & 0.084 & 0.18 & 0.24 & 1.41 \\
\hline 5 & 25 & -0.084 & 0.219 & 0.079 & 0.16 & 0.25 & 1.47 \\
\hline 5 & 26 & 0.287 & 0.175 & 0.096 & 0.14 & 0.25 & 1.53 \\
\hline 5 & 27 & 0.025 & 0.17 & 0.119 & 0.13 & 0.25 & 1.59 \\
\hline 5 & 28 & 0.239 & 0.143 & 0.111 & 0.12 & 0.25 & 1.65 \\
\hline 5 & 29 & 0.044 & 0.201 & 0.149 & 0.11 & 0.26 & 1.69 \\
\hline 5 & 30 & -0.874 & 0.46 & 0.096 & 0.11 & 0.29 & 1.77 \\
\hline 5 & 31 & -0.175 & 0.376 & 0.06 & 0.1 & 0.28 & 1.83 \\
\hline 5 & 32 & -0.09 & 0.301 & 0.045 & 0.09 & 0.28 & 1.89 \\
\hline 5 & 33 & 0.191 & 0.254 & 0.044 & 0.09 & 0.27 & 1.95 \\
\hline 5 & 34 & 0.251 & 0.328 & 0.116 & 0.08 & 0.27 & 2 \\
\hline
\end{tabular}




\begin{tabular}{|c|c|c|c|c|c|c|c|}
\hline$Q^{2}$ bin & $W$ bin & $A_{2}$ & $\sigma_{\text {stat }}$ & $\sigma_{\text {sys }}$ & $x_{\text {avg }}$ & $Q_{\text {avg }}^{2}$ & $W_{\text {avg }}$ \\
\hline 6 & 19 & -0.209 & 0.662 & 0.158 & 0.56 & 0.5 & 1.13 \\
\hline 6 & 20 & -0.05 & 0.143 & 0.053 & 0.49 & 0.5 & 1.18 \\
\hline 6 & 21 & 0.103 & 0.085 & 0.022 & 0.44 & 0.5 & 1.23 \\
\hline 6 & 22 & -0.038 & 0.107 & 0.032 & 0.39 & 0.49 & 1.29 \\
\hline 6 & 23 & 0.173 & 0.129 & 0.051 & 0.34 & 0.49 & 1.35 \\
\hline 6 & 24 & 0.345 & 0.121 & 0.063 & 0.3 & 0.49 & 1.41 \\
\hline 6 & 25 & 0.196 & 0.104 & 0.08 & 0.26 & 0.45 & 1.47 \\
\hline 6 & 26 & 0.279 & 0.087 & 0.096 & 0.23 & 0.44 & 1.53 \\
\hline 6 & 27 & 0.158 & 0.115 & 0.114 & 0.2 & 0.41 & 1.59 \\
\hline 6 & 28 & 0.028 & 0.149 & 0.105 & 0.19 & 0.44 & 1.65 \\
\hline 6 & 29 & 0.477 & 0.158 & 0.116 & 0.19 & 0.49 & 1.71 \\
\hline 6 & 30 & 0.169 & 0.158 & 0.082 & 0.18 & 0.48 & 1.77 \\
\hline 6 & 31 & -0.151 & 0.144 & 0.053 & 0.16 & 0.48 & 1.83 \\
\hline 6 & 32 & -0.011 & 0.134 & 0.041 & 0.15 & 0.47 & 1.89 \\
\hline 6 & 33 & -0.034 & 0.139 & 0.047 & 0.13 & 0.43 & 1.95 \\
\hline 6 & 34 & 0.361 & 0.243 & 0.098 & 0.11 & 0.39 & 2 \\
\hline
\end{tabular}




\begin{tabular}{|c|c|c|c|c|c|c|c|}
\hline$Q^{2}$ bin & $W$ bin & $A_{2}$ & $\sigma_{\text {stat }}$ & $\sigma_{\text {sys }}$ & $x_{\text {avg }}$ & $Q_{\text {avg }}^{2}$ & $W_{\text {avg }}$ \\
\hline 7 & 19 & -2.178 & 0.624 & 0.105 & 0.68 & 0.86 & 1.13 \\
\hline 7 & 20 & -0.22 & 0.149 & 0.051 & 0.62 & 0.84 & 1.18 \\
\hline 7 & 21 & -0.075 & 0.103 & 0.025 & 0.56 & 0.84 & 1.23 \\
\hline 7 & 22 & 0.325 & 0.128 & 0.031 & 0.51 & 0.84 & 1.29 \\
\hline 7 & 23 & 0.175 & 0.187 & 0.047 & 0.46 & 0.81 & 1.35 \\
\hline 7 & 24 & 0.518 & 0.222 & 0.055 & 0.44 & 0.89 & 1.41 \\
\hline 7 & 25 & 0.582 & 0.171 & 0.064 & 0.4 & 0.88 & 1.47 \\
\hline 7 & 26 & 0.322 & 0.18 & 0.068 & 0.36 & 0.81 & 1.53 \\
\hline 7 & 27 & 0.401 & 0.201 & 0.091 & 0.32 & 0.79 & 1.59 \\
\hline 7 & 28 & 0.327 & 0.213 & 0.078 & 0.3 & 0.77 & 1.65 \\
\hline 7 & 29 & -0.191 & 0.226 & 0.079 & 0.26 & 0.74 & 1.71 \\
\hline 7 & 30 & -0.685 & 0.217 & 0.081 & 0.25 & 0.74 & 1.77 \\
\hline 7 & 31 & -1.406 & 0.411 & 0.06 & 0.25 & 0.84 & 1.82 \\
\hline 7 & 32 & -1.479 & 0.562 & 0.068 & 0.27 & 1 & 1.89 \\
\hline 7 & 33 & 0.182 & 0.494 & 0.166 & 0.25 & 1 & 1.95 \\
\hline 7 & 34 & -0.697 & 0.647 & 0.272 & 0.24 & 1.01 & 2 \\
\hline
\end{tabular}




\begin{tabular}{|c|c|c|c|c|c|c|c|}
\hline$Q^{2}$ bin & $W$ bin & $A_{2}$ & $\sigma_{\text {stat }}$ & $\sigma_{\text {sys }}$ & $x_{\text {avg }}$ & $Q_{\text {avg }}^{2}$ & $W_{\text {avg }}$ \\
\hline 8 & 21 & -1.64 & 0.912 & 0.059 & 0.74 & 1.85 & 1.23 \\
\hline 8 & 22 & 0.385 & 0.892 & 0.071 & 0.7 & 1.88 & 1.29 \\
\hline 8 & 23 & -3.358 & 0.869 & 0.141 & 0.67 & 1.95 & 1.35 \\
\hline 8 & 24 & 0.413 & 0.809 & 0.121 & 0.63 & 1.96 & 1.41 \\
\hline 8 & 25 & -0.299 & 0.621 & 0.139 & 0.6 & 1.94 & 1.47 \\
\hline 8 & 26 & -1.596 & 0.53 & 0.166 & 0.56 & 1.93 & 1.53 \\
\hline 8 & 27 & -1.284 & 0.529 & 0.144 & 0.53 & 1.94 & 1.59 \\
\hline 8 & 28 & -0.275 & 0.449 & 0.193 & 0.51 & 1.96 & 1.65 \\
\hline 8 & 29 & -0.221 & 0.391 & 0.154 & 0.48 & 1.95 & 1.71 \\
\hline 8 & 30 & -0.3 & 0.387 & 0.172 & 0.46 & 1.95 & 1.77 \\
\hline 8 & 31 & -0.142 & 0.378 & 0.155 & 0.44 & 1.95 & 1.83 \\
\hline 8 & 32 & 0.143 & 0.363 & 0.148 & 0.41 & 1.89 & 1.89 \\
\hline 8 & 33 & -0.17 & 0.372 & 0.249 & 0.37 & 1.76 & 1.95 \\
\hline 8 & 34 & 0.034 & 0.495 & 0.272 & 0.36 & 1.74 & 2 \\
\hline
\end{tabular}


Table B.12: $A_{1}$ values for the proton, DIS ( $W>2 \mathrm{GeV}$ ) only, rebinned in $x$. These data are plotted in Figure 8.42.

\begin{tabular}{|c|c|c|c|}
\hline$x$ & $A_{1}(x)$ & $\sigma_{\text {stat }}$ & $\sigma_{\text {sys }}$ \\
\hline \hline 0.125 & 0.1798 & 0.0198 & 0.014 \\
\hline 0.175 & 0.2620 & 0.0079 & 0.016 \\
\hline 0.225 & 0.2960 & 0.0056 & 0.022 \\
\hline 0.275 & 0.3709 & 0.0062 & 0.028 \\
\hline 0.325 & 0.4273 & 0.0074 & 0.030 \\
\hline 0.375 & 0.4711 & 0.0115 & 0.030 \\
\hline 0.425 & 0.4842 & 0.0167 & 0.031 \\
\hline 0.475 & 0.5831 & 0.0231 & 0.033 \\
\hline 0.525 & 0.6157 & 0.0387 & 0.036 \\
\hline 0.575 & 0.4057 & 0.151 & 0.036 \\
\hline
\end{tabular}




\section{B.6 Structure Function Results}

Table B.13: Values of $g_{1}$ for the proton, from the EG1b data. Errors (statistical and systematic) are shown, as well as averaged kinematic data in each bin.

\begin{tabular}{|c|c|c|c|c|c|c|c|}
\hline$Q^{2}$ bin & $W$ bin & $g_{1}$ & $\sigma_{\text {stat }}$ & $\sigma_{\text {sys }}$ & $x_{\text {avg }}$ & $Q_{\text {avg }}^{2}$ & $W_{\text {avg }}$ \\
\hline 3 & 23 & -0.002 & 0.022 & 0.054 & 0.06 & 0.06 & 1.35 \\
\hline 3 & 24 & -0.062 & 0.023 & 0.055 & 0.05 & 0.06 & 1.41 \\
\hline 3 & 25 & -0.111 & 0.03 & 0.051 & 0.05 & 0.06 & 1.47 \\
\hline 3 & 26 & -0.08 & 0.035 & 0.01 & 0.04 & 0.06 & 1.53 \\
\hline
\end{tabular}

\begin{tabular}{|c|c|c|c|c|c|c|c|}
\hline$Q^{2}$ bin & $W$ bin & $g_{1}$ & $\sigma_{\text {stat }}$ & $\sigma_{\text {sys }}$ & $x_{\text {avg }}$ & $Q_{\text {avg }}^{2}$ & $W_{\text {avg }}$ \\
\hline 4 & 18 & 0.042 & 0.009 & 0.764 & 0.3 & 0.12 & 1.07 \\
\hline 4 & 19 & -0.003 & 0.006 & 0.723 & 0.26 & 0.12 & 1.1 \\
\hline 4 & 20 & -0.108 & 0.013 & 0.133 & 0.2 & 0.12 & 1.17 \\
\hline 4 & 21 & -0.245 & 0.013 & 0.089 & 0.16 & 0.12 & 1.23 \\
\hline 4 & 22 & -0.074 & 0.012 & 0.09 & 0.13 & 0.12 & 1.29 \\
\hline 4 & 23 & 0.013 & 0.012 & 0.112 & 0.11 & 0.12 & 1.35 \\
\hline 4 & 24 & 0.032 & 0.013 & 0.125 & 0.1 & 0.12 & 1.41 \\
\hline 4 & 25 & 0.04 & 0.015 & 0.11 & 0.09 & 0.12 & 1.47 \\
\hline 4 & 26 & 0.073 & 0.024 & 0.096 & 0.08 & 0.13 & 1.52 \\
\hline 4 & 27 & 0.069 & 0.028 & 0.114 & 0.07 & 0.13 & 1.59 \\
\hline 4 & 28 & -0.049 & 0.028 & 0.101 & 0.07 & 0.13 & 1.65 \\
\hline 4 & 29 & -0.054 & 0.028 & 0.139 & 0.06 & 0.13 & 1.71 \\
\hline 4 & 30 & 0.047 & 0.029 & 0.212 & 0.06 & 0.13 & 1.77 \\
\hline 4 & 31 & -0.029 & 0.03 & 0.023 & 0.05 & 0.13 & 1.83 \\
\hline
\end{tabular}




\begin{tabular}{|c|c|c|c|c|c|c|c|}
\hline$Q^{2}$ bin & $W$ bin & $g_{1}$ & $\sigma_{\text {stat }}$ & $\sigma_{\text {sys }}$ & $x_{\text {avg }}$ & $Q_{\text {avg }}^{2}$ & $W_{\text {avg }}$ \\
\hline 5 & 18 & 0.029 & 0.003 & 0.851 & 0.47 & 0.25 & 1.07 \\
\hline 5 & 19 & -0.009 & 0.003 & 0.548 & 0.42 & 0.24 & 1.1 \\
\hline 5 & 20 & -0.088 & 0.005 & 0.106 & 0.34 & 0.25 & 1.17 \\
\hline 5 & 21 & -0.197 & 0.005 & 0.037 & 0.28 & 0.25 & 1.23 \\
\hline 5 & 22 & -0.035 & 0.005 & 0.047 & 0.24 & 0.25 & 1.29 \\
\hline 5 & 23 & 0.043 & 0.005 & 0.075 & 0.21 & 0.25 & 1.35 \\
\hline 5 & 24 & 0.082 & 0.005 & 0.089 & 0.18 & 0.24 & 1.41 \\
\hline 5 & 25 & 0.159 & 0.006 & 0.076 & 0.16 & 0.24 & 1.47 \\
\hline 5 & 26 & 0.212 & 0.006 & 0.095 & 0.14 & 0.24 & 1.53 \\
\hline 5 & 27 & 0.152 & 0.006 & 0.12 & 0.13 & 0.24 & 1.59 \\
\hline 5 & 28 & 0.072 & 0.007 & 0.109 & 0.11 & 0.24 & 1.65 \\
\hline 5 & 29 & 0.071 & 0.009 & 0.152 & 0.11 & 0.24 & 1.7 \\
\hline 5 & 30 & 0.102 & 0.012 & 0.114 & 0.1 & 0.25 & 1.77 \\
\hline 5 & 31 & 0.089 & 0.012 & 0.063 & 0.09 & 0.26 & 1.83 \\
\hline 5 & 32 & 0.038 & 0.012 & 0.046 & 0.09 & 0.26 & 1.89 \\
\hline 5 & 33 & 0.052 & 0.012 & 0.044 & 0.08 & 0.26 & 1.95 \\
\hline 5 & 34 & 0.087 & 0.012 & 0.115 & 0.08 & 0.27 & 2.01 \\
\hline
\end{tabular}




\begin{tabular}{|c|c|c|c|c|c|c|c|}
\hline$Q^{2}$ bin & $W$ bin & $g_{1}$ & $\sigma_{\text {stat }}$ & $\sigma_{\text {sys }}$ & $x_{\text {avg }}$ & $Q_{\text {avg }}^{2}$ & $W_{\text {avg }}$ \\
\hline 6 & 18 & 0.015 & 0.001 & 0.368 & 0.63 & 0.49 & 1.07 \\
\hline 6 & 19 & -0.007 & 0.002 & 0.256 & 0.58 & 0.48 & 1.1 \\
\hline 6 & 20 & -0.06 & 0.003 & 0.075 & 0.5 & 0.48 & 1.17 \\
\hline 6 & 21 & -0.116 & 0.003 & 0.022 & 0.43 & 0.48 & 1.23 \\
\hline 6 & 22 & -0.018 & 0.003 & 0.033 & 0.38 & 0.48 & 1.29 \\
\hline 6 & 23 & 0.061 & 0.003 & 0.052 & 0.34 & 0.48 & 1.35 \\
\hline 6 & 24 & 0.091 & 0.003 & 0.064 & 0.3 & 0.48 & 1.41 \\
\hline 6 & 25 & 0.195 & 0.004 & 0.077 & 0.26 & 0.46 & 1.47 \\
\hline 6 & 26 & 0.253 & 0.004 & 0.095 & 0.24 & 0.46 & 1.53 \\
\hline 6 & 27 & 0.192 & 0.005 & 0.113 & 0.22 & 0.46 & 1.59 \\
\hline 6 & 28 & 0.158 & 0.006 & 0.102 & 0.2 & 0.47 & 1.65 \\
\hline 6 & 29 & 0.206 & 0.006 & 0.115 & 0.19 & 0.49 & 1.71 \\
\hline 6 & 30 & 0.184 & 0.006 & 0.083 & 0.18 & 0.48 & 1.77 \\
\hline 6 & 31 & 0.132 & 0.006 & 0.053 & 0.16 & 0.48 & 1.83 \\
\hline 6 & 32 & 0.111 & 0.006 & 0.041 & 0.15 & 0.47 & 1.89 \\
\hline 6 & 33 & 0.116 & 0.007 & 0.046 & 0.13 & 0.45 & 1.95 \\
\hline 6 & 34 & 0.131 & 0.009 & 0.099 & 0.12 & 0.45 & 2.01 \\
\hline
\end{tabular}




\begin{tabular}{|c|c|c|c|c|c|c|c|}
\hline$Q^{2}$ bin & $W$ bin & $g_{1}$ & $\sigma_{\text {stat }}$ & $\sigma_{\text {sys }}$ & $x_{\text {avg }}$ & $Q_{\text {avg }}^{2}$ & $W_{\text {avg }}$ \\
\hline 7 & 18 & 0.008 & 0.001 & 0.188 & 0.77 & 0.96 & 1.07 \\
\hline 7 & 19 & -0.001 & 0.001 & 0.143 & 0.74 & 0.96 & 1.1 \\
\hline 7 & 20 & -0.022 & 0.002 & 0.06 & 0.66 & 0.96 & 1.17 \\
\hline 7 & 21 & -0.042 & 0.002 & 0.027 & 0.6 & 0.96 & 1.23 \\
\hline 7 & 22 & 0.012 & 0.002 & 0.034 & 0.55 & 0.96 & 1.29 \\
\hline 7 & 23 & 0.051 & 0.003 & 0.055 & 0.49 & 0.93 & 1.35 \\
\hline 7 & 24 & 0.078 & 0.003 & 0.057 & 0.46 & 0.95 & 1.41 \\
\hline 7 & 25 & 0.149 & 0.004 & 0.063 & 0.42 & 0.95 & 1.47 \\
\hline 7 & 26 & 0.2 & 0.004 & 0.066 & 0.39 & 0.93 & 1.53 \\
\hline 7 & 27 & 0.163 & 0.004 & 0.09 & 0.36 & 0.94 & 1.59 \\
\hline 7 & 28 & 0.161 & 0.004 & 0.081 & 0.34 & 0.95 & 1.65 \\
\hline 7 & 29 & 0.213 & 0.005 & 0.074 & 0.31 & 0.95 & 1.71 \\
\hline 7 & 30 & 0.167 & 0.005 & 0.077 & 0.3 & 0.96 & 1.77 \\
\hline 7 & 31 & 0.148 & 0.005 & 0.057 & 0.28 & 0.99 & 1.83 \\
\hline 7 & 32 & 0.142 & 0.006 & 0.067 & 0.27 & 1 & 1.89 \\
\hline 7 & 33 & 0.157 & 0.006 & 0.166 & 0.26 & 1.01 & 1.95 \\
\hline 7 & 34 & 0.168 & 0.006 & 0.24 & 0.24 & 1.01 & 2.01 \\
\hline
\end{tabular}




\begin{tabular}{|c|c|c|c|c|c|c|c|}
\hline$Q^{2}$ bin & $W$ bin & $g_{1}$ & $\sigma_{\text {stat }}$ & $\sigma_{\text {sys }}$ & $x_{\text {avg }}$ & $Q_{\text {avq }}^{2}$ & $W_{\text {avg }}$ \\
\hline 8 & 19 & -0.001 & 0.001 & 0.273 & 0.86 & 2.07 & 1.1 \\
\hline 8 & 20 & -0.007 & 0.002 & 0.097 & 0.81 & 2.05 & 1.17 \\
\hline 8 & 21 & -0.005 & 0.002 & 0.089 & 0.76 & 2.08 & 1.23 \\
\hline 8 & 22 & 0.014 & 0.002 & 0.093 & 0.72 & 2.06 & 1.29 \\
\hline 8 & 23 & 0.03 & 0.002 & 0.157 & 0.68 & 2.06 & 1.35 \\
\hline 8 & 24 & 0.043 & 0.002 & 0.139 & 0.65 & 2.07 & 1.41 \\
\hline 8 & 25 & 0.08 & 0.002 & 0.154 & 0.61 & 2.04 & 1.47 \\
\hline 8 & 26 & 0.107 & 0.002 & 0.193 & 0.58 & 2.04 & 1.53 \\
\hline 8 & 27 & 0.083 & 0.002 & 0.155 & 0.55 & 2.04 & 1.59 \\
\hline 8 & 28 & 0.101 & 0.002 & 0.206 & 0.52 & 2.04 & 1.65 \\
\hline 8 & 29 & 0.138 & 0.003 & 0.164 & 0.49 & 2.04 & 1.71 \\
\hline 8 & 30 & 0.124 & 0.003 & 0.178 & 0.47 & 2.02 & 1.77 \\
\hline 8 & 31 & 0.107 & 0.003 & 0.158 & 0.44 & 1.99 & 1.83 \\
\hline 8 & 32 & 0.109 & 0.003 & 0.149 & 0.42 & 1.95 & 1.89 \\
\hline 8 & 33 & 0.126 & 0.003 & 0.246 & 0.39 & 1.88 & 1.95 \\
\hline 8 & 34 & 0.131 & 0.004 & 0.228 & 0.37 & 1.87 & 2.01 \\
\hline
\end{tabular}




\begin{tabular}{|c|c|c|c|c|c|c|c|}
\hline$Q^{2}$ bin & $W$ bin & $g_{1}$ & $\sigma_{\text {stat }}$ & $\sigma_{\text {sys }}$ & $x_{\text {avg }}$ & $Q_{\text {avg }}^{2}$ & $W_{\text {avg }}$ \\
\hline 9 & 20 & -0.001 & 0.001 & 0.892 & 0.9 & 4.35 & 1.16 \\
\hline 9 & 21 & 0.002 & 0.001 & 0.453 & 0.86 & 4.09 & 1.23 \\
\hline 9 & 22 & 0.005 & 0.001 & 0.331 & 0.83 & 3.91 & 1.29 \\
\hline 9 & 23 & 0.009 & 0.001 & 0.432 & 0.8 & 3.99 & 1.35 \\
\hline 9 & 24 & 0.016 & 0.001 & 0.312 & 0.77 & 3.81 & 1.41 \\
\hline 9 & 25 & 0.023 & 0.001 & 0.291 & 0.75 & 3.94 & 1.47 \\
\hline 9 & 26 & 0.042 & 0.002 & 0.344 & 0.7 & 3.5 & 1.53 \\
\hline 9 & 27 & 0.037 & 0.002 & 0.229 & 0.67 & 3.4 & 1.59 \\
\hline 9 & 28 & 0.034 & 0.003 & 0.267 & 0.67 & 3.7 & 1.65 \\
\hline 9 & 29 & 0.056 & 0.003 & 0.212 & 0.64 & 3.65 & 1.71 \\
\hline 9 & 30 & 0.05 & 0.003 & 0.216 & 0.62 & 3.65 & 1.77 \\
\hline 9 & 31 & 0.055 & 0.003 & 0.174 & 0.59 & 3.64 & 1.83 \\
\hline 9 & 32 & 0.056 & 0.003 & 0.153 & 0.57 & 3.65 & 1.89 \\
\hline 9 & 33 & 0.063 & 0.004 & 0.238 & 0.54 & 3.52 & 1.95 \\
\hline 9 & 34 & 0.075 & 0.004 & 0.198 & 0.52 & 3.45 & 2.01 \\
\hline
\end{tabular}


Table B.14: Values of $g_{1} / F_{1}$ from the EG1b data. Errors (statistical and systematic) are shown, as well as averaged kinematic values in each bin.

\begin{tabular}{|c|c|c|c|c|c|c|c|}
\hline$Q^{2}$ bin & $W$ bin & $g_{1} / F_{1}$ & $\sigma_{\text {stat }}$ & $\sigma_{\text {sys }}$ & $x_{\text {avg }}$ & $Q_{\text {avg }}^{2}$ & $W_{\text {avg }}$ \\
\hline 3 & 23 & -0.004 & 0.029 & 0.069 & 0.06 & 0.06 & 1.35 \\
\hline 3 & 24 & -0.069 & 0.026 & 0.056 & 0.05 & 0.06 & 1.41 \\
\hline 3 & 25 & -0.074 & 0.02 & 0.034 & 0.04 & 0.06 & 1.47 \\
\hline 3 & 26 & -0.049 & 0.021 & 0.006 & 0.04 & 0.06 & 1.53 \\
\hline
\end{tabular}

\begin{tabular}{|c|c|c|c|c|c|c|c|}
\hline$Q^{2}$ bin & $W$ bin & $g_{1} / F_{1}$ & $\sigma_{\text {stat }}$ & $\sigma_{\text {sys }}$ & $x_{\text {avg }}$ & $Q_{\text {avg }}^{2}$ & $W_{\text {avg }}$ \\
\hline 4 & 19 & -0.06 & 0.053 & 3.324 & 0.23 & 0.12 & 1.12 \\
\hline 4 & 20 & -0.152 & 0.016 & 0.145 & 0.19 & 0.12 & 1.18 \\
\hline 4 & 21 & -0.184 & 0.01 & 0.066 & 0.16 & 0.12 & 1.23 \\
\hline 4 & 22 & -0.089 & 0.014 & 0.099 & 0.13 & 0.12 & 1.29 \\
\hline 4 & 23 & 0.019 & 0.017 & 0.157 & 0.11 & 0.12 & 1.35 \\
\hline 4 & 24 & 0.039 & 0.016 & 0.15 & 0.1 & 0.12 & 1.41 \\
\hline 4 & 25 & 0.035 & 0.013 & 0.089 & 0.09 & 0.12 & 1.47 \\
\hline 4 & 26 & 0.052 & 0.018 & 0.07 & 0.08 & 0.13 & 1.52 \\
\hline 4 & 27 & 0.056 & 0.023 & 0.094 & 0.07 & 0.13 & 1.59 \\
\hline 4 & 28 & -0.038 & 0.021 & 0.076 & 0.07 & 0.13 & 1.65 \\
\hline 4 & 29 & -0.039 & 0.02 & 0.099 & 0.06 & 0.13 & 1.71 \\
\hline 4 & 30 & 0.037 & 0.023 & 0.164 & 0.06 & 0.13 & 1.77 \\
\hline 4 & 31 & -0.022 & 0.023 & 0.017 & 0.05 & 0.13 & 1.83 \\
\hline
\end{tabular}




\begin{tabular}{|c|c|c|c|c|c|c|c|}
\hline$Q^{2}$ bin & $W$ bin & $g_{1} / F_{1}$ & $\sigma_{\text {stat }}$ & $\sigma_{\text {sys }}$ & $x_{\text {avg }}$ & $Q_{\text {avg }}^{2}$ & $W_{\text {avg }}$ \\
\hline 5 & 19 & -0.085 & 0.025 & 2.575 & 0.38 & 0.24 & 1.13 \\
\hline 5 & 20 & -0.138 & 0.007 & 0.112 & 0.32 & 0.24 & 1.18 \\
\hline 5 & 21 & -0.161 & 0.004 & 0.031 & 0.28 & 0.24 & 1.23 \\
\hline 5 & 22 & -0.049 & 0.006 & 0.057 & 0.24 & 0.24 & 1.29 \\
\hline 5 & 23 & 0.07 & 0.008 & 0.125 & 0.2 & 0.24 & 1.35 \\
\hline 5 & 24 & 0.126 & 0.008 & 0.135 & 0.17 & 0.24 & 1.41 \\
\hline 5 & 25 & 0.167 & 0.006 & 0.08 & 0.15 & 0.24 & 1.47 \\
\hline 5 & 26 & 0.195 & 0.006 & 0.088 & 0.14 & 0.23 & 1.53 \\
\hline 5 & 27 & 0.155 & 0.006 & 0.124 & 0.12 & 0.23 & 1.59 \\
\hline 5 & 28 & 0.063 & 0.006 & 0.099 & 0.11 & 0.23 & 1.65 \\
\hline 5 & 29 & 0.057 & 0.008 & 0.127 & 0.1 & 0.24 & 1.7 \\
\hline 5 & 30 & 0.095 & 0.011 & 0.11 & 0.1 & 0.25 & 1.77 \\
\hline 5 & 31 & 0.08 & 0.011 & 0.059 & 0.09 & 0.25 & 1.83 \\
\hline 5 & 32 & 0.033 & 0.01 & 0.041 & 0.09 & 0.26 & 1.89 \\
\hline 5 & 33 & 0.043 & 0.01 & 0.037 & 0.08 & 0.26 & 1.95 \\
\hline 5 & 34 & 0.071 & 0.01 & 0.094 & 0.08 & 0.26 & 2.01 \\
\hline
\end{tabular}




\begin{tabular}{|c|c|c|c|c|c|c|c|}
\hline$Q^{2}$ bin & $W$ bin & $g_{1} / F_{1}$ & $\sigma_{\text {stat }}$ & $\sigma_{\text {sys }}$ & $x_{\text {avg }}$ & $Q_{\text {avg }}^{2}$ & $W_{\text {avg }}$ \\
\hline 6 & 19 & -0.045 & 0.021 & 1.847 & 0.54 & 0.46 & 1.13 \\
\hline 6 & 20 & -0.129 & 0.005 & 0.107 & 0.47 & 0.46 & 1.18 \\
\hline 6 & 21 & -0.133 & 0.003 & 0.024 & 0.42 & 0.46 & 1.23 \\
\hline 6 & 22 & -0.039 & 0.005 & 0.056 & 0.37 & 0.46 & 1.29 \\
\hline 6 & 23 & 0.136 & 0.006 & 0.118 & 0.33 & 0.46 & 1.35 \\
\hline 6 & 24 & 0.197 & 0.007 & 0.138 & 0.29 & 0.46 & 1.41 \\
\hline 6 & 25 & 0.293 & 0.006 & 0.113 & 0.25 & 0.44 & 1.47 \\
\hline 6 & 26 & 0.33 & 0.005 & 0.123 & 0.23 & 0.44 & 1.53 \\
\hline 6 & 27 & 0.279 & 0.007 & 0.165 & 0.21 & 0.43 & 1.59 \\
\hline 6 & 28 & 0.2 & 0.008 & 0.131 & 0.2 & 0.45 & 1.65 \\
\hline 6 & 29 & 0.237 & 0.007 & 0.137 & 0.19 & 0.47 & 1.71 \\
\hline 6 & 30 & 0.233 & 0.008 & 0.106 & 0.17 & 0.47 & 1.77 \\
\hline 6 & 31 & 0.162 & 0.007 & 0.066 & 0.16 & 0.46 & 1.83 \\
\hline 6 & 32 & 0.127 & 0.007 & 0.048 & 0.14 & 0.46 & 1.89 \\
\hline 6 & 33 & 0.122 & 0.007 & 0.049 & 0.13 & 0.44 & 1.95 \\
\hline 6 & 34 & 0.134 & 0.009 & 0.101 & 0.12 & 0.43 & 2.01 \\
\hline
\end{tabular}




\begin{tabular}{|c|c|c|c|c|c|c|c|}
\hline$Q^{2}$ bin & $W$ bin & $g_{1} / F_{1}$ & $\sigma_{\text {stat }}$ & $\sigma_{\text {sys }}$ & $x_{\text {avg }}$ & $Q_{a v q}^{2}$ & $W_{\text {avg }}$ \\
\hline 7 & 19 & 0.001 & 0.032 & 2.147 & 0.68 & 0.85 & 1.13 \\
\hline 7 & 20 & -0.099 & 0.008 & 0.186 & 0.62 & 0.84 & 1.18 \\
\hline 7 & 21 & -0.103 & 0.005 & 0.057 & 0.57 & 0.85 & 1.23 \\
\hline 7 & 22 & 0.03 & 0.007 & 0.103 & 0.52 & 0.86 & 1.29 \\
\hline 7 & 23 & 0.204 & 0.01 & 0.2 & 0.48 & 0.86 & 1.35 \\
\hline 7 & 24 & 0.293 & 0.011 & 0.206 & 0.44 & 0.9 & 1.41 \\
\hline 7 & 25 & 0.389 & 0.009 & 0.155 & 0.41 & 0.89 & 1.47 \\
\hline 7 & 26 & 0.431 & 0.008 & 0.141 & 0.37 & 0.87 & 1.53 \\
\hline 7 & 27 & 0.403 & 0.01 & 0.22 & 0.34 & 0.88 & 1.59 \\
\hline 7 & 28 & 0.336 & 0.009 & 0.166 & 0.32 & 0.89 & 1.65 \\
\hline 7 & 29 & 0.374 & 0.009 & 0.132 & 0.3 & 0.89 & 1.71 \\
\hline 7 & 30 & 0.329 & 0.009 & 0.15 & 0.28 & 0.89 & 1.77 \\
\hline 7 & 31 & 0.285 & 0.011 & 0.11 & 0.27 & 0.94 & 1.83 \\
\hline 7 & 32 & 0.254 & 0.01 & 0.115 & 0.26 & 0.95 & 1.89 \\
\hline 7 & 33 & 0.266 & 0.01 & 0.268 & 0.25 & 0.96 & 1.95 \\
\hline 7 & 34 & 0.269 & 0.009 & 0.378 & 0.23 & 0.96 & 2.01 \\
\hline
\end{tabular}




\begin{tabular}{|c|c|c|c|c|c|c|c|}
\hline$Q^{2}$ bin & $W$ bin & $g_{1} / F_{1}$ & $\sigma_{\text {stat }}$ & $\sigma_{\text {sys }}$ & $x_{\text {avg }}$ & $Q_{\text {avg }}^{2}$ & $W_{\text {avg }}$ \\
\hline 8 & 19 & -0.273 & 0.095 & 15.244 & 0.82 & 1.77 & 1.12 \\
\hline 8 & 20 & -0.157 & 0.025 & 0.979 & 0.77 & 1.74 & 1.18 \\
\hline 8 & 21 & -0.048 & 0.017 & 0.541 & 0.73 & 1.76 & 1.23 \\
\hline 8 & 22 & 0.14 & 0.017 & 0.776 & 0.69 & 1.79 & 1.29 \\
\hline 8 & 23 & 0.327 & 0.017 & 1.441 & 0.66 & 1.84 & 1.35 \\
\hline 8 & 24 & 0.407 & 0.017 & 1.08 & 0.62 & 1.87 & 1.41 \\
\hline 8 & 25 & 0.505 & 0.014 & 0.78 & 0.58 & 1.83 & 1.47 \\
\hline 8 & 26 & 0.565 & 0.012 & 0.841 & 0.55 & 1.85 & 1.53 \\
\hline 8 & 27 & 0.48 & 0.012 & 0.794 & 0.52 & 1.86 & 1.59 \\
\hline 8 & 28 & 0.455 & 0.011 & 0.833 & 0.5 & 1.87 & 1.65 \\
\hline 8 & 29 & 0.516 & 0.01 & 0.548 & 0.47 & 1.87 & 1.71 \\
\hline 8 & 30 & 0.49 & 0.01 & 0.656 & 0.45 & 1.86 & 1.77 \\
\hline 8 & 31 & 0.399 & 0.01 & 0.556 & 0.42 & 1.85 & 1.83 \\
\hline 8 & 32 & 0.358 & 0.01 & 0.477 & 0.4 & 1.81 & 1.89 \\
\hline 8 & 33 & 0.374 & 0.01 & 0.709 & 0.38 & 1.78 & 1.95 \\
\hline 8 & 34 & 0.359 & 0.01 & 0.604 & 0.35 & 1.76 & 2.01 \\
\hline
\end{tabular}




\begin{tabular}{|c|c|c|c|c|c|c|c|}
\hline$Q^{2}$ bin & $W$ bin & $g_{1} / F_{1}$ & $\sigma_{\text {stat }}$ & $\sigma_{\text {sys }}$ & $x_{\text {avg }}$ & $Q_{\text {avg }}^{2}$ & $W_{\text {avg }}$ \\
\hline 9 & 19 & -0.621 & 0.181 & 987.269 & 0.89 & 3.45 & 1.13 \\
\hline 9 & 20 & -0.252 & 0.08 & 71.14 & 0.87 & 3.38 & 1.17 \\
\hline 9 & 21 & 0.085 & 0.044 & 13.415 & 0.83 & 3.16 & 1.23 \\
\hline 9 & 22 & 0.227 & 0.04 & 10.68 & 0.8 & 3.19 & 1.29 \\
\hline 9 & 23 & 0.348 & 0.036 & 12.011 & 0.77 & 3.27 & 1.35 \\
\hline 9 & 24 & 0.471 & 0.034 & 7.003 & 0.74 & 3.26 & 1.41 \\
\hline 9 & 25 & 0.507 & 0.027 & 4.75 & 0.71 & 3.26 & 1.47 \\
\hline 9 & 26 & 0.568 & 0.026 & 4.187 & 0.68 & 3.13 & 1.53 \\
\hline 9 & 27 & 0.499 & 0.028 & 2.914 & 0.65 & 3.14 & 1.59 \\
\hline 9 & 28 & 0.431 & 0.03 & 2.975 & 0.65 & 3.41 & 1.65 \\
\hline 9 & 29 & 0.531 & 0.027 & 1.868 & 0.62 & 3.4 & 1.71 \\
\hline 9 & 30 & 0.494 & 0.028 & 1.986 & 0.6 & 3.4 & 1.77 \\
\hline 9 & 31 & 0.5 & 0.028 & 1.487 & 0.58 & 3.4 & 1.83 \\
\hline 9 & 32 & 0.464 & 0.027 & 1.182 & 0.56 & 3.43 & 1.89 \\
\hline 9 & 33 & 0.43 & 0.026 & 1.573 & 0.53 & 3.31 & 1.95 \\
\hline 9 & 34 & 0.461 & 0.026 & 1.143 & 0.51 & 3.28 & 2.01 \\
\hline
\end{tabular}


Table B.15: Values of $g_{2}$ for the proton, from the EG1b data. Errors (both statistical and systematic) are shown, with averaged kinematics in each bin.

\begin{tabular}{|c|c|c|c|c|c|c|c|}
\hline$Q^{2}$ bin & $W$ bin & $g_{2}$ & $\sigma_{\text {stat }}$ & $\sigma_{\text {sys }}$ & $x_{\text {aug }}$ & $Q_{\text {avg }}^{2}$ & $W_{\text {avg }}$ \\
\hline 4 & 19 & -0.442 & 0.423 & 0.754 & 0.3 & 0.14 & 1.1 \\
\hline 4 & 20 & -0.84 & 0.902 & 0.128 & 0.22 & 0.14 & 1.17 \\
\hline 4 & 21 & 1.263 & 1 & 0.08 & 0.18 & 0.14 & 1.23 \\
\hline 4 & 22 & -0.653 & 0.955 & 0.081 & 0.15 & 0.14 & 1.29 \\
\hline
\end{tabular}

\begin{tabular}{|c|c|c|c|c|c|c|c|}
\hline$Q^{2}$ bin & $W$ bin & $g_{2}$ & $\sigma_{\text {stat }}$ & $\sigma_{\text {sys }}$ & $x_{\text {avg }}$ & $Q_{\text {avg }}^{2}$ & $W_{\text {avg }}$ \\
\hline 5 & 19 & -0.018 & 0.086 & 0.545 & 0.43 & 0.25 & 1.1 \\
\hline 5 & 20 & -0.17 & 0.182 & 0.108 & 0.35 & 0.26 & 1.17 \\
\hline 5 & 21 & -0.029 & 0.215 & 0.035 & 0.29 & 0.26 & 1.23 \\
\hline 5 & 22 & 0.041 & 0.211 & 0.044 & 0.25 & 0.26 & 1.29 \\
\hline 5 & 23 & 0.324 & 0.228 & 0.072 & 0.21 & 0.26 & 1.35 \\
\hline 5 & 24 & -0.182 & 0.259 & 0.085 & 0.19 & 0.26 & 1.41 \\
\hline 5 & 25 & -0.242 & 0.31 & 0.075 & 0.17 & 0.26 & 1.47 \\
\hline 5 & 26 & 0.306 & 0.306 & 0.096 & 0.15 & 0.26 & 1.53 \\
\hline 5 & 27 & -0.011 & 0.295 & 0.119 & 0.14 & 0.26 & 1.59 \\
\hline 5 & 28 & 0.431 & 0.311 & 0.111 & 0.13 & 0.26 & 1.65 \\
\hline 5 & 29 & 0.051 & 0.514 & 0.149 & 0.12 & 0.27 & 1.69 \\
\hline 5 & 32 & -0.243 & 0.947 & 0.045 & 0.09 & 0.28 & 1.89 \\
\hline 5 & 33 & 0.628 & 0.92 & 0.044 & 0.09 & 0.28 & 1.95 \\
\hline
\end{tabular}




\begin{tabular}{|c|c|c|c|c|c|c|c|}
\hline$Q^{2}$ bin & $W$ bin & $g_{2}$ & $\sigma_{\text {stat }}$ & $\sigma_{\text {sys }}$ & $x_{\text {avg }}$ & $Q_{\text {avg }}^{2}$ & $W_{\text {avg }}$ \\
\hline 6 & 19 & 0.027 & 0.027 & 0.229 & 0.6 & 0.52 & 1.1 \\
\hline 6 & 20 & 0.039 & 0.046 & 0.073 & 0.52 & 0.52 & 1.17 \\
\hline 6 & 21 & 0.149 & 0.051 & 0.022 & 0.45 & 0.52 & 1.23 \\
\hline 6 & 22 & 0.017 & 0.047 & 0.032 & 0.4 & 0.52 & 1.29 \\
\hline 6 & 23 & 0.008 & 0.05 & 0.05 & 0.35 & 0.52 & 1.35 \\
\hline 6 & 24 & 0.062 & 0.056 & 0.063 & 0.32 & 0.52 & 1.41 \\
\hline 6 & 25 & 0.032 & 0.083 & 0.077 & 0.27 & 0.48 & 1.47 \\
\hline 6 & 26 & 0.076 & 0.09 & 0.095 & 0.24 & 0.46 & 1.53 \\
\hline 6 & 27 & -0.003 & 0.125 & 0.114 & 0.21 & 0.44 & 1.59 \\
\hline 6 & 28 & -0.089 & 0.193 & 0.101 & 0.2 & 0.47 & 1.64 \\
\hline 6 & 29 & 0.616 & 0.235 & 0.108 & 0.2 & 0.51 & 1.71 \\
\hline 6 & 30 & 0.129 & 0.232 & 0.082 & 0.18 & 0.51 & 1.77 \\
\hline 6 & 31 & -0.258 & 0.236 & 0.053 & 0.17 & 0.51 & 1.83 \\
\hline 6 & 32 & -0.111 & 0.258 & 0.041 & 0.16 & 0.5 & 1.89 \\
\hline 6 & 33 & -0.261 & 0.334 & 0.047 & 0.13 & 0.45 & 1.95 \\
\hline 6 & 34 & 1.021 & 0.704 & 0.097 & 0.11 & 0.39 & 1.99 \\
\hline
\end{tabular}




\begin{tabular}{|c|c|c|c|c|c|c|c|}
\hline$Q^{2}$ bin & $W$ bin & $g_{2}$ & $\sigma_{\text {stat }}$ & $\sigma_{\text {sys }}$ & $x_{\text {avg }}$ & $Q_{\text {avg }}^{2}$ & $W_{\text {avg }}$ \\
\hline 7 & 19 & -0.059 & 0.014 & 0.143 & 0.73 & 0.94 & 1.1 \\
\hline 7 & 20 & -0.019 & 0.026 & 0.061 & 0.65 & 0.93 & 1.17 \\
\hline 7 & 21 & 0.027 & 0.034 & 0.027 & 0.59 & 0.95 & 1.23 \\
\hline 7 & 22 & 0.058 & 0.032 & 0.033 & 0.54 & 0.95 & 1.29 \\
\hline 7 & 23 & -0.016 & 0.046 & 0.049 & 0.47 & 0.85 & 1.35 \\
\hline 7 & 24 & 0.045 & 0.061 & 0.056 & 0.45 & 0.92 & 1.41 \\
\hline 7 & 25 & 0.082 & 0.076 & 0.062 & 0.42 & 0.92 & 1.47 \\
\hline 7 & 26 & -0.015 & 0.109 & 0.067 & 0.37 & 0.84 & 1.53 \\
\hline 7 & 27 & 0.067 & 0.117 & 0.09 & 0.33 & 0.82 & 1.59 \\
\hline 7 & 28 & 0.138 & 0.16 & 0.078 & 0.31 & 0.81 & 1.64 \\
\hline 7 & 29 & -0.446 & 0.231 & 0.078 & 0.27 & 0.77 & 1.71 \\
\hline 7 & 30 & -0.82 & 0.217 & 0.08 & 0.25 & 0.78 & 1.77 \\
\hline 7 & 31 & -1.421 & 0.394 & 0.06 & 0.27 & 0.92 & 1.82 \\
\hline 7 & 32 & -1.539 & 0.543 & 0.078 & 0.28 & 1.06 & 1.89 \\
\hline 7 & 33 & -0.161 & 0.544 & 0.185 & 0.27 & 1.07 & 1.95 \\
\hline 7 & 34 & -0.685 & 0.78 & 0.279 & 0.25 & 1.07 & 1.99 \\
\hline
\end{tabular}




\begin{tabular}{|c|c|c|c|c|c|c|c|}
\hline$Q^{2}$ bin & $W$ bin & $g_{2}$ & $\sigma_{\text {stat }}$ & $\sigma_{\text {sys }}$ & $x_{\text {avg }}$ & $Q_{\text {avg }}^{2}$ & $W_{\text {avg }}$ \\
\hline 8 & 19 & -0.021 & 0.03 & 0.284 & 0.86 & 2.13 & 1.1 \\
\hline 8 & 20 & 0.047 & 0.076 & 0.1 & 0.81 & 2.06 & 1.17 \\
\hline 8 & 21 & -0.144 & 0.08 & 0.094 & 0.76 & 2.13 & 1.23 \\
\hline 8 & 23 & -0.268 & 0.072 & 0.173 & 0.69 & 2.14 & 1.35 \\
\hline 8 & 24 & 0.034 & 0.084 & 0.151 & 0.66 & 2.14 & 1.41 \\
\hline 8 & 25 & -0.179 & 0.104 & 0.169 & 0.62 & 2.13 & 1.47 \\
\hline 8 & 26 & -0.435 & 0.11 & 0.213 & 0.59 & 2.12 & 1.53 \\
\hline 8 & 27 & -0.273 & 0.107 & 0.164 & 0.56 & 2.11 & 1.59 \\
\hline 8 & 28 & -0.156 & 0.122 & 0.218 & 0.53 & 2.13 & 1.65 \\
\hline 8 & 29 & -0.199 & 0.139 & 0.174 & 0.5 & 2.12 & 1.71 \\
\hline 8 & 30 & -0.245 & 0.134 & 0.185 & 0.48 & 2.11 & 1.77 \\
\hline 8 & 31 & -0.188 & 0.145 & 0.162 & 0.46 & 2.11 & 1.83 \\
\hline 8 & 32 & -0.084 & 0.167 & 0.15 & 0.43 & 2.05 & 1.89 \\
\hline 8 & 33 & -0.193 & 0.222 & 0.247 & 0.38 & 1.83 & 1.95 \\
\hline 8 & 34 & -0.012 & 0.329 & 0.269 & 0.37 & 1.81 & 2 \\
\hline
\end{tabular}




\begin{tabular}{|c|c|c|c|c|c|c|c|}
\hline$Q^{2}$ bin & $W$ bin & $g_{2}$ & $\sigma_{\text {stat }}$ & $\sigma_{\text {sys }}$ & $x_{\text {avg }}$ & $Q_{\text {avg }}^{2}$ & $W_{\text {avg }}$ \\
\hline 9 & 19 & -0.052 & 0.023 & 0.854 & 0.9 & 2.88 & 1.09 \\
\hline 9 & 20 & 0.019 & 0.074 & 0.451 & 0.86 & 2.88 & 1.16 \\
\hline 9 & 21 & 0.113 & 0.083 & 0.285 & 0.82 & 2.88 & 1.24 \\
\hline 9 & 22 & -0.195 & 0.066 & 0.241 & 0.79 & 2.88 & 1.29 \\
\hline 9 & 23 & -0.168 & 0.075 & 0.323 & 0.75 & 2.88 & 1.35 \\
\hline 9 & 24 & 0.034 & 0.093 & 0.255 & 0.72 & 2.88 & 1.41 \\
\hline 9 & 25 & -0.112 & 0.103 & 0.258 & 0.69 & 2.88 & 1.46 \\
\hline 9 & 26 & -0.212 & 0.115 & 0.327 & 0.66 & 2.87 & 1.53 \\
\hline 9 & 27 & 0.003 & 0.12 & 0.217 & 0.64 & 2.87 & 1.58 \\
\hline
\end{tabular}




\section{B.7 Moments and Integrals}

Table B.16: Values of the moment $\Gamma_{1}^{p}$ from the integration of $g_{1}$. Values and systematic errors are shown for the data contributions (d) and for the data + model $(d+m)$. Statistical errors are the same in both cases.

\begin{tabular}{|c|c|c|c|c|c|c|}
\hline bin & $Q_{\text {avg }}^{2}\left(\mathrm{GeV}^{2}\right)$ & $\Gamma_{1}(\mathrm{~d})$ & $\sigma_{\text {sys }}(\mathrm{d})$ & $\Gamma_{1}(\mathrm{~d}+\mathrm{m})$ & $\sigma_{\text {sys }}(\mathrm{d}+\mathrm{m})$ & $\sigma_{\text {stat }}$ \\
\hline \hline 10 & 0.051 & -0.0122 & 0.0005 & -0.0097 & 0.0020 & 0.0026 \\
\hline 11 & 0.060 & -0.0121 & 0.0009 & -0.0087 & 0.0019 & 0.0019 \\
\hline 12 & 0.071 & -0.0157 & 0.0012 & -0.0118 & 0.0019 & 0.0017 \\
\hline 13 & 0.084 & -0.0188 & 0.0013 & -0.0144 & 0.0019 & 0.0018 \\
\hline 14 & 0.101 & -0.0144 & 0.0015 & -0.0093 & 0.0020 & 0.0019 \\
\hline 15 & 0.121 & -0.0153 & 0.0018 & -0.0095 & 0.0022 & 0.0020 \\
\hline 16 & 0.144 & -0.0115 & 0.0023 & -0.0051 & 0.0026 & 0.0019 \\
\hline 17 & 0.174 & -0.0114 & 0.0027 & -0.0045 & 0.0030 & 0.0016 \\
\hline 18 & 0.206 & -0.0064 & 0.0030 & 0.0011 & 0.0034 & 0.0014 \\
\hline 19 & 0.246 & 0.0019 & 0.0034 & 0.0109 & 0.0037 & 0.0014 \\
\hline 20 & 0.291 & 0.0072 & 0.0038 & 0.0179 & 0.0041 & 0.0011 \\
\hline 21 & 0.346 & 0.0171 & 0.0042 & 0.0297 & 0.0045 & 0.0010 \\
\hline 22 & 0.413 & 0.0261 & 0.0045 & 0.0408 & 0.0049 & 0.0010 \\
\hline 23 & 0.493 & 0.0326 & 0.0049 & 0.0499 & 0.0052 & 0.0010 \\
\hline 24 & 0.588 & 0.0416 & 0.0051 & 0.0620 & 0.0055 & 0.0010 \\
\hline 25 & 0.702 & 0.0495 & 0.0052 & 0.0736 & 0.0056 & 0.0010 \\
\hline 26 & 0.837 & 0.0575 & 0.0051 & 0.0861 & 0.0056 & 0.0010 \\
\hline 27 & 1.002 & 0.0601 & 0.0047 & 0.0943 & 0.0055 & 0.0009 \\
\hline 28 & 1.195 & 0.0618 & 0.0042 & 0.1023 & 0.0052 & 0.0009 \\
\hline 29 & 1.423 & 0.0625 & 0.0037 & 0.1104 & 0.0050 & 0.0008 \\
\hline 30 & 1.702 & 0.0595 & 0.0032 & 0.1165 & 0.0049 & 0.0007 \\
\hline 31 & 2.033 & 0.0544 & 0.0027 & 0.1215 & 0.0047 & 0.0007 \\
\hline 32 & 2.417 & 0.0454 & 0.0023 & 0.1240 & 0.0047 & 0.0006 \\
\hline 33 & 2.875 & 0.0353 & 0.0019 & 0.1265 & 0.0046 & 0.0006 \\
\hline 34 & 3.418 & 0.0256 & 0.0015 & 0.1282 & 0.0046 & 0.0005 \\
\hline 35 & 3.985 & 0.0176 & 0.0013 & 0.1290 & 0.0046 & 0.0006 \\
\hline 36 & 4.720 & 0.0070 & 0.0011 & 0.1302 & 0.0046 & 0.0005 \\
\hline & & & & & & \\
\hline
\end{tabular}


Table B.17: Values of the moment $\Gamma_{3}^{p}$ from the integration of $g_{1}$. Values and systematic errors are shown for the data contributions (d) and for the data + model $(d+m)$. Statistical errors are the same in both cases. All values except $Q^{2}$ are multiplied by a factor of 100 .

\begin{tabular}{|c|c|c|c|c|c|c|}
\hline bin & $Q_{a v q}^{2}\left(\mathrm{GeV}^{2}\right)$ & $\Gamma_{3}(\mathrm{~d})$ & $\sigma_{\text {sys }}(\mathrm{d})$ & $\Gamma_{3}(\mathrm{~d}+\mathrm{m})$ & $\sigma_{\text {sys }}(\mathrm{d}+\mathrm{m})$ & $\sigma_{\text {stat }}$ \\
\hline \hline 10 & 0.051 & -0.0059 & 0.0001 & -0.0053 & 0.0002 & 0.0018 \\
\hline 11 & 0.060 & -0.0083 & 0.0002 & -0.0069 & 0.0003 & 0.0018 \\
\hline 12 & 0.071 & -0.0164 & 0.0003 & -0.0144 & 0.0005 & 0.0024 \\
\hline 13 & 0.084 & -0.0283 & 0.0005 & -0.0254 & 0.0008 & 0.0032 \\
\hline 14 & 0.101 & -0.0323 & 0.0009 & -0.0284 & 0.0013 & 0.0045 \\
\hline 15 & 0.120 & -0.0496 & 0.0016 & -0.0444 & 0.0022 & 0.0063 \\
\hline 16 & 0.143 & -0.0511 & 0.0031 & -0.0443 & 0.0040 & 0.0080 \\
\hline 17 & 0.173 & -0.0936 & 0.0054 & -0.0877 & 0.0066 & 0.0084 \\
\hline 18 & 0.204 & -0.1193 & 0.0083 & -0.1123 & 0.0097 & 0.0096 \\
\hline 19 & 0.244 & -0.1149 & 0.0121 & -0.1070 & 0.0135 & 0.0113 \\
\hline 20 & 0.291 & -0.1451 & 0.0176 & -0.1364 & 0.0189 & 0.0122 \\
\hline 21 & 0.346 & -0.1229 & 0.0264 & -0.1139 & 0.0275 & 0.0133 \\
\hline 22 & 0.414 & -0.0895 & 0.0373 & -0.0802 & 0.0382 & 0.0145 \\
\hline 23 & 0.492 & -0.0479 & 0.0505 & -0.0385 & 0.0512 & 0.0156 \\
\hline 24 & 0.588 & 0.0462 & 0.0631 & 0.0560 & 0.0636 & 0.0168 \\
\hline 25 & 0.700 & 0.2158 & 0.0741 & 0.2264 & 0.0746 & 0.0181 \\
\hline 26 & 0.831 & 0.4026 & 0.0822 & 0.4153 & 0.0825 & 0.0212 \\
\hline 27 & 1.002 & 0.4979 & 0.0851 & 0.5176 & 0.0853 & 0.0234 \\
\hline 28 & 1.193 & 0.6833 & 0.0825 & 0.7105 & 0.0827 & 0.0216 \\
\hline 29 & 1.416 & 0.8458 & 0.0761 & 0.8886 & 0.0764 & 0.0220 \\
\hline 30 & 1.699 & 0.9557 & 0.0688 & 1.0245 & 0.0693 & 0.0217 \\
\hline 31 & 2.031 & 1.0104 & 0.0618 & 1.1203 & 0.0626 & 0.0190 \\
\hline 32 & 2.419 & 0.9903 & 0.0560 & 1.1659 & 0.0572 & 0.0168 \\
\hline 33 & 2.877 & 0.9175 & 0.0520 & 1.1964 & 0.0536 & 0.0156 \\
\hline 34 & 3.419 & 0.7833 & 0.0473 & 1.1916 & 0.0492 & 0.0164 \\
\hline 35 & 4.035 & 0.6266 & 0.0446 & 1.1861 & 0.0467 & 0.0200 \\
\hline 36 & 4.751 & 0.3245 & 0.0411 & 1.1653 & 0.0436 & 0.0193 \\
\hline & & & & & & \\
\hline
\end{tabular}


Table B.18: Values of the moment $\Gamma_{5}^{p}$ from the integration of $g_{1}$. Values and systematic errors are shown for the data contributions (d) and for the data + model $(d+m)$. Statistical errors are the same in both cases. All values except $Q^{2}$ are multiplied by a factor of $10^{4}$.

\begin{tabular}{|c|c|c|c|c|c|c|}
\hline bin & $Q_{\text {avg }}^{2}\left(\mathrm{GeV}^{2}\right)$ & $\Gamma_{5}(\mathrm{~d})$ & $\sigma_{\text {sys }}(\mathrm{d})$ & $\Gamma_{5}(\mathrm{~d}+\mathrm{m})$ & $\sigma_{\text {sys }}(\mathrm{d}+\mathrm{m})$ & $\sigma_{\text {stat }}$ \\
\hline \hline 10 & 0.051 & -0.0036 & 0.0001 & -0.0026 & 0.0001 & 0.0015 \\
\hline 11 & 0.060 & -0.0072 & 0.0001 & -0.0042 & 0.0001 & 0.0023 \\
\hline 12 & 0.071 & -0.0202 & 0.0004 & -0.0141 & 0.0004 & 0.0043 \\
\hline 13 & 0.084 & -0.0502 & 0.0004 & -0.0384 & 0.0004 & 0.0076 \\
\hline 14 & 0.101 & -0.0779 & 0.0009 & -0.0568 & 0.0009 & 0.0143 \\
\hline 15 & 0.120 & -0.1625 & 0.0019 & -0.1258 & 0.0019 & 0.0267 \\
\hline 16 & 0.143 & -0.2052 & 0.0051 & -0.1442 & 0.0052 & 0.0442 \\
\hline 17 & 0.173 & -0.5542 & 0.0137 & -0.4832 & 0.0139 & 0.0654 \\
\hline 18 & 0.204 & -0.9701 & 0.0327 & -0.8668 & 0.0332 & 0.0921 \\
\hline 19 & 0.244 & -1.2384 & 0.0653 & -1.0950 & 0.0666 & 0.1356 \\
\hline 20 & 0.291 & -2.1775 & 0.1149 & -1.9893 & 0.1178 & 0.1824 \\
\hline 21 & 0.346 & -2.6968 & 0.2084 & -2.4677 & 0.2136 & 0.2377 \\
\hline 22 & 0.414 & -3.3930 & 0.3594 & -3.1255 & 0.3672 & 0.3121 \\
\hline 23 & 0.492 & -3.9101 & 0.6224 & -3.6165 & 0.6323 & 0.3904 \\
\hline 24 & 0.588 & -4.1312 & 0.9745 & -3.8185 & 0.9860 & 0.4835 \\
\hline 25 & 0.700 & -1.3321 & 1.3950 & -1.0199 & 1.4073 & 0.5810 \\
\hline 26 & 0.829 & 2.2229 & 1.8515 & 2.5305 & 1.8637 & 0.7454 \\
\hline 27 & 1.001 & 3.2824 & 2.2448 & 3.7143 & 2.2557 & 0.9430 \\
\hline 28 & 1.193 & 9.2125 & 2.4667 & 9.6268 & 2.4760 & 0.9242 \\
\hline 29 & 1.414 & 15.0471 & 2.4596 & 15.5934 & 2.4685 & 0.9571 \\
\hline 30 & 1.698 & 20.7525 & 2.3036 & 21.4205 & 2.3163 & 1.0450 \\
\hline 31 & 2.031 & 24.6403 & 2.0892 & 25.5637 & 2.1119 & 0.9346 \\
\hline 32 & 2.419 & 27.8380 & 1.9030 & 29.1144 & 1.9392 & 0.8333 \\
\hline 33 & 2.880 & 29.2122 & 1.7945 & 31.4584 & 1.8413 & 0.7285 \\
\hline 34 & 3.417 & 28.1168 & 1.6856 & 32.0632 & 1.7441 & 0.7354 \\
\hline 35 & 4.066 & 25.2141 & 1.6358 & 32.2644 & 1.7010 & 0.8681 \\
\hline 36 & 4.787 & 15.7258 & 1.5672 & 31.6886 & 1.6412 & 0.8819 \\
\hline & & & & & & \\
\hline
\end{tabular}


Table B.19: Values of the moment $\int g_{2} d x$ from the integration of $g_{2}$. Values and systematic errors are shown for the data contributions (d) and for the data + model $(d+m)$. Statistical errors are the same in both cases.

\begin{tabular}{|c|c|c|c|c|c|c|}
\hline bin & $Q_{\text {avg }}^{2}\left(\mathrm{GeV}^{2}\right)$ & $\int g_{2} d x(\mathrm{~d})$ & $\sigma_{\text {sys }}(\mathrm{d})$ & $\int g_{2} d x(\mathrm{~d}+\mathrm{m})$ & $\sigma_{\text {sys }}(\mathrm{d}+\mathrm{m})$ & $\sigma_{\text {stat }}$ \\
\hline \hline 2 & 0.071 & 0.0489 & 0.0926 & 0.0780 & 0.0971 & 0.0466 \\
\hline 3 & 0.112 & -0.0249 & 0.0447 & -0.0076 & 0.0468 & 0.0585 \\
\hline 4 & 0.246 & -0.0084 & 0.0180 & -0.0000 & 0.0289 & 0.0283 \\
\hline 5 & 0.434 & 0.0040 & 0.0128 & 0.0181 & 0.0234 & 0.0157 \\
\hline 6 & 0.935 & -0.0310 & 0.0104 & -0.0098 & 0.0155 & 0.0174 \\
\hline 7 & 2.102 & -0.0605 & 0.0083 & -0.0423 & 0.0093 & 0.0113 \\
\hline 8 & 3.451 & -0.0238 & 0.0077 & -0.0175 & 0.0082 & 0.0085 \\
\hline
\end{tabular}

Table B.20: Values of the moment $\int x^{2} g_{2} d x$ from the integration of $g_{2}$. Values and systematic errors are shown for the data contributions (d) and for the data + model $(d+m)$. Statistical errors are the same in both cases. All values except $Q^{2}$ are multiplled by a factor of 100.

\begin{tabular}{|c|c|c|c|c|c|c|}
\hline bin & $Q_{a v g}^{2}\left(\mathrm{GeV}^{2}\right)$ & $\int x^{2} g_{2} d x(\mathrm{~d})$ & $\sigma_{\text {sys }}(\mathrm{d})$ & $\int x^{2} g_{2} d x(\mathrm{~d}+\mathrm{m})$ & $\sigma_{\text {sys }}(\mathrm{d}+\mathrm{m})$ & $\sigma_{\text {stat }}$ \\
\hline \hline 2 & 0.071 & 0.0047 & 0.0116 & 0.0295 & 0.0492 & 0.0047 \\
\hline 3 & 0.085 & 0.0031 & 0.0116 & 0.0396 & 0.0492 & 0.0215 \\
\hline 4 & 0.225 & -0.1461 & 0.0242 & -0.1457 & 0.0325 & 0.1923 \\
\hline 5 & 0.483 & 0.1037 & 0.1553 & 0.1018 & 0.1556 & 0.1451 \\
\hline 6 & 0.851 & -0.0616 & 0.1792 & -0.0736 & 0.1797 & 0.1742 \\
\hline 7 & 1.929 & -1.8138 & 0.2491 & -1.9515 & 0.2498 & 0.3812 \\
\hline 8 & 3.273 & -1.4575 & 0.3404 & -1.8756 & 0.3420 & 0.3967 \\
\hline
\end{tabular}


Table B.21: Values of the integral $d_{2}$ from the integration of $g_{1}$ and $g_{2}$ data. Values and systematic errors are shown for the data contributions (d) and for the data + model $(d+m)$. Statistical errors are the same in both cases.

\begin{tabular}{|c|c|c|c|c|c|c|}
\hline bin & $Q_{\text {avg }}^{2}\left(\mathrm{GeV}^{2}\right)$ & $d_{2}(\mathrm{~d})$ & $\sigma_{\text {sys }}(\mathrm{d})$ & $d_{2}(\mathrm{~d}+\mathrm{m})$ & $\sigma_{\text {sys }}(\mathrm{d}+\mathrm{m})$ & $\sigma_{\text {stat }}$ \\
\hline \hline 3 & 0.134 & -0.0046 & 0.0049 & -0.0042 & 0.0109 & 0.0106 \\
\hline 4 & 0.222 & -0.0073 & 0.0028 & -0.0068 & 0.0041 & 0.0065 \\
\hline 5 & 0.482 & 0.0002 & 0.0091 & 0.0005 & 0.0092 & 0.0056 \\
\hline 6 & 0.806 & -0.0132 & 0.0125 & -0.0129 & 0.0126 & 0.0085 \\
\hline 7 & 2.095 & -0.0451 & 0.0203 & -0.0527 & 0.0222 & 0.0149 \\
\hline 8 & 2.879 & -0.0334 & 0.0323 & -0.0612 & 0.0395 & 0.0145 \\
\hline
\end{tabular}


Table B.22: Values of the spin polarizability integral $\int x^{2} A_{1} F_{1} d x$. Values and systematic errors are shown for the data contributions (d) and for the data + model $(d+m)$. Statistical errors are the same in both cases. All values except $Q^{2}$ are multiplied by a factor of 100.

\begin{tabular}{|c|c|c|c|c|c|c|}
\hline bin & $Q_{\text {avg }}^{2}\left(\mathrm{GeV}^{2}\right)$ & $\int x^{2} A_{1} F_{1} d x(\mathrm{~d})$ & $\sigma_{\text {sys }}(\mathrm{d})$ & $\int x^{2} A_{1} F_{1} d x(\mathrm{~d}+\mathrm{m})$ & $\sigma_{\text {sys }}(\mathrm{d}+\mathrm{m})$ & $\sigma_{\text {stat }}$ \\
\hline \hline 10 & 0.051 & -0.0097 & 0.0001 & -0.0105 & 0.0001 & 0.0018 \\
\hline 11 & 0.060 & -0.0164 & 0.0003 & -0.0154 & 0.0003 & 0.0018 \\
\hline 12 & 0.071 & -0.0306 & 0.0003 & -0.0286 & 0.0004 & 0.0024 \\
\hline 13 & 0.084 & -0.0521 & 0.0006 & -0.0491 & 0.0006 & 0.0032 \\
\hline 14 & 0.101 & -0.0707 & 0.0010 & -0.0663 & 0.0010 & 0.0045 \\
\hline 15 & 0.120 & -0.1097 & 0.0017 & -0.1036 & 0.0017 & 0.0063 \\
\hline 16 & 0.143 & -0.1420 & 0.0031 & -0.1336 & 0.0031 & 0.0080 \\
\hline 17 & 0.173 & -0.2267 & 0.0053 & -0.2173 & 0.0053 & 0.0084 \\
\hline 18 & 0.204 & -0.3012 & 0.0080 & -0.2893 & 0.0080 & 0.0096 \\
\hline 19 & 0.244 & -0.3460 & 0.0115 & -0.3314 & 0.0115 & 0.0113 \\
\hline 20 & 0.291 & -0.4189 & 0.0169 & -0.4017 & 0.0169 & 0.0122 \\
\hline 21 & 0.346 & -0.4199 & 0.0255 & -0.4007 & 0.0256 & 0.0133 \\
\hline 22 & 0.414 & -0.3895 & 0.0365 & -0.3687 & 0.0366 & 0.0145 \\
\hline 23 & 0.492 & -0.3184 & 0.0498 & -0.2967 & 0.0499 & 0.0156 \\
\hline 24 & 0.588 & -0.1754 & 0.0626 & -0.1533 & 0.0627 & 0.0168 \\
\hline 25 & 0.700 & 0.0764 & 0.0739 & 0.0985 & 0.0740 & 0.0181 \\
\hline 26 & 0.831 & 0.3558 & 0.0821 & 0.3788 & 0.0822 & 0.0212 \\
\hline 27 & 1.002 & 0.5441 & 0.0852 & 0.5766 & 0.0852 & 0.0234 \\
\hline 28 & 1.193 & 0.8228 & 0.0826 & 0.8605 & 0.0827 & 0.0216 \\
\hline 29 & 1.416 & 1.0529 & 0.0762 & 1.1073 & 0.0765 & 0.0220 \\
\hline 30 & 1.699 & 1.2133 & 0.0688 & 1.2933 & 0.0695 & 0.0217 \\
\hline 31 & 2.031 & 1.2920 & 0.0618 & 1.4133 & 0.0630 & 0.0190 \\
\hline 32 & 2.419 & 1.2683 & 0.0561 & 1.4543 & 0.0577 & 0.0168 \\
\hline 33 & 2.877 & 1.1732 & 0.0522 & 1.4667 & 0.0542 & 0.0156 \\
\hline 34 & 3.419 & 1.0030 & 0.0476 & 1.4344 & 0.0502 & 0.0164 \\
\hline 35 & 4.035 & 0.7967 & 0.0450 & 1.3947 & 0.0479 & 0.0200 \\
\hline 36 & 4.751 & 0.4162 & 0.0418 & 1.3407 & 0.0452 & 0.0193 \\
\hline & & & & & & \\
\hline
\end{tabular}


Table B.23: Values of the forward spin polarizability $\gamma_{0}$. Values and systematic errors are shown for the data contributions (d) and for the data + model $(d+m)$. The values of $\gamma_{0}$ and its errors are given in units of $10^{-4} \mathrm{fm}$. Statistical errors are the same in both cases.

\begin{tabular}{|c|c|c|c|c|c|c|}
\hline bin & $Q_{\text {avg }}^{2}\left(\mathrm{GeV}^{2}\right)$ & $\gamma_{0}(\mathrm{~d})$ & $\sigma_{\text {sys }}(\mathrm{d})$ & $\gamma_{0}(\mathrm{~d}+\mathrm{m})$ & $\sigma_{\text {sys }}(\mathrm{d}+\mathrm{m})$ & $\sigma_{\text {stat }}$ \\
\hline \hline 10 & 0.051 & -1.1381 & 0.0090 & -1.2346 & 0.2799 & 0.2170 \\
\hline 11 & 0.060 & -1.1975 & 0.0324 & -1.1275 & 0.1250 & 0.1335 \\
\hline 12 & 0.071 & -1.3482 & 0.0267 & -1.2578 & 0.0433 & 0.1049 \\
\hline 13 & 0.084 & -1.3557 & 0.0254 & -1.2774 & 0.0294 & 0.0828 \\
\hline 14 & 0.101 & -1.0816 & 0.0229 & -1.0153 & 0.0233 & 0.0691 \\
\hline 15 & 0.120 & -0.9800 & 0.0199 & -0.9254 & 0.0199 & 0.0565 \\
\hline 16 & 0.143 & -0.7509 & 0.0154 & -0.7068 & 0.0154 & 0.0421 \\
\hline 17 & 0.173 & -0.6858 & 0.0123 & -0.6573 & 0.0123 & 0.0254 \\
\hline 18 & 0.204 & -0.5486 & 0.0094 & -0.5269 & 0.0094 & 0.0174 \\
\hline 19 & 0.244 & -0.3720 & 0.0082 & -0.3564 & 0.0082 & 0.0121 \\
\hline 20 & 0.291 & -0.2639 & 0.0069 & -0.2531 & 0.0069 & 0.0077 \\
\hline 21 & 0.346 & -0.1577 & 0.0056 & -0.1505 & 0.0056 & 0.0050 \\
\hline 22 & 0.414 & -0.0856 & 0.0043 & -0.0810 & 0.0043 & 0.0032 \\
\hline 23 & 0.492 & -0.0415 & 0.0030 & -0.0386 & 0.0030 & 0.0020 \\
\hline 24 & 0.588 & -0.0134 & 0.0020 & -0.0117 & 0.0020 & 0.0013 \\
\hline 25 & 0.700 & 0.0035 & 0.0012 & 0.0045 & 0.0012 & 0.0008 \\
\hline 26 & 0.831 & 0.0097 & 0.0006 & 0.0103 & 0.0006 & 0.0006 \\
\hline 27 & 1.002 & 0.0084 & 0.0003 & 0.0089 & 0.0003 & 0.0004 \\
\hline 28 & 1.193 & 0.0075 & 0.0002 & 0.0079 & 0.0002 & 0.0002 \\
\hline 29 & 1.416 & 0.0058 & 0.0001 & 0.0061 & 0.0001 & 0.0001 \\
\hline 30 & 1.699 & 0.0038 & 0.0000 & 0.0041 & 0.0000 & 0.0001 \\
\hline 31 & 2.031 & 0.0024 & 0.0000 & 0.0026 & 0.0000 & 0.0000 \\
\hline 32 & 2.419 & 0.0014 & 0.0000 & 0.0016 & 0.0000 & 0.0000 \\
\hline 33 & 2.877 & 0.0008 & 0.0000 & 0.0010 & 0.0000 & 0.0000 \\
\hline 34 & 3.419 & 0.0004 & 0.0000 & 0.0006 & 0.0000 & 0.0000 \\
\hline 35 & 4.035 & 0.0002 & 0.0000 & 0.0003 & 0.0000 & 0.0000 \\
\hline 36 & 4.751 & 0.0001 & 0.0000 & 0.0002 & 0.0000 & 0.0000 \\
\hline
\end{tabular}


Table B.24: Values of the integral $B_{1}$ used in hyperfine splitting calculations. Values and systematic errors are shown for the data contributions (d) and for the data + model $(d+m)$. Statistical errors are the same in both cases. All values except $Q^{2}$ are multiplied by a factor of 100 .

\begin{tabular}{|c|c|c|c|c|c|c|}
\hline bin & $Q_{\text {avg }}^{2}\left(\mathrm{GeV}^{2}\right)$ & $B_{1}(\mathrm{~d})$ & $\sigma_{\text {sys }}(\mathrm{d})$ & $B_{1}(\mathrm{~d}+\mathrm{m})$ & $\sigma_{\text {sys }}(\mathrm{d}+\mathrm{m})$ & $\sigma_{\text {stat }}$ \\
\hline \hline 10 & 0.051 & -1.1234 & 0.0567 & -0.8820 & 0.1825 & 0.2350 \\
\hline 11 & 0.060 & -1.1006 & 0.0904 & -0.7707 & 0.1661 & 0.1654 \\
\hline 12 & 0.071 & -1.3984 & 0.1118 & -1.0224 & 0.1661 & 0.1518 \\
\hline 13 & 0.084 & -1.6416 & 0.1249 & -1.2123 & 0.1704 & 0.1550 \\
\hline 14 & 0.101 & -1.2146 & 0.1442 & -0.7268 & 0.1830 & 0.1618 \\
\hline 15 & 0.121 & -1.2489 & 0.1721 & -0.6983 & 0.2071 & 0.1668 \\
\hline 16 & 0.144 & -0.9164 & 0.2090 & -0.2951 & 0.2428 & 0.1634 \\
\hline 17 & 0.175 & -0.8008 & 0.2421 & -0.1314 & 0.2760 & 0.1427 \\
\hline 18 & 0.206 & -0.2972 & 0.2749 & 0.4327 & 0.3093 & 0.1195 \\
\hline 19 & 0.247 & 0.4430 & 0.3046 & 1.3325 & 0.3390 & 0.1170 \\
\hline 20 & 0.291 & 0.9690 & 0.3323 & 2.0210 & 0.3667 & 0.0937 \\
\hline 21 & 0.346 & 1.8602 & 0.3616 & 3.1063 & 0.3962 & 0.0869 \\
\hline 22 & 0.413 & 2.6523 & 0.3884 & 4.1176 & 0.4235 & 0.0842 \\
\hline 23 & 0.493 & 3.2290 & 0.4158 & 4.9445 & 0.4525 & 0.0833 \\
\hline 24 & 0.588 & 4.0000 & 0.4372 & 6.0281 & 0.4774 & 0.0846 \\
\hline 25 & 0.703 & 4.6522 & 0.4478 & 7.0522 & 0.4949 & 0.0853 \\
\hline 26 & 0.838 & 5.3477 & 0.4414 & 8.2006 & 0.5003 & 0.0855 \\
\hline 27 & 1.003 & 5.5875 & 0.4194 & 8.9899 & 0.4956 & 0.0809 \\
\hline 28 & 1.196 & 5.7131 & 0.3821 & 9.7424 & 0.4837 & 0.0767 \\
\hline 29 & 1.424 & 5.7682 & 0.3397 & 10.5415 & 0.4723 & 0.0743 \\
\hline 30 & 1.703 & 5.4995 & 0.2940 & 11.1617 & 0.4631 & 0.0657 \\
\hline 31 & 2.033 & 5.0401 & 0.2513 & 11.6950 & 0.4575 & 0.0602 \\
\hline 32 & 2.417 & 4.2019 & 0.2104 & 11.9954 & 0.4541 & 0.0549 \\
\hline 33 & 2.875 & 3.2675 & 0.1795 & 12.2948 & 0.4535 & 0.0517 \\
\hline 34 & 3.418 & 2.3663 & 0.1402 & 12.5185 & 0.4540 & 0.0501 \\
\hline 35 & 3.983 & 1.6263 & 0.1233 & 12.6399 & 0.4551 & 0.0598 \\
\hline 36 & 4.719 & 0.6392 & 0.1057 & 12.7991 & 0.4569 & 0.0429 \\
\hline & & & & & & \\
\hline
\end{tabular}


Table B.25: Values of the integral $B_{2}$ used in hyperfine splitting calculations. Values and systematic errors are shown for the data contributions (d) and for the data + model $(d+m)$. Statistical errors are the same in both cases. All values except $Q^{2}$ are multiplled by a factor of 100 .

\begin{tabular}{|c|c|c|c|c|c|c|}
\hline bin & $Q_{\text {avg }}^{2}\left(\mathrm{GeV}^{2}\right)$ & $B_{2}(\mathrm{~d})$ & $\sigma_{\text {sys }}(\mathrm{d})$ & $B_{2}(\mathrm{~d}+\mathrm{m})$ & $\sigma_{\text {sys }}(\mathrm{d}+\mathrm{m})$ & $\sigma_{\text {stat }}$ \\
\hline \hline 2 & 0.071 & 0.0571 & 0.1386 & 0.3023 & 0.4825 & 0.0567 \\
\hline 3 & 0.086 & 0.0178 & 0.1372 & 0.3050 & 0.4753 & 0.2100 \\
\hline 4 & 0.244 & -0.3096 & 0.1753 & -0.3057 & 0.1824 & 0.4459 \\
\hline 5 & 0.505 & 0.0706 & 0.1393 & 0.0714 & 0.1404 & 0.1660 \\
\hline 6 & 0.906 & -0.1096 & 0.1209 & -0.1197 & 0.1218 & 0.1310 \\
\hline 7 & 2.096 & -0.6326 & 0.0719 & -0.6922 & 0.0725 & 0.1282 \\
\hline 8 & 3.442 & -0.2927 & 0.0907 & -0.3982 & 0.0911 & 0.0842 \\
\hline
\end{tabular}


Table B.26: Values of the leading twist integral $B_{2}^{W W}$ used in hyperfine splitting calculations. Values and systematic errors are shown for the data contributions (d) and for the data + model $(d+m)$. Statistical errors are the same in both cases. All values except $Q^{2}$ are multiplied by a factor of 100.

\begin{tabular}{|c|c|c|c|c|c|c|}
\hline bin & $Q_{a v g}^{2}\left(\mathrm{GeV}^{2}\right)$ & $B_{2}^{W W}(\mathrm{~d})$ & $\sigma_{\text {sys }}(\mathrm{d})$ & $B_{2}^{W W}(\mathrm{~d}+\mathrm{m})$ & $\sigma_{\text {sys }}(\mathrm{d}+\mathrm{m})$ & $\sigma_{\text {stat }}$ \\
\hline \hline 10 & 0.051 & 0.0551 & 0.0003 & 0.0512 & 0.0070 & 0.0162 \\
\hline 11 & 0.060 & 0.0636 & 0.0025 & 0.0556 & 0.0084 & 0.0128 \\
\hline 12 & 0.071 & 0.1010 & 0.0034 & 0.0920 & 0.0107 & 0.0134 \\
\hline 13 & 0.084 & 0.1388 & 0.0046 & 0.1285 & 0.0119 & 0.0145 \\
\hline 14 & 0.101 & 0.1279 & 0.0064 & 0.1166 & 0.0133 & 0.0167 \\
\hline 15 & 0.120 & 0.1569 & 0.0090 & 0.1449 & 0.0145 & 0.0187 \\
\hline 16 & 0.143 & 0.1336 & 0.0121 & 0.1209 & 0.0160 & 0.0190 \\
\hline 17 & 0.172 & 0.1876 & 0.0151 & 0.1790 & 0.0177 & 0.0157 \\
\hline 18 & 0.204 & 0.1878 & 0.0179 & 0.1794 & 0.0197 & 0.0148 \\
\hline 19 & 0.244 & 0.1404 & 0.0218 & 0.1325 & 0.0229 & 0.0143 \\
\hline 20 & 0.291 & 0.1336 & 0.0267 & 0.1264 & 0.0274 & 0.0128 \\
\hline 21 & 0.346 & 0.0746 & 0.0323 & 0.0681 & 0.0327 & 0.0116 \\
\hline 22 & 0.414 & 0.0156 & 0.0368 & 0.0098 & 0.0371 & 0.0106 \\
\hline 23 & 0.492 & -0.0293 & 0.0399 & -0.0347 & 0.0401 & 0.0097 \\
\hline 24 & 0.588 & -0.0995 & 0.0407 & -0.1049 & 0.0408 & 0.0090 \\
\hline 25 & 0.700 & -0.1757 & 0.0392 & -0.1813 & 0.0393 & 0.0084 \\
\hline 26 & 0.832 & -0.2374 & 0.0348 & -0.2440 & 0.0348 & 0.0085 \\
\hline 27 & 1.002 & -0.2468 & 0.0291 & -0.2559 & 0.0291 & 0.0079 \\
\hline 28 & 1.193 & -0.2713 & 0.0228 & -0.2829 & 0.0229 & 0.0065 \\
\hline 29 & 1.417 & -0.2781 & 0.0175 & -0.2940 & 0.0176 & 0.0059 \\
\hline 30 & 1.700 & -0.2631 & 0.0134 & -0.2851 & 0.0136 & 0.0049 \\
\hline 31 & 2.031 & -0.2357 & 0.0106 & -0.2654 & 0.0109 & 0.0038 \\
\hline 32 & 2.419 & -0.1954 & 0.0086 & -0.2358 & 0.0090 & 0.0030 \\
\hline 33 & 2.876 & -0.1534 & 0.0073 & -0.2074 & 0.0078 & 0.0025 \\
\hline 34 & 3.419 & -0.1110 & 0.0061 & -0.1773 & 0.0067 & 0.0023 \\
\hline 35 & 4.025 & -0.0755 & 0.0055 & -0.1520 & 0.0062 & 0.0024 \\
\hline 36 & 4.743 & -0.0330 & 0.0049 & -0.1291 & 0.0057 & 0.0020 \\
\hline & & & & & & \\
\hline
\end{tabular}




\section{Bibliography}

[1] J.D. Walecka. Electron Scattering for Nuclear and Nucleon Structure. New York: Cambridge University Press, 2001.

[2] V. Dharmawardane. "Spin Structure Functions of the Deuteron Measured with CLAS In and Above the Resonance Region." Ph.D. diss., Old Dominion University, 2004.

[3] Francis Halzen and Alan Martin. Quarks and Leptons: An Introductory Course in Modern Particle Physics. New York: John Wiley \& Sons, 1984.

[4] K. Abe, et al."Measurements of the proton and deuteron spin structure functions $g_{1}$ and $g_{2}$." Phys. Rev. D 58112003 (1998).

[5] W.M. Yao et al.(Particle Data Group). "The Review of Particle Physics.” J. Phys. G33 1 (2007).

[6] S.E. Kuhn. "Nucleon Structure Functions: Experiments and Models." Lecture notes from HUGS (Hampton University Graduate Studies at JLab) lectures (1997).

[7] David Griffiths. Introduction to Elementary Particles. New York: John Wiley \& Sons, 1987. 
[8] William R. Leo. Techniques for Nuclear and Particle Physics Experiments. Berlin: SpringerVerlag, 1994.

[9] J.J. Sakurai. Modern Quantum Mechanics. Reading, MA: Addison-Wesley, 1994.

[10] P.N. Kirk et al."Elastic Electron-Proton Scattering at Large Four-Momentum Transfer." Phys. Rev. D 863 (1973).

[11] M.E. Peskin and D.V. Schroeder. An Introduction to Quantum Field Theory. Cambridge, MA: Perseus Books Publishing, 1995.

[12] A.W. Thomas and W. Weise. The Structure of the Nucleon. Berlin: WILEY-VCH, 2001.

[13] P.E. Bosted. "Emprical Fit to the Nucleon Electromagnetic Form Factors." Phys. Rev. C 51409 (1995).

[14] J. Arrington. "Implications of the discrepancy between proton form factor measurements."Phys. Rev. C 69022201 (2004).

[15] G. Altarelli and G. Parisi. "Asymptotic freedom in parton language." Nucl. Phys. B126 298 (1977).

[16] T. Muta. Foundations of Quantum Chromodynamics. Singapore: World Scientific Publishing, 1998. 
[17] G.M. Prosperi, M. Raciti and C. Simolo. "On the running coupling constant in QCD. Prog. Part. Nucl. Phys.58 287 (2007).

[18] D. Kotlorz and A. Kotlorz. "Evolution equations for truncated moments of the parton distributions." Phys. Lett. BB644 284 (2007).

[19] O. Darrigol and F. Hayot. "Scaling Violations for the Spin-Dependent Structure Function $G_{1}$." Nucl. Phys. B141 391 (1978).

[20] A. Deur, V. Burkert, J.P. Chen and W. Korsch. "Experimental determination of the effective strong coupling constant." Phys. Lett. B650 244 (2007).

[21] A.J.G. Hey and J.E. Mandula. "Light-Cone Analysis of Spin-Dependent Deep-Inelastic Electron Scattering." Phys. Rev. D 52610 (1971).

[22] B. Lampe and E. Reya. "Spin Physics and Polarized Structure Functions." Phys. Rept.332 1 (2000).

[23] S. Kuhn and J. Chen (Ed.). GDH2004 Proceedings of the Third International Symposium on the Gerasimov-Drell-Hearn Sum Rule and its Extensions. Singapore: World Scientific Publishing, 2005.

[24] X. Ji and J. Osborne. "Generalized sum rules for spin-dependent structure functions of the nucleon.. J. Phys.G27 127 (2001). 
[25] A. Vogt "Efficient Evolution of Unpolarized and Polarized Parton Distributions with QCDPEGASUS", Comput. Phys. Commun.170 65 (2005).

[26] D. Kotlorz and A. Kotlorz. "Truncated first moment of the parton distribution - a modified approach." Eur. Phys. J.C48 457 (2006).

[27] S. Wandzura and F. Wilczek. "Sum Rules for Spin-Dependent Electroproduction-Test of Relativistic Constituent Quarks." Phys. Lett. B72 2 (1977).

[28] H. Burkhardt and W.N. Cottingham. "Sum Rules for Forward Virtual Compton Scattering." Ann. Phys.56 453 (1970).

[29] I.P. Ivanov, N.N. Nikolaev, A.V. Pronyaev and W. Schaefer. "How unitarity imposes a steep small- $x$ rise of spin structure function $g_{L T}=g_{1}+g_{2}$ and breaking of DIS sum rules." Phys. Rep.320 175 (1999).

[30] B. A. Mecking, et al."The CEBAF large acceptance spectometer (CLAS)." Nucl. Instr. Meth. A 503513 (2003).

[31] K.A. Aniol et al."Parity-violating electroweak asymmetry in ep scattering." Phys. Rev. C 69 065501 (2004).

[32] J. Pierce. "Double-Spin Asymmetry in Exclusive $\pi^{+}$Electroproduction with CLAS." Ph.D. diss., University of Virginia, 2008.

[33] N. Isgur. "Valence quark spin distribution functions."Phys. Rev. D 59034013 (2003). 
[34] F.E. Close. An Introduction to Quarks and Partons. Amsterdam: Academic Press, 1979.

[35] J. Chen. Latest Results on $g_{1}$ and $g_{2}$ at high $x$. AIP Conf. Proc. 792961 (2005).

[36] M.J. Alguard et al."Deep Inelastic Scattering of Polarized Electrons by Polarized Protons." Phys. Rev. Lett. 371261 (1976).

[37] G. Baum et al.“Measurement of Asymmetry in Spin-Dependent ep Resonance-Region Scattering." Phys. Rev. Lett. 452000 (1980).

[38] G. Baum et al."New Measurement of Deep-Inelastic ep Asymmetries." Phys. Rev. Lett. 51 1135 (1983).

[39] J. Ashman et al."A Measurement of the Spin Asymmetry and Determination of the Structure Function $g_{1}$ in Deep Inelastic Muon-Proton Scattering." Phys. Lett. B206 2 (1988).

[40] J. Ashman et al."An Investigation of the Spin Structure of the Proton in Deep Inelastic Scattering of Polarised Muons on Polarised Protons." Nucl. Phys. B328 1 (1989).

[41] B. Adeva et al."Spin Asymmetries $A_{1}$ of the proton and the deuteron in the low $x$ and low $Q^{2}$ region from polarized high energy muon scattering." Phys. Rev. D 60072004 (1999).

[42] P.L. Anthony et al."Measurements of the $Q^{2}$ dependence of the proton and neutron spin structure functions $g_{1}^{p}$ and $g_{1}^{n}$." Phys. Lett. B493 19 (2000). 
[43] A. Airapetian et al. "Precise determination of the spin structure function $g_{1}$ of the proton, deuteron and neutron." Phys. Rev. D 75012007 (2007).

[44] R. Fatemi et al. ${ }^{4}$ Measurement of the proton spin structure function $g_{1}\left(x, Q^{2}\right)$ for $Q^{2}$ from 0.15 to $1.6 \mathrm{GeV}^{2}$ with CLAS." Phys. Rev. Lett. 91222002 (2003).

[45] R. Fatemi. "The Spin Structure of the Proton in the Resonance Region." Ph.D. diss., University of Virginia, 2002.

[46] Y. Prok. "GDH Integral on the Proton from Asymmetries", Ph.D. diss., University of Virginia, 2004.

[47] Lara De Nardo. "Final Results on the Measurement of the Structure Functions $g_{1}^{p}$ and $g_{2}^{d}$ at HERMES." Proceedings of the 15th International Workshop on Deep-Inelastic Scattering and Related Subjects (DIS2007).

[48] P. Kravchenko. "Latest HERMES Results on Quark Helicity Distribution from Semi-Inclusive Deep-Inelastic Scattering." Presentation at the 11th international Conference on MesonNucleon Physics and the Structure of the Nucleon (MENU2007).

[49] P.L. Anthony et al."Prescision measurement of the proton and deuteron spin structure functions $g_{2}$ and asymmetries $A_{2}$." Phys. Lett. B55318 (2003).

[50] D.E. McNulty. "A Precise Measurement of the Spin Structure Functions $g_{2}^{p}$ and $g_{2}^{d}$ from SLAC Experiment 155x." Ph.D. diss., SLAC-Report-674 (2002). 
[51] F.R. Wesselmann, et al."Proton Spin Structure in the Resonance Region." Phys. Rev. Lett. 98 132003 (2007).

[52] M.K. Jones. "Spin Structure Functions: Proton/Deuteron Measurements in the Resonance Region." Proceedings, *Frascati 2005, Quark-Hadron Duality and the transition to pQCD*, 75 (2005).

[53] C. Leeman, D. Douglas and G. Krafft. "The Continuous Electron Beam Accelerator Facility: CEBAF at the Jefferson Laboratory." Ann. Rev. Nucl. Part. Sci. 51413 (2001).

[54] D.C. Schultz. "The Polarized Electron Gun for the SLC." Proceedings from the 3rd European Particle Accelerator Conference, Berlin, Germany (1992).

[55] C.K. Sinclair et al. "Development of a high average current polarized electron source with long cathode operational lifetime." Physical Review Special Topics - Accelerators and Beams 10 023501 (2007).

[56] H. Liu. "Space-charge effects on bunching of electrons in the CEBAF injector." Nucl. Instr. Meth. A 400213 (1997).

[57] P.K. Kloeppel. "The use of synchrotron light to monitor the beam current at CEBAF". CEBAF TN-93-102 (1993).

[58] M.L. Stutzman et al."Characterization of the CEBAF $100 \mathrm{kV}$ DC GaAs Photoelectron Gun Vacuum System." Nucl. Instr. Meth. A 574213 (2007). 
[59] R. Kazimi et al."CEBAF Injector Achieved World's Best Beam Quality for Three Simultaneous Beams with a Wide Range of Bunch Charges." JLAB-ACO-04-251. Presented at 9th European Particle Accelerator Conference (EPAC2004).

[60] D. Douglas. "Recirculator Arcs." CEBAF TN-85-0010 (1985).

[61] K. Joo. "Electron Beam Polarization Measurements using the Hall B Moller Polarimeter." Jefferson Lab Beam Physics Seminar (2002).

[62] J. Grames. "Unique Electron Polarimeter Comparison and Spin-Based Energy Measurement." JLAB-TN-01-029 (2001).

[63] CLAS online run logbook (2000-2001). http://clasweb.jlab.org/clasonline/prodruninfo/runhistory.html

[64] S. Kuhn and N. Guler. "False Asymmetry Studies for EG1b Experiment." CLAS-NOTE 2005-005 (updated 2007).

[65] C.D. Keith et al."A polarized target for the CLAS detector.", Nucl. Instr. Meth. A 501327 (2003).

[66] P.M. McKee. "Observations of radiation damage and recovery in ammonia targets." Nucl. Instr. Meth. A 52660 (2004).

[67] W. de Boer. "Dynamic Orientation of Nuclei at Low Temperatures." CERN 74-11, Laboratory I, Nuclear Physics Division (1974). 
[68] M. Borghini. “Proton Spin Orientation." CERN 68-32, Nuclear Physics Division (1968).

[69] W. Meyer. "Ammonia as a polarized solid target material-a review", Nucl. Instr. Meth. A 526 12 (2004).

[70] O.A. Rondon. "Corrections to nucleon spin structure asymmetries measured on nuclear polarized targets." Phys. Rev. C 60035201 (1999).

[71] D. Adams et al. "The polarized double cell target of the SMC." Nucl. Instr. Meth. A 43723 (1999).

[72] S. Kuhn. "Data Analysis for EG1."(analysis document)

http://ww , jlab.org/Hall-B/secure/eg1/AnalysisDoc/EG2000DataAnalyis.pdf

[73] D.R. Lide, ed. CRC Handbook of Chemistry and Physics, 78th Ed. Boca Raton, FL: CRC Press, 1997.

[74] M. Mestayer, D. Carman,et al."The CLAS drift chamber system." Nucl. Instr. Meth. A 44981 (2000).

[75] L.M. Qin et al.“Prototype studies and design considerations for the CLAS Region 2 drift chambers." Nucl. Instr. Meth. A 411265 (1998).

[76] G. Adamas et al."The CLAS Cherenkov Detector." Nucl. Instr. Meth. A 465414 (2001).

[77] E.S. Smith et al. “The time-of-flight system for CLAS.” Nucl. Instr. Meth. A 432265 (1999). 
[78] E.S. Smith, V.Dharmawardane, et al."Calibration of the CLAS TOF System." CLAS-NOTE 1999-011 (updated 2001).

[79] M. Amarian, et al."The CLAS Forward Electromagnetic Calorimeter." Nucl. Instr. Meth. A 460 239 (2001).

[80] JLab CODA Group. “Coda 1.4 Guide."

http://coda.jlab.org/

[81] Harut Avagyan, et al.“The CLAS Calibration Database.” CLAS-NOTE 2001-003 (2003).

[82] L. Elouadrhiri et al.(CLAS online working group). "CLAS Data Acquisition and Monitoring Documentation."

http://wrw.jlab.org/〜latifa/online

[83] S. Stepanyan. "Simple Event Builder (SEB) in the framework of RECSIS."

http://ww.jlab.org/^`stepanya/seb_man.html

[84] S. Boyarinov, et al.(CLAS online working group). "CLAS DAQ manual."

http://clasweb.jlab.org/online/daq/daq.html

[85] Y. Liang, et al."Measurement of $R=\sigma_{L} / \sigma_{T}$ and the separated longitudinal and transverse structure functions in the nucleon resonance region." arXiv:nucl-ex/0410027 (2004).

[86] K. Abe et al.. "Measurements of $R=\sigma_{L} / \sigma_{T}$ for $0.03<x<0.1$ and fit to world data." Phys. Lett. B452 194 (1999). 
[87] M.E. Christy and P.E. Bosted. "Empirical Fit to Precision Inclusive Electron-Proton Cross Sections in the Resonance Region." arXiv:0712.3731 (2007).

[88] P.E. Bosted and M.E. Christy, "Empirical Fit to Inelastic Electron-Deuteron and ElectronNeutron Resonance Region Transverse Cross Sections", Phys. Rev. C 77065206 (2008).

[89] N. Bianchi and E. Thomas. "Parameterisation of $\left[\sigma_{1 / 2}-\sigma_{3 / 2}\right]$ for $Q^{2} \geq 0$ and non-resonance contribution to the GDH sum rule." Phys. Lett. B444 531 (1998).

[90] S. Kuhn. "Model for the Asymmetry $A_{2}$." Technical note (2001).

http://wwr.jlab.org/Hall-B/secure/eg1/Models/A2technote.ps

[91] L.W. Mo and Y.S. Tsai. "Fadiative Corrections to Elastic and Inelastic ep and $\mu p$ Scattering." Rev. Mod. Phys. 41205 (1969).

[92] H. de Vries, C.W. De Jager and C. de Vries. "Nuclear charge-density-distribution parameters from elastic electron scattering." Atomic Data and Nuclear Data Tables. 36495 (1987).

[93] T.W. Donelly and I. Sick. "Superscaling in inclusive electron-nucleus scattering." Phys. Rev. Lett. 823212 (1999).

[94] J.E. Amaro et al."Using electron scattering superscaling to predict charge-changing neutrino cross-sections in nuclei." Phys. Rev. C 71015501 (2005).

[95] P. Bosted and R. Fersch. "Ratios of ${ }^{15} \mathrm{~N} /{ }^{12} \mathrm{C}$ and ${ }^{4} \mathrm{He} /{ }^{12} \mathrm{C}$ inclusive electroproduction crosssections in the nucleon resonance region." CLAS-ANALYSIS 2007-115 or arXiv:0712.2438v1 
[nucl-ex] (2007).

[96] P. Bosted, R. Fersch, et al. ${ }^{4}$ Ratios of ${ }^{15} \mathrm{~N} /{ }^{12} \mathrm{C}$ and ${ }^{4} \mathrm{He} /{ }^{12} \mathrm{C}$ inclusive electroproduction crosssections in the nucleon resonance region." Phys. Rev. C 78015202 (2008).

[97] P.R. Norton. "The EMC effect." Rep. Prog. Phys.66 1253 (2003).

[98] J. Gomez, et al."Measurement of the $A$ dependence of deep-inelastic electron scattering." Phys. Rev. D 494348 (1994).

[99] V. Blobel, et al."F-PACK (F - package for input/output) v 0.89/00." Institut fur Experimentalphysik, University of Hamburg (1994).

[100] A. Biselli, T. Forest, et al.(Jefferson Laboratory CVS archive/group/clas/clas_cvs/eg1_dst)

[101] R. Brun et al.“ROOT User's Guide v5.16."

http://root.cern.ch/root/doc/RootDoc.html

[102] J. Pierce, personal correspondence.

[103] L. Lyons. Statistics for nuclear and particle physicists. Cambridge: Cambridge University Press, 1986.

[104] N. Guler, personal correspondence. 
[105] M. Osipenko, A. Vlassov and M. Taiuti. "Matching between the electron candidate track and the Cherenkov counter hit." CLAS-NOTE 2004-020 (2004).

[106] P. Bosted, S. Kuhn and Y. Prok. “Raster Corrections for EG1b." CLAS-NOTE 2003-008 (2003).

[107] H. Avakian, personal correspondence.

[108] "Hall A Absolute Beam Energy Measurements"

http://hallaweb.jlab.org/equipment/beam/energy/beam_energy_table.html

and

http://hallaweb.jlab.org/equipment/beam/absol_beam.html

[109] X. Zheng. "Precision Measurement of Neutron Spin Asymmetry $A_{1}^{n}$ at Large $x_{B j}$ Using CEBAF at 5.7 GeV." Ph.D. diss., Massachusetts Institute of Technology, 2002.

[110] "Jefferson Lab HALL A Logbook"

http://www.jlab.org/ adaq/halog/html/logdir.html

[111] S. Stepanyan. "Beam energy measurement with $e p \rightarrow e p$ elastic scattering on CLAS."CLASNOTE 2002-008 (2002).

[112] Glenn F. Knoll. Radiation Detection and Measurement. New York: John Wiley \& Sons, 2000.

[113] M. Bellis. "Reliability and Limitations of GSIM." CLAS-NOTE 2002-016 (2002). 
[114] P. Bosted and H. Avakian. "Multiple Scattering and Stray Magnetic Field Corrections for Tracking in the Presence of Target Field.", CLAS-NOTE-2006-006 (2006). (Please note update to stray magnetic field corrections in this thesis.)

[115] A. Klimenko and S. Kuhn. “Momentum Corrections for E6." CLAS-NOTE-2003-005 (2003).

[116] CEBAF Hall B Conceptual Design Report. (1990).

[117] S. Kuhn, personal correspondence.

[118] P. Bosted. "Pair-Symmetric and Pion Backgrounds for EG1b." CLAS-NOTE 2004-005 (2004).

[119] T. Gehrmann and M. Stratmann. "The Bethe-Heitler Process in Polarized Photon-Nucleon Interactions." Phys. Rev. D 565839 (1997).

[120] A. Vlassov. "CLAS Cherekov Counter study"

http://www.jlab.org/ ^vlassov/cc/index.html

[121] D. G. Crabb and W. Meyer. "Solid Polarized Targets for Nuclear and Particle Physics Experiments." Annu.Rev.Nucl.Part.Sci. 4767 (1997).

[122] Yung-Su Tsai. "Radiative Corrections to Electron Scatterings." SLAC-PUB-848 (1971).

[123] T.V. Kuchto and N.M. Shumeiko. "Radiative Effects in Deep Inelastic Scattering of Polarized Leptons by Polarized Nucleons." Nucl. Phys. B219 412 (1983). 
[124] H. Olsen and L.C. Maximon. "Photon and Electron Polarization in High-Energy Bremsstrahlung and Pair Production with Screening." Phys.Rev. 114887 (1959).

[125] S. Kuhn. "Combination of different runs in EG1b." Analysis document (2006). http://www.jlab.org/Hall-B/secure/eg1/EG2000/Kuhn/CombinationOfRuns.pdf

[126] M.R. Spiegel and J. Liu. Schaum's Outlines: Mathematical Handbook of Formulas and Tables, 2nd Ed. New York: McGraw-Hill, 1999.

[127] B. Adeva et al. "Spin asymmetries $A_{1}$ and structure functions $g_{1}$ of the proton and the deuteron from polarized high energy muon scattering." Phys. Rev. D 58112001 (1998).

[128] "JLab Experiment CLAS Database."

http://clasweb. jlab.org/physicsdb/

[129] G. Baum et al. "A New Measurement of Deep-Inelastic e-p Asymmetries." SLAC-PUB-3079 (1983).

[130] "SMC results."

http://na47sun05.cern.ch/smc_results.html

[131] "SLAC Experiment E143 Home Page."

http: //wwr.slac.stanford.edu/exp/e143/home.html

[132] “HERMES Highlights and Information (Publications, Proposals, Conference Proceedings, Thesis Plots and Data, Talks and Posters)."

http://www-hermes . desy .de 
[133] “SLAC E155/E155x Experiments."

ht tp://www.slac.stanford. edu/exp/e155/home.html

[134] R. Fatemi. "Combined Results for $A_{1}$ and $g_{1}$ "

http://www.jlab.org/Hall-B/secure/eg1/Renee/data_tables/finaldata.htm

[135] Y. Prok et al."Moments of the Spin Structure Functions $g_{1}^{p}$ and $g_{1}^{d}$ for $0.05<Q^{2}<3.0 \mathrm{GeV}^{2}$." arXiv:0802.2232 [nucl-ex] (2008).

[136] J. Jourdan et al.“Update to TJNAF E-03-019: Spin Asymmetries on the Nucleon Experiment - SANE.” Jefferson Lab Hall C Proposal PR-07-003 (2006).

[137] D. Drechsel, B. Pasquini and M. Vanderhaeghen. "Dispersion relations in real and virtual Compton scattering." Phys.Rept. 37899 (2003).

[138] A. Deur et al."Experimental study of isovector spin sum rules.” Phys. Rev. D 78032001 (2008).

[139] C.E. Carlson. "Proton Structure Corrections to Hydrogen Hyperfine Splitting." arXiv:hepph/0611206. Presentation at International Workshop on Precision Physics of Simple Atomic Systems (PSAS2006).

[140] V. Nazaryan, C.E. Carlson and K. Griffioen, "New experimental constraints on polarizability corrections to hydrogen hyperfine structure", Phys. Rev. Lett. 96163001 (2006).

[141] A.C. Zemach. "Proton Structure and the Hyperfine Shift in Hydrogen." Phys.Rev.104 1771 (1956). 
[142] C.E. Carlson, V. Nazaryan and K. Griffioen. "Proton structure corrections to electronic and muonic hydrogen hyperfine splitting." arXiv:0805.2603[physics.atom-ph] (2008).

[143] J.J. Kelly. "Simple paramatrization of nucleon form factors." Phys. Rev. C 70068202 (2004).

[144] E.D. Bloom and F.J. Gilman. "Scaling, Duality, and the Behavior of Resonances in Inelastic electron-Proton Scattering." Phys. Rev. Lett. 251140 (1970).

[145] P.E. Bosted et al."Quark-hadron duality in spin structure functions $g_{1}^{p}$ and $g_{1}^{d . " ~ P h y s . ~ R e v . ~ C ~}$ 75035203 (2007).

[146] F.E. Close and W. Melnitchouk. "Symmetry breaking and quark-hadron duality in structure functions." Phys. Rev. C 68035210 (2003).

[147] O. Nachtmann. "Positivity Constraints for Anomalous Dimensions." Nucl. Phys. B63237 (1973).

[148] J. Blümlein and A. Tkabladze. "Target Mass Corrections for Polarized Structure Functions and New Sum Rules." Nucl. Phys. B553 427 (1999).

[149] S. Forte, G. Ridolfi, J. Rojo and M. Ubiali. "Borel Resummation of Soft Gluon Radiation and Higher Twists." Phys. Lett. B635 313 (2006).

[150] M. Anghinolfi et al."The GDH Sum Rule with Nearly-Real Photons and the Proton $g_{1}$ Structure Function at Low Momentum Transfer." CLAS Proposal PR-03-006 (2003). 
[151] X. Zheng, P.E. Bosted and G. Dodge. "Double Spin Asymmetries in $\pi^{ \pm}$and $K^{ \pm}$Electroproduction from Polarized $\mathrm{NH}_{3}$ and $\mathrm{ND}_{3}$ Targets Using EG4 Data." Proposal for CLAS Approved Analysis (CAA) (2007).

[152] D.G. Crabb and D.B. Bay. "The Virginia/Basel/SLAC Polarized Target: Operation and Performance During Experiment E143 at SLAC." Nucl. Instr. Meth. A 3569 (1995).

[153] O. Rondon. "SANE Spin Asymmetries on the Nucleon Experiment.", PAC31 Presentation (2007).

[154] D. de Florian and R. Sassot. "Global Analysis of Helicity Parton Densities and Their Uncertainties." arXiv:0804.0422 [hep-ph] (2008).

[155] V. Dharmawardane et al."Measurement of the $x$ - and $Q^{2}$-Dependence of the Asymmetry $A_{1}$ on the Nucleon.", Phys. Lett. B641 11 (2006).

[156] CLAS12 working group. "Hall B 12 GeV Upgrade: CLAS12 (Documentation)."

http://wws . jlab.org/Hall-B/clas12/ 